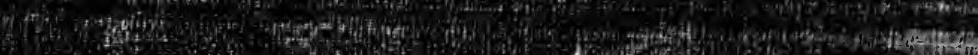

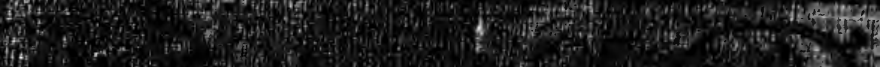
(1)

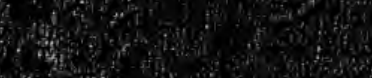

(1) if ing

(1)

in
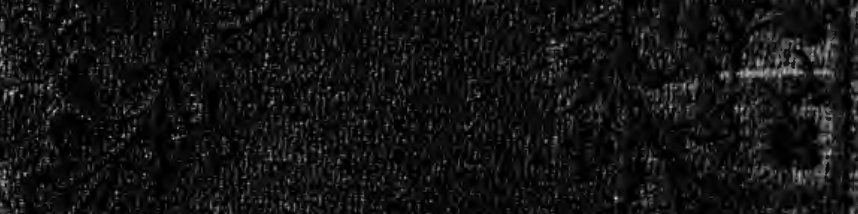

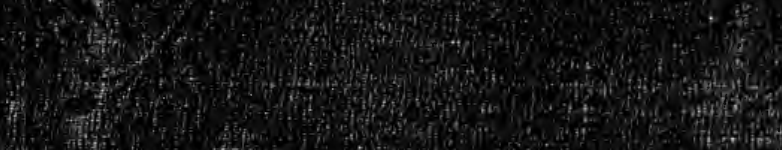

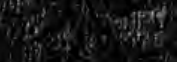

Mescitis

15.

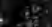

(2)

$28 a^{2}$ is

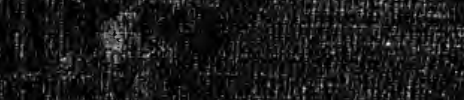

$\frac{5}{6}+x^{2}$

covest

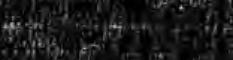

(4)

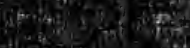

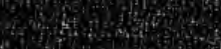

1.4.

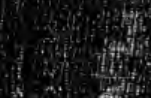

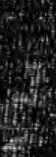

4 a 30

21:

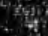

Q⿻日木

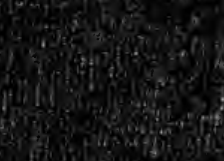

arting ing

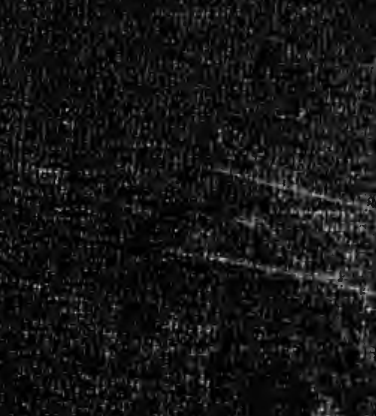

A 30

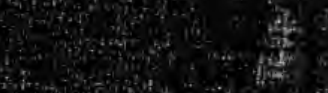

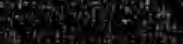

$2, \frac{2}{2}$

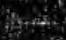

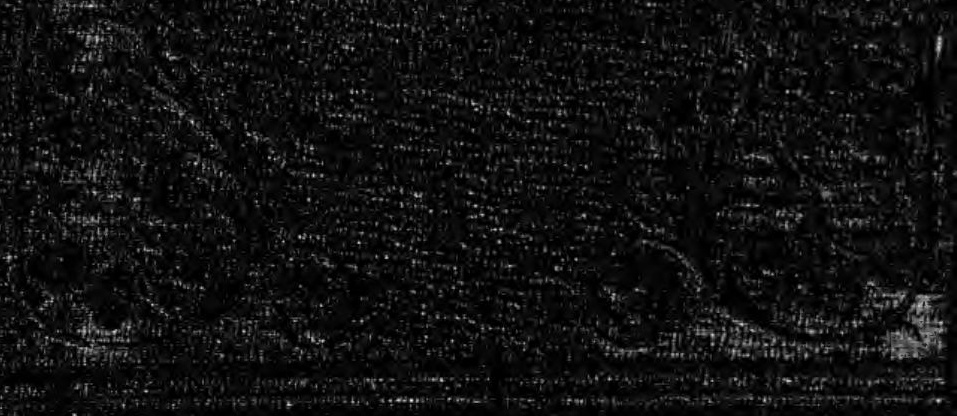




\section{BY THE SAME AUTHOR.}

\section{A HANDBOOK OF INORGANIC CHEMISTRY:}

FOR THE USE OF STUDENTS.

With Numerous Illustrations. Third Edition. 1 Vol. 5s. 6d, cloth. 


\section{G8236ha}

A HANDB $00 \mathrm{~K}$

OF

\section{ORGANIC CHEMISTRY;}

Jfor the rtse of Sturents.

BY

WILLIAM GREGORY, M.D., F.R.S.E.,

Professor of Chemistry in the University of Edinburgh.

FOURTH EDITION,

CORRECTED AND MUCH EXTENDED.

LONDON :

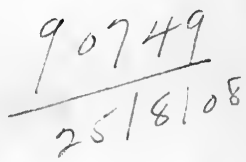

\section{WALTON AND MABERLY,}

UPPER GOWER STREeT, AND IVY LANE, PATERNOSTER ROW. 1856. 



\section{JUSTUS BARON VON LIEBIG,}

\&c. \&c. \&c.

PROFESSOR OF CHEMISTRY IN THE UNIVERSITY OF GIESSEN.

\section{My Dear Friend,}

Permit me, as a small mark of the high esteem in which I hold you, and of my sincere admiration for your achievements in Chemical Science, to inscribe to you the following pages.

If my little work possess any value as a guide to the student, it is to your writings, to your example, and to the unreserved communications with which you have at all times honoured me, that I am indebted for it.

Few men have enjoyed better opportunities of intimate personal intercourse, and of epistolary correspondence with you than I have: and few, consequently, can more confidently testify to the rare liberality with which your views on scientific subjects are communicated to your friends, or to the readiness with which your advice and assistance are rendered to your fellow labourers.

The present work is but a feeble testimony of the attachment and respect, which, after sixteen years of uninterrupted friendship, I feel for your person and character. But such as it is, it is heartily offered by

Yours ever faithfully,

WILLIAM GREGORY. 
Digitized by the Internet Archive in 2007 with funding from Microsoft Corporation 


\section{ADVERTISEMENT TO THE FOURTH EDITION.}

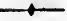

Is preparing for the press this new edition of the "Handbook of Organic Chemistry," I have found it necessary, from the rapid progress of discovery in this department of science, to make important changes in various sections, and in almost all to introduce a large amount of new matter ; so that the work is considerably increased in size.

In doing this, I have, as far as possible, endeavoured not to lose sight of the original object of the work, which was not so much to give a full and complete detail of the facts of Organic Chemistry, as to put within reach of the student a knowledge of the principles which regulate Organic Chemistry, with such facts as were necessary for illustration, or else important in themselves. It is a difficult task to give, in a small volume, any satisfactory notion of this science; but $I$ have done my best to attain this end, and I have reason to believe that the arrangement $I$ have hitherto followed has been found useful to the student.

It is well known to chemists that Gerhardt has introduced a modified notation, which will, in all probability, come finally into general use. But, as even he has not ventured to use it in his Organic Chemistry, I have thought it best to adhere to that already in use among us, at least for the present. 
For a similar reason, although Gerhardt has attempted to carry out a systematic arrangement, according to groups and series, yet he has been compelled to treat many important subjects in appendices, as if they had no place ; and this clearly shows, in my opinion, that the time for a purely systematic classification, though approaching, is not yet come. I have endeavoured to prepare the way for such an arrangement by pointing out the various relations, hitherto unsuspected, which have of late been detected between substances apparently remote, and of which more are coming to light every day.

It is unnecessary to mention in detail the improvements made in the present edition; for there is hardly a section in which some new matter has not been introduced, and some of the old corrected. But I may mention, among those where most has been altered and added, the sections on the ethylic radicals and their derivatives; on the volatile oily acids of the formylic series; on the oxalic acid series; on the oleic, or acrylic acid series ; on the artificial bases; on the natural bases; and on cellulose and its congeners, starch, sugar, gum, \&c.

A few interesting facts, which reached me too late to be inserted in their proper place, have been given in a supplement, to which the reader is referred for a brief account of the discovery, lately made by Hofmann and Cahours, of the acrylic, or rather allylic alcohol, the type of a new class of alcohols, and of its derivatives.

I beg once more to express my heartfelt gratitude for the very favourable reception which has been given to the earlier editions of this work, and to say; that I have done my utmost to render the present edition worthy of the favour which the work has hitherto enjoyed.

WILLIAM GREGORY.

114. Peinces Streít, Edinborgh, March, 1856. 


\section{PREFACE TO THE THIRD EDITION.}

Is offering to the public a new edition of my "Outlines of Organic Chemistry, for the use of Students," it is necessary to explain why it appears under a new title.

The last edition, which $I$ had taken the utmost pains to render a faithful record of the actual state of the science at the period of its publication in 1847, was already of a nature and completeness hardly indicated by the term Outlines. And the unusually favourable reception it met with, it having been adopted as a text book in the Universities of Oxford, Cambridge, and Dublin, besides many other schools, has proved that the treatment of the subject, and the arrangement of the work, are such as met and supplied an existing demand for an elementary work in a moderate compass. These considerations have urged me, on the present occasion, to do all that lay within my power to render the present edition as complete, down to the end of 1851 , as the former was down to the end of $\mathbf{1 8 4 6 .}$

But although the general plan of the work is altogether unchanged, yet the very great progress made during these five years in Organic Chemistry has not only enabled me, but has rendered it imperative on me, to dwell more fully than was possible in 1847 on many general principles of the most important character, the knowledge of which will very 
much facilitate the student's progress. In consequence of this, the title of "Outlines" would now be still more inappropriate than it was in 1847.

The reader will also find, that, while the work is considerably enlarged, this is not due to the accumulation of minute details, out of place in an elementary work, but to the absolute necessity of taking some notice, however brief, of the very numerous and important discoveries of the last few years, and of presenting to the reader the valuable deductions, so suggestive as they are of further progress, which flow from these discoveries.

For example, the brilliant discovery (predicted in 1839 by Liebig) of the new volatile bases homologous with ammonia, by Wurtz, and so admirably followed out by Hofmann in the latter part of his splendid series of papers on the artificial bases; Hofmann's own most recent discovery of the singular class of ammonium bases; the researches of a host of chemists on that striking series of volatile acids beginning with formic acid and ending, for the present, with melissic acid, and on the oils and fats generally; the continued discovery of new compounds belonging to the methylic, ethylic, and amylic series; the researches of Frankland and Kolbe on the radicals of the ethylic group, and those of Frankland on the compounds of those radicals with metals; those of $P$. Thénard on compounds of phosphorus with the same radicals; the light shed on the doctrine of substitution by these investigations, and by those of the French school, by Laurent, Gerhardt, Cahours, and others ; the admirable monograph on the bile by Strecker; the introduction of hyponitrous acid as a means of oxidising organic compounds by Piria and Strecker ; the minute study of the products of decomposition of the albuminous compounds, by Bopp, Guckelberger, and others; the many additions to our knowledge of physiological chemistry by Liebig and many other chemists; and the rapid development of the doctrine of homologous series, as a practical 
guide in research; - all these things, and many others, have rendered it necessary, even while retaining the original form of the work, to re-write almost every section of it, and, in most of them, to make large, but absolutely indispensable additions.

Thus, the section on the nutrition of plants and animals, at the end of the work, has been not only entirely rewritten, but very much enlarged, although it has been my earnest endeavour to keep descriptive details within the narrowest limits, and only to enter fully into such matters as involve general principles.

One or two alterations have been made in the arrangement. Thus, the whole of the volatile acids of the formula $\left(\mathrm{Cn}_{n} \mathrm{H}_{n}\right)_{2} \mathrm{O}_{4}$, have been placed together in the section which treats of the ethylic group of radicals, and of the acetylic group derived from them; and sugar no longer forms a supplement to ethyle, but is united to its congeners, starch, gum, and woody fibre. In all other respects the plan of the work, which has met with so cordial a reception both from teachers and students, remains unchanged.

I have largely employed tabular views as a means of illustration, and have added to the work a large table of homologous compounds belonging to the radicals ethyle, methyle, \&c., as well as to acetyle, formyle, \&c., derived from them; which table, although it extends to 17 vertical columns and 22 horizontal ones, is yet but a corner of what we might draw up, were we to have a column for every homologous series connected with the above groups of radicals. There is also another table of homologous compounds of the benzoic series. The reader will understand, that in both these large tables many compounds appear which are still unknown; but hardly a week passes without some new compound being discovered, which at once takes its place in the table. It is a good plan for the student to mark with a red line or cross such of the compounds in these 
tables as are already known. In the column of the volatile acids alone, the whole 22 compounds named in the table are known at present.

I beg leave once more to offer my grateful acknowledgments for the very favourable reception given to this work, and at the same time to express my anxious hope that the present editiou may be found, in the present more advanced state of the science, to satisfy, equally with its predecessor, the just demands of the teacher as well as of the student.

The Outlines of Inorganic Chemistry, which forms the first part of the work, as hitherto published, is now in preparation for the new (third) edition. But it will be published, as the Organic Chemistry now is, as a separate volume, as soon as it can be got ready.

UNIVERSITY OF EDINBURGH,

WILLIAM GREGORY. March, 1852. 


\section{CONTENTS.}

Introductory
Elements of the organic world:
These are nearly the same as
those of the inorganic world,
except in the order of their
relative abundance

Distinctions between organic and inorganic compounds .

The vital force modifies chemical action

Predominance of carbon in organic compounds . .

Compound organic radicals .

Advantage of assuming these .

Remarkable series of volatile oily acids of analogous properties

Many radicals now known .

Theory of types, and doctrine of substitutions

The chief types, Metal, Water, Acid, and Ammonia

Substitution not limited by the electrical character of the elements

Bases formed from ammonia by substitution

The character of a compound depends very much on the arrangement of its atoms .

Homologous series of organic compounds

Table illustrating these, facing

Explanation of table .

.22

Analogy between groups of elements and groups of homologous compounds
PAGE

Transmutation of elements conceivable, but, in all probability, not practicable . .

Decompositions and Metamorphoses of organic compounds . . . .

1. Oxidation.
a. Direct
Combustion
Eremacausis
Influence of ozone $\quad . \quad 32$

ib.

ib.

b. Indirect . . . 34

2. Action of acids . . . $i b$.

a. Of Nitric acid . . $i b$.

b. Of Hyponitrous acid. . 35

c. Of Sulphuric acid . . ib.

d. Of Phosphoric acid . $\quad 36$

3. Action of Bases . . ib.

4. Action of Heat, or, Destructive Distillation . . 37

5. Action of Ferments . . 38

Theories of Fermentation 39

Various kinds of Fermentation . . . . 42

Putrefaction . . . $i b$.

Poisons and miasms . . $i b$.

Metamorphoses of solutions of cyanogen . . 43

Liebig's definition of fermentation, putrefaction, \&c. \&c. . . . . 45

Decomposition of compounds by the galvanic current .

Artificial formation of Organic compounds . . 46

\section{8} 29

2


plex from less complex molecules $\cdot$.

Observations on the $\mathrm{Or}$ ganic acids

Existence of compound radicals in Organic acids

Hydrogen theory of acids Anhydrous Organic acids Comparison of anhydrous with true Organic acids Coupled acids . . . Arrangement of the subject . . . . . 51

COMPOUND RADICALS KNOWN OR ADMITTED.

I. AMIDE.

Its Compounds.

a. With Hydrogen . Ammonia and Ammonium

b. With Metals .

c. With Platinum, the new platinised bases $\quad 55$ Tables of these . 56,58,60 Amides, Imides, and $\mathrm{Ni}$ tryles

II. Carbonio Oxide, as a Radical Its Compounds.

a. With Oxygen, Oxalic acid, Oxalates • . .

b. With Amide, Oxamide, Oxamic acid . . .

c. With Chlorine, Phosgene gas . • • . Carbamide . . $i b$.

d. With Potassium, \&c. . . 67 Rhodizonic acid $\quad i b$. Croconic acid . $i b$. Mellitic acid . . 68 Action of heat on mellitate of Ammonia

Euchronic acid and Paramide . .

\section{Cranogen - its Compounds.}

a. With Hydrogen, Hydrocyanic acid, anhydrous . Medicinal .

b. With Oxygen . . 75

Cyanic acid . . $i b$.

Cyanates . . 76
With Cyanates of Ammonia and Urea . 77 Artificial Urea . $\quad 78$ Salts of Urea . . $i b$. Fulminic acid . . 79 Fulminates . . . $i b$. Fulminating mercury and silver . $i b$. Cyanuric acid . . 81

Cyanurates . . . 82 Cyamelide $\quad . i b$. Fulminuric acid $\quad 83$ Fulminurates . . 84 Constitution of the four preceding acids . 83 et seq.

c. With Nitrogen, Mellone - 85

$d$. With Chlorine . . . 86

e. With Bromine, Iodine, and Sulphur. . . . ib.

f. With Metals . . . 87

Cyanides . ..$i b$.

Double Cyanides . 88

IV. Ferrocyanogen. • $\quad$ - 89

Its Compounds.

a. With Hydrogen, Ferrocyanic acid . . 90

b. With metals, Ferrocyanides

Ferrocyanide of Potassium

Ferrocyanide of Iron $\quad .92$

V. Ferridcyanogen • • • 94

Its Compounds.

a. With Hydrogen, Ferridcyanic acid . . . . ib.

$b$. With Metals . . . $i b$.

Ferridcyanide of Potassium . . . . $i b$.

Nitroprussides . $\quad . \quad 95$

VI. Cobaltocyanogen . . $i b$.

With Hydrogen and Potassium . . 96

VII. Chromocyanogen • . 97

VIII. Platinocyanogen • $\cdot i b$.

Platinocyanide of Potassium .

$i b$.

IX. IRIDIOCYAN OGEN $\cdot \quad \cdot 98$

Palladiocyanogen and Manganocyanogen . $i b$.

Table of the eight preceding Radicals . . ib.

Paracyanogen . $\quad 99$ 
X. SUlphodyanogen • PAGE

Its Compounds.

a. With Hydrogen, Sulphocyanic acid . $\cdot$.

b. With Potassium and other metals . . . . $i b$. Persulphocyanic acid .101

XI. Mellone • • • 102

Its Compounds.

a. With Hydrogen, Hydromellonic acid . . . $i b$.

b. With Potassium, \&c. . 103

Products of the distillation of Sulphocyanide of ammonium

Melam

Melamine and Ammeline . $i b$.

Ammelide and Cyanuric acid

Cyameluric acid . . $\quad . \quad i b$.

Tabular view of these products . 105-107

Action of nitric acid on mellone; Cyanilic acid . 107

Sulphomellone . . . $i b$.

Hydrosulphomellonic acid .. ib. Sulphomellonides . . 108

XII. URYLE : or Cyanogen with

Carbonic oxide or Oxalyle 109

Uric acid

its oxidation. .111

by peroxide of lead . . $i b$.

Allantoine . . . $i b$.

by peroxide of manganese 112

by permanganate of potash $i b$.

by nitric acid . . . 113

1. Alloxan . . . ib.

2. Alloxanic acid . 115

3. Leucoturic acid . i ib.

4. Difluan . . . 116

5. Hydurilic acid.$\quad i b$.

6. Mesoxalic acid . . 117

7. Mykomelinic acid. . 118

8. Parabanic acid . . ib.

9. Oxaluric acid . . ib. Oxalurate of ammonia $i b$.

10. Alloxanosulphurous acid . . . 119

11. Thionuric acid . . $i b$. Thionurate of ammonia
12. Uramile $\quad . \quad .120$

13. Uramilic acid . $\quad i b$.

14. Alloxantine . . . $i b$.

Its Derivatives.

15. Dialuric acid $\quad . \quad .122$

16. Alituric acid . . . 123

17. Dilituric acid . . $i b$.

18. Murexide . . . $i b$.

19. Murexan . . 125

by ferridcyanide of potassium . . . . ib.

20. Lantanuric acid $i b$. by caustic potash . . 126

21. Hidantoic acid . $\quad i b$.

22. Uroxanic acid . $i b$.

23. Uroxile . . $i b$.

Analogy between the derivatives of uric acid and those of caffeine

$i b$.

Tabular view of some com-

pounds of Uryle . $\quad 127$

Appendix to Uric acid . . $i b$.

1. Uric or xanthic oxide . ib.

2. Cystic oxide $\quad . \quad 128$

3. Guanine . . $i b$.

4. Hyperuric acid . $i b$.

XIII. Benzoyle • • . $i b$.

1. Benzoic acid . . 129

Anhydrous . . . 131

Benzoic acid series . . $i b$.

2. Hyduret of benzoyle . 132

3. Chloride of benzoyle 133

Iodide, sulphuret, and cyanide of benzoyle . 134

4. Benzamide . . $i b$.

5. Formobenzoic acid . . ib.

6. Benzoate of hyduret of benzoyle . . . 135

7. Hippuric acid . . $i b$.

Its decompositions . $\quad .136$

$a$. into benzoic acid and glycocine . . .

b. into benzamide and carbonic acid . . 135

c. into benzoglycolic acid and ammonia. 137

Benzoglycolic acid . . $i b$.

Glycolic acid . . . . $i b$.

Series of glycocine, alanine, and leucine . . 138

Products of the transformation of the compounds of benzoyle $i b$.

$i b$. 
1. Hyposulphobenzoic acid

2. Boid $\cdot 138$

3. Nitrobenzoic acid $i b$.

4. Benzole . . . ib.

Its Derivatives.

5. Sulphobenzide . 139

6. Hyposulphobenzidic acid . . . $i b$.

7. Nitrobenzole $\cdot{ }^{*} i b$. Aniline ..$i b$. Dinitrobenzole . $i b$. Nitraniline . . . 140

8. Azobenzide . . $i b$.

9. Chloride of benzole - $i b$. 10. Chlorobenzine . . ib. Series of homologous bodies analogous to benzole . . $i b$. Table of this series . . 141

11. Benzophenone • . 142

12. Hydrobenzamide . 143

13. Benzhydramide . $i b$.

14. Azobenzoyle.$\quad \cdot i b$.

15. Azotide of benzoyle . ib.

16. Benzostilbine . . 144

17. Benzolone . . . ib.

18. Amarine . . . 145

19. Lophine . . . $i b$.

20. Benzimide . . $i b$.

21. Benzoine . $^{\circ} i b$.

22. Hydrobenzoinamide . 146

23. Benzoinam . . . $i b$.

24. Benzile . . . $i b$.

25. Benzilic acid . . 147

26. Azobenzoide - $\cdot i b$.

27. Cyanobenzile . . ib.

28. Hyduret of sulphobenzoyle.$\quad i b$.

29. Stilbene . . 148

30. Hyduret of sulphazobenzoyle . . 149

31. Bromide of benzole . ib.

32. Hydrocyanate of benzoine. .

33. Hydrocyanate of benzoyle

$i b$.

General remarks on the formation of the preceding

complex bodies . . 150

Benzoic alcohol . . 151

Appendix to Benzoyle . $\quad .152$

1. Amygdaline . . . $i b$.

2. Amygdalinic acid . $\quad i b$.
3. Distilled water of bitter almonds . . . 153

4. Laurel water $. \quad . \quad i b$.

Theory of the formation of oil of bitter almonds 153-155

Emulsine or synaptase $\cdot i b$.

Mixture recommended by

Liebig and Wöhler in-

stead of distilled water

of bitter almonds . . 155

Advantage of assuming the existence of the radical benzoyle . . . . ib.

XIV. Salicyle $\quad . \quad .156$

Its Compounds.

$a$. With Hydrogen . . $i b$. 1. Hyduret of salicyle . $i b$. Salicylurets or salicylites . 157 Salicylimide . . . $i b$. Melanic acid . . . $i b$. Parasalicyle . . . 158

$b$. With Oxygen . . . $i b$. 2. Salicylic acid . . . $i b$.

Its Derivatives.

Nitrosalicylic, or Anilic acid . . . . 159

Nitrophenesic, or Picric acid

Carbolic acid . . . ib.

c. With Chlorine . . 160

3. Chlorosalicylic acid . $i b$.

$d$. With Nitric acid . . . $i b$.

4. Nitrosalicylic acid . $i b$.

Appendix to Salicyle . 161

5. Salicine . . . $i b$.

Chlorosalicine, dichlorosalicine, and trichlorosalicine . . . 162

6. Saligenine $\cdot \quad \cdot i b$.

Chlorosaligenine, dichlorosaligenine, and trichlorosaligenine.$\quad i b$.

7. Saliretine $. \quad . \quad i b$.

8. Helicine . . . . 163

Helicoidine . . . $i b$.

Chlorobelicine . . . $i b$.

Rutiline . . . ib.

9. Phloridzine . . . ib.

Phloretine . . $i b$.

Nitrophloretic acid . . 164

Phloridzéine. . . $i b$. 
Appendix to Salicyle and sali- ${ }^{\text {PAGE }}$ cylic acid . • • 164 . Anisyle and anisic acid . . $i b$. Hyduret of anisyle . . $i b$.

Nitranisic and dinitranisic acids

Anisole. . . . . ib.

Chloranisole and bromanisole

Nitranisole, dinitranisole, and trinitranisole

Anisidine, nitranisidine, and dinitranisidine.

Nitrobenzanisidine and nitrocumanisidine

XV. Cinnamyle

Its Compounds.

a. With Hydrogen

1. Hyduret of cinnamyle Oil of cinnamon

b. With Oxygen

2. Cinnamic acid

Sulphocinnamic acid. :

Chlorocinnose

Balsam of Peru

Cinnaméine

Peruvine. . .

Cinnamole . . .

Styrole, probably identical with cinnamole.

Myroxiline . . .

Myriospermine .

Myriospermic acid . .

Balsam of Tolu . .

Tolene

Toluole

Nitrotoluole and Dinitrotoluole

Sulphotoluylic acid

Toluylic acid, homologous with benzoic acid 168 Toluidine .

XVI. Guaiacrle

Hyduret of guaiacyle

Recapitulation and general remarks on the radicals hitherto described

Phenole, Anisole or Phenometole, and Phenamylole are homologous compounds
Radicals of the general formula

$\mathrm{Cn} \mathrm{Hn}+1$ • $\cdot \quad \cdot \quad$ ib METHYLE . . . . $i b$.

Its relations , .173-175

1. Oxide of methyle . . 175

2. Hydrated oxide of methyle . . . $i b$.

3. Chloride of methyle . $\quad 177$

4. Iodide of methyle $\quad i b$.

5. Sulphuret of methyle $\cdot i b$. Hydrosulphuret of the, $i b$.

6. Cyanide of methyle, or Acetonitryle . $\quad i b$.

Salts of oxide of methyle. . I78

1. Neutral Sulphate . . ib. Sulphamate . . . ib.

2. Acid sulphate, or sulphomethylic acid $\quad .179$

3. Nitrate . . . . $i b$.

4. Carbamate . . . ib. Carbonates . . . ib.

5. Oxalate $. \quad . \quad$. ib. Oxamate . . . ib.

6. Benzoate . . . 180

7. Acetate . . . . $i b$.

8. Salicylate (oil of Gaultheria) . : . ib.

9. CarbolateorPhenometole ib.

10. Cyanate . . . 181

11. Allophanate . . . ib.

12. Cyanurate . . $i b$.

Basic compounds of Methyle 182

1. Methylamine . . $i b$. Methyloxamide . . 183 Methyloxamic acid . 184

Amide, Imide, and Nitryle bases . . . . 185

2. Dimethylamine $\cdot \quad \cdot i b$.

3. Trimethylamine $\cdot i b$.

Ammonium bases . $\quad .187$

4. Tetramethylium . . ib.

Iodide of tetramethylium $i b$.

Oxide of tetramethylium $i b$.

Hydrated oxide of ditto $i b$.

Remarks on the ammonium

bases . . . . 188

Bodies containing metals along

with methyle . . . 190

Zincomethyle . . $i b$.

Stibiomethyle . . . $i b$.

Stibiomethylium . . $i b$.

Kakodyle . . . 191

Oxide of kakodyle. . $i b$. 
Kakodylic acid . $\quad \begin{array}{r}\text { PAGK } \\ 192\end{array}$

Chloride, bromide, and iodide of kakodyle.

Sulphuret of kakodyle .

Cyanide of kakodyle

Kakoplatyle

Chloride, \&c., of kakoplatyle

Bases containing phosphorus, with methyle

Products of the oxidation of

hydrated oxide of me-

thyle

ForMYLE

Oxide of formyle .

Formic acid . .

Formiates

Chloride, bromide, iodide, \&c., of formyle

198-201

Chloroform . . $i b$.

Radicals with $\mathrm{C}_{4} \quad . \quad . \quad 201$

ETHYLE . . . . $i b$.

1. Oxide of ethyle or ether $i b$.

Its preparation . . 202

Uses of ether . . . 204

Compound ethers . $\quad i b$.

2. Hydrated oxide of ethyle or alcohol

Absolute alcohol . . 206

Spirit of wine $, \cdot, i b$.

3. Chloride of ethyle $i b$.

4. Bromide of ethyle . . $i b$.

5. Iodide of ethyle $\quad 207$

6. Sulphuret of ethyle $\quad i b$.

7. Hydrosulphuretted sulphuret of ethyle, or mercaptan . .

8. Seleniuret of ethyle .208

9. Fluoride of ethyle.$i b$.

10. Cyanide of ethyle, or propylonitryle .

Radicals containing metals along with ethyle . . 209

Zincethyle . . . $i b$.

Stibethyle $. \quad . \quad i b$.

Stibethylium .. . 210

Bismethyles . . . $i b$.

Stannethyle . . i ib.

Arsenethyle . . . 211

Arsenethylium . . . $i b$.

Plumbethyle . . $i b$.

Mercurethyle . . . $i b$.

Tellurethyle . . $i b$.
Radicals containing phosphorus

and ethyle . . . . 212

Salts of oxide of ethyle. $\quad i b$.

1. Neutral sulphate . . 213

2. Acid sulphate, or sulphovinic acid . . $i b$.

3. Acid phosphate, or phosphovinic acid . 214

4. Nitrate . . . $i b$.

5. Hyponitrite $. \quad . \quad i b$.

6. Perchlorate . . 215

7. Borates . . . . 216

8. Silicates . . . $i b$.

9. Carbonate . . . ib.

10. Double carbonate of ethyle and potash . $i b$.

11. Chlorocarbonate, or chlorocarbonic ether .217

12. Carbamate (urethane) $\ldots i b$.

13. Oxalate . . . . ib.

14. Acid oxalate, or oxalovinic acid . . 218

15. Oxamate . . . ib.

16. Sulphocarbonate, or xanthic acid . . $i b$.

17. Benzoate . . . 219

18. Hippurate . . $\cdot i b$.

19. Salicylate . . . $i b$.

20. Cyanate . . . 220

21. Ethylo-urea $\cdot \cdot^{-} i b$.

22. Allophanate of ethyle . ib.

23. Cyanurate . . 221

Oxide of ethyle and potassium . . . ib.

Bases containing ethyle $\quad i b$.

1. Ethylamine . . . $i b$. Ethyloxamide . . 223

2. Diethylamine . . 224 Methylethylamine $\cdot i b$.

3. Triethylamine . . ib. Dimethylethylamine. $i b$. Diethylomethylamine $i b$.

4. Tetrethylium . . 225

5. Methylotriethylium - $i b$. Tetrethylo-urea . . 226

Metamorphoses of the compounds of Ethyle . . ib. Oil of wine . . . ib.

Heavy oil of wine $\quad i b$. Etherole . . .227 Etherine . $i b$.

Ethionic, Isethionic, Methionic, and Althionic acids $\quad i b$. 
Products of the PAGE dation of ethyle and its derivatives 228

\section{ACETYLE.}

1. Hydrated oxide of acetyle, or aldebyde

Aldehydammonia

Thialdine and Selenaldine . 230

Alanine and its homologues $i b$.

2. Acetal

. 231

Resin of aldehyde $\quad . \quad i b$.

Elaldehyde . . . $i b$.

Metaldehyde . . . $i b$.

3. Aldehydic, Acetylous, or

Lampic acid . . 232

4. Acetic acid . . . . ib.

Anhydrous acetic acid . 234

Hydrated acetic acid . . 235

Acetates . . . $i b$.

Acetamide . . . . 239

Ethylacetamide . . $i b$.

Hyduret of acetyle.$\quad i b$.

Acetylammonium . . 240

Hydrated oxide of ditto . $i b$.

Action of chlorine, bromine,

\&c., on compounds con-

taining ethyle . . 242

1. On oxide of ethyle . . $i b$.

2. On salts of oxide of ethyle

On chloride of ethyle 245

On alcohol . . ib.

Chloral . $\cdot i b$.

On acetic acid . . ib.

Chloracetic acid $\quad i b$.

Oxychloride of acetyle 246

On sulphuret of ethyle Bromal

3. Compounds derived

from Alcohol of un. certain constitution . 247

Olefiant gas, or hyduret of acetyle

Oil of olefiant gas

Chlorides of acetyle $: i b$.

Chloretheral . . ib.

Sulphacetylic acid . 249

4. Action of bichloride of platinum on alcohol

5. Action of heat on acetic acid and acetates $\quad i b$.

Acetone . . $i b$.

Mesityle . . 250

Pteleyle .. . . $i b$.

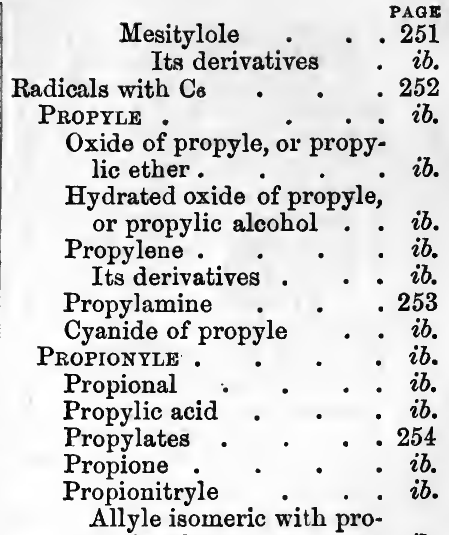

pionyle . . . . $i b$.

Radicals with $\mathrm{C}_{8} \quad . \quad . \quad .255$

Butyle . . . . $i b$.

Butylene . $\quad \bullet^{-} \quad{ }^{-} i b$.

Butylic alcohol . . 256

Butylic mercaptan . . ib.

Chloride of butyle . . $i b$.

Cyanide of butyle $\quad . \quad \quad i b$.

Butylamine . . . $i b$.

Methylopropylamine $\quad i b$.

ButYRyLE . . . 257

$\mathrm{Hyduret}$ of butyryle . . $i b$.

Hydrated oxide of butyryle, or butylic aldehyde . $\quad i b$.

Butyric acid . . . $i b$.

Anhydrous butyric acid . 258

Butyrates . . . . 259

Butyric ether . . . $i b$.

Butyronitryle . . . $i b$.

Butyramide . . . 260

Butyrone . . . $i b$.

Radicals with $\mathrm{C}_{10}$. $. \quad . \quad i b$.

AMYLE . . . . $i b$.

Oxide of amyle . . . 261

Hydrated oxide of amyle, or amylic alcohol . . $i b$.

Sulphamylic acid . $\quad i b$.

Salts of oxide of amyle . . 262

Bases containing amyle . $i b$.

1. Amylamine : . . $i b$.

Methylobutylamine $\quad i b$.

Ethylopropylamine . . $i b$.

Methylodiethylamine . $i b$.

Dimethylopropylamine . $i b$.

Amylo-urea . . . $i b$. 
PAGE

Diamylamine

262

Ethylopropylamylamine 263

Methylobutylamylamine $i b$.

3. Triamylamine . . . ib.

4. Tetramylium . $\quad i b$.

Triethylamylium . . ib.

Diethylamylamine . $\quad 264$

Methylodiethylamylium

Methylethylamylamine .

Chloride, bromide, \&c., of amyle

VALERYLE

Hydrated oxide of valeryle, or valerianic aldehyde .

Valerianic acid

Anhydrous valerianic acid.

Valerates

Valeronitryle $\cdot{ }^{\circ} 265$

Valeramide . . . . $i b$.

Valerene, hyduret of valeryle

Valerone

Radicals witb $\mathrm{C}_{12}$.

Caprotyle

Hydrated oxide of caprotyle

Cyanide of caprotyle $\quad$. $\quad 268$

Caprotylamine . . . $i b$.

Caproyle . . . . $i b$.

Hydrated oxide of caproyle . . .

Anhydrous caproic acid . $i b$.

Caproic acid . . . $i b$.

Caproates . . . 269

Caprone and Capral . . $i b$.

Nitrovalerianic acid . . $i b$.

Capronitryle . . . $i b$.

Radicals with $\mathrm{C}_{14}$. $\quad . \quad .270$

Enanthrle . . . . $i b$.

Hydrated oxide of œnanthyle, or œuanthylic alcohol

Anhydrous and hydrated œnanthylic acid

Euanthylamide . . . 271

Radicals with $\mathrm{C}_{10}$. .

OCTYLE

Hydrated oxide of octyle, or caprylic alcohol

Sulphoctylic acid . $\quad . \quad 272$

Chloride, \&c., of octyle . ib.

Octylamine

Ethyloctylamine

Capryle $i b$.

$i v$.

ib.

$i b$.

273
Hydrated oxide of capryle, or caprylic aldehyde . 273 Anhydrous caprylic acid . ib. Caprylic acid . . . $i b$. Caprylates . . . $i b$.

Caprylone and caprylal . $i b$.

Radicals with $\mathrm{C}_{18} . . . i b$. Noxyle . . . . $i b$.

Pelargyle . . . . $i b$.

Anhydrous pelargonic acid $i b$.

Pelargonic acid . . 274

Pelargone . . . . $i b$.

Hyduret of pelargyle $\quad i b$.

Radicals with $\mathrm{C}_{20}$..$\quad .275$

RUTYLE, or CAPRYLE . . $i b$.

Hydrated oxide of rutyle . ib.

Rutylic acid and rutylates. . . . $i b$.

Rutylamide . . . 276

$\mathrm{C}_{22}$ Margaritic acid . . 277

$\mathrm{C}_{24}$ Laurostearic acid . . $i b$.

$\mathrm{C}_{26}$ Cocinic acid . . . . $i b$.

$\mathrm{C}_{28}$ Myristic acid . . . $i b$.

Cso Benic acid . . . . $i b$.

Radicals with $\mathrm{C}_{32} \quad . \quad . \quad+i b$.

Cetyle . . . . $i b$.

Oxide of cetyle . . $i b$.

Hydrated oxide of cetyle, or cetylic alcohol . . ib.

Sulphocetylic acid . . 278

Chloride, \&c., of cetyle . $i b$.

Oxide of cetyle and sodium $i b$.

Tricetylamine . $\cdot i b$.

Sulphuret of cetyle . . $i b$.

Sulphhydrate of cetyle . . $i b$.

Palmityle . . . . ib.

Hydrated oxide of palmityle, or palmitic aldehyde

$i b$.

Palmitic, cetylic, or ethalic acid . . $i b$.

Palmitates . . . 280

Palmitate of cetyle, or spermaceti . . . $i b$.

Hyduret of palmityle $\cdot \dot{i b}$.

Palmitone. . . . $i b$.

Radicals with $\mathrm{C}_{34}$. . . . $i b$.

Margaric acid . . . $i b$.

Margaramide . . 282

Margarone . . . . $i b$.

Margarine . . . . $i b$.

Radicals with $\mathrm{C}_{36} \quad . \quad . \quad 283$

Stearic acid . . . . $i b$.

Stearates . . . . 284 
Stearine, the stearate of ${ }^{P A G E}$ glyceryle

• • . 285

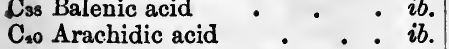

$\mathrm{C}_{42}$ Behenic acid . . . 286

$\mathrm{C}_{54}$ Cerotic acid . . . . $i b$.

Bees-wax . . . $i b$.

Chinese-wax, or cerotine $\quad i b$.

Hydrated oxide of ceryle, or ceric alcohol .

Hyduret of ceryle, or cerene

Cerotic ether . . . $i b$.

Coo Melissic acid . . 287

Hydrated oxide of myricyle, or melissic alcohol .

Chloromelal

$i b$.

Series of oxalic acid . $\quad .288$

Succinic acid . . $\quad .289$

Hyposulphosuccinic acid $\cdot i b$.

Succinates . . . . $i b$.

Succinamide, succinamic acid, and succinimide . ib.

Amber . . . . 290

Lipic acid . . . . . $i b$.

Adipic acid . . . . $i b$.

Pimelic acid . . . $i b$.

Suberic acid . $. \quad . \quad i b$.

Suberates . . . . $i b$.

Suberamide, suberanilide, \&c. . . . . 291

Sebacic acid . . . $i b$.

Sebates . . . . $i b$.

Sebacic ether Sebamide and sebamic acid 292

Table of the preceding acids of the oxalic series. $\quad . \quad i b$.

Oleic acid series $\bullet_{-} \quad . \quad i b$.

Acrylic acid . . . $i b$.

Acrylates . . . . $i b$.

Angelic acid . . . 293

Moringic acid . . . $i b$.

Oleic acid . . . . $i b$.

Oleates . . . . . 294

Action of heat on oleic acid

Action of nitrous acid on Oleic acid ; elaidic acid .

Action of nitric acid on oleic acid

Elaidic acid . . . . $i b$

Linoleic acid . . . 295

Dœglinic acid . . . $i b$.

Doglinate of oxide of dode-

- catyle
Erucic or Brassic acid

PAGE

Table of Radicals and their derivatives, forming the foundations of homologous series . 296

Remarks on this table . . . 297

Theories of Kolbe and Williamson concerning the Ethylic and Acetylic series of compounds . . . . . 298

Natural fixed Oils and Fats . . 300

Vegetable Fats . . . $i b$.

Spermaceti . . . . 301

Cholesterine . . . $i b$. Ambreine . . . $i b$.

Wax . . . . ib.

Chinese and Japan Wax : $i b$.

Cerosine . . . $i b$.

Athamantineand oreoselone $i b$.

The great majority of Fats and fixed Oils are com. pounds of Glycerine, and are called Glycerides . 302

Action of alkalies, heat, sulphuric and nitric acids upon Glycerides . .

Castor Oil . . . . $i b$

Ricinolic acid . . .303

Ricinelaidic acid . . . $i b$.

Other derivatives of Castor Oil . . . . . ib.

Drying Oils . . $\quad i b$.

Action of bases on fat oils, soaps and plasters . 304

GLYCERYLE . . . 305

Glycerine, the hydrated oxide of Glyceryle . . ib.

Acroleine . . . . $i b$.

Sulphoglyceric acid $\quad . \quad$. $i b$.

Nitroglycerine . . . 306

Glycerides . . . . $i b$.

Artificial formation of the natural oils and fats.$i b$.

Chlorhydrine . . . $i b$.

Dichlorhydrine . . . $i b$.

Monacetine . . . $i b$.

Diacetine . . . . $i b$.

Triacetine . . $\quad i b$.

Acetochlorhydrine $\quad . \quad 307$

Diethyline. . . . $i b$.

Monobutyrine $\cdot \bullet^{-} i b$.

Dibutyrine . . . $i b$.

Tributyrine . . . . $i b$.

Monovalerine . . . $i b$.

Divalerine . . . $i b$. 


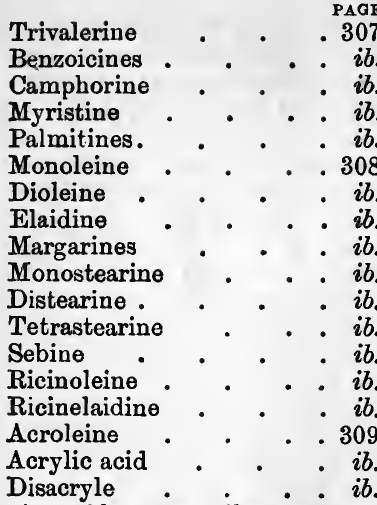

Organic acids, not oily, and soluble in water . $\quad .310$ 1. Citric acid . . . . ib. Citrates . . . . 311 Citric ether . . . $i b$. Citramide . . . $i b$. Action of heat on citric acid

Aconitic or equisetic acid ib.

Itaconic acid . . . 313

Citraconic acid . . . $i b$.

Nitrocitraconic acid $\bullet i b$.

Mesaconic acid . . . $i b$.

Citraconimide $. \quad . i b$.

Citroconanile $. \quad . \quad i b$.

Citroconanilic acid . $\quad i b$.

Itaconanilide ..$~ i b$.

2. Tartaric acid . . . 314

Nitrotartaric acid . $\quad i b$.

Tartrates . . 315

Tartrovinic acid . . $i b$.

Tartar, and tartar emetic ib.

Action of heat on tartaric acid

Anhydrous tartaric acid. $i b$.

Isomeric forms of tartaric acid

3. Paratartaric or racemic acid . . $i b$.

Inactive tartaric acid . $\quad . \quad i b$. 318

Racemic acid, separable into dextrotartaric acid (ordinary tartaric acid), and løvo-tartaric acid.

Metatartaric acid

Isotartaric acid $i b$. $i b$.

4. Malic acid . . . . ib.

Table of the preceding acids

Tartramide and tartramic acid . . . $i b$.

Pyrotartaric acid . . 320

Pyruvic acid . . . $i b$.

Malates . . . $i b$.

Malamide or asparagine . 322

Malamidic or Aspartic acid

Action of heat on malic acid

Maleic acid

Paramaleic or fumaric acid

$i b$.

Fumaramide and fumarimide

Allotropic modifications of malic and aspartic acids

Fermentation of malate of lime and asparagine. $i b$.

5. Tannic acid or Tannine . 324

Tannates . . . . i .

Resolution of tannic acid into Gallic acid and glucose

$i b$.

6. Gallic acid . . . . 326

Gallates . . . $i b$.

Action of heat on tannic and gallic acids . . $i b$.

Pyrogallic acid . . $i b$.

Metagallic acid . . . 327

Ellagic acid . . . $i b$.

Catechu and Mimotannic acid

Tanningen $\circ 328$

Tanningenic acid

Japonic and rubinic acids $i b$.

Caffeic, viridic, and boheic acids

Other products of the family of the Rubiaceæ 329

7. Meconic acid . . . ib.

Meconates . . . $i b$.

Ethylomeconic acid . $i b$.

Meconamidic acid . 331

Meconoethylomeconic acid . . . $i b$.

Biethylomeconic acid . $i b$.

8. Comenic acid . . ib.

Chlorocomenic acid . . $i b$.

Bromocomenic acid $\quad i b$.

Ethylocomenic acid ... $i b$. 
Comenamic acid . . 331

Pyromeconic acid . . ib.

Bromo and iodopyromeconic acids .

9. Kinic Acid

Kinates . . . . $i b$.

Products of the decomposition of kinic acid .

Kinone

Kinone.. 333

Hydrokinone . . . $i b$.

Chlorokinone. . $\quad i b$.

Sulphohydrokinone . $i b$.

Chlorosulphokinone $: i b$.

Volatile acids not hitherto described

334

Camphoric acid . . $\quad i b$.

Camphorates . . . $i b$.

Anhydrous camphoric acid

Sulpho-camphoric acid

Campholic acid . : ib

Borneo camphor . . $i b$.

Camphor . . $i b$.

Camphogene . . ib.

Camphrone . . . 336

Enanthic acid . . . $i b$.

Enanthic ether $\cdot v^{-} i b$.

Roccellic acid . . . $i b$.

Eugenic acid . . . $i b$.

Oil of cloves; caryophyl-

line and eugenine

Organic acids, but little

known. .537

Volatile Essential Oils. $\quad i b$.

1. Non-oxygenated Essential Oils

Oil of turpentine .

Iadyle and peucyle. $\quad .338$

Turpentinic acid . . . $i b$.

Oils of juniper, savin, and elemi . . . .

Oil of storax or styrole : Its derivatives . Nitrostyrole, chloro. styrole, \&c.

Metastyrole, or draconyle . .

Oils of lemon, orange, cedrat, and limes . .

Oil of neroli copaiva, pepper, and cubebs .

Isomeric modifications of oil of turpentine
2. Oxygenated volatile oils . 340

Oils of bitter almonds, cinnamon, spiræa, cloves, cumine, aniseed, and valerian . . . . 341

Oil of thyme . . . $i b$. Thymoïle, and thymoilole. . . . 342 Oil of carraway . . . ib. Carvene, and carvacrole $i b$. Other oxygenated volatile oils

Cumarine and cumaric acid . . . 343

3. Sulphuretted essential oils $i b$.

Oil of mustard, and its derivatives

Oil of mustard is sulpho: cyanide of allyle . . $i b$.

Thiosinuamine . . $i b$.

Sinnamine . . $i b$.

Sinapoline . . 344

Myrosine and Sinapisine . . . $i b$.

Myronic acid . 345

Oils of horseradish and alliaria

Oil of garlic, or sulphuret of allyle. . . . $i b$.

Compounds of allyle . $i b$.

Oil of hops, water-pepper, and arum . . . ib.

Sinapine, sinapic acid, and sinkaline

Concrete volatile principles allied to the essential oils

Hellenine . . . .

Nitrohellenine and hello néne . . . . $i b$.

Asarone . . . . $i b$.

Anemonine and anemonic acid . . . . $i b$.

Cantharidine . . 347

Caoutchouc, or gum elastic $i b$. RESINS

Action of heat on it . $i b$. $i b$.

Colophony, pinic, and sylvic acids

Resins of copaiva, elemi, benzoin, balsam of tolu

Toluole and its derivatives

Resin of styrax, or styracine, the cinnamate of oxide of styryle . . 349 
Hydrated oxide of styryle 349

Resins of guaiacum, lac, mastic, dragon's blood, sandarach, and jalap $i b$.

Derivatives of jalap . 350

Resins of rhubarb, copal, and peat

Resin peat $\cdot i b$. Actionous varnishes $\cdot{ }^{-} i b$. Colouring matters, non-azotised

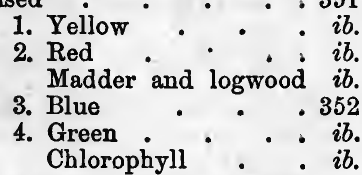
Non-azotised bitter, crystal-

line vegetable products . 353

Nitrogenised colouring mat-

ters, and allied substances 356

1. Lecanoric acid . . 357

2. Lecanoric ether . $i b$.

3. Orcine • . . 358 Orceine . . . $i b$.

4. Parellic acid . : ib.

5. Erythric acid . $i b$. Erythrelesic acid . . 359 Variolarine . . $i b$.

6. Picroerythrine.$i i b$.

7. Erythromannite $\cdot i b$.

8. Alpha orsellie acid . 360

9. Beta orsellic acid . . ib. Alpha and beta orsellesic acids . .

10. Roccelline . . . $i b$.

11. Evernic acid . . $i b$. Evernesic acid . . $i b$.

Remarks on the preceding compounds and their uses . 361

12. Usnic acid . . . ib.

13. Chrysophanic acid .362

14. Archil . . . . $i b$.

15. Litmus . . . $i b$.

16. Phloridzeine . . $i b$.

17. Indigo $\cdot \quad \cdot i b$.

White or reduced indigo . . . 364

Action of sulphuric acid on indigo . 366

Sulphoindigotic acid, \&c, $i b$.

Oxidation of indigo . . 367 Isatine
. $i b$.
PAOE

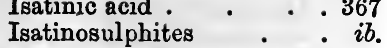

Chlorisatine . . . . $i b$.

Chlorisatinic acid . $\quad . \quad i b$.

Bichlorisatine. $\quad .368$

Bichlorisatinic acid . - ib.

Bromisatine, bromisatinic acid, \&c. \&c. $\quad$. $\quad$. 369

Tabular view of these compounds . . . . $i b$.

Aniline, chloraniline, bromaniline, \&c. \&c. . . ib.

Isatyde . . . .370

Sulphésatyde . . . $i b$.

Chlorisatyde . . $\quad i b$.

Bichlorisatyde . . . $i b$.

Sulphasatyde . . . $i b$.

Indine, indinic acid.$\quad i b$.

Hydrindine . . . 371

Nitrindine . . . . $i b$.

Chlorindine . . . ib.

Sulphisatanous acid . $i b$.

Chlorindopten . $\quad .372$

Chlorophenisic acid . . $i b$.

Trichloraniline . . . $i b$.

Chlorophenusic acid . $\quad i b$.

Chloranile $\quad$. . $i b$.

Chloranilic acid . . . $i b$.

Chloranilammon . . 373

Chloranilam . . . . ib.

Imesatine - . . . $i b$.

Imasatine.$\quad 0^{-} i b$.

Imasatinic acid . $\cdot \quad i b$.

Amasatine . . . . $i b$.

Imechlorisatinase, \&c. $\quad i b$.

Imabromisatinese . . . 374

Action of nitric acid on indigo and its derivatives . $i b$.

Nitrosalicylic or anilic acid . . . ib.

Nitrophenisic or pioric acid . . . . ib.

Action of heat and potash on indigo . . .375

Chrysanilic acid . . $i b$.

Anthranilie acid . . $i b$.

Action of heat on anthranilic acid . . . ib.

Aniline

ib.

Tabular view of some compounds derived from apiline . . . 377

Chloraniline, \&c. . , ib.

Bromaniline, \&c. . . $i b$. 
Nitraniline, \&c. $\quad . \quad \begin{array}{r}\text { PAOE } \\ .377\end{array}$

Cochineal and Carmine . 378

Carminic acid . . . ib.

Nitrococcusic acid . . $i b$.

Kermes . . . . 379

Lac dye . . . $i b$.

Action of nitric acid on aloes . . . ib.

Chrysammic acid . $\quad i b$.

Chrysolepic acid . . 380

Alkaloids or Organio Bases . $i b$.

Formation of artificial bases . 381

1. Amide bases . . . 383

2. Imide bases . . . $i b$.

3. Nitryle bases $\quad . \quad i b$.

Table of amide bases $\quad . \quad i b$.

Table of imide bases $\quad . \quad 384$

Table of nitryle bases..$i b$.

4. Ammonium bases . . $i b$.

Theory of these . . 385

Table of ammonium bases . 386

Supposed artificial formation of quinine

Methods of producing bases . 388

1. By means of ammonia - ib.

2. By destructive distillation $i b$.

3. By the action of sulphuretted hydrogen on compounds of ammonia

4. By the action of sulphuret of ammonium on azotised compounds .

5. By heating azotised compounds with potash or baryta.

6. By heating such com. pounds with acids.

7. By spontaneous metamorphosis . . .

Artificial bases . . .

a. Volatile oily bases $\quad .389$

Aniline . . . . $i b$.

Modes of formation of aniline

ib.

ib.

$i b$.

ib.

Derivatives of aniline

Analogy between aniline and ammonia illustrated by a table.

Anilic or phenylic series of bases, in a tabular form

Products of metamorphosis of aniline . . .
'PAGE

Ethylonitraniline . . 392

Diethylochloraniline . $\quad i b$.

Cyaniline . . . 393

Melaniline. . . . $i b$.

Dichloromelaniline . $i b$.

Dibromomelaniline, Diiodomelaniline, Dinitromelaniline, and Dicyanomelaniline. . $i b$.

Oxanilide . . $i b$.

Oxanilamide . . . 394

Melanoximide $\cdot \quad i b$.

Anilocyanic acid . . $i b$.

Carbanilide, Phenylourea, and Diphenylourea . . . $i b$.

Phenylo-urethane . . 395

Anilomellone . . $i b$.

Ethylophenylamine, or Ethylaniline . . . $i b$.

Diethylophenylamine 396

Triethylophenylium . . $i b$.

Methylethylamylophenylium

Ethylamylophenylamine $i b$.

Methylophenylamine $\quad i b$.

Methylethylophenylamine

Amylophenylamine $\quad i b$.

Diamylophenylamine $\cdot i b$.

Picoline, isomeric with aniline . . . $i b$.

Toluidine, homologous with aniline . . 397

Pyridine . $\quad \cdot i b$. Platinopyridine, \&c. 398

Lutidine, isomeric with toluidine . . . $i b$.

Xylidine . . . . $i b$.

Collidine . . . $i b$.

Cumidine . . . $i b$.

Parvoline . . . ib.

Cymidine . . . . $i b$.

Pyrrole bases . . 399

Quinoline . . 400

Lepidine . . . $i b$.

Cryptidine . . . $i b$.

Carmidine and Vertidine $i b$.

b. Bases from naphthaline .402

Seminaphtalidine . $\quad i b$.

Naphtalidine . . . $i b$.

c. Bases from oil of mustard . . . $i b$.

Thiosinnamine . . . ib. 


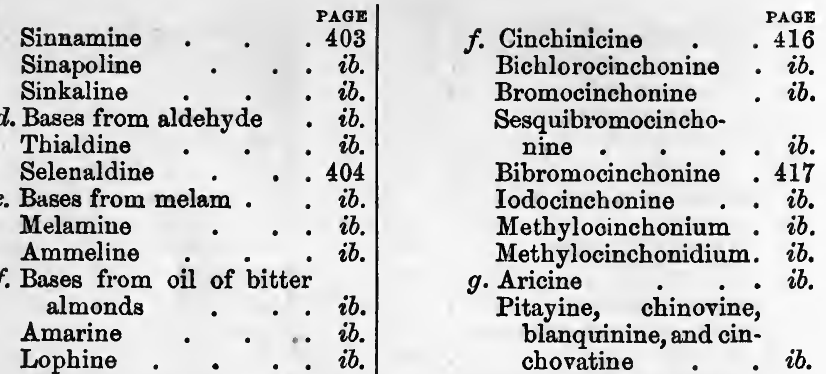

Trinitrolophyle . $\quad \because 405$

Picrine . . . $i b$.

Trinitropicryle . $\quad i b$.

g. Base from furfurole . . $i b$.

Furfurine . . . $i b$.

h. Bases from Narcotine . 406

Cotarnine and Narcogenine . . .

i. Bases from animal products . . . . ib. Glycocine . . $\quad i b$.

Alanine . . . . $i b$.

Leucine . . . 407

Sarcosine . . . . $i b$.

Kreatinine . . . $i b$.

General remarks on artificial

bases . . . . 408

Action of hyponitrous acid on

artificial bases . . . $i b$.

Natural bases or alkaloids $\quad 410$

$a$. Liquid volatile bases - $i b$.

Nicotine . . . $i b$.

Iodonicotine . . 411

Methylonicotium . $i b$.

Ethylonicotium . $\quad i b$.

Amylonicotium . . 412

Coniine . . . $i b$.

Methyloconiine . $i b$.

Ethylomethyloconium 413

Diethyloconium . . $i b$.

Sparteine • $\quad . \quad i b$.

b. Bases of cinchona bark . $i b$.

a. Quinine or Chinine $\quad i b$.

Methyloquinium . 414

Ethyloquinium . $i b$.

Herapathite or bisul-

phate of iodoquinine $i b$.
b. Quinidine
415

c. Bases of the Papaveraceæ 418

a. Morphine . . . $i b$. Its salts . . . 419

Methylomorphium . $i b$.

Ethylomorphium . 420

b. Codeine . . . ib.

Sulphocodeide . . $i b$.

Nitrocodeine . . ib.

Bromocodeine . . ib.

Tribromocodeine.$i b$.

Chlorocodeine . . ib.

Dicyanocodeine $\quad 421$

Ethylocodium . . $i b$.

c. Papaverine . . $i b$.

d. Thebaine . . . 422

e. Pseudomorphine - $i b$.

$f$. Narceine . . . $i b$.

g. Narcotine . . $i b$.

Its Derivatives.

1. Opianic acid - $i b$.

2. Opiammon . $i b$.

3. Xanthopenic acid . 423

4. Opiano-sulphurous acid . . $i b$.

5. Sulphopianic acid . $i b$.

6. Hemipinic acid $\quad i b$.

7. Cotarnine . . $i b$.

8. Opianyle $. \quad . \quad i b$.

9. Teropiammon .424

10. Humopivic acid. . $i b$.

11. Apophyllic acid . $i b$.

12. Narcogenine . $i b$.

13. Narcotinic acid $\quad i b$.

Methylonarcotine . $\mathbf{4 2 5}$

Ethylonarcotine . $i b$.

Propylonarcotine . $i b$.

c. Quinicine . . . $i b$.

d. Cinchonine. $\quad . \quad i b$.

e. Cinchonidine . . 416

$h$. Chelidonine $\quad . \quad i b$.

$i$. Chelerythrine . . ib.

k. Glaucine . . . $i b$.

l. Glaucopicrine . . ib. 
d. Alkaloids of the Solanacex, the Strychneæ, and other vegetable families 425

a. Hyoscyamine,$i b$.

b. Daturine . . . 426

c. Stramonine . . $i b$.

d. Atropine . . . ib.

e. Solanine . . $i b$.

f. Veratrine . . . ib.

g. Sabadilline . . 427

h. Colchicine . . $i b$.

$i$ Aconitine . . $i b$.

k. Delphine . . . 428

l. Staphisine . . $i b$.

m. Emetine . . . ib.

n. Chiococcine ..$i b$.

o. Viuline . . . $i b$.

p. Strychnine . . $i b$.

$q$. Brucine . . 429

$r$. Jervine . . . $i b$.

s. Curarine . .430

t. Corydaline . . $i b$.

$u$. Carapine . . $i b$.

v. Cusparine $\quad . \quad i b$.

$w$. Daphnine . . $i b$.

$x$. Bebeerine . . $i b$.

$y$. Sanguinarine $\quad i b$.

z. Azadirine . . $i b$.

aa. Capsicine . . . ib.

bb. Crotonine . . 431

cc. Buxine . . . ib.

$d d$. Apyrine . . $i b$.

ee. Cynapine . . . $i b$.

ff. Cissampeline $\cdot i b$.

gg. Oxyacanthine and Berberine $i b$.

$h h$. Surinamine and $\mathrm{Ja}$ muicine

ii. Piperine $\cdot{ }^{*} i b$. Piperidine $\cdot$.

Methylopiperidine

Ethylopiperidine

Amylupiperidino

Dimethylopiperidium

Diethylopiperidium

Piperylosulphocarbamic acid

Piperylbenzamide, \&c.

kk. Menispermine, \&c.

ll. Harmaline, and
Harmine

Leucoharmine, \&c.

432

Nitroharmine, \&ib.

Amalic acid . $: i b$

Cholestrophane . $i b$.

Caffeomurexide . 434

$n n$. Theobromine . . ib.

e. Alkaloids found in the

Animal Organism . 435

Urea and kreatinine . ib.

List of supposed alkaloids

which have not been studied $i b$.

INDIFFERENT NON-AZOTISED

COMPOUNDS. $\quad \cdot \quad \cdot i b$

1. Sugar . ..$\quad$. ib.

1. Cane sugar . . . 436

Barley sugar and caramel $i b$.

With acids . . . $i b$.

With bases and salts. 437

2. Grape sugar . . . ib. Made from starch

With acids, bases, and salts . 439

Sulphosaccharic acid. . $i b$.

Sacchulmine . . . ib.

Sacchulmic acid . . $i b$.

Glucicand melassic acids $i b$ -

Action of heat on sugar;

caramel . . . 440

Propione and propylic

acid . $i b$.

Action of nitric acid on

sugar . . . . ib.

Saccharic acid . . $i b$.

3. Sugar of milk or Lactine 441 Mucic or saccholactic acid . . . 442 Pyromucic acid $i b$.

Modified mucic acid . ib.

4. Sugar of mushrooms . $i b$.

5. Sorbine . . . . $i b$.

6. Inosite . . 443

7. Uncrystallisable sugar . ib.

ib. Vinous fermentation . . 444

Ferments. . . . $i b$.

ib. Wine . . . .445

Fuseloil . . . . . $i b$.

ib. Lactic fermentation . . . 446

Viscous fermentation . . . 447

ib. Butyric fermentation . . 448

ib. Mannite .. . . . ib.

Nitromannite $\cdot . \quad i b$. 


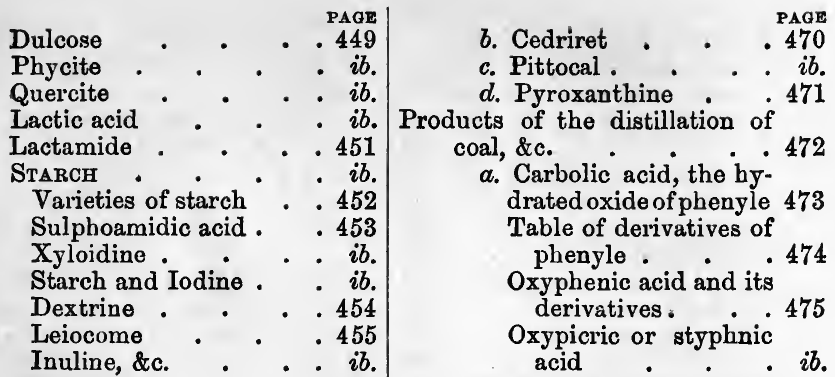

$\underset{\text { Saponine }}{\text { Lichenine }} \cdot{ }^{-} \cdot \begin{aligned} & i b . \\ & 456\end{aligned}$

Arabine . . . ib.

Mucilage • . . $i b$.

Bassorine . . . . $i b$.

Gum tragacanth . . $i b$.

Cerasine . . . .457

Pectine . . . $i b$.

Pectic acid . . . ib.

Apicine . . . 4458

Glycyrrhizine . . . $i b$.

Sarcocolline . . . $i b$.

4. WOODY FIBRE $. \quad . \quad i b$.

Cellulose . . . $i b$.

Lignone . . . . 459

Gun cotton . . $i b$.

Decay of wood . . . 460

Brown coal . . . ib.

Mould . . . . 461

Humus, \&c. . . . $i b$.

Crenicandapocrenicacids $i b$.

Products of the distillation of wood . $i b$.

1. Volatile products soluble

in water . . . . . 462

Acetic acid . . . $i b$.

Pyroxylic spirit . . $i b$.

Lignone, xylite, xylitic oil, xylitic naphtha - $i b$.

Mesitene, methole, mesite $i b$.

2. Volatile oily products inso-

luble in water . . . 463

a. Creosote . . . ib.

Its relation to carbolic acid . .465

b. Picamar . . 467

c. Capnomor - . . ib.

d. Eupion . . . .468

3. Solid products . . . $i b$.

a. Paraffine . . ib.

b. Volatile bases of coal tar . . . . 476

Aniline, picoline, lutidine, and leucoline, and pyrrole bases . ib.

c. Solid volatile products 477

a. Naphthaline . . $i b$. 1. Action of chlorine and bromine on naphthaline. . ib.

Laurent's nomenclature . $i b$.

Chlonaphtase, \&c. 478

Bronaphtase, \&c. $i b$.

Doctrine of substitutious applied to these products . . . 479

Chlonaphtese, \&c. $i b$. Bronaphtese, \&c. 481 Other analogous products $481-485$

2. Action of sulphuric acid on naphthaline

Sulphonaphthalic acid . . $i b$.

Disulphonaphthalic acid . 486

Hyposulphoglutinic acid . . $i b$.

Sulphonaphthaline, \&c. . - $i b$.

3. Action of nitric acid on naphthaline . . $i b$.

Nitronaphtase,\&c. $i b$.

Naphtiouic and thionaphtamic acids, \&c. . $\quad 487$

Naphtalase, \&c. . $i b$. 
Chloronaphtisic acid, \&c. . Phtalic acid Phtalamide and phtalimide

Nitrophtalic and clophtalisic acids $i b$.

B. Anthracene . . .490 Table of its derivatives . $i b$.

r. Metanaphthaline

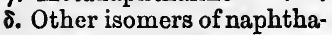
line . . $i b$

€. Chrysene . . . .491

$\zeta$. Pyrene . . . . $i b$. Ampeline and ampelic acid

Fossil resins, wax, naphtha, \&c. 492

Retinite, Hatchetine, \&c. $i b$.

Idrialine and its derivatives

Ozokerite and Fichtelite

Tekoretine, Phylloretine, \&c. Asphaltum, or mineral pitch 493 Petroleum and naphtha . $i b$. Soot and lamp-black . . 494

Compounds, vegetable and animal, containing nitrogen and sulphur, albuminous or sanguigenous compounds

The proteine theory untenable

$i b$.

1. Albumen $\quad .496$

Products of its oxidation . 498

Emulsine . . . . $i b$.

2. Fibrine . . 500

Products of its oxidation . 501

Leucine . . . $i b$.

Tyrosine . . . 502

3. Caseine. . . . . 504

Milk . . $i b$.

Products of the oxidation of caseine . . . 505

Gliadine, \&c. . . . $i b$.

Horn, hair, feathers . . $i b$.

Mucus . . . .506

Diastase . . . .507

Malt . . . . 508

Must, beer, ale, porter . $i b$.

Oil of grain and of potato spirit . . . ib.

Panification . .509
Ferment, yeast, lees of wine . . . 509

Gelatine . . . . 510

Chondrine . . . 511

Products of decomposition of gelatine . . 512

Boneblack, ivoryblack . ib.

Bile • . . . . 513

Cholic acid . . . . 514

Paracholic acid . . . $i b$.

Cholalic acid and glycocine 515

Choloidic acid . . . $i b$.

Dyslysine . . . . $i b$.

Choleic acid . . . . ib.

Cholalic acid and taurine . 516

Pig's bile . . . $i b$.

Hyocholic, cholonic, and

hyocholeic acids $\quad . \quad i b$.

Hyocholalic acid . 517

Tabular comparison of ox

and pig bile . . . ib.

Cholesterine . . 518

Biliary calculi . . . 520

Bezoar stones . . . $i b$.

Lithofellic acid . . . ib.

Brain and nervous matter.$i b$.

Cerebric acid . . . $i b$.

Milk . . . . . 521

Cheese . . . . . 523

Gastric Juice $\quad$. . . . 524

Digestion . . . . $i b$.

Pepsine does not exist $\quad .525$

Juice of flesh . . . . $i b$.

Kreatine . $\quad .526$

Proportion of it in different

kinds of flesh . . . 527

Lactic acid . . . 528

Inosinic acid . . . . $i b$.

Phosphoric acid . . $i b$.

Phosphate of potash . . $i b$.

Saliva . . . . 529

It dissolves starch..$i b$.

Pancreatic juice . . .530

Its supposed function . . $i b$.

Excrements and urine . $i b$.

Constituents of urine . $\quad i b$.

Salts of excreta compared with those of food. . 531

The peculiar odour of fæces is caused by imperfect oxidation of albumen, \&c. 532

The excreta represent the smoke, soot, and ashes 
of the animal body, considered as a furnace

Compounds occur in urine which are identical with some of those in smoke and tar

Guano

Constituents of

Theory of its action . . 534

Importance of nightsoil . $i b$.

Urinary calculi . .,$i b$.

Of uric acid . . . . $i b$.

Of urate of ammonia . 535

Of phosphate of lime . . ib.

Of phosphate of ammonia and magnesia. .

Fusible calculus . . . $i b$.

Of oxalate of lime . $\quad i b$.

Of carbonate of lime . . ib.

Of cystic and xanthic ox-

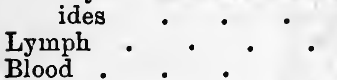

$i b$.

$i b$.

$i b$.

Serum . . . 536

Globules . . . . $i b$.

Colouring matter . . . $i b$.

Iron in the blood . . 537

Hematosine . . . $i b$.

Gases in the blood . $\quad .538$

Functions of the phosphate of soda in the blood . 539

Nutrition of plants and animals

Food of plants . . .

Germination

Endosmosis into the vegetable cell . . .

Effect of light in enabling the plant to decompose carbonic acid .

Production of malamide

, oxalic acid other acids . 542

Relation of vegetable acids to sugar

Tabular view of the production of the different groups of vegetable compounds :

Group I. Acids .

Group II. Indifferent bodies 544

Group III. Neutral, bitter, acrid, and coloured substances

Group IV. Oxygenated volatile oils and their acids 545
Group V. Oily and fatty acids, \&c. . . . 546

Group VI. Resins and camphors

Group VII. Carbohydrogens .. . .

The whole vegetative process is one of deoxidation

Carbon of plants supplied by the atmosphere, as carbonic acid

Functions of the atmosphere Balance between animal and vegetable life, as to carbon and oxygen . . $i b$.

Use of humus in the soil . 550

Nitrogen of plants also supplied by the atmosphere, as ammonia

Origin of the ammonia in air

Balance between living and dead organic matter, as to ammonia

Source of the sulphur of plants

Transformations of ammonia 552 Amides . . . . $i b$.

Malamide . . . 553

Indigo . . . . . $i b$.

Amygdaline . . . 554

Imides . . . . . $i b$.

Nitryles . . . . $i b$.

ib. Table of the formation of nitro-

genous vegetable compounds 556

Sulphurised compounds . $i b$.

Compounds with sulphur and nitrogen

Oil of mustard . . $\quad .556$

ib. Sanguigenous compounds . . 557

ib. Their formulæ . . . $i b$.

Tables, showing their derivation both from sugar, and from the ultimate constituents of the food of plants . 558

The vegetable processes exhibit deoxidation, along with the building up of more complex molecules

Mineral constituents of plants . 560

Causes of fertility and barrenness in soils $\quad . \quad i b$.

ib. Importance of phosphates .561 Uses of organic matter in manure 
Farm-yard manure is the standard manure

Sources of phosphates . . . 563

Importance of the contents of our common sewers .

Rules in using special manures

. 564

Ashes as manure . . . $i b$.

Power of carbonic acid to dissolve phosphates

Prodigious power derived from the solar rays in vegetation .

Vegetables necessarily contain the food of animals 567

Food of animals . . . $i b$. Digestion . . . 568

Changes occurring in albumen, caseine, fibrine, \&c., in the animal body, illustrated by tables.

These changes are all oxidations

Origin of the constituents of urine.

Method for estimating the amount of urea in urine

Blood albumen and egg albumen .

Fat is formed in the body from sugar . . .

Fattening of animals .

Tables illustrating the origin of fat from sugar .

The animal thus obtains oxygen from sugar .

Table, showing how the fatty acids may beformed from sugar by fermentation .

Respiration

Laws of diffusion of gases from the blood . .

Pneumic acid . . 580

Origin of the animal heat .581

Amount of it

The respiratory and sanguigenous matters in food ought to be in due proportion .

Table of the proportions of these in different articles of food

Table of the comparative value of fat and other matters as

respiratory food . . . 583

Alcohol as respiratory food . $i b$.

Mineral elements of the food . 584

The blood is alkaline . . . $i b$.

Salts of urine with vegetable and animal diet .585

Table of these . . . . 586

Importance of the mineral elements of food . . . ib.

How to dress meat and vegetables. . . . 587

Extract of meat . . . $i b$. Bread . . . . . . ib. Vegetable food . . $\quad .588$

Vegetarianiam . . . . $i b$.

Abuse of fermented liquors . 589 The animal body a furnace . . $i b$. Relations of animal and vegetable life

Circulation of the organic elements through plants and animals back to the mineral kingdom

571 Mechanical force of the body derived from the solar rays 591

572 Chemical causes of disease $\quad i b$. Diet of children . . . . 593 574 Use of tea, coffee, chocolate, \&c. 575 a necessary of life . . $i b$. ib. Conclusion . . . . .594

ib. 1. A new class of alcohols . . 595 Allylic or acrylic alcohol .596 Oxalate of allyle . . . $i b$. Chloride, \&c., of allyle . $i b$. Sulphocarbonate of allyle and potassium . . . $i b$. Other compounds of allyle . $i b$. Sulphallylic acid . . . $i b$. Allylurea . . . . $i b$. Diallylurea . . . . 597 Allylamine . . . . $i b$. Allylic series . . . $i b$.

2. A new class of acids $\quad . \quad 599$ 582 Insolinic acid . . . $i b$. 3. New phosphorised bases $\quad .600$

4. Trigenic acid . . . . 601

ib. 5. Sudoric acid $\quad . \quad . \quad . \quad i b$.

6. Thymine . . . . . 602 


\section{ERRATA.}

Page 137, line 11, for " amide," read " amides."

" 155, " 9, , " amygdaline acid," read " amygdalinic acid."

"165, , 15, ," “ $\mathrm{C}_{14} \mathrm{H}_{7} \mathrm{NO}_{4}, \mathrm{O}_{4}, "$ read " $\mathrm{C}_{14} \mathrm{H}_{7} \mathrm{NO}_{4}, \mathrm{O}_{2}$."

, 226, last line, ," “ $\mathrm{C}_{4} \mathrm{H}$," read " $\mathrm{C}_{4} \mathrm{H}_{4} . "$

, 239, line 17, , " $\mathrm{C}_{22} \mathrm{H}_{11} \mathrm{NO}_{2}$," read " $\mathrm{C}_{12} \mathrm{H}_{11} \mathrm{NO}_{4}$."

" 240, ", 9 from bottom, for " silica," read " silver."

" 241, ", 2, for " hyponitrate," read " hyponitrite."

" 242, , 18, ,, “ insoluble," read " unstable."

" 242, , 3 from bottom, for " chloride," read " chlorine."

" 256, ", 16, for " sulphobutylic acid, Bu 0, НО, $280_{3}, "$ read "sulphobutylic acid, $\mathrm{Bu} \mathrm{O,} \mathrm{HO}, 2 \mathrm{SO}_{3} . "$

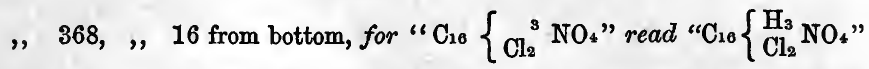

" 397, , 16 from bottom, for " (See table, p. 92)." read "(See table, p. 392)."

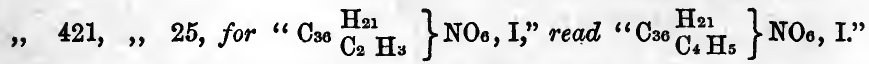

" 450, , 3 from bottom, for " $\mathrm{C}_{6} \mathrm{H} \mathrm{O}_{6}$," read " $\mathrm{C}_{6} \mathrm{H}_{6} \mathrm{O}_{6} . "$

" 503, " 14 from bottom, for " $\mathrm{C}_{18} \mathrm{~N}_{3} \mathrm{H}_{11} \mathrm{O}_{1}$," read " $\mathrm{C}_{18} \mathrm{~N}_{3} \mathrm{H}_{11} \mathrm{O}_{16}$." 


\section{ORGANIC CHEMISTRY.}

\section{INTRODUCTORY.}

Organic Chemistri is so called because it treats of the substances which form the structure of organised beings, and of their products, whether animal or vegetable. It has long been known, that all organised structures, as well as all the substances formed in or by these, are, in great part, composed of a very limited number of elements; insomuch that a large proportion of them may be described as consisting, almost exclusively, of only four simple substances, namely, Carbon, Hydrogen, Oxygen, and Nitrogen.

But while these four elements undoubtedly constitute the chief part of all organised tissues, and while such products as woody fibre, sugar, starch, gum, fat, oils, and many organic acids, contain only the first three, that is, Carbon, Hydrogen, and Oxygen, we must not forget that other elements occur in the organised kingdoms of nature; some of them, such as those of Phosphate of Lime, in large quantity; and all, whether they occur in smaller or greater proportion, as truly essential to animal and vegetable life, as the four elements above mentioned, the predominance of which characterises the organic world.

Thus, no plant ean grow, or form cells, or even fibre, without the presence of certain mineral or saline compounds, which are derived from the soil, and which, when the plant is burned, constitute its ashes. These are, Potash, Soda, Lime, Magnesia, with, occasionally, oxides of Iron and Manganese, as bases; and Silicic Acid, Phosphoric Acid, Sulphuric Acid, Chlorine, Iodine, and Fluorine, as acids and acid-radicals.

Again, the juices of all plants, and more especially their roots and seeds, contain some one or more of the compounds known by 
the names of albumen, fibrine, and caseine. Now these compounds contain small, but absolutely essential proportions of sulphur, besides earthy and alkaline phosphates.

Lastly, the bones of animals contain not only phosphate of lime, but also phosphate of magnesia and fluoride of calcium, both in very considerable quantity; and Iron is an unfailing constituent of blood.

To the four elements first mentioned, as constituting the chief mass of organic substances, we must therefore add, as no less essential, although for the most part in smaller proportion, the following metalloids, Chlorine, Iodine, Fluorine, Sulphur, Phosphorus, and Silicon; and the following metals, Potassium, Sodium, Calcium, Magnesium, Iron, and occasionally Manganese.

It thus appears that the fourteen or fifteen elements which constitute the chief mass of the mineral or inorganic world, are almost the same which occur in organised matter; the difference being chiefly this, that in inorganic nature the predominant elements, nearly in the order of their abundance, are, Oxygen, Hydrogen, Nitrogen, Silicon, Chlorine, Sodium, Aluminium, Carbon, and Iron, after which follow Potassium, Calcium, Magnesium, Sulphur, Phosphorus, Iodine, and Fluorine; while in the organic department the order is nearly as follows: Carbon, Oxygen, Hydrogen, Nitrogen, Potassium, Calcium, Phosphorus, Silicon, Sulphur, Sodium, Magnesium, Chlorine, Iron, Iodine, and Fluorine. Aluminium, so very abundant in the mineral kingdom, hardly ever occurs in organic compounds, and when it does occur, is perhaps accidental.

The above considerations are sufficient to show, that there is no essential distinction to be made between organic and inorganic Chemistry, founded on the nature of the elements concerned.

Neither is there any such distinction to be pointed out in regard to the laws of combination and decomposition which prevail in these different departments of chemistry ; for we find the same affinities operating; and although organised tissues, and their products, have, in general, a more complicated constitution than inorganic compounds, containing a larger number of equivalents of their elements, and consequently having much higher atomic weights, we cannot consider such characters as forming a valid ground of distinction.

But while we should find it very difficult, if not impossible, to draw the line between inorganic and organic Chemistry on scientific principles, we may still recognise, for convenience sake, a certain distinction, founded, first, on the origin of substances, 
whether animal and vegetable, or mineral; and secondly, on the uniform predominance of carbon in animal and vegetable matter.

In reference to the first point, it is to be observed, that, although the elements concerned are those common to the inorganic and organic kingdoms, the compounds which constitute the latter are formed under peculiar circumstances, such as, for the most part, cannot be imitated in our experiments.

It is true that chemistry has succeeded, in some cases, in forming artificially certain compounds which occur as products of organic life, such as Urea, Formic Acid, and Oil of Spiræa. But, in the first place, most, if not all of these, require for their production the aid of an organic product: thus, Formic Acid is produced from Starch, Oil of Spiræa from Salicine; and although Urea may be obtained from Cyanic Acid and Ammonia, it is doubtful if either Cyanogen or Ammonia can be obtained except from organic compounds, directly or indirectly. Secondly, it is particularly to be noticed, that we have not yet succeeded in forming, artificially, either an organised tissue, or even any one of the compounds (albumen, \&c.) of which such tissues are made. Those organic compounds which have been artificially formed, are invariably products of decomposition, or, in other words, the excretions or secretions of organised bodies: and are far less complex in their constitution than organised structures.

From these facts we draw the conclusion, that certain circumstances, of which the most important is the vital force, so modify the play of affinities in organised beings, as to produce the compounds usually termed organic, which, so far as they are capable of entering into the composition of tissues, cannot be imitated by art.

In regard to the second peculiarity of organic compounds, namely, the predominance of carbon in their composition, we observe that, as this carbon is united to the three gases, Oxygen, Hydrogen, and Nitrogen, with each of which it forms gaseous compounds, and as, further, the latter elements, among themselves, form compounds, such as water and ammonia, which are also volatile, so the action of heat on organic compounds is characteristic; producing combustion of all, save the ashes, when there is free access of air ; and charring them, or in other words, causing the separation of part of their carbon, in close vessels, while the greater part is dissipated in the form of volatile products.

Here, then, we have a ready test of organic matter, which is so characteristic, that we might almost define Organio 
Chemistry as the Chemistry of such compounds as are charred when heated to redness in close vessels. There are very few substances, indeed, of organic origin, which do not exhibit this character.

Organic Chemistry has been defined as the Chemistry of Compound Radicals; but, although we must admit the existence of many such radicals in Organic Chemistry, we cannot adopt this definition in contradistinction to that of Inorganic Chemistry, as the Chemistry of Simple Radicals, because the recent progress of science has led, or almost compelled, us to admit the existence of compound radicals in Inorganic Chemistry.

It is, perhaps, worth while to point out that all the organic compound radicals hitherto established, or supposed to exist, are compounds of carbon, if we except amidogen, which contains only hydrogen and nitrogen.

It is also proper here to state, that, under the name of organic compounds, many substances are treated of which do not occur in nature, but which have been obtained by subjecting true organic products to various influences : to that, for example, of heat, as in what is called the destructive distillation, which yields such substanoes as naphtha, naphthaline, \&c.; or to the action of chlorine or bromine, of sulphuric or nitric acids, of alkalies, \&c., by all which means whole series of new compounds are obtained. Lastly, some very interesting and important compounds are included under the term organic, which arise from the addition of elements not naturally occurring in the organic kingdom; as for example, kakodyle and its compounds, which contain arsenic as an essential constituent; and the very singular bases in which zinc, tin, and antimony are added to some of the usual elements of organic alkalies.

But while, as has just been stated, compound radicals are not exclusively characteristic of organic chemistry, we may still derive great assistance from attending to the compound radicals of organic chemistry. For while we admit the existence of such radicals in inorganic chemistry, along with simple radicals, we must bear in mind that all the organic radicals as yet discovered are compound, and many of them exceedingly complex, containing three or four elements.

It is true that we are not yet acquainted with the radicals of a very large proportion of organic compounds; such as the principal organic acids, the organic alkalies, \&c. But the known organic radicals furnish us with the means of classifying many most important substances, just as we classify the compounds of any metalloid or of any metal together. As to those groups or series of organic compounds, the radicals of which are not yet 
known, we can only class them according to analogies of pro perties, of composition, or of both.

With these introductory remarks, we shall proceed to consider the known organic compound radicals, and their derivatives.

\section{COMPOUND ORGANIC RADICALS.}

A compound radical is a substance which, although containing two or more elements, enters into combination with elementary bodies as if it were itself elementary, and in ordinary circumstances performs exactly the part of an element.

We have admitted (Inorganic Chemistry) as probable the existence of inorganic compound radicals, such as $\mathrm{SO}_{4}$, the radical of sulphuric acid, and $\mathrm{NO}_{6}$, that of nitric acid. These bodies are, in their relations to others, entirely analogous to chlorine. Thus we may represent the acids of these three radicals, with their potassium and silver salts as follows :-

\begin{tabular}{|c|c|c|c|c|}
\hline & & Acid. & $\begin{array}{l}\text { Potassium } \\
\text { Salt. }\end{array}$ & Silver Salt. \\
\hline Radicals & $\left\{\begin{array}{c}\text { Chlorine } \\
\mathrm{S} \mathrm{O}_{4} \\
\mathrm{~N} \mathrm{O}_{3}\end{array}\right.$ & $\begin{array}{l}\mathrm{H}+\mathrm{Cl} \\
\mathrm{H}+\mathrm{SO}_{4} \\
\mathrm{H}+\mathrm{NO}_{6}\end{array}$ & $\begin{array}{l}\mathrm{K}+\mathrm{Cl} \\
\mathrm{K}+\mathrm{SO}_{4} \\
\mathrm{~K}+\mathrm{NO}_{6}\end{array}$ & $\begin{array}{l}\mathrm{Ag}+\mathrm{Cl} \\
\mathrm{Ag}+\mathrm{SO}_{4} \\
\mathrm{Ag}+\mathrm{NO}_{6}\end{array}$ \\
\hline
\end{tabular}

The compound inorganic radicals, $\mathrm{SO}_{4}$ and $\mathrm{NO}_{6}$, therefore, perform exactly the part of a metalloid of the group of chlorine.

But there have also been briefly mentioned, in the First Part, certain compound organic radicals, which not only exhibit, in their relations, characters analogous to those of chlorine, but actually exist, like chlorine, in the separate state, which is not the case with $\mathrm{SO}_{4}$ and $\mathrm{NO}_{6}$, these latter being only known in combination.

The organic radicals here alluded to are Cyanogen, $\mathrm{C}_{2} \mathrm{~N}=$ $\mathrm{Cy}$, and Mellone, $\mathrm{C}_{18} \mathrm{H}_{13}=\mathrm{Me}$. They may be compared to chlorine, exactly like the two above-mentioned inorganic compound radicals. Thus,

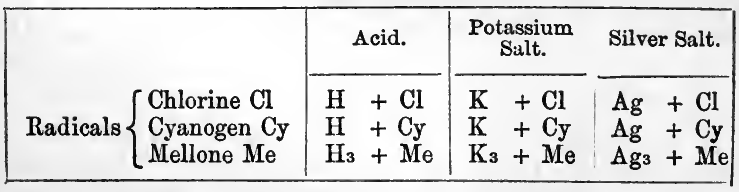

Cyanogen and Mellone are, therefore, radicals of the nature of 
the chlorine group of metalloids. The bisulphuret of eyanogen, or sulpho-cyanogen, $\mathrm{C}_{2} \mathrm{~N} \mathrm{~S}_{2}=\mathrm{Cy}_{2}$, although it contains three elements, plays the same part as chlorine or cyanogen, and forms with hydrogen the acid $\mathrm{H}+\mathrm{Cy}_{2}$, with potassium (the salt) $\mathrm{K}+\mathrm{Cy} \mathrm{S}_{2}$, and with silver (the salt) $\mathrm{Ag}+\mathrm{Cy} \mathrm{S}_{2}$.

Some compound organic radicals appear more analogous to the combustible group of metalloids, that is, to carbon, sulphur, or phosphorus; inasmuch as they form acids with oxygen, or rather with the elements of water like those metalloids, and are besides capable of entering into combination with chlorine, iodine, \&c. Such radicals are, carbonic oxide, $\mathrm{C} \mathrm{O}$, or rather a polymeric modification of it, $\mathrm{C}_{2} \mathrm{O}_{2}$; acetyle, $\mathrm{C}_{4} \mathrm{H}_{3}$; and formyle, $\mathrm{C}_{2} \mathrm{H}$. Each of these may be viewed as the radical of a powerful acid; for $\mathrm{C}_{2} \mathrm{O}_{2}+\mathrm{O}=\mathrm{C}_{2} \mathrm{O}_{3}$ is dry oxalic acid; $\mathrm{C}_{4}$ $\mathrm{H}_{3}+\mathrm{O}_{3}$ is dry acetic acid; and $\mathrm{C}_{2} \mathrm{H}+\mathrm{O}_{3}$ is dry formic acid. Again, the first forms with chlorine the compound $\mathrm{C}_{2} \mathrm{O}_{2}+\mathrm{Cl}_{2}$, called phosgene gas or chloro-carbonic acid, while the two others yield $\mathrm{C}_{4} \mathrm{H}_{3}+\mathrm{Cl}$, the chloride of acetyle, and $\mathrm{C}_{2} \mathrm{H}+\mathrm{Cl}_{3}$, the perchloride of formyle.

Further, there are organic compound radicals which play the part of metals, forming salts with chlorine, iodine, sulphur, cyanogen, \&c., and yielding, with oxygen, compounds possessing basic properties analogous to those of metallic oxides. Such radicals are, ethyle, $\mathrm{C}_{4} \mathrm{H}_{5}$, methyle, $\mathrm{C}_{2} \mathrm{H}_{3}$, and kakodyle, $\mathrm{C}_{4} \mathrm{H}_{6}$ As, besides others.

\begin{tabular}{|c|c|c|c|c|}
\hline Radicals. & $\begin{array}{c}\text { Oxygen } \\
\text { Compound. }\end{array}$ & $\begin{array}{c}\text { Chlorine } \\
\text { Compound. }\end{array}$ & $\begin{array}{l}\text { Cyanogen } \\
\text { Compound. }\end{array}$ & $\begin{array}{l}\text { Sulphur } \\
\text { Compound. }\end{array}$ \\
\hline $\begin{array}{l}\text { Ethyle } \mathrm{C}_{4} \mathrm{H}_{5}=\mathrm{Ae} \\
\text { Methyle } \mathrm{C}_{2} \mathrm{H}_{3}=\mathrm{Mt} \\
\text { Kakodyle } \mathrm{C}_{4} \mathrm{H}_{6} \mathrm{As}=\mathrm{Kd}\end{array}$ & $\begin{array}{l}\mathrm{Ae} \mathrm{O} \\
\mathrm{Mt} \mathrm{O} \\
\mathrm{Kd} \mathrm{O}\end{array}$ & $\begin{array}{l}\mathrm{Ae} \mathrm{Cl} \\
\mathrm{Mt} \mathrm{Cl} \\
\mathrm{Kd} \mathrm{Cl}\end{array}$ & $\begin{array}{l}\mathrm{Ae} \mathrm{Cy} \\
\mathrm{Mt} \mathrm{Cy} \\
\mathrm{Kd} \mathrm{Cy}\end{array}$ & $\begin{array}{l}\text { Ae S } \\
M t S \\
\text { Kd S }\end{array}$ \\
\hline
\end{tabular}

Lastly, there are some compound organic radicals, which partake of the characters of the two last groups, forming, like the acetyle group, acids and not bases with oxygen ; but yielding, with chlorine, sulphur, cyanogen, \&c., compounds analogous to those formed by the ethyle group. To this division belong Benzoyle, $\mathrm{C}_{14} \mathrm{H}_{5} \mathrm{O}_{2}=\mathrm{Bz}$; Cinnamyle, $\mathrm{C}_{18} \mathrm{H}_{8} \mathrm{O}_{2}=\mathrm{Ci}$; and several others. Benzoyle and cinnamyle, with the addition of oxygen and the elements of water, produce benzoic acid, $\mathrm{Bz} \mathrm{O}, \mathrm{HO}$, and cinnamic acid, $\mathrm{Ci} \mathrm{O}, \mathrm{HO}$. This group is characterised by forming with hydrogen certain essential oils. Thus, benzoyle yields, with hydrogen, the essential oil of bitter almonds, $\mathrm{Bz} \mathrm{H}$; cinnamyle yields the oil of cinnamon, $\mathrm{Ci} \mathrm{H}$; and salicyle, 
$\mathrm{C}_{14} \mathrm{H}_{5} \mathrm{O}_{4}=$ Sa, another radical of this group, forms with hydrogen, the oil of spiræa, $\mathrm{Sa} \mathrm{H}$.

These brief statements will serve to show that there are different kinds or groups of compound radicals, just as there are of simple ones; and further, that these compound radicals exhibit a very remarkable tendency to combine with simple radicals, and, in fact, to act the part of elementary bodies. And let us here bear in mind that the only real difference, in this point of view, between cyanogen and chlorine is this, that in the case of the former we can prove the radical to be compound, while we cannot as yet do this in the case of the latter. But, as formerly pointed out, we call chlorine, and indeed all other elements simple, only because we have not been able to show them to be compound; without having any certainty that they are really and absolutely simple. If we could not resolve cyanogen into carbon and nitrogen, we should be compelled to add it to the list of elements.

But although compound radicals usually act towards other bodies as if simple, and consequently combine generally with simple substances, they are also capable of uniting with each other. In fact, this is but another proof of their close resemblance to elementary bodies in their relations; for as simple metals, such as potassium and silver, unite with cyanogen just as with chlorine, so also such compound radicals as are analogous to metals can combine with cyanogen, itself a compound radical. Thus ethyle, methyle, benzoyle, and kakodyle, all combine with cyanogen, yielding compounds formed of two organic radicals, one playing the part of a metalloid, the other that of a metal.

Compounds of this nature furnish the very best proof and illustration of the advantages which we derive from the doctrine of compound radicals, acting like elements, whenever we are justified by facts in adopting and applying it. Thus a compound has been formed by the mutual action of a compound of kakodyle and a compound of cyanogen, the analysis of which proves that it contains carbon, hydrogen, nitrogen, and arsenic, in the relative proportions indicated by the formula $\mathrm{C}_{6} \mathrm{H}_{6} \mathrm{~N}$ As. What view are we to take of such a formula? and if we look on the compound as one formed of these four elements indiscriminately united, how are we to retain such an isolated fact in the memory? But if, on the other hand, we view it as the cyanide of kakodyle, $=\mathrm{C}_{4} \mathrm{H}_{6} \mathrm{As}+\mathrm{C}_{2} \mathrm{~N}$, or using the abbreviated notation appropriate to compound radicals, $\mathrm{Kd} \mathrm{Cy}$, we are at once enabled to retain the composition and chemical relations of the compound. Moreover, when we find that the radical, $\mathrm{Kd}\left(=\mathrm{C}_{4} \mathrm{H}_{6} \mathrm{As}\right)$ exists in a separate form, and that it forms, with oxygen, two compounds, 
Kd $\mathrm{O}$ and $\mathrm{Kd} \mathrm{O}_{3}$; with chlorine, $\mathrm{Kd} \mathrm{Cl}$; with sulphur, $\mathrm{Kd} \mathrm{S}$; and that, in short, it plays the part of a metal in all its compounds, and may in fact be separated from some of these by metals having stronger affinities than itself, we are supplied with an idea which serves to connect and to fix all these and many more analogous facts in the memory.

When we further observe, to pursue the same example, that the cyanide of kakodyle, $\mathrm{Kd} \mathrm{Cy}$, when acted on by hydrochloric acid, gives rise to hydrocyanic acid and chloride of kakodyle; and that, when acted on by potash, it yields cyanide of potassium and oxide of kakodyle, we acquire so many additional proofs of the entire analogy between simple and compound radicals in their relations to other bodies. For the two changes or reactions above mentioned are expressed by the equations, $\mathrm{Kd} \mathrm{Cy}+\mathrm{H}$ $\mathrm{Cl}=\mathrm{Kd} \mathrm{Cl}+\mathrm{H} \mathrm{Cy}$; and $\mathrm{Kd} \mathrm{Cy}+\mathrm{KO}=\mathrm{Kd} \mathrm{O}+\mathrm{K} \mathrm{Cy}$; and these equations are exactly similar to those which occur most frequently in inorganic chemistry.

The facts already ascertained with regard to those compound organic radicals, whose existence has been either established, or rendered highly probable, entitle us to conclude that all organic compounds contain one or more organic radicals, combined either with each other, or with elementary radicals. In studying, therefore, any organic product, one chief object is to determine what organic radical or radicals it contains, since the knowledge of these at once gives us a means of classification.

Thus alcohol, on the theory of compound radicals, is considered as the hydrated oxide of ethyle; ethyle being an organic radical, $\mathrm{C}_{4} \mathrm{H}_{5}$. So that alcohol, $\mathrm{C}_{4} \mathrm{H}_{6} \mathbf{O}_{2}$, is more accurately represented as $\left(\mathrm{C}_{4} \mathrm{H}_{5}\right) \mathrm{O}+\mathrm{H} \mathrm{O}$; or, if we represent ethyle, $\mathrm{C}_{4} \mathrm{H}_{5}$, by Ae, then alcohol becomes Ae $\mathrm{O}, \mathrm{H} \mathrm{O}$, hydrated oxide of ethyle; perfectly analogous to $\mathrm{K} \mathrm{O}, \mathrm{H} \mathrm{O}$, hydrated oxide of potassium, or caustic potash.

Again, benzoic ether, $\mathrm{C}_{18} \mathrm{H}_{10} \mathrm{O}_{4}$, is viewed as benzoate of oxide of ethyle, $\mathrm{C}_{4} \mathrm{H}_{5} \mathrm{O}+\mathrm{C}_{14} \mathrm{H}_{5} \mathrm{O}_{3}$; or, more briefly, $\mathrm{Ae}$ $\mathrm{O}+\mathrm{Bz} \mathrm{O}$. Here we have the basic oxide of one radical united with the acid oxide of another.

It is often by means of thus tracing the different organic radicals, that we are enabled to explain the very numerous cases of isomerism, which occur in organic chemistry. Thus the following compounds have the same composition in 100 parts :-

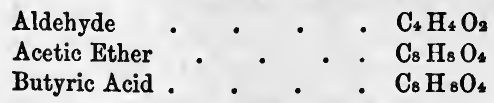

Now, aldehyde is considered to be the hydrated protoxide of 
acetyle, $\left(\mathrm{C}_{4} \mathrm{H}_{3}\right) \mathrm{O}+\mathrm{HO}$; or, abbreviated, Ac $\mathrm{O}, \mathrm{HO}$. Again, acetic ether is acetate of oxide of ethyle, $\mathrm{C}_{4} \mathrm{H}_{5} \mathrm{O}+\mathrm{C}_{4} \mathrm{H}_{3} \mathrm{O}_{3}$; or, shortly, $\mathrm{Ae} \mathrm{O}, \mathrm{Ac} \mathrm{O}_{3}$; the dry acetic acid, Ac $\mathrm{O}_{3}=\left(\mathrm{C}_{4} \mathrm{H}_{3}\right)$ $\mathrm{O}_{3}$ being a peroxide of the same radical, acetyle, $\left(\mathrm{C}_{4} \mathrm{H}_{3}=\right.$ Ac) of which aldehyde is the protoxide. Lastly, butyric acid is considered (on the older view of acids) as a hydrated acid, a compound of water with dry butyric acid; thus, $\mathrm{H} \mathrm{O}+\mathrm{C}_{8}$ $\mathrm{H}_{7} \mathrm{O}_{3}$. It is true that, in the latter case, we are not yet acquainted with the true radical of butyric acid; but we cannot doubt that, like acetic acid, it does contain a radical, butyryle, $\mathrm{C}_{8} \mathrm{H}_{7}$. These three compounds, therefore, may now be represented and distinguished as follows:-

\begin{tabular}{lll}
\multicolumn{2}{c}{ Empirical Formula. } & Rational Formula. \\
Aldehyde. & . & $\mathrm{C}_{4} \mathrm{H}_{4} \mathrm{O}_{2}=\left(\mathrm{C}_{4} \mathrm{H}_{3}\right) \mathrm{O}+\mathrm{HO}$ \\
Acetic Ether & . & $\mathrm{C}_{8} \mathrm{H}_{3} \mathrm{O}_{4}=\left(\mathrm{C}_{4} \mathrm{H}_{5}\right) \mathrm{O}+\left(\mathrm{C}_{4} \mathrm{H}_{3}\right) \mathrm{O}_{3}$ \\
Butyric Acid & . & $\mathrm{C}_{8} \mathrm{H}_{3} \mathrm{O}_{4}=\left(\mathrm{C}_{8} \mathrm{H}_{7}\right) \mathrm{O}_{3}+\mathrm{HO}$
\end{tabular}

Even in those cases in which the composition of the radical is not known, or not known with certainty, we can often trace the radical with much probability. Thus, dry oxalic acid, $\mathrm{C}_{2} \mathrm{O}_{3}$, and dry mellitic acid, $\mathrm{C}_{4} \mathrm{O}_{3}$, may be viewed as different compounds of the simple radical carbon; the latter containing just twice the proportion of carbon to the same quantity of oxygen that the former does. This is merely stated by way of illustration; for, it is at least equally probable that the true radical of oxalic acid is $\mathrm{C}_{2} \mathrm{O}_{2}$.

But in the following four acids we may trace, theoretically, the same compound radical, namely, formyle, $=\mathrm{C}_{2} \mathrm{H}$, in combination with different proportions of oxygen. Here $\mathrm{C}_{2} \mathrm{H}$ is also represented by Fo.

$$
\begin{array}{ll}
\text { Formic Acid } & \left(\mathrm{C}_{2} \mathrm{H}\right)+\mathrm{O}_{3}=\mathrm{FoO}_{3} \\
\text { Succinic Acid } & \mathrm{C}_{4} \mathrm{H}_{2} \mathrm{O}_{3}=2\left(\mathrm{C}_{2} \mathrm{H}\right)+\mathrm{O}_{3}=\mathrm{F}_{2} \mathrm{O}_{3} \\
\text { Malic Acid }: \mathrm{C}_{4} \mathrm{H}_{2} \mathrm{O}_{4}=2\left(\mathrm{C}_{2} \mathrm{H}\right)+\mathrm{O}_{4}=\mathrm{F}_{2} \mathrm{O}_{4} \\
\text { Racemic Acid } & \mathrm{C}_{4} \mathrm{H}_{2} \mathrm{O}_{5}=2\left(\mathrm{C}_{2} \mathrm{H}\right)+\mathrm{O}_{5}=\mathrm{F}_{02} \mathrm{O}_{5}
\end{array}
$$

These relations, although as yet only to be traced in the formulæ, are yet not without interest, and may, at all events, serve to aid the memory, and explain decompositions.

In like manner, it may be observed, that the following acids all contain, as hydrates, 4 eq. of oxygen : and all likewise the same number of eqs. of carbon as of hydrogen. And it is most important to remark, that the whole of this series of acids possess analogous properties. All are volatile, all but the two first are oily, and their fusing and boiling points rise, as the number of equivalents of carbon and hydrogen increases. We 
shall see that this is not the only series of the kind in organic chemistry.

Volatile and Oily Acids of the General Formula $\left(\mathrm{C}_{2} \mathrm{H}_{2}\right)_{n} \mathrm{O}_{4}$.
1. Formic Acid .
$=\mathrm{C}_{2} \mathrm{H}_{2} \mathrm{O}_{4}$
2. Acetic
3. Propylic
4. Butyric
5. Valeriani
6. Caproic
7. Enanthylic
$=\mathrm{C}_{4} \mathrm{H}_{4} \mathrm{O}_{4}$
8. Caprylic
9. Pelargonic
$=\mathrm{C}_{6} \mathrm{H}_{6} \mathrm{O}_{4}$
10. Capric
$=\mathrm{C}_{8} \mathrm{H}_{8} \mathrm{O}_{4}$
$=\mathrm{C}_{10} \mathrm{H}_{10} \mathrm{O}_{4}$
$=\mathrm{C}_{12} \mathrm{H}_{12} \mathrm{O}_{4}$
11. Margaritic
12. Laurostearic
$=\mathrm{C}_{14} \mathrm{H}_{14} \mathrm{O}_{4}$
13. Cocinic .
$\doteq \mathrm{C}_{16} \mathrm{H}_{16} \mathrm{O}_{4}$
$=\mathrm{C}_{18} \mathrm{H}_{18} \mathrm{O}_{4}$
14. Myristic
15. Benic
16. Ethalic
17. Margaric
18. Bassic
19. Balenic
20. New Acid
21. Behenic .
22. Cerotic
$=\mathrm{C}_{20} \mathrm{H}_{20} \mathrm{O}_{4}$
$=\mathrm{C}_{22} \mathrm{H}_{22} \mathrm{O}_{4}$
$=\mathrm{C}_{24} \mathrm{H}_{24} \mathrm{O}_{4}$
$=\mathrm{C}_{26} \mathrm{H}_{28} \mathrm{O}_{4}$
$=\mathrm{C}_{28} \mathrm{H}_{28} \mathrm{O}_{4}$
$=\mathrm{C}_{30} \mathrm{H}_{30} \mathrm{O}_{4}$
$=\mathrm{C}_{32} \mathrm{H}_{32} \mathrm{O}_{4}$
$=\mathrm{C}_{34} \mathrm{H}_{34} \mathrm{O}_{4}$
$=\mathrm{C}_{36} \mathrm{H}_{36} \mathrm{O}_{4}$
$=\mathrm{C}_{38} \mathrm{H}_{38} \mathrm{O}_{4}$
$=\mathrm{C}_{40} \mathrm{H}_{40} \mathrm{O}_{4}$
23. Melissic .
$=\mathrm{C}_{42} \mathrm{H}_{42} \mathrm{O}_{4}$
$=\mathrm{C}_{54} \mathrm{H}_{54} \mathrm{O}_{4}$
$=\mathrm{C}_{60} \mathrm{H}_{60} \mathrm{O}_{4}$

Here we may suppose the radical of the first acid to have been changed by the successive additions of $2,4,6,10$, or 20 , or more eqs. of carbon and hydrogen, the oxygen remaining unchanged. Or we may as readily suppose one of these acids, by losing oxygen, to pass into another. Thus we may either conceive butyric acid to be formed from acetic acid by the addition of $\mathrm{C}_{4} \mathrm{H}_{4}$; or acetic acid to give rise to butyric acid, by losing half its oxygen; for $2\left(\mathrm{C}_{4} \mathrm{H}_{4} \mathrm{O}_{4}\right)=\mathrm{C}_{8} \mathrm{H}_{8} \mathrm{O}_{8}$; and $\mathrm{C}_{8} \mathrm{H}_{8} \mathrm{O}_{8}-$ $\mathrm{O}_{4}=\mathrm{C}_{8} \mathrm{H}_{8} \mathrm{O}_{4}$.

When compound organic radicals, or their compounds, are subjected to powerful decomposing agencies, they tend to produce new and less complex radicals. Thus, when alcohol, the hydrated oxide of ethyle, is oxidised, it gives rise to aldehyde and acetic acid, which are compounds of acetyle, $\mathrm{C}_{4} \mathrm{H}_{3}$, a less complex radical than ethyle, $\mathrm{C}_{4} \mathrm{H}_{5}$. Further, when organic compounds are decomposed by a strong heat, they tend to produce compounds of simple radicals, such as carbon or hydrogen, or, at most, of the least complex radicals, such as cyanogen, $\mathrm{C}_{2} \mathrm{~N}$, and amidogen, $\mathrm{N} \mathrm{H}_{2}$. These are principles of very general application. 
It may here be observed, that while, in such cases as the supposed conversion of acetic into butyric acid, by the loss of half its oxygen, the change is from a less complex to a more complex organic compound, and while we can hardly doubt the possibility of such a result, yet the oxidation of a compound radical, that is, the addition of oxygen, appears always to produce less complex radicals or compounds.

It is often urged, as an argument against the doctrine of compound radicals, that these supposed radicals are entirely imaginary and cannot be produced. Now, it is true that a large proportion of those, whose existence is best attested, have not yet been obtained in the uncombined state; and it is even probable that some of them are only capable of existing, or rather of being preserved, when combined. But the argument founded on this fact has no cogency ; for, in the first place, some organic radicals, such as cyanogen and kakodyle, are well known in the separate state. Now cyanogen and kakodyle are, in all their relations, exactly analogous, the former to chlorine, the latter to a metal; and, if we were unable to demonstrate their compound nature, their chemical relations would compel us to classify cyanogen as an element along with chlorine, and kakodyle along with the metals ; and when we see whole series of organic compounds, in all respects analogous to those of cyanogen and kakodyle, we are entitled logically to draw the conclusion that these compounds contain similar compound radicals, even although we cannot isolate them. Secondly, in every chemical theory yet broached, many substances are admitted whose existence cannot be directly proved; but it frequently happens that the progress of discovery reveals the existence of such originally hypothetical bodies. Thus, the so-called anhydrous organic acids were, almost without exception, unknown in the separate state; they were equally imaginary with the radicals whose existence is doubted. But, as we shall see, Gerhardt, by the action of perchloride of phosphorus, has recently obtained a large number of these very anhydrous organic acids. Moreover, many inorganic acids are hypothetical. Anhydrous nitrio acid has only very recently been discovered, and no one doubts the existence of hyposulphurous acid, which yet has never been separated, either as a hydrate, or in the anhydrous state. Lastly, the progress of research is daily adding to the number of radicals known in the separate form. Within the last two or three years, ethyle and five or six other radicals of the same class, or at least bodies of the same composition as these radicals, have been discovered, besides several entirely new classes of radicals.

We conclude, therefore, that organic compound radicals exist, 
and generally play the part of elements; and we shall avail ourselves of their existence, as far as it is established, to facilitate the study, the classification, and the retention in the memory, of organic compounds.

\section{THEORY OF CHEMICAL TYPES.-DOCTRINE OF SUBSTITUTION.}

The original and ingenious researches of Laurent have led to the adoption of what is called the Theory of Types and the Law or Doctrine of Substitution, which have been supported, and in a great measure established, by Dumas and other distinguished experimenters of the French school. The views of Laurent and of Dumas were, for a time, vehemently opposed by some chemists, especially by Berzelius and Liebig; but although they have in some points been modified and restricted, the progress of discovery has gradually led to their general reception, so that some of the most striking illustrations and proofs of the law of substitution have been discovered by Dr. Hofmann, when assistant to Baron Liebig, and working under his eye.

As the subject, therefore, is no longer purely controversial, it would be wrong to omit it in an elementary work, more especially as the doctrine has now taken such a form as to facilitate very much the study of organic compounds and of their metamorphoses.

It is not easy to define a chemical type; but in inorganic chemistry we may say, for example, that one principal type is that of water, $\mathrm{H} \mathrm{O}$, in which one equivalent of an electro-positive element is united with one equivalent of an electro-negative element. Of this great type, which is also prevalent in Organic Chemistry, there are many forms or classes. Thus, hydrochloric acid, $\mathrm{H} \mathrm{Cl}$, is the type of a very numerous class of acids, the character of which is that they contain hydrogen united to a salt radical.

If for chlorine we substitute iodine, bromine, \&c, or even cyanogen, the type remains unchanged, the compound is still an acid, analogous to that which was selected as the type.

Again, common salt, $\mathrm{Na} \mathrm{Cl}$, is the type of a very large series of salts, in which a metal is united with a salt radical; and if we substitute potassium, lead, or silver for the sodium, the type is unaltered; we obtain a different salt, but still a salt of the type represented by $\mathrm{NaCl}$.

Here, then, we have the simplest types and the most obvious cases of substitution; when iodine or cyanogen is substituted for chlorine in the acid type; or when potassium, lead, or silver is substituted for sodium in the salt type; in both cases without the loss of the type.

Nay, in the salt type, represented by $\mathrm{Na} \mathrm{Cl}$, we may not only 
replace sodium by other metals, but we may also substitute iodine, bromine, \&c., or cyanogen for the chlorine, and still the type will remain unchanged. Iodide of sodium, $\mathrm{Na} \mathrm{I}$, bromide of magnesium, $\mathrm{Mg} \mathrm{Br}$, and cyanide of silver, $\mathrm{Ag} \mathrm{Cy}$, are all as good examples of the salt type, represented by $\mathrm{Na} \mathrm{Cl}$, as common salt itself is.

The whole of the compounds just alluded to are of the type of water, but this is not the only great type or series of types; for we have the type of ammonia, $\mathrm{N} \mathrm{H}_{3}$, to which belong, in inorganic chemistry, various compounds of nitrogen with metals, and which is still more frequent in organio chemistry.

Chemists have very recently come to be of opinion that many phenomena are better understood by giving to the chief types a somewhat different form. Thus, the type Metal is now often represented by $\left.\begin{array}{l}\mathrm{H} \\ \mathrm{H}\end{array}\right\}$, hydrogen having all the chemical relations of a metal, and being the representative of metals. The type Water then becomes $\left.\begin{array}{l}\mathrm{H} \mathrm{O} \\ \mathrm{H} \mathrm{O}\end{array}\right\}$; the type Acid becomes $\left.\underset{\mathrm{H} \mathrm{Cl}}{\mathrm{H} \mathrm{Cl}}\right\}$, hydrochloric acid being the representative of acids; and the type Ammonia is thus written $\mathrm{N} \underset{\mathrm{H}}{\mathrm{H}}\}$. It is found that by using such formulæ or symbols, many changes are more easily expressed. and understood. We shall frequently use them.

So far as inorganic chemistry is concerned, the study of types would serve generally to confirm and establish the electrochemical theory. At all events, we are not as yet acquainted with many exceptions to it; we do not usually find oxygen or chlorine occupying the place of $\Delta$ in a compound, or a metal playing the part of X. Even in inorganic chemistry, however, there are some examples of such interchanges. Manganese in manganic acid, $\mathrm{Mn} \mathrm{O}_{3}$, and chromium in chromic acid, $\mathrm{Cr} \mathrm{O}_{3}$, obviously represent the sulphur in sulphuric acid; and the manganese in hypermanganic acid, $\mathrm{Mn}_{2} \mathrm{O}_{7}$, represents the chlorine in perchloric acid, $\mathrm{Cl} \mathrm{O}_{7}$; while, in its other compounds, manganese acts as a metal.

In inorganic chemistry, many substances, at first sight not referable to the few types just named, are easily referred to them. Thus Oil of Vitriol, $\mathrm{H} \mathrm{O}, \mathrm{S} \mathrm{O}_{3}$, or $\mathrm{H}, \mathrm{SO}_{4}$, is of the type Acid, and may be shown to be so by the second of the formulæ here given, in which $\mathrm{SO}_{4}$ takes the place of $\mathrm{Cl}$ in hydrochloric acid. But it may also be referred to the type Water $\left.\begin{array}{r}\mathrm{H} \mathrm{O} \\ \mathrm{H}\end{array}\right\}$, thus $\left.\underset{\mathrm{SO}_{2} \mathrm{O}}{\mathrm{H}}\right\}$; in which sulphurous acid, $\mathrm{SO}_{2}$, has taken the place of one of the eq. of hydrogen, or what comes to the same thing, anhydrous sulphuric acid, $\mathrm{SO}_{3}$, is $=\mathrm{SO}_{2}, \mathrm{O}$. 
In like manner, in organic chemistry, most compounds can be referred to one or other of the chief types. For example, Ethyle, like hydrogen, is analogous to metals; and its formula, $\mathrm{C}_{4} \mathrm{H}_{5}$, is easily referred to the type Metal, $\underset{\mathrm{H}}{\mathrm{H}}\}$ thus, $\left.\underset{\mathrm{C}}{\mathrm{H}} \mathrm{H}_{4}\right\}$ where one of the atoms of hydrogen is replaced by $\mathrm{C}_{4} \mathrm{H}_{4}$.

But the researches of Laurent and Dumas have shown that in organic chemistry the substitution of one element for another, even where the type is retained, is not limited by the electrical character of the elements. Thus, in acetic acid, $\mathrm{H} \mathrm{O}, \mathrm{C}_{4} \mathrm{H}_{3} \mathrm{O}_{3}$, the 3 eq. of hydrogen in the anhydrous acid may be replaced by chlorine, giving rise to the compound $\mathrm{H} \mathrm{O}, \mathrm{C}_{4} \mathrm{Cl}_{3} \mathrm{O}_{3}$, in which the type is so little affected, that this substance, chloracetic acid, has properties highly analogous to those of acetic acid. Here it is evident that the chlorine performs the same function as the hydrogen which it replaces did; and not, as in hydrochloric acid, an opposite function.

Again, in aldehyde, $\left(\mathrm{C}_{4} \mathrm{H}_{3}\right) \mathrm{O}+\mathrm{H} \mathrm{O}$, the 3 eq. of hydrogen in the radical $\mathrm{C}_{4} \mathrm{H}_{3}$ may be replaced by 3 eq. of chlorine, and we then have chloral, $\left(\mathrm{C}_{4} \mathrm{Cl}_{3}\right) \mathrm{O}+\mathrm{H} \mathrm{O}$, a body of the same type as aldehyde.

Such cases of substitution of chlorine (iodine, bromine, \&c.) for hydrogen, and even of oxygen for hydrogen, without change of type, are very frequent; and it is this kind of substitution, so adverse to the electro-chemical theory, which is included in the theory of substitutions of Laurent. Those more usual substitutions, where one body is replaced by another of similar electric character, may be viewed as so many examples of the doctrines of equivalents, the replacing body being equivalent to that for which it is substituted, on the electro-chemical theory.

Adopting, then, the views of Laurent, we are compelled to admit that the electro-chemical theory fails when applied to cases of substitution of chlorine for hydrogen, \&c., where the type remains unaltered. This is clearly the case in acetic and chloracetic acids; and Hofmann has shown that in certain basic organic compounds, hydrogen may be replaced by chlorine, bromine, iodine, and even nitrous acid, while the new compound retains the basic type and characters.

The recent discoveries of Wurtz and Hofmann have taught us, moreover, that the hydrogen of ammonia may be partially or entirely replaced, by certain compound groups, or compound radicals, such as methyle and ethyle already mentioned, and others which belong to the same series, but stand higher in the scale, while the resulting compounds retain the basic character; and those which stand nearest to ammonia have so great a 
resemblance to it, as to have been frequently taken for ammonia, and thus overlooked, when occurring accidentally. These very remarkable and important discoveries have already thrown much light on the constitution of organic bases, and will be more fully explained when treating of that class of compounds. Here we shall content ourselves with giving a short tabular view of some of these substitution-compounds, as they are called, which, being derived from ammonia, retain the type and chemical relations of that compound.

\begin{tabular}{|c|c|c|}
\hline Ammonia & 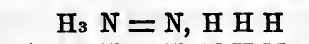 & 1 eq. of Hydrogen. \\
\hline Methylamine & $\mathrm{C}_{2} \mathrm{H}_{5} \mathrm{~N}=\mathrm{N}, \mathrm{H} \mathrm{H} \mathrm{Me}$ & $\mathrm{Me}=\mathrm{C}_{2} \mathrm{H}_{3}$ \\
\hline Ethylamine & $\mathrm{C}_{4} \mathrm{H}_{7} \mathrm{~N}=\mathrm{N}, \mathrm{H} \mathrm{H} \mathrm{Ae}$ & $\mathrm{Ae}=\mathrm{C}_{4} \mathrm{H}_{5}$ \\
\hline Propylamine & Св $\mathrm{H}_{9} \mathrm{~N}=\mathrm{N}$, H H Pr & $\mathrm{Pr}=\mathrm{C}_{8} \mathrm{H}_{7}$ \\
\hline Butylamine & $\mathrm{C}_{8} \mathrm{H}_{12} \mathrm{~N}=\mathrm{N}, \mathrm{H} \mathrm{H} \mathrm{Bu}$ & $\mathrm{Bu}=\mathrm{C}_{8} \mathrm{H}_{9}$ \\
\hline Valeramine & $\mathrm{C}_{10} \mathrm{H}_{13} \mathrm{~N}=\mathrm{N}$, H H Va & $=\mathrm{C}_{10} \mathrm{H}_{12}$ \\
\hline Caprotyl & e $\mathrm{C}_{12} \mathrm{H}$ & Cp \\
\hline
\end{tabular}

The above bases are known already. The two first are gases pungent and soluble in water, like ammonia. The rest are oils, becoming less volatile as we rise in the number of eqs. of carbon and hydrogen, but all basic. It will be seen that they correspond, in the radicals or groups which replace 1 eq. of hydrogen, with the series of radicals of which methyle and ethyle are the two first, and through these, with that other series of acid radicals, derived from them, of which Formyle and Acetyle are the two first, and which may be traced in the table of volatile acids given at page 10 .

But this is not all. For not only may one of the three eqs. of hydrogen in ammonia be thus replaced by the above six radicals, and no doubt by others of the same series, higher in the series, in which, as will be seen, $\mathrm{C}_{2} \mathrm{H}_{2}$ is added at every step; but two or even all three eqs. of hydrogen may be replaced, either by the same or by two or three different groups or radicals of this series. This has been demonstrated by Hofmann, who has produced a large number of new bases in this way. Thus we have

Ethylomethylamine

Ethylopropylamine

Methylopropylamine

Ethylobutylamine

Dimethylamine

Diethylamine

Triethylamine

Ethylomethylopropylamine
$\mathrm{C}_{6} \mathrm{H}_{\ominus} \mathrm{N}=\mathrm{N}, \mathrm{H} \mathrm{Me} \mathrm{Ae}$

$\mathrm{C}_{10} \mathrm{H}_{13} \mathrm{~N}=\mathrm{N}, \mathrm{H} \mathrm{Ae} \mathrm{Pr}$

$\mathrm{C}_{8} \mathrm{H}_{11} \mathrm{~N}=\mathrm{N}$, H Me Pr

$\mathrm{C}_{12} \mathrm{H}_{15} \mathrm{~N}=\mathrm{N}, \mathrm{H} \mathrm{Ae} \mathrm{Bu}$

$\mathrm{C}_{4} \mathrm{H}_{7} \mathrm{~N}=\mathrm{N}, \mathrm{H} \mathrm{Me} \mathrm{Me}$

$\mathrm{C}_{8} \mathrm{H}_{11} \mathrm{~N}=\mathrm{N}, \mathrm{H} \mathrm{Ae} \mathrm{Ae}$

$\mathrm{C}_{12} \mathrm{H}_{15} \mathrm{~N}=\mathrm{N}, \mathrm{Ae} \mathrm{Ae} \mathrm{Ae}$

$\mathrm{C}_{12} \mathrm{H}_{15} \mathrm{~N}=\mathrm{N}, \mathrm{MeAe} \mathrm{Pr}$

And other groups, belonging to different series, may be employed, in 
the same way, to replace the hydrogen of ammonia. Thus, aniline, $\mathrm{C}_{12} \mathrm{H}_{7} \mathrm{~N}$, is ammonia, in which 1 eq. of hydrogen is replaced by phenyle $=\mathrm{C}_{12} \mathrm{H}_{5}$, instead of methyle or ethyle. And phenyle is one of a series of which several others are known. Hofmann has produced bases, in which the hydrogen is replaced, partly by phenyle, partly by ethyle, \&c., as will be explained in the section on Artificial Organic Bases. He has also shown that the replacing group or radical may itself be changed by substitution, and yet continue to replace hydrogen, without destroying the type of the compound. Thus, in aniline, a base in which one of the three equivalents of hydrogen in ammonia is replaced by phenyle, one, two, or even three, of the equivalents of hydrogen in the phenyle itself may be replaced by chlorine, bromine, or iodine, and one has been replaced by nitrous acid.

When we can thus trace groups, such as those of methyle, ethyle, phenyle, and others, replacing hydrogen, we have not only examples of substitution without destroying the type of the compound, but we have also an additional argument for the existence of organic compound radicals; these groups being, in fact, such radicals.

We have now seen that substitution without change of type occurs both in acids, in bases, and, as is proved by the cases of aldehyde and chloral, in neutral compounds also.

Here, then, is a fact of very general occurrence, which not only proves that the electro-chemical theory of combination is inapplicable, at all events in many cases, but also tends to establish a very different view: namely, that the electric character of an element is no permanent or essential property; and that the type or character, or general properties of a compound, depend, not only on the nature, but very much on the arrangement of its elementary atoms : on the way in which they are grouped to form the compound molecule.

In the section on Isomorphism (Inorganic Chemistry), the principle was laid down that the crystalline form of certain types of salts, such as the alum-type, as well as many other properties of the compounds having those types, were the result of the similar grouping of analogous elements. We now see that, according to the law of substitution, as deduced from numerous careful observations, similarity of properties, or identity of type, is the result of similarity of grouping, even of elements not analogous, nay, of elements electrically opposed to each other. It is evident, therefore, that the arrangement of the elementary molecules to form the compound molecule is the circumstance on which depend almost exclusively the properties of the compound, or, in other words, the character of the type. 
Substitution, as has been already stated, may be either complete or partial. In chloracetic acid, and in chloral, the substitution of chlorine for the hydrogen of the radical acetyle $\mathrm{C}_{4} \mathrm{H}_{3}$ is complete. But when ether, $\left(\mathrm{C}_{4} \mathrm{H}_{5} \mathrm{O}\right)$ is acted on by chlorine, the substitution takes place by successive steps, one equivalent of hydrogen being replaced at a time, after the oxygen has also been replaced by chlorine. Thus we have, first-

\begin{tabular}{|c|c|}
\hline $\begin{array}{l}\text { ther or oxide of eth } \\
\text { then, chloride of et }\end{array}$ & $\begin{array}{ll}\left(\mathrm{C}_{4} \mathrm{H}_{5}\right)+ \\
\mathrm{C}_{4} & \left.\mathrm{H}_{5}\right)+\end{array}$ \\
\hline then, successively, & $\mathrm{Cl}_{\mathrm{Cl}}^{\mathrm{H}_{4}}+$ \\
\hline & $\mathrm{C}_{4}\left\{\begin{array}{l}\mathrm{H}_{3} \\
\mathrm{Cl}_{2}\end{array}+\right.$ \\
\hline $8+17$ & $\begin{array}{l}\mathrm{C}_{4}\left\{\begin{array}{l}\mathrm{H}_{2} \\
\mathrm{Cl}_{3}\end{array}\right. \\
\mathrm{C}_{4}\left\{\begin{array}{l}\mathrm{II} \\
\mathrm{Cl}_{4}\end{array}\right. \\
\left(\mathrm{C}_{4} \mathrm{Cl}_{5}\right)\end{array}$ \\
\hline
\end{tabular}

We thus obtain the series of compounds here indicated, in which the hydrogen is gradually replaced by chlorine, until at last we obtain the compound $\left(\mathrm{C}_{4} \mathrm{Cl}_{5}\right)+\mathrm{Cl}=\mathrm{C}_{4} \mathrm{Cl}_{6}=2 \mathrm{C}_{2} \mathrm{Cl}_{3}$, which is the perchloride of carbon. Most of these compounds have actually been obtained; and it is obvious that they may all be referred to one type. Such a series is called a series of mechanical examples of the type in question, or rather of subtypes retaining the original character although modified.

In some cases, hydrogen has been replaced partly by chlorine and partly by bromine, as we have seen it may be partly by methyle and partly by ethyle, \&c. Laurent has described two compounds derived from naphthaline by substitution, the empirical formula for both of which is the following:- $\mathrm{C}_{20} \mathrm{H}_{4} \mathrm{Cl}_{3} \mathrm{Br}$. Yet the properties of these two compounds are quite distinct, and it is certain that this difference of properties must depend on a difference in the arrangement of the elements. Now, in the formation of these two compounds we have a very beautiful proof of the existence of a difference in the arrangement: for one is produced when chlorine acts on the compound called by Laurent bronaphtése, $\mathrm{C}_{20} \mathrm{H}_{6} \mathrm{Br}_{2}$; while the other is formed when bromine is made to act on chlonaphtise, $\mathrm{C}_{20} \mathrm{H}_{5} \mathrm{Cl}_{3}$. It is obvious that in the first case 2 eq. of hydrogen and 1 eq. of bromine are replaced by chlorine; while in the second, 1 eq. of hydrogen is replaced by bromine. While, therefore, all four compounds may be deduced from the type $\mathrm{C}_{20} \mathrm{H}_{8}$, and while both the bromine and chlorine play the part of hydrogen, it is impossible to doubt that each of the 8 eq. of hydrogen has its special place in the 
compound molecule of the type, and that, in the two empirically identical formulæ above given, the 1 eq. of bromine does not replace the same eq. of hydrogen, and consequently the bromine occupies in the two compounds different positions. The same remark applies to the 3 eq. of chlorine.

We may illustrate our meaning as follows :-Let $\mathrm{C}_{20} \mathrm{H}_{8}$ be the type, and let each of the eqs. of hydrogen have a number attached indicating its place in the typical molecule. We shall then have

$$
\mathrm{C}_{20}\left\{\begin{array}{llll}
\mathrm{H}_{2} & \mathrm{H}_{2} & \mathrm{H}_{3} & \mathrm{H}_{4} \\
\mathrm{H}_{5} & \mathrm{H}_{6} & \mathrm{H}_{7} & \mathrm{H}_{8}
\end{array}\right\}
$$

Now, if we represent the two compounds above mentioned in the following manner, we can then conceive the influence of arrangement on the properties of two compounds having the same empirical formula. The first may be

$$
\mathrm{C}_{20}\left\{\begin{array}{llll}
\mathrm{H}_{1} & \mathrm{H}_{2} & \mathrm{H}_{3} & \mathrm{H}_{4} \\
\mathrm{Cl}_{5} & \mathrm{Cl}_{6} & \mathrm{Cl}_{7} & \mathrm{Br}_{8}
\end{array}\right\}
$$

and the second may be

$$
\mathrm{C}_{20}\left\{\begin{array}{llll}
\mathrm{H}_{1} & \mathrm{H}_{2} & \mathrm{H}_{3} & \mathrm{Br}_{4} \\
\mathrm{Cl}_{5} & \mathrm{Cl}_{6} & \mathrm{Cl}_{7} & \mathrm{H}_{8}
\end{array}\right\}
$$

It is only on this principle that we can explain the facts observed by Laurent; and it is easy to see that the above type, $\mathrm{C}_{20} \mathrm{H}_{8}$, will admit of innumerable modifications; for even the subtype, $\mathrm{C}_{20} \mathrm{H}_{4 .} \mathrm{Cl}_{3} \mathrm{Br}$, is capable of yielding many more than the two above given; and the change of 1 eq. produces a new subtype, equally fertile in new forms.

In fact, Laurent has actually obtained, as will be shown further on, a very large number of what we have called subtypes from the types $\mathrm{C}_{20} \mathrm{H}_{8}$, which is naphthaline, and has established the same law in reference to many other types.

The same law, no doubt, applies to the bases derived from ammonia by substitution, many of which, as may be seen in the tables above given, are isomeric, or have the same empirical formula, from a difference in the replacing groups, as diethylamine and methylopropylamine, both $=\mathrm{C}_{8} \mathrm{H}_{11} \mathrm{~N}$. But we may also have cases where the replacing group is the same, but occupies a different place in the compound molecule. Perhaps aniline and picoline, both $\mathrm{C}_{12} \mathrm{H}_{7} \mathrm{~N}$, are examples of this. Hofmann, however, has lately found that no difference of properties could be observed in two bases, formed in different ways, and in which this principle might have acted. They were identical.

The preceding observations will, I trust, be found sufficient to convey a clear general notion of the prevalent doctrines of chemical types and of substitution, as applied to Organic Chemistry. It 
is evident that the facts of substitution are of great interest, and not only enable us to classify and to remember numerous complex facts, but promise to throw much light on the molecular constitution of compound bodies. But while admitting the importance of the doctrine of substitution, it is necessary to point out that the French chemists appear to have gone too far in assuming that this doctrine is incompatible with that of compound radicals. It is certain that some compound radicals exist, and it is no less certain that by assuming their existence in other cases, we very much facilitate the study of many important series of compounds. Let us, therefore, avail ourselves of that which appears well founded in both doctrines, since there appears to be no reason why the one should be opposed to the other.

\section{HOMOLOGOUS SERIES OF ORGANIC COMPOUNDS.}

We have already had oecasion to speak of certain remarkable series of compounds, such as that of the volatile oily acids, given at p. 10. The recent progress of Organic Chemistry has shown, that such series frequently occur; nay, Gerhardt has founded on them his arrangement of organic compounds. But although it is more than probable that all may one day admit of being thus classified, at present little or nothing would be gained by adopting that arrangement, which cannot yet be carried out. Yet the properties of such groups or series as are obviously homologous are so strikingly analogous, that it is necessary for the student of Organic Chemistry to be acquainted with those which are best known.

A series of compounds is called homologous, when each member of the series differs from the others, either by a certain number of equivalents of carbon and hydrogen, or by a multiple of it, and when the properties of these different compounds, although entirely analogous, differ in degree, and that in proportion to the amount of carbon and hydrogen.

To take an example, pyroxilic spirit, alcohol, and oil of potato spirit are three bodies belonging to a homologous series. They are homologous to each other. Let us examine their formulæ.
Methylic Alcohol is
Alcohol is
$\mathrm{C}_{2} \mathrm{H}_{4} \mathrm{O}_{2}$
Propylic Alcohol is
Butylic Alcohol is
$\mathrm{C}_{4} \mathrm{H}_{6} \mathrm{O}_{2}=\mathrm{C}_{2} \mathrm{H}_{4} \mathrm{O}_{2}+\mathrm{C}_{2} \mathrm{H}_{2}$
Ce $\mathrm{H}_{8} \mathrm{O}_{2}=\mathrm{C}_{2} \mathrm{H}_{4} \mathrm{O}_{2}+2\left(\mathrm{C}_{2} \mathrm{H}_{2}\right)$
Amylic Alcohol is
$\mathrm{C}_{8} \mathrm{H}_{10} \mathrm{O}_{2}=\mathrm{C}_{2} \mathrm{H}_{4} \mathrm{O}_{2}+3\left(\mathrm{C}_{2} \mathrm{H}_{2}\right)$
$\mathrm{C}_{10} \mathrm{H}_{12} \mathrm{O}_{2}=\mathrm{C}_{2} \mathrm{H}_{4} \mathrm{O}_{2}+4\left(\mathrm{C}_{2} \mathrm{H}_{2}\right)$

In this table it is seen that methylic alcohol and alcohol differ by $\mathrm{C}_{2} \mathrm{H}_{2}$; and that, at each step higher in the scale, $\mathrm{C}_{2} \mathrm{H}_{2}$ is 
added, so that methylic and amylic alcohols differ by $\mathrm{C}_{8} \mathrm{H}_{8}=$ 4 times $\mathrm{C}_{2} \mathrm{H}_{2}$. The third and fourth compounds, propylic and butylic alcohols, have only very recently been discovered, but their existence had long been predicted.

Now, every known member of this series has analogous properties : they are neutral, volatile bodies, liquid in the lower part of the scale, above given, but solid and fusible in the higher parts where the carbon and hydrogen are in larger proportion. They are, in short, alcohols. They all yield ethers, bearing the same relation to the respective alcohols as ether does to alcohol. They all, when oxidised, yield volatile acids, bearing to the respective alcohols the same relation as formic acid to pyroxilic spirit, acetic acid to alcohol, and valerianic acid to oil of potato. In these alcohols, the boiling-point rises a certain number of degrees, about $34^{\circ} \mathrm{F}$., for each $\mathrm{C}_{2} \mathrm{H}_{2}$ added. The melting-point of these alcohols rises at each step in the series, but the precise amount of this rise has not yet been ascertained. The fact is well seen in these members of the series which, from being high in it, are solid at ordinary or at low temperatures. Common alcohol is not yet known in the solid state, but has been reduced to a thick oily state by a very intense cold. The density of the vapours of these alcohols also varies according to a fixed law, rising as the amount of $\mathrm{C}_{2} \mathrm{H}_{2}$, which is added at each step, increases. The density of the vapours varies also according to a fixed law.

It will be observed, that the ethers and the volatile acids, derived from these alcohols, form also homologous series, differing from each other by $\mathrm{C}_{2} \mathrm{H}_{2}$, or by multiples of $\mathrm{C}_{2} \mathrm{H}_{2}$. And every derivative of pyroxilic spirit, which is the beginning or starting-point of the series, is the starting-point of a parallel series, in which the composition varies as in the first; while the properties, analogous in each series, yet vary in each in degree, according to a fixed law, as the amount of $\mathrm{C}_{2} \mathrm{H}_{2}$ increases or diminishes.

When a homologous series is once ascertained in a few of its members, the ultimate discovery of the rest may be safely predicted. In the series of alcohols, few are yet known; besides the five above mentioned, not more than five or six others, much higher in the scale, some of which are of very recent discovery. The same is true of the ethers. A few years ago, only two of the acids of the series derived from alcohols by oxidation were recognised, namely, formic and acetic acids. But that series of acids is now complete up to 40 eqs. of carbon, inclusive; and three are known beyond that point, the highest in the scale yet discovered containing 60 eqs. of carbon.

It will be observed that the few homologous series above 
alluded to, have been discovered simply by the analysis of the substances, and are therefore expressions of fact. It is certain that the alcohols, the ethers, and the acids of these different series differ, in each series, by $\mathrm{C}_{2} \mathrm{H}_{2}$, or multiples of it; and that in each series the properties of all the members are analogous, yet differ in degree. We cannot doubt, therefore, that the difference in properties, so regular and graduated in each homologous series, depends on the equally regular and graduated addition of $\mathrm{C}_{2} \mathrm{H}_{2}$ to the preceding molecule.

Now, if we arrange, in a horizontal line, the lowest members of the different series-of the alcohol series, for example, the ether series, or the acid series-we have only to add, under each, in a descending vertical column, $\mathrm{C}_{2} \mathrm{H}_{2}$ at each step, to obtain the composition of the whole possible members of each series up to $40,50,60$, or $100 \mathrm{eqs}$. of carbon. And the principle of such a table is so simple, that any one who has the first horizontal line can fill it up at once.

If we take the case of alcohols, we have seen that ethers and volatile acids are derived from them. But there are many more derivations, each of which finds its place in the horizontal line, and is the beginning of a vertical or homologous series. Of course, the compounds in any horizontal column, being derived from one, such as alcohol, are not analogous to each other, but totally different in properties. But the whole members of any vertical series, in our supposed table, are so analogous as to be included under the same name, and under one general formula.

Now alcohol is not the true, theoretical foundation of the horizontal series derived from it, nor is ether, but rather the supposed radical of both, in this instance ethyle, and in the case of pyroxilie spirit, which is lowest in the scale, methyle.

The first or fundamental horizontal column will contain, therefore, methyle and its derivatives; the next, ethyle and its derivatives; standing immediately under the former, with which they are, in each vertical column, homologous. It is evident that the number of vertical or homologous series depends on that of the derivatives in the first horizontal one.

Now the derivatives of methyle are very numerous, and each is the starting-point of a series of homologous compounds. In the table, here appended, we have selected a few of the most important, extending to seventeen members or derivatives in the horizontal line, and consequently seventeen homologous series in the vertical columns.

At the head of each vertical column we have placed the general formula under which its members are included. These general 
formulæ would alone suffice to enable any one to construct the whole table.

In these general formulæ, $n$ is used to signify any given number of equivalents, and in this table, as well as generally, it stands for an even number, such as $2,4,6,8,10$, \&c. If the number of equivalents of hydrogen exceed that of those of carbon by 1 , then we write $\mathrm{Cn}_{n} \mathrm{H}_{\mathrm{n}}$, if it fall short by 1 , we write $\mathrm{C}_{n} \mathrm{H}_{n}-1$, and so on. If we wish to deduce, from the general formula, that of any particular compound, we require only to know how many equivalents of carbon that compound contains. Thus the general formula for the alcohols of this series is $\mathrm{Cn}_{n} \mathrm{H}_{n}{ }_{1} \mathrm{O}, \mathrm{HO}$ or $\mathrm{Cn}_{n} \mathrm{H}_{n}+{ }_{2} \mathrm{O}_{2}$. What is the special formula of amylic alcohol? This alcohol yields, when oxidised, valerianio acid, in which there are $10 \mathrm{eqs}$. of carbon. Now the number of eqs. of carbon is always the same in an alcohol and in the acid which it yields; we have therefore 10 eqs. of carbon. But if, in this case, $n=10, n+1$ must be $=11$, and the formula for amylic alcohol becomes $\mathrm{C}_{10} \mathrm{H}_{11} \mathrm{O}, \mathrm{HO}$, or $\mathrm{C}_{10} \mathrm{H}_{12} \mathrm{O}_{2}$.

In like manner, if we could obtain margaric alcohol, we know, since margaric acid contains 34 eqs. of carbon, that the formula of margaric alcohol must be $\mathrm{C}_{34} \mathrm{H}_{35} \mathrm{O}$, $\mathrm{HO}$, or $\mathrm{C}_{34} \mathrm{H}_{36} \mathrm{O}_{2}$.

Column 1. General formula, $\mathrm{C} n \mathrm{H}_{\mathrm{n}}+1$. This formula represents the radicals of the methyle and ethyle series. Of these, five or six have been obtained, by Frankland and by Kolbe, in the separate form, and the names of these are given in the table.

Column 2. General formula, $\mathrm{Cn} \mathrm{H}_{n}+1, \mathrm{H}$, or $\mathrm{C}_{n} \mathrm{H}^{n}+2$. This series is homologous with marsh gas, the hyduret of methyle, $\mathrm{C}_{2} \mathrm{H}_{3}, \mathrm{H}$ or $\mathrm{C}_{2} \mathrm{H}_{4}$.

Those lowest in the series are permanent gases, those above them highly volatile liquids ; those still higher oily liquids, and higher still they are fatty and waxy fusible solids. This is true also of the radicals in the preceding paragraph. It is probable that one of the bodies described as paraffine, as well as some paraffine oils, belong to this series of hydurets.

Column 3. General formula, $\mathrm{Cn}_{\mathrm{n}} \mathrm{H}^{\mathrm{n}}+1,0$. This represents the oxides of the preceding radicals, or ethers, of which ether is the type. A few only of these are known in a separate form, and a few more in combination. With acids, especially organic acids, they yield compound ethers, such as oxalio or benzoio ether. Those containing much carbon are solid.

Column 4. $\mathrm{Cn}_{\mathrm{n}} \mathrm{H}_{\mathrm{1}}, \mathrm{O}+\mathrm{HO}$, the hydrated oxides of the radicals, or alcohols, of which alcohol is the type. Like the ethers, they are volatile liquids in the first part of the scale, the boiling-point of which rises with the amount of carbon, and fusible and volatile solids when the carbon exceeds a certain 
amount; the melting and boiling-points of which, and the density of their vapours, vary according to the same law, rising a certain number of degrees for each addition of $\mathrm{C}_{2} \mathrm{H}_{2}$. The rise in the boiling-point is about $19^{\circ} \mathrm{C}$., or $34^{\circ} \mathrm{F}$., for each addition of $\mathrm{C}_{2} \mathrm{H}^{2}$.

Column 5. $\mathrm{C}_{\mathrm{n}} \mathrm{H}_{\mathrm{n}}-1$, the derived radicals, formed from those of the ethyle series by oxidation or dehydrogenation, of which acetyle is the type. They are, as yet, only known in combination. We therefore pass on to

Column 6. General formula, $\mathrm{C}_{n} \mathrm{H}_{n}-1, \mathrm{H}$ or $\left(\mathrm{C}_{n} \mathrm{H}_{n}\right)_{2}$. This series of hydurets is homologous with methylene, $\mathrm{C}_{2} \mathrm{H}, \mathrm{H}$ or $\mathrm{C}_{2} \mathrm{H}_{2}$, and ethylene or olefiant gas, $\mathrm{C}_{4} \mathrm{H}_{3}, \mathrm{H}$, or $\mathrm{C}_{4} \mathrm{H}_{4}$. The former is the hyduret of formyle, the latter the hyduret of acetyle.

These compounds much resemble those of column 2, but differ from them in being more readily acted on by chlorine, even in the dark. They are, like the others, gases when low in the scale; then, as we rise in the series, highly volatile liquids, less volatile and oily liquids, fatty and finally waxy solids. Various forms of paraffine and of paraffine oil belong to this series.

Column 7. $\mathrm{CnH}_{n-1}, \mathrm{O}+\mathrm{HO}$, the aldehydes, or hydrated oxides of the immediately preceding radicals, of which aldehyde is the type. Several are known, and they are characterised by reducing the salts of silver, so as to yield a mirror-like deposit of metal; while they pass, by oxidation, into volatile acids, which are the hydrated teroxides of the same radicals, as aldehyde does into acetic acid. It has recently been shown, by Bertagnini and others, that the aldehydes form crystallisable compounds with bisulphite of potash, soda, or ammonia. By this means we can detect the presence of aldehydes in mixtures, and even purify them from other volatile liquids.

Column 8. $\mathrm{C} \mathrm{H}_{n}-1 \mathrm{O}_{3}, \mathrm{HO}$, or $\left(\mathrm{Cn}_{n}\right)^{2} \mathrm{O}_{4}$. These are the volatile acids already alluded to, and yield the most complete series of homologous compounds yet known. They are almost all found in nature, and all but the two first are oily or fatty acids, the boiling-point of which rises about $19^{\circ} \mathrm{C}$., or $34^{\circ} \mathrm{F}$., for every step in the scale. The most abundant and important fatty aoids, such as stearic and margaric acids, cocinic and palmitic acids, and the acids of wax, belong to this series.

Column 9. $\mathrm{Cn}_{n-1} \mathrm{O}_{3}, \mathrm{NH}_{4} \mathrm{O}$, the compounds of the preceding acids with ammonia. These are here introduced, because, when deprived of 4 eqs. of water, they yield the next series.

Columns 10 and 11. $\mathrm{Cn}_{n} \mathrm{H}_{n} \mathrm{~N}$, or $\mathrm{C} \mathrm{H}_{4}+{ }_{1}, \mathrm{C}_{2} \mathrm{~N}$. The first of these formulæ, which are isomeric, namely, column 10, represents what are called nitryles, that is, compounds of nitrogen with the radical of the acid of the ammonia salt, from which the 
nitryle is derived. Thus acetate of ammonia, deprived of 4 eqs. of water, that is, of all its oxygen, and 4 eqs. of hydrogen, yields acetonitryle,

$$
\mathrm{C}_{4} \mathrm{H}_{3} \mathrm{O}_{3}, \mathrm{NH}_{4} \mathrm{O}=4 \mathrm{HO}_{4} \mathrm{C}_{4} \mathrm{H}_{3} \mathrm{~N} \text {. }
$$

The second general formula, column 11, represents the cyanide, not of the radical of the acid from which it has been formed, but of the original radical of the ethyle series, one step lower in the scale than the acid. Such a cyanide must be isomeric with the nitryle of the acid radical. For acetonitryle, $\mathrm{C}_{4} \mathrm{H}_{3} \mathrm{~N}$, is the same, empirically, as the cyanide of methyle, $\mathrm{C}_{2} \mathrm{H}_{3}+\mathrm{C}_{2} \mathrm{~N}$.

In point of fact, some of the nitryles really are such cyanides, and it is possible that all may prove to be so. But it is also possible that both compounds may exist, and therefore we have given both formulæ.

Column 12. $\mathrm{Cn}_{n} \mathrm{H}_{n} \mathrm{O}_{3}+\mathrm{Cn}_{n} \mathrm{H}_{n}+1 \mathrm{O}$, or $\mathrm{Cn} \mathrm{H}_{n} \mathrm{O}_{4}$, the compound ethers formed by the combination of one of the volatile aoids with the ether from which it is derived; of these, acetate of oxide of ethyle, or acetic ether, is the type. If we represent them by the general formula, $\mathrm{Cn}_{n} \mathrm{O}_{4}$, the amount of $\mathrm{C}$ and $\mathrm{H}$ is always exactly double of that in the hydrated acid itself. Acetic acid is $\mathrm{C}_{4} \mathrm{H}_{4} \mathrm{O}_{4}$, and acetic ether is $\mathrm{C}_{8} \mathrm{H}_{8} \mathrm{O}_{4}$. Many of this series are known and occur in nature. It must be observed, that each ether can combine not only with the acid related to it, which has the same number of eqs. of carbon, as, oxide of ethyle with acetio acid; oxide of amyle with valerianic acid, \&c. ; but also each ether can combine with all the acids, and each rcid with all the ethers of the two series. It is only, however, when the acid and ether which combine have the same number of eqs. of carbon that the relations above pointed out holds good. Thus, acetic acid combines with amylic ether, and gives the compound $\mathrm{C}_{10} \mathrm{H}_{11} \mathrm{O}, \mathrm{C}_{4} \mathrm{H}_{3} \mathrm{O}_{3}=\mathrm{C}_{14} \mathrm{H}_{14} \mathrm{O}_{4}$, which still comes under the general formula, but in which the carbon and hydrogen are not double of those in the acid or ether as in the case of acetic ether above given. The compound of acetic acid with amylic ether just named occurs in nature and can be made artificially. It gives the peculiar and agreeable flavour to the pear, while acetic ether contributes in part to that of the pineapple, along with another compound of the same class, butyric ether, that is, butyric acid combined with oxide of ethyle, $\mathrm{C}_{4} \mathrm{H}_{5}$ $\mathrm{O}, \mathrm{C}_{8} \mathrm{H}_{7} \mathrm{O}_{3}=\mathrm{Cl}_{12} \mathrm{H}_{12} \mathrm{O}_{4}$.

Column 13. $\mathrm{C}_{\mathrm{n}} \mathrm{H}_{\mathrm{n}}+_{1} \mathrm{Cl}$, the ehlorides of the radicals of the ethylic series. Chloride of ethyle is the type. Of course similar series exist for the bromides and iodides. Several of them are known, and have been used with advantage in recent researches. 
Column 14. $\mathrm{C}_{\mathrm{n}} \mathrm{H}_{\mathrm{n}}+1, \mathrm{~S}$. The sulphurets of the same radicals. These are volatile fetid liquids, so far as known.

Column 15. $\mathrm{C}_{\mathrm{n}} \mathrm{H}_{\mathrm{n}}+1, \mathrm{~s}, \mathrm{HS}$. The preceding, combined with sulphuretted hydrogen, or, as they may be called, mercaptans. The type is mercaptan, of the ethylio series, $\mathrm{C}_{4} \mathrm{H}_{5} \mathrm{~S}$, HS. These also are horribly fetid. Only a few are known as yet.

Columns 16 and 17. $\mathrm{C}_{n} \mathrm{H}_{n}+1, \mathrm{~N} \mathrm{H}_{2}$, the amides of the radicals of the ethylic series, which are isomeric, if not identical, with a very remarkable series of bases, already alluded to as derived from ammonia by the replacement of 1 eq. of its hydrogen by 1 eq. of one of these radicals. The last column, 17, contains the empirical formula of these hases, $\mathrm{Cn}_{n} \mathrm{H}_{n}+3, \mathrm{~N}$. Methylamine, $\mathrm{C}_{2} \mathrm{H}_{5} \mathrm{~N}$, or ethylamine, or $\mathrm{C}_{4} \mathrm{H}_{7} \mathrm{~N}$, is the type. Already six or seven of these bases are known.

More columns might be added, almost ad infinitum, as for example, the bisulphates of the oxides of the ethylic radicals, which act as acids, and of which sulphovinic acid is the type. But the above will suffice to explain the doctrine of homologous compounds. It will be seen that when we know the formula of a compound, belonging to this series, we can predict its general characters, physical and chemical. The former depend on its place in the vertical column to which it belongs, the latter on the general formula appropriate to that column. For example, if $\mathrm{I}$ analyse a substance, and find its formula to be $\mathrm{C}_{10} \mathrm{H}_{12} \mathrm{O}_{2}$, or $\mathrm{C}_{10} \mathrm{H}_{11} \mathrm{O}$, HO, I observe that it belongs to the general formula $\mathrm{C}_{n} \mathrm{H}_{n}+{ }_{1} \mathrm{O}$, HO, that of the alcohols. If it be $\mathrm{C}_{20} \mathrm{H}_{20} \mathrm{O}_{2}$, or $\mathrm{C}_{20} \mathrm{H}_{19} \mathrm{O}, \mathrm{HO}$, it belongs to the aldehydes, $\mathrm{CnH}_{n} \mathrm{O}_{2}$; if $\mathrm{C}_{8} \mathrm{H}_{11}$ $\mathrm{N}$, it belongs to the bases, in column 15 , and in each case $I$ know what its chemical characters will be. But the first, although an alcohol, will be less volatile than common alcohol; the second far less volatile than aldehyde, perhaps a solid at the ordinary temperature; and the third, although a base, analogous to ammonia, will be a liquid at ordinary temperature, less volatile than methylamine and ethylamine, which indeed are gases at ordinary temperatures, but easily condensed.

If the body analysed be of the formula $\mathrm{Cn}_{n} \mathrm{H}_{n} \mathrm{O}_{4}$ it must be either acid or neutral. If acid, it will be volatile and oily, or fatty, provided it contain six or more eqs. of carbon. If neutral, it will be a compound ether, see column 12, containing a volatile acid, united to an oxide of one of the radicals in column 1.

It is more than probable, that every organic compound belongs to a homologous (or vertical) series, if we could only ascertain the other members of it. And it can hardly be doubted that series will be discovered, in which the common difference or addition at each step is not $\mathrm{C}_{2} \mathrm{H}_{2}$, but some other group. Yet $\mathrm{C}_{2} \mathrm{H}_{2}$ 
appears to be the commonest, for there is another series, or rather set of homologous series, quite distinct from that here explained, in which the starting-points are very different, but the common difference is still $\mathrm{C}_{2} \mathrm{H}_{2}$. In this there is an acid series, of which the starting-point is benzoic acid, $\mathrm{C}_{14} \mathrm{H}_{6} \mathrm{O}_{4}$, and a basic series, of which the starting-point is aniline, $\mathrm{C}_{22} \mathrm{H}_{7}$ : $\mathrm{N}$; besides various other series, all of which may be included under the name of the benzoic series (just as the table above given may be called the ethylic series), in which $\mathrm{C}_{2} \mathrm{H}_{2}$ is also added at each step.

It will not escape the reader, that the foundation of the ethylic series is laid in inorganic chemistry. Thus methyle, $\mathrm{C}_{2} \mathrm{H}_{3}$, the first radical, if $\mathrm{C}_{2} \mathrm{H}_{2}$ be taken from it, yields $\mathrm{H}$, that is, hydrogen; its ether and its alcohol, minus $\mathrm{C}_{2} \mathrm{H}_{2}$, alike yield $\mathrm{HO}$, or water. Its acid, formic acid, $\mathrm{C}_{2} \mathrm{H}_{2} \mathrm{O}_{4}$, yields $\mathrm{O}_{4}$, oxygen. Formonitryle, $\mathrm{C}_{2} \mathrm{HN}$, is hydrocyanic acid, or cyanide of hydrogen; chloride of methyle, $\mathrm{C}_{2} \mathrm{H}_{3} \mathrm{Cl}$, minus $\mathrm{C}_{2} \mathrm{H}_{2}$, is hydrochloric acid; sulphuret of methyle, minus $\mathrm{C}_{2} \mathrm{H}_{2}$, is sulphuretted hydrogen, and lastly, methylamine, $\mathrm{C}_{2} \mathrm{H}_{5} \mathrm{~N}$, minus $\mathrm{C}_{2} \mathrm{H}_{2}$, is ammonia, the very type of the methylamine series of bases. Indeed, it is not easy, without analysis, to distinguish methylamine from ammonia.

Hydrogen then, is the true starting-point of the ethylic series of radicals, and ammonia of the ethylic series of volatile bases.

Such is the doctrine as exemplified in one well-marked instance of homologous compounds, a doctrine which has already done much, and is destined to do much more, in simplifying our knowledge, and enabling us to classify the immediate facts of organic chemistry.

At the meeting of the British Association in Ipswich, M. Dumas drew the attention of the Association to the extraordinary analogy between homologous groups of organic compounds and certain small groups of elementary bodies, which have long been observed. The best example is that of chlorine, bromine, and iodine, which differ from each other precisely as do three contiguous homologous compounds in the table; that is, in properties. Thus chlorine is an easily condensible gas, bromine a volatile liquid, iodine a volatile solid. In affinity, bromine is intermediate between chlorine and iodine, as it is in atomic weight. This is obviously true also of the organic group, say, for example, of methyle, ethyle, and propyle, or of formic, acetic, and propylic acids, or of methylamine, ethylamine, and propylamine. M. Dumas is said by some journals to have drawn the conclusion that, since the equivalent of bromine is the mean between those of chlorine and iodine, therefore bromine is made up of half an equivalent of chlorine 
and half an equivalent of iodine. But it is probable that he only indicated the possibility of this.

Now, although M. Dumas may have been the first to point out strongly in public the remarkable analogy between the elementary groups, chlorine, bromine, iodine, potassium, sodium, lithium, \&c., \&c., and homologous organic groups, such as methylamine, ethylamine, and propylamine, yet it must not be supposed that this had escaped the notice of chemists. The author of the present work has for several years pointed it out in his lectures; nxy, before the homologous organic groups were known, the late Dr. Turner and other teachers, including the author, constantly drew the attention to the analogy between chlorine, bromine, and iodine, as furnishing an argument for their being truly compound. But they took a different view from that of M. Dumas, and one still more analogous to what is known of homologous bodies; namely, that chlorine, bromine, and iodine had the same base or substratum, with a different addition in each case. And since the discovery of homologous bodies, it has been regarded as highly probable by the author, and mentioned as such in his lectures, that there is here also a common difference, corresponding to $\mathrm{C}_{2} \mathrm{H}_{2}$ in the ethylic series, the addition of which, in increasing quantity, converts chlorine into bromine, and bromine into iodine. It is evidently unnecessary to suppose any difference in the quality of what is added, because we see, in the organic compounds, that a difference in its quantity is amply sufficient to alter the density, the fusing and boiling points, \&c., \&c.

This has been explained fully, because M. Dumas has been said to have founded on these facts and hypotheses, an opinion that we may hope, on the principle laid down by him, to transmute one element into another. Thus we may hope to fuse one atom of chlorine and one atom of iodine into two atoms of bromine, \&c. And of course, if we could discover any metal homologous with gold, as sodium is with potassium and lithium, we might expect to convert that metal into gold.

The idea on which recent attempts at transmutation have been founded is different. It is derived from the remarkable changes produced in both simple and compound bodies by a new arrangement or grouping of the ultimate atoms into molecules of various size. Charcoal, graphite, and diamond are all carbon, yet how different! The two last crystallise in forms geometrically distinct. So does sulphur, which also exists in a third or amorphous state. Phosphorus is at one time a white, translucent, fusible, and easily combustible body; at another, a red substance, not easily fusible, and hardly combustible, save at a red 
heat, when it passes into the first state. And compounds, although of the same composition in 100 parts, may vary ad infinitum in character. Hence it was supposed that, by a new arrangement of the same atoms, a different, even although elementary substance, might be obtained; and Dr. Brown stated that he had thus converted carbon into silicon. Supposing this to be confirmed, it is easy to imagine lead or iron converted into gold. Even charcoal and diamond, although chemically identical, are physically far more different than are lead and gold, and, in short, transmutation was clearly conceivable.

But although this be the case, yet we must not forget that, until Dr. Brown's experiments be confirmed, the most widely differing forms of elementary bodies-of carbon, for example-all agree in some essential chemical properties; for example, in yielding carbonic acid when burned in oxygen gas. Whereas carbon and silicon, even in the alleged results of Dr. Brown, cannot be made to yield the same products. We have no proof, as yet, that the same atoms, differently grouped, can yield a body which has entirely new and permanent chemical properties. This is conceivable, and, strictly speaking, possible; but we have, as yet, no proof of it. And the same is true of M. Dumas's suggestion ; it is conceivable, but we have no proof that it can be done with truly elementary bodies, or even with such as are elements to us.

It is, we conceive, much more probable, that chlorine, bromine, and iodine are really homologous compounds, and not elements; and if we can discover their common difference (their $\mathrm{C}_{2} \mathrm{H}_{2}$ so to speak), we may hope to transmute them into each other. And so of all other similar groups.

But, admitting the possibility of a transmutation, it must not be forgotten, that it is almost an absurdity to suppose it easy or practicable under ordinary ciroumstances, or that it should ever become so. It must require some very great and unusual force; for if it were otherwise, if one element could easily pass into another, external nature must come to an end. Nay, if carbon could readily become silicon, how could our bodies or the vegetable world exist? In them nearly two-thirds of the weight is carbon; and if that were changed, even in part, to silicon, or to any other element, what must be the result ?

It has always appeared to us, that those bodies whioh are best known, and which resist most powerfully all our efforts to decompose or transmute them, must have, in order that nature should exist, a degree of permanence attached to them, such, that they become virtually unalterable elements, otherwise affinity could no longer act to produce the results we see. 
Even if our elements, therefore, be compound, or otherwise transmutable, it is evident that we are not intended to have the power of decomposing or transmuting them. Such is the doctrine we have for many years taught in our lectures.

Having now explained the doctrine of homologous compounds, and its bearing on the peculiar groups of elementary bodies, as well as on the idea of transmutation, we shall reserve further details till we come to describe the substances themselves which constitute the homologous series, such as the alcohols, ethers, aldehydes, volatile acids, and volatile bases.

\section{THE DECOMPOSITIONS AND METAMORPHOSES OF ORGANIC COMPOUNDS.}

Organic compounds, whether actual organised tissues, unorganised products of animal and vegetable life, or new substances artificially produced, are generally characterised by a great proneness to undergo decomposition or metamorphoses. This instability is especially marked in those compounds which contain nitrogen, not only because, containing four elements (in most cases), they are exposed to more numerous causes of change than such bodies as contain only three (carbon, hydrogen, and oxygen), but also because nitrogen is, in its relations to those three elements, the most remarkable element we know. According to the circumstances under which a change is induced nitrogen may separate uncombined, as in the ultimate analysis of organic substances, by combustion with oxide of copper or chromate of lead ; or it may combine with oxygen, yielding nitric acid, as in nitrification ; or with carbon, yielding the compound radical cyanogen, as when nitrogenised organic matter is ignited with carbonate of potash ; or with hydrogen, yielding ammonia, as when nitrogenised organic matter is ignited with hydrated caustio alkalies.

It is easy to see, therefore, that while all organic matter is prone to change, this is especially the case with nitrogenised compounds. In fact, many of these compounds cannot be kept more than a few hours without the commencement of decomposition or metamorphosis, in the shape of putrefaction or fermentation. This kind of metamorphosis will be separately considered hereafter; in the meantime it is important to observe, that when such a compound has entered into a state of decomposition, it acquires the properties of a ferment, that is, it is capable of inducing a similar metamorphosis in another compound, if placed in contact with it.

The true explanation of this fact appears to be, that the 
particles or molecules of the exciting body or ferment, being in a condition of change, and therefore in motion, communicate to the molecules of the body placed in contact with them an amount of motion sufficient to destroy the balance of the existing affinities; which in organic compounds is easily done, the chemical equilibrium being very unstable; and thus gives rise to a new play of affinities and the production of new compounds, as when sugar by contact with yeast is resolved into alcohol and carbonic acid.

But in addition to metamorphoses of the kind just alluded to, which, in the various ferments at least, commence spontaneously, air (at all events, at the commencement), moisture, and a certain temperature being the usual conditions, organic substances undergo very well-marked decompositions when exposed to the action of heat and of some powerful re-agents; and it seems advisable here to give also a general account of such decompositions, as they admit of being classified under certain heads or rules generally applicable.

We shall here, therefore, briefly describe the changes produced on organic compounds : 1 , by oxidation; 2 , by the action of acids; 3 , by the action of bases ; 4 , by the action of heat in close vessels, or the destructive distillation; and 5 , by the contact of ferments.

Oxidation : a. direct.-The direct oxidation of organio compounds takes two distinct forms. The first is the familiar one of combustion, in which the action of the atmospheric oxygen is aided by a high temperature. The results differ according to the supply of oxygen. If there be an excess of air, or of oxygen, from any source, the whole of the carbon and hydrogen is converted into carbonic acid and water, which, along with uncombined nitrogen, are the ultimate products of the action of oxygen on organic matters. But if the supply of air be deficient, the hydrogen, from its superior attraction for oxygen, is oxidised in preference to the carbon, which is deposited as smoke, soot, or lampblack.

The second form of direct oxidation is that which is commonly called decay, but which Liebig proposes to call Eremacausis (i.e., slow combustion), and which takes place when organic matter is exposed to air and moisture. In dry air it does not occur.

One of the most familiar examples of this kind of oxidation is that decay of wood by which it is slowly converted into a dark brown powder-ulmine. In this process, as De Saussure has shown, the wood absorbs oxygen, and produces an equal volume of carbonic acid along with water, and the residue-ulmine. 
As, in combustion, the oxygen combines by preference with hydrogen, so also in eremacausis there is every reason to believe that the absorbed oxygen combines with the hydrogen of the wood, and that an equivalent quantity of oxygen, also derived from the wood, is given off in the form of carbonio acid. Now, since wood may be represented as composed of carbon and the elements of water, and as water and carbonic acid are two of the products of eremacausis, it may be supposed that the water was ready formed in the wood, and that the absorbed oxygen had combined with the carbon. But it has been shown that, in presence of hydrogen, carbon does not at the ordinary temperature combine with oxygen, for which its affinity is less powerful ; and besides, in the decay of wood, the proportion of carbon in the residue (the ulmine) is constantly greater than in the wood. Thus oak wood, $\mathrm{C}_{36} \mathrm{H}_{22} \mathrm{O}_{22}$, yields in one stage of decay, ulmine, the composition of which agrees with the formula $\mathrm{C}_{35} \mathrm{H}_{20} \mathrm{O}_{20}$; and in a more advanced stage, an ulmine of the formula $\mathrm{C}_{34} \mathrm{H}_{18} \mathrm{O}_{18}$. Here we see that for every 2 eqs. of hydrogen oxidised by the air, 1 eq. of carbon and 2 eqs. of oxygen have been separated; so that the per-centage of carbon in the residue constantly increases, and the final result of eremacausis would be a residue of carbon; were it not that, as the proportion of carbon in the ulmine increases, its affinity for the other elements, strengthened by its mass, becomes too powerful to be overcome by the oxygen of the air without the aid of heat. It has been suggested, however, by Liebig, that the process of eremacausis may occur under such circumstances as to leave at last a residue of pure carbon; and the occurrence of anthracite, which is nearly pure carbon, shows that such a result is possible. The conditions necessary for this are still unknown; but if we suppose one of these conditions to be a semi-fluid state of the matter undergoing decay, and if we imagine the process to go on at a very slow rate indeed, the carbon thus eliminated might assume the crystalline form, and thus the diamond might be produced. This is a mere suggestion; but it has more probability in its favour than any other theory of the production of the diamond.

Other examples of eremacausis are, the acetification of alcohol, and the process of nitrification in which ammonia undergoes eremacausis. These, as well as other instances, will be considered in their proper place.

Eremacausis is greatly promoted by heat and by the presence of alkalies. It is, on the contrary, arrested or retarded by cold, dryness, acids, and many salts, such as corrosive sublimate, which has been used to prevent the decay of wood.

There is one circumstance connected with eremacausis, or 
decay, as above described, which is worthy of special attention. It is, that a substance, in a state of eremacausis, if placed in contact with another, which is capable of undergoing this change, speedily causes the latter to enter into the same condition of change. This effect of contact may be compared, in one sense, to that of a body in combustion, which sets fire to other bodies; but in ordinary combustion the high temperature plays an important part, while in eremacausis the effect appears to be due to the communication of motion, from the particles of the decaying body to those of the other substance, which motion, as in the case of fermentation, overturns the existing balance of affinities, unstable as it is in organic compounds, and gives rise to the formation of new products.

The process of eremacausis, or slow oxidation in the atmosphere, is one of very great practical importance, inasmuch as, by this means, the elements of dead organic matter are made to assume those forms-namely, the forms of carbonic acid, water, and ammonia-in which they are capable of contributing to the nutrition of new or growing vegetables.

It has been rendered highly probable by recent investigations, that eremacausis is always commenced, not by the ordinary oxygen of the air, but by the remarkable substance named ozone, which is now believed to be an allotropia and highly active form of oxygen. Some observers state that one form of ozone contains hydrogen, in which case, that form of it is most probably a teroxide of hydrogen ; but it is certain that in one form, at least, of what is called ozone, oxygen alone is to be found.

Ozone is formed instantaneously by lightning, so that its peculiar smell is at once perceived at a great distance from the spot where the discharge occurs. It is evident, therefore, that the air must always contain ozone. But the ozone, from its very intense action on all oxidisable matter, is consumed as fast as it is formed, so that it never accumulates. Indeed, although traces of ozone may generally be detected during, or very soon after, thunderstorms, by its turning blue, paper covered with iodide of potassium and starch, and, although it may even be detected at other times occasionally, yet it is easy to see that oxygen in so active a state must be removed from the air by the various dead organic matters in contact with it, and the vapours of organic origin diffused through it.

When finely divided phosphorus is exposed to the air, it begins at once to undergo oxidisation. Now we know that when phosphorus is placed at the bottom of a bottle, ozone is soon formed in the air about it. It is this ozone, I conceive, which oxidises the phosphorus, at least at first. The temperature gradually rises, 
the eremacausis is thereby accelerated, a higher temperature is the result, and thus action and reaction go on, till, at about $100^{\circ}$, generally in the space of one or two minutes, the phosphorus bursts into flame. I have seen this happen with solid sticks of phosphorus, half covered with water, and it seems probable that ozone is here the agent in producing the oxidation. Such also is, I believe, the origin of the spontaneous combustion of charcoal powder, of cotton or tow steeped in oil, and similar matters.

Now the same agency produces analogous results on dead organic matter, which is no sooner exposed to air and moisture than oxidation begins, due, as I conceive, to ozone, either already present in the air, or produced at the surface of the organic matter, as at the surface of phosphorus.

When the oxidation or eremacausis is once established, the temperature rises a little, and then, at a certain temperature, the process is probably carried on by the ordinary oxygen, for ozone is destroyed by a moderate degree of heat.

Bearing all this in mind, we can easily see how important the presence of ozone in the air is; we can see how it contributes to convert into the gaseous food of plants, carbonic acid, water, and ammonia, all dead organic matter; while, at the same time, we perceive that if ozone were not thus consumed as fast as it is formed, and if it could accumulate in the air, it would, from the intensity of its oxidising action, destroy or burn, not only all dead organic matter, but all living plants and animals.

Nothing can better show how very limited our knowledge of nature still is, and how much we have yet to learn, even concerning the commonest and apparently the most simple natural operations going on around us, than the fact that the important agency of ozone, nay, its very existence, were but a short time ago utterly unknown. And yet it is quite conceivable that, without the formation of ozone, and its continual consumption in the decay of dead organic matter, the whole system of animal and vegetable life might be brought to an end, simply by the accumulation of dead animals and plants. For if the commencement only of their oxidation or eremacausis depend on ozone, the absence of that body would put an end to eremacausis, and the result would be, that the supply of carbonic acid and ammonia, which the air should furnish to plants, would fail, and vegetation would languish. This, as a necessary consequence, would put an end to animal life also.

A peculiar species of eremacausis is observed in the case of the simultaneous action of oxygen and ammonia on certain colourless vegetable products, which, absorbing these gases greedily, are thus converted into nitrogenised compounds of very fine blue or 
purple colours. Of this we have examples in oroine, erythrine, and phloridzine; and there is good reason to attribute the formation of indigo, from a juice devoid of blue colour, to an action of this kind, since both oxygen and ammonia appear to be necessary to its production. The transformation of alloxantine or of uramile into murexide also depends on the simultaneous action of ammonia and oxygen.

b. Indirect Oxidation. The indirect oxidation of organje compounds may be effected in a variety of ways, as, for example, by nitric acid, the action of which we shall presently describe along with that of other acids; by hyponitrous acid; by certain salts, as by permanganate of potash, which converts sugar, for example, into oxalic acid ; or by the employment of a mixture of bichromate of potash and diluted sulphuric acid, by which means salicine may be made to yield the hyduret of salicyle (oil of spiræa); by the action of sulphuric acid, and of peroxide of manganese, as when alcohol is converted into aldehyde; or, finally, by the com-. bined action of heat and hydrated alkalies, as when indigo, heated with potash, gives rise to anthranilic acid, hydrogen being given off; or acetates, heated with baryta, yield marsh gas and carbonates. The use of fusion with caustic potash as an oxidising agent is an important means of obtaining a less perfect oxidation than is the result of some of the other processes ; and it has lately led to some very interesting discoveries in animal chemistry.

Chlorine is also used as an oxidising agent. It acts in virtue of its very strong attraction for hydrogen, decomposing water, when the nascent oxygen acts powerfully on the organic compound. A mixture of chlorate of potash with hydrochloric acid is also employed, and is a useful means of oxidation.

As a general rule, oxidation has the effect of causing the formation of compounds less complex than those organic bodies which yield them. Thus sugar, starch, and many other substances, when oxidised by nitric acid, yield oxalic acid. Now sugar and starch contain at least 12 eqs. of carbon, while oxalic acid contains not more than 4 eqs., possibly only 2 eqs. of carbon.

2. Action of Acids on Organic Compounds. This action is very various; the two acids most frequently employed are the nitric and sulphuric acids, and, as might be expected, the former acts more as an oxidising agent than the latter.

When sugar, for example, is heated with nitric acid, the latter loses oxygen, for nitrous acid is given off in enormous quantity ; while the elements of the sugar, by the action of the oxygen are made to combine so as to produce compounds of less complex radicals than that of sugar probably is. Among the products are water, carbonic acid, oxalic acid, and saccharic acid, besides 
others not yet investigated; but the three first sufficiently show the tendency of oxidation to promote the formation of less complex radicals.

When nitric acid acts on organic matters, there is generally found one acid, if not more, among the products, and in this way a large number of acids have been discovered. Examples of this are, mucic acid from gum; indigotic and nitropicric acids from indigo; margaric acid from stearic acid; suberic and succinic acids from oily acids, besides many others. It frequently happens that compounds, whether acid or neutral, formed by the action of nitric acid on organic matter, contain hyponitrous acid as a constituent, substituted for its equivalent of hydrogen. This is the case with nitrobenzide from benzine, and with nitronaphthalase, and a whole series of compounds discovered by Laurent in his study of the action of nitric acid on naphthaline. The nitropicric acid also contains nitrous acid in place of hydrogen. Some organic bases, as morphia and brucia, strike a deep red colour with nitric acid.

The action of hyponitrous acid on some organio compounds is very remarkable. A neutral or acid amide, acted on by this acid, is so oxidised as to yield the acid from which it was derived. Thus, oxamide, $\mathrm{C}_{2} \mathrm{O}_{2}, \mathrm{NH}_{2}$ acted on by hyponitrous acid, $\mathrm{N}_{3}$, yields oxalic acid, water and nitrogen, $\mathrm{C}_{2} \mathrm{O}_{2}, \mathrm{~N} \mathrm{H}_{2}+$ $\mathrm{NO}_{3}=\mathrm{C}_{2} \mathrm{O}_{3}, 2 \mathrm{HO}+\mathrm{N}_{2}$. By this means, when an amide is known, but not the corresponding acid, the latter may be obtained, as glycolic acid was from glycocine, its amide, and benzoglycolic acid from hippuric acid, which is its acid amide.

Basic amides, such as ethylamine (ethylamide), when thus oxidised, sield the corresponding alcohol, or else the ether of that alcohol combined with another portion of hyponitrous acid. In the latter case, the alcohol can be obtained from the hyponitrite of the ether. Thus, ethylamine $\mathrm{C}_{4} \mathrm{H}_{7} \mathrm{~N}+2 \mathrm{~N} \mathrm{O}_{3}=\left(\mathrm{C}_{4} \mathrm{H}_{5} \mathrm{O}, \mathrm{N} \mathrm{O}_{3}\right)$ $+2 \mathrm{H} \mathrm{O}+\mathrm{N}_{2}$; and $\left(\mathrm{C}_{4} \mathrm{H}_{5} \mathrm{O}, \mathrm{N} \mathrm{O}_{3}\right)+\mathrm{K} \mathrm{O}, \mathrm{HO}=\left(\mathrm{C}_{4} \mathrm{H}_{5} \mathrm{O}, \mathrm{HO}\right)$ $+\left(\mathrm{K} \mathrm{O}_{1} \mathrm{~N} \mathrm{O}_{3}\right)$. Hence from any volatile base we can obtain the corresponding alcohol.

When sulphuric acid is made to act on organic compounds, it chars a considerable proportion of them by virtue of its attraction for oxygen and hydrogen in the form of water. But in many cases it produces very different effects. Thus, by boiling with sulphuric acid and water, starch and lignine are converted into grape sugar. In other cases, the sulphuric acid seems to lose so much oxygen as to produce hyposulphuric acid, which enters into combination with an organic compound, forming a new acid, as when sulphuric acid acts on naphthaline, and forms sulphonaphthalic acid : or on benzoic acid, forming hyposulphobenzoic acid; 
or on alcohol under certain circumstances, when an acid is produced containing the elements of hyposulphuric acid and of a carbo-hydrogen. In other cases, the sulphuric acid combines unchanged with the organic compound, as in sulphovinic acid, which is a bisulphate of oxide of ethyle; sulphomethylic acid, and others.

Many organic compounds, heated with excess of sulphuric acid, are entirely decomposed, yielding water which combines with the acid, and other products which are disengaged. Thus oxalic acid is resolved into water, carbonic acid, and carbonic oxide : formic acid into water and carbonic oxide; alcohol into water, olefiant gas, and other products.

Several organic compounds are dissolved by sulphuric acid with the production of a fine red or purple colour. Salicine strikes a red colour with the acid, and cedriret, one of the constituents of tar, dissolves in it with a deep blue colour, as does also naphthalase.

Phosphoric acid may be employed in some cases to remove water from organic compounds, as it does not char them. Like sulphuric acid, it forms with oxide of ethyle an acid salt, known as phosphovinic aeid.

Hydrochloric acid and its congeners have no very extensive action on organic substances. With alcohol, hydrochloric acid gas yields chloride of ethyle; and a current of this gas, passed through an alcoholic solution of a fatty acid, gives rise to the compound of the fatty acid with oxide of ethyle, which would otherwise be obtained with difficulty. With oil of turpentine, oil of lemons, and some other essential oils composed of carbon and hydrogen, hydrochloric acid gas combines, forming olids resembling camphor. Pyroxanthine, a substance contained in tar, dissolves in strong hydrochloric acid with a fine and deep purple colour.

3. Action of Bases on Organic Compounds. Hydrated bases unite, of course, with organic acids; and when heated with neutral substances, they generally give rise to the formation of acids, such as acetic and oxalic acids, or even carbonic acid, oxygen being taken from the water of the base, and hydrogen being disengaged, or (if the organic body contain nitrogen), hydrogen and ammonia. This property of hydrated bases is employed as a means of converting all the nitrogen of organic compounds into ammonia, and in this form determining its quantity.

The presence of bases greatly promotes the absorption of atmospheric oxygen by organic substances. This is the reason why alkalies assist eremacausis. The same effect is very con- 
spicuous in the change which the salts of gallic acid (and some other acids) undergo when exposed to the air. A solution of an alkaline gallate absorbs oxygen very rapidly, and becomes very dark in colour, being oxidised in a far shorter time than if the acid had been uncombined. This is still better seen in pyrogallic acid, which indeed furnishes a means of determining directly the proportion of oxygen in gaseous mixtures.

4. Action of Heat on Organic Compounds in close Vessels. Thi action is known under the name of the destructive distillation. It must be considered as a combustion with a very limited supply of oxygen, that, namely, afforded by the substance itself. A very great variety of compounds is produced, many of them very interesting and useful. The destructive distillation may be considered as it affects substances containing nitrogen, and substances devoid of that element. Many products are common to both cases, but many also are confined to one case, especially to that of nitrogenised substances.

The destructive distillation of non-nitrogenised substances has been chiefly studied in the case of wood, which when heated in close vessels, yields a great variety of products : some, binary compounds, such as paraffine, naphthaline, eupione, water, carbonic oxide, carbonic acid, marsh gas, and olefiant gas : others ternary, such as acetic acid, $\mathrm{C}_{4} \mathrm{H}^{3} \mathrm{O}_{3}$; hydrated oxide of methyle or pyroxylic spirit $\left(\mathrm{C}_{2} \mathrm{H}_{3}\right) \mathrm{O}, \mathrm{HO}$; lignone, xylite, mesite, and other volatile etherial liquids, composed of the same elements as pyroxylic spirit, and very similar to it in properties; creosote; picamar; capnomore; cedriret; pittacal, and pyroxanthine, besides many other others, not yet properly investigated.

When fatty or resinous bodies are subjected to the destructive distillation, there are obtained, besides other compounds, two solid carbo-hydrogens : chrysene, $\mathrm{C}_{3} \mathrm{H}$, and pyrene, $\mathrm{C}_{10} \mathrm{H}_{2}$; which also occur among the products of the distillation of coal.

This latter distillation may serve as an example, the best known, of the action of heat on nitrogenised organic bodies; for coal contains a certain although small proportion of nitrogen. The products, besides creosote, paraffine, naphthaline, and probably several others of those obtained from wood, include much ammonia, hydrocyanio acid; some peculiar non-nitrogenised acids, as carbolic acid, $\mathrm{C}_{12} \mathrm{H}_{5} \mathrm{O}, \mathrm{HO}$ (a remarkable compound, baving an odour resembling that of creosote, and yielding, when subjected to various re-agents, an extensive series of new compounds [Runge, Laurent]); rosolic and brunolic acids; and three very remarkable series of nitrogenised bases, containing no oxygen, namely, the series of aniline, $\mathrm{C}_{12} \mathrm{H}_{7} \mathrm{~N}$, of which several are known, homologous with aniline and higher in the scale; that of picoline, 
$\mathrm{C}_{12} \mathrm{H}_{7} \mathrm{~N}$, isomeric with the first, but differing from them in properties, and that of leukoline or chinoline, $\mathrm{C}_{18} \mathrm{Hs} \mathrm{N}$, of which the other members are not yet studied. Besides these, there is a fourth series of bases, one of which was named by Runge pyrrol, and which Anderson has to some extent described as the pyrrol (pyroline) series of bases. These different bases are more abundant in the products of the distillation of the more highly nitrogenised animal matters mentioned in the next paragraph. Finally, paranaphthaline, or anthracene, $\mathrm{C}_{30} \mathrm{H}_{12}$, and coal-tar naphtha, which is used as a solvent for caoutchouc.

The distillation of animal matter, such as hoofs, horns, or bones, yields analogous results, but is characterised by the very large amount of ammonia which is obtained, animal matter being richer in nitrogen than coal is. This ammonia appears as carbonate, which salt is thus manufactured, and hence was, and occasionally still is, called salt of hartshorn. The oil from animal matter also contains several of the new bases of the ethylamine series, as well as of the series of aniline, and of those of picoline, pyroline, and loukoline, according to the experiments of Dr. Anderson.

Many organic acids, when heated in close vessels to a certain temperature, short of the destructive distillation, undergo a remarkable decomposition; carbonic acid is given off, and there remains a new acid, which is called a pyrogenous acid, or pyroacid. Thus meconic acid, at a certain temperature, yields carbonic acid and komenic acid; while komenic acid, if heated in its turn, yields carbonic acid and pyromeconic acid. We have also pyromucic, pyrotartaric, or pyroracemic and pyrocitric acids; citric acid yielding three pyro-acids, aconitic (equisetic), itaconic and citraconic acids, and malic acid also yielding two, maleic and paramaleic or fumaric acids.

From the above statements, it is obvious, that the action of heat on organic compounds gives rise to a very large number of important products, of which only the most remarkable have been named. All will be described in their proper places.

5. Action of Ferments on Organic Compounds. Of this action the best known and most important example is the fermentation of sugar, by which it is resolved into alcohol and carbonic acid.

The circumstances under which this metamorphosis occurs are these: the sugar must be dissolved, the solution must have a certain temperature, and there must bo present a ferment, such as yeast or some analogous body. In the juice of the grape a ferment, the fibrinous or caseous constituent of the juice, is naturally present; and Gay Lussac showed that the contact of atmospherical air was necessary to commence the fermentation, 
but that this contact with the atmosphere might be only for a very brief period, after which air was no longer necessary.

The ferment is always a very complex compound of nitrogen, such as fibrine and the like. Now, besides the natural tendency of all complex compounds to undergo decomposition readily, we must remember that nitrogen is a most peculiar element, which stands by itself, and whose position on the electro-chemical scale is near the middle, between the metalloids on the one hand, and the metals on the other. It has strong attractions both for oxygen and for hydrogen, yet combines with neither directly. For the metalloids in general, such as chlorine, \&c., sulphur, \&c., it has no great affinity, and this is true also of its relation to the metals. Yet it can combine with most of them, indirectly, and these compounds are usually so unstable as to be explosive. Its attraction for carbon is considerable, and carbon is another element which stands alone, without congeners. Now, such being the properties of nitrogen, it is evident that complex nitrogenised substances must, from containing this element, whose attractions are equally powerful in opposite directions, be peculiarly liable to change.

It is now doubtful whether, as Gay Lussac supposed, the admission of air to the ferment acts by oxidation in producing the change. For if the air be admitted through a tube loosely filled with cotton or asbestus, to a vessel containing grape juice or infusion of malt, no change occurs. The reason is supposed to be that the air contains the germs of minute fungi, which find in the saccharine juice a pabulum, and are there developed, and that this is the immediate origin of that decomposition of the fibrine, \&c., which enables it to excite fermentation in the sugar.

It is well known that ferment or yeast is in a great measure made up of a cellular plant, and it would appear that the germs of this plant abound in the atmosphere, and when they find a solution of fibrine, \&c., grow in it, and in so doing excite a decomposition in the fibrine, which being communicated to the sugar, causes it to ferment. The change thus induced in the fibrine has been supposed to be putrefaction, or a state analogous to it, because putrescent organic matter is found to act as a ferment on sugar. But it must be remembered that such putrescent matter, if exposed to air, will contain the germs alluded to.

It has been found, however, that certain solutions, such as infusion of meat, and also moist flesh, will putrify and become ferments even when the air admitted has passed through a tube filled with cotton or asbestus. It would seem, then, that two causes operate in producing that change in fibrine and similar bodies, by which they become ferments, although the fibrine in grape juice and infusion of malt does not undergo this change in 
air which has been filtered. In whatever way the change is produced, the fibrine- acquires the property of causing sugar to ferment under the circumstances and conditions already mentioned.

Berzelius and others conceive that the ferment acts by contact in some way not very clearly defined, by catalysis, as it is called, as they conceive sulphuric acid to do in the formation of ether from alcohol. But Liebig has proved that in this latter case the acid first combines with ether (oxide of ethyle) forming sulphovinic acid (bisulphate of oxide of ethyle), and that this compound, at a temperature rather higher than that at which it is formed, is decomposed into hydrated sulphuric acid and ether which distils over. The same chemist has pointed out many other instances of the effect of contact, even in inorganio chemistry; such as the action of oxide of silver on peroxide of hydrogen, where the former compound, by contact with the latter, not only decomposes it, causing oxygen to be rapidly given off, but is itself decomposed, losing all its oxygen; the solution in nitric acid of an alloy of platinum and silver, while platinum alone is insoluble in that acid; or the action of carbonate of silver on certain organic acids, which cause a disengagement of carbonic acid, this disengagement being attended with a partial reduction of the oxide of silver.

These, and many other more familiar cases, particularly those where a compound is decomposed with detonation in consequence of a slight touch, or gentle friction, a moderate elevation of temperature, or the contact of another substance, $(e . g$. chloride of nitrogen with oil) all tend, according to Liebig, to establish the doctrine that in certain compounds the balance of affinities is unstable, and therefore easily overturned, either by chemical or by mechanical influences.

The compounds which are capable of fermentation or any similar metamorphosis, are all of them bodies in which such an unstable equilibrium exists ; they are all, in point of fact, easily decomposed by many different agencies, such as heat, acids, bases, oxygen, chlorine, \&c. Now, we can offer no other explanation of these facts of fermentation than this, that when a body in a state of progressive change, the particles of which are consequently in a state of motion, is placed in contact with another body, the particles of which are in a state of unstable equilibrium, the amount of motion mechanically communicated to the particles of the latter from those of the former, is sufficient to overturn the existing equilibrium, and, by the formation of a new compound, to establish a new equilibrium more stable under the given circumstances. 
There is nothing unphilosophical in this explanation, and it is to be considered as the best theory of fermentation yet attempted. According to the view of Liebig, a ferment is merely a compound in a state "of decomposition, capable of setting in motion, and thereby bringing also into a state of decomposition, the particles of another compound, the existence of which depends on a nice balance of affinities.

On the other hand, the view adopted by Berzelius, according to which fermentation, and all the other phenomena of chemical change produced by contact are the results of a peculiar unknown force, the catalytic force, coming into action when certain bodies are placed in contact, appears unphilosophical, as in the first place, assuming the existence of a new force where known forces would suffice to explain the facts; and, secondly, as furnishing no real explanation, but merely acknowledging, indirectly, our inability to offer any such explanation. When we ascribe an effect to catalysis, we are only saying, in other words, that we cannot account for it; catalysis is thus merely a convenient term for all that we do not understand. And to the use of the word in this sense, namely, as a name for the agent which produces certain effects, the agent itself being unknown, there would be no objection, were it not that catalysis has been employed to account for phenomena not only different from each other, but actually of an opposite kind. For example, platinum, in causing the combination of oxygen and hydrogen, is said to act catalytically, and the action of oxide of manganese, or oxide of silver in decomposing peroxide of hydrogen, that is, in causing the separation of oxygen and hydrogen, is also called catalytic. This example proves how loosely the word has been employed, and how vague are the views which have led to its introduction.

A variety of important and interesting processes come under the head of actions caused by ferments; the production of alcohol from sugar, of oil of bitter almonds from amygdaline, and of lactic and butyric acids from cane sugar or sugar of milk, are all examples of this ; and in each of these cases the ferment is peculiar. In the case of sugar it is yeast, or gluten undergoing eremacausis and putrefaction; in the case of amygdaline it is emulsine, a peculiar modification of albumen; and in the case of cane sugar or sugar of milk, when converted into lactic or butyric acid, it is caseine, the nitrogenised constituent of the milk.

The access of air is required at first, as above explained, to cause a change in the gluten, fibrine, or other similar body, which then entering into eremacausis, or if air be excluded, into putrefaction, are capable of acting as ferments.

- In the actions induced by ferments, we are to distinguish those 
in which some external element or elements are added to those of the compound, which cases resemble ordinary decompositions, from those in which the elements of the decomposed body merely transpose themselves, producing new compounds. The latter are properly and strictly termed metamorphoses. Fermentations, in which oxygen is absorbed, are examples of eremacausis, and it has already been mentioned that a body in a state of eremacausis acts on other bodies as an excitant of the same change, that is, as a ferment.

Indeed, most ferments, whether they induce eremacausis, or a more pure metamorphosis in other bodies, are themselves in a state of eremacausis, at all events in the commencement of the change.

The subject of fermentation and ferments will be hereafter more especially considered, in connection with fermentescible compounds: here the subject is merely treated in a general way.

Putrefaction, under ordinary circumstances, partakes largely of eremacausis, and differs from the ordinary kind only in the offensive odour of some of the products, chiefly compounds of sulphur and phosphorus, as sulphuretted and phosphuretted hydrogen. When air is excluded, putrefaction goes on, provided moisture be present, and it is then a metamorphosis, giving rise, in the case of vegetable matter putrefying under water, or in the strata of mines, to gaseous products, such as marsh gas and olefiant gas, constituting with air the fire-damp, and carbonic acid, which is the choke-damp, of the miner.

Animal matter, in a state of putrefaction, as putrid flesh, blood, cheese, or wine, acts as a ferment, and is capable of causing the metamorphosis of sugar into alcohol and carbonic acid, as well as of inducing eremacausis, and also propagating a putrefactive decomposition analogous to its own. Thus, it is well known that fresh cheese, if inoculated with decaying cheese, soon passes into decay, spreading from the seat of the inoculation.

We shall, hereafter, see that it is probable that some poisons and miasmata act as ferments on the blood. The singular sausage poison of Wurtemburg is animal matter in a peculiar state of decay, and does not contain any poisonous compound, only a poisonous state or condition; and the same principle may hereafter be found to furnish the true explanation of contagions.

Fermentation, putrefaction, and eremacausis are all promoted by the same circumstances, and arrested by the same influences. Antiseptics are substances, which, by combining with the ferment, or a part of it, or even with the body to be fermented, 
prevent the continuance either of the decomposition in the ferment, or of the fermentation itself. Corrosive sublimate and arsenic, which are powerful antiseptics, combine with animal matter, and form with it stable compounds; creosote combines energetically with albumen, \&c., \&c.

In fermentation, properly so called, the elements of the ferment take no chemical share in the metamorphosis of the body acted on by the mechanical agency above explained. That body is resolved into two or more new compounds of less complex radicals. The elements of water may or may not take part in the change; when they do, as in the case of sugar, the weight of the products, in this case alcohol and carbonic acid, is equal to that of the sugar, plus a certain weight of water. As, when water is passed in vapour over carbon at a white heat, the carbon is shared between the oxygen and hydrogen, producing carbonic acid (or oxide), and carburetted hydrogen, so in the metamorphosis of sugar, and other analogous cases, we have on the one hand an oxidised compound, (in the case of sugar represented by carbonic acid,) and on the other a compound in which part of the carbon is united to all the hydrogen (in the case of sugar, the alcohol). Similar results are obtained when alcohol or acetic acid are metamorphosed by heat, and this may be viewed as a general character of the metamorphosis of non-nitrogenised bodies; namely, that the carbon is divided between the oxygen and hydrogen.

In putrefaction, again, the ferment plays a chemical part in the change, and two or more compounds, the ferment and the putrefying body or bodies, combine to give rise to new compounds, with or without the elements of water. Putrefaction is generally the characteristic transformation of nitrogenised compounds, and the very great tendency of such compounds to undergo transformations is well illustrated by the spontaneous metamorphosis of a solution of cyanogen in water. Such a solution contains the four principal elements of organic bodies; and its transformations may be said to be the only case of putrefaction which has been as yet carefully studied.

The solution after a time becomes brown and turbid, and deposits a dark matter, containing ammonia, united to a compound formed of the elements of cyanogen along with those of water. This matter being insoluble, undergoes no further ohange. Such a compound might arise from the reaction between 2 eq. cyanogen, and 4 eq. water; thus $2 \mathrm{C}_{2} \mathrm{~N}+4 \mathrm{HO}=\mathrm{NH}_{3}+\left(\mathrm{C}_{4} \mathrm{HNO}_{4}\right)$; according to some, the brown matter contains no ammonia, and is $\mathrm{C}_{4} \mathrm{~N}_{2} \mathrm{HO}=2 \mathrm{C}_{2} \mathrm{~N}+\mathrm{HO}$.

Another change is that in which water is decomposed, each 
of its elements uniting with cyanogen, and producing cyanic and hydrocyanic acids; thus: $2 \mathrm{HO}+2 \mathrm{C}_{2} \mathrm{~N}=\left(\mathrm{C}_{2} \mathrm{~N}, \mathrm{O}+\right.$ $\mathrm{HO})+\mathrm{H}, \mathrm{C}_{2} \mathrm{~N}$.

Another metamorphosis gives rise to oxalic acid and ammonia. In this case, 1 eq. cyanogen acts on 3 eq. of water; thus : $\mathrm{C}_{2} \mathrm{~N}+$ $3 \mathrm{HO}=\mathrm{N} \mathrm{H}_{3}+\mathrm{C}_{2} \mathrm{O}_{3}$.

But cyanic acid cannot exist in contact with water and other acids : it is instantly metamorphosed into bicarbonate of ammonia ; thus: $\mathrm{C}_{2} \mathrm{NO}+3 \mathrm{HO}=\mathrm{NH}_{3}+2 \mathrm{CO}_{2}$.

Towards the end of the process, when ammonia has become predominant, the cyanic acid produced undergoes a different metamorphosis. It now unites with water and ammonia, and may possibly for a time exist as hydrated cyanate of ammonia; but at all events that salt, if formed at all, is soon transformed into urea. $\mathrm{NH}_{3}+\mathrm{C}_{2} \mathrm{NO}+\mathrm{HO}=\mathrm{C}_{2} \mathrm{H}_{4} \mathrm{~N}_{2} \mathrm{O}_{2}=$ urea.

Again, the hydrocyanic acid gives rise to another brown solid body, containing cyanogen or paracyanogen (possibly mellone also), and hydrogen; and, along with this, oxalic acid, urea, and carbonic acid, by metamorphoses already described.

Lastly, the hydrocyanic acid in contact with water, and an acid or an alkali (here oxalic acid or ammonia), undergoes another metamorphosis, and is transformed into formic acid and ammonia; thus: $\mathrm{H}, \mathrm{C}_{2} \mathrm{~N}+4 \mathrm{H} \mathrm{O}=\mathrm{NH}_{3}+\mathrm{C}_{2} \mathrm{H} \mathrm{O}_{3}, \mathrm{H} \mathrm{O}$.

Thus cyanogen, a binary compound, along with water, another binary compound, gives rise to no less than eight different compounds; 1st, the black compound, containing the elements of cyanogen and those of water, possibly $\mathrm{C}_{4} \mathrm{H} \mathrm{N} \mathrm{N}_{2} \mathrm{O}$ : 2nd, ammonia, $\mathrm{N} \mathrm{H}_{3}$ : 3rd, cyanic acid, $\mathrm{H} \mathrm{O}, \mathrm{C}_{2} \mathrm{~N} \mathrm{O}$ : 4th, hydrocyanic acid, $\mathrm{H}, \mathrm{C}_{2} \mathrm{~N}$ : 5th, oxalic acid, $\mathrm{C}_{2} \mathrm{O}_{3}$ : 6th, carbonic acid: 7 th, a brown solid, containing cyanogen (paracyanogen) and hydrogen : 8th, formic acid, $\mathrm{C}_{2} \mathrm{H} \mathrm{O}_{3}, \mathrm{H} \mathrm{O}$; and in addition to these, three bodies, formed by the combination of two of the above eight, and containing all the four elements ; namely, 9th, oxalate of ammonia : 10th, bicarbonate of ammonia: 11th, urea.

This striking example is well adapted to give a clear idea of the immense variety attainable, when, instead of two binary compounds, two ternary or two quaternary compounds, along with water, are concerned; and of the slight modifications of external circumstances which are required to produce results so varied, all the above substances being produced in a liquid at the usual temperature.

Perhaps the best definition of putrefaction and of fermentation is that recently given by Liebig, in his "Letters on Chemistry."

* Third Edition, 1851. - Taylor, Walton, and Maberly. 
A putrescible body is one generally, perhaps always, nitrogenised, which, in contact with air and moisture, and at a certain temperature, undergoes a spontaneous decomposition. When in this state it is putrescent, and is a ferment. A fermentescible body is one which, by itself, or simply dissolved in water, does not undergo any decomposition; but, when in contact with a putrescent body, is resolved into new products, or enters into fermentation. As fermentation is produced by the communication of motion from the atoms, not the molecules, of the putrescent body, to the atoms of the fermentescible one, the process requires time: and the same is true of putrefaction itself. And as the ferment can only act as long as its atoms are in motion, so its power of exciting fermentation must cease as soon as its own decomposition is complete, and not before. Hence a given weight of ferment can only cause the fermentation of a limited quantity of sugar, or of any other fermentescible compound.

Decomposition of Organic Compounds by the Galvanic Current. -When organic compounds are subjected to the action of . a powerful galvanic current, they are frequently decomposed. Oxidised products are formed at the positive pole, and at the negative pole hydrogen generally appears, being possibly derived from the decomposition of water, the oxygen of which has oxidised a part of the substance.

When acetate of potash, dissolved in water, is acted on by a battery of four Bunsen's cells, hydrogen is given off at the negative pole, and at the positive pole appears a mixture of carbonic acid and methyle gases, while the potash is converted into carbonate. Thus, $2\left(\mathrm{KO}, \mathrm{C}_{4} \mathrm{H}_{3} \mathrm{O}_{3}\right)+2 \mathrm{H} \mathrm{O}=\mathrm{H}_{2}+2 \mathrm{CO}_{2}+2 \mathrm{C}_{2} \mathrm{H}_{3}+2$ $\left(\mathrm{K} \mathrm{O}, \mathrm{C} \mathrm{O}_{2}\right.$ ).

In like manner, from the potash salt of any other acid of the series to which acetic acid belongs, we can obtain the radical of the methylic series, having 2 eqs. less of carbon than the acid. As acetate of potash yields methyle, $\mathrm{C}_{2} \mathrm{H}_{3}$, so propylate of potash yields ethyle, $\mathrm{C}_{4} \mathrm{H}_{5}$; butyrate of potash yields propyle, $\mathrm{C}_{6} \mathrm{H}_{7}$, and so on.

This example will show, that by means of the galvanic current we may procure compounds which might otherwise be unattainable, and that much light may be also thrown on the constitution of organic substances.

It is hoped that the above sketch of the doctrine of the chemical changes and metamorphoses of organic compounds will suffice to enable the student to follow the individual processes and reactions, to be hereafter mentioned, which, owing to our limited space, we must treat with great brevity. 


\section{ARTIFICIAL FORMATION OF ORGANIC COMPOUNDS.}

The various processes briefly described in the preceding sections, and others, have of late greatly extended our power of artificially producing organic compounds. But as might be expected, we find it easier to destroy, by various means, the complex molecules of organic nature, than to build up, as nature does, such complex molecules from more simple ones.

The most powerful agent we have is oxidation, and by its means we can produce, from complex organic bodies, a large number of less complex products found in nature. We possess, also, the various processes of fermentation, putrefaction, and decay, by which we can produce similar results. Thus by oxidising uric acid, we can form urea, allantoine, oxalio acid, ammonia, carbonic acid, and water, all of which are formed from it in the body. By oxidising the albuminous or sanguigenous bodies, we can form such products as formic, acetic, propylic, butyric, valerianic, and benzoic acids, besides various crystalline products, such as glycocoll, leucine, and tyrosine,--the two former found in the body, the latter not yet found in nature,-as well as oil of bitter almonds, hydrocyanic acid, ammonia, and of course $\mathrm{CO}_{2}$ and $\mathrm{HO}$. By oxidising oils, we can produce many of the volatile oily acids. By oxidising sugar or starch, we can produce oxalic and formic acids. By the fermentation of sugar we can produce-first, alcohol and carbonic acid; second, lactic acid; third, gum and mannite; fourth, butyric acid; fifth, amylic alcohol, and capric acid. By the fermentation of amygdaline, we can produce oil of bitter almonds, hydrocyanic acid, sugar and formic acid. The oil of bitter almonds, by oxidation, becomes benzoic acid. Hippuric acid, boiled with hydrochloric acid, yields glycocoll and benzoic acid. By the oxidation of salicine, we form salicylic acid and also the oil of spiræa. Wood, when distilled, yields, among other products, pyroxylic spirit, or hydrated oxide of methyle; and we find in the oil of Gaultheria procumbens, salicylic acid combined with oxide of methyle. By the fermentataion of salicine, we form grape sugar and saligenine. By that of asparagine, we obtain succinic acid, which is also formed in the fermentation of malate of lime. In all these cases, and many more, complex molecules are resolved into less complex, as we approach nearer to $\mathrm{CO}_{2}, \mathrm{HO}$ and $\mathrm{NH}_{3}$.

But, although the task is more difficult, we have also made some progress in the opposite process, that of building up more complex out of less complex molecules. It is true, we have succeeded, as yet, in very few instances in thus producing natural 
products. Artificial urea, however, is a proof that it is not impossible. A solution of cyanate of ammonia passes rapidly into urea when warmed. We have already seen the results of the spontaneous action. of cyanogen and water, several of which are more complex than these compounds. The volatile bases already alluded to are in many cases formed by adding complex molecules to simpler ones, as will be described hereafter.

One very striking example of the power we have of building up more complex out of less complex molecules is afforded by the oil of bitter almonds. When this oil is acted on by ammonia, there is formed from 3 eqs: of the oil and 2 of $\mathrm{NH}_{3}, 6$ of $\mathrm{H} \mathrm{O}$ being separated, the body $\mathrm{C}_{42} \mathrm{~N}_{2} \mathrm{H}_{18}$. Here is a complex molecule. This body, hydrobenzamide, is neutral; but when boiled with potash, it is changed to an isomeric base, amarine, $\mathrm{C}_{42} \mathrm{~N}_{2} \mathrm{H}_{18}$, which much resembles natural bases. By the same process, from oil of bran or furfurole is produced by $\mathrm{NH}_{3}$, first, furfuramide, a neutral body; and when this is boiled with potash, 2 eqs. coalesce to form one of a new base, furfurine, which not only resembles the natural bases, but is actually used as an antiperiodic remedy. It is to be noted that almost all the processes by which we produce more complex from less complex molecules are processes of reduction, that is, of deoxidation. The action of ammonia tends to remove oxygen in the form of water; and a whole class of bases are obtained by the action of a very powerful reducing agent, hydrosulphuret of ammonia, on substitution products in which $H$ has been replaced by $\mathrm{NO}_{4}$. It can hardly be doubted that, in process of time, we shall discover the means of producing the natural bases, such as morphine and quinine. We already know how we could with certainty produce coniine and nicotine if we had only the corresponding carbohydrogens, bearing the same relation to these bases as benzole or hyduret of phenyle does to aniline. And these oils or carbohydrogens will very probably soon be discovered. In the same way, if we can discover, as we probably shall, the true constitution of morphine and quinine, and the substance from which the plants form them, - which will one day be done by studying the juices of these plants in all stages of growth, - we shall then have little difficulty in forming such bases. We have the principle and the process, the materials only are wanting.

ORGANIC ACIDS.

The acids met with in organic chemistry are principally compounds of carbon, hydrogen, and oxygen, although some do contain also nitrogen. They are distinguished from inorganic 
acids by their high atomic weight, and by the action of heat, which decomposes them all. As, in many of them, the oxygen they contain is a multiple by a whole number of the oxygen of the bases which neutralise them, so they are viewed as oxygen acids by those who consider true sulphuric acid to be an oxygen acid, $\mathrm{SO}_{3}$, and oil of vitriol to be its hydrate, $\mathrm{H} \mathrm{O}, \mathrm{SO}_{3}$. In the case of acetic acid, for example, the formula of which is $\left(\mathrm{C}_{4} \mathrm{H}_{3}\right) \mathrm{O}_{3}, \mathrm{HO}$, the compound $\left(\mathrm{C}_{4} \mathrm{H}_{3}\right) \mathrm{O}_{3}$, though not possessing acid properties, is called dry acetic acid, and the strong acetio acid is considered as its hydrate; and the analogy is supposed to be fortified by the fact that dry acetic acid, like dry sulphuric acid, contains 3 eqs. oxygen, and neutralises 1 eq. of base, $M 0$, containing 1 eq. of oxygen.

But a careful study of the organic acids leads us to apply to them the same theory which we have already adopted for the inorganic acids, and to consider them as compounds of hydrogen, with compound radicals, usually consisting of carbon, hydrogen, and oxygen. On this view, as oil of vitriol is $\mathrm{H}, \mathrm{SO}_{4}$, the strongest acetic acid is $\mathrm{H},\left(\mathrm{C}_{4} \mathrm{H}_{3}\right) \mathrm{O}_{4}$. It is true that this radical, ( $\left.\mathrm{C}_{4} \mathrm{H}_{3}\right) \mathrm{O}_{4}$, does not exist, or is not known in the separate form ; but the same remark applies to many other compounds, both in inorganic and in organic chemistry, which, nevertheless, are believed to exist. The supposed radicals $\mathrm{S} \mathrm{O}_{4}$ and $\mathrm{N} \mathrm{O}_{6}$, are still unknown in the separate forms.

The existence of compound radicals in organic acids is not more difficult to imagine than that of $\mathrm{S} \mathrm{O}_{4}$, the compound radical of sulphuric acid, for $\left(\mathrm{C}_{4} \mathrm{H}_{3}\right) \mathrm{O}_{4}$, only differs from it in containing three elements instead of two; indeed, as some ternary organic radicals are known in the separate form, we actually derive an argument from organic acids in favour of the existence of compound radicals in inorganic acids. Thus, the radical or organic metal, kakodyle, forms kakodylic acid.

But we find, among organic acids, a much larger proportion which are bibasic, tribasic, in short polybasic, than occurs among inorganic acids. Referring to what was said of the monobasic, bibasic, and tribasic phosphoric acids, it will be easily understood that while acetic acid, for example, is monobasic, tartaric acid, malic acid, komenic acid, alloxanic acid, and others, are bibasic; that citric acid and meconic acid are tribasic, and that saccharic acid is quinquebasic. And as the three modifications of phosphoric acid were described on the old view as monohydrated, bihydrated, and terhydrated, and on the new view, as containing, respectively, 1, 2, and 3 eqs. of hydrogen, replaceable by metals, so tartaric acid may be either $\mathrm{C}_{8} \mathrm{H}_{4} \mathrm{O}_{10}, 2 \mathrm{H} \mathrm{O}$ or $\mathrm{C}_{8} \mathrm{H}_{4} \mathrm{O}_{12}, \mathrm{H}_{2}$; malic acid may be $\mathrm{C}_{8} \mathrm{H}_{4} \mathrm{O}_{8}, 2 \mathrm{H} \mathrm{O}$ or $\mathrm{C}_{8} \mathrm{H}_{4} \mathrm{O}_{10}, \mathrm{H}_{2}$; and so on; 
while citric acid may be either $\mathrm{C}_{12} \mathrm{H}_{5} \mathrm{O}_{11}, 3 \mathrm{H} \mathrm{O}$ or $\mathrm{C}_{12} \mathrm{H}_{5} \mathrm{O}_{14}$, $\mathrm{H}_{3}$, and meconic acid, $\mathrm{C}_{14} \mathrm{H} \mathrm{O}_{11}, 3 \mathrm{H}$, or $\mathrm{C}_{14} \mathrm{H} \mathrm{O}_{14}, \mathrm{H}_{3}$; and lastly, saccharic acid may be $\mathrm{C}_{12} \mathrm{H}_{5} \mathrm{O}_{11}, 5 \mathrm{H} \mathrm{O}$; or $\mathrm{C}_{12} \mathrm{H}_{5} \mathrm{O}_{16}, \mathrm{H}_{5}$.

In such polybasic acids, there is always some hydrogen in the radical, of which it is a constituent, and some combined with the radical, and replaceable by its equivalent of metals. It is only this latter hydrogen, the amount of which affects the neutralising power of the acid.

Now, among the phenomena of the action of bases on organic acids, we have some facts which seem almost to demonstrate the existence of this replaceable hydrogen, as such, and thus to establish the new theory of acids. Thus, meconic acid, which is tribasic, forms, like tribasic phosphoric acid, three series of salts, in which 1, 2, or 3 eqs. of hydrogen are replaced by metal. But while the meconic acid, as well as the tribasic phosphoric acid, readily forms with oxide of silver, the salt in which all the hydrogen is replaced by silver; it cannot form, or forms with difficulty, a similar salt with potash, with which it forms very easily salts with 1 and 2 eqs. of metal, and 2 or 1 eqs. of hydrogen. Here we have the apparent contradiction of a weak base, oxide of silver, neutralising the acid easily and completely; while a strong base, potash, leaves it imperfectly neutralised. This cannot, I think, be accounted for on the old view, and many similar cases might be mentioned. On the new view, such apparent anomalies disappear; for since the neutralisation depends on the replacement of hydrogen by a metal, it is evident that an oxide which is easily reduced, or deprived of its oxygen by hydrogen, like oxide of silver, will most easily neutralise acids, while a difficultly reducible oxide, such as potash, cannot be so far reduced as to yield 3 eqs. of metal, so as to form the neutral salt.

Anhydrous Organic Acids,-For a long time the most important and best known organic acids were known only as hydrates, and it was supposed that the anhydrous acids could not exist in the separate state. But about the same time that anhydrous nitric acid was first obtained, Gerhardt, by a very ingenious method, obtained several anhydrous organic acids.

The method consists in acting on the hydrated acids or their salts with the perchloride of phosphorus, the terchloride of phosphorus, the oxychloride of phosphorus, or the oxychlorides of organic radicals, such as acetyle or benzoyle.

The simplest case is such as this : 1 eq. of acetate of potash, acted on by 1 eq. of oxychloride of acetyle, yields 1 eq. of chloride of potassium, and 2 eqs. of anhydrous acetic acid. 


$$
\left.\mathrm{C}_{4} \mathrm{H}_{3} \mathrm{O}_{3}, \mathrm{~K} \mathrm{O}+\mathrm{C}_{4} \mathrm{H}_{3} \mathrm{O}_{2} \text { Cl }\right\}=\mathrm{K} \mathrm{Cl}+2 \mathrm{C}_{4} \mathrm{H}_{3} \mathrm{O}_{3} \text {. }
$$

When the chlorides of phosphorus act on the acetate of potash, there are two stages in the action. In the first, phosphate of potash and oxychloride of acetyle; in the second, the latter body acts as above on a new portion of the acetate.

The anhydrous organic acids are not, strictly speaking, acid bodies, but only become acids when the elements of water are added. They are either liquid or crystallised, volatile, and on contact with water or bases, pass into the usual form. They are called, to distinguish them from the true acids, anhydrides. We are acquainted with those of many organic acids, both monobasic and bibasic.

We have already stated that many organic compounds are derived from the type water $\left.\begin{array}{r}\mathrm{H} \mathrm{O} \\ \mathrm{O}\end{array}\right\}$. Now, the true organic acids are formed from this type by the substitution of organic acid radicals for the hydrogen, when only 1 eq. of hydrogen is thus replaced. But the anhydrides are derived from the same type by the replacement of all the hydrogen by the acid radical ; or, what amounts to the same thing, they are derived from the hydrated acids when 2 eqs. lose all the basic water they contain.

Some chemists consider that the true radical of acetic acid, the true acetyle, is not $\mathrm{C}_{4} \mathrm{H}_{3}$, but $\mathrm{C}_{4} \mathrm{H}_{3} \mathrm{O}_{2}$. If water be repre-

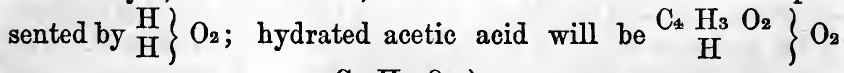
and its anhydride will be $\left.\begin{array}{lll}\mathrm{C}_{4} & \mathrm{H}_{3} & \mathrm{O}_{2} \\ \mathrm{C}_{4} & \mathrm{H}_{3} & \mathrm{O}_{2}\end{array}\right\} \mathrm{O}_{2}$, which is $=2\left(\mathrm{C}_{4} \mathrm{H}_{3} \mathrm{O}_{3}\right)$. The same view may be extended to all the organic acids, and is a very probable one.

Another remarkable fact, showing the propriety of adopting as the type of organic acids and many other bodies the type of water,

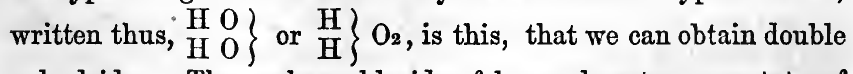
anhydrides. Thus, when chloride of benzoyle acts on acetate of potash, we obtain the anhydride

$$
\left.\begin{array}{ll}
\mathrm{C}_{4} & \mathrm{H}_{3} \mathrm{O}_{2} \\
\mathrm{C}_{14} & \mathrm{H}_{3} \mathrm{O}_{2}
\end{array}\right\} \mathrm{O}_{2},
$$

which is a compound of anhydrous acetic acid with anhydrous benzoic acid. Such compounds form, with water, a mixture of the two hydrated acids.

There are other kinds of organic acids which are what may be called coupled acids; that is to say, they contain an acid 
coupled with another body, which does not neutralise the acid, but accompanies it in all its combinations. Thus, in hyposulphonaphthalic acid, $\mathrm{C}_{20} \mathrm{H}_{7} \mathrm{~S}_{2} \mathrm{O}_{5}$, $\mathrm{H} \mathrm{O}$, we have hyposulphuric acid, $\mathrm{S}_{2} \mathrm{O}_{5}$, coupled with the body, $\mathrm{C}_{20} \mathrm{H}_{7}$, and the coupled acid neutralises exactly as much base as the hyposulphuric acid alone would do. Again, formobenzoilic acid $\mathrm{C}_{16} \mathrm{H}_{7} \mathrm{O}_{5}, \mathrm{H} \mathrm{O}$, may be viewed as a coupled acid, consisting of formic acid $\mathrm{C}_{2} \mathrm{H} \mathrm{O}_{3}, \mathrm{H} \mathrm{O}$ and oil of bitter almonds (hyduret of benzoyle) $\mathrm{C}_{14} \mathrm{H}_{6} \mathrm{O}_{2}$, which neutralises just as much base as the formic acid alone. Those coupled acids which contain hyposulphuric acid, as is often the case, are formed by the action of oil of vitriol, or of anhydrous sulphuric acid, on organic bodies, when 2 eqs. of acid, losing 1 eq. oxygen, form hyposulphuric acid; which commonly unites with the organic matter minus 1 eq. hydrogen, that hydrogen having combined with the oxygen derived from the sulphuric acid.

In the following pages we shall adopt the arrangement of Liebig, which has the advantage of uniting those substances which are naturally allied in composition. It proceeds, in the first place, on the principle of describing, under each known or admitted organic radical, all the compounds derived from, or closely connected with it. After having gone through these, it takes up the consideration of the best known organic acids, including the oily acids ; then the neutral oils, fat, and essential; the resins; the non-nitrogenous colouring matters, bitter and extractive principles, nitrogenised colouring matters and their congeners; the organic bases; starch; gum; woody fibre; destructive distillation of wood, of lignite, of coal: nitrogenised and sulphurised vegetable principles, albumen, \&c., the modifications of these in the animal kingdom: bile: nervous matter: gastric juice: saliva: excrements : urine: blood : lymph, \&c. And the whole is wound up with considerations on the nutrition of plants and animals.

This arrangement is not a scientific one, and in the present state of our knowledge such an arrangement is, I fear, unattainable. But it is very convenient, and by judicious grouping, very much facilitates the learning and the retaining in the memory of the immense mass of facts which at present constitute organic chemistry.

We proceed, therefore, to consider those organic radicals which are admitted by the best authorities, although they are not always known in the separate state. The first compound 
radicals that attract our attention are certain binary ones, already mentioned as binary compounds, but not in their character of radicals (Inorganic Chemistry); these are amide or amidogen, cyanogen, and carbonic oxide.

\section{Amide. $\mathrm{NH}_{2}=\mathrm{Ad}=16$.}

SxN. Amidogen.- It has already been mentioned that certain compounds exist, in which we can hardly doubt that this substance is present. Thus, potassium or sodium, heated in dry ammoniacal gas, disengages 1 eq. of hydrogen, forming the compound $\mathrm{K} \mathrm{N} \mathrm{H_{2 }}$ or $\mathrm{Na} \mathrm{N} \mathrm{H}$. When these are put into water, potash or soda is formed, while ammonia is set free: $\mathrm{K}, \mathrm{N} \mathrm{H}_{2}+\mathrm{H} \mathrm{O}=\mathrm{K} \mathrm{O}+\mathrm{NH}_{3}$. Again, when oxalate of ammonia, $\mathrm{NH}_{3}, \mathrm{H} \mathrm{O}, \mathrm{C}_{2} \mathrm{O}_{3}$ is heated, there are formed, water which distils over, and the compound $\mathrm{NH}_{2} \mathrm{C}_{2} \mathrm{O}_{2}$ which remains behind : thus, $\mathrm{NH}_{3}, \mathrm{HO}, \mathrm{C}_{2} \mathrm{O}_{3}=2 \mathrm{HO}+\mathrm{NH}_{2} \mathrm{C}_{2} \mathrm{O}_{2}$. The latter compound is called oxamide, and is a sparingly soluble white powder, neutral or indifferent in itself, but yielding, when digested with an alkali, ammonia, which escapes, and oxalic acid, which combines with the alkali. Or if oxamide be heated with diluted sulphuric acid, it yields ammonia which combines with the acid, and oxalic acid which crystallises in cooling. In both cases water is decomposed: thus $\mathrm{N} \mathrm{H}_{2} \mathrm{C}_{2} \mathrm{O}_{2}+2 \mathrm{HO}=\mathrm{N} \mathrm{H}_{3}$, $\mathrm{HO}+\mathrm{C}_{2} \mathrm{O}_{3}$.

Now oxamide is interesting, as being the type of a class of compounds, all of which, when heated with an alkali or an acid, yield ammonia and an acid, the ammonia and the anhydrous acid together containing just 1 or 2 eqs. of water more than the compound which, with the aid of water, has yielded them. If we consider oxamide as $\mathrm{N} \mathrm{H}_{2}+\mathrm{C}_{2} \mathrm{O}_{2}$; that is, as composed of amide (or ammonia minus hydrogen, $\mathrm{N} \mathrm{H}_{2}$ ), and the radical carbonic oxide (or oxalic acid minus oxygen, $\mathrm{C}_{2} \mathrm{O}_{2}$ ), then all its congeners are likewise compounds of amide, on the one hand, and an anhydrous organic acid, minus oxygen, on the other. On this view benzamide is benzoate of ammonia, minus water, $\left(\mathrm{N} \mathrm{H}_{3}+\mathrm{C}_{14} \mathrm{H}_{5} \mathrm{O}_{3}, \mathrm{H} \mathrm{O}\right)-2 \mathrm{H} \mathrm{O}=\mathrm{N} \mathrm{H}_{2}+\mathrm{C}_{14} \mathrm{H}_{5} \mathrm{O}_{2}$; or it is amide plus benzoyle (the radical of benzoic acid, $\mathrm{C}_{4} \mathrm{H}_{5} \mathrm{O}_{2}$ ). Therefore, when benzamide is acted on by potash, ammonia is given off, and benzoate of potash is left; and when it is heated with an acid, a salt of that acid with ammonia is obtained, while benzoio acid crystallises. There are a good many similar compounds which are called amides, and are viewed as compounds of amide.

It is obvious that the distinctive characters of amide, which is not known in the separate form, are its powerful affinity for 
hydrogen, and its equally strong tendency to combine with radicals which have a very great affinity for oxygen, sucb as those already mentioned, potassium, sodium, the radical $\mathrm{C}_{2} \mathrm{O}_{2}$, and the radical benzoyle. It is, probably, on account of its having so great an affinity for elements of the most opposite kind, that we cannot obtain it in the separate form.

With hydrogen amide forms two compounds, ammonia, Ad $\mathbf{H}$, and ammonium, Ad $\mathrm{H}_{2}$. The former may be called, in this view, hydramide. The latter, as has been already explained (Inorganic Chemistry), is considered to be a compound metal.

It is very remarkable, that, when ammonia or hydramide combines with an acid, the resulting compound is not a salt, unless the acid contain hydrogen. Thus, hydramide, with dry sulphuric acid, $\mathrm{S} \mathrm{O}_{3}$, forms a compound which is not a sulphate of ammonia, and is not a salt at all. But if, instead of $\mathrm{S} \mathrm{O}_{3}$, we employ oil of vitriol, $\mathrm{H} \mathrm{O}, \mathrm{S} \mathrm{O}_{3}$, or $\mathrm{H}, \mathrm{S} \mathrm{O}_{4}$, sulphate of ammonia, a true salt, is obtained.

Here we may conclude that $\mathrm{Ad} \mathrm{H}$, in contact with $\mathrm{H}, \mathrm{S} \mathrm{O}_{4}$, takes the hydrogen, forming ammonium, and that the resulting salt is composed of $\mathrm{Ad} \mathrm{H}_{2}+\mathrm{S} \mathrm{O} \mathrm{O}_{4}$; that is, a metal combined with the sulphuric acid radical, just as sulphate of potash is $\mathrm{K}, \mathrm{S} \mathrm{O}_{4}$. In all the true salts of ammonia, therefore, Ad $\mathrm{H}_{2}=$ Am (Ammonium) occupies the place of a metal, and may be replaced by a metal; and even if we consider sulphate of potash to be $\mathrm{KO}_{1} \mathrm{SO}_{3}$, we have only to view sulphate of ammonia as $\mathrm{N} \mathrm{H}_{4} \mathrm{O}, \mathrm{S} \mathrm{O}_{3}=\mathrm{Ad} \mathrm{H}_{2} \mathrm{O}, \mathrm{S} \mathrm{O}_{3}=\mathrm{Am} \mathrm{O}, \mathrm{S} \mathrm{O}$, that is, sulphate of oxide of ammonium.

It must be borne in mind, however, that although all the chemical relations of these compounds confirm the ammonium theory, yet neither ammonium nor its oxide are capable of existing uncombined; for ammonium, when separated, is resolved into ammonia and hydrogen : and oxide of ammorium, when separated, assumes the forms of ammonia and water.

This obviously depends on the more feeble attraction of nitrogen for the fourth eq. of hydrogen in ammonium than for the other three, and the very strong attraction of hydrogen for oxygen. $\mathrm{N} \mathrm{H}_{4}$ separates, when liberated, into $\mathrm{N} \mathrm{H}_{3}$, a very permanent compound, and $\mathrm{H}$; while $\mathrm{N} \mathrm{H}_{4} \mathrm{O}$ or $\mathrm{Am} \mathrm{O}$, which, if it could be obtained either anhydrous, or hydrated, as Am $\mathrm{O}, \mathrm{HO}$, would certainly much resemble potash $\mathrm{K} O$, or caustic potash, $\mathrm{K} \mathrm{O}, \mathrm{H} \mathrm{O}$, is resolved into $\mathrm{NH}_{3}+\mathrm{HO}$ or $\mathrm{NH}_{3}+2 \mathrm{HO}$. Perhaps the hydrated oxide of ammonium might exist at a very low temperature, or under a very great pressure, combined with intense cold. We shall see that when the four eqs. of hydrogen in ammonium are replaced by methyle or ethyle, which have less attraction for 
oxygen than hydrogen has, we obtain permanent compounds, which are hydrated bases, astonishingly analogous, both in constitution and properties, to caustic potash.

Of the chief compounds of amide, ammonia or hydramide has been previously described. It remains for us to direct attention to this substance as constantly present in the atmosphere in minute quantity, from whence it descends in the rain, being an absolutely indispensable agent in vegetation. We have already seen that the putrefaction and eremacausis of nitrogenised compounds yields carbonate of ammonia as a product. Its presence in the air is therefore certain, a priori, even although we cannot detect it until it is condensed and accumulated in rain. It is, however, absorbed from the air by almost all minerals and soils, especially aluminous and ferruginous soils, which retain it so as to prevent its being washed away by rain; hence a trace of ammonia is often found where it is not expected, and where it has been erroneously believed to have been formed by the direct union of hydrogen and nitrogen. This is the true explanation of the very remarkable and accurate experiments of Faraday, which have been confirmed by Will and Varrentrapp.

As an ingredient of manures, ammonia is most valuable. Its action on growing vegetables will be hereafter explained.

Ammonia forms a large number of compounds with the oxides of metals, the chlorides of metals, of sulphur, and of phosphorus, and finally with salts in general. These belong rather to inorganic chemistry, and the limited size of this work forbids us to describe them in detail.

But it is proper here briefly to notice the compounds formed by amide with metals, inasmuch as amide is here viewed as an organic radical, and some of these compounds are very important in reference to organic chemistry.

Amide then forms compounds with potassium, sodium, mercury, copper, silver, and platinum. The two former are of a greenish olive colour, and their action on water has been described above. The amide or amidide of mercury, $\mathrm{Hg} \mathrm{Ad}$, is not known in a separate state, but forms with bichloride of mercury the salt called white precipitate, $\mathrm{Hg} \mathrm{Ad}+\mathrm{Hg} \mathrm{Cl}_{2}$.

This salt is prepared by adding ammonia to a solution of bichloride of mercury. When boiled with potash, it yields another salt, $\mathrm{Hg} \mathrm{Ad}+\mathrm{Hg} \mathrm{Cl}_{2}+\mathrm{Hg} \mathrm{O}_{2}$. The amidide of mercury also combines with the basic sulphate, and the basic nitrates of the same metal, forming, with the basic protonitrate, the soluble mercury of Hahnemann.

The amidide of copper, $\mathrm{Ad} \mathrm{Cu}$, is only known in combination with the hyposulphate and with the nitrate of ammonia. 
The amidide of silver, $\mathrm{Ag} \mathrm{Ad}$, is known in combination with the nitrate, sulphate, seleniate and chromate of ammonia.

But it is the amidide of platinum which offers the greatest interest, as it gives rise to several very singular compounds, exhibiting the characters of very powerful bases. These remarkable substances have been studied by Gros, Reiset, Peyrone, Gerhardt, and others, but are yet far from being understood. We shall endeavour briefly to state what is known of them.

When bichloride of platinum, $\mathrm{Pt} \mathrm{Cl}_{2}$, is heated for some time, to nearly the melting point of tin, it loses half its chlorine, and is converted into protochloride, $\mathrm{Pt} \mathrm{Cl}$, which forms a powder of a dirty brownish-green colour, insoluble in water. By continued digestion in ammonia, with the aid of heat, the protochloride is first changed into a green crystalline compound, which finally dissolves entirely, forming a yellowish solution, which on evaporation deposits yellowish-white prismatic crystals. The green crystalline compound, discovered by Magnus, contains the elements of protochloride of platinum and those of ammonia, $\mathrm{Pt} \mathrm{Cl} \mathrm{N} \mathrm{H}$; the yellowish-white crystals, discovered by Reiset, contain twice as much ammonia and the elements of water, $\mathrm{Pt} \mathrm{Cl}, 2 \mathrm{~N} \mathrm{H}_{3}+$ aq.

These two compounds are very remarkable; neither of them contains ammonia as such; both are insoluble in hydrochloric and dilute sulphuric acids. But the green compound of Magnus dissolves in nitric acid, with evolution of nitrous acid, and the solution on cooling deposits white crystalline scales, while the liquid contains no ammonia. These white scales, discovered by Gros, are the nitrate of a new base, $\mathrm{Pt} \mathrm{Cl} \mathrm{N} \mathrm{H}_{6} \mathrm{O}$; which may be derived from 2 eq. of the insoluble compound of Magnus, $\mathrm{Pt}_{2} \mathrm{Cl}_{2} \mathrm{~N}_{2} \mathrm{H}_{6}$, by the loss of 1 eq. of protochloride of platinum and the addition of 1 eq. of oxygen. This base acts exactly like the basic oxide of a metal, or like oxide of ammonium, $\mathrm{N} \mathrm{H}_{4} \mathrm{O}$, combining with acids and forming neutral salts. It not only forms salts with nitric and sulphuric acids and the like, but with hydrochloric acid it yields a heavy crystalline powder, which is a chloride, bearing the same relation to the base as chloride of ammonium, $\mathrm{N} \mathrm{H} \mathrm{H}_{4} \mathrm{Cl}$ (sal ammoniac), does to oxide of ammonium. This chloride, therefore, is $\mathrm{Pt} \mathrm{Cl} \mathrm{N} \mathrm{N}_{2} \mathrm{H}_{6} \mathrm{Cl}$, and the radical of the oxide or base, and of the chloride, will be $\mathrm{Pt} \mathrm{Cl} \mathrm{N}_{2} \mathrm{H}_{6}$, corresponding to ammonium, $\mathrm{N} \mathrm{H}_{4}$. Although Gros did not isolate either the radical or its oxide, yet from the characters and composition of its salts, there can be no doubt of its existence. If we represent this radical $\mathrm{Pt} \mathrm{Cl} \mathrm{N}_{2} \mathrm{H}_{6}$ by $\mathrm{R}$, then we have 
$\mathrm{RO}=$ oxide, the base of the salts, analogous to $\mathrm{KO}$ or $\mathrm{N} \mathrm{H}_{4}, \mathrm{O}$

$\mathrm{RO}+\mathrm{SO}_{3}=\mathrm{R}+\mathrm{S} \mathrm{O}_{4}=$ sulphate, analogous to $\mathrm{K}, \mathrm{S} \mathrm{O}_{4}$

$\mathrm{RO}+\mathrm{NO}_{5}=\mathrm{R}+\mathrm{NO}_{6}=$ nitrate, analogous to $\mathrm{K}, \mathrm{N} \mathrm{O}_{6}$

$\mathrm{R} \mathrm{Cl}=$ chloride, analogous to $\mathrm{K} \mathrm{Cl}$ or $\mathrm{N} \mathrm{H}_{4}, \mathrm{Cl}$.

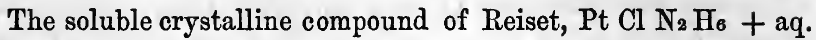
when heated to $212^{\circ}$, becomes anhydrous, and is then $\mathrm{Pt} \mathrm{Cl} \mathrm{N}$ $\mathrm{H}_{6}$, that is, it has the same composition as the radical of the base and salts of Gros. But it is not this radical ; on the contrary, it is the chloride of a different radical, $\mathrm{Pt} \mathrm{N}_{2} \mathrm{H}_{6}$, and its true formula is $\mathrm{Pt} \mathrm{N}_{2} \mathrm{H}_{6}+\mathrm{Cl}$.

This new radical is also perfectly analogous to ammonium, as may be exhibited in a tabular form as follows.

\begin{tabular}{|c|c|c|c|c|}
\hline & $\mid \begin{array}{c}\text { Chlorine } \\
\text { Compound. }\end{array}$ & Sulphate. & Nitrate. & $\begin{array}{l}\text { Double Chloride } \\
\text { with Platinum. }\end{array}$ \\
\hline 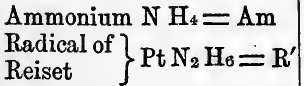 & $\begin{array}{c}\mathrm{Am} \mathrm{Cl} \\
\mathrm{R}^{\prime} \mathrm{Cl}\end{array}$ & $\begin{array}{l}\mathrm{Am} \mathrm{S} \mathrm{O} \mathrm{S}_{4} \\
\mathrm{R}^{\prime} \mathrm{S} \mathrm{O}_{4}\end{array}$ & $\begin{array}{l}\mathrm{Am} \mathrm{N} \mathrm{O}_{6} \\
\mathrm{R}^{\prime} \mathrm{NO}_{6}\end{array}$ & $\begin{array}{l}\mathrm{AmCl}+\mathrm{PtCl}_{2} \\
\mathrm{R}^{\prime} \mathrm{Cl}+\mathrm{PtCl}_{2}\end{array}$ \\
\hline
\end{tabular}

In the case of ammonium, we cannot isolate the oxide Am $\mathrm{O}$ $=\mathrm{N} \mathrm{H}_{4} \mathrm{O}$, as it instantly resolves itself into ammonia and water, $\mathrm{NH}_{3}+\mathrm{H} \mathrm{O}$. But the oxide of Reiset's platinum radical, $\mathrm{R}^{\prime} \mathrm{O}$, $=\mathrm{Pt} \mathrm{N}_{2} \mathrm{H}_{6} \mathrm{O}$, or rather its hydrate $\mathrm{Pt} \mathrm{N}_{2} \mathrm{H}_{6} \mathrm{O}+$ aq. is easily obtained from the sulphate by adding just so much baryta as will remove the sulphuric acid, and evaporating the filtered solution in vacuo, when the hydrated oxide crystallises in colourless needles. This compound is strikingly analogous to hydrate of potash : it is a powerful caustic, attracts carbonic acid from the air as strongly as potash, and exhibits all the chemical characters of one of the strongest alkalies. Few compounds are so remarkable as this base. We shall call it the base $a$.

This singular compound may be viewed as containing the elements of protoxide of platinum, ammonia and water, Pt 0 , $2 \mathrm{~N} \mathrm{H}_{3}, \mathrm{H} \mathrm{O}$. When heated to $212^{\circ}$, it loses its water and half its ammonia, leaving a compound $\mathrm{Pt} \mathrm{N} \mathrm{H} \mathrm{H}_{3} \mathrm{O}$ or $\mathrm{Pt} \mathrm{O}, \mathrm{N} \mathrm{H}_{3}$, which appears to be another new base, $b$, combining with acids and forming salts which detonate when heated. This last compound, when heated in the air, burns like tinder, and leaves metallic platinum. It is the oxide of a third radical, $b,=$ Pt $\mathrm{N} \mathrm{H}_{3}$.

With hydrochloric acid the base $a$ of Reiset yields water and the original chlorine compound, thus: $\mathrm{Pt} \mathrm{N} \mathrm{N}_{6}, \mathrm{O}+\mathrm{H} \mathrm{Cl}=$ $\mathrm{Pt} \mathrm{N}_{2} \mathrm{H}_{6}, \mathrm{Cl}+\mathrm{H} \mathrm{O}$. When this chloride is heated to from $265^{\circ}$ to $290^{\circ}$, it loses ammonia, and there is left a yellow powder, 
discovered by Peyrone, which dissolves easily in hot water, and has the composition of the green insoluble crystalline compound of Magnus, $\mathrm{Pt} \mathrm{Cl} \mathrm{N} \mathrm{H}_{3}$, of which it is an isomeric modification. Indeed, the compound of Magnus, (although insoluble in hot water,) if boiled with a saturated solution of sulphate or nitrate of ammonia, dissolves, and is deposited on cooling in yellow erystals. The yellow ammoniated protochloride of platinum of Peyrone readily dissolves in ammonia, and the solution yields fine colourless prisms of the original chlorine compound of Reiset, but apparently not containing 1 eq. of water of crystallisation, which is said to be present in the yellowish-white crystals obtained by Reiset from the green compound of Magnus.

When the yellow compound of Peyrone is acted on by nitrate of silver, it yields chloride of silver, and two new compounds containing nitric acid and platinum, one of which forms yellow octahedrons.

If the sulphate of Reiset's base, $\mathrm{Pt} \mathrm{N}_{2} \mathrm{H}_{6}+\mathrm{S} \mathrm{O}_{4}$, be acted on by iodide of barium, Ba I, there is formed sulphate of baryta, $\mathrm{Ba} \mathrm{S} \mathrm{O}_{4}$, and a protoiodide of Reiset's radical, $a, \mathrm{Pt} \mathrm{N}_{2} \mathrm{H}_{6}$, I. This iodide is soluble and crystallisable, and when boiled with water, loses ammonia, while a new iodine compound is precipitated, $\mathrm{Pt} \mathrm{N} \mathrm{H} \mathrm{H}_{3}$, I, corresponding to the chlorine compound of Peyrone and to that of Magnus, both of which are $\mathrm{Pt} \mathrm{N} \mathrm{H3} \mathrm{Cl.}$

This new iodine compound seems to be the iodide of the third radical, $b, \mathrm{Pt} \mathrm{N} \mathrm{Hs}$; for when acted on by nitrate or sulphate of silver, it yields iodide of silver, and a nitrate or sulphate of this new radical or of its oxide, $\mathrm{Pt} \mathrm{NH}_{3}, \mathrm{I}+\mathrm{Ag}, \mathrm{S} \mathrm{O}_{4}=\mathrm{PtN} \mathrm{H}_{3}$,

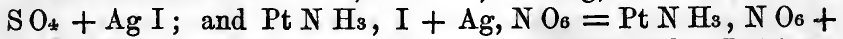
$\mathrm{Ag}$ I. These new salts may of course be represented as $\mathrm{Pt} \mathrm{N} \mathrm{H}_{3}$, $\mathrm{O}+\mathrm{S} \mathrm{O}_{3}$, and $\mathrm{Pt} \mathrm{NH}_{3}, \mathrm{O}+\mathrm{N}_{5}$. With ammonia, these salts yield the nitrate and sulphate of Reiset's base $a$; and with hydrochloric acid they yield the yellow compound of Peyrone. This would indicate that the latter compound is $\mathrm{Pt} \mathrm{N} \mathrm{H}_{3}, \mathrm{Cl}$, the chloride of the radical $\mathrm{Pt} \mathrm{NHs}$, while the green salt of Magnus may be the ammoniated protochloride of platinum $\mathrm{Pt} \mathrm{Cl}+\mathrm{NH}_{3}$, or probably $\mathrm{Pt} \mathrm{Cl} \mathrm{N} \mathrm{\textrm {H } _ { 3 }}+$ aq.

Here we have, at all events, three very remarkable compounds, which contain platinum and the elements of ammonia. There is, first, the radical $b$ last described, $\mathrm{Pt} \mathrm{NH}_{3}$; secondly, the radical of Reiset's base, $a, \mathrm{Pt} \mathrm{N}_{2} \mathrm{H}_{6}$; and thirdly, the radical of Gros's base, $\mathrm{Pt} \mathrm{Cl}, \mathrm{N}_{2} \mathrm{H}_{6}$.

Now we have given the history of these compounds somewhat minutely, because their existence throws much light on the nature of a numerous and important class of bodies, namely, the vegetable bases or alkaloids. 
It will be observed, then, that the three new radicals above described all contain nitrogen; indeed, all contain the elements of ammonia, and are in their chemical relations entirely analogous to ammonium. Thus we have

\begin{tabular}{|c|c|c|c|c|}
\hline & Radical. & Oxide. & Chloride. & Sulphate. \\
\hline & $\mathrm{NH}_{4}$ & $\mathrm{~N} \mathrm{H}_{4}$ & $\mathrm{~N} \mathrm{H}_{4}+\mathrm{Cl}$ & $\mathrm{N} \mathrm{H}_{4}$ \\
\hline $\begin{array}{l}\text { Reis } \\
\text { base }\end{array}$ & $\mathrm{Pt} \mathrm{NH}_{3}$ & $\mathrm{Pt} \mathrm{NH}_{3}+\mathrm{O}$ & $\mathrm{Pt} \mathrm{NH}_{3}+\mathrm{Cl}$ & $\mathrm{PtNH}_{3}+\mathrm{SO}_{4}$ \\
\hline $\begin{array}{l}\text { Radical of } \\
\text { Reiset's } \\
\text { base } a\end{array}$ & $\mathrm{Pt} \mathrm{N} \mathrm{N}_{6}$ & $\mathrm{Pt} \mathrm{N}_{2} \mathrm{H}_{6}+\mathrm{O}$ & $\mathrm{Pt} \mathrm{N} \mathrm{H}_{0}+\mathrm{Cl}$ & $\mathrm{Pt} \mathrm{N}_{2} \mathrm{H}_{6}+\mathrm{SO}_{4}$ \\
\hline$\left[\begin{array}{c}\text { Radical of } \\
\text { Gros's base }\end{array}\right]$ & $\mathrm{Pt} \mathrm{Cl}$ & $\mathrm{PtClN}_{2} \mathrm{H}_{6}+\mathrm{O}$ & $\mathrm{PtClN}_{2} \mathrm{H}_{6}+\mathrm{Cl}$ & $\mathrm{PtClN}_{2} \mathrm{H}_{8}+\mathrm{SO}_{4}$ \\
\hline
\end{tabular}

That the above formulæ represent in some respects truly the relation of these new bases to one another, is rendered probable by the fact that while the radical and base $b$ of Reiset differ from his radical and base $a$ by containing 1 eq. of ammonia less, and these last from those of Gros by containing 1 eq. of chlorine less, we can actually transform the salts of Reiset's base $b$ into those of his base $a$, by the addition of ammonia ; and the nitrate of Reiset's base $a$, by the addition of chlorine, yields a salt having the properties of the nitrate of Gros's base.

Now we have seen that ammonium may be viewed as a compound of amide, as $\mathrm{NH}_{2}+\mathrm{H}_{2}=\mathrm{Ad} \mathrm{H_{2 }}$. May we not therefore suppose the new radicals to be also compounds of amide? May not Reiset's radical $b$ be ammonium, in which 1 eq. of hydrogen has been replaced by 1 eq. of platinum, $\mathrm{Ad}\left\{\begin{array}{l}\mathrm{H} \\ \mathrm{Pt}\end{array}\right.$

Again, just as we have seen in acids, viewed as hydrogen compounds, analogous elements added to the radical without affecting the neutralising power of the acid, which remains the same as long as the replaceable hydrogen continues unchanged, we can suppose amide to be a basic radical, forming with hydrogen the base ammonia, but capable of taking up into the radical analogous elements without affecting the basic character of the ammonia, because we have now the hydrogen compound of an analogous, but more complex, basic radical. On this view, Reiset's radical $b$ may be the hydrogen compound of a basic radical, more complex than amide ; in fact, amidide of platinum. Its formula would then be $\mathrm{Ad} \mathrm{Pt}+\mathrm{H}$, and although on this view it should correspond to ammonia, rather than ammonium, we cannot speak positively, as this is the least known of the three. 
The other two radicals may be readily viewed as hydrogen compounds of complex amidides, as ammonium is the hydrogen compound of amidide of hydrogen.

Amide, $\mathrm{N} \mathrm{H}_{2}=\mathrm{Ad}$, with hydrogen, forms ammonia Ad, $\mathrm{H}$ and ammonium Ad H $+\mathrm{H}$. In like manner, in the radical $a$ of Reiset's salts, we have a complex amide, composed of amide and amidide of platinum, $\mathrm{Ad}+\mathrm{Pt} A \mathrm{Ad}=\mathrm{Pt} \mathrm{Ad}_{2}$, which, with 1 eq. hydrogen, may be supposed to form a compound analogous to ammonia, $\mathrm{Pt} \mathrm{Ad}, \mathrm{H}$, and with two eq. of hydrogen, actually does form the radical $a$ of Reiset, $\mathrm{Pt} \mathrm{Ad}_{2} \mathrm{H}+\mathrm{H}$, exactly analogous to ammonium. So the radical of Gros may be derived from the complex amide $\mathrm{Pt} \mathrm{Cl} \mathrm{Ad}+\mathrm{Ad}=\mathrm{Pt} \mathrm{Cl} \mathrm{Ad}$, which may form $\mathrm{Pt} \mathrm{Cl} \mathrm{Ad}+\mathrm{H}$ and $\mathrm{Pt} \mathrm{Cl} \mathrm{Ad}_{2} \mathrm{H}+\mathrm{H}$, the latter being the actual composition of the radical of Gros, corresponding to ammonium.

It may also be mentioned, that just as we may view ammoniacal salts as containing ammonia and water rather than ammonium and oxygen, so the base $b$ of Reiset may be $\mathrm{N} \mathrm{H}_{3}+\mathrm{Pt} 0$, analogous to $\mathrm{N} \mathrm{H}_{3}+\mathrm{HO}$ in the salts of ammonia. If sulphate of ammonia be $\mathrm{N} \mathrm{H}_{3}$, H $\mathrm{O}+\mathrm{S} \mathrm{O}_{3}$, the sulphate of Reiset's base $b$ will then be $\mathrm{NH}_{3}$, Pt $\mathrm{O}+\mathrm{SO}_{3}$, the protoxide of platinum here playing the part of water, or in other words, platinum playing the part of hydrogen, a substitution far from unnatural or improbable. Again, if we consider the ammoniacosulphate of copper to be $\left.{ }_{\mathrm{CuO}}^{2 \mathrm{NH}_{3}}\right\}+\mathrm{S} \mathrm{O}_{3}$, then the sulphate of Reiset's base $a$ will be $\left.{ }_{\mathrm{Pt} \mathrm{O}}^{2 \mathrm{~N}_{3}}\right\}+\mathrm{S} \mathrm{O}_{3}$, where platinum replaces copper, also a not improbable substitution. Reiset is disposed to adopt this view.

The chloride of Gros's radical, $\mathrm{Pt} \mathrm{Cl} \mathrm{N}_{2} \mathrm{H}_{6}+\mathrm{Cl}$, may be viewed as a compound of bichloride of platinum with ammonia, $\mathrm{Pt} \mathrm{Cl}_{2}+2 \mathrm{~N} \mathrm{H}_{3}$, and there is even reason to think that compounds of that radical may be obtained from the solution of bichloride of platinum in ammonia.

In a recent paper, Gerhardt has announced the discovery of a fourth platinised base, and he regards all four as representatives of ammonia, in which part of the hydrogen is replaced by platinum, which agrees generally with the views above indicated. But he supposes that in two of these four bases, the platinum exists in a polymeric modification, in which the usual equivalent of platinum replaces 2 eqs. of hydrogen, and in which, therefore, the equivalent of platinum is halved. He represents the ordinary form of platinum as usual by $\mathrm{Pt}$, and the new form by 
$\mathrm{pt}$, the latter always occurring in 2 eqs., as $\mathrm{pt}_{2}=\mathrm{Pt}$. He then gives the following table :-

$\mathrm{N}, \mathrm{H}_{2} \mathrm{H}=$ Ammonia.

$\mathrm{N}, \mathrm{H}_{2} \mathrm{Pt}=$ Platosamine (Reiset's base $b_{\text {. }}$ )

$\mathrm{N}_{2} \mathrm{H}_{5} \mathrm{Pt}=$ Diplatosamine (Reiset's base $a$.)

$\mathrm{N} \mathrm{H} \mathrm{pt} 2$ = Platinamine (new base of Gerhardt.)

$\mathrm{N}_{2} \mathrm{H}_{4} \mathrm{pt}_{2}=$ Diplatinamine (existing in the base of Gros.)

On this view diplatosamine and diplatinamine represent a double atom of ammonia $\mathrm{N}_{2} \mathrm{H}_{6}$. The chloride of Gros's base is, according to Gerhardt, the bi-hydrochlorate of diplatinamine. But as acid hydrochlorates are most unusual, this must be regarded as very doubtful. The new base platinamine forms brilliant yellow crystals, nearly insoluble. The salts are crystallisable, and many of them contain 2 eqs. of acid, for one of base.

Buckton has lately studied the action of cyanogen on diplatosamine, which is remarkable. A white crystalline compound is formed, identical with one described by Reiset as the cyanide of platosammonium, but which is really, in both cases, a platinocyanide of diplatosammonium, polymeric with the other. Its formation is as follows, from the oxide of diplatosammonium.

$$
2\left(\mathrm{Pt} \mathrm{N}_{2} \mathrm{H}_{6} \mathrm{O}\right)+4 \mathrm{H} \mathrm{Cy}=\left(\mathrm{Pt} \mathrm{N}_{2} \mathrm{H}_{6}, \mathrm{Pt} \mathrm{Cy}_{2}\right)+2 \mathrm{NH}_{4} \mathrm{Cy}+2 \mathrm{HO} .
$$

Buckton has also obtained the true cyanide of platosammonium by the action of cyanide of silver on chloride of platosammonium (hydrochlorate of platosamine), which yields chloride of silver, and yellow crystals of the new cyanide. $\mathrm{Pt} \mathrm{N} \mathrm{H}, \mathrm{Cl}+\mathrm{Ag} \mathrm{Cy}$ $=\mathrm{Pt} \mathrm{N} \mathrm{H}, \mathrm{Cy}+\mathrm{Ag} \mathrm{Cl}$. And this compound is polymeric with the preceding, for $\left(\mathrm{Pt} \mathrm{N} \mathrm{N}_{6}, \mathrm{Pt} \mathrm{Cy2}\right)=2\left(\mathrm{Pt} \mathrm{N} \mathrm{H}{ }_{3}, \mathrm{Cy}\right)$. It is evident that much remains to be done, before the history of these most remarkable platinised bases can be regarded as complete. The names proposed by Gerhardt may be adopted.

From the above remarks it will appear that every probable view which can be taken of these very interesting bases connects them with amide, ammonia or ammonium, and it is for this reason that they have been treated of in this section. Many pages might be filled with details concerning them : but we have here only indicated those points which will help to elucidate the constitution of the vegetable alkalies.

That important class of compounds not only contains nitrogen, as an essential element, but exhibits the same analogy with ammonia which we have seen to exist in the compound platinum bases. Moreover, like these bases, the alkaloids do not appear 
to contain ammonia as such; and the probability is very great that their constitution is analogous to that of the bases now described. We shall find, in treating of the alkaloids, as we have already indicated in the introductory sections, that many of them are, in all probability, derived from ammonia by substitution of compound radicals, or groups, for a part on the whole of the hydrogen in ammonia. We may therefore regard ammonia as the type or representative of a very large class of organic bases, both artificial and natural.

When a salt of ammonia loses 1 eq. of water more than reduces it to the state of an amide, there is formed a compound containing $\mathrm{N} \mathrm{H}$, that is, Imide, and such compounds are called imides; such as camphorimide and naphtalimide, from the camphorate and the naphthalate of ammonia. As an amide consists of ammonia minus 1 eq. of hydrogen and the anhydrous acid minus 1 eq. of oxygen, so an imide consists of both, the ammonia minus 2 eqs. of hydrogen, the acid minus 2 eqs. of oxygen. Imides are not frequent, but like amides when boiled with acids or bases, water is taken up, and the ammonia and acid are reproduced.

When an ammoniacal salt loses so much water that all the three eqs. of hydrogen in the ammonia, with 3 eqs. of oxygen from the anhydrous acid are given off, the compound left is called a nitryle, as containing only nitrogen from the ammonia. Thus benzoate of ammonia, $\mathrm{N}_{4} \mathrm{O}, \mathrm{C}_{4} \mathrm{H}_{5} \mathrm{O}_{3}$, when it loses 2 eqs. of water, that is 2 of hydrogen from the ammonium, $\mathrm{N} \mathrm{H}_{4}$, and two of oxygen, 1 from the oxide of ammonium, and the other from the acid, yields Benzamide, $\mathrm{C}_{14}, \mathrm{~N} \mathrm{H}_{7} \mathrm{O}_{2}$, or $\mathrm{N} \mathrm{H}_{2}, \mathrm{C}_{14} \mathrm{H}_{5} \mathrm{O}_{2}$. Were it to lose 1 more eq. of water, it would yield the true benzimide, $\mathrm{N} \mathrm{H}, \mathrm{C}_{14} \mathrm{H}_{5} \mathrm{O}=\mathrm{C}_{14} \mathrm{~N} \mathrm{H}_{6} \mathrm{O}$, which is not yet known. But when it loses still 1 eq. more of water, it actually yields benzonitryle, $\mathrm{C}_{14} \mathrm{H}_{5} \mathrm{~N}$, in forming which all the hydrogen of the ammonium has been removed, as well as all the oxygen of the anhydrous acid, and of the oxide of ammonium. Nitryles are now pretty numerous, and as all these yet known are formed from acids containing only 3 eqs. of oxygen when anhydrous, they contain themselves no oxygen, but always as much hydrogen as the anhydrous acid did. Both imides and nitryles will have to be referred to hereafter, and minute details are here unnecessary. It must be noted, however, that all or most of the known nitryles appear to be cyanogen or cyanides. Thus oxalonitryle, that is, oxalate of ammonia, minus 4 eqs. of water, is cyanogen itself, for $\mathrm{N} \mathrm{H}_{4} \mathrm{O}, \mathrm{C}_{2} \mathrm{O}_{3}-4 \mathrm{H} \mathrm{O}=$ $\mathrm{C}_{2} \mathrm{~N}$. Formonitryle is hydrocyanic acid; $\mathrm{N} \mathrm{H}_{4} \mathrm{O}, \mathrm{C}_{2} \mathrm{H} \mathrm{O}_{3}-$ $4 \mathrm{H} \mathrm{O}=\mathrm{H}, \mathrm{C}_{2} \mathrm{~N}$; acetonitryle is cyanide of methyle; $\mathrm{N} \mathrm{H}_{4} \mathrm{O}$, 
$\mathrm{C}_{4} \mathrm{H}_{3} \mathrm{O}_{3}-4 \mathrm{H} \mathrm{O}=\mathrm{C}_{2} \mathrm{H}_{3}, \mathrm{C}_{2} \mathrm{~N}$; and benzonitryle is cyanide of phenyle; $\mathrm{N} \mathrm{H}_{4} \mathrm{O}, \mathrm{C}_{14} \mathrm{H}_{5}, \mathrm{O}_{3}-4 \mathrm{H} \mathrm{O}=\mathrm{C}_{12} \mathrm{H}_{5}, \mathrm{C}_{2} \mathrm{~N}$.

II. Carbonio Oxide (as a Radical). $\mathrm{C}_{2} \mathrm{O}_{2}=28$.

Syn.: Oxalyle. - There is good reason to believe that the radical of oxalic acid is formed of 2 eqs. of carbonic oxide. It has long been known that carbonic oxide, in the sun's light, combines with chlorine to form phosgene gas or chlorocarbonic acid, $\mathrm{C} \mathrm{O}, \mathrm{Cl}$ or $\mathrm{C}_{2} \mathrm{O}_{2}, \mathrm{Cl}_{2}$. This compound may be viewed as the chloride of the radical $\mathrm{C}_{2} \mathrm{O}_{2}$, or as carbonic acid in which 1 eq. of oxygen is replaced by chlorine, $\mathrm{C}\left\{\begin{array}{l}\mathrm{O} \\ \mathrm{Cl}\end{array}\right.$, corresponding to $\mathrm{C}\left\{\begin{array}{l}\mathrm{O} \\ \mathrm{O}\end{array}\right.$ or $\mathrm{C} \mathrm{O}_{2}$ : But the existence of this radical is more securely inferred from the combinations it forms with oxygen, potassium, and amide.

\section{CARBONIC OXIDE AND OXYGEN.}

\section{OxaLio AcId. $\left(\mathrm{C}_{2} \mathrm{O}_{2}\right) \mathrm{O}+\mathrm{H} \mathrm{O}$, or $\mathrm{C}_{2} \mathrm{O}_{4}, \mathrm{H}=45$.}

This acid occurs in nature, generally in the form of an acid oxalate of potash in certain vegetable juices, such as that of oxalis acetosella, also as oxalate of lime in many lichens. It is formed artificially by the action of nitric acid on sugar, starch, and many other organic compounds; also by the action of hypermanganate of potash on sugar, \&c.

To prepare it, one part of pure starch is gently heated with eight parts of nitric acid, Sp. G. 1.20 or 1.25. A very energetic reaction ensues, and much nitrous acid is disengaged: when this slackens, heat is applied, and continued till no more red vapours appear, when the liquid, if sufficiently evaporated, deposits, on cooling, a large quantity of crystals of hydrated oxalic acid. These are dried on a porous tile, to remove the mother liquor which contains much free nitric acid, saccharic acid, and other products. The dried crystals being dissolved in a little hot water, the solution on cooling, deposits pure oxalic acid in foursided prisms, which are colourless, very acid, very soluble in hot water, moderately so in cold water. These crystals contain 3 eq. of water of crystallisation, $\mathrm{C}_{2} \mathrm{O}_{3}, \mathrm{HO}+3$ aq. When sharply heated, a part sublimes as dry acid, $\mathrm{C}_{2} \mathrm{O}_{3}, \mathrm{H} \mathrm{O}$. Oxalic acid is destroyed by heat without blackening, which serves to distinguish it from most other organic acids.

It is very poisonous, and is the cause of many fatal accidents from its similarity to Epsom salts, from which, however, it is 
easily distinguished by its very sour taste. The best antidote is prepared chalk administered in water, which forms the insoluble and inert oxalate of lime. It is easily detected by forming with lime-water, or a soluble salt of lime, if no free acid be present, the very insoluble oxalate of lime, which when dried and heated to low redness, is converted, without blackening, into carbonate of lime.

When oxalic acid, or any of its salts, is heated with oil of vitriol in excess, a brisk effervescence takes place, and the gas given off is a mixture of equal volumes of carbonic acid and carbonic oxide. This character furnishes another good means of recognising oxalic acid. The reaction is very simple, for $\mathrm{C}_{2} \mathrm{O}_{3}, \mathrm{HO}+\mathrm{H} \mathrm{O}, \mathrm{S} \mathrm{O}_{3}=$ $\left(\mathrm{SO}_{3}+2 \mathrm{HO}\right)+\mathrm{CO}+\mathrm{C} \mathrm{O}_{2}$. The sulphuric acid seizes the whole of the water, and the anhydrous oxalic acid, $\mathrm{C}_{2} \mathrm{O}_{3}$, cannot exist in the separate state.

When the oxalates of certain protoxides, as those of cobalt and nickel, are heated in close vessels, the metal is left: carbonic acid being given off; $\mathrm{Co} O, \mathrm{C}_{2} \mathrm{O}_{3}=\mathrm{CO}+2 \mathrm{C} \mathrm{O}_{2}$. Other oxalates, as that of manganese, give off carbonic acid and carbonic oxide, leaving the protoxide of the metal $\mathrm{Mn} \mathrm{O}, \mathrm{C}_{2} \cdot \mathrm{O}_{3}=\mathrm{Mn} \mathrm{O}+\mathrm{CO}$ $+\mathrm{CO}_{2}$.

Oxalic acid forms salts with bases, many of which are insoluble. The insolubility of the oxalate of lime renders oxalic acid useful as a test for lime, and as a means of separating it, and determining its quantity, in analysis. As a test, it is commonly used in the form of oxalate of ammonia. It can only detect lime in neutral or alkaline fluids, the oxalate of lime being soluble in free acids.

Recent researches tend to show that oxalic acid is bibasic, and that its true formula is double that above given, or $\mathrm{C}_{4} \mathrm{O}_{6}, 2 \mathrm{H} \mathrm{O}$. This accounts for the existence of acid oxalates and of double oxalates.

Viewed in this light, oxalic acid is the type of a very remarkable series of bibasic organic acids, to be afterwards described. This series has a curious relation to the formic or acetic series of volatile acids, from which it differs in this, that all the members of it are crystalline, soluble in water, and decomposed by heat. If to formic acid, $\mathrm{C}_{2} \mathrm{H}_{2} \mathrm{O}_{4}$, we add 2 eqs. of carbonic acid, we have $\mathrm{C}_{2} \mathrm{H}_{2} \mathrm{O}_{4}+2 \mathrm{CO}_{2}=\mathrm{C}_{4} \mathrm{H}_{2} \mathrm{O}_{8}=\mathrm{C}_{4} \mathrm{O}_{6}, 2 \mathrm{H} \mathrm{O}$, which is oxalie acid. And the same relation holds good with every acid of the oxalic series and the corresponding acid of the formic series, the latter having always 2 eqs. of carbon and 4 eqs. of oxygen less than the former.

Both these series are formed during the action of nitric acid on oils and oily acids. 
The formation of oxalic acid by the action of oxidising agents on organic matters, is a partial oxidation of their carbon; when that oxidation is complete, carbonic acid is the result. The action of nitric acid on starch or sugar is complicated, and not so well understood as to admit of being expressed in the form of an equation; but the oxidation of sugar by permanganate of potash is very simple, and is thus represented : $\mathrm{C}_{12} \mathrm{H}_{10} \mathrm{O}_{10}+6$ $\left(\mathrm{K} 0, \mathrm{Mn}_{2} \mathrm{O}_{7}\right)=6\left(\mathrm{~K} \mathrm{O}, \mathrm{C}_{2} \mathrm{O}_{3}\right)+10 \mathrm{HO}+12 \mathrm{Mn} \mathrm{O}_{2}$; that is, 1 eq. of anhydrous sugar, with 6 eqs. of the permanganate, produces 6 eqs. of oxalate of potash, 10 eqs. of water, and 12 eqs. of peroxide of manganese.

The most important oxalates are those of potash, lime, and ammonia. There are three oxalates of potash ; the neutral oxalate, $\mathrm{K} \mathrm{O}, \mathrm{C}_{2} \mathrm{O}_{3}+$ aq. ; the binoxalate, $\mathrm{K} \mathrm{O}, \mathrm{C}_{2} \mathrm{O}_{3}+\mathrm{H} \mathrm{O}, \mathrm{C}_{2} \mathrm{O}_{3}+2$ aq.; and the quadroxalate, $\mathrm{KO}, \mathrm{C}_{2} \mathrm{O}_{3}+3\left(\mathrm{H} \mathrm{O}, \mathrm{C}_{2} \mathrm{O}_{3}\right)+4$ aq. The double oxalate of potash and oxide of chromium, described by me some years since, forms crystals which are black by reflected, deep blue by transmitted, light. The solution is green and red at the same time by day-light, crimson-red by candle-light. Sir D. Brewster has described, in the Edin. Phil. Trans., the very remarkable optical properties of this salt. The oxalate of lime is $\mathrm{Ca}_{i} \mathrm{O}, \mathrm{C}_{2} \mathrm{O}_{3}+2$ aq. The oxalate of silver $\mathrm{Ag} \mathrm{O}, \mathrm{C}_{2} \mathrm{O}_{3}$, detonates when heated, yielding like several other oxalates of the noble metals, carbonic acid, and the metal. The oxalate of ammonia, $\mathrm{NH}_{4} \mathrm{O}, \mathrm{C}_{2} \mathrm{O}_{3}+$ aq. is much used as a test. It crystallises very readily. When heated, it gives rise to a very remarkable compound, namely, oxamide, which is the type of a class. We shall here consider it.

\section{Oxamide. $\mathrm{C}_{2} \mathrm{H}_{2} \mathrm{~N} \mathrm{O}_{2}=\mathrm{C}_{2} \mathrm{O}_{2}+\mathrm{N} \mathrm{H}_{2}=44$.}

When oxalate of ammonia is heated in a retort, it gives rise to a variety of products, and, among these, to a white crystalline powder, insoluble in cold water, which is oxamide. It may be formed far more abundantly by the action of ammonia in solution on oxalate of oxide of ethyle, or oxalio ether. (See oxalate of oxide of ethyle.)

The remarkable character of oxamide is, that while itself neutral, and certainly containing neither oxalic acid nor ammonia, it is easily converted into oxalic acid and ammonia by boiling it either with strong acids or strong alkalies. In this reaction, the elements of 1 eq. of water are shared between the constituents of oxamide, that is, between the radical $\mathrm{C}_{2} \mathrm{O}_{2}$ and the radical amide, $\mathrm{N} \mathrm{H}_{2}$; for oxamide is nothing more than oxalate of ammonia, $\mathrm{N} \mathrm{H}_{4} \mathrm{O}, \mathrm{C}_{2} \mathrm{O}_{3}$, minus 2 eqs. of water, or $\mathrm{N} \mathrm{H}_{2}, \mathrm{C}_{2} \mathrm{O}_{2}$. 
A very small portion of an acid, for example, is sufficient to produce this effect on a large quantity of oxamide; for if the acid we add be neutralised by the ammonia produced, a corresponding quantity of oxalic acid is set free, and acts as any other acid would do. A minute proportion of an acid, therefore, here appears to exert its influence on an unlimited portion of oxamide, but this is only in appearance. The fact, however, that the presence of a little oxalic acid enables oxamide to decompose water and to produce ammonia and oxalic acid, is very important, and tends to throw light on many similar changes in the organic kingdom, where the agency is not so apparent.

But oxamide is not the only product of the action of heat on oxalate of ammonia; for besides carbonic acid, carbonic oxide, hydrocyanic acid, water, ammonia, and oxamide, all of which are or may be formed, there is produced, when the heat is so regulated that a honey yellow residue remains in the retort, a new acid, called oxamic acid, which constitutes that residue. It is mixed with a little oxamide, which is, however, left undissolved by water, in which the oxamic acid dissolves.

Oxamic acid forms soluble and crystallisable salts with lime, baryta, ammonia, and oxide of silver. Acids precipitate it from the saturated solution of its compound with ammonia, as a white sparingly soluble powder, the composition of which is $\mathrm{C}_{4} \mathrm{H}_{2} \mathrm{NO}_{5}+$ aq. Although an acid, this compound exhibits all the relations of a compound of amide or amidogen, being converted by the action of alkalies at a high temperature into oxalic acid and ammonia. It differs, however, from oxamide in yielding 2 eq. oxalic acid and 1 eq. ammonia; for 1 eq. of oxamic acid, plus $3 \mathrm{eq}$. of water, contains the elements of

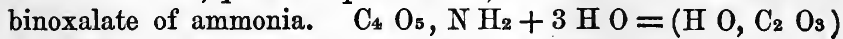
$+\left(\mathrm{N} \mathrm{H}_{4} \mathrm{O}, \mathrm{C}_{2} \mathrm{O}_{3}\right)$. This also explains its production;

for 2 eq. Oxalate of Ammonia $2\left(\mathrm{~N} \mathrm{H}_{4} \mathrm{O}, \mathrm{C}_{2} \mathrm{O}_{3}\right)=\mathrm{C}_{4} \mathrm{H}_{8} \mathrm{~N}_{2} \mathrm{O}_{8}$ when acted on by heat,

Yield 1 eq. ammonia

3 eq. water

1 eq. anbydrous oxamic acid

$$
\begin{aligned}
& =\mathrm{H}_{3} \mathrm{~N} \\
& =\mathrm{H}_{3} \mathrm{O}_{3} \\
& =\mathrm{C}_{4} \mathrm{H}_{2} \mathrm{~N} \mathrm{O}_{5}
\end{aligned}
$$

$$
\text { Together . - . } \mathrm{C}_{4} \mathrm{H}_{8} \mathrm{~N}_{2} \mathrm{O}_{8}
$$

Oxamic acid is certainly a very remarkable compound, being an acid amidide, or at least admitting of being so regarded, $\mathrm{C}_{4} \mathrm{O}_{5}, \mathrm{~N} \mathrm{H}_{2}$. There are many other examples of acid amidides, and we shall soon come to one, namely euchronic acid, which is highly analogous to oxamic acid: being formed by the action of heat on mellitate of ammonia, along with a neutral amidide, 
paramide, similar to oxamide; and as oxamic acid yields acid oxalate, so euchronic acid yields acid mellitate of ammonia when long boiled with water. Cyanic acid may also be considered as, in some sense, an acid amidide; for $\mathrm{C}_{2} \mathrm{~N} \mathrm{O}, \mathrm{H} \mathrm{O}=\mathrm{C}_{2} \mathrm{O}_{2}, \mathrm{~N} \mathrm{H}$; and $\mathrm{C}_{2} \mathrm{O}_{2}, \mathrm{NH}+2 \mathrm{HO}=\mathrm{N} \mathrm{H}_{3}, 2 \mathrm{C} \mathrm{O}_{2}$; or, as is well known, cyanic acid, in contact with water, produces bicarbonate of ammonia.

Oxamic acid may further be viewed as a coupled oxalic acid, the adjunct in which is oxamide; for $\mathrm{C}_{4} \mathrm{O}_{5} \mathrm{~N} \mathrm{H}_{2}$ is equal to $\mathrm{C}_{2} \mathrm{O}_{3}+\mathrm{C}_{2} \mathrm{O}_{2}, \mathrm{~N} \mathrm{H}_{2}$. Berzelius adopts this view, and is, generally speaking, favourable to the idea of coupled acids.

An alcoholio solution of oxalic ether, when ammonia is cautiously added till a precipitate of oxamide just begins to appear, yields a very beautifully crystallisable compound, long known as oxamethan, which is nothing else than oxamate of oxide of ethyle (see salts of oxide of ethyle). Its composition

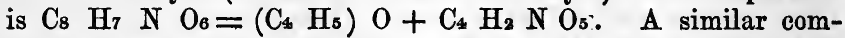
pound exists with oxide of methyle, and was formerly called oxamethylan.

\section{C $\triangle$ RBonio AcId. $\mathrm{C} \mathrm{O}_{2}=22$.}

This acid has been already described, and it is introduced here merely because it is formed by the complete oxidation of carbonic oxide; $\mathrm{C}_{2} \mathrm{O}_{2}+\mathrm{O}_{2}=2 \mathrm{C} \mathrm{O}_{2}$.

\section{CARBONIC OXIDE AND CHLORINE.}

Chlorocarbonic Acid. C $\left\{\begin{array}{l}0 \\ \mathrm{Cl}\end{array}\right.$ or $\mathrm{C} \mathrm{O} \mathrm{Cl}=49 \cdot 5$.

Syn.: Phosgene gas. When equal volumes of chlorine and carbonio oxide are mixed and exposed to the sun's rays, they combine to form a colourless gas, of a pungent disagreeable smell which acts strongly on the eyes. Sp. G. of the gas, 3.399. When dissolved in water, it decomposes it, producing carbonic and hydrochloric acids. With alcohol and methylic alcohol it produces very remarkable compound ethers, to be afterwards described.

With ammonia, this acid forms sal ammoniac, and a white volatile crystalline substance, which is carbamide, $\mathrm{C} 0, \mathrm{~N} \mathrm{H}_{2}$,

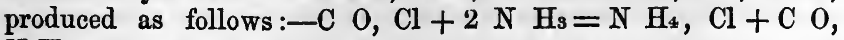
$\mathrm{N} \mathrm{H}$.

Under the influence of the mineral acids, carbamide yields ammonia and carbonic acid, $\mathrm{C} \mathrm{O}, \mathrm{N} \mathrm{H}_{2}+\mathrm{H} \mathrm{O}=\mathrm{C} \mathrm{O}_{2}+\mathrm{N} \mathrm{H}_{3}$. 
The chlorocarbonic acid may be considered as carbonic acid $\mathrm{C}+\left\{\begin{array}{l}0 \\ 0\end{array}\right.$ in which half the oxygen has been replaced by its equivalent of chlorine, $\mathrm{C}+\left\{\begin{array}{l}0 \\ \mathrm{Cl}\end{array}\right.$

\section{CARBONIC OXIDE WITH POTASSIUM.}

\section{Oxycarburet of Potassium : Rhodizonic Acid.}

When potassium is heated in carbonic oxide gas, combination takes place, and a dark olive powder is formed, composed of carbonic oxide and potassium, in the proportions of $\mathrm{C}_{7} \mathrm{O}_{7}+\mathrm{K}_{3}$, or $7 \mathrm{C} 0+3 \mathrm{~K}$. This substance is formed in large quantity in the preparation of potassium from carbonate of potash and charcoal, and is the source of great loss as well as inconvenience. No such compound is formed with sodium, for which reason that metal may be more cheaply prepared than potassium.

The oxycarburet of potassium, if heated in the air, takes fire, but if exposed to moist air, or placed in water, it is converted into the potash salt of a new acid, rhodizonic acid, hydrogen being disengaged, $\mathrm{C}_{7} \mathrm{O}_{7} \mathrm{~K}_{3}+3 \mathrm{H} \mathrm{O}=\mathrm{C}_{7} \mathrm{O}_{7}, 3 \mathrm{KO}+\mathrm{H}_{3}$. As this hydrogen, however, is not pure, but contains carbon, the reaction is probably more complicated.

All the salts of rhodizonic acid are deep red, and when in crystals, reflect a green light. The rhodizonate of potash, when heated in solution in water, undergoes a very remarkable change, yielding free potash, oxalate of potash and croconate of potash, the latter being the salt of another new acid containing the same elements as rhodizonic acid in different proportions; this salt is $\mathrm{C}_{5} \mathrm{O}_{4}, \mathrm{~K} \mathrm{O}$ or $\mathrm{C}_{5} \mathrm{O}_{5}, \mathrm{~K}$. The composition of rhodizonate of potash explains this reaction perfectly, for $\mathrm{C}_{7} \mathrm{O}_{7}+3 \mathrm{~K} \mathrm{O}=\mathrm{K} 0$ $+\mathrm{K} 0, \mathrm{C}_{2} \mathrm{O}_{3}+\mathrm{K} \mathrm{O}, \mathrm{C}_{3} \mathrm{O}_{4}$.

\section{Croconic Acid. $\mathrm{C}_{5} \mathrm{O}_{4}, \mathrm{HO}$ ? or $\mathrm{C}_{5} \mathrm{O}_{5}, \mathrm{H}$.}

This acid is named from the yellow colour of its salts. It is obtained from the croconate of potash, prepared as above, by the action of fluosilicic acid, which separates the potash. The acid is yellow, soluble in water and alcohol, and crystallises easily. All its salts are likewise yellow.

The rhodizonic acid, $\mathrm{C}_{7} \mathrm{O}_{7}, 3 \mathrm{H} 0$, may be viewed as a tribasic hydrogen acid, $\mathrm{C}_{7} \mathrm{O}_{10} \mathrm{H}_{3}$ : the croconic acid may also be viewed both as a hydrated oxygen acid, $\mathrm{C}_{5} \mathrm{O}_{4}, \mathrm{H} \mathrm{O}$, and as a hydrogen acid, $\mathrm{C}_{5} \mathrm{O}_{5}, \mathrm{H}$. In this last form it connects itself with earbonic oxide, as it may be $5 \mathrm{C} \mathrm{O}+\mathrm{H}$. The same remark applies to 
another remarkable acid, containing the same elements, namely, the mellitio acid.

Mellitic Acid. $\mathrm{C}_{4} \mathrm{O}_{3}, \mathrm{HO}$ or $\mathrm{C}_{4} \mathrm{O}_{4}, \mathrm{H}$.

This acid occurs, combined with alumina, in a very rare mineral, probably of organic origin, the mellite or honeystone. The acid is soluble, very sour, and permanent, not being altered by boiling nitric or sulphuric acids, nor by a heat of nearly $580^{\circ}$. The general formula of its salts, when dried at $212^{\circ}$, is $\mathrm{M} 0$, $\mathrm{C}_{4} \mathrm{O}_{4} \mathrm{H}$, or $\mathrm{H} \mathrm{O}, \mathrm{C}_{4} \mathrm{O}_{4} \mathrm{M}$. The salt of silver, however, at $212^{\circ}$, loses 1 eq. of water, and is left as $\mathrm{C}_{4} \mathrm{O}_{3}, \mathrm{Ag} \mathrm{O}$, or $\mathrm{C}_{4} \mathrm{O}_{4}, \mathrm{Ag}$. According to the latter formula, the radical in this salt, heated to $212^{\circ}$, is a form of carbonic oxide, $\mathrm{C}_{4} \mathrm{O}_{4}=4 \mathrm{C} 0$.

The crystallised acid $\mathrm{C}_{4} \mathrm{O}_{3}, \mathrm{H} \mathrm{O}$, or $\mathrm{C}_{4} \mathrm{O}_{4}, \mathrm{H}$, appears to unite with most bases without the separation of water, generally observed when salts are formed. And although the silver salt would seem to contain a different radical, yet it yields, when decomposed, the original mellitic acid.

The mellitate of silver may also be looked on as oxalate of silver, $\mathrm{Ag} \mathrm{O}, \mathrm{C}_{2}^{-} \mathrm{O}_{3}$, plus 2 eq. of carbon in the acid $\mathrm{Ag} \mathrm{O}_{4} \mathrm{C}_{4} \mathrm{O}_{3}$.

The mellitate of ammonia, $\mathrm{N} \mathrm{H}_{4} \mathrm{O}, \mathrm{C}_{4} \mathrm{H}_{3}$, when heated in a retort, yields several new and remarkable products. When this salt, $\mathrm{N} \mathrm{H}_{3}, \mathrm{H} \mathrm{O}, \mathrm{C}_{4} \mathrm{O}_{3}=\mathrm{C}_{4} \mathrm{H}_{4} \mathrm{~N} \mathrm{O}_{4}$ is heated to $320^{\circ}$, it gives off ammonia and water, and there remains a mixture of two new compounds; a soluble one which contains euchronic acid, in oombination with ammonia, and an insoluble one which is called paramide.

Paramide is a yellow solid, like elay. Its most remarkable character is, that, when long boiled with water, it is converted into bimellitate of ammonia. This is the character of an amide,

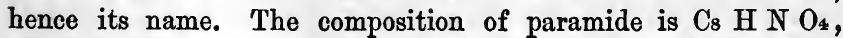
which readily explains both its formation, and its conversion into bimellitate of ammonia.

If from bimellitate of ammonia, $\mathrm{N} \mathrm{H}_{4} \mathrm{O}, \mathrm{C}_{4} \mathrm{O}_{3}+\mathrm{H} \mathrm{O}, \mathrm{C}_{4} \mathrm{O}_{3}=\mathrm{C}_{8} \mathrm{H}_{5} \mathrm{NO}_{3}$ we subtract 4 eq. of water . . . . . . $\mathrm{H}_{4} \mathrm{O}_{4}$

there will remain

Paramide $=\mathrm{C}_{8} \mathrm{H} \mathrm{NO}_{4}$

and of course, when converted into bimellitate of ammonia by long boiling, it merely takes up again these 4 eq. of water.

The soluble compound, euchronate of ammonia, when its solution is acted on by hydrochloric acid, deposits a white crystalline

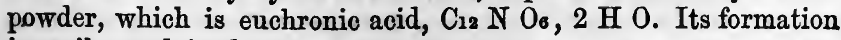
is easily explained; for 
if from 3 eq. of mellitate of ammonia $=3\left(\mathrm{C}_{4} \mathrm{H}_{4} \mathrm{NO}_{4}\right)=\mathrm{C}_{12} \mathrm{H}_{12} \mathrm{~N}_{3} \mathrm{O}_{12}$ we subtract 6 eq. water and 2 eq. ammonia there will remain anhydrous euchronic acid

$$
=\frac{\mathrm{H}_{12} \mathrm{~N}_{2} \mathrm{O}_{6}}{\mathrm{~N} \mathrm{O}_{6}}
$$

We can now see that the action of heat on 7 eq. of mellitate of ammonia gives rise to the following substances:-

$\begin{array}{ccc}\begin{array}{c}2 \text { eq. Paramide } \\ 1 \text { eq. Euchronic Acid }\end{array} & = & \mathrm{C}_{16} \mathrm{H}_{2} \mathrm{~N}_{2} \mathrm{O}_{3} \\ 4 \text { eq. Ammonia } & = & \mathrm{C}_{12} \mathrm{~N} \mathrm{O}_{6} \\ 14 \text { eq. Water } & = & \mathrm{H}_{12} \mathrm{~N}_{4} \\ 7 \text { eq. mellitate of ammonia } & = & \frac{\mathrm{H}_{14} \mathrm{O}_{14}}{\mathrm{C}_{28} \mathrm{H}_{28} \mathrm{~N}_{7} \mathrm{O}_{28}}\end{array}$

When euchronic acid is boiled with water, it is dissolved and converted into an acid mellitate of ammonia.

$\begin{array}{ccc}\begin{array}{c}1 \text { eq. Euchronic Acid } \\ \text { and } 6 \text { eq. Water }\end{array} & = & \mathrm{C}_{12} \mathrm{NO}_{6} \\ \text { Together } & \text {. } & \frac{\mathrm{H}_{6} \mathrm{O}_{6}}{\mathrm{C}_{12} \mathrm{H}_{6} \mathrm{NO}_{12}} \\ \begin{array}{c}3 \text { eq. Mellitic Acid } \\ \text { and } 1 \text { eq. Ammonia }\end{array} & =\frac{\mathrm{C}_{12} \mathrm{H}_{3} \mathrm{O}_{12}}{\mathrm{H}_{3} \mathrm{~N}} \\ \text { Together } & = & \frac{\mathrm{C}_{12} \mathrm{H}_{6} \mathrm{NO}_{12}}{}\end{array}$

Euchronic acid is deoxidised by a plate of zinc, yielding a powder of a fine deep blue colour, which dissolves in ammonia or potash, with a splendid tint of purple. The blue powder is an inferior oxide of the same nitrogenised radical, which, combined with more oxygen, forms euchronic acid. The whole subject of mellitic acid and euchronic acid is most interesting, but mellitic acid is so rare that it is very difficult to find material for the investigation. As mellitic acid, like succinic acid, is of organic origin, and contains only 1 eq. of hydrogen less, and 1 eq. of oxygen more than succinic acid, we may hope to be enabled to obtain it artificially.

$$
\text { III. Cranogen. } \mathrm{C}_{2} \mathrm{~N}=\mathrm{Cy}=26 \text {. }
$$

This very important compound has already been mentioned as a compound of carbon and nitrogen : but we have now to consider it in its far more important character of a compound radical. In fact, it was the first compound radical discovered, and the discovery of cyanogen by Gay-Lussac has proved more fertile in results than any other discovery yet made in Organic Chemistry. 
As cyanogen acts exactly like an element, we shall represent it by the symbol $\mathrm{Cy}$, rather than by $\mathrm{C}_{2} \mathrm{~N}$; using the latter only where the elements of cyanogen, and not itself, enter into changes and reactions.

Cyanogen is formed when animal matter is ignited along with carbonate of potash in close or covered iron vessels. The cyanogen being a gas, and combustible, would be dissipated, and in open vessels burned, were it not that it enters into combination with potassium derived from the carbonate, forming cyanide of potassium, $\mathrm{K} \mathrm{Cy}$, a salt not altered by a red heat in close vessels. As this salt, however, is decomposed by the action of water, yielding carbonate of potash and of ammonia, hydrogen being set free, $\left(\mathrm{K}, \mathrm{C}_{2} \mathrm{~N}+5 \mathrm{H} \mathrm{O}=\mathrm{K} \mathrm{O}, \mathrm{C} \mathrm{O}_{2}+\mathrm{N} \mathrm{H}_{3}\right.$, $\mathrm{H} \mathrm{O}, \mathrm{C} \mathrm{O}_{2}+\mathrm{H}$ ), it is necessary to convert the cyanide of potassium into a more stable compound. This is effected by the addition of iron, or of sulphuret of iron, the latter of which is formed by the mutual action of the sulphate of potash (always present in potashes), carbon, and the iron of the vessel. The iron, or its sulphuret, is readily dissolved by the aqueous solution of cyanide of potassium, yielding cyanide of iron, $\mathrm{Fe} \mathrm{Cy}$, and sulphuret of potassium $\mathrm{K} \mathrm{S}$, for $\mathrm{K} \mathrm{Cy}+\mathrm{Fe} \mathrm{S}=\mathrm{Fe} \mathrm{Cy}+\mathrm{K} \mathrm{S}$. The elements of the cyanide of iron then form, with cyanide of potassium, the very permanent double cyanide, well known as prussiate of potash, properly ferrocyanide of potassium, which forms large and pure transparent yellow crystals. From this compound, all the other compounds of cyanogen, and cyanogen itself, are prepared. Its empirical formula is $\mathrm{Fe} \mathrm{Cy}, 2 \mathrm{~K} \mathrm{Cy}+3$ $\mathrm{H} \mathrm{O}$, or $\mathrm{Fe} \mathrm{K}_{2} \mathrm{Cy}_{3}+3 \mathrm{H} \mathrm{O}$. At $212^{\circ}$ it loses all the water, and then contains only iron, potassium, and cyanogen $\mathrm{Fe}+\mathrm{K}_{2}+\mathrm{Cy}_{3}$. It may be conveniently viewed as a compound of cyanide of iron with cyanide of potassium; but we shall see hereafter that its rational formula is probably very different, and that it is a compound of potassium with a new radical, ferrocyanogen.

Cyanogen gas is best prepared by heating dried bicyanide of mercury, when the gas is given off, a part, however, assuming the solid form, and remaining behind as a black matter, paracyanogen, isomeric with cyanogen; or by heating a mixture of 6 parts dried ferrocyanide of potassium and 9 parts bichloride of mercury, when chloride of potassium is formed by the action of the bichloride on the cyanide of potassium of the ferrocyanide, and the cyanide of mercury, if formed, is at once decomposed by the heat. $\mathrm{Fe} \mathrm{Cy,} 2 \mathrm{~K} \mathrm{Cy}+\mathrm{Hg} \mathrm{Cl}_{2}=\mathrm{Fe} \mathrm{Cy}, 2 \mathrm{~K} \mathrm{Cl}+\mathrm{Hg}+\mathrm{Cy}_{2}$. The cyanide of iron is not altered. The gas may be collected over mercury, but is absorbed by water, with which it produces the various changes which have been already explained. 
It has a very pungent and peculiar smell, is colourless and transparent; and may be liquefied by a pressure of about 4 atmospheres. It is combustible, and burns with a beautiful pink or purplish flame, edged with yellow.

Cyanogen forms with hydrogen an acid, the hydrocyanic, $H$ $\mathrm{Cy}$; with oxygen and the elements of water, four acids, $\mathrm{Cy} \mathrm{O}$, $\mathrm{H} \mathrm{O}$; $\mathrm{Cy}_{2} \mathrm{O}_{2}, 2 \mathrm{H} \mathrm{O}$ : and $\mathrm{Cy}_{3} \mathrm{O}_{3}, 3 \mathrm{H} \mathrm{O}$; of which the first is cyanic, the second fulminic, and the third is cyanuric acid. The fourth acid has very recently been discovered by Liebig, which is isomerio with cyanuric acid. It is called fulminuric acid, because it is derived from fulminic acid.

Cyanogen combines with chlorine; with sulphur it forms a new radical $\mathrm{Cy} \mathrm{S}_{2}$, sulphocyanogen; and with metals it forms salts, perfectly analogous with chlorides, such as $\mathrm{K} \mathrm{Cy}, \mathrm{Fe} \mathrm{Cy}$, $\mathrm{Ag} \mathrm{Cy}, \mathrm{Hg} \mathrm{Cy}_{2}$, \&c. In short it plays exactly the part of a simple radical, and were it not easily decomposable, we should at once class it with chlorine as an element.

\section{CYANOGEN AND HYDROGEN.}

Hydrocyanic or Prussic Acid. H $\mathrm{Cy}=27$.

This acid may be obtained by a great variety of processes; but the easiest, most economical, and most certain, is to act on the ferrocyanide of potassium with diluted sulphuric acid. The process requires to be slightly modified, according as our object is to prepare the dry or anhydrous acid, or the diluted aqueous solution of it used in medicine.

1. Anhydrous Acid.-To prepare this acid, 15 parts of powdered ferrocyanide are to be distilled at a gentle heat, with a mixture of 9 parts of oil of vitriol, and 9 of water, and the product is to be received in a convenient receiver placed in a freezing mixture, and containing 5 parts of chloride of calcium in small lumps. As soon as liquid enough has distilled to cover the chloride, the distillation is stopped, and the hydrocyanic acid, deprived of water by the chloride of calcium, is to be decanted into a dry and well-stopped bottle. It may also be obtained by distilling, under similar circumstances, cyanide of potassium with dilute sulphuric acid. In both cases the acid is formed by the reaction of sulphuric acid on cyanide of potassium, or its elements, $\mathrm{K} \mathrm{Cy}+\mathrm{H}, \mathrm{S} \mathrm{O}_{4}=\mathrm{K}, \mathrm{SO}_{4}+\mathrm{H} \mathrm{Cy}$. Dry hydrocyanic acid is a limpid and colourless liquid, of Sp. G. 0.6967 at $66^{\circ}$; at $59^{\circ}$ it becomes a fibrous mass, in consequence of the presence of a trace of water; and at $80^{\circ}$ it boils ; the density of its vapour is 0.9476 . It is inflammable, and has a very peculiar and disagreeable smell 
and taste. It is the most energetic poison known, one drop introduced into the mouth being sufficient to destroy an animal of considerable size. When pure, it is often soon spontaneously decomposed, depositing a dark brown solid; a trace of sulphurio acid is said to cause it to keep perfectly; but we have seen various specimens of it, all prepared alike, some of which have kept well for years, while others have deposited much brown solid matter, and burst the tubes by the gas disengaged. When in contact with the strong mineral acids and water, it is decomposed, being converted into ammonia and formic acid; $\mathrm{H}, \mathrm{C}_{2} \mathrm{~N}+$ $4 \mathrm{HO}=\mathrm{NH}_{3}, \mathrm{HO}+\mathrm{C}_{2} \mathrm{HO}_{3}$.

2. Medicinal or diluted Hydrocyanic Acid.-This may be prepared by simply diluting the anhydrous acid with the required proportion of water, adding, for example, 97 grains of water to 3 of the acid, to obtain an acid of 3 per cent.; which is about the average strength used in medicine. In round numbers, to one part, by weight, of dry acid, $32 \frac{1}{3}$ parts of water, by weight, are to be added: or, to 1 volume of anhydrous acid, $22 \frac{1}{2}$ volumes of water. But as it is troublesome to prepare the anhydrous acid, it is best to distil 2 parts of ferrocyanide, with 1 of sulphuric acid and 2 of water, to dryness in a chloride of calcium bath,

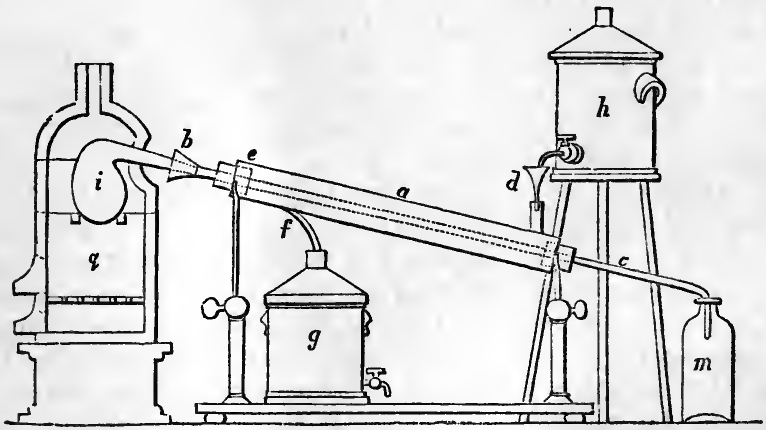

condensing in a Liebig's apparatus (see woodcut), in the receiver of which 2 more parts of water are placed. By this means we obtain $4 \frac{1}{2}$ parts of an acid, not anhydrous, but far too strong for use, containing from 15 to 20 per cent of dry acid. Its precise strength is ascertained, and it is reduced to the standard strength, in the following simple manner :-

Any convenient quantity, say 50 or 100 grains, is weighed out, being added to a counterpoised vessel containing about 2 
drachms of nitrate of silver, dissolved in 2 or 3 ounces of water. Let us suppose that we have dropped into this vessel 70 grains of our acid. This will be entirely converted into cyanide of silver, but we make sure by testing with a drop of nitrate of silver. The precipitate is then collected on a filter, well washed, dried at $212^{\circ}$ on a weighed filter, and the increase in weight of the filter gives the weight of the cyanide of silver formed. Now this cyanide is formed as follows: $\mathrm{H} \mathrm{Cy}+(\mathrm{Ag} 0$, $\left.\mathrm{N} \mathrm{O}_{5}\right)=\mathrm{Ag} \mathrm{Cy}+\left(\mathrm{HO}_{2} \mathrm{NO}_{5}\right)$. Therefore, 1 eq. of hydrocyanic acid, $\mathrm{H} \mathrm{Cy}=27$ produces 1 eq. cyanide of silver $\mathrm{Ag} \mathrm{Cy}=$ 134; or 1 grain of anhydrous hydrocyanic acid will yield almost exactly 5 grains of cyanide of siiver; for 23: 134:: $1: 4 \cdot 9$. We may, therefore, safely assume that the weight of the cyanide of silver, divided by 5 , gives the weight of anhydrous acid present with sufficient accuracy for all practical purposes. Now, we have used 70 grains of our dilute acid, the strength of which we wish to know. Let us suppose that our filter weighs, when empty, 20 grains, and with the cyanide of silver, dried at $212^{\circ}$ till it ceases to lose weight, 100 grains. The difference, or 80 grains, is the weight of cyanide of silver obtained from 70 grains of our acid. Dividing this by 5 , we have 16 grains as the weight of anhydrous acid contained in the 70 grains, and consequently combined with 54 of water.

Now, if we wish to state the per-centage of this acid, we obtain $16+100$

it by the calculation, $70: 16:: 100: x$, and $x=\frac{16+100}{}=22 \cdot 85$, 70

so that our acid contains 22.85 per cent. of anhydrous acid. But if our only object be to reduce the acid to a standard strength, say that of 3 per cent., this last calculation is unnecessary, and we can proceed as follows : acid of 3 per cent. contains 3 grains of dry acid and 97 of water; therefore, to find how much water is to be added to 16 grains of anhydrous acid, $3: 97:: 16: x$, $97+16$

and $x=\frac{}{3}=517 \cdot 3$ grains of water, which must be added

to 16 grains of anhydrous acid, to bring it to the same strength. But our 70 grains of acid contain already, with the 16 of anhydrous acid, 54 grains of water, consequently we have only to add to these 70 grains $517 \cdot 3-54=463 \cdot 3$ grains of water, and the same quantity of water for every 70 grains of the same acid. Of course, it is easy to calculate the water necessary for 1 or more ounces of the acid, when we bave once found it for any given quantity. I have here supposed 70 grains, but with 50 or 100 the calculation is easier, and with a drachm by weight 
(60 grains), we have simple data for calculating how much water is required for any number of ounces or drachms, troy, of acid.

This beautiful and simple method of preparing the medicinal hydrocyanic acid, and ascertaining its precise strength, has been minutely described, because of its practical importance. It is so simple an operation, that any one may very soon learn to ascertain the strength of hydrocyanic acid, and it is very exact. Besides, no other method of obtaining a medicinal acid of uniform strength ought to be trusted to; and we ought never to attempt to obtain the acid of the standard strength by distillation, although many methods are given for this. I have never seen any one of these yield twice the same result; whereas by the above method we can prepare acid of exactly the same strength any number of times, and the acid prepared from the ferrocyanide, by sulphuric acid, keeps perfectly well. Of course, when we have added the calculated quantity of water to reduce the acid, it is proper to asccrtain its strength once more, to make sure that we have made no error in our calculation. If it be acid of 3 per cent., it will yield 15 grains of cyanide of silver from 100 of acid.

There are two other methods which deserve to be mentioned, as, with pure materials and careful manipulation, they yield, in a few minutes, a standard acid. The first is that of Dr. Clarke, who adds to 1 eq. cyanide of potassium dissolved in water, 2 eq. Tartaric acid, which separates the potassium as cream of tartar, while diluted hydrocyanic acid remains dissolved. For every 100 grains of water, $7 \frac{1}{3}$ grains of cyanide of potassium and $16 \frac{1}{2}$ of crystallised tartaric acid, are required. This is an excellent extemporaneous process, if we have pure cyanide of potassium, but that salt does not keep well, and even in its preparation it is apt to be decomposed, at least partially. It is, besides, an expensive salt. The other is the process of Everett, who suspends cyanide of silver in water, and adds an equivalent of hydrochloric acid. $\mathrm{AgCy}+\mathrm{H} \mathrm{Cl}=\mathrm{AgCl}+\mathrm{H} \mathrm{Cy}$. This is also a good extemporaneous process, 15 grains of $\mathrm{Ag}$ Cy being used for every 100 of water, and 4 grains of dry $\mathrm{H} \mathrm{Cl}$, that is, about 12 grains of acid Sp. G. 1.21, being added. This process is also expensive, although the silver is not lost; but the chief objection is, that it is difficult to add the precise amount of hydrochloric acid which is necessary, and that an excess causes, pro tanto, a conversion of the hydrocyanic acid into formic acid and ammonia.

The medicinal acid has the smell and taste of the anhydrous, and is very poisonous, the average dose safe for an adult being 1 to 2 drops in a glass of water. It is much used as a sedative 
and anodyne, but, unless its strength and dose be perfectly known, it is a dangerous remedy. Fatal accidents have occurred from prescriptions, found, after experience, to act favourably, being made up in another place, or by the same druggist with a fresh stock; this fresh stock being exactly of the standard strength, while the previous acid had lost so much by keeping that the dose had been of necessity increased. There, danger actually arose from a too weak acid having been used. Hence the necessity for the great exactness, for which rules are given above. In cases of poisoning by this acid, now unfortunately of very frequent occurrence, there is seldom time to administer an antidote; but when life is not extinct, we may confidently rely on the antidotes we possess. The best is the administration of two solutions, one of mixed sulphate of protoxide and peroxide of iron, and the other of carbonate of potash, as recommended by Messrs. Smith, Edinburgh, ${ }^{*}$ by which the acid still in the stomach is rendered insoluble, being converted into Prussian blue. The symptoms already produced are best combated by ammonia inspired from a sponge, or taken, diluted, internally, and by other diffusable stimulants; also by the cold affusion.

Hydrocyanic acid is very easily recognised by its smell, and by its forming Prussian blue if acted on, in solution, successively, by proto-persulphate of iron, by potash, and by an excess of hydrochloric acid. The first two tests form the Prussian blue; the last, dissolving the excess of oxide of iron, brings the blue into view. Any liquid, suspected to contain hydrocyanio acid, ought first to be distilled with the addition of a little dilute sulphuric acid, and the tests applied to the first ounce that comes over. Nitrate of silver produces a white cloud of cyanide of silver, exactly like the chloride in appearance.

Hydrocyanic acid, with metallic oxides, give rise to water and metallic cyanides. $\mathrm{H} \mathrm{Cy}+\mathrm{M} \mathrm{O}=\mathrm{H} \mathrm{O}+\mathrm{M}$ Cy: or $3 \mathrm{H} \mathrm{Cy}+$ $\mathrm{MO}_{3}=3 \mathrm{H} \mathrm{O}+\mathrm{M} \mathrm{Cys}_{\text {. }}$

\section{CYANOGEN AND OXYGEN.}

1. Cranic Acid. Cy $\mathrm{O}, \mathrm{HO}=\mathrm{Cy} \mathrm{O}_{2}, \mathrm{H}=43$.

A monobasic acid; is formed when dry cyanide of potassium is heated in the air, when oxygen is absorbed, and cyanate of potash is produced. $\mathrm{K} \mathrm{Cy}+\mathrm{O}_{2}=\mathrm{KO}, \mathrm{Cy} \mathrm{O}$ or $\mathrm{K}, \mathrm{Cy} \mathrm{O}_{2}$.

When a stronger acid is added to this salt, the cyanic acid is set free, but immediately decomposes with water, producing ammonia, which unites with the strong acid used, and carbonic 
acid which escapes as gas. $\mathrm{C}_{2} \mathrm{~N} \mathrm{O}, \mathrm{HO}+2 \mathrm{H} \mathrm{O}=\mathrm{N} \mathrm{H}_{3}+2$ $\mathrm{C} \mathrm{O}_{2}$. The carbonic acid oarries with it a little cyanic acid, which forms dense white vapours, and has a pungent acid smell like that of strong acetic acid. Under all circumstances, free cyanic acid, in contact with water, is instantly destroyed.

It may, however, be obtained in the anhydrous state, according to the formula $\mathrm{Cy}_{2}+\mathrm{H}$, or as monohydrated acid, if viewed as $\mathrm{Cy} \mathrm{O}, \mathrm{H} \mathrm{O}$, by distilling cyanuric acid, $\mathrm{Cy}_{3} \mathrm{O}_{6}+\mathrm{H}_{3}$, or $\mathrm{Cys}_{\mathrm{O}} \mathrm{O}+3 \mathrm{H} \mathrm{O}$. This acid is polymeric with cyanic acid, and when heated, 1 eq. cyanuric acid splits up into 3 eq. cyanio acid, which appears in the receiver as a volatile, pungent, very corrosive acid liquid. This acid only keeps for a very short time, even in the freezing mixture in which it is condensed. If removed from the cold, it soon becomes turbid, then hot, boils violently, and with violent shocks, and is converted into a solid dense white body, like porcelain, quite insoluble and indifferent.

This is Cyamelide, another polymeric compound, containing the same elements in the same proportions, hut differently arranged, possibly $\mathrm{C}_{2} \mathrm{O}_{2}+\mathrm{NH}$, that is, bicarbimide, for it yields, under the influence of water and strong acids, carbonic acid and ammonia, $\left(\mathrm{C}_{2} \mathrm{O}_{2}, \mathrm{NH}+2 \mathrm{H} \mathrm{O}=2 \mathrm{C} \mathrm{O}_{2}+\mathrm{NH}_{3}\right)$ just as cyanic acid does. When distilled, it is reconverted into cyanic acid, another proof that it is polymeric with that acid.

\section{CYaNATES.}

The salts of cyanic acid are all distinguished by the action on them of strong acids, which eause disengagement of carbonic acid, while ammonia may now be detected in the liquid. The cyanates of potash, ammonia, \&c., are soluble; those of lead, silver, \&c., insoluble.

Cyanate of Potash is best formed by the oxidation of Liebig's cyanide of potassium, * which may easily be effected by adding litharge in proper quantity to the melted salt, $\mathrm{K} \mathrm{Cy}+2 \mathrm{PbO}$. $=\mathrm{K}$, $\mathrm{Cy}_{2}+\mathrm{Pb}_{2}$. The cooled mass is powdered and boiled with alcohol of 80 per cent., which on cooling deposits pure crystals of cyanate of potash, very similar to chlorate of potash. Or dried ferrocyanide of potassium, mixed with half its weight of peroxide of manganese, may be gently heated, spread out on an iron plate, when it burns like tinder, partly at the expense of the oxide of manganese, partly in the oxygen of the air. It is well stirred till every part has glowed, and the cold mass is treated with alcohol as above.

\footnotetext{
* The formation of this salt will be described below.
} 
Cyanate of potash must be kept in sealed tubes, for in phials occasionally opened it is soon changed into bicarbonate of potash, ammonia being given off. $\mathrm{K}, \mathrm{C}_{2} \mathrm{~N} \mathrm{O}_{2}+3 \mathrm{HO}=$ $\left(\mathrm{K} 0,2 \mathrm{C} \mathrm{O}_{2}\right)+\mathrm{N} \mathrm{H}_{3}$ : Triturated with dried oxalic acid, this salt yields oxalate of potash and cyamelide. When acetic acid is added to a concentrated freshly made solution of cyanate of potash, the latter being in excess, there is deposited the acid cyanurate of potash,

$$
\mathrm{Cy}_{3} \mathrm{O}_{6}\left\{\begin{array} { l } 
{ \mathrm { K } } \\
{ \mathrm { H } _ { 2 } }
\end{array} \text { or } \mathrm { Cy } _ { 3 } \mathrm { O } _ { 3 } \left\{\begin{array}{rl}
\mathrm{K} & \mathrm{O} \\
2 \mathrm{H} \mathrm{O} & \mathrm{O}
\end{array}\right.\right.
$$

CYANATE OF AMMONIA.

a. basic. When dry ammonia and the vapour of cyanic acid are mixed, they form a light white solid, which is a cyanate of ammonia, containing more ammonia than is required for a neutral salt. This salt gives off ammonia when treated with alkalies, and cyanic acid when treated with sulphuric acid. But if dissolved in water, and the solution digested and evaporated, it yields crystals, which, although containing the elements of cyanic acid, ammonia, and water, exhibit neither of these characters of a cyanate, but are found to possess all the properties of urea, a product of the animal system.

b. Anomalous cyanate of ammonia, or Urea, $\mathrm{C}_{2} \mathrm{~N}_{2} \mathrm{H}_{4} \mathrm{O}_{2}=$ $\left(\mathrm{C}_{2} \mathrm{~N} \mathrm{O}, \mathrm{H} \mathrm{O}, \mathrm{N} \mathrm{H}_{3}\right)$. This remarkable compound exists in large proportion in healthy urine, and is extracted from it by evaporating it about $200^{\circ}$ to a thin syrup, and adding about an equal volume of colourless nitric acid, Sp. G. 1.35, quite free from nitrous acid, which forms a very copious crystallisation of nitrate of urea, while the colouring matter is destroyed with brisk effervescence. If cold be applied, the colouring matter resists, and the nitrate is then very dark and very difficult to purify; cold ought, therefore, not to be used, and the nitrate of urea is deposited nearly white, having only a clear yellow tint. It is dissolved in water, after being recrystallised, and neutralised by potash or baryta. The whole is then gently evaporated to dryness, after separating as much nitrate of potash or of baryta as possible, and the dry mass digested in alcohol, which dissolves only the urea, and by spontaneous evaporation yields it in large transparent prismatic crystals. Should these not be colourless, the digestion of their aqueous solution with a little permanganate of potash, which has no action on urea, destroys the colouring matter entirely. Should an excess of that salt be added, a few drops of alcohol will instantly destroy it, and the filtered liquor will yield snow-white crystals of urea. 
But although urea may thus be obtained (or by the action of oxalio acid on the urine, which forms a sparingly soluble oxalate of urea) in any quantity from urine, it is found much easier to prepare it artificially from cyanate of ammonia. Liebig recommends the following process, which I have found to succeed perfectly : 28 parts of dried ferrocyanide of potassium, and 14 of peroxide of manganese, are mixed in powder and calcined, as above described, on a flat iron plate at a very low red heat, suffcient to keep up the glow which takes place. When this is over, the cold mass, powdered, is acted on by a moderate quantity of cold water, which dissolves the cyanate of potash. This is filtered off, and set aside. A fresh portion of cold water being added to the powder, to wash it, is also filtered, and in this liquid are now dissolved $20 \frac{1}{2}$ parts of sulphate of ammonia, and the solution is added to the first filtered solution of the cyanate. A large quantity of sulphate of potash is deposited, which is strained off, and the filtered liquid now containing, with some sulphate of potash, all the cyanate of ammonia, is evaporated to dryness, during which process the cyanate of ammonia is transformed into urea. The dry mass is digested in alcohol, which dissolves only the urea, and yields it from the first chemically pure and in any quantity, if the operation be carefully performed. Urea thus obtained is far cheaper than that extracted from urine.

The artificial production of urea from cyanate of ammonia was discovered by Wöhler. It was, with the exception of oxalic acid, and of hydrocyanic acid, the first example of an organic product artificially formed, although many other cases are now known.

Urea forms 4-sided prisms, resembling nitre in appearance, and also in their taste, which is saline and cooling, exactly like that of nitre. It is soluble both in water and alcohol. When heated, it melts, gives off much ammonia, and finally solidifies, being in a great measure converted into ammonia and cyanuric acid.

Urea belongs to the class of organic bases, for it forms crystallisable compounds with several acids, such as nitric, oxalic, and acetic acids.

The nitrate, when impure, crystallises in scales of a high lustre ; when pure, it forms opaque prisms, or a crystalline powder. It is sparingly soluble in cold water, but very soluble in hot water. Formula, ( $\mathrm{C}_{2} \mathrm{~N}_{2} \mathrm{H}_{4} \mathrm{O}_{2}, \mathrm{H} \mathrm{O}, \mathrm{N} \mathrm{O}_{5}$ ).

The oxalate forms long transparent prisms, very sparingly soluble. Formula, $\left(\mathrm{C}_{2} \mathrm{~N}_{2} \mathrm{H}_{4} \mathrm{O}_{2}, \mathrm{H} \mathrm{O}, \mathrm{C}_{2} \mathrm{O}_{3}\right)=\mathrm{C}_{4} \mathrm{~N}_{2} \mathrm{H}_{5} \mathrm{O}_{6}$.

The acetate $I$ have obtained as a mass of prismatic crystals, exceedingly soluble in water. Formula, probably $\left(\mathrm{C}_{2} \mathrm{~N}_{2} \mathrm{H}_{4} \mathrm{O}_{2}\right.$, $\left.\mathrm{H} \mathrm{O}, \mathrm{C}_{4} \mathrm{H}_{3} \mathrm{O}_{3}\right)=\mathrm{C}_{6} \mathrm{~N}_{2} \mathrm{H}_{8} \mathrm{O}_{6}$. 
Although urea combines with pure nitric acid, it is instantaneously decomposed by hyponitrous acid, yielding ammonia, water, and equal volumes of carbonic acid and nitrogen gases. $\mathrm{C}_{2} \mathrm{~N}_{2} \mathrm{H}_{4} \mathrm{O}_{2}+\mathrm{N}_{3}=\mathrm{NH}_{3}+\mathrm{HO}+2 \mathrm{CO}_{2}+\mathrm{N}_{2}$. When acted on by strong acids, or alkalies, with the aid of heat, urea takes up the elements of water, and forms carbonate of ammonia, $\mathrm{C}_{2} \mathrm{~N}_{2} \mathrm{H}_{4} \mathrm{O}_{2}+2 \mathrm{H} \mathrm{H}=2\left(\mathrm{~N} \mathrm{H}_{3}, \mathrm{C} \mathrm{O} \mathrm{O}_{2}\right)$. It may therefore be regarded as a double molecule of carbamide for $\mathrm{C}_{2} \mathrm{~N}_{2} \mathrm{H}_{4} \mathrm{O}_{2}=2\left(\mathrm{~N} \mathrm{H}_{2}, \mathrm{C} 0\right)$. When urine is left in contact with the mucus usually suspended in it, the mucus entering into decomposition excites in the urea such a reaction with the elements of water, as very soon to convert the whole urea into carbonate of ammonia. Hence the reason why urine so soon becomes alkaline, even if acid when voided. But if filtered from the mucus as soon as passed, it keeps unchanged, in clean vessels, for a long period.

\section{Fulminio Acid. $\mathrm{Cy}_{2} \mathrm{O}_{2}, 2 \mathrm{HO}=\mathrm{Cy}_{2} \mathrm{O}_{4}, \mathrm{H}_{2}=86$.}

A bibasic acid, unknown in the separate form. It is obtained in combination with oxide of mercury, or oxide of silver, by treating alcohol with the nitrates of these metals, and free nitric acid. A violent effervescence takes place, dense white vapours are disengaged, and a crystalline powder is deposited, which is fulminate of mercury or of silver. Both detonate powerfully by heat, friction, or percussion.

In the above reaction there are first formed, on the one hand, hyponitrous acid; on the other, aldehyde, and formic and oxalic acids. The fulminic acid is the result of a reaction between oxide of ethyle (ether) and hyponitrous acid, in presence of oxide of mercury or oxide of silver. $2 \mathrm{~N} \mathrm{O}_{3}+\left(\mathrm{C}_{4} \mathrm{H}_{5}\right) \mathrm{O}=\mathrm{C}_{4} \mathrm{~N}_{2} \mathrm{O}_{2}+$ $5 \mathrm{H} \mathrm{O}=\mathrm{Cy}_{2} \mathrm{O}_{2}, 2 \mathrm{H} \mathrm{O}+3 \mathrm{H} \mathrm{O}$.

This acid cannot be isolated, being instantly decomposed when deprived of a fixed base. It forms two series of salts : neutral, with 2 eq. of fixed base, $\mathrm{Cy}_{2} \mathrm{O}_{2}, 2 \mathrm{M} \mathrm{O}$; and acid, with 1 eq. of fixed base and 1 of water, $\mathrm{Cy}_{2} \mathrm{O}_{2} ;,\left\{\begin{array}{ll}\mathrm{H} \mathrm{O} \\ \mathrm{M} \mathrm{O}\end{array}\right.$. It also forms salts with 1 eq. of two different bases, of which one is always easily reducible, as oxide of silver, mercury, and copper, while the other may be difficult to reduce, such as baryta, potash, \&c.

The fulminates of silver and mercury, $\mathrm{Cy}_{2} \mathrm{O}_{2}, 2 \mathrm{Ag} \mathrm{O}$, and $\mathrm{Cy}_{2} \mathrm{O}_{2}, 2 \mathrm{Hg} \mathrm{O}$, are examples of the first class. The acid fulminate of zino $\mathrm{Cy}_{2} \mathrm{O}_{2}\left\{\begin{array}{l}\mathrm{H} \mathrm{O} \\ \mathrm{ZnO}\end{array}\right.$, formerly supposed to be fulminic acid, is an example of the second; and the double 
fulminates of copper and silver, and of potash and silver, $\mathrm{Cy}_{2} \mathrm{O}_{2}\left\{\begin{array}{ll}\mathrm{Cu} & \mathrm{O} \\ \mathrm{Ag} & \mathrm{O}\end{array}\right.$, and $\mathrm{Cy}_{2} \mathrm{O}_{2}\left\{\begin{array}{ll}\mathrm{K} & \mathrm{O} \\ \mathrm{Ag} & \mathrm{O}\end{array}\right.$ are examples of the third. No neutral fulminates exist with 2 eq. of a difficultly reducible oxide, such as potash, soda, baryta, \&c. : nor do any acid fulminates occur with such bases. These very remarkable facts evidently point to some relation, not yet understood, between the oxygen of the base in a salt and the acid of that salt. It will be seen that the hydrated acid is polymeric with cyanic acid, for $\mathrm{Cy}_{2} \mathrm{O}_{2}, 2 \mathrm{H} \mathrm{O}=2$ (Cy O, $\mathrm{H} \mathrm{O}$ ).

Liebig recommends to dissolve 3 parts of mercury in 36 parts of nitric acid, of Sp. G. 1·34, without heat, in a large flask, capable of containing about 20 times the volume of the acid. The nitrous acid vapours which are formed remain in the flask. The solution is then poured into another vessel, containing 17 parts of alcohol, and the mixture at once returned to the flask, where it is to be well agitated with the red vapours, which it in great measure absorbs.

After 5 or 10 minutes, bubbles of gas begin to rise, and a heavy, highly refractive liquid appears in the bottom of the flask. This is mixed with the rest by agitation, and after a short time, the liquid becomes dark from the separation of a little metallic mercury. A very turbulent reaction now begins, giving out thick white vapours in large quantity, with traces of nitrous vapours. The action is now to be moderated by the gradual addition of $\mathbf{1 7}$ parts more of the same alcohol. The dark colour now disappears, and the crystals of the fulminate of mercury begin to separate. At first they are in part brought to the surface by the gas disengaged, but the addition of the alcohol causes them to settle, and the liquid is easily decanted from them, when the whole is cold. No heat is applied externally during this process, which yields the whole mercury in the form of fulminate, amounting to 4.6 parts from 3 of mercury, and purer than by the usual process.

This salt, and the fulminate of silver, prepared in the same way, are very dangerous compounds, exploding by slight friction or percussion, as well as when heated, and sometimes from very slight causes, almost spontaneously. An explosion of this kind caused the death of a distinguished chemist, Mr. Hennell, who was preparing a large quantity of fulminating mercury, to be used for percussion caps, in the open air. Caustic alkalies precipitate from the solution of these fulminates half the metal, forming double fulminates; and chlorides also only precipitate half the silver. Nitric acid, added to the solution, causes a deposit of acid fulminate of silver, 


$$
\mathrm{Cy}_{2} \mathrm{O}_{2}\left\{\begin{array} { l l } 
{ \mathrm { H } } & { \mathrm { O } } \\
{ \mathrm { Ag } } & { \mathrm { O } }
\end{array} \text { or } \mathrm { Cy } _ { 2 } \mathrm { O } \mathrm { O } _ { 4 } \left\{\begin{array}{l}
\mathrm{H} \\
\mathrm{Ag}
\end{array}\right.\right.
$$

Hydrochloric acid added to fulminate of silver, gives rise to water, chloride of silver, hydrocyanic acid, and a new acid, chlorohydrocyanic acid $\mathrm{C}_{2} \mathrm{~N} \mathrm{Cl}_{5}, \mathrm{H}_{2}$. Thus-

$$
\mathrm{C}_{4} \mathrm{~N}_{2} \mathrm{O}_{2}, 2 \mathrm{Ag} \mathrm{O}+7 \mathrm{HCl}=4 \mathrm{HO}+2 \mathrm{AgCl}+\mathrm{C}_{2} \mathrm{NH}+\mathrm{C}_{2} \mathrm{~N} \mathrm{Cl}_{5} \mathrm{H}_{2} \text {. }
$$

\section{Cranurio Aaid. $\mathrm{Cy}_{3} \mathrm{O}_{3}, 3 \mathrm{HO}=\mathrm{Cy}_{3} \mathrm{O}_{6}, \mathrm{H}_{3}=129$.}

A tribasic acid discovered among the products of the distillation of uric acid. It is formed when solid chloride of cyanogen $\mathrm{Cy}_{3}$ $\mathrm{Cl}_{3}$ acts on water; $\mathrm{Cy}_{3} \mathrm{Cl}_{3}+6 \mathrm{H} \mathrm{O}=3 \mathrm{H} \mathrm{Cl}+\mathrm{Cy}_{3} \mathrm{O}_{6}, \mathrm{H}_{3}$. It is also formed when urea is heated so as to expel its ammonia. According to what has been stated of the composition of urea, it ought to yield cyanic acid when deprived of its ammonia; but at that temperature 3 eq. of cyanic acid, $3(\mathrm{Cy} \mathrm{O}, \mathrm{H} \mathrm{O}$ ), coalesce to form 1 eq. of cyanuric acid, $\mathrm{Cy}_{3} \mathrm{O}_{3}, 3 \mathrm{H} \mathrm{O}$. When, again, acetic acid is added to cyanate of potash, in quantity insufficient to decompose the whole, there is deposited a salt which is an acid cyanurate of potash, $\mathrm{Cy}_{3} \mathrm{O}_{3}\left\{\begin{array}{l}\mathrm{K} \mathrm{O} \\ 2 \mathrm{H} \mathrm{O} .\end{array}\right.$

Finally, cyanuric acid is obtained by dissolving melame, ammeline, ammelide and melamine (see those substances) in sulphuric acid, and diluting and heating the solution, until it yields no precipitate with ammonia. On evaporation and cooling, the acid forms prismatic crystals, which are $\mathrm{Cy}_{3} \mathrm{O}_{3}, 3 \mathrm{H} \mathrm{O}+4 \mathrm{aq}$. When dissolved in hot nitric or hydrochloric acid, it is deposited, on cooling, in anhydrous octahedrons. When it has been prepared by heating urea it is purified by dissolving it in oil of vitriol, and adding nitric acid, drop by drop, till the colour is entirely destroyed. An equal bulk of water is then added, and, on cooling, pure cyanuric acid is obtained in crystals.

Cyanuric acid has a weak acid taste, and is sparingly soluble in cold water, more soluble in hot water. Unlike cyanic and fulminic acids, it is very permanent in the uncombined state. When heated in close vessels, it is entirely volatilised in the form of cyanic acid, of which 3 eq. are exactly equal to 1 eq. of cyanuric acid.

As cyanuric acid is formed when urea (cyanate of ammonia) is decomposed by heat, and when cyanate of potass is acted on by acetic acid, and as cyanuric acid, when heated, is resolved into cyanic acid, we have every reason to think that in the former cases, 3 eqs. of cyanic acid coalesce to form one of cyanurio acid; and that, in the latter case, 1 eq. of cyanuric acid is broken up into 3 eqs. of cyanic acid. 3 (Cy $\mathrm{O}, \mathrm{H} \mathrm{O})=\mathrm{Cy}_{3} \mathrm{O}_{3}, 3 \mathrm{H} \mathrm{O}$. 
Like other tribasic acids, cyanurio acid forms three series of salts, according to the formula :-

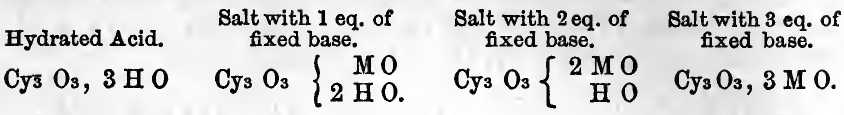

The salts with 1 and 2 eqs. of fixed base are acid; that with 3 eqs. is neutral. With potash and other similar oxides, only the two acid salts are known; with oxide of silver, the salts with 2 and 3 eqs. of oxide.

\section{The three following salts, namely,}

$\begin{array}{ccc}\text { Cyanate of Silver, } & \text { Fulminate of Silver, } & \text { Cyanurate of Silver, } \\ \mathrm{Cy} \mathrm{O}, \mathrm{Ag} \mathrm{O} ; & \mathrm{Cy}_{2} \mathrm{O}_{2}, 2 \mathrm{Ag} \mathrm{O} ; & \mathrm{Cy}_{3} \mathrm{O}_{3}, 3 \mathrm{Ag} \mathrm{O}\end{array}$

have exactly the same composition in 100 parts; and yet they are in properties entirely dissimilar ; this can only be accounted for by some such difference in their formulæ as is exhibited above. The acids of these salts are all mutually convertible: for when fulminate of silver is decomposed by a salt of ammonia, the fulminate of ammonia is transformed, like the cyanate, into urea; urea when heated yields cyanuric acid; and cyanuric acid when distilled is transformed into cyanic acid. All these circumstances favour the belief that all three are polymeric compounds of cyanogen, although Wöhler considers cyanuric acid to be bibasic, and its formula to be $\mathrm{C}_{6} \mathrm{~N}_{3} \mathrm{H} \mathrm{O}, 2 \mathrm{H}$.

$$
\text { Cyamelide; } \mathrm{C}_{2} \mathrm{O}_{2}+\mathrm{N} \text { H. (? Bicarbimide.) }
$$

But this is not the end of these transformations; for when cyanic acid is left to itself it becomes turbid and hot, and is soon converted with great heat, into an opaque white solid body, cyamelide, which has no acid properties; and which is also obtained as a white insoluble powder, when fused cyanate of potash is triturated with dried oxalic acid, in which case oxalate of potash is formed, and the cyanic acid, at the moment of being set free, is transformed into cyamelide. Cyamelide is neutral and insoluble in water and acids. It dissolves into aqua potassæ, ammonia being evolved, and the solution yields cyanurate of potash. Heated by itself it yields cyanic acid, which again passes into cyamelide; and when heated with strong sulphuric acid it forms sulphate of ammonia, while carbonic acid escapes: this action is identical with that which occurs when cyanic acid is acted on by water and by acids. All these observations prove that cyamelide is another isomeric modification of cyanic, fulminic, or cyanuric acid. 'Its probable formula is $\mathrm{C}_{2} \mathrm{O}_{2}+\mathrm{N} \mathrm{H}$, 
and this, with 2 eqs. H 0 , yields bicarbonate of ammonia, just as cyanic acid, the formula of which is the same, does. The action of potash is probably this. $4\left(\mathrm{C}_{2} \mathrm{O}_{2} \mathrm{~N} \mathrm{H}\right)+3 \mathrm{HO}+4 \mathrm{~K} \mathrm{O}=$ $\mathrm{C}_{6} \mathrm{~N}_{3} \mathrm{O}_{6},\left\{\begin{array}{r}2 \underset{\mathrm{H}}{\mathrm{K}} \mathrm{O} \\ \mathrm{O}\end{array}\right.$ cyamelide, 3 eqs. water, and 4 eqs. potash, yield 1 eq. cyanurate of potash, 2 eqs. carbonate of potash, and 1 eq. ammonia.

The three oxygen acids of cyanogen may be fitly compared with the three phosphorio acids; and as we have hitherto made use of the older view of these acids, that which makes them hydrated oxygen acids, we shall, in this comparison, adopt the other theory, and speak of them as hydrogen acids.

$\begin{array}{llcc}\text { Acids of Cyanogen } & \begin{array}{l}\text { Monobasic. } \\ \text { Acids of Phosphorus }\end{array} & \begin{array}{c}\text { Bibasic. } \\ \mathrm{Cy}_{2} \mathrm{O}_{4}+\mathrm{H}_{2}\end{array} & \begin{array}{c}\text { Tribasic. } \\ \mathrm{Cy}_{3} \mathrm{O}_{6}+\mathrm{H}_{3}\end{array} \\ \text {. } & \mathrm{P} \mathrm{O}_{6}+\mathrm{H} & \mathrm{P} \mathrm{O}_{7}+\mathrm{H}_{2} & \mathrm{P} \mathrm{O}_{8}+\mathrm{H}_{3}\end{array}$

In the cyanic acids, both the radical and the replaceable hydrogen being doubled and trebled, the capacity of saturation increases in the same ratio. In the phosphorio acids, the capacity of saturation also increases with the replaceable hydrogen, but a great addition to the radical has no corresponding effect in increasing either the replaceable hydrogen or the neutralising power. Thus, if to tribasic phosphoric acid $\mathrm{P} \mathrm{O}_{8}+\mathrm{H}_{3}$ we add 2 eqs. of $\mathrm{PO}_{5}=\mathrm{P}_{2} \mathrm{O}_{10}$ we obtain $\mathrm{P}_{3} \mathrm{O}_{18}+\mathrm{H}_{3}=3\left(\mathrm{P} \mathrm{O}_{6}+\mathrm{H}\right)$; that is, the acid becomes monobasic, but the quantity of base neutralised is the same after that addition as before. Notwithstanding this difference, however, the analogy between the monobasic, bibasic, and tribasic acids of cyanogen and phosphorus, as well as that between their salts, is most interesting and worthy of attention. As we believe that the phosphoric acids contain three different radicals, $\mathrm{P} \mathrm{O}_{6}, \mathrm{P} \mathrm{O}_{7}$ and $\mathrm{P} \mathrm{O}_{8}$, so we may admit that the three cyanio acids have each a distinct radical ; only in this case the three radicals are alike in composition, and differ in their relative weight: the first being $\mathrm{C}_{2} \mathrm{~N}=\mathrm{Cy}$; the second $\mathrm{C}_{4} \mathrm{~N}_{2}$ $=\mathrm{Cy}_{2} ;$ and the third $\mathrm{C}_{6} \mathrm{~N}_{3}=\mathrm{Cy}_{3}$; or, in other words, these radicals are cyanogens, differing in molecular constitution, or three different allotropic or polymeric forms of cyanogen. The relations of chlorine to cyanogen, and the existence of paracyanogen, countenance this idea.

Liebig has lately shown that from the salts of fulminic acid, by boiling with soluble chlorides, there are obtained salts of a new acid, the composition of which is the same as that'of cyanuric acid, but which is monobasic. It is called fulminuric acid, and its formula is Cys $\mathrm{Os}_{3}, 3 \mathrm{H} \mathrm{O}=\mathrm{Cys}_{3} \mathrm{H}_{2} \mathrm{O}_{s}, \mathrm{HO}$, or $\mathrm{C}_{6} \mathrm{H}_{2} \mathrm{Ns}_{s} \mathrm{O}_{s}, \mathrm{HO}$. 
The fulminurate of potash is got by boiling fulminate of mercury with water and chloride of potassium. The fulminate after a time dissolves, and subsequently a new reaction takes place, yellow hydrated oxide of mercury being separated. When the liquid has lost its turbid appearance, and the oxide has been entirely deposited, the whole is filtered, and the filtered liquid on evaporation yields crystals of fulminurate of potash. There are two stages in the process. In the first, 3 eqs. of fulminate of mercury and 3 of chloride of potassium yield 3 of fulminate of potash and 3 of chloride of mercury. Liebig represents fulminic acid as isomeric with cyanic acid, and therefore he writes fulminate of mercury, Cy $\mathrm{O}, \mathrm{Hg} \mathrm{O}$. Now,

$$
\begin{aligned}
& 3(\mathrm{Cg} \mathrm{O}, \mathrm{Hg} \mathrm{O})+3 \mathrm{~K} \mathrm{Cl}= \\
= & 3(\mathrm{Cg} \mathrm{O}, \mathrm{K} \mathrm{O})+3 \mathrm{Hg} \mathrm{Cl} .
\end{aligned}
$$

$\mathrm{He}$ also, like many foreign authors, makes the eq. of mercury 100, and consequently peroxide of mercury, as above, $\mathrm{Hg} \mathrm{O}$. I retain his formulæ, because $I$ think that this remarkable reaction is far more simply explained on his view than on ours.

In the second stage, 2 eqs. of water take a share, and

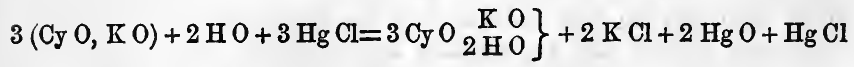

That is to say, 3 eqs. of fulminate of potash yield 1 eq. of fulminurate of potash, and the 2 eqs. of potash displaced by water act on 2 eqs. of chloride of mercury (bichloride as we call it), forming 2 eqs. of chloride of potassium and 2 eqs. of oxide of mercury, the third eq. of chloride of mercury remaining unchanged.

The fulminurates of soda, ammonia, '\&c., are obtained in the same way, using the chlorides of sodium and ammonium, \&c.

The fulminurates crystallise beautifully, and have a very high dispersive power, and consequently a lustre and play of colours similar to that of the diamond. They are not explosive, but are decomposed by heat with a rapidity short of explosive.

The formulæ of cyanuric and fulminuric acids, which are isomeric, differ in the amount of basic water or replaceable hydrogen.

Cyanuric acid $\mathrm{C}_{6} \mathrm{~N}_{3} \mathrm{O}_{3}, 3 \mathrm{H} \mathrm{O}$

or $\mathrm{C}_{6} \mathrm{~N}_{3} \mathrm{O}_{6}, \mathrm{H}_{3}$

Fulminuric acid $\mathrm{C}_{6} \mathrm{H}_{2} \mathrm{~N}_{3} \mathrm{O}_{5}, \mathrm{H} \mathrm{O}$

or $\mathrm{C}_{6} \mathrm{H}_{2} \mathrm{~N}_{3} \mathrm{O}_{6}, \mathrm{H}$

The.former is tribasic, the latter monobasic. The fulminate of 
potash will be exactly isomeric with the acid cyanurate of potash with 1 eq. of potassium and 2 of basic water.

We must here state that the true formula of fulminic acid is still uncertain ; for while Liebig makes it $\mathrm{Cy} \mathrm{O}, \mathrm{H} \mathrm{O}$, the same as cyanic acid, many chemists consider it, as we have done, to be double of this, $\mathrm{Cy}_{2} \mathrm{O}_{2}, 2 \mathrm{H} \mathrm{O}$, and to be bibasic.

\section{CYANOGEN AND NTTROGEN.}

Mellone $=\mathrm{C}_{18} \mathrm{~N}_{13}=\mathrm{Me}=290$.

This compound was discovered by Liebig, and by him named Mellone, and its formula was said to be $\mathrm{C}_{6} \mathrm{~N}_{4}=\mathrm{Cy}_{3}+\mathrm{N}$, that is, 3 eqs. of cyanogen, and 1 eq. of nitrogen, united to form a compound radical. Gerhardt maintained that it also contained hydrogen, but Liebig denied this, as its compound with potassium could be formed from substances containing no hydrogen, and the proportion of hydrogen found by analysis was both very small and variable, depending on accidental moisture. The latest researches of Liebig have confirmed these facts, but have proved that the true formula of mellone is $\mathrm{C}_{18} \mathrm{~N}_{13}$, that is, 3 times as much as the former one, with the addition of 1 eq. more of nitrogen. For 3 times $\mathrm{Ce}_{3} \mathrm{~N}_{4}$ is $\mathrm{C}_{18} \mathrm{~N}_{12}$. Mellone may, therefore, be regarded as composed either of $\mathrm{C}_{18}+\mathrm{N}_{13}$, or of $\mathrm{Cy}_{9}+\mathrm{N}_{4}$. In the former case it is a compound of carbon and nitrogen in most unusual proportions, such as are found in no other binary compound. In the latter case it is a compound of 9 eq. of cyanogen with 4 of nitrogen, which is equally unprecedented. The researches of Liebig, however, leave no doubt that such a compound really exists, whichever view be taken of its constitution. It is probable that future researches will show that its constitution involves some law at present unknown, and that we may learn the true constitution of so very strange a compound.

Mellone is not known in the separate state, at least not in a perfectly pure form. But when sulphocyanogen is decomposed by heat, a substance is left, which seems to be impure mellone, in the form of a yellowish-grey powder. When further heated, it is resolved chiefly into cyanogen and nitrogen gases, but it contains also some hydrogen, which is expelled in combination when the heat is first applied.

Liebig has shown, however, that mellone has all the chemical relations of a compound radical, in the same way as cyanogen, sulphocyanogen, ferrocyanogen or ferridcyanogen. It forms an acid with hydrogen, and salts with metals.

Mellone, like ferridcyanogen, is a tribasic radical; for the 
hydromellonio acid is $\mathrm{C}_{18} \mathrm{~N}_{13} \mathrm{H}_{3}=\mathrm{Me} \mathrm{H}_{3}$, and, in its salts, this hydrogen is partially or entirely replaced by metals.

As a radical, we shall briefly dèscribe it and its chief compounds, after those of cyanogen.

\section{CYANOGEN AND CHLORINE.}

Cyanogen forms two chlorides: one gaseous, but compressible, obtained by the action of chlorine gas on dry hydrocyanic acid; the other solid, formed by a spontaneous transformation of the former when kept in the liquid form in sealed tubes. Both are volatile, and both contain cyanogen and chlorine, equivalent for equivalent. But the vapour of the solid chloride is three times denser than that of the other : and, moreover, the solid chloride, in contact with water, produces hydrochloric and cyanuric acids : while the gaseous chloride, under the same circumstances, gives rise to hydrochloric acid, and cyanic acid, the latter, with water, at once passing into bicarbonate of ammonia, so that the final result is sal ammoniac and carbonic acid.

These considerations prove that the two chlorides are polymeric: that the first or gaseous chloride is $\mathrm{Cy} \mathrm{Cl}$; and that the solid chloride is $\mathrm{Cy}_{3} \mathrm{Cl}_{3}$, and is formed by the junction into one group of 3 eq. of the other; and we are consequently justified in extending this view to cyanic and cyanuric acids, and in supposing the vapour of the latter to be three times more condensed than the vapour of cyanio acid: and the cyanogen of cyanuric acid to be the cyanogen of the solid chloride, and three times denser than ordinary cyanogen.

\section{CYANOGEN WITH BROMINE AND IODINE.}

With these elements cyanogen readily combines when cyanide of mercury is distilled with bromine or iodine: The bromide and iodide are both volatile, and crystallisable, pungent, and poisonous.

To judge by the density of their vapours, these compounds are $\mathrm{Cy} \mathrm{Br}$ and $\mathrm{Cy}$ I, corresponding to the liquid chloride, which is also very pungent and irritating to the eyes.

\section{CYANOGEN AND SULPHUR.}

Bisulphuret of Cyanogen. $\mathrm{Cy}_{2} \mathrm{~S}_{2}=58$.

When sulphocyanide of potassium (see that salt) is acted on by chlorine or by dilute nitric acid, there is obtained an orangeyellow powder, which contains sulphur and cyanogen, and which 
is supposed by some to be bisulphuret of cyanogen, by others to contain hydrogen. This question will be considered hereafter; for whether this be so or not, there is a compound, possibly that compound, of sulphur and cyanogen, which acts as a radical, and is hence called sulphocyanogen. It will be described separately, since it is far more important as a radical than as a compound of cyanogen.

CYANOGEN AND METALS.

With metals, cyanogen forms compounds which in many cases are analogous to the chlorides of the same metal. When the metal is easily reducible, such as silver, mercury, or palladium, the cyaniàe is formed by the action of hydrocyanic acid on the oxide, or its salts; $\mathrm{MO} O \mathrm{H} C \mathrm{Cy}=\mathrm{HO}+\mathrm{M} \mathrm{Cy}$. In the case of difficultly reducible metals, such as potassium, hydrocyanic acid seems to combine with the oxide, not being able to reduce it, until another cyanide is added, which tends to form a double cyanide.

Cyanide of Potassium, $=\mathrm{K} \mathrm{Cy}$ is best formed by heating to whiteness in close vessels the ferrocyanide of potassium, a salt which may be viewed as containing cyanide of iron and cyanide of potassium. The former is converted into insoluble carburet of iron; the latter remains unchanged, and may be mechanically picked out, or dissolved in hot alcohol of 60 p.c., which deposits it on cooling. Or, an alcoholic solution of pure and dry hydrocyanic acid is added to an alcoholic solution of potash : or, lastly, the vapours of hydrocyanic acid, formed on the process given at p. 71, are conducted into an alcoholic solution of potash, kept cool, when the salt is deposited.

It is white, and crystallisable in cubes. It has no smell when pure, but when exposed to moist air, smells of hydrocyanic acid. It is very poisonous. When heated, it fuses easily, and is not altered by heat in close vessels. Heated in the air, it absorbs oxygen, and is converted into cyanate of potash $\mathrm{K} \mathrm{Cy}+\mathrm{O}_{2}=$ $\mathrm{K} \mathrm{O}$, Cy $\mathrm{O}$. It is very soluble and deliquescent, and its solution, attracting carbonic acid from the air, gives off hydrocyanic acid: hence its smell.

Cyanide of Sodium is analogous to the preceding salt.

Cyanide of Zinc, $\mathrm{Zn} \mathrm{Cy}$, is obtained by adding hydrocyanio acid to acetate of zinc. It is a snow-white insoluble powder, which is used in medicine.

Cyanide of Iron.-Protocyanide of iron, $\mathrm{Fe} \mathrm{Cy}$, has not been isolater. There appears to exist a percyanide, also not yet isolated, $\mathrm{Fe}_{2} \mathrm{Cy}_{3}$; and, according to Pelouze, there may be obtained an intermediate cyanide, $\mathrm{Fe}_{3} \mathrm{Cy}_{4}$, analogous to the magnetic oxide of iron, as a green powder. 
But although the eyanides of iron are little known, there are some very important compounds, especially those of two radicals, which contain those elements, namely, cyanogen and iron, along with hydrogen, and with metals. These will be described after we have gone over the simple cyanides of the metals.

Cyanide of Cobalt, Co $\mathrm{Cy}$, is obtained as a brownish-white precipitate, by adding hydrocyanic acid to the acetate of cobalt. Cobalt also forms compounds analogous to those of iron above alluded to, with cyanogen, \&c.

Bicyanide of Mercury, $\mathrm{Hg}$ Cy”, is easily obtained by dissolving red oxide of mercury in very dilute hydrocyanic acid, till the smell of the acid is destroyed. The liquid, rendered neutral, if necessary, by a few. drops of hydrocyanic acid, yields fine crystals, on evaporation and cooling. Or it may be made by the action of three parts of persulphate of mercury on two of ferrocyanide of potassium dissolved in fifteen of hot water, which is boiled and filtered, and the filtered liquid on cooling deposits the bicyanide.

It forms regular prismatic crystals, permanent in the air, of a horrible metallic taste, soluble in water and alcohol. It is used for making cyanogen, also to prepare hydrocyanic acid by one process, and to yield the bromide and iodide of cyanogen.

Cyanide of Silver, Ag Cy.-Formed by the action of hydrocyanic acid or cyanide of potassium on nitrate of silver. It is exactly similar to the chloride, white, curdy, insoluble in water or acids, soluble in ammonia. It is decomposed by hydrochloric acid, yielding hydrocyanic acid.

Cyanide of Palladium, Pd Cy.-The affinity of cyanogen for palladium is most powerful, and hydrocyanic acid, or a soluble cyanide, when added to a salt of oxide of palladium, precipitates the cyanide as a grey powder. This property serves for separating palladium from other metals.

Cyanides of Gold.-The protocyanide, $\mathrm{Au} \mathrm{Cy}$, is formed by adding cyanide of potassinm in excess to protochloride of gold, till a clear solution is formed, and then adding hydrochloric acid, which precipitates the protocyanide as a bright yellow powder, very permanent, and soluble in eyanide of potassium.

The tercyanide, Au Cys, is formed by the action of cyanide of potassium on terchloride of gold, and appears as whitishyellow precipitate, soluble in cyanide of potassium, insoluble in acids.

DOUBLE CYANIDES OF THE METALS.

The insoluble metallic cyanides, such as those of iron, cobalt, 
copper, silver, gold, platinum, and others, dissolve readily in cyanide of potassium, or sodium, forming crystallisable double cyanides, which are not affected by soluble carbonates nor by chlorides, but are generally decomposed by acids, which precipitate the insoluble cyanide. If these double cyanides, which may be $2 \mathrm{~K} \mathrm{Cy}+\mathrm{m} \mathrm{Cy}$ ( $\mathrm{m}$ Cy being an insoluble metallic cyanide) are added to the solution of the oxide of another metal, forming also an insoluble cyanide, this latter metal takes the place of the potassium, and a new double insoluble cyanide is the result. Thus $(2 \mathrm{~K} \mathrm{Cy}+\mathrm{mCy})+2 \mathrm{MO}=2 \mathrm{KO}+(2 \mathrm{M} \mathrm{Cy}+\mathrm{m} \mathrm{Cy})$. (Here $\mathrm{m}$ may be iron, and $\mathrm{M}$ copper or lead.) Now, as these double cyanides, thus precipitated, are of various and often characteristic colours, the ferrocyanide of potassium, $2 \mathrm{~K} \mathrm{Cy}+\mathrm{Fe} \mathrm{Cy}$, is extensively used as a test for metals.

The protocyanide of iron, the percyanide of iron, the percyanide of cobalt, and perhaps some others, not only form double salts with the elements of cyanide of potassium, but also produce very remarkable acids, by combining with the elements of hydrocyanic acid, that is, with hydrogen replacing the potassium of the double salt. Thus ferrocyanic acid is $2 \mathrm{H} \mathrm{Cy}+\mathrm{Fe} \mathrm{Cy}$, and so on. In these compounds the hydrocyanic acid is no longer poisonous, and it is therefore highly probable that these acids, and their corresponding salts, really contain new and very peculiar radicals. The assumption of these radicals will alone enable us to classify or remember these compounds.

\section{Ferrooyanogen. $\quad \mathrm{Cy}_{3} \mathrm{Fe}=\mathrm{Cfy}=106$.}

We assume, then, the existence of this radical as the basis of ferrocyanide of potassium, or prussiate of potash. It is bibasic, combining with 2 eq. of hydrogen or of metals. The following formulæ express the composition of ferrocyanic acid and of ferrocyanide of potassium, \&c.

Ferrocyanic Acid $=\mathrm{Cfy}, \mathrm{H}_{2}=\mathrm{Cy} 3 \mathrm{Fe}, \mathrm{H}_{2}=2 \mathrm{H} \mathrm{Cy}+\mathrm{Fe}$ Cy. Ferrocyanide of Potassium $=\mathrm{Cfy}, \mathrm{K}_{2}=\mathrm{Cy}_{3} \mathrm{Fe}, \mathrm{K}_{2}=2 \mathrm{~K} \mathrm{Cy}+\mathrm{Fe} \mathrm{Cy}$. Iron $=3 \mathrm{Cfy}, \mathrm{Fe}_{4}=3 \mathrm{Cy}_{3} \mathrm{Fe}, \mathrm{Fe}_{4}=9 \mathrm{Cy}+7 \mathrm{Fe}$.

It will be seen, by the two first compounds, that we may consider them either as compounds of the radical Cfy with hydrogen or metals, or as double cyanides. In the third, which is the formula of prussian blue, we see that the iron exists in two different states, in one of which it cannot be detected by the usual tests. That form is the radical Cfy, which exists also in the two preceding compounds. 


\section{Ferrocyanic Acid. Cfy, $\mathrm{H}_{2}=108$.}

This very interesting compound is obtained by mixing a cold saturated solution of ferrocyanide of potassium with one quarter its volume of strong hydrochloric acid, and agitating the mixture with half its bulk of pure ether; the ether rises to the surface, carrying with it, suspended, a white crystalline substance, which, when washed with ether and dried, is ferrocyanic acid. It is soluble in water and alcohol, has a decidedly acid taste and reaction, and decomposes the alkaline carbonates, forming, with carbonate of potash, the ferrocyanide of potassium. The production of this compound by the action of hydrochlorio acid on ferrocyanide of potassium is very easily explained. Cfy, $\mathrm{K}_{\mathbf{2}}+$ $2 \mathrm{H} \mathrm{Cl}=\mathrm{Cfy}, \mathrm{H}_{2}+2 \mathrm{~K} \mathrm{Cl}$.

Although this compound may be represented as a combination of cyanide of iron with hydrocyanic acid, $\mathrm{Fe} \mathrm{Cy}+2 \mathrm{H} \mathrm{Cy}$, there is every reason to believe that it contains no hydrocyanic acid, as such. For not only is it far more strongly acid, (and protocyanide of iron cannot be supposed to give acid properties to hydrocyanic acid,) but it is totally destitute of all poisonous properties, and both it and its salts may be taken internally without further effect than the laxative action common to most neutral salts.

Ferrocyanic acid, being bibasic, forms two kinds of salts: in the first the 2 eq. of hydrogen are replaced by 2 eq. of potassium, sodium, \&c.; in the second, they are replaced by equivalents of two different metals: such as potassium and barium. An example of the first class is, Cfy $+K_{2}$; of the second Cfy $\begin{cases}\mathrm{K} & \text { Of all these compounds, by far the most }\end{cases}$ important is the ferrocjanide of potassium, or prussiate of potash, from which all the compounds of cyanogen may be prepared, and which is manufactured on the large scale, being also used in the arts.

Ferrocyanide of Potassium. SrN. Prussiate of Potash. Anhydrous, Cfy $K_{2}, 184 \cdot 4$. Crystallised, it is Cfy, $\mathrm{K}_{2}+3 \mathrm{HO}=$ 211.4. -This valuable salt is prepared by fusing animal matter, such as dried blood, hoofs, hair, horns, \&c., or the animal charcoal remaining after such matters have been distilled to obtain carbonate of ammonia, with potashes or pearlash, in iron vessels, as long as gas is disengaged. The melted mass is then withdrawn from the fire, and when cold, lixiviated with water, the solution digested with the insoluble part, filtered and evaporated, when it deposits, on cooling, Jellow crystals ; which by a second solution and crystallisation become quite pure. 
The essential points of this process are, first, the presence of as much nitrogen as possible in the animal matter: hence fresh, dried, uncalcined animal matter is far preferable to animal oharcoal : secondly, the presence of metallic iron or sulphuret of iron: the former is either directly derived from the vessels, which are rapidly corroded, or added as filings; the latter is formed from the iron of the vessels by the action of bisulphuret of potassium, which arises from the decomposition, by charcoal at a red heat, of the sulphate of potash contained in the potashes or pearlash; hence pure carbonate of potash is not adapted to this process. Thirdly, the exclusion of the air as far as possible, in order to prevent the oxidation and destruction of the cyanide of potassium formed.

The explanation of the process is very simple and beautiful, and we are indebted for it to the researches of Liebig. When animal matter is heated along with potash, cyanogen is formed, and combining with the potassium (set free at the same time by the action of carbon on potash) produces cyanide of potassium, a salt which is not decomposed by a red heat in closed vessels. At the same time metallic iron and sulphuret of iron are present in the mixture, but not a trace of the ferrocyanide, previous to the action of water, for the very obvious reason, that the ferrocyanide is decomposed by a red heat, into cyanide of potassium and carburet of iron, nitrogen being disengaged.

If the cooled mass be now digested in water, the cyanide of potassium dissolves either the metallic iron with disengagement of hydrogen, or the sulphuret of iron, and the filtered liquid now contains the ferrocyanide.

Direct experiments have shown that cyanide of potassium. dissolves either iron, oxide of iron, or sulphuret of iron, producing the ferrocyanide; $3 \mathrm{~K} \mathrm{Cy}+\mathrm{Fe}+\mathrm{H} \mathrm{O}=(2 \mathrm{~K} \mathrm{Cy}+$ $\mathrm{Fe} \mathrm{Cy})+\mathrm{KO}+\mathrm{H}$. Or, $3 \mathrm{~K} \mathrm{Cy}+\mathrm{Fe} \mathrm{S}=(2 \mathrm{~K} \mathrm{Cy}+\mathrm{Fe} \mathrm{Cy})$ $+\mathrm{KS}$. And it has also been shown by Liebig that if the fused mass be lixiviated with alcohol, the residue, when treated with water, yields no ferrocyanide, while the alcohol contains none, but only cyanide of potassium.

Taking these facts into consideration, perhaps the best method would be to use pure carbonate of potash, free from sulphate, by which means no sulphuret of potassium would be formed, and the iron vessels would not suffer as they now do. The cyanide of potassium, being dissolved in water and filtered, should then be placed in contact with iron turnings in flat open vessels, when the third part of the potassium being oxidised by the air, the iron is rapidly dissolved, and a quantity of ferrocyanide obtained equal to that indicated by the cyanide of potassium present. 
It appears from the researches of Runge, that while the preceding theory of the formation of this salt is true of what takes place in experiments on the small scale, yet when it is manufactured on a large scale, different results are obtained. If iron be added to the materials before ignition, Runge finds that the ignited mass does not yield to alcohol any cyanide of potassium, and that the insoluble residue contains ferrocyanide ready-formed, which is curious, since we know that a strong red heat decomposes it.

The contradiction between the results of Runge and those of Liebig must depend partly on the influence of the larger mass in Runge's case, but chiefly, no doubt, on the temperature employed.

It is stated by Runge that when the ignited mass is dissolved in iron vessels, the vessels are not attacked, because the ferrocyanide already exists; but it is a well-known fact, that when animal matter is calcined with potash, the mass dissolved in water in iron vessels, rapidly corrodes them with disengagement of hydrogen, while the ferrocyanide is formed from the cyanide of potassium.

The ferrocyanide of potassium forms large honey-yellow, transparent, fiat, quadrangular prisms, derived from a rhombic octahedron. It is very soluble in water, and forms precipitates in almost all metallic solutions, many of which are characteristic. Thus, with solutions of zinc and lead, it gives a white precipitate, with those of copper a chestnut brown, with those of peroxide of iron a deep blue, with protoxide of iron a pale blue, with those of barium and calcium whitish-yellow crystalline precipitates. The lead precipitate is $\mathrm{Cfy}, \mathrm{Pb}_{2}$; that of copper $\mathrm{Cfy}, \mathrm{Cu}_{2}$; those of barium and calcium

Cfy $\left\{\begin{array}{l}\mathrm{K} \\ \mathrm{Ba}\end{array}\right.$ and Cfy $\left\{\begin{array}{l}\mathrm{K} \\ \mathrm{Ca}\end{array}\right.$; and that of zinc, $2 \mathrm{Cfy}+\left\{\begin{array}{l}\mathrm{Zn} 3 \\ \mathrm{~K}\end{array}\right.$ $\mathrm{aq}=\mathrm{Cfy}, \mathrm{Zn}_{2}+\mathrm{Cfy}\left\{\begin{array}{l}\mathrm{Zn} \\ \mathrm{K}\end{array}+6 \mathrm{aq}\right.$. That of protoxide of iron is 2 Cfy $\left\{\begin{array}{l}\mathrm{Fe} 3 \\ \mathrm{~K}\end{array}=\mathrm{Cfy}, \mathrm{Fe}_{2}+\mathrm{Cfy}\left\{\begin{array}{l}\mathrm{Fe} \\ \mathrm{K}\end{array}\right.\right.$. All of these formulæ are easily referred to the general formula of the ferrocyanides, Cfy, $\mathrm{M}_{2}$.

Ferrocyanide of Iron.-But the most remarkable of all these compounds is that formed with persalts of iron, namely prussian blue. It is produced when ferrocyanide of potassium comes in contact with perchloride, or any salt of peroxide of iron. Now as ferrocyanogen is a bibasic radical, 1 eq. of it corresponds to 2 eq. of potassium, hydrogen, oxygen, chlorine, \&c.; and as perchloride of iron, $\mathrm{Fe}_{2} \mathrm{Cl}$, contains $3 \mathrm{eq}$. of chlorine, $1 \frac{1}{2} \mathrm{eq}$. of ferrocyanide of potassium will be required to decompose 1 eq. of it, or, to avoid fractions, 3 eq. of ferrocyanide Cfys $\mathrm{K}_{6}=3$ (Cfy, 
$\mathrm{K}_{2}$ ), are required for 2 eq. perchloride of iron, $2 \mathrm{Fe}_{2} \mathrm{Cl}_{3}=\mathrm{Fe}_{4}$ Clo. The result is, Cfys $\mathrm{K}_{6}+\mathrm{Fe}_{4} \mathrm{Cl}_{6}=6 \mathrm{~K} \mathrm{Cl}+\mathrm{Cfy}_{3} \mathrm{Fe}_{4}$; and this last is the true formula of prussian blue, although, from its tendency to combine both with ferrocyanide of potassium and with oxide of iron, its analysis offers great difficulties. The formula $\mathrm{Cfy}_{3} \mathrm{Fe}_{4}$ or $\mathrm{Fe}_{4} \mathrm{Cfy}_{3}$ shows that prussian blue corresponds to peroxide and perchloride (sesquioxide and sesquichloride) of iron, $\mathrm{Fe}_{2} \mathrm{O}_{3}$ and $\mathrm{Fe}_{2} \mathrm{Cl}_{3}$, for Cfy being bibasic is equivalent to $\mathrm{Cl}_{2}$ or $\mathrm{O}_{2}$, and consequently $\mathrm{Fe}_{4} \mathrm{Cfy}_{3}$ is equivalent to $\mathrm{Fe}_{4} \mathrm{Cl}_{6}$ or $\mathrm{Fe}_{4} \mathrm{O}_{6}$, that is, to $2 \mathrm{Fe}_{2} \mathrm{Cl}_{3}$ and $2 \mathrm{Fe}_{2} \mathrm{O}_{3}$. It must be admitted to be a very strong argument in favour of the existence of ferrocyanogen, as a bibasic radical, according to the theory of Liebig, that prussian blue, in all other theories the most complex and anomalous compound in the whole range of chemistry, becomes quite normal and one of a series.

If we make $\mathrm{fe}=\frac{2}{3}$ of $\mathrm{Fe}$, then prussian blue may be represented by the formula $\mathrm{Cfy}_{\mathrm{fe}}=\mathrm{Fe} \mathrm{Cy}, 2 \mathrm{fe} \mathrm{Cy}$ just as ferrocyanide of potassium is Cfy, $\mathrm{K}_{2}=\mathrm{Fe} C \mathrm{Cy}+\mathrm{K} \mathrm{Cy}$. Now there is reason to believe that iron exists with the two different atomic weights indicated by $\mathrm{Fe}=28$ and $\mathrm{fe}=18.66$. This view simplifies still more the formula of prussian blue, and shows that it is a salt of the same type as the ferrocyanide of potassium.

Ferrocyanide of potassium is employed to yield cyanogen and all its compounds. We have already seen how cyanogen, hydrocyanic acid, cyanide of potassium, cyanate of potash, urea, cyanic and cyanuric acids, and ferrocyanic acid are obtained from it. Cyanide of potassium, for testing, and to be used as a flux and in analysis, is best prepared, according to Liebig, by heating 8 parts of the dry ferrocyanide with 3 of pure earbonate of potash in an iron vessel till the fused mass is colourless. It is then poured off from the sponge of reduced iron, and kept in wellstoppered bottles. The reaction is as follows (representing the ferrocyanide, for convenience, as a double cyanide of iron and potassium $): 2(\mathrm{Fe} \mathrm{Cy}, 2 \mathrm{~K} \mathrm{Cy})+2\left(\mathrm{~K} \mathrm{O}, \mathrm{C} \mathrm{O}_{2}\right)=\mathrm{Fe}_{2}+5 \mathrm{~K} \mathrm{Cy}$ $+\mathrm{KO}, \mathrm{Cy} \mathrm{O}+2 \mathrm{COz}$. Hence the resulting salt contains 1 eq. cyanate of potash to 5 eq. cyanide of potassium. This very useful preparation, known as Liebig's cyanide of potassium, is not quite pure, containing cyanate of potash; but this does not interfere with its use.

Another very remarkable compound is produced by the action of a current of chlorine gas on the solution of ferrocyanide, if transmitted until the solution ceases to produce prussian blue with perchloride of iron, yielding only a brownish-green colour, 
but no precipitate. The liquid now gives, on evaporation, beautiful deep hyacinth-red crystals of a new salt, the ferridcyanide of potassium, discovered by Gmelin. This salt contains a new radical, ferrideyanogen.

\section{v. Ferridotanogen. $\mathrm{Cy}_{6} \mathrm{Fe}_{2}=\mathrm{Cfdy}=212$.}

This radical has not yet been isolated. It is formed by the coalescence of 2 eq. of ferrocyanogen, and is tribasic. It forms an acid with hydrogen, and salts with metals.

$$
\text { Perridcyanic Acid, }\left(\mathrm{Cy}_{6} \mathrm{Fe}_{2}\right)+\mathrm{H}_{3}=\left(2 \mathrm{Cfy}+\mathrm{H}_{3}\right)=\mathrm{Cfdy}, \mathrm{H}_{3}=215 \text {. }
$$

This acid is obtained from the lead salt $\mathrm{Cfdy}, \mathrm{Pbs}$, by the action of sulphuric acid. It is soluble. in water, and by the action of sulphuretted hydrogen, is converted into ferrocyanic acid: $2 \mathrm{Cfy}, \mathrm{H}_{3}+\mathrm{H} \mathrm{S}=2\left(\mathrm{Cfy}, \mathrm{H}_{2}\right)+\mathrm{S}$. With bases it forms salts; as with potash the ferridcyanide of potassium.

Ferridcyanide of Potassium. Srn. Red prussiate of potash.Its preparation has been described above. Its formula is $2 \mathrm{Cfy}$ $+K_{3}=C f d y, K_{3}$; and it is quite anhydrous. Like the yellow prussiate, it forms precipitates with most metallic solutions, many of which are characteristic. With salts of peroxide or with perchloride of iron, it only strikes a brown or green colour, but with protochloride or salts of protoxide, it forms prussian blue. As the radical is tribasic, 1 eq. of it ought to be equivalent to 3 eq. of oxygen, chlorine, \&c., and if we suppose the potassium in the ferridcyanide replaced by its equivalent of iron, we should have $\mathrm{Cfdy}, \mathrm{Fe}_{3}=\mathrm{Fe}_{5} \mathrm{Cy}_{6}$; for $\mathrm{K}_{3} \mathrm{Cfy}_{2}+3 \mathrm{Fe} \mathrm{O}=$ $\mathrm{Fe}_{3} \mathrm{Cfy}_{2}+3 \mathrm{KO}$; and $\mathrm{Fe}_{3} \mathrm{Cfy}_{2}=\mathrm{Fe}_{6} \mathrm{Cy}_{6}$. But, instead of this compound, there is formed prussian blue, the same we have above described as being formed by the action of yellow prussiate on peroxide of iron. In fact, when the red prussiate is added to a solution of a salt of protoxide, or to protochloride of iron, yellow prussiate is formed along with prussian blue and a salt of potash. Bearing in mind, as in all the above explanations, that 1 eq. ferrideyanogen Cfdy is equal to 2 eq. ferrocyanogen Cfy, and that, consequently, the red prussiate, Cfdy, $K_{3}$ may be equally well represented as $2 \mathrm{Cfy}, \mathrm{K}_{3}$, then we have $2\left(2 \mathrm{Cfy}, \mathrm{K}_{3}\right)+4$ $\left(\mathrm{Fe} \mathrm{O}, \mathrm{S} \mathrm{O}_{3}\right)=\left(3 \mathrm{Cfy}+\mathrm{Fe}_{4}\right)+\left(\mathrm{Cfy}, \mathrm{K}_{2}\right)+4\left(\mathrm{~K} \mathrm{O}, \mathrm{S} \mathrm{O}_{3}\right)$.

The ferridcyanide of potassium may be viewed as ferrocyanide of potassium, plus a certain amount of ferrocyanogen ; 2 (Cfdy, $\mathrm{K}_{3}$ ) $=3\left(\mathrm{Cfy}, \mathrm{K}_{2}\right)+\mathrm{Cfy}$.

With salts of lead, ferridcyanide of potassium forms the ferridcyanide of lead, Cfdy, $\mathrm{Pbs}$. 
According to some, the compound Cfdy, $\mathrm{Fe}_{3}$ is really formed, and is another kind of prussian blue. They represent it as a double cyanide $3 \mathrm{Fe} \mathrm{Cy}+3 \mathrm{fe} \mathrm{Cy} \cdot 1$

It is probable that this is the compound which is known as Turnbull's blue. But, as we have seen, in certain circumstances ordinary prussian blue may be obtained by the action of the ferridcyanide on salts of iron.

When hydrocyanic acid is prepared from the ferrocyanide by diluted sulphuric acid, a white salt separates, which is Cys $\mathrm{Fe}_{2} \mathrm{~K}$ $=\operatorname{Cfy}\left\{\begin{array}{l}\mathrm{Fe} \\ \mathrm{K}\end{array}\right.$. This, by nitric acid and other ordinary agents, is converted into another deep blue salt, which is derived from 2 eqs. of the white salt by the loss of 1 eq. of potassium, just as the ferridcyanide is from the ferrocyanide. $2 \mathrm{Cy}_{3} \mathrm{Fe}_{2} \mathrm{~K}=\mathrm{Cy}_{6} \mathrm{Fe}_{4} \mathrm{~K}_{2}$, and $1 \mathrm{eq}$. $K$ being removed by the action of air, chlorine, or nitrio acid, there is left the blue compound $\mathrm{Cy}_{6} \mathrm{Fe}_{4} \mathrm{~K}$, which is $\left.=\operatorname{Cfdy} \begin{array}{l}\mathrm{Fe}_{2} \\ \mathrm{~K}\end{array}\right\}$, a ferridcyanide of iron and potassium.

Thus, the blue salt, heated with a solution of the ferrocyanide of potassium, yields the ferridcyanide of potassium, and the white salt from which the blue one was obtained. This is the best process for making the ferrideyanide, because the product is very pure, and the white salt can be again oxidised into blue salt, and this again used any number of times.

Nitro-prussides. - When the ferrocyanide, or ferrideyanide, of potassium is acted on by nitric acid a new acid is formed, which combines with bases, forming salts, called by Playfair, who discovered them, nitro-prussides. The reaction is very complicated. The new salts, when soluble, form beautiful ruby-red crystals, as is the case with those of potassium and sodium. The insoluble salts have various colours. Playfair deduced from his analysis the complicated formula $\mathrm{Fe}_{5} \mathrm{Cy}_{12}, 3 \mathrm{~N} \mathrm{O}, \mathrm{Na}_{5}+10 \mathrm{H} \mathrm{O}$ for the salts of sodium. But he also suggested that it might probably be simpler, $\mathrm{Fe}_{2} \mathrm{Cy}_{5}, \mathrm{~N} \mathrm{O}, \mathrm{Na}_{2}+4 \mathrm{H} \mathrm{O}$. Kyd has shown the latter to be the more correct. We may compare these salts (M standing for any metal) with the ferrocyanides and ferridcyanides, as follows, if we double the formula of the ferrocyanides :

$$
\begin{aligned}
& \mathrm{Fe}_{2} \mathrm{Cy}_{6}{ }^{\circ}+\mathrm{M}_{4}=\text { Ferrocyanides. } \\
& \mathrm{Fe}_{2} \mathrm{Cy}_{6}+\mathrm{M}_{3}=\text { Ferridcyanides. } \\
& \left.\mathrm{Fe}_{2} \begin{array}{l}
\mathrm{Cy} 5 \\
\mathrm{NO}
\end{array}\right\}+\mathrm{M}_{2}=\text { Nitro-prussides. }
\end{aligned}
$$

With caustic potash, the nitro-prusside of potassium yields a new salt, as a yellow crystalline powder. Its formula is 
probably $\mathrm{Fe}_{2} \mathrm{Cy}_{5}, \mathrm{~N} \mathrm{O}, \mathrm{K}_{2}+2 \mathrm{~K} \mathrm{O}+4 \mathrm{HO}$. With sulphuret of sodium, the nitro-prusside of sodium forms a new compound, which dissolves in water with a splendid purple colour, which seems to be $\mathrm{Fe}_{2} \mathrm{Cy}_{5}, \mathrm{NO}, \mathrm{Na}_{2}+\mathrm{Na} \mathrm{S}+3 \mathrm{H} \mathrm{O}$. These singular compounds, in which we see protoxide of nitrogen replacing cyanogen, and sulphuret of sodium replacing water, cannot be regarded as fully understood. They are therefore merely noticed, and the reader is referred for details to Dr. Playfair's paper in the "Philosophical Transactions."

\section{VI. Сobaltoctanogen. Су6 $\mathrm{Cog}_{2}=\mathrm{Cky}=21 \cdot 5$.}

Not yet isolated, but known in combination with hydrogen, potassium, \&c. It is analogous to ferridcyanogen in constitution, and like it, is tribasic.

Cobaltocyanic acid, $\mathrm{Cky}_{3}=219 \cdot 52$. - Obtained by the action of sulphuric acid on cobaltocyanide of lead, Cky, $\mathrm{Pb}$. It forms silky filaments, which are deliquescent and strongly acid.

Cobaltocyanide of Potassium, Cky K.-Is obtained by acting on a salt of oxide of cobalt with solution of cyanide of potassium and hydrocyanic acid, when hydrogen is given off and the new salt is obtained in crystals. The protocyanide of cobalt, precipitated on the first addition of cyanide of potassium redissolves in an excess of that salt, forming a compound $2 \mathrm{Co} \mathrm{Cy}+\mathrm{K} \mathrm{Cy}$, or $2 \mathrm{Co} \mathrm{Cy}+3 \mathrm{~K} \mathrm{Cy}$. At all events there is enough of cyanide of potassium present to form the latter compound. The hydrocyanic acid, being now added, yields 1 eq. of cyanogen, converting the 2 eq. of protocyanide into 1 eq. of sesquicyanide of cobalt, while hydrogen is given off: $2 \mathrm{Co} \mathrm{Cy}+\mathrm{H} \mathrm{Cy}=\mathrm{Co}_{2} \mathrm{Cy}_{3}+\mathrm{H}$. Lastly, the sesquicyanide $\mathrm{Co}_{2} \mathrm{Cy}_{3}$, with the 3 eq. of cyanide of potassium, $3 \mathrm{~K} \mathrm{Cy}$, produces the cobaltocyanide of potassium, Cy6 $\mathrm{Co}_{2}+\mathrm{K}_{3}=\mathrm{Cky}, \mathrm{K}_{3}$. The crystals are isomorphous with those of the red prussiate of potash; they are yellow, soluble; their solution is not altered by acids, and gives, in solution of protoxide of cobalt, a beautiful rose-coloured precipitate, analogous probably to prussian blue; possibly, however, it may be $\mathrm{Cky}$, Co3. It precipitates many other metallio solutions, such as those of lead and silver.

Cobaltocyanide of potassium is a singularly permanent salt, resisting the action of the strongest acids; which is, in itself, a sufficient proof that it cannot contain cyanide of potassium as such. With the salts of nickel it forms a green precipitate, Cky, $\mathrm{Ni}_{3}$, which is insoluble in boiling dilute acids. This property has been applied by Liebig to the separation of cobalt from nickel in analysis. All other metals being remored, an excess of potash is 
first added, and then hydrocyanic acid till the precipitate at first formed is dissolved, and the whole is then boiled. Hydrochloric acid is now added, and if no nickel be present, it produces no change, because it has no action on cobaltocyanide of potassium. But if nickel be present (of course by this time as cyanide) it is converted into chloride, and this is instantly precipitated by the cobaltocyanide of potassium as cobaltocyanide of nickel. Should there be more cobalt than nickel present, the whole nickel is precipitated, and the precipitate, acted on by potash, leaves the nickel as peroxide, while the cobalt is dissolved as cobaltocyanide, and may be determined along with the portion not precipitated for want of nickel. If, on the other hand, there be more nickel than cobalt, all the cobalt is contained in the green precipitate of cobaltocyanide of nickel, and may be dissolved by potash, and its quantity determined, while the nickel left by the potash as peroxide may be added to that left in the liquid for want of cobalt. Such is an outline of this very beautiful and refined method, which gives most accurate results.

\section{Chromocyanogen. Cyo $\mathrm{Cr}_{2}=$ Cry.}

This radical is little known. It is analogous to the two preceding, forming with hydrogen an acid, Cry, $\mathrm{H}_{3}$, and with potassium a yellow crystallisable salt, $\mathrm{Cry}_{3}, \mathrm{~K}_{3}$, which precipitates metallic solutions.

\section{Puatinocyanogen. Cy2 $\mathrm{Pt}=\mathrm{Cpy}=151 \cdot 7$.}

This radical is not known in the separate state. It forms with hydrogen a crystallisable acid of a gold or copper colour and metallic lustre, $\mathrm{Cpy} \mathrm{H}_{2}$, which is very soluble and deliquescent. This acid is powerful, decomposes the carbonates, and produces platinocyanides. Platinocyanide of potassium, Cpy, $\mathrm{K}_{\mathbf{2}}$, is easily obtained by heating spongy platinum, or still better, as I have found, finely divided platinum black, to low redness with twice its weight of dried ferrocyanide of potassium, and lixiviating with water; or by dissolving protochloride of platinum in hot solution of cyanide of potassium. It forms crystals, yellow and metallic by transmitted, blue by reflected light. By the action of this salt on protonitrate of mercury, a cobalt-blue precipitate is formed, which, when heated in the fluid, becomes white, and is then pure platinocyanide of mercury, $\mathrm{Cpy}, \mathrm{Hg}_{2}$ (?). This salt, acted on by sulphuretted hydrogen, yields the platinocyanic acid. A solution of platinocyanide of potassium acted on by chlorine, yields beautiful copper-like crystals of a new salt, which is either 
a double cyanide, $2 \mathrm{~K} \mathrm{Cy}+\mathrm{Pt}_{2} \mathrm{Cys}+5 \mathrm{H} \mathrm{O}$; or the potassium salt of a new radical, $\mathrm{Pt}_{2} \mathrm{Cy}_{5}, \mathrm{~K}_{2}+5 \mathrm{H} \mathrm{O}$ (Knop). Recent researches have greatly increased the number of platinocyanides, and it appears that many of these are isomeric or polymeric with one another.

The platinocyanides of barium, strontium, and calcium, are easily obtained by the action of platinocyanic acid on these bases, and crystallise readily with a beautiful greenish-yellow colour, or in some cases green and red with metallic lustre.

\section{Iridiooyanogen. Cys $\mathrm{Ir}=$ Ciy.}

This radical has not been isolated: it forms, with hydrogen, iridiocyanic acid $\mathrm{Cy}_{3}, \mathrm{Ir}, \mathrm{H}_{2}$, which is obtained by the action of sulphuretted hydrogen on iridiocyanide of lead $\mathrm{Cys} \mathrm{Ir}, \mathrm{Pbs}$.

Iridiocyanide of Potassium, Сys $\mathrm{Ir}, \mathrm{K}_{2}$, is obtained by the action of protochloride of iridium on cyanide of potassium. It forms colourless crystals; its solution gives, with salts of peroxide of iron, a deep indigo colour.

There appears to be a series of similar compounds formed by cyanide of palladium. The palladiocyanide of potassium corresponds to the platinocyanide, and its formula is $\mathrm{Cy}_{2} \mathrm{Pd}, \mathrm{K}$.

There is also reason to believe that manganese forms a manganocyanogen, corresponding to ferridcyanogen, Су6 $\mathbf{M n}_{2}=$ Cmy. The manganocyanide of potassium is probably $\mathrm{Cy}_{6} \mathrm{Mn}_{2}+$ $\mathrm{K}_{\mathbf{s}}=\mathrm{Cmy}, \mathrm{K}_{\mathbf{s}}$.

From what has been stated in the preceding pages, it will be seen that cyanogen has a very great tendency to form cyanides containing 2 or 3 metals, and likewise cyanides containing one of these metals and hydrogen in the place of the other. As these latter compounds are very powerful acids, we are naturally led to consider them as hydrogen acids, in which the hydrogen is combined with radicals. This view has been adopted above, and we have seen reason to admit the following radicals :-

$$
\begin{aligned}
& \text { Platinocyanogen } \mathrm{Cpy}=\mathrm{Cy}_{2} \mathrm{Pt} \\
& \text { Palladiocyanogen } \mathrm{Cpdy}=\mathrm{Cy}_{2} \mathrm{Pd} \\
& \text { Ferrocyanogen } \mathrm{Cfy}=\mathrm{Cy}_{3} \mathrm{Fe} \\
& \text { Iridiocyanogen } \mathrm{Ciy}=\mathrm{Cy}_{3} \mathrm{Ir} \\
& \text { Ferridcyanogen }=\mathrm{Cfdy}=\mathrm{Cy}_{6} \mathrm{Fe}_{2} \\
& \text { Cobaltocyanogen }=\mathrm{Cky}=\mathrm{Cy}_{6} \mathrm{Co}_{2} \\
& \text { Chromocyanogen }=\text { Cry }=\mathrm{Cy}^{6} \mathrm{Cr}_{2} \\
& \text { Manganocyanogen }=\mathrm{Cmy}=\mathrm{Cy}_{6} \mathrm{Mn}_{2}
\end{aligned}
$$

It will be observed that there are three different formulæ among these radicals, namely, $\mathrm{Cy}_{2} \mathrm{M}$; Суз $\mathrm{M}$; and $\mathrm{Cy}_{6} \mathrm{M}_{2}$; the first 
monobasic, the second bibasic, the third tribasic. No other view can at present be given of these compounds, of their acids, and of their salts, which is at once so satisfactory, so consistent, and so advantageous for the learner, as being adapted to assist the memory. It is true that the acids may be viewed as compounds of cyanide of a metal with cyanide of hydrogen (hydrocyanic acid), and their salts as compounds of two metallic cyanides. Thus, ferrocyanic acid, $\mathrm{Cys} \mathrm{Fe}+\mathrm{H}_{2}$, may be said to be $2 \mathrm{H} \mathrm{Cy}+\mathrm{Fe} \mathrm{Cy}$, and ferrocyanide of potassium, $2 \mathrm{~K} \mathrm{Cy}+\mathrm{Fe} \mathrm{Cy}$. Again, ferridcyanic acid may be $3 \mathrm{H} \mathrm{Cy}+\mathrm{Fe}_{2} \mathrm{Cy}_{3}$, and its potassium salt $3 \mathrm{~K} \mathrm{Cy}+\mathrm{Fe}_{2} \mathrm{Cy}_{3}$ : while platinocyanic acid and its potassium salt may be $\mathrm{H} \mathrm{Cy}+\mathrm{Pt} \mathrm{Cy}$ and $\mathrm{K} \mathrm{Cy}+\mathrm{Pt} \mathrm{Cy}$.

But the strong acid properties and harmless nature of these acids, and the remarkable permanence, both of the acids and of the salts, are entirely inconsistent with the presence of so weak an acid and so frightful a poison as hydrocyanic acid, or of bodies so easily decomposed as hydrocyanic acid and cyanide of potassium. Besides, there are numerous double cyanides, such as $\mathrm{K} \mathrm{Cy}, \mathrm{Zn} \mathrm{Cy}$; $\mathrm{K} \mathrm{Cy}, \mathrm{Cd} \mathrm{Cy} ; \mathrm{K} \mathrm{Cy}, \mathrm{Cu}_{2} \mathrm{Cy}$, \&c. \&c., which act as such ; being easily decomposed, and exhibiting no indications of containing radicals like those above described. We shall therefore not dwell on any other view, and merely allude here to the true double cyanides, as belonging more to the history of the metals, and less to that of the organic radicals.

It should here be mentioned, that the cubic copper-coloured crystals, found in the slag of iron furnaces, and regarded as metallic titanium, have been found by Wöhler to be a compound of cyanide of titanium with nituret of titanium.

\section{PARACYANOGEN.}

As an appendix to the metallic cyanides, we may here mention this compound, which is left behind as a dark-brown powder, when cyanide of mercury is heated in a retort. As oyanogen and mercury alone are given off, we should expect the salt to be dissipated by heat entirely; but this not being the case, it is evident that the residue, if it contain no mercury, must have the same composition as cyanogen, and be, in short, an isomerio modification of it-a solid cyanogen. Again, when cyanide of silver is heated, it gives off part of its cyanogen; it then glows, and, if soon removed from the fire, yields a peculiar residue, which is only partly dissolved by nitric acid. The insoluble residue appears to contain silver and cyanogen in the proportion of $\mathrm{Ag} \mathrm{Cys}$, and it is probable that the cyanogen here is in the solid modification, of which 1 eq. is supposed to be formed by 3 eq. of cyanogen. 
Whether, therefore, we admit paracyanogen as a separate radical or not, the two residues just mentioned contain carbon and nitrogen in the proportions to form cyanogen. It is also possible that some such compound may exist in cast iron and steel, which appear to contain nitrogen as well as carbon.

\section{CYANOGEN AND SULPHUR.}

\section{Sulphootanogen. $\quad \mathrm{Cy} \mathrm{S}_{2}=\mathrm{Csy}=58$.}

SYN. Bisulphuret of Cyanogen.-When ferrocyanide of potassium is heated with sulphur, there is formed a new salt, the formula of which is $\mathrm{Cy} \mathrm{S}_{\mathbf{2}}+\mathrm{K}$. This is sulphocyanide of potassium, which appears to contain the radical $\mathrm{Cy} \mathrm{S}_{2}$, or Csy. We cannot say that this radical is known in the free state, but by the action of chlorine on sulphocyanide of potassium there is formed a bright orange powder, which contains sulphocyanogen, mixed with some other bodies. Like the preceding radicals, sulphocyanogen, with hydrogen, forms a peculiar acid, the sulphocyanic or hydrosulphocyanic acid.

Hydrosulphocyanic Acid-Cy $\mathrm{S}_{2}, \mathrm{H}=\mathrm{Csy} \mathrm{H}$-is obtained by passing sulphuretted hydrogen gas through sulphocyanide of lead, $\mathrm{C}$ sy, $\mathrm{Pb}$, suspended in water. The solution thus formed is highly acid, and has the odour of acetic acid. It strikes a blood-red colour with salts of peroxide of iron, and this property is found in all soluble sulphocyanides. The formula of this acid corresponds to that of cyanic acid, $\mathrm{Cy}_{2}, \mathrm{H}$; and it may be viewed as cyanic acid, the oxygen of which has been replaced by sulphur. With metallic oxides, it forms the sulphocyanides of the metals; Cy $\mathrm{S}_{2}, \mathrm{H}+\mathrm{M} \mathrm{O}=\mathrm{Cy} \mathrm{S}_{2}, \mathrm{M}+\mathrm{H} \mathrm{O}$.

Sulphocyanide of Potassium-Cy $\mathrm{S}_{2}, \mathrm{~K}=\mathrm{Csy}, \mathrm{K}$.-The best. process for obtaining this salt is to melt at a gentle heat (only raised at the end to low redness) 46 parts of dried ferrocyanide of potassium, 32 of sulphur, and 17 of pure carbonate of potash. The mass when cold is boiled with water, and the solution, being filtered and evaporated, deposits striated prismatic crystals of the salt, very similar in appearance, and in taste also, to nitre.

If not quite pure, it is purified by solution in alcohol and recrystallisation. In this process, the whole cyanogen of the ferrocyanide is first converted into cyanide of potassium, and then, by the taking up of sulphur, into sulphocyanide; while the iron is converted into sulphuret. - As 1 eq. ferrocyanide contains 3 eq. of cyanogen and 2 of potassium, 1 eq. of carbonate of potash is added, and the 3 eq. of cyanide of potassium thus obtained take 
up 6 eq. of sulphur to form the new salt. $3 \mathrm{~K} \mathrm{Cy}+\mathrm{S}_{8}=$ 3 ( $\left.\mathrm{Cy} \mathrm{S}_{2}, \mathrm{~K}\right)$.

Sulphocyanide of potassium causes precipitates in some metallic solutions, but as many metallio sulphocyanides are soluble, the greater number of metals are not precipitated by this salt. With salts of peroxide of iron it strikes an intense blood-red colour, but causes no precipitate. With acetate of lead it gives yellow crystals, with subacetate a white precipitate, and with salts of suboxide of copper also an insoluble white subsulphocyanide of copper. Sulphocyanide of silver is precipitated as a curdy white solid, when sulphocyanide of potassium is added to nitrate of silver. The other sulphocyanides are soluble.

When sulphocyanic acid is set free from its salts, by diluted acids, and exposed to heat, it is resolved, with the aid of the elements of water, into carbonic acid, bisulphuret of carbon, and

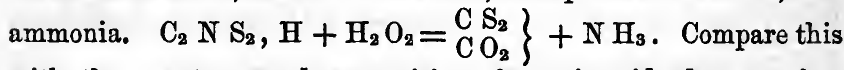
with the spontaneous decomposition of cyanic acid when set free

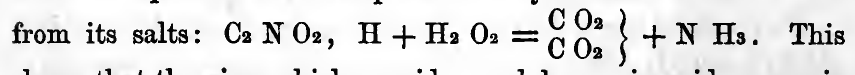
shows that the view which considers sulphocyanic acid as cyanic acid the oxygen of which has been replaced by sulphur, is confirmed by the similarity in the decomposition of these two acids; which, in this point of view, may be said to belong to the same type.

When sulphocyanide of potassium is mixed with 6 or 8 volumes of strong hydrochloric acid, hydrocyanio acid is given off, and a new crystalline acid is deposited, which contains more sulphur, and may be called (hydro) persulphocyanic acid. 3 (Cy S2, H), that is, 3 eq. of sulphocyanic acid lose $\mathrm{Cy} \mathrm{H,} 1$ eq. of hydrocyanic acid, and there remain 2 eq. of the compound $\mathrm{Cy}_{\mathbf{S}} \mathrm{S}_{3}, \mathrm{H}$, or persulphocyanic acid. The formula of its salts is $\mathrm{Cy}_{\mathbf{3}}, \mathrm{M}$. When the acid is dissolved in ammonia, it soon deposits sulphur, and the liquid retains a now compound $\mathrm{Cy}_{2} \mathrm{~S}_{5}, \mathrm{H}_{2}\left(=2 \mathrm{Cy} \mathrm{S}_{3}, \mathrm{H}-\mathrm{S}\right)$ combined with ammonia: on adding an acid, persulphocyanic acid is reproduced and deposited, while sulphocyanic acid remains in the solution: $\mathrm{Cy}_{2} \mathrm{~S}_{5}, \mathrm{H}_{2}= \begin{cases}\mathrm{Cy}_{\mathrm{s}}, \mathrm{H} \\ \mathrm{Cy} \mathrm{S}_{2}, \mathrm{H} \text {. }\end{cases}$

When solution of sulphocyanide of potassium is acted on by chlorine, or by nitric acid, a bright orange-yellow powder is deposited, which was long supposed to be a sulphocyanogen : but it now appears to be a compound or mixture of sulphocyanogen, sulphocyanic acid, and water, in the proportions $3 \mathrm{Cy} \mathrm{S}_{2}+\mathrm{Cy} \mathrm{S}_{2}$, $\mathrm{H}+\mathrm{H} \mathrm{O}=\mathrm{C}_{6} \mathrm{~N}_{3} \mathrm{~S}_{6}+\left(\mathrm{C}_{2} \mathrm{~N} \mathrm{~S}_{2}, \mathrm{H}\right)+\mathrm{H} 0=\mathrm{C}_{8} \mathrm{~N}_{4} \mathrm{~S}_{8} \mathrm{H}_{2} 0$. This yellow compound undergoes a very remarkable change 
when heated: it gives off bisulphuret of carbon, sulphur, and a little persulphocyanic acid, and there is left in the retort a grayishyellow powder, containing no sulphur, oxygen, nor hydrogen, and not decomposed by a low red heat. By a strong red heat it is dissipated, yielding a mixture of 3 vol. cyanogen to 1 vol. nitrogen.

This residue is the substance already alluded to as crude or impure mellone. Being impure its composition cannot be expressed by a formula. Mellone, which is a new radical, will be presently considered as such.

If the orange-yellow compound which yields it be thrown into melted sulphocyanide of potassium, the mellone actually seizes the potassium, expelling the sulphocyanogen, which is resolved into bisulphuret of carbon, sulphur, cyanogen, and nitrogen, all of which escape with effervescence. This is because mellone is not only a powerful radical, but also capable of resisting a strong heat.

\section{Melione. $\mathrm{Me}=\mathrm{C}_{18} \mathrm{~N}_{13}=\mathrm{Cy}_{9} \mathrm{~N}_{4}=290$.}

This radical is not fully known, not having been obtained in a state of purity. We have already considered it as a compound of cyanogen with nitrogen, and explained the facts which lead to the adoption of the very strange formula now found to belong to it. There is reason to believe that mellone is a solid, capable of resisting a pretty high temperature, but ultimately dissipated in the forms of cyanogen and nitrogen gases. It seems to be present in the residue left when sulphocyanogen, or the yellow body so called, is heated nearly to redness, and the residue obtained by heating the sulphocyanide of ammonium was supposed to be mellone pure, or nearly so. But this does not appear to be the case, although mellone is probably present in this residue as well as in that previously mentioned.

But the compounds of mellone are better known:

Hydromellonic Acid- $\mathrm{Me} \mathrm{H}_{3}=\mathrm{C}_{18} \mathrm{~N}_{13}, \mathrm{H}_{3}$.-This acid is obtained in solution by decomposing a solution of mellonide of mercury in dilute hydrocyanic acid by sulphuretted hydrogen. The solution of the acid tastes sour, and decomposes the carbonates. With potash it forms mellonide of potassium. When evaporated to dryness, even in vacuo, the acid is in great part decomposed, and a residue is left, which is nearly insoluble, but still contains a little of the acid.

This acid is tribasic, forming with potash three salts, $\mathrm{Me} \mathrm{K}_{3}$, or mellonide of potassium; $\left.\mathrm{Me} \frac{\mathrm{H}}{\mathrm{K}_{2}}\right\}$ or soluble acid mellonide, and $\left.\mathrm{Me} \underset{\mathrm{K}}{\mathrm{H}_{2}}\right\}$ or insoluble acid mellonide. 
Mellonide of Potassium-Me $\mathrm{K}_{3}=\mathrm{C}_{18} \mathrm{~N}_{18} \mathrm{~K}_{3}$. -This salt may be obtained by fusing sulphur with ferrocyanide of potassium, when it is produced along with the sulphocyanide; or by fusing the sulphocyanide with the chlorides of antimony or bismuth; or with melam, or with the residue left when melam is heated, that is, crude mellone. The changes are not fully understood, but bisulphuret of carbon is expelled, and the residue boiled with water, yields the mellonide, which is deposited on cooling in the form of very slender acicular crystals, interwoven into a mass, like those of sulphate of quinine. This is mellonide of potassium with 10 eq. of water of crystallisation $\mathrm{Me} \mathrm{K}_{3}+10$ aq. It formerly occurred to Liebig (to whom we are indebted for our knowledge of its composition and properties, though Gmelin first observed it,) with 5 eq. of water, but in all his later researches it has crystallised with 10 eq. It is very soluble in hot water, less so in cold water, insoluble in alcohol. With hydrochloric acid it yields the insoluble acid mellonide, $\mathrm{Me} \underset{\mathrm{K}}{\mathrm{H}}\}$, and with acetio acid the soluble acid salt $\mathrm{Me} \underset{\mathrm{H}}{\mathrm{K}}\}+6$ aq.

A boiling solution of the mellonide, mixed with nitrate of silver, gives a white precipitate of mellonide of silver, $\mathrm{Me} \mathrm{Ag}_{3}$. With corrosive sublimate it yields insoluble mellonide of mercury $\mathrm{Me} \mathrm{Hgs,} \mathrm{and} \mathrm{with} \mathrm{a"} \mathrm{salt} \mathrm{of} \mathrm{lead} \mathrm{it} \mathrm{yields} \mathrm{insoluble} \mathrm{mellonide}$ of lead $\mathrm{Me}, \mathrm{Pb}_{3}$.

When boiled with caustic potash in excess, mellonide of potassium is decomposed, ammonia is given off, and a new acid, cyamelurio acid, is formed. At this period the liquid, on the addition of sal ammoniac, yields a precipitate of ammelide, but if longer boiled, the ammelide disappears, and the addition of acetic acid separates another new acid, melanurio acid. If the boiling be still further continued, ammonia is continually given off, and acetic acid at last causes a precipitate of acid cyanurate of potash. The ultimate products of this reaction are therefore ammonia, ammonia and cyanuric acid. The relation of the intermediate products to this one are easily explained.

Cyameluric acid is $\mathrm{C}_{12} \mathrm{~N}_{7} \mathrm{O}_{6}, \mathrm{H}_{3}$, and its potash salt is $\mathrm{C}_{12} \mathrm{~N}_{7}$ $\mathrm{O}_{6}, \mathrm{~K}_{3}$. Melanuric acid is $\mathrm{C}_{12} \mathrm{H}_{8} \mathrm{~N}_{8} \mathrm{O}_{8}$.

2 eq. of mellonide of potassium with 18 eq. of water yield 2 eq. of cyamelurate of potash, 1 eq. of ammelide, $\mathrm{C}_{22} \mathrm{~N}_{8} \mathrm{H}_{8} \mathrm{O}_{9}$, and 3 eq. of ammonia.

Ammelide, by taking up 2 eq. of water, and losing 1 eq. of ammonia, is converted into melanuric acid, and 1 eq. of this acid, with 4 eq. of water, is resolved into 2 eq. of cyanuric acid and 2 eq. of ammonia. 
PRODUCTS OF THE DISTILLATION OF SUIPHOCYANIDE OF AMMONIUM.

As an appendix to sulphocyanogen and its derivative mellone, we may consider the remarkable results of the action of heat on sulphocyanide of ammonium, $\mathrm{N} \mathrm{H}_{4}, \mathrm{C}_{2} \mathrm{~N} \mathrm{~S}_{2}$.

When this salt is heated, bisulphuret of carbon, ammonia, and sulphuretted hydrogen are expelled, and a grayish residue is left, which Liebig calls melam. Its formula is $\mathrm{C}_{12} \mathrm{~N}_{11} \mathrm{H}_{\theta}$, and it gives rise to a series of compounds, which are curiously related together, and which are also related to those we have just mentioned.

When boiled with sulphuric acid, it yields ammonia and cyanuric acid. Taking up 12 eq. of water, it produces 2 eq. of cyanuric acid and 5 eq. of ammonia.

When melam is acted on by boiling with potash, a series of new compounds is obtained. The first is melamine, which is deposited in crystals when the alkaline solution cools. Melamine contains no oxygen, but is an artificial organic base, neutralising acids, and forming salts. Its formula is $\mathrm{C}_{6} \mathrm{~N}_{6} \mathrm{H}_{6}=\mathrm{C}_{6} \mathrm{~N}_{4}+\mathrm{N}_{2} \mathrm{H}_{6}$; that is, it contains the elements of 1 eq. mellone, and 2 eq. ammonia.

The second new body is obtained as a white powder, when the alkaline solution which has deposited melamine, is supersaturated with acetic acid. It is called ammeline, and is also a base, although weaker than melamine. Its formula is $\mathrm{C}_{6} \mathrm{~N}_{5} \mathrm{H}_{5} \mathrm{O}_{2}=\mathrm{C}_{6} \mathrm{~N}_{4}+\mathrm{NH}_{3}$ $+2 \mathrm{H} \mathrm{O}$, or 1 eq. mellone, 1 eq. ammonia, and 2 eq. water. It forms a crystallisable salt, with nitric acid.

It may here be observed, that melam, $\mathrm{C}_{12} \mathrm{~N}_{11} \mathrm{H}_{\theta}$, with 2 eq. water, $\mathrm{H}_{2} \mathrm{O}_{2}$, contains the elements of 1 eq. melamine, $\mathrm{C}_{6} \mathrm{~N}_{6} \mathrm{H}_{6}$, and 1 eq. ammeline, $\mathrm{C}_{6} \mathrm{~N}_{5} \mathrm{H}_{5} \mathrm{O}_{2}$.

When either melamine or ammeline is dissolved in strong sulphuric acid, or melam in nitric acid, and the solution mixed first with two vol. of water, and then with four of alcohol, a white powder is obtained, resembling ammeline, but having the formula $\mathrm{C}_{12} \mathrm{~N}_{8} \mathrm{H}_{8} \mathrm{O}_{6}=2 \mathrm{C}_{6} \mathrm{~N}_{4}+\mathrm{NH}_{3}+6 \mathrm{H} \mathrm{O}$, or 2 eq. mellone, 1 eq. ammonia, and 6 eq. water. It is called ammelide, and has rather the characters of an acid than of a base.

Melamine, by the action of hydrochloric acid, aided by heat, is transformed into ammeline, giving off ammonia, while water is taken up. $\mathrm{C}_{6} \mathrm{~N}_{6} \mathrm{H}_{6}+\mathrm{H}_{2} \mathrm{O}_{2}=\mathrm{C}_{6} \mathrm{~N}_{6} \mathrm{H}_{8} \mathrm{O}_{2}$, and $\mathrm{C}_{6} \mathrm{~N}_{6} \mathrm{H}_{8}$ $\mathrm{O}_{2}-\mathrm{N} \mathrm{H}_{3}=\mathrm{C}_{6} \mathrm{~N}_{5} \mathrm{H}_{5} \mathrm{O}_{2}$.

Melam, also, when treated in the same way, yields ammeline and ammonia. $\mathrm{C}_{12} \mathrm{~N}_{11} \mathrm{H}_{9}+\mathrm{H}_{4} \mathrm{O}_{4}=\mathrm{C}_{12} \mathrm{~N}_{11} \mathrm{H}_{13} \mathrm{O}_{4}$; and $\mathrm{C}_{12} \mathrm{~N}_{11} \mathrm{H}_{13} \mathrm{O}_{4}-\mathrm{N} \mathrm{H}_{3}=\mathrm{C}_{12} \mathrm{~N}_{10} \mathrm{H}_{10} \mathrm{O}_{4}=2\left(\mathrm{C}_{6} \mathrm{~N}_{5} \mathrm{H}_{5} \mathrm{O}_{2}\right)$.

All these substances may be resolved, by the action of acids 
into cyanuric acid and ammonia. It appears that they are all, that is-melam, melamine, and ammeline, first converted into ammelide, and that ammelide is the source of the cyanuric acid and ammonia.

It is obvious that they are all elosely related to each other, and to cyanuric acid. That they are also related to mellone is probable, because when heated they leave a yellow residue, which is converted by a stronger heat into cyanogen and nitrogen; which, in short, is mellone. All these compounds may be represented as tribasic cyanurate of ammonia, minus water, or water and ammonia. Thus,

1 eq. anhydrous cyanurate of ammonia, $\mathrm{Cy}_{3} \mathrm{O}_{3}, 3 \mathrm{~N} \mathrm{H}_{3}=\mathrm{C}_{6} \mathrm{~N}_{6} \mathrm{H}_{9} \mathrm{O}_{3}$

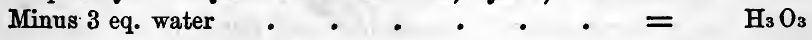

Yields 1 eq. melamine . . . . . $=\mathrm{C}_{6} \mathrm{~N}_{6} \mathrm{H}_{6}$

1 eq. anhydrous cyanurate of ammonia . $\quad=\mathrm{C}_{6} \mathrm{~N}_{6} \mathrm{H}_{9} \mathrm{O}_{3}$

$\left.\begin{array}{rl}\text { Minus } 1 \text { eq. water } & =\mathrm{H}_{0} \\ , \quad 1 \text { eq. ammonia } & =\mathrm{H}_{3} \mathrm{~N}\end{array}\right\} \mathrm{NH}_{4} \mathrm{O} . .=\mathrm{N} \mathrm{H}_{4} \mathrm{O}$

Yields 1 eq. ammeline : . . . . $=\overline{=\mathrm{C}_{6} \mathrm{~N}_{5} \mathrm{H}_{5} \mathrm{O}_{2}}$

2 eq. anhydrous cyanurate of ammonia $\cdot .=\mathrm{C}_{12} \mathrm{~N}_{12} \mathrm{H}_{18} \mathrm{O}_{6}$

Minus 1 eq. ammonia $=$
,$\quad 6$ eq. water $\mathrm{H}_{3}$

Yields 1 eq. melam . . . . . $=\mathrm{C}_{12} \mathrm{~N}_{11} \mathrm{H}_{9}$

2 eq. anhydrous cyanurate of ammonia . . $=\mathrm{C}_{12} \mathrm{~N}_{12} \mathrm{H}_{18} \mathrm{O}_{6}$

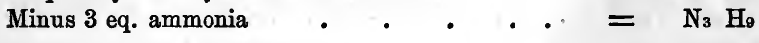

Yields 1 eq. ammelide . . . . $=\overline{\mathrm{C}_{12} \mathrm{~N}_{9} \mathrm{H}_{9} \mathrm{O}_{8}}$

When the mass remaining in the retort when urea is heated, and formerly believed to be cyanuric acid or cyanurate of ammonia, is acted on by acids, it yields cyanuric acid, and ammonia is found in the solution; but if it be boiled with water, an insoluble snow-white powder is obtained, which agrees with ammelide in almost all its properties. Its formula, however, is $\mathrm{C}_{12} \mathrm{~N}_{8} \mathrm{H}_{8} \mathrm{O}_{8}$, and it may also be derived from cyanurate of ammonia, as follows :-

1 eq. anhydrous tribasic cyanurate of ammonia,

Minus 2 eq. ammonia $\mathrm{Cy}_{3} \mathrm{O}_{3}, 3 \mathrm{~N} \mathrm{H}_{3}$

and

Plus 1 eq. water. $\quad$. $\quad$.

$=\mathrm{C}_{6} \mathrm{~N}_{6} \mathrm{H}_{9} \mathrm{O}_{3}$

$=\mathrm{N}_{2} \mathrm{H}_{6}$

$\mathrm{C}_{6} \mathrm{~N}_{4} \mathrm{H}_{3} \mathrm{O}_{3}$

Yields 1 eq. of the new product from urea $=\mathrm{C}_{6} \mathrm{~N}_{4} \mathrm{H}_{4} \mathrm{O}_{4}$ Or, $\mathrm{C}_{12} \mathrm{~N}_{8} \mathrm{H}_{8} \mathrm{O}_{3}$. 
This product, therefore, is intermediate between ammelide and cyanurio acid. It is now called melanuric acid.

To be transformed into hydrated cyanuric acid, it has only to lose 1 eq. ammonia, and to gain 2 eq. water. Its formation from. urea is very easily understood; for 4 eq. urea, minus 2 eq. carbonic acid and 4 eq. ammonia, will give this compound :-

4 eq. urea
Minus 2 eq. carbonic acid $\mathrm{C}_{2} \mathrm{O}_{4}$
," 4 eq. ammonia

1 eq. of the new compound

$$
\begin{array}{r}
\mathrm{N}_{4} \mathrm{H}_{12} \\
\mathrm{C}_{8} \mathrm{~N}_{8} \mathrm{H}_{10} \mathrm{O}_{8} \\
\mathrm{C}_{2} \mathrm{~N}_{4} \mathrm{H}_{12} \mathrm{O}_{4}
\end{array}
$$

C6 $\mathrm{N}_{4} \mathrm{H}_{4} \mathrm{O}_{4}$

In order to render still more obvious the relation of these compounds, melam excepted, to cyanuric acid and among each other, let us express the hypothetical compound $\mathrm{N} H$ by $\mathbf{M}_{2}, \mathbf{M}$, therefore, standing for $\frac{1}{2}$ eq. $\mathrm{NH}$. We then have-

$$
\begin{aligned}
& \text { Cyanuric acid, } \mathrm{C}_{6} \mathrm{~N}_{3} \mathrm{H}_{3} \mathrm{O}_{6} \quad . \quad . \quad .=\mathrm{Cys} \mathrm{O}_{6}+\mathrm{H}_{3} \\
& \text { Melamine, } \mathrm{C}_{6} \mathrm{~N}_{6} \mathrm{H}_{6}{ }^{\circ} \quad . \quad . \quad . \quad=\mathrm{Cy}_{3} \mathrm{M}_{6}+\mathrm{H}_{3} \\
& \text { Ammeline, } \mathrm{C}_{6} \mathrm{~N}_{5} \mathrm{H}_{5} \mathrm{O}_{2} \cdot . \quad . \quad .=\mathrm{Cy}_{3}\left\{\begin{array}{l}
\mathrm{M}_{4} \\
\mathrm{O}_{2}
\end{array}+\mathrm{H}_{3}\right. \\
& \text { Ammelide, } \mathrm{C}_{6} \mathrm{~N}_{4} \frac{1}{2} \mathrm{H}_{4} \frac{1}{2} \mathrm{O}_{3} \quad . \quad . .=\mathrm{Cy}_{3}\left\{\begin{array}{l}
\mathrm{M}_{3} \\
\mathrm{O}_{3}
\end{array}+\mathrm{H}_{3}\right. \\
& \text { Melanuric acid, } \mathrm{C}_{6} \mathrm{~N}_{4} \mathrm{H}_{4} \mathrm{O}_{4} \quad . . \quad=\mathrm{Cy}_{3}\left\{\begin{array}{l}
\mathrm{M} \\
\mathrm{O}_{4}
\end{array}+\mathrm{H}_{3}\right. \\
& \text { Cyanuric acid . . . . . . }=\mathrm{Cys} \mathrm{O} \mathrm{O}_{3}+\mathrm{H}_{3} *
\end{aligned}
$$

Here we see the change in properties accompanying the gradual substitution of $\mathrm{M}$ for $\mathrm{O}$. At one end of the series is melamine, a base, containing no oxygen; at the other, cyanuric acid, a highly oxygenised acid: while ammeline is a weak base, and the remaining ones are neutral, or have gradually increasing acid properties.

Cyameluric acid, already mentioned, is related to the compounds here described. Its formula, $\mathrm{C}_{12} \mathrm{~N}_{7} \mathrm{O}_{6} \mathrm{H}_{3}$, differs from that of melanuric acid, by containing 1 eq. of ammonia and 2 eq. of water less; and like the other compounds when boiled with acids, it yields ammonia and cyanurio acid, the elements of water contributing to the change.

If a compound be supposed, in the preceding table, between melanuric and cyanurio acids, its composition referred to $12 \mathrm{eq}$.

* In this table some of the formulas are only half of what we have previously given, but it is only intended to show the relations among these compounds, not their exact equivalents. 
of carbon, would be $\mathrm{C}_{12} \mathrm{~N}_{7} \mathrm{H}_{7} \mathrm{O}_{10}$, which is cyameluric acid+ 4 eq. of water. This acid, however, has not been shown to be formed from melam when boiled with acids, but is produced, as already mentioned, when melamide of potassium is boiled with excess of potash, a process in which ammelide and melanuric acid are also formed. There can be no doubt, therefore, that cyameluric acid belongs to this remarkable series of compounds, which are basic, neutral or acid, are all derived from mellone or melam, and all, when boiled with acids, yield cyanurio acid and ammonia.

Before quitting these compounds, it is proper to point out that as sulphocyanic acid corresponds to cyanic acid, sulphur being substituted for oxygen, so sulphocyanide of ammonium corresponds precisely in the same way to urea; for urea is $\mathrm{C}_{2} \mathrm{~N}_{2} \mathrm{H}_{4} \mathrm{O}_{2}$, and sulphocyanide of ammonium is (see above) $\mathrm{C}_{2} \mathrm{~N}_{2} \mathrm{H}_{4} \mathrm{~S}_{2}$. That this analogy is not imaginary we have seen in the similarity of the action of heat on both.

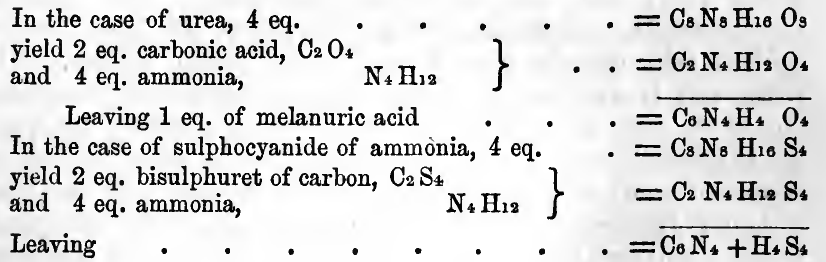

The analogy only fails in this, that the bisulphuret of carbon and ammonia, instead of uniting to form one compound, are resolved into several, among which are sulphuretted hydrogen, and a solid substance, probably containing mellone.

When impure or crude mellone is boiled with nitrio acid a new acid is formed crystallising in octahedrons, which, when redissolved in water, form pearly scales. Liebig, who alone has studied it, found its formula and all its reactions exactly like those of cyanuric acid, and called it cyanilic acid: 1 eq. mellone and 6 eq. water $\mathrm{C}_{6} \mathrm{~N}_{4}+\mathrm{H}_{6} \mathrm{O}_{6}$, are equal to 1 eq. cyanilic (or cyanuric) acid, and 1 eq. ammonia $\left(\mathrm{C}_{6} \mathrm{~N}_{3} \mathrm{O}_{6} \mathrm{H}_{3}\right)+\mathrm{N} \mathrm{H}_{3}$. Further experiments are required to establish cyanilio acid as an independent acid.

Sulphomellone. Hydrosulphomellonic acid.

According to the researches of Jamieson, the orange-yellow powder, commonly called sulphocyanogen, and which, at p. 100, is 
said to contain 3 eq. of bisulphuret of cyanogen, 1 eq. of hydrosulphocyanic acid, and 1 eq. water, really consists of the elements of 1 eq. of each of these three substances, Cy $\mathrm{S}_{2}+\mathrm{Cy} \mathrm{S}_{2}, \mathrm{H}$ $+\mathrm{H} \mathrm{O}=\mathrm{C}_{4} \mathrm{~N}_{2} \mathrm{H}_{2} \mathrm{O} \mathrm{S}_{4}$, how arranged we know not.

When dissolved to saturation in hydrosulphuret of sulphuret of potassium, $\mathrm{K} \mathrm{S}, \mathrm{H} \mathrm{S}$, the elements of $4 \mathrm{eq}$. sulphocyanogen, $4 \mathrm{Cy} \mathrm{S} \mathrm{S}_{2}=\mathrm{C}_{8} \mathrm{~N}_{4} \mathrm{~S}_{8}$, along with 4 eq. of $\mathrm{K} \mathrm{S}, \mathrm{H} \mathrm{S}$, give rise to 7 eq. sulphuretted hydrogen, $7 \mathrm{H} \mathrm{S} ; 1$ eq. pentasulphuret of potassium $\mathrm{K} \mathrm{S}_{5} ; 2$ eq. carbonate of potash, $2\left(\mathrm{KO}, \mathrm{C} \mathrm{O}_{2}\right)$ and 1 eq. sulphomellonide of potassium, $\mathrm{C}_{6} \mathrm{~N}_{4} \mathrm{H}_{3} \mathrm{~S}_{4}, \mathrm{~K}$.

When this salt is decomposed by an acid, as hydrochloric acid, a simple exchange takes place. $\mathrm{C}_{6} \mathrm{~N}_{4} \mathrm{H}_{3} \mathrm{~S}_{4} \mathrm{~K}+\mathrm{H} \mathrm{Cl}=\mathrm{K} \mathrm{Cl}$ $+\mathrm{C}_{6} \mathrm{~N}_{4} \mathrm{H}_{31} \mathrm{~S}_{4} ; \mathrm{H}$. The latter formula represents the hydrosulphomellonic acid, which is a sparingly soluble white crystalline powder. It combines readily with the alkalies, and decomposes the carbonates of the alkaline earths when boiled with them. All the sulphomellonides of the metals of the alkalies and alkaline earths are soluble and crystallise beautifully. The general formula for the anhydrous salts is $\mathrm{C}_{6} \mathrm{~N}_{4} \mathrm{H}_{3} \mathrm{~S}_{4} \mathrm{M}$; but the crystallised salts contain from 2 to 6 eq. of water besides. The silver salt is anhydrous. The supposed radical of this acid, and of its salts, is not yet known in a separate state; but when the sulphomellonide of potassium is acted on by chlorine, there is separated a white powder, which seems to be sulphomellone, although Jamieson has not yet been able to ascertain that point. But the acid and its salts are rendered very simple by assuming the existence of this radical, which agrees with all the facts yet known, and makes the sulphomellonides analogous in composition, as they are in their relations, in their origin, and in their properties, to chlorides, bromides, iodides, fluorides, cyanides, and mellonides.

Having now concluded our sketch of the compounds derived from that of sulphur and cyanogen, it only remains to mention, that cyanogen forms one or two compounds with sulphuretted hydrogen, and that sulphocyanic acid forms a compound with the same gas. These compounds, however, are as yet too little known to permit of their being clearly laid down.

Cyanogen does not form any compound of importance with phosphorus or the remaining metalloids. But there is a very interesting and important series of compounds, in which we may conceive, with some probability, a radical to exist formed of the elements of cyanogen and those of oxalyle or carbonic oxide, $\mathrm{C}_{2} \mathrm{O}_{2}$. This is the series of compounds derived from uric acid, and consequently closely connected with urea, which we have 
seen to be derived from cyanate of ammonia, and, through cyanic acid, from cyanurio acid, which connects it again with mellone, melam, and sulphocyanogen.

We shall first briefly describe the uric acid itself, as the starting-point of an extensive series of products. The radical supposed to exist in these compounds will be better understood if described after we have become acquainted with them.

\section{CYANOGEN WITH CARBONIC OXIDE.}

\section{URYLE.}

1. URIo Actd. $\mathrm{C}_{10} \mathrm{~N}_{4} \mathrm{H}_{4} \mathrm{O}_{6}=\mathrm{C}_{10} \mathrm{~N}_{4} \mathrm{H}_{3} \mathrm{O}_{5}+\mathrm{H} \mathrm{O}$. Or $\mathrm{C}_{10} \mathrm{~N}_{4} \mathrm{H}_{2} \mathrm{O}_{4} 2 \mathrm{H} \mathrm{O}$; or $\mathrm{C}_{5} \mathrm{~N}_{2} \mathrm{H} \mathrm{O}_{2}, \mathrm{HO}$ (Bensch).

SxN. Urilic Acid.-Lithic Acid.-Occurs in small quantity in the healthy urines of man and quadrupeds, and in much larger quantity in the urine of birds, whether carnivorous or herbivorous, as in the pigeon and hawk tribes. In the urine of birds it forms the white part, in the form of urate of ammonia, and it is still found as such in guano-a substance produced by the long-continued action of the air on the urine (or excrement, for they are voided together) of sea-fowl. The only excrement of serpents, as, for example, of the boa-constrictor, is a white semisolid mass, which soon dries, and is pure acid urate of ammonia. In serpents, which are all carnivorous, it is very remarkable that the whole excreta (except occasionally hair and feathers, which pass undigested,) should take the form of urate of ammonia. In diseased urine, uric acid is often deposited on cooling, and generally of a reddish colour; it also constitutes the most frequent form of gravel and of calculus, when deposited within the bladder. Acid urate of soda is found in the chalk-stones of gouty patients; and it is well known that gout is a disease closely allied to calculus of this kind.

It is best obtained from the excrement (or urine) of the boa, which is powdered, and dissolved in 40 parts of boiling water by the gradual addition of caustic potash, till the liquid is decidedly alkaline. The uric acid forms urate of potash, which dissolves, while the ammonia escapes. The hot liquid, being filtered to separate impurities (and with the above proportion of water it filters rapidly, while with less it crystallises on the filter and chokes it up), is mixed with a decided excess of hydrochloric acid, when the uric acid is set free, and being insoluble, is deposited, at first, as a very bulky gelatinous hydrate, which in a few moments spontaneously loses water and shrinks into a crystalline heavy precipitate. This is well washed with cold 
water and dried in the air, when it forms a shining powder, composed of distinct but minute crystals. If made from a cold saturated solution of urate of potash, the crystals are much larger, but contain 17.5 per cent. of water; in this case 4 eq. of water are expelled at $212^{\circ}$, leaving the same substance as that precipitated from a hot solution, which, when dried in the air, loses no weight at $212^{\circ}$. The latter is $\mathrm{C}_{10} \mathrm{~N}_{4} \mathrm{H}_{3} \mathrm{O}_{5}+\mathrm{H} \mathrm{O}$ $=\overline{\mathrm{U}} \mathbf{r}+\mathrm{H} \mathrm{O}$; the large crystals are hydrate, Ur, $\mathrm{H} \mathbf{O}+$ 4 aq.

If pure white fragments of the urine of the boa have been used, the above simple process yields uric acid chemically pure, even when the solution in potash has had a decided yellow colour. This uric acid is snow-white, and is entirely dissipated by heat, leaving no trace of ashes. But if the boa's urine have been impure, or if calculi have been employed to yield uric acid, in both of which cases the alkaline solution is brown, often very dark, and yields a coloured uric acid, or again if we wish to extract uric acid from guano, we must first purify the urate of potash by evaporating the alkaline solution till it crystallises in a mass, or passing carbonic acid through it to neutralise the free potash, when the acid urate of potash is deposited, and is washed on a filter with cold water, in which it is very sparingly soluble, till it is quite white. It is then dissolved in boiling water, and decomposed by hydrochloric acid as before. I have described thus minutely the preparation of pure and colourless uric acid, because none of the very interesting products derived from it can be obtained if we employ uric acid with even a very slight tinge of colour. The presence of a mere trace of the colouring matter of urine I have found to exert a most remarkable influence on the oxidation of uric acid by nitric acid, an influence which I can only compare to that of a ferment in causing a peculiar decomposition to take place.

Uric acid requires 15,000 parts of cold and nearly 2000 of hot water for solution, and its solution reddens litmus. It forms salts with bases, especially with the alkalies and alkaline earths, all of which are insoluble or sparingly soluble.

The salts of uric acid have been lately examined by Bensch, according to whom the common urates are acid salts, while the eq. of the acid is only half that above given, or $\mathrm{C}_{5} \mathrm{~N}_{2} \mathrm{H}_{2} \mathrm{O}_{3}=$ $\mathrm{C}_{5} \mathrm{~N}_{2} \mathrm{H} \mathrm{O}_{2}+\mathrm{H} \mathrm{O}$. The neutral salts are $\mathrm{C}_{5} \mathrm{~N}_{2} \mathrm{H} \mathrm{O}_{2}+\mathrm{M} \mathrm{O}$, and the acid salts are $\mathrm{C}_{5} \mathrm{~N}_{2} \mathrm{H} \mathrm{O}_{2}, \mathrm{MO}+\mathrm{C}_{5} \mathrm{~N}_{2} \mathrm{H} \mathrm{O} \mathrm{O}_{2}, \mathrm{H} \mathrm{O}$. Bensch has obtained the neutral salts of potash and soda, which are much more soluble than the well-known urates of these bases, acid urates, according to Bensch. The neutral urate of potash dissolves in 35 parts of hot water, is alkaline, and is converted 
into the acid salt, both by water and carbonio acid. The acid salt requires 85 parts of boiling water for solution. The urates of soda are less soluble, and the acid urate of ammonia, the only salt formed with this base, dissolves in about 1600 parts of water at $60^{\circ}$, and 240 parts at $212^{\circ}$.

The only other urates hitherto examined by Bensch are the acid salts of magnesia, lime, baryta, strontia, lead, and copper. It would appear that, adopting his formula, the uric acid has a most remarkable tendency to form acid salts, and as it appears also to form double salts, it is not easy to see why he halves the formula of Liebig for the hydrated acid; since the above characters are those which distinguish bibasic acids, and it would appear more consistent with the newly observed relations of the acid, to express the formula of Liebig, $\mathrm{C}_{10} \mathrm{~N}_{4} \mathrm{H}_{4} \mathrm{O}_{6}=\mathrm{C}_{10} \mathrm{~N}_{4} \mathrm{H}_{3} \mathrm{O}_{5}+\mathrm{H} \mathrm{O}$ by the following bibasic form $\mathrm{C}_{10} \mathrm{~N}_{4} \mathrm{H}_{2} \mathrm{O}_{4}+2 \mathrm{H} \mathrm{O}$ than by the monobasio form $2\left(\mathrm{C}_{5} \mathrm{~N}_{2} \mathrm{H} \mathrm{O}_{2}, \mathrm{H} \mathrm{O}\right)$. If we adopt the bibasic formula, then the general formulæ for the neutral and acid salts, as above given by Bensch, will become, respectively, $\mathrm{C}_{10} \mathrm{~N}_{4} \mathrm{H}_{2} \mathrm{O}_{4}$ $+2 \mathrm{M} \mathrm{O}$, and $\left.\mathrm{C}_{10} \mathrm{~N}_{4} \mathrm{H}_{2} \mathrm{O}_{4}+\underset{\mathrm{H} \mathrm{O}}{\mathrm{M}}\right\}$.

\section{PRODUCTS OF THE OXIDATION OF URIC ACID.}

Uric acid is very permanent under ordinary circumstances, but is readily oxidised by powerful oxidising agents, such as peroxide of lead, peroxide of manganese, permanganate of potash, and nitric acid.

1. Oxidation of uric acid by peroxide of lead.-If uric acid be mixed with twenty parts of boiling water, and peroxide of lead added in small portions to the liquid kept boiling, the brown colour of the oxide disappears, and a heavy white powder is formed. When we have added about two parts of the oxide for one of uric acid, or, at all events, when the oxide begins not to lose its brown colour, the hot liquid is to be filtered, and on cooling it deposits a number of hard brilliant white crystals, of which more are obtained on evaporation. The mother liquid at last crystallises in a mass of very soluble prismatic crystals. These last are pure urea: the first crystals are Allantoine, and the powder is oxalate of lead, mixed with a little carbonate, and with the excess of peroxide. Hence the products of this oxidation are, Urea, Allantoine, and Oxalic Acid. After describing allantoine, we shall be able to explain the reaction.

Allantoine.- $\mathrm{C}_{4} \mathrm{~N}_{2} \mathrm{H}_{3} \mathrm{O}_{3}$.-SrN. Allantoic Acid.-This body was first observed in the allantoic fluid of the fotal calf, which is, in fact, the urine of the fotal animal. When this fluid is evapo- 
rated, it deposits crystals of allantoine, formerly called allantoic acid, which, however, is not an acid. Its occurrence in the allantoic fluid-that is, as an ingredient in urine-and its artificial production from uric acid by a process of oxidation, are facts of very great interest when viewed in connection. It is best obtained from uric acid, as above described, or, as Wöhler has recently shown, from the urine of calves by evaporation, when it crystallises out. It is a very indifferent, or neutral substance, and forms few compounds; only one, with oxide of silver, has been described, the formula of which is $\mathrm{C}_{8} \mathrm{~N}_{4} \mathrm{H}_{5} \mathrm{O}_{5}+\mathrm{Ag} \mathrm{O}=2$ eq. allantoine, minus 1 eq. water, and plus 1 eq. oxide of silver.

When boiled with alkalies, it is resolved into ammonia which escapes, and oxalic acid which combines with the alkali. In fact, both allantoine, $\mathrm{C}_{4} \mathrm{~N}_{2}+\mathrm{H}_{3} \mathrm{O}_{3}$, and oxalate of ammonia, $\mathrm{C}_{2} \mathrm{O}_{3}+\mathrm{N} \mathrm{H}_{4} \mathrm{O}$ may be represented as formed of cyanogen and water, and it is obvious that the addition of $3 \mathrm{eq}$. of water to 1 eq. of allantoine 'gives oxalate of ammonia, for $\mathrm{C}_{4} \mathrm{~N}_{2}+\mathrm{H}_{6} \mathrm{O}_{6}$ $=2\left(\mathrm{C}_{2} \mathrm{O}_{3}+\mathrm{N} \mathrm{H}_{3}\right)$.

We can now explain the formation of allantoine.

1 eq. uric acid

Plus 2 eq. oxygen (from 2 eq. $\left.\mathrm{Pb} \mathrm{O}_{2}\right)=\mathrm{F}_{3} \mathrm{O}_{2}$
and 3 eq. water

Together . $. \quad \cdot \quad \cdot \quad=\mathrm{C}_{10 \mathrm{~N}_{4} \mathrm{H}_{7} \mathrm{O}_{11}}$

Are equal to 1 eq. urea . . . . . $=\mathrm{C}_{2} \mathrm{~N}_{2} \mathrm{H}_{4} \mathrm{O}_{2}$

2 eq. oxalic acid . . . . . $=\mathrm{C}_{4} \quad \mathrm{Oe}_{\mathrm{e}}$

1 eq. allantoine $\quad$. . . $=\mathrm{C}_{4} \mathrm{~N}_{2} \mathrm{H}_{3} \mathrm{O}_{3}$

Together $\quad=\mathrm{C}_{10} \mathrm{~N}_{4} \mathrm{H}_{7} \mathrm{O}_{12}$

Or, in the form of an equation,

$\mathrm{C}_{10} \mathrm{~N}_{4} \mathrm{H}_{4} \mathrm{O}_{6}+\mathrm{O}_{2}+\mathrm{H}_{3} \mathrm{O}_{3}=\mathrm{C}_{2} \mathrm{~N}_{2} \mathrm{H}_{4} \mathrm{O}_{2}+2 \mathrm{C}_{2} \mathrm{O}_{3}+\mathrm{C}_{4} \mathrm{~N}_{2} \mathrm{H}_{3} \mathrm{O}_{3}$.

That allantoine is closely related to uric acid and urea, further appears from the fact that 1 eq. uric acid, 1 eq. urea, and 1 eq. water, added together, are exactly equal to the sum of 3 eq. allantoine. $\mathrm{C}_{10} \mathrm{~N}_{4} \mathrm{H}_{4} \mathrm{O}_{6}+\mathrm{C}_{2} \mathrm{~N}_{2} \mathrm{H}_{4} \mathrm{O}_{2}+\mathrm{H} \mathrm{O}=\mathrm{C}_{12} \mathrm{~N}_{6} \mathrm{H}_{9} \mathrm{O}_{9}$ $=3\left(\mathrm{C}_{4} \mathrm{~N}_{2} \mathrm{H}_{3} \mathrm{O}_{3}\right)$.

2. Oxidation of uric acid by peroxide of manganese.-This is performed much as the preceding, and there appear to be produced compounds, partly the same as those from peroxide of lead, partly different. Of the latter, one at least is crystallisable, but has not been sufficiently examined. The subject requires investigation.

3. By permanganate of potash.-In this oxidation also, some products appear, which are obtained by peroxide of lead, such as 
urea; and, in some forms of the experiment at all events, oxalic acid; but I have also observed the formation of a new acid, containing nitrogen, the precise nature and composition of which is not yet ascertained.

4. By nitric acid. - This mode of oxidation of uric acid has been minutely studied by Liebig and Wöhler, and they have shown that it yields a very large number of new and important products, among which is again found urea, and also, under certain circumstances, oxalic acid. The changes are best traced when colourless nitric acid of a considerable concentration, of Sp. G. 1.45 for example, is employed.

1. Alloxan.-When uric acid is added, in small portions, to this acid, it is dissolved with a gentle and uniform effervescence, due to the escape of pure carbonic acid and nitrogen gases, without any trace of the red vapours of nitrous acid. Heat is also developed, so that no external heat is required, and it may even be necessary to moderate the reaction by placing the vessel in cold water. If too much uric acid be added at once, or if the mixture be allowed to get too hot, a violent reaction ensues, accompanied by copious red fumes, after which the experiment cannot succeed, and must be recommenced with fresh materials. The presence of a trace of the colouring matter of the urine infallibly causes this violent reaction, even with a much weaker nitric acid, and thus prevents us from obtaining the desired result, even to a small extent. In all these cases, the whole seems to be converted into oxalate and carbonate of ammonia. When the operation is properly managed, and a little practice makes it quite easy, there appear in the warm liquid, after a certain quantity of uric acid has been dissolved, granular crystals of the new compound, alloxan. If more uric acid be added, it is still decomposed, and when the warm liquid (at about $120^{\circ}$ ) contains a great many crystals, it is allowed to cool, when the quantity of crystals greatly increases. They are now thrown on a filter stopped with asbestus, and when they have drained, the acid liquor still in their pores is displaced by a little ice-cold water, which is added till the droppings have only a slight acid taste. The crystals are now dissolved on the funnel with water at $120^{\circ}$, and the filtered solution evaporated at that or even a lower temperature, till, on being set aside, it deposits large transparent crystals of hydrated alloxan, which are chemically pure. The mother liquid of these crystals, being gently evaporated, yields more, and the final mother liquid, which is now rather acid, from nitric acid, is added to the original acid mother liquor, to be used for other purposes. By the above process, I have constantly obtained, without difficulty, upwards of from 90 to 105 
parts of hydrated alloxan, quite pure, from 100 of uric acid, besides what remains in the mother liquid, and cannot be extracted in that form. Not more than $2 \mathrm{oz}$. or $3 \mathrm{oz}$. of nitric acid should be used in one operation, and this quantity will decompose about one-third of its weight of uric acid, or more.

The crystals of hydrated alloxan, when heated to $212^{\circ}$, lose about 27 per cent. of water, $=6$ eq. The dry or anhydrous alloxan, which may also be obtained in crystals when a saturated solution is evaporated in a warm place, is composed of $\mathrm{C}_{8} \mathrm{~N}_{2}$ $\mathrm{H}_{4} \mathrm{O}_{10}$, which explains its formation from uric acid; for $\mathrm{C}_{10} \mathrm{~N}_{4}$ $\mathrm{H}_{4} \mathrm{O}_{6}+\mathrm{O}_{2}+\mathrm{H}_{4} \mathrm{O}_{4}=\mathrm{C}_{8} \mathrm{~N}_{2} \mathrm{H}_{4} \mathrm{O}_{10}+\mathrm{C}_{2} \mathrm{~N}_{2} \mathrm{H}_{4} \mathrm{O}_{2}$ : that is, uric acid plus 2 eq. oxygen and 4 eq. water, yields 1 eq. alloxan and 1 eq. urea.

The urea, when formed, is in contact with hyponitrous acid (derived from nitric acid by the separation of 2 eq. oxygen,), and is immediately decomposed by it, yielding oxide of ammonium, which combines with some free nitric acid, carbonic acid, and nitrogen, which two last escape as gases. $\mathrm{C}_{2} \mathrm{~N}_{2} \mathrm{H}_{4} \mathrm{O}_{2}+\mathrm{N} \mathrm{O}_{3}$ $=\mathrm{N} \mathrm{H}_{4}, \mathrm{O}+2 \mathrm{C} \mathrm{O}_{2}+\mathrm{N}_{2}$. At the end of the operation, therefore, the acid liquid, which has deposited crystals of alloxan, contains nothing but alloxan, nitrate of ammonia, and free nitric acid. We may therefore express the final result as follows: $\mathrm{C}_{10} \mathrm{~N}_{4} \mathrm{H}_{4} \mathrm{O}_{6}+2\left(\mathrm{H} \mathrm{O}, \mathrm{N} \mathrm{O}_{5}\right)+2 \mathrm{H} \mathrm{O}=\mathrm{C}_{8} \mathrm{~N}_{2} \mathrm{H}_{4} \mathrm{O}_{10}+$ $\left(\mathrm{N} \mathrm{H}_{4} \mathrm{O}, \mathrm{N} \mathrm{O}_{5}\right)+2 \mathrm{C} \mathrm{O}_{2}+\mathrm{N}_{2}$.

Alloxan is very soluble in water, also in alcohol. Its solution stains the skin pink, and gives to it a heavy sickly odour. Its taste is peculiar and almost acidulous ; but, although it reddens litmus, it has not the chemical characters of an acid. It is a very remarkable substance from the numerous transformations which it undergoes, when subjected to the action of different re-agents.

By the action of soluble fixed alkalies, it is converted into a powerful acid, alloxanic acid : by the action of ammonia it yields another acid, mykomelinic acid; boiled with peroxide of lead, it is converted into urea and carbonic acid: by boiling with nitric acid it is changed into a new and powerful acid, parabanic acid; by the action of sulphuretted hydrogen and other deoxidising agents, it yields a new compound, alloxantine; with hydrosulphuret of ammonia it gives a new salt called dialurate of ammonia; with sulphurous acid it combines, forming a compound acid; alloxano-sulphurous acid: and with sulphite of ammonia it forms another new salt called thionurate of ammonia. Such are the compounds formed by the direct action of re-agents on alloxan ; but many others are produced by the action of re-agents on these singly or jointly. Thus, when alloxan and alloxantine are both 
present in a hot solution, ammonia causes the development of a deep purple colour, and the deposition, on cooling, of the gold green crystals of murexide; acids acting on thionurate of ammonia produce thionuric acid, uramile and uramilic acid; acids acting on murexide produce murexan; acids acting on dialurate of ammonia separate dialuric acid; ammonia, acting on parabanic acid, converts it into a new acid, oxaluric acid; and by the action of heat on alloxanate of baryta, another new acid, mesoxalic acid, is produced. Alloxanic acid, when heated, yields leucoturic acid and difuun; and alloxantine when boiled with hydrochloric acid yields alituric and dilituric acids. We shall endeavour briefly to trace the formation and the relations of these remarkable products.

2. Alloxanic Acid.-Its formula $\mathrm{C}_{8} \mathrm{~N}_{2} \mathrm{H}_{2} \mathrm{O}_{8}+2 \mathrm{H} 0$. It is therefore isomeric with alloxan, and differs from it in this, that 2 eq. water have become basic, and replaceable by metallic oxides. It is a bibasic acid. It is formed when solution of alloxan is mixed with barytio water, as long as the white precipitate first formed redissolves with a gentle heat. When it begins to be permanent, a drop or two of alloxan is added to clear all up; and on cooling, alloxanate of baryta is deposited in small white crystals. From this salt the acid is obtained by adding sulphuric acid, so as to separate all the baryta. The acid solution on evaporating yields crystals of alloxanic acid. The acid, when neutralised by ammonia, forms, with nitrate of silver, a white precipitate, which, when boiled, becomes yellow, and is reduced with effervescence. When the solutions of its salts, with baryta, lime, and strontia, are boiled, they become turbid, depositing carbonates, while urea and a mesoxalate remain dissolved. The formula of the neutral alloxanates is $\mathrm{C}_{8} \mathrm{~N}_{2} \mathrm{H}_{2} \mathrm{O}_{8}, 2 \mathrm{M} \mathrm{O}+$ aq.;

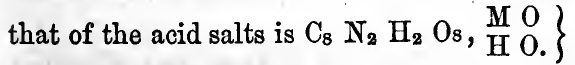

Schlieper has lately studied the salts of alloxanic acid, and has, confirmed the view above given. But he has also examined the products of decomposition of this acid, which are remarkable.

When a solution of alloxanic acid is boiled to dryness, and pretty strongly heated in a capsule, carbonic acid is given off; and, when at last the residue ceases to yield any gas, and flows smoothly, the addition of water causes the separation of a crystalline powder in small quantity, which is leucoturic acid; while the liquid contains a neutral body, difluan.

3. Leucoturic acid is white and crystalline, sparingly soluble, and not decomposed by nitric acid. It is dissolved by ammonia, with which it forms a crystallisable salt, and by potash, which, however, soon transforms it into oxalio acid and ammonia. Its 
composition is $\mathrm{C}_{6} \mathrm{~N}_{2} \mathrm{H}_{3} \mathrm{O}_{6}=\mathrm{C}_{6} \mathrm{~N}_{2} \mathrm{H}_{2} \mathrm{O}_{5}+\mathrm{H} \mathrm{O}$. It contains the elements of 2 eq. cyanogen $\mathrm{C}_{4} \mathrm{~N}_{2}, 1$ eq. oxalic acid $\mathrm{C}_{2} \mathrm{O}_{3}$, and 3 eq. water, $\mathrm{H}_{3} \mathrm{O}_{3}$. With three more eqs. of water, it is resolved, when acted on by potash, into oxalic acid and ammonia.

$$
\mathrm{C}_{6} \mathrm{~N}_{2} \mathrm{H}_{3} \mathrm{O}_{6}+3 \mathrm{H} \mathrm{O}=2\left(\mathrm{NH}_{3}\right)+3\left(\mathrm{C}_{2} \mathrm{O}_{3}\right) \text {. }
$$

4. Difluan, the more soluble product of the decomposition of alloxanic acid, is precipitated from the aqueous solution by absolute alcohol as a flocculent mass, which in vacuo dries up to a light bulky white powder, which, when exposed to the air, very rapidly deliquesces; hence its name. It is an indifferent body, which is dissolved by potash, and rapidly decomposed in contact with that base. Its composition is, $\mathrm{C}_{6} \mathrm{~N}_{2} \cdot \mathrm{H}_{4} \mathrm{O}_{5}$, thus containing 1 eq. of hydrogen more, and 1 eq. of oxygen less, than leucoturic acid. When it is resolved into oxalio acid and ammonia, oxygen is probably absorbed from the air, unless hydrogen be given off, or some other new product formed. Thus $\mathrm{C}_{6} \mathrm{~N}_{2} \mathrm{H}_{4} \mathrm{O}_{5}+2 \mathrm{H} \mathrm{O}$ $+\mathrm{O}_{2}=3\left(\mathrm{C}_{2} \mathrm{O}_{3}\right)+2\left(\mathrm{~N} \mathrm{H}_{3}\right)$. This reaction has not been fully studied; but oxalic acid and ammonia are certainly produced.

Schlieper has observed a third product, along with leucoturic acid and difluan, which appears to contain 1 eq. of hydrogen more and 1 eq. of oxygen less, than difluan. This, however, is not established, from the small amount of the substance obtained. The three formulæ exhibit the inverse variations in the hydrogen and oxygen.

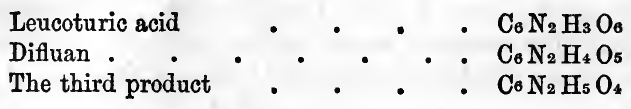

The formation of leucoturic acid and of difluan from alloxanic acid is very simple.

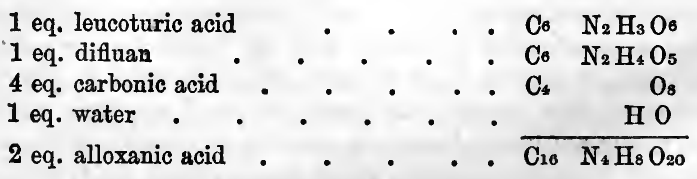

5. Hydurilic Acid.-In the preparation of alloxan, Schlieper, on one occasion obtained a new body, which proved to be the ammonia salt of a new acid, hydurilic acid, $\mathrm{C}_{12} \mathrm{~N}_{3} \mathrm{H}_{5} \mathrm{O}_{11}$. It is a bibasic acid, $=\mathrm{C}_{22} \mathrm{~N}_{3} \mathrm{H}_{3} \mathrm{O}_{9}+2 \mathrm{H} \mathrm{O}$. It forms minute whit: 
prisms, sparingly soluble in cold water. It may be viewed as a compound of water and uryle $\left(\mathrm{C}_{8} \mathrm{~N}_{2} \mathrm{O}_{4}\right)$, for $3\left(\mathrm{C}_{8} \mathrm{~N}_{2} \mathrm{O}_{4}\right)+$ $10 \mathrm{H} \mathrm{O}=2\left(\mathrm{C}_{12} \mathrm{~N}_{3} \mathrm{H}_{5} \mathrm{O}_{11}\right)$. Hence its name.

It is evidently formed by an incomplete oxidation of uric acid, but Schlieper was not able to reproduce it.

With nitric acid, it yields a new acid, Nitrohydurilic acid, of very similar external character, the empirical formula of which seems to be, $\mathrm{C}_{8} \mathrm{~N}_{3} \mathrm{H}_{2} \mathrm{O}_{14}$. This is equal to

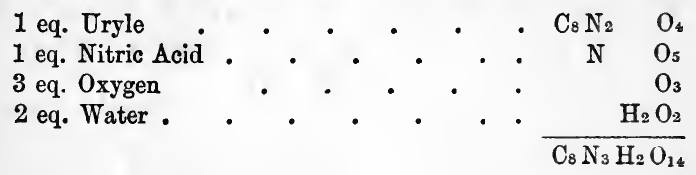

And it is formed from

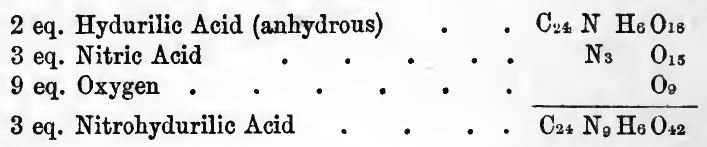

Schlieper has further detected, in the same operation, in which he observed the hydurilie acid, another new acid, of which, however, little is known. Its empirical formula was found to be, $\mathrm{C}_{10} \mathrm{~N}_{2} \mathrm{H}_{5} \mathrm{O}_{9}$. It has not been named.

6. Mesoxalic Acid.-Obtained in combination with baryta, by boiling alloxanate of baryta ; or combined with oxide of lead, by adding alloxan in solution, to a boiling solution of acetate of lead. The baryta salt is pale yellow, and sparingly soluble; the lead salt white and insoluble. The former is $\mathrm{C}_{3} \mathrm{O}_{4}\left\{\begin{array}{l}\mathrm{Ba} \mathrm{O} \\ \mathrm{H} \mathrm{O}\end{array}\right.$ the latter $\mathrm{C}_{3} \mathrm{O}_{4}, 2 \mathrm{~Pb} \mathrm{O}$. The acid may be obtained from either of these salts; it crystallises, is very sour, and is probably bibasic, and has, also probably, the formula $\mathrm{C}_{3} \mathrm{O}_{4}, 2 \mathrm{H} \mathrm{O}$. In that case, the anhydrous acid is very remarkable as a new compound of carbon and oxygen, of the same class as mellitic and oxalic acids; hence the name. It is characterised by forming, when neutralised by ammonia, with nitrate of silver, a yellow precipitate, which, when heated, is reduced with brisk effervescence. This is evidently the cause of the reaction of alloxanic acid, above mentioned, with nitrate of silver. Mesoxalic acid deserves and requires a very careful investigation. Its formation from alloxan or alloxanic acid, if its formula be $\mathrm{C}_{3} \mathrm{O}_{4}$, is very easily explained: for 1 eq. alloxan, 
minus 1 eq. urea, gives 2 eq. mesoxalio acid. $\mathrm{C}_{8} \mathrm{~N}_{2} \mathrm{H}_{4} \mathrm{O}_{20}$

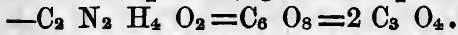

7. Mykomelinic Acid- $\mathrm{C}_{8} \mathrm{~N}_{4} \mathrm{H}_{5} \mathrm{O}_{5}$-is formed when ammonia acts on solution of alloxan; when a yellowish gelatinous precipitate of mykomelinate of ammonia soon appears. This boiled with dilute sulphuric acid yields a similar yellowish precipitate, which, when dry, forms a powder sparingly soluble in cold, more readily in hot, water. It is decidedly acid. It is mykomelinic acid formed by the reaction of 2 eq. of ammonia on 1 eq. alloxan. $\mathrm{C}_{8} \mathrm{~N}_{2} \mathrm{H}_{4} \mathrm{O}_{10}+2 \mathrm{~N} \mathrm{H}_{3}=\mathrm{C}_{8} \mathrm{~N}_{4} \mathrm{H}_{5} \mathrm{O}_{5}+5 \mathrm{H} 0$. It would appear to differ from allantoine; only by 1 eq. of water ; for 2 eq. of allantoine are $\mathrm{C}_{8} \mathrm{~N}_{4} \mathrm{H}_{6} \mathrm{O}_{6}$.

8. Parabanic Acid- $\mathrm{C}_{6} \mathrm{~N}_{2} \mathrm{O}_{4}+2 \mathrm{H} 0$-is formed when alloxan or uric acid is heated with an excess of nitric acid, and the solution concentrated until on cooling it forms a soft crystalline mass. This is dried on a tile, and the dry crystals are purified by solution in hot water, filtration, and recrystallisation. A large quantity of parabanic acid may easily be obtained from the acid mother liquors of alloxan. When pure, the acid is beautifully white and crystallised, very acid, and very soluble. It is characterised by its great permanence in the free state, for it may be boiled with nitric acid, as its preparation shows, and at the same time by its extreme proneness to change in contact with bases. Thus, if neutralised with ammonia in the warm solution, it deposits, on cooling, a crystallised salt, which is oxalurate of ammonia. The same change takes place with all bases except oxide of silver, so that the parabanate of silver is the only salt of this very powerful and remarkable acid which can be obtained. To be converted into oxaluric acid, parabanic acid only requires 3 eq. of water. The production of parabanic acid is very simple. 1. From uric acid. $\mathrm{C}_{10} \mathrm{~N}_{4} \mathrm{H}_{4} \mathrm{O}_{6}+\mathrm{O}_{4}$ $+\mathrm{H}_{2} \mathrm{O}_{2}=\mathrm{C}_{2} \mathrm{~N}_{2} \mathrm{H}_{4} \mathrm{O}_{2}+2 \mathrm{C} \mathrm{O}_{2}+\mathrm{C}_{6} \mathrm{~N}_{2} \mathrm{O}_{4}, 2 \mathrm{H} 0$. 2. From alloxan. $\mathrm{C}_{8} \mathrm{~N}_{2} \mathrm{H}_{4} \mathrm{O}_{10}+\mathrm{O}_{2}=2 \mathrm{C} \mathrm{O}_{2}+2 \mathrm{HO}+\mathrm{C}_{6}$ $\mathrm{N}_{2} \mathrm{O}_{4}, 2 \mathrm{H} 0$.

9. Oxaluric Acid, $-\mathrm{C}_{6} \mathrm{~N}_{2} \mathrm{H}_{3} \mathrm{O}_{7}+\mathrm{H}$ 0.-Formed by the action of bases on parabanic acid. $\mathrm{C}_{6} \mathrm{~N}_{2} \mathrm{O}_{4}+\mathrm{KO}+3 \mathrm{H} \mathrm{O}$ $=\mathrm{C}_{6} \mathrm{~N}_{2} \mathrm{H}_{3} \mathrm{O}_{7}, \mathrm{KO}$. The acid is obtained by adding dilute sulphurio acid to a hot saturated solntion of oxalurate of ammonia, prepared by the action of ammonia on parabanic acid. On cooling, the oxaluric acid is deposited as a heavy white powder. When long boiled in water, it is decomposed into oxalate of urea and free oxalic acid. In fact, it oontains the elements of 2 eq. oxalio acid and 1 eq. urea. $2 \mathrm{C}_{2} \mathrm{O}_{3}+\mathrm{C}_{2} \mathrm{~N}_{2} \mathrm{H}_{4} \mathrm{O}_{2}=\mathrm{C}_{6} \mathrm{~N}_{2} \mathrm{H}_{3}{ }^{2} \mathrm{O}_{7}, \mathrm{H} \mathrm{O}$. It is also characterised by forming with oxide of silver a white salt, which dissolves in hot water, and orystallises beautifully on cooling. 
The oxalurate of ammonia $-\mathrm{N} \mathrm{H}_{4} \mathrm{O}+\mathrm{C}_{6} \mathrm{~N}_{2} \mathrm{H}_{3} \mathrm{O}_{7}$-is formed whenever a solution of alloxan, or alloxantine in water, or of uric acid in nitric acid, is evaporated with excess of ammonia; and where colouring matter is present, as when ammonia is made to act on the acid in the mother liquors of alloxan, the oxalurate is often deposited in radiated hemispherical concretions, which sometimes attain the size of an inch or two in diameter, and are very hard. When decolorised by animal charcoal, it forms small, soft, flexible needles. I have found that this salt, when exposed to heat in a retort, yields ammonia, hydrocyanic acid, and much oxamide, besides water, and perhaps other products, while a dark residue is left.

10. Alloxanosulphurous Acid.-When to a cold saturated solution of alloxan there is added a strong solution of sulphurous acid in water, till the smell of the acid, which at first disappears, becomes permanent, and the mixture is now neutralised by the addition of a strong solution of potash, avoiding excess of the latter, it soon deposits on standing, as I have found, regular and beautiful erystals, perfectly transparent, and of a strong vitreous lustre. These are the potash salt of a new acid, which may be called Alloxanosulphurous acid. The acid is not yet known in the separate form, but it appears to contain 1 eq. of alloxan, united to two of sulphurous acid. This acid requires investigation.

11. Thionuric Acid- $\mathrm{Cs}_{8} \mathrm{~N}_{3} \mathrm{H}_{7} \mathrm{~S}_{2} \mathrm{O}_{14}=\left(\mathrm{C}_{8} \mathrm{~N}_{3} \mathrm{H}_{5} \mathrm{O}_{8}, 2 \mathrm{SO}_{2}\right)+$ $2 \mathrm{HO}$-is formed when sulphite of ammonia, with excess of base, is added to solution of alloxan, and the whole boiled for a few minutes, or until crystals appear in the hot liquid. On cooling it forms a semi-solid mass, from the separation of a large quantity of thionurate of ammonia in beautiful silvery crystals, which are to be washed with cold water, and dried on a tile. From this salt thionurate of lead is prepared, and this, being decomposed by sulphuric acid, yields thionuric acid. The acid is crystallisable, but very soluble. It is bibasie, and contains the elements of 1 eq. alloxan, 1 eq. ammonia, and 2 eq. sulphurous acid, not however as such, for the elements of 2 eq. water have assumed the basio form.

Its most striking character is, that when its solution is heated it becomes turbid from the deposition of a new compound, uramile, and in the liquid sulphuric acid may now be found, which was not previously present. $\mathrm{Cs}_{8} \mathrm{~N}_{3} \mathrm{H}_{5} \mathrm{O}_{8}+2 \mathrm{~S} \mathrm{O}_{2}=$ $\mathrm{C}_{8} \mathrm{~N}_{3} \mathrm{H}_{5} \mathrm{O}_{6}+2 \mathrm{~S} \mathrm{O}_{3}$; so that the sulphurous acid obtains oxygen from the rest of the acid, and becomes sulphurio acid, leaving uramile, $\mathrm{Cs} \mathrm{N}_{3} \mathrm{H}_{5} \mathrm{O}_{6}$.

Thionurate of Ammonia-( $\left.\mathrm{Cs}_{8} \mathrm{~N}_{3} \mathrm{H}_{5} \mathrm{O}_{8}, 2 \mathrm{~S} \mathrm{O}_{2}\right)+2 \mathrm{NH}_{4} \mathrm{O}$ +2 aq. - is formed as above described. When its solution is 
mixed with 1 eq. of hydrochloric acid, half the ammonia is removed, and by evaporation we obtain acid thionurate of ammonia in minute silky needles. But when the hot solution of thionurate of ammonia is mixed with an excess of acid, the thionuric acid is set free and instantly decomposed, uramile being deposited. Little is known of the other thionurates.

12. Uramile, $\mathrm{C}_{8} \mathrm{~N}_{3} \mathrm{H}_{5} \mathrm{O}_{6}$.- Its formation has been described above. It occurs either as a crystalline powder, or in dendritic or feathery crystallisations, of very beautiful aspect. It dissolves in ammonia and potash, and the solution absorbs oxygen, becoming purple, and depositing green crystals of murexide, or of potassium-murexide. When boiled with peroxide of mercury, and a very little ammonia, it is also converted into murexide. Boiled with caustio potash, or with dilute acids, it is said to yield uramilio acid.-Nitric acid re-converts it into alloxan.

13. Uramilic Acid.-Obtained, by Liebig and Wöhler, by evaporating acid thionurate of ammonia, or uramile, with dilute sulphuric acid; also, it is said, by boiling uramile with potash. It appeared to these chemists as fine prisms, very soluble in water, and its analysis indicated the formula $\mathrm{C}_{16} \mathrm{~N}_{5} \mathrm{H}_{10} \mathrm{O}_{15}$; which might be derived from 2 eq. uramile by the loss of 1 eq. ammonia, and the addition of 3 eq. water. $2\left(\mathrm{C}_{8} \mathrm{~N}_{3} \mathrm{H}_{5} \mathrm{O}_{6}\right)+$ $2 \mathrm{H} \mathrm{O}-\mathrm{N} \mathrm{H} \mathrm{H}_{3}=\mathrm{C}_{16} \mathrm{~N}_{5} \mathrm{H}_{10} \mathrm{O}_{15}$. But this acid has not been again obtained, and its existence is still doubtful.

14. Alloxantine, $\mathrm{C}_{8} \mathrm{~N}_{2} \mathrm{H}_{5} \mathrm{O}_{10}$.-Obtained in large quantity by diluting the acid mother liquid of alloxan with 3 or 4 parts of water, and passing a current of sulphuretted hydrogen through it. In a short time sulphur is deposited, and then white crystals of alloxantine. When a large quantity has formed, it is collected with the sulphur, on a filter, washed with a little cold water, and the filter with its contents then boiled with a large quantity of water. The solution filtered while hot, and with the addition of a few drops of hydrochloric acid, deposits, on cooling, a large crop of pure crystals of alloxantine. The acid liquid, filtered from the first deposit, often, on standing a day or two, deposits a large additional quantity of alloxantine. This always happens if too much sulphuretted hydrogen has been used; for that converts the alloxantine partially into dialuric acid, which is more soluble, but by absorbing oxygen from the air, is reconverted into alloxantine, and thus deposited.

Alloxantine may also be obtained by deoxidising a pure solution of alloxan, either by sulphuretted hydrogen, or by other deoxidising agents : or by heating a solution of alloxan to the boiling-point, either by itself or with the addition of dilute 
mineral acids, when alloxantine is formed and deposited on cooling. But the process above given for converting into alloxantine the alloxan of the acid mother liquor, which cannot be purified by crystallisation, is so productive, and yields alloxantine so pure, that, if we have to prepare alloxan, we need never be at a loss for alloxantine.

The formation of alloxantine from alloxan by sulphuretted hydrogen is easily explained, for these compounds only differ by 1 eq. hydrogen, which the alloxan takes from sulphuretted hydrogen. Oxidising agents, by converting this hydrogen into water, readily reconvert alloxantine into alloxan. $\mathrm{C}_{8} \mathrm{~N}_{2} \mathrm{H}_{5} \mathrm{O}_{10}$ $+\mathrm{O}=\mathrm{HO}+\mathrm{Cr}_{8} \mathrm{~N}_{2} \mathrm{H}_{4} \mathrm{O}_{10}$.

When the crystals of alloxan, with 7 eq. of water of crystallisation, which are not so permanent as the usual forms, are kept in a slightly moist state (or perhaps when moistened by the separation of part of the water of crystallisation at a certain temperature) they undergo a gradual change, the result of which is the formation of a large quantity of pure alloxantine, along with at least one other substance, more soluble in water and crystallisable. This substance has acid properties, but I have not been able to complete its examination. The production of alloxantine from alloxan in this case is not yet accounted for, nor do we know what becomes of that portion of the alloxan which yields hydrogen to the rest, to convert it into alloxantine. The new body has many properties in common both with alloxan and alloxantine, but it must differ from them in composition.

Alloxantine forms white, hard, brilliant crystals, which never exceed a certain small size. It is very sparingly soluble in cold water, much more so in hot water. Its solution is characterised by giving with solution of baryta a deep violet precipitate, which, with excess of baryta, changes to white; and by instantly reducing nitrate of silver, forming a black powder of silver, the alloxantine passing into alloxan, or oxaluric acid. The crystals of alloxantine, heated to $300^{\circ}$, lose 3 eq. of water.

In the preparation of alloxan, it is necessary, as has been stated, to be very careful that the first solution of the crystals formed in the nitric aoid should not be heated too strongly, because, as this solution contains free nitric acid, alloxantine is formed at a certain temperature; and besides, even a pure solution of alloxan, if boiled, is partly converted into alloxantine. The action of diluted nitric and other mineral acids on alloxan is to produce, from 2 eq. alloxan, 1 eq. alloxantine, 3 eq. oxalio acid, 1 eq. ammonia, and 1 eq. cyanic acid, the latter, with 3 eq. water, producing bicarbonate of ammonia. When solution of alloxan is boiled alone, it is converted into alloxantine, parabanio 
acid, and carbonic acid. $3\left(\mathrm{C}_{8} \mathrm{~N}_{2} \mathrm{H}_{4} \mathrm{O}_{10}\right)=2\left(\mathrm{C}_{8} \mathrm{~N}_{2} \mathrm{H}_{5} \mathrm{O}_{10}\right)+$ $\mathrm{C}_{6} \mathrm{~N}_{2} \mathrm{H}_{2} \mathrm{O}_{6}+2 \mathrm{CO}_{2}$.

In all these, or in similar cases, the presence and the relative proportion of alloxantine contained in alloxan at any period, may be judged of by the colour of the precipitate formed in baryta. If pure white, no alloxantine is present; if slightly pink, it is present in small quantity; if deep violet, all, or nearly all, the alloxan has been couverted into alloxantine.

It is when both alloxan and alloxantine are present, that the addition of ammonia produces the deep purple colour, and the green crystals of murexide. When ammonia acts on alloxantine alone, it gives rise to uramile, and, finally, to oxalurate of ammonia.

One most remarkable change which alloxantine undergoes is that caused by the further action of sulphuretted hydrogen. If that gas be passed through a hot solution of alloxantine, sulphur is precipitated, and an acid liquid is obtained, which, if neutralised by carbonate of ammonia, forms a salt in soft white silky crystals, the dialurate of ammonia. Alloxantine, by the action of hydrogen, which removes 1 eq. oxygen, is converted into dialuric acid.

15. Dialuric Acid, $\mathrm{C}_{8} \mathrm{~N}_{2} \mathrm{H}_{3} \mathrm{O}_{7}+\mathrm{HO}=\mathrm{C}_{8} \mathrm{~N}_{2} \mathrm{H}_{4} \mathrm{O}_{8}$-produced by the action of sulphuretted hydrogen on alloxantine, $\mathrm{C}_{8} \mathrm{~N}_{2} \mathrm{H}_{2} \mathrm{O}_{10}+\mathrm{H} \mathrm{S}=\mathrm{S}+2 \mathrm{HO}+\mathrm{C}_{8} \mathrm{~N}_{2} \mathrm{H}_{4} \mathrm{O}_{8}$. It is best obtained, in combination with ammonia, by adding a slight excess of hydrosulphuret of ammonia to a solution of alloxan or alloxantine, when a copious crystalline precipitate appears. This, when boiled, dissolves in the liquid, and on cooling is deposited in minute silky prisms, which are white, but in drying become pink or even deep red. They should be washed on the filter, first with diluted hydrosulphuret of ammonia, then with alcohol, to which a little hydrosulphuret has been added; and lastly, with pure alcohol, and dried by pressure in blotting-paper and in the vacuum of the air-pump. They may thus be obtained white, or very nearly so ; and when once quite dry, they are permanent. When this salt is dissolved in hot and moderately strong hydrochloric acid, crystals of dialuric acid are deposited. on cooling. These crystals resemble somewhat those of alloxantine, but are larger, and not so brilliant. Their solution, and the crystals themselves under water, absorb oxygen, and are soon changed into alloxantine, from which dialuric acid only differs by 1 eq. oxygen and 1 eq. water.

Dialuric acid is a powerful acid. Its salts are insoluble or sparingly soluble, and only permanent in the dry state. The dialurate of ammonia, above described, is the most interesting. 
16. Alituric Acid.-This acid is formed when alloxantine is boiled with hydrochloric acid, and was discovered by Schlieper. It is soluble in 15 or 20 parts of hot water, and is deposited on cooling as a bulky crystalline powder. It is not altered by nitric acid. Its formula is $\mathrm{C}_{6} \mathrm{~N}_{2} \mathrm{H}_{3} \mathrm{O}_{4}=\mathrm{C}_{6} \mathrm{~N}_{2} \mathrm{H}_{2} \mathrm{O}_{3}+\mathrm{H} \mathrm{O}$. When heated with potash, ammonia is disengaged, and the addition of an acid causes a precipitate of a new body, apparently composed of $\mathrm{C}_{18} \mathrm{~N}_{3} \mathrm{H}_{8} \mathrm{O}_{18}+\mathrm{KO}$. Such a body might be formed from 3 eq. alituric acid +4 eq. oxygen +2 eq. water, the whole-1 eq. ammonia.

17. Dilituric Acid.-This is found in the liquid obtained when the preceding acid is purified from alloxantine by nitric acid, in combination with ammonia. The acid retains the ammonia with such force that it has not yet been obtained in a separate form: The ammoniacal salt is $\mathrm{C}_{8} \mathrm{~N}_{3} \mathrm{HO}_{8}+\mathrm{NH}_{4} \mathrm{O}+\mathrm{HO}$; and the acid is supposed to be bibasic, $\mathrm{C}_{8} \mathrm{~N}_{3} \mathrm{H} \mathrm{O}_{8}+2 \mathrm{HO}$.

It is probably a coupled acid, for it contains the elements of 3 eq. cyanic acid, 2 eq. carbonic acid, and 3 eq. water. $3\left(\mathrm{C}_{2}\right.$ $\mathrm{N} \mathrm{O})+2 \mathrm{C} \mathrm{O}_{2}+3 \mathrm{H} \mathrm{O}=\mathrm{C}_{8} \mathrm{~N}_{3} \mathrm{H}_{3} \mathrm{O}_{10}$. The silver salt explodes when heated, which is rather favourable to this view.

18. Murexide. SrN. Purpurate of Ammonia.-Formed, as already mentioned, when ammonia acts on a solution containing both alloxan and alloxantine, which explains its production when ammonia is added to the solution of urio acid in dilute nitrio acid, after evaporation to a certain extent; also, when uramile or murexan is boiled, with red oxide of mercury or oxide of silver, in water, with a few drops of ammonia, or when uramile or murexan is dissolved in ammonia and exposed to the atmosphere; and in a great variety of circumstances from all the preceding compounds, or nearly all of them.

On the small scale, 4 grains of alloxantine and 7 grains of hydrated alloxan, are dissolved together in $\frac{1}{2} \mathrm{oz}$. by measure of water by boiling, and the hot solution added to $\frac{1}{6} \mathrm{oz}$. by measure of a saturated or nearly saturated solution of carbonate of ammonia, the latter being cold. This mixture has exactly the proper temperature for the formation of murexide; and it does not, owing to its small bulk, remain too long hot. It instantly becomes intensely purple, while carbonic acid is expelled; and as soon as it begins to cool, the beautiful green and metalliclooking crystals of murexide appear. As soon as the liquid is cold, these may be collected, washed with a little cold water, and dried on filtering-paper. I have obtained them, by the above process, and on this small scale, of from 2 to 3 lines in length. When made with larger quantities, the crystals are always smaller, owing, probably, to some effect of the slower cooling of 
the larger mass of liquid, as continued heat is not favourable to their formation. If we do not care about having the finest crystals, we may prepare murexide in large quantity by adding solution of alloxan to a boiling solution of alloxantine, and cautiously adding cold solution of carbonate of ammonia, till the mixture has become nearly black, and the green crystals begin to appear. The vessel being removed from the fire, deposits a very large quantity of murexide. In these processes, the residual liquid is still coloured, and is alkaline from excess of ammonia: if kept, it loses the red colour, becomes yellowish, and if evaporated, yields much alloxanate of ammonia in crystals.

Murexide is one of the most beautiful products of chemistry: the crystals are metallic green by reflected light, like the cantharides fly or the gold beetle, and deep red by transmitted light. Their solution is deep purplish red, and they dissolve in potash with the most splendid purplish blue colour that can be imagined; this, however, soon disappears. When their solution is acted on by a dilute mineral acid, it is decolorised, and deposits a shining scaly crystalline powder, of a pale yellow colour, which is murexan. The same compound is obtained when acids are added to the solution of murexide in potash, after the purple tint has disappeared on digestion in a gentle heat.

The composition of murexide is uncertain, and there are different views of its constitution. According to some it is a salt of ammonia; and this view is supported by the fact that, with salts of baryta and oxides of lead and silver, it yields purple salts, which, according to Fritzsche, contain the same acid that in murexide is combined with ammonia, and which may be called purpuric acid. But murexide is not a compound of ammonia with the purpuric acid of Prout, for when that body (murexan) is dissolved in ammonia, it only forms murexide by absorbing oxygen from the air, and yields other compounds at the same time. Again, the action of sulphuretted hydrogen is inconsistent with the view of murexide being a salt of ammonia, and in many of its relations it more resembles a neutral body-such as a compound of amide. Its products of decomposition are very numerous, and altogether the subject is one of much difficulty. Possibly there may be two substances similar in appearance, but distinct in constitution; one a salt of ammonia, the other an indifferent body, or an amidide. The great discrepancy in the results of analysis, as obtained by Liebig and Wöhler on the one hand, and Fritzsche on the other, as well as some differences in the properties ascribed to it by different chemists, lead to some such conclusion. The formula considered by Liebig and Wöhler 
the most probable, all things considered, but not established, is $\mathrm{C}_{22} \mathrm{~N}_{3} \mathrm{H}_{6} \mathrm{O}_{8}$; another somewhat less probable, is $\mathrm{C}_{20} \mathrm{~N}_{8} \mathrm{H}_{10} \mathrm{O}_{14}$. Both of these will enable us to account for its production in different oircumstances. The formula of Fritzsche, which agrees with his analysis, is $\mathrm{C}_{16} \mathrm{~N}_{6} \mathrm{H}_{8} \mathrm{O}_{11}=\mathrm{N} \mathrm{H}_{3}+\mathrm{C}_{26} \mathrm{~N}_{5} \mathrm{H}_{6} \mathrm{O}_{11}$. The salt formed with nitrate of silver is, $\mathrm{C}_{26} \mathrm{~N}_{5} \mathrm{H}_{5} \mathrm{O}_{11}+\mathrm{Ag} . \mathrm{O}$, which would exhibit the unusual phenomenon of ammonia, instead of oxide of ammonium, being replaced by oxide of silver; and the baryta compound is $\mathrm{C}_{16} \mathrm{~N}_{5} \mathrm{H}_{5} \mathrm{O}_{11}+\mathrm{HO}+\mathrm{BaO}$. Admitting the formulæ of Fritzsche for the silver and barium compounds to be correct, these are not demonstrated to be salts of purpuric acid; but besides this, his formula for murexide does not enable us to explain its production in any case. In these circumstances, we shall not attempt to explain the formation of murexide, further than to point out, that it appears to require the presence of a compound intermediate between alloxan and alloxantine (the former losing oxygen, the latter gaining it), and of ammonia : and that it is not the only product.

19. Murexan. Syv. Purpuric Acid. $\mathrm{C}_{6} \mathrm{~N}_{2} \mathrm{H}_{4} \mathrm{O}_{5}($ ?)-Formed by the action of acids on murexide, but along with several other products. It appears of a shining powder, composed of seales generally pale yellow, sometimes pale brown, never quite white. It is insoluble in water or nearly so, but the liquid filtered from it has always a peculiar opalescent aspect and play of colours. It dissolves in potash and ammonia, and the solutions become purple, by absorbing rapidly oxygen from the air, and finally deposit green crystals. When boiled with peroxide of mercury, water, and a little ammonia, it yields murexide. It dissolves in oil of vitriol, and is precipitated unchanged by water. In all these characters, except in its external aspect, it coincides entirely with uramile, and it is not impossible that it may hereafter be found to be uramile, disguised by the presence of a foreign substance. For the present, however, its analysis compels us to distinguish it from uramile.

The following products have been described by Schlieper and Städeler. They include a fifth mode of oxidation of uric acid.

20. Lantanuric Acid. $\mathrm{C}_{6} \mathrm{~N}_{2} \mathrm{H}_{4} \mathrm{O}_{6}, \mathrm{H}$ O, or $\mathrm{C}_{22} \mathrm{~N}_{4} \mathrm{H}_{8} \mathrm{O}_{12}, 2 \mathrm{H} 0$. According to Schlieper, uric acid, when acted on by a mixture of ferridcyanide of potassium (red prussiate of potash) and caustic potash, is oxidised, and yields this acid in combination with potash. In the first instance allantoine and carbonio acid are formed, $\mathrm{C}_{10} \mathrm{~N}_{4} \mathrm{H}_{4} \mathrm{O}_{6}+\mathrm{O}_{2}+2 \mathrm{H} \mathrm{O}=2\left(\mathrm{C}_{4} \mathrm{~N}_{2} \mathrm{H}_{3} \mathrm{O}_{3}\right)+2 \mathrm{C} \mathrm{O}_{2}$. The allantoine, in contact with potash, takes up 2 eqs. of water, and is then resolved into lantanuric acid and urea. $2\left(\mathrm{C}_{4} \mathrm{~N}_{2} \mathrm{H}_{3} \mathrm{O}_{3}\right)$ 
$+2 \mathrm{H} \mathrm{O}=\mathrm{C}_{8} \mathrm{~N}_{4} \mathrm{H}_{8} \mathrm{O}_{8} ;$ and $\mathrm{C}_{8} \mathrm{~N}_{4} \mathrm{H}_{8} \mathrm{O}_{8}=\mathrm{C}_{2} \mathrm{~N}_{2} \mathrm{H}_{4} \mathrm{O}_{2}+$ $\mathrm{C}_{6} \mathrm{~N}_{2} \mathrm{H}_{4} \mathrm{O}_{6}$.

21. Hidantoic Acid. $\mathrm{C}_{8} \mathrm{~N}_{4} \mathrm{H}_{8} \mathrm{O}_{8}, \mathrm{H} 0$. According to Schlieper this acid is formed when allantoine is dissolved in caustic potash, and its formation is the first stage in the process described in the preceding section. It may be obtained in combination with oxide of lead, by dissolving allantoine in cold solution of caustic potash, and allowing the solution to stand about two days. After the addition of acetic acid in excess and water, acetate of lead causes a precipitate of the lead salt. The anhydrous acid is polymeric with oxamide, but the atoms are obviously arranged in a different manner, since, when boiled with alkalies, it yields no oxalic acid.

22. Uroxanic Acid. $\mathrm{C}_{10} \mathrm{~N}_{3} \mathrm{H}_{9} \mathrm{O}_{14}$, or $\mathrm{C}_{10} \mathrm{~N}_{3} \mathrm{H}_{7} \mathrm{O}_{12}, 2 \mathrm{HO}$. (Städeler). When a solution of uric acid in excess of caustic potash is left to itself, it deposits by degrees, along with acid urate of potash, crystals of the potash salt of this new acid. The acid crystallises from its solution in hot water in tetrahedra or in irregular feathery prisms. It is formed from uric acid by the addition of 8 eqs. of water, and the separation of 1 eq. of ammonia. Thus,

$$
\begin{aligned}
& \text { Uric Acid. } \\
& \left(\mathrm{C}_{10} \mathrm{~N}_{4} \mathrm{H}_{4} \mathrm{O}_{6}\right)+8 \mathrm{H} \mathrm{O}=\left(\mathrm{C}_{10} \mathrm{~N}_{3} \mathrm{H}_{9} \mathrm{O}_{14}\right)+\mathrm{N} \mathrm{H}_{3} .
\end{aligned}
$$

23. Uroxile. $\mathrm{C}_{8} \mathrm{~N}_{3} \mathrm{H}_{7} \mathrm{O}_{8}$. (Städeler). When uroxanic acid is heated to $212^{\circ}$, it loses carbonic acid and water, and uroxile is left as a yellowish coherent mass.

$$
\begin{aligned}
& \text { Uroxanic Acid. } \\
& \mathrm{C}_{10} \mathrm{~N}_{3} \mathrm{H}_{9} \mathrm{O}_{14}=2 \mathrm{H} \mathrm{Oroxile.} \\
& \mathrm{O}+2 \mathrm{C} \mathrm{O}_{2}+\left(\mathrm{C}_{8} \mathrm{~N}_{3} \mathrm{H}_{7} \mathrm{O}_{8}\right) \text {. }
\end{aligned}
$$

Uroxile contains 2 eqs. of water more than uramile, and it is isomeric with dialurate of ammonia; but it is quite distinct from that salt.

The recent very interesting researches of Rochleder on caffeine or theine have led to the discovery of some very remarkable relations between some of the products of oxidation of caffeine and those of uric acid just described. Liebig had long ago pointed out that the action of caffeine on the body probably depended on the fact that its composition was related to that of uric acid; and Rochleder has obtained, among various other curious products, one analogous to alloxantine, and another equally analogous to murexide. These will be afterwards described, and we shall then see that it would appear that alloxan, alloxantine, murexide, \&c., have their homologues in the Caffeine series, the investigation of which will doubtless throw much light on the true constitu- 
tion of these compounds. We shall return to this subject under caffeine.

Having now described the numerous products of the oxidation of uric acid by nitric acid, we are prepared to understand the nature of the radical supposed to be common to most of these compounds.

URYLE. Ul. $=\mathrm{C}_{8} \mathrm{~N}_{2} \mathrm{O}_{4}=4 \mathrm{CO}+2 \mathrm{C}_{2} \mathrm{~N}$.

Srv. Cyanoxalic Acid. - This radical is unknown in the separate form. It contains the elements of 4 eq. carbonic oxide or 2 eq. oxalyle $\left(\mathrm{C}_{2} \mathrm{O}_{2}\right)$, and 2 eq. cyanogen. Hence the name cyanoxalic acid. Assuming it to exist, we have the following series :-

\begin{tabular}{|c|c|c|c|c|c|c|}
\hline & ational formulæ. & Names. & & Empiric & 1 forr & ulæ. \\
\hline UI & +1 eq. urea & $=$ Uric Acid & $=$ & $\mathrm{C}_{10} \mathrm{~N}_{4}$ & $\mathrm{H}_{4}$ & 08 \\
\hline U1 & $+\mathrm{O}+5 \mathrm{HO}$ & $=$ Alloxantine & $=$ & & $\mathrm{H}_{5}$ & $\mathrm{O}_{10}$ \\
\hline UI & $+\mathrm{O}_{2}+4 \mathrm{HO}$ & $=$ Alloxan & $=$ & $\mathrm{C}_{8} \mathrm{~N}_{2}$ & $\mathrm{H}_{*}$ & $\mathrm{O}_{10}$ \\
\hline UI & $+4 \mathrm{H} \mathrm{O}$ & $=$ Dialuric Acid & $=$ & $\mathrm{C} 8 \mathrm{~N}_{2}$ & $\mathrm{H}_{4}$ & $\mathrm{O}_{8}$ \\
\hline $\mathrm{Ul}_{3}$ & $+10 \mathrm{HO}$ & $=$ Hydurilic acid & $=2$ & $\left(\mathrm{C}_{12} \mathrm{~N}_{3}\right.$ & $\mathrm{H}_{5}$ & $\left.\mathrm{O}_{11}\right)$ \\
\hline U & $\begin{aligned} & +\mathrm{NO}_{5}+\mathrm{O}_{3} \\
+ & 2 \mathrm{HOO}\end{aligned}$ & $\left.=\begin{array}{l}\text { Nitrohydurilic } \\
\text { acid }\end{array}\right\}$ & $=$ & $\mathrm{C}_{8} \mathrm{~N}_{3}$ & $\mathrm{H}_{2}$ & $0_{14}$ \\
\hline 0 & $+\mathrm{NH}_{3}+2 \mathrm{H}$ & $=$ Uramile & $=$ & $\mathrm{C}_{8} \mathrm{~N}_{3}$ & $\mathrm{H}_{5}$ & Oo \\
\hline & $\begin{aligned} & +\mathrm{O}_{2}+4 \mathrm{HO} \\
+ & \mathrm{H} \mathrm{H}_{3}+2 \mathrm{~S} \mathrm{O}_{2}\end{aligned}$ & $=$ Thionuric acid & $=$ & $\mathrm{C} \mathrm{N}_{3} \mathrm{~N}_{3}$ & $\mathrm{H}_{7}$ & $\mathrm{O}_{14}$ \\
\hline
\end{tabular}

The ready conversion of these compounds one into another is a strong argument for the existence of the radical uryle. But the rational formulæ above given do not represent what we suppose to be the actual arrangement; they only point out by what simple means, as the addition or removal of oxygen, or ammonia, or water, the elements of the new compounds might be supplied. The other derivatives of uric acid are probably compounds of different radicals: thus, parabanic and oxaluric acids each contain only 6 eq. of carbon, and cannot therefore be compounds of uryle.

The above brief sketch of the products derived from uric acid, will serve to show the inexhaustible variety of new products, which one complex body may yield. Notwithstanding all that has been done, we must consider this subject as merely opened up, and a further prosecution of it will infallibly lead to many interesting and valuable results.

APPENDIX TO URIC ACID.

1. Uric or Xanthic Oxide.-This is a very rare ingredient of urinary calculi. Its formula is $\mathrm{C}_{5} \mathrm{~N}_{2} \mathrm{H}_{2} \mathrm{O}_{2}$, which, taken 
double, differs from uric acid only by 2 eq. oxygen. Hence its name of uric oxide. It is soluble in potash and precipitated by acids as a.white powder. It dissolves in nitric acid, and the solution evaporated to dryness leaves a yellow residue; hence the name of xanthic oxide. It is said to occur in small quantities in some kinds of guano:

2. Cystic Oxide, $\mathrm{C}_{6} \mathrm{NH}_{6} \mathrm{O}_{4} \mathrm{~S}_{2}$.-Another very rare form of calculus. It dissolves both in acids and alkalies, and has the characters of an organic base, forming crystalline compounds with acids. It is remarkable from the large quantity of sulphur it contains.

3. Guanine, $\mathrm{C}_{10} \mathrm{~N}_{5} \mathrm{H}_{5} \mathrm{O}_{2}$.-This compound has been discovered quite recently in guano by Unger, who at first supposed it to be identical with uric or xanthic oxide, but subsequently found it to be a distinct compound. It seems to be a base, somewhat analogous to urea; for it unites with hydrochloric, sulphuric, and nitric acids, forming crystallisable salts, among which are acid salts, with hydrochloric and nitric acids; a very rare phenomenon.

Guanine is a white powder, insoluble in water. Like other organic bases, it forms a double salt when its hydrochlorate is mixed with bichloride of platinum. This salt crystallises in fine orange yellow crystals the formula of which is $\left(\mathrm{C}_{10} \mathrm{~N}_{5} \mathrm{H}_{5} \mathrm{O}_{2}\right.$, $\mathrm{H} \mathrm{Cl})+2 \mathrm{Pt} \mathrm{Cl}_{2}+4 \mathrm{H} \mathrm{O}$. It is remarkable that guanine forms no basic salts, as most weak bases do, but only neutral and acid salts.

Hyperuric Acid (?).-This acid was also discovered by Unger, who obtained it by acting on guanine with a mixture of hydrochloric acid and chlorate of potash. Its formula, according to Unger's experiments, is $\mathrm{C}_{30} \mathrm{~N}_{4} \mathrm{H}_{5} \mathrm{O}_{9}$, which differs from uric acid by 1 eq. water, and 2 eq. oxygen; hence the name. Unger conjectures it, from the analysis of the silver salt, to be bibasic $\mathrm{C}_{10} \mathrm{~N}_{4} \mathrm{H}_{3} \mathrm{O}_{7}+2 \mathrm{H} \mathrm{O}$; but the want of material has prevented him from fully investigating this curious acid; and he marks its formula, and even its name as doubtful.

The acid forms short rhombic prisms, colourless and of brilliant lustre. When heated, it yields hydrated cyanic acid, water, and a residue of carbon. This might occur with the formula above given, for $\mathrm{C}_{10} \mathrm{~N}_{4} \mathrm{H}_{5} \mathrm{O}_{0}=4\left(\mathrm{C}_{2} \mathrm{~N} \mathrm{O}, \mathrm{H} 0\right)$ $+\mathrm{HO}+\mathrm{C}_{2}$.

XIII. Benzorir. $\mathrm{Bz}=\mathrm{C}_{24} \mathrm{H}_{5} \mathrm{O}_{2}$.

This is the radical of Benzoic Acid, of oil of bitter almonds, and of an extensive series of compounds. The radical is not yet 
known with certainty in the separate form, although a compound exists, having the same composition. (See Benzile.) We shall describe first the benzoic acid, and afterwards the other compounds derived from, or connected with it.

\section{Benzoic Acid. $\mathrm{C}_{14} \mathrm{H}_{5} \mathrm{O}_{3}, \mathrm{HO}=\mathrm{Bz} \mathrm{O}, \mathrm{HO}$, or $\mathrm{Bz} \mathrm{O}_{2}, \mathrm{H}$.}

This acid is found in gum benzoin, mixed with some resins; and it also occurs in the urine of herbivorous animals, under certain ciroumstances. It is also formed by the oxidation of the oil of bitter almonds. It may be obtained from benzoin by sublimation, the powdered gum being gently heated on an iron plate forming the bottom of a broad and short cylinder, the top of which is covered with bibulous paper pasted tightly down to the sides, while another cylinder slides over the upper end of the first, to prevent the escape of the acid. The vapours of the acid, which is very volatile, pass through the paper, and forming crystals, are there retained, falling on its upper surface. The following method, however, is far more productive, as.in the process of sublimation some of the acid is always decomposed. Benzoin is dissolved in strong alcohol, and to the hot solution there is added hydrochloric acid in quantity sufficient to precipitate the resin; the whole is then distilled. The benzoio acid passes over under the form of benzoic ether (benzoate of oxide of ethyle): and when the greater part of the liquid has been distilled off, water is added to the residue, and distilled as long as any ether passes over with it. When this ceases the hot water remaining in the retort is filtered, and on cooling deposits part of the benzoic acid in crystals. The benzoic ether and all the distilled liquors are now treated with caustic potash, until all the ether is decomposed, and the solution, now containing benzoate of potash, is heated to boiling, and supersaturated with hydrochloric acid. On cooling it deposits the benzoic acid in erystals. By this means the whole benzoic acid of the benzoin is obtained.

When the urine of the cow is evaporated to a small bulk, and hydrochloric acid added in excess, a quantity of crystals is deposited. These are sometimes impure benzoio acid alone, sometimes a mixture of that acid, and of hippuric acid. Ether dissolves the former, and leaves the latter. The ether being distilled off, the benzoic acid is purified by combining it with lime, and precipitating the acid by the addition of hydrochlorio acid; or by dissolving it in boiling water, and adding nitrio acid, which destroys the colouring matter.

Benzoic acid is also formed, as will be explained hereafter, by 
boiling hippurio acid with strong hydrochloric acid. Lastly, benzoic acid is a very frequent product of the oxidation of nitrogenised or albuminous organic compounds. Its radical is, therefore, one of the products of the decomposition of very complex organic molecules.

Benzoic acid forms fine, light, prismatic erystals, or flexible pearly scales. When pure, it has no smell, but by heat it acquires the odour of benzoin or of vanilla, and as commonly prepared it has a very pleasant odour derived from the presence of some foreign compound which accompanies the acid, and is not easily separated from it. It is very fusible and volatile, and its vapours are very irritating, provoking cough. It is inflammable, burning with smoke. It is sparingly soluble in cold water, more so in boiling water; it dissolves also in alcohol and ether.

With bases it forms salts, many of which are crystallisable. Their general formula is $\mathrm{Bz} \mathrm{O}, \mathrm{M} \mathrm{O}$, or $\mathrm{Bz} \mathrm{O}_{2}, \mathrm{M}$. When the alkaline and earthy benzoates are heated in close vessels, they yield carbonates, while new products distil over, such as benzone, benzole, naphthaline, \&c. The benzoate of peroxide of iron, $3 \mathrm{BzO}+\mathrm{Fe}_{2} \mathrm{O}_{3}$, has a reddish white colour, and is insoluble. Benzoic acid, in the form of benzoate of ammonia, is therefore sometimes used as a means of separating peroxide of iron from some other bases. But its use requires many precautions, and it is quite inapplicable if alumina, glucina, yttria, or zirconia be present. Benzoate of silver, $\mathrm{Bz} \mathrm{O}, \mathrm{Ag} \mathrm{O}$, is sparingly soluble, and when formed in hot solutions crystallises on cooling.

Benzoic acid appears to be the starting-point, or lowest member of a series of homologous acids, of which two more are known, namely, the Toluylic and Cuminic Acids. It will be seen by the following table, that the common difference here, as in the series to which Formic, Acetic, and Valerianic acids, and the other volatile oily acids belong, is $\mathrm{C}_{2} \mathrm{H}_{2}$. And in this, as in the former case, the analogy in properties is as remarkable as the analogy in composition. The acids of the benzoic series are crystalline, volatile, and when heated with bases, yield aromatic oily compounds, which are carbohydrogens, homologous, like the acids from which they are derived, of which benzole is the type. From these oils various compounds are derived, of which we shall only here mention a remarkable series of homologous bases, that of which Aniline or Phenylamine is the type. 


\section{Benzorc Serres.}

$\begin{array}{rlllll}\text { Benzoic Acid } & \mathrm{C}_{14} \mathrm{H}_{6} & \mathrm{O}_{4} & \text { Benzole } \mathrm{C}_{12} \mathrm{H}_{6} & \text { Aniline } & \mathrm{C}_{12} \mathrm{H}_{7} \cdot \mathrm{N} \\ \text { Toluylic Acid } & \mathrm{C}_{16} \mathrm{H}_{8} \mathrm{O}_{4} & \text { Toluole } \mathrm{C}_{14} \mathrm{H}_{8} & \text { Toluidine } & \mathrm{C}_{14} \mathrm{H}_{9} \mathrm{~N} \\ \text { (?) } & \mathrm{C}_{18} \mathrm{H}_{10} \mathrm{O}_{4} & \text { Xylole } \mathrm{C}_{16} \mathrm{H}_{10} & \text { Xylidine } & \mathrm{C}_{16} \mathrm{H}_{12} \mathrm{~N} \\ \text { Cuminic Acid } & \mathrm{C}_{20} \mathrm{H}_{12} \mathrm{O}_{4} & \text { Cumole } \mathrm{C}_{18} \mathrm{H}_{12} & \text { Cumidine } & \mathrm{C}_{18} \mathrm{H}_{13} \mathrm{~N}\end{array}$

It will be seen that one acid, that with 18 eqs. of carbon, is not yet discovered, but it will probably soon be formed, as the corresponding members of the two other series are already known. We shall return to the benzoic series hereafter. The table here given is merely a specimen, to illustrate the nature of the group. There are many more series, connected with the three above given.

Anhrdrous Benzoic Aoid.

$$
\left.\mathrm{C}_{14} \mathrm{H}_{5} \mathrm{O}_{3}, \text { or } \mathrm{C}_{28} \mathrm{H}_{10} \mathrm{O}_{6}={\stackrel{\mathrm{C}}{\mathrm{C}_{14} \mathrm{H}_{5} \mathrm{O}_{3}}}_{\mathrm{H}_{5} \mathrm{O}_{3}}^{\mathrm{H}_{3}}\right\}
$$

This remarkable compound was discovered by Gerhardt, and is the type of a numerous class of compounds, the anhydrous organio acids, or anhydrides, for they are not true acids.

The anhydrous benzoic acid or benzoate of benzoyle, may be formed in various ways; either by the action of chloride of benzoyle on benzoate of soda, or by that of chloride of benzoyle on oxalate of potash; or by that of oxychloride of phosphorus on benzoate of soda. The first process is as follows,

$$
\left.\mathrm{C}_{14} \mathrm{H}_{5} \mathrm{O}_{2}, \mathrm{Cl}+\mathrm{Na} \mathrm{O}, \mathrm{C}_{14} \mathrm{H}_{5} \mathrm{O}_{3}=\mathrm{NaCl}+\begin{array}{l}
\mathrm{C}_{14} \mathrm{H}_{5} \mathrm{O}_{3} \\
\mathrm{C}_{14} \mathrm{H}_{5} \mathrm{O}_{3}
\end{array}\right\}
$$

In the second, we have,

$$
\left.\mathrm{C}_{4} \mathrm{O}_{8} \mathrm{~K}_{2}+2\left(\mathrm{C}_{14} \mathrm{H}_{5} \mathrm{O}_{2}, \mathrm{Cl}\right)=2 \mathrm{~K} \mathrm{Cl}+{\stackrel{\mathrm{C}}{\mathrm{C}_{14} \mathrm{H}_{5} \mathrm{O}_{5} \mathrm{O}_{3}}}_{\mathrm{C}_{3}}^{\mathrm{C}_{3}}\right\}+2 \mathrm{CO}+2 \mathrm{C} \mathrm{O}_{2}
$$

In the third, chloride of benzoyle and phosphorous acid are first formed by the action of the oxjchloride of phosphorus on the benzoic acid, and this subsequently acts on another portion of benzoate of soda, as in the first process.

In all these processes, the materials are gradually mixed in a large flask, and then heated, with constant agitation, to a temperature of about $265^{\circ}$ or $270^{\circ} \mathrm{F}$., till the smell of chloride of benzoyle has disappeared. The mass, when cold, is washed with cold water, to remove the chloride of potassium, and the insoluble residue is the anbydride. It may be purified by dissolving it in the smallest possible quantity of hot alcohol, which, on cooling, deposits it in brilliant oblique prisms. There is always 
a loss, from the formation of benzoic ether, and if too much alcohol be used, but little of the acid will be obtained. When the quantity is considerable, it is best purified by distillation. It distils at a high temperature, about $590^{\circ} \mathrm{F}$., yielding a colourless oil, which, on cooling, crystallises in acute rhombs or acicular prisms. It melts at $107^{\circ} \mathrm{F}$., and on cooling, crystallises like sulphur and bismuth, so that by pouring off the remaining fluid when the solidification is half finished, very fine crystals may be obtained.

The solutions of this anhydride in alcohol or ether are perfectly neutral, but it easily passes into benzoic acid when boiled with water, especially if a base be present.

With ammonia it forms benzamide; with aniline, benzanilide. With caustic potash and other bases it is converted into benzoates.

We shall see that many other anhydrides or anhydrous acids, as they are called, having the composition of anhydrous acids, but no acid properties, may be formed in the same way, and have analogous properties. The methods generally pursued are those here described, only using the salts of other acids instead of those of benzoic acid.

The double formula above given for this anhydride is derived from the type water, thus written, $\left.\begin{array}{l}\mathrm{H} O \\ \mathrm{H}\end{array}\right\}$ which corrresponds to

$$
\left.\begin{array}{l}
\mathrm{C}_{14} \mathrm{H}_{5} \mathrm{O}_{2}, \\
\mathrm{C}_{14} \mathrm{H}_{5} \mathrm{O}_{2}, \mathrm{O}
\end{array}\right\}
$$

Now the occurrence of double anhydrides is in favour of the double formulæ. We shall see that anhydrides may be formed containing 1 eq. of one anhydride and 1 eq. of another; for example, the anhydrous aceto-benzoic acid, (or acetate of benzoyle, or benzoate of acetyle, for it may be named by all these names) which is $\mathrm{C}_{28} \mathrm{H}_{8} \mathrm{O}_{6}$ evidently $\left.\begin{array}{llll}\mathrm{C}_{4} & \mathrm{H}_{5} & \mathrm{O}_{2}, & 0 \\ \mathrm{C}_{3} & \mathrm{O}_{2}, & 0\end{array}\right\}$ corresponding to the type water, thus $\left.\begin{array}{ll}\mathrm{H} & \mathrm{O} \\ \mathrm{O}\end{array}\right\}$.

\section{Hyduret of Benzoyle. $\mathrm{C}_{14} \mathrm{H}_{6} \mathrm{O}_{2}=\mathrm{Bz} \mathrm{H}$.}

Syn. Essential oil of bitter almonds. - When bitter almonds, after being macerated with water for a day or two, are distilled with the water, there is obtained a fragrant oily liquid, heavier than water, which contains, besides hyduret of benzoyle, benzoic acid, hydrocyanic acid, and benzoine, a solid compound isomeric with the hyduret of benzoyle. To purify it, this oily liquid is 
distilled along with a mixture of protochloride of iron and slaked lime, which retain the two acids, and the benzoine remaining behind, the pure hyduret passes over.

It is a colourless transparent liquid, of a high refractive power. It has a peculiar and very powerful smell, and it is on this account that the crude oil is so much used in perfumery. Its odour has been compared to that of hydrocyanic acid, but this has arisen from the fact that the crude oil contains both; for on comparing the two, both pure, no great similarity can be perceived. It ought, however, to be borne in mind that the commercial oil is highly poisonous, because it contains hydrocyanio acid, though the hyduret of benzoyle is not poisonous. Hyduret of benzoyle boils at $356^{\circ}$.

When exposed to the air, it absorbs 2 eq. of oxrgen, and is converted into pure crystallised benzoic acid. $\mathrm{BzH}+\mathrm{O}_{2}=\mathrm{Bz}$ $\mathrm{O}, \mathrm{H} \mathrm{O}$. Heated with caustic potash in close vessels, it yields benzoate of potash and hydrogen gas, which is disengaged. $\mathrm{K}, \mathrm{H} \mathrm{O}+\mathrm{BzH}=\mathrm{K} \mathrm{O}, \mathrm{BzO}+\mathrm{H}$. It is still more easily converted into benzoate of potash by an aleoholic solution of potash; the alcohol here swims above the salt, and holds in solution an oily matter not yet examined.

I have found that the benzoate of potash, formed by the action of an alcoholic solution of potash in the crude oil of bitter almonds, is very soluble in alcohol, from which it crystallises in cooling a hot saturated solution, in a mass of fine silvery or pearly scales. The benzoate of potash, made directly from benzoic acid, is very sparingly soluble in hot alcohol, and erystallises differently. The cause of this difference is still unknown; but it may depend on a difference in the amount of water of crystallisation, or on the absence of such water in one of the salts ; or on one salt being neutral, the other acid, in constitution. I did not find, however, any such difference, so far as I have examined them.

When hyduret of benzoyle is mixed with a little hydrocyanio acid, and placed in contact with aqua potassæ, lime water, or baryta water, it is gradually converted into the solid crystalline compound, isomeric with itself, which is called benzoine. When mixed with aqua ammoniæ, and gently heated, it produces a new compound, hydrobenzamide. With chlorine and bromine, if dry, it yields chloride and bromide of benzoyle, with hydrochloric and hydrobromic acids; if water be present, benzoic acid is likewise formed, part of which combines with some unchanged hyduret of benzoyle.

3. Chloride of benzoyle, formed by the action of dry chlorine on the hyduret, is a colourless liquid, of a strong disagreeable odour. 
It is formed as follows: $\mathrm{BzH}+\mathrm{Cl}_{2}=\mathrm{BzCl}+\mathrm{H} \mathrm{Cl}$. With the alkalies it yields benzoate of the alkali, and chloride of the metal: $\mathrm{BzCl}+2 \mathrm{~K} \mathrm{O}=\mathrm{K}, \mathrm{Bz} \mathrm{O}+\mathrm{K} \mathrm{Cl}$. With dry ammonia it yields benzamide: with alcohol it produces benzoic ether and hydrochloric acid. $\mathrm{Ae} \mathrm{O}, \mathrm{H} \mathrm{O}+\mathrm{Bz} \mathrm{Cl}=\mathrm{Ae} \mathrm{O}, \mathrm{Bz}$ $\mathrm{O}+\mathrm{H} \mathrm{Cl}$. When acted on by metallic bromides, iodides, sulphurets, or cyanides, it produces metallic chlorides, and bromide, iodide, sulphuret or cyanide of benzoyle. When it acts on the salts of organic acids, it gives rise to anhydrous organic acids or anhydrides (see above). The bromide of benzoyle is a crystalline solid, in other respects analogous to the chloride. The iodide and sulphuret of benzoyle are also crystallisable: the cyanide is a liquid having an odour like that of cinnamon.

\section{Benzamide. $\mathrm{C}_{14} \mathrm{~N} \mathrm{H}_{7} \mathrm{O}_{2}=\mathrm{C}_{14} \mathrm{H}_{5} \mathrm{O}_{2}+\mathrm{NH}_{2}=\mathrm{Bz}$ Ad.}

This compound is formed when dry ammonia acts on chloride of benzoyle, $\mathrm{Bz} \mathrm{Cl}+\mathrm{N} \mathrm{H}_{2}, \mathrm{H}=\mathrm{Bz}, \mathrm{N} \mathrm{H}_{2}+\mathrm{H} \mathrm{Cl}$ : also when hippuric acid (which see), is boiled with peroxide of lead, and when anhydrous benzoic acid is acted on by ammonia. When prepared from the chloride, it is accompanied by sal ammoniac, formed by the hydrochloric acid produced, with the excess of ammonia. This is removed by cold water; and the benzamide being dissolved in hot water, crystallises on cooling. It forms fine soft needles or pearly scales, very fusible and volatile. Like other amidides, it yields ammonia when boiled with alkalies, while a benzoate is formed.

5. Formobenzoilic Acid. $\left\{\begin{array}{c}\mathrm{C}_{2} \mathrm{H} \mathrm{O}_{3}+\mathrm{C}_{14} \mathrm{H}_{6} \mathrm{O}_{2}+\mathrm{HO}=\mathrm{F}_{0} \mathrm{O}_{3}, \\ \mathrm{Bz} \mathrm{H}+\mathrm{H} \mathrm{O} .\end{array}\right.$

This compound, which is a striking example of a coupled acid, is obtained by adding to distilled water some crude oil of bitter almonds, which always contains hydrocyanic acid (see Amygdaline), and evaporating to dryness along with some hydrochloric acid. From the dry mass, ether dissolves the new acid, which it deposits as a crystalline powder by evaporation. In this process, the hydrocyanic acid, under the influence of hydrochlorio acid, is converted, along with the elements of water, into formic acid and ammonia. The latter combines with the hydrocyanic acid, the former with the hyduret of benzoyle, yielding formobenzoilic acid. With bases this acid forms salts, in which the quantity of base neutralised is exactly that which would be neutralised by the formic acid alone. Their formula is $\mathrm{MO}+\mathrm{Fo} \mathrm{O}_{3}, \mathrm{Bz} \mathrm{H}$; by which it is seen that the hyduret of benzoyle has entered into the radical of the acid, without altering its power of saturation; 
and perhaps the rational formula of the acid ought rather to be, $\left(\mathrm{F}_{0} \mathrm{O}_{4}, \mathrm{Bz} \mathrm{H}\right)+\mathrm{H}$; and that of the salts, $\left(\mathrm{Fo} \mathrm{O}_{4}, \mathrm{Bz} \mathrm{H}\right)+\mathrm{M}$; which exhibits this view still more clearly.

\section{Benzoate of Hyduret of Benzoyle. $\mathrm{C}_{42} \mathrm{H}_{18} \mathrm{O}_{8}=\mathrm{Bz} \mathrm{O}$, $\mathrm{H} \mathrm{O}+2 \mathrm{Bz} \mathrm{H}$.}

This compound is formed when moist chlorine is passed through the oil of bitter almonds. Hydrated benzoic acid is formed, which unites with the unchanged hyduret. The action of moist chlorine in producing hydrated benzoic acid is as follows: $\mathrm{Bz} \mathrm{H}+2 \mathrm{H} \mathrm{O}+\mathrm{Cl}_{2}=2 \mathrm{H} \mathrm{Cl}+\mathrm{BzO}, \mathrm{H} \mathrm{O}$. The new compound is crystalline, insoluble in water, soluble in alcohol and ether. It is volatile without decomposition. An alcoholio solution of potash dissolves it, and converts it into benzoate of potash.

\section{Hippuric Acid. $\mathrm{C}_{18} \mathrm{NH}_{8} \mathrm{O}_{5}+\mathrm{H} \mathrm{O}$.}

This acid is found in very considerable quantity in the urine of herbivorous animals, such as the horse and cow, more especially when stall-fed. It has also been lately discovered by Liebig in human urine. It is easily obtained by boiling down to a small bulk the fresh urine of the horse or cow, after adding an excess of milk of lime, and acidulating with hydrochloric acid. On standing, the liquid deposits brown crystals of hippuric acid, which may be decolorised by boiling with excess of lime, and enough of boiling water to dissolve all the hippurate of lime, and adding to the strained solution hydrochloric acid in excess, when the hippuric acid is deposited. A repetition of this process renders it quite white. If benzoio acid be present, it is removed by ether.

The pure acid forms pretty large semi-opaque four-sided prisms, sparingly soluble in cold water, very soluble in hot water, and in alcohol. When heated, it melts, and gives off benzoic acid, benzoate of ammonia, and an oily matter, which has a very fragrant odour like that of the tonka bean. By nitric acid it is converted into benzoic acid. Heated with peroxide of manganese and sulphuric acid, it yields ammonia, carbonic acid, and benzoic acid; boiled with peroxide of lead, it yields benzamide and carbonic acid.

With bases it forms salts, most of which are soluble and crystallisable.

Hippuric acid may be viewed in two ways ; first, as a compound of benzamide with an acid, $\mathrm{C}_{4} \mathrm{H} \mathrm{O}_{3}$ (fumaric or aconitic acid ?) and it is remarkable that Schwartz, who has lately studied the hippurates, observed a very great similarity to the fumarates; as if the hyduret of benzoyle were simply taken up into the 
molecule of these salts, without much affecting their properties ; secondly, as composed of hyduret of benzojle, hydrocyanic acid, and formic acid. Either view readily accounts for its easy decomposition into benzoic acid and other products. As an ingredient of the urine, this acid is important; and we shall hereafter see that benzoic acid, taken into the system, appears in the urine as hippuric acid.

But Dessaignes has recently shown, that, when hippuric acid is boiled with stronger acids for some time, it is decomposed, and yields benzoic acid, which crystallises on cooling; while the mother liquid, on further evaporation, yields fine crystals of the salts formed by the combination of glycocine with the acid employed. In fact, glycocine, according to Dessaignes and to Horsford, is a basis quite analogous to urea; and its salts, with the mineral acids, form double salts, and appear to act as acids, which is also the case with urea. If to

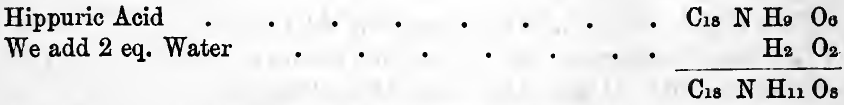
And from the sum deduct 1 eq. hydrated benzoic acid $\mathrm{C}_{14} \mathrm{H}_{6} \mathrm{O}_{4}$ There remains . . . . . . $\overline{\mathrm{C}_{4} \mathrm{~N} \mathrm{H}_{5} \mathrm{O}_{4}}$

And this is the formula of glycocine, Hippuric acid, therefore, may be regarded as composed of anhydrous benzoic acid and anhydrous glycocine; as a coupled benzoic acid, in which glycocine is the copula, which does not affect the neutralising power of the benzoic acid. Hippuric acid, when acted on by nitric acid, yields a new acid, nitrohippuric acid, very similar to hippuric acid. Its formula is $\mathrm{C}_{18} \mathrm{~N}_{3} \mathrm{H}_{8} \mathrm{O}_{10}$, and it is hippuric acid, in which 1 eq. of hydrogen is replaced by $\mathrm{N} \mathrm{O}_{4}$, or nitrous acid.

We have seen that hippuric acid may be regarded as constituted in three ways; namely, as

Fumaric Acid.

Benzamide $\mathrm{C}_{14} \mathrm{NH}_{7} \mathrm{O}_{2}+\mathrm{C}_{4} \mathrm{H} \mathrm{O}_{3}, \mathrm{HO}=\mathrm{C}_{18} \mathrm{NH}_{8} \mathrm{O}_{5}, \mathrm{H} \mathrm{O}$.

Formic Acid.

$\left.\begin{array}{c}\text { Hyduret of } \\ \text { Benzoyle }\end{array}\right\} \mathrm{C}_{14} \mathrm{H}_{6} \mathrm{O}_{2}+\mathrm{C}_{2} \mathrm{~N} \mathrm{H}+\mathrm{C}_{2} \mathrm{H} \mathrm{O}_{3} \mathrm{HO}=\mathrm{C}_{18} \mathrm{~N} \mathrm{H}_{8} \mathrm{O}_{5}, \mathrm{HO}$.

Glycocine, dry.

Benzoic acid, dry $\mathrm{C}_{14} \mathrm{H}_{5} \mathrm{O}_{3}+\mathrm{C}_{4} \mathrm{NH}_{4} \mathrm{O}_{3}=\mathrm{C}_{18} \mathrm{~N} \mathrm{H}_{8} \mathrm{O}_{5}, \mathrm{H} \mathrm{O}$.

But the recent researches of Socoloff and Strecker tend to show that a fourth arrangement is the true one. They regard hippuric acid as the amide of a new acid, benzoglycolic acid, $\mathrm{C}_{18} \mathrm{H}_{8} \mathrm{O}_{8}=$ $\mathrm{C}_{28} \mathrm{H}_{7} \mathrm{O}_{7}, \mathrm{HO}$. Supposing this acid to act on ammonia, the 
result would be, as with amides generally, $\mathrm{C}_{18} \mathrm{H}_{8} \mathrm{O}_{8}+\mathrm{N} \mathrm{H}_{3}=$ $2 \mathrm{H} \mathrm{O}+\mathrm{C}_{18} \mathrm{~N} \mathrm{H}_{8} \mathrm{O}_{5} ; \mathrm{HO}$. On this view, the name and constitution of hippuric acid will be benzoglycolamidic acid $=\mathrm{C}_{1} 8 \mathrm{H}_{7} \mathrm{O}_{6}$ $\mathrm{N} \mathrm{H}_{2}$, and the addition of 2 eqs. of water will suffice to convert it into benzoglycolate of ammonia, as is the case with other amides. When it is heated with acids, however, a different result ensues; benzoic acid being formed on the one hand, and glycocine on the other. The reason of this will presently appear.

Benzoglycolic Acid, $\mathrm{C}_{18} \mathrm{H}_{7} \mathrm{O}_{7}, \mathrm{HO}=\mathrm{C}_{18} \mathrm{H}_{8} \mathrm{O}_{8}$. This acid is formed when hippuric acid is acted on by hyponitrous acid, an agent which generally reproduces from amide the acids which had yielded them. $\mathrm{C}_{18} \mathrm{~N} \mathrm{H}_{9} \mathrm{O}_{6}+\mathrm{NO}_{3}=\mathrm{HO}+\mathrm{N}_{2}+\mathrm{C}_{88} \mathrm{H}_{8} \mathrm{O}_{8}$. This reaction is of itself a strong argument in favour of the idea that hippuric acid is the amide of benzoglycolic acid. This acid is orystalline, sparingly soluble in cold water, soluble in alcohol and ether. It is decomposed by heat, and even by boiling with water. Its salts are generally soluble and crystallisable. When boiled with acids, it is rapidly decomposed, yielding benzoic acid and a new acid, glycolic acid.

Glycolic Acid, $\mathrm{C}_{4} \mathrm{H}_{3} \mathrm{O}_{5}, \mathrm{HO}=\mathrm{C}_{4} \mathrm{H}_{4} \mathrm{O}_{6}$. This acid is formed in the manner just described. Thus, $\mathrm{C}_{18} \mathrm{H}_{8} \mathrm{O}_{8}+2 \mathrm{HO}=$ $\mathrm{C}_{14} \mathrm{H}_{6} \mathrm{O}_{4}+\mathrm{C}_{4} \mathrm{H}_{4} \mathrm{O}_{6}$. When purified, it forms a syrup, like lactic acid, which is decomposed by heat. In fact, glycolic acid is homologous with, and perfectly analogous to, lactio acid, $\mathrm{C}_{6} \mathrm{H}_{6} \mathrm{O}_{6}$. Benzoglycolic acid is composed of dry benzoic and dry glycolic acid. $\mathrm{C}_{18} \mathrm{H}_{8} \mathrm{O}_{8}=\mathrm{C}_{14} \mathrm{H}_{5} \mathrm{O}_{3}+\mathrm{C}_{4} \mathrm{H}_{3} \mathrm{O}_{5}$. And glycocine (glycocoll) is the amide of glycolic acid. $\mathrm{C}_{4} \mathrm{H}_{4} \mathrm{O}_{6}+$ $\mathrm{NH}_{3}=\mathrm{C}_{4} \mathrm{NH}_{5} \mathrm{O}_{4}+2 \mathrm{HO}$. We can now see how it is that hippuric acid, although it be the amide of benzoglycolic acid, does not yield that acid and ammonia, when boiled with acids, but benzoic acid and glycocine. The benzoglycolic acid is at first formed, along with ammonia; but it is immediately decomposed into benzoic and glycolic acid, and the latter, with the ammonia, forms water and glycocine.

It has been stated that glycolic acid is the lowest member of a homologous series of acids, of which the next is lactic acid. The only other member known is the fifth, and that has only been discovered, not named nor studied. Besides this, we have glycocine as the first member of a homologous series of bases, of which alanine is the second, and leucine the fifth. And in like manner, benzoglycolic and hippuric acids must be the lowest or first members of two other homologous series of acids. Of the former of these two, the second member is known, namely, benzolactic acid, which is quite analogous to benzoglycolic acid. Thus we have 
Glycocine Series.

Alanine Series.

Leucine Series.

Glycolic acid $\mathrm{C}_{4} \mathrm{H}_{4} \mathrm{O}_{6} \quad$ Lactic acid $\mathrm{C}_{6} \mathrm{H}_{8} \mathrm{O}_{6} \quad$ Leucic acid $\mathrm{C}_{12} \mathrm{H}_{12} \mathrm{O}_{6}$ Glycocine $\mathrm{C}_{4} \mathrm{NH}_{5} \mathrm{O}_{4}$ Alanine $\mathrm{C}_{6} \mathrm{~N} \mathrm{H}_{7} \mathrm{O}_{4}$ Leucine $\mathrm{C}_{12} \mathrm{~N} \mathrm{H}_{13} \mathrm{O}_{4}$

$\left.\left.\begin{array}{c}\text { Benzoglyco- } \\ \text { lic acid }\end{array}\right\} \mathrm{C}_{18} \mathrm{H}_{8} \mathrm{O}_{8} \begin{array}{c}\text { Benzolac- } \\ \text { tic acid }\end{array}\right\} \mathrm{C}_{20} \mathrm{H}_{10} \mathrm{O}_{8}$

$? \quad \mathrm{C}_{26} \mathrm{H}_{16} \mathrm{O}_{8}$

Hippuric acid $\mathrm{C}_{18} \mathrm{~N} \mathrm{H}_{9} \mathrm{O}_{6} ? \quad \mathrm{C}_{20} \mathrm{NH}_{11} \mathrm{O}_{6} ? \mathrm{C}_{26} \mathrm{~N} \mathrm{H}_{17} \mathrm{O}_{6}$

Of the intermediate series, with $\mathrm{C}_{8}$ and $\mathrm{C}_{10}$, as well as of those with $\mathrm{C}_{14}$ and upwards (in the first acid and base, corresponding to glycolic acid and glycocine) nothing is known; but these will in time be discovered.

\section{PRODUCTS OF THE TRANSFORMATION OF THE COMPOUNDS OF}

\section{BENZOYLE.}

1. Hyposulphobenzoic Acid. $\mathrm{C}_{14} \mathrm{H}_{4} \mathrm{O}_{3}+\mathrm{S}_{2} \mathrm{O}_{5}+2 \mathrm{HO}$.

A bibasio acid. Formed when anhydrous sulphurio acid acts on crystallised benzoic acid. $\mathrm{C}_{24} \mathrm{H}_{5} \mathrm{O}_{3}, \mathrm{HO}+2 \mathrm{~S} \mathrm{O}_{3}=\mathrm{C}_{14}$ $\mathrm{H}_{4} \mathrm{O}_{3}+\mathrm{S}_{2} \mathrm{O}_{5}+2 \mathrm{H} \mathrm{O}$. The acid is soluble and crystallisable, and forms with baryta a soluble and crystallisable salt, from which the acid may be obtained by the action of sulphurio acid. It forms two series of salts, one with 2 eq. of fixed base, the other with 1 eq. of fixed base and 1 eq. of water.

\section{Bromobenzoic Acid. $\mathrm{C}_{28} \mathrm{H}_{9} \mathrm{Br} \mathrm{O}_{8}+2 \mathrm{HO}$.}

A bibasic acid. When the vapour of bromine is allowed to act on benzoate of silver at the ordinary temperature, there is produced this acid, along with bromide of silver and hydrobromic acid. 2 eq. of benzoate of silver and 4 eq. of bromine yield 1 eq. bromobenzoic acid, 1 eq. hydrobromic acid, and 2 eq. bromide of silver. $2\left(\mathrm{C}_{24} \mathrm{H}_{5} \mathrm{O}_{3} \mathrm{Ag} \mathrm{O}\right)+\mathrm{Br}_{4}=\mathrm{C}_{28} \mathrm{H}_{8} \mathrm{Br}$. $\mathrm{O}_{8}+\mathrm{H} \mathrm{Br}+2 \mathrm{Ag} \mathrm{Br}$. Ether dissolves the acid and deposits it on evaporation, in a confused mass of crystals, very sparingly soluble in water. When the acid crystallises, it takes up 2 eq. of water. With bases it forms salts, which are generally soluble and crystallisable. Their general formula is $\mathrm{C}_{28} \mathrm{H}_{9} \mathrm{Br}$ $\mathrm{O}_{8}, 2 \mathrm{M} \mathrm{O}$.

\section{Nitrobenzoic Acid. $\mathrm{C}_{14} \mathrm{H}_{4} \mathrm{NO}_{7}$, H $\mathrm{O}$.}

Formed when nitric acid acts on benzoic acid. It is a crystalline volatile acid, and contains the elements of benzoic acid, in which 1 eq. hyponitric or nitrous acid has been substituted for 1 eq. hydrogen. $\mathrm{C}_{14} \mathrm{H}_{5} \mathrm{O}_{3}-\mathrm{H}+\mathrm{N} \mathrm{O}_{4}$ $=\mathrm{C}_{14} \begin{cases}\mathrm{H}_{4} & \mathrm{O}_{3} ; \text { and the basic water of the benzoic acid unites } \\ \mathrm{N} \mathrm{O}_{4} & \text { and }\end{cases}$ with the new acid as with the old. 


\section{Benzole. $\mathrm{C}_{12} \mathrm{H}_{6}$.}

Srx. Benzine. Benzene. Phene.-Occurs in the volatile liquids condensed from oil gas; but is best obtained in a state of purity by distilling 1 part of crystallised benzoio acid with 3 of slaked lime. It is a limpid, colourless liquid, of an agreeable etherial odour. Its Sp. G. is 0.85 ; it boils at $186^{\circ}$, and at $32^{\circ}$ it becomes "solid. It is insoluble in water, soluble in alcohol and ether. In its formation, 1 eq. of benzoic acid yields 2 eq. carbonic acid and $1 \mathrm{eq}$. benzole, the carbonic acid uniting with the lime, $\mathrm{C}_{14} \mathrm{H}_{5} \mathrm{O}_{3}, \mathrm{HO}=\mathrm{C}_{12} \mathrm{H}_{6}+2 \mathrm{C} \mathrm{O}_{2}$.

5. Sulphobenzide, $\mathrm{C}_{2} \mathrm{H}_{5} \mathrm{~S} \mathrm{O}_{2}$. When anhydrous sulphuric acid acts on benzole, a viscid mass is formed, from which, by the addition of water, is separated a new compound, which may be dissolved and crystallised by means of ether. $\mathrm{C}_{12} \mathrm{H}_{6}+$ $\mathrm{S} \mathrm{O}_{3}=\mathrm{HO}+\mathrm{C}_{12} \mathrm{H}_{5}, \mathrm{~S} \mathrm{O}_{2}$. Sulphobenzide is quite neutral. 6. Hyposulphobenzidic Acid.-This acid is found in the liquid from which the preceding compound has been deposited. Its formula is $\mathrm{C}_{22} \mathrm{H}_{5} \mathrm{~S}_{2} \mathrm{O}_{5},+0$. It may be viewed as formed by the action of 2 eq. of dry sulphuric acid on 1 eq. of benzole, $\mathrm{C}_{12} \mathrm{H}_{6}+2 \mathrm{~S} \mathrm{O}_{3}=\mathrm{C}_{12} \mathrm{H}_{5}, \mathrm{~S}_{2} \mathrm{O}_{5} \mathrm{H} \mathrm{O}$; or as formed by the combination of sulphobenzide with oil of vitriol, $\mathrm{C}_{12} \mathrm{H}_{5} \mathrm{~S} \mathrm{O}_{2}$ $+\mathrm{H} \mathrm{O}, \mathrm{S} \mathrm{O}_{3}$. Either view readily explains its formation. The acid is best obtained pure from its salt with oxide of copper (which crystallises very easily), by the action of sulphuretted hydrogen. It is very soluble and may be crystallised. It has a very acid taste, and neutralises bases, forming crystallisable salts. 7. Nitrobenzole, $\mathrm{C}_{12} \mathrm{H}_{5} \mathrm{~N} \mathrm{O}_{4}$. Syn. Nitrobenzide.-Formed when benzole is dissolved to saturation in fuming nitric acid, and water added to the hot solution. On cooling, the nitrobenzole falls to the bottom as a heavy oil. It is, at $60^{\circ}$, a yellow liquid, very sweet to the taste, with an odour like that of cinnamon; it boils at $434^{\circ}$, and solidifies at $37^{\circ}$. Its Sp. G. is 1.209 . It is insoluble in water, soluble in alcohol and ether. It is formed from 1 eq. benzole and 1 eq. nitric acid, $\mathrm{C}_{12} \mathrm{H}_{6}+\mathrm{N}_{5}=$ $\mathrm{H} \mathrm{O}+\mathrm{C}_{12} \mathrm{H}_{5}, \mathrm{~N} \mathrm{O}_{4}$. By the action of reducing agents, such as sulphuretted hydrogen, nitrobenzole loses the whole of its oxygen, and taking up 2 eqs. of hydrogen, it is transformed into aniline, $\mathrm{C}_{12} \mathrm{~N} \mathrm{H}_{7}$. (See Aniline.) Nitrobenzole may be viewed as benzole, $\mathrm{C}_{12} \mathrm{H}_{6}$, in which 1 eq. hydrogen is replaced by nitrous acid, $\left.\mathrm{C}_{22} \underset{\mathrm{N}_{3}}{\mathrm{H}_{3}}\right\}$ while in aniline this eq. of hydrogen is replaced by amide; $\left.\mathrm{C}_{22} \mathrm{H}_{7} \mathrm{~N}=\mathrm{C}_{22} \underset{\mathrm{H}_{5}}{\mathrm{~N}_{2}}\right\}$. By the further action of nitric acid, nitrobenzide is converted into dinitrobenzole, $\mathrm{C}_{12} \mathrm{H}_{4}$ $\left.\mathrm{N}_{2} \mathrm{O}_{8}=\mathrm{C}_{22} \mathrm{H}_{4} \mathrm{~N}_{4}\right\}$ in which a second eq. of hydrogen is 
replaced by nitrous acid. This compound, acted on by hydrosulphuret of ammonia, yields nitraniline, a base, the formula of which is $\left.\mathrm{C}_{22} \underset{\mathrm{N} \mathrm{O}_{4}}{\mathrm{H}_{6}}\right\} \mathrm{N}$, corresponding to aniline $\mathrm{C}_{12} \mathrm{H}_{7} \mathrm{~N}$. 8. Azobenzide, $\mathrm{C}_{12} \mathrm{H}_{5} \mathrm{~N}$.-This compound is formed when an alcoholic solution of nitrobenzide is distilled with dry hydrate of potash. After the alcohol has distilled, the azobenzide volatilises, forming large red crystals, fusible at $150^{\circ}$, boiling at $380^{\circ}$. In the production of this compound alcohol takes a part, and there are formed, besides azobenzide, aniline, water, and oxalio acid, which remains combined with the potash, $2\left(\mathrm{C}_{12} \mathrm{H}_{5} \mathrm{~N} \mathrm{O}_{4}\right)$ $+\mathrm{C}_{4} \mathrm{H}_{6} \mathrm{O}_{2}=\mathrm{C}_{22} \mathrm{H}_{5} \mathrm{~N}+\mathrm{C}_{22} \mathrm{H}_{7} \mathrm{~N}+\mathrm{C}_{2} \mathrm{O}_{3}+3 \mathrm{HO}$. 9. Chloride of Benzole, $\mathrm{C}_{22} \mathrm{H}_{6} \mathrm{Cl}_{6}$.-Formed when chlorine gas and benzole are exposed to the sun's rays. It is a colourless crystalline solid. 10. Chlorobenzine, $\mathrm{C}_{12} \mathrm{H}_{3} \mathrm{Cl}_{3}$.-Obtained by distilling the preceding compound with hydrate of lime, as a colourless oily liquid.-Bromine forms with benzole analogous compounds.

Benzole, as already hinted, is the starting-point of a series of homologous compounds, the general formula of which is $\mathrm{C}_{n} \mathrm{H}_{n-6}$, and the common difference, added at each step, is $\mathrm{C}_{2} \mathrm{H}_{2}$. These compounds are all fragrant aromatic volatile oily liquids, or as we rise in the scale, fusible solids. They all admit of their hydrogen being replaced, apparently to the extent of 1, 2, or 3 eqs., by chlorine, bromine, nitrous acid, and amide, and in the last case they give rise to bases, of which aniline or phenylamine, $\mathrm{C}_{12} \mathrm{H}_{7} \mathrm{~N}$, or $\left.\mathrm{C}_{12} \mathrm{~N}_{5} \mathrm{H}_{2}\right\}$, is the type. Here we see amide combined with the group $\mathrm{C}_{12} \mathrm{H}_{5},\left(\mathrm{C}^{12} \mathrm{H}_{6}\right.$ (benzole) minus $\mathrm{H}_{\text {.) }}$ Hence aniline may be regarded as ammonia $\mathrm{N} \mathrm{H}_{2}, \mathrm{H}$, in which the last eq. of hydrogen is replaced by $\mathrm{C}_{2} \mathrm{H}_{5}$. To the group we give the name phenyle, and hence aniline is also called phenylamine. Benzole may be regarded as the hyduret of phenyle. $\mathrm{Cl}_{12} \mathrm{H}_{5}, \mathrm{H}$. Of course the homologues of benzole, namely toluole, xylole, and cumole, as they yield bases homologous with aniline, give rise to a series of groups or radicals, homologous with phenyle, and the general formula of which is $\mathrm{C}_{n} \mathrm{H}_{n} \rightarrow 7$. Since benzole, although derived from benzoic acid, which contains 14 eqs. of carbon, has itself only 12 eqs. of carbon, it cannot contain the radical benzoyle, but is itself a new radical, derived from it ; just as acetyle, derived from ethyle, contains 2 eqs. of hydrogen less than ethyle. The following table will show some of the derivations of benzole, and indicate the homologous series to which each belongs. Only the compounds connected with benzole are here named, the formulæ only of those higher in the series being given :- 


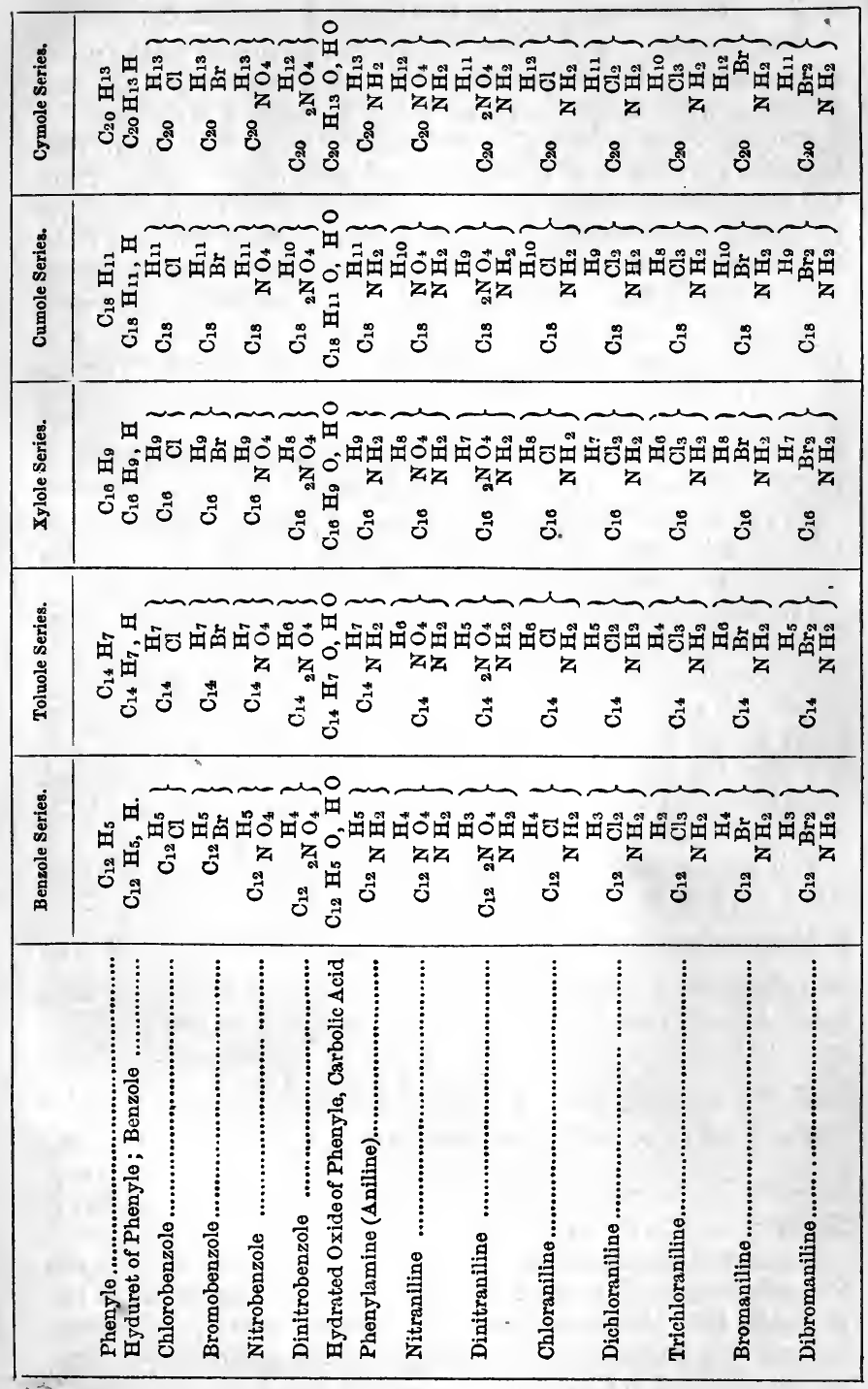




\section{Benzophenone. $\mathrm{C}_{26} \mathrm{H}_{10} \mathrm{O}_{2}=\mathrm{C}_{12} \mathrm{H}_{5}+\mathrm{C}_{14} \mathrm{H}_{5} \mathrm{O}_{2}$.}

This compound, which may be regarded as formed by the union of the radicals phenyle and benzoyle, is obtained, along with other products, when benzoate of lime is distilled with a little lime. A red liquid passes over, which is rectified. These then distil, first benzole or hyduret of phenyle, mixed with other bodies; then, the temperature rapidly rises till it reaches $600^{\circ} \mathrm{F}$., the receiver is changed, and the liquid, which distils between $600^{\circ}$ and $620^{\circ}$, is collected apart. It is nearly pure benzophenone, and on standing concretes into a mass of crystals. It is purified by recrystallisation in a mixture of alcohol and ether. It forms very fine large transparent crystals, which are rhombic prisms. It melts at $115^{\circ} \mathrm{F}$, and boils at $600^{\circ}$; is insoluble in water, soluble in alcohol, and still more in ether.

Its formation from benzoate of lime is thus explained, $2(\mathrm{Ca} \mathrm{O}$, $\left.\mathrm{C}_{14} \mathrm{H}_{5} \mathrm{O}_{3}\right)=2\left(\mathrm{Ca} \mathrm{O}, \mathrm{C} \mathrm{O}_{2}\right)+\mathrm{C}_{26} \mathrm{H}_{10} \mathrm{O}_{2}$.

The presence of benzole is due to a secondary decomposition, for benzoic acid, with excess of lime, yields benzole; and benzophenone, with caustic alkalies, also yields benzole and benzoate.

But along with the benzophenone are found two carbohydrogens, which are isomeric or polymeric with naphthaline. These are both crystalline, fusible and volatile, but differ in their solubility in alcohol and ether. Naphthaline is $\mathrm{C}_{20} \mathrm{H}_{8}$, and if we suppose these bodies to be, one $\mathrm{C}_{20} \mathrm{H}_{8}$, isomeric with naphthaline, and the other $\mathrm{C}_{30} \mathrm{H}_{12}$, polymeric with naphthaline, we can understand their formation from benzophenone. For $2\left(\mathrm{C}_{26} \mathrm{H}_{10} \mathrm{O}_{2}\right)=2 \mathrm{C} \mathrm{O}_{2}$ $+\mathrm{C}_{20} \mathrm{H}_{8}+\mathrm{C}_{30} \mathrm{H}_{12}$.

With nitric acid, benzophenone yields a new compound, in which 2 eqs. of hydrogen are replaced by 2 eqs. of Nitrous Acid. This

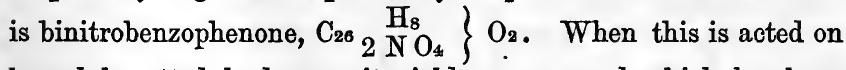
by sulphuretted hydrogen, it yields a compound which has been called flavine, from its yellow colour, but which is really diphenyle-urea, that is urea in which 2 eqs. of hydrogen are replaced by 2 eqs. of phenyle. $\left.\mathrm{C}_{26} \underset{2 \mathrm{NO}_{4}}{\mathrm{H}_{8}}\right\} \mathrm{O}_{2}+12 \mathrm{H} \mathrm{S}=\mathrm{S}_{12}+8 \mathrm{HO}$ $+\mathrm{C}_{26} \mathrm{H}_{12} \mathrm{~N}_{2} \mathrm{O}_{2}$. This last formula is that of the new body, for urea is $\mathrm{C}_{2} \mathrm{H}_{4} \mathrm{~N}_{2} \mathrm{O}_{2}$, and diphenyle-urea is $\left.\left.\mathrm{C}_{2} \underset{2}{\mathrm{H}_{2}} \mathrm{C}_{22} \mathrm{H}_{5}\right)\right\}$ $\mathrm{N}_{2} \mathrm{O}_{2}=\mathrm{C}_{26} \mathrm{H}_{12} \mathrm{~N}_{2} \mathrm{O}_{2}$.

The mixed liquid obtained by distilling benzoate of lime was formerly described as benzone $\mathrm{C}_{13} \mathrm{H}_{5} \mathrm{O}$; a formula in itself improbable, from the occurrence of an odd number of eqs. of carbon. For our present more exact knowledge of these curious products, we are indebted to Chancel. 


\section{Hydrobenzamide. $\mathrm{C}_{42} \mathrm{H}_{18} \mathrm{~N}_{2}$.}

When 1 vol. hyduret of benzoyle and 20 vol. of strong aqua ammoniæ are exposed in a hermetically sealed vessel to a temperature of from $105^{\circ}$ to $120^{\circ}$, it is converted after a time into a crystalline mass, which is to be washed with ether. The residue dissolved in alcohol, yields, by spontaneous evaporation, regular crystals of hydrobenzamide. In its formation, 3 eq. hyduret of benzole, and 2 eq. ammonia, produce 1 eq. hydrobenzamide and 6 eq. water. $3\left(\begin{array}{lll}\mathrm{C}_{14} & \mathrm{H}_{6} & \mathrm{O}_{2}\end{array}\right)+2 \mathrm{~N} \mathrm{H} \mathrm{H}_{3}=\mathrm{C}_{42} \mathrm{H}_{18} \mathrm{~N}_{2}+$ $6 \mathrm{H} \mathrm{O}$.

Hydrobenzamide, by the action of heat, or by boiling with caustic potash, is converted into the base amarine, isomeric with it.

Nitric acid acts upon hydrobenzamide, producing nitrohydrobenzamide, in which 5 eqs. of hydrogen are replaced by 5 of nitrous acid, $\mathrm{C}_{42} \underset{\left.(\mathrm{N} \mathrm{O})_{4}\right)_{5}}{\mathrm{H}_{13}} \mathrm{~N}_{2}$.

When this substance is boiled with potash, it is transformed into nitramarine, a base, isomeric with it, and which is amarine, with 5 eqs. of nitrous acid replacing 5 eqs. of hydrogen.

If, in preparing hydrobenzamide, we employ the crude oil of bitter almonds, we obtain a yellow resinous mass, which is a mixture of hydrobenzamide, benzhydramide, azobenzoyle, and azotide of benzoyle, all of them discovered by Laurent. 13. Benzhydramide is isomerio with hydrobenzamide, but is not converted, like the former, into hyduret of benzoyle and sal ammoniac by the action of hydrochloric acid. 14. Azobenzoyle, $\mathrm{C}_{42} \mathrm{H}_{15} \mathrm{~N}_{2}$, is much less soluble in alcohol than the preceding. It is derived from benzoyle as follows: $-3\left(\mathrm{C}_{14} \mathrm{H}_{5} \mathrm{O}_{2}\right)+2 \mathrm{~N} \mathrm{H}_{3}=\mathrm{C}_{42} \mathrm{H}_{15}$ $\mathrm{N}_{2}+6 \mathrm{H} \mathrm{O}$. 15. Azotide of benzoyle, $\mathrm{C}_{14} \mathrm{H}_{5} \mathrm{~N}$, is quite insoluble in boiling alcohol. It may be derived from anhydrous

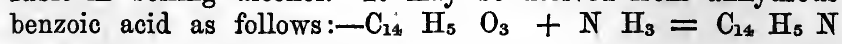
$+3 \mathrm{H} \mathrm{O}$.

It may here be mentioned that aniline, which is analogous to ammonia in constitution and relations, also acts on hyduret of benzoyle, and gives rise to a new hody, hydrobenzanilide, analogous to hydrobenzamide. 1 eq. of the hyduret, and 1 eq. of aniline, losing 2 eqs. of water, yield 1 eq. of hydrobenzanilide, or benzoylanilide, as it is called. $\mathrm{C}_{14} \mathrm{H}_{6} \mathrm{O}_{2}+\mathrm{C}_{12} \mathrm{H}_{7} \mathrm{~N}=2 \mathrm{H} \mathrm{O}+$ $\mathrm{C}_{26} \mathrm{H}_{11} \mathrm{~N}$. No doubt, methylamine and ethylamine, which are so much more analogous to ammonia, will yield analogous products.

Even urea, a substance which, though feebly basic, is yet not analogous to ammonia in constitution, acts in a somewhat similar 
way on the hyduret of benzoyle, yielding water and a new product, benzoyle-ureide, $\mathrm{C}_{50} \mathrm{H}_{28} \mathrm{~N}_{8} \mathrm{O}_{8}$. Thus, $3\left(\mathrm{C}_{44} \mathrm{H}_{6} \mathrm{O}_{2}\right)+$ $4 \mathrm{C}_{2} \mathrm{H}_{4} \mathrm{~N}_{2} \mathrm{O}_{2}=6 \mathrm{H} \mathrm{O}+\mathrm{C}_{30} \mathrm{H}_{28} \mathrm{~N}_{8} \mathrm{O}_{8}$.

\section{Benzostilbine. $\mathrm{C}_{31} \mathrm{H}_{11} \mathrm{O}_{2}$.}

Rochleder has shown that when hydrobenzamide is heated with fused potash, till the mass begins to blacken, three new substances are formed; namely, a yellow oil in very small quantity, and two solid crystalline compounds, Benzostilbine and Benzolone. Benzostilbine is best obtained by heating only till the mass becomes like gamboge. It is then purified by solution in alcohol, which dissolves it readily when the yellow oil is present. When the oil is destroyed by a little chlorine, the benzostilbine is immediately deposited in small colourless crystals of silvery lustre, which are insoluble in water, sparingly soluble in aloohol, and soluble with a blood-red colour in oil of vitriol, from which water precipitates it unchanged.

\section{Benzolone. $\mathrm{C}_{11} \mathrm{H}_{4} \mathrm{O}$.}

This compound is formed in greatest quantity when the mixture of hydrobenzamide with potash is heated till it becomes blackish brown. The mass is then washed with water, and dissolved in oil of vitriol, to which it gives a splendid red colour. The addition of weak alcohol now causes the benzolone to separate in small crystals.

It is obvious that the two preceding compounds contain the whole carbon of the hydrobenzamide, without any of its nitrogen, and they may both be derived from it by the addition of 3 eq. of water, and the elimination of 2 eq. ammonia,

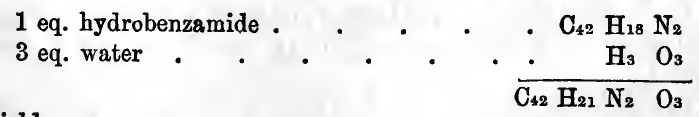

may yield

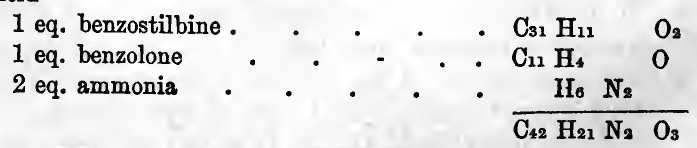

It would appear, however, that benzolone is also formed from benzostilbine, which is first produced, and then decomposed by the increased heat. Benzostilbine in fact contains the elements of 2 eq. benzolone, with an excess of carbon and hydrogen, which are partly given off in the form of carburetted hydrogen, and 
hydrogen, water also being decomposed, the oxygen ' of which combines with part of the carbon to form earbonic acid, which combines with the potash.

\section{Amarine. $\mathrm{C}_{ \pm 2} \mathrm{H}_{18} \mathrm{~N}_{2}$.}

When the hyduret of benzoyle, or rather, the oil of bitter almonds, is acted on by ammonia and alcohol, there is found an organic base, to which Laurent, its discoverer, has given the name of amarine. Its formula is $\mathrm{C}_{42} \mathrm{H}_{18} \mathrm{~N}_{2}$, and it is, therefore, isomeric, or polymeric, with hydrobenzamide. It is a wellmarked base, and we shall return to it as such.

\section{Lophine. $\mathrm{C}_{46} \mathrm{H}_{16} \mathrm{~N}_{3}$.}

When hydrobenzamide is subjected to the action of heat in close vessels, there is formed, among other products, a new organic base, which Laurent has named lophine. It has distinct basic properties, and will be again referred to in the section on organic bases. Its furmula is $\mathrm{C}_{46} \mathrm{H}_{16} \mathrm{~N}_{3}$.

The formation of this and of the preceding compound is very interesting, as proving the derivation of bodies having the high atomic weight of the organic bases from bodies of lower equivalents : these bodies, as well as the isomeric hydrobenzamide and benzhydramide, being all derived from the hyduret of benzoyle, $\mathrm{C}_{14} \mathrm{H}_{6} \mathrm{O}_{2}$. There may be some doubt as to the atomic weight of indifferent bodies, such as hydrobenzamide; but there is hardly any as to that of the bases above mentioned, which are evidently formed by the coalescence of 3 or more eqs. of the hyduret of benzoyle, since 1 eq. contains 42 or 46 eqs. of carbon ${ }_{2}$ while the hyduret only contains 14 eqs. of that element.

\section{Benzimide. $\mathrm{C}_{28} \mathrm{NH}_{11} \mathrm{O}_{4}$.}

According to Laurent, this compound is found in the orude oil of bitter almonds. It is crystallisable, and appears to be decom posed by acids into benzoic acid, and ammonia. It may be derived from anhydrous bibenzoate of ammonia by the separation of 2 eq. of water. $\mathrm{C}_{28} \mathrm{H}_{10} \mathrm{O}_{6}+\mathrm{NH}_{3}=\mathrm{C}_{28} \mathrm{Hn}$ $\mathrm{NO}_{4}+2 \mathrm{H} \mathrm{O}$. But this is not probable. A compound precisely similar is obtained when an alcoholic solution of potash is added to a mixture of hyduret of benzoyle and strong hydrocyanic acid; but this compound yields with acids ammonia and hyduret of benzoyle.

\section{Benzoine. $\mathrm{C}_{28} \mathrm{H}_{12} \mathrm{O}_{4}$.}

Polymerio with hyduret of benzoyle. It is formed when an alcoholic solution of potash, or sulphuret or cyanide of potassium, 
acts on the crude oil of bitter almonds; containing hydrocyanio acid. It separates in a congeries of small crystals, insoluble in water, soluble in alcohol. It may be volatilised without change. Sulphuric acid dissolves it with a violet colour. Hydrate of potash melted with it, forms a benzoate of potash, with disengagement of hydrogen. It dissolves with a violet colour in a hot alcoholic solution of potash, and is converted by boiling with it into benzilic acid. When its vapour is passed through a redhot tube, it is converted into hyduret of benzoyle, or, at least, into an oil smelling like that compound, and passing into benzoic acid on exposure to the air. By the action of chlorine it loses hydrogen, and is converted into Benzile, a compound having the composition of the radical benzoyle.

The action of hydrocyanic acid in promoting the formation of benzoine is not yet explained; but it is certain that from pure hyduret of benzoyle we cannot procure it, while the addition of hydrocyanic acid ensures its formation. It is, probably, formed by the simple coalescence of 2 eqs. hyduret of benzoyle, and the derivatives of benzoine most likely agree with it in containing 28 eqs. of carbon.

\section{Hydrobenzoinamide.}

Syx. Benzoinamide.-Isomeric, or polymeric with hydrobenzamide. It is formed by exposing a mixture of benzoine and ammonia to a moderate heat; and appears as a white tasteless powder, volatile without decomposition.

\section{Benzoinam. $\mathrm{C}_{56} \mathrm{~N}_{2} \mathrm{H}_{24} \mathrm{O}_{2}$.}

When a mixture of ammonia, alcohol, and benzoine, is left for several months in a closed bottle, there are formed many products, among which are the preceding compound, benzoinamide, and a new substance, benzoinam, which is with difficulty purified. It forms small white needles, the formula of which is $\mathrm{C}_{56} \mathrm{~N}_{2} \mathrm{H}_{24}$ $\mathrm{O}_{2}$. It is evidently formed by the coalescence of 2 eq. benzoine (itself produced by the coalescence of 2 eq. hyduret of benzoyle), while ammonia is taken up, and water separated. Thus 2 eq. benzoine $=\mathrm{C}_{56} \mathrm{H}_{24} \mathrm{O}_{8}$, plus 2 eq. ammonia $\mathrm{N}_{2} \mathrm{H}_{6}$, and minus 6 eq. water, $\mathrm{H}_{6} \mathrm{O}_{6}$, are equal to $\mathrm{C}_{56} \mathrm{~N}_{2} \mathrm{H}_{24} \mathrm{O}_{2}$.

Benzoinam dissolves in acids, and is precipitated by alkalies; it seems, therefore, to have the characters of a weak base.

\section{Benzile. $\mathrm{C}_{28} \mathrm{H}_{10} \mathrm{O}_{4}$.}

SrN. Benzoyle.-It is formed by the action of chlorine gas on melted benzoine, or by heating benzoine with nitric acid. When cold the mass is boiled with alcohol, which on cooling deposits 
benzile in crystals, which are yellow six-sided prisms; insoluble in water, soluble in alcohol and ether, melting at $195^{\circ}$, and volatile without decomposition. An alcoholic solution of potash dissolves it with a violet colour, and converts it into benzilio acid. Benzile has the composition in 100 parts of the supposed radical benzoyle, but there is good reason to believe its equivalent to be twice as high.

\section{Benzilic Acid. $\mathrm{C}_{28} \mathrm{H}_{11} \mathrm{O}_{3}+\mathrm{HO}$.}

Formed when benzile or benzoine is dissolved in a hot alcoholic solution of potash, and boiled until the violet colour at first produced has disappeared, and is no longer restored by a fresh portion of potash. To the boiling solution of benzilate of potash hydrochloric acid is added in excess, and on cooling the benzilic acid is deposited in colourless brilliant crystals, fusible at $248^{\circ}$, not volatile, but yielding, when heated, benzoic acid, and purple vapours. Sulphuric acid dissolves it with a bright crimson colour, Its formation is explained as follows :-1 eq. of benzile takes up 2 eqs. of water, one of which is incorporated in the acid; while the other is replaceable by bases. $\mathrm{C}_{28} \mathrm{H}_{10} \mathrm{O}_{4}+$ $2 \mathrm{H} \mathrm{O}=\mathrm{C}_{28} \mathrm{H}_{11} \mathrm{O}_{5}+\mathrm{H} \mathrm{O}$. Benzilate of potash forms large transparent crystals, soluble in water and alcohol.

26. Azobenzoide- $-\mathrm{C}_{84} \mathrm{H}_{38} \mathrm{~N}_{5}$ ? Obtained by adding ammonia to the oil produced when bitter almonds are distilled per descensum, and dissolving away by means of ether all other products. A white powder, decomposed by heat. Its formula is doubtful.

27. Cyanobenzile.-Formed when an alcoholic solution of benzile is warmed with $\frac{1}{3}$ of its volume of concentrated hydrocyanic acid. It is deposited in large transparent crystals, the composition of which is not yet known.

\section{Hyduret of Sulphobenzoyle. $\mathrm{C}_{14} \mathrm{H}_{5} \mathrm{~S}_{2}, \mathrm{H}$.}

One vol. of crude essence of bitter almonds is dissolved in 8 or 10 of alcohol, and gradually mixed with 1 vol. of hydrosulphuret of ammonia. After a time, the mixture deposits a fine white powder, formed of grains smaller than those of starch, which give to the fingers a very persistent odour of garlic. It is insoluble in water and alcohol. Ether liquifies it, but a few drops of alcohol restore its solid form. It may be considered as the hyduret of a new radical, in which the oxygen of benzoyle has been replaced by sulphur. When heated it melts, and if now allowed to cool, forms first a transparent plastic mass, and afterwards a brittle glass. If kept melted for some time, it crystallises, but is now altered. 


\section{Stilbene. $\mathrm{C}_{28} \mathrm{H}_{12}$.}

When the preceding compound is strongly heated, it gives off a large quantity of sulphuretted hydrogen, and a little of a liquid, apparently bisulphuret of carbon. Continuing the heat, there distil over, first a substance crystallising in pearly scales, stilbene; and later, a compound, crystallising in needles, called by Laurent, the discoverer, sulphessale. To obtain the stilbene pure, the first crystals are dissolved in boiling alcohol, which leaves undissolved the other body, and on cooling deposits stilbene in tables. These being dissolved in hot ether, form, by slow evaporation, remarkably fine crystals, with the pearly lustre of stilbite; hence the name. It is fusible and volatile, and combines with chlorine and bromine. Nitric acid decomposes it, giving rise to several new products. Chromic acid attacks it with violence, and reproduces hyduret of benzoyle.

Chloride of stilbene is formed when chlorine is passed through melted stilbene. It appears in two isomeric modifications, $a$ and $b$, both of which have the formula $\mathrm{C}_{28} \mathrm{H}_{12}, \mathrm{Cl}_{2}$, but crystallise in different forms. By the action of a boiling alcoholic solution of potash, each loses 1 eq. of hydrochloric acid (or its elements), and thus they produce two isomeric modifications of a new compound $\mathrm{C}_{28}\left\{\begin{array}{l}\mathrm{H}_{11} \\ \mathrm{Cl} \text {, which Laurent calls Chlostilbase; }\end{array}\right.$ and in which 1 eq. of the hydrogen of stilbene is replaced by chlorine. One of these is called Chlostilbase $a$, the other Chlostilbase $b$. Both are oily liquids, but they are distinguished by the action of bromine, which combines with both, producing again two isomeric compounds, both crystallisable, but in entirely different forms. Their empirical formulæ are, $a \mathrm{C}_{28}\left\{\begin{array}{l}\mathrm{H}_{11} \\ \mathrm{Cl}\end{array}\right.$ and $b \mathrm{C}_{28}\left\{\begin{array}{l}\mathrm{H}_{11} \\ \mathrm{Cl}+\mathrm{Br}_{2} .\end{array}\right.$ ference between these two compounds, but there must be a difference. It is, perhaps, that the particular atom of hydrogen replaced by chlorine is not the same in each, or it may be, that while one of them is represented by the formula above given, the other is $\left.\mathrm{C}_{28} \mathrm{Cl}_{\mathrm{Br}}^{\mathrm{H}_{10}}\right\}$, H $\mathrm{Br}$. Or, again, one of them may be $\left.\mathrm{C}_{28} \begin{array}{r}\mathrm{H}_{10} \\ \mathrm{Br}_{2}\end{array}\right\}, \mathrm{H} \mathrm{Cl}$. All these expressions, and many more, come under the same general formula.

Along with chloride of stilbene is formed another compound, chloride of chlostilbase, analogous to these bromides of chlostilbase. It is a crystallisable solid: formula, $\mathrm{C}_{2}\left\{\begin{array}{l}\mathrm{H}_{11} \\ \mathrm{Cl}\end{array}\right.$ 
Bromine combines with stilbene, forming the bromide of stilbene, $\mathrm{C}_{28} \mathrm{H}_{12}, \mathrm{Br}_{2}$, which is a white powder.

When stilbene is boiled with nitric acid, it forms several compounds not yet fully examined. Among these are Nitrostilbase, Nitrostilbèse, and Nitrostilbic acid. The latter, according to Laurent, is $\mathrm{C}_{28} \mathrm{H}_{11} \mathrm{~N} \mathrm{O}_{14}$.

30. Hyduret of Sulphazobenzoyle, $\left(\mathrm{C}_{14} \mathrm{H}_{5} \mathrm{~S}_{\frac{4}{3}} \mathrm{~N}_{\frac{2}{3}}\right) \mathrm{H}$, or rather $\mathrm{C}_{42} \mathrm{H}_{18} \mathrm{~S}_{4} \mathrm{~N}_{2}$, is generally formed along with hyduret of sulphobenzoyle. It seems to be hyduret of benzoyle, in which the 2 eqs. oxygen of the benzoyle are replaced partly by sulphur, partly by nitrogen. Besides these compounds, Laurent has described a hydrosulphuret of azobenzoyle, with the strange formula $\mathrm{C}_{14} \mathrm{H}_{6} \mathrm{~S} \mathrm{~N}_{\frac{3}{4}}$. Ought it not rather to be $\mathrm{C}_{14} \mathrm{H}_{6} \mathrm{~S} \mathrm{~N}$, or $\mathrm{C}_{56} \mathrm{H}_{24} \mathrm{~S}_{4} \mathrm{~N}_{3}$ ?

\section{Bromide of Benzole. $\mathrm{C}_{12} \mathrm{H}_{6} \mathrm{Br} 6$.}

This compound is formed as a white insoluble powder, when bromine acts on benzole. An alcoholic solution of potash removes hydrobromic acid (or its elements), and causes the separation of a white crystalline body, $\mathrm{C}_{12}\left\{\begin{array}{l}\mathrm{H}_{3} \\ \mathrm{Br}_{3}\end{array}\right.$ which Laurent calls bromobenzinise; and which is formed by substitution of bromine for half the hydrogen of benzole. The bromide of benzole may, therefore, be $\mathrm{C}_{22}\left\{\begin{array}{l}\mathrm{H}_{3} \\ \mathrm{Br}_{3}\end{array}+3 \mathrm{H} \mathrm{Br}\right.$.

32. Hydrocyanate of benzoine. $-\mathrm{C}_{46} \mathrm{~N}_{2} \mathrm{H}_{18} \mathrm{O}_{4}$, is formed as a light crystalline matter, when oil of bitter almonds is mixed with one-fourth its volume of dry hydrocyanic acid, and warmed with its own volume of aqua potassæ, Sp. G. 1·25, diluted with six parts of alcohol. It arises from the action of 3 eqs. hyduret of benzoyle and 2 eqs. hydrocyanio acid: $3\left(\mathrm{C}_{24} \mathrm{H}_{6} \mathrm{O}_{2}\right)+2\left(\mathrm{C}_{2} \mathrm{NH}\right)=$ $\mathrm{C}_{46} \mathrm{~N}_{2} \mathrm{H}_{18} \mathrm{O}_{4}+2 \mathrm{HO}$.

33. Hydrocyanate of benzile, $\mathrm{C}_{14} \mathrm{H}_{5} \mathrm{O}_{2}+\mathrm{C}_{2} \mathrm{NH}=\mathrm{C}_{16} \mathrm{~N} \mathrm{H}_{6}$ $\mathrm{O}_{2}$, is formed by direct combination when a hot alcoholic solution of benzile is mixed with an equal bulk of anhydrous hydrocyanic acid. It forms large colourless crystals. Possibly the body called Cyanobenzile mentioned above, No. 27, may be identical with this compound.

The above is but a brief and imperfect notice of the numerous compounds which have been obtained from oil of bitter almonds by the various transformations and decompositions above mentioned. Probably no substance has yielded so rich a harvest of discovery as this remarkable oil, and for our knowledge of the new products we are indebted to Liebig and Wöhler, Mitscherlich, 
Zinin, Rochleder, but above all, to Laurent, who has discovered by far the greater part of them.

In the recent work of Gerhardt on Organic Chemistry, the reader will now find the most complete account of all these compounds, as well as of many others which are not here inserted, for want of space, and also because those which have been noticed include the chief products of the principal reactions, and will suffice to give a notion of the principles which regulate such transformations.

The study of these compounds has shed great light, and will, in future, shed much more, on the origin of the more complex organic molecules. In several instances, we see 2 or 3 eqs. of hyduret of benzoyle coalescing to yield 1 eq. of a new and more complex substance, either with or without a change in the relative proportion of oxygen and hydrogen, and with or without the addition of nitrogen. According to Laurent, compounds exist in which 4, 6, and even 9 eqs. of the hyduret have coalesced.

It was mentioned, in a former part of this work, that our power of artificially forming bodies which occur as products of organic life, was limited chiefly to the decomposition or splitting up of complex molecules, such as those of sugar, salicine, uric acid, or fibrine, into less complex molecules.

But, although this is still true of the actual products of organic life which have been imitated in the laboratory, the facts above described show that, under certain circumstances, we can, from the less complex molecules, build up more complex ones, so as to produce, if not the actual products of organic life, yet substances entirely analogous to them, and equally complex.

Thus, the artificial bases, amarine, lophine, and benzoinam, have equivalents higher than those of the vegetable alkalies, morphia and quinine; and we have almost demonstrative evidence that these three compounds arise from the reverse process to that which yields urea, formic, oxalic, and salicylic acids. The latter are obtained by processes of oxidation, which tend to split up the more complex molecules into less complex ones: the former are, on the contrary, built up out of the less complex molecules, by processes of reduction. There are other cases, of recent discovery, which establish the same principle.

Now we know, that the distinguishing feature of vegetable life is the power of reduction possessed by plants, which easily deprive carbonic acid of its oxygen, an operation requiring a prodigious chemical force.

We are, therefore, warranted in concluding that we shall in time succeed, by means of the various processes of reduction known to chemists, (and possibly of new methods also), in pro- 
ducing, for example, the vegetable alkalies, morphine, quinine, and strychnine.

This consideration gives to the compounds of the Benzoyle series, and their derivatives, a high degree of interest, far surpassing that which would attach to them as a mere list of new compounds, each possessing a distinct composition and distinct properties.

\section{BENZOIC ALCOHOL.}

The hyduret of benzoyle may be considered as the aldehyde of benzoic acid, bearing to that acid the same relation as aldehyde does to acetic acid. This has been already alluded to ; but some additional facts have rendered this view more probable. We have already seen that the hyduret agrees with aldehydes in general in forming crystalline compounds with the bisulphites of potash or ammonia.

Now Cannizzaro has shown that the oily liquid which separates when the hyduret is acted on by an alcoholic solution of potash, has the formula $\mathrm{C}_{14} \mathrm{H}_{8} \mathrm{O}_{2}$, which has the same relation to the hyduret as alcohol has to aldehyde, and he therefore calls it benzoic alcohol. It is a colourless oily liquid, heavier than water, and highly refractive, boiling about $400^{\circ} \mathrm{F}$. Its rational formula, on the supposition that it is an alcohol, will be $\mathrm{C}_{24} \mathrm{H}_{7} \mathrm{O}, \mathrm{HO}$, and the relations of the acid, the aldehyde and the alcohol, may be thus compared with those of acetic acid, aldehyde, and alcohol.

\section{Benzoyle series.}

$\begin{array}{ll}\text { Aldehyde } & \mathrm{C}_{14} \mathrm{H}_{6} \mathrm{O}_{2} \\ \text { Acid } & \mathrm{C}_{14} \mathrm{H}_{6} \mathrm{O}_{4}\end{array}$
Acetyle series.

$\mathrm{C}_{4} \mathrm{H}_{4} \mathrm{O}_{2}$

$\mathrm{C}_{4} \mathrm{H}_{4} \mathrm{O}_{4}$

$\mathrm{C}_{4} \mathrm{H}_{6} \mathrm{O}_{2}$

This relation is not found in the formula only; for benzoic alcohol is converted into hyduret of benzoyle, its aldehyde, by oxidising agents, just as alcohol is into aldehyde; and the aldehyde, by oxidation, passes into the acid in both series.

It. would appear, therefore, that hyduret of benzoyle possesses two different kinds of characters. We have seen that it exhibits those of a compound of hydrogen with the radical benzoyle, which may be made also to combine with chlorine and other bodies. And on the other hand, it has the composition and chemical relations of an aldehyde, and in this view, is not a compound of hydrogen with benzoyle, $\mathrm{H}+\mathrm{C}_{14} \mathrm{H}_{5} \mathrm{O}_{2}$, but the hydrated oxide of a different radical, $+\left(\mathrm{C}_{14} \mathrm{H}_{5}\right) \mathrm{O}, \mathrm{H} \mathrm{O}$, the radical here being a carbo-hydrogen $\mathrm{C}_{14} \mathrm{H}_{5}$, analogous to acetyle $\mathrm{C}_{4} \mathrm{H}_{3}$.

It is important to bear in mind, that a compound may not only be represented by two different formulæ, but may exhibit in its 
chemical relations two sets of characters corresponding to the different formulæ.

Gerhardt calls the benzoic alcohol hydrate of toluenyle, the latter name, toluenyle, being that of a supposed radical, $\mathrm{C}_{14} \mathrm{H}_{7} \mathrm{O}$. It will be seen that his formula is the same as that given above for the alcohol, namely $\mathrm{C}_{14} \mathrm{H}_{7} \mathrm{O}, \mathrm{HO}$; only he regards it as the hydrate of an oxygenated radical, while Cannizzaro considers it as the hydrated oxide of the carbo-hydrogen $\mathrm{C}_{14} \mathrm{H}_{7}$ analogous to ethyle, and homologous with phenyle; thus $\left(\mathrm{C}_{14} \mathrm{H}_{7}\right) 0$, HO. So that two different views may be taken of the constitution of the benzoic alcohol as well as of its aldehyde.

\section{APPENDIX TO BENZOYLE.}

\section{Amygdaline. $\mathrm{C}_{40} \mathrm{~N} \mathrm{H}_{27} \mathrm{O}_{22}$.}

Is found in bitter almonds, in the leaves of the cherry laurel, and probably in the kernels of all the bitter species of amygdalus and prunus, as the peach and plum. To obtain it, bitter almonds are pounded and forcibly pressed between warm iron plates to remove the fat oil (oil of almonds). The marc or residue is boiled with alcohol of 94 per cent., and the tinctures distilled off in the water-bath to the consistence of syrup. This liquid, which contains amygdaline and sugar, is diluted with water, mixed with yeast, and set aside. When the fermentation is over, the whole is filtered, and again evaporated to a syrup, which being mixed with a large excess of cold alcohol (of 94 per cent.), or with half its bulk of ether, deposits the amygdaline as a white crystalline powder. This is pressed in folds of bibulous paper, and finally purified by repeated erystallisation from boiling alcohol. It forms crystalline scales very soluble in water, very sparingly soluble in cold alcohol, but more soluble in hot alcohol. It has a bitter taste. When heated it emits an odour like that of May blossom, and leaves a bulky coal.

When distilled with nitrio acid, or other oxidising agents, it is resolved into ammonia, hyduret of benzoyle, benzoic acid, formic acid, and carbonic acid. Caustic alkalies convert it into ammonia and amygdalinic acid: permanganate of potash converts it into cyanate and benzoate of potash.

\section{Amygdalinic Acid. $\mathrm{C}_{40} \mathrm{H}_{26} \mathrm{O}_{24}+\mathrm{HO}$.}

Prepared by boiling amygdaline with baryta as long as ammonia is given off, and then removing the baryta from the soluble amygdalinate of that base by sulphuric acid. By eva- 
poration it yields a transparent amorphous mass, which has a very pleasant acid taste. Nitric acid, and other oxidising agents, convert it into hyduret of benzoyle, with formic and benzoic acid. Its salts are almost all soluble; their formula is $\mathrm{C}_{40} \mathrm{H}_{26}$ $\mathrm{O}_{24}+\mathrm{MO}$.

\section{Distilled Water of Bitter Almonds.}

Expressed bitter almonds are made into a thin cream with water, and this distilled in the heat of a chloride of calcium bath, till a quantity of water has passed over equal in weight to the almonds before being pressed. The distilled water is milky, from suspended oil of bitter almonds, and smells both of hyduret of benzoyle and of hydrocyanic acid. When freshly prepared, it contains little more than 1 grain of hydrocyanic acid per ounce; but its strength diminishes by keeping, and as it is difficult to obtain it of uniform strength even when fresh, it is not a good form of administering hydrocyanic acid. It is remarkable that nitrate of silver does not detect the hydrocyanio acid, unless ammonia is added.with the nitrate, and after a time neutralised by nitric acid. It is used in medicine, especially on the Continent, and is poisonous.

\section{Laurel Water.}

Obtained by distilling two parts of fresh leaves of prunus laurocerasus with water till three parts have passed over. It exactly resembles the preceding water, and is equally uncertain, and equally poisonous.

\section{THEORY OF THE FORMATION OF HYDURET OF BENZOYLE FROM BITTER ALMONDS.}

Bitter almonds contain, like sweet almonds, a large quantity of an albuminous or caseous matter, called emulsine or synaptase, along with abundance of a mild fat oil, the oil of almonds, very similar to olive oil. But in addition to these, the bitter almonds contain 4 or 5 per cent. of amygdaline, which is not present in sweet almonds.

Now, if the amygdaline be removed by boiling alcohol, the residue, when distilled with water, does not yield a trace of the volatile oil of bitter almonds. Again, if the residue of the bitter almonds, after the fat oil has been pressed out, be heated to suoh a point as to coagulate the emulsine, before water is added, the distillation also yields no volatile oil, even although the amygdaline be present.

These facts prove that the production of the volatile oil of bitter almonds depends on the presence, first, of amygdaline; secondly. 
of soluble emulsine; and that it is the result of the mutual action of these bodies on each other. This is further demonstrated by the fact, that if amygdaline be placed in contact with the emulsine or synaptase of sweet almonds, and water, distillation of the mixture now yields the oil abundantly.

When the solution of 10 parts of amygdaline in 100 of water is added to a solution of 1 part of synaptase in 10 of water, mutual decomposition at once takes place: the liquid acquires the odour of hyduret of benzoyle and of hydrocyanic acid, and when distilled yields the crude oil of bitter almonds, which is a mixture of these two compounds. The residue of the distillation contains sugar in such quantity, that it is probable the elements of the synaptase have contributed to form it; and when this sugar is destroyed by fermentation, a fixed acid is found. If the synaptase has been coagulated, it has not the slightest action on amygdaline.

When the expressed bitter almonds are moistened with water, the very same reaction occurs; and if enough water be present to dissolve the oil as it is formed, the whole amygdaline disappears in a short time. But if the expressed almonds be thrown into boiling water, the synaptase coagulates; and can then produce no change in the amygdaline. To obtain the full proportion of oil, 1 part of expressed almonds should be macerated for twenty-four hours with 20 parts of water at about $102^{\circ}$, and then distilled.

100 parts of amygdaline produce 47 of crude oil, and these 47 parts of crude oil contain almost exactly 6 of anhydrous hydrocyanic acid; so that 17 grains of amygdaline dissolved in $1 \mathrm{oz}$. of emulsion of sweet almonds, yields a mixture containing 1 grain of dry hydrocyanic acid, and consequently of the same strength as the distilled water professes to be. This mixture has, besides, the advantage of containing the hyduret of benzole present in the distilled water, to which is perhaps owing the superiority of the distilled water of bitter almonds on laurel leaves over mere diluted hydrocyanic acid, a superiority which, according to the continental physicians, is very decided and obvious. The above simple recipe, given by Liebig and Wöhler, is admirably adapted for extemporaneous use, and the mixture ought never to be made in a larger quantity at a time, as, like the distilled water, it alters by keeping.

In the above remarkable decomposition, we have a very beautiful example of a metamorphosis in which the elements of two bodies take a share; but as the whole of the products are not yet exactly known, and even the composition of the emulsine or synaptase is uncertain, we cannot explain the whole change with 
precision. We know, however, that from 1 eq. amygdaline, $\mathrm{C}_{40} \mathrm{~N}$ $\mathrm{H}_{27} \mathrm{O}_{22}$, the following compounds may be derived:-

1 eq. hydrocyanic acid . . . . . $\mathrm{C}_{2} \mathrm{NH}$

2 eqs. hyduret of benzoyle . . . . . $\mathrm{C}_{28} \mathrm{H}_{12} \mathrm{O}_{4}$

$\frac{1}{2}$ eq. sugar . $. \quad . \quad . \quad . \quad . \quad \mathrm{C}_{6} \mathrm{H}_{5} \mathrm{O}_{5}$

2 eqs. formic acid $\quad . \quad$. $\quad . \quad$. $C_{4} \mathrm{H}_{2} \mathrm{O}_{6}$

7 eqs. water . . . . . $\mathrm{H}_{7} \mathrm{O}_{7}$

1 eq. amygdaline . . . . . . $\overline{\mathrm{C}_{40} \mathrm{NH}_{27} \mathrm{O}_{22}}$

Also, 1 eq. amygdaline acid, $\mathrm{C}_{40} \mathrm{H}_{26} \mathrm{O}_{24}$, may yield :-

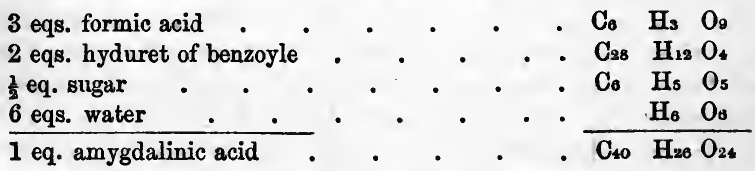

Now, we can trace all these products among the results of this transformation; and, it is probable, not only that there is more sugar than can be accounted for by the amygdaline, but also that other products, not yet known, are formed: as, for example, the fixed acid above alluded to. The emulsine or synaptase, which produces this remarkable change in amygdaline, in which it itself participates, contains nitrogen, is soluble in water, coagulable by heat, and in short very analogous both to albumen and caseine, along with which we shall again notice it. In the almond it appears to be accompanied by albumen.

We have seen, above, that the assumption of the existence of the radical benzoyle brings a number of compounds into a more easily understood form, and very materially aids the memory by enabling us to classify these compounds as analogous to others better known. Other views may be taken of this series of compounds: for example, according to Dumas, hyduret of benzoyle may be represented as a compound of benzoic acid with a carbohydrogen : $2 \mathrm{C}_{44} \mathrm{H}_{5} \mathrm{O}_{3}+\left(\mathrm{C}_{4} \mathrm{H}_{5}\right) \mathrm{H}_{3}$, benzoic acid being the teroxide and the carbo-hydrogen the terhyduret of the body, $\mathrm{C}_{14} \mathrm{H}_{5}$ : for $2\left(\mathrm{C}_{14} \mathrm{H}_{5}\right) \mathrm{O}_{3}+\left(\mathrm{C}_{14} \mathrm{H}_{5}\right) \mathrm{H}_{3}=\mathrm{C}_{42} \mathrm{H}_{18} \mathrm{O}_{6}=3$ $\left(\mathrm{C}_{14} \mathrm{H}_{6} \mathrm{O}_{2}\right)=3 \mathrm{Bz} \mathrm{H}$. The same view might be extended to some of the other compounds of benzoyle, but it is complex, and cannot well be applied to benzamide, hydrobenzamide, and several others. Again, according to Mitscherlich, benzoic acid is $\mathrm{C}_{12} \mathrm{H}_{6}:+2 \mathrm{C} \mathrm{O}_{2}$; that is, benzole plus 2 eqs. carbonic acid; while a dry benzoate would contain, united to the base, the hypothetical body benzide, $\mathrm{C}_{22} \mathrm{H}_{5}$, and the equally hypothetioal 
anhydrous oxalic acid, $\mathrm{C}_{2} \mathrm{O}_{3}$. These views appear both to be in all respects inferior to that which we have adopted, and which must be retained, until a better shall be proposed. If I understand rightly some expressions in a paper by Laurent, that chemist considered hyduret of benzoyle, $\mathrm{C}_{14} \mathrm{H}_{6} \mathrm{O}_{2}$, as the oxide of a radical benzene; but it does not appear whether it is $\left(\mathrm{C}_{14} \mathrm{H}_{6}\right)+\mathrm{O}_{2}$, or $\left(\mathrm{C}_{14} \mathrm{H}_{6} \mathrm{O}\right)+\mathrm{O}$. The term oxide of benzene, however, may be applied to one of the isomeric modifications of the hyduret.

It has already been stated that benzoic acid is the startingpoint of a series of homologous acids. One of these, toluylic acid, will be described further on, after the cuminic acid, and under the balsam of tolu, with both of which it is connected; but a third, namely, the cuminic acid, agrees still more closely with benzoic acid, and especially in the existence of a compound, analogous to and homologous with hyduret of benzoyle. This is the oxygenated oil of cumine, $\mathrm{C}_{20} \mathrm{H}_{12} \mathrm{O}_{2}$, which may be viewed as hyduret of cumyle, $\mathrm{C}_{20} \mathrm{H}_{11} \mathrm{O}_{2}, \mathrm{H}$. When oxidised by nitric acid, it yields cuminio acid, $\mathrm{C}_{20} \mathrm{H}_{12} \mathrm{O}_{3}, \mathrm{H} \mathrm{O}$ or $\mathrm{C}_{20} \mathrm{H}_{12} \mathrm{O}_{4}$, which is crystalline and volatile, like benzoio acid. When heated with bases, it yields cumole, $\mathrm{C}_{18} \mathrm{H}_{12}$, homologous with benzole; which, like benzole, also yields substitution-products, as in the fourth column of the table, p. 141, in which chlorine, bromine, nitrous acid, and amide are substituted for part of its hydrogen. Details are unnecessary, as the analogy of properties with the corresponding compounds of the benzoic series is remarkable, and is the chief point of interest. Cumole, however, is an ingredient of the crude oil of cumine, and is best obtained from that source. It is also, like benzole, toluole, and xylole, found in the oil of tar.

\section{Salicyle. $\mathrm{C}_{14} \mathrm{H}_{5} \mathrm{O}_{4}=\mathrm{Sa}$.}

This is the hypothetical radical of a remarkable series of compounds; and, as such, belongs to the same group as benzoyle. Its most interesting compound is the hyduret of salicyle, which we shall therefore first consider.

\section{Hyduret of Salicyle. $\mathrm{C}_{14} \mathrm{H}_{5} \mathrm{O}_{4}, \mathrm{H}=\mathrm{Sa} \mathrm{H}$.}

SrN. Salicylous Acid.-This compound is found as the chief ingredient in the essence of meadowsweet, that is, the essential oil obtained by distilling the flowers of spiraa ulmaria with water. It is probable that, like the essence of bitter almonds, it is formed by the metamorphosis of a compound or compounds 
present in the flowers. The crude essence is distilled with aqua potassæ, which combines with the hyduret, and an oil distils over which seems to be a carbo-hydrogen. The salt of potash being now redistilled with a slight excess of dilute sulphuric acid, yields the pure hyduret of salicyle.

It may also be obtained by distilling one part of salicine, one part of bichromate of potash, two and a half of oil of vitriol, and twenty of water, together. The salicine is dissolved in part of the water, and the acid diluted with the rest. The mixture is then made in a retort, and after the effervescence which takes place is over, the whole is distilled, and yields the hyduret, to the amount of one-fourth of the salicine employed.

Hyduret of salicyle is an oily colourless liquid, having a fragrant aromatic odour and a burning taste. Its Sp. G. is $1 \cdot 1731$, and it boils about $380^{\circ}$. With chlorine and bromine it forms new substitution-compounds. With bases it forms salicylurets, water being separated. $\mathrm{H} \mathrm{Sa}+\mathrm{MO}=\mathrm{H} \mathrm{O}+\mathrm{M} \mathrm{Sa}$, so that it has the characters of an acid.

Salicyluret of ammonium, $\mathrm{Sa}, \mathrm{N} \mathrm{H}_{4}$, is formed when concentrated ammonia is poured upon hyduret of salicyle. It is a yellow solid, having a faint odour of roses. When moist, this salt is decomposed spontaneously, becoming black, and giving off ammonia and an odour like that of roses. With dry ammoniacal gas, hyduret of salicyle forms the compound $3 \mathrm{Sa}$ $\mathrm{H}+2 \mathrm{~N} \mathrm{H}_{3}$.

Salicylimide, $\mathrm{C}_{42} \mathrm{~N}_{2} \mathrm{H}_{18} \mathrm{O}_{6}$, is formed when caustic ammonia is added, drop by drop, to a solution of 1 vol. hyduret of salicyle in 3 vol. alcohol, and the small yellow crystals which first formed are dissolved by a gentle heat. On standing, salicylimide appears in the form of golden yellow brilliant prisms. It is formed from 3 eqs. hyduret of salicyle and 2 eqs. of ammonia, by the separation of 6 eqs. water, $3\left(\mathrm{C}_{14} \mathrm{H}_{6} \mathrm{O}_{4}\right)+2 \mathrm{~N} \mathrm{Hs}-6 \mathrm{H} \mathrm{O}=$ $\mathrm{C}_{42} \mathrm{~N}_{2} \mathrm{H}_{18} \mathrm{O}_{6}$. As it is no longer soluble in the liquid from which it was first deposited, it is probable that the yellow salt first formed, which dissolved in the alcohol by aid of a gentle heat, was salicyluret of ammonium, which, by excess of ammonia, was converted into salicylimide.

The salts of the hyduret of salicyle or hydrosalicylic acid, are constituted, for the most part, according to the formula Sa M. The potassium salt $\mathrm{Sa} \mathrm{K}$, when exposed to the air in a moist state, becomes first green, then black. When the change is complete, water dissolves acetate of potash, and lesves a black matter, melanic acid, $\mathrm{C}_{10} \mathrm{H}_{4} \mathrm{O}_{5}: 1$ eq. of salicyluret of potassium, 2 eqs. water, and 3 eqs. oxygen, contain the element of 1 eq. acetate of potash, and 1 eq. melanic acid, $\mathrm{C}_{14} \mathrm{H}_{5} \mathrm{O}_{4}, \mathrm{~K}+$ 
$2 \mathrm{H} \mathrm{O}+\mathrm{O}_{3}=\mathrm{C}_{4} \mathrm{H}_{3} \mathrm{O}_{4}, \mathrm{~K}+\mathrm{C}_{10} \mathrm{H}_{4} \mathrm{O}_{5}$. Melanic acid combines with bases.

Ettling and Stenhouse have shown that when the salicyluret of copper, $\mathrm{Cu} \mathrm{Sa}=\mathrm{C}_{14} \mathrm{H}_{3} \mathrm{O}_{4}+\mathrm{Cu}$, and the benzoate of copper, $\mathrm{Cu} \mathrm{O}, \mathrm{C}_{14} \mathrm{H}_{5} \mathrm{O}_{3}$, which is isomeric with it, are distilled, several new products are formed. Among these are salicylic acid and two crystalline neutral compounds.

Parasalicyle is a product of the action of heat on salicyluret (salicylite) of copper. Its formula is $\mathrm{C}_{14} \mathrm{H}_{5} \mathrm{O}_{3}$, and it has the composition of anhydrous benzoic acid, or of salicylous acid, (hyduret of salicyle viewed as an oxygen acid), as it exists in its salts. The copper salt from which it is formed may be represented either as $\mathrm{C}_{14} \mathrm{H}_{5} \mathrm{O}_{4}+\mathrm{Cu}$, or as $\mathrm{Cu} \mathrm{O}, \mathrm{C}_{14} \mathrm{H}_{5} \mathrm{O}_{3}$. Parasalicyle is fusible and volatile, and only attacked by strong acids.

When benzoate of copper is distilled, there is formed, besides salicylic acid, a neutral volatile crystalline compound, which contains 1 eq. oxygen less than parasalicyle, and the formula of which is consequently $\mathrm{C}_{14} \mathrm{H}_{3} \mathrm{O}_{2}$. It is therefore isomeric with benzile, and has the composition of the radical benzoyle. Indeed, as it yields benzoic acid when acted on by potash, while benzile yields benzilic acid, it may very probably be that radical. It has not, however, been named by Ettling, who discovered it.

\section{Salicylic Acid. ( $\left.\mathrm{C}_{14} \mathrm{H}_{5} \mathrm{O}_{4}\right) \mathrm{O}, \mathrm{H} \mathrm{O}=\mathrm{Sa} \mathrm{O}, \mathrm{HO}=\mathrm{Sa} \mathrm{O}_{2}, \mathrm{H}$.}

Formed when hyduret of salicyle is heated with hydrate of potash till the mass loses its brown colour. Hydrogen is given off, and salicylate of potash is formed. Hydrochloric acid, added to the solution of this salt, causes the deposition of crystals of salicylic acid.

The same acid is formed. when coumarine, the stearoptene or camphor of the tonka bean, is acted on by caustic potash. Moreover the essential oil of winter-green, or Gaultheria procumbens, appears, by the researches of Cahours and Gerhardt, to be the salicylate of oxide of methyle. The acid, therefore, is most readily obtained, by gently heating this oil with a slight excess of potash, and afterwards adding an acid, when the salicylic acid, being very sparingly soluble, is deposited. It is easily purified by recrystallisation, and sometimes forms crystals one or two inches in length.

Salicylic acid erystallises in tufts of slender prisms, very like benzoic acid. It may be sublimed without decomposition. The formation of this acid from hyduret of salicyle is very simple: $\mathrm{C}_{4} \mathrm{H}_{5} \mathrm{O}_{4}, \mathrm{H}+\mathrm{K} \mathrm{O}, \mathrm{H} \mathrm{O}=\mathrm{C}_{14} \mathrm{H}_{5} \mathrm{O}_{5}, \mathrm{KO}+\mathrm{H}_{2}$. When 
the salicylate of potash is decomposed by an acid, the salicylic acid takes up 1 eq. of basic water, and separates as $\mathrm{C}_{\mathbf{1 4}} \mathrm{H}_{5} \mathrm{O}_{5}$, $\mathrm{H} \mathrm{O}$, or $\mathrm{C}_{14} \mathrm{H}_{5} \mathrm{O}_{6}, \mathrm{H}$. The decompositions of this acid are very interesting, connecting it with several other series of organio compounds.

When it is acted on by nitric acid, it is first converted into indigotic acid, or nitrosalicylic acid, $\mathrm{C}_{24} \mathrm{H}_{4} \mathrm{~N} \mathrm{O}_{9}, \mathrm{H} \mathrm{O}$, along with other products; and by the continued action of nitric acid, there is finally produced the remarkable bitter acid, called carbazotic, nitropicric or nitrophenisic acid, $\mathrm{C}_{12} \mathrm{H}_{2} \mathrm{~N}_{3} \mathrm{O}_{13}, \mathrm{HO}$. Both these acids are formed from indigo by the action of nitric acid.

When salicylic acid is distilled with caustic baryta, it yields carbonate of baryta, and a heavy oily liquid, which is the acid known as carbolic acid (Runge), and hydrate of phenyle (Laurent), the formula of which is $\mathrm{C}_{12} \mathrm{H}_{6} \mathrm{O}_{2}=\mathrm{C}_{12} \mathrm{H}_{5} \mathrm{O}, \mathrm{H} \mathrm{O}$. The production of carbolic acid from salicylic acid is easily understood; it is, in fact, analogous to that of benzine from benzoic acid. $\mathrm{C}_{14} \mathrm{H}_{5} \mathrm{O}_{5}, \mathrm{HO}+2 \mathrm{BaO}=2\left(\mathrm{BaO}, \mathrm{C} \mathrm{O}_{2}\right)+\mathrm{C}_{12} \mathrm{H}_{6} \mathrm{O}_{2}$. As the action with baryta is so violent as to destroy a great part cf the acid, it is found better to heat rapidly salicylic acid mixed with powdered glass, when it is resolved into carbolic and carbonic acids.

Now it is very remarkable, that carbolic acid, which is one of the chief ingredients of the oil of coal-tar, a product, therefore, of destructive distillation, is also converted by the action of nitric acid into nitropicric acid. This would indicate that when salicylic acid is acted on by nitric acid, it yields, first, some compound containing the same radical as carbolic and nitropicric acid, and that this is further oxidised into the latter acid. We shall hereafter see that the probable origin of carbolic acid is benzole, $\mathrm{C}_{22} \mathrm{H}_{6}$, from which, by substitution and oxidation, carbolic, and nitropicric acids, and a whole series of compounds, may be formed. Benzole is itself the hyduret of the radical phenyle, $\mathrm{C}_{12} \mathrm{H}_{5}$. The hydrated oxide of this is carbolio acid. Nitropicric, or nitrophenisic acid is carbolic acid, in which 3 eqs. of hydrogen are replaced by $3 \mathrm{~N} \mathrm{O}_{4}$.

On the whole, from its relation to hyduret of salicyle and to salicine (from which it may also be formed by the action of caustic potash), from its analogy in formation, constitution, and properties to benzoic acid, from its occurrence in nature in the first compound of methyle not artiticially produced, and from its ready convertibility into indigotic acid, carbolic acid and nitropicric acid, salicylic acid is a compound of very great interest. 
The salts of salicylic acid have the general formula $\mathrm{C}_{14} \mathrm{H}_{5} \mathrm{O}_{5}$, M O, or $\mathrm{C}_{14} \mathrm{H}_{5} \mathrm{O}_{6}, \mathrm{M}$. The salicylate of oxide of methyle, $\mathrm{C}_{2}$ $\mathrm{H}_{3} \mathrm{O}+\mathrm{C}_{14} \mathrm{H}_{5} \mathrm{O}_{5}$, occurs in the essential oil of Gaultheria procunibens, and is much used in perfumery. It gives rise to a number of remarkable products, when subjected to the action of nitric acid, chlorine, bromine, alkalies, \&c.; but all these things will be treated of in their proper places.

3. Chlorosalicylic Acid. $\mathrm{C}_{14} \mathrm{H}_{5}\left\{\begin{array}{l}\mathrm{O}_{4} \\ \mathrm{Cl}\end{array}\right.$, or $\mathrm{C}_{14} \mathrm{H}_{5} \mathrm{O}_{4}, \mathrm{Cl}$.

When dry chlorine is made to act on dry hyduret of salicyle this compound is formed, along with hydrochloric acid; it crystallises readily, and may be sublimed unchanged. Its formation is entirely analogous to that of chloride of benzoyle, and it might be viewed as chloride of salicyle, $\mathrm{C}_{44} \mathrm{H}_{5} \mathrm{O}_{4}, \mathrm{Cl}=\mathrm{Sa} \mathrm{Cl}$. But its properties are those of an acid, and hence we are compelled to consider it as salicylic acid, $\mathrm{C}_{14} \mathrm{H}_{5} \mathrm{O}_{5}$, in which $1 \mathrm{eq}$. oxygen has been replaced by 1 eq. chlorine, the type remaining unchanged. Hence, after this substitution, it is still an acid, and its true formula is $\mathrm{C}_{14} \mathrm{H}_{5}\left\{\begin{array}{l}\mathrm{O}_{4} \\ \mathrm{Cl}\end{array}\right.$, an acid of the type of anhydrous salicylic acid.

With ammonia it forms a new compound, chlorosalicylimide. As in the action of ammonia on hyduret of salicyle, so in this case, 2 eqs. ammonia act on 3 of the acid, and 6 of water are separated. The formula of chlorosalicylimide, which is a yellow, insoluble solid, is $\mathrm{C}_{42} \mathrm{H}_{15} \mathrm{~N}_{2} \mathrm{Cl}_{3} \mathrm{O}_{6}$; for $3\left(\mathrm{C}_{14} \mathrm{H}_{5} \mathrm{Cl} \mathrm{O}_{4}\right)+$ $2 \mathrm{~N} \mathrm{H}_{3}=6 \mathrm{H} \mathrm{O}+\mathrm{C}_{42} \mathrm{H}_{15} \mathrm{Cl}_{3} \mathrm{~N}_{2} \mathrm{O}_{6}$. It is, in fact, salicylimide in which 3 eqs. chlorine have been substituted for 3 eqs. hydrogen.

With bromine and iodine, hyduret of salicyle yields the two analogous compounds, bromosalicylic acid, and iodosalicylic acid; $\mathrm{C}_{14} \mathrm{H}_{5}\left\{\begin{array}{l}\mathrm{O}_{4} \\ \mathrm{Br}\end{array}\right.$ and $\mathrm{C}_{14} \mathrm{H}_{5}\left\{\begin{array}{l}\mathrm{O}_{4} \\ \mathrm{I}\end{array}\right.$.

\section{Nitrosalicylic Acid. $\mathrm{C}_{14} \mathrm{H}_{5} \mathrm{NO}_{10}=\mathrm{C}_{14} \mathrm{H}_{4} \mathrm{NO}_{8}, \mathrm{HO}$.}

SrN. Anilic acid, Indigotic acid.-This acid is formed by the action of nitric acid on hyduret of salicyle, or still better, on salicylic acid. It forms yellow prisms, and with bases, yields yellow detonating salts. Ammonia colours it blood-red, and perchloride of iron cherry-red. It is, according to the above formula, derived from the analysis of Piria, salicylic acid, in which 1 eq. nitrous (hyponitric) acid is substituted for 1 eq. hydrogen. But its characters, and those of its salts, are such as to convince us 
that it is identical with indigotic, or anilic acid; under which names we shall have again to treat of it.

\section{APPENDIX TO SALICYLE.}

\section{Salicine. $\mathrm{C}_{26} \mathrm{H}_{18} \mathrm{O}_{14}=\mathrm{C}_{12} \mathrm{H}_{10} \mathrm{O}_{10}+\mathrm{C}_{14} \mathrm{H}_{8} \mathrm{O}_{4}$.}

This compound ocours in the bark of all such willows as are bitter, such as Salix helix, S. amygdalina, \&c. It is extracted by boiling with water, decolorising the decoction with litharge, removing the lead dissolved by sulphuric acid and sulphuret of barium, and evaporating to a syrup. The salicine crystallises on standing, in fine scales of a silky lustre, which have a very pure, bitter taste, and are highly febrifuge. It is neutral, soluble in water and alcohol, insoluble in ether. Oil of vitriol colours it blood-red. Distilled with bichromate of potash and sulphuric acid, it yields formic and carbonic acids, hyduret of salicyle, and a resinous matter.

\section{Saligenine. $\mathrm{C}_{14} \mathrm{H}_{8} \mathrm{O}_{4}$.}

The recent researches of Piria have very greatly extended our knowledge of salicine. The formula above given for salicine is deduced from his analyses, and he has shown that by contact with synaptase, salicine is decomposed, being resolved into sugar, $\mathrm{C}_{12} \mathrm{H}_{10} \mathrm{O}_{10}$ (which takes up 4 eqs. of water and appears as grape sugar, $\mathrm{C}_{12} \mathrm{H}_{14} \mathrm{O}_{14}$ ) and a new body, saligenine, $\mathrm{C}_{14} \mathrm{H}_{8}$ $0_{4}$. When the metamorphosis is complete, which, with 200 parts of water, and 3 parts of synaptase for 50 of salicine, and a temperature of $160^{\circ}$, takes place in 24 hours, most of the saligenine separates in crystals, being very sparingly soluble in cold water, and the rest is obtained by agitating the solution with ether, which dissolves the saligenine. The aqueous liquid is now found to contain only grape sugar, and the residue of the synaptase.

Saligenine melts at $180^{\circ}$, and forms on cooling a crystalline mass. It appears to form a oombination with potash, but when it is heated with that alkali, hydrogen is disengaged and salicylic acid is formed. Several oxidising agents convert saligenine into hyduret of salicyle, from which it differs only by containing 2 eqs. of hydrogen more. Aqueous solutions of perehloride of iron and of salts of peroxide of iron strike an indigo blue with saligenine; but this colour is not formed when alcoholio or ethereal solutions are used. The blue oompound has not been separated. When heated to a certain point, or boiled with acids, saligenine is converted into saliretine, a body which is also 
obtained when salieine is boiled with dilute acids, and which is no doubt in this latter case derived from the saligenine contained in the salicine. Saliretine will be described presently.

As it appears certain from the experiments of Piria that salicine is composed of saligenine and sugar, the products of various reactions on salicine will be generally those on a mixture of saligenine and sugar. It will therefore be better to describe in connection the substances produced from saligenine, and those formed from salicine.

By the action of chlorine on salicine, there are formed three compounds analogous to salicine, in which 1,2 , and 3 eqs. of hydrogen are replaced by chlorine.

These are :-

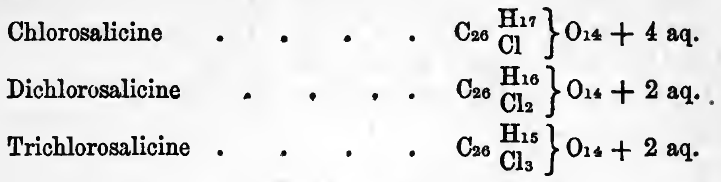

These compounds are all erystallised, and all of them, like salicine, are decomposed by fermentation, in contact with synaptase, into sugar and a body derived from saligenine.

Thus we have:-

Chlorosalicine $=$ Sugar + Chlorosaligenine,

$\left.\left.\left.\mathrm{C}_{26} \underset{\mathrm{Cl}}{\mathrm{C}}\right\}\right\} \mathrm{O}_{14}=\mathrm{C}_{22} \mathrm{H}_{10} \mathrm{O}_{10}+\mathrm{C}_{14} \underset{\mathrm{Cl}}{\mathrm{H}_{7}}\right\} \mathrm{O}_{4}$;

Dịchlorosalicine $=$ Sugar + Dichlorosaligenine,

$\left.\left.\mathrm{C}_{26} \begin{array}{l}\mathrm{H}_{16} \\ \mathrm{Cl}_{2}\end{array}\right\} \mathrm{O}_{14}=\mathrm{C}_{12} \mathrm{H}_{10} \mathrm{O}_{10}+\mathrm{Cl}_{14} \underset{\mathrm{Cl}_{2}}{\mathrm{H}_{8}}\right\} \mathrm{O}_{4}$;

and

Trichlorosalicine $=$ Sugar + Trichlorosaligenine,

$\left.\left.\mathrm{C}_{26} \begin{array}{l}\mathrm{H}_{15} \\ \mathrm{Cl}_{3}\end{array}\right\} \mathrm{O}_{14}=\mathrm{C}_{22} \mathrm{H}_{10} \mathrm{O}_{10}+\mathrm{C}_{14} \underset{\mathrm{Cl}_{3}}{\mathrm{H}_{5}}\right\} \mathrm{O}_{4}$.

Chlorosaligenine forms very fine crystals, and is singularly like saligenine in its outward characters. It colours oil of vitriol, however, green, whereas saligenine colours it red, as does also salicine, The two other compounds are obtained with much greater difficulty by the action of synaptase on Dichlorosalicine and Trichlorosalicine, owing to the very sparing solubility of these compounds. But their existence has been established.

\section{Saliretine.}

When saligenine is boiled with diluted acid, or heated beyond its melting point, it is changed into a resinous substance, which differs from saligenine only by 2 eqs. water. Its empirical for- 
mula is $\mathrm{C}_{14} \mathrm{H}_{6} \mathrm{O}_{2}$, and therefore it is isomeric or polymeric with hyduret of benzoyle.

It appears that Chlorosaligenine, Dichlorosaligenine, and Trichlorosaligenine also yield resins when boiled with acids, and these resins are in all probability derived from saliretine, and composed of $\left.\left.\left.\mathrm{C}_{14} \underset{\mathrm{Cl}}{\mathrm{Cl}}\right\} \mathrm{O}_{2}, \mathrm{Cl}_{14} \underset{\mathrm{Cl}_{2}}{\mathrm{H}_{4}}\right\} \mathrm{O}_{2}, \mathrm{C}_{14} \underset{\mathrm{Cl}_{3}}{\mathrm{H}_{3}}\right\} \mathrm{O}_{2}$. If so, they will be named Chlorosaliretine, Dichlorosaliretine, and Trichlorosaliretine.

8. Helicine. $\mathrm{C}_{26} \mathrm{H}_{16} \mathrm{O}_{14}=\mathrm{C}_{12} \mathrm{H}_{10} \mathrm{O}_{10}+\mathrm{C}_{14} \mathrm{H}_{6} \mathrm{O}_{4}$.

This compound is formed when salicine is acted on by diluted nitric acid. When pure it forms small white needles, which contain, in addition to twice the above formula, 3 eqs. of water of crystallisation. Helicine contains the elements of sugar and hyduret of salicyle, and is actually resolved into them by synaptase, and in other ways. It is no doubt formed by the oxidation of the saligenine producing hyduret of salicyle, which then unites with the sugar to form helicine.

Helicine, when heated to $347^{\circ}$, is converted into an insoluble resinous mass.

When the oxidation of salicine is carried further, the sugar is destroyed, and yields formic and carbonic acids; but when the oxidation is insufficient to form helicine, there is produced a body, helicoidine, the empirical formula of which is $\mathrm{C}_{52} \mathrm{H}_{34} \mathrm{O}_{28}+$ $3 \mathrm{H} \mathrm{O} ;=2$ eqs. sugar, $\mathrm{C}_{24} \mathrm{H}_{20} \mathrm{O}_{20} ; 1$ eq. hyduret of salicyle, $\mathrm{C}_{14} \mathrm{H}_{6} \mathrm{O}_{4}$; and 1 eq. saligenine, $\mathrm{C}_{14} \mathrm{H}_{8} \mathrm{O}_{4}$.

By the action of chlorine, helicine yields chlorohelicine $\left.\mathrm{C}_{26} \underset{\mathrm{Cl}}{\mathrm{H}_{15}}\right\} \mathrm{O}_{14}$, which is formed of sugar and chloride of salicyle, $\left.\mathrm{C}_{12} \mathrm{H}_{10} \mathrm{O}_{10}+\mathrm{C}_{14} \underset{\mathrm{Cl}}{\mathrm{H}}\right\} \mathrm{O}_{4}$. With bromine it yields a similar compound.

Rutiline is the name given to the red compound formed by the action of oil of vitriol on salicine. When pure it is of a deep reddish-brown colour : acids change it to a bright red, alkalies to a deep violet.

\section{Phloridzine. $\mathrm{C}_{42} \mathrm{H}_{29} \mathrm{O}_{24}=\mathrm{C}_{42} \mathrm{H}_{23} \mathrm{O}_{18}+6 \mathrm{H} \mathrm{O}$ ?}

This is a substance, very analogous to salicine, which occurs in the bark of the roots of the apple, pear, plum, \&c. It is . extracted in the same way as salicine, and resembles it much; forming small scales, soluble in hot water, and in alcohol, very bitter, and powerfully febrifuge. When boiled with dilute sulphuric acid, it yields a resinous compound, phloretine, 
analogous to saliretine, along with grape sugar. Phloretine is $\mathrm{C}_{30} \mathrm{H}_{15} \mathrm{O}_{10}=\mathrm{C}_{30} \mathrm{H}_{14} \mathrm{O}_{9}, \mathrm{H} \mathrm{O}$ ? and $\mathrm{C}_{30} \mathrm{H}_{15} \mathrm{O}_{10}+\mathrm{C}_{12} \mathrm{H}_{14}$ $\mathrm{O}_{14}=\mathrm{C}_{42} \mathrm{H}_{29} \mathrm{O}_{24}$. According to Stas, however, phloretine is $\mathrm{C}_{6} \mathrm{H}_{3} \mathrm{O}_{2}$, or double or quadruple of this. The compound of phloretine with oxide of lead he found to be $\mathrm{C}_{22} \mathrm{H}_{5} \mathrm{O}_{3}, 2 \mathrm{~Pb} \mathrm{O}$; which would indicate, for hydrated phloretine, $\mathrm{Ci2}_{22} \mathrm{H}_{5} \mathrm{O}_{3} 2 \mathrm{HO}=$ $\mathrm{C}_{12} \mathrm{H}_{6} \mathrm{O}_{4}, \mathrm{HO}=\mathrm{C}_{12} \mathrm{H}_{7} \mathrm{O}_{5}$ ?

By the action of nitric acid on phloridzine, there is formed a puce-coloured acid, nitrophloretic acid, which, according to Piria, is $\mathrm{C}_{30} \mathrm{H}_{12} \mathrm{NO}_{15}$; according to Stas, $\mathrm{C}_{24} \mathrm{H}_{10} \mathrm{~N} \mathrm{O}_{12}$.

Phloridzéine. $\mathrm{C}_{42} \mathrm{H}_{29} \mathrm{~N}_{2} \mathrm{O}_{26}$ ? When moist phloridzine is exposed to air and ammonia, it is transformed into a deep red compound, which dissolves in ammonia, and may be precipitated by acids. It is equal to phloridzine, plus 8 eqs. oxygen, and 2 eqs. ammonia. When dissolved in ammonia, and dried in vacuo, it leaves a purple mass, with coppery lustre, which communicates to water a splendid blue colour. This is a compound of phloridzéine and 1 eq. ammonia. The formation of this blue pigment is a very good example of that kind of eremacausis with the aid of ammonia, by which indigo, litmus, orchil, \&c., are produced from colourless bodies.

When salicine was supposed to be $\mathrm{C}_{42} \mathrm{H}_{29} \mathrm{O}_{22}$, phloridzine appeared to differ from it by only 2 eqs. oxygen; but as that formula for salicine, according to Piria, is erroneous, it is most probable that the formula of phloridzine will require to be changed. It is therefore marked as doubtful along with its derivatives.

\section{APPENDIX TO SALICXLE AND SALICYLIC ACID.}

Anisyle and Anisic Acid.

The stearoptene of oil of anise, as well as the oil of estragon, yields, when oxidised by nitric acid, a crystalline acid, in some respects analogous to cinnamic acid. This is anisic acid, $\mathrm{C}_{16} \mathrm{H}_{8}$ $\mathrm{O}_{6}=\mathrm{C}_{16} \mathrm{H}_{7} \mathrm{O}_{5}, \mathrm{HO}$. Like benzoio and cinnamic acid, it is supposed to be derived from an oil analogous to the hyduret of benzoyle. This would be hyduret of anisyle, $\mathrm{C}_{16} \mathrm{H}_{8} \mathrm{O}_{4}=$ $\mathrm{C}_{16} \mathrm{H}_{7} \mathrm{O}_{4}, \mathrm{H}$.

Anisio acid, when heated with bases, yields an oil, anisole, $\mathrm{C}_{14}$ $\mathrm{H}_{8} \mathrm{O}_{2}$, the acid having lost 2 eqs. of carbonic acid. The acid itself is homologous with salicylic acid, just as anisole is with phenole or carbolio acid, the difference being $\mathrm{C}_{2} \mathrm{H}_{2}$.

The hyduret of anisyle is not known in the separate form, and the oil of anise or estragon is believed to be $\mathrm{C}_{20} \mathrm{H}_{12} \mathrm{O}_{2}$, which is 
nearly the formula of oil of cinnamon, and of oil of cumine. But these oils are evidently not identical, but only isomerio or polymeric, since the action of nitric acid in each case yields a different result. It is probable that anisic acid belongs to a homologous series, differing from the benzoic as well as from the cinnamic series.

Anisic acid yields, when acted on by a mixture of nitric and sulphuric acids, two substitution products, nitranisic acid,

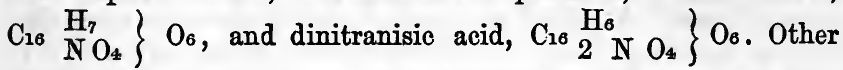
substitution products have been obtained by Cahours, both from the acid, and from anisole. The latter body, anisole, $\mathrm{C}_{44} \mathrm{H}_{8} \mathrm{O}_{2}$ is homologous with phenole or hydrated oxide of phenyle (carbolic acid). From it are obtained chloranisole, bromanisole, and three nitro-compounds, nitranisole, dinitranisole, and trinitranisole. Nitranisole, $\left.\mathrm{C}_{14} \mathrm{H}_{7} \mathrm{O}_{4}\right\} \mathrm{O}_{4}$, when acted on by sulphuret of ammonium, yields the base, anisidine, $\mathrm{C}_{14} \mathrm{H}_{9} \mathrm{~N} \mathrm{O}_{2}$; and dinitranisole yields the base nitranisidine, $\mathrm{C}_{4} \mathrm{H}_{8} \mathrm{~N}_{2} \mathrm{O}_{6}$, in which 1 eq. of the hydrogen of anisidine is replaced by 1 eq. of nitrous acid. In like manner, trinitranisole yields the base dinitranisidine, in which 2 eqs. of hydrogen are replaced by nitrous acid. When nitranisidine is acted on by chloride of benzoyle and chloride of cinnamyle, two new bases are formed, nitrobenzanisidine, $\mathrm{C}_{28} \mathrm{H}_{12} \mathrm{~N}_{2} \mathrm{O}_{8}$, and nitrocumanisidine, $\mathrm{C}_{32} \mathrm{H}_{14} \mathrm{~N}_{2} \mathrm{O}_{8}$. In these two remarkable products, which approach closely to natural bases, benzoyle and cinnamyle have been substituted for 1 eq. of hydrogen in nitranisidine.

\section{Cinnamyle. $\mathrm{C}_{18} \mathrm{H}_{7} \mathrm{O}_{2}=\mathrm{Ci}$.}

The radical of essence of cinnamon, but unknown in a separate form.

1. Hyduret of Cynnamyle. $\mathrm{Ci} \mathrm{H}=\mathrm{C}_{18} \mathrm{H}_{8} \mathrm{O}_{2}=\mathrm{C}_{18} \mathrm{H}_{7} \mathrm{O}_{2}, \mathrm{H}$.

This is the purified essence, or oil of cinnamon. The oil of commerce contains, besides, cinnamic acid and two resins, all of which have been produced from the original oil, $\mathrm{C}_{20} \mathrm{H}_{11} \mathbf{O}_{2}$, by the addition to 3 eqs. of it, of 8 eqs. oxygen. $3\left(\mathrm{C}_{20} \mathrm{H}_{11} \mathrm{O}_{2}\right)+$ $\mathrm{O}_{8}=\mathrm{C}_{18} \mathrm{H}_{8} \mathrm{O}_{4}$ (cinnamic acid) $+\mathrm{C}_{12} \mathrm{H}_{5} \mathrm{O}$ (a resin); + $\mathrm{C}_{30}$ $\mathrm{H}_{15} \mathrm{O}_{4}$ (another resin) $+5 \mathrm{HO}$. With less oxygen, hyduret of cinnamyle, $\mathrm{C}_{18} \mathrm{H}_{8} \mathrm{O}_{2}$, is formed, along with the resins, so that the oil of commerce contains, besides the original oil, all the compounds above mentioned.

The hyduret of cinnamyle, $\mathrm{Ci} \mathrm{H}$, is a fragrant oil. It forms, 
with nitric acid, a crystalline compound, $\mathrm{Ci} \mathrm{H}+\mathrm{N} \mathrm{O}_{5}$, which, when mixed with water, is resolved into its constituents, hyduret of cinnamyle and nitric acid. When the hyduret is exposed to the air, it absorbs oxygen, producing cinnamic acid, $\mathrm{Ci} \mathrm{O}, \mathrm{HO}=$ $\mathrm{C}_{18} \mathrm{H}_{7} \mathrm{O}_{3}, \mathrm{HO}$.

The fresh oil of cinnamon is, as has been stated, $\mathrm{C}_{20} \mathrm{H}_{11} \mathrm{O}_{2}$. With 6 eqs. oxygen from the air, it yields hyduret of cinnamyle and the two resins: with 2 eqs. more, the hyduret passes into cinnamic acid. With oil of vitriol, 3 eqs. of the fresh oil $=\mathrm{C}_{60}$ $\mathrm{H}_{33} \mathrm{O}_{6}$, lose 3 eqs. water, and form two resins, one $=\mathrm{C}_{30} \mathrm{H}_{15} \mathrm{O}$; the other $=\mathrm{C}_{30} \mathrm{H}_{15} \mathrm{O}_{2}$. With hydrochloric acid, it yields an oil and two resins; one, $\mathrm{C}_{20} \mathrm{H}_{8} \mathrm{O}$ : the other $\mathrm{C}_{14} \mathrm{H}_{6} \mathrm{O}$.

\section{Cinnamic Acid. $\mathrm{C}_{18} \mathrm{H}_{7} \mathrm{O}_{3}+\mathrm{HO}=\mathrm{Ci} \mathrm{O}$, $\mathrm{H} \mathrm{O}$.}

Formed by exposing oil of cinnamon to the air. It is most easily obtained by dissolving the oil of Balsam of Peru in an alcoholic solution of potash, evaporating to dryness, dissolving in hot water, and adding to the solution of cinnamate of potash an excess of hydrochloric acid. It crystallises very readily, and may be sublimed. By the action of nitric acid, cinnamic acid is converted into hyduret of benzoyle, and into an acid very similar to benzoic acid, if not identical with it. When cinnamic acid is added to cold nitric acid, it forms an acid, C18 $\mathrm{H}_{7} \mathrm{~N} \mathrm{O}_{8}=\mathrm{C}_{18} \mathrm{H}_{8} \mathrm{O}_{4}-\mathrm{H}+\mathrm{N} \mathrm{O}_{4}$; that is, cinnamic acid, in which 1 eq. of nitrous acid is substituted for 1 of hydrogen; $\mathrm{C}_{18}\left\{\begin{array}{l}\mathrm{H}_{6} \\ \mathrm{NO}_{4}\end{array} \mathrm{O}_{3}, \mathrm{HO}\right.$. This is nitrocinnamic acid. Its salts detonate when heated. With oxide of ethyle, it forms a crystallisable ether, $\mathrm{Ae} 0, \mathrm{C}_{18} \mathrm{H}_{6} \mathrm{NO}_{7}$. When oil of cinnamon is mixed with fuming sulphuric acid, there is formed, according to Herzog, a new acid, sulphocinnamic acid, the formula of which is $\mathrm{Cl}_{18} \mathrm{H}_{6} \mathrm{O}_{2}, 2 \mathrm{SO}_{3}+2 \mathrm{H} \mathrm{O}=\mathrm{Cl}_{18} \mathrm{H}_{8} \mathrm{O}_{10} \mathrm{~S}_{2}$. It is bibasic, and almost all its salts are soluble.

When oil of cinnamon is acted on by nitric acid with the aid of heat, it yields benzoic and nitrobenzoic acids. By the action of chlorine, oil of cinnamon is converted into several new

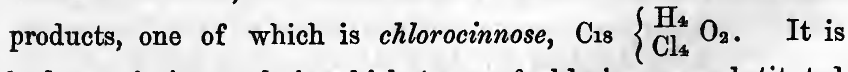
hyduret of cinnamyle in which 4 eqs. of chlorine are substituted for 4 eqs. of hydrogen.

Balsam of Peru contains compounds connected with cinnamyle. The principal is an oil, cinnameine, which, when boiled with alkalies, yields cinnamic acid and a neutral oily body, peruvine. 
Cinnameine has been ascertained by Scharling to have the formula $\mathrm{C}_{36} \mathrm{H}_{18} \mathrm{O}_{4}$, and to contain cinnamic acid, $\mathrm{C}_{18} \mathrm{H}_{7} \mathrm{O}_{3}$, united to a new ether, $\mathrm{C}_{18} \mathrm{H}_{11} \mathrm{O}$. When boiled with potash, cinnameine yields on the one hand cinnamate of potash, and on the other peruvine, $\mathrm{C}_{18} \mathrm{H}_{12} \mathrm{O}_{2}$, the latter compound being the alcohol corresponding to the new ether, and formed from it by the addition of 1 eq. of water.

Cinnumole, $\mathrm{C}_{16} \mathrm{H}_{8}$, a liquid bearing the same relation to cinnamic acid that benzole does to benzoic acid, is formed when cinnamio acid is distilled with baryta. It is, like benzole, formed of earbon and hydrogen in the proportion of two eqs. of the former to one of the latter; but its density and boiling-point prove that its formula is $\mathrm{C}_{16} \mathrm{~Hz}$. The following equation shows its formation :

$$
\underbrace{\mathrm{C}_{18} \mathrm{H}_{8} \mathrm{O}_{4}}_{\text {Cinnamic acid. }}+2 \mathrm{Ba} \mathrm{O}=2\left(\mathrm{Ba} \mathrm{O}, \mathrm{C} \mathrm{O}_{2}\right) \underbrace{\mathrm{C}_{16} \mathrm{H}_{8}}_{\text {Cinnamole. }}
$$

Cinnamole is isomerio, or perhaps identical with styrole, an oil obtained by distillation from storax, to be afterwards described. Dragon's blood also yields styrole by distillation. The ohief difference between cinnamole and styrole is, that the former is not, like the latter, converted by a heat of $392^{\circ}$, in sealed tubes, into a solid compound, metastyrole, the formula of which is $\mathrm{C}_{14}$ $\mathrm{H}_{7}$. But the presence of some impurity in cinnamole may prevent the change.

According to Richter, Balsam of Peru contains two distinct oils, myroxiline, insoluble in alcohol, and myriospermine, soluble in alcohol. With an alcoholio solution of potash, myriospermine yields an acid resembling cinnamic acid, but different from it, myriospermic acid.

BALSAM OF TOLU.

Tolene; Toluole.

According to Deville, Balsam of Tolu contains benzoic acid and a body isomeric with hyduret of benzoyle; also cinnameine, and a liquid carbohydrogen, tolene, the formula of which is $\mathrm{C}_{24} \mathrm{H}_{18}$; besides several resins, and another liquid carbohydrogen, analogous to, and homologous with, benzole, the name given to which is toluole, and its formula $\mathrm{C}_{14} \mathrm{H}_{8}$. It boils at $226^{\circ}$, and its Sp. G. is 0.87 at $65^{\circ} \mathrm{F}$. When acted on by nitric acid, it yields two new compounds, in which the hydrogen is partly replaced by nitrous acid. These are, 1st, nitrotoluole, $\mathrm{C}_{14} \mathrm{H}_{7} \mathrm{NO}_{4}$, which is liquid; and 2nd, dinitrotoluole, $\mathrm{C}_{14} \mathrm{H}_{6} \mathrm{~N}_{2} \mathrm{O}_{8}$, which is a crsstalline solid. By the action of chlorine on toluole, Deville has also 
obtained a series of compounds in which the hydrogen is replaced by chlorine; these compounds, however, chiefly occur in combination with hydrochloric acid. Toluole dissolves in forming sulphuric acid, and forms a crystalline mass of sulphotoluylic acid, the composition of which, according to Deville, is $\mathrm{C}_{14} \mathrm{H}_{7} \mathrm{~S}_{2} \mathrm{O}_{5}$; that is, 1 eq. of hydrogen seems to be replaced by 1 eq. hyposulphuric acid. It is analogous to the sulphonaphthalic or hyposulphonapththalic acid.

By the tables already given at pp. 131 and 141, it will be seen that toluole is not only homologous with benzole, but also yields a whole series of derivatives, two of which are mentioned in the preceding paragraphs, homologous with those of benzole. Another point of analogy is, that both benzole and toluole occur in the oil of tar. Toluylic acid, $\mathrm{C}_{16} \mathrm{H}_{8} \mathrm{O}_{4}$, which corresponds to benzoic acid, is not known as a natural product, but has been obtained by the oxidation of cymole, $\mathrm{C}_{20} \mathrm{H}_{14}$, the highest known number of the benzole series. It is very analogous to benzoic acid, and, like it, is a hydrate, its true formula being $\mathrm{C}_{16} \mathrm{H}_{7} \mathrm{O}_{3}, \mathrm{HO}$.

Besides nitrotoluole and dinitrotoluole, the table also contains the base, toluidine, homologous with aniline, which is formed by the action of hydrosulphuret of ammonia on nitrotoluole, just as aniline is from nitrobenzole, as will be explained in the section on artificial bases. Toluidine, like aniline, admits of the partial replacement of its hydrogen by chlorine, bromine, or nitrous acid, thus giving rise to new bases, the formulæ of which are given in the table already referred to.

\section{GUATACYte. $\mathrm{C}_{14} \mathrm{H}_{7} \mathrm{O}_{4}$ ?}

\section{Hyduret of Guaiate. $\quad \mathrm{C}_{14} \mathrm{H}_{8} \mathrm{O}_{4}=\mathrm{C}_{14} \mathrm{H}_{7} \mathrm{O}_{4}+\mathrm{H}$ ?}

When the resin, or gum-resin, called Guaiacum, is distilled, there is obtained, among other products, a colourless oily liquid, which has acid properties. According to Deville, it is analogous to the hyduret of salicyle, which is also an acid, and is sometimes called salicylous acid. Indeed, Sobrero, who first obtained the acid from Guaiacum, called it pyroguaiacic acid. If viewed as a hydrogen acid, it will be the hyduret of guaiacyle, $\mathrm{C}_{14} \mathrm{H}_{7} \mathrm{O}_{4}+\mathrm{H}$, and this agrees with the formula dedueed from analysis $\mathrm{C}_{24} \mathrm{H}_{8}$ .04 , which is that of saligenine. The aoid corresponding to salicylic acid has not been formed, but will probably hereafter be obtained. Its formula will be $\mathrm{C}_{14} \mathrm{H}_{8} \mathrm{O}_{6}=\mathrm{C}_{14} \mathrm{H}_{7} \mathrm{O}_{5}, \mathrm{HO}$. But Thierry has obtained another acid, which he calls guaiacic acid, the formula of whioh is $\mathrm{C}_{12} \mathrm{H}_{8} \mathrm{O}_{6}=\mathrm{C}_{12} \mathrm{H}_{7} \mathrm{O}_{3}, \mathrm{HO}$. Deville has also described another oil, which is neutral, and seems to be derived from the last acid, as benzole from benzoic 
acid, its formula is $\mathrm{C}_{10} \mathrm{H}_{8} \mathrm{O}_{2}$; and it is derived from the acid $\mathrm{C}_{12} \mathrm{H}_{8} \mathrm{O}_{8}$ by the separation of 2 eqs. carbonic acid; for $\mathrm{C}_{22} \mathrm{H}_{8}$ $\mathrm{O}_{6}-2 \mathrm{C} \mathrm{O}_{2}=\mathrm{C}_{10} \mathrm{H}_{8} \mathrm{O}_{2}$. This neutral oil has been called guaiacéne.

In the preceding pages we have considered a large number of compound radicals, constituting chiefly the group of radicals analogous in their relations to chlorine; namely, cyanogen; ferrocyanogen and its numerous congeners; sulphocyanogen; and mellone. We have only described one radical, amide, which has a tendency to produce basic compounds, such as ammonia, ammonium, and the platinised bases; and one carbonic oxide or oxalyle, the chief tendency of which is to form acid compounds, such as oxalic, carbonic, rhodizonic, croconic, and mellitic acids. Finally, we have studied three; namely, benzoyle, salicyle and cinnumyle, whose characteristic is to form essences or fragrant volatile compounds, with hydrogen, acids with oxygen, and peculiar compounds with chlorine, iodine, \&c. This last group would appear to have a strong tendency to form acid compounds: for not only is an acid formed by the union of salicyle and oxygen, but the hyduret of salicyle is a decided acid, and may oe represented as salicylous acid, $\mathrm{C}_{14} \mathrm{H}_{5} \mathrm{O}_{3}, \mathrm{H} \mathrm{O}$, isomerio with benzoic acid, instead of hyduret of salicyle, $\mathrm{C}_{4} \mathrm{H}_{5} \mathrm{O}_{4}, \mathrm{H}$. Further, the chloride, bromide, and iodide of salicyle, although they contain salicyle, plus those elements, are all strong acids, and appear to be, in fact, anhydrous salicylic acid with 1 eq. of chlorine, \&c., substituted for 1 eq. of oxygen. It is evident that this group of radicals, so well characterised by their hydrogen compounds, does not correspond exactly to any simple radical, but has characters common to different groups of elementary radicals, being, however, analogous in more points to carbon, sulphur, and phosphorus than to any other elements. In studying the decomposition of the compounds of salicyle, we have met with the very remarkable fact of the production from that radical of a series of compounds : namely, anilic acid, nitropicric acid, carbolic acid, \&c., probably derivatives of a totally different radical, phenyle; which radical is also met with in numerous other decompositions, and especially in the decomposition of indigo by nitric acid, by alkalies, and by heat, and in the destructive distillation of coal. This is an important consideration, as every day's experience tends to identify with each other the products of decomposition of different and apparently quite unconnected organic compounds, even in cases where these products have been described as different. Another very important fact which has come under our notice is the occurrence of salicylate of oxide of methyle as the chief ingredient in the oil 
of Gaultheria. Not only is the occurrence of salicylic acid interesting, since this acid was only known before as an artificial product, but the existence, in this oil, of oxide of methyle, hitherto only known as the product of destructive distillation, goes far to confirm the theory which admits this radical methyle, and others similar to it. All the properties of the oil of Gaultheria entirely agree with the doctrine of its containing two radicals, methyle and salicyle; the former oxidised, as a base: the latter, also oxidised, as an acid. It is remarkable, however, that even this compound plays the part of an acid, and forms crystalline salts with the alkalies into which it enters unchanged. This is so well marked a character that the oil has been called gaultheric acid, and the salts gaultherates.

We have also, in the course of the preceding pages, become acquainted with one remarkable series of homologous groups, namely, the benzoic series. We have seen that two acids are known, toluylic and cuminic acids, homologous with benzoic acid, and of the general formula $\mathrm{C}_{n} \mathrm{H}_{n}-8 \mathrm{O}_{4}$; these acids are perfectly analogous in properties. Of the neutral carbohydrogens, derived from these acids by the separation of 2 eqs. of carbonic acid, and the formula for which is $\mathrm{C}_{n} \mathrm{H}_{n}-6$, we know not only benzole, toluole, and cumole, but two more, the acids of which are not yet known, namely xylole and cymole. All five are found in tar, and they all yield analogous substitution products, including bases of a very remarkable kind, of which aniline is the type, and which also yield other basic products of substitution.

It is highly probable that both cinnamic and anisic acids belong each to a different series, of which few other members are as yet known. Indeed, anisic acid, $\mathrm{C}_{16} \mathrm{H}_{8} \mathrm{O}_{6}$, is homologous with salicylic acid, $\mathrm{C}_{14} \mathrm{H}_{6} \mathrm{O}_{6}$. Benzoic acid and its homologues yield, by the loss of 2 eqs. of carbonic acid, $\left(\mathrm{C}_{2} \mathrm{O}_{4},\right)$ carbohydrogens, because these acids contain only 4 eqs. of oxygen. The same is true of cinnamic acid, and of its homologues, should any be discovered. But anisio and salicylic acids, containing 6 eqs. of oxygen, necessarily yield, on the removal of $\mathrm{C}_{2} \mathrm{O}_{4}$, not carbohydrogens, but oils containing 2 eqs. of oxygen, namely, anisole, $\mathrm{C}_{14} \mathrm{H}_{8} \mathrm{O}_{2}$, and phenole or carbolic acid (perhaps better, salicole) $\mathrm{C}_{12} \mathrm{H}_{6} \mathrm{O}_{2}$; in which, as in benzole, toluole, xylole, cumole, cymole, and cinnamole (styrole), the carbon is to the hydrogen as 2 to 1.

But although in one point of view anisole is homologous with phenole, yet, according to Cahours, it is not a true homologue of that compound, and is not analogous to it in properties. Cahours has discovered two other oils, phenetole, $\mathrm{C}_{16} \mathrm{H}_{10} \mathrm{O}_{2}$, and phena- 
mylole, $\mathrm{C}_{22} \mathrm{H}_{16} \mathrm{O}_{2}$, which are perfectly homologous with anisole, while all three are neutral, whereas phenole is an acid, and is called carbolic acid. According to Cahours, anisole is the carbolate of oxide of methyle; phenetole, the carbolate of oxide of ethyle, and phenamylole the carbolate of oxide of amyle, as may be seen in the following formulæ:-

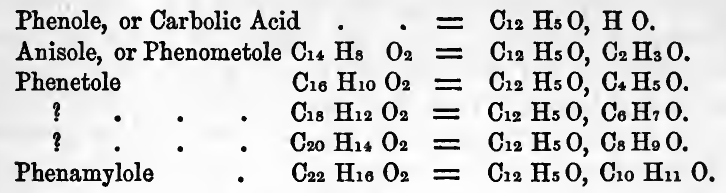

In proof of this view it may be stated, that as phenole is formed when salicylic acid, $\mathrm{C}_{14} \mathrm{H}_{6} \mathrm{O}_{4}$, is heated with bases, so anisole, phenetole, and phenamjlole are formed when the salicylates of the oxides of methyle, ethyle, and amyle are respectively heated with bases. It would appear, therefore, that the three latter bodies, anisole and its homologues, are not homologous with phenole, but are isomeric with its homologues. Since onisic acid, $\mathrm{C}_{16} \mathrm{H}_{8} \mathrm{O}_{6}$, is homologous with salicylic acid, we should expect it to yield a body strictly homologous to phenole, but on the contrary it yields anisole, properly phenometole; the same body as is obtained from the salicylate of oxide of methyle, by heating with bases, the salicylate of methyle being isomerio with anisic acid. We here see what is not uncommon, that two isomeric compounds, when exposed to the same cause of change, yield products which are not isomeric but identical. The following equations will make this clear;

$\mathrm{C}_{16} \mathrm{H}_{8} \mathrm{O}_{6}=\mathrm{C}_{16} \mathrm{H}_{7} \mathrm{O}, \mathrm{H} \mathrm{O}$, yields $2 \mathrm{C} \mathrm{O}_{2}$ and

Anisic Acid.

$\mathrm{C}_{14} \mathrm{H}_{8} \mathrm{O}_{2}=\mathrm{C}_{22} \mathrm{H}_{5} \mathrm{O}, \mathrm{C}_{2} \mathrm{H}_{3} \mathrm{O}$

Anisole, or Carbolate of Methyle.

$\mathrm{C}_{16} \mathrm{H}_{8} \mathrm{O}_{6}=\mathrm{C}_{14} \mathrm{H}_{5} \mathrm{O}_{5}, \mathrm{C}_{2} \mathrm{H}_{3} \mathrm{O}$, yields $2 \mathrm{C} \mathrm{O}_{2}$, and

Salicylate of Methyle.

$\mathrm{C}_{14} \mathrm{H}_{8} \mathrm{O}_{2}=\mathrm{C}_{12} \mathrm{H}_{5} \mathrm{O}, \mathrm{C}_{2} \mathrm{H}_{3} \mathrm{O}$

Anisole, or Carbolate of Methyle.

The production of phenetole and phenamylole is quite analogous to the last of these equations, ethyle and amyle being substituted for methyle. Both phenetole and phenamylole, like anisole, yield substitution products, among which are two new 
bases, phenetidine and phenamylidine, homologous with anisidine. The latter has not been described, but Cahours has obtained, from dinitrophenamylole the substitution base nitrophenamylidine, homologous with nitranisidine.

Cahours has obtained the three compounds, anisole, phenetole, and phenamylole, by the action of carbolate of potash on the iodides of methyle, ethyle, and amyle, which leaves no doubt as to their true constitution. They are, as already stated, the carbolates of the oxides of methyle, ethyle, and amyle, as represented in the first column. Were the three lower compounds acids, truly homologous with carbolic acid, they would be as in the second column.

$\begin{array}{ll}\text { Carbolic Acid } & \mathrm{C}_{12} \mathrm{H}_{5} \mathrm{O}, \mathrm{H} \mathrm{O}=\mathrm{C}_{22} \mathrm{H}_{5} \mathrm{O}, \mathrm{H} \mathrm{O} \\ \text { Anisole, or Phenometole } \mathrm{C}_{22} \mathrm{H}_{5} \mathrm{O}, \mathrm{Me} \mathrm{O}=\mathrm{C}_{14} \mathrm{H}_{7} \mathrm{O}, \mathrm{H} \mathrm{O} \\ \text { Phenetole } & \mathrm{C}_{12} \mathrm{H}_{5} \mathrm{O}, \mathrm{Ae} \mathrm{O}=\mathrm{C}_{16} \mathrm{H}_{9} \mathrm{O}, \mathrm{H} \mathrm{O} \\ \text { Phenamylole } & \mathrm{C}_{22} \mathrm{H}_{5} \mathrm{O}, \mathrm{Ayl} \mathrm{O}=\mathrm{C}_{22} \mathrm{H}_{15} \mathrm{O}, \mathrm{H} \mathrm{O}\end{array}$

We now proceed to consider that group of electropositive radicals, to which ethyle belongs, and which, in their chemical characters, are quite analogous to metals, and in some respects to hydrogen, which, as well as metals, they are capable of replacing. There are a considerable number of them, but we shall first describe such as are known of that remarkable series of homologous radicals beginning with methyle and ethyle, and we shall afterwards notice those radicals of this group which are not homologous with methyle and ethyle.

But besides these, we shall have to notice certain electronegative radicals, derived from the electropositive ones of the ethyle series, and forming acids by uniting with oxygen. Of these, which are not yet known in the separate form, the type is formyle, derived from methyle. We shall treat of them under the radicals, from which they are respectively derived, and not in a separate section.

Under the radicals of the methyle and ethyle series, we shall also introduce the new radicals formed by the combination of these with phosphorus, and with metals, such as zinc, antimony, arsenic. A strict arrangement is not yet attainable, but it will be found, that with the aid of homologous series and of the doctrine of substitution, these numerous compounds, (our account of which must necessarily be very brief,) will be rendered more intelligible to the student and more easily remembered than hitherto. We begin, then, with methyle, the first organic radical of the homologous series to which it belongs. 


\section{RAdioAls of the General formola, $\mathrm{C}$ Hn +1 .}

\section{Methyle. $\mathrm{C}_{2} \mathrm{H}_{3}=\mathrm{Me}$.}

This radical was first obtained by Frankland and Kolbe, as a product of the action of potassium on cyanide of ethyle. It was soon after observed by Kolbe among the products of the electrolytic decomposition of acetic acid; and by Frankland, among the products of the action of zinc, aided by heat and under pressure, on iodide of ethyle, and by the action of water on the new radical zincethyle. In all these cases it is accompanied by other products, all of which are not exactly known; but in the third of them it seems to be formed in the following manner: iodide of ethyle, $\mathrm{C}_{4} \mathrm{H}_{5} \mathrm{I}$, acted on by zinc, yields iodide of zinc, $\mathrm{Zn} \mathrm{I}$, and ethyle, $\mathrm{C}_{4} \mathrm{H}_{5}$; and a part of the ethyle is resolved into methyle and elayle, thus : $\mathrm{C}_{4} \mathrm{H}_{5}=\mathrm{C}_{2} \mathrm{H}_{3}+\mathrm{C}_{2} \mathrm{H}_{2}$.

Methyle is a gas, of Sp. G. 1.0365 , which at $0^{\circ} \mathrm{F}$. is not liquid, and which has resisted a pressure of 20 or 30 atmospheres. It is combustible, burning with a bluish flame and little light. It has an ethereal smell, and is not absorbed by water, but alcohol absorbs it. It has certainly the composition $\mathrm{C}_{2} \mathrm{H}_{3}$, but we cannot be certain that it is the radical methyle, or that it may not be only isomeric with that body. Since, however, its production by the electrolysis of acetic acid is attended by that of oxide of methyle, we may conclude, for the present, that it is the true radical.

Methyle exists in nature, but not uncombined, so far as we yet know. Its oxide is found in the oil of Gaultheria procumbens, combined with salicylic acid. But oxide of methyle is more abundant and better known as one of the chief ingredients, in the form of hydrate, of the more volatile product of the distillation of wood.

Methyle is chiefly interesting as the lowest or first member of a very remarkable group of homologous radicals, the formulæ of a number of which are given in Column 1 of the table of homologous compounds. Immediately above it in the series (below it in the column) stands ethyle, $\mathrm{C}_{4} \mathrm{H}_{5}$, which differs from it by $\mathrm{C}_{2} \mathrm{H}_{2}$, and the same increase, of $\mathrm{C}_{2} \mathrm{H}_{2}$, is made at each step. Now, if we subtract $\mathrm{C}_{2} \mathrm{H}_{2}$ from methyle, instead of adding them to it, we obtain $\mathrm{H}$, or hydrogen, which is therefore the inorganic starting-point of this organic series. And we shall find that methyle and its homologues agree in many points with hydrogen. Thus they all combine, although not directly, with oxygen, chlorine, bromine, iodine, sulphur, cyanogen, \&c., in the same proportions by volume as hydrogen does; they may be substituted 
for hydrogen in ammonia without changing the type of that base; and as hydrogen forms compounds with certain metals, tellurium, arsenic, and antimony, so also do methyle and ethyle. In one point of view, therefore, we may regard all the radicals of the methyle series as homologues of hydrogen. But we must remember that in another they differ; for while the oxide of hydrogen is neutral, that of methyle is basic; the chlorides, \&c., of hydrogen are strong acids, while the chlorides, \&c., of methyle are neutral ethers.

Another circumstance must be mentioned here. Most of the known compounds of carbon and hydrogen which are volatile have such a density in the form of gas, that their formula represents 4 vols.; that is, the densities of their elements, added together in the proportions indicated by the formula, represent the weight of 4 vols. of the compound, and this sum must be divided by 4 to give the density of the compound, which is the weight of 1 vol. But methyle and its homologues, if examined in reference to their density, are found to represent only 2 vols., a somewhat unusual occurrence. Thus methyle, $\mathrm{C}_{2} \mathrm{H}_{3}$, represents not 4 , but 2 volumes of the gas, which is therefore twice as heavy as it should be, if it followed the law of 4 volumes. For this reason, Hofmann and others are disposed to think that the formula of methyle should be doubled, which would give $\mathrm{C}_{4} \mathrm{H}_{6}$, and this, calculated for 4 volumes, would agree with the actual density of the gas. But it is certain, that many things in the history of methyle agree better with the formula $\mathrm{C}_{2} \mathrm{H}_{3}$ than with $\mathrm{C}_{4} \mathrm{H}_{6}$, for the latter would make the oxide $\mathrm{C}_{4} \mathrm{H}_{6} \mathrm{O}_{2}$, the chloride $\mathrm{C}_{4} \mathrm{H}_{6} \mathrm{Cl}_{2}$, \&o., and would make it necessary to double the quantities of all those substances which act on it, thus yielding very improbable formulæ. The two opinions may be reconciled by supposing that, as Frankland has recently shown, the methyle produced in the processes above mentioned is not identical in all of them. The methyle obtained from cyanide of ethyle by potassium, and that formed by the action of water on zincethyle, $\mathrm{C}_{4} \mathrm{H}_{5} \mathrm{Zn}$, is not true methyle, but a polymeric compound, $\mathrm{C}_{4} \mathrm{H}_{6}$, homologous with marsh-gas (methylole or formène, $\mathrm{C} \mathrm{H}_{2}$ or rather $\mathrm{C}_{2} \mathrm{H}_{4}$ ): while the gas produced in the electrolysis of acetic acid is the true methyle, $\mathrm{C}_{2} \mathrm{H}_{3}$. The former may be supposed to be produced by the coalescence of 2 atoms of the latter into one double molecule. The same remarks apply to ethyle, and the other radicals homologous with methyle, and will not, therefore, need to be repeated when treating of these.

When zincethyle, $\mathrm{C}_{4} \mathrm{H}_{5} \mathrm{Zn}$, a body to be described hereafter, is acted on by water, there is formed oxide of zinc, and a gas is 
given off, which, as already mentioned, is either methyle, $\mathrm{C}_{2} \mathrm{H}_{3}$, or the polymeric body, $\mathrm{C}_{4} \mathrm{H}_{6}$, most probably the latter. Its formation is very simple, $\mathrm{C}_{4} \mathrm{H}_{5} \mathrm{Zn}+\mathrm{H} \mathrm{O}=\mathrm{ZnO}+2\left(\mathrm{C}_{2} \mathrm{H}_{3}\right)$; or $\mathrm{C}_{4} \mathrm{H}_{5} \mathrm{Zn}+\mathrm{H} \mathrm{O}=\mathrm{Zn} \mathrm{O}_{3}+\mathrm{C}_{4} \mathrm{H}_{6}$. If we adopt the latter formula, we may regard it as hyduret of ethyle, $\mathrm{C}_{4} \mathrm{H}_{5}, \mathrm{H}$; and the corresponding compound of methyle, which is formed when zincomethyle is acted on by water, will be $\mathrm{C}_{2} \mathrm{H}_{4}$. Its formation will be as follows: $\mathrm{C}_{2} \mathrm{H}_{3} \mathrm{Zn}+\mathrm{H} \mathrm{O}=\mathrm{ZnO}+2\left(\mathrm{CH}_{2}\right)$; or $\mathrm{C}_{2} \mathrm{H}_{3} \mathrm{Zn}+\mathrm{H} \mathrm{O}=\mathrm{C}_{2} \mathrm{H}_{4}$. The latter formula will become $\mathrm{C}_{2} \mathrm{H}_{3}, \mathrm{H}$, or hyduret of methyle. Adopting this view, we have the two parallel and polymeric series, in each of which the members are homologous :-

$\begin{array}{llll} & \mathrm{C}_{2} \mathrm{H}_{4}=\mathrm{C}_{2} \mathrm{H}_{3}, \mathrm{H}, \text { Hyduret of Methyle. } \\ \mathrm{C}_{2} \mathrm{H}_{3} \text { Methyle } & \mathrm{C}_{4} \mathrm{H}_{6}=\mathrm{C}_{4} \mathrm{H}_{5}, \mathrm{H}, \text { Hyduret of Ethyle. } \\ \mathrm{C}_{4} \mathrm{H}_{5} \text { Ethyle } & \mathrm{C}_{8} \mathrm{H}_{10}=\mathrm{C}_{8} \mathrm{H}_{9}, \mathrm{H}, \text { Hyduret of Butyle. } \\ \mathrm{C}_{6} \mathrm{H}_{7} \text { Propyle } & \mathrm{C}_{12} \mathrm{H}_{14}=\mathrm{C}_{12} \mathrm{H}_{13}, \mathrm{H}, \text { Hyduret of Caproyle, }\end{array}$

and so on. It is highly probable that in each case both compounds exist, except in the first hyduret, that of methyle, which has no corresponding polymeric body in the first column, since such a compound would be $\mathrm{C} \mathrm{H}_{1}$, an impossible formula.

Having now explained the relations of methyle, we proceed to its compounds.

\section{Oxide of Methyle. $\mathrm{C}_{2} \mathrm{H}_{3} \mathrm{O}=\mathrm{Me} \mathrm{O}$.}

This compound is obtained, like ether (oxide of ethyle) when pyroxilic spirit, which is the hydrated oxide of methyle, is distilled with its own volume of oil of vitriol; it escapes as an inflammable gas. Like oxide of ethyle, it is a base and neutralises acids. It even forms a neutral sulphate, more easily than oxide of ethyle. It is worthy of notice, that oxide of methyle is polymeric with alcohol; for $\mathrm{C}_{4} \mathrm{H}_{8} \mathrm{O}_{2}=2\left(\mathrm{C}_{2} \mathrm{H}_{3} \mathrm{O}\right)$ : so that these two bodies have the same composition, in 100 parts; that is, the same relative proportions of the same elements, but a very different absolute amount : the equivalent of alcohol being twice as heavy as that of oxide of methyle. The constitution of these compounds, moreover, is different, for one is a hydrate, $\mathrm{C}_{4} \mathrm{H}_{5} \mathrm{O}+$ $\mathrm{H} 0$; while the other is an anhydrous oxide, $\mathrm{C}_{2} \mathrm{H}_{3} \mathrm{O}$.

\section{Hydrated Oxide of Methyle. $\mathrm{C}_{2} \mathrm{H}_{3} \mathrm{O}, \mathrm{H} \mathrm{O},=\mathrm{Me} \mathrm{O}, \mathrm{H} \mathrm{O}$.}

Srn. Methylic Alcohol. Pyroxilic Spirit. This compound is one of the chief products of the destructive distillation of wood, and is found in the watery portion along with acetic acid, 
acetone, acetate of oxide of methyle, and several other ethereal liquids, besides portions of the oily matter of the tar dissolved in them.

By rectification with chloride of calcium, the pyroxilic spirit which combines with that salt, is separated from several other liquids which distil over in the heat of the vapour-bath. The residue, if mixed with its own bulk of water, and again heated in the vapour-bath, now gives off the pyroxilic spirit, which is still mixed with water. It is purified by rectification with quicklime, which also destroys any acetate of methyle that may be present.

Pure hydrated oxide of methyle is a liquid very similar to alcohol, having the same density and the same degree of inflammability. Its odour is peculiar and ethereal. It boils at about $140^{\circ}$ or $150^{\circ}$.

When heated with peroxide of manganese, water and sulphuric acid, it yields various products, among which the chief are, formic acid and formomethylal. It is decomposed by nitric acid, yielding oxalic acid, and by chlorine, yielding new products. It dissolves resins, and is used in making varnishes. It forms with baryta a crystalline compound $\mathrm{BaO}+\mathrm{Me} \mathrm{O}, \mathrm{H} \mathrm{O}$; and, with chloride of calcium, another crystalline body in large hexagonal tables, $\mathrm{CaCl}+2(\mathrm{Me} \mathrm{O}, \mathrm{H} \mathrm{O})$.

By the action of platinum powder, pyroxilic spirit is oxidised into formic acid, which bears the same relation to it that acetic acid does to alcohol.

Methylic alcohol is the true type and starting-point of the series of alcohols to which it belongs, although common or ethylic alcohol has been longer known, and is the body from which the series (column 3 of the table) is named.

As is always the case with two contiguous members of a homologous series, the properties of the two alcohols, ethylic and methylic, are in the highest degree analogous. The chief differences are in the boiling-point and the density of the vapour, which are both higher in ethylic than in methylic alcohol. The boiling-points differ by $34^{\circ} \mathrm{F}$. All the other properties are so similar, that methylic alcohol is used for most of the purposes for which we employ alcohol ; for spirit lamps, as a solvent for resins, \&c. ; and recently government has permitted alcohol, mixed with $\frac{1}{10}$ th part of methylic alcohol, under the name of methylated spirit, to be used, free of duty, in various manufactures. This addition of methylic alcohol does not interfere with the solvent power of the alcohol, and has only the effect of making it so unpalatable that it cannot be used as a drink, the taste of methylic alcohol being unpleasant. 
3. Chloride of Methyle, $\mathrm{C}_{2} \mathrm{H}_{3}, \mathrm{Cl}=\mathrm{Me} \mathrm{Cl}$, is a gas of an ethereal smell, inflammable, of Sp. G. 1.1737. It is formed by the action of sulphuric acid and chloride of sodium on pyroxilic spirit. By the action of chlorine, aided by the sun's rays, it yields several new compounds containing chlorine.

4. Iodide of Methyle, $\mathrm{C}_{2} \mathrm{H}_{3}, \mathrm{I}=\mathrm{Me} \mathrm{I}$, is obtained by distilling 12 parts of pyroxilic spirit, 8 of iodine, and 1 of phosphorus. It is a liquid boiling between $102^{\circ}$ and $122^{\circ}$. The chloride and iodide of methyle are now used in researoh.

5. Sulphuret of Methyle, $\mathrm{C}_{2} \mathrm{H}_{3} \mathrm{~S}=\mathrm{Me} \mathrm{S}$, is best formed by the action of a current of chloride of methyle on sulphuret of potassium dissolved in alcohol. $\mathrm{Me} \mathrm{Cl}+\mathrm{KS}=\mathrm{Me} \mathrm{S}+\mathrm{K} \mathrm{Cl}$. It is a mobile liquid, of a very offensive alliaceous odour, boiling at $104^{\circ}$. Its $\mathrm{Sp}$. G. in the liquid form is 0.845 ; in the form of vapour, it is $2 \cdot 115$. With chlorine it gives rise to several new compounds. The hydrosulphuret of sulphuret of methyle (corresponding to mercaptan) I obtained by distilling the double sulphate of methyle and potash with the hydrosulphuret of sulphuret of potassium. (K $0, \mathrm{Me} \mathrm{O}_{2} 2 \mathrm{~S} \mathrm{O}_{3}$ ) $+\mathrm{H} \mathrm{S}, \mathrm{K} \mathrm{S}=2$ $\left(\mathrm{K} \mathrm{O}_{3} \mathrm{~S} \mathrm{O}_{3}\right)+\mathrm{H} \mathrm{S}, \mathrm{Me} \mathrm{S}$. Its formula is $\mathrm{H} \mathrm{S}, \mathrm{Me} \mathrm{S}=\mathrm{C}_{2} \mathrm{H}_{3}$, $\mathrm{S}+\mathrm{H} \mathrm{S}$. It is a colourless liquid, lighter than water, which boils at $70^{\circ}$, and acts on the oxides of mercury and lead exactly as mercaptan,does. Its odour is most offensive, resembling that of leeks highly concentrated.

6. Cyanide of Methyle. Sxn. Acetonitryle. $\mathrm{C}_{4} \mathrm{H}_{3} \mathrm{~N}=\mathrm{C}_{2} \mathrm{H}_{3}$, $\mathrm{C}_{2} \mathrm{~N}=\mathrm{Me} \mathrm{Cy}$, is formed when the double sulphate of potash and methyle is distilled with cyanide or ferrocyanide of potassium. It is purified by digestion with red oxide of meroury, and rectifcation. It is a colourless, mobile, volatile liquid, of a slightly alliaceous, penetrating smell, analogous to that of putrid fish, and somewhat stupifying. It is very inflammable. When heated with potash, it yields ammonia and acetate of potash-

$$
\mathrm{C}_{4} \mathrm{H}_{3} \mathrm{~N}+\mathrm{KO}+3 \mathrm{HO}=\mathrm{NH}_{3}+\mathrm{KO}, \mathrm{C}_{4} \mathrm{H}_{3} \mathrm{O}_{3} \text {. }
$$

It has lately been discovered that cyanide of methyle may be obtained by the action of heat, aided by anhydrous phosphoric acid, on acetate of ammonia.

$$
\mathrm{C}_{4} \mathrm{H}_{3} \mathrm{O}_{3}, \mathrm{~N} \mathrm{H}_{4} \mathrm{O}+\mathrm{PO}_{5}=\mathrm{PO}_{5}, 4 \mathrm{HO}+\mathrm{C}_{4} \mathrm{H}_{3} \mathrm{~N} \text {. }
$$

Here we see the cyanide of methyle formed from a compound in which no methyle is present; but, according to the usual view, acetyle, $\mathrm{C}_{4} \mathrm{H}_{3}$, a radical derived by oxidation or dehydrogenation from ethyle. If we could obtain the oxide of methyle, or its hydrate, from the cyanide, then, as we can obtain the cyanide 
from acetate of ammonia, we should be able to form the yet unknown alcohols, analogous with pyroxilio spirit and alcohol, from the volatile acids of the formic and acetic series, of which so many are known, while of the alcohols we know very few as yet. We should, then, from acetic acid obtain hydrated oxide of methyle, or methylic alcohol, from propylic acid ordinary alcohol, from butyric acid propylic alcohol, and so on. But we cannot yet produce methylic alcohol from the cyanide of methyle, although it is probable we shall soon find the means of doing so.

It may here be noticed that the liquids formed by heating the ammonia salts of acetic acid and its homologues with anhydrous phosphoric acid, were at first supposed to be a new class of compounds, and were called nitryles, as explained in the description of the table of homologous compounds. The above liquid, $\mathrm{C}_{4} \mathrm{H}_{3} \mathrm{~N}$, was called acetonitryle, because it contains acetyle, $\mathrm{C}_{4} \mathrm{H}_{3}$, and nitrogen. But it has been found to be identical with cyanide of methyle, and to yield cyanide of potassium, when acted on by that metal, which almost proves it to contain cyanogen. It is not certain, however, that there may not exist a class of nitryles, which are not identical, but only isomeric with the cyanides. The reader will observe, with the aid of the table just referred to, that these remarks apply not only to methyle and acetic acid, but to their homologues.

\section{SALTS OF OXIDE OF METHYLE.}

1. Neutral Sulphate. $\mathrm{Me} \mathrm{O}, \mathrm{S} \mathrm{O}$, is obtained when pyroxilic spirit is distilled with a large excess of sulphuric acid. It forms an oily liquid, of a slightly alliaceous smell. It boils at $370^{\circ}$. Boiling water decomposes it into acid sulphate and hydrate of oxide of methyle. When heated with chlorides, cyanides, \&c., it yields the compound of methyle with chlorine, cyanogen, \&c.; with a salt of benzoic, succinic, or other organic acid, it yields benzoate, \&c., of oxide of methyle. Ammonia converts it into sulphamethylane.

When a current of ammonia is made to act on the neutral sulphate of methyle, there is produced a crystalline compound, $\mathrm{C}_{2} \mathrm{H}_{5} \mathrm{~N} \mathrm{~S}_{2} \mathrm{O}_{6}$, which has been called sulphamethylane, and may be viewed as oxamethylane, in which sulphamide, $\mathrm{S} \mathrm{O}_{2}, \mathrm{~N} \mathrm{H}_{2}$, has been substituted for oxamide, $\mathrm{C}_{2} \mathrm{O}_{2}, \mathrm{~N} \mathrm{H}_{2}$; or $\mathrm{S} \mathrm{O}_{2}$ for $\mathrm{C}_{2} \mathrm{O}_{2}$. It may also be considered, if oxamethylane be the oxamate of oxide of methyle, $\mathrm{C}_{2} \mathrm{H}_{3} \mathrm{O}+\mathrm{C}_{4} \mathrm{H}_{2} \mathrm{~N} \mathrm{O}_{5}$, as composed of oxide of methyle and a peculiar acid, formed of hyposulphuric acid and amide, or rather of sulphuric acid and sulphamide, and which may be called sulphamic acid; and its formula will be $\mathrm{C}_{2} \mathrm{H}_{3} \mathrm{O}+$ 
$\left(\mathrm{S}_{2} \mathrm{O}_{5}, \mathrm{~N} \mathrm{H}_{2}\right)$, or $\mathrm{C}_{2} \mathrm{H}_{3} \mathrm{O}+\left(\mathrm{S} \mathrm{O}_{3}+\mathrm{N} \mathrm{H}_{2}, \mathrm{~S} \mathrm{O}_{2}\right)$. On this view, sulphamethylane is the sulphamate of oxide of methyle.

2. Bisulphate of Oxide of Methyle. Srv. Sulphomethylic Acid. $\mathrm{H} \mathrm{O}, \mathrm{Me} \mathrm{O}, 2 \mathrm{~S} \mathrm{O}_{3}$, is perfectly analogous to sulphovinic acid, and forms double salts, such as that of potash, $\mathrm{K} \mathrm{O}, \mathrm{Me} \mathrm{O}, 2 \mathrm{~S} \mathrm{O}_{3}$, which are often called sulphomethylates, and correspond exactly to the sulphovinates. The acid sulphate itself may be obtained in crystals, which are very soluble and very acid. It is best obtained by the action of hot water on the neutral sulphates. The double salts, or sulphomethylates, are of no particular importance. They crystallise readily.

3. Nitrate of Oxide of Methyle, $\mathrm{Me} \mathrm{O}_{2} \mathrm{~N} \mathrm{O}_{5}$, is obtained when pyroxilic spirit is distilled with nitrate of potash and sulphuric acid. It is an oily liquid, the vapour of which if heated beyond $248^{\circ}$ explodes violently.

The neutral carbonate of methyle is not known; but double carbonates, analogous to those of ethyle with alkalies, may be prepared in the same way as those compounds.

4. Carbamate of Oxide of Methyle. Srv. Urethylane, $\mathrm{C}_{4} \mathrm{H}_{5}$ $\mathrm{N} \mathrm{O}_{4}=\mathrm{C}_{2} \mathrm{H}_{3} \mathrm{O}, \mathrm{C}_{2} \mathrm{NH}_{2} \mathrm{O}_{3}$. This compound is formed when chlorocarbonic acid acts on pyroxylic spirit, and the product, $\mathrm{C}_{4} \mathrm{H}_{3} \mathrm{Cl} \mathrm{O}_{4}$, is mixed with ammonia. The oily product first produced, $\mathrm{C}_{4} \mathrm{H}_{3} \mathrm{Cl} \mathrm{O}_{4}$, is a compound of oxide of methyle with an acid, $\left.\mathrm{C}_{2} \mathrm{Cl}_{\mathrm{Cl}}^{\mathrm{O}_{3}}\right\}$, and may be represented thus, $\left.\mathrm{C}_{2} \mathrm{H}_{3} \mathrm{O}, \mathrm{C}_{2} \underset{\mathrm{Cl}}{\mathrm{O}_{3}}\right\}$. When it acts on ammonia, the latter is decomposed, 1 eq. of its hydrogen taking the chlorine of the oil, while the chlorine is replaced by amide, and the resulting beautifully crystalline compound is the carbamate of oxide of methyle, $\left.\mathrm{C}_{2} \mathrm{H}_{3} \mathrm{O}, \mathrm{C}_{2} \mathrm{Cl}_{3}\right\}+\mathrm{N}$ $\left.\mathrm{H}_{3}=\mathrm{H} \mathrm{Cl}+\mathrm{C}_{2} \mathrm{H}_{3} \mathrm{O}, \mathrm{C}_{2} \underset{\mathrm{NH}_{2}}{\mathrm{O}_{3}}\right\}$. This last group, $\mathrm{C}_{2} \underset{\mathrm{N} \mathrm{H}_{2}}{\mathrm{O}}$ is bicarbamide, or carbamic acid, an acid amidide. The carbamate is isomeric with glycocine.

5. Oxalate of Oxide of Methyle, $\mathrm{Me} \mathrm{O}_{2} \mathrm{C}_{2} \mathrm{O}_{3}=\mathrm{C}_{4} \mathrm{H}_{3} \mathrm{O}_{4}$, is obtained in a manner analogous to that in which oxalic ether is prepared. It forms a crystalline solid, soluble in alcohol and pyroxilic spirit, which deposit it in large crystals. By the action of dry ammonia, it is converted into oxamethylane (analogous to oxamethane), which is the oxamate of oxide of methyle, $\mathrm{C}_{6} \mathrm{H}_{5} \mathrm{~N}$ $\mathrm{O}_{6}=\mathrm{C}_{2} \mathrm{H}_{3}, \mathrm{O}+\mathrm{C}_{4} \mathrm{H}_{2} \mathrm{~N} \mathrm{O}_{5}$. Liquid ammonia converts it into oxamide, as is the case with oxalic ether, only here methylic and not ethylic alcohol is formed at the same time. $\mathrm{C}_{2} \mathrm{H}_{3}, \mathrm{O}, \mathrm{C}_{2} \mathrm{O}_{3}$ $+\mathrm{NH}_{3}=\mathrm{C}_{2} \mathrm{H}_{3} \mathrm{O}, \mathrm{H} \mathrm{O}+\mathrm{C}_{2} \mathrm{O}_{2} \mathrm{NH}_{2}$. This is perhaps the easiest way of obtaining oxamide in large quantity. 
Bisulphuret of carbon acid acts on pyroxilic spirit exactly as on alcohol, producing analogous compounds. (See sulphocarbonate of oxide of ethyle.)

6. Benzoate of Oxide of Methyle, $\mathrm{Me} \mathrm{O}, \mathrm{Bz} \mathrm{O}$, is best obtained by distilling dry benzoate of lime or soda with neutral sulphate of methyle. It is an oily liquid of a balsamic odour, analogous in other respects to benzoic ether.

7. Acetate of Oxide of Methyle, $\mathrm{Me} \mathrm{O}, \mathrm{Ac}_{3}$, is obtained in the same way as acetic ether, which it resembles. It occurs in considerable quantity in raw pyroxilic spirit, and even in that which has only been purified by rectification. When quicklime is used in the rectification, it is destroyed, yielding an additional quantity of the pure hydrate of oxide of methyle. It is very volatile and inflammable, and for most purposes its presence in the wood spirit is not at all injurious. It is isomeric with formiate of oxide of ethyle : for, $\mathrm{C}_{2} \mathrm{H}_{3} \mathrm{O}+\mathrm{C}_{4} \mathrm{H}_{3} \mathrm{O}_{3}=\mathrm{C}_{4} \mathrm{H}_{5} \mathrm{O}+\mathrm{C}_{2} \mathrm{H} \mathrm{O}_{3}$.

8. Salicylate of Oxide of Methyle, $\mathrm{C}_{26} \mathrm{H}_{8} \mathrm{O}_{6}=\mathrm{C}_{2} \mathrm{H}_{3} \mathrm{O}, \mathrm{C}_{44} \mathrm{H}_{5}$ $\mathrm{O}_{5}$, exists ready formed, as the volatile oil of Gaultheria procumbens, or Wintergreen. It is very fragrant, and plays the part of an acid, combining with alkalies. When mixed with them, however, salicylates are formed, and pyroxilic spirit set free. It is a very interesting compound, as it contains two substances previously only known as artificial products; namely, salicylic acid and oxide of methyle.

9. Phenomethole. SrN. Anisole: Carbolate of Oxide of Methyle. $\mathrm{C}_{14} \mathrm{H}_{8} \mathrm{O}_{2}=\mathrm{C}_{2} \mathrm{H}_{3} \mathrm{O}, \mathrm{C}_{22} \mathrm{H}_{5} \mathrm{O}$. This compound, apparently homologous with hydrated carbolic acid, $\mathrm{C}_{12} \mathrm{H}_{6} \mathrm{O}_{2}$, is obtained by heating with baryta the preceding compound, or anisic acid, $\mathrm{C}_{26} \mathrm{H}_{7} \mathrm{O}_{5}, \mathrm{H} \mathrm{O}$, which is isomeric with it. $\mathrm{C}_{26} \mathrm{H}_{8} \mathrm{O}_{6}$ $+2 \mathrm{BaO}=2\left(\mathrm{BaO}, \mathrm{C} \mathrm{O}_{2}\right)+\mathrm{C}_{14} \mathrm{H}_{8} \mathrm{O}_{2}$. Cahours has shown that this compound, which bears to anisic acid the same relation as phenole or carbolic acid does to salicylic acid, is really the carbolate of oxide of methyle, or hydrated carbolic acid, in which 1 eq. of water is replaced by 1 eq. of oxide of methyle. It is, therefore, not truly homologous with carbolic acid, but is a compound of that acid with oxide of methyle, and is consequently the first member of a series of homologous compounds, thus,

1. Phenomethole $\mathrm{C}_{14} \mathrm{H}_{8} \mathrm{O}_{2}=\mathrm{C}_{2} \mathrm{H}_{3} \quad \mathrm{O}, \mathrm{C}_{12} \mathrm{H}_{5} \mathrm{O}$.

2. Phenethole $\mathrm{C}_{16} \mathrm{H}_{10} \mathrm{O}_{2}=\mathrm{C}_{4} \mathrm{H}_{5} \mathrm{O}, \mathrm{C}_{12} \mathrm{H}_{5} \mathrm{O}$.

3. Phenopropylole $\mathrm{C}_{18} \mathrm{H}_{12} \mathrm{O}_{2}=\mathrm{C}_{6} \mathrm{H}_{7} \mathrm{O}, \mathrm{C}_{12} \mathrm{H}_{5} \mathrm{O}$.

4. Phenobutylole $\mathrm{C}_{20} \mathrm{H}_{14} \mathrm{O}_{2}=\mathrm{C}_{8} \mathrm{H}_{9} \mathrm{O}, \mathrm{C}_{12} \mathrm{H}_{5} \mathrm{O}$.

5. Phenamylole $\mathrm{C}_{22} \mathrm{H}_{18} \mathrm{O}_{2}=\mathrm{C}_{10} \mathrm{H}_{11} \mathrm{O}, \mathrm{C}_{12} \mathrm{H}_{5} \mathrm{O}$.

The compounds Nos. 3 and 4 have perhaps not yet been formed, 
but as propylic and butylic alcohols and ethers are now known, their formation may be with certainty predicted. The names given in the table are analogous to that of phenole (carbolic acid) with which these compounds are apparently or empirically homologous, as carbolic acid differs from No. 1 by $\mathrm{C}_{2} \mathrm{H}_{2}$. These compounds, however, cannot be truly homologous with carbolic acid, because they are not acids but neutral ethers, and their true names are, in full, carbolate of oxide of methyle, or of ethyle, propyle, butyle, amyle, and so on.

When salicylate of oxide of methyle is acted on by nitric acid, it yields nitrosalicylate of oxide of methyle, $\mathrm{C}_{2} \mathrm{H}_{3} \mathrm{O}$, $\left.\mathrm{C}_{14}{\underset{\mathrm{NO}}{4}}_{\mathrm{NO}_{4}}^{\mathrm{H}_{4}}\right\} \mathrm{O}_{5}$, in yellow silky prisms. With ammonia, this compound forms, like the corresponding ethyle compound (which see), nitrosalicylamide.

The mucate of oxide of methyle is a crystalline solid, analogous in its preparation and properties to the corresponding salt of ethyle.

10. Cyanate of Oxide of Methyle, $\mathrm{C}_{4} \mathrm{~N} \mathrm{H}_{3} \mathrm{O}_{2}=\mathrm{C}_{2} \mathrm{H}_{3} \mathrm{O}, \mathrm{C}_{2} \mathrm{~N}, \mathrm{O}$. This compound was obtained by Wurtz, by distilling cyanate of potash with sulphomethylate of potash (double sulphate of methyle and potash). It is a volatile liquid, of a very penetrating smell. With ammonia it yields a crystalline product, $\mathrm{C}_{4} \mathrm{~N}_{2} \mathrm{H}_{6} \mathrm{O}_{2}$, homologous with urea, and which may be called methylo-urea, since it is urea, in which 1 eq. of hydrogen is replaced by 1 eq. of methyle. Cyanate of oxide of methyle, in contact with water, is resolved into carbonic acid and a new compound, dimethylo-urea, isomeric with ethylo-urea,

$$
2\left(\mathrm{C}_{4} \mathrm{~N} \mathrm{H}_{3} \mathrm{O}_{2}\right)+2 \mathrm{HO}=2 \mathrm{CO}_{2}+\mathrm{C}_{6} \mathrm{~N}_{2} \mathrm{H}_{8} \mathrm{O}_{2} \text {. }
$$

Here we have another series of homologous compounds, not included in the table; but, indeed, every compound of methyle, and every derivative from it yet known, is, in like manner, the starting-point of a homologous series, and it will be seen that the table, large though it is, admits of enormous extension horizontally.

11. Allophanate of Oxide of Methyle, $\mathrm{C}_{6} \mathrm{~N}_{2} \mathrm{H}_{6} \mathrm{O}_{6}$. When the vapours of cyanic acid are brought in contact with pyroxilic spirit, this compound is formed. It is $\mathrm{C}_{2} \mathrm{H}_{3} \mathrm{O}+\mathrm{C}_{4} \mathrm{~N}_{2} \mathrm{H}_{3} \mathrm{O}_{5}$. The latter group is allophanic acid, the production of which will be explained further on, under ethyle.

12. Cyanurate of Oxide of Methyle, $\mathrm{C}_{12} \mathrm{~N}_{3} \mathrm{H}_{9} \mathrm{O}_{6}=3 \mathrm{C}_{2} \mathrm{H}_{3} \mathrm{O}$, $\mathrm{C}_{6} \mathrm{~N}_{3} \mathrm{O}_{3}$. This compound, according to Wurtz, is formed when sulphomethylate of potash is heated with cyanurate of potash. It is a crystalline solid, fusible at $284^{\circ}$, and volatile at $563^{\circ}$. It is insoluble in water, soluble in alcohol and ether. 
Many other salts of oxide of methyle might be enumerated did space permit; but those already given suffice to show that oxide of methyle replaces the water of hydrated acids, just as oxide of potassium does; or rather, that methyle replaces the hydrogen of hydrated or of hydrogen acids, as metals do, yielding neutral compounds.

Before proceeding to the products derived by oxidation or other modes of decomposition from oxide of methyle or pyroxilic spirit, it may be as well here to point out the existence of compounds, in which methyle replaces the hydrogen of ammonia, without destroying its basic characters, compounds which are among the most remarkable which have been of late years discovered, and the discovery of which has very greatly added to our knowledge of the organic bases. They are here introduced, because the first of them was obtained from some of the salts described in the immediately preceding paragraphs, namely, from the cyanate of oxide of methyle, from the methylo-urea, and from the cyanurate, polymeric with the cyanate.

1. Methylamine. $\left.\mathrm{C}_{2} \mathrm{H}_{5} \mathrm{~N}=\mathrm{N} \underset{\mathrm{Me}}{\mathrm{H}_{2}}\right\}$ : According to Wurtz, this compound is obtained by heating cyanate of oxide of methyle with potash. Bearing in mind that hydrated cyanic acid (from which this cyanate only differs in containing 1 eq. methyle, $\mathrm{C}_{2} \mathrm{H}_{3}$ in place of 1 eq. hydrogen) is decomposed by potash, yielding carbonate of potash and ammonia, this change is readily explained by the following equations :

$\mathrm{C}_{2} \mathrm{~N} \mathrm{O}, \mathrm{HO}+2(\mathrm{~K} \mathrm{O}, \mathrm{H} \mathrm{O})=2\left(\mathrm{C} \mathrm{O}_{2}, \mathrm{KO}\right)+\mathrm{N} \mathrm{H}_{3}$

Hydrated Cyanic Acid.

$\left.\mathrm{C}_{2} \mathrm{~N} \mathrm{O}, \mathrm{Me} \mathrm{O}+2(\mathrm{~K} \mathrm{O}, \mathrm{HO})=2\left(\mathrm{C} \mathrm{O}_{2}, \mathrm{~K} \mathrm{O}\right)+\mathrm{N}_{\mathrm{Me}}^{\mathrm{H}_{2}}\right\}$

Cyanate of Oxide of Methyle.

Methylamine.

Since the cyanurate of oxide of methyle, which, as we have seen, is tribasic, is polymeric with the cyanate, its equivalent being exactly 3 times greater, we have only to multiply the last equation by 3 to explain the production of methylamine from the cyanurate. From methylo-urea it is obtained as follows, comparing the action with that of urea :

$$
\begin{aligned}
& \underbrace{\mathrm{C}_{2} \mathrm{~N}_{2} \mathrm{H}_{4} \mathrm{O}_{2}}+2(\mathrm{~K} \mathrm{O}, \mathrm{H} \mathrm{O})=2\left(\mathrm{C} \mathrm{O}_{2}, \mathrm{~K} \mathrm{O}\right)+\mathrm{N} \mathrm{H}_{3}+\mathrm{N} \mathrm{H}_{3} \\
& \text { Urea. } \\
& \left.\mathrm{C}_{4} \mathrm{~N}_{2} \mathrm{H}_{6} \mathrm{O}_{2}+2(\mathrm{~K} \mathrm{O}, \mathrm{H} \mathrm{O})=2\left(\mathrm{C} \mathrm{O}_{2}, \mathrm{~K} \mathrm{O}\right)+\mathrm{N} \mathrm{H}_{3}+\mathrm{N}_{\mathrm{Me}}^{\mathrm{H}_{2}}\right\}
\end{aligned}
$$


These equations show, that, as in the compounds employed, hydrogen has been replaced by methyle, $\mathrm{C}_{2} \mathrm{H}_{3}$, so in the products the same substitution continues. And if we regard ammonia as the hyduret of amide, $\mathrm{N} \mathrm{H}_{2}, \mathrm{H}$, then methylamine is methylamide, $\mathrm{N} \mathrm{H}_{2}, \mathrm{Me}=\mathrm{N} \mathrm{H}_{2}, \mathrm{C}_{2} \mathrm{H}_{3}=\mathrm{C}_{2} \mathrm{H}_{5} \mathrm{~N}$.

Methylamine has also been observed among the products of decomposition of several organio compounds, such as morphine, codeine, caffeine, \&c., and is probably a constituent of many natural products. It is found also in the oil obtained by distillation from animal matter.

Methylamine is a condensible gas, of Sp. G. 1.075. At or a little below $32^{\circ}$, it forms a mobile liquid. It has a very pungent smell, so like that of ammonia, that it has certainly been frequently taken for ammonia, when accidentally formed, and thus overlooked. It is, of all known gases, the most soluble in water. At $50^{\circ}$ water takes up about 1150 times its volume of the gas. The smell of the gas and of its solution, although closely resembling that of ammonia, has yet something empyreumatic and peculiar. Methylamine is distinguished from ammonia by the property of kindling at a candle, and burning with a yellowish flame. Ammonia can only be kindled in oxygen gas.

Methylamine is a very powerful base. It neutralises the strongest acids, and forms thick white vapours with the volatile acids, like ammonia. It acts on metallic solutions as, for example, those of silver and copper, exactly like ammonia. The salts of methylamine are closely analogous to those of ammonia, and the bichlorides of platinum and mercury, and the terchloride of gold, form double salts, resembling the corresponding compounds of ammonia. Nay, when methylamine acts on protochloride of platinum, it gives rise to a series of bases perfectly homologous with those described under ammonia, but which our limited space forbids us to describe in detail.

When sulphate of methylamine is evaporated along with cyanate of potash, methylo-urea is obtained. It would appear that the body thus obtained has all the properties of a urea, and that the compound already described under that name is perhaps only an isomeric modification of it.

When heated strongly, methylamine is decomposed, yielding cyanide of ammonium, hydrocyanic acid, marsh-gas, and hydrogen. Thus:

$$
3\left(\mathrm{C}_{2} \mathrm{H}_{5} \mathrm{~N}\right)=\mathrm{N} \mathrm{H}_{4}, \mathrm{C}_{2} \mathrm{~N}+\mathrm{H}, \mathrm{C}_{2} \mathrm{~N}+\mathrm{C}_{2} \mathrm{H}_{4}+\mathrm{H}_{8} .
$$

Methyloxamide, $\mathrm{C}_{4} \mathrm{~N} \mathrm{H}_{4} \mathrm{O}_{2}$. When the oxalate of methylamine, $\mathrm{C}_{2} \mathrm{H}_{5} \mathrm{~N}, \mathrm{H} \mathrm{O}, \mathrm{C}_{2} \mathrm{O}_{3}$, is heated, it yields methyloxamide, 
homologous with oxamide. But this compound, like oxamide, is better obtained by the action of the base itself on oxalic ether.

$$
\underbrace{\mathrm{C}_{4} \mathrm{H}_{5} \mathrm{O}, \mathrm{C}_{2} \mathrm{O}_{3}}_{\text {Oxalic Ether. }}+\mathrm{C}_{2} \mathrm{H}_{5} \mathrm{~N}=\mathrm{C}_{4} \mathrm{H}_{6} \mathrm{O}_{2}+\mathrm{C}_{4} \mathrm{~N} \mathrm{H} \mathrm{H}_{4} \mathrm{O}_{2} .
$$

Methyloxamic acid, $\mathrm{C}_{6} \mathrm{~N} \mathrm{H}_{4} \mathrm{O}_{5}, \mathrm{H} \mathrm{O}$, is obtained, like oxamic acid, by heating the binoxalate of the base.

$$
\underbrace{\mathrm{C}_{2} \mathrm{H}_{5} \mathrm{~N}, \mathrm{H} \mathrm{O}, \mathrm{C}_{2} \mathrm{O}_{3}+\mathrm{C}_{2} \mathrm{O}_{3}, \mathrm{H} \mathrm{O}}_{\text {Binoxalate of Methylamine. }}=2 \mathrm{H} \mathrm{O}+\underbrace{\mathrm{C}_{6} \mathrm{~N} \mathrm{H}_{4} \mathrm{O}_{5}, \mathrm{H} \mathrm{O}}_{\text {Methyloxamic } \mathrm{Acid}} .
$$

These two products are homologous with, and perfectly analogous to, oxamide and oxamic acid. Methyloxamide, however, is soluble in water.

It is hardly necessary to add, that for every neutral amide, such as oxamide, benzamide, \&o., as well as for every acid amide, such as oxamic acid, carbamic acid, \&c., we may expect to have a corresponding compound, in which 1 eq. of hydrogen is replaced by methyle, or, what is the same thing, amidogen, $\mathrm{N}, \mathrm{HH}$, is replaced by methylamidogen, $\mathrm{N}, \mathrm{H} \mathrm{Me}$.

Methylamine, when acted on by chlorine, bromine, and iodine, yields substitution products, which as yet have been little studied. Those of the corresponding base, ethylamine, appear to have lost the basic character, as will be explained further on. Here it is only necessary to state that methylamine does yield similar products.

We have seen that methylamine is the first member of a series of bases, homologous with ammonia; and we may even regard ammonia itself as an organic base, and the true starting-point of the series, as hydrogen is of the series of radicals to which methyle belongs. The series will be seen in the last column of the table of homologous compounds, and it will be observed that, viewed as the starting-point of that series, ammonia is to be represented as hydramide, $\mathrm{N} \mathrm{H}_{2} \mathrm{H}$; and that it is this last atom of bydrogen which is replaced by methyle and its congeners, so that we have

$$
\begin{aligned}
& \text { Ammonia } \quad \mathrm{H}_{3} \mathrm{~N}=\mathrm{N} \mathrm{H}_{2}, \mathrm{H} \\
& \text { Methylamine } \mathrm{C}_{2} \mathrm{H}_{5} \mathrm{~N}=\mathrm{N} \mathrm{H}_{2}, \mathrm{Me} \\
& \text { Ethylamine } \mathrm{C}_{4} \mathrm{H}_{7} \mathrm{~N}=\mathrm{N} \mathrm{H}_{2}, \mathrm{Ae} \text { \& \&. }
\end{aligned}
$$

At each step $\mathrm{C}_{2} \mathrm{H}_{2}$ is added-or, what is the same thing, in each base, amide, $\mathrm{N} \mathrm{H}_{2}$, is combined with methyle, $\mathrm{C}_{2} \mathrm{H}_{3}$, ethyle, $\mathrm{C}_{4}$ $\mathrm{H}_{3}$, \&c., \&c., these radicals differing by $\mathrm{C}_{2} \mathrm{H}_{2}$. This may be called the first series of bases, homologous with ammonia, or amide bases, since in them we have amide, $\mathrm{N} \mathrm{H}_{2}$, plus the 
radicals. The general formula of this series is either, Ad. (amide) $+\mathrm{C}_{n} \mathrm{H}_{n}+1$; or $\mathrm{Cn}_{n} \mathrm{H}_{n}+3 \mathrm{~N}$. We shall see that a considerable number are known of this first or methylic series of amide bases, as well as several of a second or phenylio series also of amide bases, of which aniline (phenylamine) is the type. And doubtless other series will be discovered, still of amide bases.

But there are already known some series of bases, homologous with ammonia, yet not amide bases, for our knowledge of which we are indebted to Hofmann. The first of these is that of the imide bases of the methylio radicals. The name imide is given to the compound $\mathrm{N} \mathrm{H}$, or amide minus hydrogen, which exists, combined, in certain derivatives of ammonia.

Now, to understand the formation of imide bases, ammonia must be viewed as $\mathrm{NH}, \mathrm{H} H$; the group $\mathrm{NH}$ being imide; and we have then to replace the 2 eqs. of hydrogen beyond it by methyle, ethyle, \&c. This may be done in the case of methyle by causing bromide of methyle to act on methylamine.

$$
\mathrm{Me} \mathrm{Br}+\mathrm{N} H, \mathrm{H}, \mathrm{Me}=\mathrm{H} \mathrm{Br}+\mathrm{N} \text { H, Me, Me. }
$$

2. Dimethylamine.-The new base is called dimethylamine. It has not yet been studied, but the corresponding ethyle base, diethylamine, has been obtained, and will be described under that head. Its formula must be $\mathrm{C}_{4} \mathrm{H}_{7} \mathrm{~N}$, and it is isomeric with ethylamine.

Hofmann has, however, obtained and described some other imide bases, in which, while the first eq. of hydrogen is replaced by methyle, the second is replaced by a different radical, such as ethyle or phenyle. Of these the best known, as yet, is Methylaniline or Methylophenylamine, $\mathrm{N} \mathrm{H}, \mathrm{Ph} \mathrm{Me}$, which is an imide base, formed from methylamine by the substitution of 1 eq. phenyle, $\mathrm{C}_{12} \mathrm{H}_{5}$, for 1 eq. of hydrogen. This base will be described under phenyle. Many similar bases will,no doubt, soon be known.

3. Trimethylamine. - But this is not all, for Hofmann has shown that a third series of bases, homologous with ammonia, may be obtained, in which the third eq. of hydrogen is also replaced by a radical of the methylic class, or of any other similar class. These are called nitryle bases, to imply that in them only the nitrogen of the ammonia is left. Ammonia may be viewed as $\mathrm{N}+\mathrm{H}, \mathrm{H}, \mathrm{H}$; and if we suppose all these eqs. of hydrogen replaced by methyle we have trimethylamine, $\mathrm{N}+\mathrm{Me}, \mathrm{Me}, \mathrm{Me}$. This base certainly exists, and has been found in the brine of pickled herrings, to which a salt of this base gives its peculiar flavour. The base, in this case, is $\mathrm{C}_{6} \mathrm{H}_{\theta} \mathrm{N}$, and was at first supposed to be propylamine, $\mathrm{NH}_{2}, \mathrm{Pr}=\mathrm{NH}_{2}, \mathrm{C}_{6} \mathrm{H}_{7}$; but it has been shown to be really tri- 
methylamine, which is isomeric with propylamine. Hofmann has since shown that trimethylamine and its salts possess the peculiar flavour observed in the brine above alluded to, in which trimethylamine has probably been formed by a transformation, possibly a kind of putrefaction of some animal compound contained in the fish.

The imide base, methylethylamine, $\mathrm{NH}, \mathrm{Me} \mathrm{Ae}=\mathrm{C}_{6} \mathrm{H}_{9} \mathrm{~N}$, is isomeric with both trimethylamine and propylamine, but is quite distinct from both, although, like them, it is an oily base, analogous to ammonia.

As we rise higher in the series, we shall find that the number of imide and nitryle bases, isomeric with any amide base, steadily increases. The reason of this is, that, since all these classes have the same general formula $\mathrm{Cn}_{n} \mathrm{H}_{n}+3 \mathrm{~N}$, so when the number of eqs. of carbon increases, there must be more ways of making it up by different combinations of radicals differing by 2 eqs. of carbon, or by multiples of two. And as, for each of the three eqs. of hydrogen in ammonia, any radical of the formula $\mathrm{Cn} \mathrm{Hn}+1$ may be substituted, all such combinations must yield $\mathrm{Cn}_{n} \mathrm{H}_{n}+3 \mathrm{~N}$ whatever the amount of $\mathrm{C}$ may be, so long as radicals of the formula $\mathrm{Cn} \mathrm{Hn}+{ }_{1}$ only are used. When radicals of the phenylic series are introduced, this law no longer holds, because in that series the formula of the radicals is $\mathrm{C}_{n} \mathrm{H}_{n}-7$, and that of the bases formed hy these radicals must be $\mathrm{Cn} \mathrm{H}_{n}{ }_{5} \mathrm{~N}$, so long as these radicals alone are used. And of course, where radicals of both series replace the hydrogen of ammonia, the formula must vary, and cannot be reduced to a general one. Ethylophenylamine, for example, which is $\mathrm{NH}, \mathrm{Ae} \mathrm{Ph}$, is $=\mathrm{C}_{16} \mathrm{H}_{10} \mathrm{~N}$, while methylophenylamine, $\mathrm{N} \mathrm{H}, \mathrm{Me} \mathrm{Ph}$, is $\mathrm{C}_{14} \mathrm{H}_{8} \mathrm{~N}$. These two, and others like them, would come under the formula $\mathrm{Cn}_{\mathrm{n}} \mathrm{H}_{n}-{ }_{6} \mathrm{~N}$. But such a base as ethylodiphenylamine, $\mathrm{N} \mathrm{Ae} \mathrm{Ph} P h=\mathrm{C}_{28} \mathrm{H}_{15} \mathrm{~N}$, and its congeners, would require the general formula $\mathrm{Cn}_{n} \mathrm{H}_{n}-13 \mathrm{~N}$; and diethylophenylamine, $\mathrm{N}$, Ae $\mathrm{Ae} \mathrm{Ph}=\mathrm{C}_{20} \mathrm{H}_{15} \mathrm{~N}$, would come under the general formula $\mathrm{C}_{n} \mathrm{H}_{n}-{ }_{5} \mathrm{~N}$, as would also its congeners. But the general formula $\mathrm{Cn}_{\mathrm{n}}+\mathrm{H}_{\mathrm{N}} \mathrm{N}$ will, as has been said above, include all bases, whether amide, imide, or nitryle bases, in which the hydrogen of ammonia is replaced by 1,2 , or 3 equivalents of the radicals of the methylic or ethylic series, $\mathrm{Cn} \mathrm{Hn}+1$. Triethylamine has been described by Hofmann.

There are many other nitryle bases, in which 2 eqs. of hydrogen are replaced by one radical, and 1 eq. by another, or all three by different radicals. Thus methylethylaniline, $\mathrm{N}, \mathrm{Me}, \mathrm{Ae}, \mathrm{Ph}=$ $\mathrm{C}_{18} \mathrm{H}_{13} \mathrm{~N}$, has been obtained, with many others, by Hofmann. These will be described in their proper places.

Thus it will be seen that we are acquainted with three dif- 
ferent forms of substitution bases, derived from ammonia, and all homologous with it. If $R$ represent any one radical, then we have
1. Amide bases.
2. Imide bases.
$\mathrm{N} \mathrm{H}+\mathrm{R}$.
$\mathrm{N} \mathrm{H}+\mathrm{R}$.
3. Nitryle bases.
$N+R R R$.

And if $X, Y, Z$, represent any three radicals, we have
1. Amide bases.
2. Imide bases.
3. Nitryle bases.
$\mathrm{N} \mathrm{H}+\mathrm{X}(\mathrm{Y}$ or $\mathrm{Z})$,
$\mathrm{NH}+\mathrm{X} \mathrm{Y}$ (or Y Z or X Z)
$\mathrm{N}+\mathrm{XYZ}$ or $\mathrm{X} X \mathrm{Z}$ or Y Y Z, \&c.

But even this is not all ; for Hofmann, by studying the action of iodide of methyle on ammonia, has obtained a new base of the most remarkable composition and properties, which is the starting point of a new and very extensive series of homologous compounds and substitution products.

4. Tetramethylium, $\mathrm{N}, \mathrm{Me}_{4}=\mathrm{C}_{8} \mathrm{H}_{12} \mathrm{~N}$, is obtained, in combination with iodine, when iodide of methyle acts on ammonia. Along with it, however, are formed the iodide of ammonium, and the hydriodates of methylamine, dimethylamine, and trimethylamine (which last three compounds may be also called iodides of methylium, dimethylium, and trimethylium). The new compound, like ammonium, cannot be obtained in the separate form, but its iodide crystallises; and when this salt is decomposed by oxide of silver, there is formed, along with the insoluble iodide of silver, the hydrated oxide of tetramethylium, which is soluble, and when evaporated in vacuo, yields a deliquescent crystalline mass, which, like potash, attracts carbonio acid from the air. The oxide is a powerful base, almost caustic, and bitter. Its salts crystallise. This new base, which corresponds to hydrated oxide of ammonium, or ammonia plus 2 eqs. of water, $\mathrm{N} \mathrm{H}_{3}, \mathrm{H} \mathrm{O}+$ $\mathrm{H} \mathrm{O}$, or $\mathrm{N} \mathrm{H}_{4} \mathrm{O}, \mathrm{H} \mathrm{O}$, and to hydrate of potash, $\mathrm{K} \mathrm{O}, \mathrm{H} \mathrm{O}$, has all the characters of a strong alkali; whereas hydrated oxide of ammonium does not differ from ammonia and water. The new base has therefore no exact analogue. It belongs to a series quite distinct from that of methylamine, \&c.; for these all correspond to ammonia, while the radical of this base corresponds to ammonium. Triethylamine, plus water, is a true analogue of ammonia plus water, but in the new base we have new characters. It is not volatile, like methylamine and its congeners, which, like ammonia, refuse to yield the oxides or hydrated oxides of their supposed metals. We should expect, from the analogy with ammonia, that on separating the hydrated oxide of tetramethylium 
from its salts, it would resolve itself into trimethylamine and methylic alcohol, but it does not; it forms a permanent, nonvolatile hydrate. The following table shows the relations of the base to ammonia :-

$\begin{array}{lll}\mathrm{NH}_{3}=\text { Ammonia } & \mathrm{NMe}_{3} & \text { = Methylammonia } \\ & & \text { (Trimethylamine) } \\ \mathrm{N} \mathrm{H}_{4}=\text { Ammonium } & \mathrm{N} \mathrm{Me}_{4} & \text { = Tetramethylium } \\ \mathrm{N} \mathrm{H}_{4} \mathrm{O}=\text { Oxide of ditto. } & \mathrm{N} \mathrm{Me}_{4} \mathrm{O} \text { = Oxide of ditto } \\ \mathrm{N} \mathrm{H}_{4} \mathrm{O}+\mathrm{H} \mathrm{O}=\text { Hydrated Oxide of do. } & \mathrm{N} \mathrm{Me}_{4} \mathrm{O}, \mathrm{HO}=\text { Hydrated Oxide }\end{array}$
of ditto.

This base acts on metallic solutions like potash, and converts oils into soap when boiled with them. The chloride of tetramethylium forms with the chlorides of mercury, gold, and platinum, double salts perfectly analogous to those of potassium and ammonium. Thus,

Double Chloride of Platinum and Ammonium $=\mathrm{N} \mathrm{H}_{4} \mathrm{Cl}+\mathrm{Pt} \mathrm{Cl}_{2}$. Ditto Ditto and Tetramethylium $=\mathrm{N} \mathrm{Me} 4 \mathrm{Cl}+\mathrm{Pt} \mathrm{Cl}_{2}$.

Here, then, we have a totally unexpected new series of compounds, in which the 4 eqs. of hydrogen in ammonium are all replaced by methyle and its homologues, as well as by other radicals, and in these, the supposed metals corresponding to ammonium, although not yet known in the separate state, yet yield what ammonium does not, permanent hydrated oxides. And the 4 eqs. of hydrogen may be replaced by one, by two, by three, or by four different radicals, as will be seen further on.

The best character of the hydrated oxides of these homologues of ammonium is that they are not volatile, but are decomposed by heat, and that they are powerful bases. Their existence leads to the suspicion, that there may be a base, oxide or hydrated oxide of ammonium, different from the hypothetical one, which is simply ammonia plus water. If such a compound exist, it will probably not be volatile without decomposition, and it may possibly be capable of assuming the separate form, under intense cold, combined with great pressure. But we must not theorise too far, nor allow ourselves to be carried away by analogy. Hitherto the fourth atom of hydrogen in ammonium, $\mathrm{N} \mathrm{H}_{4}$, or its oxide, $\mathrm{N} \mathrm{H}_{4} \mathrm{O}$, has been regarded as being quite distinct from the rest; when ammonia, or its salts, took up an atom of water, the hydrogen was supposed to convert the ammonia into ammonium, which then took up the oxygen to form the oxide. It was thought that trimethylamine, $\mathrm{N}, \mathrm{Me}_{3}$, in which the 3 eqs. of hydrogen 
were replaced, was the limit of this substitution, and that we should have

$$
\left.\begin{array}{l}
\mathrm{NH}_{3}+\mathrm{H}=\text { Ammonium } \quad \mathrm{N} \mathrm{H}_{4}, \text { and } \\
\mathrm{NMe}_{3}+\mathrm{H}=\text { Trimethylium } \mathrm{Ne}_{\mathrm{H}}^{\mathrm{Me}}
\end{array}\right\}
$$

and the action of trimethylamine on acids corresponds exactly to this view. It takes up 1 eq. of water, and forms, probably, the oxide of trimethylium $\left.\mathrm{N}_{\mathrm{H}}^{\mathrm{Mes}}\right\} 0$, which, like oxide of ammonium, is resolved, when separated, into water and trimethylamine.

$$
\begin{aligned}
& \mathrm{N} \mathrm{H}_{4} \mathrm{O}=\mathrm{HO}+\mathrm{NH}_{3} \\
& \left.\mathrm{~N} \underset{\mathrm{H}}{\mathrm{Me}_{3}}\right\} \mathrm{O}=\mathrm{H} \mathrm{O}+\mathrm{N} \mathrm{Me}_{3} \text {. }
\end{aligned}
$$

On this view, hydrochlorate of methylamine is chloride of trimethylium, just as hydrochlorate of ammonia is chloride of ammonium; and sulphate of trimethylamine is sulphate of oxide of trimethylium, as sulphate of ammonia is sulphate of oxide of ammonium.

But now we find that the fourth eq. of hydrogen can also be replaced by methyle, \&c., and that the new metal $\mathrm{N} \mathrm{Me}_{4}$ is produced, which forms an oxide and a hydrate of totally new properties. Hofmann found it impossible to replace any more of the hydrogen in the new body, showing that the true limit of substitution is ammonium, $\mathrm{NH}_{4}$, and not ammonia, $\mathrm{NH}_{3}$. Yet when the fourth eq. of hydrogen is replaced, the base, which is now a hydrated oxide, loses the character of volatility which it had retained as long as only 3 eqs. were replaced. This proves that there is a difference, although not to the extent formerly supposed, between the fourth eq. of hydrogen in ammonium and the other three. Probably the true cause of the instability of oxide of ammonium and of those analogous compounds which like it, have 1 eq. of hydrogen, the fourth, derived from water, is this, that the tendency of their 1 eq. of oxygen to form water with 1 eq. of hydrogen is so strong as to overturn the equilibrium of the oxides; whereas, in tetramethylium, the affinity of the oxygen for the fourth atom of methyle is not sufficient to do this.

We have dwelt somewhat fully on these new compounds, not to detail their individual properties, which our space forbids, but to explain the important general laws which regulate their formation, and which have an important bearing on the theory of the formation of natural organic bases. The reader will bear in mind that we have four series of bases derived from ammonia and ammonium; three of which are volatile like ammonia, 
namely, the amide, imide, and nitryle bases, while the fourth is not volatile without decomposition; these are called ammonium bases. It was necessary here to explain their nature; but we shall return to the general considerations under the head of organic bases.

Zincomethyle, $\mathrm{C}_{2} \mathrm{H}_{3} \mathrm{Zn}$. When iodide of methyle is heated along with zinc in a sealed tube, a white crystalline product is obtained. When this product is distilled in an atmosphere of hydrogen, it yields a volatile liquid of a very fetid smell, the vapours of which are very poisonous. It takes fire spontaneously on contact with the air, and burns with a bright greenish blue flame, depositing a large quantity of zinc and oxide of zinc. This is zincomethyle, apparently a new radical. It was discovered by Frankland. In contact with water, it is decomposed with ignition, yielding oxide of zinc, and hyduret of methyle (marsh-gas), $\mathrm{C}_{2} \mathrm{H}_{3} \mathrm{Zn}+\mathrm{H} \mathrm{O}=\mathrm{Zn} \mathrm{O}+\left(\mathrm{C}_{2} \mathrm{H}_{3}, \mathrm{H}\right)$.

It would appear that tin, and probably also, bismuth, forms a similar radical, but of these hardly anything is yet known.

Stibiomethyle, $\mathrm{C}_{6} \mathrm{H}_{9}, \mathrm{Sb}=\mathrm{Sb} \mathrm{Mes}_{3}$. This compound is formed when iodide of methyle is heated with an alloy of potassium and antimony. It is a volatile liquid of an offensive odour, spontaneously inflammable in air, insoluble in water, soluble in alcohol and ether. It is a compound radical of very powerful affinities, combining energetically with the elementary bodies. It takes up 2 eqs. of oxygen, chlorine, \&c., and its oxide is basic (Landolt). The corresponding ethyle radical and its compounds will be described farther on.

Stibiomethylium, $\mathrm{C}_{8} \mathrm{H}_{12} \mathrm{Sb}=\mathrm{Sb} \mathrm{Me}_{4}$. According to Landolt, when iodide of methyle is added to stibiomethyle, there is formed the crystalline iodide of a compound radical, analogous to ammonium, which he hence calls stibiomethylium. Sb $\mathrm{Mes}_{3}+\mathrm{Me} \mathrm{I}=\mathrm{Sb} \mathrm{M}_{4}, \mathrm{I}$.

By the action of oxide of silver on this iodide, the oxide is formed. $\mathrm{Sb} \mathrm{Me}_{4} \mathrm{I}+\mathrm{Ag} \mathrm{O}=\mathrm{AgI}+\mathrm{Sb} \mathrm{Me}_{4} \mathrm{O}$. This is obtained as a hydrate, in the form of a white crystalline mass, $\mathrm{Sb} \mathrm{Me4}_{\mathrm{O}}$, H O, which in alkaline characters may be compared to caustic potash. It may be volatilised by careful heating, but if suddenly heated is decomposed, yielding vapours which take fire spontaneously. It forms, with acids, salts which are analogous to, and isomorphous with, those of oxides of potassium and ammonium.

Some very remarkable componnds are known, in which arsenic, and arsenic and platinum are combined with methyle. We shall now mention these.

When acetate of potash is heated along with arsenious acid, 
a very remarkable liquid is obtained, which is the oxide of a new radical. This liquid, which is spontaneously inflammable, and has a most offensive alliaceous smell, has been long known in an impure state, under the names of the liquor of Cadet, and alkarsine. Bunsen, by a long series of the most profound and persevering researches, established its true character as the oxide of the radical kakodyle. He has even succeeded in obtaining the radical itself in the separate state, and in establishing the most perfect analogy between that radical and a metal, in all its chemical relations.

Kakodyle, $\mathrm{C}_{4} \mathrm{H}_{6} \mathrm{As}=\mathrm{As} \mathrm{Mez}_{2}$. The radical is best obtained from the chloride of kakodyle, $\mathrm{Kd} \mathrm{Cl}$, by the action of zinc at $212^{\circ}$. Chloride of zinc is formed, and kakodyle is set free. It is rectified in an apparatus filled with carbonic acid gas, to prevent decomposition. It is a clear liquid, refracting light strongly. When cooled, it crystallises in large square prisms, and acquires, when pure, the appearance of ice. Its smell is insupportably offensive, and its vapour is highly poisonous. The two latter characters belong to all the compounds of kakodyle, with hardly an exception. Kakodyle is spontaneously inflammable in the air; a rod moistened with it instantly takes fire when exposed to the air. It forms two distinct oxides: the protoxide, $\mathrm{Kd} \mathrm{O}$ (alkarsine), and kakodylic acid, $\mathrm{Kd} \mathrm{O}_{3}$.

Kakodyle may be viewed, either as a compound radical, acting as an element, or as a coupled compound, the affinities of which depend on one part, the other being merely a copula. Kolbe regards it as arsenic, coupled with methyle, the latter not affecting the affinities of the metal. But since arsenic always takes 3 and 5 eqs. of the elements, while all the compounds of kakodyle except kakodylic acid, take only 1 eq., it is more probable that the active part of the compound is 1 eq. methyle, $\mathrm{Me}$, coupled with $\mathrm{Me} \mathrm{As.} \mathrm{Its} \mathrm{formula} \mathrm{will} \mathrm{then} \mathrm{be} \mathrm{(Me} \mathrm{As)} \mathrm{Me,}$ and its oxides will correspond to those of manganese, thus,
Mn 0 Protoxide.
(Me As) Me, $\mathrm{O}$ Protoxide. $\mathrm{Mn}_{3}$ Acid.
( $\mathrm{Me} \mathrm{As}) \mathrm{Me}, \mathrm{O}_{3}$ Acid.

The chloride, bromide, iodide, sulphuret, \&c., correspond perfectly with those of manganese, but not with those of arsenic.

Oxide of Kakodyle. As $\mathrm{Me}_{2} \mathrm{O}=\mathrm{Kd} 0$. This is the chief ingredient of the liquor of Cadet; it is purified by repeated rectifications in an atmosphere of carbonic acid, and is, when pure, a limpid ethereal liquid, refracting light powerfully; it boils at about $300^{\circ}$, and at- $9^{\circ}$ it crystallises in white scales of a satiny lustre. Its smell is most offensive, and its taste very 
nauseous. If placed on the skin it causes violent itching, and if taken internally it is a most energetic poison. It is sparingly soluble in water, more soluble in alcohol and ether. Like kakodyle, it takes fire when exposed to the air. When left under water, it gradually disappears, being for the most part converted into kakodylic acid. The production of oxide of kakodyle is very simple : 2 eqs. dry acetic acid and 1 eq. arsenious acid yield 4 eqs. carbonic acid and 1 eq. oxide of kakodyle. $2\left(\mathrm{C}_{4} \mathrm{H}_{3} \mathrm{O}_{3}\right)$ $+\mathrm{As}_{\mathrm{s}} \mathrm{O}_{3}=4 \mathrm{C} \mathrm{O}_{2}+\mathrm{C}_{4} \mathrm{H}_{6} \mathrm{As} \mathrm{O}$.

Kakodylic acid, Kd Os. Formed by the gradual oxidation of the protoxide, under water. It forms oblique four-sided prisms, brittle, and of a glassy lustre. They have no smell, and are soluble in water and alcohol. Its salts do not crystallise. Many reducing agents convert it into the protoxide by removing 2 eqs. of oxygen. It is not in the least poisonous.

There appears to be an intermediate oxide, $\mathrm{Kd} \mathrm{O}_{2}$; but it has not been obtained in a state of purity.

Chloride of Kakodyle, $\mathrm{Kd} \mathrm{Cl}=\mathrm{C}_{4} \mathrm{H}_{6} \mathrm{As}, \mathrm{Cl},=\mathrm{Kd} \mathrm{Cl}$, is obtained by heating a compound of oxide of kakodyle and corrosive sublimate along with hydrochlorio acid: $\mathrm{Kd} \mathrm{O}, \mathrm{Hg}$ $\mathrm{Cl}_{2}+\mathrm{H} \mathrm{Cl}=\mathrm{Kd} \mathrm{Cl}+\mathrm{H} \mathrm{O}+\mathrm{HgCl}_{2}$. It is a volatile, horribly fetid liquid, the vapour of which attacks strongly the lining membrane of the nose, and provokes a flow of tears. When exposed to the air, it deposits crystals of an exychloride of kakodyle, $\mathrm{Kd} \mathrm{O}+3 \mathrm{Kd} \mathrm{Cl}$. The iodide, bromide, and fluoride of kakodyle, are in all points analogous to the chloride; and form, when exposed to the air, oxyiodide, oxybromide, \&c.

Sulphur forms with kakodyle three compounds; the protosulphuret, $\mathrm{Kd} \mathrm{S}$, is obtained by distilling chloride of kakodyle with hydrosulphuret of sulphuret of barium : $\mathrm{Kd} \mathrm{Cl}+\mathrm{Ba} \mathrm{S}, \mathrm{H} \mathrm{S}=$ $\mathrm{Kd} \mathrm{S}+\mathrm{BaCl}+\mathrm{H} \mathrm{S}$. It is a clear, volatile, very fetid liquid, heavier than water. It dissolves sulphur, forming the bisulphuret $\mathrm{Kd} \mathrm{S}_{2}$, which is a very permanent compound. The persulphuret, $\mathrm{Kd} \mathrm{S}_{3}$, is a sulphur acid, and forms sulphur salts, which are very permanent, with the sulphurets of highly basic metals. The sulphur salt of kakodyle and lead, $\mathrm{Pb} \mathrm{S}, \mathrm{Kd} \mathrm{S}$, crystallises beautifully.

Cyanide of Kakodyle, $\mathrm{Kd} \mathrm{Cy}=\mathrm{C}_{4} \mathrm{H}_{6} \mathrm{As}, \mathrm{C}_{2} \mathrm{~N}$, is formed by distilling bicyanide of mercury with water and oxide of kakodyle. When pure, it forms large brilliant crystals, very fusible and volatile. The vapour of this compound is so poisonous as to be in the highest degree dangerous to the experimenter.

Kakoplatyle. Kd, $\mathrm{Pt} \mathrm{O}, \mathrm{H} \mathrm{O}$. This radical is not yet known in a separate form, and it would seem probable that the formula here given is rather that of an oxide, possibly of a hydrated 
oxide. If it be the radical, it contains the elements of kakodyle, protoxide of platinum, and water. It is, in all probability, a somewhat complex coupled compound, in which, perhaps, from the aspect of its salts, platinum is the active element.

Chloride of Kakoplatyle, $\mathrm{C}_{4} \mathrm{H}_{7} \mathrm{As} \mathrm{Pt} \mathrm{O}_{2}, \mathrm{Cl}$. This compound is formed when an alcoholic solution of bichloride of platinum is added to a similar solution of chloride of kakodyle, when a reddish-brown precipitate is formed, which, being boiled with water, gives a solution from which, on cooling, needles of the new compound are deposited. Bromide of kakoplatyle and iodide of kakoplatyle may be formed from the chloride, and are analogous to it. The former appears in large yellow crystals, the latter in golden micaceous scales.

When the chloride is acted on by sulphate of silver, there is obtained, along with chloride of silver, the sulphate of the oxide of kakoplatyle, $\mathrm{C}_{4} \mathrm{H}_{7} \mathrm{As} \mathrm{Pt} \mathrm{O}_{3}, \mathrm{~S} \mathrm{O}_{3}$. It forms white crystalline grains. The compounds described are :-

\begin{tabular}{|c|c|}
\hline Radical, Kakoplats & $\mathrm{D}+\mathrm{O} \mathrm{C}+\mathrm{Cl}$ \\
\hline Chloride of do., anhydrous & $\mathrm{Pt} 0, \mathrm{Kd}+\mathrm{Cl}$ \\
\hline Chloride, hydrated & $\mathrm{Pt} \mathrm{O}, \mathrm{Kd}+\mathrm{Cl}+\mathrm{H}$ \\
\hline Chloride, ammoniated & $\mathrm{Pt} 0, \mathrm{Kd}+\mathrm{Cl}+\mathrm{N} \mathrm{H}_{3}$ \\
\hline Oxide, hydrated & $+\mathrm{O}+\mathrm{HO}$ \\
\hline Sulphate, hydrated & O, $\mathrm{H} 0)+$ \\
\hline
\end{tabular}

It is to be particularly borne in mind that this radical, whose basic character is quite obvious, contains two metals, arsenic and platinum, quite foreign, in general, to organic compounds. But it cannot be considered as having yet been fully investigated.

The existence of kakodyle itself, and the perfect analogy which may be traced between it and the simple metals, in their relations to all other substances, render the results of the researches of Bunsen, which have been so very briefly described in this work, of the very highest importance to the theory of organic compounds, and especially to that of compound radicals. Kakodyle was the first electro-positive radical ever isolated; but the progress of discovery has made known a large and increasing number of such radicals.

Methyle and Phosphorus.-It will be remembered that phosphuretted hydrogen, $\mathrm{P} \mathrm{H}_{3}$, is in some respects analogous to ammonia, and possesses basic properties. Paul Thenard has shown that there are three compounds of phosphorus and hydrogen, namely, $\mathrm{P}_{2} \mathrm{H}, \mathrm{P} \mathrm{H}_{2}$, and $\mathrm{P} \mathrm{H}_{3}$. The same chemist has obtained new bases, in which the hydrogen of these compounds is replaced by methyle. Thus, we have 


$$
\begin{aligned}
& \mathrm{P}_{2} \mathrm{H} \text { and } \mathrm{P}_{2} \mathrm{Me}=\mathrm{C}_{2} \mathrm{H}_{3} \mathrm{P}_{2} \\
& \mathrm{P} \mathrm{H}_{2} \text { and } \mathrm{P} \mathrm{Me} 2=\mathrm{C}_{4} \mathrm{H}_{6} \mathrm{P} \\
& \mathrm{P} \underset{\mathrm{H}}{\mathrm{H}}\} \text { and } \mathrm{P} \underset{\mathrm{Me}}{\mathrm{H}}\}=\mathrm{C}_{2} \mathrm{H}_{5} \mathrm{P}
\end{aligned}
$$

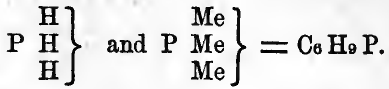

The compound $\mathrm{P} \mathrm{Me}_{2}=\mathrm{C}_{4} \mathrm{H}_{6} \mathrm{P}$ is a liquid of a most insupportably fetid smell, and very poisonous, obviously corresponding to kakodyle, $\mathrm{C}_{4} \mathrm{H}_{6} \mathrm{As}$. It is spontaneously inflammable, but when slowly oxidised yields a crystalline acid product not yet examined. It combines with 1 eq. of hydrochloric acid to form a very stable crystallisable salt. With two equivalents of the acid, it yields a liquid compound of little stability; and when acted on by excess of the acid, it yields the hydrochlorate of the base $\mathrm{P} \mathrm{Mes,} \mathrm{and}$ the compound $\mathrm{P}_{2} \mathrm{Me}$.

The first hydrochlorate, $\mathrm{P} \mathrm{H}_{2} \mathrm{Me}, \mathrm{H} \mathrm{Cl}$, when heated with water, yields an acid liquid, and a new gas, which appears to be basic, but when oxidised is converted into another acid. This gas appears to be $\mathrm{P} \mathrm{H}_{2} \mathrm{Me}$.

The body $\mathrm{P}_{2} \mathrm{Me}$, is a yellow solid, insoluble in water: but the compound $\mathrm{P} \mathrm{Me}_{3}$ is a powerful base, not spontaneously inflammable, which by absorbing oxygen yields a new acid product.

It will be seen that there is opened up here, as well as in the preceding section of those compounds in which metals are added to methyle, \&c., a new and extensive field of discovery. It cannot be doubted that many other radicals may be substituted for methyle, and in all varieties of proportion, or that other bodies will be found to play the same part, as zinc, antimony, arsenic, and phosphorus. Indeed, tellurium, tin and mercury, are already known to do so.

\section{PRODUCTS OF THE OXIDATION OF HYDRATED OXIDE OF METHYLE.}

When hydrated oxide of methyle is oxidised by means of platinum powder, it is finally converted into pure formic acid. There is evidently, therefore, the same relation between methyle and formic acid, as between ethyle and acetic acid; and on comparing the formulæ of pyroxilic spirit and of formic acid, $\mathrm{C}_{2} \mathrm{H}_{3} \mathrm{O}, \mathrm{H} \mathrm{O}$, and $\mathrm{C}_{2} \mathrm{H} \mathrm{O}_{3}, \mathrm{H} \mathrm{O}$, we perceive that the former, to be converted into the latter, must have lost 2 eqs. of hydrogen, and taken up 2 eq. of oxygen. This is exactly what 
takes place with alcohol, and there can be no doubt that the pyroxilio spirit yields an intermediate compound, exactly analogous to aldehyde, although this has not yet been isolated. Such a compound would be the hydrated protoxide of a derived radical formyle, analogous to acetyle, of which formic acid is the hydrated peroxide; and its formula would be $\left(\mathrm{C}_{2} \mathrm{H}\right) \mathrm{O}+\mathrm{H} \mathrm{O}$. We shall, therefore, assume the existence of this radical, and proceed to describe its compounds, which are quite analogous to those of acetyle.

Formyle, $\mathrm{C}_{2} \mathrm{H}=\mathrm{Fo}$. This radical is unknown in a separate form, as are its protoxide and deutoxide, corresponding to aldehyde and aldehydic acid. But when hydrated oxide of methyle is distilled with sulphuric acid, water, and peroxide of manganese, a volatile liquid is obtained, which is a mixture of formiate of oxide of methyle, and another liquid called methylal. When purified this latter has the formula $\mathrm{C}_{8} \mathrm{H}_{8} \mathrm{O}_{4}$, which indicates, that it is composed of $\mathrm{C}_{2} \mathrm{H} \mathrm{O}, \mathrm{H} \mathrm{O}$, or hydrated oxide of formyle, and 2 eqs. of oxide of methyle, $2\left(\mathrm{C}_{2} \mathrm{H}_{3} \mathrm{O}\right)$. This compound, briefly, Fo $0, \mathrm{H} \mathrm{O}+2 \mathrm{Me} 0$, corresponds to acetal in the series of ethyle; acetal being Ac $\mathrm{O}, \mathrm{H} \mathrm{O}+\mathrm{Ae} 0$.

Although not yet known in the separate form, as methyle is, yet the existence of formyle may be assumed, as a means of classifying a number of facts. It will be observed that formyle, $\mathrm{C}_{2} \mathrm{H}$, is methyle, $\mathrm{C}_{2} \mathrm{H}_{3}$, minus 2 eqs. of hydrogen. Formyle is therefore regarded as a derived radical, derived from methyle by the action of oxygen, which removes 2 eqs. of hydrogen; and it is the starting-point of a series of such derived radicals, differing from those of the methyle series by containing 2 eqs. of hydrogen less. These derived radicals (see column 4 of the table of homologous compounds) are distinguished by forming hydrated protoxides or aldehydes (column 5) and teroxides, which are strong acids, the volatile acids, $\mathrm{C}_{n} \mathrm{H}_{n} \mathrm{O}_{4}$ of column 6. The properties of the aldehydes will be detailed under acetyle, because the hydrated oxide of formyle is hardly known. But formic acid is the starting-point of the remarkable series of homologous volatile acids, and on this account deserves particular attention. It will be seen that the two first acids of this series are very powerful, and, although volatile, not at all oily; these are formic and acetic acids. But, as the quantity of carbon and hydrogen increases, the acids become by degrees less powerful, and acquire the character- of oils, and finally of fats. The third in the scale is slightly oily, and less soluble in water than the two first. This is propylic acid, $\mathrm{C}_{6} \mathrm{H}_{6} \mathrm{O}_{4}$. The fourth, butyric acid, $\mathrm{C}_{8} \mathrm{H}_{8} \mathrm{O}_{4}$, is more oily and still less soluble; the fifth, valerianic acid, $\mathrm{C}_{10} \mathrm{H}_{10} \mathrm{O}_{4}$, is hardly soluble, and very oily, and the acid with 20 eqs. of carbon, capric 
acid, is a solid fat at ordinary temperatures, as are all above it in the scale. Another character of the radicals of the formyle series is that of forming terchlorides, the first of which, terchloride of formyle, or chloroform, has very valuable properties. Perhaps other compounds, homologous with chloroform, may also be found useful when they shall have been studied.

\section{Formic Acid. $\mathrm{C}_{2} \mathrm{H} \mathrm{O}_{3}, \mathrm{H} \mathrm{O}=\mathrm{F}_{0} \mathrm{O}_{3}, \mathrm{HO}$.}

This remarkable acid occurs in the red ant, Formica rufa, and may be obtained, in a diluted and impure state, by infusing these insects in water. Its production from pyroxilic spirit has been described above. It may also be prepared by distilling a mixture of starch or sugar with peroxide of manganese, water, and sulphuric acid; and it is formed under a great variety of circumstances from many organic compounds, especially when their oxidation is carried to a point somewhat short of complete oxidation, the formula of this acid being very simple, and approaching nearly to that of carbonic acid, the ultimate product of the oxidation of their carbon.

To prepare the hydrated acid pure and concentrated, the dry formiate of lead, $\mathrm{PbO}, \mathrm{FoO}_{3}$, is decomposed by sulphuretted hydrogen gas, and the vapour of the formic acid condensed in a well-cooled receiver. It is boiled for a few moments to expel any sulphuretted hydrogen. It is a clear liquid of Sp. G. 1.235, fuming slightly, and has a very pungent acid smell. Below $32^{\circ}$ it crystallises in brilliant scales. It boils at $212^{\circ}$, and its vapour is inflammable, burning with a blue flame. This is probably owing to the formation of carbonic oxide, for the acid $\mathrm{C}_{2} \mathrm{H} \mathrm{O}_{3}$, $\mathrm{H} \mathrm{O}$, contains the elements of 2 eqs. carbonic oxide, $\mathrm{C}_{2} \mathrm{O}_{2}$, and 2 eqs. of water, $\mathrm{H}_{2} \mathrm{O}_{2}$.

With 1 eq. of water it forms the second hydrate, $\mathrm{Fo}_{3}+$ $2 \mathrm{H} \mathrm{O}$, which much resembles the first in properties. Its boiling point is $221^{\circ}$, and its Sp. G. is $1 \cdot 110$.

Both these hydrates are highly corrosive ; a drop of either on any delicate portion of the skin causes a severe burn, which blisters, suppurates, and is very painful and difficult to heal. In this respect, the formic acid can only be compared to hydrofluoric and hydrated cyanic acids.

A weaker acid is obtained by distilling formiate of soda, lime, or lead, with sulphuric acid, previously diluted with half its weight of water. Ten parts of formiate of lime, 8 of oil of vitriol, and 4 of water, yield 9 of formic acid, of Sp. G. 1.075.

The salts of the acid are best prepared from the weak and impure acid obtained by distilling a mixture of 10 parts of 
starch, 37 of peroxide of manganese, 30 of oil of vitriol, and 30 of water. These materials yield 3.35 parts of formic acid, such that 100 parts neutralise 15 of dry carbonate of soda. From this acid, the formiate of lead may be easily prepared and purified; and from it, by the addition of carbonate of soda, formiate of soda may be obtained.

Formic acid is easily recognised by the action of sulphuric acid, which decomposes, without blackening, both it and its salts, causing the disengagement of pure carbonic oxide. It also reduces the oxides of all the noble metals, and is itself oxidised into carbonic acid. Formic acid not only reduces the oxides, but also, in most cases, the soluble salts, of the noble metals. 1 eq. formic acid can reduce 2 eqs. of a protoxide, such as that of silver, $2 \mathrm{Ag} \mathrm{O}+\mathrm{C}_{2} \mathrm{H} \mathrm{O}_{3}=\mathrm{Ag}_{2}+\mathrm{HO}+2 \mathrm{C} \mathrm{O}_{2}$; or 1 eq. of a deutoxide, such as that of mercury; $\mathrm{Hg} \mathrm{O} \mathrm{O}_{2}+\mathrm{C}_{2} \mathrm{H} \mathrm{O} \mathrm{O}_{3}=$ $\mathrm{Hg}+\mathrm{HO}+2 \mathrm{CO}_{2}$.

Formic acid is a very powerful acid, and forms salts with bases, all of which are soluble. They are generally similar to the acetates; but yet quite distinct. Many metallic formiates, when heated in close vessels, give off carbonic acid and carbonic oxide, leaving the metal reduced: others give off carbonic oxide, leaving the oxide.

Formiate of ammonia, $\mathrm{N} \mathrm{H}_{4} \mathrm{O}, \mathrm{C}_{2} \mathrm{H} \mathrm{O}_{3}$, contains the elements of hydrocyanic acid and water, $\mathrm{C}_{2} \mathrm{~N} \mathrm{H}+4 \mathrm{HO}$; and is converted into these compounds when its vapour is passed through a red-hot tube. Formiate of oxide of ethyle, prepared like the acetate, is a volatile ethereal liquid, with a peculiar aromatic smell. The corresponding salt of oxide of methyle is quite analogous. Formiate of potash is very deliquescent. Formiate of soda is also very soluble, but may be obtained in crystals. It is a very powerful reducing agent, both in a moist and dry way. In the former, it reduces the noble metals; in the latter, at a red heat, by virtue of the carbonic oxide it gives off, it reduces most of the reducible metals, such as lead, copper, antimony, arsenic, cobalt, nickel, \&c. Formiate of lead is sparingly soluble in cold water, and is therefore easily purified, and serves to prepare formic acid and formiate of soda. Formiate of deutoxide of mercury and formiate of protoxide of mercury both exist. When red oxide of mercury is dissolved in cold formic acid, the former salt is produced; but the slightest heat causes an effervescence, while formiate of the protoxide is deposited in silvery scales like the acetate. These, when warmed, are decomposed with effervescence and deposition of metallic mercury. Formiate of silver resembles the acetate; but is very easily decomposed by heat, the metal being reduced. 
COMPOUNDS OF FORMYLE WITH CHLORINE, ETC.

When chlorine or hypochlorites act on oxide of methyle, hydrated oxide of methyle, and chloride of methyle, a great variety of new compounds are produced, corresponding in most cases to the compounds obtained by the action of chlorine, \&c., on alcohol, ether, and chloride of ethyle. Our space forbids us to give the details of these decompositions; but we may mention that two of the compounds formed by the action of chlorine on the compounds of ethyle and acetyle, may be viewed as protochloride and bichloride of formyle. These are $\mathrm{C}_{4} \mathrm{H}_{2} \mathrm{Cl}_{2}=2\left(\mathrm{C}_{2} \mathrm{H}\right.$, $\mathrm{Cl})$; and $\mathrm{C}_{4} \mathrm{H}_{2} \mathrm{Cl}_{4}=2\left(\mathrm{C}_{2} \mathrm{H}, \mathrm{Cl}_{2}\right)$.

But the perchloride or terchloride of formyle is certainly known, and has of late, as chloroform, acquired a high practical value.

Terchloride of formyle. Sys. Chloroform. $\mathrm{C}_{2} \mathrm{H} \mathrm{Cl}_{3}=\mathrm{F}_{0}$ $\mathrm{Cl}_{3}$. This compound is best obtained by distilling pure alcohol with water and bleaching powder. The product is well washed with water and rectified, after being agitated for a short time with oil of vitriol, which removes traces of water and destroys certain oils which are usually present in small quantity. The rectified chloroform should have the Sp. G. $1 \cdot 49$ to $1 \cdot 50$, and should not colour oil of vitriol when shaken with it, unless to a very trifling extent. If it colours the acid strongly, or if, when evaporated on the hand, it leaves an unpleasant odour, it is not fit for use, and must be again washed and reotified. It may be prepared also from pure pyroxilic spirit, but alcohol yields it purer.

Chloroform is a transparent, colourless, volatile liquid, of Sp. G. 1.5: it has a peculiar heavy sweetish smell, and when inhaled from the hand, from a sponge, or handkerchief, soon produces insensibility to pain, and indeed a sort of coma. But if too sparingly or timidly applied, the effect often stops at the stage of excitement, similar to that produced by inhaling the nitrous oxide, which latter, being a gas, cannot conveniently be administered in a full dose, since one of its first effects is to relax the muscles of the mouth which grasp the tube, and thus admit air. But chloroform may be held near the mouth on a handkerchief, or a sponge, and so much given as to carry the patient beyond the stage of excitement into that of coma and entire insensibility to pain.

For the introduction of this valuable remedy we are indebted to Dr. Simpson, and although ether, benzole, and many other liquids can produce insensibility to pain, chloroform is of all the most powerful, as well as the most manageable. Of course great 
care must be taken to ensure its purity, for the oils which accompany it when first formed are very deleterious, and in administering it, one person should do nothing but watch the pulse and respiration of the patient, and remove the chloroform if necessary. With due precaution, chloroform is very safe; and this precaution will prevent its being used in cases where its use is contra-indicated by disease of the heart, or by marked tendency to apoplexy. In proof of its safety, it may be mentioned that in Edinburgh, where it must now have been used in many more than 100,000 cases, no instance of a fatal result has occurred. Deaths have occurred elsewhere, but it is probable either that the chloroform was not pure, or that it was used in cases unfit for it, or finally, that due care was not taken to prevent an overdose. That all these things can be avoided is proved by the results of the Edinburgh practice.

It has been found, of late, that although chloroform may be obtained which is quite permanent under ordinary circumstances, - for I have some which I made more than ten years ago which is still quite unaltered-yet it often, perhaps generally, happens, that exposure to the direct rays of the sun, or even to strong diffused daylight, causes a decomposition, by which chlorine is set free, the liquid becomes yellowish, and it acquires of course the suffocating smell of chlorine, so that it cannot be used for inhalation. It is probable that this depends on the purity of the chloroform, for I have found that those portions which I had purified with the greatest care were the soonest decomposed by sunlight. It may, however, also depend on the presence of traces of substances used in purifying it, which may promote decomposition; though, in the case of prussic acid, we find that a trace of sulphuric rather has the effect of rendering it more permanent.

This point is not yet cleared up, but, however it may be, it is easy to preserve chloroform unchanged, by keeping it in a rather dark place, or by storing it, "either in bottles of dark-coloured glass, or in bottles covered with dark paper. Even in clear glass, however, it usually keeps well in the ordinary light of a room into which the sun does not directly shine.

It has been doubted whether the protoxide of nitrogen can produce insensibility to pain, and whether its action resembles that of chloroform. I can only state, that I have seen many cases among those who have inhaled the gas, where insensibility to pain was perfectly developed. This has occurred, not only in such as were rendered apparently insensible to all surrounding objects for a short time, which is now and then seen, but also in several who were, while under the influence of the gas, violently 
excited and prone to laughter and muscular exertion. Some of these have struck wood or stone with their hands, till these were covered with blood, and have not even known this, till the effect passed off, not having felt any inducement to look at their hands. But I have also tested the insensibility to pain in various ways.

In some cases, as already hinted, there was produced the same half comatose state as is produced by a full dose of chloroform. These were very sensitive subjects, who had got a full dose of the gas before the muscles of their lips relaxed. But such cases are rare.

On the other hand, chloroform, in insufficient quantity to cause the coma, often produces laughter, singing, and violent muscular efforts, exactly like the gas; and when to this we add that the taste of the gas is almost the same as that of chloroform, and that it produces the very same thrilling warmth over the whole system as chloroform and ether do, as I can testify from personal experience, it appears reasonable to conclude that their action is alike, and that the differences observed are only those of an earlier or a later stage of that action, the former being the rule in the case of the gas, from the mechanical causes I have specified, the latter being the rule in the case of chloroform or ether, of which a.full dose is easily administered.

When acted on by chlorine, perchloride of formyle yields perchloride of carbon, $\mathrm{C}_{2} \mathrm{Cl}_{4}=\mathrm{C}_{2} \mathrm{Cl} \mathrm{Cl}_{3}$, just as perchloride of acetyle, $\mathrm{C}_{4} \mathrm{H}_{3}, \mathrm{Cl}_{3}$, yields sesquichloride of carbon, $\mathrm{C}_{4} \mathrm{Cl}_{6}=$ $\mathrm{C}_{4} \mathrm{Cl}_{3}, \mathrm{Cl}_{3}$.

Perbromide of formyle is obtained from bromal. Periodide of formyle, obtained by the action of alcohol on iodine and potash, is a yellow crystalline, volatile solid, having an odour analogous to that of saffron.

When chlorine acts on oxide of methyle $\mathrm{C}_{2} \mathrm{H}_{3} \mathrm{O}$, it produces, by substitution of chlorine for hydrogen, the compounds

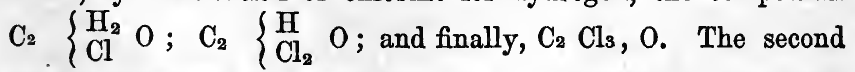
may be considered as formic acid, $\mathrm{C}_{2} \mathrm{H}_{3}$, in which two-thirds of the oxygen are replaced by chlorine, $\mathrm{C}_{2} \mathrm{H}\left\{\begin{array}{l}\mathrm{O} \\ \mathrm{Cl}_{\mathbf{2}}\end{array}\right.$. It will then be an oxychloride of formyle, analogous to oxychloride of acetyle.

When chlorine acts on chloride of methyle, $\mathrm{C}_{2} \mathrm{H}_{3} \mathrm{Cl}$, three compounds are formed by substitution. These are, 1st, $\mathrm{C}_{2} \mathrm{H}_{2}$ $\mathrm{Cl}_{2}=\mathrm{C}_{2}\left\{\begin{array}{l}\mathrm{H}_{2} \\ \mathrm{Cl}\end{array}, \mathrm{Cl} ; 2 \mathrm{nd}\right.$, perchloride of formyle, $\mathrm{C}_{2} \mathrm{H} \mathrm{Cl}=$ $\mathrm{C}_{2}\left\{\begin{array}{l}\mathrm{H} \\ \mathrm{Cl}_{2}\end{array}, \mathrm{Cl}\right.$; and 3rd, as before explained, perchloride of carbon, $\mathrm{C}_{2} \mathrm{Cl}_{4}=\mathrm{C}_{2} \mathrm{Cl}_{3}, \mathrm{Cl}$. 
The action of chlorine on sulphuret of methyle, $\mathrm{C}_{2} \mathrm{H}_{3} \mathrm{~S}$,

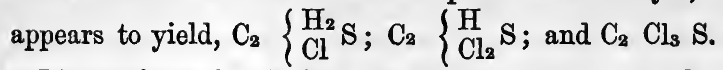

The action of chlorine on oxalate, benzoate, and acetate of oxide of methyle is quite analogous to its action on the corresponding compounds of ethyle, producing the oxalate, benzoate, and acetate of oxychloride of formyle, $\mathrm{C}_{2} \mathrm{O}_{3}+\mathrm{C}_{2} \mathrm{H}\left\{\begin{array}{l}\mathrm{O} \\ \mathrm{Cl}_{2}\end{array}\right.$; $\mathrm{C}_{14} \mathrm{H}_{5} \mathrm{O}_{3},+\mathrm{C}_{2} \mathrm{H}\left\{\begin{array}{l}0 \\ \mathrm{Cl}_{2}\end{array}\right.$; and $\mathrm{C}_{4} \mathrm{H}_{3} \mathrm{O}_{3}+\mathrm{C}_{2} \mathrm{H}\left\{\begin{array}{l}0 \\ \mathrm{Cl}_{2}\end{array}\right.$. These curious results, like those which precede them, are here merely indicated, as we shall explain the principle of their formation under the heads of ethyle and acetyle.

In some specimens of raw pyroxilic spirit there occurs a large proportion of a peculiar volatile liquid, which has been called lignone or xylite. As its constitution is quite uncertain, although it is believed to contain some compound of oxide of methyle, we shall state what is known of it when treating of the products of the dry distillation of wood.

\section{Ethyle. $\mathrm{C}_{\boldsymbol{t}} \mathrm{H}_{5}=\mathrm{Ae}$.}

This, the second radical of the methylic series (see table of homologous compounds) is obtained when iodide of ethyle is acted on by zinc, in closed tubes, at a high temperature, $\mathrm{C}_{4} \mathrm{H}_{5} \mathrm{I}+\mathrm{Zn}=\mathrm{Zn}$ $\mathrm{I}+\mathrm{C}_{4} \mathrm{H}_{5}$. Part of the ethyle combines with another portion of zinc, to form zincethyle; part of it is resolved into methyle and elayle, thus $\mathrm{C}_{4} \mathrm{H}_{5}=\mathrm{C}_{2} \mathrm{H}_{3}+\mathrm{C}_{2} \mathrm{H}_{2}$; and part of it escapes unchanged. It is a colourless gas of a faint ethereal smell, burning with a bright white flame; its Sp. G. is $2 \cdot 00394$. At $32^{\circ}$, and under the ordinary pressure, it is not condensed ; but under a pressure of $2 \frac{1}{4}$ atmospheres it forms a mobile liquid. Alcohol absorbs it.

Ethyle is, in all respects, analogous to methyle, but, according to the law which regulates homologous series, its density is higher, while its volatility is less. Methyle is gaseous under a pressure of 20 atmospheres and upwards, but ethyle is easily condensed, and under the ordinary pressure, boils at $23^{\circ} \mathrm{F}$. At the same time we must not forget that this compound, although it has the formula of the radical ethyle, may, like methyle, be possibly only an isomeric modification of the true radical. It does not exhibit strong affinities, and cannot be made directly to combine with oxygen, chlorine, \&c. But assuming it to be the radical, with which it is at all events isomeric, we shall now consider its compounds.

\section{Oxide of Ethyle. $\mathrm{C}_{4} \mathrm{H}_{5} \mathrm{O}=\mathrm{Ae} \mathrm{O}$.}

Srn. Ether.-Sulphuric Ether. This compound is found in 
nature in small quantity, not uncombined, but in combination with acids, chiefly with acids of the formic and acetic series, $C_{n}$ $\mathrm{H}_{n} \mathrm{O}_{4}$, so often mentioned. These compounds are neutral ethers, and have usually a very fragrant odour. The flavour of the pineapple, melon, and various other fruits, depends on the presence of such compounds.

Oxide of ethyle is obtained artificially from alcohol, its hydrate, by heating it gently along with sulphuric or phosphoric acid, which removes the water, or at all events causes the separation of the ether from the water. The best process is as follows: 5 parts of alcohol, or at least 90 per cent., are mixed with 9 of sulphuric acid, and the mixture introduced into a retort, where it is rapidly heated to the boiling-point, and kept at that point, while by means of a bent tube passing through the cork which stops the tubulure of the retort, and furnished with a stop-cock, fresh alcohol is allowed to enter the retort so as to keep the liquid constantly at the original level, flowing in exactly as fast as the ether, \&c., distils over. The products are condensed in a powerful Liebig's refrigeratory; they consist chiefly of ether, with water in such proportion as would convert the ether into alcohol; and a very little alcohol, sometimes none at all. The operation may be advantageously continued until 31 parts of alcohol, at 90 per cent., have flowed into the retort, and of course an equal volume of ether and water has distilled over.

This process, as to its final result, may be thus expressed, Ae $\mathrm{O}$, $\mathrm{HO}+2\left(\mathrm{H} \mathrm{O}_{2} \mathrm{~S} \mathrm{O}_{3}\right)=2\left(\mathrm{H} \mathrm{O}_{2} \mathrm{~S} \mathrm{O}_{3}\right)+\mathrm{HO}+\mathrm{Ae} \mathrm{O}$; that is, the action of heat and of oil of vitriol has caused the separation of the ether and the water, which, together, constitute alcohol. And it is to this view of the change, which he considers one of decomposition by contact, that Berzelius applies his theory of a catalytic force. But in reality, the process consists of two stages: the first is the formation of bisulphate of oxide of ethyle (sulphovinic acid) $\mathrm{Ae} \mathrm{O}, \mathrm{H} \mathrm{O}, 2 \mathrm{~S} \mathrm{O}$; and the second is the decomposition of this by heat into $\mathrm{Ae} \mathrm{O}$ and $\mathrm{H} \mathrm{O}, 2 \mathrm{~S} \mathrm{O}_{3}$. The bisulphate is formed when a mixture of 2 eqs. oil of vitriol, 1 eq. alcohol, and from 1 to 3 eqs. of water, is heated to about $285^{\circ}$; and at almost precisely the same temperature, the bisulphate is decomposed, especially if the liquid be kept in steady ebullition. Now, the bisulphate contains anhydrous sulphuric acid, ether, and water, $2 \mathrm{~S} \mathrm{O}+\mathrm{Ae} \mathrm{O}+\mathrm{H} \mathrm{O}$; and when decomposed, the sulphuric acid seizes the water, thus preventing the ether from uniting with it to reproduce alcohol. The ether, therefore, distils over : but as, when the bisulphate was formed, ether, Ae 0 , displaced water, $\mathrm{H} \mathrm{O}$, from half the oil of vitriol, so now, when the bisulphate is decomposed, and whilc its water is 
retained by the 2 eqs. of anhydrous acid, the vapours of ether traverse a liquid containing oil of vitriol diluted, both with the water displaced from the other part of it by the ether, and with the water of the alcohol (which at 90 per cent. amounts to 2 eqs. for 1 of ether, one combined with the ether to form the alcohol, the other serving to dilute it). Now, a sulphuric acid, thus far diluted, and heated to a temperature even short of $285^{\circ}$, gives off water, and therefore the vapour of ether in passing through this acid, becomes saturated with the vapour of water, without combining with it, and thus ether and water distil over together. At the surface of the boiling or effervescing liquid, however, the ether produced by the decomposition of the bisulphate is brought into contact with water in the nascent state, also derived from that decomposition, and in this manner, according to Liebig, a little alcohol is reproduced, and distils over with the ether, the ether being produced in the body of the liquid, the alcohol only at the surface. It is certain that a little alcohol generally accompanies the ether, even in the most successful operation; but it is not easy to see how, according to the above explanation, the sulphuric acid which, in the body of the liquid, is able to prevent the ether from combining with water, should fail to do so at the surface. The ether and water, it would appear, are equally in the nascent state in both situations. While, therefore, we admit Liebig's very beautiful explanation of the facts connected with the production of ether, we cannot feel the same certainty in regard to his explanation of the simultaneous occurrence of alcohol. It will now be seen how little necessity there is for resorting to the mysterious agency of catalysis; for the change is not one due to contact alone, but, on the contrary, one depending on strong affinities very nicely balanced, and influenced to a very great extent by the degree of heat employed. The idea that the contact of oil of vitriol caused the ether and water of alcohol to separate, arose from the circumstance that the formation of the bisulphate of ethyle takes place at a temperature quite close to that at which it is decomposed, and that the formation of the bisulphate in this process had been overlooked.

The crude ether is mixed with an alcoholic solution of potash, so as to render it alkaline, and distilled in the vapour-bath, as long as the Sp. G. of the product does not exceed 0.725 at $80^{\circ}$. The ether is then digested for a few days with chloride of calcium, or quicklime, and rectified once more with one of these substances. When pure, oxide of ethyle is a colourless, very mobile, highly refracting liquid, of Sp. G. $0 \cdot 725$ at $60^{\circ}$. It is very volatile, boiling at $76^{\circ}$, and producing intense cold by 
its evaporation. It has a pungent, cooling, aromatio taste ; and a penetrating agreeable odour. It is very combustible, and its vapour is apt to form dangerous explosive mixtures with air. When oxidised slowly, it yields aldehyde, aldehydic acid, acetic and formic acids.

Ether is used in medicine as a diffusible stimulant, and in chemistry as a solvent, especially of organic matters, such as fats, fat oils, essential oils, resins, some acids, and some bases. Its vapour, when mixed with air and inhaled, produces a species of coma, or at least, in most cases, insensibility to pain; and this method is now resorted to to facilitate the performance of severe surgical operations. In using ether, great care must be taken, on account of its great volatility, not to approach a light to it, which would inflame the vapour and might cause dangerous accidents. Care must also be taken to have air enough mixed with the vapour of ether, which, if inhaled alone, would cause asphyxia.

In its relations to other bodies it exhibits the characters of a base, neutralising acids, although it does not combine directly with them, and forming compounds which are subject to the laws of double decomposition, like salts of inorganic bases. These salts of oxide of ethyle are, commonly, ethereal liquids ; many of them crystallising at low temperatures, and a good many being solid and crystalline at ordinary temperatures. They are often called ethers, with the name of the acid; as acetic ether, benzoic ether, \&c. Those ethers which contain organic acids are for the most part fragrant, and many fruits owe their fragrance to the presence of the ethers of organic acids. The melon and pine-apple, as well as the apple, have exactly the odour of some such ethers. The formula for the salts of oxide of ethyle corresponds to that for the salts of potash, \&c. Thus, as $\mathrm{K} O, \overline{\mathrm{A}}$ represents acetate, and $\mathrm{K} 0, \overline{\mathrm{Bz}}$ benzoate of potash, so Ae $0, \bar{A}$, and $\mathrm{Ae} 0, \overline{\mathrm{Bz}}$, represent the acetate and benzoate of oxide of ethyle.

The analogy between ether and metallic protoxides is further shown in the action of both on hydrochloric acid and its congeners ; for while $\mathrm{K} \mathrm{O}$ with $\mathrm{H} \mathrm{Cl}$ yields $\mathrm{H} \mathrm{O}$ and $\mathrm{K} \mathrm{Cl}$, so $\mathrm{Ae} \mathrm{O}+\mathrm{H} \mathrm{Cl}$ $=\mathrm{H} \mathrm{O}+\mathrm{Ae} \mathrm{Cl}$ : that is, ether, with hydrochloric acid, yields water and chloride of ethyle. The same is true of the bromide, iodide, \&c., and by proper means both the cyanide of ethyle, Ae $\mathrm{Cy}$, and the sulphuret of ethyle, $\mathrm{Ae} \mathrm{S}$, may be obtained. In short, we cannot better connect and classify the numerous facts now known in regard to ether, than by adopting the view which considers it as the basic oxide of ethyle, a compound radical, very analogous to a metal. 
2. Hydrated Oxide of Ethyle. Ae $\mathrm{O}, \mathrm{H} \mathrm{O}=\mathrm{C}_{4} \mathrm{H}_{5} \mathrm{O}, \mathrm{H} \mathrm{O}$. SyN. Alcohol.-This compound is formed when ether and water meet in the nascent state, as we shall see occurs when some of the acid salts of ethyle are heated, or otherwise decomposed. But, practically, it is produced entirely from sugar by fermentation. The juice of the grape, or any other saccharine juice, or an infusion of malt, when exposed to the air for a short time, and then to a temperature of from $40^{\circ}$ to $85^{\circ}$, soon enters into fermentation, and a large quantity of carbonic acid is given off, while the sugar totally disappears, and alcohol is found in its place. A pure solution of sugar in water does not ferment, but on the addition of yeast it does so. The juice of the grape and the infusion of malt both contain, besides sugar, some body which plays the part of yeast, or ferment. This is, in the grape-juice, fibrine, and in the malt, gluten, both of which readily enter into putrefaction when exposed to air and moisture, and being in this state, their particles in motion, this motion is communicated to the particles of sugar, and the existing equilibrium of affinities being thereby disturbed, new compounds are formed, in this case alcohol and carbonic acid. The subject of fermentation will be afterwards fully discussed: in the mean time it is to be observed, that any similar substance, in a state of putrefaction, will induce the fermentation of sugar; as, for example, putrefying flesh, blood, milk, cheese, white of egg, urine, \&c., \&c., and that none of these ferments contribute to the production of the alcohol, or carbonic acid, but yield ammonia and other products. Crystallised cane sugar, $\mathrm{C}_{12} \mathrm{H}_{11} \mathrm{O}_{11}$ requires the elements of 1 eq. of water to yield 2 eqs. alcohol, $\mathrm{C}_{8} \mathrm{H}_{12} \mathrm{O}_{4}$ and 4 eqs. carbonic acid, $4 \mathrm{C} \mathrm{O}_{2}=\mathrm{C}_{4} \mathrm{O}_{8}$; and grape sugar, $\mathrm{C}_{12} \mathrm{H}_{14} \mathrm{O}_{14}$, produces, besides the alcohol and carbonic acid, 2 eqs. of water. Thus we have $\mathrm{C}_{12} \mathrm{H}_{11} \mathrm{O}_{11}+\mathrm{H} \mathrm{O}=2\left(\mathrm{C}_{4} \mathrm{H}_{6} \mathrm{O}_{2}\right)+4 \mathrm{C} \mathrm{O}_{2}$; and $\mathrm{C}_{12} \mathrm{H}_{14} \mathrm{O}_{14}$ $=2\left(\mathrm{C}_{4} \mathrm{H}_{6} \mathrm{O}_{2}\right)+4 \mathrm{C} \mathrm{O} \mathrm{O}_{2}+2 \mathrm{H} \mathrm{O}$.

From the fermented liquid, which, in the case of the grapejuice, is wine, in that of malt, beer, ale, or wort, the alcohol is separated by distillation, and being more volatile than water, it predominates in the first portions distilled. These constitute, when from wine, brandy; when from a fermented infusion of malt, whiskey; and when from fermented solution of molasses, rum. In these forms it still contains 30,40, or more per cent. of water, and a little volatile odoriferous oil. It is again rectified, and the first portions distilled are colourless, and go by the name of spirits of wine. After another rectification they are called rectified spirits of wine. They now contain only alcohol, with from 10 to 20 per cent. of water, which is removed by digesting the spirit with quicklime, and distilling; or by 
rectifying over dried carbonate of potash, or chloride of calcium. Pure or absolute alcohol is a colourless mobile fluid of Sp. G. 0.795 at $60^{\circ}$, and boiling at $173^{\circ}$. It has a burning taste, and a pleasant fruity smell. Rectified spirit of wine, that of 90 per cent., is very similar to it, having the Sp. G. 0.825 to 0.836 : ordinary spirit of wine, at 70 per cent., has the Sp. G. $0 \cdot 880$. Pure alcohol has never been frozen, but, by a most intense artificial cold, it has been brought to the state of a viscid oil, proving that a still more intense cold would solidify it. It is very combustible, and produces, in burning, a very intense heat. Hence spirit of wine is much used for lamps for chemical purposes. Alcohol has a strong attraction for water, and on this account acts as an antiseptic, preserving animal and vegetable substances from putrefaction. The strength of alcohol is ascertained by its specific gravity; and all chemical works contain tables of the relation between the density of the spirit and the per-centage.

Alcohol is a powerful diffusible stimulant, and has intoxicating properties. It is much used as a solvent, for many, indeed most vegetable acids and bases, for volatile oils, for resins, and for many salts, even inorganic. Most deliquescent salts are soluble in alcohol, as are the caustic alkalies and iodine. Acids act on alcohol, producing the compound ethers. Solutions made with proof spirit (a mixture of equal volumes of rectified spirit of wine and of water, Sp. G. 0.960) are called tinctures.

3. Chloride of Ethyle, $\mathrm{Ae} \mathrm{Cl}=\mathrm{C}_{4} \mathrm{H}_{5}, \mathrm{Cl}$, is prepared by saturating alcohol with hydrochloric acid gas, and distilling the mixture in the vapour-bath, collecting the product in a wellcooled receiver. It is formed as follows: $\mathrm{Ae} \mathrm{O}, \mathrm{H} \mathrm{O}+\mathrm{H} \mathrm{Cl}=$ $\mathrm{Ae} \mathrm{Cl}+2 \mathrm{H} \mathrm{O}$. It is dried by digestion with chloride of calcium. It is a colourless liquid, Sp. G. 0.874 , boiling at $52^{\circ}$, of an aromatic odour, slightly alliaceous. It is now employed in research. When exposed to the combined action of chlorine and the sun's rays, it yields by substitution a whole series of chlorinised ethers, of which series one extremity is ether $\left(\mathrm{C}_{4} \mathrm{H}_{5}\right) \mathrm{O}$; and the other, perchloride of carbon $\left(\mathrm{C}_{4} \mathrm{Cl}_{5}\right) \mathrm{Cl}=2 \mathrm{C}_{2} \mathrm{Cl}_{3}$. With an alcoholic solution of protosulphuret of potassium, $\mathrm{K} \mathrm{S}$, it gives chloride of potassium and sulphuret of ethyle. $\mathrm{Ae} \mathrm{Cl}+\mathrm{K} \mathrm{S}=\mathrm{Ae} \mathrm{S}+\mathrm{K}$ Cl. With hydro-sulphuret of sulphuret of potassium, it yields mercaptan, which is alcohol, in which all the oxygen has been replaced by sulphur. Ae $\mathrm{Cl}+\mathrm{K} \mathrm{S}, \mathrm{H} \mathrm{S}=\mathrm{K} \mathrm{Cl}+\mathrm{Ae} \mathrm{S}, \mathrm{H} \mathrm{S}$; the latter, mercaptan, being analogous to $\mathrm{Ae} \mathrm{O}, \mathrm{H} \mathrm{O}$.

4. Bromide of Ethyle, formed by distilling bromine with alcohol and phosphorus. There are first formed when the materials are mixed, phosphorous and hydrobromic acids, and the latter, when 
heated with the alcohol, decomposes it, yielding $\mathrm{Ae} \mathrm{Br}$, which resembles the chloride. It is used in research.

5. Iodide of Ethyle, Ae I, formed by a similar process, is analogous to the two preceding compounds, and is also used in research.

6. Sulphuret of Ethyle, $\mathrm{Ae} \mathrm{S}$, is prepared, as above stated, from the chloride by sulphuret of potassium. It is a colourless liquid, boiling at $167^{\circ}$, of a strong offensive alliaceous smell.

7. Hydrosulphuret of Sulphuret of Ethyle, or Mercaptan, Ae $\mathrm{SH} \mathrm{S}=\mathrm{C}_{4} \mathrm{H}_{6} \mathrm{~S}_{2}$ or $\mathrm{C}_{4} \mathrm{H}_{5}, \mathrm{~S}+\mathrm{H} \mathrm{S}$. This very remarkable compound is formed when a solution of the double sulphate of lime and oxide of ethyle (sulphovinate of lime) of Sp. G. 1.28, is distilled with its own bulk of a solution of potash of the same density, previously saturated with sulphuretted hydrogen, and converted into $\mathrm{K} \mathrm{S}, \mathrm{H} \mathrm{S}$. The volatile product, after digesting it with a little oxide of mercury and chloride of calcium, to remove sulphuretted hydrogen and water, is mercaptan. Its formation is thus explained: $\left(\mathrm{Ca} \mathrm{O}, \mathrm{S} \mathrm{O}_{3}+\mathrm{Ae} \mathrm{O}, \mathrm{S} \mathrm{O} \mathrm{O}_{3}\right)+\mathrm{K} \mathrm{S}$, $\mathrm{H} \mathrm{S}=\mathrm{CaO}, \mathrm{S} \mathrm{O}_{3}+\mathrm{K} \mathrm{O}, \mathrm{S} \mathrm{O}+\mathrm{Ae} \mathrm{S}, \mathrm{H} \mathrm{S}$. Pure mercaptan is a colourless liquid, very mobile, boiling at $97^{\circ}$, of Sp. G. 0.842. It has a most penetrating and offensive odour of onions, as it were concentrated, which adheres obstinately to the hair or clothes, so that it is most unpleasant to experiment upon. As above mentioned, it is formed from alcohol by substitution of sulphur for oxygen; and as alcohol is the hydrate of oxide of ethyle, Ae $0, \mathrm{H} \mathrm{O}$, mercaptan is the hydrosulphuret of the sulphuret of ethyle, Ae S, H S. The sulphuret of ethyle, Ae S, corresponds to the oxide, ether, Ae 0 .

Mercaptan acts strongly on some metallic oxides, especially those of the noble metals, such as mercury, gold, platinum, \&c. The metal takes the place of the hydrogen of the sulphuretted hydrogen in mercaptan; thus $\mathrm{MO}+(\mathrm{Ae} \mathrm{S}, \mathrm{H} \mathrm{S})=\mathrm{H} \mathrm{O}+(\mathrm{Ae}$ $\mathrm{S}, \mathrm{M} \mathrm{S})$. The red oxide of mercury is acted on by mercaptan, and converted into a white crystalline compound, called the mercaptide of mercury; oxide of gold forms a gelatinous white mercaptide; and oxide of lead yields lemon-yellow crystals of mercaptide of lead, Ae S, Pb S.

Mercaptan may also be viewed as $H+A e S_{2}$, in which case the above metallic compounds will have the general formula $\mathrm{M}+\mathrm{Ae} \mathrm{S}_{\mathbf{2}}$. Here the supposed radical, the mercaptum of Zeise, is bisulphuret of ethyle; so that on either view mercaptan is connected with ethyle.

Zeise has described, under the name of Thialic oil or ether, another very fetid compound, which seems to be $\mathrm{Ae} \mathrm{S}_{3}$, or persulphuret of ethyle. 
8. Seleniuret of Ethyle is a volatile alliaceous offensive liquid, formed when sulphuret of ethyle and potash is distilled with seleniuret of potassium. Sulphocyanide of potassium, alcohol and sulphuric acid, when dissolved together, yield a most offensive volatile liquid, supposed to be, or at all events to contain, sulphocyanide of ethyle. It will be observed that all these compounds of sulphur with ethyle and similar bodies are characterised by odours resembling that of garlic, but so intense and penetrating as to be insupportable. This character is observed in all volatile organic compounds of sulphur, whether artificial, as the above, or natural, as oils of garlic, assafetida, horseradish, \&c.

9. Fluoride of Ethyle, $\mathrm{C}_{4} \mathrm{H}_{5} \mathrm{~F}=\mathrm{AeF}$ - -Srn. Hydrofuoric Ether. This compound is formed when hydrofluoric acid acts on absolute alcohol, kept cold. It is a mobile, very volatile liquid, the odour of which recals that of radish. It burns with a blue flame, disengaging hydrofluoric acid. It must be kept, as well as prepared, in vessels of lead, silver, or platinum, as it acts on glass.

10. Cyanide of Ethyle, $\mathrm{Ae} \mathrm{Cy}=\mathrm{C}_{6} \mathrm{H}_{5} \mathrm{~N}$, is obtained when cyanide or ferrocyanide of potassium is heated with sulphovinate of potash (double sulphate of potash and ethyle). It is a volatile liquid of a peculiar slightly alliaceous odour, like that of putrid fish, with an analogy to hydrocyanic acid. It is very stupefying when inhaled, and no doubt poisonous. When heated with potash, it yields ammonia and propylate of potash.

$$
\underbrace{\mathrm{C}_{6} \mathrm{H}_{5} \mathrm{~N}}_{\text {Cyanide of Ethyle. }}+\mathrm{K} \mathrm{O}+3 \mathrm{H} \mathrm{O}=\mathrm{N} \mathrm{\textrm {H } _ { 3 }}+\underbrace{\mathrm{K} \mathrm{O}, \mathrm{C}_{6} \mathrm{H}_{5} \mathrm{O}_{3}}_{\text {Propylate of Potash. }} .
$$

Cyanide of ethyle appears to be identical with propylonitryle, a compound formed when propylate of ammonia is heated with anhydrous phosphoric acid, which deprives it of all its oxygen in the shape of water.

$$
\mathrm{NH}_{4} \mathrm{O}, \mathrm{C}_{6} \mathrm{H}_{5} \mathrm{O}_{3}+\mathrm{PO}_{5},=\mathrm{PO}_{5}, 4 \mathrm{HO}+\mathrm{C}_{6} \mathrm{H}_{5} \mathrm{~N} \text {. }
$$

If, therefore, we could obtain oxide or hydrated oxide of ethyle from the cyanide, which as yet we cannot do, we should have the means of producing ether and alcohol, which have 4 eqs. of carbon, from the acid, with 6 eqs. of carbon. And in like manner, from the acid, with 8 eqs. of carbon, we should be able to form the ether and alcohol with 6 eqs., from the acid with 10 eqs., the ether and alcohol with 8 eqs. of carbon; and so forth. It can hardly be doubted that we shall some day succeed in doing this. 
EThyle, With Metals, PHOSPhoRUs, \&c.

Zincethyle, $\mathrm{C}_{4} \mathrm{H}_{5} \mathrm{Zn}=\mathrm{Ae} \mathrm{Zn}$.- This compound is obtained by distilling the crystalline product formed by the action of zinc on iodide of ethyle (Frankland). It is a volatile, fetid, poisonous, and highly inflammable liquid, and like zincmethyle, appears to be an electropositive radical, although its compounds have not yet been examined minutely. In contact with water, it is decomposed, yielding oxide of zinc and a carbohydrogen of the formula $\mathrm{Cn}_{n} \mathrm{H}_{n}+{ }_{2}$. Thus $\mathrm{C}_{4} \mathrm{H}_{5} \mathrm{Zn}+\mathrm{H} \mathrm{O}=\mathrm{ZnO}+\mathrm{C}_{4} \mathrm{H}_{6}$. This body, $\mathrm{C}_{4} \mathrm{H}_{6}$, is most probably hyduret of ethyle, $\mathrm{C}_{4} \mathrm{H}_{5}, \mathrm{H}$, or Ae $\mathrm{H}$. But it may also be the radical methyle, for $\mathrm{C}_{4} \mathrm{H}_{6}=$ $2\left(\mathrm{C}_{2} \mathrm{H}_{3}\right)$. The latter view, however, has been shown by Frankland to be less probable, and we shall consider the body $\mathrm{C}_{4} \mathrm{H}_{6}$ as the hyduret of ethyle, while the compound $\mathrm{C}_{2} \mathrm{H}_{3}$, or the radical methyle, polymeric with it, is that formed in the electrolytic decomposition of acetio acid.

Stibethyle, $\mathrm{Sb} \mathrm{C}_{12} \mathrm{H}_{15}=\mathrm{Sb} \mathrm{Ae}_{3}=\mathrm{Sbæ}$. This radical is obtained when the alloy of antimony and potassium is heated with iodide of ethyle. It is a very mobile, highly refracting liquid, of an offensive alliaceous odour, which, when exposed to the air, first forms a thick white smoke, and then takes fire, burning with a very luminous white flame. It boils at $302^{\circ}$, and its Sp. G. is $1 \cdot 324$, that of its vapour $7 \cdot 499$. It is heavier than water, and soluble in alcohol or ether. When it is slowly oxidised, it yields two compounds. The first is a viscid transparent body, and is an oxide of stibethyle, $\mathrm{Sb} \mathrm{Ae}, \mathrm{O}_{2}=\mathrm{Sb} \circledast$ $\mathrm{O}_{2}$. The other is a compound of antimonious acid with oxide of stibethyle. This compound does not crystallise, and its solutions when warmed become thick and gelatinous, and dry up into a friable porcelain-like mass. It has a very bitter taste, and gives, with sulphuretted hydrogen, a light yellow precipitate.

The oxide of the radical, stibethyle, forms with acids crystallisable salts. Both the oxide and its salts are very bitter. The nitrate forms fine large rhombohedral crystals, very soluble in water. Its formula is $\mathrm{Sb} \mathrm{Ae}_{3} \mathrm{O}_{2}, 2 \mathrm{~N} \mathrm{O}_{5}=\mathrm{Sb}_{2} \mathrm{O}_{2}, 2 \mathrm{~N} \mathrm{O}$. The sulphate is $\mathrm{Sb} \mathrm{Ae}_{3} \mathrm{O}_{2}, 2 \mathrm{~S} \mathrm{O}=\mathrm{SbæO}_{2}, 2 \mathrm{~S} \mathrm{O}_{3}$.

Sulphuret of Stibethyle, Sbæ $\mathrm{S}_{2}$, forms small vithite crystals, of a brilliant silvery lustre, alliaceous smell, and bit er, hepatic taste. It is soluble in water, and precipitates metall: solutions, like sulphuret of potassium. The seleniuret Sbæ Ses, resembles the sulphuret. The iodide, Sbæ I crystallises beautifully. It is fusible, and in its reactions resembles iodide of potassium. Bromide of stibethyle $\mathrm{Sbæ} \mathrm{Br}_{2}$, is a transparent liquid, of $\mathrm{Sp} . \mathrm{G}$. 
1.953, which crystallises when exposed to cold. The chloride is a liquid of Sp. G. 1:54. All these compounds exhibit the reaction of metallic salts, chlorides, bromides, \&c.

Stibethyle is remarkable for its very strong affinities. In fact, it combines directly with almost all the non-metallic elements, and with so much energy, that it generally takes fire, and it is therefore necessary to employ indirect or slow methods of forming its compounds. Its affinities are almost, if not quite, as powerful as those of potassium. Löwig and Schweizer, who discovered it, are of opinion that it is a coupled radical, of the formul $\mathrm{Sb}$ $\mathrm{Ae}, \mathrm{Ae}_{2}$, and that this is the reason why it combines with 2 eqs. of oxygen, sulphur, \&c. ; its affinities depending on the 2 eqs. of ethyle, and not on the copula Sb Ae.

Stibethylium, $\mathrm{Sb} \mathrm{Ae}=\mathrm{C}_{16} \mathrm{H}_{20} . \mathrm{Sb}$. This compound metal, corresponding to ammonium, and having antimony instead of nitrogen, and ethyle instead of hydrogen, is analogous to stibiomethylium. It is unknown in the separate form, but its iodide is obtained by the action of iodide of ethyle on stibethyle. This oxide, being acted on by oxide of silver, yields the hydrated oxide of stibethylium analogous to caustic potash. It has not been yet fully studied.

Bismethyles. There are two compounds of bismuth with ethyle. Bismotriethyle, $\mathrm{C}_{12} \mathrm{H}_{15} \mathrm{Bi}$, is formed when iodide of ethyle acts on an alloy of bismuth and potassium. It cannot be distilled, and is purified by solution in ether, adding water, and distilling off the ether in close vessels filled with carbonic acid gas. The new radical remains under the water as a yellow oil, very easily decomposed, both by air and moisture. Its density is 1.82 , and it ferments in the air, taking fire after a short exposure. Its taste and smell are unpleasant. When heated, it is decomposed, and at a certain point it explodes with violence. Its compounds with chlorine, iodine, \&c., are very unstable.

When it is acted on by the bichloride of mercury, there are formed chloride of mercurethyle, and chloride of a new radical bismethyle, $\mathrm{C}_{4} \mathrm{H}_{5} \mathrm{Bi}$. The iodide of this radical forms very fine hexagonal crystals. The other compounds are unstable.

Stannethyle, $\mathrm{Sn} \mathrm{Ae}=\mathrm{C}_{4} \mathrm{H}_{5} \mathrm{Sn}$, is analogous to zincethyle, and is obtained in the same way.

It forms an oxide, $\mathrm{Sn} \mathrm{Ae} \mathrm{O}$, which is a bulky, insoluble, white powder. The chloride, bromide, and iodide all crystallise easily, and are more or less soluble in water. The nitrate of the oxide also crystallises. The other salts are generally insoluble, as is also the sulphuret. 
Löwig states, that there exist besides stannethyle, several other compounds, such as $\mathrm{Sn}_{2} \mathrm{Ae}_{2}, \mathrm{Sn}_{4} \mathrm{Ae}_{4}, \mathrm{Sn}_{4} \mathrm{Ae}_{3}, \mathrm{Sn}_{2} \mathrm{Ae}_{3}, \mathrm{Sn}_{4}$ $\mathrm{Ae}_{5}, \mathrm{Sn}_{6} \mathrm{Ae}_{4}$, all of which, like stannethyle, are compound metals in their chemical relations. They form oxides, chlorides, bromides, iodides, \&o., many, if not all of which, as well as the salts of the oxides, crystallise well.

It will be observed, that some of these formulø are multiples of that of stannethyle. These, as well as the other formulæ, are strange, and it is probable that these compounds are not yet fully understood.

Arsenodiethyle, Aez As, is homologous with kakodyle or Arsenobimethyle, $\mathrm{Me}_{2}$ As. It is formed when arsenic acts on iodide of ethyle, and is obtained as an iodide, $\mathrm{Ae}_{2} \mathrm{As}, \mathrm{I}$. It resembles kakodyle in all its characters and relations.

Arsenethyle $\mathrm{Ae}_{3} \mathrm{As}$, is formed along with the preceding, and is analogous to Stibethyle. It is also a volatile fetid liquid. Its oxide is an oily liquid, its sulphuret is crystallisable. The chloride is not known in a separate form, but is volatile and attacks the eyes violently. The iodide forms yellow flocks, easily decomposed.

Arsenethylium, $\mathrm{Ae}_{4} \mathrm{As}$ or $\mathrm{As} \mathrm{Ae}_{4}$, analogous to ammonium and stibethylium, is formed by the action of iodide of ethyle on arsenethyle, as an iodide. The hydrated oxide is a strong base, the chloride and iodide are crystalline.

Plumbethyle, $\mathrm{Pb}_{2} \mathrm{Ae}_{3}$, is obtained as iodide, when iodide of ethyle acts on an alloy of sodium and lead. There appear to be several compounds of lead with ethyle, but the one above named is a volatile liquid, insoluble in water. It is oxidised by exposure to air. The hydrated oxide is a strong base, which crystallises. Its formula is not fixed, but as it is caustic, attracts carbonic acid from the air, and in other respects resembles caustic potash, and as the chloride and bromide contain only 1 eq. of salt radical, it is probably $\left(\mathrm{Pb}_{2} \mathrm{Ae}_{3}\right) \mathrm{O}, \mathrm{H} \mathrm{O}$. The chloride and bromide crystallise, as do the sulphate, nitrate, and carbonate of the oxide.

Mercurethyle, $\mathrm{Hg}_{2} \mathrm{Ae}$, is formed when iodide of ethyle acts on mercury. The hydrated oxide, probably $\mathrm{Hg}_{2} \mathrm{Ae} \mathrm{O}, \mathrm{H} \mathrm{O}$ is a powerful caustic base, like caustic potash. The chloride, bromide, and iodide crystallise.

Tellurethyle, $\mathrm{C}_{4} \mathrm{H}_{5} \mathrm{Te}=\mathrm{Te}$ Ae. This radical is obtained when sulphovinate of baryta is distilled with telluret of sodium (Wöhler). It is a deep red liquid, heavier than water, in which it is sparingly soluble. It has a very offensive smell, and is poisonous. Its oxide, Te Ae 0 , is a crystallisable base, forming salts with acids. Its chloride, $\mathrm{Te} \mathrm{Ae} \mathrm{Cl}$, is 
a colourless liquid, of a disagreeable odour volatile without decomposition.

Mallet, who has ascertained the true nature of tellurethyle, has also obtained another compound, which is either bitelluret of ethyle $\mathrm{Ae} \mathrm{Te}_{2}$, and probably also a radical, or else a compound analogous to mercaptan, $\mathrm{C}_{4} \mathrm{H}_{5} \mathrm{Te}, \mathrm{H} \mathrm{Te}$, which only differs from the other by 1 eq. hydrogen. It is a very offensive liquid.

Thenard has also indicated the existence of compounds of phosphorus and ethyle, corresponding to those described under methyle, but has not yet described them. It is evident that an immense number of new compounds, containing metals, phosphorus, and other elements, along with organic groups, and having, like those here described, very remarkable properties, must very soon be discovered.

Salts of Oxide of Ethyle.

Oxide of Ethyle forms both neutral and acid salts. The neutral salts are not at ordinary temperatures decomposed by other salts, like inorganic saline compounds. Thus an alcoholic solution of chloride of calcium does not cause any precipitate in an alcoholic solution of oxalate of oxide of ethyle or oxalic ether. But they are easily decomposed by contact with hydrated alkalies, the acid uniting with the alkali, while the oxide of ethyle separates as hydrate, that is, as alcohol. Thus oxalic ether, $\mathrm{C}_{2} \mathrm{O}_{3}$ Ae $\mathrm{O}$, with hydrate of potash, $\mathrm{K} \mathrm{O}, \mathrm{H} \mathrm{O}$, yields oxalate of potash, $\mathrm{KO}, \mathrm{C}_{2} \mathrm{O}_{3}$, and hydrate of oxide of ethyle, $\mathrm{Ae} \mathrm{O}, \mathrm{H} \mathrm{O}$.

Oxide of ethyle has a very great tendency to form double salts, in which there are 2 eqs. of the acid, 1 eq. of a base, and 1 eq. oxide of ethyle. In these salts the acid, as in the neutral salts, cannot be detected by the usual tests; and indeed they may be viewed as simple salts, containing a compound acid, of which oxide of ethyle is a constituent, united to the inorganic base. Thus the double sulphate of ethyle and potash, $\mathrm{K} \mathrm{O}, \mathrm{S} \mathrm{O}_{3}+\mathrm{Ae} \mathrm{O}_{2} \mathrm{~S} \mathrm{O}_{3}$, may be viewed as sulphovinate of potash $\mathrm{K} \mathrm{O}+\mathrm{Ae} 0,2 \mathrm{~S} \mathrm{O}_{3}$; and sulphovinic acid is, on this view, when separated, $\mathrm{H} \mathrm{O+}$ $\mathrm{Ae} 0,2 \mathrm{~S} \mathrm{O}_{3}$.

The acid salts of ethyle are on one view double salts, as, for example, the double sulphate formed of sulphate of ethyle, Ae O, $\mathrm{S} \mathrm{O}_{3}$, with sulphate of water, $\mathrm{H} \mathrm{O}, \mathrm{S} \mathrm{O}_{3}$. On the other view, they are compound or coupled acids, and the above example becomes, as mentioned in the last paragraph, sulphovinic acid, $\mathrm{H} \mathrm{O}+\left(\mathrm{Ae} \mathrm{O}_{2} 2 \mathrm{~S} \mathrm{O}_{3}\right)$, the hydrate of a compound of anhydrous sulphuric acid with ether. These acid salts are decomposed, by boiling with water, into alcohol which distils over, and hydrated 
acids which remain behind. When distilled with the salts of volatile acids, they yield the ethers of those acids; formiate and acetate of ethyle may be thus obtained. When an acid salt of ethyle is heated with acids not volatile, it often happens that the ethers of these acids are obtained : this is the case with the fatty acids and with some others.

1. Neutral sulphate of Oxide of Ethyle, $\mathrm{Ae} \mathrm{O}, \mathrm{S} \mathrm{O}_{3}$, is formed when anhydrous sulphurio acid acts on pure ether. It is a neutral oil, which may be distilled, but is easily decomposed by too strong a heat. Its taste is like that of oil of peppermint. In contact with water, it yields alcohol and two acids, which are isomeric, namely, sulphovinic acid, or acid sulphate of ethyle, and isethionic acid.

$$
\begin{aligned}
& 4\left(\mathrm{Ae} \mathrm{O}_{2} \mathrm{~S} \mathrm{O}_{3}\right)+4 \mathrm{H} \mathrm{O}=2 \mathrm{~S} \mathrm{O}_{3}, \mathrm{Ae} \mathrm{OH} \mathrm{O}+\mathrm{Ae} \mathrm{O}, \mathrm{S} \mathrm{O}_{3}, \mathrm{HO}, \mathrm{S} \mathrm{O}_{3}+ \\
& \text { Neutral Sulphate. } \\
& \text { Isethionic Acid. } \\
& \text { Sulphovinic Acid. } \\
& 2 \text { ( } \mathrm{Ae} \mathrm{O}, \mathrm{H} \mathrm{O}) \text {. } \\
& \text { Alcohol. }
\end{aligned}
$$

2. Acid sulphate of Oxide of Ethyle, $\mathrm{H} \mathrm{O}, \mathrm{S} \mathrm{O}_{3}+\mathrm{Ae}_{\mathrm{O}} \mathrm{O}, \mathrm{S} \mathrm{O}_{3}$, is also called sulphovinic acid, $\mathrm{H} \mathrm{O}+(\mathrm{Ae} \mathrm{O}, 2 \mathrm{~S} \mathrm{O})_{3}$. This acid salt is formed when the vapour of ether is conducted into oil of vitriol, or when oil of vitriol is mixed with alcohol and heated to a certain point. To obtain it pure, the double sulphate of ethyle and baryta (sulphovinate of baryta) in solution, is decomposed by sulphuric acid, and the filtered liquid is a solution in water of the acid sulphate. It has a very sour taste, and cannot be concentrated by evaporation, whether at the ordinary temperature or with the aid of heat, without being decomposed into alcohol and sulphuric acid. It forms, with most bases, crystallisable double salts, which are all soluble, so that, for example, the addition of baryta causes no precipitate if the acid be pure. As the acid sulphate itself is called sulphovinic acid, so these double salts are called sulphovinates. It is because all these salts are soluble, that the usual test cannot detect the sulphuric acid they contain. When, however, their solutions are boiled with a little hydrochloric acid, alcohol is given off, and then the sulphuric acid may be detected as usual. All these salts are decomposed by heat, yielding, according to the temperature, double sulphate of ether and etherole, alcohol, sulphurous acid, olefiant gas, and a sulphate as residue, mixed with charcoal. When heated with hydrated alkalies, they yield sulphates and alcohol. The double sulphate of ethyle and potash crystallises in shining scales, which are the anhydrous salt, $\mathrm{K} \mathrm{O}, \mathrm{S} \mathrm{O}_{3}+\mathrm{Ae} \mathrm{O}, \mathrm{S} \mathrm{O}_{3}$. The baryta salt contains 2 eqs. of water of crystallisation, and forms beautiful tabular 
crystals, as do also the salt of lime, and the salt of lead, both of which likewise contain 2 eqs. of water. These three salts are all composed according to the formula, $\mathrm{M} \mathrm{O}, \mathrm{S} \mathrm{O}_{3}+\mathrm{Ae} \mathrm{O}, \mathrm{S} \mathrm{O}_{3}+2$ aq. There exist several compounds apparently isomeric with sulphovinic acid, which will be noticed further on.

3. Acid Phosphate of Oxide of Ethyle, or phosphovinic acid, $\mathrm{PO}_{5}\left\{\begin{array}{l}\mathrm{Ae} \mathrm{O} \\ 2 \mathrm{HO},\end{array}\right.$ is formed in the same way as sulphovinic acid, and obtained pure from the double salt of baryta, $\mathrm{P} \mathrm{O}_{5}\left\{\begin{array}{l}\mathrm{Ae} \mathrm{O} \\ 2 \mathrm{Ba} \mathrm{O}\end{array}\right.$ $+12 \mathrm{H} \mathrm{O}$. It is a tolerably permanent acid, decomposed only by a high temperature. With bases it forms double salts, in which the 2 eqs. water of the acid are replaced by 2 eqs. of a protoxide. The baryta salt, the formula of which is given above, crystallises in pearly scales.

4. Nitrate of Oxide of Ethyle, $\mathrm{C}_{4} \mathrm{H}_{5} \mathrm{O}, \mathrm{N} \mathrm{O}_{5}=\mathrm{Ae} \mathrm{O}, \mathrm{N} \mathrm{O}_{5}$. Sxn. Nitric Ether. When 2 fluid-ounces of alcohol, and 1 fluidounce of pure nitric acid, of Sp. G. 1.4, are distilled together, with the addition of 10 or 20 grains of urea, to destroy any nitrous or hyponitrous acid, the distillation proceeds calmly and smoothly, and the distilled liquor contains water, alcohol, and nitrate of ethyle, which partly separates, towards the end of the process, as a heavy oily stratum, and is more completely separated by the addition of water. It is a colourless liquid, of Sp. G. 1.112, which boils at $185^{\circ}$, and is inflammable, burning with a bright white flame. It is quite insoluble in water, but very soluble in alcohol ; and it possesses a pleasant smell and a sweet taste. An alcoholic solution of potash converts it into alcohol and pure nitrate of potash.

5. Hyponitrite of Oxide of Ethyle, $\mathrm{Ae} \mathrm{O}, \mathrm{N} \mathrm{O}_{3}$. Srr. Nitrous Ether. This is best prepared in a state of purity when a current of hyponitrous acid vapours, derived from starch and nitric acid, is passed through weak alcohol, the product being condensed in Liebig's refrigeratory. The ether is washed with water, and dried by means of chloride of calcium. The whole apparatus must be kept cold, otherwise the action is too violent, and the results very complex. When nitrous ether is made by the usual processes, in which ordinary nitric acid is mixed with alcohol, the product always contains a large proportion of aldehyde, and, in fact, very litfle of the true ether. The action in this case is as follows: $2\left(\mathrm{C}_{4} \mathrm{H}_{6} \mathrm{O}_{2}\right)+\mathrm{N} \mathrm{O}_{5}=\mathrm{C}_{4} \mathrm{H}_{3} \mathrm{O}, \mathrm{HO}$ (aldehyde), + $3 \mathrm{H} \mathrm{O}+\left(\mathrm{C}_{4} \mathrm{H}_{5}, \mathrm{O}+\mathrm{N} \mathrm{O}_{3}\right)$. The pure hyponitrous ether, prepared by Liebig's process, given above, is a pale yellow liquid, boiling at $62^{\circ}$, of Sp. G. 0.947. It has a very agreeable odour of rennet-apples. With an alcoholic solution of potash, it yields 
alcohol, and pure hyponitrite of potash. The sweet spirit of nitre, or spiritus atheris nitrosi of the pharmacopœia, is a solution of the impure hyponitrous ether in alcohol.

6. Perchlorate of Oxide of Ethyle, $\mathrm{C}_{4} \mathrm{H}_{5} \mathrm{O}, \mathrm{Cl} \mathrm{O}_{7}=\mathrm{Ae} \mathrm{O}$, $\mathrm{Ce}_{77}$. Srv. Perchloric Ether. This ether is prepared by distilling a mixture of 1 part of perchlorate of baryta with 1 part of crystallised ethylosulphate (sulphovinate) of baryta. The mixture must be in a small retort, and must not exceed 1 drachm in weight; and it is heated gradually in an oil bath. A U-shaped tube serves as receiver, and is kept cold by a freezing mixture. $\mathrm{Up}$ to $212^{\circ}$, and as long as the water of crystallisation has not been expelled, no explosion need be feared. The retort is then gently heated to not beyond $340^{\circ} \mathrm{F}$., at which point the action is completed. In the receiver is found the new ether, under a stratum of water; the latter is removed, cautiously, by means of slips of bibulous paper, without touching the receiver with the hand, because a slight motion may cause explosion.

Perchloric ether is a liquid heavier than water, and insoluble in it, of an agreeable odour, and a taste at first sweet, then pungent, like cinnamon. It neither boils nor explodes when heated to $212^{\circ}$ with care. But a stronger heat or friction, or percussion, will cause it to explode, which it does with greater violence than any known compound. It is very apt to explode from causes so slight that it appears to do so without any cause, and is therefore extremely dangerous. In making and experimenting on it, the operator must be protected by very thick gloves, and a strong mask with thick glass eye-holes.

This dangerous compound can only be kept safely when it is mixed with a sufficient quantity of absolute alcohol, which dissolves it.

A little of this solution, on the addition of water, deposits the ether, which collects below in a drop of size proportioned to the quantity employed. If the water be decanted off with care, and the last portion of it taken up by paper, the drop of ether may be made to detonate on a plate or capsule, by touching it with a hot body, or by a slight blow with a hammer. The smallest drop inevitably shatters the vessel on which it rests.

The cause of the explosion is evidently twofold. First, the compound is unstable, from its complexity, its empirical formula being $\mathrm{C}_{4} \mathrm{H}_{5} \mathrm{Cl} \mathrm{O}_{8}$. Secondly, the hydrogen has a very strong aftinity both for oxygen and chlorine, and the carbon for oxygen, and there is oxygen enough present to convert the whole into gaseous compounds.

For $\mathrm{C}_{4} \mathrm{H}_{5} \mathrm{Cl} \mathrm{Os}=\mathrm{H} \mathrm{Cl}+4 \mathrm{H} \mathrm{O}+4 \mathrm{C} \mathrm{O}$, so that it is entirely resolved into hydrochloric acid, steam, and carbonic oxide gas, 
and as this takes place instantaneously, much heat is developed, which heats and expands to a high degree the gases formed.

7. Borates of Oxide of Ethyle.-There appear to be several borates of oxide of ethyle, or boracic ethers. The neutral borate is $3 \mathrm{Ae} \mathrm{O}+\mathrm{B} \mathrm{O}_{3}$, corresponding to a terhydrate of the acid, $3 \mathrm{HO}+$ $\mathrm{B} \mathrm{O}_{3}$. It is obtained by the action of chloride of boron on absolute alcohol, and is a mobile liquid, of a peculiar pleasant odour, and a warm bitter taste. Its density is 0.8849 ; that of its gas is $5 \cdot 14$, corresponding to 4 vols. for the formula above given. It boils at $246^{\circ} \mathrm{F}$., and is decomposed by water, which dissolves it, yielding boracic acid and alcohol.

Another borate is the biborate of ethyle, $\mathrm{Ae} \mathrm{O}, 2 \mathrm{~B} \mathrm{O}$; which is formed by the action of boracic acid, previously fused to expel all water, on absolute alcohol. It is a viscid liquid of an ethereal smell and a pungent taste.

8. Silicates of Oxide of Ethyle.-These are also several silicic ethers. That formed by the action of chloride of silicon on absolute alcohol, is $4 \mathrm{Ae} 0+4 \mathrm{Si} 0$. It is a limpid ether, of a pleasant odour, and pungent taste, of Sp. G. 0.933 at $68^{\circ} \mathrm{F}$., insoluble in water, which gradually decomposes it. It boils at about $330^{\circ} \mathrm{F}$., and the density of its gas is $7 \cdot 32=4$ vols. for the above formula.

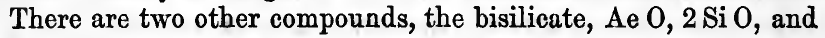
the quadrisilicate, Ae 0, $4 \mathrm{Si} 0$. The former is a liquid, of Sp. G. 1.079 , boiling at $662^{\circ} \mathrm{F}$. When left in contact with water, it is slowly decomposed, and the silicic acid is deposited as a hydrate which is transparent and glassy, but contracts ultimately to a mass of hyaline quartz, sufficiently hard to scratch glass, and which in some cases is true hydrophane; that is, like that mineral, it becomes transparent in water.

The quadrisilicate is a glassy mass, like amber, softening by heat, and when still more strongly heated, yielding silicic acid, and the first ether or protosilicate. It is soluble in alcohol, ssider, and the two preceding ethers.

9. Carbonate of Oxide of Ethyle, $\mathrm{Ae} \mathrm{O}, \mathrm{C} \mathrm{O}_{2}$. Srn. Carbonic Ether. When oxalic ether is acted on by potassium, there are formed several products, one of which is this ether. When pure it is an aromatic liquid, of Sp. G. 0.975 , boiling at $260^{\circ}$. An alcoholic solution of potash converts it into alcohol and carbonate of potash. Chlorine acts on it, forming products to be described when we treat of the action of chlorine on ethers generally.

10. Double Carbonate of Ethyle and Potash, $\mathrm{K} \mathrm{O}, \mathrm{C} \mathrm{O}_{2}+\mathrm{Ae} \mathrm{O}$, $\mathrm{C} \mathrm{O}_{2}$, is formed when dry carbonic acid gas is passed through an alcoholic solution of fused potash. A saline mass is obtained, from which, after washing with ether, alcohol dissolves the double salt, leaving carbonate and bicarbonate of potash. The double salt 
forms pearly scales, which are decomposed by water into alcohol and bicarbonate of potash.

11. Chlorocarbonic Ether, $\mathrm{C}_{6} \mathrm{H}_{5} \mathrm{Cl}_{4}=\mathrm{C}_{4} \mathrm{H}_{5}, \mathrm{O}+\mathrm{C}_{2}\left\{\begin{array}{l}\mathrm{O}_{3} \\ \mathrm{Cl}\end{array}\right.$ ? is formed when absolute alcohol is placed in contact with chlorocarbonic acid gas. It appears as an oily liquid, of Sp. G. 1.133, boiling at $200^{\circ}$. It is formed as follows : 2 eqs. of chlorocarbonic acid and 1 of alcohol, losing 1 eq. hydrochloric acid, yield 1 eq. of the new ether. $\mathrm{C}_{2} \mathrm{O}_{2} \mathrm{Cl}_{2}+\mathrm{C}_{4} \mathrm{H}_{6} \mathrm{O}_{2}=\mathrm{H} \mathrm{Cl}+\mathrm{C}_{6} \mathrm{H}_{6} \mathrm{Cl} \mathrm{\textrm {O } _ { 4 }}$. It may be viewed as a compound of oxide of ethyle, with a peculiar acid $\mathrm{C}_{2}\left\{\begin{array}{l}\mathrm{O}_{3} \\ \mathrm{Cl}\end{array}\right.$; or as carbonic ether, $\mathrm{C}_{5} \mathrm{H}_{5} \mathrm{O}_{3}$, plus 1 eq. chlorocarbonic acid, $\mathrm{C}\left\{\begin{array}{l}\mathrm{O} \\ \mathrm{Cl}\end{array}\right.$.

12. Carbamate of Oxide of Ethyle, Carbamic Ether, $\mathrm{C}_{6} \mathrm{H}_{7} \mathrm{~N} \mathrm{O}_{4}$, SrN. Urethane, is formed by the action of ammonia on the preceding compound, along with sal-ammoniac, from which it is separated by being sublimed, or rather distilled, when it passes over at a gentle heat, as a liquid which crystallises on cooling. It is very soluble in water and alcohol, and yields very large crystals. It may be viewed as chlorocarbonic ether, in which amide, $\mathrm{N} \mathrm{H}_{2}$, has been substituted for the chlorine, $\mathrm{C}_{4} \mathrm{H}_{5}$ $0+\mathrm{C}_{2}\left\{\begin{array}{l}\mathrm{O}_{3} \\ \mathrm{~N} \mathrm{H}_{2}\end{array}\right.$. It is formed as follows: $\mathrm{C}_{6} \mathrm{H}_{5} \mathrm{Cl} \mathrm{O}_{4}+2 \mathrm{~N} \mathrm{H}_{3}$, $=\left(\mathrm{N} \mathrm{H}_{3}, \mathrm{H} \mathrm{Cl}\right)+\mathrm{C}_{6} \mathrm{H}_{7} \mathrm{~N} \mathrm{O}_{4}$. Berzelius, with much probability, considers it as a compound of oxide of ethyle with an acid, not yet known in the separate form, containing the elements of carbonic acid and carbamide, just as oxamic acid does those of oxalic acid and oxamide. On this view its rational formula will be $\mathrm{C}_{4} \mathrm{H}_{5} \mathrm{O}+\mathrm{C} \mathrm{O}_{2}, \mathrm{C} \mathrm{ON} \mathrm{H}=\mathrm{C}_{6} \mathrm{~N} \mathrm{H}_{7} \mathrm{O}_{4}$.

Oxide of Ethyle. Carbamic Acid.

It is remarkable that three other compounds, quite distinct in properties, have the same empirical formula as urethane or carbamic ether. These are lactamide, or anhydrous lactate of ammonia $\mathrm{C}_{6} \mathrm{H}_{4} \mathrm{O}_{4}+\mathrm{N} \mathrm{H}_{3}$; sarcosine, $\mathrm{C}_{6} \mathrm{~N} \mathrm{H}_{7} \mathrm{O}_{4}$, a new base, derived from kreatine, and alanine, a compound homologous with glycocine.

13. Oxalate of Oxide of Ethyle, Ae $\mathrm{O}_{1} \mathrm{C}_{2} \mathrm{O}_{3}$. SYN. Oxalic Ether. This ether is formed by distilling 4 parts of superoxalate of potash, 5 of oil of vitriol, and 4 of alcohol at $90 \mathrm{p}$. c., mixing the product with 4 times its bulk of water, and washing with water the ether which separates, until all free acid is removed. The ether is then rectified. It is a colourless liquid, of Sp. G. $1 \cdot 093$, boiling at $364^{\circ}$. It has an aromatic smell. If 
pure, it may be kept under water; but if a trace of alcohol or of oxalic acid be present, it is soon resolved into oxalic acid and alcohol when in contact with water. Fixed alkalies cause the same change. When an excess of ammonia is added to it oxamide is formed: where the ether is in excess, there is formed a substance in beautiful pearly tables, formerly called oxamethane, but now proved to be oxamate of ethyle. These two reactions are easily explained. In the first case, $\mathrm{Ae} O, \mathrm{C}_{2} \mathrm{O}_{3}+$ $\left.\mathrm{N} \mathrm{H}_{3}=(\mathrm{Ae} \mathrm{O}, \mathrm{H} \mathrm{O})+\mathrm{C}_{2} \mathrm{O}_{2}, \mathrm{~N} \mathrm{H}_{2}\right)$. In the second, half the ether undergoes the above change, and the other half combines with the oxamide formed. $\mathrm{Ae} \mathrm{O}, \mathrm{C}_{2} \mathrm{O}_{3}+\mathrm{C}_{2} \mathrm{O}_{2}, \mathrm{~N} \mathrm{H}_{2}=\mathrm{Ae} \mathrm{O}$, $\mathrm{C}_{4} \mathrm{~N} \mathrm{H}_{2} \mathrm{O}_{5}$. Chlorine acts on oxalio ether, giving rise to products which will be hereafter described along with the results of the action of chlorine on other ethers.

14. Acid Oxalate of Oxide of Ethyle. Srn. Oxalovinic Acid. When to an alcoholic solution of oxalic ether there is added enough of an alcoholic solution of potash or soda to decompose the half of the ether, double salts are obtained, of the formula $\mathrm{M} \mathrm{O}, \mathrm{C}_{2} \mathrm{O}_{3}+\mathrm{Ae} \mathrm{O}, \mathrm{C}_{2} \mathrm{O}_{3}$. When the alcoholio solution of the double oxalate of ethyle and potash is treated by fluosilicic acid there is obtained the acid oxalate of ethyle, $\mathrm{H} \mathrm{O}, \mathrm{C}_{2} \mathrm{O}_{3}+\mathrm{Ae} \mathrm{O}$, $\mathrm{C}_{2} \mathrm{O}_{3}$, which is often called oxalovinic acid. The salt of potash, $\mathrm{K} 0, \mathrm{C}_{2} \mathrm{O}_{3}+\left(\mathrm{Ae} \mathrm{O}, \mathrm{C}_{2} \mathrm{O}_{3}\right.$ ) (oxalovinate of potash), forms crystalline scales, soluble in alcohol. The oxalovinate of baryta is extremely soluble, and may be used to furnish the other oxalovinates, by acting with it on the soluble sulphates of different bases.

15. Oxamate of Oxide of Ethyle, $\mathrm{C}_{8} \mathrm{~N} \mathrm{H}_{7} \mathrm{O}_{6}=\mathrm{Ae} 0, \mathrm{C}_{4}$ $\mathrm{N} \mathrm{H}_{2} \mathrm{O}_{5}=\mathrm{Ae} \mathrm{O}, \mathrm{C}_{2} \mathrm{O}_{2}+\mathrm{Ad}, \mathrm{C}_{2} \mathrm{O}_{2}$, is formed, as above stated, when ammonia is cautiously added to an alcoholic solution of oxalio ether, until a white powder (oxamide) begins to appear. The liquid now yields fine pearly tabular crystals, formerly called oxamethane. It now appears to be oxamate of ethyle, but may also be viewed as oxalate of ethyle, plus oxamide. By an excess of ammonia, it is converted into alcohol and oxamide. The action of ammonia on oxalio ether has been explained above.

16. Sulphocarbonate of Ethyle and Water, $\mathrm{Ae} \mathrm{O}, \mathrm{H} \mathrm{O}, 2 \mathrm{C} \mathrm{S}$. When bisulphuret of carbon is added to a strong alcoholic solution of potash, a salt is obtained, in colourless or yellow needles, which is a double sulphocarbonate of ethyle and potash, $\mathrm{K} \mathrm{O}, \mathrm{C} \mathrm{S}_{2}+\mathrm{Ae} \mathrm{O}, \mathrm{C} \mathrm{S}_{2}$. When this salt is acted on by diluted sulphuric or hydrochloric acid, there is obtained a heavy oily liquid. This is the acid compound in question, $\mathrm{H} \mathrm{O}, \mathrm{C} \mathrm{S}_{2}+$ Ae $0, \mathrm{C} \mathrm{S}_{2}$, formerly called xanthic acid, from the yellow colour 
of its salts. With bases it gives rise to double salts, like that of potash, just mentioned, which were called xanthates. The salt of protoxide (suboxide) of copper is lemon-yellow.

17. Benzoate of Oxide of Ethyle, or Benzoic Ether, $\mathrm{Ae} \mathrm{O}, \mathrm{Bz}$ 0 , is best formed by distilling 4 parts of alcohol, 2 of benzoic acid, and 1 of strong hydrochloric acid. The ether distils over with alcohol, from which water separates it. When pure, it is an oily, colourless liquid, of a faint agreeable aromatic odour, and an acrid, spicy taste. Its Sp. G. is 1.054 , and it boils at about $410^{\circ}$. Chlorine decomposes it, giving rise to several products, probably by substitution.

18. Hippurate of Oxide of Ethyle, or Hippuric Ether, $\mathrm{C}_{4} \mathrm{H}_{5}$, $\mathrm{O}+\mathrm{C}_{18} \mathrm{~N} \mathrm{H}_{8} \mathrm{O}_{5}$, is formed by passing a current of hydrochloric acid gas through a solution of hippuric acid in alcohol, and heating the mixture for some time near to its boiling point. The addition of water separates a thick, heavy oil, which when purified from alcohol and hydrochloric acid, and placed in vacuo, along with sulphuric acid, forms a solid crystalline mass, composed of silky needles. It is decomposed, like other ethers, by alkalies, and by boiling with water.

19. Salicylate of Oxide of Ethyle, $\mathrm{C}_{4} \mathrm{H}_{5}, \mathrm{O}+\mathrm{C}_{14} \mathrm{H}_{5}, \mathrm{O}_{5}$, is obtained by distilling 2 parts of alcohol, $1 \frac{1}{2}$ of salicylic acid, and 1 of sulphuric acid. When purified from alcohol, acid, and water, it is a colourless oily fluid, having a sweet smell like that of the corresponding compound of methyle, which occurs naturally in the oil of Gaultheria procumbens. It is heavier than water, and boils at $437^{\circ}$. Like the oil of Gaultheria, it plays the part of an acid, forming with bases crystallised soluble salts. When exposed to a high temperature with caustic baryta, it yields carbonic acid, and an oil analogous to that obtained from the methyle compound, which is phenetole, $\mathrm{C}_{16} \mathrm{H}_{10} \mathrm{O}_{2}$, or carbolate of oxide of ethyle. When fuming nitric acid is added, drop by drop, to the salicylic ether, it dissolves it with a deep red colour: water now separates an oil, which soon concretes into a solid mass, which when dissolved in hot alcohol, yields, on cooling, yellow silky needles. These are indigotate or anilate, properly nitrosalicylate of oxide of ethyle, $\mathrm{C}_{4} \mathrm{H}_{3}, \mathrm{O}+\mathrm{C}_{44} \mathrm{~N} \mathrm{H}_{4} \mathrm{O}_{9}$. By the further action of nitric acid, carbazotic or nitropicric acid is obtained. The nitrosalicylic ether dissolves in potash and soda, apparently like the salicylic ether, playing the part of an acid. Nitrosalicylic ether does not dissolve in ammonia; left in contact within close vessels, it finally disappears, alcohol is reproduced, and there is formed a new product, nitrosalicylamide, $\mathrm{C}_{14} \mathrm{~N}_{2} \mathrm{H}_{6}$ $\mathrm{O}_{8}$, which, when pure, forms brilliant yellow crystals. When boiled with potash, nitrosalicylamide yields nitrosalicylate of 
potash, and gives off ammunia; for $\mathrm{C}_{14} \mathrm{~N}_{2} \mathrm{H}_{6} \mathrm{O}_{8}+2 \mathrm{H} \mathrm{O}=$ $\mathrm{N} \mathrm{H}_{3}+\mathrm{C}_{44} \mathrm{H}_{4} \mathrm{~N} \mathrm{O} \mathrm{O}_{0} \mathrm{HO}$. Bromine acts on salicylic ether, producing two compounds ; monobromuretted salicylic ether, $\mathrm{C}_{88} \mathrm{H}_{9} \mathrm{Br} \mathrm{O}_{6}=\mathrm{C}_{4}\left\{\begin{array}{l}\mathrm{H}_{4} \\ \mathrm{Br}\end{array}\right.$ salicylic ether, $\mathrm{C}_{18} \mathrm{H}_{8} \mathrm{Br}_{2} \mathrm{O}_{6}=\mathrm{C}_{4}\left\{\begin{array}{l}\mathrm{H}_{3} \\ \mathrm{Br}_{2}\end{array} \mathrm{O}+\mathrm{C}_{24} \mathrm{H}_{5} \mathrm{O}_{5}\right.$. The former crystallises in fine needles, the latter in large pearly scales, which, when melted, form, on cooling, a most beautiful crystallisation, formed of large and perfect cubes, like those of bismuth.

20. Cyanate of Oxide of Ethyle. SrN. Cyanic Ether. Ae O, Cy $\mathrm{O}=\mathrm{C}_{6} \mathrm{~N} \mathrm{H}_{5} \mathrm{O}_{2}$. Obtained by Wurtz by heating sulphovinate with cyanate of potash. It is a very pungent, volatile liquid. The Sp. G. of its vapour is $2 \cdot 4$. It forms, with ammonia, a crystalline body, $\mathrm{C}_{6} \mathrm{~N}_{2} \mathrm{H}_{8} \mathrm{O}_{2}=\mathrm{N} \mathrm{H}_{3}+\mathrm{C}_{6} \mathrm{~N} \mathrm{H}_{5} \mathrm{O}_{2}$. The constitution of this compound is uncertain. In contact with water, cyanic ether yields carbonic acid and a new compound, $2\left(\mathrm{C}_{6} \mathrm{~N} \mathrm{H}_{5} \mathrm{O}_{2}\right)+2 \mathrm{HO}=2 \mathrm{C} \mathrm{O}_{2}+\mathrm{C}_{10} \mathrm{~N}_{2} \mathrm{H}_{12} \mathrm{O}_{2}$. The latter body is crystalline, soluble in alcohol and water. It is either butylo-urea (a body homologous with urea), or diethylo-urea, which is isomeric with that compound.

21. Ethylo-urea, $\mathrm{C}_{6} \mathrm{~N}_{2} \mathrm{H}_{8} \mathrm{O}_{2}$, is formed when cyanate of potash is heated in solution with sulphate of ethylamine. The substance thus formed is truly homologous with urea and methylo-urea. Its formation corresponds to that of urea from cyanate of potash and sulphate of ammonia, $\mathrm{K} \mathrm{O}, \mathrm{C}_{2} \mathrm{~N} \mathrm{O}+\mathrm{C}_{4} \mathrm{H}_{7} \mathrm{~N}, \mathrm{H} \mathrm{O}, \mathrm{S} \mathrm{O}_{3}=$ $\mathrm{K} \mathrm{O}, \mathrm{S} \mathrm{O}_{3}+\mathrm{C}_{6} \mathrm{~N}_{2} \mathrm{H}_{8} \mathrm{O}_{2}$.

22. Allophanate of Oxide of Ethyle. This compound is formed when the vapour of hydrated cyanic acid is brought in contact with a mixture of ether and alcohol. It forms brilliant prisms, which may be purified from cyamelide by being dissolved in boiling alcohol, or in boiling water. Its formula is $\mathrm{C}_{8} \mathrm{~N}_{2} \mathrm{H}_{8} \mathrm{O}_{6}$ $=\mathrm{C}_{4} \mathrm{H}_{5} \mathrm{O}, \mathrm{C}_{4} \mathrm{~N}_{2} \mathrm{H}_{3} \mathrm{O}_{5}$. It was formerly supposed to consist of 2 eqs. hydrated cyanuric acid, 3 eqs. oxide of ethyle, and 3 eqs. of water; and it is, in fact, resolved by heat into alcohol and cyanuric acid. But Liebig and Wöhler have shown that it is composed of oxide of ethyle and of a new acid, namely, anhydrous allophanic acid, $\mathrm{C}_{4} \mathrm{~N}_{2} \mathrm{H}_{3} \mathrm{O}_{5}$. The former view was quite anomalous, since oxide of ethyle unites only with anhydrous acids; and the latter, therefore, brings the compound into the ordinary category of compound ethers.

Allophanic acid is unknown in the hydrated or separate state, the formula of which would be $\mathrm{C}_{4} \mathrm{~N}_{2} \mathrm{H}_{3} \mathrm{O}_{5}, \mathrm{H} \mathrm{O}=\mathrm{C}_{4} \mathrm{~N}_{2}$ $\mathrm{H}_{4} \mathrm{O}_{6}$. It forms crystallisable salts with baryta, potash and 
soda; but when we attempt to isolate it, it is resolved into urea and carbonic acid, $\mathrm{C}_{4} \mathrm{~N}_{2} \mathrm{H}_{4} \mathrm{O}_{6}=\mathrm{C}_{2} \mathrm{~N}_{2} \mathrm{H}_{4} \cdot \mathrm{O}_{2}+2 \mathrm{C} \mathrm{O}_{2}$. Allophanic acid is produced by the action of 2 eqs. of hydrated cyanic acid on 1 eq. of alcohol. The water of the alcohol, added to the cyanic acid, produces the new acid, which combines with the oxide of ethyle. Thus, $2\left(\mathrm{C}_{2} \mathrm{~N} \mathrm{O}, \mathrm{H} \mathrm{O}\right)+\mathrm{C}_{4} \mathrm{H}_{5} \mathrm{O}, \mathrm{H} \mathrm{O}=$ Hydrated Cyanic Acid.

Alcohol.

$\underbrace{\mathrm{C}_{4} \mathrm{H}_{5} \mathrm{O}}+\underbrace{\mathrm{C}_{4} \mathrm{~N}_{2} \mathrm{H}_{3} \mathrm{O}_{5}}$.

Oxide of Ethyle. Allophanic Acid.

23. Cyanurate of Oxide of Ethyle. $\mathrm{Cy}_{3} \mathrm{O}_{3}, 3 \mathrm{Ae} \mathrm{O}=\mathrm{C}_{18} \mathrm{~N}_{3}$ $\mathrm{H}_{15} \mathrm{O}_{6}$ (Wurtz). SrN. Cyanuric Ether. This compound is obtained by heating sulphovinate of potash with tribasic cyanurate of potash. It forms brilliant crystals, melting at $185^{\circ}$, and boils at about $529^{\circ}$. The density of its vapour is 7.4. It is soluble in alcohol and ether.

Limpricht has discovered a compound, $\mathrm{C}_{14} \mathrm{~N}_{3} \mathrm{H}_{11} \mathrm{O}_{6}$, which may be viewed as a cyanuric ether, with 2 eqs. of oxide of ethyle and one of basic water, $\mathrm{C}_{6} \mathrm{~N}_{3} \mathrm{O}_{3}+\begin{array}{r}2 \mathrm{Ae} \\ \mathrm{H}\end{array}$ opinion that neither this nor the preceding compound are true ethers, because they do not yield cyanuric acid and alcohol when heated with potash, but carbonic acid and ethylamine. If not true cyanuric ethers, they are at least isomerio with such compounds.

Oxide of Ethyle and Potassium:- $\left.\mathrm{C}_{4} \mathrm{H}_{5} \mathrm{O}, \mathrm{KO}=\underset{\mathrm{K}}{\mathrm{C}_{4}}\right\}_{\mathrm{O}^{\circ}}^{\mathrm{H}_{5}}$

SxN.-Alcoholate of Potash. This body is formed when potassium acts on pure anhydrous alcohol:

$$
\left.\left.\underset{\mathrm{H}}{\mathrm{C}_{4} \mathrm{H}_{5}}\right\}_{\mathrm{O}}^{\mathrm{O}}+\mathrm{K}=\underset{\mathrm{K}}{\mathrm{C}_{4} \mathrm{H}_{5}}\right\}_{\mathrm{O}}^{\mathrm{O}}+\mathrm{H} \text {. }
$$

It forms large transparent crystals. When acted on by chloride or iodide of ethyle, oxide of ethyle is reproduced, thus :

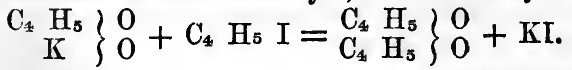

\section{BASES CONTAINING ETHYLE.}

1. Ethylamine, $\mathrm{C}_{4} \mathrm{H}_{7} \mathrm{~N}=\mathrm{NH}_{2} \mathrm{Ae}$. This base, discovered by Wurtz, is homologous with methylamine, and is obtained exactly in the same way, by heating cyanic ether, cyanuric ether, or ethylo-urea with potash. 
$\underbrace{\mathrm{C}_{4} \mathrm{H}_{5} \mathrm{O}, \mathrm{C}_{2} \mathrm{~N} \mathrm{O}}_{\text {Cyanic Ether. }}+2(\mathrm{~K}, \mathrm{H} 0)=2\left(\mathrm{~K} \mathrm{O}_{1} \mathrm{C} \mathrm{O}_{2}\right)+\underbrace{\mathrm{C}_{4} \mathrm{H}_{7} \mathrm{~N}}_{\text {Ethylamine. }}$.

We have only to treble this equation to explain the formation of ethylamine from cyanuric ether. The following equation explains its production from ethylo-urea.

$$
\underbrace{\mathrm{C}_{6} \mathrm{~N}_{2} \mathrm{H}_{8} \mathrm{O}_{2}}_{\text {Ethylo-urea. }}+2(\mathrm{~K} \mathrm{O}, \mathrm{H} \mathrm{O})=2\left(\mathrm{~K} \mathrm{O}_{,} \mathrm{C} \mathrm{O}_{2}\right)+\mathrm{N} \mathrm{H}_{3}+\underbrace{\mathrm{C}_{4} \mathrm{H}_{7} \mathrm{~N}}_{\text {Ethylamine. }} .
$$

Ethylamine may be obtained by other processes. Hofmann has produced it by the action of bromide of ethyle on ammonia.

$$
\mathrm{N} \mathrm{H}_{3}+\mathrm{C}_{4} \mathrm{H}_{5} \mathrm{Br}=\mathrm{H} \mathrm{Br}+\mathrm{C}_{4} \mathrm{H}_{7} \mathrm{~N} \text {. }
$$

Strecker has discovered another method of obtaining it. When neutral sulphate of oxide of ethyle is acted on by dry ammonia, there is formed the ammonia salt of a new acid, sulphethamic acid. When this salt is boiled with carbonate of baryta till all ammonia is expelled, and the residue distilled with potash, ethylamine is obtained. The sulphethamic acid $\left(\mathrm{C}_{16} \mathrm{~N} \mathrm{H}_{23} \mathrm{O}_{4}\right.$, $4 \mathrm{~S} \mathrm{O}_{3}$ ) yields, when decomposed, several different products, and as all of these are not yet known, the change cannot be represented in the form of an equation.

Lastly, ethylamine is found in the basic oil of coal tar, and in the basic part of animal oil, produced by the distillation of animal matter; and it appears also to occur among the products of the oxidation of some vegetable products. At all events, other bases of this series, such as methylamine, propylamine, and butylamine are so produced. It is probable that many sources of ethylamine remain to be discovered.

Ethylamine is a volatile liquid, boiling at $68^{\circ} \mathrm{F}$. Its density in the liquid form is 0.6964 at $45^{\circ} \mathrm{F}$. The density of its gas is 1.57 to $1 \cdot 60$. The density of its vapour is 1.5568. It mixes readily with water, developing heat. It has a very pungent ammoniacal smell, with something putrid or empyrcumatic. This is natural, since the smell of empyreumatic animal oil is made up of those of ammonia, methylamine, ethylamine, \&c., besides other oils, such as kreosote. It is also probable that in the putrefaction of animal matter, not only ammonia, but also methylamine and ethylamine, are formed. Ethylamine has no doubt long been overlooked, from its great resemblance to ammonia. It is powerfully rubefacient, and even caustic. It takes fire on the contact of a light, and burns with a yellowish flame.

It is a very strong base, being the second in the series of 
methylamine. Its rational formula is $\mathrm{Ad} \mathrm{Ae}$, or $\mathrm{N} \mathrm{H}_{2} \mathrm{Ae}$, which may be thus compared to ammonia, $\mathrm{Ad} \mathrm{H}$, or $\mathrm{N} \mathrm{H}_{2}, \mathrm{H}$.

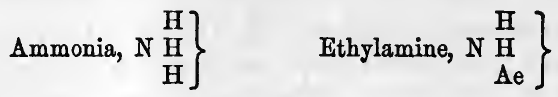

Like methylamine, it is an amide base of the general formula $\mathrm{N} \mathrm{H}_{2}+\mathrm{R}$, or Ad $\mathrm{R} ; \mathrm{R}$ being hydrogen, or any radical of the methylic series, or even of other series, such as that of phenyle. It expels ammonia from its salts, with the aid of heat, and acts on metallic solutions very much as ammonia and methylamine do. An excess of it dissolves alumina even more readily than ammonia does.

The salts of ethylamine resemble those of ammonia and methylamine. It forms double chlorides with the chlorides of mercury, gold, and platinum. The latter salt forms golden yellow crystals, more soluble than the corresponding compound of ammonia.

Ethylamine acts like ammonia and methylamine on the protochloride of platinum, and gives rise to two new platinised bases, probably to as many as ammonia. Wurtz compares as follows the two bases of Reiset with their homologues containing ethylamine and methylamine.

Ammonia series. Methyle series.

Base a. $\mathrm{N}\left\{\begin{array}{l}\mathrm{NH}_{2} \\ \mathrm{Pt}\end{array}\right\}$
Ethyle series.

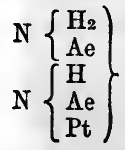

$$
\begin{aligned}
& \text { Base b. } \left.\mathrm{N} \underset{\mathrm{Pt}}{\mathrm{H}_{2}}\right\} \\
& \mathrm{N} \underset{\mathrm{Pt}}{\mathrm{Me}}\}
\end{aligned}
$$

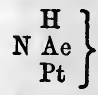

It cannot be doubted that all the other platinised bases will also find homologues in the ethyle and methyle series; and farther, that similar compounds may be formed with the other members of the series, homologous with methyle and ethyle.

When ethylamine is acted on by oxalic ether, there is formed ethyloxamide, homologous with oxamide. Its formula is $\mathbf{C}_{6}$ $\mathrm{N} \mathrm{H}_{6} \mathrm{O}_{2}$. It is soluble and crystallisable, and represents oxamide, in which 1 eq. of hydrogen is replaced by ethyle. Its formation is as follows :

$$
\underbrace{\mathrm{C}_{4} \mathrm{H}_{5} \mathrm{O}, \mathrm{C}_{2} \mathrm{O}_{3}}_{\text {Oxalate of Ethyle. }}+\mathrm{C}_{4} \mathrm{H}_{7} \mathrm{~N}=\underbrace{\mathrm{C}_{4} \mathrm{H}_{6} \mathrm{O}_{2}}_{\text {Alcohol. }}+\underbrace{\mathrm{C}_{6} \mathrm{~N} \mathrm{H}_{6} \mathrm{O}_{2}}_{\text {Ethyloxamide. }} .
$$


The acid amide, corresponding to oxamic acid, probably exists, but has not yet been described, as in the case of methylamine it has.

2. Diethylamine, $\mathrm{C}_{8} \mathrm{H}_{11} \mathrm{~N}=\mathrm{N} \mathrm{H}, \mathrm{Ae}_{2}$. This is an imide base; that is, ammonia, in which 2 eqs. of hydrogen are replaced by ethyle. It is formed by the action of bromide of ethyle on ethylamine, thus :

\section{$\mathrm{C}_{4} \mathrm{H}_{7} \mathrm{~N}+\mathrm{C}_{4} \mathrm{H}_{5} \mathrm{Br}=\mathrm{HBr}+\mathrm{C}_{8} \mathrm{H}_{11} \mathrm{~N}$.}

It is a volatile inflammable oil, soluble in water, and strongly basic. Its salts have been little examined.

If bromide of ethyle were made to act on methylamine, or bromide of methyle on ethylamine, it is quite certain that the result would be the formation of an imide base, ethylomethylamine or methylethylamine, $\mathrm{C}_{6} \mathrm{H}_{9} \mathrm{~N}$, in which the 2 eqs. of hydrogen are replaced by two different radicals.

$$
\mathrm{C}_{4} \mathrm{H}_{5} \mathrm{Br}+\mathrm{C}_{2} \mathrm{H}_{5} \mathrm{~N}=\mathrm{H} \mathrm{Br}+\mathrm{C}_{6} \mathrm{H}_{9} \mathrm{~N}=\mathrm{N} \mathrm{H}, \mathrm{MeAe} \text {. }
$$

We shall see further on that several similar imide bases have thus been formed.

3. Triethylamine, $\mathrm{C}_{12} \mathrm{H}_{15} \mathrm{~N}=\mathrm{N} \mathrm{Ae}_{3}$. This is a nitryle base, or ammonia, in which all 3 eqs. of hydrogen are replaced by ethyle. It is obtained by the action of bromide of ethyle on diethylamine, thus :

$$
\begin{aligned}
\mathrm{NH}, \mathrm{Ae}_{2}+\mathrm{Ae} \mathrm{Br} & =\mathrm{H} \mathrm{Br}+\mathrm{NAes}, \text { or } \\
\mathrm{C}_{8} \mathrm{H}_{11} \mathrm{~N}+\mathrm{C}_{4} \mathrm{H}_{5} \mathrm{Br} & =\mathrm{HBr}+\mathrm{C}_{12} \mathrm{H}_{15} \mathrm{~N} .
\end{aligned}
$$

It is a light, volatile, inflammable liquid, but less so than diethylamine, and it has strong basic properties: The double chloride of triethylamine and platinum is very soluble, and forms large and fine aurora-red crystals.

It is evident that we may expect to obtain other nitryle bases, in which both methyle and ethyle are present, such as diethylomethylamine, $\mathrm{N}, \mathrm{Me} \mathrm{Ae}_{2}$, and dimethylethylamine, $\mathrm{N}, \mathrm{Me}_{2} \mathrm{Ae}$. But these, as well as trimethylamine, $\mathrm{N} \mathrm{Me}_{3}$, are not yet known. We shall find that various nitryle bases, however, are already known, in which ethyle is associated with other radicals to be afterwards described, namely, amyle, $\mathrm{C}_{10} \mathrm{H}_{11}$, and phenyle, $\mathrm{C}_{12} \mathrm{H}_{5}$.

The three ethyle bases above described, ethylamine, diethylamine, and triethylamine, are all formed when bromide of ethyle acts on ammonia. In them, the 3 eqs. of hydrogen are successively replaced by ethyle, while the type of ammonia remains entire. For all these bases are volatile, and in the highest degree 
analogous to ammonia. But the action does not stop here, for a fourth compound is formed, along with the three preceding, and in this, as we have seen under methyle, the type is changed, the base is no longer volatile, but its basic properties are stronger than ever.

4. Tetrethylium, $\mathrm{NAe}_{4}$, is formed along with the three preceding bases, in the action of bromide of ethyle on ammonia, or still better, when triethylamine is acted on by iodide of ethyle.

\section{$\mathrm{NAe}+\mathrm{Ae} I=\mathrm{NAe}_{4} \mathrm{I}$.}

The compound $\mathrm{N} \mathrm{Ae}$ I is the iodide of tetrethylium, and when this is decomposed by oxide of silver, iodide of silver and oxide of tetrethylium are formed. $\mathrm{N} \mathrm{Ae}_{4} \mathrm{I}+\mathrm{Ag} \mathrm{O}=\mathrm{AgI}+$ $\mathrm{N} \mathrm{Ae}_{4} \mathrm{O}$. The oxide dissolves in water, and by evaporation is obtained as a caustic hydrate in crystals, which, like caustic potash, absorb water and carbonic acid from the air. The group $\mathrm{N} \mathrm{Ae} e_{4}=\mathrm{C}_{16} \mathrm{H}_{20} \mathrm{~N}$, is really in its chemical relations an organio metal, perfectly analogous to ammonium and to potassium. The oxide resembles potash, and has at once the caustic taste of that alkali and the bitterness of quinine. It saponifies the oils just as potash does, and in all its reactions, on metallic solutions as well as on organic compounds, it closely resembles potash.

Under the corresponding methyle compound the analogies and the differences between that compound and its oxide on the one hand, and ammonium and its oxide on the other, have been fully pointed out.

When heated, the hydrated oxide is resolved into triethylamine, water and olefiant gas, $\mathrm{N} \mathrm{Ae}_{4} \mathrm{O}, \mathrm{H} \mathrm{O}=\mathrm{N} \mathrm{Ae}_{3}+$ $2 \mathrm{H} \mathrm{O}+\mathrm{C}_{4} \mathrm{H}_{4}$.

The salts of this base are crystallisable, but most of them are deliquescent. The double chloride of tetrethylium and platinum, $\mathrm{N} \mathrm{Ae} e_{4} \mathrm{Cl}, \mathrm{Pt} \mathrm{Cl}_{2}$, instead of being more soluble than the corresponding salt of triethylamine, is, like those of potassium and ammonium, sparingly soluble.

5. Methylotriethylium, $\mathrm{C}_{14} \mathrm{H}_{1 \mathrm{~s}} \mathrm{~N}=\mathrm{N} \mathrm{MeAe}$, is formed when iodide of methyle acts on triethylamine. It corresponds in characters to tetrethylium, and its oxide is a powerful bitter base, yielding crystallisable salts with acids. It is decomposed by heat, but the products have not been studied. It will, no doubt, yield water, a carbohydrogen, and either triethylamine, or methylodiethylamine.

The four ethylised bases, when acted on by chlorine and bromine, yield substitution-products, in which, however, the basic character is lost. This is evidently because the chlorine or 
bromine introduced does not replace the basic hydrogen of ammonia, but some of the hydrogen of the ethyle. Wurtz has described dichlorethylamine, $\mathrm{C}_{4} \mathrm{H}_{5} \mathrm{Cl}_{2} \mathrm{~N}=\mathrm{N} \mathrm{H}_{2}, \mathrm{C}_{4} \mathrm{Cl}_{2} \mathrm{H}_{3}$, and dibromethylamine, $\left.\mathrm{NH}_{2}, \mathrm{C}_{4} \underset{\mathrm{Br}_{2}}{\mathrm{H}_{3}}\right\}$. Hofmann has obtained corresponding substitution-products from tetrethylium, in which the hydrogen is partially replaced by chlorine, bromine, and iodine ; they crystallise beautifully, but are not basic.

According to Hofmann, when cyanic acid is added to the hydrated oxide of tetrethylium, there is obtained a crystalline body, which is an urea, in which the 4 eqs. of hydrogen are replaced by 4 eqs. of ethyle.

\section{$\mathrm{C}_{2} \mathrm{~N}_{2} \mathrm{H}_{4} \mathrm{O}_{2}=$ Urea. $\mathrm{C}_{2} \mathrm{~N}_{2} \mathrm{Ae}_{4} \mathrm{O}_{2}=\mathrm{C}_{18} \mathrm{~N}_{2} \mathrm{H}_{20} \mathrm{O}_{2}=$ Tetrethylo-urea.}

We have thus seen that ethylised compounds exist, all of which are basic, but which belong to four different classes of bases; three of these, the amide, imide and nitryle bases, being volatile homologues of ammonia, and the fourth, or ammonium bases, homologues of oxide of ammonium, but differing from it in this, that while ammonium is resolved into ammonia and hydrogen, and oxide of ammonium seems to be identical with ammonia plus water, these ammonium bases have acquired new and permanent characters, ceasing to be volatile without decomposition, and exactly resembling oxide of potassium.

\section{METAMORPHOSES OF THE COMPOUNDS OF ETHYLE.}

When ether or alcohol is passed in vapour through a red-hot tube, it yields aldehyde, water, olefiant gas, and marsh-gas, $3\left(\mathrm{C}_{4} \mathrm{H}_{5} \mathrm{O}\right)=\mathrm{C}_{4} \mathrm{H}_{4} \mathrm{O}_{2}+\mathrm{HO}+3 \mathrm{C}_{2} \mathrm{H}_{2}+\mathrm{C}_{2} \mathrm{H}_{4}$ : or $2\left(\mathrm{C}_{4} \mathrm{H}_{5} \mathrm{O}\right)$ $=\mathrm{C}_{4} \mathrm{H}_{4} \mathrm{O}_{2}+\mathrm{C}_{2} \mathrm{H}_{2}+\mathrm{C}_{2} \mathrm{H}_{4}$. By the action of chloride of zine on alcohol, there are formed water, and two liquid carbohydrogens, $\mathrm{C}_{8} \mathrm{H}_{7}$ and $\mathrm{C}_{8} \mathrm{H}_{9}$; together $\mathrm{C}_{16} \mathrm{H}_{16}$, that is, olefiant gas, or an isomeric modification of it. Now ether and alcohol both contain the elements of water and of olefiant gas; for alcohol is $\mathrm{C}_{4} \mathrm{H}_{6} \mathrm{O}_{2}$ $=2 \mathrm{H} \mathrm{O}+\mathrm{C}_{4} \mathrm{H}_{4}$; and ether is $\mathrm{C}_{4} \mathrm{H}_{5} \mathrm{O}=\mathrm{H} \mathrm{O}+\mathrm{C}_{4} \mathrm{H}_{4}$.

In the manufacture of ether there occur two liquids, one of which is called oil of wine, which is $\mathrm{C}_{16} \mathrm{H}_{15}$, or very nearly the proportions of olefiant gas, according to the only analysis we have. The other is called the sweet or heavy oil of wine, and is a compound of sulphuric acid with ether, and a body having the same composition in 100 parts as olefiant gas. Sweet oil of wine is $2 \mathrm{~S} \mathrm{O}^{3}+\mathrm{Ae} \mathrm{O}+\mathrm{C}_{4} \mathrm{H}_{4}$; and as this body, $\mathrm{C}_{4} \mathrm{H}$, is 
called etherole, the compound is named the double sulphate of oxide of ethyle and of etherole. This latter is produced, along with sulphovinic acid, from the reaction of 4 eqs. dry sulphuric acid and 3 eqs. ether. $4 \mathrm{~S} \mathrm{O}_{3}+3 \mathrm{Ae} \mathrm{O}=\left(2 \mathrm{~S} \mathrm{O}_{3}, \mathrm{Ae} \mathrm{O}, \mathrm{H} \mathrm{O}\right)$ $+\left(2 \mathrm{~S} \mathrm{O}_{3}, \mathrm{Ae} \mathrm{O}_{2} \mathrm{C}_{4} \mathrm{H}_{4}\right)$. The same compound is formed when sulphovinate of lime is heated, but is accompanied by alcohol, sulphurous acid, olefiant gas, and a residue of sulphate of lime and charcoal. $2\left(\mathrm{Ca} \mathrm{O}, \mathrm{Ae} \mathrm{O}, 2 \mathrm{~S} \mathrm{O}_{3}\right)=\left(2 \mathrm{~S} \mathrm{O}_{3}, \mathrm{Ae}\right.$ $\left.0, \mathrm{C}_{4} \mathrm{H}_{4}\right)+\mathrm{HO}+2\left(\mathrm{CaO}, \mathrm{S} \mathrm{O}_{3}\right)$; and again, $2(\mathrm{Ca} \mathrm{O}, \mathrm{Ae} \mathrm{O}$, $\left.2 \mathrm{~S} \mathrm{O}_{3}\right)=2\left(\mathrm{Ca} 0, \mathrm{~S} \mathrm{O}_{3}\right)+2 \mathrm{~S} \mathrm{O}_{2}+\mathrm{C}_{4} \mathrm{H}_{6} \mathrm{O}_{2}+\mathrm{C}_{2} \mathrm{H}_{2}+\mathrm{C}_{2}$ $+2 \mathrm{H} 0$.

The sweet oil of wine is an oily liquid, quite neutral. It boils at $536^{\circ}$, and has the Sp. G. 1'133. When heated with water it gives off etherole as an insoluble oil, while the liquid contains pure sulphovinic acid. When the etherole, thus separated, is exposed to cold, it deposits crystals of etherine, a compound isomeric with etherole and with olefiant gas.

ETHIONIC, ISETHIONIC, METHIONIC, AND ALTHIONIC ACIDS.

These acids are formed by the action of sulphuric acid on ether and alcohol under various circumstances. When anhydrous acid, $\mathrm{S} \mathrm{O}_{3}$, acts on alcohol, or when olefiant gas is absorbed by that dry acid, there is formed a compound, $2 \mathrm{~S} \mathrm{O}_{3}+\mathrm{C}_{4} \mathrm{H}_{4}$, in crystals, which, when put into cold water, produce ethionic acid, $2 \mathrm{~S} \mathrm{O}_{3}$, $\mathrm{C}_{4} \mathrm{H}_{5} \mathrm{O}$. When this solution is heated, 2 eqs. of sulphurio acid and 1 of alcohol separate from one-half, and there is formed, from the other half, isethionic acid, $2 \mathrm{~S} \mathrm{O}_{3}, \mathrm{C}_{4} \mathrm{H}_{5} \mathrm{O}$, or rather $\mathrm{S}_{2} \mathrm{O}_{5}$, $\mathrm{C}_{4} \mathrm{H}_{5} \mathrm{O}_{2}$. Ethionate of baryta, formed by adding baryta to the acid before boiling, is $2 \mathrm{~S} \mathrm{O}_{3}, \mathrm{C}_{4} \mathrm{H}_{5} \mathrm{O}, \mathrm{Ba} \mathrm{O}$. From it all the other ethionates may be made. The salts of isethionic acid, like those of ethionic acid, have the same composition in 100 parts as the sulphovinates; but as they contain hyposulphuric acid, their formula is $\mathrm{S}_{2} \mathrm{O}_{5}, \mathrm{C}_{4} \mathrm{H}_{5} \mathrm{O}_{2}+\mathrm{MO}$. They crystallise with remarkable facility. When, in acting on ether with anhydrous sulphuric acid, the mixture gets too hot, there is formed a new acid, methionic acid, the baryta salt of which is $\mathrm{S}_{2} \mathrm{C}_{2} \mathrm{H}_{3} \mathrm{O}_{7}, \mathrm{Ba}$ 0 , or $\mathrm{S}_{2} \mathrm{O}_{5} \mathrm{C}_{2} \mathrm{H}_{3} \mathrm{O}_{2}+\mathrm{Ba} \mathrm{O}$. This acid apparently bears the same relation to oxide of methyle, $\mathrm{C}_{2} \mathrm{H}_{3} \mathrm{O}$, as isethionic does to ether, $\mathrm{C}_{4} \mathrm{H}_{5} \mathrm{O}$. When oil of vitriol in great excess is heated with alcohol, olefiant gas is given off, and the residue is found to contain an acid, the salts of which have the very same composition as the sulphovinates, but differ in crystalline form. This acid is called althionic acid. It is highly probable that the althionates are mixtures or compounds of sulphovinates, with 
isethionates, just as the salts of ethionic acid appear to contain sulphovinates and isethionates or compounds isomerio with these. The two compounds which seem to be distinct and independent are sulphovinic acid, ( $\mathrm{Ae} \mathrm{O}, \mathrm{H} \mathrm{O}, 2 \mathrm{~S} \mathrm{O}_{3}$ ) and isethionic acid, isomeric with it, but probably arranged as ( $\left.\mathrm{H} \mathrm{O}, \mathrm{Ae} \mathrm{O}_{2} \mathrm{~S}_{2} \mathrm{O}_{5}\right)$; the salts of the former being strictly double sulphates of ethyle and bases $\left(\mathrm{M} \mathrm{O}_{2} \mathrm{SO}_{3}+\mathrm{Ae} \mathrm{O}_{2} \mathrm{~S} \mathrm{O}_{3}\right)$ : and those of the latter being $\mathrm{M} \mathrm{O}+\left(\mathrm{Ae} \mathrm{O}_{2}, \mathrm{~S}_{2} \mathrm{O}_{5}\right)$. Whatever explanation may be given of the fact, it is a fact, that the ethionic and althionic acids and their salts have the same empirical composition as the sulphovinic and isethionic acids and their salts, at least according to the best analyses we have.

\section{PRODUCTS OF THE OXIDATION OF ETHYLE AND ITS DERIVATIVES.}

The oxidation of alcohol and of ether may be effected in a great variety of ways, and the products are rather numerous, varying according to the amount of oxygen taken up. Thus we have, first aidehyde, then acetic acid, formic acid, oxalic acid, and finally carbonic acid and water. The first effect of oxidation is to destroy the radical ethyle, giving rise to a new and less complex radical, acetyle, $=\mathrm{C}_{4} \mathrm{H}_{3}$. Afterwards we obtain compounds of the still less complex radical, formyle, $=\mathrm{C}_{2} \mathrm{H}$, and lastly, compounds of the simple radicals carbon and hydrogen.

We shall here consider, first, the radical acetyle and its compounds: bearing in mind that, while derived from the basic radical ethyle, acetyle has no basic characters whatever, but is, on the contrary, a most distinctly acidifiable radical, homologous with formyle.

AcETYLE. $\quad \mathrm{C}_{4} \mathrm{H}_{3}=$ Ac.

Acetyle is the second member of the series of electro-negative radicals, of which formyle is the first. These are derived from the methyle or electro-positive radicals, by oxidation, which removes 2 eqs. of hydrogen; thus ethyle, $\mathrm{C}_{4} \mathrm{H}_{5}+\mathrm{O}_{2}=$ Acetyle, $\mathrm{C}_{4} \mathrm{H}_{3}+2 \mathrm{H} \mathrm{O}$.

Neither formyle, acetyle, nor any of the homologous radicals of the series, are yet known in the separate state, but they are easily obtained in the form of their teroxides, which are the volatile acids of the former series, or, in some instances, in that of their protoxides, which are the aldehydes. They are also obtained in combination with chlorine, with amide, and other substances. 
1. Hydrated Oxide of Acetyle or Aldehyde. ( $\left.\mathrm{C}_{4} \mathrm{H}_{3}\right) \mathrm{O}, \mathrm{HO}=\mathrm{Ac} \mathrm{O}, \mathrm{H}$.

Aldehyde is formed when ether or alcohol is passed through a red-hot tube, or when ether or alcohol are oxidised by nitric acid, or by ohlorine, \&c. In these cases it is not pure; but it may be obtained quite pure by distilling 2 parts of the compound of aldehyde and ammonia dissolved in 2 parts of water, along with a mixture of three parts of oil of vitriol and 4 of water, and rectifying at a temperature of about $80^{\circ}$, over chloride of calcium.

It is a clear, colourless liquid, of a peculiar and powerful ethereal odour, of Sp. G. $0 \cdot 79$ at $65^{\circ}$, and boiling at $70^{\circ}$. It mixes in all proportions with water, alcohol, and ether, and is neutral and inflammable. In contact with the atmosphere it rapidly absorbs oxygen, passing into hydrated acetic acid: for $\mathrm{C}_{4} \mathrm{H}_{3}$, $\mathrm{O}+\mathrm{HO}$, with $\mathrm{O}_{2}$, at once produce $\mathrm{C}_{4} \mathrm{H}_{3}, \mathrm{O}_{3}+\mathrm{H} \mathrm{O}$; or Ac $\mathrm{O}$, $\mathrm{HO}+\mathrm{O}_{2}=\mathrm{Ac} \mathrm{O}_{3}, \mathrm{HO}$. Aldehyde is a constant ingredient of the nitrous ether of the pharmacopœias.

When heated with caustic potash, it is rapidly converted into the brown matter called resin of aldehyde. If gently heated with oxide of silver and water, part of the oxide is reduced without effervescence, coating the glass tube with a bright surface of silver, while the water is found to contain a salt of silver, the acid of which contains less oxygen than acetic acid: this is aldehydic or lampic acid, $\mathrm{C}_{4} \mathrm{H}_{3} \mathrm{O}_{2}, \mathrm{H} \mathrm{O}$, or $\mathrm{Ac}_{2}, \mathrm{H} \mathrm{O}$. The solution of aldehydate of silver, if filtered and heated to boiling, again deposits metallic silver, while the aldehydic acid becomes acetic.

When long kept, even in sealed tubes, aldehyde is transformed into two polymeric modifications, namely, metaldehyde, a hard crystalline inodorous solid, and elaldehyde, which is liquid.

Aldehydite of ammonia. SxN. Aldehydammonia. $\mathrm{C}_{4} \mathrm{H}_{3}, \mathrm{O}+$ $\mathrm{NH}_{3}+\mathrm{H} \mathrm{O}$. Aldehyde has no basic characters, and rather exhibits a tendency to the acid character, in combining with ammonia, as it does directly, to form a crystallised compound. To prepare it, as the substance from which aldehyde is obtained, 6 parts of oil of vitriol, 4 of water, 4 of alcohol, and 6 of peroxide of manganese, in fine powder, are distilled together. The crude product is twice rectified over chloride of calcium ; it is now aldehyde, containing a little water, alcohol, and acetic and formic ethers; and this liquid, when mixed with ether, and saturated with ammoniacal gas, yields crystals of the new compound, which are washed with ether. These crystals become brown on being kept, even in close vessels, and acquire the smell of burnt feathers. 
If perfectly pure, however, I find them to keep well for a year or more. They dissolve in water and alcohol, but hardly in ether. Nitrate of silver forms, in the concentrated solution, a precipitate, which, when heated, is reduced.

Thialdine. Liebig and Wöhler have very recently discovered that when an aqueous solution of aldehydammonia is acted on by sulphuretted hydrogen, a little ammonia being previously added, crystals of a new compound are deposited, which has basic properties, and has been called Thialdine. It is produced by the action of 3 eqs. of aldehydammonia on 6 eqs. of sulphuretted hydrogen. Thus-

$$
\begin{aligned}
& 3 \text { eqs. aldehydammonia . } \mathrm{C}_{12} \mathrm{H}_{21} \mathrm{~N}_{3} \mathrm{O}_{6} \\
& \text { and } 6 \text { eqs. sulphuretted hydrogen } \quad \mathrm{H}_{\mathrm{o}} \mathrm{S}_{\mathrm{b}} \\
& \overline{\mathrm{C}_{22} \mathrm{H}_{27} \mathrm{~N}_{3} \mathrm{O}_{6} \mathrm{~S}_{6}} \\
& \text { yield } 1 \text { eq. Thialdine . . . . } \mathrm{C}_{12} \mathrm{H}_{13} \mathrm{~N} \mathrm{~S}_{4} \\
& 6 \text { eqs. water . . . . } \mathrm{H}_{6} \mathrm{O}_{6} \\
& 2 \text { eqs. sulphuret of ammonium } \quad \mathrm{H}_{8} \mathrm{~N}_{2} \mathrm{~S}_{2} \\
& \mathrm{C}_{12} \mathrm{H}_{27} \mathrm{~N}_{3} \mathrm{O}_{6} \mathrm{~S}_{6}
\end{aligned}
$$

Thialdine is almost insoluble in water, but very soluble in alcohol and ether. It is deposited, by spontaneous evaporation, from its ethereal solution, in large and beautiful crystals, resembling camphor. It is very volatile and fusible, has an unpleasant aromatic smell, and is a powerful base, neutralising the strongest acids, and forming beautiful crystalline salts. When heated with hydrate of lime, thialdine yields leucoline or quinoline, one of the volatile bases of coal tar.

When aldehydammonia is acted on by seleniuretted hydrogen, an analogous basic compound, selenaldine, is formed; and it appears probable, from the experiments of Liebig and Wöhler, that telluretted hydrogen is capable also of yielding a similar compound.

According to Strecker, when aldehydammonia is acted on by hydrocyanic acid, and an excess of hydrochloric acid, there. is formed a crystalline product, soluble in water, to which he has given the name of Alanine. It is homologous with glycocoll or glycocine, and with leucine.

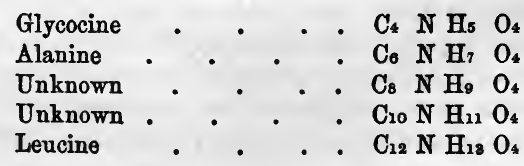


Its formation, from aldehyde and hydrocyanic acid, is as follows: $\mathrm{C}_{4} \mathrm{H}_{4} \mathrm{O}_{2}+\mathrm{C}_{2} \mathrm{NH}+2 \mathrm{H} \mathrm{O}_{2}=\mathrm{C}_{6} \mathrm{~N} \mathrm{H}_{7} \mathrm{O}_{4}$.

Alanine is isomeric with lactamide, urethane, and sarcosine; but all four compounds are distinet. Lactamide, when boiled with acids or bases, yields lactic acid and ammonia, but alanine does not. When acted on by hyponitrous acid, however, alanine is converted into lactic acid, nitrogen being given off.

$$
\underbrace{\mathrm{C}_{6} \mathrm{~N} \mathrm{H}_{7} \mathrm{O}_{4}}_{\text {Alanine. }}+\mathrm{N}_{3}=\mathrm{H} \mathrm{O}+\mathrm{N}_{2}+\underbrace{\mathrm{C}_{6} \mathrm{H}_{6} \mathrm{O}_{6}}_{\text {Lactic Acid. }} .
$$

We shall see farther on other instances in which hyponitrous acid produces very definite and remarkable transformations. Lactic acid may be regarded as formic acid, coupled with aldehyde, just as formobenzoic acid is formic acid, coupled with hyduret of benzoyle,

$$
\mathrm{C}_{2} \mathrm{H} \mathrm{O}_{3}, \mathrm{H} \mathrm{O}+\mathrm{C}_{4} \mathrm{H}_{4} \mathrm{O}_{2}=\mathrm{C}_{6} \mathrm{H}_{5} \mathrm{O}_{5}, \mathrm{H} \mathrm{O} \text {. }
$$

\section{Acetal. $\mathrm{C}_{8} \mathrm{H}_{9} \mathrm{O}=\mathrm{C}_{4} \mathrm{H}_{3}, \mathrm{O}+\mathrm{C}_{4} \mathrm{H}_{5} \mathrm{O}+\mathrm{HO}=\mathrm{AcO}+\mathrm{AeO}+\mathrm{H} \mathrm{O}$.}

Is formed by the action of the oxygen of the air on the vapour of alcohol, under the influence of the black powder of platinum. It is a colourless very mobile liquid, Sp. G. 0.825 , boiling at $203^{\circ}$. It might, according to its composition, be a compound of 3 eqs. oxide of ethyle, and 1 eq. acetic acid; for $3\left(\mathrm{C}_{4} \mathrm{H}_{5}, \mathrm{O}\right)+$ $\mathrm{C}_{4} \mathrm{H}_{3} \mathrm{O}_{3}=\mathrm{C}_{16} \mathrm{H}_{18} \mathrm{O}_{6}=2\left(\mathrm{C}_{8} \mathrm{H}_{9} \mathrm{O}_{3}\right)$. But the action of potash which forms resin of aldehyde, and of oil of vitriol, which blackens and thickens as it does aldehyde, indicate pretty certainly the presence of aldehyde; and we therefore prefer the formula above given, which makes acetal a compound of aldehyde and oxide of ethyle.

The resin of aldehyde, formed by the action of potash on aldehyde, is little known. Elaldehyde, an isomerio form of aldehyde, which is liquid at ordinary temperatures, but solid at $32^{\circ}$, has a formula exactly triple that of aldehyde, $\mathrm{C}_{12} \mathrm{H}_{12} \mathrm{O}_{6}$. Metaldehyde, the other isomeric modification, which, like the preceding, spontaneously forms in aldehyde, when kept, has, no doubt, a similar relation in its formula to that of aldehyde: but its precise formula is not yet known. The density of its vapour would settle the point. It forms very hard prisms, which sublime at $248^{\circ}$ without melting. It is worthy of notice that aldehyde, like the corresponding chlorine compound, formed by substitution, chloral, $\mathrm{C}_{4} \mathrm{Cl}_{3} \mathrm{O}+\mathrm{H} \mathrm{O}$, undergoes very easily, and even spontaneously, these very singular metamorphoses. 
This indicates a relation in the constitution of these bodies which cannot be overlooked.

\section{Acetylous Acid. $\left(\mathrm{C}_{4} \mathrm{H}_{3}\right) \mathrm{O}_{2}, \mathrm{HO}=\mathrm{Ac}_{2}, \mathrm{HO}$.}

Srv. Aldehydic Acid. Lampic Acid. It has already been stated that this acid is obtained in combination with oxide of silver, when aldehyde is gently heated with excess of that oxide in water. The solution, when the silver has been separated by sulphuretted hydrogen, contains the acetylous acid very diluted. It is very easily decomposed, especially by heat, into acetic acid and a brown resin, like that of aldehyde. When the acetylite or aldehydate of silver is decomposed by baryta, so as to precipitate all the oxide of silver, and the acetylite of baryta is now heated with the precipitated oxide of silver, the metal is reduced, and acetate of baryta is now found in the solution. This demontrates the relation of acetylous to acetic acid: for $\mathrm{Ba} \mathrm{O}, \mathrm{Ac}_{2}+$ $\mathrm{Ag} \mathrm{O}=\mathrm{BaO}, \mathrm{Ac} \mathrm{O}_{3}+\mathrm{Ag}$. This acid is one chief ingredient of the acid produced by the slow combustion of ether in the lamp with a spiral of platinum wire on the wick, the platinum continuing red-hot, but no flame appearing, and which acid is called lampic acid.

\section{Acetic Acid. $\mathrm{C}_{4} \mathrm{H}_{3}, \mathrm{O}_{3}+\mathrm{HO}=\mathrm{Ac}_{3}, \mathrm{HO}$.}

Srn. Acetylic Acid. Pyroligneous Acid. Vinegar. This important acid is formed in two principal ways : first, by the oxidation of alcohol; and, secondly, by the destructive distillation of wood. Wine, beer, and other fermented liquors, if exposed to the air, under certain circumstances, undergo what is erroneously termed the acetous fermentation; that is, they attract oxygen from the air, undergo eremacausis of the alcohol they contain, and, after a time, contain no alcohol, but in its place acetic acid; they are, in fact, converted into vinegar. The ultimate change is very simple: $\mathrm{C}_{4} \mathrm{H}_{6} \mathrm{O}_{2}+\mathrm{O}_{4}=\mathrm{C}_{4} \mathrm{H}_{3} \mathrm{O}_{3}$, $3 \mathrm{H} \mathrm{O}=\mathrm{Ac}_{3}, \mathrm{HO}+2$ aq. But we have already seen that there are intermediate steps in the process. The first effect of the oxygen is to remove from the alcohol, or rather from the ethyle in it, 2 eqs. of hydrogen, thus leaving the radical acetyle, $\mathrm{C}_{4} \mathrm{H}_{3}$, in the place of the ethyle $\left(\mathrm{C}_{4} \mathrm{H}_{5}\right) \mathrm{O}, \mathrm{H} \mathrm{O}+\mathrm{O}_{2}=$ $\left(\mathrm{C}_{4} \mathrm{H}_{3}\right) \mathrm{O}, \mathrm{H} \mathrm{O}+2$ aq. In this stage, alcohol is simply converted into aldehyde, while 2 eqs. of water are formed. In the next stage, the hydrated protoxide of acetyle (the aldehyde), or rather the radical $\mathrm{C}_{4} \mathrm{H}_{3}$, takes up 2 additional equivalents of oxygen, and thereby becomes acetic or acetylic acid $\left(\mathrm{C}_{4} \mathrm{H}_{3}\right) \mathrm{O}$, $\mathrm{H} \mathrm{O}+\mathrm{O}_{2}=\left(\mathrm{C}_{4} \mathrm{H}_{3}\right) \mathrm{O}_{3}, \mathrm{HO}$. 
Such being the action of the oxygen of the air on alcohol, it is obvious that the process of acetification is no fermentation, but a case of eremacausis, or slow combustion. But, as was formerly explained, the state of eremacausis is induced by contact of a body in that state, or even in the state of fermentation or putrefaction, and the presence of a ferment is required to commence the process of eremacausis of alcohol. Pure alcohol, exposed to air alone, is not acetified; but if its vapour, mixed with air, come in contact with platinum powder, eremaoausis is induced. Hence, if alcohol be placed in a flat basin under a bell jar, beside a small flat dish containing platinum black, the bell jar is, in a few seconds, filled with the pungent smell of aldehyde, and in an hour or two the acetification is nearly complete. Here the platinum, by virtue of its singular power of causing gases or vapours to unite on its surface, acts as a ferment, or, as it may be called, an excitant, inducing the slow combustion, and acting as a carrier of oxygen from the air to the alcohol.

In wine or beer, there is present an actual ferment in the shape of gluten or fibrin, at least in all cases where the vinous fermentation has not decomposed the whole of the ferment. In these cases, exposure to the air for a short time causes the decomposition of the gluten, \&c., to recommence, and this state of decomposition, consisting in part, no doubt, of eremacausis, being mechanically communicated in the shape of motion to the particles of alcohol, slow combustion commences, and continues till every trace of alcohol has been acetified, when the process is arrested for want of fuel, that is, of alcohol. Where, in the fermentation of wine (as sherry), or of beer (as Bavarian beer), all ferment has been destroyed or removed, these liquors do not become sour when exposed to air; and if we wish to acetify them, we must add yeast or some other ferment. So, also, when we wish to make strong vinegar by the acetification of brandy or of whiskey, we have to add a ferment, such as yeast, and expose the mixture to a certain temperature in open vats. By carefully attending to all these principles, the process of acetification may be very much abridged. The following is the rapid process now followed on the Continent. There is made a mixture of 1 part of alcohol at 80 p. c., 4 to 6 parts of water, and $\frac{1}{1000}$ of a ferment, such as vinegar, honey, or must of beer. A large, high barrel is packed with twigs or shavings of beech, previously soaked in strong vinegar, and holes are drilled in the middle and upper part of the barrel to admit a free circulation of air. The mixture is now warmed to from $75^{\circ}$ to $80^{\circ}$, and made to trickle slowly upon the shavings and through the barrel, thus exposing an immense surface to the air. The temperature rises rapidly to 
$95^{\circ}$ or $105^{\circ}$, and if a proper supply of air be given, continues at that point during the operation. When the mixture has been three or four times passed through the barrel, it is found perfectly acetified: this may take place in from 24 to 36 hours. Should the supply of fresh air, that is, of oxygen, be deficient, much aldehyde is produced, which, from its volatility, is carried off as vapour, and lost. This was long a source of great loss to the makers, and the cause could not be traced, until Liebig, by the discovery of aldehyde, explained it, and showed how to avoid the loss, by giving a due supply of air. The manufacturer now obtains, as nearly as can be expected, the theoretical quantity of vinegar from his spirits. Any aromatic substance, or essential oil, or even a trace of wood vinegar (contaminated with kreosote, $\& c$.$) , will arrest the progress of acetification.$

The peculiar pleasant smell of good vinegar, in addition to that of pure diluted acetic acid, is owing to the presence of acetio ether. Distilled vinegar is a tolerably pure but weak acetio acid; but to obtain acetic acid pure and strong, we must have recourse to the salts of acetic acid, which, when distilled with moderately strong sulphuric acid, yield pure acetic acid, mixed with more or less water.

The pyroligneous acid is contaminated with pyroxylic spirit and with oil of tar. When combined with soda, lime, or oxide of lead, these salts may be easily purified by crystallisation, and by heating them so far as to expel or destroy the oily impurities. The pure salts, distilled with sulphuric acid, yield acetic acid, identical with that from true vinegar.

Anhydrous Acetic Acid, $\mathrm{C}_{4} \mathrm{H}_{3} \mathrm{O}_{3}$, or $\left.\begin{array}{l}\mathrm{C}_{4} \mathrm{H}_{3} \mathrm{O}_{3} \\ \mathrm{C}_{4} \mathrm{H}_{3} \mathrm{O}_{3}\end{array}\right\}$. The anhydrous acetic acid, or anhydride of acetic acid, is obtained by gradually adding oxychloride of phosphorus to fused acetate of potash, and returning several times the liquid which distils over, till it contains no more oxychloride of acetyle, which is at first formed. Or the oxychloride of acetyle, obtained in the first distillation, is allowed to act on the fused acetate of potash.

$$
\mathrm{C}_{4} \mathrm{H}_{3} \mathrm{O}_{2}
$$

The anhydride is a colourless mobile refractive liquid, of a pungent odour, like that of the hydrated acid, with something of hawthorn blossom. Its density at $45^{\circ} \mathrm{F}$. is 1.073 , or the same as that of the hydrated acid. It boils at $290^{\circ} \mathrm{F}$., and the density of its gas is 3.47 .

It is converted into the hydrated acid by contact with water. 
This anhydride forms double anhydrides with those of other acids, such as benzoic, salicylic, and cuminic acids.

The aceto-benzoic anhydride is formed when chloride of benzoyle acts on acetate of potash.

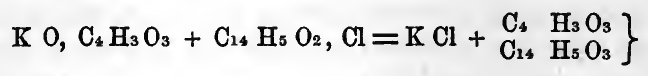

Hydrated Acetic Acid, radical Vinegar, or Crystallisable Acetic Acid, $\mathrm{Ac}_{3}, \mathrm{HO}$, is obtained by distilling 3 parts of dry powdered acetate of soda with $9 \cdot 7$ of oil of vitriol, as pure and concentrated as possible : $\frac{1}{8}$ of the acid distils over by the heat spontaneously developed in the mixture; a gentle heat expels the rest. The product is rectified and exposed to a cold of $23^{\circ}$ or $24^{\circ}$, when crystals of the hydrate are formed in a weaker liquid. The crystals are allowed to drain, and then melted, and again exposed to cold. The crystals of this second operation are generally free from all superfluous water. At temperatures below $60^{\circ}$, hydrated acetic is solid ; at $62^{\circ}$ or $63^{\circ}$ it melts, forming a liquid which sometimes continues liquid at a much lower temperature, and then crystallises from some very trifling cause. The Sp. G. of the liquid is 1.063 ; it boils at $248^{\circ}$; has a pungent, peculiar, but agreeable smell, and a burning acid taste. It raises a blister on the skin, and soon produces a painful sore, like a mineral acid. It is miscible in all proportions with water, alcohol, and ether. It dissolves camphor and essential oils ; and the aromatic vinegar is a solution in it of camphor, with a little oil of lemons and bergamot, \&c. Strong acetic acid, in this form, is used as a diffusible stimulant, applied to the nostril in faintness or sickness. It may be used, also, externally as a very powerful rubefacient and epispastic. The hydrated acid is combustible. It is decomposed by anhydrous sulphuric acid, yielding a new acid, sulphacetic acid: also by chlorine, yielding, by substitution, chloracetic acid. The vapour of acetic acid, passed through a red-hot tube, yields carbonic acid and acetone, $\mathrm{C}_{3} \mathrm{H}_{3}$ 0 , or $\mathrm{C}_{6} \mathrm{H}_{6} \mathrm{O}_{2}$ : the same transformation occurs when acetic acid is heated with bases. Acetic acid is, as already mentioned, the second of the remarkable volatile acids of the general formula $\mathrm{C}_{n} \mathrm{H}_{n} \mathrm{O}_{4}$.

The salts of acetic acid are, almost without exception, soluble in water: the acetates of silver and protoxide of mercury are sparingly soluble. The formula for the neutral acetates is $\mathrm{MO}$, $\mathrm{C}_{4} \mathrm{H}_{3} \mathrm{O}_{3}$, or $\mathrm{M}, \mathrm{C}_{4} \mathrm{H}_{3} \mathrm{O}_{4}$. There are hardly any acid salts : but a considerable number of basic salts, as basic acetates of lead and copper.

Acetate of Oxide of Ethyle, or Acetic Ether, $\mathrm{Ae} 0, \mathrm{Ae} \mathrm{O}_{3}$, is 
easily prepared by distilling 10 parts of acetate of soda, 16 of oil of vitriol, and 6 of alcohol. The product is rectified over lime and chloride of calcium. It may also be obtained by distilling any sulphovinate with strong acetic acid. In either case, acetic acid is brought in contact with nascent ether, and combines with it. Acetic ether is a colourless liquid, of a refreshing odour, very combustible. It boils at $165^{\circ}$; its Sp. G. is 0.89 . It is easily decomposed by alkalies, yielding an acetate and alcohol. Acids also decompose it. It is always present, in small quantity, in wine vinegar, which owes its flavour to this compound.

Acetate of Ammonia. There is a well-known febrifuge and diaphoretic remedy called the spirit of Mindererus, which is a diluted solution of acetate of ammonia, formed by neutralising distilled wine vinegar with carbonate of ammonia. A more uniform preparation, although stronger than that usually employed here, is made by neutralising 6 parts of aqua ammoniæ, Sp. G. 0.96, with strong acetic acid, and adding enough water to make up 24 parts. This, being uniform, can easily be reduced if desirable. There is an acid acetate of ammonia, which forms deliquescent needles. Acetate of potash, $\mathrm{KO}, \mathrm{Ac} \mathrm{O}_{3}$, is obtained as a fibrous crystalline mass, very deliquescent, which has a warm saline taste. It is wuch used as a diuretic. When heated, with arsenious acid, it yields oxide of kakodyle, or arsenomethyle, a compound metal or basic radical, of most remarkable composition and characters, $\mathrm{C}_{4} \mathrm{H}_{6} \mathrm{As}$. It has been already fully described with its compounds, under Methyle. Acetate of Soda, Na O, Ac $\mathrm{O}_{3}+6$ aq., is formed from pyroligneous acid, and is the form in which the acid is brought in order to be purified from oil of tar. The salt is melted at a moderate heat, and roasted, then redissolved, filtered through charcoal, evaporated, again melted, and so on, until it becomes snow-white. 10 parts of the crystals of the salt, after the first fusion, while still slightly coloured, being distilled with 6 of oil of vitriol, yield what is called wood vinegar, sufficiently pure for use, but requiring 7 waters to reduce it to the average strength of wine vinegar. Acetates of baryta, strontia, and lime, all crystallise readily. The first is used as a test; the last in the manufacture of acetic acid and all other acetates from pyroligneous acid. Acetate of alumina, $\mathrm{Al}_{2} \mathrm{O}_{3}, 3$ Ac $\mathrm{O}_{3}$, prepared by mixing solutions of alum and acetate of lead (or of baryta) is very soluble. It is much used in the above form, containing sulphate of potash, as a most valuable mordant in dyeing and calico-printing. When heated it deposits an insoluble basic salt, which adheres tenaciously to the cloth, and afterwards combines firmly with the colouring matter. The pure acetate of alumina, formed from sulphate of alumina and acetate 
of baryta, is not so decomposed by heat, but requires the presence of a neutral salt. Mr. Crum has minutely studied the acetates of alumina, and has described several remarkable compounds, the details of which our space compels us to omit. Acetate of manganese, $\mathrm{Mn} \mathrm{O}, \mathrm{Ac} \mathrm{O}_{3}$, formed by acting on sulphate of manganese by acetate of lime, is much used in calico-printing, as it gives, with bleaching liquor, a rich bronze-brown. Acetate of zinc, $\mathrm{Zn} 0, \mathrm{Ac}_{3},+3$ aq., is used in medicine and pharmacy. Acetate of protoxide of iron, $\mathrm{Fe} \mathrm{O}, \mathrm{Ac} \mathrm{O}_{3}$, is used as a mordant. Acetate of peroxide of iron, $\mathrm{Fe}_{2} \mathrm{O}_{3}, 3 \mathrm{Ac} \mathrm{O}_{3}$, is formed by precipitating acetate of lead with persulphate of iron. It has the same valuable properties as acetate of alumina, depositing a basic salt, when heated with neutral salts, and is much prized as a mordant. Acetate of lead: a. neutral, Syn. Sugar of lead, is best prepared by dissolving litharge in acetic acid, and crystallising. It has a sweet astringent taste, and is much used as an astringent and styptic in diarrhoea, dysentery, and various hæmorrhages. It is poisonous, especially where it forms carbonate: it ought therefore never to be given without abundance of vinegar being taken by the patient. The crystals are $\mathrm{Pb} 0$, $\mathrm{Ac}_{3}+3$ aq. b. sesquibasic, $3 \mathrm{~Pb} \mathrm{O}+2 \mathrm{Ac}_{3}$, soluble pearly scales. c. tribasic or subacetate of lead is formed in crystals by mixing 1 vol. of aqua ammoniæ with 5 of a cold saturated solution of the neutral salt, and setting it aside. It forms long needles. The solution, or Goulard's extract, is made by digesting 7 parts of litharge with 6 of sugar of lead and 30 of water, till the oxide, which is not dissolved, has become white. It is much used as a lotion, and to precipitate gum, organic acids, albumen, caseine, extractive matter, \&c., from organic mixtures, and solutions. Its formula is $3 \mathrm{PbO}+\mathrm{Ac} \mathrm{O}_{3}$. d. sexbasic, formed by adding the last or any of the previous acetates of lead to an excess of ammonia. It forms a crystalline powder; very sparingly soluble, which is $6 \mathrm{PbO}+\mathrm{Ac} 0 s$. It usually exists in white-lead, along with carbonate of lead.

Acetate of Copper : a. neutral; appears in two forms : as dark green oblique rhombio prisms, becoming opaque in air, $\mathrm{Cu} \mathrm{O}$, Ac $\mathrm{O}_{3}+$ aq. ; and as dark blue transparent crystals of great beauty, $\mathrm{Cu} \mathrm{O}, \mathrm{Ac}_{3}+5$ aq. These latter, heated to $86^{\circ}$, lose 4 eqs. of water, and fall to a powder of the green salt. b. bibasic or verdigris, $2 \mathrm{Cu} O+\mathrm{Ac}_{3}+6$ aq. $=\mathrm{Cu} 0, \mathrm{Ac}_{3}, 5$ aq. $+\mathrm{Cu}$ $0, \mathrm{HO}$. Verdigris is prepared by a tedious process, and is seldom pure, containing usually different basic acetates. c. sesquibasic, $3 \mathrm{Cu} \mathrm{O}, 2 \mathrm{Ac} \mathrm{O}_{3}+6$ aq. d. tribusic, $6 \mathrm{Cu} 0,2 \mathrm{Ao} \mathrm{O}_{3},+3$ aq. The two last occur in the verdigris of commerce. All these salts are poisonous. 
Schweinfurt or Vienna Green is a double salt, formed of acetate and arsenite of copper, $\mathrm{Cu} \mathrm{O}, \mathrm{Ac}_{3}+3\left(\mathrm{Cu} \mathrm{O}, \mathrm{As} \mathrm{O}_{3}\right)$; is formed when 10 parts of verdigris, suspended in water, are left to digest for 24 hours, after mixing them with a hot solution of 8 parts of arsenious acid in 100 of water. A dirty green precipitate first appears, which, on standing, changes to a most beautiful green, much used as a paint.

Acetate of Protoxide of Mercury is obtained, in sparingly soluble silvery scales, by adding acetate of potash to protonitrate of mercury, both hot. It blackens when exposed to light. It is used in medicine, especially on the Continent.

Acetate of Silver, $\mathrm{Ag} \mathrm{O}, \mathrm{Ac} \mathrm{O}_{3}$, very much resembles the preceding salt; it is occasionally used as a test. It is very sparingly soluble.

When acetate of ammonia is heated with excess of anhydrous phosphoric acid, it loses all its oxygen in the form of water, and there distils over a volatile liquid, which is acetonitryle, $\mathrm{C}_{4} \mathrm{H}_{3} \mathrm{~N}$, or cyanide of methyle, $\mathrm{C}_{4} \mathrm{H}_{3}+\mathrm{C}_{2} \mathrm{~N}=\mathrm{Me} \mathrm{Cy}$. Its formation is very simple, for $\left(\mathrm{N} \mathrm{H}_{4} \mathrm{O}, \mathrm{C}_{4} \mathrm{H}_{3} \mathrm{O}_{3}\right)+\times \mathrm{P} \mathrm{O}_{5},=\times \mathrm{P} \mathrm{O}_{5}, 4 \mathrm{HO}$ $+\mathrm{C}_{4} \mathrm{H}_{3} \mathrm{~N}$. All the acids of the series $\mathrm{C}_{2} \mathrm{H}_{4} \mathrm{O}_{4}$ yield similar products, when their ammonia salts are heated with anhydrous phosphoric acid, or when their amides are treated in the same way. Formonitryle, $\mathrm{C}_{2} \mathrm{H} \mathrm{N}$, is cyanide of hydrogen, or hydrocyanic acid, and it is well known that formiate of ammonia, when heated, yields water and hydrocyanic acid. Acetonitryle, the next member of the series, is identical with cyanide of methyle, and in this view, being a neutral compound, is the first of the homologous series of the nitryles. It will be seen that the nitryle (or nitrogen compound) of any radical of the formyle series is identical with the cyanide of the radical one step lower in the ethyle series. Thus acetonitryle, $\mathrm{C}_{4} \mathrm{H}_{3}, \mathrm{~N}$, is identical with cyanide of methyle, $\mathrm{C}_{2} \mathrm{H}_{3}$, $\mathrm{C}_{2} \mathrm{~N}$. It is possible that, higher in the scale, some of the nitryles may be not identical, but only isomeric, with the corresponding cyanides. But such as are hitherto known appear to be identical with those cyanides. Hence, in some cases, the only or the best method of obtaining a compound of any given radical of the ethyle series, is to decompose the ammonia salt of the acid or teroxide of the radical one step higher in the formyle or acetyle series, since a large number of these acids, of the formula $\mathrm{Cn} \mathrm{Hn}_{4}$ or $\mathrm{Cn}_{n}-\mathrm{O}_{3}, \mathrm{H} \mathrm{O}$, are known. For example, butyrate of ammonia will yield butyronitryle, $\mathrm{Cs}_{8} \mathrm{H}_{\ominus} \mathrm{N}$, which is cyanide of propyle, $\mathrm{C}_{6} \mathrm{H}_{7}, \mathrm{C}_{2} \mathrm{~N}$. And from this body we may hope to obtain the oxide and hydrated oxide of propyle, the propylic ether and propylic alcohol which have lately been discovered by Chancel in the alcohol from wine or grape juice, and described by him. But 
we are not yet able to do this; for the cyanides, when heated with bases, regenerate the acids from which they were obtained, just as if they were really nitryles, and not cyanides. This is strange, since the chlorides of the same radicals readily yield, with bases, the hydrated oxides. Some method, however, will doubtless be found of effecting the desired transformation.

Acetamide, $\mathrm{C}_{4} \mathrm{H}_{3} \mathrm{O}_{2}, \mathrm{~N} \mathrm{H}_{2}$. This compound is easily obtained by the action of aqueous ammonia on the acetate of oxide of ethyle (acetic ether), and is quite analogous to oxamide. It is a white crystalline fusible solid, which, when boiled with acids or bases, takes up 2 eqs. of water, forming acetic acid and ammonia.

When ethylamine acts on acetio ether there is formed a compound, ethylacetamide, which is acetamide with 1 eq. of ethyle replacing 1 of hydrogen in the amide $\left.\mathrm{C}_{4} \mathrm{H}_{3} \mathrm{O}_{2}, \mathrm{~N}_{\mathrm{Ae}}^{\mathrm{H}}\right\}=\mathrm{C}_{8} \mathrm{H}_{9}$ $\mathrm{N} \mathrm{O}_{2}$. It is a syrupy liquid.

When anhydrous acetio acid acts on cyanic ether, there is formed ethylodiacetamide, which is $\mathrm{C}_{12} \mathrm{H}_{11} \mathrm{~N} \mathrm{\textrm {O } _ { 2 }}$, probably $\left.\mathrm{N} \underset{\mathrm{C}_{4} \mathrm{H}_{3} \mathrm{O}_{2}}{\mathrm{Ae}}\right\}, \mathrm{C}_{4} \mathrm{H}_{3} \mathrm{O}_{2}$.

Compounds analogous to ethylacetamide are obtained when methylamine, amylamine, phenylamine, and many other analogous bases, act on acetic ether.

Hyduret of Acetyle, $\mathrm{C}_{4} \mathrm{H}_{3}, \mathrm{H}=\mathrm{C}_{4} \mathrm{H}_{4}$. This compound is described farther on as olefiant gas. It is produced in many decompositions of alcohol, acetic acid, acetates, ether, \&c., especially by heat. It is a member of a homologous series, of which the hyduret of formyle is the first. These carbohydrogens, along with the hydurets of the methylic radicals, occur in the oils of wood and coal tars, and resemble each other in physical properties, in the same way as homologous compounds usually do.

Gerhardt and other chemists regard the true radical of acetic acid, not as $\mathrm{C}_{4} \mathrm{H}_{3}$, acetyle, but as $\mathrm{C}_{4} \mathrm{H}_{3} \mathrm{O}_{2}$, which they call acetyle, analogous to benzoyle $\mathrm{C}_{14} \mathrm{H}_{5} \mathrm{O}_{2}$. On this view, which is not at all improbable, acetic acid will be $\left(\mathrm{C}_{4} \mathrm{H}_{3} \mathrm{O}_{2}\right) \mathrm{O}, \mathrm{H} \mathrm{O}$; oxychloride of acetyle will be chloride of acetyle, and so on. The formulæ are thus quite as simple in this way as in that we have given, but either will answer the purpose of fixing the facts in the memory.

Gerhardt is also disposed to consider acetic acid as formic acid, in which 1 eq. of hydrogen is replaced by methyle, and to call it methyloformic acid. The formula of formic acid, according to

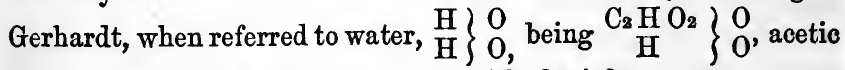

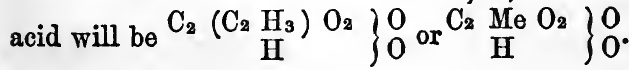


In like manner he supposes all the acids above acetic acid in the series to be formic acid, with an ethylic radical in place of the hydrogen of the radical $\mathrm{C}_{2} \mathrm{H} \mathrm{O}_{2}$ (Gerhardt's formyle).

Since acetic acid is homologous with formic acid, it is evident that it is the same thing whether we suppose the ethylic radicals to replace hydrogen in Gerhardt's formyle, or another series of radicals, the acetylic or formylic, also carbohydrogens, to replace each other, so that, 2 eqs. of water being the type, $\mathrm{H}\}$ have, $\left(\mathrm{Fo}_{\mathrm{O}}=\mathrm{C}_{2} \mathrm{H}, \mathrm{Ac}=\mathrm{C}_{4} \mathrm{H}_{3}, \mathrm{Me}=\mathrm{C}_{2} \mathrm{H}_{3}\right)$.

$$
\begin{aligned}
& \text { Acids. }
\end{aligned}
$$

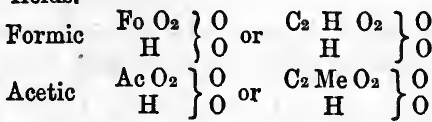

Both formulæ suffice to explain the similarity of these acids, but perhaps the view of Gerhardt will be found to agree better with some of the phenomena, although the facts we are now to mention almost demonstrate that acetyle exists, and forms various compounds.

Acetylammonium. Natanson has recently shown that acetyle, $\mathrm{C}_{4} \mathrm{H}_{3}$, is capable of replacing 1 eq. of hydrogen in ammonium, $\mathrm{N} \mathrm{H}_{4}$, yielding a new radical or compound metal, acetylammonium. $\left.\mathrm{N}_{\mathrm{C}_{4} \mathrm{H}_{3}}^{\mathrm{H}_{3}}\right\}=\mathrm{C}_{4} \mathrm{H}_{6} \mathrm{~N}$. This is unknown in the separate state, but its hydrated oxide is a base.

Hydrated Oxide of Acetylammonium, $\mathrm{C}_{4} \mathrm{H}_{6} \mathrm{~N} \mathrm{O}, \mathrm{H} \mathrm{O}$, is best obtained from the oil of olefiant gas, $\mathrm{C}_{4} \mathrm{H}_{4} \mathrm{Cl}_{2}$. When this oil, which is the hydrochlorate of the chloride of acetyle, $\mathrm{C}_{4} \mathrm{H}_{3} \mathrm{Cl}$, $\mathrm{H} \mathrm{Cl}$, is heated $302^{\circ}$ in a sealed tube, with five times its bulk of the strongest aqua ammoniæ, it is dissolved and a yellow liquid obtained, which contains sal ammoniac and the chloride of the new radical, $\left.\mathrm{C}_{4} \mathrm{H}_{3} \mathrm{Cl}, \mathrm{H} \mathrm{Cl}+2 \mathrm{~N} \mathrm{H}_{3}=\mathrm{N} \mathrm{H}_{4} \mathrm{Cl}+\mathrm{N}_{\mathrm{C}_{4} \mathrm{H}_{3}}^{\mathrm{H}_{3}}\right\} \mathrm{Cl}$. After the sal ammoniac has been separated by crystallisation, the mother liquid, which contains the chloride of acetylammonium, is acted on by moist oxide of silica, evaporated to dryness to expel the excess of ammonia; the residue, dissolved in water, treated with sulphuretted hydrogen to remove some silver dissolved by the new base, and the filtered liquid is now a solution of the hydrated oxide of acetylammonium. It is carefully neutralised by sulphuric acid, the sulphate decomposed by baryta water, taking care to avoid excess of baryta, and from the mass alcohol dissolves the new base.

Neither the hydrated oxide, nor its salts can be obtained in 
orystals, but their constitution is demonstrated by their re-actions. Thus, when to the solution of oxide, hyponitrate of silver is added, with a little sulphuric acid, and heat applied, aldehyde is regenerated.

$$
\left.\begin{array}{c}
\mathrm{C}_{4} \mathrm{H}_{3} \\
\mathrm{H}_{3}
\end{array}\right\} \mathrm{NO}, \mathrm{HO}+\mathrm{N} \mathrm{O}_{3}=\mathrm{C}_{4} \mathrm{H}_{3} \mathrm{O}, \mathrm{HO}+3 \mathrm{HO}_{2}+\mathrm{N}_{2}
$$

This exactly corresponds to the action of hyponitrous acid on ethylamine, when alcohol is regenerated, and proves that the new compound contains acetyle replacing hydrogen.

With nitrate of silver and ammonia, the base or its salts yield a mirror-like deposit of silver, proving that here also aldehyde, that is, hydrated oxide of acetyle, is regenerated.

The hydrated oxide, when its solution is dried up, forms a tough yellow amorphous mass. Its solution has a strong alkaline reaction, and a feeble caustic taste. When boiled, it has the peculiar odour of alkaline leys. The new base expels ammonia from its salts and attracts carbonic acid from the air. It is, therefore, like the hydrated oxide of tetrethylium, analogous in constitution to hydrated oxide of ammonium, and in properties, to hydrated oxide of potassium or caustic potash. It is, however, from the negative character of acetyle, a weak base, and its salts with the stronger acids, are all somewhat acid.

These researches of Natanson are not yet completed, but we have given here the first part of them, because they have an important bearing on the question of the real existence of the negative radicals, formyle, acetyle, and their homologues. It is evident that the existence of the base just described, is strongly in favour of the view which admits these radicals. We may expect, not only that many bases homologous with this one, and containing radicals higher in the scale, will be obtained, but also that similar compounds may be formed, in which two, three, or all four of the equivalents of hydrogen in ammonium are replaced by formyle, acetyle, \&c., or partly by one, and partly by another of these radicals, or even partly by these, and partly by ethylic or phenylic radicals.

It might be expected that as hydrated oxide of tetrethylium, when heated, yields triethylamine and alcohol, $\mathrm{N} \mathrm{Aes} \mathrm{O}, \mathrm{H} \mathrm{O}=$ $\mathrm{N} \mathrm{Aes}+\mathrm{Ae} \mathrm{O}, \mathrm{H} \mathrm{O}$, the hydrated oxide of acetylammonium should yield ammonia and aldehyde when heated,

$$
\left.\underset{\mathrm{C}_{4} \mathrm{H}_{3}}{\mathrm{H}_{3}}\right\} \mathrm{NO}, \mathrm{HO}=\mathrm{NH}_{3}+\mathrm{C}_{4} \mathrm{H}_{3} \mathrm{O}, \mathrm{HO} \text {. }
$$

This, perhaps, takes place, but at the same time the base is charred, so that a further decomposition cannot be avoided. 
Natanson has not yet been able to obtain any volatile bases analogous to ethylamine, \&c., containing acetyle, as replacing the hydrogen of ammonia. And from the circumstances that in the action of ammionia on the oil of olefiant gas, we obtain, not hydrochlorate of acetylamine, $\left.\mathrm{N}_{\mathrm{C}_{4} \mathrm{H}_{3}}^{\underset{\mathrm{H}_{2}}{\mathrm{H}_{2}}}\right\}, \mathrm{H} \mathrm{Cl}$, but chloride of acetylammonium, $\left.\mathrm{N}_{\mathrm{C}_{4} \mathrm{H}_{3}}^{\mathrm{H}_{3}}\right\} \mathrm{Cl}$, he draws the conclusion that the true fundamental bases are the ammonium bases, such as oxide of ammonium, or of tetrethylium, and that the volatile or ammonia bases are merely products of their decomposition. $\mathrm{He}$ infers also, that the ammonium theory of ammoniacal compounds is the only true one. But it is not easy to see how the facts prove these inferences; for oxide of ammonium is unknown in the free state, and his own base does not yield, when heated, the products which we should anticipate. It would seem rather that something in the relations of nitrogen and hydrogen renders ammonia $\mathrm{N} \mathrm{H}_{3}$, as well as its numerous homologues, very permanent: while oxide, or hydrated oxide of ammoniun $\mathrm{N} \mathrm{H}_{4} \mathrm{O}$, $\mathrm{H} \mathrm{O}$, is so insoluble as not to be known in the free state, on account of the attraction of oxygen for the fourth eq. of hydrogen. In the ammonium bases, the fourth eq. being ethyle, methyle, acetyle, or other similar radicals, has no such strong tendency to combine with oxygen, and therefore the compound molecule is more permanent.

It is worthy of remark, that hydrated oxide of acetylammonium is isomeric with aldehydammonia; for,

$$
\begin{aligned}
& \text { Aldehydammonia. Hydrated Oxide of } \\
& \left.\mathrm{C}_{4} \mathrm{H}_{3} \mathrm{O}, \mathrm{H} \mathrm{O}, \mathrm{N} \mathrm{H}_{3}=\mathrm{N}_{\mathrm{C}_{4} \mathrm{H}_{3}}^{\mathrm{H}_{3}}\right\} \mathrm{O}, \mathrm{HO}
\end{aligned}
$$

the empirical formula of both being $\mathrm{C}_{4} \mathrm{H}_{7} \mathrm{~N} \mathrm{\textrm {O } _ { 2 }}$. The differences in their properties are accounted for by the rational formulæ above given, while the fact that they agree in some points, as in reducing the salts of silver, is also accounted for by both containing acetyle, and yielding aldehyde when decomposed.

\section{ACTION OF CHLORINE, BROMINE, ETC., ON ETHYLE, ACETYLE, AND THEIR DERIVATIVES.}

\section{Oxide of Ethyle and Chlorine.}

When dry ohloride is made to act on ether, with the aid of the sun's rays, there are produced several compounds. The first is a heavy oily liquid, oxychloride of acetyle, $\mathrm{C}_{4} \mathrm{H}_{3}\left\{\begin{array}{l}\mathrm{O} \\ \mathrm{Cl}_{2}\end{array}\right.$; which is 
dry acetic acid, with 2 eqs. of oxygen replaced by 2 eqs. of chlorine. With water, it forms acetio and hydrochloric acids. When the action is pursued, there is obtained a compound, $\mathrm{C}_{4} \mathrm{Cl}_{5} \mathrm{O}$, which is ether, in which all the hydrogen is replaced by chlorine. The first compound may be connected with this one by being viewed as ether in which hydrogen is partially replaced by chlorine, $\mathbf{C}_{\boldsymbol{4}}$ $\left\{\mathrm{H}_{3}\right.$ O. Finally there is obtained a crystallisable solid compound, $\mathrm{C}_{4} \mathrm{Cl}_{6}$, which may be represented as $\mathrm{C}_{4} \mathrm{Cl}_{5}, \mathrm{Cl}$, showing that the oxygen of ether is also here replaced by chlorine. This last body is chloride of carbon, $\mathrm{C}_{2} \mathrm{Cl}_{3}$, for $\mathrm{C}_{4} \mathrm{Cl}_{6}=2\left(\mathrm{C}_{2} \mathrm{Cl}_{3}\right)$. Besides these compounds, there are others formed at the same time, more difficult to separate, and which are doubtless the interme-. diate links of the chain of substitutions, beginning with ether, $\mathrm{C}_{4} \mathrm{H}_{5}, 0$, and ending with chloride of carbon, $\mathrm{C}_{4} \mathrm{Cl}_{5}, \mathrm{Cl}$. The other members of the series which are more easily obtained by the action of chlorine on chloride of ethyle are $\mathrm{C}_{4} \mathrm{H}_{3}, \mathrm{Cl}$,

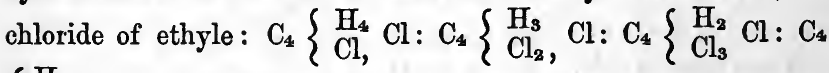
$\left\{\begin{array}{l}\mathrm{H} \\ \mathrm{Cl}_{4}\end{array}, \mathrm{Cl}\right.$; and as the compound $\mathrm{C}_{4} \mathrm{Cl}_{5} \mathrm{O}$ is formed, it is probable that there is another series beginning with ether, and ending with $\mathrm{C}_{4} \mathrm{Cl}_{5}, \mathrm{O}$, in which the oxygen remains unchanged. The above are not all cases of substitution with preservation of the type; for the compound $\mathrm{C}_{4}\left\{\begin{array}{l}\mathrm{H}_{3} \\ \mathrm{Cl}_{2},\end{array}\right.$, may very likely be $\mathrm{C}_{4} \mathrm{H}_{3}\left\{\begin{array}{l}\mathrm{O} \\ \mathrm{Cl}_{2}\end{array}\right.$ belonging to the type of acetic acid, rather than that of ether. These compounds are as yet but little known; it is obvious that the simultaneous occurrence of so many similar compounds must render the study of them exceedingly complicated and difficult.

The body, $\mathrm{C}_{4} \mathrm{H}_{3} \mathrm{~S}_{\mathrm{Cl}_{2}}^{\mathrm{O}}$, or oxychloride of acetyle, when acted on by sulphuretted hydrogen, yields two new compounds, in which its chlorine is partially or entirely replaced by sulphur. Both are crystalline: one, the oxysulphuret of acetyle, $\mathrm{C}_{4} \mathrm{H}_{3}$ $\left\{\begin{array}{l}0 \\ \mathrm{~S}_{2}\end{array}\right.$, forms large colourless prisms: the other, oxychlorosulphuret of acetyle, $\mathrm{C}_{4} \mathrm{H}_{3}\left\{\begin{array}{l}\mathrm{O} \\
\mathrm{S} \\
\mathrm{Cl} \text {, forms yellow tabular crystals. }\end{array}\right.$

2. Salts of Oxide of Ethyle with Chlorine.

When chlorine acts on these salts, or ethers, their oxide of ethyle is acted on as if separate, but in many cases the acids remain combined with the new chlorinised compounds; or the 
acids also are acted on, and the products derived from them combine with those derived from the ether.

Acetic Ether yields a compound $\mathrm{C}_{8} \mathrm{H}_{6} \mathrm{Cl}_{2} \mathrm{O}_{4}$, which may be viewed as $\mathrm{C}_{4} \quad \mathrm{H}_{3}\left\{\begin{array}{l}\mathrm{O} \\ \mathrm{Cl}_{2}\end{array}+\mathrm{C}_{4} \mathrm{H}_{3} \mathrm{O}_{3}\right.$, that is, acetate of the oxychloride of acetyle. When the compound is further exposed to the action of chlorine at a high temperature, it yields a series of compounds, in which its hydrogen is gradually replaced by chlorine, till the compound $\mathrm{C}_{8} \mathrm{Cl}_{8} \mathrm{O}_{4}$ is left, which is called perchloruretted acetic ether. Acetic ether is $\mathrm{C}_{8} \mathrm{H}_{8} \mathrm{O}_{4}$; and we have the acetate of oxychloride of acetyle $\mathrm{C}_{8}\left\{\begin{array}{l}\mathrm{H}_{6} \\ \mathrm{Cl}_{2}\end{array} \mathrm{O}_{4}\right.$; then $\mathrm{C}_{8}\left\{\begin{array}{l}\mathrm{H}_{5} \\ \mathrm{Cl}_{3}\end{array} \mathrm{O}_{4}: \mathrm{Cl}_{8}\left\{\begin{array}{l}\mathrm{H}_{4} \\ \mathrm{Cl}_{4}\end{array} \mathrm{O}_{4}: \mathrm{C}_{8}\left\{\begin{array}{l}\mathrm{H}_{3} \\ \mathrm{Cl}_{5}\end{array} \mathrm{O}_{4}: \mathrm{C}_{8}\left\{\begin{array}{l}\mathrm{H}_{2} \\ \mathrm{Cl}_{6}\end{array} \mathrm{O}_{4}: \mathrm{C}_{8}\right.\right.\right.\right.$

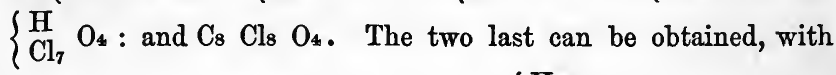
certainty, pure: the others, after $\mathrm{C}_{8} \cdot\left\{\mathrm{H}_{6} \mathrm{Cl}_{2} \mathrm{O}_{4}\right.$, are so mixed that it is very difficult to obtain them pure enough for analysis.

When benzoic ether, Ae $\mathrm{O}, \mathrm{Bz} \mathrm{O}$, is acted on by chlorine, it loses 2 eqs. hydrogen, and 1 eq. oxygen, and takes up 3 eqs. chlorine, producing a compound which may be viewed as containing chloride of benzoyle and oxychloride of acetyle, $\mathrm{Bz} \mathrm{Cl}$ $+\mathrm{C}_{4} \mathrm{H}_{3}\left\{\begin{array}{l}\mathrm{O} \\ \mathrm{Cl}_{2}\end{array}=\mathrm{C}_{18} \mathrm{H}_{8} \mathrm{Cl}_{3} \mathrm{O}_{3}\right.$.

Oxalic Ether, exposed to the action of chlorine, under the influence of the direct rays of the sun, loses all its hydrogen, which is replaced by chlorine. $\left(\mathrm{C}_{4} \mathrm{H}_{5}\right) \mathrm{O}, \mathrm{C}_{2} \mathrm{O}_{3}$ thus becomes $\left(\mathrm{C}_{4} \mathrm{Cl}_{5}\right) \mathrm{O}, \mathrm{C}_{2} \mathrm{O}_{3}$. The latter is called chloroxalic ether. It is a crystallisable solid, fusible at $288^{\circ}$. Dry ammonia acts on it as on oxalic ether, producing chloroxamethane, a crystalline compound analogous to oxamethane. It was formerly stated that

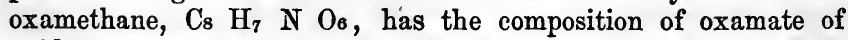
oxide of ethyle $\left(\mathrm{C}_{4} \mathrm{H}_{5}\right) \mathrm{O}+\mathrm{C}_{4} \mathrm{H}_{2} \mathrm{~N} \mathrm{O}_{5}$; or of oxalic ether plus oxamide $\left(\mathrm{C}_{4} \mathrm{H}_{5}\right) \mathrm{O}, \mathrm{C}_{2} \mathrm{O}_{3}+\mathrm{N} \mathrm{H}_{2}, \mathrm{C}_{2} \mathrm{O}_{2}$. In like manner, chloroxamethane represents chloroxalic ether plus oxamide: $\left(\mathrm{C}_{4}\right.$ $\left.\mathrm{Cl}_{5}\right) \mathrm{O}, \mathrm{C}_{2} \mathrm{O}_{3}+\mathrm{N} \mathrm{H}_{2}, \mathrm{C}_{2} \mathrm{O}_{2}=\mathrm{C}_{8} \mathrm{H}_{2} \mathrm{Cl}_{5} \mathrm{~N} \mathrm{O}_{6}$. When chloroxamethane is left in contact with ammonia, it takes up 2 eqs. of water, and forms a new salt, chloroxalovinate of ammonia, $\mathbf{C}_{4}$ $\mathrm{Cl}_{5} \mathrm{O}, \mathrm{N} \mathrm{H}_{4} \mathrm{O}, 2 \mathrm{C}_{2} \mathrm{O}_{3}$, which is very deliquescent. From the corresponding salt of soda the chloroxalovinic acid may be obtained, which may of course be viewed as an acid oxalate of the compound $\mathrm{C}_{4} \mathrm{Cl}_{5}{ }_{5} \mathrm{O}$. Its formula is $\mathrm{C}_{4} \mathrm{Cl}_{5} \mathrm{O}, \mathrm{C}_{2} \mathrm{O}_{3}+\mathrm{H} \mathrm{O}, \mathrm{C}_{2} \mathrm{O}_{3}$. By the action of alcohol on chloroxalic ether, there is formed a neutral oil, $\mathrm{C}_{8} \mathrm{Cl}_{5} \mathrm{O}_{7}$, which contains the elements of anhydrous 
chloroxalovinic acid, $\mathrm{C}_{4} \mathrm{Cl}_{5} \mathrm{O}, 2 \mathrm{C}_{2} \mathrm{O}_{3}$; and when dissolved in potash, yields chloroxalovinate of potash.

Carbonic Ether, by the action of chlorine, yields two products : 1st, Bichloruretted carbonic ether, $\mathrm{C}_{4}\left\{\begin{array}{l}\mathrm{H}_{3} \\ \mathrm{Cl}_{2}\end{array}\right.$ $\mathrm{O}_{3}$; and 2nd, Perchloruretted carbonic ether, $\mathrm{C}_{4} \mathrm{Cl}_{5} \mathrm{O}, \mathrm{C} \mathrm{O}$ $=\mathrm{C}_{5} \mathrm{Cl}_{5} \mathrm{O}_{3}$. The former is an oily liquid; the latter crystallisable.

Chloride of Ethyle, $\left(\mathrm{C}_{4} \mathrm{H}_{5}\right) \mathrm{Cl}$, when acted on by chlorine, yields a very remarkable series of products, in which the hydrogen is gradually replaced by chlorine, as mentioned at p. 17, to which I refer for the formulæ. I shall only here mention that the compound there represented as $\mathrm{C}_{4}\left\{\begin{array}{l}\mathrm{H}_{4} \\ \mathrm{Cl}\end{array}, \mathrm{Cl},=\mathrm{C}_{4} \mathrm{H}_{4} \mathrm{Cl}_{2}\right.$, corresponds to aldehyde, and is, therefore, probably $\left(\mathrm{C}_{4} \mathrm{H}_{3}\right.$ $\mathrm{Cl}+\mathrm{H} \mathrm{Cl}$, just as aldehyde is $\left(\mathrm{C}_{4} \mathrm{H}_{3}\right) \mathrm{O}+\mathrm{H} \mathrm{O}$. In like manner, the compound $\mathrm{C}_{4}\left\{\begin{array}{l}\mathrm{H}_{3} \\ \mathrm{Cl}_{2}\end{array}, \mathrm{Cl}=\mathrm{C}_{4} \mathrm{H}_{3} \mathrm{Cl}_{3}\right.$, corresponds to dry acetic acid, $\mathrm{C}_{4} \mathrm{H}_{3} \mathrm{O}_{3}$. The action of potash on these two compounds confirms this view, according to which the former is hydrochlorate of protochloride, and the latter perchloride, of acetyle.

When alcohol, the hydrated oxide of ethyle, is subjected to the long-continued action of chlorine, aided by the sun's rays, there is formed, after a very tedious operation, a remarkable compound called chloral, the empirical formula of which is $\mathrm{C}_{4} \mathrm{H}$ $\mathrm{Cl}_{3} \mathrm{O}_{2}=\mathrm{C}_{4} \mathrm{Cl}_{3} \mathrm{O}, \mathrm{H} \mathrm{O}$. This compound represents aldehyde or hydrated oxide of acetyle, in which the hydrogen of the acetyle has been replaced by chlorine. It is an oily liquid, boiling at $199^{\circ}$, of Sp. G. 1·502. Like aldehyde, chloral, when kept, is spontaneously converted into an insoluble solid compound, which has the same composition as chloral itself. In contact with water, chloral is soon converted into a solid hydrate, which dissolves in a larger quantity of water. It contains 1 eq. chloral and 2 eqs. water. When heated with caustic alkalies, chloral produces formiate of the alkali and perchloride of formyle, $\mathrm{C}_{4} \mathrm{H} \mathrm{Cl} \mathrm{Cl}_{3} \mathrm{O}_{2}+$ $\mathrm{H} \mathrm{O}, \mathrm{KO}=\left(\mathrm{C}_{2} \mathrm{H}\right) \mathrm{O}_{3}, \mathrm{KO}+\left(\mathrm{C}_{2} \mathrm{H}\right) \mathrm{Cl}_{3}$. The perchloride of formyle, in contact with the alkali, is partly decomposed, yielding chloride of the metal.

According to Städeler, chloral is formed when starch is heated with hydrochloric acid and peroxide of manganese.

Pure Acetic Acid, when acted on by chlorine and the sun's rays, is converted into a crystallisable acid, the chloracetic acid, $\mathrm{C}_{4} \mathrm{Cl}_{3} \mathrm{O}_{3} \mathrm{H} \mathrm{O}$. As acetic acid may be considered to be aldehyde plus 2 eqs. oxygen, or hydrated peroxide of acetyle, so chloracetio 
acid is chloral plus 2 eqs. oxygen, or hydrated peroxide of $\mathrm{C}_{4} \mathrm{Cl}_{3}$, which may be called chloracetyle. Chloracetic acid forms tabular crystals, fusible at $113^{\circ}$, boiling at $390^{\circ}$. The density of the liquefied acid at $113^{\circ}$ is 1.617 . When heated with excess of potash, it yields first carbonic acid and perchloride of formyle, $\mathrm{C}_{4} \mathrm{Cl}_{3} \mathrm{O}_{3}$, $\mathrm{HO}+2 \mathrm{KO}=2\left(\mathrm{~K} \mathrm{O}, \mathrm{C} \mathrm{O}_{2}\right)+\mathrm{C}_{2} \mathrm{H} \mathrm{Cl}_{3}$. The perchloride of formyle is partly converted by another portion of potash, into formiate of potash and chloride of potassium. $\mathrm{C}_{2} \mathrm{H} \mathrm{Cl}_{3}+4 \mathrm{~K} \mathrm{O}$ $=3 \mathrm{~K} \mathrm{Cl}+\mathrm{K} \mathrm{O}, \mathrm{C}_{2} \mathrm{H} \mathrm{O}$.

With bases, chloracetic acid forms salts which are very analogous to the acetates; and it is very important here to observe, that both in chloral and chloracetic acjd, the substitution of chlorine for all the hydrogen of the radical (acetyle) of aldehyde and acetic acid, has not affected the general chemical characters of the compounds; that, in other words, the original type has been retained. We have also seen, in the preceding pages, among the products of the action of chlorine on oxide of ethyle and on the salts of oxide of ethyle, that oxide of ethyle, $\mathrm{C}_{4} \mathrm{H}_{5} \mathrm{O}$, is converted into oxide of chlorethyle, $\mathrm{C}_{4} \mathrm{Cl}_{5} \mathrm{O}$, without the type being altered: the oxide of chlorethyle forming with the acids previously combined with oxide of ethyle, compounds perfectly analogous to the ethers from which they are obtained.

Oxychloride of Acetyle, $\left.\mathrm{C}_{4} \mathrm{H}_{3} \begin{array}{l}\mathrm{O}_{2} \\ \mathrm{Cl}\end{array}\right\}$. When oxychloride of phosphorus is gradually added to fused acetate of potash, a liquid distils over, which is the oxychloride. It is a volatile liquid, boiling at $131^{\circ}$, of a pungent suffocating odour. It is used in the preparation of anhydrous acetic acid, and other compounds.

The Sulphuret of Ethyle is readily acted on by chlorine, and yields a yellow oily liquid, of Sp. G. 1.673, boiling at $320^{\circ}$, of a most fetid odour, the formula of which is $\mathrm{C}_{4}\left\{\begin{array}{l}\mathrm{H} \\ \mathrm{Cl}_{4}\end{array}\right.$ S. Here 4 eqs. of hydrogen of the compound, $\mathrm{C}_{4} \mathrm{H}_{5} \mathrm{~S}$, are replaced by chlorine.

Heavy Muriatic Ether is an oily liquid, formed by the action of moist chlorine on alcohol. It is obviously a mixture, and probably contains aldehyde, chloride of ethyle, chloral, and products intermediate between aldehyde and chloral.

Bromal, $\mathrm{C}_{4} \mathrm{Br}_{3} \mathrm{O}, \mathrm{H} \mathrm{O}$. This compound, analogous to chloral, is formed by the action of bromine on alcohol. It forms a hydrate with 3 eqs. water. By caustic alkalies it is resolved into formic acid, which combines with the alkali, and perbromide of formyle.

Iodine does not, so far as is known, produce a compound corresponding to chloral and bromal; but a solution of iodine in 
alcohol, treated with an alcoholic solution of potash, yields formiate of potash and periodide of formyle, $\mathrm{C}_{2} \mathrm{H} \mathrm{I}_{3}$.

By the action of chlorine on alcohol, holding in solution hydrocyanic acid on a metallic cyanide, there is produced a crystalline compound, the empirical formula of which appears to be $\mathrm{C}_{16} \mathrm{H}_{14} \mathrm{~N}_{2} \mathrm{Cl}_{2} \mathrm{O}_{8}$. This is equal to 3 eqs. aldehyde, 2 eqs. chloride of cyanogen, and 2 eqs. water; but the true nature of this compound is unknown.

3. Compounds derived from Alcohol, but of uncertain constitution.

Olefiant Gas. Srn. Hyduret of Acetyle. Ethylene. $\mathrm{C}_{4} \mathrm{H}_{4}=$ $\mathrm{C}_{4} \mathrm{H}_{3}, \mathrm{H}=\mathrm{Ac} \mathrm{H}$. This well-known compound is generally present in coal gas, oil gas, and, in general, in all gaseous mixtures produced by the action of heat on organic substances. It is best obtained pure by heating 1 part of alcohol with 6 or 7 of oil of vitriol, adding as much sand as will convert the whole into a paste, which prevents the frothing that would otherwise occur. There is produced some ether, then sweet oil of wine, and lastly, a mixture of sulphurous acid and olefiant gases. By passing the gas through milk of lime, the sulphurous acid is removed, and by then passing it through oil of vitriol, the ether, alcohol, and water which may be present, are likewise separated. Pure olefiant gas has been already described (see Part I.): here we shall attend to its combinations, premising that it is the second in a homologous series of earbohydrogens, of which methylene, or hyduret of formyle, $\mathrm{C}_{2} \mathrm{H}_{2}=\mathrm{C}_{2} \mathrm{H}, \mathrm{H}$, is the first. It is absorbed by anhydrous sulphuric acid, forming the crystalline compound formerly mentioned, $2 \mathrm{~S} \mathrm{O}_{3}+\mathrm{C}_{4} \mathrm{H}_{4}$, which, in contact with water, produces ethionic aeid. When mixed with its own volume of chlorine, both gases are condensed into a liquid, the composition of which is $\mathrm{C}_{4} \mathrm{H}_{4} \mathrm{Cl}_{2}$. This is the oily compound, from which the gas was called olefiant gas : the oil is often called the oil of the Dutch chemists, having been discovered by an association of chemists in Holland.

When mixed with 2 vol. of chlorine, and set fire to, the whole of the carbon of the gas is deposited in the solid form or as smoke, while all the hydrogen forms hydrochloric acid, $\mathrm{C}_{4} \mathrm{H}_{4}+\mathrm{Cl}_{4}=$ $4 \mathrm{H} \mathrm{Cl}+\mathrm{C}_{4}$.

The oil of olefiant gas, or of the Dutch chemists, $\mathrm{C}_{4} \mathrm{H}_{4} \mathrm{Cl}_{2}$, may be viewed as composed of hydrochloric acid, and a chloride of acetyle: $\mathrm{H} \mathrm{Cl}+\mathrm{C}_{4} \mathrm{H}_{3}^{-} \mathrm{Cl}$. When acted on by an alcoholie solution of potash, chloride of potassium and water are formed, and a new compound separates, which is the protochloride of acetyle, $\mathrm{C}_{4}$ $\mathrm{H}_{3} \mathrm{Cl}$. It is gaseous at ordinary temperatures, has an alliaceous 
smell, and burns, like all similar chlorinised compounds, with a dark red flame, edged with green. At $0^{\circ}$ it condenses into a liquid.

When this protochloride of acetyle is acted on by perchloride of antimony, it yields, among other products, a liquid, boiling at $240^{\circ}$, which is $\mathrm{C}_{4} \mathrm{H}_{3} \mathrm{Cl}_{3}$, and therefore has the same composition as perchloride of acetyle, formerly mentioned. But the action of potash, dissolved in alcohol, proves that these two compounds are distinct, and that the one now under consideration is $\mathrm{C}_{4} \mathrm{H}_{2}$ $\mathrm{Cl}_{2}+\mathrm{H} \mathrm{Cl}$. At all events it yields chloride of potassium, water, and a very volatile liquid, $\mathrm{C}_{4} \mathrm{H}_{2} \mathrm{Cl}_{2}=2\left(\mathrm{C}_{2} \mathrm{H}, \mathrm{Cl}\right)$, or in other words, protochloride of formyle.

By continuing the action of chlorine, there is obtained a compound $\mathrm{C}_{4} \mathrm{H}_{2} \mathrm{Cl}_{4}=\mathrm{Cl}_{4} \mathrm{H} \mathrm{Cl}_{3}+\mathrm{H} \mathrm{Cl}$; which, with potash, yields the body $\mathrm{C}_{4} \mathrm{H} \mathrm{Cl}_{3}$; and the final result of this action is the protochloride of carbon, $\mathrm{C}_{4} \mathrm{Cl}_{4}=4 \mathrm{C} \mathrm{Cl}$; which, however, unites with chlorine to produce the sesquichloride, $\mathrm{C}_{4} \mathrm{Cl}_{4}+\mathrm{Cl}_{2}=\mathrm{C}_{4} \mathrm{Cl}_{6}$ $=2 \mathrm{C}_{2} \mathrm{Cl}_{3}$.

The Perchloride of Acetyle has been already mentioned as a product of the action of chlorine on ether: it is $\mathrm{C}_{4} \mathrm{H}_{3} \mathrm{Cl}_{3}=$ $\mathrm{Ac} \mathrm{Cl}_{3}$.

The Oil of Olefiant $\mathrm{Gas}, \mathrm{C}_{4} \mathrm{H}_{4} \mathrm{Cl}_{2}$, which may be considered the hydrochlorate of chloride of acetyle, $\mathrm{C}_{4} \mathrm{H}_{3}, \mathrm{Cl}+\mathrm{H} \mathrm{Cl}$, is best prepared by passing olefiant gas into perchloride of antimony, as long as it is absorbed. The mixture, if distilled, yields the oil in question. It is purified by alternate distillation with water and sulphuric acid, and finally drying it with chloride of calcium. It is a very mobile liquid, of a pleasant ethereal smell, and a very sweet taste; it boils at $180^{\circ}$, is insoluble in water, soluble in alcohol and ether.

When subjected to the action of chlorine, it yields hydrochloric acid, and products rich in chlorine. Among these are, the hydrochlorate of chloride of formyle, $\mathrm{C}_{2} \mathrm{H} \mathrm{Cl}+\mathrm{H} \mathrm{Cl}$, which distils at $240^{\circ}$, and the bichloride of formyle, $\mathrm{C}_{2} \mathrm{H}, \mathrm{Cl}_{2}$, which distils at $275^{\circ}$. This last is finally converted into sesquichloride of carbon; for $\mathrm{C}_{2} \mathrm{H}, \mathrm{Cl}_{2}+\mathrm{Cl}_{2}=\mathrm{C}_{2} \mathrm{Cl}_{3}+\mathrm{H} \mathrm{Cl}$.

Chloretheral is the name given by D'Arcet to a compound formed by the action of chlorine on olefiant gas, containing both alcohol and ether. Its empirical formula is $\mathrm{C}_{4} \mathrm{H}_{4} \mathrm{Cl} \mathrm{O}$; so that it may be aldehyde, plus oil of olefiant gas ; $\mathrm{C}_{4} \mathrm{H}_{3} \mathrm{O}, \mathrm{H} \mathrm{O}+$ $\mathrm{C}_{4} \mathrm{H}_{3} \mathrm{Cl}, \mathrm{H} \mathrm{Cl}=2\left(\mathrm{C}_{4} \mathrm{H}_{4} \mathrm{Cl} \mathrm{O}\right)$; or oxychloride of acetyle, plus

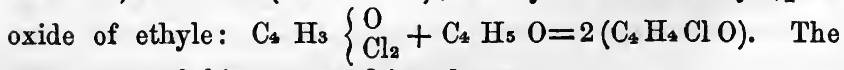
true nature of this compound is unknown.

Bromine forms, with olefiant gas, a liquid compound analogous to the oil of olefiant gas. Its formula is $\mathrm{C}_{4} \mathrm{H}_{3}, \mathrm{Br}+\mathrm{H} \mathrm{Br}$. 
Iodine forms, with olefiant gas, a solid compound, which would appear to be $\mathrm{C}_{4} \mathrm{H}_{3}, \mathrm{H}+\mathrm{I}_{2}$ rather than $\mathrm{C}_{4} \mathrm{H}_{3}$ ', $\mathrm{I}+\mathrm{H} \mathrm{I}$.

Anhydrous Sulphuric Acid absorbs olefiant gas, producing a white crystalline solid, $2 \mathrm{~S} \mathrm{O} \mathrm{O}_{3}+\mathrm{C}_{4} \mathrm{H}_{4}$; which, when dissolved in water, forms with 1 eq. of water, ethionic acid, $2 \mathrm{~S} \mathrm{O} 3+\mathrm{C}_{4} \mathrm{H}_{5} \mathrm{O}$ $=\mathrm{C}_{4} \mathrm{H}_{5} \mathrm{O}_{2}+\mathrm{S}_{2} \mathrm{O}_{5}$. The original crystalline compound has been called sulphacetylic acid.

\section{Action of Bichloride of Platinum on Alcohol.}

This action is very complex, yielding aldehyde, chloride of ethyle, chloride of acetyle, and other volatile compounds, along with a salt, composed of chloride of platinum and chloride of acetyle. It is possible that 3 eqs. of oxide of ethyle, with 4 eqs. of bichloride of platinum, may yield 1 eq. aldehyde, 1 eq. water, 4 eqs. hydrochloric acid, and 2 eqs. of the new salt. $3\left(\mathrm{C}_{4} \mathrm{H}_{5} \mathrm{O}\right)+$ $4 \mathrm{Pt} \mathrm{Cl}_{2}=\mathrm{C}_{4} \mathrm{H}_{3} \mathrm{O}, \mathrm{H} \mathrm{O}+\mathrm{H} \mathrm{O}+4 \mathrm{H} \mathrm{Cl}+2\left(\mathrm{C}_{4} \mathrm{H}_{3} \mathrm{Cl}+\mathrm{Pt}_{2} \mathrm{Cl}\right)$. Zeise considers the salt to be $\mathrm{C}_{4} \mathrm{H}_{4}+2 \mathrm{Pt} \mathrm{Cl}$, which formula differs from the preceding in containing 1 eq. hydrogen more. Malaguti supposes it to be $\mathrm{C}_{4} \mathrm{H}_{3} \mathrm{O}+2 \mathrm{Pt} \mathrm{Cl}$; but Zeise has shown that it contains no oxygen. It does not crystallise, but forms a gummy mass, spontaneously decomposing when kept.

When a solution of bichloride of platinum in alcohol is digested with a little hydrochloric acid, and chloride of potassium, the alcohol distilled off, and the residue neutralised by carbonate of potash, a yellow crystallisable salt is obtained, which contains the preceding compound, plus 1 eq. chloride of potassium, $\mathrm{C}_{4} \mathrm{H}_{3} \mathrm{Cl}+$ $\mathrm{Pt}_{2} \mathrm{Cl}+\mathrm{K} \mathrm{Cl}$. Similar double salts are formed with chloride of sodium and chloride of ammonium.

These double salts form with ammonia a yellow precipitate, which is $\mathrm{C}_{4} \mathrm{H}_{3} \mathrm{Cl}, \mathrm{Pt} \mathrm{Cl}+\mathrm{N} \mathrm{H}$.

\section{Action of Heat on Acetic Acid and the Acetates.}

Acetone, Sxn. Pyroacetic Spirit, Mesitic Alcohol;-Formula $\mathrm{C}_{3} \mathrm{H}_{3} \mathrm{O}$,-is formed when acetic acid is passed through a tube heated to low redness, along with carbonic acid, carbonic oxide, and carburetted hydrogen: also when the acetate of an alkali or alkaline earth is exposed to heat, when a carbonate is left, and acetone distils over. It is best prepared by distilling a mixture of 2 parts of crystallised acetate of lead, and 1 part quicklime. Its formation is easily explained: for anhydrous acetic acid, $\mathrm{C}_{4} \mathrm{H}_{3} \mathrm{O}_{3}$, contains the elements of 1 eq. carbonic acid, and 1 eq. acetone. $\mathrm{C}_{4} \mathrm{H}_{3} \mathrm{O}_{3}=\mathrm{C} \mathrm{O}_{2}+\mathrm{C}_{3} \mathrm{H}_{3} \mathrm{O}$. Acetone is also formed in the distillation of sugar, of citric acid, of tartario 
acid, \&c. It is purified by rectification, until its boiling-point becomes constant, at $100^{\circ}$. It is a clear and colourless liquid, of Sp. G. 0.7921, and has a peculiar smell and a pungent taste. It is miscible with water, alcohol, and ether, in all proportions; and is separated from water by the addition of caustic potash, chloride of calcium, or other salts insoluble in acetone.

Heated with hypochlorite of lime, it is converted into carbonio acid and perchloride of formyle. When prepared by the distillation of acetates, acetone is accompanied by an oily liquid, $\mathrm{C}_{10} \mathrm{H}_{8} \mathrm{O}$.

Acetone contains, in 3 eqs., the elements of 1 eq. carbonic ether and 1 eq. olefiant gas (hyduret of acetyle). $\mathrm{C}_{4} \mathrm{H}_{5} \mathrm{O}, \mathrm{C} \mathrm{O}_{2}+$ $\mathrm{C}_{4} \mathrm{H}_{3}, \mathrm{H}=\mathrm{C}_{9} \mathrm{H}_{9} \mathrm{O}_{3}=3\left(\mathrm{C}_{3} \mathrm{H}_{3} \mathrm{O}\right)$; or, in 4 eqs., we have the elements of 1 eq. acetic ether, and 1 eq. hyduret of acetyle: $\mathrm{C}_{4} \mathrm{H}_{5} \mathrm{O}, \mathrm{C}_{4} \mathrm{H}_{3} \mathrm{O}_{3}+\mathrm{C}_{4} \mathrm{H}_{3} \mathrm{H}=\mathrm{C}_{12} \mathrm{H}_{12} \mathrm{O}_{4}=4\left(\mathrm{C}_{3} \mathrm{H}_{3} \mathrm{O}\right)$. Kane considers acetone to be $\mathrm{C}_{6} \mathrm{H}_{6} \mathrm{O}_{2}=\mathrm{C}_{6} \mathrm{H}_{5} \mathrm{O}, \mathrm{H} \mathrm{O}$; that is, the hydrated oxide of a radical $\mathrm{C}_{6} \mathrm{H}_{5}$, which he calls mesityle. In this view, mesityle is isomeric with proponyle, but analogous to ethyle; acetone is analogous to alcohol, and $\mathrm{C}_{6} \mathrm{H}_{5} \mathrm{O}$, the oxide of mesityle, to ether. But although Kane has obtained this compound, $\mathrm{C}_{6} \mathrm{H}_{5} \mathrm{O}$, and also another, $\mathrm{C}_{6} \mathrm{H}_{5} \mathrm{Cl}$, his chloride of mesityle, and although he has likewise formed double salts containing sulphuric acid and the elements $\mathrm{C}_{6} \mathrm{H}_{5} \mathrm{O}$, yet the analogy is far from being established. It has not yet been found possible to reproduce acetone, the alcohol, from the supposed ether of the series, as we can reproduce alcohol from the salts of oxide of ethyle. Moreover, in these double salts, the body $\mathrm{C}_{6} \mathrm{H}_{5} \mathrm{O}$, does not act as a base, but is only coupled with the acid, as naphthaline in sulphonaphthalic acid. We shall not, therefore, enter into minute details of the theoretical views alluded to. It is sufficient to enumerate the supposed radical mesityle, $\mathrm{C}_{6} \mathrm{H}_{5}$; its oxide, $\mathrm{C}_{6} \mathrm{H}_{5} \mathrm{O}$, oxide of mesityle; its hydrated oxide, $\mathrm{C}_{6} \mathrm{H}_{5} \mathrm{O}, \mathrm{H} \mathrm{O}$ (acetone); the chloride and iodide of mesityle, $\mathrm{C}_{6} \mathrm{H}_{5} \mathrm{Cl}$ and $\mathrm{C}_{6} \mathrm{H}_{5} \mathrm{I}$; the acid sulphate of oxide of mesityle, $\mathrm{C}_{6} \mathrm{H}_{5} \mathrm{O}, \mathrm{H} \mathrm{O}, 2 \mathrm{~S} \mathrm{O}_{3}$ (sulphomesitylic acid); the double salts of this sulphate, the formula of which is $\mathrm{C}_{6} \mathrm{H}_{5} \mathrm{O}, \mathrm{H} \mathrm{O}, 2 \mathrm{M} \mathrm{O}$, $2 \mathrm{~S} \mathrm{O}_{3}$; and a compound discovered by Zeise, containing oxide of mesityle with chloride of platinum, $\mathrm{C}_{6} \mathrm{H}_{5} \mathrm{O}, \mathrm{Pt} \mathrm{Cl}$.

The action of nitric acid on acetone gives rise to a new product : nitrite of oxide of pteleyle, $\mathrm{C}_{6} \mathrm{H}_{3} \mathrm{O}, \mathrm{N} \mathrm{O}_{3}$; phosphoric acid appears to form a compound acid with acetone; and when phosphorus, iodine, and acetone are distilled together, another acid is obtained, which appears to contain hypophosphorous acid.

When chlorine acts on acetone, it produces a liquid, $\mathrm{C}_{6} \mathrm{H}_{4}$ $\mathrm{Cl}_{2} \mathrm{O}_{2}$, which is called mesitic chloral. 
Mesitylole, $\mathrm{C}_{18} \mathrm{H}_{12}$, is obtained when acetone is distilled with fuming sulphuric acid. It is an oily liquid, boiling at $327^{\circ}$. Its formation is as follows:-

\section{3 eqs. acetone $=3\left(\mathrm{C}_{6} \mathrm{H}_{6} \mathrm{O}_{2}\right)$ yield $6 \mathrm{H} 0+\mathrm{C}_{18} \mathrm{H}_{12}$}

Mesitylole, when acted on by chlorine, bromine, and nitrous acid, yields substitution products, as in the following table:-

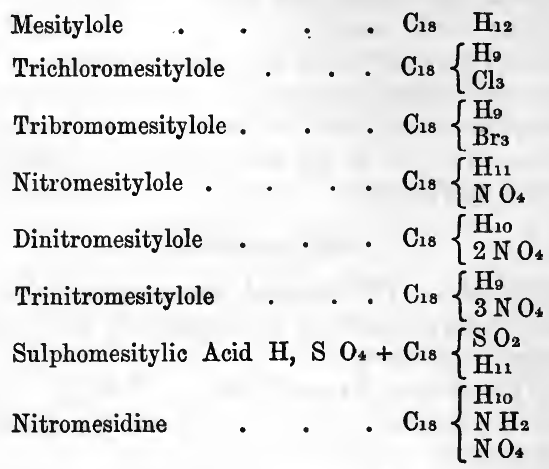

The acid is described by Hofmann. The base was obtained by Maule, from dinitromesitylole by the action of sulphuretted hydrogen. Thus:-

$$
\mathrm{C}_{18}\left\{\begin{array}{l}
\mathrm{H}_{10} \\
2 \mathrm{~N} \mathrm{O}_{4}
\end{array}+6 \mathrm{H} \mathrm{S}=4 \mathrm{HO}+\mathrm{S}_{6}+\mathrm{C}_{18}\left\{\begin{array}{l}
\mathrm{H}_{10} \\
\mathrm{~N} \mathrm{H}_{2} \\
\mathrm{~N} \mathrm{O}_{4}
\end{array}\right.\right.
$$

In all probability nitromesitylole would yield, by the same process, the base mesidine $\mathrm{C}_{18}\left\{\begin{array}{l}\mathrm{H}_{11} \\ \mathrm{~N} \mathrm{H}_{2}\end{array}\right.$. The reader will observe, that mesitylole is isomeric with cumole, mesidine with cumidine, and nitromesidine with nitrocumidine. But the two series of compounds are quite distinct. (See table, p. 141.)

We have now gone through, very briefly, the known compounds of methyle and ethyle, the two first members of the homologous series of electropositive radicals of the formula $\mathrm{C}_{n} \mathrm{H}_{n}+1$. Of this group several are already known higher in the scale. But we have also become acquainted with certain electronegative radicals of another series, derived from those of the methyle series, namely, formyle and acetyle, and with the hydrated protoxides and acid 
teroxides of these last. Now, both of these likewise belong to homologous series, to those of the aldehydes, and of the volatile acids of the formula (as hydrates) $\mathrm{C}_{n} \mathrm{H}_{n} \mathrm{O}_{4}(\mathrm{n}=2,4,6,8,10$, \&c.) Now although we know but few of the compounds homologous with methyle or ethyle, with ether, or with alcohol, yet we know a large number homologous with formic and acetic acids, from the existence of which we conclude as to the existence of the others. Thus the acid next above acetic acid, $\mathrm{C}_{4} \mathrm{H}_{4} \mathrm{O}_{4}$, is propylic acid, $\mathrm{C}_{6} \mathrm{H}_{6} \mathrm{O}_{4}$. Its immediate radical, propionyle, $\mathrm{C}_{6} \mathrm{H}_{5}$, must be homologous with acetyle, $\mathrm{C}_{4} \mathrm{H}_{3}$; but as acetyle is derived from ethyle, so propionyle, $\mathrm{C}_{6} \mathrm{H}_{5}$, must be derived from a radical homologous with ethyle, propyle, $\mathrm{C}_{6} \mathrm{H}_{7}$, and of this radical the oxide or propylic ether, $\mathrm{C}_{6} \mathrm{H}_{7} \mathrm{O}$, and the hydrated oxide or propylic alcohol, $\mathrm{C}_{6} \mathrm{H}_{7} \mathrm{O}, \mathrm{H} \mathrm{O}$, are respectively homologous with common ether and alcohol.

Radicals with $\mathrm{C}_{6}$.

Propyle, $\mathrm{C}_{6} \mathrm{H}_{7}$. This radical, homologous with methyle, is formed when butyric acid is decomposed by galvanism, but has not been much examined.

Oxide of Propyle, or Propylic Ether, $\mathrm{C}_{6} \mathrm{H}_{7}, \mathrm{O}$. This oxide is analogous to that of ethyle, and has been found to exist by Chancel, but is little known. It is an ethereal liquid.

Hydrated Oxide of Propyle, $\mathrm{C}_{6} \mathrm{H}_{7} \mathrm{O}, \mathrm{H} \mathrm{O}=\mathrm{Pr} \mathrm{O}, \mathrm{H} \mathrm{O}$. Srn. Propylic Alcohol.-Discovered in 1853 by Chancel among the products of the fermentation of the marc, or expressed grapes. When the fermented liquor is distilled to yield brandy, there is obtained an oily liquid, less volatile than alcohol, which is a mixture of several alcohols, higher in the series, with ethylic alcohol. The most abundant of these is the amylic alcohol, but the propylic and butylic alcohols, and apparently the caproic alcohol, possibly, also, the œnanthylic alcohol, are present. The propylic alcohol is found, along with alcohol, and a little methylic alcohol, in the most volatile part of this mixture. Some compound ethers are also present, which are decomposed by solution of potash, and boiling, yielding these alcohols and acids, the latter remaining with the potash. The propylic alcohol is purified by rectification. It is a volatile, rather fragrant liquid, very similar to alcohol, except in having a higher boiling point, namely $206^{\circ} \mathrm{F}$., and in not being miscible with water in all proportions. In fact, in this alcohol, the oily character begins to appear, evidently because the amount of carbon and hydrogen is so large in proportion to the oxygen.

In all its chemical relations it resembles alcohol, and it is unnecessary to give minute details. 
The radical, propyle, combines with chlorine, bromine, iodine, cyanogen, sulphur, \&c., forming compounds analogous to those of ethyle.

Propylamine, $\mathrm{C}_{6} \mathrm{H}_{9} \mathrm{~N}=\mathrm{N} \mathrm{H}_{2} \mathrm{Pr}$. This is a volatile base, homologous with ammonia and methylamine. It occurs in Dippel's animal oil, and may no doubt be formed by distilling the cyanate of oxide of propyle, or by the action of ammonia on iodide of propyle. (See Methylamine and Ethylamine.) It is an oily liquid, of a strong peculiar ammoniacal odour, which has all the characters of a powerful volatile base of the type ammonia.

Propylamine has the same formula as trimethylamine and ethylomethylamine, but is a different substance from both. It is said to be formed when narcotine or codeine is heated with potash or lime and potash.

Dipropylamine and Tripropylamine are not yet known.

Propyle appears to combine with arsenic, and will no doubt combine with other metals, as ethyle does.

Cyanide of Propyle, $\mathrm{C}_{6} \mathrm{H}_{7}, \mathrm{C}_{2} \mathrm{~N}=\mathrm{Pr} \mathrm{Cy}$. This compound is the same with butyronitryle, and is obtained when butyramide is heated with anhydrous phosphoric acid. It resembles cyanide of ethyle, but is less volatile.

Propionyle, $\mathrm{C}_{6} \mathrm{H}_{5}=$ Pro. This is a radical, homologous with acetyle, and is unknown in the free state. It is isomerio with Kane's mesityle, and also with allyle, but is distinct from both, even if the formula of mesityle were not doubtful. Its compounds are homologous with those of acetyle.

Propional, or Propylic Aldehyde, $\mathrm{C}_{6} \mathrm{H}_{5} \mathrm{O}, \mathrm{H} \mathrm{O}=\mathrm{C}_{6} \mathrm{H}_{6} \mathrm{O}_{2}$ $=$ Pro $0, \mathrm{H} 0$. This compound is among the products of the oxidation of albuminous substances, but has not yet been much studied. It is a volatile oily liquid, having all the characters of an aldehyde, and when oxidised yielding propylic acid.

Hydrated Propylic Acid, $\mathrm{C}_{6} \mathrm{H}_{5} \mathrm{O}_{3}, \mathrm{H} \mathrm{O}=\mathrm{C}_{6} \mathrm{H}_{6} \mathrm{O}_{4}=\mathrm{Pro}_{3} \mathrm{O}_{3}$, $\mathrm{H} \mathrm{O}$. This acid, the next above acetic acid in the series of volatile acids $\left(\mathrm{C}_{n} \mathrm{H}_{n}\right)_{2} \mathrm{O}_{4}$, is remarkable as being the first of these acids which exhibits oily properties. It is best obtained by heating cyanide of ethyle with an alcoholic solution of potash. The reaction is very simple. $\mathrm{C}_{4} \mathrm{H}_{5}, \mathrm{C}_{2} \mathrm{~N}+\mathrm{KO}+3 \mathrm{H} \mathrm{O}=$ $\mathrm{C}_{6} \mathrm{H}_{5} \mathrm{O}_{3}, \mathrm{~K} \mathrm{O}+\mathrm{N} \mathrm{H}_{3}$. When the propylate of potash thus obtained is distilled with sulphuric acid, propylic acid passes over. It very much resembles acetic acid, but is less volatile, and much less miscible with water, on which it floats in oily drops when the water is saturated with the acid. It occurs in several other processes, as when metacetone or propione is oxidised, when sugar is heated with potash, and also among the products of the destructive distillation of wood. 
It is also formed in some fermentations, as in the fermentation of tartrate of lime, that of glycerine, and it is among the products of the action of nitric acid on oils. It is also formed when peas or lentils are allowed to putrefy under water. Lastly, it is produced when angelic acid is heated with caustio potash.

Propylic acid boils at $284^{\circ}$, and only dissolves to a certain extent in water. Any excess floats in the solution as oily drops. Its odour is acid and pungent, resembling those of acetic and butyric acids.

Its salts resemble the acetates, but have something of a fatty character. The propylate of oxide of ethyle is a fragrant ether. There exists a nitro-compound, nitropropylic acid, in which 1 eq. of the hydrogen of propylic acid is replaced by nitrous acid, $\mathrm{N}_{4}$. Its formula is $\left.\mathrm{C}_{6} \underset{\mathrm{N} \mathrm{O}_{4}}{\mathrm{H}_{4}}\right\} \mathrm{O}_{3}, \mathrm{H} \mathrm{O}$.

Propione, $\mathrm{C}_{5} \mathrm{H}_{5} \mathrm{O}$, or $\mathrm{C}_{10} \mathrm{H}_{10} \mathrm{O}_{2}$. This compound, homologous with acetone, is formed when propylate of baryta is heated, and, along with acetone, when sugar is heated with lime. When oxidised it yields propylic acid, which leads us to prefer the formula $\mathrm{C}_{10} \mathrm{H}_{10} \mathrm{O}_{2}$. It is a fragrant oily volatile liquid, and has much analogy with acetone. It was formerly called metacetone, and hence propylic acid was called metacetic acid.

Propionitryle, or Cyanide of Ethyle, $\mathrm{C}_{6} \mathrm{H}_{5} \mathrm{~N}=\mathrm{C}_{4} \mathrm{H}_{5}, \mathrm{C}_{2} \mathrm{~N}$, is formed when propylate of ammonia is heated with anhydrous phosphoric acid. It has been already described as cyanide of ethyle.

Hyduret of Propionyle, $\mathrm{C}_{6} \mathrm{H}_{5}, \mathrm{H}=\mathrm{C}_{6} \mathrm{H}_{6}$. This is a carbohydrogen homologous with hyduret of ethyle or olefiant gas. It is a gas much like olefiant gas, and absorbed, like it, by chlorine. It is formed when amylic alcohol is passed through a red-hot tube.

With chlorine, bromine, \&c., it yields products analogous to those of olefiant gas.

Propylamide, $\mathrm{C}_{6} \mathrm{H}_{5} \mathrm{O}_{2}, \mathrm{~N} \mathrm{H}_{2}$, is analogous to acetamide. It is formed when ammonia acts on propylate of oxide of ethyle. When heated with anhydrous phosphoric acid, it yields propionitryle, which is cyanide of ethyle.

Gerhardt has proposed to name the electropositive radicals of the methylic series, standing above ethyle in the list, in a way which indicates their place in the series. Thus, he names propyle, trityle, as being the third member of the series; butyle, the fourth, will be tetryle; amyle, the fifth, will be pentyle, and so on. It is not unlikely that this nomenclature may be adopted for these radicals, while those of the formylic series shall retain names derived from the acids in which they are supposed to exist. 
It will be observed, that all the compounds of propyle, $\mathrm{C}_{6} \mathrm{H}_{7}$, and of its derived radical, propionyle, $\mathrm{C}_{6} \mathrm{H}_{5}$, which, so far as known, have been briefly described in the preceding pages, correspond perfectly to those of methyle and ethyle, and of their derivatives, formyle and acetyle. And it cannot be doubted, that we shall in time discover for every compound of methyle or of formyle, a corresponding one of propyle and propionyle, as we have of ethyle and acetyle. The properties of these compounds may even now be confidently predicted to a great extent. Thus, we shall have a zincopropyle, a stibiopropyle, \&c., with properties closely resembling those of the ethyle compounds.

Before quitting this subject, it may here be mentioned, that oil of garlic, $\mathrm{C}_{6} \mathrm{H}_{5} \mathrm{~S}$, and oil of mustard, $\mathrm{C}_{6} \mathrm{H}_{5} \mathrm{C}_{2} \mathrm{~N} \mathrm{~S}$, contain a radical allyle, $\mathrm{C}_{6} \mathrm{H}_{5}$, isomeric with propionyle, but not identical with it. At least the oxide of allyle, which is known, does not possess the properties which we should expect to find in oxide of propionyle. It is probable that allyle and propionyle may prove to be isomeric only.

\section{Radicals with $\mathrm{C}_{8}$.}

Butyle, $\mathrm{C}_{8} \mathrm{H}_{9}=\mathrm{Bu}$. Srs. Valyle. -This radical, discovered by Kolbe, is obtained among the products of the decomposition, by the galvanic current, of valerianic or valeric acid, $\mathrm{C}_{10} \mathrm{H}_{10} \mathrm{O}_{4}$ : thus; $\mathrm{C}_{10} \mathrm{H}_{10} \mathrm{O}_{4}=\mathrm{C}_{8} \mathrm{H}_{9}+2 \mathrm{C} \mathrm{O}_{2}+\mathrm{H}$. It is a colourless transparent liquid, of an agreeable ethereal smell, and a taste at first slight, afterwards burning. It is insoluble in water, soluble in alcohol and ether. It boils at $226^{\circ} \mathrm{F}$., is inflammable, and burns with a luminous but smoky flame. Its density, in the liquid state, at $64^{\circ}$, is 0.694 ; that of its vapour is 4.053 .

When oxidised by nitric acid, it yields butyric acid and nitropropylic acid. In the formation of butyle by the electrolysis of valerate of potash, it appears at the positive pole, and as the oxygen of decomposed water meets it there, it is oxidised in a peculiar way, yielding oxide of butyle, which combines with a portion of the valerianic acid, forming valerianate of oxide of butyle, $\mathrm{C}_{8} \mathrm{H}_{9} \mathrm{O}$, $\mathrm{C}_{10} \mathrm{H}_{9} \mathrm{O}_{3}=\mathrm{C}_{18} \mathrm{H}_{18} \mathrm{O}_{4}$. If this compound could be obtained in sufficient quantity, it would enable us to ascertain the properties of the hydrated oxide of butyle, or butylic alcohol, and probably also of the oxide of butyle or butylic ether. Kolbe was only able to obtain evidence of the existence of the butylic alcohol, formed when the above compound is heated with alcoholic solution of potash, $\mathrm{C}_{8} \mathrm{H}_{9} \mathrm{O}, \mathrm{C}_{10} \mathrm{H}_{9} \mathrm{O}_{3}+\mathrm{K} \mathrm{O}, \mathrm{H} \mathrm{O}=\mathrm{K} \mathrm{O}, \mathrm{C}_{10} \mathrm{H}_{9}$ $\mathrm{O} s+\mathrm{C}_{8} \mathrm{H}_{9} \mathrm{O}, \mathrm{HO}$. He has further obtained the carbohydrogen, $\mathrm{C}_{8} \mathrm{H}_{8}$, homologous with olefiant gas and with propylene. It is called butylene, and in properties, and in its relation to chlorine, 
\&c., resembles its homologues. It occurs in the oil of oil gas, probably also in that of coal gas.

But Wurtz has found, among the products of the fermentations of potato starch, the butylic alcohol.

Hydrated oxide of butyle (tetryle), $\mathrm{C}_{8} \mathrm{H}_{\theta} \mathrm{O}, \mathrm{H} \mathrm{O}=\mathrm{Bu} \mathrm{O}, \mathrm{HO}$. Syn. Butylic alcohol. Found in the oil of potato spirit. These oils contain much amylic alcohol. But if the more volatile portions be collected separately, and purified by rectification, then are obtained alcohol, traces of propylic alcohol, and a certain quantity of butylic alcohol. When pure, this compound boils at $233^{\circ}$, and is a liquid, lighter than water, in which it dissolves only sparingly, the excess floating in oily drops... Its odour is analogous both to that of alcohol and of amylic alcohol. When fused with potash, it is converted into butyrate of potash, hydrogen being given off. With oil of vitriol it forms an acid sulphate of oxide of butyle, or sulpho-butylic acid, $\mathrm{Bu} \mathrm{O}, \mathrm{H} \mathrm{O}, 28 \mathrm{Os}$.

The oxide of butyle, or butylic ether, is not yet well known.

Hydrosulphuret of sulphuret of butyle, or butylic mercaptan, $\mathrm{Bu} \mathrm{S}, \mathrm{H} \mathrm{S}=\mathrm{C}_{8} \mathrm{H}_{9} \mathrm{~S}, \mathrm{H} \mathrm{S}$, seems to be formed when sulphur is heated with olive or almond oil. At least a mixture is then obtained, which contains a liquid, having a most offensive garlic odour, and, like mercaptan, forming compounds which crystallise with salts of mercury and some other metals. The butylic mercaptan, however, has not been obtained in a state of perfect purity.

When the sulpho-butylate of potash is distilled with cyanate of potash, there is obtained a mixture of cyanate and cyanurate of oxide of butyle. And when this mixture is heated with potash, butylamine is formed.

Chloride of butyle, $\mathrm{C}_{8} \mathrm{H}_{9} \mathrm{Cl},=\mathrm{Bu} \mathrm{Cl}$, is formed when perchloride of phosphorus acts on butylic alcohol. It is as yet little known.

Cyanide of Butyle, $\mathrm{C}_{10} \mathrm{H}_{9} \mathrm{~N}=\mathrm{C}_{8} \mathrm{H}_{9}, \mathrm{C}_{2} \mathrm{~N}$, is identical with valeronitryle, which see.

Butylamine, $\mathrm{C}_{8} \mathrm{H}_{11} \mathrm{~N}=\mathrm{N} \mathrm{H}_{2}, \mathrm{C}_{8} \mathrm{H}_{9} .-$ This base has been discovered by Anderson in the basic part of Dippel's animal oil, along with ethylamine, methylamine, propylamine, and other bases of the same series, besides some bases of other series. This is an amide base, and it is isomeric with two imide bases, namely, diethylamine and methylopropylamine, and also with a nitryle base, namely, ethylodimethylamine. The following table will show this :-

Butylamine

Diethylamine

Methylopropylamine

Ethylodimethylamine

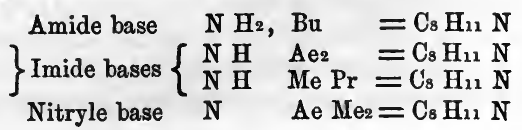


All these bases are distinct, and the same law of isomeric variety prevails throughout this series of bases, the variety increasing as we rise in the scale, two new isomeric forms being added at each step.

Butylamine is less volatile than propylamine, oily, and powerfully basic. It is still, however, so volatile, that Anderson first named it petinine, from its volatility. It will probably be found to occur in various decompositions, and, like ethylamine, to yield substitution-products with chlorine, \&o.

We are not yet acquainted with any radicals in which butyle, $\mathrm{C}_{8} \mathrm{H}_{9}$, is united with metals; but we cannot doubt that such compounds will be discovered, homologous with kakodyle, stibethyle, \&c. Indeed a compound, containing arsenic, has been derived from butyric acid, but its nature is not yet exactly known.

Butyryle, $\mathrm{C}_{8} \mathrm{H}_{7}$. This radical is not yet known in the separate form. It is, however, homologous with acetyle, formyle, and propionyle. Its hydrated oxide, or butyraldehyde, called also butyral, homologous with aldehyde, is known.

Hyduret of butyryle, $\mathrm{C}_{8} \mathrm{H}_{7}, \mathrm{H}=\mathrm{C}_{8} \mathrm{H}_{8}$. Syn. Butylene, Tetrylene. This compound, homologous with olefiant gas, and with hyduret of propionyle, is found among the products of the decomposition of oils by a red heat, or of the action of a mixture of lime and potash, aided by heat, on oily aeids of high atomic weight. It is a gas at the ordinary temperature, but condensible by cold. It boils below $32^{\circ}$. The density of the gas is 1.926. It yields, with chlorine and bromine, compounds analogous to those yielded by olefiant gas. There can be no doubt that this substance, butylene, is a constant ingredient, both of coal and oil gases, and also of the more volatile parts of the oil of wood, coal, or oil tars.

, Hydrated Oxide of Butyryle, $\mathrm{C}_{8} \mathrm{H}_{7} \mathrm{O}, \mathrm{H} \mathrm{O}=\mathrm{C}_{8} \mathrm{H}_{8} \mathrm{O}_{2}$. This compound was found by Guckelberger, among the products of the oxidation of fibrine, albumens, \&c., by sulphurio acid and peroxide of manganese, or bichromate of potash. Chancel has also obtained a compound of the same composition by distilling the butyrate of lime, and which he calls butyral; but it appears that the latter is isomeric only, not identical, with the former, which is the true aldehyde of butyric acid. It is perfectly analogous to aldehyde in its relation to potash, to oxide of silver, to ammonia, and to oxygen, yielding, when oxidised, butyric acid. It boils at $156^{\circ}$ to $160^{\circ}$, and its density at $60^{\circ}$ is 0.8 . Its compound with ammonia seems to yield, with sulphuretted hydrogen, a base, homologous with thialdine.

Butyric Acid, $\mathrm{Cs}_{8} \mathrm{H}_{7} \mathrm{O}_{3}, \mathrm{H} \mathrm{O}$. This acid, the hydrated 
teroxide of butyryle, is the fourth in the series of volatile oily acids $\left(\mathrm{Cn} \mathrm{H}_{n}\right)_{2} \mathrm{O}_{4}$. It exists in butter, in small quantity, combined with oxide of glyceryle (oxide of lipyle), to which compound the flavour of butter is chiefly to be ascribed, the great mass of butter being tasteless and inodorous.

Anhydrous Butyric Acid, $\mathrm{C}_{8} \mathrm{H}_{7} \mathrm{O}_{3}$, is obtained by the action of oxychloride of butyryle on butyrate of potash, $\mathrm{C}_{8} \mathrm{H}_{7} \mathrm{O}_{3}, \mathrm{KO}$ $\left.\left.+\mathrm{C}_{8} \mathrm{H}_{7} \stackrel{\mathrm{O}_{2}}{\mathrm{Cl}}\right\}\right\}=\mathrm{KCl}+2 \mathrm{C}_{8} \mathrm{H}_{7} \mathrm{O}_{3}$.

It is a mobile, highly refractive liquid, lighter than water, boiling at $372^{\circ} \mathrm{F}$., of a pleasant ethereal odour. It is converted, by contact with water, into the hydrated acid. With ammonia it forms butyramide, and with aniline butyranilide.

To prepare the hydrated acid, sugar is dissolved in 4 parts of water, and some pressed curd, which has been exposed to the air for a time, is added along with a quantity of prepared chalk. The whole mass is kept at a temperature of from $76^{\circ}$ to $85^{\circ} \mathrm{F}$. After some days, the whole becomes solid by the formation of lactate of lime; but if the operation be continued, and water added to supply the place of that lost by evaporation, it again becomes liquid, the lactate disappears, and butyrate of lime is formed. With 4 lbs. of sugar I have seen the change complete in a fortnight. At other times it has lasted six weeks. This depends on changes of temperature; for, with a steady heat of $85^{\circ}$ or even $90^{\circ}$, it proceeds rapidly.

The mass is warmed, passed through a cloth, and the residue well washed; the liquors, united, are evaporated, and the butyrate crystallises on cooling from the concentrated solution.

The salt is then dissolved in water, and to this solution, or to the original solution when of a proper volume, carbonate of soda is added, which converts the butyrate of lime into butyrate of soda, and carbonate of lime. To this solution of butyrate of soda, sulphuric acid, previously diluted with its own weight of water, is added in excess, and the greater part of the butyric acid rises to the surface as an oil, while some remains dissolved in the liquid. The oil is drawn off, and the liquid distilled as long as it yields any butyric acid. This is added to the rest, and the whole purified by rectification.

Butyric acid may also be made from starch, jelly, or boiled potatoes crushed and suspended in water, using flesh as the ferment, with chalk as before to combine with the acid as it is formed. This mixture succeeds better on a small seale than the other, being less dependent on a uniform temperature.

Hydrated butyric acid is a colourless liquid, very mobile, of an odour like that of acetic acid and that of rancid butter mixed, 
and a burning acid taste. It boils about $250^{\circ} \mathrm{F}$, or a little lower. It may be crystallised by a very intense cold. The density of the liquid is 0.98 , but mixed with an equal volume of water it becomes heavier, the density being then 1.00287. The density of its gas is $3 \cdot 7$. It is only soluble to a certain extent in water, and any excess appears as an oil.

The production of the acid is thus explained :

$$
\underbrace{\mathrm{C}_{12} \mathrm{H}_{12} \mathrm{O}_{12}}_{\text {Sugar. }}+\mathrm{Ca} \mathrm{O}, \mathrm{C} \mathrm{O}_{2}=(\underbrace{\mathrm{Ca} \mathrm{O}, \mathrm{C}_{8} \mathrm{H}_{7} \mathrm{O}_{3}}_{\text {Butyrate of lime. }})+\mathrm{H} \mathrm{O}+5 \mathrm{C} \mathrm{O}_{2}+\mathrm{H}_{4} \text {, }
$$

and it is therefore accompanied by carbonic acid and hydrogen gases. When distilled with sulphuric acid, this salt yields the hydrated butyric acid, $\mathrm{C}_{8} \mathrm{H}_{7} \mathrm{O}_{3}, \mathrm{H} \mathrm{O}=\mathrm{C}_{8} \mathrm{H}_{8} \mathrm{O}_{4}$, which is polymeric with aldehyde, $\mathrm{C}_{4} \mathrm{H}_{4} \mathrm{O}_{2}$, and isomeric with acetio ether, $\mathrm{C}_{4} \mathrm{H}_{5} \mathrm{O}, \mathrm{C}_{4} \mathrm{H}_{3} \mathrm{O}_{3}$.

The salts of butyric acid are like those of acetic and propylic acids, but have more of the character of soaps, as the acid is more oily. They do not readily moisten with water. The butyrate of lime crystallises readily, as does that of baryta.

Butyrate of Oxide of Ethyle, or Butyric Ether, $\mathrm{C}_{4} \mathrm{H}_{5} \mathrm{O}$, $\mathrm{C}_{8} \mathrm{H}_{7} \mathrm{O}_{3}=\mathrm{C}_{12} \mathrm{H}_{12} \mathrm{O}_{4}$, is a very fragrant ether, and appears to constitute the chief part of the flavouring matter of the pineapple, melon, strawberry, and some other fruits. The presence of this ether, in small proportions, gives to spirits the flavour of old rum, and hence no doubt the reason why the best old rum is called pine-apple rum. Butyric ether dissolved in 6 parts of alcohol is now used as essence of pine-apple. When rum is made by the fermentation of molasses, the freshly-distilled spirit has much less flavour than after long keeping. In fact it contains but little of the ether, and a small quantity of free butyric acid, which, on keeping, gradually combines with oxide of ethyle and increases the flavour. Some very highly-flavoured wines owe their flavour to the presence of traces of acetic and butyric ethers. There is a nitrobutyrio acid, $\mathrm{C}_{8} \mathrm{H}_{6} \mathrm{~N} \mathrm{O}, \mathrm{HO}$.

Butyronitryle, $\mathrm{C}_{8} \mathrm{H}_{7} \mathrm{~N}=\mathrm{C}_{6} \mathrm{H}_{7}, \mathrm{C}_{2} \mathrm{~N}$. SrN. Cyanide of Propyle. This is an oily liquid of Sp. G. 0.795, boiling at $245^{\circ}$.

If we could obtain from this compound the hydrated oxide of propyle, or propylic alcohol, that substance might be had in any desired quantity, and would yield, no doubt, a whole series of compounds, homologous with those derived from alcohol. But this we cannot yet do, for when cyanide of propyle is boiled with potash, butyrate of potash is obtained, a circumstance rather favouring the idea that it is not really a cyanide, but a compound of nitrogen with butyryle, a true nitryle. On the other hand, with potassium it yields cyanide of potassium. Further researches 
must determine whether it be a true cyanide or not. Its formation is as follows: $\left(\mathrm{N} \mathrm{H}_{4} \mathrm{O}, \mathrm{C}_{8} \mathrm{H}_{7} \mathrm{O}_{3}\right)+\times \mathrm{PO}_{5}=\mathrm{xP} \mathrm{O}_{5}, 4 \mathrm{H} \mathrm{O}$ $+\mathrm{C}_{8} \mathrm{H}_{7} \mathrm{~N}$. Butyronitryle is also found among the products of the oxidation of albuminous compounds.

Butyramide, $\mathrm{C}_{8} \mathrm{H}_{7} \mathrm{O}_{2}, \mathrm{~N} \mathrm{H}_{2}$, is formed when butyrate of oxide of ethyle (butyric ether) is heated with ammonia in closed tubes. ( $\left.\mathrm{C}_{4} \mathrm{H}_{5} \mathrm{O}, \mathrm{C}_{8} \mathrm{H}_{7} \mathrm{O}_{3}\right)+\mathrm{N} \mathrm{H}_{3}=\mathrm{C}_{4} \mathrm{H}_{5} \mathrm{O}, \mathrm{H} \mathrm{O}+$ $\mathrm{C}_{8} \mathrm{H}_{7} \mathrm{O}_{2}, \mathrm{~N} \mathrm{H}_{2}$. Heated with dry phosphoric acid, butyramide yields butyronitryle, only 2 eqs. of water being separated. $\mathrm{C}_{8} \mathrm{H}_{7} \mathrm{O}_{2}, \mathrm{~N} \mathrm{H}_{2}+\mathrm{P} \mathrm{O}_{5}=\times \mathrm{P} \mathrm{O}_{5}, 2 \mathrm{HO}+\mathrm{C}_{8} \mathrm{H}_{7} \mathrm{~N}$.

Butyrone, $\mathrm{C}_{7} \mathrm{H}_{7} \mathrm{O}$ or $\mathrm{C}_{14} \mathrm{H}_{14} \mathrm{O}_{2}$, is homologous with acetone and propione, and resembles them closely, but is, of course, less volatile than propione. It is formed when butyrate of baryta is exposed to heat. $\mathrm{C}_{8} \mathrm{H}_{7} \mathrm{O}_{3}, \mathrm{BaO}=\mathrm{BaO}, \mathrm{C} \mathrm{O}_{2}+\mathrm{C}_{7} \mathrm{H}_{7} \mathrm{O}$. The formula $\mathrm{C}_{14} \mathrm{H}_{14} \mathrm{O}_{2}$, however, seems to be the true one. It is isomeric with the aldehyde of œnanthic acid, or cenanthal, the hydrated oxide of œnanthyle, $\mathrm{C}_{14} \mathrm{H}_{13} \mathrm{O}, \mathrm{H} \mathrm{O}$. But these two compounds are not identical.

We have now seen that all the known compounds of butyle, $\mathrm{C}_{8} \mathrm{H}_{9}$, and of butyryle, $\mathrm{C}_{8} \mathrm{H}_{7}$, correspond perfectly to those of methyle and formyle, of ethyle and acetyle, of propyle and propionyle. In the ease of the radicals with 6 eqs. and of those with 8 eqs. of carbon, the names given to the two series, one positive, the other negative, are nearly similar. Thus we have

Original, or electro-positive radicals.

Methyle $\mathbf{C}_{2} \mathrm{H}_{3}$

Ethyle $\mathbf{C}_{4} \mathbf{H}_{5}$

Propyle $\mathrm{C}_{6} \mathrm{H}_{7}$

Butyle $\mathrm{C}_{8} \mathrm{H}_{9}$, \&c.
Derived, or electro-negative radicals.

Formyle $\mathrm{C}_{2} \mathrm{H}$

Acetyle $\mathrm{C}_{4} \mathrm{H}_{3}$

Propionyle $\mathrm{C}_{6} \mathrm{H}_{5}$

Butyryle $\mathrm{C}_{8} \mathrm{H}_{7}$, \&c.

The reason of this is, that in the two latter cases we were not till lately acquainted with the alcohols and ethers, but only with the derived acids. Hence the name of the radicals in the first or positive series was, in these cases, taken from the acids, and the same is continued as we ascend in the scale. We shall give, however, as in the above instances, the shortest and simplest names to the original or positive radicals, while the derived ones will have modified names, if possible, connected with the acid to which they belong, as propionyle from propionic (now called propylic) acid. In the next case, however, as the alcohol has long becn known, we have, as in the cases of methyle and ethyle, quite different names for the original and the derived radicals.

Radicals with Cio.

Amyle. Valeryle.

Amyle, $\mathrm{C}_{10} \mathrm{H}_{11}=$ Ayl. This radical was discovered by 
Frankland, as a product of the action of zinc on iodide of amyle, and subsequently obtained by Brazier and Gossleth among the products of decomposition of caproio acid, $\mathrm{C}_{22} \mathrm{H}_{12} \mathrm{O}_{4}$, by the galvanic current. $\mathrm{C}_{12} \mathrm{H}_{12} \mathrm{O}_{4}=\mathrm{C}_{2} \mathrm{O}_{4}+\mathrm{H}+\mathrm{C}_{10} \mathrm{H}_{11}$. It is an oily liquid, boiling at $311^{\circ}$, and is homologous with methyle, ethyle, \&c., being the fifth in that series.

Oxide of Amyle, $\mathrm{C}_{10} \mathrm{H}_{11} \mathrm{O}=\mathrm{Ayl}$. 0. Srn. Amylic Ether, is obtained when chloride of amyle is acted on by an alcoholic solution of potash. It boils at $233^{\circ}$. By the action of sulphuric acid on amylic alcohol there is obtained a compound isomeric with it, but less volatile. Oxide of amyle is homologous with oxides of methyle, ethyle, \&c., and forms, like them, compounds with almost all acids.

Hydrated Oxide of Amyle. Srv. Amylic Alcohol. Fuseloil. $\mathbf{C}_{10}$ $\mathrm{H}_{12} \mathrm{O}, \mathrm{H} \mathrm{O}=\mathrm{C}_{10} \mathrm{H}_{12} \mathrm{O}_{2}$. This compound, which is a true alcohol, perfectly homologous with methylic and ethylic alcohols, is the fifth member of the series of alcohols. It is formed, along with ethylic or common alcohol, in the fermentation of potato or grain starch under certain circumstances, which are not fully understood. It is also formed in the fermentation of grape juice, along with common alcohol and propylic alcohol, as well as other alcohols, higher in the series. Its formation evidently depends on a peculiar fermentation of sugar. Thus, we may suppose 5 eqs. of sugar to yield 4 eqs. of amylic alcohol, 12 of water, and 20 of carbonic acid. $5\left(\mathrm{Cl2}_{12} \mathrm{H}_{12} \mathrm{O}_{12}\right)=4\left(\mathrm{C}_{10} \mathrm{H}_{12} \mathrm{O}_{2}\right)$ $+12 \mathrm{H} \mathrm{O}+2 \mathrm{C} \mathrm{O}_{2}$. But the amylic fermentation may be one in which other compounds, not yet ascertained, are formed. The oil of potato or grain-spirit, which distils over in the latter part of the process, contains alcohol, amylic alcohol, œnanthio acid, capric acid, and margaric acid. Whether these acids are formed in the amylic fermentation is not yet known.

Amylic alcohol is a colourless, somewhat oily, liquid, very little soluble in water, and boiling at $270^{\circ}$. It has a powerful but unpleasant odour, and when inhaled produces spasmodic constriction in the air-passages or bronchi, and nausea. It has an acrid nauseous taste. At $-4^{\circ}$ it crystallises. When heated with potash, it is oxidised, hydrogen being given off, and valerate of potash is formed. Heated with anhydrous phosphoric acid, it yields the carbohydrogen $\mathrm{C}_{10} \mathrm{H}_{10}$, homologous with olefiant gas. This is called amylene or valerene. (See Valerene.)

The salts of oxide of amyle are ethereal compounds, very similar to those of oxide of ethyle, but, of course, much less volatile. The nentral sulphate is unknown, but the acid sulphate or sulphamylic acid is quite analogous to sulphovinic acid.

Sulphamylic Acid, $\mathrm{SO}_{3}$, Ayl $\mathrm{O}+\mathrm{SO}_{3} \mathrm{HO}$. This compound 
is formed exactly like sulphovinic acid (which see). Its salts are soluble, but have somewhat of a fatty feel. Their formula is $\mathrm{S} \mathrm{Os,} \mathrm{Ayl} \mathrm{O+S} \mathrm{Os,} \mathrm{M} \mathrm{O.} \mathrm{There} \mathrm{is} \mathrm{another} \mathrm{acid,} \mathrm{the} \mathrm{hyposul-}$ phamylic acid, the formula of which is $\mathrm{C}_{10} \mathrm{H}_{11}, \mathrm{~S}_{2} \mathrm{O}_{5}, \mathrm{H} \mathrm{O}$, or Ayl $\mathrm{S}_{2} \mathrm{O}_{5}, \mathrm{HO}$.

The hyponitrite, the nitrate, the carbonate, the benzoate, the oxalate, the acetate, the cyanate, the cyanurate, and many other salts of oxide of amyle, have been studied, and are quite analogous to those of oxide of ethyle, \&c. Some of the compounds of oxide of amyle with the acids of the acetic series are very fragrant ethers, and give the flavour to some fruits. This is the case in jargonel pears, the essence of which is a compound of this kind, or a mixture of two or three such compounds, but chiefly the acetate of oxide of amyle, which is now made and used as essence of pears, and has the perfect flavour of that fruit.

Carbamate of Oxide of Amyle, $\mathrm{C}_{12} \mathrm{~N} \mathrm{H}_{13} \mathrm{O}_{4}$, or amylo-urethane, is prepared like the corresponding ethyle compound, to which it is quite analogous.

1. Amylamine, $\mathrm{C}_{10} \mathrm{H}_{13} \mathrm{~N}=\mathrm{N} \mathrm{H} \mathrm{H}_{2}$ Ayl, homologous with methylamine, \&c., is obtained by heating the cyanate or cyanurate of amyle, or amylo-urea, with potash. To take the first process, that from the cyanate, we have $\mathrm{C}_{10} \mathrm{H}_{11} \mathrm{O}, \mathrm{C}_{2} \mathrm{~N} \mathrm{O}+2$ (K $\mathrm{K}$, $\mathrm{H} \mathrm{O})=2\left(\mathrm{~K} \mathrm{O}_{1} \mathrm{C} \mathrm{O}_{2}\right)+\mathrm{C}_{10} \mathrm{H}_{13} \mathrm{~N}$. It is a liquid, boiling at $203^{\circ}$, with a density, at $64^{\circ}$, of 0.7503 . It is a powerful base, with a strong ammoniacal smell and burning taste, miscible with water in every proportion. It precipitates almost all metallic solutions. Its salts much resemble those of ammonia. It produces, with oxalic ether, the compound amyloxamide, $\mathrm{C}_{22} \mathrm{H}_{12}$ $\mathrm{N} \mathrm{O}_{2}$; homologous with oxamide, methyloxamide, \&c. The double salt of amylamine with chloride of platinum is much more soluble than the ammonia salt.

Amylo-urea, $\mathrm{C}_{12} \mathrm{~N} \mathrm{H}_{14} \mathrm{O}_{2}$. Cyanate of potash with sulphate of amylamine, yields amylo-rerea, $\mathrm{K} \mathrm{O}, \mathrm{C}_{2} \mathrm{NO}+\mathrm{C}_{10} \mathrm{H}_{13} \mathrm{~N}, \mathrm{H} \mathrm{O}$, $\mathrm{S} \mathrm{O}=\mathrm{K} \mathrm{O}, \mathrm{SO}_{3}+\mathrm{C}_{12} \mathrm{NH}_{14} \mathrm{O}_{2}$.

Amylamine is found among the basic products of the distillation of auimal matter.

The formula $\mathrm{C}_{10} \mathrm{H}_{13} \mathrm{~N}$, represents not only amylamine, but several other bases isomeric with it. Such are methylobutylamine, $\mathrm{N} \mathrm{H}, \mathrm{Me} \mathrm{Bu}$; ethylopropylamine, $\mathrm{N} \mathrm{H}, \mathrm{Ae} \mathrm{Pr}$; methylodiethylamine, $\mathrm{N}, \mathrm{Me} \mathrm{Ae}_{2}$; dimethylopropylamine, $\mathrm{N}, \mathrm{Mez} \mathrm{Pr}$, all of which differ in properties, but all are volatile bases, and all have the empirical formula $\mathrm{C}_{10} \mathrm{H}_{13} \mathrm{~N}$.

2. Diamylamine, $\mathrm{C}_{20} \mathrm{H}_{23} \mathrm{~N}=\mathrm{NH}, \mathrm{Ayl}_{2}$, is formed by the action of iodide of amyle on ammonia, but still better by the action of bromide of amyle on amylamine. It is a light oil of a peculiar 
aromatic smell and burning taste. It is strongly basic, and boils about $340^{\circ}$. It forms salts with acids, analogous to those of ammonia, but less soluble, except the double salt of hydrochlorate of amylamine and bichloride of platinum, which is more soluble than the ammonia compound, This base is isumeric with a large number, such as ethylopropylamylamine, N Ae $\mathrm{Pr}$ Ayl, methylobutylamylamine, N, Me Bu Ayl, \&c. \&c.; since the hydrogen of ammonia, if replaced by any one, two, or three of the radicals hitherto described, methyle, ethyle, propyle, butyle, and amyle, will yield a base of the formula $\mathrm{C}_{20} \mathrm{H}_{13} \mathrm{~N}$, provided the carbon of the radical or radicals employed does not exceed $\mathrm{C}_{20}$ when added together. We may have (omitting the hydrogen) $\mathrm{C}_{2}, \mathrm{C}_{8}, \mathrm{C}_{10}=\mathrm{C}_{20}$; or, $\mathrm{C}_{4}, \mathrm{C}_{6}, \mathrm{C}_{10}=\mathrm{C}_{20}$; or, $\mathrm{C}_{4}, \mathrm{C}_{8}, \mathrm{C}_{8}=\mathrm{C}_{20}$; or, $\mathrm{C}_{6}, \mathrm{C}_{6}, \mathrm{C}_{8}=\mathrm{C}_{20}$, and the same holds true in every case. Besides, similar isomeric bases may be obtained by using, in addition to the radicals with $\mathrm{C}=2,4,6,8$, or 10 , those with $\mathrm{C}=12,14,16,18$, and 20.

3. Triamylamine, $\mathrm{C}_{30} \mathrm{H}_{33} \mathrm{~N}=\mathrm{N}, \mathrm{Ayl}_{3}$, is obtained by the action of bromide of amyle on diamylamine, or by heating the hydrated oxide of tetramylium, to be presently described. It resembles diamylamine, but boils at about $498^{\circ}$. It is, of course, isomerie with a still greater number of possible bases than the preceding, inasmuch as the permutations of $\mathrm{C}_{2}, \mathrm{C}_{4}, \mathrm{C}_{6}, \mathrm{C}_{8}, \mathrm{C}_{10}$, \&c., up to 30 , are more numerous than with $\mathrm{C}_{20}$ only. Several such isomeric bases have already been produced.

4. Tetramylium, N, Ayl , and its oxide, N, $\mathrm{Ayl}_{4} \mathrm{O}_{3}$ are homologous with the corresponding methyle and ethyle compounds, and are prepared in the same way, by the action of iodide of amyle on triamylamine, or on ammonia. The iodide of tetramylium is crystallisable, very bitter, and its formula is $\mathbf{C}_{\mathbf{4 0}}$ $\mathrm{H}_{44} \mathrm{~N}, \mathrm{I}=\mathrm{N}$ Ayl4, $\mathrm{I}$. With oxide of silver, it yields iodide of silver, and the oxide of tetramylium, the hydrate of which is very bitter, but less soluble than the corresponding ethyle compound. The hydrate appears as an oil which crystallises, and its solution deposits on standing crystals of an inch or more in length and thickness, which are compound of the hydrate with water of crystallisation. The hydrate, when heated, is resolved into triamylamine, water, and amylene or valerene, thus: $\mathrm{N} \mathrm{Ayl}_{\mathrm{i}} \mathrm{O}$, $\mathrm{HO}=\mathrm{N} \mathrm{Ayl} 3+2 \mathrm{H} \mathrm{O}+\mathrm{C}_{10} \mathrm{H}_{10}$.

Triethylamylium, $\mathrm{C}_{22} \mathrm{H}_{26} \mathrm{~N}=\mathrm{N}$, Ae Ayls. This compound, in which 3 eqs. of the hydrogen of ammonium are replaced by ethyle and one by amyle, forms, like the last, a crystalline bitter iodide, and a bitter basic bydrated oxide, which is a powerful ammonium base. When heated, it yields a new base, diethylamylamine, water, and ethylene, or $\mathrm{C}_{\mathbf{4}} \mathrm{H}_{4}$. 
Diethylamylamine, $\mathrm{C}_{18} \mathrm{H}_{21} \mathrm{~N}=\mathrm{N}, \mathrm{Ae}_{2}$, Ayl, is formed as above described. It is not so powerful a base as some of its congeners, but is yet distinctly and strongly basic, of the nitryle series, corresponding to $\mathrm{N} \mathrm{H}_{3}$.

Methylodiethylamylium, $\mathrm{C}_{20} \mathrm{H}_{24} \mathrm{~N}=\mathrm{N}, \mathrm{Me} \mathrm{Ae}_{2}$ Ayl, is a new ammonium compound, obtained as iodide, when iodide of methyle acts on the preceding. Its oxide is a powerful base, like the others of this class. When heated it yields a new volatile methyle base (methylethylamylamine), water, and ethylene.

Methylethyiamylamine, $\mathrm{C}_{16} \mathrm{H}_{19} \mathrm{~N},=\mathrm{N}$, $\mathrm{Me} \mathrm{Ae} \mathrm{Ayl,} \mathrm{is} \mathrm{a} \mathrm{vola-}$ tile liquid base, boiling at $275^{\circ}$, and neutralising acids.

The chloride, bromide, iodide, cyanide, sulphuret, hydrosulphuretted sulphuret, and sulphocyanide of amyle, are prepared exactly in the same way as the corresponding eompounds of ethyle, and have analogous properties. The analogy is indeed so perfect that we need not describe these compounds in detail. The cyanide is identical with capronitryle. (See Caproic Acid.)

No compounds of amyle, corresponding to zincethyle, to kakodyle, to stibiomethyle, are yet fully known, although Frankland has shown that zincamyle does exist; but it is hardly to be doubted that such compounds may be formed, and that they will prove analogous to those already known.

Valeryle, $\mathrm{C}_{10} \mathrm{H}_{9}$; hydrated oxide of valeryle, $\mathrm{C}_{10} \mathrm{H}_{0} \mathrm{O}, \mathrm{H} \mathrm{O}$. This radical, the fifth in the series of formyle, $\mathrm{Cn}_{n}-1$, is not known in the separate form; but its hydrated protoxide, valeraldehyde or valeral, appears to be one of the products of the oxidation. of albuminous matter. It is a volatile liquid, having all the characteristic properties of an aldehyde, yielding valerianic acid when oxidised, and forming a crystalline compound with ammonia. Its formula is $\mathrm{C}_{10} \mathrm{H}_{9} \mathrm{O}, \mathrm{H} \mathrm{O}=\mathrm{C}_{10} \mathrm{H}_{10} \mathrm{O}_{2}$, and it is polymeric with capric acid, $\mathrm{C}_{20} \mathrm{H}_{20} \mathrm{O}_{4}$, and with valerate of oxide of amyle, $\mathrm{C}_{10} \mathrm{H}_{11} \mathrm{O}, \mathrm{C}_{10} \mathrm{H}_{9} \mathrm{O}_{3}$. Valerianic aldehyde, or as it is called for brevity, valeral, may be obtained more easily by acting on amylic alcohol with sulphuric acid and bichromate of potash.

It is a mobile liquid, boiling at $227^{\circ} \mathrm{F}$, of Sp. Gr. 0.820 ; the density of its gas is $\mathbf{2} \cdot 96$.

It forms, with nitric acid, a substitution-product analogous to nitropropylic acid. With ammonia it forms a crystalline compound analogous to aldehydammonia.

If valeral were to act on hydrocyanic acid, it would probably yield a substance homologous with glycocine and alanine. This has not been tried, but such a substance is known. It is leucine, $\mathrm{C}_{12} \mathrm{H}_{13} \mathrm{~N} \mathrm{O}_{4}$; to be afterwards described, as a product of the action of potash on animal matters.

Vulerianic Acid, $\mathrm{C}_{10} \mathrm{H}_{9} \mathrm{O}_{3}, \mathrm{H} \mathrm{O}=\mathrm{C}_{10} \mathrm{H}_{10} \mathrm{O}_{4}$. This acid, 
the fifth in the series, $\left(\mathrm{Cn}_{n} \mathrm{H}_{n}\right)_{2} \mathrm{O}_{4}$, is found in nature in the oil of valerian; also, in small proportion, combined with oxide of lipyle, in train oil, and the oil of the cetacea generally, which owe their smell to this latter compound. It is found also in the seeds of Viburnum opulus, and occurs as a frequent product along with the preceding compound, and many of their homologues, among the products of oxidation of animal matters and of fat oils. It is formed, besides, when amylic alcohisl is heated with caustic potash, and when valeral is oxidised. I have also seen it produced by a spontaneous change in salted butter, ill prepared, and in jam made with greengage plums, which had been kept for some time. It is an oily liquid of Sp. G. 0.944 , and boiling at $270^{\circ}$, with a strong odour of valerian. Its salts are soluble, and have a sweetish taste and fatty aspect. Valerate of oxide of ethyle, or valeric ether, is a fragrant compound, occurring in some vegetable products.

The anhydrous valerianic acid $\mathrm{C}_{10} \mathrm{H}_{9} \mathrm{O}_{3}$, is formed by the action of oxychloride of phosphorus on valerate of potash. It is a limpid oil, very mobile, of Sp. G. 0.934 ; with an agreeable smell of apples when pure, but by contact with water it is hydrated, and acquires an intense odour of valerian, which is that of the hydrated acid. It boils about $440^{\circ} \mathrm{F}$., and the density of its gas is 6.23. Its vapour attacks both the eyes and the respiratory organs.

The salts of valerianic acid resemble the butyrates, but have more of a soapy character. Some of them are used in medicine.

Valerate of oxide of ethyle, $\mathrm{C}_{4} \mathrm{H}_{5} \mathrm{O}, \mathrm{C}_{10} \mathrm{H}_{9} \mathrm{O}_{3}$, or valerianic ether, is fragrant, and occurs, it is said, in some fruits.

Valerate of oxide of amyle, $\mathrm{C}_{10} \mathrm{H}_{11} \mathrm{O}, \mathrm{C}_{10} \mathrm{H}_{9} \mathrm{O}_{3}=\mathrm{C}_{20} \mathrm{H}_{20} \mathrm{O}_{4}$, is homologous with acetic ether, isomeric with capric acid, and polymeric with valeral. It is a fragrant ether, and gives to the apple its peculiar grateful flavour. We have seen that the pear is flavoured by the acetate of oxide of amyle, $\mathrm{C}_{10} \mathrm{H}_{11} \mathrm{O}, \mathrm{C}_{4} \mathrm{H}_{3} \mathrm{O}_{3}$, and that butyric ether, $\mathrm{C}_{4} \mathrm{H}_{5} \mathrm{O}, \mathrm{C}_{8} \mathrm{H}_{7} \mathrm{O}_{3}$, contributes to the flavour of the pine-apple. All these compounds, and many others of similar properties, contain an oxide homologous with ether, or ether itself, and an acid homologous with acetic acid, or acetic acid itself.

Valerate of Ammonia, $\mathrm{N} \mathrm{H}_{4} \mathrm{O}, \mathrm{C}_{10} \mathrm{H}_{9} \mathrm{O}_{3}$, under certain circumstances loses 2 eqs. of water and yields valeramide. Thus, when valerate of oxide of ethyle is left in contact with strong ammonia, valeramide and alcohol are formed. $\mathrm{C}_{4} \mathrm{H}_{5} \mathrm{O}, \mathrm{C}_{10} \mathrm{H}_{9} \mathrm{O}_{3}+\mathrm{NH}_{4}$ $\mathrm{O}=\mathrm{C}_{4} \mathrm{H}_{5} \mathrm{O}, \mathrm{HO}+\mathrm{HO}+\mathrm{C}_{10} \mathrm{NH}_{11} \mathrm{O}_{2}$. This latter body, valeramide, forms large crystals.

Valeronitryle, $\mathrm{C}_{10} \mathrm{H}_{9} \mathrm{~N}$, is formed when valeramide is heated 
with anhydrous phosphoric acid. It is identical with cyanide of butyle, $\mathrm{C}_{8} \mathrm{H}_{9}, \mathrm{C}_{2} \mathrm{~N}$. Valeronitryle is found among the products of the oxidation of albuminous compounds. It is a volatile, aromatic liquid, which, like the other nitryles, yields, with potassium, cyanide of potassium, hydrogen, and a carbohydrogen ; with potash, ammonia, and the acid; in this case valerianic acid.

Hyduret of Valeryle, $\mathrm{C}_{10} \mathrm{H}_{10}=\mathrm{C}_{10} \mathrm{H}_{9}, \mathrm{H}$, or valerene, is obtained as one of the ploducts of the action of zinc on iodide of amyle, along with hyduret of amyle, $\mathrm{C}_{10} \mathrm{H}_{12}$, and amyle $\mathrm{C}_{10} \mathrm{H}_{11}$. 2 eqs. amyle, $\mathrm{C}_{20} \mathrm{H}_{22}$, yield 1 eq. valerene, $\mathrm{C}_{10} \mathrm{H}_{10}$, and 1 eq. hyduret of amyle, $\mathrm{C}_{10} \mathrm{H}_{12}$. The former of these is homologous with olefiant gas, and may be hyduret of valeryle, $\mathrm{C}_{10} \mathrm{Ho}, \mathrm{H}$. The latter is homologous with marsh gas, $\mathrm{C} \mathrm{H}_{\mathbf{x}}$, and its rational formula is $\mathrm{C}_{10} \mathrm{H}_{11}, \mathrm{H}$. Both are volatile, oily, combustible liquids.

Valerone, $\mathrm{C}_{9} \mathrm{H}_{9} \mathrm{O}$, or rather $\mathrm{C}_{18} \mathrm{H}_{18} \mathrm{O}_{2}$, homologous with acetone, is formed when valerianic acid is heated with excess of baryta. It is less volatile than butyrone, which it resembles. It is isomeric with the aldehyde of pelargonic acid, or pelargonal, $\mathrm{C}_{18} \mathrm{H}_{19} \mathrm{O}$, $\mathrm{H} \mathrm{O}$.

It will be seen that the analogy of homologous compounds is perfectly observed, so far as they are known, in the compounds of amyle and valeryle. Only the most important of these have been mentioned, and that very briefly; but, next to methyle and formyle, ethyle and acetyle, these radicals, amyle and valeryle, are, in this series, those by much the best known, and are particularly interesting, from the very perfect analogy they present to the others just named. The additions of $3\left(\mathrm{C}_{2} \mathrm{H}_{2}\right)$, and of $4\left(\mathrm{C}_{2} \mathrm{H}_{2}\right)$, to the compounds of ethyle and acetyle, of methyle and formyle, respectively, has, as will be observed, only produced a complete though of course slightly modified copy of those compounds; and with the doctrine of homologous series, we might have predicted nearly all the properties of the compounds with $\mathrm{C}_{10}$, from our knowledge of those with $\mathrm{C}_{2}$ and $\mathrm{C}_{4}$. The series with $\mathrm{C}_{10}$ is at present far more complete than those with any higher number. As we rise in the scale we shall find, as a general rule, fewer compounds known, but in every case, up to $\mathrm{C}_{40}$, and in some beyond that point, we are acquainted with the acid, homologous with acetic acid.

\section{Radicals with $\mathbf{C}_{12}$.}

Caprotyle. Caproyle.

As in the case of $\mathrm{C}_{2}, \mathrm{C}_{4}, \mathrm{C}_{10}, \mathrm{C}_{6}$ and $\mathrm{C}_{8}$, we have admitted as highly probable, two radicals in each case, one of the formula $\mathrm{Cn} \mathrm{Hn}_{n}+$, or the methylic series, and the other with the 
formula $\mathrm{C}_{\mathrm{n}} \mathrm{H}_{\mathrm{n}}-\mathbf{1}$, or the formylio series; so also we admit two corresponding radicals with $\mathrm{C}_{12}$, namely $\mathrm{C}_{12} \mathrm{H}_{13}$ and $\mathrm{C}_{12}$ Hi1. As the latter is the radical of an acid named before its nature was known, the caproic acid, we shall call it caproyle $=$ $\mathrm{C}_{12} \mathrm{H}_{11}=\mathrm{Cpr}$. The former we shall call caprotyle $=\mathrm{C}_{12} \mathrm{H}_{13}=$ Cpt, inserting here the letter $t$, to indicate that it belongs to the series of ethyle and methyle, in which $t$ occurs, while the two names show that the radicals are closely connected. This must be attended to, as considerable confusion has arisen from both having been named Caproyle.

Caprotyle $=\mathrm{C}_{12} \mathrm{H}_{13}=\mathrm{Cpt}$, is obtained when œnanthylate of potash $\mathrm{C}_{14} \mathrm{H}_{14} \mathrm{O}_{3}, \mathrm{~K} \mathrm{O}$, is decomposed by galvanism. $\mathrm{K}, \mathrm{C}_{14}$ $\mathrm{H}_{13} \mathrm{O}_{3}+\mathrm{HO}=\mathrm{KO}, 2 \mathrm{CO}_{2}+\mathrm{H}+\mathrm{C}_{12} \mathrm{H}_{13}$. Probably a part of it is decomposed into caprotene, or caproylene, $\mathrm{C}_{12} \mathrm{H}_{12}$, homologous with olefiant gas, and hyduret of caprotyle, $\mathrm{C}_{12} \mathrm{H}_{14}=\mathrm{Cl}_{12}$ $\mathrm{H}_{13}, \mathrm{H}$, homologous with marsh gas, for $2\left(\mathrm{C}_{12} \mathrm{H}_{13}\right)=\mathrm{C}_{12} \mathrm{H}_{12}+$ $\mathrm{C}_{12} \mathrm{H}_{14}$. This radical, called also hexyle, as being the sixth in the series, is a fragrant oil, boiling at $395^{\circ} \mathrm{F}$. It is not decomposed by sulphuric acid, but is attacked by chlorine. When boiled with a mixture of nitric and sulphuric acid, it is converted into a volatile acid, probably caproic acid. Of the compounds of caprotyle, homologous with those of ethyle, we already know the alcohol, or hydrated oxide from which no doubt the ether or oxide may be formed, and the acid sulphate, as well as the volatile base caprotylamine. As yet, the chloride, bromide, iodide, and sulphuret, have not been studied. Of the derivatives of the corresponding negative radical, caproyle, we know the hydrated oxide or aldehyde, and the hydrated teroxide or acid.

Hydrated oxide of caprotyle. Caprotylic or Caproic alcohol, $\mathrm{C}_{12} \mathrm{H}_{13} \mathrm{O}, \mathrm{HO}$, is obtained from the less volatile parts of the inferior brandy made from the marc or expressed residue of the grape in the South of France. This less volatile portion consists chiefly of alcohol and amylic alcohol, with smaller proportions of methylic, propylic, butylic, and caproic alcohols, and from it the propylic alcolol is obtained. In the rectitication of the latter, the caproic alcohol accumulates in the least volatile parts, which are by repeated rectitications, or fractionated distillation, separated into a more volatile part, containing much amylic and little or no caproic alcohol, and a less volatile part, in which caproic alcohol predominates. Faget, who detected it, only obtained enough to prove that it exists, and has all the properties to be expected in an alcohol of its place in the series. It is at tirst mixed with a less volatile liquid, possibly an alcohol, or a mixture of alcohols, higher in the scale, and can only be got pure by often repeated rectifications, which are continued till a liquid is obtained, which 
has a constant boiling-point of from $300^{\circ}$ to $320^{\circ} \mathrm{F}$. In these operations the quantity rapidly diminishes as the purification proceeds, and the final product is but small.

It is an oily mobile liquid, of an agreeable aromatic vinous odour and warm pungent taste, having great transparency and a high refractive power; of Sp. G. 0.833 at $32^{\circ}$. The density of its gas is 3.53 . It boils between $300^{\circ}$ and $320^{\circ}$.

When heated with caustic potash, it is converted into caproate of potash, hydrogen being disengaged.

With oil of vitriol it forms the acid sulphate of oxide of caprotyle, or sulphocaprotylic acid, which, with potash, forms a salt crystallising in scales. In all these points caproic alcohol corresponds to its homologues, methylic, ethylic, propylio, butylic and amylic alcohols.

Cyanide of caprotyle. This compound has been little studied, but is the same with œnanthonitryle, $\mathrm{C}_{14} \mathrm{H}_{13} \mathrm{~N}$, which will, in all probability, be formed when œnanthylamide is heated with anhydrous phosphoric acid. Like the nitryles already mentioned, cenanthonitryle is a cyanide, and its true formula will be, $\mathrm{C}_{12} \mathrm{H}_{13}$, $\mathrm{C}_{2} \mathrm{~N}$.

Caprotylamine, $\mathrm{C}_{12} \mathrm{H}_{15} \mathrm{~N}=\mathrm{N} \mathrm{H}_{2}$ Cpt. SxN. Hexylamine. This base, homologous with methylamine, \&c., has not been much studied, but will no doubt soon be formed from the alcohol. It seems to occur in the oil obtained by distilling bones, in which methylamine, ethylamine, butylamine, and probably amylamine, are also present. It is similar to amylamine, but less volatile.

Caproyle, $\mathrm{C}_{12} \mathrm{H}_{11}=\mathrm{Cp}$. This radical, homologous with formyle, \&c., is not known in a separate form ; but we are acquainted with its hydrated oxide, or aldehyde, and with its teroxide, or caproic acid.

Hydrated oxide of caproyle, $\mathrm{C}_{12} \mathrm{H}_{12} \mathrm{O}_{2}=\mathrm{C}_{12} \mathrm{H}_{11} \mathrm{O}, \mathrm{H} \mathrm{O}$. Srn. Caproic aldehyde. Caproal. This aldehyde is among the products of the distillation of caproate of baryta. It has the characters of an aldehyde, but has not yet been fully studied.

Anhydrous caproic acid, $\mathrm{C}_{22} \mathrm{H}_{11} \mathrm{O}_{3}$. This anhydride is obtained when oxychloride of phosphorus acts on the caproate of baryta, exactly as in the case of anhydrous acetic or butyric acids. It is an oil lighter than water, of a disagreeable odour, which is converted into the hydrated acid by contact with water. It is perfectly neutral, as are the anhydrides in general.

Hydrated caproic acid, $\mathrm{Cl}_{12} \mathrm{H}_{12} \mathrm{O}_{4}=\mathrm{C}_{12} \mathrm{H}_{11} \mathrm{O}_{3}, \mathrm{HO}$. This acid occurs, combined with oxide of lipyle in cow's and goat's butter and goat's fat, whence its name, and in cocoa-nut oil ; it is also found among the products of the oxidation of albuminous matter, and of fat oils, especially of oleic acid. Enanthole, or 
œnanthal, $\mathrm{C}_{14} \mathrm{H}_{14} \mathrm{O}_{2}$, when oxidised, yields this acid among other products. But it is best obtained from the cyanide of amyle or capronitryle, $\mathrm{C}_{12} \mathrm{H}_{11} \mathrm{~N}$, by boiling it with an alcoholic solution of potash. The caproate of potash is distilled with sulphurio acid, and yields caproic acid.

It is very inflammable; its Sp. G. is 0.931 at $60^{\circ}$; it is not congealed by a cold of $16^{\circ} \mathrm{F}$. The pure acid fumes in the air, and boils at about $390^{\circ} \mathrm{F}$. It is very sparingly soluble in water. Its taste is acid and pungent, with a sweetish after taste, and it has a strong acid smell, resembling that of vinegar mixed with that of rancid butter and that of sweat. There is no doubt that it is formed in the animal "body; for not only does it occur in butter, and, as the smell indicates; in the cutaneous transpiration, but it has been detected in some urinary calculi in man. It has also been found in Limburg cheese; which is half putrescent, and is probably one of the products of putrefaction generally:

Its salts have more of a soapy character than those of butyric or valerianic acid, and have the rancid smell of the acid. The caproate of glycerine or of lipine, or, as it is called, caproine, which is one of the odorous constituents of butter; 'has a smell which when diluted with air is pleasant, but it is easily decomposed, especially in contact with albuminous matters, and the acid, with others analogous to it, being set free, contributes to give the rancid flavour to stale butter.

The caproate of methyle has an odour approaching that of the acid, but the caproate of oxide of ethyle is a very fragrant ether, having the odour of melon or pine-apple in a high degree. The caproate of oxide of amyle has a less pleasant odour, and boils at about $412^{\circ}$.

Caprone, $\mathrm{C}_{11} \mathrm{H}_{11} \mathrm{O}$, or rather $\mathrm{C}_{22} \mathrm{H}_{22} \mathrm{O}_{2}$, is obtained by heating caproic acid with baryta. It resembles valerene, but is less volatile. It is accompanied by a portion of the compound $\mathrm{C}_{12} \mathrm{H}_{12}$ $\mathrm{O}_{2}$, which is capraldehyde or capral.

Caprone, acted on by nitric acid, yields a nitro-acid, namely, nitrovalerianic acid, $\left.\mathrm{C}_{10} \underset{\mathrm{N}_{3}}{\mathrm{H}_{4}}\right\} \mathrm{O}_{3}, \mathrm{H} \mathrm{O}$.

Caproate of ammonia, and caproylamide, heated with anhydrous phosphoric acid, will probably yield capronitryle, $\mathrm{C}_{12} \mathrm{H}_{11}$ $\mathrm{N}$, already mentioned as cyanide of amyle, and the best source of caproic acid. But it is best obtained by heating sulphamylate of potash with cyanide of potassium. In this process, some cyanate of oxide of amyle is also formed, so that when the mixed product is heated with potash, amylamine is expelled, and caproate of potash remains behind. This is the best method of obtaining amylamine. 


\section{Radicals with $\mathrm{C}_{14}$. \\ Heptyle. GEnanthyle.}

There can be no doubt of the existence of a radical $\mathrm{C}_{14} \mathrm{H}_{15}$, analogous to, and homologous with, methyle, \&c., and which, being the seventh in the series, may be called heptyle. But neither the radical, nor any of its compounds, are yet known with certainty, although some chemists have described what they supposed to be the hydrated oxide, or œnanthylic alcohol. It appears, however, that this was really the next or caprylic (octylic) alcohol. It is possible that the œnanthylic alcohol may occur, with the butylic, amylic, and caproic alcohols, or the less volatile part of the liquid obtained from the fermentation of the mare or expressed residue of the grape. There is some liquid present, less volatile than caproio aloohol, but it has not been obtained in sufficient quantity to purify it. We may expect some day to find the means of causing sugar to ferment so as to yield this alcohol as the chief product.

Of the derived or negative radical, œnanthyle, $\mathrm{C}_{14} \mathrm{H}_{13}$, homologous with formyle, \&c., we know the hydrated oxide or aldehyde, and the hydrated teroxide or œnananthylic acid.

Hydrated oxide of enanthyle, $\mathrm{C}_{14} \mathrm{H}_{13} \mathrm{O}, \mathrm{H} \mathrm{O}=\mathrm{C}_{14} \mathrm{H}_{14} \mathrm{O}_{2}$. SrN. Enanthole. Enanthal. This compound, the aldehyde of œnanthylic acid, is obtained by the distillation of castor oil. It is an oily liquid, of a somewhat aromatic smell. When oxidised, it yields œenanthylio and caproic acids, perhaps also valerianic, butyric, propylic, acetic, and formic acids. Enanthole may possibly be $\mathrm{C}_{28} \mathrm{H}_{28} \mathrm{O}_{4}=\mathrm{C}_{14} \mathrm{H}_{15} \mathrm{O}, \mathrm{C}_{14} \mathrm{H}_{13} \mathrm{O}_{3}$.

When fused with potash, it yields œnanthylate of potash and hydrogen. With the alkaline bisulphites it forms crystalline compounds, which is a character of the aldehydes. It also forms a crystalline compound with ammonia, and reduces the salts of silver, producing a mirror-like deposit of the metal.

When agitated with nitric acid at $32^{\circ}$, it is converted, if the mixture be left in the cold, into a beautiful crystalline solid, isomeric or polymeric with œnanthal, called metœnanthal.

Enanthal is best obtained by acting on the liquid formed by distilling castor oil with bisulphite of soda. A mass of crystals is formed. These are purified by means of alcohol, and decomposed by diluted sulphuric acid, when the cenanthal separates. Its density is 0.8271 at $64^{\circ} \mathrm{F}$, and that of its gas is 4.178 . It boils at $312^{\circ}$ to $317^{\circ}$. It is oxidised by the contact of air into œnanthylic acid.

Anhydrous cenanthylic acid, $\mathrm{C}_{14} \mathrm{H}_{13} \mathrm{O}_{3}$. Formed by the action of oxychloride of phosphorus on cenanthylate of potash. 
A limpid, neutral oil, of Sp. G. 0.92 at $51^{\circ}$. It has a rancid smell, probably from being converted into the hydrated acid by the moisture of the air.

Enanthylic acid. $\mathrm{C}_{14} \mathrm{H}_{13} \mathrm{O}_{3}, \mathrm{H} \mathrm{O}$, is obtained by the oxidation of œnanthal, and also by that of oleic acid and of albuminous matter, and oils in general by means of nitric acid. It may easily be got by distilling castor oil with nitric acid, when it distils over. It is an oily acid, less volatile than caproic acid, boiling at $298^{\circ}$, and its salts are fatty in aspect. It has an aromatic smell with some rancidity, and a burning taste. The œnanthates of oxides of ethyle and methyle are fragrant ethereal compounds. None of its other compounds and derivatives have yet been much examined. It is remarkable that butter, which contains the acids with 12, 16, and 20 eqs. of carbon, never contains the intermediate acids with 14, and 18 eqs. of carbon, so that œnanthylic acid is not found in butter.

Enanthylamide, $\mathrm{C}_{14} \mathrm{H}_{25} \mathrm{~N} \mathrm{O}_{2}=\mathrm{N} \mathrm{H}_{2}, \mathrm{C}_{14} \mathrm{H}_{13} \mathrm{O}_{2}$, is formed when ammonia acts on anhydrous œnanthylio acid, or on œnanthylic ether. It crystallises in scales. If heated with dry phosphoric acid, it will probably yield œnanthylonitryle, that is, cyanide of caprotyle, $\mathrm{C}_{14} \mathrm{H}_{13} \mathrm{~N}=\mathrm{C}_{12} \mathrm{H}_{13}, \mathrm{C}_{2} \mathrm{~N}$.

The base, corresponding to methylamine, which might be called heptylamine, is not yet known, but there can be no doubt as to its existence. - Its formula will be, of course, $\mathrm{C}_{14} \mathrm{H}_{17} \mathrm{~N}=$ $\mathrm{N} \mathrm{H}_{2}, \mathrm{C}_{34} \mathrm{H}_{15}$.

Enanthylic ether, or œnanthylate of oxide of ethyle, Cis $\mathrm{H}_{18} \mathrm{O}_{4}=\mathrm{C}_{4} \mathrm{H}_{5} \mathrm{O}, \mathrm{C}_{14} \mathrm{H}_{13} \mathrm{O}_{3}$, is a fragrant ether, having a sweetish taste, but leaving on the palate a disagreeable impression.

Radicals with $\mathrm{C}_{16}$.

Octyle. Capryle.

There are under this head two radicals, which may be called octyle, $\mathrm{C}_{16} \mathrm{H}_{17}$, and capryle, $\mathrm{C}_{16} \mathrm{H}_{16}$, corresponding to methyle $\mathrm{C}_{2} \mathrm{H}_{3}$ and formyle $\mathrm{C}_{2} \mathrm{H}$.

Octyle, $\mathrm{C}_{16} \mathrm{H}_{17}=$ Oct, is not known in the separate form, but will probably be formed in the action of the galvanic current on pelargonate of potash or on caprate of potash.

The oxide of octyle, $\mathrm{C}_{16} \mathrm{H}_{17} \mathrm{O}$, has not been described, but exists combined with sulphuric acid, as we shall see.

Hydrated oxide of octyle, $\mathrm{C}_{16} \mathrm{H}_{17} \mathrm{O}, \mathrm{H} \mathrm{O}$. SxN. Octylic or caprylic alcohol. This compound is obtained, according to Bouis, by distilling ricinoleic acid with caustic potash, or by distilling castor oil with the same base. It is purified by rectification. It is a colourless oily liquid, staining paper like volatile oils, 
insoluble in water, soluble in alcohol and ether. It has a pleasant aromatic smell. Its Sp. G. is 0.823 at $66^{\circ}$ It boils at $356^{\circ}$.

With sulphuric acid it forms the sulphoctylic acid, or acid sulphate of octyle, homologous with sulphovinic acid. The formula of this acid is $\mathrm{C}_{16} \mathrm{H}_{17} \mathrm{O}, \mathrm{H} \mathrm{O}, 2 \mathrm{~S} \mathrm{O}$, and with bases it forms salts of the formula $\mathrm{C}_{16} \mathrm{H}_{17} \mathrm{O}, \mathrm{M} \mathrm{O}, 2 \mathrm{~S} \mathrm{O}_{3}$.

Heated with excess of sulphuric acid, octylic alcohol yields the carbohydrogen, octylene, $\mathrm{C}_{16} \mathrm{H}_{16}$, homologous with olefiant gas, which is also found in the naphtha of coal tar, and in native naphtha.

With nitric acid, this alcohol is oxidised, yielding pimelic, lipic, succinic and butyric acids, with other products.

Chloride of octyle is formed when dry hydrochloric acid acts on octylio alcohol. It is a very fragrant ether. Formula $\mathrm{C}_{16}$ $\mathrm{H}_{17} \mathrm{Cl}$.

Bromine also forms, with the alcohol, the bromide, and iodine the iodide of octyle, when phosphorus is added, as in preparing the iodide or bromide of ethyle. They resemble the chloride.

It appears from the latest experiments of Bouis, that chloride of octyle (which he calls chloride of capryle, unfortunately, since the name capryle belongs to the radical of caprylic acid) when acted on by sodium, yields either the radical octyle, $\mathrm{C}_{16} \mathrm{H}_{17}$, or caprylene, $\mathrm{C}_{16} \mathrm{H}_{16}$, which is the hyduret of eapryle, $\mathrm{C}_{16} \mathrm{H}_{15}, \mathrm{H}$; according to the temperature employed.

Octylamine. The base octylamine (erroneously caprylamine) will undoubtedly be formed, if sulphocaprylate of potash be distilled with cyanate of potash, and the product distilled with caustic potash. Its formula is $\mathrm{C}_{16} \mathrm{H}_{19} \mathrm{~N}=\mathrm{N} \mathrm{H}_{2}, \mathrm{C}_{16} \mathrm{H}_{17}$, and it most probably exists in the distilled oil of bones, along with the other bases of the same series.

But Bouis has obtained this base by Hofmann's process of heating iodide of octyle with an alcoholic solution of ammonia in a tube hermetically sealed. There is formed a crystalline iodide of octylium, $\mathrm{N} \mathrm{H_{2 }}, \mathrm{Cl}_{16} \mathrm{H}_{17}, \mathrm{H}+\mathrm{I}$, (or hydriodate of octylamine) which, decomposed by potash, yields the new base, as a liquid, lighter than water, colourless, limpid, with an ammoniacal odour, recalling that of mushrooms. It boils between $341^{\circ}$ and $347^{\circ} \mathrm{F}$. It forms salts with acids, and is a strong base.

When iodide of ethyle acts on octylamine, there is formed the hydriodate of a new base, ethyloctylamine, $\mathrm{C}_{16} \mathrm{H}_{18}\left(\mathrm{C}_{4} \mathrm{H}_{5}\right) \mathrm{N}$, $\mathrm{H}$ I. In this base, one of the two remaining atoms of the hydrogen of ammonia, the third having been replaced by octyle, is replaced by ethyle, so that its rational formula is $\mathrm{N} \mathrm{H}, \mathrm{Ae}$, 
Oct, and it is an imide base, analogous to ethylomethylamine, $\mathrm{N} \mathrm{H,} \mathrm{Me} \mathrm{Ae.}$

Capryle, $\mathrm{C}_{16} \mathrm{H}_{15}$. Of this, the derived negative radical, we know the hydrated oxide, the acid, and the hyduret.

Hydrated Oxide of Capryle, $\mathrm{C}_{16} \mathrm{H}_{15} \mathrm{O}, \mathrm{H} .0$. SYN. Caprylic Aldehyde. Caprylal. This compound is little known, but is contained in the product of the action of heat on caprylate of baryta.

Anhydrous Caprylic Acid, $\mathrm{C}_{16} \mathrm{H}_{15} \mathrm{O}_{3}$, is formed when oxychloride of phosphorus acts on caprylate of baryta. It is a limpid mobile oil, feeling fatty to the touch, of an offensive nauseous smell, especially when it is converted by the moisture of the air into the hydrated acid. It boils at $536^{\circ} \mathrm{F}$.

Hydrated Caprylic Acid, $\mathrm{C}_{16} \mathrm{H}_{15} \mathrm{O}_{3}, \mathrm{H} \mathrm{O}=\mathrm{C}_{16} \mathrm{H}_{16} \mathrm{O}_{4}$. This acid occurs combined with oxide of lipyle, in butter, the flavour of which depends in part on this compound. It is also obtained from the oil of coco, and is among the volatile products. of the oxidation of many organic substances, especially oils, by nitric acid and other oxidising agents. It is an oily liquid, having a rancid odour of sweat, which is especially strong when it is warmed. It congeals at $50^{\circ}$, and the crystals do not melt till near $60^{\circ}$.

Its salts have a good deal of the character of soaps, and are insoluble in water, with the exception of those of the alkalies and alkaline earths.

Caprylate of baryta, when heated, yields caprylone, homologous with acetone, the formula of which is $\mathrm{C}_{30} \mathrm{H}_{30} \mathrm{O}_{2}$. At the same time, caprylal, or the caprylic aldehyde, seems to be formed.

\section{Radicals with $\mathbf{C}_{18}$.}

Nonyle. Pelargyle.

There can be no doubt of the existence of two series of compounds with 18 eqs. of carbon, one corresponding to the derivations of ethyle, and in which we should suppose the radical $\mathrm{C}_{18}$ $\mathrm{H}_{19}$, or nonyle; the other corresponding to those of acetyle, in which the radical is supposed to be $\mathrm{C}_{18} \mathrm{H}_{17}$, or pelargyle. As yet, no compounds of the nonylic series are known, although we may expect nonylic alcohol soon to be discovered. Of the pelargylic series we know the hyduret and the acid.

Anhydrous Pelargonic Acid, $\mathrm{C}_{18} \mathrm{H}_{17} \mathrm{O}_{3}$. Formed when oxychloride of phosphorus acts on pelargonate of baryta. It is an oil, lighter than water, of a rancid smell, which becomes aromatic when the acid is placed in boiling water. At $32^{\circ}$ it congeals. 
Hydrated Pelargonic Acid, $\mathrm{C}_{18} \mathrm{H}_{17} \mathrm{O}_{3}, \mathrm{H} \mathrm{O}$. This acid was first observed in the volatile oil of Pelargonium roseum, but may be obtained more easily by acting on the oil of rue, Ruta graveolens, with its volume of nitric acid, such as is made by mixing equal volumes of strong acid and water. It is also found among the volatile products of the oxidation of oils and fats by nitric acid. It is a colourless acid oil, of an odour resembling that of butyric acid, easily solidified by cold, sparingly soluble in water, very soluble in alcohol and ether. Perchloride of phosphorus attacks it violently, and converts it into oxychloride of pelargyle,

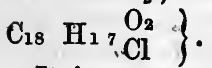

It is supposed by some that the manthic acid of Liebig and Pelouze, which is not identical with the cnanthylic acid obtained from castor oil, is pelargonic acid. In that case the onanthic ether of wine. would be pelargonic ether.

The salts of this acid resemble those of the analogous acids. That of baryta is less soluble than the valerate and œnanthylate, but more soluble than the rutate (caprate, See below).

The pelargonate of ethyle is formed when hydrochloric acid gas is passed through a solution of pelargonic acid in alcohol. It is a colourless oil, boiling at about $240^{\circ}$. It has a peculiar, stupifying, vinous odour, and seems to be in some instances the cnanthic ether of wine, which, in other wines, appears to be œnanthic, or perhaps œnanthylic ether. No doubt pelargonic and œnanthylie ethers are very similar in properties, being contiguous members of a homologous series.

Pelargonic acid forms with deutoxide of nitrogen, a new acid, $\mathrm{C}_{18} \mathrm{H}_{17} \mathrm{O}_{3}, 2 \mathrm{~N} \mathrm{O}_{2}, \mathrm{HO}$, which is an oily liquid, of an odour quite different from that of pelargonic acid. It forms salts with bases, which crystallise.

Oxychloride of pelargyle, already noticed, is an oily liquid, heavier than water, of a suffocating odour if fuming in the air. With alcohol it forms pelargonate of ethyle.

Pelargone, $\mathrm{C}_{34} \mathrm{H}_{34} \mathrm{O}_{2}$, analogous to acetone, is formed when pelargonate of baryta is heated.

Hyduret of Pelargyle, $\mathrm{C}_{18} \mathrm{H}_{18}=\mathrm{C}_{18} \mathrm{H}_{17} \mathrm{H}$. Srn. Nonylene, is obtained by the distillation of hydroleic or metoleic acids. It is a colourless oil, insoluble in water, soluble in alcohol and ether. It has a penetrating odour, and boils at $240^{\circ}$. The density of its gas is 4.488 . It is homologous with the hyduret of ethyle or olefiant gas, and, like it, is acted on by chlorine, forming the compound, $\mathrm{C}_{18} \mathrm{H}_{17} \mathrm{Cl}, \mathrm{HCl}$, homologous with the oil of olefiant gas. 


\section{Radicals with $\mathrm{C}_{20}$.}

\section{Capryle, or Rutyle.}

Here also two series of compounds undoubtedly exist, corresponding to the ethylic and acetylio series; but as yet no compounds of the former, which may be called the decatylic series, are known. Of the latter, the aldehyde and the acid are well known.

Hydrated Oxide of Rutyle (capryle), $\mathrm{C}_{2 \mathrm{O}} \mathrm{H}_{10} \mathrm{O}, \mathrm{H} \mathrm{O}=\mathrm{C}_{20} \mathrm{H}_{2 \mathrm{O}}$ $\mathrm{O}_{2}$. This aldehyde is found as the principal constituent of the volatile oil of rue, Ruta graveolens. It is purified by rectification, and is then an oily liquid of a strong disagreeable odour, boiling at $445^{\circ} \mathrm{F}$. The density of its gas is 5.83. Like the other aldehydes, it forms crystalline compounds with ammonia and with the bisulphites of the alkalies, and reduces the ammoniacal nitrate of silver, yielding a mirror-like deposit of silver. By. nitric acid it is oxidised into the corresponding acid.

Like other aldehydes, rutylic aldehyde presents isomeric or polymeric modifications. When the purified oil of rue is dissolved in thrice its volume of alcohol, and saturated with hrdrochloric acid gas, a brown, fuming mass is obtained. After distilling off the more volatile parts, water is added to the residue, which separates an oil, having a fragrant odour quite different from that of the rutylic aldehyde. On standing, it solidifies at a temperature at which the oil of rue is still quite liquid, and the crystals melt at $56^{\circ}$. This substance has exactly the same composition in $\mathbf{1 0 0}$ parts as rutylic aldehyde or purified oil of rue.

The stearoptene, or solid essence of mint, has also the same composition, but different properties.

Hydrated Rutylic Acid (capric acid), $\mathrm{C}_{20} \mathrm{H}_{19} \mathrm{Os}, \mathrm{H} \mathrm{O}=\mathrm{C}_{20}$ $\mathrm{H}_{20} \mathrm{O}_{4}$. This acid was first found by Chevreul in small quantity in butter, and especially in the butter and fat of the goat, along with caproic, caprylic, and butyric acids. Hence it was named capric acid, from caper a goat. But as both caproic and caprylic acids are thus named, it is better, to aroid confusion, to call this one rutylic acid, and its radical rutyle, and the more so, as the acid is best obtained by oxidising the oil of rue or rutylic aldehyde. In butter, and those fats where it occurs, it is combined with oxide of glyceryle or lipyle, forming, like the other oily and fat acids, a neutral oil, rutyline or rutylate of glycerine (lipine), which is one of the substances giving to the fat of the goat its peculiar strong odour.

The rutylic acid is also found in the oil of grain, along with 
amylic and butylic (probably also propylic) alcohols and margaric acid. It is one of the volatile products of the oxidation of oils, especially oily acids by nitric acid; and it occurs in the oil or fat of coco, in the same form as in butter and goats' fat.

It is a crystalline solid at the ordinary temperature, being the first of the formylic series of acids which is so, though pelargonic acid is easily frozen. It melts at about $85^{\circ}$; is insoluble in cold water, soluble in alcohol and ether. Boiling water dissolves a little, and deposits it on cooling in crystals.

The rutylates have a soapy character, and many of them are insoluble in water.

Rutylate of ethyle, $\mathrm{C}_{4} \mathrm{H}_{5} \mathrm{O}, \mathrm{C}_{20} \mathrm{H}_{19} \mathrm{O}_{3}=\mathrm{C}_{24} \mathrm{H}_{24} \mathrm{O}_{4}$. SxN. Rutylic Ether. Capric Ether. This is formed when hydrochloric acid gas is passed throngh an alcoholic solution of the acid. It is an oily volatile liquid, of a very agreeable odour of fruits. Its density is 0.862 . By ammonia in excess it is converted into Rutylamide, $\mathrm{NH}_{2}, \mathrm{Cl}_{18} \mathrm{H}_{19} \mathrm{O}_{2}=\mathrm{C}_{18} \mathrm{H}_{21} \mathrm{NO}_{2}$. It is a crystalline substance. Probably, if heated with anhydrous phosphoric acid it will yield Rutylonitryle, that is, cyanide of nonyle, $\mathrm{C}_{20} \mathrm{H}_{19} \mathrm{~N}$ $=\mathrm{C}_{18} \mathrm{H}_{19}, \mathrm{C}_{2} \mathrm{~N}$.

Rutylic acid is interesting, as the first truly fatty acid of the series $\left(\mathrm{C}_{n} \mathrm{H}_{n}\right)_{2} \mathrm{O}_{4}$. Yet, although solid at ordinary temperatures, it is perfectly analogous to the acids of this series hitherto described. In these, from formic to capric acid, the boiling point has steadily risen as we rose in the scale, while the melting point has also risen in the same way. The oily character first appears in propylic acid, $\mathrm{C}_{6}$, and the acids with $\mathrm{C}_{8}$ to $\mathrm{C}_{16}$ are more and more oily as we go on. Pelargonic acid, $\mathrm{C}_{18}$, although a liquid, is easily congealed by a moderate degree of cold, and capric acid requires a heat of $80^{\circ}$ to melt it. All the acids above $\mathrm{C}_{20}$ are likewise fatty acids, and their melting point rises with the amount of carbon. It is said that, in this series, the boiling point rises $19^{\circ} \mathrm{C}$, or $66.5^{\circ} \mathrm{F}$, for each addition of $\mathrm{C}_{2} \mathrm{H}_{2}$, but we do not yet know exactly how many degrees the melting point rises at each step. This uncertainty depends, perhaps, on the existence of isomeric acids, with different melting points: but, however this may be, the melting point rises as we rise in the scale.

Of the members of the two series of radicals, containing more than 20 eqs. of carbon, we know only the acids of the series $\left(\mathrm{C}_{n} \mathrm{H}_{n}\right)_{2}+\mathrm{O}_{4}$, save in a few instances in which one or two other compounds, belonging to the series of the alcohols and ethers, are also known. We shall, therefore, merely give a brief catalogue of the remaining acids, adding, in these cases, what is known of the other compounds. 
- $\mathrm{C}_{22}$. Margaritic Acid, $\mathrm{C}_{22} \mathrm{H}_{21} \mathrm{O}_{3}, \mathrm{H} \mathrm{O}=\mathrm{C}_{22} \mathrm{H}_{22} \mathrm{O}_{4}$. This is a crystalline fatty acid, less fusible than the last. It is obtained from castor oil, in which it is combined with oxide of glyceryle or lipyle, by saponification. Its alkaline salts are true soaps. It is now considered doubtful whether this acid have the composition here given.

$\mathrm{C}_{24}$. Laurostearic Acid, $\mathrm{C}_{24} \mathrm{H}_{23} \mathrm{O}_{3}, \mathrm{H} \mathrm{O}=\mathrm{C}_{24} \mathrm{H}_{24} \mathrm{O}_{4}$. A crystalline fatty acid, obtained by saponification from the fat of the berries of Laurus nobilis. Forms soaps with the alkalies, and a fragrant ether with oxide of ethyle.

$\mathrm{C}_{26}$. Cocinic Acid, $\mathrm{C}_{26} \mathrm{H}_{25} \mathrm{O}_{3}, \mathrm{H} \mathrm{O}=\mathrm{C}_{26} \mathrm{H}_{26} \mathrm{O}_{4}$. Like the preceding, but less fusible. It is found in the fat of the cocoa nut, combined with oxide of glyceryle. Cocinate of oxide of ethyle is a liquid, with a very strong fragrance of apples.

$\mathrm{C}_{28}$. Myristic Acid, $\mathrm{C}_{28} \mathrm{H}_{27} \mathrm{O}_{3}, \mathrm{H} \mathrm{O}=\mathrm{C}_{28} \mathrm{H}_{28} \mathrm{O}_{4}$. A crystalline fatty acid, found in the fat of the seeds of Myristica moschata, or nutmeg. It melts at $118^{\circ}$. Its compound with oxide of lipyle is the chief ingredient of the fat of nutmeg. The myristate of oxide of ethyle is an oily liquid.

C30. Benic Acid, $\mathrm{C}_{30} \mathrm{H}_{20} \mathrm{O}_{3}, \mathrm{H} \mathrm{O}=\mathrm{C}_{30} \mathrm{H}_{30} \mathrm{O}_{4}$. Found by Walter in a certain variety of oil of Ben or Behen. It resembles the preceding, but melts at $125^{\circ}$. The same acid is said to exist, in small quantity, in spermaceti : and the acid of the oil of Stillingia sebifera, or stillistearic acid, appears to have the same formula.

Radicals with $\mathbf{C}_{32}$.

Cetyle $\mathrm{C}_{32} \mathrm{H}_{33}$, and Palmityle $\mathrm{C}_{32} \mathrm{H}_{31}$.

These radicals both form a good many compounds, some of which are very abundant in nature.

Cetyle, $\mathrm{C}_{32} \mathrm{H}_{33}=\mathrm{Ct}$, has not been described in the separate state, but will certainly prove to be a solid, crystalline, fusible, and volatile, being homologous with ethyle, amyle, \&c.

Oxide of Cetyle, Cs2 $\mathrm{H}_{33}$ 0, homologous with oxide of ethyle, is obtained when iodide of cetyle is acted on by the oxide of cetyle and sodium, $\left.\underset{\mathrm{Na}}{\mathrm{C}_{32} \mathrm{H}_{33}}\right\} \begin{aligned} & \mathrm{O} \\ & \mathrm{O}\end{aligned}$ It forms brilliant scales, insoluble in water, soluble in alcohol and ether, melting at $131^{\circ}$, boiling at $572^{\circ}$.

Hydrated Oxide of Cetyle, $\mathrm{C}_{32} \mathrm{H}_{33}$ O, H O, Srn. Cetylic Alcohol. Ethal. This alcohol is obtained from cetine or purified spermaceti, which is composed of palmitic acid (see below) and oxide of cetyle. The cetine is saponified by boiling with an alcoholic solution of potash, when palmitate of potash and. 
hydrated oxide of cetyle are formed. The palmitic acid is precipitated by means of chloride of barium, as palmitate of baryta; the precipitate, to which the new alcohol adheres, is acted on by alcohol which dissolves the ethal. It is purified by solution in ether.

Ethal, or cetylic alcohol, is a white waxy solid, which crystallises from its solution in alcohol or ether in shining scales. It melts at $112^{\circ}$, and may be distilled unchanged. It even passes over with the vapour of water. It is tasteless and inodorous, insoluble in water, soluble in alcohol and ether. It is in all respects homologous with alcohol, forming, with sulphuric acid, an acid sulphate, sulphocetylic acid; and the salts of this acid correspond to the sulphovinates.

Chloride of Cetyle, $\mathrm{C}_{32} \mathrm{H}_{33} \mathrm{Cl}$, is formed by the action of perchloride of phosphorus on cetylic alcohol. It is an oily liquid, lighter than water.

Bromide of Cetyle, formed by the action of bromine and phosphorus on cetylic alcohol, is a fusible solid. Formula $\mathrm{C}_{32} \mathrm{H}_{33} \mathrm{Br}$.

Iodide of Cetyle, $\mathrm{C}_{32} \mathrm{H}_{33} \mathrm{I}$, formed in the same way as the bromide, is also a crystallisable, fusible solid. With dry ammonia it yields tricetylamine.

Oxide of Cetyle and Sodium, $\left.\underset{\mathrm{Na}}{\mathrm{C}_{32} \mathrm{H}_{33}}\right\} \underset{\mathrm{O}}{\mathrm{O}}$ is formed when sodium acts on cetylic alcohol. It is a solid body, melting at $230^{\circ}$.

Tricetylamine, $\left.\mathrm{N}, \begin{array}{l}\mathrm{C}_{32} \\ \mathrm{C}_{32} \\ \mathrm{C}_{32} \mathrm{H}_{33} \\ \mathrm{H}_{33}\end{array}\right\}$ or $\mathrm{N} \mathrm{Cts,} \mathrm{homologous} \mathrm{with} \mathrm{am-}$ monia, $\left.\begin{array}{r}\mathrm{N} \\ \mathrm{H}\end{array}\right\}$ or $\mathrm{N} \mathrm{H}_{3}$, is obtained by the action of dry ammonia on fused iodide of cetyle. It dissolves in hot alcohol, and on cooling crystallises in acicular crystals, fusible at $103^{\circ}$. It is a base, though not a powerful one, and some of its salts crystallise. Its empirical formula is $\mathrm{C}_{96} \mathrm{H}_{99} \mathrm{~N}$, and, notwithstanding the prodigious difference between this and the formula of ammonia, $\mathrm{N} \mathrm{H}$, it yet possesses all the essential characters of a base homologous with ammonia, being volatile, though solid at ordinary temperatures, and apt to be partially destroyed by the heat required to distil it; it combines with bases, forming salts, and yields a double salt with chloride of platinum, corresponding to that formed by ammonia. All this is explained by the rational formula, $\mathrm{N} \mathrm{Ct}_{3}$, for this is the type of ammonia, $\mathrm{N} \mathrm{H}_{3}$, and tricetylamine is exactly parallel to trimethylamine, $\mathrm{N} \mathrm{Me}$. The bases with only 1 and 2 eqs. of cetyle are not yet known, but 
will certainly be more analogous to ammonia than this one, just as methylamine, $\left.\mathrm{N}_{\mathrm{Ne}}^{\mathrm{H}_{2}}\right\}$ is liker to ammonia than dimethylamine $\mathrm{N} \underset{\mathrm{Mez}}{\mathrm{H}}$ \}or trimethylamine, $\mathrm{N}$ Mes; so like indeed, as not to be easily distinguished from it. The formulæ of these bases will be, $\left.\mathrm{C}_{32} \mathrm{H}_{35} \mathrm{~N}=\mathrm{N}_{\mathrm{Ct}}^{\mathrm{H}_{2}}\right\}$ and $\left.\mathrm{C}_{64} \mathrm{H}_{67} \mathrm{~N}=\mathrm{N} \underset{\mathrm{Ct}_{2}}{\mathrm{H}}\right\}$.

Sulphuret of Cetyle, $\mathrm{C}_{32} \mathrm{H}_{33} \mathrm{~S}$, is formed when an alcoholic. solution of protosulphuret of potassium acts on chloride of cetyle. It is a crystalline solid, fusible at $137^{\circ} \mathrm{F}$, very soluble in ether.

Sulphhydrate of Cetyle, Cs2 $\mathrm{H}_{33} \mathrm{~S}, \mathrm{H}$ S. SrN. Cetylic Mercaptan is formed as the sulphuret, using sulphhydrate of potassium, $\mathrm{K} \mathrm{S}, \mathrm{H} \mathrm{S}$, and chloride of cetyle. It is a crystalline solid, melting at $113^{\circ}$. Its alcoholic solution causes, after- a time, precipitates in solutions of silver and mercury.

Palmityle, $\mathrm{C}_{32} \mathrm{H}_{31}=\mathrm{Pl}$, is not yet known in the unoombined state.

Hydrated Oxide of Palmityle, $\mathrm{C}_{32} \mathrm{H}_{31} \mathrm{O}, \mathrm{H} \mathrm{O}=\mathrm{C}_{32} \cdot \mathrm{H}_{32} \mathrm{O}_{2}$. Srn. Palmitic aldehyde. Cetylic aldehyde. It is obtained by. oxidising cetylic alcohol, as aldehyde is from alcohol. It is a solid, melting at $216^{\circ} \mathrm{F}$. It is but little known as yet.

Hydrated Palmitic Acid, $\mathrm{C}_{22} \mathrm{H}_{31} \mathrm{O}_{3}, \mathrm{H} \mathrm{O}=\mathrm{C}_{32} \mathrm{H}_{32} \mathrm{O}_{4}$. SxN. Cetylic Acid. Ethalic Acid. This acid is very abundant innature. Combined with glycerine, in the form of a neutral fat, called palmitine, it is the chief ingredient in palm oil. ${ }^{\circ}$ Combined with oxide of cetyle, it forms cetine, the principal constituent of spermaceti. In bees' wax it occurs, combined with oxide of myricyle (see below), as myricine, that part of purified wax which boiling alcohol does not dissolve. It is found also. in butter and in human fat as palmitine, mixed however with other neutral fats.

It may be formed artificially by heating with caustic potask. either cetylic alcohol or oleic acid (see below).

In palm oil, when long kept, the palmitine is more or less decomposed, the palmitic acid and the glycerine being both set free. It is best obtained by saponifying palm oil with caustic soda, and decomposing the soap with hydrochloric or tartaric acid. The fatty acid mass which separates, is dissolved in hot alcohol, which, on cooling, deposits crystals of palmitic acid, the oleic acid of the palm oil remaining dissolved. By pressing the crystals in bibulous paper, and repeating the process of crystallisation with fresh alcohol, the acid is obtained pure; which is known by its melting at $143.5^{\circ} \mathrm{F}$. The melted acid, in cooling, forms a mass of crystalline scales. It is insoluble in water, very 
soluble in alcohol and ether. When cautiously heated, it may be volatilised without residue and unchanged.

The palmitates of the alkalies are regular soaps. The other palmitates are insoluble. The characters of a fatty acid, and those of soaps in its salts with potash and soda, are perfectly seen in palmitic acid; and yet it is perfectly homologous with acetic acid, in which none of these characters appear. It is acid, volatile, and in all its chemical relations runs parallel with acetic acid. The general formula of the palmitates is $\mathrm{C}_{32} \mathrm{H}_{31}$ $\mathrm{O}_{3}, \mathrm{MO}$.

Palmitate of ethyle, or palmitic ether, is a solid, fusible at $76^{\circ}$ $F$. and the palmitate of amyle is similar.

Palmitate of Cetyle is cetine, the pure solid crystalline mass of spermaceti. It forms beautiful silvery scales, fusible at $112^{\circ}$, and at $684^{\circ}$ it volatilises unchanged. Its formula is $\mathrm{C}_{32} \mathrm{H}_{33} \mathrm{O}$, $\mathrm{C}_{32} \mathrm{H}_{31} \mathrm{O}_{3}=\mathrm{C}_{64} \mathrm{H}_{64} \mathrm{O}_{4}=\mathrm{Ct} \mathrm{O}, \mathrm{Pl} \mathrm{O}$.

It was for some time supposed, that the palmitic acid of spermaceti differed from that of palm oil. But it has been proved, not only that these two are identical, but that the same acid occurs in human fat, butter, wax, and other fatty bodies, so that it is one of the most important fatty acids.

Palmitate of Myricyle, $\mathrm{C}_{60} \mathrm{H}_{61} \mathrm{O}, \mathrm{C}_{32} \mathrm{H}_{31} \mathrm{O}_{3}=\mathrm{C}_{92} \mathrm{H}_{92} \mathrm{O}_{4}$, is the part of wax insoluble in hot alcohol. It melts at $162^{\circ}$ when quite pure.

Hyduret of Palmityle, $\mathrm{C}_{32} \mathrm{H}_{31}, \mathrm{H}=\mathrm{C}_{32} \mathrm{H}_{32}$. SrN. Cetylene. Cetene. Palmitene. Is homologous with olefiant gas, and is obtained by heating cetylic alcohol with anhydrous phosphoric acid. It is an oily liquid, boiling at $527^{\circ} \mathrm{F}$. The density of its gas is 8.007. It is insoluble in water, soluble in alcohol, ether, \&c., and burns with a pure white flame. It is formed also when cetine is distilled.

From the heavy or sweet oil of wine when acted on by water, there separates a liquid, the light oil of wine, which seems to be palmitine, boiling at the same point. In the cold it yields crystals of the same composition.

Palmitone, $\mathrm{C}_{62} \mathrm{H}_{62} \mathrm{O}_{2}$, is homologous with acetone. It is formed when palmitic acid is heated with excess of lime. It is a crystalline solid, easily fusible.

\section{Radicals with $\mathrm{C}_{34}$.}

Of this series, we know only compounds of the negative radical $\mathrm{C}_{34} \mathrm{H}_{33}$, those of the positive radical $\mathrm{C}_{34} \mathrm{H}_{35}$ being as yet undiscovered.

Margaric Acid, $\mathrm{C}_{34} \mathrm{H}_{33} \mathrm{O}_{3}, \mathrm{HO}=\mathrm{Cs}_{4} \mathrm{H}_{34} \mathrm{O}_{4}$. This acid is one of the most abundant and important of the fatty acids. 
Combined with glycerine, as margarine, it occurs in many fats, in human fat, goose fat, butter, and some other animal fats, and in vegetable fats, such as olive oil. The acid may be extracted from soap made of these fats, but as it is mixed with much oleic acid, it is better to prepare it by distilling either tallow or crude stearic acid. In the latter case, the product is well squeezed and purified by solution in alcohol, and crystallisation. If prepared from pure stearic acid by nitric acid, it is pure from the first. It belongs to the series $\left(\mathrm{C}_{n} \mathrm{H}_{n}\right)+\mathrm{O}_{4}$, and it is probably in consequence of its composition, that, like the other acids of that series, it is volatile without decomposition.

Margaric acid is a white solid fat, of distinct acid properties, fusible at $140^{\circ}$, very soluble in hot aleohol and in ether. It instantly combines with bases, decomposing the carbonates and forming perfect soaps with potash and soda. The neutral margarates of potash and soda are decomposed by the addition of much water, depositing the acid margarates in pearly scales. Margarate of glycerine, or margarine, is found pure in the solid part of human fat or of olive oil. It dissolves in hot alcohol and crystallises on cooling. Margarate of oxide of ethyle is a white fusible solid.

The general formula of the neutral margarates is $\mathrm{Cs}_{34} \mathrm{H}_{3 s}$ $\mathrm{O}_{3}, \mathrm{MO}$.

Most chemists adopt the formula here given for margaric acid, but Heintz contends that margaric acid is not a single acid, but a mixture, or rather a compound of palmitic acid (Css) with stearic acid ( $\left.\mathrm{C}_{36}\right)$. He states, that if margaric acid, melting at $140^{\circ}$, be subjected to further purification, it yields palmitic acid, which melts at $144^{\circ}$. It is certainly remarkable that the melting point of margaric acid ( $\left.\mathrm{C}_{34}\right)$, should be lower than that of palmitic acid ( $\left.\mathrm{C}_{32}\right)$; and it is a very frequent occurrence that a mixture or compound of two similar substances differing in fusibility, such as two metals, or two fats, is more fusible than either. Heintz also states that, by mixing 9 or 10 parts of palmitic acid with 1 part of stearic acid, an acid is obtained having the melting point and crystalline form of margaric acid.

On the other hand Chevreul, who first described margaric and stearic acids, is distinguished for his extreme accuracy, and certainly did not neglect the purification of his margaric acid; yet he could not obtain it, although he endeavoured to do so, with a higher melting point than $140^{\circ}$. Many other chemists have confirmed his results, and the numerous analyses of margaric acid and its salts agree well with the formula $\mathrm{C}_{\mathbf{3 4}} \mathrm{H}_{\mathbf{3 4}} \mathrm{O}_{4}$. No doubt a mixture of 1 eq. of palmitic acid with 1 eq. of stearic acid would have the same composition, for $\mathrm{C}_{32} \mathrm{H}_{32} \mathrm{O}_{4}+\mathrm{C}_{36} \mathrm{H}_{36} \mathrm{O}_{4}=\mathrm{C}_{68}$ 
$\mathrm{H}_{68} \mathrm{O}_{8}=2\left(\mathrm{C}_{34} \mathrm{H}_{34} \mathrm{O}_{4}\right)$. But Heintz found it necessary to use 9 or 10 parts of palmitic to 1 of stearic acid, which must yield, on analysis, a result hardly differing from that obtained with palmitic acid.

The probability is, that both facts are true, and that there exists an acid with the formula $\mathrm{C}_{34} \mathrm{H}_{34} \mathrm{O}_{4}$, homologous with acetic, butyric, and palmitic acids, and the others of the series; this is margaric acid, which Chevreul and others have examined.

But as two contiguous members of a homologous series are always very similar, margaric acid must, and indeed does, very closely resemble palmitic acid on the one side, and stearic acid on the other. And nothing is more likely than the occurrence of a mixture of palmitic and stearic acids, both of them, as well as margaric acid, being abundant and widely diffused. Such a mixture must still more closely resemble margaric acid; and such a mixture has probably been examined by Heintz. It is even probable that the same kind of fat may at one time yield margaric acid and at another the stearic palmitic acid of Heintz. For we know that in butter, at one time we obtain both butyric and caproic acids, at another time neither of these, but a new acid intermediate between them, which is made up of 1 eq. of each, $\mathrm{C}_{8} \mathrm{H}_{8} \mathrm{O}_{4}+\mathrm{C}_{12} \mathrm{H}_{12} \mathrm{O}_{4}=\mathrm{C}_{20} \mathrm{H}_{20} \mathrm{O}_{8}=2$ ( $\left.\mathrm{C}_{10} \mathrm{H}_{10} \mathrm{O}_{4}\right)$. This acid, which Lerch calls vaccinic acid, forms salts different from those of butyric and caproic acids, but, when heated, it is resolved into these two acids. Yet this does not interfere with the existence of an acid of the formula $\mathrm{C}_{10} \mathrm{H}_{10} \mathrm{O}_{4}$, for that is the formula of valerianic acid, which is quite distinct from butyric, vaccinic or caproic acids.

In like manner, stearopalmitic acid may exist, and will be analogous to vaccinic acid, and very similar to margaric acid, while margaric acid also exists and corresponds to valerianic acid, stearic acid corresponding to the caproic acid of the above illustration.

Margaramide, $\mathrm{C}_{34} \mathrm{H}_{35} \mathrm{NO}_{2}=\mathrm{NH}_{2} \mathrm{C}_{34} \mathrm{H}_{33} \mathrm{O}_{3}$, is formed when dry ammoniacal gas acts on fats or oils containing margarine. It is a white crystalline solid, which is fusible, soluble in alcohol or ether, and decomposed by boiling with alkalies, yielding ammonia and margaric acid.

Margarone.-This is a solid white crystalline body, formed when margaric acid is heated with excess of lime.

Margarine.-This is a compound of margaric acid with oxide of glyceryle, which is very abundant in nature, and is indeed the form in which the acid chiefly occurs. It is a neutral fat, and with alkalies, yields a soap of the alkali, or margarate, and glycerine or hydrated oxide of glyceryle. Its precise composition is 
not certainly known, but we shall return to it under the head of neutral fats and oils which are compounds of glycerine with fatty or oily acids.

\section{Radicals with $\mathrm{C}_{30}$.}

Here also no compounds of the positive radical $\mathrm{C}_{36} \mathrm{H}_{37}$ are known; but the acid of the negative radical Cse $\mathrm{H}_{3 s}$ is the wellknown stearic acid.

Hydrated Stearic Acid, $\mathrm{C}_{36} \mathrm{H}_{35} \mathrm{O}_{3}$, HO? This is, perhaps, the most important and most abundant of the fatty acids. It exists, in combination with glycerine, as stearine, in beef and mutton fat, and in several vegetable fats, such as the butter of cacao. To obtain it, mutton suet is saponified by boiling with potash, and the purified soap decomposed by an acid, when a mixture of stearic and oleic acids, the latter in small proportion, rises to the surface. It is strongly pressed between warm plates, so as to get rid of the oleic acid in great part, and it is finally purified by solution in hot alcohol, and crystallisation, repeated till its melting point is constant at $167^{\circ}$. Or the stearic acid of commerce, which is nearly pure, may be purified by means of alcohol. Or again, tallow may be mixed with half its weight of oil of vitriol, and the mass melted in hot water, which removes a compound of sulphuric acid with glycerine, while the stearic acid rises to the surface and is to be purified as above. Finally, pure stearine, if saponified, and the soap acted on by an acid, yields at once pure stearic acid.

Stearic acid is a white solid, fusible at $167^{\circ}$, and on cooling forming brilliant white needles. It may be reduced to powder, and is, like all fat acids, insoluble in water, soluble in alcohol and ether. It burns like wax, and is used in the formation of improved candles.

The stearic acid of which candles are made (called wax candles, and in many points a good and cheap substitute for candles of real wax), is made by saponifying tallow or suet with lime and hot water, aided by a current of steam. The saponified mass, consisting of soaps of lime, with excess of lime, is now stirred up in the water and added to diluted sulphuric acid, and the mixture warmed by steam. The fatty acids are set free and rise to the surface as an oil, which on cooling forms a solid fat. This is strongly pressed in a hydraulic press to remove the liquid acid, oleic acid, and the cake thus obtained is again pressed between warm iron plates, to expel more of the liquid acid. The solid residue is now nearly pure, if tallow or suet have alone been used; but if other fats, such as lard or palm oil, have been added, as is 
often the case, the stearic acid is contaminated with margaric or palmitic acid, or both.

Stearic acid may, if heated in small quantities, be volatilised unaltered, a character belonging to all the acids below it in the series. When heated too strongly, or in large masses, it is decomposed, yielding carbohydrogens, water, \&c. The product thus obtained was at one time supposed to be margaric acid, but it is only a mixture of stearic acid with carbohydrogens and water, possibly with some margaric or palmitio acid also.

Steario acid was long supposed to be bibasic, and to have a formula double at least of that now given. But it is evident, from the perfect analogy in properties between it and palmitio and margaric acids, that it must belong to the series of monobasio volatile acids, homologous with acetio acid.

Its neutral salts, therefore, will have the formula $\mathrm{C}_{36} \mathrm{H}_{35} \mathrm{O}_{3}$, $\mathrm{M} O$, and in its acid salts, the formula of the hydrated acid will be added to that of the neutral salt.

Heated with anhydrous phosphoric acid, stearic acid loses 2 eqs. of water, and yields a waxy solid, of the formula $\mathrm{C}_{36} \mathrm{H}_{34} \mathrm{O}_{2}$, not yet named.

Heated with quick lime, stearic acid yields another fusible crystalline solid, along with liquid carbohydrogens. The solid product is called stearone. It forms pearly scales, fusible at $169^{\circ}$. Its composition is not accurately known, but it contains oxygen. Margaric acid seems to yield the same compound.

The neutral stearates of the alkalies are perfect soaps. They dissolve in from 10 to 20 parts of hot water; the addition of a large quantity of water decomposes them into acid stearates which are deposited, and basic stearates which remain dissolved. For the same reason a hot solution of a neutral stearate becomes gelatinous on cooling, from the separation of the acid salt. Acid stearate of oxide of ethyle, $\overline{\mathrm{St}}, \mathrm{Ae} \mathrm{O}+\overline{\mathrm{St}}, \mathrm{HO}$, and neutral stearate of the same base, $\overline{\mathrm{St}}, \mathrm{Ae} \mathrm{O}$, are both white crystalline fusible solids; as is likewise the stearate of oxide of methyle, $\overline{\mathrm{st}}$, Mt 0.

Stearine, the chief ingredient of suet and tallow, is a stearate of oxide of glyceryle, but we shall mention it, with other analogous compounds, under glycerine. When boiled with alkalies, stearine, like all other fats, is saponified : that is, the steario acid combines with the alkali, forming soap, and glycerine is separated. Pure stearine is obtained by pressing tallow between hot plates, and afterwards dissolving in hot ether, which on cooling deposits the stearine. It is like wax in appearance when it has been melted, and it may be powdered. It crystallises from its solution in hot ether in pearly scales. 
Stearine is nsually said to melt at $143.5^{\circ}$. But it has been shown by Duffy that stearine may be obtained by reiterated crystallisations from ether, the melting point of which is so high as $147 \cdot 5^{\circ}$, and that the same stearine can exist in several states, each with a different melting point. Thus stearine melting at $147 \cdot 5^{\circ}$, if heated to $150^{\circ}$, will solidify at $145.5^{\circ}$ or $146^{\circ}$. But if it be heated to $152^{\circ}$, it will not solidify till it cools to about $124^{\circ}$, and now it has recovered its first melting point of $147 \cdot 5^{\circ}$. Again, that which has only been heated to $150^{\circ}$, which solidifies at $146^{\circ}$, is opaque and friable, instead of being translucent, and in this state it only melts at the high temperature of $151^{\circ}$ or even $157^{\circ}$.

There are thus three states of the same stearine; one $\alpha$, melting at $124^{\circ}$, another $\beta$, at $147.5^{\circ}$, and a third $\gamma$, at $151^{\circ}$; that which solidifies at $124^{\circ}$ does so rapidly ; while that which solidifies at $146^{\circ}$ congeals very slowly. They differ somewhat in appearance, and also, to a small degree, in density. The Sp. G. of $\alpha$ has been found to be, at $60^{\circ}, 0.9867$; that of $\beta, 1.0101$; and that of $\gamma, 1.0178$.

These modifications have some analogy with the allotropic states of certain elements.

Stearate of lead is an insoluble fusible soap, or, as it is called, a plaster. The same is true of margarate of lead, and in general of the compounds of lead with fat acids.

When margarine or stearine are distilled, they yield the very acrid vapours of acroleine, a product derived from the glyeerine contained in these fats; but pure stearic and margaric acids yield not a trace of acroleine. Neither do they yield any sebacic acid among the products of their distillation, this acid being derived exclusively from oleic acid.

Bassic Acid, a fatty acid found in the oil of Bassia latifolia, was supposed to be a different acid from stearic acid, when the latter was regarded as isomeric with margaric acid. But as we have now adopted for stearic acid the formula $\mathrm{C}_{36} \mathrm{H}_{36} \mathrm{O}_{4}$, which is also that of bassic acid, it is evident that bassio acid is simply steario acid from a new source, and probably slightly modified by the presence of a trace of some other acid, which makes it rather more fusible.

$\mathrm{C}_{38}$. Balenic Acid, $\mathrm{C}_{38} \mathrm{H}_{37} \mathrm{O}_{3}, \mathrm{HO}=\mathrm{C}_{88} \mathrm{H}_{38} \mathrm{O}_{4}$. This is a fatty acid, melting at $164^{\circ}$, and is said to be found in the usual combination, in some forms of whale oil.

$\mathrm{C}_{40}$. Arachidic Acid, $\mathrm{C}_{40} \mathrm{H}_{39} \mathrm{O}_{3}, \mathrm{H} \mathrm{O}=\mathrm{C}_{40} \mathrm{H}_{40} \mathrm{O}_{4} \cdot$ This acid is found in the usual form of a neutral glyceride, in the oil of Arachis hypogaa, or earth nut. When purified from the oleic acid and other acids which accompany it, it is a solid white fat, crystallising in brilliant scales, fusible at $167^{\circ}$. It is sparingly soluble in cold alcohol, but very soluble in boiling absolute 
alcohol and in ether. The arachidate of ethyle is a tough crystalline substance, melting about $131^{\circ}$.

$\mathrm{C}_{42}$. Behenic Acid, $\mathrm{C}_{42} \mathrm{H}_{41} \mathrm{O}_{3}, \mathrm{H} \mathrm{O}=\mathrm{C}_{42} \mathrm{H}_{42} \mathrm{O}_{4}$. -This acid was found by Völcker in the oil of Moringa oleifera, or oil of Ben or Behen, as it is called, which is very similar to olive oil, and has the advantage of not soon becoming rancid. Hence it is much used for hair oil, and is the basis of Macassar oil. The oil contains oxide of lipyle or glycerine, united to behenic acid and one or two other fatty acids, which are obtained by saponification, as usual. Behenic acid is a crystalline fatty acid, melting at $173^{\circ}$. Like stearic acid, it may be powdered. Behenic ether $\mathrm{C}_{4} \mathrm{H}_{5} \mathrm{O}$, $\mathrm{C}_{\overline{4} 2} \mathrm{H}_{41} \mathrm{O}_{3}$, is a crystalline solid, melting at about $120^{\circ}$. Up to this point, namely $\mathrm{C}_{\mathbf{4 2}}$, the series of volatile, oily, and fatty acids, from formic acid to behenic acid, is complete. Beyond $\mathrm{C}_{\mathbf{4 2}}$, two acids are known, and in each case the alcohol of the corresponding series is also known.

$\mathrm{C}_{54}$. Cerotic Acid, $\mathrm{C}_{54} \mathrm{H}_{53} \mathrm{O}, \mathrm{H} \mathrm{O}=\mathrm{C}_{54} \mathrm{H}_{54} \mathrm{O}_{4}$. This acid was recently discovered by Brodie, as an ingredient of beeswax, and, what is unusual, in the free state. It may be obtained by dissolving wax in hot alcohol, and repeating the operation with the substance deposited on cooling, till its melting point rises to $162^{\circ}$. After this it is purified by repeated crystallisations from ether, till the melting point rises to $171^{\circ}$, which is that of the acid. It was formerly supposed to be a neutral fat, in the impure state, and was called cerine, but Brodie has proved that it is a volatile acid, and easily combines with bases. Its proportion in wax is about one-fifth of the whole.

Chinese wax contains cerotio acid, combined with oxide of ceryle, yielding Cerotine, a body homologous with formiate of methyle, and others of that series. Thus:

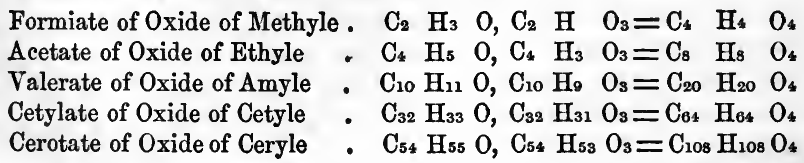

I When heated with potash, cerotine yields cerotate of potash, and the hydrated oxide of ceryle, $\mathrm{C}_{54} \mathrm{H}_{55} \mathrm{O}, \mathrm{H} \mathrm{O}=\mathrm{C}_{54} \mathrm{H}_{56} \mathrm{O}_{2}$. This is the ceric alcohol. It is a waxy crystallisable solid, melting at $207^{\circ}$. With sulphuric acid it forms a solid like wax, which is the sulphate of ceryle. The anhydrous oxide, or ceric ether, is not known in a separate form, but the carbo-hydrogen of the olefiant gas series, or cerene $=\mathrm{C}_{54} \mathrm{H}_{54}$, has been obtained as a solid, wax-like body, as also a compound of sulphuric acid 
with cerotine, $\mathrm{C}_{54} \mathrm{H}_{55} \mathrm{O}, \mathrm{H} \mathrm{O}, \mathrm{S} \mathrm{O}_{3}$. The aldehyde of cerotic acid is unknown, but Brodie has obtained a substitution-product derived from it. The aldehyde, or cerotal, would be $\mathrm{C}_{54} \mathrm{H}_{54} \mathrm{O}_{2}$, and the chlorine compound, chlorocerotal, is $\mathrm{C}_{54}\left\{\begin{array}{ll}\mathrm{H}_{41} \\ \mathrm{Cl}_{13}\end{array} \mathrm{O}_{2}\right.$. Cerene also yields with chlorine the products $\mathrm{C}_{54}\left\{\begin{array}{l}\mathrm{H}_{35} \\ \mathrm{Cl}_{10}\end{array}\right.$,

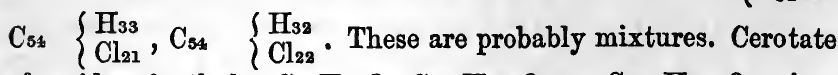
of oxide of ethyle, $\mathrm{C}_{4} \mathrm{H}_{5} \mathrm{O}, \mathrm{C}_{54} \mathrm{H}_{53} \mathrm{O}_{3}=\mathrm{C}_{58} \mathrm{H}_{58} \mathrm{O}_{4}$, is a fusible solid.

Ceo. Melissic Acid, $\mathrm{C}_{60} \mathrm{H}_{59} \mathrm{O}_{3}, \mathrm{H} \mathrm{O}=\mathrm{C}_{60} \mathrm{H}_{60} \mathrm{O}_{4}$. When wax is boiled with alcohol, a portion remains insoluble. This was formerly called myricine. It melts at $147^{\circ}$. It is easily saponified, and yields an acid, and the hydrate of the oxide with which the acid was combined. These are separated by crystallisation from alcohol. The acid is palmitic acid, which, combined with oxide of myricyle, constitutes the chief part of myricine. But when the hydrated oxide of myricyle $\mathrm{C}_{60} \mathrm{H}_{61}$, or myricic alcohol is heated with lime and potash, it yields hydrogen gas, and melissate of the base. The acid, which is melissic acid, $\mathrm{C}_{60} \mathrm{H}_{59} \mathrm{O}_{3}{ }^{\prime}, \mathrm{H} \mathrm{O}=\mathrm{C}_{60} \mathrm{H}_{60} \mathrm{O}_{4}$, when separated, is a crystalline waxy substance, melting at $192^{\circ}$.

Hydrated Oxide of Myricyle. Syn. Melissine. Melissic Alcohol, $\mathrm{C}_{60} \mathrm{H}_{62} \mathrm{O}_{2}=\mathrm{C}_{60} \mathrm{H}_{61} \mathrm{O}, \mathrm{H} \mathrm{O}$. The oxide of the positive radical $\mathrm{C}_{60} \mathrm{H}_{61}$, is found in myricine, which forms about four-fifths of bees-wax, combined with palmitic acid. Separated as above, the oxide forms the hydrate, which is a waxy body, melting at $184^{\circ}$. It is a true alcohol, and like common alcohol, it yields a carbohydrogen, homologous with olefiant gas. This is called melene, and its formula is $\mathrm{C}_{60} \mathrm{H}_{60^{\circ}}$. This body and cerene, $\mathrm{C}_{54} \mathrm{H}_{54}$, have the properties of paraffine, and it is probable that several others, homologous with these, have been included under that name. The anhydrous oxide of the negative radical melissyle, $\mathrm{C}_{60}$ $\mathrm{H}_{59}$, is not known, nor the hydrate of the oxide of the negative radical $\mathrm{C}_{60} \mathrm{H}_{59}$, the melissic aldehyde. But Brodie obtained a substitution-product, belonging to the latter, namely, chloromelal, $\left.\mathrm{C}_{60} \begin{array}{l}\mathrm{H}_{45} \\ \mathrm{Cl}_{15}\end{array}\right\} \mathrm{O}_{2}$.

It will be seen that even here, at the very highest known part of the series, the analogy of homologous compounds prevails. We have cerotic and myricic alcohols, analogous to methylic and ethylic alcohols, while amylic alcohol lies nearer to the latter, and cetylic alcohol lies between it and the highest ones. And for every one of all the known alcohols, we have the acid, while we can trace the 
analogy also in the series of the ethers, the aldehydes and the bases, as well as the carbo-hydrogens and other derivatives. In the acids alone, however, as we have seen, is the series at all complete, but the fact of the analogy continuing in the acids, alcohols, \&c., so high as we have seen it, is sufficient to convince us that all the intermediate compounds, in the large table, are attainable. In fact, new ones are every day discovered.

We have still, however, two very remarkable series to mention, which have only been recognised within a short period. These are, the oxalic acid series of bibasic acids, and the oleic acid series of monobasic oily acids, neither of which are volatile without alteration.

\section{SERIES OF OXALIC ACID.}

The general formula of this series is $\mathrm{C}_{n} \mathrm{H}_{n}-{ }_{4} \mathrm{O}_{6}, 2 \mathrm{H} \mathrm{O}$, and the acids of it are bibasic. It was stated, under oxalic acid, that it was probably, from its tendency to form acid salts and double salts, a bibasic acid. This is now ascertained, and the formula of oxalic acid is consequently $\mathrm{C}_{4} \mathrm{O}_{6}, 2 \mathrm{H} \mathrm{O}=$ $\mathrm{C}_{4} \mathrm{H}_{2} \mathrm{O}_{8}$. As there are 4 eqs. of carbon, of course the anhydrous acid cannot here contain hydrogen, but the next member of the series will be $\mathrm{C}_{6} \mathrm{H}_{2} \mathrm{O}_{6}, 2 \mathrm{HO}=\mathrm{C}_{6} \mathrm{H}_{4} \mathrm{O}_{8}$. This one, however, has not yet been obtained. The acids of this series are crystallisable, soluble in water, and have an acid taste. They are decomposed by heat, and generally yield among the products, carbonic acid, and a volatile acid of the formylic series.

This is exactly what their general formula might lead us to expect; for it will be seen, that the hydrated acids of this series contain the elements of a volatile acid with 2 eqs. of carbonic acid. Thus oxalic acid, $\mathrm{C}_{4} \mathrm{H}_{2} \mathrm{O}_{8}$, contains the elements of formic acid, $\mathrm{C}_{2} \mathrm{H}_{2} \mathrm{O}_{4}$, and $\mathrm{C}_{2} \mathrm{O}_{4}$, that is, 2 eqs. of carbonic acid, $2\left(\mathrm{CO}_{2}\right)$. Now oxalio acid, when heated, yields formic and carbonic acids. Oxalic acid having been already described, we have here only briefly to notice the other known acids of this series.

We must first, however, mention, that another fact shows the close relation between these non-volatile bibasic acids and the volatile monobasic acids of the formylic series. We have mentioned that many of the latter are formed when nitric acid acts on oils or on any of these oily volatile acids, or on oleic acid. Now, along with the volatile acids thus formed, among which are acetic, propylic, butyric, valerianic, caproic, œnanthylic, caprylic and pelargonic acids, there are formed several acids of the oxalic series, which remain in the liquid after the volatile acids have 
been distilled off. Among these are oxalic, succinic, suberic, lipic, adipic and pimelic acids. They would seem to be formed by the union of nascent carbonic acid with the nascent volatile acids.

Succinic Acid, $\mathrm{C}_{8} \mathrm{H}_{4} \mathrm{O}_{6}, 2 \mathrm{H} \mathrm{O}=\mathrm{C}_{8} \mathrm{H}_{6} \mathrm{O}_{8}=\overline{\mathrm{S}} 2 \mathrm{H} \mathrm{O}$. This acid exists ready formed in amber, and may be obtained by distilling that body. But the mother liquor of the suberic acid, formed from margaric acid, \&c., by nitric acid, contains a large quantity of succinic acid, along with a little suberic acid. The mixture, being dried up, is acted on by ether, which dissolves the suberic acid, leaving the succinic acid; it is finally purified by sublimation. Succinic acid is also formed from malic acid by fermentation, as will be hereafter explained.

It forms regular crystals, which may be easily sublimed, but not without some change, for it loses water. The formula of the sublimed crystals is ( $\left.\mathrm{C}_{8} \mathrm{H}_{4} \mathrm{O}_{6}\right)+\mathrm{H} \mathrm{O}$; but by repeated sublimation it may be obtained anhydrous. The first hydrate $\overline{\mathrm{S}}, 2 \mathrm{H} 0$, melts at $356^{\circ}$, and boils at $455^{\circ}$, subliming, however, slowly at $284^{\circ}$. The sublimed hydrate, $2 \mathrm{Su}+\mathrm{H} 0$, melts at $320^{\circ}$, and boils at $468^{\circ}$; and the anhydrous acid melts at $257^{\circ}$, and boils at $482^{\circ}$.

By the action of anhydrous sulphuric acid, it yields a new acid, apparently $\mathrm{C}_{8} \mathrm{H}_{2} \mathrm{~S}_{2} \mathrm{O}_{10}, 4 \mathrm{H} \mathrm{O}$, hyposulphosuccinio acid.

The constitution of the succinates is bibasic. The most recent researches of Fehling, who has twice examined these salts with care, lead to the general formulæ of $\mathrm{C}_{8} \mathrm{H}_{4} \mathrm{O}_{6}, 2 \mathrm{M} \mathrm{O}$, for the neutral, and $\mathrm{C}_{8} \mathrm{H}_{4} \mathrm{O}_{6}, \mathrm{H} \mathrm{O}, \mathrm{M} \mathrm{O}$ for the acid salts. The succinates of lead present some anomalies. The succinate of methyle, $\mathrm{C}_{8} \mathrm{H}_{4} \mathrm{O}_{6}, 2 \mathrm{Mt} \mathrm{O}$, is a volatile crystalline solid. Succinute of ethyle, $\mathrm{C}_{8} \mathrm{H}_{4} \mathrm{O}_{6}, 2 \mathrm{Ae} 0$, is an oily liquid, boiling at $417^{\circ} \mathrm{F}$. When this compound is acted on by ammonia, it yields succinamide, $\mathrm{C}_{8} \mathrm{H}_{4} \mathrm{O}_{4}, 2 \mathrm{~N} \mathrm{H}_{2}$, which is succinate of ammonia, minus 4 eq. of water. It is a crystalline solid, having the usual properties of an amide. There exists also an acid amide, or succinamic acid, $\mathrm{C}_{8} \mathrm{H}_{5} \mathrm{O}_{6}, \mathrm{~N} \mathrm{\textrm {H } _ { 2 }}$, which is acid succinate of ammonia, minus 2 eq. of water. And there is a third amide, succinimide, $\mathrm{C}_{8} \mathrm{H}_{5} \mathrm{~N} \mathrm{O}_{4}$, which is acid succinate of ammonia, minus 4 eq. of water. Succinamic acid is only known in combination with oxide of silver. Succinimide is formed when ammonia acts on anhydrous succinic acid. It forms beautiful rhombic tabular crystals, very soluble in water. With aniline (phenylamine) succinic acid yields anilides analogous to the amides just mentioned.

Succinic acid and its ethers and amides, yield substitution-products with chlorine, which our limits prevent us from more fully describing. 
The origin of amber is very uncertain; but it is most probably derived from some resin, formerly liquid or soft. It may possibly have arisen from the slow oxidation of a fatty matter, as we see succinic acid formed from fats by oxidation. Amber is a clear brittle yellow solid, becoming electric by friction. It is for the most part insoluble in all menstrua. When heated, it yields succinic acid and a volatile oil, and there is left a large proportion of a matter which may be called bituminous, and forms the principal part of the amber.

Lipic Acid, $\mathrm{C}_{20} \mathrm{H}_{6} \mathrm{O}_{6}, 2 \mathrm{H} \mathrm{O}=\mathrm{C}_{20} \mathrm{H}_{8} \mathrm{O}_{8}$. This acid is homologous with oxalic and succinic acids. It is found among the products of the oxidation of oils or oily acids by nitric acid along with other acids both of the oxalic and formic series. It is crystalline, soluble in water. Lipic acid has not been much studied.

Adipic Acid, $\mathrm{C}_{22} \mathrm{H}_{8} \mathrm{O}_{6}, 2 \mathrm{H} \mathrm{O}=\mathrm{C}_{22} \mathrm{H}_{10} \mathrm{O}_{8}$. This acid is found with the preceding ones among the products of the action of nitric aoid on oils and oily acids. It is also crystalline, and soluble in water, but has not been minutely examined. Adipate of ethyle has a strong fragrance of rennet apples.

Pimelic Acid, $\mathrm{C}_{14} \mathrm{H}_{10} \mathrm{O}_{6}, 2 \mathrm{H} \mathrm{O}=\mathrm{C}_{14} \mathrm{H}_{12} \mathrm{O}_{8}$. Found with the preceding acids. It appears in the form of minute crystalline grains. It melts at about $237^{\circ} \mathrm{F}$, and volatilises at a higher temperature. It is very soluble in hot water. When heated with potash, hydrogen is given off, and the residue, acted on by sulphuric acid, yields a volatile acid, apparently valerianic acid, or a mixture of it with others of the same series.

Suberic Acid, $\mathrm{C}_{16} \mathrm{H}_{12} \mathrm{O}_{6}, 2 \mathrm{HO}=\mathrm{C}_{16} \mathrm{H}_{14} \mathrm{O}_{8}=\overline{\mathrm{Su}}, 2 \mathrm{H} \mathrm{O}$. This acid is formed when cork is oxidised by nitric acid, but especially when nitric acid acts on stearic acid, margaric acid, oleic acid, and other fatty bodies. The acid solution, obtained by boiling stearic or margaric acid with nitric acid till it is entirely dissolved, is evaporated to one half, and on cooling deposits a large quantity of suberic acid, which is easily purified by crystallisation.

It forms small granular crystals, fusible, when moist, at $130^{\circ}$, when dried, at $248^{\circ}$, volatile at a higher temperature, and subliming in the form of long needles. It is sparingly soluble in cold water, very soluble in hot water, in alcohol, and ether.

The general formula of the suberates is $\overline{\mathrm{Su}}, 2 \mathrm{M} 0$. The suberate of oxide of ethyle is prepared like the ethers of all the fatty acids, by passing hydrochloric acid gas through the alcoholic solution of the acid. When suberate of lime is distilled, it yields, among other oily products, a liquid boiling at $366^{\circ}$, the formula of which is $\mathrm{C}_{16} \mathrm{H}_{14} \mathrm{O}_{2}$, or $\mathrm{C}_{14} \mathrm{H}_{12} \mathrm{O}_{2}$. It is converted into suberic 
acid by the action of the air and of nitric acid. The nature of this compound is not yet ascertained.

Suberic acid gives rise to suberamide, $\mathrm{C}_{16} \mathrm{H}_{12} \mathrm{O}_{4}, 2 \mathrm{~N} \mathrm{H}=$ $\mathrm{C}_{16} \mathrm{H}_{16} \mathrm{~N}_{2} \mathrm{O}_{4}$, formed when ammonia acts on suberate of ethyle to suberanilide, an analogous compound, formed by the action of aniline (phenylamine) on suberic ether; and suberanilic acid, an acid anilide, formed along with the preoeding compound. The suberamic acid is not yet known.

Sebacic Acid, $\mathrm{C}_{20} \mathrm{H}_{16} \mathrm{O}_{6}, 2 \mathrm{H} \mathrm{O}=\mathrm{C}_{20} \mathrm{H}_{18} \mathrm{O}_{8}$. This, the next known acid of the oxalic series, and as yet the highest in the series, is formed in the distillation of oleic acid and of all oils containing that acid. It is soluble in hot water, sparingly soluble in cold water, and therefore when the products of the distillation are boiled with water, the filtered liquid deposits, on cooling, nearly the whole of the sebacio acid, in light feathery masses of crystals somewhat resembling sublimed benzoic acid. The presence of this acid among the products of distillation of oils is a sure proof and a delicate test of the presence of oleine or of oleic acid in the oil distilled.

A more productive process is to heat ricinolic acid (see castor oil) with half its weight of caustic potash, or castor oil may be at once treated in the same manner. Hydrated oxide of octyle, or caprylic alcohol, distils over, and sebate of potash is left.

Ricirolic acid. Sebate of potash. Caprylic acid. $\mathrm{C}_{36} \mathrm{H}_{34} \mathrm{O}_{6}+2(\mathrm{~K} \mathrm{O}, \mathrm{H} \mathrm{O})=\mathrm{C}_{20} \mathrm{H}_{16} \mathrm{O}_{6}, 2 \mathrm{~K} \mathrm{O}+\mathrm{C}_{16} \mathrm{H}_{18} \mathrm{O}_{2}+\mathrm{H}_{2}$.

The sebate, decomposed by sulphuric acid, yields the acid in abundance.

Sebacic acid melts at $81^{\circ}$, and is volatilised at a higher temperature. Its vapour irritates strongly the respiratory organs. When fused with potash, the residue yields, by the action of sulphuric acid, a volatile oily acid, or a mixture of such acids. Nitric acid acts on it slowly, and converts it into succinio acid according to some, while others allege that it yields pyrotartaric acid, $\mathrm{C}_{10} \mathrm{H}_{8} \mathrm{O}_{8}$. It will be observed that pyrotartaric acid has the same formula as lipic acid, and as it is also bibasic, it may really be identical with lipic acid, or they may only be isomeric. But it is easy to see how nitric acid should form both lipic, or pyrotartaric and succinio acids from sebacic acid, just as it forms from margaric or capric acid, volatile acids of the same series, lower in the scale, by removing part of the carbon.

The sebates are either neutral $\mathrm{C}_{20} \mathrm{H}_{16} \mathrm{O}_{6}, 2 \mathrm{MO}$, or acid, $\mathrm{C}_{20} \mathrm{H}_{10} \mathrm{O}_{0}, \mathrm{HO}, \mathrm{MO}$.

Sebate of ethyle, or sebacic ether, $\mathrm{C}_{20} \mathrm{H}_{16} \mathrm{O}_{6}, 2 \mathrm{A \theta} 0$, is a U 2 
volatile ether, which has a strong and pleasant fragrance of pine apple.

When sebacic ether is acted on by ammonia, it yields sebamide $\mathrm{C}_{20} \mathrm{H}_{20} \mathrm{~N}_{2} \mathrm{O}_{4}$, which is neutral sebate of ammonia, minus 4 eq. of water; and sebamic acid, $\mathrm{C}_{20} \mathrm{H}_{19} \mathrm{NO}_{6}$, which is acid sebate of ammonia minus 2 eq. of water. These compounds have the reactions of amides.

We have now mentioned all the known acids of the oxalic series, and it will be seen by the following table, that they correspond each to a volatile acid of the formylic series, containing 2 eq. of carbon less.

Thus we see that if we add to the acids of the formylic series 2 eq. of carbonic acid, we have the formula of the acids of the oxalic series.

Monobasic Volatile Acids.

Bibasic Acids.

\begin{tabular}{|c|c|c|}
\hline $\begin{array}{l}\text { Formic Acid } \\
\text { Acetic Acid } \\
\text { Propylic Acid }\end{array}$ & $\begin{array}{lllll}\mathrm{C}_{2} & \mathrm{H}_{2} & \mathrm{O}_{4}+2 \mathrm{C} \mathrm{O}_{2}=\mathrm{C}_{4} & \mathrm{H}_{2} & \mathrm{O}_{8} \\
\mathrm{C}_{4} & \mathrm{H}_{4} & \mathrm{O}_{4}+2 \mathrm{C} \mathrm{O}_{2}=\mathrm{C}_{6} & \mathrm{H}_{4} & \mathrm{O}_{8} \\
\mathrm{C}_{8} & \mathrm{H}_{6} & \mathrm{O}_{4}+2 \mathrm{C} \mathrm{O}_{2}=\mathrm{C}_{8} & \mathrm{H}_{6} & \mathrm{O}_{8}\end{array}$ & $\begin{array}{l}\text { Oxalic Acid } \\
\text { Unknown } \\
\text { Succinic Acid }\end{array}$ \\
\hline Butyric Acid & $2 \mathrm{C} \mathrm{O}_{2}=\mathrm{C}_{10} \mathrm{H}_{8}$ & $\begin{array}{l}\text { Lipic Acid } \\
\text { Pyrotartari }\end{array}$ \\
\hline $\begin{array}{l}\text { Valerianic Acid } \\
\text { Caproic Acid } \\
\text { Enanthylic Acid } \\
\text { Caprylic Acid } \\
\text { Pelargonic Acid }\end{array}$ & $\begin{array}{l}\mathrm{O}_{4}+2 \mathrm{C} \mathrm{O}_{2}=\mathrm{C}_{12} \mathrm{H}_{1} \\
\mathrm{O}_{4}+2 \mathrm{C} \mathrm{O}_{2}=\mathrm{C}_{14} \mathrm{H}_{1} \\
\mathrm{O}_{4}+2 \mathrm{C} \mathrm{O}_{2}=\mathrm{C}_{16} \mathrm{H}_{2} \\
\mathrm{O}_{4}+2 \mathrm{C} \mathrm{O}_{2}=\mathrm{C}_{18} \mathrm{H}_{1} \\
\mathrm{O}_{4}+2 \mathrm{C} \mathrm{O}_{2}=\mathrm{C}_{20} \mathrm{H}_{1}\end{array}$ & $\begin{array}{l}\text { Adipic Acid } \\
\text { Pimelic Acid } \\
\text { Suberic Acid } \\
\text { Unknown } \\
\text { Sebacic Acid. }\end{array}$ \\
\hline
\end{tabular}

It cannot be doubted that the bibasic acids, corresponding to acetic and œnanthylic acids, as well as others higher in the scale, will in time be discovered. We have already pointed out that pyrotartaric acid is either isomeric or identical with lipic acid, and an acid produced by the action of nitric acid on rhodeoretic acid (a body formed by the action of bases on the resin of jalap), which has been called ipomic acid, is isomeric at least, and probably identical, with sebacic acid. Oxalic acid, as is well known, occurs frequently in nature.

\section{OLEIC ACID SERIES.}

General formula $\mathrm{Cn} \mathrm{Hn}_{2} \mathrm{O}_{4}$, or $\mathrm{Cn}_{n} \mathrm{Hn}_{3} \mathrm{O}_{3}, \mathrm{H} \mathrm{O}$.

Of this series a few acids are known. They all contain 2 eqs. of carbon more than the corresponding acid of the formylic series.

Acrylic Acid, $\mathrm{C}_{6} \mathrm{H}_{3} \mathrm{O}_{3}, \mathrm{H} \mathrm{O}=\mathrm{C}_{6} \mathrm{H}_{4} \mathrm{O}_{4}$. This acid is formed by the oxidation of acroleine (See under glycerine), by means of oxide of silver. Acrylic acid is a limpid liquid, of a pungent but 
agreeable acid odour, not unlike that of acetic acid. It is miscible with water in all proportions, and boils at a temperature above $212^{\circ}$. Its salts are monobasio, and resemble the acetates and formiates. The acrylate of silver detonates when heated. Acrylic acid is easily oxidised and decomposed. Acrylic ether is not known in a state of purity, but appears to have the odour and taste of radish.

Angelic Acid, $\mathrm{C}_{10} \mathrm{H}_{7} \mathrm{O}_{3}, \mathrm{HO}$, is found in the root of angelica and of other umbelliferæ. It is extracted by water, then combined with potash, evaporated to expel volatile oils, and the residue distilled with sulphurio acid and water. At first, acetic and valerianic acids pass over, then angelic acid, whioh condenses in the neck of the retort. Water is added to the residue, and the distillation continued as long as any angelic acid passes over with the water.

The acid may also be obtained by the action of caustic potash on oil of camomile, taking care to avoid too strong a heat, as the acid is itself decomposed by potash.

It forms fine crystals, fusible at $113^{\circ}$ F., boiling at $374^{\circ}$. Heated with potash, it yields acetate and propylate of potash, and hydrogen gas.

Moringic Acid, $\mathrm{C}_{30} \mathrm{H}_{27} \mathrm{O}_{3}, \mathrm{H} \mathrm{O}=\mathrm{C}_{30} \mathrm{H}_{28} \mathrm{O}_{4}$. This acid occurs, along with benic, behenic, and margario acids, in the oil of ben, which is that of the fruit of Moringa nux behen, or $\mathbb{M}$. oleifera. It is a liquid, and slightly acid, very soluble in alcohol. At $32^{\circ}$ it solidifies. It is but little known.

Oleic Acid, $\mathrm{C}_{36} \mathrm{H}_{33} \mathrm{O}_{3}, \mathrm{H} \mathrm{O}=\mathrm{C}_{36} \mathrm{H}_{34} \mathrm{O}_{4}$. This is the most important and abundant acid of the series named from it. It occurs in almost all the animal and vegetable oils which are not drying oils, in the form of oleine, (a compound of the acid with glycerine) as their chief constituent, and in almost all similar vegetable fats in smaller proportion.

It is best obtained pure from almond oil, which is saponified by putash, the soap decomposed by an acid, and the mixed acids thus obtained are digested with half their weight of oxide of lead, with which they all combine. Ether now dissolves the acid oleate of lead, leaving the margarate or stearate of lead undissolved. Hydrochloric acid is added to the clear solution, and the oleic acid dissolves in the ether, while chloride of lead is deposited. The ether being distilled off, the acid is left, but is still coloured. By exposure to cold, the pure acid crystallises, and is expressed in the cold. Or the coloured acid is converted into oleate of baryta, which is purified by means of alcohol, and the pure salt decomposed by an acid.

Pure oleic acid is liquid above $57^{\circ} \mathrm{F}$., colourless, congealing in 
cold weather. When melted, it does not solidify till cooled to $40^{\circ}$; when solid, it does not melt till warmed to $57^{\circ}$. It rapidly absorbs oxygen from the air and becomes brown.

It is decomposed by heat, which is the case with all the acids of this series at all high in the scale. Among the products are acetic, caprylic, and capric acids, probably others of that series, but chiefly sebacic acid, the presence of which in the matter obtained by distilling any oil, is a sure test of the presence of oleine in that oil.

By contact with nitrous acid oleic acid is converted into elaidic acid, which is isomerio with it.

When fused with caustic potash, oleic acid is resolved into acetic and palmitic acids and hydrogen gas.

Oleic Acid.

Palmitate of Potash. Acetate of Potash.

$\mathrm{C}_{36} \mathrm{H}_{34} \mathrm{O}_{4}+2(\mathrm{~K} \mathrm{O}, \mathrm{H} \mathrm{O})=\mathrm{C}_{32} \mathrm{H}_{31} \mathrm{O}_{3}, \mathrm{~K} \mathrm{O}+\mathrm{C}_{4} \mathrm{H}_{3} \mathrm{O}_{3}, \mathrm{~K} \mathrm{O}+\mathrm{H}_{2}$.

Oleic acid, mixed with a good deal of margaric or stearic acid, is produced in large quantity in the manufacture of stearic acid for candles. This impure oleic acid is the liquid part expressed from the mass of oily acids which is formed when tallow suet or lard is saponified by lime and the soap decomposed by sulphuric acid. It may be purified as above directed; but in its impure state it is much used in making soaps.

When boiled with nitric acid, oleic acid yields many acids both of the formylic and oleic series, all of course lower in the scale than itself. Succinic and suberic acids are especially abundant in this process.

The oleates have the characters of soaps or plasters, according as they are soluble or insoluble in water. Oleate of potash is a soft soap, and is the chief ingredient of Naples soap. Oleate of soda, like soda soaps in general, is hard. Oleate of lead is an excellent plaster, being very fusible and adhesive. Oleate of ethyle is an oily liquid.

Sulphuric acid, when it acts on oils, forms an acid, sulpholeic acid, along with a sulphomargaric acid. These acids are not fully known. The former is said, by contact with water, to yield metoleic acid, and when boiled, to form another acid, called hydroleic acid. The composition of these acids is not ascertained.

Elaidic Acid. This acid, as already stated, is isomeric with oleic acid, and is formed from it by contact with nitrous or hyponitrous acid. It is solid, fusible at $113^{\circ} \mathrm{F}$. For this reason, oleic acid is solidified by contact with nitrous acid; and oleine, or oils containing oleine, are also rapidly solidified by 
contact with nitrous acid, or with a solution of nitrate of mercury, which yields nitrous acid. Elaidic acid may be distilled unaltered.

Oleine, the liquid principle of most natural oils and fats, and elaidine, into which it is converted by nitrous acid, are compounds of the acids with glycerine.

Oleic Acid of drying oils. Linoleic Acid. This acid, which occurs, combined with glycerine, in linseed oil, walnut oil, and the other drying oils, is different from oleic acid, though resembling it in external properties. It has been said to be $\mathrm{C}_{46} \mathrm{H}_{39} \mathrm{O}_{6}$, but this is highly doubtful.

The acid itself, and its glyceride or linoleine, attract oxygen from the air, and dry up into a resinous mass. Hence their use in painting.

Doeglinic Acid, $\mathrm{C}_{38} \mathrm{H}_{36} \mathrm{O}_{4}=\mathrm{C}_{38} \mathrm{H}_{35} \mathrm{O}_{3}, \mathrm{H} \mathrm{O}$, is formed in the oil of Balana rostrata, where it is combined, according to Scharling, not with glycerine, but with the oxide of the radical $\mathrm{C}_{24} \mathrm{H}_{25}$, which may be called dodecatyle, homologous with ethyle. When this oil is saponified, there are obtained the acid, which is oily, liquid at $62^{\circ}$, congealing several degrees above $32^{\circ}$; and the hydrated oxide of dodecatyle, or dodecatylio alcohol, $\mathrm{C}_{24} \mathrm{H}_{25} \mathrm{O}$, $\mathrm{H} \mathrm{O}=\mathrm{C}_{24} \mathrm{H}_{26} \mathrm{O}_{2}$.

Erucic Acid, or Brassic Acid, $\mathrm{C}_{44} \mathrm{H}_{42} \mathrm{O}_{4}=\mathrm{C}_{44} \mathrm{H}_{4 \mathrm{i}} \mathrm{O}_{3}, \mathrm{H} \mathrm{O}$. This acid is found in the fat oil of mustard, obtained by expressing the seeds, also in the oil of colza, which is obtained from the seeds of another cruciferous plant, Brassica campestris oleifera. It is solid, melts about $90^{\circ} \mathrm{F}$., and crystallises in needles from its alcoholic solution. The oil contains another acid, which is liquid at the ordinary temperature, but is different from oleic acid; most probably an acid of the oleic series. It is very probable that these acids, or others homologous with them, may be found in the seeds of other cruciferæ.

Let us briefly recapitulate the different classes of compounds treated of in this section, and represented in the table. This will best be done in a tabular form, and the reader will bear in mind that the vertical list here given corresponds to the horizontal line of the table of homologous compounds, which is only a selection from it, and that each number here given is the starting-point of a series of homologous compounds, the formulæ of which are obtained by simply adding $\mathrm{C}_{2} \mathbf{H}_{2}$ successively to the formulæ of the methyle compounds, here taken as the lowest in the series.

Now, when it is considered that all the above compounds, are known in the methyle and formyle series, and all in that of ethyle and acetyle; that each of these 39 compounds is the 


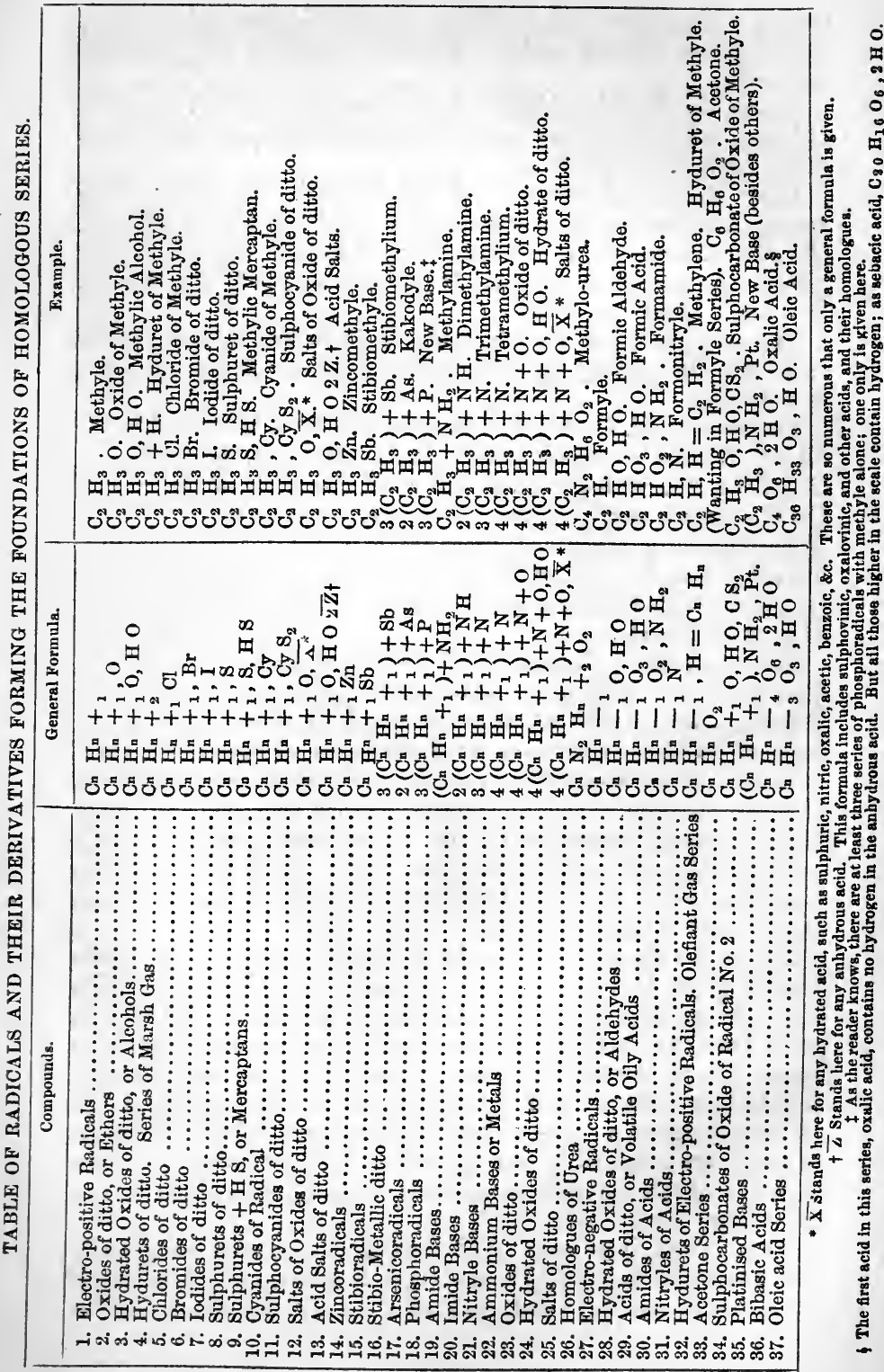


starting-point of a homologous series, like that of the fatty and oily acids, No. 29, which have been described; and that several of the numbers, namely, Nos. 12, 14, 15, 16, 17, 18, 19, 20, 21, 25 , and 35 being bases, combine with all acids, and that each salt thus formed is also the starting-point of a similar series; that the bodies under Nos. 13 (of which there are several, such as oxalovinic, tartrovinic, phosphovinic, and carbovinio acids, not mentioned in the table) and 29 , being acid, combine with all bases, and that each salt thus formed is the starting-point of a new homologous series; that the radicals and bases, from No. 14 to No. 22, all admit of substitution of one radical for another, yielding new bases, and consequently new salts ad infinitum; and finally, that probably every oompound in the table admits of the substitution of chlorine, bromine, iodine, and nitrous acid for part of its hydrogen, thus yielding other inexhaustible series of new compounds, it will be seen, that even this formidable list, and the large table, are nothing more than short extracts from the real list of possible and probable compounds, producible by art, besides the infinite variety of nature. And yet, bearing in mind the laws of homologous compounds, and of substitutions, the whole of this immense mass of formulæ become easily intelligible, insomuch that with a knowledge of one such series as has just been partially exemplified, any one may construct for himself a table including all, which, however, if printed at full length, even in the abbreviated shape of formulæ, would fill a volume. And all this refers only to the compounds connected with, or derived from, one series of radicals, that of methyle and ethyle. Nature, however, uses not only these, but many others, as yet less known to us, to produce her organised products. We trust that the study of this section will enable the student to grasp the principle, and to see his way through the seeming chaos of organio chemistry.

Of those radicals which do not belong to the series of ethyle and methyle (the best known), those most interesting are phenyle, $\mathrm{C}_{12} \mathrm{H}_{5}$, and its homologues, as given in the table, p. 141, with some of their derivatives. As these may all be derived from the oil of tar, we shall describe them and their derivatives under that head. We shall then see that these radicals agree with those of the methyle series in replacing hydrogen in ammonia, and in giving rise to a large number of bases and other interesting products.

Before quitting the subject of the radicals of the methyle and ethyle series, $\mathrm{Cn}_{\mathrm{n}} \mathrm{H}_{n}+1$, and their derivatives, it must be mentioned that different views have been taken of these compounds, besides that here adopted. Some consider alcohol and ether to be compounds of $\mathrm{C}_{4} \mathrm{H}_{4}$, or ethylene, with water: but this view 
is now generally abandoned. But the views taken by Kolbe, and those of Williamson, must be noticed. Kolbe admits the existence of the methyle series of radicals, but not that of the derived acid radicals of the formyle series, $\mathrm{C}_{n} \mathrm{H}_{n}-1$. According to him the acids of the formic series are coupled oxalic acids, the copula being in each case a radical of the methyle series. The following table exhibits together the view already adopted in this work, and that of Kolbe, for the six first acids of the series, $\left(\mathrm{Cn}_{\mathrm{n}} \mathrm{H}_{n}\right)_{2} \mathrm{O}_{4}$ :-

Kolbe.

Formic Acid $\mathrm{C}_{2} \mathrm{H}, \mathrm{O}_{3}, \mathrm{HO}=\mathrm{H}, \mathrm{C}_{2} \mathrm{O}_{3}, \mathrm{HO}=\mathrm{C}_{2} \mathrm{H}_{2} \mathrm{O}_{4}$ Acetic do. $\mathrm{C}_{4} \mathrm{H}_{3}, \mathrm{O}_{3}, \mathrm{HO}=\mathrm{C}_{2} \mathrm{H}_{3}, \mathrm{C}_{2} \mathrm{O}_{3}, \mathrm{HO}=\mathrm{C}_{4} \mathrm{H}_{4} \mathrm{O}_{4}$ Propylic do. $\mathrm{C}_{6} \mathrm{H}_{5}, \mathrm{O}_{3}, \mathrm{HO}=\mathrm{C}_{4} \mathrm{H}_{5}, \mathrm{C}_{2} \mathrm{O}_{3}, \mathrm{H} \mathrm{O}=\mathrm{C}_{6} \mathrm{H}_{6} \mathrm{O}$. Butyric do. $\mathrm{C}_{8} \mathrm{H}_{7}, \mathrm{O}_{3}, \mathrm{HO}=\mathrm{C}_{6} \mathrm{H}_{7}, \mathrm{C}_{2} \mathrm{O}_{3}, \mathrm{HO}=\mathrm{C}_{8} \mathrm{H}_{8} \mathrm{O}_{4}$ Valerianic do. $\mathrm{C}_{10} \mathrm{H}_{9}, \mathrm{O}_{3}, \mathrm{HO}=\mathrm{C}_{8} \mathrm{H}_{9}, \mathrm{C}_{2} \mathrm{O}_{3}, \mathrm{HO}=\mathrm{C}_{10} \mathrm{H}_{10} \mathrm{O}_{4}$ Caproic do. $\mathrm{C}_{12} \mathrm{H}_{14}, \mathrm{O}_{3}, \mathrm{HO}=\mathrm{C}_{10} \mathrm{H}_{11}, \mathrm{C}_{2} \mathrm{O}_{3}, \mathrm{HO}=\mathrm{C}_{12} \mathrm{H}_{12} \mathrm{O}_{4}$

It will be seen that in the first column the acid radicals formyle, acetyle, propionyle, butyryle, valeryle, and caproyle, are supposed to be united to 3 eqs. of oxygen and 1 of water; while in the second, hydrated oxalic acid, $\mathrm{C}_{2} \mathrm{O}_{3}, \mathrm{H} \mathrm{O}$, is supposed to be coupled, first with hydrogen, and then with the homologues of hydrogen, the basic radicals methyle, ethyle, propyle, butyle, and amyle. It is evident that either view sufficiently accounts for the great analogy of these acids among each other, and that the latter perhaps accounts better for the fact, that the ammonia salts of these radicals yield the cyanides of the basic radicals they are supposed to contain. Thus acetate of ammonia yields cyanide of methyle, propylate of ammonia, cyanide of ethyle, \&c. On the whole, it is impossible, as yet, to give to the one view a decided preference over the other; and it will be seen that Kolbe's view may easily be applied to all compounds supposed, on the other theory, to contain formyle, acetyle, and the radicals of that series. Kolbe has, indeed, with much ingenuity, extended it to all these and many analogous products, such as aldehyde, including those of substitution, chloral, chloracetic acid, and the chlorinised ethers generally. But our limited space forbids us to enter into these details, and we therefore stop, recommending to the student to bear in mind, that not only the doctrine of compound radicals, but also that of coupled compounds, probably, has a great share in the production of organic bodies.

Williamson follows Berzelius in not regarding alcohol as the hydrated oxide of ethyle, $\mathrm{C}_{4} \mathrm{H}_{5} \mathrm{O}, \mathrm{HO}=\mathrm{C}_{4} \mathrm{H}_{6} \mathrm{O}_{2}$, but as having only half this atomic weight, $\mathrm{C}_{2} \mathrm{H}_{3} \mathrm{O}$; and in consequence of some very remarkable discoveries of his own, he 
finds it necessary to halve the equivalent of hydrogen, and to write the formula of water $\mathrm{H}_{2} \mathrm{O}$, and that of alcohol $\mathrm{C}_{2} \mathrm{H}_{6} \mathrm{O}$. He supposes alcohol to be water, in which one half of the hydrogen is replaced by the body $\mathrm{C}_{2} \mathrm{H}_{5}$ (which, with the double or usual equivalent of hydrogen, would be $\mathrm{C}_{2} \mathrm{H}_{2} \frac{1}{2}$, hence the reason for halving the eq. of hydrogen), thus: water $\underset{H}{H}\} 0$; alcohol $\left.\underset{\mathrm{C}_{2}}{\mathrm{H}} \mathrm{H}_{5}\right\} 0$. According to this view, ether is water in which both the eqs. of hydrogen (that is, all the hydrogen) have been replaced by $\mathrm{C}_{2} \mathrm{H}_{5}$; water $\left.\underset{\mathrm{H}}{\mathrm{H}}\right\} 0$; ether $\left.\begin{array}{l}\mathrm{C}_{2} \\ \mathrm{C}_{2} \mathrm{H}_{5}\end{array}\right\} 0=\mathrm{C}_{4}$ $\mathrm{H}_{10} \mathrm{O}\left(=\right.$, in the ordinary form, $\mathrm{C}_{4} \mathrm{H}_{5} \mathrm{O}$ ).

We have already explained that if, without altering the atomic weight of hydrogen, we represent water thus: $\underset{H}{H}\} \stackrel{0}{0}$; alcohol

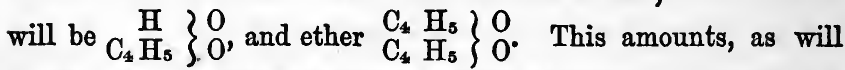
be perceived, to the same thing as Williamson's view, and it must be borne in mind, that many other facts render it probable that the true formula for water is a double one, or that the molecule of water, which is the most frequent type of organic compounds, contains 2 at. of hydrogen and 2 of oxygen.

The strongest arguments for this view are derived from the fact, observed by Williamson, that alcohol is converted into ether by the action of iodide of ethyle on a solution of potash in alcohol. According to the usual view, this change would be thus represented: $\mathrm{C}_{4} \mathrm{H}_{5} \mathrm{O}, \mathrm{KO}+\mathrm{C}_{4} \mathrm{H}_{5} \mathrm{I}=\mathrm{KI}+2\left(\mathrm{C}_{4} \mathrm{H}_{5} \mathrm{O}\right)$, the potash being supposed to have replaced the water of alcohol. Williamson supposes one-sixth of the hydrogen in alcohol to have been replaced by potassium, thus: $\mathrm{C}_{2} \mathrm{H}_{6} \mathrm{O}+\mathrm{KO}=\mathrm{HO}+$ $\left.\mathrm{C}_{2} \underset{\mathrm{K}}{\mathrm{H}} \mathrm{H}_{5}\right\} \mathrm{O}$ (halving the eq. of potassium). Then this compound, acted on by iodide of ethyle, which he makes $\mathrm{C}_{2} \mathrm{H}_{5} \mathrm{I}$ (halving the eq. of iodine also), yields ether with it, thus: $\left.{ }_{\mathrm{C}_{2}}^{\mathrm{H}_{\mathrm{K}}}\right\} \mathrm{O}+$

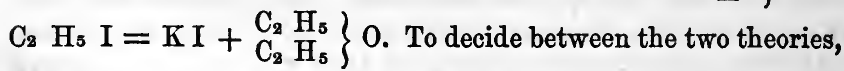
Williamson tried to replace one of the eqs. of hydrogen by methyle. If the ordinary view were right, he should have obtained a mixture of oxide of ethyle and oxide of methyle, by causing iodide of methyle to act on the potash compound of ether, $\mathrm{C}_{4} \mathrm{H}_{5} \mathrm{O}, \mathrm{KO}+\mathrm{C}_{2} \mathrm{H}_{3} \mathrm{I}=\mathrm{KI}+\mathrm{C}_{4} \mathrm{H}_{5} \mathrm{O}+\mathrm{C}_{2} \mathrm{H}_{3} \mathrm{O}$ (using the ordinary equivalents); but instead of this, he obtained a new alcohol, $\mathrm{C}_{3} \mathrm{Hs}_{3} \mathrm{O}$. This he explains as follows. In his 
notation, iodide of methyle is $\mathrm{CH}_{3} \mathrm{I}$. Hence $\left.\mathrm{C}_{2} \frac{\mathrm{H}_{5}}{\mathrm{~K}}\right\} 0+$ $\left.\mathrm{C} \mathrm{H}_{3} \mathrm{I}=\mathrm{KI}+\mathrm{C}_{\mathrm{C}}^{\mathrm{C}_{2} \mathrm{H}_{3}}\right\}$ o. This latter body, $\mathrm{C}_{3} \mathrm{H}_{8} \mathrm{O}$, in ordinary notation, $\mathrm{C}_{3} \mathrm{H}_{4} \mathrm{O}$ or $\mathrm{C}_{6} \mathrm{H}_{8} \mathrm{O}_{2}$, is a very remarkable new alcohol, and Williamson has obtained others of the same class, which, however, as well as the first, have not yet been fully described. These remarkable researches will probably hereafter lead to modifications of our views on many points; but, in the meantime, we shall adhere to the usual view, which, for the moment, is the best attainable, but which, like all theories, must in time give place to such as better agree with all known facts. A theory in chemistry is nothing more than such a view as groups together the largest number of facts at a given time, and has no pretensions to absolute truth, nor to be more than a guide to the enquirer, and an aid to his memory.

\section{NATURAL FIXED OILS AND FATS.}

We have already mentioned, under the oily acids of the formylic and oleic series, that the natural fixed oils and fats are neutral compounds of these acids with, for the most part, the basic compound glycerine, or oxide of glyceryle (to be presently described), and in a few cases with basic oxides of the series of oxide of ethyle. These neutral oils and fats are named according to the acids they contain. Thus, stearine, margarine, palmitine, oleine, caprine, valerine, and butyrine, are the compounds of glycerine with stearic, margaric, palmitic, oleic, capric, valerianic, and butyric acids. Cetine is the palmitate of oxide of eetyle; Chinese wax is the ceretate of oxide of ceryle. The part of bees-wax insoluble in alcohol is palmitate of myricyle, and the oil of the balæna rostrata, or beaked whale, contains with other oils the doglinate of oxide of dodecatyle.

The chief liquid fat oils and drying oils of the vegetable kingdom have already been mentioned. In the animal kingdom, there are fish oils, characterised by containing valerate of glycerine, or valerine, which gives the smell of train oil, and occurs also in cod-liver oil, \&c. \&c.

The solid oils or fats of the vegetable kingdom are, butter of eacao (Theobroma cacao); of nutmeg (Myristica moschata); of cocoa-nut (Cocos nucifera); of laurel (Laurus nobilis); palm-oil (Avoira elais : Elais Guianensis); galam butter (Bassia butyracea); and some others. Those of the animal kingdom are tallow, or suet, butter, hog's lard, human fat, \&c. 
Spermaceti is a peculiar fat found in the head of Physeter macrocephalus. When purified from a small quantity of a liquid oil, it constitutes cetine, which is a compound of oxide of cetyle with cetylic acid, and yields the hydrated oxyde of cetyle, or ethal, and cetylic acid, as formerly explained, when boiled with potash. Cetine crystallises beautifully when melted or when dissolved in hot alcohol.

Cholesterine is a fat found in bile, and also, in small proportion, in the blood, and in much larger quantity as an ingredient of cerebral matter. It forms the chief ingredient of biliary calculi. It dissolves in hot alcohol, crystallising on cooling in silvery scales, but cannot be saponified by boiling with potash. It will be described more fully under the head of bile. When acted on by nitric acid, it yields a new acid, cholesteric acid, which contains nitrogen, probably as nitrous acid.

Ambreine, a fat analogous to cholesterine, is found in ambergris. It yields, with nitric acid, ambreic acid. Castorine is a similar fat found in castoreum.

Wax is another peculiar fatty body, the origin of which is derived from flowers, whence it is collected by the bee. It melts at about $150^{\circ}$. It is a mixture of two fats, cerotic acid, and palmitate of oxide of melissyle, as formerly explained; the former soluble, the latter insoluble, in hot alcohol. When heated with nitric acid, wax is almost entirely converted into succinic acid.

Chinese wax consists of cerotate of oxide of ceryle alone.

Japan, or tree wax, is a true fat, composed of palmitic acid and glycerine without oleine. When acted on by nitric acid it yields, first pimelic and adipic acids, and then succinic acid, as in the case of spermaceti.

We shall return to the subject of the composition of the fixed oils under glycerine, the basic principle which they all contain.

Cerosine is the name given to a waxy substance occasionally found on the surface of the sugar-cane. It is not saponifiable, and appears to contain $\mathrm{C}_{48} \mathrm{H}_{48} \mathrm{O}_{2}$. It is probably an aldehyde, or if its formula be doubled, $\mathrm{C}_{96} \mathrm{H}_{96} \mathrm{O}_{4}$, it may be a compound ether.

Athamantine, from the root of Athamanta oreoselinum, is a crystalline fat-like body, containing valerianic acid, united to a body, oreoselone, which supplies the place of glycerine in the neutral athamantine. Oreoselone is $\mathrm{C}_{14} \mathrm{H}_{5} \mathrm{O}_{3}$, that is, isomeric with dry benzoic acid. Athamantine is $\mathrm{C}_{24} \mathrm{H}_{15} \mathrm{O}_{7}=\mathrm{C}_{14} \mathrm{H}_{5} \mathrm{O}_{3}$ (1 eq. oreoselone) $+\mathrm{C}_{10} \mathrm{H}_{10} \mathrm{O}_{4}$ (1 eq. valerianic acid). Athamantine combines with hydrochloric acid, and the compound, when boiled with water, deposits crystals, which are oreoselone 
plus water $=\mathrm{C}_{14} \mathrm{H}_{6} \mathrm{O}_{4}$, and isomeric with crystallised benzoic acid.

These neutral oils or fats never occur singly, for suet and tallow consist of stearine, margarine, and oleine ; lard and human fat of margarine and oleine; olive and almond oils of the same two fats in different proportions ; butter of margarine or palmitine, oleine, and small quantities of caprine, capryline, caproine, and butyrine; train oil of oleine, with a little of some solid fat and a little valerine, as does also cod-liver oil; palm-oil of palmitine and oleine, and so on.

The compounds of glycerine, which constitute the great majority of fats and fixed oils, are called glycerides. They are all saponified by boiling with potash or soda, which unite with the acids to form soaps, while the glycerine is set free, taking up the elements of water.

The glycerides of the fatty acids high in the scale, above $\mathrm{C}_{20}$, are devoid of taste and smell when pure, but by long exposure to air are in part decomposed, the acids being to some extent liberated, when they acquire a rancid taste and smell.

Those of the acids lower in the scale have generally some smell and taste: thus the flavour of butter is due to the small properties of butyrine, caproine, \&c., which it contains; and that of train oil and cod-liver oil arises from the presence of valerine.

The 'glycerides are generally insoluble in water, but very soluble in ether or turpentine, sparingly soluble in cold alcohol.

They are all decomposed by heat, those which contain oleic acid yielding sebacic acid, and all glycerides whatever yielding the pungent suffocating vapours of acroleine. (See below.)

Ammonia not only saponifies the glycerides, but also acts on the acids, forming amides, such as margaramide.

Sulphuric acid decomposes them, producing sulphoglycerie acid, and compounds of sulphuric acid with the fatty acids, which are not stable, and are spontaneously decomposed by contact with water.

The action of nitric acid on the glycerides is violent, and it oxidises both the glycerine and the acid. The products of the latter action, as mentioned under the various acids, are chiefly carbonic acid and various acids both of the formylic and oxalic series, especially succinic and suberic acids.

It is unnecessary to describe individually the numerous oils, both animal and vegetable, all of which have the general characters here mentioned. But one oil is so peculiar that it deserves a special notice. This is castor-oil, the oil of Ricinus communis.

Castor-oil is a glyceride, or rather a mixture of glycerides, the acids of which are in part peculiar. When saponified, and the 
soap decomposed by an acid, there is obtained an oily acid liquid, chiefly formed of ricinolic acid, $\mathrm{C}_{36} \mathrm{H}_{34} \mathrm{O}_{8}=\mathrm{C}_{36} \mathrm{H}_{33} \mathrm{O}_{5}, \mathrm{H} \mathrm{O}$, and a small portion of a solid acid, apparently a mixture of stearic and palmitic acids.

Ricinolic acid is an oily liquid which by intense cold congeals into spherical concretions. Its salts with the alkalies are soaps. The ricinolate of ethyle is oily. Ricinolamide is a crystalline body.

When heated, ricinolic acid is deoomposed, yielding hydrated oxide of œnanthyle or œnanthylic aldehyde, with some œnanthylic acid.

When heated with caustic potash, ricinolic acid is resolved into hydrated oxide of octyle, or caprylic alcohol, sebacic acid, and hydrogen.

Ricinolic acid. Sebate of Potash. Caprylic alcohol. $\mathrm{C}_{36} \mathrm{H}_{34} \mathrm{O}_{6}+2(\mathrm{~K} \mathrm{O}, \mathrm{H} \mathrm{O})=\mathrm{C}_{20} \mathrm{H}_{16} \mathrm{O}_{6}, 2 \mathrm{KO}+\mathrm{C}_{16} \mathrm{H}_{18} \mathrm{O}_{2}+\mathrm{H}_{2}$

By contact with hyponitrous acid, ricinolio acid is transformed into a solid acid isomerio with it, ricinelaidic acid. It is decomposed by heat in the same way as ricinolic acid.

Castor-oil, when distilled alone or with potash, yields the same products as ricinolic acid, along with those formed by the action of heat on glycerine and on the solid acids of the oil. By hyponitrous acid, or nitrate of mercury, castor-oil, or rather the ricinoline in it, is converted into the solid ricinelaidine, which has a waxy consistence.

\section{DRYING OILS.}

These oils, of which linseed oil, walnut oil, and hempseed oil are the most important, contain an oleine, the acid of which, as before stated, differs from common oleic acid. They attract oxygen rapidly from the air, and dry into a varnish. This change is promoted by first heating the oil with litharge, which removes mucus, \&c. The presence of some foreign substance in small quantity seems, however, to be favourable to the desiccation of these oils.

The fixed or fat oils in general are apt to become rancid when kept. This is caused by the presence of traces of albuminous matters, which act as ferments, and cause the glycerides to be gradually resolved into free glycerine and free acids; and as most fats contain a little of the glycerides of some of the more volatile oily acids, such as butyric, caproic, valerianic, caprylic or capric acids, and as these acids, when free, have a strong rancid odour, 
oils which have been thus decomposed become rancid. Pure stearine, margarine, palmitine, or oleine, do not undergo this change, which is particularly apt to occur in butter, lard, and olive-oil, because these fats and oils all contain some albumen, fibrine or caseine.

\section{ACTION OF BASES ON FAT OILS. SOAPS AND PLASTERS.}

When fat oils are boiled with solution of caustic alkalies, they are gradually dissolved in the water, if there be not too great an excess of alkali present, forming ropy or gelatinous solutions, which gelatinise on cooling. These are solutions of soaps, that is, potash and soda salts of the fatty acids, along with the glycerine set free. In order to have the soaps in a solid form, the solutions are boiled down, and when the alkali reaches a certain concentration, the soap becomes insoluble, and rises to the surface in a soft, half melted state. This is drawn off into moulds, and the mass formed on cooling is soap. Another method of causing the soap to separate from the water in which it is dissolved, consists in adding sea-salt, which at once coagulates the soap, converting it into a soap of soda, if it be a soap of potash. Of course, the glycerine, in both cases, is carried off in the mother liquid. Such is the theory of soap-making, which is very simple, depending on the affinity between the alkalies and the fat acids ; on the solubility in water of the alkaline stearates, margarates, oleates, palmitates, \&c. ; and, finally, on the power of a certain amount of free alkali or of sea-salt to coagulate the soap and render it insoluble in the liquid in which it swims, and which in fact runs off its surface as water does off the surface of fat, while yet the soap retains perfectly its solubility in pure water.

The soaps of lime, baryta, \&c., are insoluble in water, and have no detergent power: hence the waste occasioned by using hard, that is, calcareous, water for washing. All the salts of lime in such water must first be entirely precipitated in the form of curdy flocculi before any soap can be dissolved so as to act as a detergent.

The soaps of potash are soft, compared with those of soda, which are called hard soaps. White soap is a stearate with some oleate of soda. Naples soap is oleate and margarate of potash. Common soft soap is chiefly oleate of potash, but as it is made from whale oil or seal oil, it contains also valerate of potash, derived from the valerine, which gives it a disagreeable smell.

Castile soap is oleate and margarate of soda, coloured by metallic oxides, chiefly oxides of iron, in such a way as to give the desired 
mottled appearance. Much and excellent soap is now made of palm oil, and is, therefore, palmitate of soda.

Soaps are soluble in alcohol, forming tincture of soap, which is an admirable liniment for bruises, and is much used along with laudanum, as tincture of soap and opium; also with camphorated spirit, forming opodeldoo.

Plasters are soaps of certain metallic oxides, chiefly oxide of lead, which are insoluble in water, but fusible, and possess useful properties, Litharge plaster is made by boiling 5 parts of finely powdered oxide of lead with 9 parts of olive oil and some water, till the combination is complete. It is plastio at ordinary temperatures, and melts when heated. When solution of acetate of lead is added to solution of soap, plaster, that is, oleate and margarate of lead, is precipitated. When prepared in this way it becomes hard. White-lead plaster, made with carbonate of lead, is very plastic and fusible, and is much used. Iron plaster and mercurial plaster are of small importance.

\section{GhyorRyle, $\quad \mathrm{C}_{6} \mathrm{H}_{7}=\mathrm{Gl}$.}

This is the hypathetical radical of glycerine, a basio compound which exists in all neutral fat oils combined with oily acids.

Hydrated Oxide of Glyceryle. $\mathrm{C}_{6} \mathrm{H}_{7} \mathrm{O}_{5}+\mathrm{HO}=\mathrm{C}_{6} \mathrm{H}_{8} \mathrm{O}_{6}$.

SxN. Glycerine. To obtain it, olive oil is converted into plaster by long boiling with litharge and water. When the plaster is completely formed, the glycerine is found dissolved in the water. It is purified from lead by sulphuretted hydrogen, and is then concentrated in the vapour bath and finally in vacuo. When pure, it forms a viscid syrup, colourless or slightly yellow. It has a decided sweet taste, and its Sp. G. is 1י252. By the action of heat it is decomposed, yielding a peculiar volatile compound, acroleine, which attacks the eyes most powerfully.

With sulphuric acid, glycerine forms an acid sulphate $\mathrm{C}_{6} \mathrm{H}_{7} \mathrm{O}_{5}$, $\mathrm{H} 0,2 \mathrm{~S} \mathrm{O}_{3}$; which forms double salts, analogous to the sulphovinates, the formula of which is, $\mathrm{M} \mathrm{O}, \mathrm{C}_{6} \mathrm{H}_{7} \mathrm{O}_{5}, 2 \mathrm{~S} \mathrm{\textrm {O } _ { 3 }}$.

In contact with yeast, a solution of glycerine undergoes fermentation, yielding much propylic acid and a little formic and acetic acid. In this process the glycerine is dehydiated.

$$
\begin{aligned}
& \text { Glycerine. } \\
& \mathrm{C}_{6} \mathrm{Hs}_{6}-2 \mathrm{H} \mathrm{O}=\mathrm{C}_{6} \mathrm{H}_{6} \mathrm{O}_{4} \text {. }
\end{aligned}
$$

When gently heated with potash, glycerine yields acetic and formic acids and hydrogen.

With anhydrous phosphoric acid glyoerine loses 4 eq. of water, forming acroleine, $\mathrm{C}_{6} \mathrm{H}_{4} \mathrm{O}_{2}$. 
With hydrated acids it unites, forming compounds, which may be called Glycerides, many of which have the properties of fixed oils.

A mixture of nitric and sulphuric acids converts glycerine into a yellow oil, of a sweet aromatic taste, but which, when a drop is placed on the tongue, causes subsequently a severe migraine. This is nitroglycerine, a liquid so explosive, that if paper, moistened with one drop of it, be placed on an anvil and struck by a hammer, it explodes with a deafening report. It is glycerine, in which 3 eq. of hydrogen have been replaced by 3 eq. of $\mathrm{N}_{4}$, and its formula, therefore, is $\left.\begin{array}{c}\mathrm{C}_{6} \mathrm{H}_{5} \\ 3 \mathrm{NO}_{4}\end{array}\right\} \mathrm{O}_{6}$.

\section{GLYCERIDES. COMPOSITION OF THE FIXED OILS.}

For a long time it was found impossible to cause glycerine again to unite with the oily acids with which it had been found combined in fixed oils, such as stearine, margarine, or oleine. But Pelouze and Gelis succeeded in reproducing butyrine, and Berthelot has shown that nearly all acids may be made to combine with glycerine, the elements of water being always separated, if kept in contact with it at a somewhat high temperature. Or the acid may be added to the glycerine, and hydrochloric acid gas passed through the mixture. The compounds thus formed are called Glycerides, and are of an oily character, and neutral. They are saponified by the alkalies.

The artificial glycerides commonly contain 1 eq. of acid to 1 eq. of glycerine; but the natural ones often contain 3 or 4 eq. of acid to 1 eq. of glycerine. Some of these have been also formed artificially. They are all named, for brevity, from the acid, as stearine, oleine, \&c.

Chlorhydrine is the hydrochlorate $\mathrm{C}_{6} \mathrm{H}_{8} \mathrm{O}_{6}+\mathrm{H} \mathrm{Cl}-2 \mathrm{H} \mathrm{O}=$ $\mathrm{C}_{6} \mathrm{H}_{7} \mathrm{Cl} \mathrm{\textrm {O } _ { 4 }}$. It is a neutral oil of a sweet and pungent taste, and a cooling smell, boiling at $440^{\circ} \mathrm{F}$. There are, in all, three compounds of glycerine with hydrochloric acid. That just described is monochlorhydrine. Dichlorhydrine is $\mathrm{C}_{6} \mathrm{H}_{6} \mathrm{Cl}_{2} \mathrm{O}_{2}=$ $\mathrm{C}_{6} \mathrm{H}_{8} \mathrm{O}_{6}+2 \mathrm{H} \mathrm{Cl}-4 \mathrm{H} \mathrm{O}$. Epichlorhydrine is $\mathrm{C}_{6} \mathrm{H}_{5} \mathrm{Cl} \mathrm{O}_{2}$ $=\mathrm{C}_{6} \mathrm{H}_{8} \mathrm{O}_{6}+\mathrm{H} \mathrm{Cl}-4 \mathrm{H} \mathrm{O}$. The former is an ethereal neutral oil, boiling at $353^{\circ}$. The latter is a heavy ethereal oil, boiling at $256^{\circ}$.

Acetines. There are likewise three of these :

Monacetine, $\mathrm{C}_{20} \mathrm{H}_{10} \mathrm{O}_{8}=\mathrm{C}_{8} \mathrm{H}_{8} \mathrm{O}_{6}+\mathrm{C}_{4} \mathrm{H}_{4} \mathrm{O}_{4}-2 \mathrm{HO}$.

Diacetine, $\mathrm{C}_{14} \mathrm{H}_{12} \mathrm{O}_{10}=\mathrm{C}_{6} \mathrm{H}_{8} \mathrm{O}_{6}+2\left(\mathrm{C}_{4} \mathrm{H}_{4} \mathrm{O}_{4}\right)-4 \mathrm{H} \mathrm{O}$.

Triacetine, $\mathrm{C}_{18} \mathrm{H}_{14} \mathrm{O}_{12}=\mathrm{C}_{6} \mathrm{H}_{8} \mathrm{O}_{6}+3\left(\mathrm{C}_{4} \mathrm{H}_{4} \mathrm{O}_{4}\right)-6 \mathrm{H} \mathrm{O}$.

They are neutral oils, of a somewhat ethereal odour. Some form 
of acetine, probably triacetine, seems to form part of some natural oils, which yields some acetic acid when saponified.

Acetochlorhydrine is a neutral mobile oil, of an odour like that of acetic ether. It is $\mathrm{C}_{10} \mathrm{H}_{9} \mathrm{Cl} \mathrm{O}_{6}=\mathrm{C}_{6} \mathrm{H}_{8} \mathrm{O}_{6}+\mathrm{C}_{4} \mathrm{H}_{4} \mathrm{O}_{4}+\mathrm{H} \mathrm{Cl}$ $-4 \mathrm{H} 0$.

Diethyline is formed when 1 eq. of glycerine acts on 2 eq. of alcohol, 4 of water being separated. It is a limpid oil, of a pungent ethereal smell, and its formula is $\mathrm{C}_{14} \mathrm{H}_{16} \mathrm{O}_{6}=\mathrm{C}_{6} \mathrm{H}_{8} \mathrm{O}_{6}+$ $2\left(\mathrm{C}_{4} \mathrm{H}_{6} \mathrm{O}_{2}\right)-4 \mathrm{H} \mathrm{O}$.

Butyrines. There are three of these, formed in the same way as the acetines:

Monobutyrine, $\mathrm{C}_{14} \mathrm{H}_{14} \mathrm{O}_{8}=\mathrm{C}_{6} \mathrm{H}_{8} \mathrm{O}_{6}+\mathrm{C}_{8} \mathrm{H}_{8} \mathrm{O}_{5}-2 \mathrm{H} \mathrm{O}$.

Dibutyrine, $\mathrm{C}_{22} \mathrm{H}_{20} \mathrm{O}_{10}=\mathrm{C}_{6} \mathrm{H}_{8} \mathrm{O}_{6}+2\left(\mathrm{C}_{8} \mathrm{H}_{8} \mathrm{O}_{4}\right)-4 \mathrm{H} 0$.

Tributyrine, $\mathrm{C}_{30} \mathrm{H}_{28} \mathrm{O}_{12}=\mathrm{C}_{6} \mathrm{H}_{8} \mathrm{O}_{6}+3\left(\mathrm{C}_{8} \mathrm{H}_{8} \mathrm{O}_{4}\right)-6 \mathrm{H} 0$.

They are neutral oils, of a strong odour, and a pungent bitterish taste. Probably tributyrine is that which is present in butter in small quantity, and contributes to its flavour. There is also a butyrochlorhydrine.

Valerines. There are three of these; but as they are formed in the same way as those already mentioned, from 1 eq. glycerine with 1, 2, and 3 eq. of the acid, and the separation of 2,4 , and 6 eqs. of water, it is unnecessary to repeat all the details. Their empirical formulæ are :

Monovalerine, $\mathrm{C}_{16} \mathrm{H}_{10} \mathrm{O}_{8}$.

Divalerine, $\mathrm{C}_{26} \mathrm{H}_{24} \mathrm{O}_{10}$.

Trivalerine, $\mathrm{C}_{38} \mathrm{H}_{32} \mathrm{O}_{12}$.

They are also neutral oils, of an unpleasant odour. Trivalerine appears to be the compound which gives the peculiar smell to train oil, cod-liver oil, and similar oils. There is also a valerochlorhydrine.

Benzoicines. Of these there are two, Monobenzoicine $\mathrm{C}_{20} \mathrm{H}_{12} \mathrm{O}_{8}$, and Tribenzoicine $\mathrm{C}_{48} \mathrm{H}_{20} \mathrm{O}_{12}$. The former is a semi-solid transparent resinoid mass ; the latter crystallises in fine needles. There is also a benzochlorhydrine, $\mathrm{C}_{20} \mathrm{H}_{11} \mathrm{Cl} \mathrm{O}_{6}$.

Camphorine. Camphoric acid forms, with glycerine, this compound, not yet analysed. It is a viscid mass like thick turpentine.

Myristine. This is the natural fat of the oil of nutmeg, and is formed by 4 eq. of myristic acid and 1 eq. of glycerine, minus 6 eq. of water. Formula, $\mathrm{C}_{118} \mathrm{H}_{114} \mathrm{O}_{16}$.

Palmitines. There are three, formed from 1, 2, and 4 eq. of palmitic acid respectively with 1 eq. of glycerine, water being in 
each case separated; 2 eqs. in the two first, 6 eqs. in the third. These compounds are monopalmitine, $\mathrm{C}_{38} \mathrm{H}_{38} \mathrm{O}_{8}$; dipalmitine, $\mathrm{C}_{70} \mathrm{H}_{70} \mathrm{O}_{12}$; and tetrapalmitine, $\mathrm{C}_{134} \mathrm{H}_{130} \mathrm{O}_{16}$. They are all fatty oils, and the last-named is the natural fat of palm oil.

Oleines. There are two, Monoleine, $\mathrm{C}_{42} \mathrm{H}_{40} \mathrm{O}_{8}$, and Dioleine, $\mathrm{C}_{78} \mathrm{H}_{74} \mathrm{O}_{12}$. The former is an oil, neutral and congealing at $60^{\circ}$. The latter also crystallises about the same temperature. It is not precisely known what is the formula of natural oleine; probably it is trioleine or tetroleine.

Elaidine, formed from elaidic acid and glycerine, or by the action of hyponitrous acid on oleine, is a solid fat, isomeric with oleine.

Margarines. Monomargarine, $\mathrm{C}_{40} \mathrm{H}_{40} \mathrm{Os}$, is a fat, melting at $133^{\circ}$. Tetramargarine is also a fat, and seems to be the natural compound. It is $\mathrm{C}_{142} \mathrm{H}_{138} \mathrm{O}_{18}$. It melts at $127^{\circ}$ and solidifies at $106^{\circ}$.

Stearines. Berthelot has formed three of these by heating stearic acid with glycerine in sealed tubes for some time. Monostearine, $\mathrm{C}_{44} \mathrm{H}_{44} \mathrm{O}_{8}$, is a neutral fat, fusible at $143^{\circ}$, and volatilising unchanged in vacuo, If kept for a long time in contact with concentrated hydrochloric acid at $212^{\circ}$, the acid and glycerine separate, which is the case also with most of the artificial glycerides.

Distearine, $\mathrm{C}_{78} \mathrm{H}_{78} \mathrm{O}_{12}$, is also a crystalline fat, melting at $136^{\circ}$.

Tetrastearine, $\mathrm{C}_{250} \mathrm{H}_{146} \mathrm{O}_{16}$, is also a crystalline fat, and is the natural stearine. It is best obtained from suet, tallow, or cacao butter, by acting on the solid part of these fats, after expressing as much oleine as possible with hot ether, which on cooling returns in solution oleine and margarine, leaving stearine, which is pure when its melting-point becomes constant at about $147^{\circ}$. But, as has been already mentioned, stearine has several meltingpoints, depending, no doubt, on allotropic modifications,

Stearine, when pure, that is, the natural or tetrastearine, is harder than wax, and may be reduced to powder. It forms beautiful scaly crystals from its solution in hot ether, which dissolves it; but it is only sparingly soluble in cold ether or in alcohol.

Sebine, $\mathrm{C}_{32} \mathrm{H}_{30} \mathrm{O}_{16}$, which is formed from 1 eq. of sebacic acid and 2 eq. of glycerine, minus 4 eq. of water, is a crystalline solid.

Ricinoleine is the chief constituent of castor oil. Its formula is not yet ascertained.

Ricinelaidine is a solid fat, isomeric with the preceding, and formed from it by the action of hyponitrous acid. 


\section{ACROLEINE,}

Or Hydrated Oxide of Acryle, $\mathrm{C}_{6} \mathrm{H}_{4} \mathrm{O}_{2}=\mathrm{C}_{6} \mathrm{H}_{8} \mathrm{O}_{6}-4 \mathrm{H} \mathrm{O}$.

We have seen that glycerine, when it combines with acids, loses 2,4 , or 6 eqs. of water, and it is probable that the true base or glycerine is $\mathrm{C}_{6} \mathrm{H}_{4} \mathrm{O}_{2}$, that is, glycerine minus 4 eqs. of water.

When glycerine, or any oil containing it, is distilled, there are formed vapours which attack the eyes and organs of respiration most violently. These are the vapours of acroleine, $\mathrm{C}_{8} \mathrm{H}_{4} \mathrm{O}_{2}$, which is best obtained by heating glycerine with anhydrous phosphoric acid. As it is very easily oxidised, it must be puritied by rectification in an atmosphere of carbonic acid. When pure, it is a limpid colourless liquid, of high refractive powers, which affects the eyes so painfully as to be quite intolerable. In preparing it, as it is very volatile, the uncondensed vapours must not be suffered to escape into the room, otherwise the operator must leave it; but they ought to be sent into the chimney. A few drops of it, in a large room, will cause a whole assembly to shed abundant tears, with a most distressing sensation. It is a mere trace of this substance which gives its painful pungency to the vapour of a candle when blown out so as to leave the wick red-hot. It has a burning taste. It is lighter than water, and boils at $125^{\circ}$. It is rapidly oxidised in the air, and converted into acrylic acid, $\mathrm{C}_{6} \mathrm{H}_{4} \mathrm{O}_{4}$, already described. Acroleine does not keep, even in close vessels, being converted either into a white amorphous, inodorous powder, called disacryle, or into a resinous mass, resin of disacryle. The former is probably isomeric with acroleine; the latter is said to be $\mathrm{C}_{20} \mathrm{H}_{13} \mathrm{O}_{6}$, but this is a doubtful formula.

We can now understand how heat acts on oils or fats. All such as contain glycerine, a large majority of them, yield acroleine, of which a mere trace is easily detected by the action on the eyes and its smell. Those which contain oleic acid, also a large majority of natural fat oils, yield sebacic acid, which is easily detected by boiling the product with water and filtering. The liquid on cooling will deposit sebacic acid, if oleic acid have been present.

The other products are oily acids of the formylic series, which are volatile, water, and carbohydrogens.

Having now described the best known radicals, and the series connected with them, we shall proceed to consider briefly those compounds the radicals of which are but little or not at all known. 
We shall begin with an important class of compounds, namely, the more abundant organic or vegetable acids, not oily or fatty in character.

\section{ORGANIC ACIDS, NOT OILY.}

1. Citrio Acid. $\mathrm{C}_{12} \mathrm{H}_{5} \mathrm{O}_{11}, 3 \mathrm{H} \mathrm{O}=\overline{\mathrm{Ci}}, 3 \mathrm{H} \mathrm{O}$.

This acid is found in many vegetable juices, especially those of sour fruits, as the lemon, lime, orange, red currant, \&c. It is extracted by adding chalk to the acid juice, by which means an insoluble citrate of lime is formed. This is decomposed by diluted sulphuric acid with the aid of heat, and the solution, filtered from the sulphate of lime, gives on evaporation and cooling crystals of citric acid, consisting of $\overline{\mathrm{Ci}}, 3 \mathrm{H} \mathrm{O}+$ aq. A slight excess of sulphuric acid promotes the crystallisation.

It forms large transparent crystals, very soluble in water, of a very strong and agreeable acid taste. A diluted solution is soon decomposed, becoming mouldy. By spontaneous evaporation of a saturated solution, crystals may be obtained, which are $\overline{\mathrm{Ci}}, 3 \mathrm{H} \mathrm{O}+2 \mathrm{aq}$. At $212^{\circ}$, these lose the 2 eqs. of water of crystallisation. The other crystals, above mentioned, do not lose water at $212^{\circ}$, but melt at $256^{\circ}$; and when heated beyond $300^{\circ}$, both kinds are decomposed. Heated with oil of vitriol in excess, citric acid is decomposed, yielding,

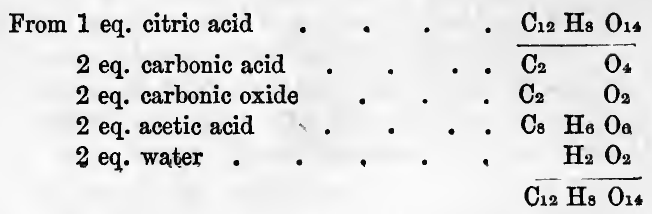

In like manner, when fused with oaustic potash, citric acid is resolved into 2 eqs. of acetic acid, 2 eqs. oxalic acid, and 2 eqs. water.

When oitric acid is added to lime-water, the liquid remains clear, but when heated becomes turbid, and deposits citrate of lime. This character serves to distinguish eitric acid from most other vegetable acids.

\section{SALTS OF CITRIC ACID.}

Citric acid is tribasic, and forms three series of neutral salts, that is, of salts with 3 eqs. of base, whether fixed base or basic 
water. It also forms basie salts, of the formula $\overline{\mathrm{Ci}}, 3 \mathrm{M} \mathrm{O}+$ $\mathrm{M} \mathrm{O}$ or $\overline{\mathrm{Ci}}, 3 \mathrm{M} \mathrm{O}+\mathrm{MO}+\mathrm{aq}$. These basic salts correspond in constitution to the two forms of crystallised acid.

When a dry citrate, with 3 eqs. of fixed base, is decomposed by an alcoholic solution of hydrochloric acid, so that no more water is presented to the citric acid than the 3 eqs. of basic water derived from the hydrogen of the hydrochloric acid and the oxygen of the base, there are formed, from 3 eqs. dry citric acid, 2 eqs. of the acid with 1 eq. of water of crystallisation, and 1 eq. of the hydrated aconitic acid. $3\left(\mathrm{C}_{12} \mathrm{H}_{5} \mathrm{O}_{11}, 3 \mathrm{H} \mathrm{O}\right)=$ $2\left(\mathrm{C}_{12} \mathrm{H}_{5} \mathrm{O}_{11}, 3 \mathrm{H} \mathrm{O}+\right.$ aq. $)+\mathrm{C}_{12} \mathrm{H}_{3} \mathrm{O}_{9}, 3 \mathrm{H} \mathrm{O}$. This is the same change which takes place in citric acid when heated to a certain point, water being given off.

Citrate of Oxide of Ethyle, $\overline{\mathrm{Ci}}, 3 \mathrm{Ae} \mathrm{O}$, is an oily liquid of Sp. G. 1·142. By alkalies it is converted into citrates and alcohol. With ammonia it yields citramide $\mathrm{C}_{22} \mathrm{H}_{11} \mathrm{~N}_{3} \mathrm{O}_{8}$, which is tribasic citrate of ammonia, minus 6 eqs. of water. With aniline, citric acid yields four compounds, neutral and acid anilides, corresponding to neutral and acid amides and imides, although, of the latter, only citramide is known in a state of purity.

Citrate of Potash occurs in three forms. $\overline{\mathrm{Ci}}, 3 \mathrm{~K} 0$; $\overline{\mathrm{Ci}}$, $\left\{\begin{array}{r}2 \mathrm{~K} \mathrm{O} \\ \mathrm{H} \mathrm{O}\end{array}\right.$ and $\overline{\mathrm{Ci}}\left\{\begin{array}{r}\mathrm{K} \mathrm{O} \\ 2 \mathrm{H} \mathrm{O}\end{array}\right.$ with difficulty.

Citrate of Soda also forms three salts. 1. $\overline{\mathrm{CI}}, 3 \mathrm{NaO}+11$ aq. This salt forms large regular crystals. 2. $\overline{\mathrm{Ci}}, 2 \mathrm{Na} \mathrm{O}, \mathrm{H} \mathrm{O}$; formed by adding to a solution of the preceding salt half as much citric acid as it already contains. It forms, by evaporation, needles of a very pleasant subacid taste. 3. $\overline{\mathrm{Ci}}, \mathrm{Na} \mathrm{O}, 2 \mathrm{H} \mathrm{O}$. Formed by adding to a solution of No. 1, as much citric acid as it already contains. This salt does not crystallise in water, but forms a gummy mass. A saturated alcoholic solution, however, deposits crvstalline grains. Citrate of Baryta forms two varieties. 1. $\overline{\mathrm{C}_{1}}, 3 \mathrm{BaO}+7 \mathrm{aq}$. which falls when citrate of soda is added to chloride of barium. 2. $2 \overline{\mathrm{Ci}}\left\{\begin{array}{c}5 \mathrm{BaO} \\ \mathrm{H} \mathrm{O}\end{array}+7\right.$ aq. $=\overline{\mathrm{Ci}}\left\{\begin{array}{c}2 \mathrm{BaO} \\ \mathrm{H} \mathrm{O}\end{array}+\overline{\mathrm{Ci}}, 3 \mathrm{BaO}+7\right.$ aq. This is deposited on cooling, when a boiling solution of citrate of soda is added to a boiling solution of chloride of barium and free citric acid. Citrate of lime also yields two salts. 1. neutral, $\overline{\mathrm{Ci}}, 3 \mathrm{Ca} O$ +4 aq. formed by mixing chloride of calcium and citrate of soda. It is insoluble. 2. basic, $\overline{\mathrm{Ci}}, 3 \mathrm{CaO}+\mathrm{CaO}+$ aq. formed when citric acid is heated with excess of lime-water. 
The citrate of lime formed from lemon, or currant-juice, by chalk, is an impure mixture of the basic and neutral salts. Citrates of lead. 1. $\overline{\mathrm{Ci}}, 3 \mathrm{~Pb} 0+$ aq. 2 . $\overline{\mathrm{Ci}}, 2 \mathrm{~Pb}, \mathrm{H} \mathrm{O}+2$ aq. 3. basic, $\overline{\mathrm{Ci}}, 3 \mathrm{~Pb} 0+3 \mathrm{~Pb} 0$. 4. also basic, $\overline{\mathrm{Ci}}$, $3 \mathrm{PbO}+\mathrm{PbO}+\mathrm{aq}$. These are all sparingly soluble or insoluble, and are decomposed by washing. Citrate of copper is basic, $\overline{\mathrm{Ci}}, 3 \mathrm{Cu} \mathrm{O}+\mathrm{Cu} \mathrm{O}$. Citrate of silver is a brilliant white, insoluble powder, $\overline{\mathrm{Ci}}, 3 \mathrm{Ag} \mathrm{O}+\mathrm{aq}$. It loses its water under $80^{\circ}$. Citrate of antimony and potash is a double salt, $\overline{\mathrm{Ci}}, \mathrm{Sb}_{2} \mathrm{O} \mathrm{s}+\overline{\mathrm{Ci}}, 3 \mathrm{~K} \mathrm{O}+5 \mathrm{aq}$. It forms hard, brilliant, white prisms, which lose their water at $212^{\circ}$.

\section{ACTION OF HEAT ON CITRIC ACID.}

The first effect of heat on crystallised citric acid is to melt it, and the next, to expel the water of crystallisation. The acid, if now dissolved, crystallises unchanged. But if the heat be continued, there are given off gas and inflammable vapours, and the residue is no longer citric acid but hydrated aconitic acid.

When the heat is increased, other products appear, particularly two new acids : but these are derived from aconitic acid. They are itaconic and citraconic acids.

\section{Aconitic Acid. $\mathrm{C}_{12} \mathrm{H}_{6} \mathrm{O}_{12}=\mathrm{C}_{12} \mathrm{H}_{3} \mathrm{O}_{9}, 3$ Hं 0 .}

Srv. Equisetic Acid. This acid occurs native in Aconitum napellus and in Equisetum fluviatile. It is formed by the action of heat on citric acid, as above. To obtain it, citric acid is heated till it ceases to give off inflammable vapours, and the residue, dissolved in alcohol, is treated with hydrochloric acid gas, which causes the formation of aconitate ether. The addition of water causes this to separate, and by caustic potash it is converted into aconitate of potash. From this aconitate of lead is prepared, and this salt, decomposed by sulphuretted hydrogen, yields the acid.

It forms indistinct crystals; and the acid thus prepared has the same composition as that from aconite, and that from equisetum. They are, in fact, identical. The aconitic acid, when heated, yields itaconic and citraconic acids.

Aconitic acid, according to the present state of our knowledge, is tribasic, and forms, like citric acid, three series of salts, with 1,2 , or 3 eqs. of fixed base, and 2 or 1 eq. of water in the two first. The aconitates are not important. Aconitate of ethyle is an oily liquid of an aromatic smell and bitter taste.

When aconitic acid (from citric acid) is heated to from $356^{\circ}$ 
to $392^{\circ}$, it boils and yields a mixture of two acids; itaconic acid, which condenses in crystals, and eitraconio acid, which appears as an oily liquid. When the distillation is very rapid, itaconic acid predominates; when it is slow, there is more citraconic acid. These two acids have both the formula $\mathrm{C}_{10}$ $\mathrm{H}_{6} \mathrm{O}_{8}=\mathrm{C}_{10} \mathrm{H}_{4} \mathrm{O}_{6}, 2 \mathrm{H} 0$.

Itaconic Acid is readily purified by solution in hot water, as it crystallises with great facility. It is soluble in water, alcohol, and ether. When heated it is resolved into water and anhydrous citraconic acid. It is a bibasic acid, and its formula is as above given. The formula of its salts is $\mathrm{C}_{10} \mathrm{H}_{4} \mathrm{O}_{6}, 2 \mathrm{M} \mathrm{O}$. It also forms acid salts, the formula of which is $\mathrm{C}_{20} \mathrm{H}_{11} \mathrm{O}_{6}$, $\left.\begin{array}{l}\text { HO } \\ \text { MO }\end{array}\right\}$. The itaconates are not of special interest.

Citraconic Acid is formed when the preceding acid is distilled, and then appears, in the anhydrous state, as a limpid oily liquid. It distils unaltered at $410^{\circ}$, and volatilises slowly at much lower temperatures. It attracts moisture from the air, forming a crystalline hydrate, which, when heated, is again resolved into water and anhydrous acid. The formula of the anhydrous acid is $\mathrm{C}_{10} \mathrm{H}_{4} \mathrm{O}_{6}=\overline{\mathrm{C}_{\mathrm{C}}}$ : that of the hydrate, $\mathrm{C}_{10} \mathrm{H}_{4}$ $\mathrm{O}_{6}, 2 \mathrm{H} \mathrm{O}=\mathrm{Ct}, 2 \mathrm{HO}$. It forms, like the preceding, both neutral and acid salts ; and produces, with oxide of silver, a neutral salt with water of crystallisation; an apparently anomalous case. The formation of these two acids takes place as follows: -1 eq. of aconitic acid, $\mathrm{C}_{12} \mathrm{H}_{6} \mathrm{O}_{12}$, yields 1 eq. itaconic acid, and 2 eqs. carbonic acid, $2 \mathrm{C} \mathrm{O}_{2}$. The itaconic acid, when formed, is partially resolved into water and anhydrous citraconic acid.

Gottlieb has recently observed, that citraconic acid, but not itaconic acid, yields with nitric acid a new isomeric acid, mesaconic acid, $\mathrm{C}_{10} \mathrm{H}_{4} \mathrm{O}_{6}, 2 \mathrm{H} \mathrm{O}$; for it, as well as the preceding acids, appear, from his researches, to be bibasic. Mesaconic acid forms minute crystals, sparingly soluble in water. Its production is obscure, but only part of the citraconic acid undergoes the transformation; another part is converted into oxalic acid, and into a

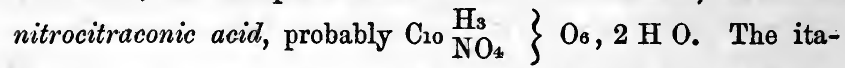
conate, citraconate, and mesaconate of ethyle are oily aromatic liquids. The last has a very pleasant odour of fruits.

Citraconic acid, like other bibasic acids, readily forms an imide, citraconimide, $\mathrm{C}_{10} \mathrm{H}_{5} \mathrm{~N}_{4}=\mathrm{NH}+\mathrm{C}_{10} \mathrm{H}_{4} \mathrm{O}_{4}$. It also forms, with great facility, the corresponding compound with aniline, citraconanile, $\mathrm{C}_{22} \mathrm{~N}_{9} \mathrm{O}_{4}=\mathrm{N} \mathrm{C}_{22} \mathrm{H}_{5}+\mathrm{C}_{10} \mathrm{H}_{4} \mathrm{O}_{4}$; as well as the acid anilide, citraconanilic acid, $\mathrm{C}_{22} \mathrm{~N} \mathrm{H}_{10} \mathrm{O}_{5}, \mathrm{H} O$. Itaconic acid forms the anilide, itaconanilide, $\mathrm{C}_{34} \mathrm{H}_{16} \mathrm{~N}_{2} \mathrm{O}_{4}=$ 
$2\left(\mathrm{~N} \mathrm{C}_{12} \mathrm{H}_{5}\right), \mathrm{C}_{10} \mathrm{H}_{0} \mathrm{O}_{4}$, analogous to the amide. The tendency to form imides, nitryles, and compounds corresponding to these, with aniline, marks the bibasio acids, while the monobasic only form amides and corresponding anilides. Mesaconic acid seems to form compounds like those of citraconic acid, but these are easily changed into the compounds of the latter, and are therefore difficult to study; but the numbers of amides and anilides formed by these isomerio acids is very great.

Mesaconic acid, according to Pebal, forms acid salts with lead, silver, and barium, a sufficient proof of its bibasic character. It forms a neutral ether, $2 \mathrm{Ae} \mathrm{O}, \mathrm{C}_{10} \mathrm{H}_{4} \mathrm{O}_{6}$. According to some, lipic acid, which we have mentioned as one of the oxalio series of bibasic acids, is isomeric with these three acids, and its formula is $\mathrm{C}_{10} \mathrm{H}_{6} \mathrm{O}_{8}=\mathrm{C}_{10} \mathrm{H}_{4} \mathrm{O}_{6}, 2 \mathrm{H} \mathrm{O}$; instead of $\mathrm{C}_{10} \mathrm{H}_{8} \mathrm{O}_{8}=\mathrm{C}_{10}$ $\mathrm{H}_{6} \mathrm{O}_{6}, 2 \mathrm{HO}$. The mode of preparation of lipic acid, however, and its occurrence with so many of the acids of the oxalic acid series, are favourable to the latter-mentioned formula.

\section{Tartaric Acid. $\mathrm{C}_{8} \mathrm{H}_{4} \mathrm{O}_{10}, 2 \mathrm{H} \mathrm{O}=\overline{\mathrm{T}}, 2 \mathrm{H}$.}

This acid occurs in the juice of the grape as acid tartrate of potash; also in many other plants. It is prepared from tartrate of lime, exactly as citric acid is from citrate of lime. Tartrate of lime is obtained by the action of chalk on acid tartrate of potash, or cream of tartar.

Tartaric acid orystallises in large rhombic prisms, transparent and colourless. They are very soluble in water, and have a pleasant acid taste. When boiled with alcohol, tartario acid forms acid tartrate of oxide of ethyle. A high temperature decomposes tartario acid, giving rise to several new products.

An excess of potash, aided by heat, transforms it into acetate and oxalate of potash, $\mathrm{C}_{8} \mathrm{H}_{4} \mathrm{O}_{10}, 2 \mathrm{H} \mathrm{O}=\mathrm{C}_{4} \mathrm{H}_{3} \mathrm{O}_{3}, \mathrm{HO}+$ $2\left(\mathrm{C}_{2} \mathrm{O}_{3}, \mathrm{H} \mathrm{O}\right)$. By peroxide of manganese and sulphurio acid it is converted into formic acid, carbonic acid, and other products. There is some relation between tartaric and formio acids: for if formic acid be $\mathrm{Fo}_{2}\left(\mathrm{Fo}=\mathrm{C}_{2} \mathrm{H}\right)$, tartaric acid is $2\left(\mathrm{Fo}_{2} \mathrm{O}_{5}\right)$.

When finely powdered tartaric acid is mixed with $4 \frac{1}{2}$ times its weight of pure hydrated nitric acid, it rapidly dissolves, and if an equal volume of oil of vitriol be added, the mass soon forms a stiff jelly like starch jelly. It is placed between two porous bricks under a bell jar, to remove the greater part of the sulphuric acid; when there is left a light silky mass, forming in the air. It is purified by dissolving it in lukewarm water, and cooling to $32^{\circ}$, when the solution deposits a large quantity of fine interwoven silky needles, which render the whole semisolid. This is pressed 
in a funnel, when the liquid runs down, and the crystals, which have shrunk remarkably, are dried by pressure in bibulous paper. They are nitrotartaric acid, and appear to be formed by the combination of tartaric acid with nitric acid.

The new acid is very unstable, and when its solution is left to itself, or acted on by sulphuretted hydrogen, or made to combine with bases, a new acid is formed, which appears to be homologous with malic acid. There is first formed an acid, not yet analysed, in large crystals, which, when heated, yields another crystalline acid, $\mathrm{C}_{6} \mathrm{H}_{4} \mathrm{O}_{10}$. This would be homologous with malic acid, which, as we shall see, is $\mathrm{C}_{8} \mathrm{H}_{6} \mathrm{O}_{10}$. The salt of silver of the new acid is $\mathrm{C}_{6} \mathrm{H}_{2} \mathrm{O}_{8}, 2 \mathrm{Ag} \mathrm{O}$, so that it appears to be bibasic, as malic acid is.

Tartaric acid preoipitates lime-water white, but an excess dissolves the precipitate. In solution of potash, if the aoid be added in excess, it causes a crystalline deposit of cream of tartar, which, where the potash is in very minute proportion, is rendered more visible by the addition of alcohol.

Tartaric acid is a bibasic acid, and forms two series of salts: 1. neutral, $\overline{\mathrm{T}}, 2 \mathrm{M} \mathrm{O} ; 2$ acid, $\mathrm{T}, \mathrm{M} \mathrm{O}, \mathrm{H} \mathrm{O}$. It forms also two kinds of double salts : in one the 2 eqs. of fixed base are different protoxides, $\mathrm{T},\left\{\begin{array}{l}\mathrm{M} \mathrm{O} \\ \mathrm{m} \mathrm{O}\end{array}\right.$; in the other, one of the equivalents of fixed base is replaced by 1 eq. of a sesquioxide; $\overline{\mathrm{T}},\left\{\begin{array}{l}\mathrm{MO} O \\ \mathrm{~m}_{2} \mathrm{O}_{3}\end{array}\right.$. This latter kind may be considered basic, since the sesquioxide, $\mathrm{m}_{2}$ $\mathrm{O}_{3}$, usually neutralises as much acid as 3 eqs. of protoxide. Tartaric acid is remarkable for its tendency to form double salts.

Among the neutral tartrates are, tartrate of ammonia, $\overline{\mathrm{T}}$, $2 \mathrm{NH}_{4} \mathrm{O}+2$ aq.; tartrate of potash, or soluble tartar, $\mathrm{T}$, $2 \mathrm{KO}$; tartrate of soda, T, $2 \mathrm{NaO}+4$ aq. ; tartrate of lime, $\mathrm{T}$, $2 \mathrm{Ca} 0+8$ aq., \&c. \&c.

Among the acid salts are, acid tartrate of ethyle, or tartrovinic acid, T, Ae $\mathrm{O}, \mathrm{H} \mathrm{O}$ - a crystallisable compound: acid tartrate of potash, or tartar, $\mathrm{T}, \mathrm{K} \mathrm{O}, \mathrm{HO}$. This is the principal compound of tartaric acid. It exists in the juice of the grape, dissolved by the aid of the sugar present, and when that sugar is converted into alcohol, in which the tartar is insoluble, it is deposited on the sides of the fermenting casks. When purified it is quite white, and is called oream of tartar. It is much used in medicine as a safe and mild laxative. When caloined in a covered crucible it leaves a mixture of carbonate of potash and charcoal, called black flux. Henoe carbonate of potash is called salt of tartar. Like all the tartrates, cream of tartar, when heated, gives off a very 
peculiar smell of burnt vegetable matter, peculiar to tartaric and racemic acids and their salts.

Among the very numerous double tartrates may be mentioned the tartrate of potash and ammonia, $\mathrm{T}, \mathrm{K} 0, \mathrm{~N} \mathrm{H}_{4} \mathrm{O}$ : the tartrate of potash and oxide of ethyle, $\mathrm{T}, \mathrm{Ae} \mathrm{O}, \mathrm{K} \mathrm{O}$; tartrate of potash and boracic acid, $\overline{\mathrm{T}}, \mathrm{KO}, \mathrm{B} \mathrm{O}_{3}$; this is the soluble cream of tartar used as a laxative on the continent:-tartrate of potash and soda, $\mathrm{T}, \mathrm{KO}, \mathrm{NaO}+10 \mathrm{aq}$. This is the salt of Seignette or Rochelle salt. It crystallises in very large transparent prisms, and is used as a mild laxative:-tartrate of potash and peroxide of iron; $\overline{\mathrm{T}}, \mathrm{K} \mathrm{O}, \mathrm{Fe}_{2} \mathrm{O}_{3}$; this is the tartarised iron of the pharmacopoias; tartrate of potash and antimony, $\mathrm{T}, \mathrm{K} \mathrm{O}, \mathrm{Sb}_{2} \mathrm{O}_{3}$. This is tartar emetic, one of the most valuable remedies. It must be considered as a basic salt; for $\mathrm{Sb}_{2} \mathrm{O}_{3}$, here substituted for $\mathrm{H} \mathrm{O}$ or $\mathrm{K} \mathrm{O}$, in short for a protoxide, requires for its neutralisation an additional equivalent of tartaric acid. It then yields the compound $2 \pi+\left\{\begin{array}{l}\mathrm{K} \mathrm{O} \\ \mathrm{Sb}_{2} \mathrm{O}_{3}\end{array}\right.$ which is neutral, since the bases contain 4 eqs. of oxygen for 2 eqs. of acid.

Tartar emetic is formed when 3 parts of oxide of antimony and 4 of cream of tartar are ground together, and made into a thin cream with water, which is heated to $158^{\circ}$, till a portion, tried separately, dissolves in 15 parts of cold water. When this is the case, 6 or 8 parts of water are added, and the whole boiled for half an hour. The liquid, filtered white hot, deposits, on cooling, crystals of tartar emetic. It forms white brilliant crystals which soon become opaque. It is soluble in 14 or 15 parts of cold water and 2 parts of boiling water. The crystals are $\pi, \mathrm{K} \mathrm{O}_{,} \mathrm{Sb}_{2} \mathrm{O}_{3},+$ 2 aq.

When heated, the crystals first lose the 2 eqs. of water of crystallisation; and when the heat rises to $390^{\circ}, 2$ more eqs. of water are given off, without the acid being destroyed. The salt is then $\mathrm{Cs}_{8} \mathrm{H}_{2} \mathrm{O}_{10}+\left\{\begin{array}{l}\mathrm{K} \mathrm{O} \\ \mathrm{Sb} \mathrm{O}\end{array}\right.$. That is to say, 2 eqs. of oxygen, from the oxide of antimony, have been expelled along with 2 of hydrogen from the acid. It has been already shown that tartar emetic $\mathrm{C}_{12} \mathrm{H}_{4} \mathrm{O}_{10}+\left\{\begin{array}{l}\mathrm{KO}_{2} \\ \mathrm{Sb}_{2} \mathrm{O}_{3}\end{array}\right.$, contains 2 eqs. of oxygen in the bases, more than is required for a neutral salt; and it is apparently these 2 eqs. of oxygen which are thus expelled as water. If we bear in mind that $\mathrm{Sb}_{2} \mathrm{O}_{3}$ is the equivalent of $3 \mathrm{~K} \mathrm{O}$; or in other words, that $\mathrm{Sb}_{\frac{2}{3}}$ is equivalent to $\mathrm{K}$ or to $\mathrm{H}$, we can then see that the tartar emetic heated to $390^{\circ}$ is analogous in composition to neutral tartrate of potash. 


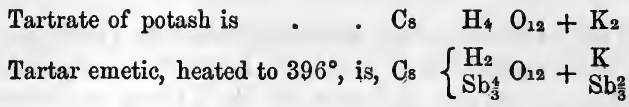

In this point of view the $2 \mathrm{Sb}$ are divided, $\frac{4}{3} \mathrm{Sb}$ replacing hydrogen in the radical, and $\frac{2}{3} \mathrm{Sb}$ replacing potassium in the base,

The neutral tartrate of potash and antimony, $2 \pi,\left\{\begin{array}{l}\mathrm{K} \mathrm{O} \\ \mathrm{Sb}_{2} \mathrm{O}_{3}\end{array}+7\right.$ aq., is always formed in the mother liquors of tartar emetic. It is also formed when tartar emetic is dissolved in tartaric acid.

Tartar emetic forms a double salt with cream of tartar, $\bar{T}$, $\mathrm{K} 0, \mathrm{Sb}_{2} \mathrm{O}_{3}+3(\mathrm{~T}, \mathrm{~K} \mathrm{O}, \mathrm{H} \mathrm{O})$. It orystallises in scales,

\section{ACTION OF HEAT ON TARTARIC ACID,}

When tartario acid is heated, it melts, and gives rise to several curious acid products, most of which are isomeric with the acid. That first formed, at $350^{\circ} \mathrm{F}$., is metatartaric acid, $\mathrm{C}_{8} \mathrm{H}_{6} \mathrm{O}_{12}$. When the heat is continued, this changes to isotartaric acid, $\mathrm{C}_{8} \mathrm{H}_{6} \mathrm{O}_{12}$. The former of these acids is bibasic, and its salts resemble those of tartario acid, but are more soluble, The latter is monobasic, so that its neutral salts have the same composition as the acid tartrates. The heat being continued, the isotartaric acid loses water and forms the soluble anhydrous tartaric acid, $\mathrm{C}_{8} \mathrm{H}_{4} \mathrm{O}_{10}$; and a higher temperature converts this last into an insoluble modification of the anhydrous acid. Lastly, a still greater degree of heat causes the formation of pyrotartaric acid, $\mathrm{C}_{10} \mathrm{H}_{3} \mathrm{O}_{8}$, and of pyruvic acid, $\mathrm{C}_{6} \mathrm{H}_{4} \mathrm{Oa}_{4}$. The former of these acids is bibasic, the latter monobasic. It is unnecessary to enter into minute details concerning the salts of these acids, but the reader will find what is known of them in Gerhardt's Organic Chemistry,

When the tartrates, especially tartrate of lime, are made to undergo fermentation, by contact with water and a ferment, there is gradually formed a large quantity of propylic acid, $\mathrm{C}_{6} \mathrm{H}_{6} \mathrm{O}_{4}$, which is found in the residue as propylate of lime.

Paratartaric or Racemic Acid, $\mathrm{Cs}_{8} \mathrm{H}_{6} \mathrm{O}_{12}, 2 \mathrm{aq} .=\mathrm{Cs}_{4} \mathrm{O}_{10}$, $2 \mathrm{H} 0+2$ aq.

This is another isomeric form of tartaric acid, which occurs along with it in the juice of the grape, and as the acid paratartrate of potash is more soluble than the bitartrate, the former salt accumulates in the mother liquids of cream of tartar. When separated, the acid crystallises with 2 eqs. of water of crystallisation, whereas tartaric acid crystallises without any water in this form. The crystals of paratartaric acid lose their water of crys- 
tallisation at $212^{\circ}$, and when further heated, yield the same products as tartaric acid. This acid is less soluble in water than tartaric acid, so that, in a solution of both, the paratartaric acid is first deposited.

The salts of this acid are very similar to those of tartaric acid, but yet distinct. Neither the acid nor its salts have any action on the plane of polarisation.

There is still another isomeric modification of tartaric acid, which is called passive or inactive tartaric acid, because it also has no power to cause the plane of polarisation to deviate.

Now tartaric acid and its salts possess this power in a high degree, and it is chiefly in this, and in the form of the crystals, that the different modifications can be distinguished from one another.

Common tartaric acid and its salts form hemihedral crystals, all of which have the faces developed on one side, and they cause the plane of polarisation to deviate very strongly to the right. Hence this acid has been called dextrotartaric or dextroracemic acid.

We have seen that paratartaric acid has no action on the plane of polarisation. But when we form the double paratartrate of soda and ammonia, large and fine crystals are obtained, all of which are hemihedral, and they are found to be no longer paratartrates but tartrates, for they have a powerful action on the plane of polarisation.

But the remarkable fact is, that these crystals are of two kinds, one hemihedral to the right, and causing deviation to the right, the other hemihedral to the left, and causing deviation to the left. These crystals are in all respects, but these, identical, but they cannot be superposed one on the other; being symmetrical, they are so related, that if one be held before a mirror, the image will represent the other corresponding.

Now if these crystals be separated, and the acid extracted from each, it will be found that the acid from the right-hand crystals also form right-hand crystals, and that from the left-hand ones yield left-hand crystals.

The former are crystals of common or dextrotartaric acid, the latter are those of left-hand or lævotartaric acid, which makes one more isomeric form of tartaric acid.

We now see that, while common tartaric acid is dextrutartaric acid, paratartaric acid is a compound of it with an equal weight of lævotartaric acid. And when it is converted into the double salt of soda and ammonia, paratartaric acid splits up into the two acids, dextrotartaric and lævotartaric acids, each of which forms its own double salt, the one dextrohemihedral, the other lævohemi- 
hedral. We see also how paratartaric acid has no action on the plane of polarisation. In fact, if we mix concentrated solutions of dextrotartaric and lævotartaric acids, heat is disengaged, and a crystalline deposit is formed, which is found to be pure paratartaric or racemic acid.

The paratartrates have the same formulæ as the tartrates, and, as already mentioned, resemble them in the highest degree. We need not therefore repeat these formulæ, but it may here be mentioned, that the paratartrates are never hemihedral, and have no action on the plane of polarisation. It is only when, as in the case of the double salt of soda and ammonia above alluded to, the acid is resolved into its constituent acids, that hemihedral crystals are obtained, and these are no longer paratartrates.

Metatartaric Acid has the aspect of a transparent gum, and is very deliquescent. It forms salts which have a different form from that of the corresponding tartrates, and are much more soluble. In the fused state, it causes a powerful deviation to the right, but loses that property when solidified. It is bibasic.

Isotartaric Acid also forms a transparent vitrous mass, very soluble and deliquescent. It appears to be monobasic, so that its neutral salts have the composition of the bitartrates, $\mathrm{C}_{8} \mathrm{H}_{5} \mathrm{O}_{11}$ $\left.\mathrm{MO}=\mathrm{Cs}_{4} \mathrm{O}_{10} \underset{\mathrm{MO}}{\mathrm{HO}}\right\}$.

It is to Pasteur that we are indebted for our present knowledge of the very remarkable physical and optical characters of the various modifications of tartaric acid. His researches have opened up a new field of investigation, for they show in a very marked way the extent to which the physical characters of a compound may be altered without any change in its composition; a result which can only be accounted for by some change in the position or arrangement either of the molecules or of the atoms forming these molecules. The acids of this section may be summed up as follows:-

Anhydrous tartaric acid, soluble $=\mathrm{C}_{8} \mathrm{H}_{4} \mathrm{O}_{10}$. Anhydrous tartaric acid, insoluble $=\mathrm{C}_{8} \mathrm{H}_{4} \mathrm{O}_{10}$. Dextrotartaric acid, $\mathrm{C}_{8} \mathrm{H}_{8} \mathrm{O}_{12}=\mathrm{C}_{8} \mathrm{H}_{4} \mathrm{O}_{10}, 2 \mathrm{H} \mathrm{O}$. Lævotartaric acid, $\mathrm{C}_{8} \mathrm{H}_{8} \mathrm{O}_{12}=\mathrm{C}_{8} \mathrm{H}_{4} \mathrm{O}_{10}, 2 \mathrm{H} \mathrm{O}$. Paratartaric acid, $\mathrm{C}_{8} \mathrm{H}_{8} \mathrm{O}_{12}=\mathrm{C}_{8} \mathrm{H}_{4} \mathrm{O}_{10}, 2 \mathrm{HO}$. Metatartaric acid, $\mathrm{C}_{8} \mathrm{H}_{8} \mathrm{O}_{12}=\mathrm{Cs}_{8} \mathrm{H}_{4} \mathrm{O}_{10}, 2 \mathrm{H} \mathrm{O}$. Isotartaric acid, $\mathrm{C}_{8} \mathrm{H}_{8} \mathrm{O}_{1_{2}}=\mathrm{C}_{8} \mathrm{H}_{5} \mathrm{O}_{11}, \mathrm{H} \mathrm{O}$.

Tartaric and Paratartaric acids both form compounds with the oxides of ethyle and methyle. Some of them are neutral, others acid ethers, corresponding to the neutral and acid salts with fixed base. 
Tartaric acid forms also two amides; one neutral, tartramide, $\mathrm{C}_{8} \mathrm{H}_{8} \mathrm{~N}_{2} \mathrm{O}_{8}$, which exhibits a right-hand and left-hand form; and the other acid, tartramic acid, $\mathrm{C}_{8} \mathrm{H}_{7} \mathrm{NO}_{10}$.

Pyrotartaric Acid, $\mathrm{C}_{10} \mathrm{H}_{8} \mathrm{O}_{8}=\mathrm{C}_{10} \mathrm{H}_{6} \mathrm{O}_{6}, 2 \mathrm{H} \mathrm{O}$. This acid, which has been already alluded to as probably being one of the oxalic series of bibasic acids, is formed when tartaric acid is distilled. It is best obtained, however, by distilling cream of tartar. The product consists of an oily and an aqueous liquid. The latter, filtered and evaporated, yields the acid in crystalline grains. The operation is more productive, if the tartar or tartaric acid be first mixed with powdered pumice-stone.

Pyrotartaric acid is bibasic, and its salts are $\mathrm{C}_{10} \mathrm{H}_{6} \mathrm{O}_{6}, 2 \mathrm{M} \mathrm{O}$ or $\left.\mathrm{C}_{10} \mathrm{H}_{6} \mathrm{O}_{6} \mathrm{M} \mathrm{O}_{\mathrm{O}}^{\mathrm{M}}\right\}$. The pyrotartrate of ethyle, $\mathrm{C}_{10} \mathrm{H}_{6} \mathrm{O}_{6}, 2 \mathrm{Ae} \mathrm{O}$ is an aromatic oily liquid.

Pyruvic Acid, $\mathrm{C}_{6} \mathrm{H}_{4} \mathrm{O}_{6}=\mathrm{C}_{6} \mathrm{H}_{3} \mathrm{O}_{5}, \mathrm{H}$ O. This acid is also found in the distillation of tartaric acid or of tartrates along with acetic acid, pyrotartaric acid and oily liquids. When pure the pyruvic acid forms a syrup, which does not crystallise. It is very aoid and also acrid and bitter; it may be distilled, but is partially decomposed in the process so as to become brawn. It is soluble in water, alcohol, and ether. Its salts have the formula $\mathrm{C}_{6} \mathrm{H}_{3} \mathrm{O}_{5}, \mathrm{MO}$. Many of them orystallise well. It seems to form an acid salt with soda, which would seem to indicate that the acid is bibasic. The pyruvates commonly exist in two forms, one crystalline, the other gummy. The crystalline form is changed inta the other by a gentle heat.

\section{Malic Acid. $\mathrm{C}_{8} \mathrm{H}_{4} \mathrm{O}_{8}, 2$ H $\mathrm{O}=\overline{\mathrm{M}}_{2} 2$ Н $\mathrm{O}$.}

This acid is of very frequent occurrence in acid fruits, as in the apple, and especially in the unripe berries of Sorbus aucuparia, or mountain-ash. The best method of extracting it is to express these berries when they begin to turn red, and to add to the strained liquid a thin milk of lime so as not entircly to neutralise the acid. On heating, neutral malate of lime separates, and is removed by a skimmer. To the mother liquid more milk of lime is added cautiously, so as to produce an additional quantity of salt. The malate of lime is washed with cold water, and dissolved with the aid of heat in a mixture of 1 part of nitric acid, and 10 of water. On cooling, acid malate of lime is deposited in regular crystals, which are almost always colourless, They are rendered quite pure by a solution in hot water, and crystallisation. From this salt by the addition of acetate of lead, malate of lead is precipitated as a curdy white solid, which, if 
left in the liquid, changes into shining silky orystals. These, which are pure malate of lead, being decomposed by sulphuretted hydrogen, yield the acid, which, when evaporated to a syrup, forms a granular crystalline mass, deliquescent in the air, of a strong but agreeable acid taste. When the crystallised acid is kept for some time at a heat of $280^{\circ}$, it melts, and the melted acid is gradually filled with crystals. Cold water removes the unchanged malic aoid, which, if again heated, undergoes the same change, till at length all the malic acid is converted into these crystals, which are paramaleic or fumaric acid.

If malic acid be distilled by a sharp heat, a great part passes over in the form of a volatile crystallisable acid, the maleic acid. At a certain period of the distillation, if the retort be removed from the fire, the boiling residue having become turbid and thick, it suddenly becomes quite solid, and is found to consist of fumaric acid.

Malic acid is bibasic; in proof of which, it forms acid salts with lime, magnesia, and oxide of zinc, which monobasic acids never do. There are two series of malates: 1 . neutral, $\overline{\mathrm{M}}, 2 \mathrm{M} 0 ; 2$. acid, $\overline{\mathrm{M}}, \mathrm{M} \mathrm{O}, \mathrm{H} \mathrm{O}$. Most of the malates are soluble in water, but not in alcohol. Lime water neutralised by malic acid continues clear, whether cold or hot, which serves to distinguish it irom tartaric, citric, racemic, and oxalic acids.

Acid Malate of Ammonia, $\overline{\mathrm{M}}, \overline{\mathrm{N}} \mathrm{H}_{4} \mathrm{O}, \mathrm{H} \mathrm{O}$, is best formed by neutralising with ammonia one of two equal portions of malic acid (as prepared from the crude malate of lead by diluted sulphuric acid), and then adding the other portion and evaporating to a syrup. On cooling, large and very regular crystals of the acid salt are deposited, which are easily decolorised by animal charcoal. This is an excellent method of purifying malic acid, when it is much contaminated with other substances. Acid malate of lime, $\overline{\mathrm{M}}, \mathrm{Ca} \mathrm{O}, \mathrm{H} \mathrm{O}+6$ aq., is prepared as above described. It forms very regular and pure crystals, soluble in their own weight of boiling water, but requiring 20 parts of cold water. When malic acid is saturated with chalk, an acid liquid is obtained, which, when boiled, deposits the neutral malate of lime, $\overline{\mathrm{M}}, 2 \mathrm{Ca} \mathrm{O}$. The malate of lead $\overline{\mathrm{M}}, 2 \mathrm{~Pb} \mathrm{O}+6 \mathrm{aq}$, is remarkable for changing when left in the liquid in which it has been formed, from a curdy white precipitate to a mass of fine silky needles. In hot water this salt melts into a mass like pitch in consistence. Acid malate of copper, $\overline{\mathrm{M}}, \mathrm{Cu} \mathrm{O}, \mathrm{H} \mathrm{O}+2$ aq., forms splendid large crystals of a tine cobalt blue colour. There appears to be a basic malate of copper, $\overline{\mathrm{M}}, 2 \mathrm{Cu} \mathrm{O}+\mathrm{Cu} \mathrm{O}+6 \mathrm{aq}$, , which forms green crystals. Mralate of silver, $\mathrm{M}, 2 \mathrm{Ag} 0$, is a white powder, soluble in boiling water. The other malates are analogous to these, and possess little interest. 
Malamide, $\mathrm{C}_{8} \mathrm{~N}_{2} \mathrm{H}_{10} \mathrm{O}_{8}$. This substance, which occurs in asparagus, and in Althaa officinalis, has been called asparagine. It is formed from neutral malate of ammonia, as follows, $\mathrm{C}_{8} \mathrm{H}_{4}$ $\mathrm{O}_{8}, 2 \mathrm{~N} \mathrm{H}_{4} \mathrm{O}=2 \mathrm{H} \mathrm{O}+\mathrm{C}_{8} \mathrm{~N}_{2} \mathrm{H}_{10} \mathrm{O}_{8}$. It is best obtained from vetches or pease, grown in the dark, or etiolated, by evaporating the expressed juice, when it forms large transparent crystals. When acted on by hyponitrous acid, it yields malic acid, thus: $\mathrm{C}_{8} \mathrm{~N}_{2} \mathrm{H}_{10} \mathrm{O}_{8}+2 \mathrm{~N} \mathrm{O}_{3}=4 \mathrm{H} \mathrm{O}+\mathrm{N}_{4}+\mathrm{C}_{8} \mathrm{H}_{4} \mathrm{Os}_{3}, 2 \mathrm{H} \mathrm{O}$.

Malamidic Acid, $\mathrm{C}_{8} \mathrm{~N} \mathrm{H}_{7} \mathrm{O}_{8}$. SYN. Aspartic Acid. This compound is formed along with ammonia when malamide is boiled with potash or with acids. Its origin from malic acid is similar to that of all acid amides. Acid malate of ammonia, losing 2 eqs. of water, yields malamidic acid. $\left(\mathrm{C}_{8} \mathrm{H}_{4} \mathrm{O}_{8}, \mathrm{H} \mathrm{O}, \mathrm{N} \mathrm{H}_{4} \mathrm{O}\right)=$ $2 \mathrm{HO}+\mathrm{C}_{8} \mathrm{~N} \mathrm{H}_{7} \mathrm{O}_{8}$. This compound also yields malic acid, when acted on by hyponitrous acid. $\mathrm{C}_{8} \mathrm{~N} \mathrm{H}_{7} \mathrm{O}_{8}+\mathrm{N} \mathrm{O}_{3}=\mathrm{HO}+$ $\mathrm{N}_{2}+\mathrm{C}_{8} \mathrm{H}_{4} \mathrm{O}_{8}, 2 \mathrm{H} \mathrm{O}$.

Maleic Acid, $\mathrm{C}_{8} \mathrm{H}_{2} \mathrm{O}_{6}, 2 \mathrm{H} \mathrm{O}=\overline{\mathrm{Ma}}, 2 \mathrm{H} 0$, is prepared as above mentioned, by distilling malic acid. This acid is bibasic, but has the same composition, in 100 parts, as aconitic, or equisetic acid. It forms crystals, which are very soluble in water, alcohol, and ether. When heated sharply, it yields water, and a white volatile solid, melting at $134^{\circ}$, and boiling at $350^{\circ}$, which appears to be anhydrous maleic acid. When the hydrated acid is kept melted for some time, it is changed, exactly as malic acid is, into fumaric acid. Hydrated maleic acid has precisely the same composition as anhydrous malic acid, which at once explains its formation.

When heated more strongly, maleic acid loses water, and is converted into anhydrous maleic acid, $\mathrm{C}_{8} \mathrm{H}_{2} \mathrm{O}_{8}$.

The general formula of its salts is $\overline{\mathrm{Ma}}, 2 \mathrm{M} \mathrm{O}$ for the neutral, and $\overline{H a}, \mathrm{M} \mathrm{O}, \mathrm{HO}$ for the acid maleates. It forms an acid maleate of silver, $\overline{\mathrm{Ma}}, \mathrm{Ag} \mathrm{O}, \mathrm{H} \mathrm{O}$.

Fumaric or Paramaleic Acid, $\mathrm{C}_{8} \mathrm{H}_{2} \mathrm{O}_{6}, 2 \mathrm{H} \mathrm{O}=\overline{\mathrm{Fu}}, \mathrm{H} 0$, is formed as above stated, by heating either malic or maleic acids to their melting-point, and keeping them melted for a long time. It occurs in Fumaria officinalis, and in Iceland moss. It forms micaceous scales requiring 200 parts of cold water for solution. It is soluble in alcohol.

It is a bibasic acid, isomeric with maleic acid, and which at once explains its formation from malic, or from maleic acid. Its salts are sparingly soluble. The fumarate of oxide of ethyle is a heavy oily liquid of an aromatic smell of fruits. When this ether, $\overrightarrow{\mathrm{Fu}}$, $2 \mathrm{Ae} \mathrm{O}$, is acted on by aqua ammoniæ, it forms a white insoluble powder, which is fumaramide, $\mathrm{C}_{8} \mathrm{H}_{6} \mathrm{~N}_{2} \mathrm{O}_{4}$. This body has all the characters of a compound amide. 
When the bimalate of ammonia is heated, there is formed a brick-coloured powder, which seems to be fumarimide, $\mathrm{C}_{8} \mathrm{H}_{3} \mathrm{~N} \mathrm{O}_{4}$. By the action of boiling hydrochloric acid, this substance is converted into aspartic acid.

Malic acid, like tartaric acid, exists in two isomeric, or rather allotropic modifications, which agree in chemical characters, but differ in regard to polarised light. The active or common malic acid, causes deviation of the plane of polarisation to the left. Its salts cause deviation, some to the right, others to the left. The inactive malic acid, or passive, as it is also called, has no action of this kind.

Malamide, or asparagine, and malamidic or aspartic acid, also cause deviation, but with asparagine, it is to the left when dissolved in water or alkalies, to the right when dissolved in acids. The aspartic acid has two allotropic forms, active and passive. That formed from asparagine deviates to the left or right, according as it is dissolved in bases or in acids. The passive acid, which is formed by heating bimalate of ammonia to $392^{\circ}$, and boiling the residue with hydrochloric acid, has no action on the plane of polarisation.

When malate of lime, maleate of lime, or asparagine (malamide) are allowed to ferment, in contact with water and yeast, succinic acid is formed.

From malic acid, it is thus formed :-

Malic Acid.
$3\left(\mathrm{C}_{8} \mathrm{H}_{8} \mathrm{O}_{10}\right)=2\left(\mathrm{C}_{8} \mathrm{H}_{6} \mathrm{O}_{8}\right)+\mathrm{C}_{4} \mathrm{H}_{4} \mathrm{O}_{4}+4 \mathrm{C} \mathrm{O}_{2}+2 \mathrm{H} \mathrm{O}$.

The other processes are explained as easily. Asparagine yields succinate of ammonia by taking up water and hydrogen, derived from a portion that is destroyed.

$$
\begin{aligned}
& \text { Asparagine. } \\
& \mathrm{C}_{8} \mathrm{H}_{8} \mathrm{~N}_{2} \mathrm{O}_{6}+2 \mathrm{H} \mathrm{O}+\mathrm{H}_{2}=\mathrm{C}_{8} \mathrm{H}_{4} \mathrm{O}_{6}, 2 \mathrm{~N} \mathrm{H}_{4} \mathrm{O} \text {. }
\end{aligned}
$$

The maleic acid probably first takes up water, and is reconverted into malic acid, which then ferments.

Under certain circumstances, the fermentation of malate of lime yields, not only carbonic acid, but hydrogen gas, and the succinate of lime, if kept in contact with the ferment, also exhibits this result. This is due to a different fermentation, in which either malic or succinic acid is converted into butyric acid.

Malic Acid. Butyric Acid.

$2\left(\mathrm{C}_{8} \mathrm{H}_{8} \mathrm{O}_{10}\right)=\mathrm{C}_{8} \mathrm{H}_{8} \mathrm{O}_{4}+8 \mathrm{C} \mathrm{O}_{2}+\mathrm{H}_{4}$. 
Succinic Acid. Butyric Acid.

$3\left(\mathrm{C}_{8} \mathrm{H}_{6} \mathrm{O}_{8}\right)=2\left(\mathrm{C}_{8} \mathrm{H}_{8} \mathrm{O}_{4}\right)+8 \mathrm{C} \mathrm{O}_{2}+\mathrm{H}_{2}$.

By this process, succinic acid may be obtained in any quantity from the impure malate of lime, as it is got by heating the juice of the mountain-ash berries with milk of lime; and if required, butyric acid might also be thus procured in abundance.

\section{Tannic Acid. $\mathrm{C}_{54} \mathrm{H}_{22} \mathrm{O}_{34}$.}

Srn. Quercitannic Acid. Gallotannic Acid. Tannine. This acid occurs chiefly in oak-bark and in nut-galls, an excrescence

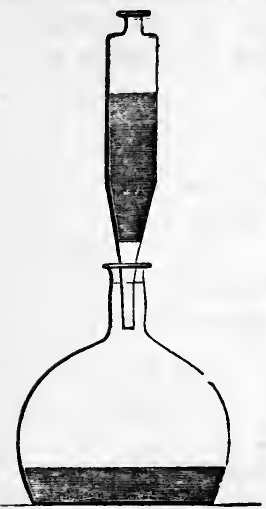
on oak leaves caused by the attacks of an insect, which apparently pierces the leaf in order to deposit its eggs. It has lately been stated that the tannic acid of oakbark differs from that of nut-galls, and does not, like it, yield gallic acid.

To obtain it, coarsely-powdered nutgalls are acted on, in an apparatus of displacement, by ether, free from alcohol, but saturated with water. When the other, after being left some time in contact with the powder, is allowed to drop into the lower vessel, it separates into two strata of liquid, the lower of which is a pure solution of tannic aoid in water, which is drawn off and dried up after being washed with ether. The dry mass is re-dissolved in water, and again dried up in vacuo.

Tannic acid thus obtained is nearly white, and not at all crsstalline. It is very soluble in water, and has a most astringent taste without bitterness. It is soluble in weak alcohol, but hardly soluble in ether. The aqueous solution, if exposed to the air, absorbs oxygen, produces an equal volume of carbonic acid, and is converted into gallic and ellagic acids. The addition of the mineral acids to a solution of tannic acid, causes a precipitate, which is composed of tannic aoid and the aoid employed (sulphuric, $\& c$.$) , and which is very soluble in pure water. The precipitate$ formed by sulphuric aoid, in a hot solution, dissolves in hot diluted sulphuric acid, and when this solution has been boiled a short time, it contains no tannic acid, the whole being converted into gallic acid and grape sugar.

Tannic acid combines with animal gelatine, forming an insoluble curdy precipitate. A piece of prepared skin, introduced 
into a solution of tannic acid, absorbs the acid, and is converted into leather. When heated, tannic acid is converted into metagallic and pyrogallic acids.

Tannic acid and its salts strike a deep blue, nearly black colour with persalts of iron; and it likewise causes a precipitate in the solutions of most of the vegetable bases.

The composition of the salts of tannic acid is but little known. That of ammonia appears to contain $1 \mathrm{eq}$. of base, while those of potash, soda, and baryta seem to contain 2 eq., and those of iron and lead 3 eq. of base for the above formula, $\mathrm{C}_{54}$ \& $\&$. It is possible that tannic acid may be tribasic, in which case we may have three kinds of tannates, $\mathrm{C}_{64} \mathrm{H}_{22} \mathrm{O}_{33}, \mathrm{MO}$; $\mathrm{C}_{64} \mathrm{H}_{20} \mathrm{O}_{32}$, $2 \mathrm{MO}$ and $\mathrm{C}_{54} \mathrm{H}_{19} \mathrm{O}_{31}, 3 \mathrm{M} \mathrm{O}$; and the acid itself may be $\mathrm{C}_{54} \mathrm{H}_{19} \mathrm{O}_{31}, 3 \mathrm{H} 0$. The salts with 1 and 2 eq. of fixed base may of course also be written $\left.\mathrm{C}_{54} \mathrm{H}_{19} \mathrm{O}_{31}, 2 \mathrm{MO} \mathrm{HO}\right\}$ and $\mathrm{C}_{54} \mathrm{H}_{19} \mathrm{O}_{31}$, $2 \mathrm{M}$
$\mathrm{H}$

The most interesting fact yet known in the history of tannio acid is its resolution-when its solution is exposed to the air, or more rapidly when boiled with diluted sulphuric or hydrochloric acids-into gallic acid and glucose, or grape sugar. This transformation is easily explained, for

$$
\begin{aligned}
& \text { Tannic Acid. } \\
& \mathrm{C}_{54} \mathrm{H}_{22} \mathrm{O}_{34}+8 \mathrm{H} \mathrm{O}=3\left(\mathrm{C}_{14} \mathrm{H}_{6} \mathrm{O}_{10}\right)+\mathrm{C}_{12} \mathrm{H}_{12} \mathrm{O}_{12}
\end{aligned}
$$

This is a kind of fermentation, but it is not promoted-indeed, it seems rather to be retarded-by the addition of yeast or other ferments. The infusion of nut-galls seems to contain some ferment which assists in the change, but that infusion, like a solution of pure tannic acid, absorbs oxygen, and gives off carbonic acid, and we do not yet know how these facts are connected with the production of gallic acid and glucose. When, by exposure to air, this change has taken jlace, yeast and other ferments then cause the glucose to ferment, yielding alcohol and carbonic acid. The accuracy of the equation above given of the transformations of tannic acid is proved by the proportions of gallic acid and of glucose obtained; for we get 87 parts of gallic acid and 22 of glucose from 100 of tannic acid.

The uses of tannic acid are important. In oak-bark and sumach it is used in the manufacture of leather, which is animal membrane or gelatine combined with tannic acid. Mixed with salts of iron, the tannic acid of nut-galls yields ink. Pure tannic acid, as well as nut-galls, is used in medicine as a powerful astringent. 


\section{Gallic Acid. $\mathrm{C}_{14} \mathrm{H}_{6} \mathrm{O}_{10}=\mathrm{C}_{14} \mathrm{H}_{3} \mathrm{O}_{7}, 3 \mathrm{H} \mathrm{O}$.}

This aoid exists in the seeds of mango, and is formed as above described by the deoomposition of tannic acid. The best method is to boil tannic acid with sulphuric acid diluted with 8 parts of water, or to keep it near the boiling point for some hours, replacing the water which evaporates. The liquid, on evaporation, yields a large crop of crystals nearly pure, on cooling. When coloured, gallio acid is purified from colouring matter by combining it with oxide of lead, and decomposing the gallate of lead, suspended in water, by sulphuretted hydrogen; the sulphuret of lead acts as a decolorising agent.

Pure gallic acid forms beautiful prisms of a silky lustre, and a slight yellowish colour, of the formula $\mathrm{C}_{14} \mathrm{H}_{3} \mathrm{O}_{7}, 3 \mathrm{H} \mathrm{O}+2 \mathrm{aq}$. It is sparingly soluble in cold water, requiring 100 parts, but dissolves in 3 parts of boiling water. Solutions of the acid and its salts strike a black colour with persalts and protopersalts of iron. When exposed to the air, the solution of gallic acid absorbs oxygen, and becomes dark-coloured; this change is very rapid in the presence of alkalies, so that the alkaline gallates, especially if the alkali be in excess, are rapidly decomposed, and become nearly black.

When dissolved in hot oil of vitriol, and precipitated from the cold solution by water, gallio acid is obtained in a peculiar form, called rufigallio aoid, which is $\mathrm{Cl}_{4} \mathrm{H}_{4} \mathrm{O}_{8}$, that is, gallic acid, minus $2 \mathrm{H} \mathrm{O}$. This acid is a reddish-brown orystalline powder, which might be used in dyeing, as it yields colours on cloth like those from madder. When heated it forms a sublimate of fine red prisms, whioh call to mind alizarine, the crystalline matter found in madder.

By the action of heat, crystallised gallio aoid yields, like tannic acid, pyrogallio and metagallic acids.

The gallates are little known. As in the oase of other tribasic aoids there are salts with 1 eq., 2 eq., or 3 eq. of fixed base. There are also acid salts and basio salts; but they are very easily decomposed by the action of the air. The recent investigations of Büchner, jun., have shown that both the tannates and gallates are in some respects anomalous, and require further study.

When tannio, or gallic aoid, is heated by a sharp fire, carbonic acid, water, and pyrogallic aeid distil over, while a dark solid remains in the retort, which is metagallic aeid.

Pyrogallic Acid, $\mathrm{C}_{22} \mathrm{H}_{6} \mathrm{O}_{6}$, is best obtained by heating the aqueous extract of galls in the same way as gum benzoin is heated 
to yield benzoio acid. The crystals collect on the upper surface of the paper diaphragm, quite pure. It forms shining seales of a hitter and astringent taste; fusible at $240^{\circ}$, volatile at $410^{\circ}$. It is converted by a stronger heat into metagallic acid. It is formed from gallic acid, by the loss of 2 eq. carbonio acid. If acid at all, it is a very feeble acid, and in an alkaline solution it absorbs oxygen very rapidly, so that it may be used for the analysis of air. Pyrogaliic acid is also used in photography.

Metagallic Acid, $\mathrm{C}_{22} \mathrm{H}_{3} \mathrm{O}_{3}, \mathrm{HO}$ ? $\mathrm{C}_{24} \mathrm{H}_{8} \mathrm{O}_{8}$ ? is produced as above mentioned from tannic, gallic, and pyrogallio acids. It is a black powder, insoluble in water, soluble in alkalies. Of its salts little is known. It differs from pyrogallic aoid only by the elements of water.

When an infusion of nut-galls has been so long exposed to the air, that all tannic acid has disappeared, the gallic acid is found mixed with an insoluble, or sparingly soluble powder, which is a new acid, ellagic acid. Its composition is, $\mathrm{C}_{28} \mathrm{H}_{6} \mathrm{O}_{16}$ +4 aq., and when dried at $240^{\circ}, \mathrm{C}_{28} \mathrm{H}_{6} \mathrm{O}_{18}=\mathrm{C}_{28} \mathrm{H}_{4} \mathrm{O}_{14}, 2 \mathrm{H} \mathrm{O}$. It has not been muoh studied, and we do not know its actual atomic weight. When heated, it yields greenish yellow vapours, which condense into crystals of the same colour, insoluble in water, alcohol, or ether; soluble in sulphurio acid and in alkalies.

This acid is said to occur in the root of tormentilla vulgaris : and is also the chief constituent of bezoar stones, which are a species of animal oonoretion. It seems to be a bibasic acid, but its salts are little known.

Tannic acid, and the substances derived from it, occur in a good many plants, besides those of the genus quercus; the infusions of all of which are recognised by their striking a bluishblaok with persalts of iron. But the astringent taste, and the property of tanning, or combining with animal gelatine, are found in many plants, such as cinchona, kino, catechu, pinus, \&c. These are distinguished by giving, with persalts of iron, either a dark green or a grey colour. It has not been proved that they contain tannio aoid, but Geiger has shown that these different oolours may occur even when the same tanning principle is present, ard that the green is owing, at all events frequently, to the presence of free acid, while the addition of chalk, in some cases, ohanges the green to the characteristic bluish-black due to tannio acid. There are, however, some reasons for admitting more than one tanning or astringent principle, and it is supposed by some that there are several different tannic acids homologous with each other, which is far from improbable. The whole subject requires investigation. 


\section{Catechu. Mimotannic Acid.}

When catechu, the dried extract of mimosa catechu, is acted on by cold water, it yields a soluble matter very similar to tannic acid, if not iủentical with it when pure. It is, however, contaminated by some compound which causes it to redden when exposed to air. It does not appear to yield the same products when heated, as tannic acid does; but this is uncertain, and may be caused by the presence of impurities. Berzelius proposes to call this tannic acid mimotannic acid, from mimosa, to distinguish it from the tannic acid of galls, which he calls quercitannic acid, from quercus.

The portion of catechu insoluble in cold water contains a peculiar compound, called catechine or tanningenic acid. It is soluble in hot water, and when pure forms a white silky crystalline powder, which is said to be composed of $\mathrm{C}_{15} \mathrm{H}_{6} \mathrm{O}_{6}$. When heated, it is said to be transformed into (mimo?) tannic acid. By the action of caustic potash it yields a black acid, japonic acid, $\mathrm{C}_{22} \mathrm{H}_{4} \mathrm{O}_{4}, \mathrm{H} \mathrm{O}$ ? Carbonate of potash converts it into a red acid, rubinic acid, $\mathrm{C}_{18} \mathrm{H}_{6} \mathrm{O}_{4}$ (?) in the anhydrous state. The hydrated acid is said to have the same composition as japonic acid, possibly therefore $\mathrm{C}_{18} \mathrm{H}_{6} \mathrm{O}_{6}$ ? But as the japonic acid, on the same authority, Svanberg, in combining with silver forms a salt $\mathrm{C}_{24} \mathrm{H}_{8} \mathrm{O}_{7}, \mathrm{Ag} \mathrm{O}$, in which 2 eqs. of the acid have lost 2 eqs. of water and gained only 1 eq. of oxide of silver, it is evident that our knowledge of these compounds is very imperfect.

Rochleder has shown that in coffee, tea, and other plants of the family of the Rubiaceæ, there exists a remarkable series of acids.

Caffeotannic or Caffeic acid, $\mathrm{C}_{34} \mathrm{H}_{8} \mathrm{O}_{7}$ is found in coffee, and has some resemblance to tannic acid. Viridic acid, $\mathrm{C}_{14} \mathrm{H}_{7} \mathrm{O}_{8}$, is also found in coffee and in Paraguay tea. Boheic acid, $\mathrm{C}_{4} \mathrm{H}_{6} \mathrm{O}_{8}$, occurs in tea, and is also in some respects similar to tannic acid. Now, all these plants produce caffeine, and, as will be seen under the head of caffeine, that body contains a group with $\mathrm{C}_{12}$. The above acids seem to contain also such a group, $\mathrm{C}_{12} \mathrm{H}_{6} \mathrm{O}_{5}$, which coupled with formic aldehyde, with formic acid, and with oxalie acid, forms these acids.

$$
\begin{array}{ll}
\mathrm{C}_{22} \mathrm{H}_{6} \mathrm{O}_{5}+\mathrm{C}_{2} \mathrm{H}_{2} \mathrm{O}_{2}=\mathrm{C}_{14} \mathrm{H}_{8} \mathrm{O}_{7} \\
\mathrm{C}_{12} \mathrm{H}_{6} \mathrm{O}_{5}+\mathrm{C}_{2} \mathrm{H} & \mathrm{O}_{3}=\mathrm{C}_{14} \mathrm{H}_{7} \mathrm{O}_{8} \\
\mathrm{C}_{12} \mathrm{H}_{6} \mathrm{O}_{5}+\mathrm{C}_{2} & \mathrm{O}_{3}=\mathrm{C}_{14} \mathrm{H}_{6} \mathrm{O}_{8}
\end{array}
$$

It is worthy of remark that caffeine appears to contain 
cyanogen and methylamine, both closely related to formic acid. The same family of plants yields another series of acids, hitherto little studied, with $\mathrm{C}^{\mathbf{2 4}} \mathrm{Hs}$ and a variable amount of oxygen, but not resembling tannio acid. Both series readily lose 2 eqs. of carbon in some form, and probably therefore contain a group with $\mathrm{C}^{12}$. There is a third series of acids, with $\mathrm{C}_{12}$, found in this family, as citric acid, $\mathrm{C}_{12} \mathrm{H}_{5} \mathrm{O}_{11}, 3 \mathrm{H} \mathrm{O}$, and chinovic acid, $\mathrm{C}_{12} \mathrm{H}_{9} \mathrm{O}_{3}, 3 \mathrm{H} \mathrm{O}$. Lastly, this large and important family yields bodies like quinine, ciuchonine, and alizarine, \&c., with 20 eqs. - of carbon. It appears that Rochleder has modified the formulæ above given, and now makes caffeio acid, $\mathrm{C}_{28} \mathrm{H}_{16} \mathrm{O}_{14}$, while Gerhardt proposes for it the formula $\mathrm{C}_{70} \mathrm{H}_{38} \mathrm{O}_{34}$. It is quite evident that we must wait for more decisive results.

\section{Meconic Acid. $\mathrm{C}_{14} \mathrm{H}_{11}, 3 \mathrm{H} \mathrm{O}=\overrightarrow{\mathrm{Me}}, 3$ H $\mathrm{O}$.}

A tribasic acid, found only in opium, the dried juice of papaver somniferum. To prepare it, the crude meconate of lime, obtained in the manufacture of muriate of morphia, is mixed with 20 parts of boiling water, and 3 parts of strong hydrochloric acid added to the mixture, which must be removed from the fire, and not boiled after the acid has been added. On cooling, acid meconate of lime is deposited in shining crystals, which are collected on a cloth filter, squeezed, and treated a second time with the same quantities of acid and hot water. The strained acid liquid contains a large but variable proportion of sulphate of lime, always present, sometimes even to the extent of $\frac{1}{2}$ or $\frac{3}{4}$ in the crude meconate of lime. Hence the advantage of using so much hydrochloric acid, which also renders the meconic acid less soluble. This time the crystals are meconic acid, still much coloured. They are collected and squeezed as before, and to make sure that all lime is removed, a third time dissolved in 20 parts of hot water and 2 of hydrochloric acid. The addition of the acid not only removes the last traces of lime, but causes the meconic acid to crystallise almost entirely out of the liquid, it being nearly insoluble in diluted acid. The crystals, washed with a little cold water, and dried at the ordinary temperature, are now pure from everything but colouring matter, and when heated to redness leave' no residue.

To get rid of the colour, the crystals are now mixed with warm water, and caustic potash gradually added, so as nearly, but not quite, to neutralise the acid. As soon as the point of neutralisatiou is reached, the reddish colour changes to green, and so much potash must be added, that any further quantity would produce the green colour. The whole is then beated in the water bath, 
till all is dissolved, hot water being added if necessary. (Were the potash now in excess, the whole acid would be decomposed into oxalic and carbonic aoids.) On cooling, the meconate of potash, $\overline{M_{e}}\left\{\begin{array}{l}2 \mathrm{KO} \\ \mathrm{HO}\end{array}\right.$, erystallises, forming a semi-solid mass, which is to be squeezed out. The colour is carried off for the most part in the mother liquid, which is very dark, and the squeezed salt after a second, or if necessary, a third solution in hot water, crystallisation, and squeezing, is snow-white. This purified meconate of potash is then acted on by pure hydrochloric acid, exactly as recommended for the meconate of lime, and after the third operation yields perfectly pure and white meconic acid in beautiful silvery scales, which, to remove any traces of the acid mother liquid adhering to them, may be once more dissolved in the smallest possible quantity of hot water, avoiding a heat of $212^{\circ}$, which decomposes the acid; the pure acid is deposited on cooling, as $\overline{\mathrm{Me}}, 3 \mathrm{H} \mathrm{O}+6$ aq.

Meconic acid, when gently heated, loses 6 eq. of water of crystallisation. It is soluble in water and in alcohol. When boiled, its solution becomes coloured, produoing comenio acid, carbonic acid, and a dark brown colouring matter. If boiled with hydrochloric acid, it is resolved into comenic acid and carbonic acid, without the production of colouring matter. When the dry acid is heated to $250^{\circ}$, the same change takes place. When heated with excess of aqua potassæ, meconic acid is entirely decomposed into oxalic acid, carbonic acid, and a dark colouring matter. Its distinguishing oharacteristic is that of oausing, in persalts of iron, a deep blood-red oolour, but no precipitate.

It forms three series of salts, like other tribasic acids. Thus, there are three mecanates of potash : 1. acid, $\overline{\mathrm{Me}}\left\{\begin{array}{l}\mathrm{K} \mathrm{O} \\ 2 \mathrm{H} \mathrm{O}\end{array} ; 2\right.$. neutral, above mentioned, $\overline{M e}\left\{\begin{array}{l}2 \mathrm{~K} \mathrm{O} \\ \mathrm{H} \mathrm{aq} .\end{array}\right.$ Both of these orystallise. 3. tribasic, $\overline{\mathrm{Me}} 3 \mathrm{~K} 0$. This is yellow, and does not crystallise. There are also three meconates of soda; two of lime, acid and neutral or bibasic, and two of silver, bibasic $\overline{\mathrm{Me}}$, $2 \mathrm{Ag} \mathrm{O}, \mathrm{H} \mathrm{O}$, and tribasic $\overline{\mathrm{Me}}, 3 \mathrm{Ag} \mathrm{O}$. The meconate of peroxide of iron is very soluble, of an intense blood-red colour, but as it cannot be obtained pure or crystallised, its composition is still unknown. Mr. How has recently obtained the following compounds :

Bibasic meconate of ammonia, $\mathrm{H} \mathrm{O}, 2 \mathrm{~N} \mathrm{H}_{4} \mathrm{O}, \mathrm{C}_{14} \mathrm{H} \mathrm{O}_{11}$ Monobasic ditto $2 \mathrm{H} \mathrm{O}, \mathrm{N} \mathrm{H}_{4} \mathrm{O}, \mathrm{C}_{14} \mathrm{H} \mathrm{O}_{11}$ Ethylomeconic acid . $\quad 2 \mathrm{H} \mathrm{O}, \mathrm{C}_{4} \mathrm{H}_{5} \mathrm{O}, \mathrm{C}_{14} \mathrm{H} \mathrm{O}_{11}$ and its salts. 
Meconamidic acid . . . $9 \mathrm{H} \mathrm{O}, \mathrm{C}_{84} \mathrm{H}_{24} \mathrm{~N}_{7} \mathrm{O}_{13}+6$ aq. and its salts. Meconoethylomeconic acid . $3 \mathrm{H} \mathrm{O}, \mathrm{C}_{24} \mathrm{H} \mathrm{O}_{11}+2 \mathrm{H} \mathrm{O}, \mathrm{C}_{4} \mathrm{H}_{5} \mathrm{O}, \mathrm{C}_{14}$

Biethylomeconic acid . . $\mathrm{H} \mathrm{O}, 2 \mathrm{C}_{4} \mathrm{H}_{5} \mathrm{O}, \mathrm{C}_{14} \mathrm{H} \mathrm{O}_{12}$ and its salts.

\section{Comenic Acid. $\mathrm{C}_{12} \mathrm{H}_{2} \mathrm{O}_{8}, 2 \mathrm{H} \mathrm{O}=\overline{\mathrm{Co}}, 2 \mathrm{H} \mathrm{O}$.}

This acid is formed as above described, from meconio acid by the action of heat, or of heat and acid combined. 1 eq. dried meconic acid, $\mathrm{C}_{14} \mathrm{HO}_{11}+3 \mathrm{HO}=\mathrm{C}_{14} \mathrm{H}_{4} \mathrm{O}_{14}$, yields 1 eq. comenic acid, $\mathrm{C}_{12} \mathrm{H}_{2} \mathrm{O}_{8}, 2 \mathrm{H} \mathrm{O}$, and 2 eqs. oarbonic acid, $2 \mathrm{C} \mathrm{O}_{2}$. The acid is readily obtained by boiling meconate of lime with an excess of diluted hydrochloric acid. It is deposited on cooling in coloured crystals, which may be decolorised by recrystallisation with the aid of animal charooal. The pure aoid has a slight yellow tinge, and is very sparingly soluble in cold water. When heated, it is resolved into carbonic acid, pyromeconic acid, and a small quantity of a third substance, paracomenic acid, which, in some few points, differs from comenic acid, but has the same composition, and in many points is so similar, that it may possibly turn out to be essentially the same.

Comenic acid forms two series of salts, with 1 and 2 eq. of fixed base respectively. With persalts of iron it forms a deep red solution, which deposits black crystals of unknown composition. Mr. How has also studied this acid, and obtained the following compounds, besides a large number of its salts, which he has described :

Chlorocomenic acid $\left.2 \mathrm{H} 0, \mathrm{C}_{12} \underset{\mathrm{Cl}}{\mathrm{H}}\right\} \mathrm{O}_{8}+3$ aq., and its salts.

Bromocomenic acid $\left.2 \mathrm{H} \mathrm{O}, \mathrm{C}_{12} \underset{\mathrm{Br}}{\mathrm{H}}\right\} \mathrm{O}_{8}+3$ aq., and its salts.

Ethylocomenic acid $\mathrm{H} \mathrm{O}, \mathrm{C}_{4} \mathrm{H}_{5} \mathrm{O}, \mathrm{C}_{12} \mathrm{H}_{2} \mathrm{O}_{3}$, and its salts. Comenamic acid $\mathrm{H} \mathrm{O}, \mathrm{C}_{12} \mathrm{H}_{4} \mathrm{~N} \mathrm{O}_{7}+4$ aq., and its salts.

Pyromeconic Acid, $\mathrm{C}_{10} \mathrm{H}_{3} \mathrm{O}_{5}, \mathrm{H} \mathrm{O}$, is obtained as a crystalline sublimate by heating meconic or comenic acid. In fact, 1 eq. comenic acid, $\mathrm{C}_{12} \mathrm{H}_{4} \mathrm{O}_{10}$, contains the elements of $1 \mathrm{eq}$. pyromeconic acid, $\mathrm{C}_{10} \mathrm{H}_{4} \mathrm{O}_{6}$, and 2 eqs. carbonic acid, $2 \mathrm{C} \mathrm{O}_{2}$. It forms very soluble four-sided prisms, rather styptic to the taste; the solution of which forms with persalts of iron a crystalline salt of a fine red colour, the powder of which is like vermilion. This salt is $\mathrm{Fe}_{2} \mathrm{O}_{3}+3 \mathrm{C}_{10} \mathrm{H}_{3} \mathrm{O}_{5}$. Pyromeconic acid is a feeble acid, but Mr. Brown has recently described several of its salts, as well as two substitution products, bromopyromeconic and iodopyromeconic 
acids. It has the same composition as pyromucic acid, but is distinct from that compound.

9. Kinic Acid. $\mathrm{C}_{7} \mathrm{H}_{4} \mathrm{O}_{4}, 2 \mathrm{H} \mathrm{O}$ ? or $\mathrm{C}_{14} \mathrm{H}_{11} \mathrm{O}_{11}, \mathrm{H} \mathrm{O}$ ?

This very remarkable acid occurs in cinchona bark. It is obtained in the manufacture of sulphate of quinine, in the form of kinate of lime, from which the lime is easily separated by means of oxalic acid. The liquid filtered from the oxalate of lime yields, on evaporation, the kinic acid in crystals. The lime may also be removed by sulphuric acid, and any adhering sulphate of lime separated by alcohol.

The salts of kinic acid are somewhat anomalous. Thus there is a salt of lead, $\mathrm{C}_{7} \mathrm{H}_{4} \mathrm{O}_{4} 2 \mathrm{~Pb} \mathrm{O}$, and a salt of copper, $\mathrm{C}_{7} \mathrm{H}_{4} \mathrm{O}_{4}$, $\mathrm{Cu} \mathrm{O}, \mathrm{H} \mathrm{O}$, while the crystals of kinic acid are $\mathrm{C}_{7} \mathrm{H}_{6} \mathrm{O}_{6}$. All this would lead to the conclusion that the acid was a bibasic one, $\mathrm{C}_{7} \mathrm{H}_{4} \mathrm{O}_{4}, 2 \mathrm{H} \mathrm{O}$. But the kinate of lime, and the kinate of silver, both quite neutral salts, are $\mathrm{C}_{14} \mathrm{H}_{11} \mathrm{O}_{11}, \mathrm{Ca} \mathrm{O}$, and $\mathrm{C}_{14} \mathrm{H}_{11}$ $\mathrm{O}_{11}, \mathrm{Ag} \mathrm{O}$, as if the acid were monobasic, $\mathrm{C}_{14} \mathrm{H}_{11} \mathrm{O}_{11}, \mathrm{H} \mathrm{O}=$ $\mathrm{C}_{14} \mathrm{H}_{12} \mathrm{O}_{12}$. If we assume the acid to be quadribasic, we can then bring all the above salts into one series.

$$
\begin{aligned}
& \text { Thus,-Kinic acid would be } \quad . \quad \mathrm{C}_{14} \mathrm{H}_{8} \mathrm{O}_{8}, 4 \mathrm{HO} \\
& \text { Kinate of lead . } \quad . \quad \mathrm{C}_{14} \mathrm{H}_{8} \mathrm{O}_{8}, 4 \mathrm{~Pb} \mathrm{O} \\
& \text { Kinate of copper . . . } \mathrm{C}_{14} \mathrm{H}_{8} \mathrm{O}_{8}\left\{\begin{array}{l}
2 \mathrm{Cu} \mathrm{O} \\
2 \mathrm{H} \mathrm{O}
\end{array}\right. \\
& \text { Kinate of lime . . . } \mathrm{C}_{14} \mathrm{H}_{8} \mathrm{O}_{8}\left\{\begin{array}{l}
\mathrm{CaO}_{3} \mathrm{O} \\
3 \mathrm{HO}
\end{array}\right. \\
& \text { Kinate of silver . . . } \mathrm{C}_{14} \mathrm{H}_{8} \mathrm{O}_{8}\left\{\begin{array}{l}
\mathrm{Ag} \mathrm{O} \\
3 \mathrm{H} \mathrm{O} .
\end{array}\right.
\end{aligned}
$$

But the objection to this view is that, if this be the true constitution of the acid, the salts of lime and silver ought to be very acid, instead of being quite neutral.

According to Gerhardt, kinic acid is $\mathrm{C}_{28} \mathrm{H}_{20} \mathrm{O}_{20}, 2 \mathrm{H} \mathrm{O}$, and is bibasic. There are reasons which render this formula probable, but the subject requires investigation.

\section{PRODUCTS OF THE DECONPOSITION OF KINIC ACID.}

When kinic acid or kinate of lime is distilled with diluted sulphuric acid and peroxide of manganese, there is obtained a new compound called kinone, as a sublimate of fine golden yellow crystals, soluble in water, and very volatile, having a pungent smell in the state of vapour. Their composition is $\mathrm{C}_{25} \mathrm{H}_{8} \mathrm{Os}$. When acted on by reducing agents, it takes up 2 and 4 eqs. of 
hrdrogen, forming two new compounds, green and white hydrokinone. The green hydrokinone, $\mathrm{C}_{25} \mathrm{H}_{10} \mathrm{O}_{8}$, is one of the most beautiful compounds known to chemists, forming long prisms of the most brilliant gold-green metallic lustre, surpassing those of murexide in beauty. It is best formed by adding a few drops of sulphurous acid to a solution of kinone. When an excess of sulphurous acid is used, the white hydrokinone, $\mathrm{C}_{25} \mathrm{H}_{12} \mathrm{O}_{8}$, is formed, which crystallises in six-sided prisms. When acted on by oxidising agents, the solution of white hydrokinone becomes dark-red, nearly black, and almost immediately deposits the splendid erystals of the green compound. The latter is also formed by simply mixing solutions of kinone and white hydrokinone, being intermediate in composition between those bodies. Wöhler, to whom we are indebted for most of our knowledge in regard to these very curious compounds, has described a series of bodies obtained from the above by the action of hydrochloric acid, chlorine, and sulphuretted hydrogen, The following tabular view contains the names and composition of these substances, as far as we yet know them.

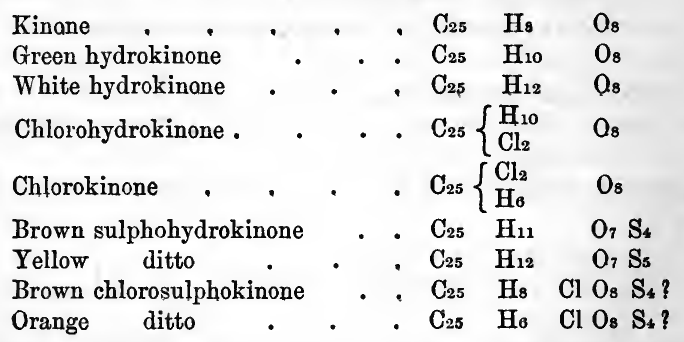

It will be observed, that in all these formulæ the carbon remains unaltered, and that several are instances of pure substitution, as $\mathrm{C}_{25} \mathrm{H}_{8} \mathrm{O}_{8}$, compared with $\mathrm{C}_{25}\left\{\begin{array}{l}\mathrm{H}_{6} \\ \mathrm{Cl}_{2}\end{array} \mathrm{O}_{8}\right.$; and $\mathrm{C}_{25} \mathrm{H}_{12} \mathrm{O}_{8}$, compared with $\mathrm{C}_{25}\left\{\begin{array}{l}\mathrm{H}_{10} \mathrm{O}_{8} . \mathrm{We}_{e} \text { can also see } \\ \mathrm{Cl}_{2}\end{array}\right.$ the relation of the sulphohydrokinones to kinone if we express them as follows:- $\mathrm{C}_{25} \mathrm{H}_{8}\left\{\begin{array}{l}\mathrm{O}_{7} \\ \mathrm{~S}+3 \mathrm{H} \mathrm{S} \text {; and } \mathrm{C}_{25} \mathrm{H}_{8}\end{array}\right.$ $\left\{\begin{array}{l}\mathrm{O}_{7} \\ \mathrm{~S}\end{array}\right.$

We have given the formulæ of these curious products according to Wöhler. But the progress of organic chemistry has taught us to look with suspicion on formulæ with an odd number of eq. of carbon, and Gerhardt, whose labours have aided so much in 
determining the true formulæ of many substances, proposes for kinic acid, as already mentioned, the formula $\mathrm{C}_{28} \mathrm{H}_{22} \mathrm{O}_{22}$; and for kinone $\mathrm{C}_{22} \mathrm{H}_{4} \mathrm{O}_{4}$. Hydrokinone becomes $\mathrm{C}_{12} \mathrm{H}_{6} \mathrm{O}_{4}$; green hydrokinone, $\mathrm{C}_{12} \mathrm{H}_{4} \mathrm{O}_{4}+\mathrm{C}_{22} \mathrm{H}_{6} \mathrm{O}_{4}=\mathrm{C}_{24} \mathrm{H}_{10} \mathrm{O}_{8}$. The chlorokinones, according to him, are

Chlorokinone $\mathrm{C}_{12}\left\{\begin{array}{l}\mathrm{H}_{3} \\ \mathrm{Cl}^{2}\end{array} \mathrm{O}_{4}\right.$
Bichlorokinone. $\mathrm{C}_{12}\left\{\begin{array}{l}\mathrm{H}_{2} \\ \mathrm{Cl}_{2} \mathrm{O}_{4}\end{array}\right.$
Trichlorokinone $\mathrm{C}_{12}\left\{\begin{array}{l}\mathrm{H}_{\mathrm{Cl}_{3}} \mathrm{O}_{4} \\ \text { Perchlorokinone or Chloranile }\end{array} \mathrm{C}_{12} \mathrm{Cl}_{4} \mathrm{O}_{4}\right.$.

The other compounds come to be represented by equally simple formulæ.

When kinic acid is decomposed by heat, it yields carbolic acid, benzoic acid, hyduret of salicyle, benzine (hyduret of phenyle), carbonic acid, and water. These changes are easily explained by Gerhardt's formula ; for

$$
\begin{gathered}
\text { Kinic Acid. } \\
\mathrm{C}_{28} \mathrm{H}_{22} \mathrm{O}_{22}
\end{gathered}=\underset{\text { and }}{2\left(\mathrm{C}_{12} \mathrm{H}_{6} \mathrm{O}_{2}\right)}+4 \mathrm{C} \mathrm{O}_{2}+10 \mathrm{H} \mathrm{O}
$$

Kinic Acid. Hydrokinone. Benzoic Acid.

$\mathrm{C}_{28} \mathrm{H}_{22} \mathrm{O}_{22}=\mathrm{C}_{22} \mathrm{H}_{6} \mathrm{O}_{4}+\mathrm{C}_{14} \mathrm{H}_{6} \mathrm{O}_{4}+\mathrm{C}_{2} \mathrm{O}_{4}+10 \mathrm{HO}$.

Again, as benzoic acid and hyduret of salicyle are isomeric, we have

$$
\begin{aligned}
& \begin{array}{l}
\text { Benzoic Acid or Hyd. } \\
\text { of Salicyle. }
\end{array} \\
& \mathrm{C}_{14} \mathrm{H}_{6} \mathrm{O}_{4}=\mathrm{C}_{12} \mathrm{Henzole.}_{8}+2 \mathrm{C} \mathrm{O}_{2} .
\end{aligned}
$$

These considerations tend to render Gerhardt's formulæ more probable than those of Wöhler; but for the present, and until farther researches, we shall retain both.

We have now briefly to notice a few acids of no great importance, which in some respects stand between the oily or fatty acids and those we have just mentioned.

Camphoric Acid, $\mathrm{C}_{20} \mathrm{H}_{16} \mathrm{O}_{8}=\mathrm{C}_{20} \mathrm{H}_{14} \mathrm{O}_{6}, 2 \mathrm{H} \mathrm{O}$.-This acid is formed by the action of nitric acid on camphor. It forms crystalline scales, sparingly soluble in cold water, very soluble in alcohol and ether, fusible at $158^{\circ}$. These are the hydrated acid, which, if distilled, is resolved into water and anhydrous camphoric acid. The camphorates are not peculiarly interesting. With oxide of ethyle, camphoric acid forms two compounds: 1. neutral, or camphoric ether, $\mathrm{C}_{20} \mathrm{H}_{14} \mathrm{O}_{6}, 2 \mathrm{Ae} \mathrm{O}$, an oily liquid, of a bitter taste and nauseous smell. 2. acid, $\mathrm{C}_{20} \mathrm{H}_{14} \mathrm{O}_{6}, \mathrm{Ae} \mathrm{O}, \mathrm{H} \mathrm{O}$, also 
called camphovinic acid, as it forms double salts analogous to the sulphovinates.

Anhydrous Camphoric Acid, $\mathrm{C}_{20} \mathrm{H}_{14} \mathrm{O}_{6}$, is solid, crystalline and volatile, and with dry ammonia forms a compound from which potash disengages no ammonia: with liquid ammonia it yields camphoramate of ammonia. Its action on oxide of ethyle has not been studied; but the subject deserves investigation. Camphoric acid yields an acid amide, camphamic acid, which, however, has not been much studied.

By the action of sulphuric acid on anhydrous camphoric acid, there is formed, with disengagement of carbonic oxide, a new acid, sulphocamphoric acid, $\mathrm{C}_{18} \mathrm{H}_{16} \mathrm{~S}_{2} \mathrm{O}_{12}+4$ aq. $=\mathrm{C}_{18} \mathrm{H}_{14}$ $\mathrm{S}_{2} \mathrm{O}_{10}, 2 \mathrm{H} \mathrm{O}+4 \mathrm{aq}$. This acid is crystallisable, and forms crystallisable salts of the formula $\mathrm{C}_{18} \mathrm{H}_{14} \mathrm{~S}_{2} \mathrm{O}_{10}, 2 \mathrm{M} \mathrm{O}$.

\section{CAMPHOR.}

There are two kinds of eamphor: that of Japan, or common camphor, $\mathrm{C}_{20} \mathrm{H}_{16} \mathrm{O}_{2}$; and that of Bornen, $\mathrm{C}_{20} \mathrm{H}_{18} \mathrm{O}_{2}$. The properties of common camphor, and its peculiar smell are well known. Its Sp. G. is 0.985 to 0.996 ; it is very volatile, evaporating at ordinary temperatures. Small fragments of camphor, on the surface of water, evaporate more rapidly, with rotatory movements. It dissolves in alcohol, and is precipitated by water. When distilled with anhydrous phosphoric acid, it yields a carbohydrogen, called camphogen, $\mathrm{C}_{20} \mathrm{H}_{14}$.

When camphor is passed in va pour over a heated mixture of hydrates of potash and lime, it yields a new acid, campholic acid, $\mathrm{C}_{20} \mathrm{H}_{17} \mathrm{O}_{3}, \mathrm{H} \mathrm{O}$. Nitric acid, with the aid of heat, converts camphor into camphoric acid. The essence or oil of camphor of commerce is $\mathrm{C}_{20} \mathrm{H}_{16} \mathrm{O}=1$ eq. camphor minus 1 eq. oxygen.

Borneo camphor occurs in small crystalline fragments. Its odour is different from that of common camphor. I find that, if wrapped in paper, a distinctly alliaceous odour traverses the paper, and may thus be detected. Heated with phosphoric acid, it yields a carbohydrogen, $\mathrm{C}_{20} \mathrm{H}_{16}$; and this is also the composition of the essence which accompanies Borneo camphor. This essence is, therefore, Borneo camphor, $\mathrm{C}_{20} \mathrm{H}_{18} \mathrm{O}_{2}$, minus 2 eqs. water.

Common camphor is produced by the Laurus Camphora; Borneo camphor is the produce of Dryobalanops Camphora, and is so highly prized by the Japanese, as a remedy for rheumatism, that it is not found in the markets of Europe.

Camphogen, $\mathrm{C}_{20} \mathrm{H}_{14}$, is the name given to the carbohydrogen 
obtained when common camphor is distilled with dry phosphoric acid. It occurs naturally in the oil of cumin. When acted on by sulphuric acid, it forms a new acid, sulphocamphic or hyposulphocamphic acid, $\mathrm{C}_{20} \mathrm{H}_{13} \mathrm{~S}_{2} \mathrm{O}_{5}, \mathrm{HO}$.

When camphor is passed over red-hot lime, another new compound is formed, namely, camphrone, $\mathrm{C}_{30} \mathrm{H}_{21} \mathrm{O}$ ? At a white heat camphor yields naphthaline, carburetted hydrogen, and carbonic oxide. 2 eqs. camphor contain the elements $\left(\mathrm{C}_{40} \mathrm{H}_{32} \mathrm{O}_{4}\right)$ of 1 eq. naphthaline, $\mathrm{C}_{20} \mathrm{H}_{8}$; olefiant gas, $2 \mathrm{C}_{4} \mathrm{H}_{4}$; marsh gas, $8 \mathrm{C} \mathrm{H}_{2}$; and carbonic oxide, $4 \mathrm{C} \mathrm{O}$.

Enanthic Acid. $\mathrm{C}_{14} \mathrm{H}_{13} \mathrm{O}_{3}, \mathrm{HO}$. This acid, in combination with oxide of ethyle, forming œnanthio ether, is found in wine, in the oil of grain spirit, and in some other fermented liquors. It is, as œnanthic ether, the cause of that peouliar odour of wine which adheres so remarkably to vessels in which wine has been kept, and enables us at once to say that an empty bottle or cask has contained wine. To obtain the acid, the ether is decomposed by caustic potash, and the enanthate of potash distilled with dilute sulphuric aoid. The hydrated acid is semi-solid like butter, and is, in fact, a fat oil, insoluble in water, soluble in alcohol and ether.

Of its salts, the onanthate of oxide of ethyle, œnanthic ether, is best known. It is a colourless liquid, of a peculiar vinous smell, which, when strong, has a stupifying effect. When hydrated œnanthio aoid is distilled, it yields water, and anhydrous acid, more solid than the hydrate. Enanthic acid contains 1 eq. of oxygen less than œnanthylic acid, but its formula must be considered as somewhat doubtful. Some think that the onanthic ether is either œnanthylio ether, caprylic ether, or pelargonic ether, and consequently that the acid is either œnanthylic, caprylic, or pelargonic acid.

Roccellic Acid, $\mathrm{C}_{17} \mathrm{H}_{15} \mathrm{O}_{3}, \mathrm{HO}$ ? This acid occurs in roccella tinctoria. It is crystallisable, insoluble in water, soluble in alcohol and ether. It has most of the properties of a fat acid, and its salts with the alkalies resemble soaps.

Eugenic Acid, $\mathrm{C}_{20} \mathrm{H}_{12} \mathrm{O}_{4}$ ? or $\mathrm{C}_{20} \mathrm{H}_{13} \mathrm{O}_{5}$ ? This acid is found in cloves, along with a neutral oil, $\mathrm{C}_{10} \mathrm{H}_{8}$, or $\mathrm{C}_{20} \mathrm{H}_{18}$. The latter is separated by potash, and the eugenic acid obtained by distilling the salt of potash with dilute sulphuric acid. It is an oily liquid, of Sp. G. 1.079, having the strongest odour of cloves. It forms crystallisable salts with bases, and among them an acid salt of potash, $2 \mathrm{C}_{20} \mathrm{H}_{12} \mathrm{O}_{4}+\mathrm{KO}+\mathrm{HO}$ ?

Cloves, likewise, contain two crystallisable compounds :1. Caryophyline, which forms yellow prisms; of the formula $\mathrm{C}_{20} \mathrm{H}_{16} \mathrm{O}_{2}$, an oxide, therefore, of the neutral oil of cloves. 
2. Eugenine, which forms yellow pearly scales, the composition of which is the same as that of eugenic acid.

It only remains here to mention the names of a number of acids, little known, or doubtful, which are found in the vegetable kingdom. Such are achilleic, atropic, anacardic, bebiric, boletic, caincic, carmufellic, cetraric, cevadic, chinovic, cocognidic, columbic, coneic, crameric, crotonic, digitalic, euphorbic, fungic, hederic, igasuric, ipecuanic, lactucic, lichenostearic, menispermic, papaveric, rheadic, polygalic, robinic, rutinic, solanic, tanacetic acids, and others. It is probable that many of them will prove to be identical with some of the better known acids, and that others are mixtures. Chelidonic acid, found by Lerch in Chelidonium majus, is analogous to meconic acid, and is said to be $\mathrm{C}_{14} \mathrm{H}_{5} \mathrm{O}_{13}$ $=\mathrm{C}_{14} \mathrm{H}_{2} \mathrm{O}_{10}, 3 \mathrm{H} \mathrm{O}$. The formulp given for several of the others are doubtful.

\section{VOLATILE OR ESSENTIAL OILS.}

These oils are so called because they are obtained by distillation of vegetables, generally along with water, and because, having, in most cases, the concentrated odour of the plant, they are usually salled essences. Most of them exist ready-formed in the plant, which owes its smell to them; but some, as oil of bitter almonds and oil of spiræa, are formed by a kind of fermentation, excited, in the case of the former, as already stated, by the contact of amygdaline, emulsine, and water.

Many plants, when cut, yield balsams, which are mixtures of essential oils and resins. In many essential oils a crystalline matter is deposited, called a camphor or stearoptene. They are all soluble in alcohol. Many absorb oxygen from the air and become acid, as oil of sinnamon. They are violently acted on by nitric acid and iodine, chlorine, bromine, \&c.

They may be divided into three kinds: 1st, those containing only carbon and hydrogen, as oil of turpentine; 2nd, those containing also oxygen, as oil of cloves; $3 \mathrm{rd}$, those containing sulphur, as oil of garlic.

\section{Non-Oxygenated Essential Oils.}

Almost every one of these (which constitute a rery numerous class of oils), as yet accurately analysed, has been found to contain carbon and hydrogen in the proportion $\mathrm{C}_{10} \mathrm{H}_{8}$, or, what is the same thing, $\mathrm{C}_{5} \mathrm{H}_{4}$, or $\mathrm{C}_{20} \mathrm{H}_{16}$. The following are the most important:-

Oil of turpentine, $\mathrm{C}_{10} \mathrm{H}_{8}$, or $\mathrm{C}_{20} \mathrm{H}_{16}$, is obtained by distilling, with water, turpentine, the juice exuding from many species of 
pinus. Rosin, resin, or colophonium, remains in the retort. The oil has a peculiar smell, and burns with a smoky flame. Its Sp. G. is 0.86 . It boils at $312^{\circ}$. Strong nitric acid sets fire to it, and it is also decomposed with flame by chlorine. It dissolves sulphur, phosphorus, and fat oils. When exposed to hydrochloric acid gas, it combines with it, forming a white crystalline solid like camphor, and a liquid compound. The solid is $\mathrm{C}_{20} \mathrm{H}_{17}$ $\mathrm{Cl}=\mathrm{C}_{20} \mathrm{H}_{16}, \mathrm{H} \mathrm{Cl}$. When heated with lime, it yields a pure oil, dadyle, $\mathrm{C}_{20} \mathrm{H}_{16}$. The liquid hydrochlorate, heated with lime, yields another pure oil, peucyle, rather more volatile than dadyle, but having the same composition. Oil of turpentine would seem to be composed of peucyle and dadyle, both $\mathrm{C}_{20} \mathrm{H}_{16}$; the former giving a liquid, the latter a solid compound, with hydrochloric acid.

Nitric acid, hy long boiling, converts oil of turpentine into an acid, turpentinic acid, $\mathrm{C}_{14} \mathrm{H}_{9} \mathrm{O}_{7}, \mathrm{H} \mathrm{O}$ ?

Oil of turpentine is used in medicine, internally, as a vermifuge, especially in cases of the larger worms, such as tania; externally, as an excellent rubefacient and counter-irritant. In the arts it is much prized as a solvent for resins in making varnishes.

Oil of juniper has the same composition as oil of turpentine, but possesses its own peculiar odour, which it communicates to alcohol in gin. This oil is diuretic.

Oil of savin has the same composition. It is also diuretic. Oil of elemi has the same composition, and a pleasant odour. Oil of storax, or styrole, is a very remarkable oil, of the formula $\mathrm{C}_{16} \mathrm{H} 8$, and therefore isomeric with cinnamole, and probably identical with it. It has a pleasant aromatic smell, boils at $295^{\circ}$, and evaporates at all temperatures. When acted on by nitric acid, chlorine, or bromine, it yields substitution products; nitrostyrole, $\left.\mathrm{C}_{16} \mathrm{~N} \mathrm{O}_{4} \mathrm{H}_{7}\right\}$, chlorostyrole, $\left.\mathrm{C}_{16} \underset{\mathrm{Cl}}{\mathrm{C}}\right\}$, and bromostyrole, $\left.\mathrm{C}_{16} \begin{array}{l}\mathrm{H}_{7} \\ \mathrm{Br}\end{array}\right\}$; the first is crystalline; the second appears to be liquid, but is hardly known except in combination with hydrochloric acid, as an oil, $\left.\mathrm{C}_{16} \mathrm{H}_{8} \mathrm{Cl}_{2}=\mathrm{C}_{16} \underset{\mathrm{Cl}}{\mathrm{Cl}}\right\}$ \} $\mathrm{H} \mathrm{Cl}$. The third is crystalline, and as it has a very peculiar smell, its formation is a good test of the presence of styrole. A similar compound is obtained, probably the same, by the action of bromine on cinnamole.

When styrole is heated in a sealed tube to $398^{\circ}$, it is quickly changed into a transparent solid mass like glass. The same change occurs, but more slowly, at $212^{\circ}$, and even at lower temperatures, and by exposure to the sun's rays. The glassy com- 
pound is metastyrole, which has the same composition in 100 parts as styrole, but its formula is $\mathrm{C}_{14} \mathrm{H}_{7}$. It yields with nitric acid

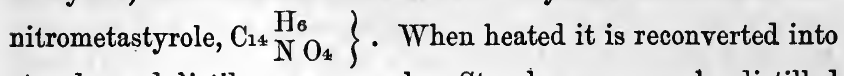
styrole, and distils over as such. Styrole can never be distilled. without a part of it changing into metastyrole, which, however, if the heat be increased, passes over as styrole.

When dragon's blood, a resinous substance, is distilled, it yields two oils, dracyle and draconyle. The latter is metastyrole, but it is not stated whether the former be styrole, although this is probable. There are probably many oils of the same series as styrole and metastyrole, that is $\mathrm{Cn} \times 2 \mathrm{Hn}$.

Oil of lemons has the probable composition, $\mathrm{C}_{5} \mathrm{H}_{4}$. Like oil of turpentine it is composed of two isomeric oils, citrene and citrylene, which combine with hydrochloric acid, forming a liquid and a solid compound, decomposed by heating with lime. The solid camphor seems to be, $\mathrm{C}_{10} \mathrm{H}_{9} \mathrm{Cl}=\mathrm{C}_{10} \mathrm{H}_{8}, \mathrm{H} \mathrm{Cl}$. The oils of cedro, cedrat, oranges, and limes, are all essentially identical with oil of lemons. Oil of neroli, or of orange-flower, is quite distinct, having the odour of the flower, while the others have that of the rind of the fruit. Its composition is not accurately known.

Oil of copaiva is another isomeric form of oil of turpentine, which it very much resembles, forming a camphor with hydrochloric acid. It is diuretic, and much used in affections of the bladder and urethra. Oils of pepper and of cubebs are still of the same composition in 100 parts, although the latter is supposed to be $\mathrm{C}_{15} \mathrm{H}_{12}$.

We have already alluded to the remarkable homologous series of carbohydrogens, of which benzole, or hyduret of phenyle, $\mathrm{C}_{22} \mathrm{H}_{6}$, is the type. One of these occurs as a natural oil. Thus, oil of cumine is a mixture of cuminole or hyduret of oumyle, $\mathrm{C}_{20} \mathrm{H}_{12} \mathrm{O}_{2}$, a compound homologous with hyduret of benzoyle, and a carbohydrogen, $\mathrm{C}_{20} \mathrm{H}_{14}$, called cymene, or hyduret of cymyle, homologous with benzole. This carbohydrogen has also been named hyduret of thymyle, because, in the essential oil of thyme, there is found a substance, $\mathrm{C}_{20} \mathrm{H}_{14} \mathrm{O}_{2}$, or hydrated oxide of thymyle, which is homologous with hydrated oxide of phenyle or carbolic acid. The carbohydrogen, toluole, or hyduret of toluyle, $\mathrm{C}_{14} \mathrm{H}_{8}$, homologous with benzole or hyduret of phenyle, is obtained by distilling balsam of tolu, and is also found in the oil of tar, and in the oil which separates from crude pyroxylic spirit on the addition of water. It is therefore a product of destructive distillation, and is accompanied by its homologues, the oils $\mathrm{C}_{12} \mathrm{H}_{6}, \mathrm{C}_{16} \mathrm{H}_{10}, \mathrm{C}_{18} \mathrm{H}_{12}$, and $\mathrm{C}_{20} \mathrm{H}_{14}$, which, as formerly ex- 
plained under the benzoic series, are derived from a series of acids homologous with benzoic acid, and yield a series of volatile bases, homologous with aniline, or phenylamine, besides many substitution products with chlorine, bromine, iodine, or nitrous acid.

It has recently been shown that a very large number of natural essential oils have the same composition as oil of turpentine, while yet many of them possess distinct properties. Oil of turpentine itself is found to exhibit many isomerio modifications, which differ remarkably in density and volatility; one of them, for example, isoterebenthene, boiling at $352^{\circ}$, while another, metaterebenthene, boils at about $693^{\circ} \mathrm{F}$. Both of these forms have a strong action on polarised light, causing the plane of polarisation to deviate to the left. These two modifications are produced by the continued action of heat on the common oil in closed vessels.

By contact with a very small proportion of oil of vitriol, oil of turpentine is changed into certain modifioations which have no action on polarised light. One of these, terebene, boils at $320^{\circ}$, and forms a liquid compound with hydrochloric acid. Another, colophene, whioh is supposed to have the formula $\mathrm{C}_{40} \mathrm{H}_{32}$, is colourless when the light which passes through it falls directly on the eye, but in other directions appears of a deep indigo-blue, and forms with hydrochlorio acid a deep blue compound.

Of the different sorts of commercial turpentine, or pine balsam, some cause the plane of polarisation to deviate to the left, while the oil extracted from them causes deviation to the right. Others cause deviation to the right, while the oil they yield causes deviation to the left.

Oil of turpentime forms, by contact with water, and exposure to cold, or still better, when 4 parts of the oil, 3 of alcohol, and 1 of nitric acid are left together for a month or six weeks, a crystalline hydrate, $\mathrm{C}_{20} \mathrm{H}_{16}+4 \mathrm{H} \mathrm{O}+2$ aq. This has been called terpine. It forms large and beautiful crystals, which are soluble in 200 parts of cold water, and 22 parts of boiling-water. It melts at about $218 \mathrm{~F}$., and loses water. When rapidly heated, the 2 eq. of water of crystallisation are expelled, leaving the dry hydrate, $\mathrm{C}_{20} \mathrm{H}_{16}, 4 \mathrm{H} \mathrm{O}$, which melts at $302^{\circ}$ and sublimes at $490^{\circ}$, passing over unchanged.

\section{Oxygenated Essential Oils.}

The principal oils of this class have been already considered, their radicals being known. These are oil of bitter almonds, or 
hyduret of benzoyle; oil of spiraa, or hyduret of salicyle; oil of cinnamon, or hyduret of cinnamyle; oil of cloves (eugenic acid); oil of cumine, or hyduret of cumyle; oil of aniseed, the solid part of which is $\mathrm{C}_{20} \mathrm{H}_{12} \mathrm{O}_{2}$, and with nitric acid yields anisic acid, and other compounds already described at p. 164 ; oil of valerian, chiefly valerianic acid, \&c. The essence of valerian, according to Gerhardt, generally contains several compounds, especially if old. When fresh, it contains no valerianic acid, but an oil, valerole, which is crystallisable, and soon passes into valerianic acid in the air. This oil is $\mathrm{C}_{12} \mathrm{H}_{10} \mathrm{O}_{2}$, and is isomeric with metacetone, also with Kane's oxide of mesityle, and with oxide of allyle (see oil of garlic, p. 345). Besides valerole, the essence contains a carbohydrogen, bornéène, $\mathrm{C}_{10} \mathrm{H}_{8}$, identical with the oil obtained from borneo camphor; and finally a camphor, which is identical with borneo camphor.

Oil of cinnamon, according to Mulder, is, when quite fresh, $\mathrm{C}_{20} \mathrm{H}_{11} \mathrm{O}_{2}$. It rapidly attracts oxygen, and $3\left(\mathrm{C}_{20} \mathrm{H}_{11} \mathrm{O}_{2}\right)+$ $\mathrm{O}_{8}=1$ eq. cinnamic acid, 1 eq. resin alpha, $\mathrm{C}_{12} \mathrm{H}_{5} \mathrm{O}, 1$ eq. resin beta, $\mathrm{C}_{30} \mathrm{H}_{25} \mathrm{O}_{4}$, and 6 eqs. water, $\mathrm{H}_{6} \mathrm{O}_{6}$. With hydrochloric acid it yields two different resins, $\mathrm{C}_{20} \mathrm{H}_{8} \mathrm{O}$, and $\mathrm{C}_{44} \mathrm{H}_{7}$ 0 , besides other products. With oil of vitriol it yields two more resins, $\mathrm{C}_{30} \mathrm{H}_{15} \mathrm{O}_{2}$, and $\mathrm{C}_{30} \mathrm{H}_{25} \mathrm{O}$, which together are equal to 3 eqs. of the oil minus 3 eqs. water. With nitric acid the fresh oil forms a crystalline compound, $\mathrm{C}_{18} \mathrm{H}_{8} \mathrm{~N} \mathrm{O}_{7}=\mathrm{C}_{28} \mathrm{H}_{8} \mathrm{O}_{3}+$ $\mathrm{N}_{3}+\mathrm{H} \mathrm{O}$. With water this body yields hyduret of cinnamyle, $\mathrm{C}_{28} \mathrm{H}_{8} \mathrm{O}_{2}$. If dissolved in sulphuric acid and mixed with water it gives cinnamic acid, $\mathrm{C}_{18} \mathrm{H}_{7} \mathrm{O}_{3}$. Along with the crystals, nitric acid yields a red oil, which, with water, gives another oil, $\mathrm{C}_{15} \mathrm{H}_{7} \mathrm{O}_{2}$.

Oil of anise, $\mathrm{C}_{20} \mathrm{H}_{12} \mathrm{O}_{2}$, yields with bromine a compound in fine crystals, $\mathrm{C}_{20}\left\{\begin{array}{l}\mathrm{H}_{9} \\ \mathrm{Cl}_{3}\end{array} \mathrm{O}_{2}\right.$. When acted on by strong acids, or by the chlorides of tin or antimony, oil of anise is converted into an isomeric body, anisoine, analogous to benzoine.

Oil of cumine contains not only hyduret of cumyle, already mentioned, but the carbo-hydrogen, cymene, $\mathrm{C}_{20} \mathrm{H}_{14}$, homologous with benzole, $\mathrm{C}_{12} \mathrm{H}_{6}$. As benzole is hyduret of phenyle, $\mathrm{C}_{12} \mathrm{H}_{5}$, $\mathrm{H}$, so cymene is hyduret of thymyle, $\mathrm{C}_{20} \mathrm{H}_{13}, \mathrm{H}$. And as phenyle yields a hydrated oxide, $\mathrm{C}_{12} \mathrm{H}_{5} \mathrm{O}, \mathrm{H} \mathrm{O}$, or $\mathrm{C}_{12} \mathrm{H}_{6} \mathrm{O}_{2}$, which is carbolic acid, so thymyle yields the hydrated oxide of thymyle, already mentioned as a homologue of carbolic acid, and as occurring in oil of thyme. There is therefore a relation between the oil of cumine and that of thyme, and the oil of Monarda punctata contains the same oxygenated compound, $\mathrm{C}_{20} \mathrm{H}_{14} \mathrm{O}_{2}$.

This compound, when acted on by sulphuric acid and peroxido 
of manganese, yields a new compound, thymoïle, $\mathrm{C}_{24} \mathrm{H}_{16} \mathrm{O}_{4}$, which is very remarkable, as being homologous with kinone. Like kinone, it a yellow solid crystallising in scales, and volatile, and, when acted on by reducing agents, takes up hydrogen, forming a body, $\mathrm{C}_{24} \mathrm{H}_{18} \mathrm{O}_{4}$, homologous with white hydrokinone, which has been called thymoillole. It forms colourless crystals, and when mixed in due proportion with thymoïle an intermediate compound is formed, homologous with the green hydrokinone, which appears in brilliant, bronze-coloured crystals of high metallic lustre, the solution of which is deep red. Its formula is $\mathrm{C}_{48} \mathrm{H}_{34} \mathrm{Os}_{8}=\mathrm{C}_{24}$ $\mathrm{H}_{16} \mathrm{O}_{4}+\mathrm{C}_{24} \mathrm{H}_{18} \mathrm{O}_{4}$; and it is of course formed whenever thymoille is only half reduced or hydrogenated. In short, in their formation, characters, and composition, thymoïle and its derivatives present a most striking and beautiful, though unexpected example of the parallelism of homologous compounds, from their perfect analogy with kinone and its derivatives. The chief difference lies in the starting-point, for while kinone is formed from kinic aoid, thymoille is obtained from the hydrated oxide of thymyle, a body apparently of very different properties.

The Oil of carraway contains, along with an oil, carvene, isomeric with oil of turpentine, an oxygenated oil, carvole, which is $\mathrm{C}_{20} \mathrm{H}_{14} \mathrm{O}_{2}$, that is, isomeric with the hydrated oxide of thymyle, but an entirely different substance. It boils at $282^{\circ} \mathrm{F}$., and forms with sulphuretted hydrogen a crystalline compound. By the action of caustic potash or of anhydrous phosphoric acid, it is in part converted into another isomeric modification, whioh boils at $450^{\circ} \mathrm{F}$., and has a disagreeable smell and a very persistent acrid taste. It has been called carvacrole.

Of the remaining oils of this class may be mentioned the oils of dill, of fennel, of parsley, of coriander, of pimpernel, of peppermint ( $\mathrm{C}_{10} \mathrm{H}_{10} \mathrm{O}$, or $\mathrm{C}_{20} \mathrm{H}_{20} \mathrm{O}_{2}$; this oil yields several new compounds with chlorine), of marjoram, of lavender, rosemary, basil, rue $\left(\mathrm{C}_{20} \mathrm{H}_{20} \mathrm{O}_{2}\right.$, or the aldehyde of capric acid, along with a carbohydrogen), cascarilla, chamomile (containing an oil, $\mathrm{C}_{10} \mathrm{H}_{8} \mathrm{O}_{2}$, and a carbohydrogen, $\mathrm{C}_{20} \mathrm{H}_{16}$ ), wormwood, tea, cardamom, nutmeg, cajeput, rhodium, rose (otto or attar of roses), bergamot, saffron, sassafras, and sueet bay $\left(\mathrm{C}_{20} \mathrm{H}_{16} \mathrm{O}\right)$. Of these, little certain is known, and almost all require a careful study. The oil of sassafras, $\mathrm{C}_{10} \mathrm{H}_{5} \mathrm{O}_{2}$, when cooled, deposits very large and beautiful crystals, measuring $1 \frac{1}{2}$ inch on the side. With bromine, the solid essence yields orystals, composed of $\mathrm{C}_{10} \mathrm{H} \mathrm{Br}_{4} \mathrm{O}_{2}$.

The oil or essence of Semen contra is said to be $\mathrm{C}_{18} \mathrm{H}_{15} \mathrm{O}_{2}$. That of Artemisia dracunculus, or essence of estragon, yields, when treated with sulphuric acid, anisoine, identioal with that of oil of anise, and in fact contains the same oxygenated oil (stea- 
roptene of anise), along with a different carbohydrogen. Iaurent has obtained from essence of estragon a series of new compounds. $\mathrm{He}$ represents the essence by $\mathrm{C}_{32} \mathrm{H}_{20} \mathrm{O}_{3}$ : but Gerhardt has shown that it is identical with the oil of anise, and that draconic acid and dracole are the same as anisic acid and anisole. The acid is $\mathrm{C}_{44} \mathrm{H}_{7} \mathrm{O}_{5}, \mathrm{H} \mathrm{O}$.

The concrete essence of the tonka bean is called coumarine. It is very fragrant, and its formula is $\mathrm{C}_{18} \mathrm{H}_{6} \mathrm{O}_{4}$. Potash changes it first into cumaric acid, $\mathrm{C}_{18} \mathrm{H}_{7} \mathrm{O}_{5}, \mathrm{H} \mathrm{O}$, and afterwards into salicylic acid; and hot nitric acid converts it into nitropicric acid. Cold nitric acid produces a white volatile crystalline solid, $\mathrm{C}_{18}\left\{\begin{array}{l}\mathrm{H}_{5} \\ \mathrm{~N} \mathrm{O}_{4}\end{array} \mathrm{O}_{4}\right.$. Coumarine also combines with chloride of antimony, forming yellow crystals.

\section{Sulphuretted Essential Oils.}

This class of oils is distinguished by a pungent peculiar smell, and acrid burning taste, as in oil of mustard, or an intense alliaceous odour, as in oil of garlic or of onions. The more important of them have been lately investigated, and have yielded very striking results.

Essence of mustard is prepared from mustard-seed in the same way as oil of bitter almonds from that seed. The seed is macerated with water and afterwards distilled, when it yields an oil of a most remarkable nature, containing not only sulphur, but also nitrogen. The pure oil is colourless, of Sp. G. 1.010, and boils at $298^{\circ}$ or $300^{\circ}$. Its formula is $\mathrm{C}_{8} \mathrm{H}_{3} \mathrm{~N} \mathrm{~S}_{2}$, so that it contains no oxygen, and it is, as we shall see, the sulphocyanide of allyle, $\mathrm{C}_{6}$ $\mathrm{H}_{5}, \mathrm{C}_{2} \mathrm{~N} \mathrm{~S}_{2}$. With ammonia it forms a crystalline compound, which is, in fact, an organic base or alkali, Thiosinnamine $=\mathrm{C}_{8}$ $\mathrm{H}_{8} \mathrm{~N}_{2} \mathrm{~S}_{2}$. This is a bitter compound, which forms, like nearly all organic bases, crystalline compounds with chloride of platinum and chloride of mercury. Gerhardt regards it as sulphocyanide of ammonium, in which 1 eq. of hydrogen is replaced by allyle; $\left.\mathrm{N}_{\mathrm{C}_{6} \mathrm{H}_{5}}^{\mathrm{H}_{3}}\right\} \mathrm{C}_{2} \mathrm{~N} \mathrm{~S}_{2}$.

Thiosinnamine, acted on by dry oxide of lead or of mercury, loses all its sulphur, forming a new base, sinnamine $=\mathrm{C}_{8} \mathrm{H}_{6} \mathrm{~N}_{2}$ $=\mathrm{C}_{8} \mathrm{H}_{8} \mathrm{~N}_{2} \mathrm{~S}_{2}-2 \mathrm{H} \mathrm{S}$. It is, therefore, Thiosinnamine, minus 2 eqs. sulphuretted hydrogen, which have acted on the oxide of lead, forming water and sulphuret of lead. Sinnamine forms definite compounds with chlorides of mercury and platinum. It is a powerful base, and very bitter to the taste. It may be regarded as ammonia, in which 2 eg. of hydrogen are replaced, one by allyle, the other by cyanogen, $\mathrm{N} \mathrm{H}, \mathrm{C}_{6} \mathrm{H}_{5}, \mathrm{C}_{2} \mathrm{~N}$. 
When oil of mustard is acted on by moist hydrated oxide of lead, it loses both sulphur and carbon, in the proportion $\mathrm{C} \mathrm{S}_{2}$, forming sulphuret of lead and carbonate of lead, along with a new base, sinapoline, which dissolves in hot water, in alcohol, and in ether. Its formula is $\mathrm{C}_{14} \mathrm{H}_{12} \mathrm{~N}_{2} \mathrm{O}_{2}$; and it is formed from 2 eqs. oil of mustard, with 6 eqs. oxide of lead and 2 eqs. water, as follows: $2\left(\mathrm{C}_{8} \mathrm{H}_{5} \mathrm{~N} \mathrm{~S}_{2}\right)+6 \mathrm{PbO}+2 \mathrm{H} \mathrm{O}=\mathrm{C}_{14} \mathrm{H}_{12}$ $\mathrm{N}_{2} \mathrm{O}_{2}+4 \mathrm{~Pb} \mathrm{~S}+2\left(\mathrm{~Pb} \mathrm{O}, \mathrm{C} \mathrm{O}_{2}\right)$. This base may be regarded as diallylurea; that is, urea in which 2 eqs. of hydrogen are replaced by allyle. Urea is $\mathrm{C}_{2} \mathrm{H}_{4} \dot{\mathrm{N}}_{2} \mathrm{O}_{2}$, and diallylurea is $\left.\left.\mathrm{C}_{2} \underset{2}{\mathrm{H}_{2}} \mathrm{C}_{5}\right)\right\} \mathrm{N}_{2} \mathrm{O}_{2}$.

When oil of mustard is acted on by an alcoholic solution of potash, it produces carbonate of potash, and two new compounds, the elements of $8 \mathrm{eqs}$. of water being taken up.

Thus,

$$
\begin{aligned}
3\left(\mathrm{C}_{8} \mathrm{H}_{5} \mathrm{~N} \mathrm{~S}_{2}\right) & =\mathrm{C}_{8} \mathrm{H}_{7} \mathrm{~N} \mathrm{~S}_{4} \\
+8 \mathrm{HO} & +\mathrm{C}_{14} \mathrm{H}_{14} \mathrm{~N}_{2} \mathrm{~S}_{2} \mathrm{O}_{2} \\
& +2 \mathrm{C} \mathrm{O}_{2} .
\end{aligned}
$$

The body $\mathrm{C}_{8} \mathrm{H}_{7} \mathrm{~N} \mathrm{~S}_{4}$ is a compound of oil of mustard with sulphuretted hydrogen, and is a strong sulphur acid, forming crystallicable salts with the metallic sulphurets. It may be represented by $\mathrm{C}_{8} \mathrm{H}_{5} \mathrm{~N} \mathrm{~S}_{2}, 2 \mathrm{H} \mathrm{S}$, and its salts as $\mathrm{C}_{8} \mathrm{H}_{5} \mathrm{~N} \mathrm{~S}_{2}$, H S, M S. Gerhardt considers it as sulphocarbonic acid, in which 1 eq. of hydrogen is replaced by allyle. But Will has produced and analysed many of the sulpho-salts of this acid, which renders his view preferable. He has also shown, that by the action of alcoholic solutions of the sulphurets of potassium, sodium, ammonium, \&c., saturated with sulphuretted hydrogen, on oil of mustard, the whole of the oil may be converted into the salt of the new acid with the sulphuret of the metal employed. In other words, the oil of mustard takes up 1 eq. of $\mathrm{H} \mathrm{S}$, and 1 eq. of metallic sulphuret. The oil of mustard itself also combines with 2 eqs. of several metallic sulphurets.

The oil, $\mathrm{C}_{14} \mathrm{H}_{14} \mathrm{~N}_{2} \mathrm{~S}_{2} \mathrm{O}_{2}$, has not been fully studied. It is said to form, from 2 eqs. of it, losing 1 eq. of ammonia, another oil, $\mathrm{C}_{23} \mathrm{H}_{25} \mathrm{~N}_{3} \mathrm{~S}_{4} \mathrm{O}_{4}$, which resembles the essence of mustard in its general characters, but is not much known.

It will be observed, that all the facts here mentioned lead to the admission of the radical allyle, $\mathrm{C}_{6} \mathrm{H}_{5}$, of which the oil or essence of mustard is the sulpho-cyanide. We shall see presently that other compounds of allyle are known.

Oil of mustard contains an indifferent nitrogenised body, myrosine, which, analogous to emulsine, causes the formation of the essential oil after maceration of the seed with water and fermentation. The fermentation of myrosine is prevented in the 
same way as that of emulsine, namely by coagulation. The seeds also contain a crrstalline body, sinapisine, resembling a fat. The substance, which, along with myrosine, yields the oil, appears to be myronic acid, or rather myronate of potash, a body not yet fully studied. The seeds of Sinapis alba contain the myrosine, as sweet almonds contain emulsine ; but, being destitute of myronic acid or myronate of potash, as sweet almonds are of amygdaline, they yield none of the oil.

It has very recently been shown by Hubatka and Wertheim, that the essential oils of Cochlearia armoracia (horse-radish), Cochlearia officinalis, and Alliaria officinalis, consist almost entirely of oil of mustard, although the latter oil has also a very strong smell of oil of garlic, an oil which has not been discovered in it.

The essential oil of garlic, from the bulbs of Allium sativum, is a peculiar sulphurised compound. Wertheim has lately studied it, and shown that it is the sulphuret of allyle, and its formula is $\mathrm{C}_{6} \mathrm{H}_{5}, \mathrm{~S}=\mathrm{All} \mathrm{S}$. The crude oil contains a little of a higher sulphuret, possibly All $S_{2}$, and also some of the oxide of allyle, C6 $\mathrm{H}_{5} \mathrm{O}=\mathrm{All} \mathrm{O}$, which is an oily liquid of an offensive smell. The radical allyle appears to enter into numerous combinations, and, among others, Wertheim analysed the following: the sulphuret, or pure oil of garlic, All S ; the compounds of that sulphuret with the sulphurets of platinum, palladium, and silver, 5 All $\mathrm{S}+6 \mathrm{Pt} \mathrm{S}_{2} ; 2$ All $\mathrm{S}+3 \mathrm{Pt} \mathrm{S}_{2} ; 2$ All $\mathrm{S}+3 \mathrm{Pd} \mathrm{S}$; and $\mathbf{x}$ All $\mathrm{S}+\mathrm{Ag} \mathrm{S}(?)$; double compounds with the sulphurets and chlorides of mercury and platinum ; 3 (All $\left.\mathrm{S}+\mathrm{Pt} \mathrm{S}_{2}\right)+$ (All $\mathrm{Cl}+\mathrm{Pt} \mathrm{Cl} 2)$; and (All S $+2 \mathrm{Hg} \mathrm{S})+(\mathrm{All} \mathrm{Cl}+2 \mathrm{Hg} \mathrm{Cl})$; and lastly, nitrate of the oxides of silver and allyle, (All $\mathrm{O}+\mathrm{Ag} \mathrm{O}$ ) $+\mathrm{N}_{5}$. Our space does not permit us to do more than point out the existence of these curious compounds.

The essential oil of assafotida appears to consist of at least two oils, one of which, if not both, contains sulphur. It has a very offensive odour. It does not combine with ammonia like the oil of mustard. Dr. Douglas Maclagan finds, as might be expected from the odour, that one of the oils it contains is sulphuret of allyle.

The essential oils of hops, of water-pepper, and of Arum maculatum, are believed to contain sulphur.

The seeds of Sinapis alba or white mustard have been shown by Babo and Herschbrunn to contain a new base, sinapine, which is $\mathrm{C}_{32} \mathrm{H}_{23} \mathrm{~N} \mathrm{O}_{10}$. By the action of mineral alkalies, it is resolved into a new acid, sinapic acid, and another new base, sinkaline. Thus :-

Sinapine. Sinapic acid. Sinkaline. $\mathrm{C}_{32} \mathrm{H}_{23} \mathrm{NO}_{10}+2 \mathrm{HO}=\mathrm{C}_{22} \mathrm{H}_{12} \mathrm{O}_{10}+\mathrm{C}_{10} \mathrm{H}_{13} \mathrm{~N} \mathrm{O}_{2}$ 
Sinapine, which exists in the white mustard as hydrosulphocyanate, cannot be obtained pure, from its tendency to decomposition. Its solution is yellow. It is a decided base, and its salts are crystallisable, very soluble, and rather more stable than the base itself. The hydrosulphocyanate, which exists in white mustard, may be obtained in crystals, which are small needles, grouped in very voluminous bundles. This salt was formerly called sinapine, and sulphosinapisine, when its composition was unknown. Its solution, when boiled with alkalies, yields sinapic acid and sinkaline.

Sinapic acid forms small prisms, sparingly soluble in cold water or cold alcohol, but very soluble in boiling alcohol. Its salts are not very stable.

Sinkaline is formed along with sinapic acid, when sinapine or its hydrosulphocranate is boiled with potash or baryta. It is obtained as a crystalline mass, soluble in water. It is a strong base, precipitating all metallic oxides. Its salts are very soluble, even deliquescent. The double chloride of platinum and sinkaline forms splendid orange crystals.

CONCRETE VOLATILE PRINCIPLES ALLIED TO THE ESSENTIAL OILS.

There are several substances which may be classed under this head; such as Hellenine, from Inula helenium, which is a volatile erystalline solid, $\mathrm{C}_{15} \mathrm{H}_{10} \mathrm{O}_{2}$. With nitric acid it yields nitrohellenine, $\mathrm{C}_{15}\left\{\begin{array}{l}\mathrm{H}_{9} \\ \mathrm{~N} \mathrm{O}_{4}\end{array} \mathrm{O}_{2}\right.$. When distilled with anhydrous phosphoric acid, hellenine loses 2 eqs. water, yielding hellenene, a carbohydrogen, $\mathrm{C}_{15} \mathrm{H}_{6}$. With chlorine it yields the compound

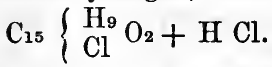

Asarone, from Asarum europaum, is a volatile solid, having a remarkable tendency to crystallise in beautifully defined forms, and also to pass into the amorphous condition, from which it may be again brought into the crystalline state. Schmidt has studied its crystallisation under the microscope, and has obtained results which are most interesting in reference to the formation of crystals in general. I must refer to his elaborate paper in the "Annalen der Chemie und Pharmacie," for February, 1845. Its composition is $\mathrm{C}_{20} \mathrm{H}_{13} \mathrm{O}_{5}$.

Anemonine, from various species of Anemone, is a volatile, crystallisable solid, the formula of which is $\mathrm{C}_{5} \mathrm{H}_{2} \mathrm{O}_{2}$. It forms with oxide of lead a compound, $3\left(\mathrm{Cs}_{5} \mathrm{H}_{2} \mathrm{O}_{2}\right)+\mathrm{Pb} \mathrm{O}$. With bases it yields anemonic acid, the composition of which is unknown. 
Cantharidine, the active principle of Spanish flies, is a volatile, acrid solid, the composition of which is $\mathrm{C}_{10} \mathrm{H}_{6} \mathrm{O}_{4}$.

The following plants, Epidendron vanilla, Quassia amara, Tanghinia Madagascariensis, Primula Auricula, and Primula veris, contain concrete volatile essences, not yet analysed.

\section{CAOUTCHOUC, OR GUM ELASTIC.}

Caoutchouc is a substance sui generis, which in composition approaches more nearly to the essential oils than to any other class of compounds. It is the coagulated or inspissated juice of many tropical trees, the chief of which is Siphonia elastica (Iatropha elastica, Hevea guianensis). The juice, as it flows from the tree, is made to dry on moulds of clay, which are afterwards broken out, leaving a bottle of caoutchouc. It is generally blackened by smoke, but when pure it is white and transparent, it is highly elastic, and the freshly cut surfaces adhere strongly if pressed together. It is insoluble in water, alcohol, and acids : but it dissolves in ether, naphtha, coal-tar naphtha, bisulphuret of carbon, and essential oils. Its solutions in ether and coal-tar naphtha, when dried up, leave the caoutchoue in an elastic state. On this principle water-proof cloth is made. Caoutchouc is much used in chemical operations to form flexible connecting tubes.

When exposed to heat, caoutchouc first melts, and then distils, yielding a mixture of several oily liquids, all of which, as well as pure caoutchouc itself, are carbohydrogens. Some of these oils boil at $90^{\circ}$, others at $680^{\circ}$, and at intermediate points. I found that one highly rectified oil which boiled at $96^{\circ}$, and had the composition of olefiant gas, when acted on by sulphuric acid, yielded an oil which boiled at $428^{\circ}$, and had the same composition. But most of these oils have the composition of oil of turpentine, $\mathrm{C}_{5} \mathrm{H}_{4}$ or $\mathrm{C}_{10} \mathrm{H}_{8}$. One of these, called caoutchine, gives with chlorine an oil, $\mathrm{C}_{10} \mathrm{Hs}+\mathrm{Cl}$.

\section{RESINS.}

Resins are generally found along.with essential oils, and many of these oils, by the action of the air, are converted into resins. In this change, the essential oils lose a part of their hydrogen, which is converted into water, and take up some oxygen besides. In fact, the resins, as a class, are acid bodies. They are insoluble in water, but become soft in boiling water. They dissolve in alcohol, and often crystallise from that solvent.

The acid resins combine with bases; their saits with the alkalies are called resinous soaps. The resins are not volatile, 
although very inflammable. They are purified from essential oils by distilling off the latter along with water ; but, as thus obtained, they are generally mixtures of several resins.

Turpentine and Colophony, or Common Resin. Turpentine is the semifluid juice which exudes from many species of Pinus. When distilled with water, it yields oil of turpentine, Cró $\mathrm{Hs}$ or $\mathrm{C}_{40} \mathrm{H}_{32}$, while colophony or resin remains behind, which is $\mathrm{C}_{10}$ $\mathrm{H}_{7} \mathrm{O}$, or $\mathrm{C}_{40} \mathrm{H}_{28} \mathrm{O}_{4}$, or more accurately, $\mathrm{C}_{40} \mathrm{H}_{30} \mathrm{O}_{4}$. Here the oil, $\mathrm{C}_{40} \mathrm{H}_{32}$, has lost 2 eqs. hydrogen, replaced by 2 eqs. oxygen, $\mathrm{C}_{4} \mathrm{O} \mathrm{H}_{30} \mathrm{O}_{2}$, and this compound, like aldehyde, has taken up 2 eqs. of oxygen to form the acid resin, $\mathrm{C}_{40} \mathrm{H}_{30} \mathrm{O}_{4}$.

Culophony contains two different resins: resin alpha, or pinic acid, and resin beta, or sylvic acid. The latter is said to be $\mathrm{C}_{20}$ $\mathrm{H}_{15} \mathrm{O}_{2}$, the former polymeric with it, $\mathrm{C}_{40} \mathrm{H}_{30} \mathrm{O}_{4}$; and their properties are very similar, being those of colophony which is formed of them. The sylvic acid is crystallisable.

When distilled with lime, colophony yields two oily liquids, resineone, $\mathrm{C}_{26} \mathrm{H}_{23} \mathrm{O}$, and resinone, $\mathrm{C}_{10} \mathrm{H}_{9} \mathrm{O}$.

The resin of copaiva is $\mathrm{C}_{40} \mathrm{H}_{30} \mathrm{O}_{4}$, according to Rose ; but there is some reason to believe that it is isomeric with the preceding. A variety of it has occurred, containing $\mathrm{C}_{4.0} \mathrm{H}_{34} \mathrm{O}_{8}$, and when combined with oxide of lead, $\mathrm{C}_{40} \mathrm{H}_{28} \mathrm{O}_{6}$. This resin crystallises.

The resin of elemi contains two resins, one crystallisable. Both are said to be $\mathrm{C}_{20} \mathrm{H}_{16} \mathrm{O}_{2}$. Anime also contains two resins. Euphorbium yields a resin having the same composition as elemi. Benzoin contains, besides benzoic acid and a volatile oil, three resins, alpha, $\mathrm{C}_{70} \mathrm{H}_{42} \mathrm{O}_{14}$; beta, $\mathrm{C}_{40} \mathrm{H}_{22} \mathrm{O}_{9}$; and ganma, $\mathrm{C}_{30}$ $\mathrm{H}_{20} \mathrm{O}_{5}$. The resin alpha contains the sum of the other two, and by long boiling with carbonate of soda, which dissolves the resin gamma alone, is resolved into them.

Balsam of Tolu contains, besides essential oil, benzoic and cinnamic acids, and a carbohydrogen, $\mathrm{C}_{24} \mathrm{H}_{18}$, a resin, $\mathrm{C}_{18} \mathrm{H}_{10} \mathrm{O}_{5}$. It contains the elements of benzoio ether, plus 1 eq. oxygen. When the balsam is distilled, per se, it actually yields benzoic ether, along with a new carbohydrogen, called benzoéne or toluole, $\mathrm{C}_{14} \mathrm{H}_{8}$, homologous with benzole, $\mathrm{C}_{12} \mathrm{H}_{6}$. This last compound yields with sulphuric acid a new acid, $\mathrm{C}_{14} \mathrm{H}_{7} \mathrm{~S}_{2} \mathrm{O}_{5}+3 \mathrm{HO}$; and, with nitric acid, two new nitrogenised compounds, $\mathrm{C}_{14}\left\{\begin{array}{l}\mathrm{H}_{7} \\ \mathrm{NO}_{4}\end{array}\right.$, called nitrotoluole, analogous to nitrobenzole, and another, binitrotoluole, $\mathrm{C}_{14} \mathrm{H}_{6}+2 \mathrm{~N} \mathrm{O.4}$. The former is isomeric with salicylamide, and with anthranilic acid, and, like the latter body, when heated with lime, yields aniline. When acted on by hydrosulphuret of ammonia, it yields another artificial base, toluidine. With chlorine, toluole also yields several new products. Balsam 
of Peru has already been mentioned, along with balsam of Tolu, under cinnamyle.

Styracine, the resin of styrax, is $\mathrm{C}_{24} \mathrm{H}_{11} \mathrm{O}_{2}$ (?) When acted on by nitric acid, it yields the products of decomposition of cinnamic acid. When fluid storax is distilled with water, it yields a remarkable oil, styrole, $\mathrm{C}_{16} \mathrm{H}_{8}$, which has been already described as oil of storax.

When liquid storax is distilled with carbonate of soda, styrole distils over, and in the residue is found cinnamate of soda and a crystalline body, styracine. The occurrence of cinnamic acid along with styrole in storax, is a strong argument in favour of the identity of styrole and cinnamole. Styracine is $\mathrm{C}_{36} \mathrm{H}_{16} \mathrm{O}_{4}$, and is a compound of cinnamic acid, $\mathrm{C}_{18} \mathrm{H}_{7} \mathrm{O}_{3}$, with the body $\mathrm{C}_{18} \mathrm{H}_{9} \mathrm{O}$, the oxide of a radical, which may be called styryle, $\mathrm{C}_{18} \mathrm{H}_{9}$, in which the carbon is to the hydrogen, as in benzole, $\mathrm{C}_{12} \mathrm{H}_{6}$, styrole, $\mathrm{C}_{16} \mathrm{H}_{8}$, and metastyrole, $\mathrm{C}_{14} \mathrm{H}_{7}$, or as 2 to 1 . When styracine is heated with solution of potash, cinnamate of potash is left, and there distils over the hydrated oxide of styryle, $\mathrm{C}_{18} \mathrm{H}_{9} \mathrm{O}, \mathrm{HO}=\mathrm{C}_{18} \mathrm{H}_{10} \mathrm{O}_{2}$, which is a true alcohol of the cinnamic series, bearing to hydrated cinnamic acid, $\mathrm{C}_{18} \mathrm{H}_{8} \mathrm{O}_{4}$, the same relation as alcohol does to acetic acid. It will be remembered that balsam of Peru yields a compound, cinnameine, analogous to styracine, containing cinnamic acid combined with the oxide of another radioal, $\mathrm{C}_{18} \mathrm{H}_{11}$, and yielding an alcohol, $\mathrm{C}_{18} \mathrm{H}_{12} \mathrm{O}_{2}$. It is probable that we shall hereafter find all the acids of the benzoic and cinnamic series to form such oxides or ethers, and hydrated oxides or alcohols.

The hydrated oxide of styryle, called also styrone, exists in two forms, as an oil and in a crystalline form, of the same composition. Styraoine, the cinnamate of oxide of styryle, exhibits also two isomeric or allotropic modifications. Styrone is easily oxidised into cinnamic acid, as alcohol is into acetio acid.

\section{$\mathrm{C}_{4} \mathrm{H}_{6} \mathrm{O}_{2}+\mathrm{O}_{4}=\mathrm{C}_{4} \mathrm{H}_{4} \mathrm{O}_{4}+2 \mathrm{HO}$; and $\mathrm{C}_{18} \mathrm{H}_{10} \mathrm{O}_{2}+\mathrm{O}_{4}=\mathrm{C}_{18} \mathrm{H}_{8} \mathrm{O}_{4}+2 \mathrm{HO}$ \\ Alcohol. Acetic Acid.}

The resin of guaiacum is remarkable for its tendency to become blue by the contact of many different substances. It contains two resins, but their composition is not ascertained. Lac contains four resins, beside colouring matter, Dammara, mastic, dragon's blood, and sandarach, are resins much used in making varnishes.

Jalap contains two resins: one, a soft resin soluble in ether, $\mathrm{C}_{42} \mathrm{H}_{34} \mathrm{O}_{18}$; and an acid resin insoluble in ether, which, from striking a fine red colour with sulphuric acid, is called rhodeoretine, 
$\mathrm{C}_{42} \mathrm{H}_{35} \mathrm{O}_{20}$. When combined with bases, it takes up 1 eq. water, forming hydrorhodeoretine, very similar to rhodeoretine, but soluble in water, $\mathrm{C}_{42} \mathrm{H}_{36} \mathrm{O}_{21}$. When rhodeoretine is acted on by hydrochloric acid, it is resolved into grape sugar, $\mathrm{C}_{22} \mathrm{H}_{1}$ $\mathrm{O}_{12}$, and an oily liquid rhodeoretinole, $\mathrm{C}_{30} \mathrm{H}_{10} \mathrm{Os}$. This reaction places rhodeoretine near to salicine and phloridzine. On the other hand, if we compare 7 eqs. of starch, $7\left(\mathrm{C}_{12} \mathrm{H}_{10} \mathrm{O}_{10}\right)=$ $\mathrm{C}_{84} \mathrm{H}_{70} \mathrm{O}_{70}$ with 2 eqs. rhodeoretine, $2\left(\mathrm{C}_{42} \mathrm{H}_{35} \mathrm{O}_{20}\right)=\mathrm{C}_{84} \mathrm{H}_{70}$ $\mathrm{O}_{40}$, we can see how this resin may be formed from starch, \&c. by deoxidation. Rhubarb contains 3 resins, aporetine, phooretine, and erythroretine. The two first are both $\mathrm{C}_{26} \mathrm{H}_{8} \mathrm{O}_{7}$; the third is $\mathrm{C}_{2} \mathrm{H}_{9} \mathrm{O}_{7}$. They are accompanied by an intensely yellow crystallisable acid, chrysophanic acid, $\mathrm{C}_{10} \mathrm{H}_{8} \mathrm{O}_{3}$ or $\mathrm{C}_{40} \mathrm{H}_{32} \mathrm{O}_{12}$. This latter substance is also found in lichens, such as Parmelia parietina, Squamaria elegans, \&c. Copal, which of all the resins is the most insoluble, is said to contain five resins, Copal varnish is made by adding hot oil of turpentine to copal fused at a gentle heat.

Turf or peat contains several resinous bodies, examined by Mulder. In the turf of Friesland he found 4 resins; alpha, $\mathrm{C}_{30} \mathrm{H}_{40} \mathrm{O}_{9} ;$ beta, $\mathrm{C}_{77} \mathrm{H}_{67} \mathrm{O}_{9} ;$ gamma, $\mathrm{C}_{104} \mathrm{H}_{94} \mathrm{O}_{9}$; and delta, $\mathrm{C}_{131} \mathrm{H}_{121} \mathrm{O}_{9}$. A lighter kind of turf from another locality vielded two resins; alpha, $\mathrm{C}_{35} \mathrm{H}_{28} \mathrm{O}_{5}$; and gamma, $\mathrm{C}_{90} \mathrm{H}_{84} \mathrm{O}_{6}$.

Resinous varnishes are made, by dissolving resins in oil of turpentine and other essential oils; or in drying oils. Spirit varnishes are made by dissolving resins in very strong alcohol.

\section{ACTION OF HEAT ON RESINS.}

When resins are distilled in close vessels, they yield a great deal of gas of a high illuminating power, and many volatile liquid compounds of carbon and hydrogen.

Pinic acid yields, when heated, colopholic acid. Colophony yields resineine, an oil, $\mathrm{C}_{20} \mathrm{H}_{15} \mathrm{O}$; also retinaphtha, $\mathrm{C}_{14} \mathrm{H}_{8}$, identical with toluole or toluene, which with chlorine forms a compound $\mathrm{C}_{14} \mathrm{H}_{6} \mathrm{Cl}_{2}$; retinyléne, $\mathrm{C}_{18} \mathrm{H}_{12}$, isomeric with cumole or cumene, which with sulphuric acid yields an acid, isomeric with sulphocumenic acid, $\mathrm{C}_{18} \mathrm{H}_{11} \mathrm{~S}_{2} \mathrm{O}_{5}, \mathrm{HO}$; retinole, $\mathrm{C}_{32} \mathrm{H}_{16}$; and finally a solid product, retisteréne, fusible at $152^{\circ}$, having the same composition as naphthaline, $\mathrm{C}_{20} \mathrm{H}_{8}$. 


\section{COLOURING MATTERS CONTAINING NO NITROGEN.}

1. Yellow colouring matters.

The following are the most important of the yellow vegetable colouring matters, many of which are used in dyeing.

Curcumine, from the root of Curcuma longa, is resinous, and is dissolved by alkalies, which change it to brown. Hence it is used as a test for alkalies, under the name of turmeric. Gamboge yellow is extracted from gamboge, the dried juice of Garcinia gambogia. It is resinous and powerfully purgative. Annotto or Anatto is obtained from the seeds of Bixa orellana and Metella tinctoria. Carotine is the colouring matter of the carrot, Daucus carota. Rhubarberine is a name formerly given to the yellow acid of rhubarb, now called chrysophanic acid, which is found also in lichens as above stated. It has great colouring power, and yields a fine violet with alkalies. It is fusible and volatile. Formula, $\mathrm{C}_{10} \mathrm{H}_{8} \mathrm{O}_{3}$. From occurring in Parmelia parietina, it has also been called parietine and parietinic acid. Luteoline, the colouring principle of Reseda luteola or Woad, is volatile and crystallisable. Quercitrine, from the bark of Quercus tinctoria, is crystalline, and its composition is $\mathrm{C}_{16} \mathrm{H}_{8} \mathrm{O}_{9}, \mathrm{HO}$. Other yellow colouring matters are Morine, from Morus tinctoria; Safflower yellow, from Carthamus tinctorius; Polychroite from Saffron; Morindine, from Morinda citrifolia, which resembles the Rubian of madder, and yields morindone, a red body like alizarine. Perhaps morindine is identical with rubian. There are other colouring matters of less interest.

\section{Red colouring matters.}

Draconine, or Dragon's blood, is a red gum resin, from Dracana draco. It is much used to colour varnishes. Santaline, the colouring matter of Pterocarpus santalinus, is also resinous, and has an intense red colour. Anchusine, from Anchusa tinctoria, is the source of the colour of alkanet; it is resinous, and yields violet vapours when heated. Carthamine is the red colouring matter of safflower, Carthamus tinctorius. It is a very fine and intense red, much used for dyeing rose colour, for pink saucers, and for rouge, at least the rouge végétale.

Madder, the root of Rubia tinctorium, contains three different red colouring matters, madder-purple, red, and orange. All three are volatile; and the sublimed crystals of madder-red, which are of a fine orange-red colour, are called alizarine. This is the substance which yields the turkey-red dye. With alkalies it yields 
purple or violet colour; with acids, yellow. When dissolved in hot water or alcohol, alizarine yields rose-coloured solutions. The composition of alizarine is $\mathrm{C}_{20} \mathrm{H}_{10} \mathrm{O}_{10}=\mathrm{C}_{20} \mathrm{H}_{6} \mathrm{O}_{6}+4$ aq., in the state of hydrate. According to Rochleder, madder contains, besides alizarine, a yellow crystalline acid, $\mathrm{C}_{72} \mathrm{H}_{4} \mathrm{O}_{40}$, which, when heated with acids or alkalies, yields sugar, $\mathrm{C}_{22} \mathrm{H}_{10}$ $\mathrm{O}_{10}$, and alizarine. Schunck, who had previously observed this fact, called the yellow body rubiane. Notwithstanding the labours of many excellent chemists, the subject of madder has not yet been cleared up.

Hamatoxyline is the red of logwood, Hamatoxylum Campechi. anum. It is soluble in water and alcohol, and yields orange crystals, which give to water a red colour, brightened by acids and turned to a violet or blue by alkalies. With alum, logwood yields various shades of violet; with an iron mordant, grey and black. Black oloth and hats are dyed with it, which is the reason they are reddened by acids. According to Erdmann, pure hæmatoxyline is pale yellow, and is coloured red by the atmosphere. Its formula he found to be $\mathrm{C}_{40} \mathrm{H}_{17} \mathrm{O}_{15}$. When acted on by ammonia, it yields hamateine, which is dark-red, and forms with excess of ammonia a splendid purple matter. Hæmateine is $\mathrm{C}_{40} \mathrm{H}_{15} \mathrm{O}_{16}$; and the purple compound with ammonia is $\mathrm{C}_{40} \mathrm{H}_{15}$ $\mathrm{O}_{16}+2 \mathrm{~N} \mathrm{H}_{3}+$ aq. Braziliwood and Camwood yield colouring matters very similar to hæmatoxyline, if not identical with it. The red of China nova bark is $\mathrm{C}_{12} \mathrm{H}_{6} \mathrm{O}_{5}$.

Many flowers contain a red colouring matter, which is turned green by alkalies, and is very fugitive. It is soluble in water and alcohol.

\section{Blue colouring matters.}

These are chiefly found in flowers and fruits. They are very closely allied to the red of flowers and fruits, which are no doubt often derived from them by the action of acids. They are all turned green by alkalies, and red by acids. Such blue colouring matters as are more permanent contain nitrogen, and will be considered hereafter.

\section{Green colouring matter. Chlorophyll.}

This is the green of leaves. It is of a nature allied to that of wax, soluble in ether and alcohol, insoluble in water. It is very neutral or indifferent in its relations to other substances.

Polychrome is the name given to a peculiar crystalline principle found in some vegetables, such as quassia. It gives to water the quality of exhibiting a curious play of colours, among which blue predominates, like that of the opal, when the solution is viewed by reflected light; 1 part will give this property to $1,500,000$ of 
water. Its formula is said to be $\mathrm{C}_{16} \mathrm{H}_{8} \mathrm{O}_{9}, \mathrm{H} \mathrm{O}$, which is the same as that of quercitrine, and contains 2 eqs. of oxygen more than the aporetine of rhubarb.

\section{NON-AZOTISED VEGETABLE COMPOUNDS, THF NATURE OF WHICH IS NOT YET ASCERTAINED.}

In this subdivision may be included a number of compounds, most of which crystallise and have'a bitter taste, but are neutral and cannot yet be referred to any particular series of compounds. Salicine, phloridzine, and rhodeoretine, which would formerly have been here described, are now treated of along with substances allied to them. The substances now to be briefly mentioned have usually been termed the bitter and extractive principles of plants.

Gentianine, from Gentiana lutea, forms yellow needles, very bitter. Menyanthine, from Menyanthes trifoliata, is bitter, but does not crystallise. Athamantine, a bitter and acrid crystalline substance found in Athamanta oreoselinum, is $\mathrm{C}_{48} \mathrm{H}_{30} \mathrm{O}_{14 .}$ When combined with hydrochloric acid and boiled, it is resolved into oreoselone and valerianic acid. Thus :

$$
\mathrm{C}_{48} \mathrm{H}_{30} \mathrm{O}_{14}=\underbrace{2\left(\mathrm{C}_{10} \mathrm{H}_{10} \mathrm{O}_{4}\right)}_{\text {Valerianio acid. }}+\underbrace{\mathrm{C}_{28} \mathrm{H}_{10} \mathrm{O}_{6}}_{\text {Oreoselone. }} .
$$

Absinthine, from Artemisia absinthium, or wormwood, is a semi-crystalline mass, very bitter, soluble in alcohol. Tanacetine, from Tanacetum vulgare, is very similar to it. Santonine is a beautifully crystallisable compound, obtained from Artemisia contra. It is soluble in alcohol, bitter to the taste, volatile, and coloured yellow by the action of light. Populine, from the bark and leaves of Populus tremula, forms white crystals, of a sweetish and acrid taste, coloured red by sulphuric acid. It may possibly be connected with salicine. Liriodendrine is a crystalline bitter substance, from the bark of Liriodendron tulipifera. Picrolichenine is an intensely bitter crystalline compound, found in the lichen Variolaria amara. It is powerfully febrifuge. In contact with ammonia and without the access of air, it is changed into a reddish-yellow matter, which finally deposits yellow crystals, not bitter. With access of air, it yields with ammonia a deep red very soluble matter. Cetrarine is another bitter principle, from the lichen, Cetraria Islandica, or Iceland moss. It is coloured deep blue by hydrochloric acid. It it is said also to be febrifuge. Ilicine, from Ilex aquifolium, forms brownish-yellow crystals, very bitter and febrifuge. Syringine is the bitter principle of the lilac, Syringa vulgaris. It is 
crystalline and soluble in alcohol. Daphnine is a bitter crystalline substance, obtained from Daphne Mezereon. Hesperidine is a crystalline body found in the spongy envelope of oranges and lemons. Elaterine is the active principle of Momordica elaterium, is crystalline, bitter, and very purgative. Its formula is $\mathrm{C}_{20}$ $\mathrm{H}_{14} \mathrm{O}_{5}$ (?) Colocynthine, the active principle of colocynth, is amorphous, intensely bitter and purgative. Bryonine, from Bryonia alba and dioica, is similar in its properties. Mudarine is the emetic principle of Calotropis mudarii; it is a brown amorphous matter, the solution of which in water gelatinises at $95^{\circ}$, and becomes again liquid on cooling. Scillitine is the bitter of Scilla maritima. It is amorphous, bitter, purgative, and emetic. Cathartine is the bitter purgative prinoiple of senna. Antiarine, $\mathrm{C}_{28} \mathrm{H}_{20} \mathrm{O}_{10}$, is the active principle of the poison called Upas antiar. It is, when introduced into a wound, especially along with soluble matters, such as sugar, a most powerful poison, and, hitherto, no means are known by which its fatal action can be arrested. Digitaline, the active principle of Digitalis, formerly supposed to be a base, is now said to be non-azotised, and to have the formula $\mathrm{C}_{20} \mathrm{H}_{18} \mathrm{O}_{8}$. It excites violent sneezing, and is very poisonous, if taken internally.

Zanthopicrine is a bitter crystalline substance from the bark of Zanthoxylum Clava Herculis. It has been little studied. Picrotoxine, the bitter principle of Menispermum cocculus (Cocculus indicus), forms white prisms. The composition of these is said to be $\mathrm{C}_{10} \mathrm{H}_{6} \mathrm{O}_{4}$, but some recent researches seem to show that picrotoxine is a vegetable base, and contains nitrogen, like all that class of compounds. Cantharidine, the active principle of cantharides, forms rhombic scales, which are said to be $\mathrm{C}_{20} \mathrm{H}_{6} \mathrm{O}_{4}$, the same formula as that above given for picrotoxine. Columbine is a crystalline bitter substance, obtained from columbo, the root of Menispermum palmatum, and somewhat analogous to picrotoxine. Phloridzine, from the bark of the root of the apple, pear, cherry, plum, \&c., resembles salicine, and is, according to Strecker, $\mathrm{C}_{42} \mathrm{H}_{24} \mathrm{O}_{20}+4 \mathrm{aq}$. By boiling with acids, it is resolved into grape sugar and phloretine.

$$
\mathrm{C}_{42} \mathrm{H}_{24} \mathrm{O}_{20}+2 \mathrm{H} \mathrm{O}=\underbrace{\mathrm{C}_{12} \mathrm{H}_{12} \mathrm{O}_{12}}_{\text {Grape Sugar. }}+\underbrace{\mathrm{C}_{30} \mathrm{H}_{14} \mathrm{O}_{10}}_{\text {Phloretine. }} .
$$

By the action of air and ammonia, it yields a blue compound, phloridzeine, $\mathrm{C}_{42} \mathrm{H}_{30} \mathrm{~N}_{2} \mathrm{O}_{26}$. Quassine is a yellow, crystalline, and very bitter substance from the wood of Quassia amara. Its formula is said to be $\mathrm{C}_{20} \mathrm{H}_{12} \mathrm{O}_{6}$. Quercetrine, from quercetron bark, has been lately shown to be $\mathrm{C}_{36} \mathrm{H}_{18} \mathrm{O}_{20}+2$ aq. It seems 
to be homologous with phloridzine, and like it, when boiled with acids, yields grape sugar, and a new body, quercetine,

$$
\mathrm{C}_{30} \mathrm{H}_{18} \mathrm{O}_{20}+2 \mathrm{H} \mathrm{O}=\underbrace{\mathrm{C}_{12} \mathrm{H}_{12} \mathrm{O}_{12}}_{\text {Grape Sugar. }}+\underbrace{\mathrm{C}_{24} \mathrm{H}_{8} \mathrm{O}_{10}}_{\text {Quercetine. }} .
$$

Thus quercetine is homologous with phloretine. Lupuline, the bitter principle of hops, is not crystallisable. Lactucine is a crystalline resinoid bitter substance, from the juice of Lactuca virosa (Lactucarium). It has anodyne properties. Ergotine is an uncrystallised brown powder, extracted from ergot of rye by hot alcohol, after the fatty matters have been removed by ether. It appears to be poisonous, and is probably the active matter of the ergot. Porphyroxine is a crystallisable substance found in Bengal opium. Its solution in diluted acids becomes red when boiled. It requires further investigation. Saponine is a peculiar principle, found in the root of Saponaria officinalis. It is white, amorphous, and has a taste first sweet, then styptic, and finally acrid. It is a powerful sternutatory. It is soluble in water, and its solution, even when much diluted, froths when agitated, like a solution of soap. The root is used as a detergent. When acted on by alkalies, it is converted into an acid, saponic acid, $\mathrm{C}_{26} \mathrm{H}_{23} \mathrm{O}_{12}$. Smilacine is a crystalline substance, found in Smilax sarsaparilla. Its composition is $\mathrm{C}_{15} \mathrm{H}_{13} \mathrm{O}_{5}$. In China nova there is found a substance very analogous to smilacine, the composition of which is $\mathrm{C}_{15} \mathrm{H}_{12} \mathrm{O}_{4}$ : that is, smilacine, minus 1 eq. water. Seneguine is an acrid and astringent substance, found in Polygala senega. It excites sneezing powerfully. Formula, $\mathrm{C}_{22} \mathrm{H}_{18} \mathrm{O}_{11}$. Guaiacine appears to be the active principle of guaiacum. It is acrid and bitter. Plumbagine, extracted from the root of Plumbago europae, forms yellow prisms, the taste of which is first sweet and styptic, then acrid and hot. The yellow colour of its aqueous solution is turned cherry-red by alkalies. Cyclamine is a crystalline matter from the root of Cyclamen europaum. It is very acrid, purgative, and emetic. Peucedanine is a very acrid crystalline principle derived from the root of Peucedanum officinale. Formula $\mathrm{C}_{24} \mathrm{H}_{12} \mathrm{O}_{6}$. When heated with potash, it is resolved into angelic acid and oreoselone.

Angelic Acid. Oreoselone.

$$
\mathrm{C}_{24} \mathrm{H}_{12} \mathrm{O}_{6}+2 \mathrm{H} \mathrm{O}=\mathrm{C}_{10} \mathrm{H}_{3} \mathrm{O}_{4}+\mathrm{C}_{14} \mathrm{H}_{6} \mathrm{O}_{4} \text {. }
$$

Imperatorine, $\mathrm{C}_{24} \mathrm{H}_{12} \mathrm{O}_{5}$, is a crystallisable compound, obtained from the root of Imperatoria ostruthium. It is very acrid and styptic. Phillyrine, from the bark of various species of Phillyrea, crystallises in silvery scales, which are bitter. Fraxinine, from the bark 
of Fraxinus excelsior, is a crystallisable bitter principle. Tanghine is a similar substance from Tanghinia Madagascariensis. It is poisonous. Melampyrine is a tasteless, neutral, crystalline substance, from Melampyrum nemorosum. Meconine is a neutral, crystalline compound contained in opium. It is soluble in water, alcohol, and ether. It is acrid to the taste, fusible and volatile. Formula $\mathrm{C}_{10} \mathrm{H}_{5} \mathrm{O}_{4}$. With nitric acid it yields nitromeconine or nitromeconic acid, $\mathrm{C}_{20} \mathrm{H}_{9} \mathrm{~N} \mathrm{O}_{12}$. As meconine may be $\mathrm{C}_{20}$ $\mathrm{H}_{10} \mathrm{O}_{8}$, nitromeconine is $\mathrm{C}_{20}\left\{\begin{array}{l}\mathrm{H}_{9} \\ \mathrm{~N} \mathrm{O}_{4}\end{array} \mathrm{O}_{8}\right.$; in which 1 eq. hydrogen is replaced by 1 eq. nitrous acid. Chlorine transforms meconine into crystals containing chlorine, which, however, by the action of alkalies, yield an acid free from chlorine, mechloic acid, $\mathrm{C}_{14} \mathrm{H}_{7} \mathrm{O}_{10}$ (?).

Cubebine, $\mathrm{C}_{34} \mathrm{H}_{16} \mathrm{O}_{10}$, is a crystalline compound contained in cubebs. Olivile, $\mathrm{C}_{22} \mathrm{H}_{9} \mathrm{O}_{4}$, is a crystallisable acrid substance, found in the gum of the olivc-tree. Olivine is another crystalline matter found on the leaves of the same plant. It is bitter. Cnicine is a crystalline matter, found in Centaurea benedicta, and in the numerous family of the Cynarocephalea. It is neutral and bitter, and very similar to columbine. Its formula is $\mathrm{C}_{40} \mathrm{H}_{26} \mathrm{O}_{14}$ (?), and in some respects it approaches to salicine and phloridzine; but it requires further investigation. Limonine, or Limone, a bitter crystalline matter found in the seeds of oranges, lemons, \&c., has not yet been fully examined. Angelicine is a crystallised compound found in angelica root.

Besides the above, which have merely been briefly catalogued for want of space, at least an equal number of substances, chiefly crystallisable, and either bitter, acrid, or in a few cases tasteless, have been extracted by various chemists from many different genera and species of plants, but have been so little examined hitherto that we cannot safely describe them as distinct and peculiar.

\section{NITROGENISED COLOURING MATTERS, AND ALIIED SUBSTANCES.}

There are several fine and valuable colours, which contain nitrogen as an essential element, and probably in the form of ammonia or amide. Such colours are archil, litmus, and cudbear, derived from certain species of lichens; and indigo, derived from the juice of various plants, especially different species of indigofera. All these colours are derived from colourless compounds, frequently of an acid nature, by the combined action of air and ammonia. Of these colourless substances, the most important are, lecanoric acid, erythric acid, orsellic acid, evernic acid, and orcine. 
These acids are easily extracted by the following process (Stenhouse). The lichens cut in small pieces are moistened with water, and after remaining half an hour, slaked lime is added, and the mixture allowed to stand for a time. It is then placed in a vessel with a double bottom, the upper being perforated, and the liquid displaced by cautious addition of water, as long as that which drops gives a deep purple-red colour with bleaching liquor, a character belonging to all the acids which yield archil. The solution is then supersaturated with hydrochloric acid, and a gelatinous precipitate falls, which is washed and dried. The acids are extracted from it by weak alcohol, without boiling, which would form other compounds.

1. Lecanoric Acid occurs in Lecanora tartarea, Gyrophora pustulata, and other lichens. Its formula, according to Schunck, is $\mathrm{C}_{18} \mathrm{H}_{9} \mathrm{O}_{9}=\mathrm{C}_{18} \mathrm{H}_{8} \mathrm{O}_{8}, \mathrm{HO}$. According to Stenhouse, it is $\mathrm{C}_{36} \mathrm{H}_{18} \mathrm{O}_{15}=\mathrm{C}_{36} \mathrm{H}_{17} \mathrm{O}_{14}, \mathrm{H} \mathrm{O}$. It forms minute white crystals insoluble in water, soluble in alcohol and ether. When heated to $212^{\circ}$ it becomes anhydrous, $\mathrm{C}_{18} \mathrm{H}_{8} \mathrm{O}_{8}$. When heated with alkalies, such as baryta, a carbonate is deposited, and a sweet substance remains dissolved, which crystallises on evaporation. This is orcine, which is formed along with carbonic acid, according to the following equation:-

$$
\underbrace{\mathrm{C}_{18} \mathrm{H}_{8} \mathrm{O}_{8}}_{\text {Anhydrous lecanoric acid. }}=2 \mathrm{C} \mathrm{O}_{2}+\underbrace{\mathrm{C}_{16} \mathrm{H}_{8} \mathrm{O}_{4}}_{\text {Anhydrous orcine. }}
$$

The same change takes place when lecanoric acid is boiled with water, and for this reason orcine alone is obtained when the lichens are extracted by that solvent. According to Stenhouse, the change is not quite so simple.

By the combined action of air and ammonia, lecanoric acid is gradually changed into a deep rich blue or purple mass. Here orcine is first produced, and by the action of ammonia on that compound, orceine, the blue compound, is formed. When heated, lecanoric acid yields a sublimate of anhydrous orcine. When boiled with alcohol, it is gradually converted into the following compound.

2. Lecanorate of Oxide of Ethyle. SYN. Pseudoerythrine of Heeren; Erythrine of Kane.-This compound is formed when lecanoric acid and the lichens containing it are boiled with alcohol. It is soluble in hot water, from which it crystallises on cooling, and the solution, after long boiling, contains orcine. It may be sublimed unchanged, and when boiled with alkalies it yields alcohol, carbonic acid, and orcine. Its true nature was discovered by Schunck, who also first detected lecanoric acid. Its formula is $\mathrm{C}_{22} \mathrm{H}_{13} \mathrm{O}_{9}=\mathrm{C}_{4} \mathrm{H}_{5} \mathrm{O}+\mathrm{C}_{38} \mathrm{H}_{8} \mathrm{O}_{8}$. 
Lecanorate of Oxide of Methyle is analogous to the preceding compound.

3. Orcine forms large transparent crystals, is very soluble in water, and has a sweet taste. When anhydrous, it may be distilled unchanged. When mixed with ammonia, and exposed to the air, it gradually acquires a deep blood-red colour, forming a nitrogenised colouring matter, orceine, soluble in ammonia with a deep red, in fixed alkalies with a rich violet colour, the formula of which is $\mathrm{C}_{16} \mathrm{~N} \mathrm{H}_{9} \mathrm{O}_{7}$. The production of anhydrous orcine from lecanoric acid, by boiling with baryta, or even by boiling with water, has been described above. But the crystals of orcine, as deposited from its aqueous solution, are a hydrate, $\mathrm{C}_{16} \mathrm{H}_{11} \mathrm{O}_{7}=\mathrm{C}_{16} \mathrm{H}_{8} \mathrm{O}_{4}+3 \mathrm{H} \mathrm{O}$. It is this hydrate, which, with the addition of 5 eqs. of oxygen and 1 eq. of ammonia, yields orceine and water.

$$
\underbrace{\mathrm{C}_{16} \mathrm{H}_{11} \mathrm{O}_{7}}_{\text {Hydrated orcine. }}+\mathrm{O}_{5}+\mathrm{NH}_{3}=\underbrace{\mathrm{C}_{16} \mathrm{~N} \mathrm{H}_{9} \mathrm{O}_{7}}_{\text {Orceine. }}+5 \mathrm{HO}
$$

Orcine forms with oxide of lead a compound, the formula of which is $\mathrm{C}_{16} \mathrm{H}_{8} \mathrm{O}_{4}+3 \mathrm{~Pb} 0$.

4. Parellic Acid.-This acid accompanies lecanoric acid. It forms either minute crystals, the formula of which is $\mathrm{C}_{21} \mathrm{H}_{8} \mathrm{O}_{10}$, or large hydrated crystals, which are transparent and of great density. The formula of the latter is $\mathrm{C}_{21} \mathrm{H}_{9} \mathrm{O}_{11}=\mathrm{C}_{21} \mathrm{H}_{8} \mathrm{O}_{10}$, H 0 . When heated to $212^{\circ}$, both kinds lose water and become $\mathrm{C}_{21} \mathrm{H}_{7} \mathrm{O}_{9}$, which is probably the formula of the anhydrous acid.

With ammonia, parellic acid becomes brown, and when heated with fixed alkalies, it yields carbonic acid, and other products, not investigated.

5. Erythric Acid. SrN. Orcino-lecanoric acid. This acid is found in Parmelia roccella, and Roccella tinctoria, the latter being the lichen which yields the best archil. When heated, it yields a sublimate of orcine, and when boiled with alkalies, it is resolved into carbonic acid and orcine. When boiled with alcohol, it yields lecanorate of oxide of ethyle. All these facts indicate a close relation to lecanoric acid, and Schunck, who discovered the erythric acid, has pointed out that its formula, $\mathrm{C}_{34} \mathrm{H}_{19} \mathrm{O}_{15}$, represents a compound of 1 eq. lecanoric acid and 1 eq. orcine. It is probably therefore a coupled acid, orcinolecanoric acid.

$$
\underbrace{\mathrm{C}_{18} \mathrm{H}_{8} \mathrm{O}_{8}}+\underbrace{\mathrm{C}_{16} \mathrm{H}_{11} \mathrm{O}_{7}}=\underbrace{\mathrm{C}_{34} \mathrm{H}_{19} \mathrm{O}_{15}}
$$

Lecanoric acid. Hydrated orcine. Orcino-lecanoric acid.

It combines with 4 eqs. of oxide of lead, three of which probably unite with the orcine, and the fourth with the lecanoric 
acid. When boiled with alcohol, it is resolved into lecanorio acid, which unites with oxide of ethyle, and orcine, which is found in solution. It must also yield orcine in large quantity when boiled with baryta, in which case it loses 2 eqs. of carbonio acid, and takes up 3 eqs. of water.

$$
\underbrace{\mathrm{C}_{34} \mathrm{H}_{19} \mathrm{O}_{15}}_{\text {Erythric acid. }}+3 \mathrm{H} \mathrm{O}=2 \underbrace{\left(\mathrm{C}_{16} \mathrm{H}_{11} \mathrm{O}_{7}\right)}_{\text {Hydrated orcine. }}+2 \mathrm{C} \mathrm{O}_{2}
$$

In other words, the orcine ready formed is separated; and the lecanoric acid undergoes the change formerly described.

Erythric or orcino-lecanoric acid is the most important of all the principles found in lichens; some of them, treated as Stenhouse recommends, yield 12 per cent. of the crude acid; hence their superior value. But Stenhouse states the formula of this acid to be $\mathrm{C}_{20} \mathrm{H}_{11} \mathrm{O}_{10}=\mathrm{C}_{20} \mathrm{H}_{10} \mathrm{O}_{9}, \mathrm{H} \mathrm{O}$ : and if so, the production of orcine from it cannot be so simple as above stated. Stenhouse states that when erythric acid is boiled with lime or baryta, it yields picroerythrine, and a new acid, erythrelesic acid, which, when boiled with water, yields carbonic acid and orcine. Like lecanoric acid, erythric acid readily forms ethers when boiled with alcohol or pyroxilic spirit, but these appear to contain not erythric but erythrelesic acid, and it would seem therefore probable, that in Schunck's mode of extracting erythric acid, by boiling the lichen with water, it had been converted into erythrelesic acid, and that therefore this latter acid is the same as Schunck's erythric acid, while the true erythric acid is that of Stenhouse.

Variolarine is the name given to a substance obtained by Robiquet from Variolaria dealbata. It has been little studied, and is probably identical with some of the compounds above described.

6. Picroerythrine: SxN. Erythrin-bitter of Heeren. Amarythrine of Kane.-This compound is formed when erythric acid, or the lichens containing it, are boiled with water, and is the cause of the bitter taste of the decoction. When boiled with baryta it yields orcine, erythromannite, and carbonate of baryta, and when heated, it yields a sublimate of orcine. This is easily explained by its formula, which is $\mathrm{C}_{34} \mathrm{H}_{24} \mathrm{O}_{20}$, that is, erythric acid plus 5 eqs. of water; but, according to Stenhouse, it is $\mathrm{C}_{34} \mathrm{H}_{23} \mathrm{O}_{20}$, and he also discovered the formation of erythromannite when picroerythrine is boiled with bases.

7. Erythromannite. This very curious substance is formed along with a little orcine and carbonic acid, when picroerythrine is boiled with baryta. It is soluble both in water and alcohol, 
and forms very large colourless crystals, of a sweet taste. It is very analogous in its properties to mannite, and its formula is $\mathrm{C}_{11} \mathrm{H}_{14} \mathrm{O}_{11}$, while that of mannite is $\mathrm{C}_{6} \cdot \mathrm{H}_{7} \mathrm{O}_{6}$ or $\mathrm{C}_{12} \mathrm{H}_{14} \mathrm{O}_{12}$. It is not impossible that these two bodies may be homologous; and that their true formulæ are yet unknown. Stenhouse at first gave to erythromannite the formula $\mathrm{C}_{10} \mathrm{H}_{13} \mathrm{O}_{10}$, nor has any reason been given for changing it to $\mathrm{C}_{11} \mathrm{H}_{14} \mathrm{O}_{12}$. If the latter be correct, however, the difference between it and that of mannite is $\mathrm{C} \mathrm{O}$, and it is possible that homologous series may exist in which $\mathrm{C} \mathrm{O}$ is the common difference; but it is more probable that the difference may really be $\mathrm{C} \mathrm{H}$, or $\mathrm{C}_{2} \mathrm{H}_{2}$. Like mannite, erythromannite forms with nitric acid a compound, in which 5 eqs. of water are replaced by 5 of nitric acid.

8. Alpha Orsellic Acid. Found by Stenhouse in Roccella tinctoria, from South America. It is crystallisable, nearly insoluble in cold water, soluble in hot water. Its formula is $\mathrm{C}_{32} \mathrm{H}_{16} \mathrm{O}_{14}=\mathrm{C}_{32} \mathrm{H}_{15} \mathrm{O}_{13}, \mathrm{H} \mathrm{O}$. With bleaching liquor it forms a deep but fugitive blood-red colour, as do all the acids which yield orcine and archil. Its solution in ammonia also becomes of a deep red colour when exposed to the air. When the neutral alpha orsellate of baryta is boiled with water, a new acid is formed, alpha orsellesic acid, $\mathrm{C}_{16} \mathrm{H}_{9} \mathrm{O}_{8}=\mathrm{C}_{16} \mathrm{H}_{8} \mathrm{O}_{7}, \mathrm{H} \mathrm{O}$. When this acid is boiled with water, it yields carbonic acid and colourless orcine. With bleaching liquor it gives the same fugitive violet colour as orcine, not the blood-red of orsellic acid. It combines with oxide of ethyle, but orsellic acid does not.

9. Beta Orsellic Acid is found in the same lichen from the Cape. It is prepared like the other acids, and closely resembles the preceding in all its relations. It is intermediate in properties between alpha orsellic and erythric acids, but nearer to the former. Its formula is $\mathrm{C}_{34} \mathrm{H}_{18} \mathrm{O}_{15}=\mathrm{C}_{34} \mathrm{H}_{17} \mathrm{O}_{14}, \mathrm{H} \mathrm{O}$. It yields, like the preceding acid, a beta orsellesic acid, not yet analysed, which is capable of forming ethers, and when boiled, yields colourless orcine.

10. Roccelline. This body is formed along with the preceding. It is neutral, yields no red colour with bleaching liquor, and its formula is $\mathrm{C}_{38} \mathrm{H}_{17} \mathrm{O}_{15}$.

11. Evernic Acid. Found in Evernia prunastri. This acid resembles the others, but is not coloured red by bleaching liquor. It however yields orcine when heated, and gives a red colour with ammonia on exposure to the air, but slowly. Its formula is $\mathrm{C}_{34} \mathrm{H}_{13} \mathrm{O}_{14}=\mathrm{C}_{34} \mathrm{H}_{15} \mathrm{O}_{13}, \mathrm{H} 0$. When boiled with bases, it yields evernesic acid, and orcine. Evernesic acid yields no red or purple with bleaching liquor or ammonia. Its formula is $\mathrm{C}_{18} \mathrm{H}_{10}$ $\mathrm{O}_{8}=\mathrm{C}_{18} \mathrm{H}_{8} \mathrm{O}_{7}, \mathrm{H} \mathrm{O}$, very near to Schunok's formula for leca- 
noric acid, from which, however, it is quite distinet. It combines with oxide of ethyle.

It will be seen that all the above acids, as found in lichens, namely, lecanoric, erythric, alpha orsellie, beta orsellic, and evernic acids, yield red dyes with ammonia (orceine or archil dyes), and strike a blood-red but fugitive colour with bleaching liquor. But all of them, when their neutral salts are boiled, yield new acids, such as erythrelesic and the two orsellesio acids, (lecanoric acid no doubt yields one of these, or a similar acid), which, save in the case of evernesic acid, strike a violet colour with bleaching liquor. Now, these last acids, when boiled with water, yield colourless orcine and carbonic acid, and this is the best method of obtaining orcine, the true origin of the dyes formed with ammonia. If orcine be required in large quantity, and slightly coloured, which does not interfere with its properties, it is best obtained by extracting the lichens with milk of lime in the cold, passing carbonic acid through the filtered solution as long as carbonate of lime is formed, after boiling it for some hours, and evaporating it to a small bulk. The filtered liquid is to be evaporated to dryness, and the residue boiled in strong spirits, which, on standing, deposit abundance of orcine in reddish crystals. These may be further purified by solution in ether and evaporation in vacuo, when large crystals are deposited with little colour, apparently anhydrous orcine. Orcine gives, with bleaching liquor, the same fugitive violet colour as erythrelesic and orsellesic acids, \&c.; and, as has been already stated, it yields, with ammonia, on exposure to air, the deep red oreeine. For our knowledge of these singular compounds we are chiefly indebted to Schunck and to Stenhouse. The latter recommends that the acids of the lichens should be extracted by his process, with lime and hydrochlorio acid, on the spot, and the dried precipitate of crude acid imported instead of the bulky lichens, which contain from 2 to 12 per cent. only of available colouring matter. The process is so easy, and the value of the crude acids-probably $1000 l$. per ton-so great, that the recommendation of Stenhouse will probably be adopted.

12. Usnic Acid.-This acid is found in many lichens, such as Lichen rangiferinus, and in many species of Usnea, Ramalina, Parmelia, Biatora, Lecidea, Alectora, Evernia, and Cladonia. It is extracted by Stenhouse's process, and forms sulphur-yellow crystals, insoluble in water and alcohol. Like the other acids of the lichens, it yields coloured compounds under the influence of air and ammonia or fixed alkalies; but these appear to contain no nitrogen, and are not blood-red or purple, but red. It cannot be made to yield orcine, so far as is yet known. Its formula is 
$\mathrm{C}_{38} \mathrm{H}_{17} \mathrm{O}_{14}$, which corresponds to 2 eqs. of lecanoric acid, plus $\mathrm{C}_{2} \mathrm{H}_{2}$, and minus $\mathrm{O}_{2}$.

13. Chrysophanic Acid.-This is another crystallisable acid, found in Parmelia parietina. It forms golden-yellow crystals, and with alcoholic solutions of potash and ammonia yields a splendid red colour. Its formula is said to be $\mathrm{C}_{20} \mathrm{H}_{8} \mathrm{O}_{6}$, which might be naphthaline, $\mathrm{C}_{20} \mathrm{H}_{8}$, plus 6 eqs. of oxygen. Its salts are blue or violet when dry, red in solution. This acid has also been found in rhubarb by Schlossberger and Doepping. This and the preceding acid were discovered in the lichens by Rochleder and Heldt. If these two acids are employed in dyeing, they yield colours quite different from those given by the white acids previously described.

14. Archil contains, according to Kane, two blue compounds,

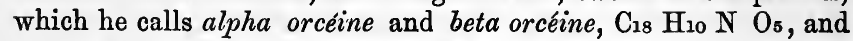
$\mathrm{C}_{18} \mathrm{H}_{10} \mathrm{~N} \mathrm{O}_{8}$; besides a third of an acid nature, erythroleic acid, $\mathrm{C}_{26} \mathrm{H}_{22} \mathrm{O}_{8}$.

15. Litmus contains, according to Kane, a red fluid, erythroleine, $\mathrm{C}_{26} \mathrm{H}_{22} \mathrm{O}_{4}$; and three solids, of a purple colour, erythrolitmine, $\mathrm{C}_{26} \mathrm{H}_{23} \mathrm{O}_{18}$; azolitmine, which contains nitrogen, and is the principal constituent of litmus; and spaniolitmine, $\mathrm{C}_{18}$ $\mathrm{H}_{7} \mathrm{O}_{16}$. According to Gélis, litmus contains three colouring matters, one soluble in ether, which is orange-red; one soluble in alcohol, blood-red, and one soluble in water. The second is the chief ingredient of the dye. All give blue compounds with alkalies.

16. Phloridzeine is the deep blue compound formed from phloridzine by the action of air on ammonia. Its formula is $\mathrm{C}_{42} \mathrm{H}_{30}$ $\mathrm{N}_{2} \mathrm{O}_{26}$.

\section{Indigo.}

This valuable dye has been long known; but it is only since 1827 that its chemical relations have been accurately studied. No substance in the whole range of chemistry has yielded a greater variety of most interesting products: and the study of the metamorphoses of indigo has already thrown much light on the laws of the decomposition of organic substances. For what is known on this subject, we are indebted, first, in point of time, to Chevreul, Runge, Walter Crum, Liebig, Berzelius, and Dumas; more lately, and in an especial manner, to the comprehensive researches of Erdmann, Fritzsche, and Laurent; the last of whom has made known several interesting series of new compounds derived from indigo, and has both confirmed and extended, as well as corrected, in some cases, the previous 
results obtained by Erdmann; still more recently, Hofmann and Muspratt have greatly extended our knowledge of the derivatives of indigo.

Indigo is obtained from various plants, chiefly of the genus Indigofera, as $I$. tinctoria, anil, argentea, \&c., but also of other genera, as Nerium, Isatis, Pergularia, Gymnema, Polygonum, Tephrosia, Amorpha, and others. In the juice of the plants, it exists in the furm of a colourless soluble compound, probably a compound of white indigo with an alkali. When exposed to the air, it is converted into the blue compound, and becomes at the same time insoluble, just as in an artiticial solution of white or reduced indigo in an alkali. The manufacture of indigo is not thoroughly understood, but it would appear that ammonia, as well as air, contributes to the formation of the colour, probably by converting into white indigo some compound present in the fresh juice, the nature of which, however, is still uncertain. In the Antilles and in the East Indies, the leaves are made to ferment in water, during which much ammonia is formed, and the indigo is found in the soluble state, ready to become blue and insoluble by absorbing oxygen. But in North America, the dried leaves are infused in warm water, or boiled with water, till the liquid becomes green, when, on exposure to the air, it deposits blue insoluble indigo. Here the same change must, in great part at least, have taken place during the drying, as during the fermentation of the other process.

The indigo of commerce is a deep blue powder, often cohering in cakes, and exhibiting, when polished by rubbing with the nail or any hard substances, a coppery colour and lustre. It is tasteless and inodorous, insoluble in water, and nearly so in alcohol and ether. It may be purified by treating it successively with boiling diluted sulphuric acid and with water, which remove a glutinous matter; with aqua potassæ, at a gentle heat, which dissolves a brown colouring matter, and with boiling alcohol, which takes up a red colouring matter. When fresh alcohol becomes no longer red, but blue, the indigo is as pure as it can be made by such means.

To purify it still further, it is digested with water, lime, and grape, or starch sugar, which deoxidises, or reduces the indigo, while the lime combines with the reduced indigo, forming a soluble compound, of a wine-yellow tint. This being filtered into dilute hydrochloric acid, which removes the lime, deposits pure indigo as a blue powder. Cloth steeped in the above solution of indigo, and exposed to the air, is quickly dyed blue, as the indigo, at the moment of being rendered insoluble, combines with the fibre of the cloth, to which it adheres very firmly, so that it 
cannot be washed away. If indigo, grape sugar, soda, and alcohol, be digested together in proper proportions, a yellow solution is obtained, which, when exposed to air, deposits pure indigo, in crystals. (Fritzsche.)

Pure indigo, when rapidly heated on a slip of platinum, volatilises, yielding purple vapours, which condense in purple crystals on a cold surface. These crystals are called indigotine: but they are nothing else than sublimed indigo, and have all the chemical characters of pure indigo. When distilled, indigo yields, among other products common to all nitrogenised organic matters, a very curious oily liquid, of powerfully basic properties, and forming salts with acids which crystallise with singular facility. 'This base is aniline, phenylamine, or phenamine, $\mathrm{C}_{22} \mathrm{H}_{7} \mathrm{~N}$, in which 1 of the 3 eqs. of hydrogen in ammonia is replaced by phenyle, $\mathrm{C}_{12} \mathrm{H}_{5}$ : thus $\mathrm{N} \mathrm{H}_{2}+\mathrm{C}_{12} \mathrm{H}_{5}=\mathrm{C}_{22} \mathrm{H}_{7} \mathrm{~N}$. It is found in the oil of coal-tar, as well as in the products of distillation of many nitrogenised bodies.

Oil of vitriol dissolves indigo with a deep blue colour, forming two blue acids. This solution is much used in dyeing. Nitric acid, chloric acid, chromie acid, chlorine and bromine, all dissolve indigo, giving rise to oxygenised and chlorinised or brominised products, all of which are yellow and orange-coloured. When boiled with strong aqua potassæ, indigo is also oxidised and dissolved in the form of new acids.

When placed in contact with deoxidising or reducing agents, such as protosalts of iron, tin, and manganese, or honey and grape sugar, along with an alkali such as soda or lime, indigo is decolorised and dissolved in combination with the alkali. The addition of diluted hydrochloric acid, air being carefully excluded, precipitates reduced or white indigo.

White or reduced indigo, $\mathrm{C}_{16} \mathrm{H}_{6} \mathrm{~N} \mathrm{O}_{2}$, obtained as above described, is a crystalline powder of a dirty white, which, if washed with water previously boiled to expel air, and cooled in closed vessels, and dried in vacuo, is bluish externally, but grey within. The blue parts being removed, the remainder is reduced indigo. When moist, it very rapidly passes into blue indigo, oxygen being absorbed; when dry, the change is more slowly effected. It is insoluble in water and acids, very soluble in alkaline solutions; its solutions, if exposed to the air, deposit pure indigo blue, as a powder.

The first accurate analysis of indigo blue was made by Walter Crum; and his results have been confirmed by all succeeding experimenters. The formula now adopted for indigo, as agreeing best with its numerous metamorphoses, is one first proposed by Dumas, namely, $\mathrm{C}_{26} \mathrm{H}_{5} \mathrm{~N} \mathrm{O}_{2}$. This formula is the same with 
that of cyanide of benzoyle, or of benzile, $\mathrm{C}_{14} \mathrm{H}_{5} \mathrm{O}_{2}+\mathrm{C}_{2} \mathrm{~N}=$ $\mathrm{C}_{16} \mathrm{H}_{3} \mathrm{~N} \mathrm{O}_{2}$.

The relation of white indigo to blue indigo is, according to one view, the same as that of hyduret of be nzoyle to benzoyle, or of alloxantine to alloxan. Thus we have

Benzoyle $\mathrm{C}_{14} \mathrm{H}_{5} \quad \mathrm{O}_{2}$ Hyduret of Benzoyle $\mathrm{C}_{14} \mathrm{H}_{5} \quad \mathrm{O}_{2}+\mathrm{H}$

Alloxan $\mathrm{Cs} \mathrm{H}_{4} \mathrm{~N}_{2} \mathrm{O}_{10}$ Alloxantine . . $\mathrm{Cs}_{8} \mathrm{H}_{4} \mathrm{~N}_{2} \mathrm{O}_{10}+\mathrm{H}$ Indigo blue $\mathrm{C}_{16} \mathrm{H}_{5} \mathrm{~N}_{2}$ Indigo white $\mathrm{O}_{2} \mathrm{C}_{16} \mathrm{H}_{5} \mathrm{~N} \mathrm{O}_{2}+\mathrm{H}$

According to another hypothesis, white indigo is the hydrate of an inferior degree of oxidation of the same radical of which blue indigo is the higher oxide. Blue indigo, on this view, is $\mathrm{C}_{16} \mathrm{H}_{3} \mathrm{~N}+\mathrm{O}_{2}$, and white indigo is a hydrate, $\mathrm{C}_{16} \mathrm{H}_{5} \mathrm{~N}+\mathrm{O}+\mathrm{HO}$. This latter view is the more probable, not only in regard to indigo, but also in regard to alloxan, for, assuming uryle to be $\mathrm{C}_{8} \mathrm{~N}_{2} \mathrm{O}_{4}=$ $\mathrm{Ul}$, alloxan will be $\mathrm{Ul}+\mathrm{Os}+4 \mathrm{H} \mathrm{O}$, and alloxantine will be $\mathrm{Ul}$ $+0+5 \mathrm{H} 0$.

The action of grape sugar, in reducing indigo, tells in favour of the latter hypothesis. For if white indigo is blue indigo plus hydrogen, this hydrogen is derived from water, the oxygen of which must have combined with the hydrogen of the sugar (to convert the sugar into formic acid, which is produced in this operation). Here, then, we should have oxygen leaving hydrogen to combine with hydrogen, or, in other words, water produced and decomposed at the same time, which is in the highest degree improbable. To demonstrate this, let the radical of indigo be assumed to be anyle $=\mathrm{C}_{16} \mathrm{H}_{5} \mathrm{~N}=\mathrm{An}$ : let blue indigo be $\mathrm{An}_{2}$, and white indigo $\mathrm{An}_{2}, \mathrm{H}$ or An $\mathrm{O}, \mathrm{H} \mathrm{O}$. Then 12 eqs. of blue indigo, 12 of water, and 1 of grape sugar, act on each other, and, according to the view which makes white indigo the hyduret of blue indigo, the reaction is as follows: 12 An $\mathrm{O}_{2}+12 \mathrm{H} \mathrm{O}+$ $\mathrm{C}_{12} \mathrm{H}_{12} \mathrm{O}_{12}=12\left(\mathrm{An} \mathrm{O}_{2}, \mathrm{H}\right)+6\left(\mathrm{C}_{2} \mathrm{H} \mathrm{O} \mathrm{O}_{3}\right)+6 \mathrm{H} \mathrm{O}$. In this explanation it is evident that 6 eqs. of oxygen have quitted hydrogen to combine with hydrogen. On the other view the reaction is as follows, free from any such absurdity : $12 \mathrm{An}_{2}+$ $12 \mathrm{H} \mathrm{O}+\mathrm{C}_{12} \mathrm{H}_{12} \mathrm{O}_{12}=12 \mathrm{An}, \mathrm{H} \mathrm{O}+6\left(\mathrm{C}_{2} \mathrm{H} \mathrm{O} \mathrm{O}_{3}\right)+6 \mathrm{H} \mathrm{O}$. Here the 12 eqs. of deutoxide of anyle (blue indigo) lose 12 eqs. oxygen, which are replaced by 12 eqs. water; and the 12 eqs. oxygen acting on the sugar, 6 eqs. take 6 eqs. hydrogen, forming water, and 6 eqs. convert the residue into formic acid. The student will bear in mind that $\mathrm{C}_{2} \mathrm{H} \mathrm{O}_{3}$ is the formula of anhydrous formic acid, and $\mathrm{C}_{12} \mathrm{H}_{12} \mathrm{O}_{12}$ that of dry grape sugar.

We shall therefore consider white indigo as the hydrated protoxide of anyle $\left(\mathrm{C}_{16} \mathrm{H}_{5} \mathrm{~N}, \mathrm{O}+\mathrm{H} \mathrm{O}\right)$, and blue indigo as the 
anhydrous deutoxide $\left(\mathrm{C}_{16} \mathrm{H}_{5} \mathrm{~N}, \mathrm{O}_{2}\right)$. White indigo forms compounds with bases, in which, no doubt, its hydratic water is replaced by an equivalent of metallic oxide, as in the general formula, $\mathrm{C}_{26} \mathrm{H}_{5} \mathrm{~N}, \mathrm{O}+\mathrm{M} \mathrm{O}$.

\section{ACTION OF SULPHURIC ACID ON INDIGO.}

When powdered pure indigo is added to 15 parts of oil of vitriol, and gently warmed, a deep blue solution is formed, which mixes perfectly with water. But if only 8 or 10 parts of acid are used, the addition of water causes the deposition of a purple powder, while a blue solution is obtained. The purple powder, which, althongh insoluble in dilute acid, is soluble in pure water, is sulphopurpuric acid; the blue solution contains two acids, sulphoindigotic and hyposulphoindigotic acids. When neutralised with potash, these acids form salts, which separate from the liquid, when it is saturated with any alkaline salt, such as acetate or carbonate of potash. The two blue salts may be separated from each other by alcohol, but the composition of the hyposulphoindigotate of potash is not known. The sulphoindigotate appears to be strictly a hyposulphoindigotate, and its formula is in all probability, $\mathrm{C}_{16} \mathrm{H}_{4} \mathrm{~N} \mathrm{O}_{2}, \mathrm{~S}_{2} \mathrm{O}_{5}+\mathrm{KO}$. Here the indigo has lost 1 eq. of hydrogen, and the 2 eqs. sulphuric acid 1 eq. oxygen. Dumas's view, according to which the salt is a double sulphate, analogous to sulphovinate of potash, $\mathrm{Cl6}_{\mathbf{6}} \mathrm{H}_{4}$ $\mathrm{N} \mathrm{O}, \mathrm{S} \mathrm{O}_{3}+\mathrm{K} 0, \mathrm{~S} \mathrm{O}_{3}$, is not supported by the chemical relations of these substances. Dumas conjectured that indigo blue was analogous to alcohol, and that its formula was $\mathrm{C}_{16} \mathrm{H}_{4} \mathrm{~N}, 0$ $+\mathrm{H} \mathrm{O}$, the body $\mathrm{C}_{16} \mathrm{H}_{4} \mathrm{~N}, \mathrm{O}$ being analogous to oxide of ethyle. But, as already stated, this view is far-fetched, and does not agree with the chemical relations of indigo. It would make, for example, white indigo $\mathrm{C}_{16} \mathrm{H}_{4} \mathrm{~N}, \mathrm{O}+\mathrm{H}+\mathrm{H} \mathrm{O}$ or $\mathrm{C}_{16} \mathrm{~N} \mathrm{H}_{4}+$ $2 \mathrm{H} \mathrm{O}$, both most improbable formulæ.

The blue solution of indigo in oil of vitriol, if diluted and digested with flannel or woollen cloth, is entirely deprived of blue colour, while the cloth is so effectually dyed that the colour cannot be washed out. It can, however, be dissolved from the cloth by carbonate of ammonia, and by this means the sulphoindigotates of ammonia, and from these the other salts of the blue acids, are prepared.

The sulphopurpuric acid, according to Dumas, contains the elements of 2 eqs. sulphuric acid and 2 eqs. indigo, and neutralises only 1 eq. of base. But the indigo in it has probably lost hydrogen (while the acid has lost oxygen), or hydrogen and oxygen. 


\section{PRODUCTS OF THE OXIDATION OF INDIGO.}

Isatine, $\mathrm{C}_{16} \mathrm{H}_{5} \mathrm{~N} \mathrm{O}_{4}$. This interesting compound, which is blue indigo, plus 2 eqs. oxygen, is formed by digesting indigo along with water, sulphuric acid, and bichromate of potash, or by heating indigo with weak nitric acid. It dissolves, and the solution on evaporation deposits aurora-red crystals of isatine, sparingly soluble in cold water, more soluble in hot water, and in alcohol. By the action of chlorine, it yields two compounds in which hydrogen is partially replaced by chlorine. It may be volatilised if heated on a plate of metal. When acted on by a strong solution of potash, isatine is dissolved with an intense violet colour, which on addition of water and evaporation changes to yellow, and the liquid deposits pale yellow crystals, which contain potash, united to a new acid, isatinic acid, formed from isatine by the addition of 1 eq. water. When separated from its salts by stronger acids, isatinic acid is at once resolved into isatine and water; but if isatinate of lead be decomposed by sulphuretted hydrogen, and the filtered solution evaporated spontaneously in vacuo, the acid is obtained as a white flocculent powder, which, when dissolved in boiling water, instantly becomes red, and the solution on cooling deposits crystals of isatine. Isatinic acid is $\mathrm{C}_{16} \mathrm{H}_{6} \mathrm{~N} \mathrm{O}_{5}, \mathrm{H} \mathrm{O}$. Its salts have the formula, $\mathrm{C}_{16} \mathrm{H}_{6} \mathrm{~N} \mathrm{O}_{5}, \mathrm{MO}$. The violet-coloured compound first formed when isatine acts on potash, is a compound of isatine and potash, which, when heated with water, soon passes into isatinate of potash.

With sulphurous acid, or rather sulphites, isatine forms salts of the formula, $\mathrm{C}_{16} \mathrm{H}_{5} \mathrm{~N} \mathrm{O}_{4}, 2 \mathrm{~S} \mathrm{O}_{2}+\mathrm{MO}$; which may be formed also by the action of sulphurous acid on isatinates.

By the action of chlorine, isatine is converted into two compounds, chlorisatine and bichlorisatine. When chlorine is passed through isatine or indigo suspended in water, both these compounds are formed, and they are separated by crystallisation, chlorisatine being the least soluble of the two.

Chlorisatine, $\mathrm{C}_{16}\left\{\begin{array}{l}\mathrm{H}_{4} \\ \mathrm{Cl}_{4}\end{array} \mathrm{~N} \mathrm{O}_{4}\right.$, forms transparent orange-yellow 4-sided prisms, isomorphous with isatine, and very analogous to it in all respects. When acted on by potash, there is first formed a deep red solution, which when heated soon becomes yellow, and on cooling deposits brilliant pale yellow crystals of chlorisatinate of potash, $\mathrm{C}_{16}\left\{\begin{array}{l}\mathrm{H}_{5} \\ \mathrm{Cl}\end{array} \mathrm{N} \mathrm{O}_{5}, \mathrm{~K} \mathrm{O}\right.$, a salt perfectly 
analogous to isatinate of potash, and containing an acid, chlorisatinic acid, which is chlorisatine, plus 1 eq. water, $\mathrm{C}_{16} \mathrm{H}_{5} \mathrm{Cl}$ $\mathrm{N} \mathrm{O}_{5}$. Like isatinic acid, when separated from its salts it is speedily resolved into chlorisatine and water. Chlorisatinate of silver, $\mathrm{C}_{16}\left\{\begin{array}{l}\mathrm{H}_{5} \\ \mathrm{Cl} \mathrm{N} \mathrm{O}_{5}, \mathrm{Ag} \mathrm{O} \text { forms yellow crystals, soluble }\end{array}\right.$ in hot water. Chlorisatinate of baryta, $\mathrm{C}_{16}\left\{\begin{array}{l}\mathrm{H}_{5} \\ \mathrm{Cl}^{2}\end{array} \mathrm{~N} \mathrm{O}_{5}, \mathrm{Ba} \mathrm{O}\right.$, +3 aq. forms golden yellow tables. Chlorisatinate of lead, $\mathrm{C}_{16}$ $\left\{\begin{array}{l}\mathrm{H}_{3} \\ \mathrm{Cl}\end{array}\right.$ potash by nitrate of lead, forms a gelatinous yellow precipitate, which soon becomes flocculent, acquiring a splendid searlet colour. The red salt is crystalline, the yellow amorphous. Chlorisatinate of copper forms at first a brownish yellow bulky precipitate, which soon changes to a heavy granular blood-red powder.

Like the isatinates, the chlorisatinates combine with sulphurous acid, forming salts of the formula, $\mathrm{C}_{16}\left\{\begin{array}{l}\mathrm{H}_{4} \\ \mathrm{Cl}\end{array} \mathrm{N} \mathrm{O}_{4}\right.$, $2 \mathrm{~S} \mathrm{O}_{2}+\mathrm{MO}$. In short, the analogy between isatine and chlorisatine, isatinates and chlorisatinates, \&c., is such as to furnish a very beautiful example of the substitution of chlorine for hydrogen, while the type or chemical character of the compound is unaffected. In bichlorisatine, we shall see an additional example of the same truth.

Bichlorisatine, $\mathrm{C}_{16}\left\{\begin{array}{l}\mathrm{H}_{3} \\ \mathrm{Cl}_{2}\end{array} \mathrm{~N} \mathrm{O}_{4}\right.$, is formed along with the preceding compound. It is more soluble than chlorisatine, but is otherwise remarkably similar to it. With aqua potassæ it first forms a deep red solution (here, as in the case of isatine and chlorisatine, a compound of it with potash), which, when heated, changes to yellow, and on evaporation yields yellow scales of a salt composed of potash and bichlorisatinic acid. The acid may be separated by stronger acids as a yellow powder, which when dissolved and warmed is resolved into bichlorisatine and water. Its formula is $\mathrm{C}_{16}\left\{\begin{array}{l}\mathrm{H}_{4} \\ \mathrm{Cl}_{2}\end{array} \mathrm{~N} \mathrm{O}_{5}, \mathrm{H} \mathrm{O}\right.$. The salt of baryta, $\mathrm{C}_{16}\left\{\begin{array}{l}\mathrm{H}_{4} \\ \mathrm{Cl}_{2}\end{array}\right.$ salt of copper is at first bulky and brown, but soon becomes greenish-yellow and crystalline, and finally, a heavy granular powder of a fine carmine-red colour.

Bichlorisatine with sulphites forms compounds analogous to those above mentioned of isatine and chlorisatine. In this case 
also, therefore, the type is perfectly retained, although 2 eqs. of the hydrogen of isatine have been replaced by chlorine.

Bromine acts on isatine, and forms two compounds, bromisatine and bibromisatine, entirely analogous to chlorisatine and bichlorisatine, forming bromisatinic and bibromisatinic acids, and also compounds with sulphites analogous to those just mentioned. We have, therefore, from isatine and isatinic acid, by substitution, the following compounds :

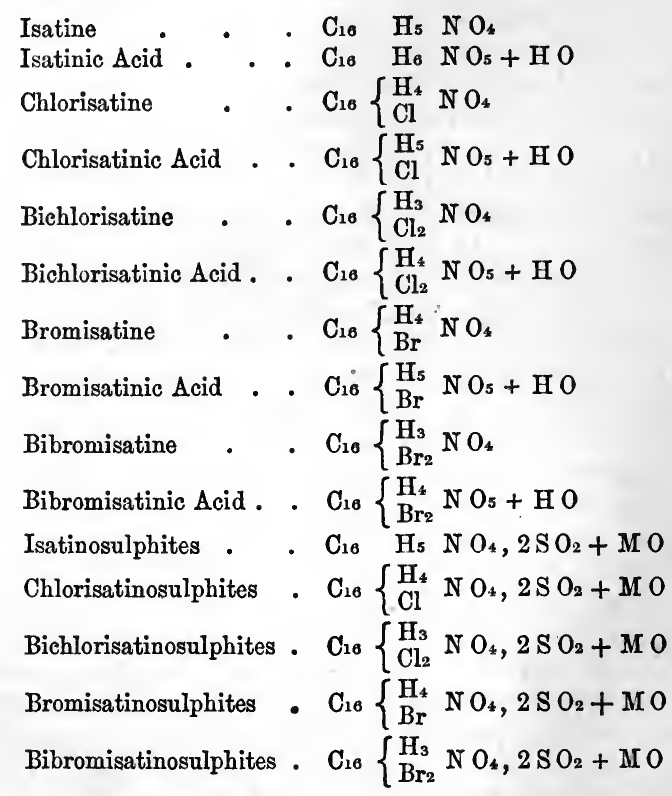

Hofmann has lately shown that isatine when heated with potash, yields aniline (see Aniline), and that chlorisatine, bromisatine, \&c., when treated in the same way, yield compounds of the type of aniline, in which hydrogen is replaced by chlorine, bromine, or both. Some of these compounds retain the basic character, and are therefore very interesting, as the first examples of such chlorinised or brominised bases. A table of the compounds derived by substitution from aniline is given under that substance. Here we have only space to mention that chloraniline, dichloraniline, bromaniline, and dibromaniline, are crystalline volatile bases, forming crystallisable salts, and very analogous 
to aniline: while in trichloraniline, tribromaniline, chlorodibromaniline, the basic character is lost.

Isatyde, $\mathrm{C}_{16} \mathrm{H}_{6} \mathrm{~N} \mathrm{O}_{4}=\mathrm{C}_{26} \mathrm{H}_{5} \mathrm{~N} \mathrm{O}_{4}, \mathrm{H}$, is formed when an alcoholic solution of isatine is acted on by sulphuret of ammonium. It is a grey crystalline powder, and may be considered to represent isatine plus 1 eq. hydrogen.

Sulphésatyde, $\mathrm{C}_{16} \mathrm{H}_{6} \mathrm{~N}\left\{\begin{array}{l}\mathrm{O}_{2} \\ \mathrm{~S}_{2}\end{array}\right.$ is isatyde, in which 2 eqs. oxygen are replaced by sulphur. It is formed by the action of sulphuretted hydrogen on isatine dissolved in alcohol, and is a greyish-yellow amorphous powder. Sulphisatine is the name of a compound obtained in the same way by Erdmann, which he considers to be different from sulphésatyde.

Chlorisatyde, $\mathrm{C}_{16}\left\{\begin{array}{l}\mathrm{H}_{5} \\ \mathrm{Cl} \mathrm{N}_{4}\end{array}\right.$ is a white powder somewhat crystalline, obtained by the action of sulphuret of ammonium on chlorisatine. It is perfectly analogous to isatyde. By the action of sulphuretted hydrogen on chlorisatine, a compound is formed which is $\mathrm{C}_{26}\left\{\begin{array}{l}\mathrm{H}_{5} \\ \mathrm{Cl}\end{array} \mathrm{N} \mathrm{S}_{4}\right.$; that is chlorisatyde, in which all the oxygen is replaced by sulphur.

Bichlorisatyde, $\mathrm{C}_{16}\left\{\begin{array}{l}\mathrm{H}_{4} \\ \mathrm{Cl}_{2}\end{array} \mathrm{~N}_{4}\right.$ and bibromisatyde, $\mathrm{C}_{16}\left\{\begin{array}{l}\mathrm{H}_{4} \\ \mathrm{Br}_{2}\end{array}\right.$ $\mathrm{N} \mathrm{O}_{4}$, are perfectly analogous, in formation and properties, to chlorisatyde.

Sulphasatyde, $\mathrm{C}_{16} \mathrm{H}_{6} \mathrm{~N}\left\{\begin{array}{l}\mathrm{O}_{3} \\ \mathrm{~S}\end{array}\right.$ is formed by the action of potash on sulphésatyde, from which it differs in having only 1 eq. oxygen replaced by sulphur. It is a white crystalline powder.

Indine, $\mathrm{C}_{16} \mathrm{H}_{6} \mathrm{~N} \mathrm{O}_{2}$ is a crystallised substance, of a beautiful rose colour, formed by the action of potash on sulphésatyde, along with the preceding: or by the action of potash on sulphasatyde or isatyde. In the last case, isatinate of potash is also formed, thus, $3\left(\mathrm{C}_{16} \mathrm{H}_{6} \mathrm{~N} \mathrm{O}_{4}\right)+2 \mathrm{~K} \mathrm{O}=2(\mathrm{~K} 0$, $\left.\mathrm{C}_{16} \mathrm{H}_{6} \mathrm{~N} \mathrm{O}_{5}\right)+\mathrm{C}_{16} \mathrm{H}_{6} \mathrm{~N} \mathrm{O}_{2}$. It is sulphésatyde minus 2 eqs. sulphur, and is also isomeric with white indigo. It is decomposed by nitric acid, and by bromine, which give rise to new products.

When indine, moistened with alcohol, is covered with a lukewarm solution of potash, it forms a black solution which in a few moments becomes a semisolid mass of black needles, which are a compound of potash with indine, or rather with indinic acid, an acid formed from indine, like isatinic acid from isatine, by the assimilation of 1 eq. water, and which is very easily again resolved into indine and water. 
Hydrindine is a yellow crystalline compound formed by heating indine, sulphasatyde, or isatyde with potash. Its composition is $\mathrm{C}_{32} \mathrm{H}_{13} \mathrm{~N}_{2} \mathrm{O}_{5}$, that is, 2 eqs. indine plus 1 eq. water : and when strongly heated, it is resolved into indine and water. It is not composed of these substances, however, for it forms with potash a white salt, hydrindinate of potash. The acid in this salt appears to be formed, like some of those already described, by the addition of water to hydrindine; its formula is probably $\mathrm{C}_{26} \mathrm{H}_{15}$ $\mathrm{N}_{2} \mathrm{O}_{7}, 2 \mathrm{H} \mathrm{O}$.

Nitrindine, $\mathrm{C}_{16} \mathrm{H}_{4} \mathrm{~N}_{2} \mathrm{O}_{7}$, is a beautiful violet-coloured powder formed by the action of nitric acid on indine and hydrindine. It is indine, in which 2 eqs. hydrogen are replaced,

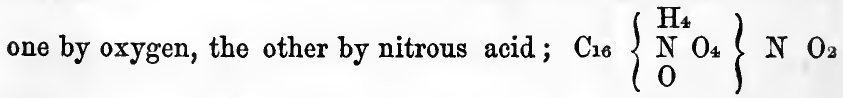

\section{Chlorindine, $\mathrm{C}_{16}\left\{\begin{array}{l}\mathrm{H}_{5} \\ \mathrm{Cl}\end{array}\right\} \mathrm{N} \mathrm{O}_{2}+2 \mathrm{HO}$, is a powder of a dirty} violet colour, formed by the action of heat on chlorisatyde. Analogous compounds are obtained from bichlorisatyde and bibromisatyde. Bichlorindine is like chlorindine. Bibromindine is a very dark red, and dissolves in alcohol with a fine purple colour.

The action of potash on isatyde appears to be the type of its action on chlorisatyde, bromisatyde, bichlorisatyde, and bibromisatyde. When isatyde is acted on by potash, it yields isatine (or isatinate of potash); indine (or indinate of potash), and hydrindine (or hydrindinate of potash). 6 eqs. isatyde, $6\left(\mathrm{Cl}_{16}\right.$ $\left.\mathrm{H}_{6} \mathrm{NO}_{4}\right)$ are equal to 4 eqs. isatine, $4\left(\mathrm{C}_{16} \mathrm{H}_{5} \mathrm{~N} \mathrm{O}_{4}\right)+2$ eqs. indine, $2\left(\mathrm{C}_{16} \mathrm{H}_{6} \mathrm{NO}_{2}\right)+4$ eqs. water: or they are equal to 4 eqs. isatine, $4\left(\mathrm{C}_{16} \mathrm{H}_{5} \mathrm{~N} \mathrm{O}_{4}\right)+1$ eq. hydrindine, $\left(\mathrm{C}_{32} \mathrm{H}_{13} \mathrm{~N}_{2}\right.$ $\left.\mathrm{O}_{5}\right)+3$ eqs. water. Both changes probably occur, and the three compounds, isatine, indine, and hydrindine, alike take up the elements of water to form the acids, which, to avoid confusion, are not here expressed. Now there is good reason to believe that precisely analogous changes occur when potash acts on bichlorisatyde and on bibromisatyde, each yielding three corresponding compounds and the three acids derived from these. The reader, by strictly following the analogy of the formulæ given above for the action of potash on isatyde, will easily be able to construct the equations for the other analogous cases.

When sulphésatyde is acted on by bisulphite of ammonia; there is formed among other products not fully investigated, a salt formed of ammonia united to a new acid, sulphisatanous acid, quite different from the acid in the salts formed when isatine is 
acted on by sulphites. This new acid is $\mathrm{C}_{16} \mathrm{H}_{6} \mathrm{~N} \mathrm{O}_{3}, 2 \mathrm{SO}_{2}+$ $\mathrm{H} \mathrm{O}$, or perhaps rather $\mathrm{C}_{16} \mathrm{H}_{6} \mathrm{~N}\left\{\begin{array}{l}\mathrm{O}_{2} \\ 2 \mathrm{~S} \mathrm{O}_{2}\end{array}\right\}+\mathrm{H} \mathrm{O}$ : that is sulphésatyde, in which the 2 eqs. of sulphur have been replaced by 2 eqs. sulphurous acid.

The action of bisulphite of ammonia on sulphésatyde sometimes gives rise to the formation of different products : among others, to an insoluble white powder, isatan, $\mathrm{C}_{16} \mathrm{H}_{6} \mathrm{~N} \mathrm{O}_{3}$, which, when heated, yields isatine and indine; $3\left(\mathrm{C}_{6} \mathrm{H}_{6} \mathrm{~N} \mathrm{O}_{3}\right)=$ $\mathrm{C}_{16} \mathrm{H}_{5} \mathrm{~N} \mathrm{O}_{4}+2\left(\mathrm{C}_{16} \mathrm{H}_{6} \mathrm{~N} \mathrm{O}_{2}\right)+\mathrm{H} \mathrm{O}$. Both indine and nitrindine, when acted on by bisulphite of ammonia, appear to produce compounds analogous to those derived from sulphésatyde.

Chlorindopten is the name given by Erdmann to a volatile crystalline substance, formed along with chlorisatine and bichlorisatine, when chlorine acts on indigo. When the chlorinised mass is distilled with water, this substance passes over in white crystals, which are acid, and evidently a mixture of two substances. When this chlorindopten is heated with potash, a neutral substance passes over, in white crystals similar to the original ones; this is trichloraniline; while the potash retains an acid of a disagreeable odour, chlorophenisic acid.

Chlorophenisic acid, $\mathrm{C}_{12} \mathrm{H}_{2} \mathrm{Cl}_{3} \mathrm{O}, \mathrm{H} \mathrm{O}$, is separated from its potash salt by acids, as a white flocculent matter of a very disagreeable odour. Laurent has identified it with the acid derived from coal-tar by the action of chlorine, and makes its formula, $\mathrm{C}_{12}\left\{\begin{array}{l}\mathrm{H}_{2} \\ \mathrm{Cl}_{3}\end{array}\right\} \mathrm{O}, \mathrm{H} \mathrm{O}$, that is, trichlorocarbolic acid. The volatile white crystals are trichloraniline, $\mathrm{C}_{22}\left\{\begin{array}{l}\mathrm{H}_{4} \\ \mathrm{Cl}_{3}\end{array}\right\} \mathrm{N}$ (Hofmann).

By the further action of chlorine on chlorisatine or bichlorisatine, dissolved in alcohol, new compounds are formed, among which are chlorinised chlorindopten, which, like chlorindopten, is a mixture apparently of trichloraniline, with an acid, $\mathrm{Cl}_{2} \mathrm{Cl}_{5} \mathrm{O}$, $\mathrm{H} \mathrm{O}$; which is the chlorophenusic acid of Laurent. This acid is accompanied by chloranile, $\mathrm{Cl}_{12} \mathrm{Cl}_{4} \mathrm{O}_{4}$, a neutral body in volatile golden-yellow scales, soluble in hot alcohol, which is also derived from the oil of coal-tar, or rather from the hydrate of phenyle or carbolic acid of that oil, from which chlorophenisic and chlorophenusic acids are obtained.

Chloranile dissolves in weak potash with a deep purple colour, and the solution deposits dark purplish-red crystals, composed of potash and a new acid, chloranilic acid. This acid forms scarlet or yellow crystals, according as it contains water of crystallisation or not. Its formula is $\mathrm{C}_{22} \mathrm{Cl}_{2} \mathrm{O}_{6}, 2 \mathrm{H} \mathrm{O}$, or $\mathrm{C}_{6} \mathrm{Cl} \mathrm{O}_{3}, \mathrm{H} \mathrm{O}$. 
When chloranile is acted on by aqua ammoniæ, it is dissolved, forming a blood-red solution, which deposits chesnut-brown crystals of chloranilammon, $\mathrm{C}_{6} \mathrm{H}_{3} \mathrm{~N} \mathrm{Cl} \mathrm{O}_{3}+4$ aq. $=\mathrm{C}_{6} \mathrm{Cl} \mathrm{O}_{3}+$ $\mathrm{NH}_{3}+4$ aq. It dissolves in water with a purple colour, and when a saturated solution is mixed with hydrochloric acid, it deposits very brilliant black needles of great length. These are a new compound, chloranilam, $\mathrm{Cl}_{12} \mathrm{Cl}_{2} \mathrm{H}_{3} \mathrm{~N} \mathrm{O}_{6}=\mathrm{C}_{12} \mathrm{Cl}_{2} \mathrm{O}_{6}+$ $\mathrm{N} \mathrm{H}_{3}$; that is, 2 eqs. of chloranilammon, $2\left(\mathrm{C}_{6} \mathrm{Cl} \mathrm{O}_{3}, \mathrm{~N} \mathrm{H}_{3}\right)=$ $\mathrm{C}_{12} \mathrm{Cl}_{2} \mathrm{O}_{6}, \mathrm{~N}_{2} \mathrm{H}_{6}$; minus 1 eq. ammonia, $\mathrm{N} \mathrm{H}_{3}$. Both these compounds give precipitates with metallio solutions, which are the same from both, but distinct from those formed by chloranilic acid or its salts. Chloranilammon, according to Laurent, is the ammonia salt of an acid amide. Chloranilam is the acid amide itself.

By the action of ammonia on isatine there are produced several new compounds, varying with the strength of the ammonia, and the menstruum employed. In these compounds oxygen is replaced by amide, $\mathrm{N} \mathrm{H}_{2}$, or imide, which is here $\frac{1}{2} \mathrm{~N} \mathrm{H}=\mathrm{Im}$.

Imesatine is formed when dry ammonia is passed through an alcoholic solution of isatine. It forms fine deep yellow crystals, the formula of which is $\mathrm{C}_{16} \mathrm{H}_{6} \mathrm{~N}_{2} \mathrm{O}_{2}=\mathrm{C}_{16} \mathrm{H}_{5} \mathrm{~N}\left\{\begin{array}{l}\mathrm{O}_{2} \\ \mathrm{Im}_{2}\end{array}\right.$.

Imasatine is formed when aqua ammoniæ acts on a solution of isatine in alcohol. It is a greyish-yellow crystalline substance, the formula of which is $\mathrm{C}_{16} \mathrm{H}_{5} \mathrm{~N}\left\{\begin{array}{l}\mathrm{O}_{3} \\ \mathrm{Im}^{2}\end{array}\right.$ ?

Imasatinic acid is formed along with the preceding, and is dissolved along with ammonia. By the addition of an acid it is precipitated as a beautiful scarlet erystalline powder, soluble in hot alcohol, which deposits it in splendid tabular crystals similar to the sublimed periodide of mercury. It dissolves sparingly in acids with a violet colour, and these solutions deposit violet crystals. Its formula is $\mathrm{C}_{16} \mathrm{H}_{5} \mathrm{~N}\left\{\begin{array}{l}\mathrm{O}_{3} \\ \mathrm{Im}\end{array}\right\}+\mathrm{HO}$.

Amasatine is formed along with the two preceding bodies. It has a fine yellow colour, and dissolves in acids with a violet colour, apparently passing into imasatinic acid. Its formula is $\mathrm{C}_{16} \mathrm{H}_{5} \mathrm{~N}$ $\left\{\begin{array}{l}\mathrm{O}_{3} \\ \mathrm{Ad}\end{array}\right\}+\mathrm{H} \mathrm{O}\left(\mathrm{Ad}=\mathrm{N} \mathrm{H_{2 }}=\right.$ amide$)$.

The analogy between chlorisatine, \&c., and isatine, holds in regard to the action of ammonia on them. By the action of dry ammonia on an alcoholic solution of chlorisatine there is formed a yellow crystalline compound, analogous to imesatine. It is called Imechlorisatinase, and its formula is $\mathrm{C}_{16}\left\{\begin{array}{l}\mathrm{H}_{4} \\ \mathrm{Cl}\end{array}\right\} \mathrm{N}\left\{\begin{array}{l}\mathrm{O}_{2} \\ \mathrm{Im} \text {. }\end{array}\right.$ 
Imachlorisatinase is analogous to imasatine. Its formula is $\mathrm{Cr}_{16}$ $\left\{\begin{array}{l}\mathrm{H}_{4} \\ \mathrm{Cl}\end{array}\right\} \mathrm{N}\left\{\begin{array}{l}\mathrm{O}_{3} \\ \mathrm{Im}:\end{array}\right.$ It forms brownish-yellow crystals.

Imabromisatinese, formed by the action of dry ammonia on

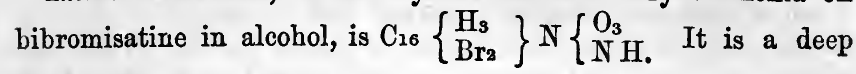
orange crystalline powder.

We have now briefly run over the catalogue of the very remarkable compounds derived from indigo by the action of sulphuric acid and bichromate of potash, which produces isatine, and by the action of chlorine either on indigo or on isatine, of bromine on the same, and of potash, ammonia, sulphuretted hydrogen and sulphuret of ammonium on the products of these actions. The nomenclature of these compounds is in a very imperfect state, and requires reformation, but this cannot be effected until the substances themselves have been more thoroughly studied. It is most important to observe, that by or through chloranile and the chlorophenisic acids, the series to which indigo belongs connects itself with that of carbolio acid or hydrate of phenyle (to be afterwards described), and this again with the series of salicyle. We have now to mention one or two products of the action of nitric acid on indigo, which are common to all these series, and, like chloranile, seem likely to be very frequently met with as products of the decomposition of organic compounds.

Anilic Acid. Srn. Indigotic Acid, Nitrosalicylic Acid, $\mathrm{C}_{14} \mathrm{H}_{4}$ $\mathrm{N} \mathrm{O}_{9}, \mathrm{H} \mathrm{O}$.- This acid is formed by the long-continued action of weak nitric acid on indigo. It is also formed in the preparation of isatine, if the action be pushed too far. It is identical with nitrosalicylic acid, obtained by the action of nitric acid on salicylic acid or on salicine. It forms fine yellowish-white prisms, which are light and bulky, and shrink much in drying. It is fusible and volatile. By the action of strong nitric acid it is converted into oxalic and picric acids. It requires 1000 parts of cold water for solution. Its salts crystallise well, and their general formula is $\mathrm{C}_{14} \mathrm{H}_{4} \mathrm{~N} \mathrm{O}, \mathrm{MO}$. The anilate of oxide of methyle is obtained as a crystalline compound by the moderated action of nitric acid on the salicylate of oxide of methyle (oil of Gaultheria). The anilate of oxide of ethyle is exactly similar.

Picric Acid, Syn. Carbazotic Acid, Nitropicric Acid, Nitrophenisic Acid, $\mathrm{C}_{22}\left\{\begin{array}{ll}\mathrm{H}_{2} & \\ 3 \mathrm{~N} & \mathrm{O}_{4}\end{array}\right\} \mathrm{O}, \mathrm{H} \mathrm{O}$. This acid is formed by the action of nitric acid on anilic acid, indigo, salicine, salicylic acid, hydrate of phenyle or carbolio acid, coumarine, silk, aloes, and other substances. It is most easily formed from carbolic 
acid, salicine, or oil of Gaultheria, by the action of an excess of fuming nitric acid assisted by heat. It is purified by solution in hot water and recrystallisation. It forms pale yellow, or even white scales of a silvery lustre. They dissolve in hot water with a strong yellow colour, and a very bitter taste. The acid is fusible and volatile. Its salts crystallise most readily, and all explode when heated. When these salts are put in contact with lime and green vitriol, blood-red solutions are formed, containing the lime salt of a new acid. The picrate of potash is so sparingly soluble, especially in alcohol, that an alcoholic solution of picric acid may be used as a test for potash.

Picric acid is interesting as occurring among the products of the decomposition by nitric acid of so many different substances. It is easily derived from the series of phenyle, that is, from carbolic acid, $\mathrm{C}_{12} \mathrm{H}_{5} \mathrm{O}$, H 0 . Derived from this, we have Chlorophenesic acid, $\mathrm{C}_{12}\left\{\begin{array}{l}\mathrm{H}_{3} \\ \mathrm{Cl}_{2}\end{array}\right\} \mathrm{O}, \mathrm{H}$ O, Chlorophenisic acid, $\mathrm{C}_{12}\left\{\begin{array}{l}\mathrm{H}_{2} \\ \mathrm{Cl}_{3}\end{array}\right\} \mathrm{O}, \mathrm{HO}$, Chlorophenusic acid, $\mathrm{C}_{12} \mathrm{Cl}_{5} \mathrm{O}, \mathrm{H} \mathrm{O}$, and Picrio acid, $\mathrm{C}_{12}\left\{\begin{array}{l}\mathrm{H}_{2} \\ 3 \mathrm{~N} \mathrm{O}_{4}\end{array}\right\} \mathrm{O}, \mathrm{H} \mathrm{O}$. It is, therefore, carbolic acid, in which 3 eqs. of hydrogen are replaced by 3 eqs. of nitrous acid.

When indigo is heated with concentrated potash, there are formed two new acids: chrysanilic acid, the composition of which is uncertain, and anthranilic acid, $\mathrm{C}_{14} \mathrm{H}_{7} \mathrm{~N} \mathrm{O}_{4}=\mathrm{C}_{14}$ $\mathrm{H}_{6} \mathrm{~N} \mathrm{O}_{3}, \mathrm{H} \mathrm{O}$. The latter is purified in the form of anthranilate of potash, and the acid separated by an excess of acetic acid. It forms transparent yellow scales, which, however, when quite pure, are colourless. It is derived from blue indigo, $\mathrm{C}_{16} \mathrm{H}_{5}$ $\mathrm{N}_{2}$, by the loss of 2 eqs. carbon and the addition of 2 eqs. water.

When anthranilic acid is mixed with powdered glass, and rapidly heated, it is resolved into carbonic acid, and an oily liquid, which is aniline, a very powerful base, devoid of oxygen, $\mathrm{C}_{12} \mathrm{H}_{7} \mathrm{~N}$. This metamorphosis is very simple: $\mathrm{C}_{14} \mathrm{H}_{7} \mathrm{~N} \mathrm{O}_{4}=$ $2 \mathrm{C} \mathrm{O}_{2}+\mathrm{C}_{12} \mathrm{H}_{7} \mathrm{~N}$. Since aniline is obtained in many other cases of decomposition of organic matters by heat, it becomes a subject of great interest.

Aniline is recognised by its property of striking a deep violet blue colour with chloride of lime. It obtained the name kyanol from this property. Its other name, crystalline, indicates its great tendency to form crystallisable salts with acids.

The recent researches of $\mathrm{A}$. W. Hofmann have greatly extended our knowledge of this remarkable compound. He has shown that it is best obtained by heating isatine with potash, thus $\mathrm{C}_{16} \mathrm{~N} \mathrm{H}_{5} \mathrm{O}_{4}+4(\mathrm{~K} \mathrm{O}, \mathrm{H} \mathrm{O})=\mathrm{C}_{22} \mathrm{H}_{7} \mathrm{~N}+4\left(\mathrm{~K} \mathrm{O}, \mathrm{C} \mathrm{O}_{2}\right)+\mathrm{H}_{2}$. 
But he has also proved that its formation is not confined to the products of the decomposition of indigo, it being formed when other substances, isomeric with anthranilic acid, are exposed to heat along with bases, such as lime or baryta. Such substances are salicylamide and nitrotoluole, both of which have the empirical formula, $\mathrm{C}_{4} \mathrm{H}_{7} \mathrm{~N} \mathrm{O}_{4}$. The former yields little, but the latter is entirely resolved into aniline and carbonic acid. $\mathrm{He}$ has also fully identified aniline with the crystalline of Unverdorben, a base occurring with others among the products of distillation of animal matter, and in coal-tar. It is worthy of remark that a close connection may be traced between aniline and carbolic acid (hydrate of phenyle). The latter is $\mathrm{C}_{12} \mathrm{H}_{5} \mathrm{O}, \mathrm{H} \mathrm{O}$. The carbolate of ammonia, $\mathrm{C}_{22} \mathrm{H}_{5} \mathrm{O}, \mathrm{N} \mathrm{H}_{4} \mathrm{O}$, minus 2 eqs. water would yield an amide, phenylamide, which would be $\mathrm{C}_{22} \mathrm{H}_{5} \mathrm{~N} \mathrm{H}_{2}=$ $\mathrm{C}_{12} \mathrm{H}_{7} \mathrm{~N}$; and this is aniline. Now in Hofmann's experiment above mentioned, in which salicylamide was heated with lime, it did not yield much aniline, but, on the other hand, it furnished a large quantity of carbolic acid. But perhaps the simplest view is that which regards aniline as an amide base, that is, ammonia, in which 1 eq. of hydrogen is replaced by phenyle, $\mathrm{C}_{22} \mathrm{H}_{5}$. Ammonia is $\mathrm{NH}_{2}+\mathrm{H}$, and aniline or phenylamine is $\mathrm{N}_{2}+$ $\mathrm{C}_{22} \mathrm{H}_{5}$. It is therefore analogous to ethylamine, \&c., but belongs to another series, and like ethylamine, it has several homologues.

It is further to be noticed that carbolic acid (hydrate of phenyle) and aniline (phenylamine) occur together in coal-tar; and that all the substances which either yield one or other of these are also converted into picric acid, $\mathrm{C}_{12}\left\{\begin{array}{l}\mathrm{H}_{2} \\ 3 \mathrm{~N} \mathrm{O}_{4}\end{array}\right\} \mathrm{O}, \mathrm{H} \mathrm{O}$, by the action of nitric acid. Thus carbolic acid, indigo, salicine, and salicylic acid, are all transformed into picric acid by excess of nitric acid. The compounds of phenyle, and many of its derivatives, will be described among the products of destructive distillation.

Hofmann has shown that isatine, when distilled with potash, yields aniline; and that chlorisatine, so analogous in all respects to isatine, undergoes a similar decomposition, yielding a new base, chloraniline; also that other compounds may be formed containing more chlorine, but still belonging to the same series; that bromisatine also yields a base, bromaniline; finally, that by the action of ammonia and sulphuretted hydrogen on binitrobenzide or dinitrobenzole, there is formed a base, nitraniline, in which 1 eq. of hydrogen is replaced by 1 eq. of nitrous acid, and that in this base 2 eqs. of hydrogen may further be replaced by bromine, yielding a nentral body. The following are the formulæ of these 
new compounds, which, it will be observed, all correspond with aniline, and are simply derived from it by substitution.

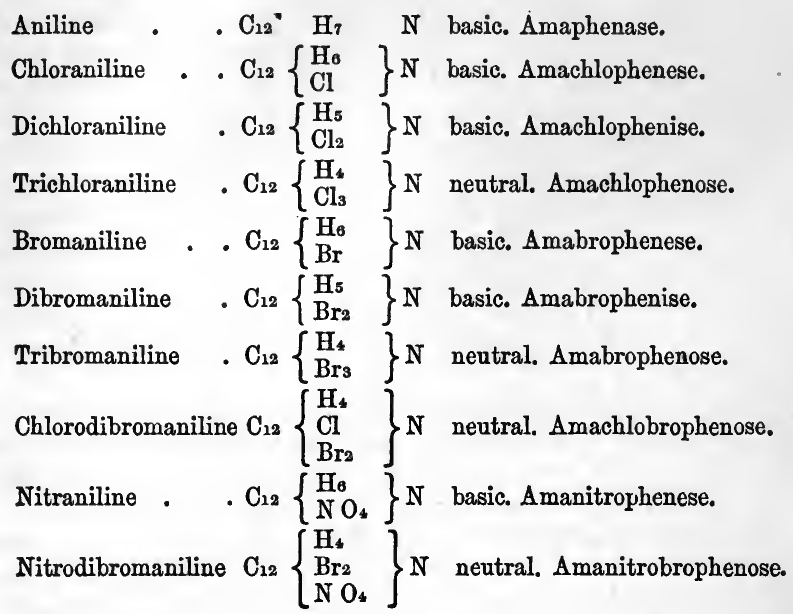

Our space will not permit us to enter into any details concerning the preparation and properties of these interesting compounds; but it may be remarked that chloraniline by the action of hydrochloric acid and chlorate of potash yields chloranile; and that the same substance, when passed over lime (hydrated?) at a low red heat, yields aniline, thus affording additional proof that all these compounds belong to one series, and are different subtypes of one general type. Nor must it be forgotten, that in the case of aniline, chloraniline, and bromaniline, we have chlorine and bromine substituted for hydrogen in a basic compound, without affecting its basic characters. This was the first known example, already followed by a multitude of others, of a base formed by substitution from another base, although similar facts, in regard to acids and neutral bodies, had long been known.

It is very well worthy of remark that a certain amount of chlorine or bromine, usually so antagonistio to hydrogen, does not, when substituted for that element, destroy the basic character; but that the chlorine and bromine appear to assume the function of hydrogen. A large proportion, however, of these elements does efface the basic character.

But the existence of nitraniline is even more remarkable. In 
this body 1 eq. of hydrogen in aniline has been replaced by 1 eq. of a powerful acid, nitrous acid, and yet the compound is decidedly basic, and retains the type of aniline.

Nitraniline forms fine golden yellow prisms, and with acids yields crystallisable salts. When acted on by bromine, it yields nitrodibromaniline, a neutral compound in which 2 more eqs. of hydrogen are removed and replaced by bromine.

We have here considered aniline chiefly in reference to its derivation from indigo and isatine; and the other derived bases of the same type, as also connected with indigo through chlorisatine, \&c. Under the head of organic bases, a few pages further on, we shall describe more particularly the properties of aniline, and some others of its derivatives, and others of them will fall to be noticed under the products of destructive distillation.

Carmine. This name has been given to the colouring matter of cochineal, which is nitrogenised, and may be obtained in dark red crystalline grains, very soluble in water and alcohol. It forms with alumina a beautiful red lake, well known as carmine.

The colouring matter of cochineal has been studied with great care by Warren de la Rue. It is extracted by boiling water precipitated by acidulated acetate of lead, the precipitate well washed, decomposed by sulphuretted hydrogen, and the red solution thus obtained again treated with lead and sulphuretted hydrogen. It is now dried up at a heat not exceeding $94^{\circ} \mathrm{F}$., and $\frac{7}{8}$ of the dry mass dissolved in boiling absolute alcohol. The remaining $\frac{1}{8}$ is dissolved in water, precipitated by acetate of lead, and the precipitate added to the alcoholic solution. By this means, the last traces of phosphoric acid are separated as phosphate of lead. Ether is now added to the alcoholio solution, which throws down some azotised matter, with a little of the colouring substance. This precipitate is re-dissolved in alcohol, and precipitated a second time by ether, when the oolouring matter first carried down remains dissolved. This solution is added to the other, the whole distilled, and the residue dried in vacuo. This is the pure colouring matter, or carminic acid. It is a friable mass of a dark purple brown, giving a fine red powder, soluble in water and alcohol, but hardly in pure ether. It dissolves also in acids. Its formula is $\mathrm{C}_{28} \mathrm{H}_{14} \mathrm{O}_{16}$. Its solution is precipitated by alkalies, and also by alum if a little ammonia be added, yielding fine lakes.

Nitric acid of Sp. Gr. 1.4, converts it into oxalio acid and nitrococcusic acid, which is $\left.\mathrm{C}_{16} \mathrm{H}_{6} \mathrm{~N} \mathrm{O}_{4}\right\} \mathrm{O}_{6}+2$ aq., apparently derived from an acid $\mathrm{C}_{16} \mathrm{H}_{8} \mathrm{O}_{6}$ by replacement of 3 eq. of hydrogen by 3 eq. of $\mathrm{NO}_{4}$. It forms yellow tables, soluble in water, and 
colouring the skin yellow. Its salts are also yellow, and detonate when heated. The silver salt is soluble in water.

It will be remembered that under anisic acid, $\mathrm{C}_{16} \mathrm{H}_{8} \mathrm{O}_{6}$, we mentioned two acids, nitranisic and dinitranisic acid, in which 1 and 2 eq. of hydrogen were respectively replaced by 1 and 2 eq. of $\mathrm{NO}_{4}$. The acid just described has the formula of trinitranisic acid, and there is nothing in its properties inconsistent with the idea that it may be that substanoe, which apparently is not formed by the action of nitric acid on oil of anise, or oil of estragon.

The finest crimson and scarlet dyes are made from cochineal, according as the mordant is alumina or tin.

Kermes is a dye-stuff similar in its origin and properties to cochineal, but inferior in beauty of tint. It is derived from Coccus ilicis.

The red colouring matter of stick-lac, which is also used as a dye, has similar properties. Lac is produced on certain plants by the puncture of an insect.

\section{ACTION OF NITRIC ACID ON ALOES.}

This action so much resembles, in some points, the action of nitric acid on indigo, that it may be properly mentioned here. Aloes is the well-known inspissated juice of certain species of $a l o e$, and is very bitter and purgative. The nature of its active principle is still unknown: but when heated with nitrio acid it yields a yellow bitter substance, which is converted, by the further action of the acid, into two crystallisable acids, chrysammic and chrysolepic acid. The artificial bitter of aloes appears to be formed of two acids, aloetic and aloeretinic acids, which form red salts, but the composition of which is not exactly ascertained.

Chrysammic Acid, $\mathrm{C}_{15} \mathrm{H} \mathrm{N}_{2} \mathrm{O}_{12}, \mathrm{H} \mathrm{O}$, is obtained as a yellow precipitate, when water is added to the solution obtained by heating aloes with excess of nitric acid. It is purified by being combined with potash, and this salt, after recrystallisation, is dissolved in hot water and decomposed by diluted nitric acid. The chrysammic acid is deposited as a powder formed of goldenyellow shining scales. Its solution is of a fine purple. All its salts are crystallisable and of a deep red colour, frequently with green reflection, like murexide. The chrysammate of ammonia forms dark green crystals, which, when dissolved and acted on by nitrio acid, deposit brilliant black scales, which are not chrysammic acid, but are transformed into it when boiled with acids or bases. The solution of chrysammate 
of ammonia gives with metallic salts peculiar precipitates distinct from those formed with the same salts by chrysammate of potash.

Chrysolepic Acid, $\mathrm{C}_{12} \mathrm{H}_{2} \mathrm{~N}_{3} \mathrm{O}_{13}, \mathrm{H} \mathrm{O}$, has the formula and many of the properties of picric acid; but it is said by Schunck, who discovered it, to be different. It is darker in colour, and its salt with potash is much more soluble than picrate of potash. It would, however, appear that the two acids are essentially the same; for the salts are in general very similar, and all explode when heated. Now that picric acid is recognised as so frequent a product of the action of nitric acid, we have no difficulty in understanding its occurrence here.

\section{ALKALOIDS OR ORGANIC BASES.}

These names are given to a class of nitrogenised organic compounds which, in their relations, are quite analogous to ammonia, or to oxide of ammonium. They are to be distinguished from such basic oxides as oxide of ethyle, oxide of methyle, \&c., which contain no nitrogen, and, although they form neutral compounds with acids, yet exist in a peculiar state in these compounds, which cannot be decomposed, like ordinary salts, by double decomposition. Thus, oxalate of oxide of ethyle does not precipitate with nitrate of lime, and chloride of ethyle does not decompose nitrate of silver. But the case is quite different with the alkaloids; for their salts undergo the same decompositions as those of ammonia.

Most of the alkaloids are found in vegetable juices, seeds, or roots; these are called vegetable alkalies, and they are generally the active principles of the plants, for the most part poisonous, in which they are found. But of late, organic bases quite analogous to those produced by nature, have been formed in a variety of processes; as, for example, the singular bases containing platinum, described at pp. 55 to 60 ; the bases containing arsenic, or arsenic and platinum, mentioned at p. 193; the bases of coal-tar, of which aniline, formed in several different processes, is one; the bases, chloraniline, \&c., derived from aniline; the bases derived from oil of mustard, (see p. 343); those derived from the decomposition of natural alkaloids, as chinoline and cotarnine; those formed by the action of potash on melam, as melamine and ammeline; those produced by the action of ammonia on oil of bitter almonds and analogous bodies, such as amarine, lophine, picrine, and furfurine; those formed by the action of sulphuret of ammonium on certain nitrogenised bodies, as aniline from nitrobenzole, naphthalidine from nitro- 
naphthalese, thialdine from aldehydammonia, \&c.; those derived from kreatine by the action of acids and bases, namely, kreatinine and sarcosine; and such as are formed by the action of acids and bases on other animal compounds, of which glycocine is an example, being formed from gelatine by the action of potash, and from hippuric acid by the action of hydrochloric acid. The number of artificial bases has of late been very greatly augmented by the discoveries of Wurtz and of Hofmann, who have shown that an almost unlimited number of such bases, homologous with ammonia, may be obtained. Some of these have been already mentioned under the benzoic and ethylic series of compounds, and many more remain to be noticed. Most of these artificially formed bases are of very recent discovery, and it is evident that they must throw much light on the theory of the production of the natural alkaloids, and that the careful study of this part of the subject will, in all probability, eventually lead to the artificial formation of the natural organic bases.

The alkaloids possess, for the most part, very decided basic properties; when dissolved they act on vegetable colours like the inorganic alkalies; and they neutralise the strongest acids completely, generally forming crystallisable salts. Most of them, at the ordinary temperature, are expelled from their salts by ammonia, but many of them, at the heat of boiling water, expel ammonia from its salts, owing to the volatility of the latter alkali.

Their basic properties are not derived from the oxygen they contain, for no variation in the amount of that element affects their neutralising power. On the other hand, there is every reason to believe that their basic character depends on the nitrogen they contain; for they all, without exception, contain nitrogen, though several are devoid of oxygen. Moreover, most, if not all, of those which have been formed artificially, are prepared with the aid of ammonia, or some compound of ammonia, or amidogen. Before proceeding to describe them, we shall premise some general observations on the formation and constitution of artificial organic bases.

\section{ON THE FORMATION AND CONSTITUTION OF ARTIFICIAL ORGANIC BASES.}

Within the last few years, this is the department of organic chemistry which has made the greatest progress, and has yielded the most surprising results. Several theories had been started as to the constitution of the natural alkaloids, and Berzelius warmly advocated one of these, namely, that the alkaloids were 
coupled compounds, in which ammonia was coupled with other compounds. Taking aniline, $\mathrm{C}_{12} \mathrm{H}_{7} \mathrm{~N}$, as an example (for aniline, although an artificial base, is closely analogous to nicotine and coneine, which are natural bases), Berzelius conceived aniline to be ammonia coupled with $\mathrm{C}_{12} \mathrm{H}_{4}$; thus $\left(\mathrm{C}_{12} \mathrm{H}_{4}\right)+$ $\mathrm{N} \mathrm{H}_{3}=\mathrm{C}_{12} \mathrm{H}_{7} \mathrm{~N}$. $\mathrm{He}$ supposed its neutralising power to depend on its ammonia just as, in a coupled acid, the attached copula has no influence on the neutralising power. As all bases contained nitrogen and hydrogen, this view was possible, although none of them gave any signs of containing ammonia ready formed. On this and on other accounts, Liebig objected to this view, and proposed another, namely, that the organic bases were bodies in which amide, $\mathrm{N} \mathrm{H}_{2}$, was combined with some compound, replacing the third equivalent of hydrogen in ammonia. To this he was led by considering ammonia as the hyduret of amidogen or amide; as $\mathrm{NH}_{2}+\mathrm{H}=\mathrm{Ad} \mathrm{H}=\mathrm{N} \mathrm{H}_{3}$. Hence he viewed aniline as amide plus phenyle, $\mathrm{C}_{12} \mathrm{H}_{5} . \mathrm{N} \mathrm{H}_{2}+\mathrm{C}_{12} \mathrm{H}_{5}=\mathrm{Ad} \mathrm{Ph}=\mathrm{C}_{12}$ $\mathrm{H}_{7} \mathrm{~N}$. And in a memoir published in 1839 , he ventured to predict, that means would be found of substituting other compound radicals for the third atom of hydrogen in ammonia. Nay, he went so far as to say, that if ever the radicals, ethyle and methyle, should be thus substituted for hydrogen, the resulting compounds would be, without the least doubt, bodies perfectly analogous in properties to ammonia; that is, powerful volatile bases, but less volatile than ammonia. A more remarkable prediction was never ventured in chemistry, and it has been absolutely fulfilled; for, in 1849, Wurtz obtained the predicted compounds, methylamine, $\mathrm{C}_{2} \mathrm{H}_{5} \mathrm{~N}=\mathrm{Ad} \mathrm{Me}$, and ethylamine, $\mathrm{C}_{4}$ $\mathrm{H}_{7} \mathrm{~N}=\mathrm{Ad} \mathrm{Ae}$, and found them to be precisely what Liebig had anticipated ten years before; indeed, so like ammonia, that it is now certain that these bases had frequently occurred, and been taken for ammonia, from the resemblance of their smell to that of ammonia. This remarkable discovery of Wurtz has led to the further discovery, both by himself, by Hofmann, and others, of a vast number of similar bases. Besides this, in the interval between the prediction and its fulfilment, many others had been discovered, chiefly by Hofmann, all of which tended to establish Liebig's views. Such were the substitution bases from aniline (see p. 376), the bases homologous with aniline, toluidine, cumidine, \&c., and others, so that the theory of Liebig became at last firmly established. But the march of discovery did not stop here, for Hofmann soon ascertained that it applied only to one class, a very large class of artificial bases, and that bases could be formed, in which no amide was or could be present. In short, it appeared that not only the third eq. of hydrogen in am- 
monia, but with it one or both of the others, might be replaced by very many radicals, without destroying the basic character. Hofmann thus established the existence of three distinct series of bases, all derived from ammonia by substitution, and all analogous to it in properties, being volatile bases. If we make $X, Y$, and $Z$ any three compound radicals, whether of the same or of different series, then we have

1. Amide bases . $\mathrm{NH}_{2}+\mathrm{R}$ ( $\mathrm{R}$ being either hydrogen or a compound

Ammonia . . $\mathrm{NH}_{2}+\mathrm{H}$ radical.)

New bases . . $\mathrm{NH}_{2}+\mathrm{X}$, or $\mathrm{NH}_{2}+\mathrm{Y}$, or $\mathrm{NH}_{2}+\mathrm{Z}$

2. Imide bases . $\mathrm{NH}+\mathrm{R}_{2}(\mathrm{NH}=$ Imide)

Ammonia . . $\mathrm{NH}+\mathrm{H}_{2}$

New bases . . N H $+\mathrm{XY}$, or $\mathrm{NH}+\mathrm{YZ}$, or $\mathrm{NH}+\mathrm{XZ}$

3. Nitryle bases . $\mathrm{N}+\mathrm{R}_{3}$

Ammonia . $\mathrm{N}+\mathrm{H}_{3}$

New bases . . N + XYZ, or N + XXZ, or N + YYZ; or N $+\mathrm{XZZ}$, or $\mathrm{N}+\mathrm{YZZ}$; or $\mathrm{N}+\mathrm{YXX}$; or- $-\mathrm{N}+\mathrm{Y}$ Y X.

Here is at once a wide extension of the original view of Liebig as to the constitution of organic bases; and, whatever be their real constitution, bases are now known, belonging to each of the above series: that is, bases, the formula of which may be thus interpreted, and which are really obtained from compounds believed to contain the radicals supposed to be present in the bases.

Of the amide bases, many are known. The following are a few :-

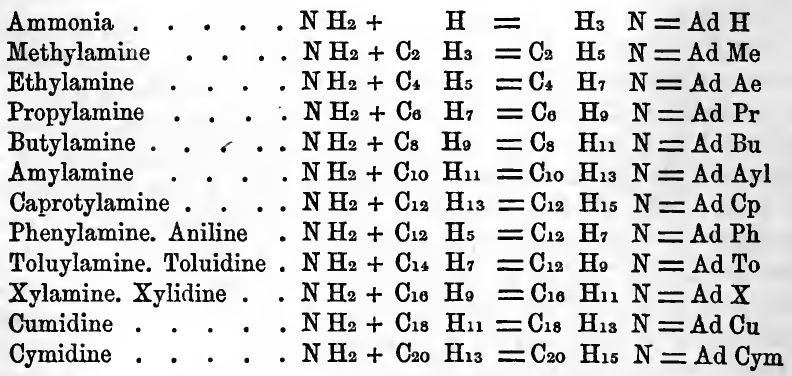

All these are known, and many more will soon be formed, especially in the ethyle series. Indeed, Fridau has obtained the compound, eetylamine, $\mathrm{C}_{32} \mathrm{H}_{35} \mathrm{~N}=\mathrm{N} \mathrm{H}_{2}+\mathrm{C}_{34} \mathrm{H}_{33}=\mathrm{Ad} \mathrm{Ctl}$, although it has no basic characters, or very feeble ones. 
The following are the names of a few imide bases :-

Ammonia . $\mathrm{NH}+\mathrm{H}_{2}$

Dimethylamine . $\mathrm{NH}+2\left(\mathrm{C}_{2} \mathrm{H}_{3}\right)$

$=\mathrm{H}_{3} \mathrm{~N}=\mathrm{Id}, \mathrm{H}_{2}$

Diethylamine. . $\mathrm{NH}+2\left(\mathrm{C}_{4} \mathrm{H}_{5}\right)$

$=\mathrm{C}_{4} \mathrm{H}_{7} \quad \mathrm{~N}=\mathrm{Id}, \mathrm{Me}_{2}$

Diamylamine .

. $\mathrm{NH}+2\left(\mathrm{C}_{10} \mathrm{H}_{11}\right)$

$=\mathrm{C}_{8} \mathrm{H}_{11} \mathrm{~N}=\mathrm{Id}, \mathrm{Ae}_{2}$

Diphenylamine

. $\mathrm{NH}+2\left(\mathrm{C}_{12} \mathrm{H}_{5}\right)$

$=\mathrm{C}_{20} \mathrm{H}_{23} \mathrm{~N}=\mathrm{Id}, \mathrm{Ayl}_{2}$

$=\mathrm{C}_{24} \mathrm{H}_{11} \mathrm{~N}=\mathrm{Id}, \mathrm{Ph}_{2}$

Methylophenylamine $\mathrm{NH}+\mathrm{C}_{2} \mathrm{H}_{3}+\mathrm{C}_{12} \mathrm{H}_{5}=\mathrm{C}_{14} \mathrm{H}_{9} \quad \mathrm{~N}=\mathrm{Id}, \mathrm{Me} \mathrm{Ph}$

Fthylophenylamine. $\mathrm{NH}+\mathrm{C}_{4} \mathrm{H}_{5}+\mathrm{C}_{12} \mathrm{H}_{5}=\mathrm{C}_{16} \mathrm{H}_{11} \mathrm{~N}=\mathrm{Id}, \mathrm{AePh}$

Amylophenylamine. $\mathrm{NH}+\mathrm{C}_{10} \mathrm{H}_{11}+\mathrm{C}_{12} \mathrm{H}_{5}=\mathrm{C}_{22} \mathrm{H}_{17} \mathrm{~N}=\mathrm{Id}$, Ayl Ph

All the above have been discovered and described by Hofmann. They are all volatile bases, analogous to ammonia.

Of nitryle bases the following will serve as an example :-

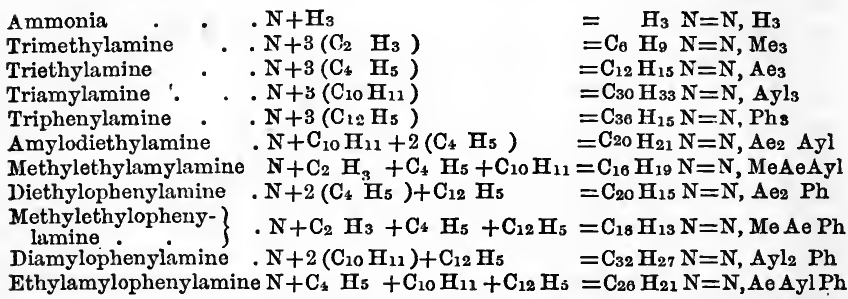

All of these, except triphenylamine, have been already obtained by Hofmann. It will be seen that the whole three series may be included under one head, namely, that of bases, corresponding to ammonia, in which one, two, or all three of the eqs. of hydrogen in ammonia have been replaced by one, two, or three different radicals; and that the amide, imide, and nitryle bases are the subdivisions of this group. Now, there are two strong reasons for believing such to be the constitution of these hodies; 1st, that they are all analogous in properties to ammonia, and 2ndly, that they are obtained by the action of ammonia on compounds of the radicals, methyle, ethyle, amyle, and phenyle; so that their formation and properties could be, and were predicted. It is obvious, therefore, that Liebig's view is only true of one of the subdivisions, the amide bases, and must be extended so as to include the two others.

But Hofmann has gone further still. He has discovered that, to the nitryle bases, such as triethylamine, $\mathrm{N}+3\left(\mathrm{C}_{4} \mathrm{H}_{5}\right)=$ $\mathrm{C}_{12} \mathrm{H}_{15} \mathrm{~N}$, another equivalent of ethyle, \&c., may be added, and a new series of bases obtained, corresponding, not to ammonia, but to oxide of ammonium, $\mathrm{N} \mathrm{H}_{4} \mathrm{O}$, and resembling, in their 
properties, oxide of potassium or potash, much more than ammonia, as they are not volatile without decomposition. Oxide of ammonium is the same thing as ammonia plus water; and if, as is probable, oxide of ammonium does exist in the salts of ammonia, such as sulphate of ammonia, $\mathrm{N} \mathrm{H}_{4} \mathrm{O}, \mathrm{S} \mathrm{O} \mathrm{O}_{3}$, it is resolved into ammonia and water, as soon as separated. Such is also the case with the metals belonging to the three first series of ammonia bases (for being analogous to ammonia, they form, with hydrogen, metals like ammonium). Thus, to take one example, triethylamine, with hydrochloric acid, forms chloride of triethylium, just like ammonia with the same acid.

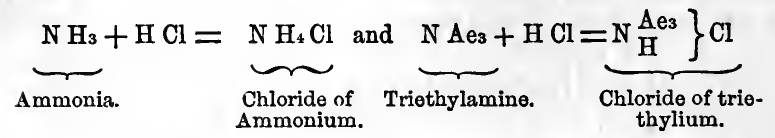

And in sulphate of triethylamine, we have the oxide of the metal, as in sulphate of ammonia.

$$
\underbrace{\mathrm{NH}_{4} \mathrm{O}, \mathrm{S} \mathrm{O}_{3}}_{\text {Sulphate of Oxide of Ammonium. }} \underbrace{\left.\mathrm{N} \begin{array}{l}
\mathrm{Ae} \\
\mathrm{H}
\end{array}\right\} \mathrm{O}, \mathrm{S} \mathrm{O}_{3}}_{\text {Sulphate of Oxide of triethylium. }}
$$

And this oxide, like that of ammonium, is resolved when separated into water and triethylamine.

But with the new or fourth series of bases it is different.

\begin{tabular}{|c|c|c|}
\hline $\begin{array}{l}\text { Ammonia } \\
\text { Ammonium : } \\
\text { Oxide of ditto } \\
\text { Chloride of ditto }\end{array}$ & $\begin{array}{l}\text { - } \mathrm{NH}_{3} \\
\text { - } \mathrm{NH}_{4} \\
\text { - } \mathrm{NH}_{4} \mathrm{O} \\
\text {. } \mathrm{NH}_{4} \mathrm{Cl}\end{array}$ & $\begin{array}{l}\text { Triethylamine } \\
\text { Tetrethylium } \\
\text { Oxide of ditto } \\
\text { Chloride of ditto }\end{array}$ \\
\hline
\end{tabular}
The oxide of the metal is not resolved into other products, when separated from the acid, but can exist in a separate form. We have, first,

While oxide of ammonium is instantly resolved into ammonia and water, oxide of tetrethylium is permanent, and much resembles potash.

Here, then, is a totally new series of bases representing, not ammonia, but oxide of ammonium, and therefore containing oxygen. If oxide of tetrethylium were as unstable as oxide of ammonium, it should yield triethylamine and ether, as oxide of ammonium does ammonia and water; thus: $\mathrm{N} \mathrm{H}_{4} \mathrm{O}=\mathrm{N} \mathrm{H}_{3}+$ $\mathrm{H} \mathrm{O}$. $\mathrm{N} \mathrm{Ae}_{4} \mathrm{O}=\mathrm{N} \mathrm{Ae}+\mathrm{Ae} 0$. Evidently, in the former case, the affinity of oxygen for hydrogen breaks up the oxide; but in the latter the affinity of oxygen for ethyle is not powerful enough to do so at the ordinary temperature. But where an atom 
of hydrogen is present in the same place as the fourth atom in ammonium, it is separated as water, as we have seen in triethylium, the oxide of which, like that of ammonium, cannot exist in the separate form. Thus,

$$
\left.\mathrm{NH}_{4} \mathrm{O}=\mathrm{NH} \mathrm{H}_{3}+\mathrm{HO} ; \mathrm{N}_{\mathrm{H}}^{\mathrm{Ae}_{3}}\right\} \mathrm{O}=\mathrm{NAe}_{3}+\mathrm{HO}
$$

These considerations explain how it happens that the oxides of the new organic metals, analogous to ammonium, are permanent. And it is worthy of notice, as a strong proof that these new compounds represent ammonium in which hydrogen has been entirely replaced by one, two, three, or even four different radicals, that it has not been found possible to replace by these radicals more hydrogen than 4 equivalents, evidently the four of ammonium, $\mathrm{N} \mathrm{H}_{4}$. Should any further replacement of hydrogen by ethyle, for example, occur, then either the group will be broken up, or the replacement will affect the radical itself; that is, if in tetrethylium we could replace an atom of hydrogen by ethyle, it would be an atom in one of the atoms of ethyle, which one might then become $\mathrm{C}_{4}\left\{\begin{array}{l}\mathrm{H}_{4} \\ \mathrm{Ae} .\end{array}\right.$ But this is not very likely. The following table gives the hydrated oxides of the bases already discovered in the ammonium series :-

Hydrated oxide of

\begin{tabular}{|c|c|c|c|}
\hline & & & \\
\hline Ammonium & & $\mathrm{N} \cdot \mathrm{H}_{4} \mathrm{O}, \mathrm{HO}$ & $=\mathrm{N} \mathrm{H}_{3}, 2 \mathrm{HO}$ \\
\hline Tetramethylium & & $\mathrm{NMe}_{4} \mathrm{O}, \mathrm{HO}$ & $=\mathrm{Cs} \mathrm{H}_{12} \mathrm{~N}, \mathrm{O}, \mathrm{HO}$ \\
\hline Tetrethylium & & $\mathrm{NAe}_{4} \mathrm{O}, \mathrm{H} \mathrm{O}$ & $=\mathrm{C}_{16} \mathrm{H}_{20} \mathrm{~N}, \mathrm{O}, \mathrm{HO}$ \\
\hline Methylotriethylium & & $\mathrm{N} \mathrm{Me} \mathrm{Aes} \mathrm{O}, \mathrm{H} O$ & $=\mathrm{C}_{14} \mathrm{H}_{18} \mathrm{~N}, \mathrm{O}, \mathrm{HO}$ \\
\hline Amylotriethylium & & 3 Ayl O, Н 0 & $=\mathrm{C}_{22} \mathrm{H}_{26}$ \\
\hline Tetramylium & - & 0 & $=\mathrm{C}_{40} \mathrm{H}_{4}$ \\
\hline Methylodiethylamylin & & $\mathrm{e}_{2} \mathrm{Ayl}$ & 10 \\
\hline friethylonylivim & & . $\mathrm{N} \mathrm{Ae}_{3} \mathrm{Ph} \mathrm{O}, \mathrm{HO}$ & $=\mathrm{C}_{24} \mathrm{H}_{20}$ \\
\hline 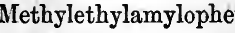 & & & \\
\hline
\end{tabular}

The general character of these bases is well marked. Like oxide of ammonium, and unlike the ammonia bases, they all have an even number of equivalents of hydrogen. The hydrates crystallise and are very soluble in water. They cannot be distilled without decomposition. Like potash, they attract carbonic acid from the air, have a caustic taste, and saponify oils. But they, and all their salts, are very bitter. Their iodides, chlorides, \&c., contain the compound metals in union with iodine, \&c., and resemble iodide of potassium. Thus hydriodio acid aots on the oxide of tetrethylium, yielding water and the iodide, just as it acts on oxide of potassium.

$\mathrm{KO}, \mathrm{HO}+\mathrm{HI}=\mathrm{KI}+2 \mathrm{HO}$; and $\mathrm{NMe}_{4} \mathrm{O}, \mathrm{HO},+\mathrm{HI}=\mathrm{NMe}_{4} \mathrm{I}+2 \mathrm{HO}$ 
Here, then, it will be seen, we have four large series of bases, evidently representing, three of them ammonia, the fourth, oxide of ammonium; and it is highly probable that many of the natural bases belong to these four series. As, however, the ammonia bases of all three series are volatile, so we can only at present refer to that class the volatile natural bases, such as coniine and nicotine. But the ammonium bases agree with the non-volatile natural bases in four points : first, in being fixed; second, in containing oxygen; third, in having a bitter taste; and fourth, in yielding volatile bases when decomposed by heat. Some of the formulx in the last column of the last table approach very nearly to those of natural fixed organic bases. In fact, an attempt was made by Hofmann to form artificially a natural base, quinine. The formula of quinine, according to the best analyses, is $\mathrm{C}_{20} \mathrm{H}_{12} \mathrm{~N} \mathrm{O}_{2}=\mathrm{C}_{20} \mathrm{H}_{11} \mathrm{~N} \mathrm{O}$, H O. When heated, it yields a volatile base, quinoline. Now this was supposed to be $\mathrm{C}_{28} \mathrm{H}_{8} \mathrm{~N}$, and quinine to differ from it by $\mathrm{C}_{2} \mathrm{H}_{4} \mathrm{O}_{2}$. And the hydrated oxide of tetramethylium, a bitter fixed base, $\mathrm{C}_{8} \mathrm{H}_{18}$ $\mathrm{N}_{2}=\mathrm{C}_{8} \mathrm{H}_{12} \mathrm{~N} \mathrm{O}, \mathrm{H} \mathrm{O}$, yields, when heated, a volatile base, trimethylamine, $\mathrm{C}_{6} \mathrm{H}_{8} \mathrm{~N}$; the difference being, as before, $\mathrm{C}_{2} \mathrm{H}_{4}$ $\mathrm{O}_{2}$. Now as trimethylamine may be reconverted into the hydrated oxide of tetramethylium by the action of iodide of methyle on it, and of oxide of silver on the iodide thus produced, the question was asked, may not quinoline, which is found in coal-tar, yield quinine in the same way? In point of fact, the action of iodide of methyle on quinoline does yield a crystallised iodide, apparently similar to the hydriodate of quinine, but as recent researohes show, not even isomeric with it; the true formula of quinoline being $\mathrm{C}_{28} \mathrm{H}_{7} \mathrm{~N}$. Still we may hope to succeed in process of time in forming artificially the natural bases. For further details concerning quinoline and its homologues see pp. 400, et seq.

But we must not suppose that Liebig's theory, even as extended by Hofmann, so as to include, besides amide bases, imide, nitryle and ammonium bases, includes all the possible modes of formation of organic bases. Some may be, as Berzelius imagined, coupled ammonias, some coupled amide, imide, nitryle, or ammonium bases, or substitution products derived from these. And it must be borne in mind, that even of these four series of bases we have only learned to know those containing the four radioals, methyle, ethyle, amyle, and phenyle, while there may be hundreds of similar radicals. The field has been opened; it remains for us to cultivate it. Of the bases mentioned above, many have been already described under the ethylic series of radicals, and the remainder will be noticed under aniline or phenylamine, further on.

Besides the methods employed for the production of the bases of c c 2 . 
the four classes above mentioned, chiefly the action of the bromides or iodides of methyle, ethyle, \&c., on ammonia, there are various other processes which yield artificial organic bases, which must here be briefly noticed. Bases, then, are formed:-

1. By the action of ammonia on organic products, aided by other processes. Thus ammonia forms bases, as we have seen, with oil of bitter almonds and furfurole, when aided by the subsequent action of potash: with oil of mustard; and probably in other instances.

2. In the destructive distillation of nitrogenised animal and vegetable matters, such as albuminous compounds, natural bases, indigo, and coal. In Dippel's animal oil are found bases of three, if not four, distinct series ; those of methylamine, \&c., of aniline, of picoline, isomeric with that of aniline, and the series of pyrrole bases (Anderson). Indigo yields aniline; morphine, narcotine, codeine, quinine, cinchonine, strychnine and pelosine, yield volatile bases when heated; and the oil of coal-tar contains various bases of the methylamine series, of that of aniline, and perhaps of others.

3. By the action of sulphuretted hydrogen on compounds of ammonia. Thialdine, a very remarkable base, is formed by the action of sulphuretted hydrogen on aldehydammonia.

4. By the action of sulphuretted hydrogen, or of sulphuret of ammonium on substitution compounds containing $\mathrm{N} \mathrm{O}_{4}$; as when aniline is formed in this way from nitrobenzole, nitraniline from dinitrobenzole, or toluidine from nitrotoluole.

5. By heating nitrogenised compounds with potash or baryta; as when aniline is formed by heating isatine with potash, chloraniline by heating chlorisatine with potash, melamine and ammeline by heating melam with potash, and sarcosine by heating kreatine with baryta.

6. By heating organic compounds with acids ; as when glycocine is formed by boiling hippuric acid with hydrochloric acid; kreatinine from kreatine; and alanine from a mixture of aldehydammonia, hydrocyanic acid, and hydrochloric acid.

7. Lastly, by spontaneous metamorphosis, as when hydrated cyanate of ammonia passes into urea, or cyanate of methylamine into methylo-urea, when their solutions are evaporated.

Of all of those methods, examples will be given under the individual bases. Of the production of the four classes of bases, amide, imide, nitryle, and ammonium bases, with the radicals of the ethyle series, numerous examples have already been given.

It is to be observed, that we have already succeeded in forming artificially three natural bases, all of animal origin, namely, urea, glycocine, and kreatinine. In other cases, as Hofmann's 
new base, methylo-quinoline, turns out not to be quinine, we have only, as yet, produced bases closely analogous to natural bases. Thus furfurine, an artificial base, is used, like quinine, as an anti-periodic: and the bitter ammonium bases of Hofmann will most probably be found powerful remedies, possibly of that class.

Lastly, in the formation of artificial bases, we have succeeded in several, indeed in many cases, in imitating the natural process of building up complex atoms or molecules from such as are less complex. This is seen in the long list of Hofmann's bases; and also in furfurine, produced by the coalescence of 2 eqs. of furfuramide; and amarine, formed by the coalescence of 3 eqs. of oil of bitter almonds along with ammonia. It has hitherto been found comparatively easy to break up or resolve into simpler forms the complex natural organic compounds; but now we begin, like nature, to construct the complex from the more simple: perhaps, in its ultimate results, the most important step yet made in advance in organic chemistry. Our methods are still coarse and violent compared with those of nature, which are gentle but irresistible, and infinitely varied in their results; but yet we are able to imitate nature, and to replace one group by another, and thus to form a series of artificial products, running parallel, in many instances, with the natural ones, and exhibiting precisely similar properties.

We shall now briefly describe the artificial bases, in so far as they have not hitherto been mentioned, and we shall group them according to their properties and the sources whence they are derived.

\section{(a.) Volatile oily artificial Alkaloids.}

Of this class of bases we have already described a large number, under the radicals, methyle, ethyle, \&c., which see. We now proceed to a base, already frequently mentioned as a product of many decompositions. This is aniline, which name we shall use for shortness.

Aniline, $\mathrm{C}_{22} \mathrm{H}_{7} \mathrm{~N}=\mathrm{N} \mathrm{H}_{2}, \mathrm{C}_{12} \mathrm{H}_{5}=\mathrm{Ad} \mathrm{Ph}$. Sxw. Phenylamine, Phenamine, Phenamide, Kyanole, Crystalline, Benzidam. This base, which is most properly called phenylamine or phenamine, has already been described as derived from indigo, anthranilic acid, and isatine, and as produced also by the action of bases aided by heat on nitrotoluole (protonitrobenzoene). It is further produced, along with other bodies, when nitrobenzole (nitrobenzide) is distilled with alcohol and potash, and when the same compound is acted on by sulphuret of ammonia. Lastly, it occurs in coal-tar, and in the oil obtained by the destructive distillation of animal matter. 
We shall give here, in one view, the equations which explain the production of aniline, as no other compound admits of being produced in so great a variety of ways, and all these equations are instructive.

$$
\text { 1. } \underbrace{\mathrm{C}_{14} \mathrm{NH}_{6} \mathrm{O}_{3}, \mathrm{HO}}_{\text {Anthrauilic acid }}=2 \mathrm{CO}_{2}+\underbrace{\mathrm{C}_{12} \mathrm{H}_{7} \mathrm{~N}}_{\text {Aniline. }}
$$

2. $\underbrace{\mathrm{C}_{16} \mathrm{~N} \mathrm{H}_{5} \mathrm{O}_{4}}+4(\mathrm{~K} \mathrm{O}, \mathrm{HO})=4\left(\mathrm{~K} \mathrm{O}, \mathrm{CO}_{2}\right)+\underbrace{\mathrm{C}_{12} \mathrm{H}_{7} \mathrm{~N}}+\mathrm{H}_{2}$ Isatine.

3. $\underbrace{\mathrm{C}_{14}\left\{\begin{array}{l}\mathrm{H}_{7} \\ \mathrm{~N} \mathrm{O}_{4}\end{array}\right\}}_{\text {Nitrotoluole. }}+2 \mathrm{BaO}=2\left(\mathrm{Ba} 0, \mathrm{CO}_{2}\right)+\underbrace{\mathrm{C}_{12} \mathrm{H}_{7} \mathrm{~N}}_{\text {Aniline. }}$

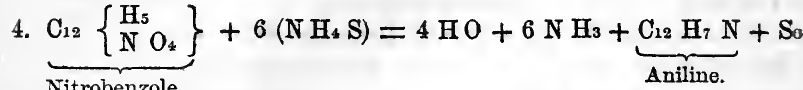

5. $\underbrace{2 \mathrm{C}_{12}\left\{\begin{array}{l}\mathrm{H}_{5} \\ \mathrm{~N} \mathrm{O}_{4}\end{array}\right\}}_{\text {Nitrobenzole. }}+\underbrace{\mathrm{C}_{4} \mathrm{H}_{6} \mathrm{O}_{2}}_{\text {Alcohol. }}+2(\mathrm{~K} \mathrm{O}, \mathrm{H} \mathrm{O})=\underbrace{\mathrm{C}_{12} \mathrm{H}_{5} \mathrm{~N}}_{\text {Azobenzide. }}+$

$\mathrm{C}_{12} \mathrm{H}_{7} \mathrm{~N}+2 \mathrm{C}_{2} \mathrm{O}_{3}, \mathrm{KO}+6 \mathrm{Ho}$.

Aniline. Oxalate of Potash.

These equations will give the reader some idea of the great variety of processes by which one organic base may be formed, and it is probable that every one of these processes may, if applied to other compounds, give rise to other bases. In fact, this is already the case, as will be seen further on, with the processes Nos. 2, 3, and 4.

Besides the above sources of aniline there is another, namely, the destructive distillation of azotised matter. That of indigo is explained by No. 1, as anthranilic acid is probably first formed and then destroyed. But aniline is perhaps best obtained from the basic oil of coal-tar. The crude oil of coal-tar is well agitated with hydrochloric acid, which takes up the basio oils in an impure state. They are separated from the acid by ammonia, and consist chiefly of aniline and leucoline. The former is found in that part of the oil which, when it is rectified, passes at near $360^{\circ}$, and it is purified by rectification till it distils at that temperature, combining it with oxalic acid, purifying the oxalate by crystallisation, and distilling the pure salt with potash.

Aniline is a colourless oil, highly refracting, of Sp. G. 1.020. It has a buruing taste, and a pleasant vinous smell when pure. It turns the delicate purple of the dahlia to green, but does not act on turmeric. A drop of it on fir-wood, moistened with 
hydrochloric acid, strikes a deep yellow colour. It produces a deep but fugitive purple with a solution of bleaching powder. It neutralises acids, forming salts which have a very great tendency to crystallise, hence the name crystalline. In combining with oxygen acids, aniline, like ammonia, takes up 1 eq. of water, but it combines direetly like ammonia with hydrogen acids. Like ammonia, also, its chloride forms double salts with bichloride of platinum and other analogous salts.

Nitric acid converts aniline into nitrophenisic (picric or nitropioric) acid; and by the action of chlorine, and of chlorate of potash with hydrochloric acid, it is converted into chlorophenisic and chlorophenusic acids, chloranile and trichloraniline. Bromine produces tribromaniline. These transformations prove that aniline belongs to the series of phenyle, as formerly explained. In fact, it has the composition of phenylamide, for $\mathrm{C}_{12} \mathrm{H}_{7} \mathrm{~N}=\mathrm{C}_{12}\left\{\begin{array}{l}\mathrm{H}_{5} \\ \text { Ad }\end{array}\right\}$.

The analogy of aniline with ammonia extends beyond what has been above stated; for as ammonia forms, under certain circumstances, such compounds as oxamide and carbamide, the latter only known in combination, so does aniline, under similar circumstances, yield analogous compounds, oxanilide and carbanilide. It even yields formanilide, the analogous compound to which, formamide, is not known. It also forms acid anilides, such as carbanilic acid analogous to carbamic acid, \&c. A compound has also been obtained in which the oxygen of carbanilide is replaced by sulphur. (Gerhardt. Hofmann.)

Hofmann has also discovered that cyanate of aniline spontaneously passes into anilo-urea, as eyanate of ammonia does into urea, and cyanate of methylamine into methylo-urea. $\mathrm{N} \mathrm{H}_{4} \mathrm{O}, \mathrm{C}_{2} \mathrm{NO}=\mathrm{C}_{2} \mathrm{~N}_{2} \mathrm{H}_{4} \mathrm{O}_{2}$, and $\mathrm{C}_{22} \mathrm{H}_{8} \mathrm{~N} \mathrm{O}, \mathrm{C}_{2} \mathrm{NO}=$ $\mathrm{C}_{14} \mathrm{~N}_{2} \mathrm{H}_{8} \mathrm{O}_{2}$.

It , has already been mentioned that by heating chlorisatine, bromisatine, \&c., with potash, bases and neutral compounds are obtained in which the hydrogen of aniline is replaced by chlorine and bromine, and that by the action of sulphuretted hydrogen and ammonia on dinitrobenzole, another base, nitraniline, is formed, in which 1 eq. of hydrogen is replaced by 1 eq. of nitrous acid. A table of these last compounds having been already given, we shall here give only a tabular view of the analogy between aniline and ammonia.

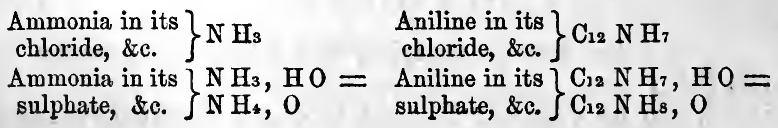


Ammonia plati- $\mathrm{N} \mathrm{H}_{4} \mathrm{Cl}+$ num salt, \&c. $\mathrm{Pt} \mathrm{Cl}_{2}$

Oxamide $\left\{\begin{array}{l}\mathrm{NH}_{2} \mathrm{C}_{2} \mathrm{O}_{2}= \\ \left(\mathrm{N} \mathrm{H}_{4} \mathrm{O}, \mathrm{C}_{2} \mathrm{O}_{3}\right)-2 \mathrm{H} \mathrm{O}\end{array}\right.$ Oxanilide $\left\{\begin{array}{l}\mathrm{C}_{12} \mathrm{~N} \mathrm{H}_{6} \mathrm{C}_{2} \mathrm{O}_{2}= \\ \left(\mathrm{C}_{12} \mathrm{~N} \mathrm{H}_{8} \mathrm{O}, \mathrm{C}_{2} \mathrm{O}_{3}\right) \\ -2 \mathrm{HO}\end{array}\right.$

Oxamic acid $\left\{\begin{array}{l}\mathrm{C}_{4} \mathrm{H}_{3} \mathrm{~N} \mathrm{O}_{6}= \\ \mathrm{C}_{2} \mathrm{O}_{2}, \mathrm{~N} \mathrm{H}_{2}+\mathrm{C}_{2} \mathrm{O}_{3} \mathrm{HO} O\end{array}\right.$ Oxanilic Acid $\left\{\begin{array}{l}\mathrm{C}_{16} \mathrm{NH}_{7} \mathrm{O}_{6} \\ =\mathrm{C}_{14} \mathrm{NH}_{6} \mathrm{O}_{2} \\ +\mathrm{C}_{2} \mathrm{O}_{3}, \mathrm{HO}\end{array}\right.$
Aniline plati- $\mathrm{C}_{12} \mathrm{~N} \mathrm{H}_{8}, \mathrm{Cl}+$ num salt, \&c. $\} \mathrm{Pt} \mathrm{Cl}_{2}$

$$
\text { Urea }\left\{\begin{array} { l } 
{ \mathrm { C } _ { 2 } \mathrm { N } _ { 2 } \mathrm { H } _ { 4 } \mathrm { O } _ { 2 } = } \\
{ ( \mathrm { N } \mathrm { H } _ { 4 } \mathrm { O } , \mathrm { C } _ { 2 } \mathrm { NO } ) }
\end{array} \quad \text { Anilo-urea } \quad \left\{\begin{array}{l}
\mathrm{C}_{14} \mathrm{~N}_{2} \mathrm{H}_{8} \mathrm{O}_{2} \overline{\overline{(}} \\
\left(\mathrm{C}_{12} \mathrm{~N} \mathrm{H}_{8} \mathrm{O}, \mathrm{C}_{2} \mathrm{NO}\right)
\end{array}\right.\right.
$$

It is impossible, after considering the facts exhibited in the above table, to doubt the perfect analogy existing, in all their chemical relations, between ammonia and aniline.

This analogy is at once explained by regarding both as amide bases, ammonia as amide + hydrogen, $\mathrm{Ad} \mathrm{H}$, aniline as amide + phenyle, $\mathrm{Ad} \mathrm{Ph}$. Phenyle is $\mathrm{C}_{22} \mathrm{H}_{5}$. And as phenyle is only one of a series of homologous radicals, the phenylic series, $\mathrm{Cn}_{n} \mathrm{H}_{n}-7$, so aniline is the first of a series of homologous bases, in which amide is combined with those radicals.

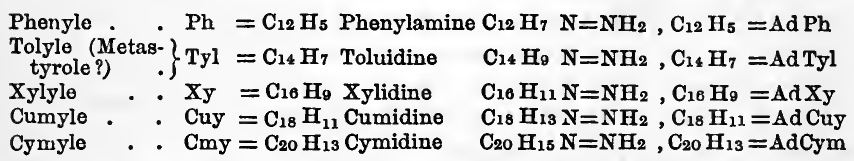

These bases will be briefly noticed in their proper places.

Although aniline is perfectly analogous to ammonia, yet from its complex nature it necessarily yields products of decomposition or metamorphosis such as ammonia cannot yield. We have already seen that it yields substitution compounds, chiefly basic, namely, chloraniline, dichloraniline, trichloraniline, bromaniline, dibromaniline, tribromaniline, and nitraniline, in which part of the hydrogen of the phenyle is replaced by chlorine, \&c. ; and it has been ascertained that the bases in the above table homologous with aniline, are capable of yielding similar substitution products. Hofmann has also obtained bases, in which ethyle is substituted for part of the hydrogen of chloraniline and nitraniline, namely, ethylonitraniline, $\left.\begin{array}{l}\mathrm{H} \\ \mathrm{Ce}_{12} \\ \left.\mathrm{CH}_{4}, \mathrm{H}_{4}\right)\end{array}\right\} \mathrm{N}$; and diethylochloraniline, $\mathrm{Ae}$ Ae $\mathrm{C}_{23}\left(\mathrm{H}_{4} \mathrm{Cl}\right)$ N. But in studying the action of cyanogen on aniline, which he expected to yield products in which cyanogen 
was substituted for hydrogen, he obtained entirely different results.

Cyaniline. When cyanogen gas is made to act on an alcoholic solution of aniline, crystals are formed, which contain several bodies. One of these is a base, cyaniline, which is $\mathrm{C}_{14} \mathrm{H}_{7} \mathrm{~N}_{2}=$ $\mathrm{C}_{12} \mathrm{H}_{7} \mathrm{~N}+\mathrm{C}_{2} \mathrm{~N}$. Here the cyanogen has combined with aniline to form the new base. It forms brilliant silvery scales, very sparingly soluble in any liquid. It forms salts with acids, which are permanent in the dry state, but easily decomposed in solution.

Melaniline. When gaseous chloride of cyanogen acts on aniline, there is formed the hydrochlorate of this base. The base itself is erystalline, and has the formula $\mathrm{C}_{26} \mathrm{H}_{13} \mathrm{~N}_{3}$. It is thus formed-

2. $\underbrace{\left(\mathrm{C}_{12} \mathrm{H}_{7} \mathrm{~N}\right)}_{\text {Aniline. }}+\underbrace{\mathrm{C}_{2} \mathrm{~N} \mathrm{Cl}}_{\text {Chloride of Cyanogen. }}=\underbrace{\mathrm{C}_{26} \mathrm{H}_{13} \mathrm{~N}_{3}}_{\text {Melaniline. }}+\mathrm{H} \mathrm{Cl}$

It may be regarded as aniline, coupled with a compound, which is aniline, in which 1 eq. of hydrogen has been replaced by cyanogen. Thus,

$$
\left.\mathrm{C}_{26} \mathrm{H}_{13} \mathrm{~N}_{3}=\mathrm{C}_{12} \mathrm{H}_{7} \mathrm{~N}+\mathrm{C}_{12} \frac{\mathrm{H}_{2}^{6}}{\mathrm{C}_{2}} \mathrm{~N}\right\} \mathrm{N} \text {. }
$$

Melaniline is very sparingly soluble in water, but soluble in alcohol and ether. It forms crystallisable salts with acids. With chlorine it yields the hydrochlorate of a new base, dichloromelaniline, $\left.\mathrm{C}_{26} \mathrm{Hl}_{21} \mathrm{Cl}_{2}\right\} \mathrm{N}_{3}$; with bromine, dibromomelaniline $\left.\begin{array}{ll}\mathrm{C}_{26} & \left.\begin{array}{l}\mathrm{H}_{11} \\ \mathrm{Br}_{2}\end{array}\right\} \mathrm{N}_{3} \text {; and with iodine, diiodomelaniline, } \mathrm{C}_{26} \underset{\mathrm{I}_{2}}{\mathrm{H}_{11}}\end{array}\right\} \mathrm{N}_{3}$. With nitric acid it yields the base dinitromelaniline, $\left.\mathrm{C}_{26}{ }_{2} \mathrm{H} \mathrm{O}_{11}\right\}$ $\mathrm{N}_{3}$; and with cyanogen, it forms a compound in which 1 eq. of melaniline is united with 2 of cyanogen, analogous to cyaniline, and called dicyanomelaniline; but this is not, like the other four, a substitution product. It may be viewed as composed of 1 eq. cyaniline, 1 eq. of the compound $\left.\mathrm{C}_{12} \frac{\mathrm{H}_{6}}{\mathrm{Cy}}\right\}$, supposed to exist in melaniline, and 2 eqs. of cyanogen, as if each of the groups in melaniline took up, like aniline itself, 1 eq. of cyanogen. Its formula is $\mathrm{C}_{30} \mathrm{H}_{13} \mathrm{~N}_{5}=\mathrm{C}_{28} \mathrm{H}_{13} \mathrm{~N}_{3}+2 \mathrm{C}_{2} \mathrm{~N}=\left(\mathrm{C}_{14} \mathrm{H}_{7} \mathrm{~N}_{2}+\right.$ $\left.\left.\mathrm{C}_{2} \mathrm{~N}\right)+\left(\mathrm{C}_{12} \underset{\mathrm{C}_{2} \mathrm{~N}}{\mathrm{H}}\right\} \mathrm{N}+\mathrm{C}_{2} \mathrm{~N}\right)$. It is a feeble base.

With diluted acids, cyaniline yields various products, among which are oxamide, oxanilide, analogous to oxamide, and a 
compound of both, oxanilamide. Oxanilide is $\mathrm{C}_{22} \mathrm{H}_{6} \mathrm{~N}, \mathrm{C}_{2}$ $\mathrm{O}_{2}$, and oxanilamide is $\mathrm{C}_{12} \mathrm{H}_{6} \mathrm{~N}, \mathrm{C}_{2} \mathrm{O}_{2}+\mathrm{H}_{2} \mathrm{~N}, \mathrm{C}_{2} \mathrm{O}_{2}=\mathrm{C}_{16} \mathrm{~N}_{2}$ $\mathrm{H}_{8} \mathrm{O}_{4}$.

When dicyanomelaniline, $\mathrm{C}_{30} \mathrm{H}_{13} \mathrm{~N}_{5}$, is boiled with acids, it undergoes a remarkable change, and yields a yellow product, $\mathrm{C}_{30} \mathrm{H}_{11} \mathrm{~N}_{3} \mathrm{O}_{4}$. Thus, $\mathrm{C}_{30} \mathrm{H}_{13} \mathrm{~N}_{5}+4 \mathrm{H} \mathrm{O}+2 \mathrm{H} \mathrm{Cl}=2$ $\left(\mathrm{N} \mathrm{H}_{4} \mathrm{Cl}\right)+\mathrm{C}_{30} \mathrm{H}_{11} \mathrm{~N}_{3} \mathrm{O}_{4}$. It may be regarded as binoxalate of melaniline, minus 4 eqs. of water. This is the composition of an imide, and the compound has been named Melanoximide. When heated, the body yields, among other products, a volatile liquid, boiling at $35.3^{\circ}$, of a very pungent odour, $\mathrm{C}_{14} \mathrm{~N} \mathrm{H}_{5} \mathrm{O}_{2}$, which bears to hydrated cyanic acid the same relation as aniline does to ammonia. $\mathrm{C}_{22} \mathrm{H}_{7} \mathrm{~N}=\mathrm{N} \mathrm{H}_{3}+\mathrm{C}_{12} \mathrm{H}_{4} . \mathrm{C}_{14} \mathrm{~N} \mathrm{H}_{5} \mathrm{O}_{2}=$ $\mathrm{C}_{2} \mathrm{~N} \mathrm{O}, \mathrm{H} \mathrm{O}+\mathrm{C}_{12} \mathrm{H}_{4}$. Hence it is named Anilocyanic Acid. With acids and bases it is resolved into aniline and carbonic acid, just as cyanic acid into ammonia and carbonic acid, $\mathrm{C}_{44} \mathrm{~N} \mathrm{H}_{5}$ $\mathrm{O}_{2}+2 \mathrm{H} \mathrm{O}=\mathrm{C}_{22} \mathrm{H}_{7} \mathrm{~N}+2 \mathrm{C} \mathrm{O}_{2}$. In short, it is hydrated cyanic acid, in which the hydrogen of its basic water is replaced by phenyle, $\mathrm{C}_{12} \mathrm{H}_{5}$, or it is the cyanate of oxide of phenyle.

\section{$\mathrm{C}_{2} \mathrm{NO}, \mathrm{HO}$ \\ Hydrated Cyanic Acid. \\ $\mathrm{C}_{2} \mathrm{~N} \mathrm{O}, \mathrm{C}_{12} \mathrm{H}_{5} \mathrm{O}$ \\ Cyanate of oxide of phenyle.}

As cyanic acid with water yields, first ammonia and carbonic acid, and then by the action of this on another portion of the acid, cyanate of ammonia or urea, so anilocyanic acid, with water, yields first aniline and carbonic acid, and secondly cyanate of aniline, or 2 atoms of carbanilide, analogous to urea, which is 2 atoms of carbamide.

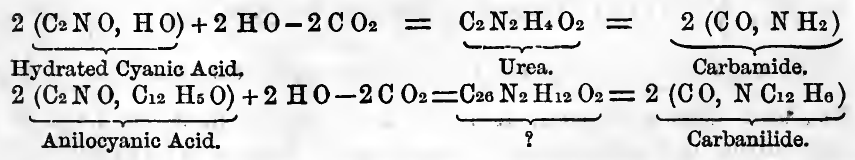

Carbanilide is therefore urea, in which 2 eqs. of hydrogen are replaced by phenyle, $\mathrm{C}_{12} \mathrm{H}_{5}$, diphenylo-urea ; but it is uncertain whether it may not be only polymeric with the true diphenylourea, that is, whether 1 eq. of the latter may not resolve itself into 2 eqs. of carbanilide. There is another compound, carbamidecarbanilide, in which 1 eq. of hydrogen in urea is replaced by 1 of phenyle. This is phenylo-urea, and is formed when anilocyanic acid acts on ammonia. $\mathrm{C}_{2} \mathrm{~N} \mathrm{O}, \mathrm{H} \mathrm{O}+\mathrm{N} \mathrm{H}_{3}=\mathrm{C}_{2} \mathrm{H}_{4} \mathrm{~N}_{2} \mathrm{O}_{2}$, and $\mathrm{C}_{2} \mathrm{~N} \mathrm{O}, \mathrm{C}_{12} \mathrm{H}_{5} \mathrm{O}+\mathrm{N} \mathrm{H}_{3}=\mathrm{C}_{14} \mathrm{~N}_{2} \mathrm{H}_{8} \mathrm{O}_{2}$.

When cyanic acid acts on alcohol it yields two compounds, 
urethane or carbamate of oxide of ethyle, and allophanate of oxide of ethyle, according as 1 or 2 eqs. of the acid act on 1 of alcohol. Hofmann fiuds that anilocyanic acid produces with the known alcohols compounds analogous to urethane, and probably also compounds analogous to allophanio ether. As urethane contains carbamic acid, so the phenylo-urethane must contain an acid, in which 1 eq. of hydrogen in carbamic acid is replaced by phenyle. Now carbamic acid is $\mathrm{C}_{2} \mathrm{~N} \mathrm{H}_{2} \mathrm{O}_{3}, \mathrm{HO}$, and the new acid must be $\left.\mathrm{C}_{2} \mathrm{~N}_{\mathrm{C}_{12} \mathrm{H}_{5}}^{\mathrm{H}}\right\} \mathrm{O}_{3}, \mathrm{H} \mathrm{O}=\mathrm{C}_{14} \mathrm{~N} \mathrm{H}_{6}$ $\mathrm{O}_{3} ; \mathrm{H} \mathrm{O}=\mathrm{C}_{14} \mathrm{~N} \mathrm{H}_{7} \mathrm{O}_{4}$. This is the formula of anthranilic or carbanilic acid, but whether the acid in phenylo-urethane or carbanilate of oxide of ethyle be identical or only isomeric with anthranilic acid from indigo is not yet known.

The reader will not fail to observe, that as the cyanates of oxides of ethyle, methyle, \&c., when heated with potash, yield ethylamine, methylamine, \&c., so we may expect the anilocyanates or phenyle-cyanates of the oxides of ethyle, \&c., to yield with potash a new series of bases, in which 1 eq. of the hydrogen of aniline is replaced by ethyle, methyle, \&c. Here also aniline will probably retain its analogy to ammonia, but the investigation is very difficult, from the long, tedious, and expensive processes necessary to obtain first aniline, then melaniline, dicyanomelaniline, and anilocyanic acid, with which last it must be commenced.

The reader is referred to Dr. Hofmann's papers for many interesting details and ingenious speculations, which we are compelled here to omit.

When melaniline is heated it yields, among other products, a body called aniline-mellone, which, however, combines with aniline to yield the compound $\mathrm{C}_{54} \mathrm{H}_{25} \mathrm{~N}_{7}=3\left(\mathrm{C}_{12} \mathrm{H}_{7} \mathrm{~N}\right)+\mathrm{C}_{18}$ $\mathrm{H}_{4} \mathrm{~N}_{4}$. The latter group is anilomellone. Laurent has obtained a compound $\mathrm{C}_{30} \mathrm{H}_{11} \mathrm{~N}_{5}$, which is $=\mathrm{C}_{12} \mathrm{H}_{7} \mathrm{~N}+\mathrm{C}_{18} \mathrm{H}_{4} \mathrm{~N}_{4}$. Anilomellone, or phenylomellone, $\mathrm{C}_{18} \mathrm{H}_{4} \mathrm{~N}_{4}$, is mellone, $\mathrm{C}_{6} \mathrm{~N}_{4}$, coupled with $\mathrm{C}_{12} \mathrm{H}_{4}$, or phenyle, minus $\mathrm{H}$.

Besides the above derivatives from aniline, there are many in which phenyle is substituted for hydrogen; as for example, volatile bases, like those of the series of ethylamine, aniline itself being the first of them; in the others, we have the hydrogen of ammonia replaced to the extent of two, three, or four eqs., either by phenyle, or by phenyle in part, and in part by one or more of the ethylic radicals. Of these, the following are known :-

Ethylophenylamine or Ethylaniline, $\mathrm{C}_{16} \mathrm{H}_{11} \mathrm{~N}=\mathrm{N}, \mathrm{H}$ Ae. $\mathrm{Ph}$. 
This base is obtained by the action of bromide of ethyle on aniline. $\mathrm{N} \mathrm{H} 2 \mathrm{Ph}+\mathrm{Ae} \mathrm{Br}=\mathrm{H} \mathrm{Br}+\mathrm{NH}$ Ae $\mathrm{Ph}$. Its empirioal formula is $\mathrm{C}_{16} \mathrm{H}_{11} \mathrm{~N}$. It much resembles aniline.

Diethylophenylamine, $\mathrm{C}_{20} \mathrm{H}_{15} \mathrm{~N}=\mathrm{N}, \mathrm{Ae}_{2} \mathrm{Ph}$, is formed when the preceding base is acted on by bromide of ethyle. It resembles the foregoing.

Triethylophenylium. The iodide of this compound is formed when iodide of ethyle acts on the preceding base, $\mathrm{N} \mathrm{Ae} 2 \mathrm{Ph}+$ $\mathrm{Ae} \mathrm{I}=\mathrm{N}$ Aes $\mathrm{Ph}$, I. It forms, like the other ammonium bases, a hydrated oxide, which is a powerful base.

Methylethylamylophenylium. This sesquipedalian word denotes an ammonium compound, the iodide of which is formed when iodide of methyle acts on ethylamylophenylamine, $\mathrm{Me} \mathrm{I}+\mathrm{N} \mathrm{Ae}$ Ayl $\mathrm{Ph}=\mathrm{N}, \mathrm{Me}$ Ae Ayl $\mathrm{Ph}+\mathrm{I}$. Its hydrated oxide is, like the preceding one, a powerful base.

Ethylamylophenylamine, $\mathrm{N}, \mathrm{Ae} \mathrm{Ayl} \mathrm{Ph}$, is obtained by the action of bromide of amyle on ethylophenylamine, or of the bromide of ethyle on amylophenylamine. $\mathrm{N}, \mathrm{H} \mathrm{Ae} \mathrm{Ph}+\mathrm{Ayl} \mathrm{Br}=\mathrm{N}$, Ae Ayl Ph $+\mathrm{H} B r$, or $\mathrm{N}, \mathrm{H}$ Ayl $\mathrm{Ph}+\mathrm{Ae} \mathrm{Br}=\mathrm{N}$, Ae Ayl Ph + $\mathrm{H} \mathrm{Br}$.

Methylophenylamine, $\mathrm{N}, \mathrm{H} \mathrm{Me} \mathrm{Ph} ;$ Methylethylophenylamine, $\mathrm{N}, \mathrm{Me} \mathrm{Ae} \mathrm{Ph;Amylophenylamine,} \mathrm{N,} \mathrm{H} \mathrm{Ayl} \mathrm{Ph;} \mathrm{Diamylophe-}$ nylamine, $\mathrm{N}$, Ayl $\mathrm{Ph}$; are all formed by means of analogous processes, and are all volatile bases. It is singular that the two last-named have a strong and fragrant smell of roses.

Aniline or phenylamine, and all its derivatives, contain the radical phenyle; but this same radical is found in many other compounds, as will be explained under the head of hydrated oxide of phenyle or carbolic acid, among the products of the distillation of coal, and, as has been already noticed, under benzoyle, salicyle, and indigo.

Picoline, $\mathrm{C}_{22} \mathrm{H}_{7} \mathrm{~N}$, is another volatile oily base, isomeric with aniline, lately discovered in coal-tar by Dr. T. Anderson. It is found in the more volatile portion of the basic oil, boiling at $272^{\circ}$. It has no action on bleaching-liquid or on fir-wood. It is a powerful base; and as it has a strong penetrating odour, it is probably identical with the odorine of Unverdorben, which, however, was not obtained in a state of purity. Under Naphthaline, we shall return to the isomerism of aniline and picoline. Anderson finds that picoline is one of a series of bases isomeric and parallel with that to which aniline belongs.

Pyridine, $\mathrm{C}_{10} \mathrm{H}_{5} \mathrm{~N}$, is the lowest of the picoline series, and 
there is no corresponding base in the aniline series. It is more volatile than picoline, but resembles it in many characters. (Anderson.)

Toluidine, $\mathrm{C}_{14} \mathrm{H}_{9} \mathrm{~N}$. This base, the second of the homologous series of aniline, is formed by the action of sulphuretted hydrogen and ammonia on nitrotoluole, as aniline is from nitrobenzole. It is derived from the fundamental compound of the series, toluole, $\mathrm{C}_{14} \mathrm{H}_{8}$, as aniline is from benzole or phène, $\mathrm{C}_{12} \mathrm{H}_{6}$.

$$
\underbrace{\mathrm{C}_{14}\left\{\begin{array}{l}
\mathrm{H}_{7} \\
\mathrm{~N}_{4}
\end{array}\right\}}_{\text {Nitrotoluole. }}+6 \mathrm{~N} \mathrm{H}_{4} \mathrm{~S}=6 \mathrm{NH}_{3}+4 \mathrm{H} \mathrm{O}^{\mathrm{O}}+\underbrace{\mathrm{C}_{14} \mathrm{H}_{\vartheta} \mathrm{N}+\mathrm{S} \mathrm{S}_{8}}_{\text {Toluidine. }}
$$

Toluidine is, in taste and smell, very similar to aniline; and, although solid and crystalline at the ordinary temperature, it melts at $104^{\circ}$ to a highly refracting oil, which boils at $388^{\circ}$. It also turns fir-wood yellow, with the aid of hydrochlorio acid. It belongs, therefore, evidently to the class of volatile oily bases, none of which, as we have seen, contain oxygen.

With bromine, toluidine yields a crystalline body, $\mathrm{C}_{24}\left\{\begin{array}{l}\mathrm{Hr}_{3} \\ \mathrm{Br}_{3}\end{array} \mathrm{~N}\right.$; and chlorine appears to form an analogous compound. These products correspond to tribromaniline and trichloraniline.

Lutidine, $\mathrm{C}_{14} \mathrm{H}_{9} \mathrm{~N}$, is isomerio with toluidine, but homologous with picoline rather than aniline. It is found in the oil of Dippel, along with aniline, picoline, and bases of the ethylamine series. (Anderson.)

Xylidine. This base is formed from the carbohydrogen xylole, $\mathrm{C}_{16} \mathrm{H}_{10}$, homologous with benzole and toluole (see table, p. 92). It is first converted into nitroxylole, $\left.\mathrm{C}_{10} \mathrm{NO}_{\mathrm{O}_{4}}^{\mathrm{H}}\right\}$, and this, acted on by sulphuret of ammonium, yields the base. (See process for Aniline, No. 4, p. 390.) It has been little studied, but would seem by its properties to belong to the series of aniline and toluidine.

Collidine, $\mathrm{C}_{16} \mathrm{H}_{11} \mathrm{~N}$. Anderson has found in Dippel's animal oil a base isomeric with xylidine, which he has thus named. It resembles lutidine. It belongs to the series of pyridine, picoline, and lutidine, which runs parallel to that of aniline.

Anderson has lately ascertained, that the picoline series of bases are nitryle bases, and that, by the action of iodide or bromide of methyle or ethyle, they yield ammonium compounds. Thus

Pyridine is $\mathrm{N}, \mathrm{C}_{10} \mathrm{H}_{5}$ Ethylopyridium is $\mathrm{N}\left\{\begin{array}{l}\mathrm{C}_{10} \mathrm{H}_{4} \\ \mathrm{C}_{4} \mathrm{H}_{5}\end{array}\right.$ Picoline is $\mathrm{N}, \mathrm{C}_{22} \mathrm{H}_{7}$ Ethylopicolium is $\mathrm{N}\left\{\begin{array}{l}\mathrm{C}_{22} \mathrm{H}_{6} \\ \mathrm{C}_{3} \mathrm{H}_{5}\end{array}\right.$

Pyridine and picoline correspond to ammonia, $\mathrm{N} \mathrm{H}_{3}$, ard are 
volatile. The two new compounds correspond to ammonium, $\mathrm{N} \mathrm{H}_{4}$, and to tetrethylium, and are not volatile.

Anderson has also obtained, from pyridine and picoline, fine platinised bases, such as .

$$
\begin{aligned}
& \text { Platinopyridine } \\
& \text { Platosopyridine }
\end{aligned} \text {. }^{-} \quad \cdot \quad \cdot \begin{aligned}
& \mathrm{C}_{10} \mathrm{H}_{3} \mathrm{Pt} \mathrm{N} \\
& \mathrm{C}_{10} \mathrm{H}_{4} \mathrm{Pt} \mathrm{N}
\end{aligned}
$$

Cumidine, $\mathrm{C}_{18} \mathrm{H}_{13} \mathrm{~N}$. One of the products derived from oil of cumine is cumole, $\mathrm{C}_{18} \mathrm{H}_{12}$; and it is also found in the oil of coal-tar, with its homologues, benzole, toluole, and xylole. It is converted into nitrocumole, $\left.\mathrm{C}_{18} \mathrm{H} \mathrm{O}_{11} \mathrm{~N}_{4}\right\}$, and this, as usual, into the base. Cumidine is a beautiful crystalline base, fusible, and, when melted, resembling aniline. It yields, like aniline and toluidine, substitution products with chlorine, bromine, nitrous acid, \&c.; and it has been ascertained that both toluidine and cumidine behave, with cyanogen, as aniline does, and no doubt yield a whole series of new products analogous to those described under aniline.

Parvoline, $\mathrm{C}_{18} \mathrm{H}_{13} \mathrm{~N}$. This base, parallel, in the pyridine series, to cumidine in the aniline series, has been found by Williams in the naphtha of shale.

Cymidine, $\mathrm{C}_{20} \mathrm{H}_{15} \mathrm{~N}$. Oil of cumine yields another carbohydrogen, cymole, $\mathrm{C}_{20} \mathrm{H}_{14}$, homologous with benzole, and present in the oil of coal-tar. Nitrocymole, from which the base is prepared, is formed when cymole and fuming nitric acid previously cooled in a freezing mixture, are brought together. Cymidine is oily, and boils at about $482^{\circ} \mathrm{F}$. It is the highest known member of the anilic series of bases.

We have now seen that in the series of aniline five, and in that of pyridine, five bases have been discovered; the lowest in the aniline, and the highest in the pyridine series being yet unknown. We have

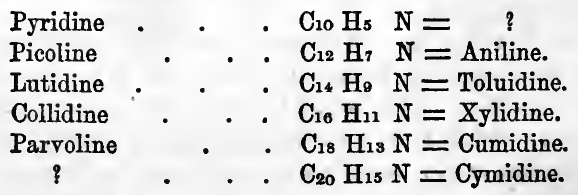

We have already seen that aniline and its homologues are amide bases, in which 'phenyle, \&c., take the place of the third eq. of hydrogen. It now appears that the pyridine series are nitryle bases, in which $\mathrm{H}_{3}$ is replaced by $\mathrm{C}_{10} \mathrm{H}_{4}, \mathrm{C}_{12} \mathrm{H}_{6}$, and so on, whether $\mathrm{C}_{10} \mathrm{H}_{4}$ and the like be single radicals, equivalent to $\mathrm{H}_{3}$, or made up of two or three radicals. The difference of properties 
between these two series of volatile bases is now easily understood. In fact, picoline, though isomeric with aniline, could not, from its constitution, have the same properties.

It may here be noted that Anderson's new compounds, ethylopyridium and ethylopicolium, considered as compound ammoniums, are isomeric with lutidine and collidine, being $\mathrm{C}_{14} \mathrm{H}_{9} \mathrm{~N}$ and $\mathrm{C}_{16}$ $\mathrm{H}_{11} \mathrm{~N}$. The corresponding methyle compound of pyridine will be isomeric with picoline or with aniline, $\mathrm{C}_{12} \mathrm{H}_{7} \mathrm{~N}$, and the methyle compound of picoline will be $\mathrm{C}_{14} \mathrm{H}_{9} \mathrm{~N}$ isomeric with lutidine, toluidine, and ethylopyridium; yet all these will be totally distinct compounds. This is like what we have seen of the numerous cases of isomerism in the volatile bases of the ethylic series.

Pyrrole bases. In Dippel's oil, besides bases of the picoline and ethylamine series, there are bases of another series, characterised by yielding a red resinous matter when decomposed. According to Anderson, they contain bases of the picoline series, coupled with a body which gives rise to the red matter when separated.

Greville Williams has examined in detail the volatile bases from the distillation of coal (coal tar), of cinchonine, and of Dorsetshire shale, and his results, compared with those of Anderson obtained with the oil from the distillation of bones (that is, from gelatine) or Dippel's animal oil, indicate very striking analogies between the results in substances of origin so widely different. Thus we have-

In Dippel's oil -

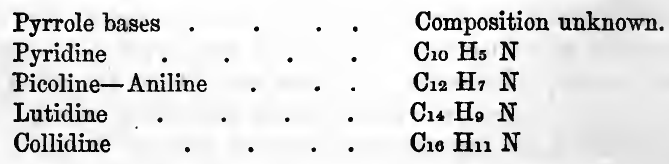

In Dorsetshire shale oil :-

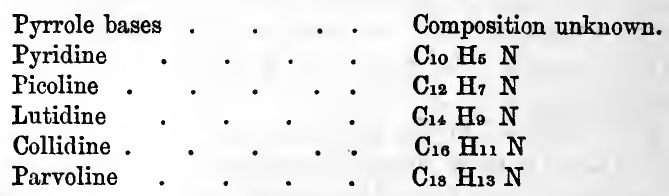

The absence of aniline and its homologues is here very remarkable. 
In the oil from cinchonine :-

$\begin{array}{ll}\text { Pyrrole bases } & \text { Composition unknown. } \\ \text { Pyridine } \mathrm{C}_{10} \mathrm{H}_{5} \mathrm{~N} & \\ \text { Picoline } \mathrm{C}_{12} \mathrm{H}_{7} \mathrm{~N} & \text { Quinoline } \mathrm{C}_{18} \mathrm{H}_{7} \mathrm{~N} \\ \text { Lutidine } \mathrm{C}_{14} \mathrm{H}_{9} \mathrm{~N} & \text { Lepidine } \mathrm{C}_{20} \mathrm{H}_{9} \mathrm{~N} \\ \text { Collidine } \mathrm{C}_{16} \mathrm{H}_{11} \mathrm{~N} & \end{array}$

Here aniline and its homologues are still absent, but we find quinoline and lepidine, homologous bases, of a third series, for our knowledge of which, beyond quinoline, we are indebted to Williams, who has also greatly extended and corrected our notions of quinoline itself.

In coal tar we find:-

$\begin{array}{ll}\text { Pyrrole bases } & \text { Composition unknown. } \\ \text { Pyridine } \mathrm{C}_{10} \mathrm{H}_{5} \mathrm{~N} & \\ \text { Picoline } \mathrm{C}_{12} \mathrm{H}_{7} \mathrm{~N} & \text { Quinoline } \mathrm{C}_{18} \mathrm{H}_{7} \mathrm{~N} \\ \text { Lutidine } \mathrm{C}_{14} \mathrm{H}_{8} \mathrm{~N} & \text { Lepidine } \mathrm{C}_{20} \mathrm{H}_{9} \mathrm{~N} \\ \text { Collidine } \mathrm{C}_{16} \mathrm{H}_{12} \mathrm{~N} & \text { Cryptidine } \mathrm{C}_{22} \mathrm{H}_{12} \mathrm{~N}\end{array}$

Aniline $\mathrm{C}_{12} \mathrm{H}_{7} \mathrm{~N}$

Here Williams finds a third member of the quinoline series, and aniline is abundant, though, as yet, none of its homologues, toluidine, xylidine, cumidine or cymidine, have been found in coal tar. As the corresponding carbohydrogens, however, benzole, toluole, xylole, cumole and cymole, do occur in coal tar, it is probable that these bases will ultimately be found there.

Williams has also obtained from the shale oil, traces of two bases, not yet isolated. One of these, vertidine, strikes a beautiful emerald green colour with bleaching liquor, and seems to be isomeric with lutidine. This base appears also to occur in small proportion in Dippel's oil. The other base is called carmidine, and was obtained, when a portion consisting chiefly of lutidine, was passed as vapour over red hot lime. It gives a fine red with fir wood and hydrochloric acid, and a bluish green with bleaching liquor. Its composition is not yet ascertained. There is, perhaps, some relation between these two bases, but there appears to be at least one peculiar base, though we do not know to which series it belongs, and it may possibly be one of a new series altogether.

I am indebted to the kindness of Mr. Williams for the use of the MS. of a most interesting paper by him, not yet published, from which, and from his earlier papers, I have extracted the above results.

But it is proper also here to mention that Williams has deter- 
mined the true formula of quinoline, $\mathrm{Cl}_{18} \mathrm{H}_{7} . \mathrm{N}$, and has shown that the basic oil from cinchonine contains not only quinoline and its homologue lepidine, but also the pyridine series and the pyrole series. The formula of quinoline being as abore stated, it is evident that the supposed conversion of quinoline from coaltar into quinine or a base isomeric with it, by the action of iodide of methyle, followed by that of oxide of silver, must be erroneous, since that requires the formula $\mathrm{C}_{18} \mathrm{H}_{8} \mathrm{~N}$, and it is remarkable that we seldom, if ever, find in these volatile bases an even number of atoms of hydrogen to 1 atom of nitrogen. Mr. Williams has found that both quinoline and lepidine form bases with the iodides of ethyle and methyle, but that as quinoline, \&c., are nitryle bases, the new ones are ammonium bases, corresponding to tetramethylium, and, like it, only known in the forms of iodide, hydrated oxide, or platinum salt.

The hydrated oxide of methyloquinolium, does not undergo, when heated with potash, the same decomposition as that of tetramethylium, but apparently a more complicated one. A volatile base is given off, acting strongly on the eyes, and the solution of the fixed base, as soon as formed, begins to give off a volatile base, probably methylamine. It is, therefore, totally different from quinine. It remains to be ascertained what the fixed base was, the iodide of which Hofmann supposed to be isomeric with quinine.

With iodide of ethyle, quinoline yields hydriodate of ethyloquinoline, or iodide of ethyloquinolium, from which the hydrated oxide is obtained by oxide of silver. This hydrated oxide is decomposed by heat, yielding a most splendid crimson colour, which dries up with emerald green metallic lustre. This colour, which passes also into purple and blue of very fine tints, is due apparently to the formation of some new basic compound. The corresponding compounds derived from lepidine, exhibit similar characters. When the iodide of ethyloquinolium is acted on by sulphate of silver, a colourless solution is formed with a precipitate of iodide of silver. The filtered liquid on evaporation acquires an indigo blue colour, and dries up with a coppery metallic colour, like that of indigo when polished. This reminds one of the characters of Reichenbach's pittacal obtained by the action of baryta on the heavy oil of wood tar, and therefore possibly basic.

Lepidine $\left(\mathrm{C}_{20} \mathrm{H}_{9} \mathrm{~N}\right)$ is homologous with the quinoline $\left(\mathrm{C}_{18} \mathrm{H}_{7} \mathrm{~N}\right)$ of Williams; both give with acids beautifully crystalline salts, and resemble one another in properties. Lepidine boils at about $510^{\circ} \mathrm{F}$. The bichromate is a fine salt erystallising in goldenyellow needles nearly an inch long. 


\section{b. Bases derived from Naphthaline.}

These bases are formed in a manner quite analogous to that in which aniline is produced from benzole, and toluidine from toluole. The carbohydrogen is first converted, by the action of nitric acid, into a compound in which $\mathrm{H}$ is replaced by $\mathrm{N} \mathrm{O}_{4}$ : and the new product, according to the ingenious process devised by Zinin, is dissolved in alcohol, and acted on by ammonia and sulphuretted hydrogen.

Seminaphtalidine, or azonaphtylamine, $\mathrm{C}_{20} \mathrm{H}_{10} \mathrm{~N}_{2}$, is obtained when nitronaphtalèse is treated by Zinin's process.

$$
\underbrace{\mathrm{C}_{20}\left\{\begin{array}{l}
\mathrm{H}_{6} \\
2 \mathrm{~N} \mathrm{O}_{4}
\end{array}\right\}}_{\text {Nitronaphtalese. }}+12 \mathrm{~N} \mathrm{H}_{4} \mathrm{~S}=8 \mathrm{H} \mathrm{O}+12 \mathrm{~N} \mathrm{H}_{3}+\mathrm{S}_{12} \underbrace{+\mathrm{C}_{20} \mathrm{H}_{10} \mathrm{~N}_{2}}_{\text {Seminaphtalidine. }}
$$

It forms reddish-yellow prisms, of metallic lustre, insoluble in water, soluble in alcohol and ether, and yielding crystallisable salts with acids.

Naphtalidine, or naphtylamine, $\mathrm{C}_{20} \mathrm{H}_{9} \mathrm{~N}$, is produced, in a similar way, from nitronaphtalase.

$$
\underbrace{\mathrm{C}_{20}\left\{\begin{array}{l}
\mathrm{H}_{7} \\
\mathrm{~N}_{4}
\end{array}\right\}}_{\text {Nitronaphtalase. }}+6 \mathrm{~N} \mathrm{H}_{4} \mathrm{~S}=4 \mathrm{H} \mathrm{O}+6 \mathrm{~N} \mathrm{H}_{3}+\mathrm{S}_{6}+\underbrace{\mathrm{C}_{20} \mathrm{H}_{9} \mathrm{~N}}_{\text {Naphtalidine. }}
$$

It appears in the form of colourless crystals, soluble in alcohol and ether, and combines with acids, yielding crystallisable salts.

This alkali is ammonia, in which 1 eq. of hydrogen is replaced by naphtyle, $\mathrm{C}_{20} \mathrm{H}_{7}$, and may be thus written; $\mathrm{N} \mathrm{H}_{2}, \mathrm{C}_{20} \mathrm{H}_{7}$; or $\left.\begin{array}{l}\mathrm{H} \\ \mathrm{N} \\ \mathrm{C}_{20} \mathrm{H}_{7}\end{array}\right\}$

Piria has lately given a new process for obtaining it, which consists in the metamorphoses of the salts of thionaphtamic acid. This acid contains the elements of naphtylamine with 2 eqs. of anhydrous sulphuric acid, $\mathrm{C}_{20} \mathrm{H}_{9} \mathrm{~N}, \mathrm{~S}_{2} \mathrm{O}_{6}$, and when its salts are acted on by dilute sulphuric acid, aided by heat, sulphate of naphtylamine is deposited in crystals. This salt, distilled with lime, yields the base.

\section{c. Bases derived from Oil of Mustard.}

These bases have already been briefly described under the head of Oil of Mustard. They are as follows :

1. Thiosinnamine, $\mathrm{C}_{8} \mathrm{H}_{8} \mathrm{~N}_{2} \mathrm{~S}_{2}$, formed by the action of ammonia on oil of mustard. 


$$
\underbrace{\mathrm{C}_{8} \mathrm{H}_{5} \mathrm{~N} \mathrm{~S}_{2}}_{\text {Oil of Mustard. }}+\mathrm{N} \mathrm{H}_{3}=\underbrace{\mathrm{C}_{8} \mathrm{H}_{8} \mathrm{~N}_{2} \mathrm{~S}_{2}}_{\text {Thiosinnamine. }}
$$

According to Hinterberger, thiosinnamine forms new bases, in which 1 eq. of its hydrogen is replaced by methyle, ethyle, amyle, phenyle, and naphtyle $\left(\mathrm{C}_{20} \mathrm{H}_{7}\right)$. These bases, except the two last, do not crystallise, but they form salts which do, especially the chloroplatinates.

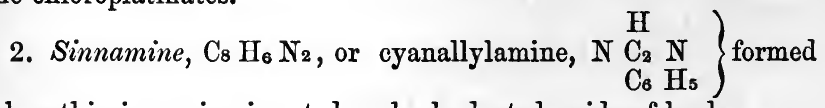
when thiosinnamine is acted on by hydrated oxide of lead.

$$
\underbrace{\mathrm{C}_{8} \mathrm{H}_{8} \mathrm{~N}_{2} \mathrm{~S}_{2}}_{\text {Thiosinnamine. }}+2(\mathrm{~Pb} \mathrm{O}, \mathrm{H} 0)=2 \mathrm{~Pb} \mathrm{~S}+4 \mathrm{H} \mathrm{O}^{0}+\underbrace{\mathrm{C}_{8} \mathrm{H}_{6} \mathrm{~N}_{2}}_{\text {Sinnamine. }}
$$

3. Sinapoline, $\mathrm{C}_{14} \quad \mathrm{H}_{12} \quad \mathrm{~N}_{2} \mathrm{O}_{2}$, formed when oil of mustard is acted on by baryta or oxide of lead.

$$
2\left(\mathrm{C}_{8} \mathrm{H}_{5} \mathrm{~N} \mathrm{~S}_{2}\right)+6 \mathrm{PbO}+2 \mathrm{HO}=2\left(\mathrm{~Pb} \mathrm{O}, \mathrm{C} \mathrm{O}_{2}\right)+4 \mathrm{~Pb} \mathrm{~S}+
$$

Oil of Mustard.

$$
\underbrace{\mathrm{C}_{14} \mathrm{H}_{12} \mathrm{~N}_{2} \mathrm{O}_{2}}_{\text {Sinapoline. }}
$$

4. Sinkaline, $\mathrm{C}_{10} \mathrm{H}_{13} \mathrm{~N} \mathrm{O}_{2}$, formed, along with sinapic acid, from sinapine, the peculiar base of white mustard, by the addition of water.

$$
\underbrace{\mathrm{C}_{22} \mathrm{H}_{23} \mathrm{~N} \mathrm{O}_{10}}_{\text {Sinapine. }}+2 \mathrm{H} \mathrm{O}=\underbrace{\mathrm{C}_{22} \mathrm{H}_{12} \mathrm{O}_{10}}_{\text {Sinapic Acid. }}+\underbrace{\mathrm{C}_{10} \mathrm{H}_{13} \mathrm{~N} \mathrm{O}_{2}}_{\text {Sinkaline. }}
$$

The above equations will serve to explain the formation of these remarkable compounds, all of which, it will be seen, are produced by the action of bases, and one of which contains sulphur, an element hitherto only known to occur in one other organic base, namely, thialdine.

\section{d. Bases derived from Aldehyde.}

These bases are of recent discovery, and have been briefly mentioned under Aldehyde. For the convenience of the reader, we here repeat the equation which illustrates their formation.

$$
3 \underbrace{\left(\mathrm{N} \mathrm{H}_{3}, \mathrm{C}_{4} \mathrm{H}_{4} \mathrm{O}_{2}\right.}_{\text {Aldehjdammonia. }})+6 \mathrm{HS}=2\left(\mathrm{~N} \mathrm{H}_{4}, \mathrm{~S}\right)+6 \mathrm{H} \mathrm{O}+\underbrace{\mathrm{C}_{12} \mathrm{H}_{13} \mathrm{~N} \mathrm{~S}_{2}}_{\substack{\text { Thialdine. } \\ \text { D D } 2}}
$$


Selenaldine, $\mathrm{C}_{12} \mathrm{H}_{13} \mathrm{~N}$ Se2, is formed by a perfectly analogous process.

\section{e. Bases derived from Melam.}

These bases have been already described, but we shall here repeat the equation, for the sake of easy reference. When melam is boiled with potash it yields two bases, melamine and ammeline.

$$
\underbrace{\mathrm{C}_{12} \mathrm{~N}_{11} \mathrm{H}_{9}}_{\text {Melam. }}+2(\mathrm{~K} \mathrm{O}, \mathrm{HO})=\underbrace{\mathrm{C}_{6} \mathrm{H}_{6} \mathrm{~N}_{6}}_{\text {Melamine. }}+\underbrace{\mathrm{C}_{6} \mathrm{~N}_{5} \mathrm{H}_{5} \mathrm{O}_{2}}_{\text {Ammeline. }}+2 \mathrm{KO} .
$$

\section{f. Bases derived from oil of bitter almonds.}

Two of these bases have also been mentioned, and another has since been described. We shall now, as was promised under Amarine and Lophine, give a brief account of these compounds.

Amarine, $\mathrm{C}_{42} \mathrm{H}_{18} \mathrm{~N}_{2}$, is formed from hydrobenzamide, with which it is isomeric or polymeric, by boiling the latter compound with potash. The formation of hydrobenzamide is thus explained :

$$
3 \underbrace{\left(\mathrm{C}_{14} \mathrm{H}_{6} \mathrm{O}_{2}\right.}_{\text {Hyduret of Benzoyle. }})+2 \mathrm{~N} \mathrm{H}_{3}=6 \mathrm{H} \mathrm{O}+\underbrace{\mathrm{C}_{42} \mathrm{H}_{18} \mathrm{~N}_{2}}_{\text {Hydrobenzamide. }}
$$

When hydrobenzamide, a neutral compound, is boiled with potash, a new arrangement of particles takes place, and a base is the result. It is possible that hydrobenzamide is really = $\frac{\mathrm{C}_{42} \mathrm{H}_{18} \mathrm{~N}_{2}}{3}$; or, if we represent $\frac{\mathrm{N}}{3}\left(\frac{1}{3}\right.$ of $\mathrm{N}$ ) by $\mathrm{Az}$, then hydrobenzamide will be $\mathrm{C}_{14} \mathrm{H}_{6} \mathrm{Az}_{2}$; that is hyduret of benzoyle, in which $\mathrm{O}_{2}$ is replaced by $\mathrm{Az}_{2}$. In this case 3 eqs. of hydrobenzamide, $\mathrm{C}_{14} \mathrm{H}_{6} \mathrm{Az}_{2}$, coalesce to form 1 eq. of amarine; $3\left(\mathrm{C}_{14} \mathrm{H}_{6} \mathrm{Az}_{2}\right)=\mathrm{C}_{42} \mathrm{H}_{18} \mathrm{~N}_{2}$.

Amarine forms fine white needles, soluble in hot alcohol, insoluble in water. It has all the characters of a powerful organic base.

Lophine, $\mathrm{C}_{46} \mathrm{H}_{16} \mathrm{~N}_{2}$, is formed when hydrobenzamide is distilled. Ammonia is given off, then a fragrant oil, and a mass is left, from which ether removes a substance not yet examined, leaving the lophine undissolved. Lophine is soluble in alcohol with the aid of acids, and is precipitated by ammonia. It forms fine silky crystals, which possess all the characters of a base. We cannot as yet represent its formation accurately, because the accompanying products have not yet been properly investigated; 
but it is evident that if hydrobenzamide be $\mathrm{C}_{42} \mathrm{H}_{18} \mathrm{~N}_{2}$, then 2 eqs. at least must be required to yield 1 of lophine; and if hydrobenzamide be $\mathrm{C}_{14} \mathrm{H}_{6} \mathrm{Az}_{2}$, as suggested under Amarine, less than 4 eqs. could not furnish 1 of lophine.

By the action of hot nitric acid, lophine yields a yellow crystalline compound, trinitrolophyle, the formula of which is $\mathrm{C}_{46}\left\{\begin{array}{lll}\mathrm{H}_{13} & \\ 3 \mathrm{~N}_{4}\end{array}\right\} \mathrm{N}_{2}$.

Picrine, Syn. Picryle, $\mathrm{C}_{42} \mathrm{H}_{15} \mathrm{~N} \mathrm{O}_{4}$, is formed when the mass produced by acting on oil of bitter almonds by sulphuret of ammonium is distilled. It is one of a large number of products not fully examined, so that its formation cannot be represented in the usual way. It must be produced, however, by the coalescence of 3 eqs. of the oil. It forms colourless octahedrons, insoluble in water. It appears to have the properties of a weak base.

By the action of nitric acid, picrine is converted into a yellow crystalline powder, trinitropicryle, $\mathrm{C}_{42}\left\{\begin{array}{l}\mathrm{H}_{12} \\ 3 \mathrm{~N} \mathrm{O}_{4}\end{array}\right\} \mathrm{N} \mathrm{\textrm {O } _ { 4 }}$.

The formation of the three preceding bases is very important, in reference to the views expressed in the general remarks on the artificial bases, as they are good examples of the power of building up complex molecules from such as are less complex.

\section{g. Base derived from Furfurole.}

Furfurole, $\mathrm{C}_{15} \mathrm{H}_{6} \mathrm{O}_{6}$, is a volatile oil obtained by heating bran with sulphuric acid. When this oil is acted on by ammonia, it yields a crystalline compound, furfurolamide, analogous to hydrobenzamide. Its formula is $\mathrm{C}_{15} \mathrm{NH}_{6} \mathrm{O}_{3}$.

$$
\underbrace{\mathrm{C}_{15} \mathrm{H}_{6} \mathrm{O}_{6}}_{\text {Furfurole. }}+\mathrm{NH}_{3}=3 \mathrm{HO}+\underbrace{\mathrm{C}_{15} \mathrm{H}_{6} \mathrm{~N} \mathrm{O}_{3}}_{\text {Furfurolamide. }}
$$

Furfurine, $\mathrm{C}_{30} \mathrm{H}_{12} \mathrm{~N}_{2} \mathrm{O}_{6}$, is a base, formed when furfurolamide is dissolved in hot potash, just as amarine is formed from hydrobenzamide. Here 2 eqs. of furfurolamide coalesce to form 1 eq. of the base.

Furfurine is a powerful base, soluble in hot water, and crystallisable. It expels ammonia from its salts when heated with them, and forms crystalline salts with acids. In composition it approaches nearly to several vegetable alkaloids, and has the bitter taste and other properties of quinine. Nitrate of furfurine is used as an antiperiodic. 


\section{h. Bases derived from Narcotine.}

These bases, Cotarnine, $\mathrm{C}_{25} \mathrm{H}_{13} \mathrm{~N} \mathrm{O}_{6}$, and Narcogenine, $\mathrm{C}_{36}$ $\mathrm{H}_{19} \mathrm{~N} \mathrm{O}_{10}$, will be presently described under Narcotine.

\section{i. Bases derived from Animal products.}

Glycocine. SrN. Glycocoll, Sugar of gelatine, $\mathrm{C}_{4} \mathrm{~N} \mathrm{H}_{5} \mathrm{O}_{4}-$ This remarkable compound was first obtained among the products of the decomposition of gelatine by boiling with potash or with acids. But Dessaignes has lately discovered that it is best prepared by heating hippuric acid with hydrochloric acid, when water is taken up, and benzoic acid is the only other product.

$$
\underbrace{\mathrm{C}_{18} \mathrm{~N} \mathrm{H}_{9} \mathrm{O}_{6}}_{\text {Hydrated hippuric acid. }}+2 \mathrm{HO}=\underbrace{\mathrm{C}_{14} \mathrm{H}_{6} \mathrm{O}_{4}}_{\text {Hydrated benzoic acid. }}+\underbrace{\mathrm{C}_{4} \mathrm{~N} \mathrm{H}_{5} \mathrm{O}_{4}}_{\text {Hydrated glycocine. }}
$$

It forms large transparent erystals, soluble in water, and very sweet. It has the properties of a base, and neutralises acids, forming beautifully crystallised salts; but Horsford has shown that it also combines with bases and neutral bodies. By its composition, it is closely related to many different compounds, and probably plays an important part in the animal organism.

Glycocoll is one of a series of homologous compounds, of which two others are known. One of these is alanine, already mentioned under aldehydammonia, where a table of these compounds is given. Glycocine may be supposed to be formed from hydrated oxide of formyle, or formic aldehyde, by the action of hydrocyanic acid. Thus, $\mathrm{C}_{2} \mathrm{H} \mathrm{O}, \mathrm{HO}+\mathrm{C}_{2} \mathrm{NH}+2 \mathrm{H} \mathrm{O}_{2}=\mathrm{C}_{4} \mathrm{~N} \mathrm{H}_{5} \mathrm{O}_{4}$.

Alanine, $\mathrm{C}_{6} \mathrm{~N} \mathrm{H}_{7} \mathrm{O}_{4}$, differs from glycocine by $\mathrm{C}_{2} \mathrm{H}_{2}$. It is formed when aldehydammonia is acted on by hydrocyanio and hydrochloric acids. The latter takes the ammonia, and the aldehyde and hydrocyanic acid act on each other with the aid of water; $\mathrm{C}_{4} \mathrm{H}_{3} \mathrm{O}, \mathrm{HO}+\mathrm{C}_{2} \mathrm{NH}+2 \mathrm{HO}=\mathrm{C}_{6} \mathrm{~N} \mathrm{H}_{7} \mathrm{O}_{4}$. Alanine is in all respects analogous to glycocine, and like it, exhibits both basic, acid, and even neutral characters. Its most remarkable character, however, is that when acted on by hyponitrous acid, it yields lactic acid, $\mathrm{C}_{6} \mathrm{~N} \mathrm{H}_{7} \mathrm{O}_{4}+\mathrm{N} \mathrm{O}_{3}=\mathrm{H} \mathrm{O}+$ $\mathrm{N}_{2}+\mathrm{C}_{6} \mathrm{H}_{5} \mathrm{O}_{5}, \mathrm{H} \mathrm{O}$. It is probable that lactic acid is only one of a homulogous series of acids, and that glycocine, treated by hyponitrous acid, may yield the acid below lactic acid, which would be $\mathrm{C}_{4} \mathrm{H}_{3} \mathrm{O}_{5}, \mathrm{HO}=\mathrm{C}_{4} \mathrm{H}_{4} \mathrm{O}_{6}$. Now this acid has actually been obtained from hippuric acid by Socoloff and Strecker, and is called Glycolic acid. Its formation from glycocine is as follows: 
$\mathrm{C}_{4} \mathrm{NH}_{3} \mathrm{O}_{4}+\mathrm{N} \mathrm{O}_{3}=2 \mathrm{HO}_{4}+\mathrm{C}_{4} \mathrm{H}_{4} \mathrm{O}_{6}$. It resembles lactic acid, and will be more fully described along with that acid. Here we may state that glycolic acid and lactic acid may be regarded as formic acid, coupled with formic aldehyde and acetic aldehyde, and that glycocine and alanine may be regarded as amides of these coupled acids. Thus we have

Glycolic Acid $\mathrm{C}_{4} \mathrm{H}_{3} \mathrm{O}_{5}, \mathrm{HO}=$

Lactic Acid $\mathrm{C}_{6} \mathrm{H}_{5} \mathrm{O}_{5}, \mathrm{HO}=$

Glycocine . $\mathrm{C}_{4} \mathrm{~N} \mathrm{H}_{5} \mathrm{O}_{4}=\mathrm{N} \mathrm{H}_{4} \mathrm{O}+\mathrm{C}_{4} \mathrm{H}_{3} \mathrm{O}_{5}-2 \mathrm{HO}=$ Glycolamide Alanine. . $\mathrm{C}_{6} \mathrm{~N} \mathrm{H}_{7} \mathrm{O}_{4}=\mathrm{NH}_{4} \mathrm{O}+\mathrm{C}_{6} \mathrm{H}_{5} \mathrm{O}_{5}-2 \mathrm{HO}=$ Lactamide

But yet alanine is not identical with lactamide, and is isomeric not only with that body, but with sarcosine, and with carbamate of oxide of ethyle or urethane.

Leucine, $\mathrm{C}_{22} \mathrm{~N} \mathrm{H}_{13} \mathrm{O}_{4}$, the only other homologue of glycocine yet known, may be viewed as the amide of an acid containing 1 eq. of the aldehyde of valerianic acid coupled with formic acid. This acid is, therefore, $\mathrm{C}_{2} \mathrm{H}_{2} \mathrm{O}_{4}+\mathrm{C}_{10} \mathrm{H}_{10} \mathrm{O}_{2}$. Leucine is obtained by the action of potash on animal matter, and resembles glycocine and alanine. It crystallises, and combines both with acids and bases. It will probably yield with hyponitrous acid, the acid $\mathrm{C}_{12} \mathrm{H}_{11} \mathrm{O}_{5}, \mathrm{H} \mathrm{O}$, resembling lactic acid; and other products, homologous with those derived from glycocine or connected with it, such as hippuric acid (which see).

Sarcosine, $\mathrm{C}_{6} \mathrm{~N} \mathrm{H}_{7} \mathrm{O}_{4}$. - This base is formed, along with urea, when kreatine is boiled with baryta ; but the urea is resolved into carbonic acid and ammonia.

$$
\mathrm{C}_{8} \mathrm{~N}_{3} \mathrm{H}_{11} \mathrm{O}_{6}+2 \mathrm{BaO}+2 \mathrm{HO}=2\left(\mathrm{BaO}, \mathrm{CO}_{2}\right)+2 \mathrm{NH}_{3}+
$$

Hydrated Kreatine.

$$
\underbrace{\mathrm{C}_{6} \mathrm{~N} \mathrm{H}_{7} \mathrm{O}_{4}}_{\text {Sarcosine. }}
$$

Sarcosine forms right rhombic prisms, soluble in water, fusible, and volatile at a heat not much beyond $212^{\circ}$. It neutralises acids, and has all the characters of an alkaloid. It is isomeric with urethane, or carbamate of oxide of ethyle, with lactamide or anhydrous lactate of ammonia, and, as above mentioned, with alanine.

Kreatinine, $\mathrm{C}_{8} \mathrm{~N}_{3} \mathrm{H}_{7} \mathrm{O}_{2}$. - This base occurs in the juice of flesh, and in urine (Liebig); but it was first observed as a product of the action of acids on kreatine. When kreatine is boiled with hydrochloric acid, 4 eqs. of water are given off, and the new base is found combined with the acid. 


$$
\underbrace{\mathrm{C}_{8} \mathrm{~N}_{3} \mathrm{H}_{11} \mathrm{O}_{6}}_{\text {Kreatine. }}+\mathrm{H} \mathrm{Cl}=4 \mathrm{HO}+\underbrace{\left(\mathrm{C}_{8} \mathrm{~N}_{3} \mathrm{H}_{7} \mathrm{O}_{2}+\mathrm{H} \mathrm{Cl}\right.}_{\text {Hydrochlorate of Kreatinine. }})
$$

Kreatinine is separated from the hydrochlorate by means of oxide of lead. It forms prisms, soluble in water and alcohol. It combines with acids, yielding crystallisable salts, and it has all the characters of a powerful organic base.

Kreatinine is important, as occurring in the animal organism. Under the urine and the juice of flesh we shall return to it. The substance discovered by Pettenkofer in urine, is, according to Liebig, a mixture of kreatinine with kreatine.

We have now gone over the artificial organic bases, so far as they have not been mentioned in previous sections. It will be seen that these compounds exhibit a great variety in their composition, as well as in their properties, and that they are formed in many different processes. But they all agree in containing nitrogen, and may be divided into two classes, according to the presence or absence of oxygen. The non-oxygenised bases are liquids or fusible solids, volatile without decomposition, and, whether as amidic, imidic, or nitrylic bases, may be regarded as representatives of ammonia. The oxygenised bases, on the other hand, are destroyed by heat, and generally yield one or more volatile bases when heated. We see, also, that the greater number of artificial oxygenised bases, such as picrine, furfurine, and the hydrated oxides of tetramethylium and its congeners, are bitter to the taste. These are to be referred to oxide of ammonium as the type of their composition, but as that is not permanent, to the hydrated oxide of tetramethylium as the type of their properties. This class of bases is likely to admit of useful application in medicine, as is already the case with furfurine, and as the bitter taste and strong affinities of the oxides of tetramethylium and its congeners renders probable in their case. The mild bases, such as kreatinine, sarcosine, glycocine, and alanine, and the sulphurised bases from oil of mustard, have probably a somewhat different constitution.

\section{ACTION OF HYPONITROUS ACTD ON ARTIFICIAL BASES.}

It is necessary here to notice the remarkable change produced on compounds of the olass of organic bases, and on others derived from ammonia, such as amides, \&c., by hyponitrous acid. The reaction is attended by the formation of water, of free nitrogen, 
and of an oxidised product. We have mentioned some examples of it incidentally, as that of the conversion of alanine into lactic acid; but this peculiar method of oxidation seems destined very much to extend our power of artificially producing or reproducing organic bodies. Piria has shown that oxamide, succinamide, butyramide, and asparamide or malamide, are thus converted into oxalic, succinic, butyric, and malic acids. Let $\mathrm{N} \mathrm{H}_{2} \mathrm{X}$ be $=$ any amide. Then $\mathrm{N}_{2} \mathrm{X}+\mathrm{N} \mathrm{O}_{3}=2 \mathrm{H} \mathrm{O}+\mathrm{N}_{2}+\mathrm{XO}$. And in the case of oxamide we have $\mathrm{N} \mathrm{H}_{2}, \mathrm{C}_{2} \mathrm{O}_{2}+\mathrm{N} \mathrm{O}_{3}=$ $2 \mathrm{HO}+\mathrm{N}_{2}+\mathrm{C}_{2} \mathrm{O}_{3}$. Strecker has found that hippuric acid, glycocine, alanine, and leucine, are thus converted into the acids $\mathrm{C}_{18} \mathrm{H}_{8} \mathrm{O}_{5}, \mathrm{C}_{4} \mathrm{H}_{4} \mathrm{O}_{6}, \mathrm{C}_{6} \mathrm{H}_{6} \mathrm{H}_{6}$, and $\mathrm{C}_{12} \mathrm{H}_{12} \mathrm{O}_{6}$. Now it is important to know what is the result of the action of hyponitrous acid on the bases. Hunt found that aniline, when its hydrochlorate is warmed with hyponitrite of silver, yields chloride of silver, nitrogen, and hydrated oxide of phenyle, exactly as might be expected. $\mathrm{C}_{12} \mathrm{H}_{7} \mathrm{~N}, \mathrm{H} \mathrm{Cl}+\mathrm{Ag} \mathrm{O}, \mathrm{N} \mathrm{O}_{3}=$ $\mathrm{Ag} \mathrm{Cl}+\mathrm{N}_{2}+\mathrm{C}_{12} \mathrm{H}_{5} \mathrm{O}, \mathrm{HO}+2 \mathrm{HO}$. Now this compound bears to aniline the same relation as alcohol does to ethylamine, and it was therefore to be expected that ethylamine with $\mathrm{N}_{3}$ would yield alcohol, $\mathrm{C}_{4} \mathrm{H}_{7} \mathrm{~N}+\mathrm{N} \mathrm{O}_{3}=\mathrm{HO}+\mathrm{N}_{2}=\mathrm{C}_{4} \mathrm{H}_{5} \mathrm{O}, \mathrm{HO}$. But Hofmann has found the reaction to be somewhat different; 2 eqs. of $\mathrm{N}_{3}$ act on 1 eq. of ethylamine, and give rise to hyponitrite of oxide of ethyle, $\mathrm{C}_{4} \mathrm{H}_{7} \mathrm{~N}+2 \mathrm{~N} \mathrm{O}_{3}=\left(\mathrm{C}_{4} \mathrm{H}_{5} \mathrm{O}, \mathrm{N} \mathrm{O} \mathrm{O}_{3}\right)$ $+\mathrm{N}_{2}+2 \mathrm{H} \mathrm{O}$. Here, then, we have formed from the base, not the hydrated oxide, but the anhydrous oxide of the radical ethyle, which has combined with the second eq. of $\mathrm{N} \mathrm{O}_{3}$. But from the hyponitrite the alcohol or hydrated oxide may be obtained, by the action of sulphohydrate of potassium, $\left(\mathrm{C}_{4} \mathrm{H}_{5} \mathrm{O}, \mathrm{N} \mathrm{\textrm {O } _ { 3 }}\right)+6$ $(\mathrm{K} \mathrm{S}, \mathrm{H} \mathrm{S})=\left(\mathrm{C}_{4} \mathrm{H}_{5} \mathrm{O}, \mathrm{H} \mathrm{O}\right)+\mathrm{N} \mathrm{H}_{3}+2 \mathrm{H} \mathrm{O}+6 \mathrm{~K} \mathrm{~S}_{2}$. Hence it will be possible, if we can obtain any base, the alcohol of which is unknown, to obtain the alcohol as above. Hofmann has done so with amylamine and propylamine, as well as ethylamine, or has shown that it may be done, although he has not yet described the propylic alcohol. But there can now be no doubt, that if we could obtain propylamine, butylamine, caprotylamine, or any of the bases of that series, in considerable quantity, as we may one day be able to do, from the oil of tar or animal oil, we shall then be able to obtain the hyponitrites, and from these the hydrates, of the oxides, $\mathrm{C}_{6} \mathrm{H}_{7} \mathrm{O}, \mathrm{C}_{8} \mathrm{H}_{9} \mathrm{O}, \mathrm{C}_{12} \mathrm{H}_{13} \mathrm{O}$, \&c.; that is, the propylic, butylic, and caprotylio alcohols. In general, the action of hyponitrous acid on all the known artificial bases should be carefully studied.

Let us now turn to the natural organic bases. 


\section{Natural Alkaloids or Vegetable Bases.}

The alkaloids occur in combination, generally with vegetable acids; and they are separated from these combinations by the same means which are employed in the case of inorganic bases, modified in each case, according as the alkaloid is soluble or insoluble in water and other solvents, fixed or volatile when heated. Thus quinine, morphine, and strychnine, are separated by adding to their soluble salts, lime, ammonia, or magnesia, which form soluble saits with the acids which are present, while the alkaloids, being insoluble, are precipitated ; codeine, being soluble in ether as well as water, is first set free by potash, and ether being added to the aqueous liquid, is agitated with it, and rises to the surface, carrying the codeine along with it, and the same process applies to other alkaloids. Lastly, coniine, nicotine, and other volatile alkaloids, are obtained by distilling their salts with an excess of liquor potassæ.

The alkaloids, like ammonia, combine with hydrogen acids, forming salts, without the addition of water or its elements being necessary; some, also, like ammonia, refuse to combine with anhydrous oxygen acids, requiring 1 eq. of water to form dry salts. Their hydrochlorates, like sal ammoniac, form double salts with the bichlorides of platinum and mercury.

The salts of most of the alkaloids are precipitated as tannates by infusion of galls.

The alkaloids are generally decomposed by chlorine, bromine, and iodine, forming substitution-products, some of which have been described. They are also decomposed by nitric acid, some of them with a deep red colour.

Some of them, such as quinine, strychnine, \&c., when heated with strong caustic potash, yield oily compounds, which are also bases, such as quinoline, methylamine, ethylamine, \&c.

We shall now briefly describe the individual alkaloids, dividing them into groups, according to their characters.

\section{a. Liquid Volatile Bases.}

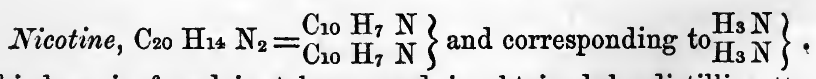
This base is found in tobacco, and is obtained by distilling the concentrated infusion of the leaves along with potash. The distilled liquid, which contains nicotine, water, and ammonia, is neutralised by sulphuric acid and the neutral solution dried up. Alcohol then dissolves the sulphate of nicotine, leaving undissolved the sulphate of ammonia. The pure sulphate, distilled with potash, 
yields pure nicotine, which appears as an oily, limpid, colourless liquid, having a weak smell of tobacco. Its Sp. G. is 1.048. It is decidedly alkaline, and mixes with water, alcohol, and ether. It is highly poisonous. With acids it forms salts which crystallise with difficulty. The hydrochlorate of nicotine combines with bichloride of platinum, forming a double salt, which yields large, regular, orange-red crystals, of the formula $\mathrm{C}_{20} \mathrm{H}_{14} \mathrm{~N}_{2}, 2(\mathrm{H} \mathrm{Cl}$, $\mathrm{Pt} \mathrm{Cl}_{2}$ ). It is probable that nicotine, besides being found in the fresh leaves of tobacco, is produced in larger quantity during the fermentation to which the leaves are subjected in the manufacture of tobacco; and there is also reason to believe that it is produced by the action of heat on tobacco, as in smoking, and that, from the comparative simplicity of its formula, it will be found among the products of the distillation of organic compounds containing nitrogen.

The Comte de Bocarmé, lately executed for the murder of his wife's brother, poisoned him by pouring into his mouth Nicotine, of which he had prepared, it is said, several pints.

According to Kekule and Planta, nicotine represents two molecules of ammonia $\left.\mathrm{N} \mathrm{H}_{3} \mathrm{NH}_{3}\right\}$ in which $\mathrm{H}_{3}$ is replaced by the group $\mathrm{C}_{10} \mathrm{H}_{7}$. It is no doubt from this peculiar constitution that nicotine does not yield compounds analogous to amides and anilides.

The salts of nicotine, especially its double salts, crystallise well.

Iodonicotine, $2 \mathrm{C}_{20} \mathrm{H}_{14} \mathrm{~N}_{2}+\mathrm{I}_{3}$ is formed when ethereal solutions of iodine and nicotine are mixed. It forms fine ruby-red acicular crystals, fusible at $212^{\circ}$. It is a base, and with hydrochlorio acid forms a salt, which crystallises in fine scales of a pale ruby-red.

As in nicotine, the 3 eq. of hydrogen of ammonia are all replaced by the group $\mathrm{C}_{10} \mathrm{H}_{7}$, so we cannot obtain any volatile imide or amide bases by substituting ethyle, methyle, \&c., for hydrogen. But we can obtain ammonium bases by adding methyle to nicotine, forming a group like ammonium or tetrethylium.

Hydrated oxide of Methylonicotium, $\left.\mathrm{N}_{\mathrm{C}_{2} \mathrm{H}_{3}}^{\mathrm{C}_{2} \mathrm{H}_{7}}\right\} 0, \mathrm{H} \mathrm{O}$, is formed when iodide or bromide of methyle acts on nicotine, and the iodide or bromide of methylonicotium thus formed is decomposed by oxide of silver. The hydrate does not crystallise, but forms a viscid mass, soluble in water, caustic, alkaline, and very bitter. Its salts are soluble.

Hydrated Oxide of Ethylonicotium $\left.\mathrm{N}_{\mathrm{C}_{4}}^{\mathrm{C}_{10} \mathrm{H}_{7}} \mathrm{H}_{5}\right\} 0, \mathrm{H} \quad \mathrm{O}$, is formed like the preceding base. This base forms a radiated crystalline mass, very soluble in water, alkaline, caustic, and 
bitter, attracting carbonic acid from the air, as does also the preceding one. In short, both of them, like Hofmann's ammonium bases, very much resemble caustic potash, and are at the same time bitter like quinine.

\section{Hydrated Oxide of Amylonicotium, $\left.\mathrm{N} \underset{\mathrm{C}_{10} \mathrm{H}_{11}}{\mathrm{C}_{10} \mathrm{H}_{7}}\right\} 0, \mathrm{H} \quad 0$, is} obtained like the two preceding bases, and has similar characters.

It will be seen, that while nicotine itself represents a double molecule of ammonia, the $\mathrm{H}_{3}$ of each alone being replaced by the group $\mathrm{C}_{10} \mathrm{H}_{7}$, in these new bases, this group occurs singly. This is ascertained by the neutralising power. $\mathrm{C}_{20} \mathrm{H}_{14} \mathrm{~N}_{2}$ (Nicotine) neutralises as much acid as $2 \mathrm{~N} \mathrm{H}$ H $_{3}$ but $\left.\mathrm{N}_{\mathrm{C}_{4} \mathrm{H}_{5}}^{\mathrm{C}_{3} \mathrm{H}_{7}}\right\}$, $\mathrm{H} \mathrm{O}$, or $\mathrm{C}_{14} \mathrm{H}_{13} \mathrm{~N} \mathrm{O}, \mathrm{H} \mathrm{O}$, the hydrated oxide of ethylonicotium, neutralises the same weight of acid as $\mathrm{N} \mathrm{H}_{3}$ or $\mathrm{N} \mathrm{H}_{4} \mathrm{O}, \mathrm{HO}$.

Coniine. Srn. Conia, $\mathrm{C}_{16} \mathrm{H}_{15} \mathrm{~N}$. This base occurs in the hemlock, Conium maculatum, and is extracted by a process quite analogous to that above described for nicotine. It is also an oily liquid, boiling at $338^{\circ}$, highly poisonous, and easily decomposed. Its taste and smell are both very acrid and disagreeable, and somewhat analogous to those of nicotine. Its salts are acrid and poisonous, crystallising with difficulty. As it is the active principle of the conium, coniine, either pure or as a salt, ought to be used instead of the extract or tincture, which are very variable.

Kekule and Planta have shown that the coniine, extracted from hemlock, is a mixture of two or more homologous bases, namely, coniine, $\mathrm{C}_{16} \mathrm{H}_{15} \mathrm{~N}$; methyloconiine, $\mathrm{C}_{18} \mathrm{H}_{17} \mathrm{~N}$; and perhaps ethyloconiine, $\mathrm{C}_{20} \mathrm{H}_{10} \mathrm{~N}$. Both the two latter bases may be formed from coniine, and as they are still homologous with $\mathrm{N} \mathrm{H}_{3}$ and volatile, it is clear that coniine has not, like nicotine, all the 3 eqs. of hydrogen replaced, but only part of them; according to Kekulé and Planta, 2 eqs. are replaced by the group $\mathrm{C}_{16} \mathrm{H}_{14}$. The new bases are therefore nitryle bases, like ethylodimethylamine and others, while coniine is an imide base.

The salts of coniine are soluble, but do not in general crystallise easily.

Coniine appears to form substitution-products with chlorine and bromine, which crystallise, but are not well known as yet.

Methyloconiine, $\left.\mathrm{N}_{\mathrm{C}_{2} \mathrm{H}_{3}}^{\mathrm{C}_{16} \mathrm{H}_{14}}\right\}=\mathrm{C}_{18} \mathrm{H}_{17} \mathrm{~N}$, is found in the coniine of commerce, and may be obtained by the action of iodide of methyle on coniine, or by that of heat on hydrated oxide of ethylomethyloconium. It is very similar to coniine.

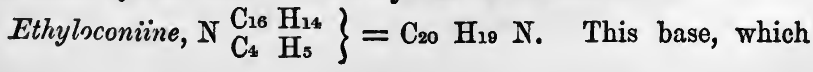


may perhaps occur with the two preceding, in some specimens of coniine, to judge by the discordant results of the analyses made when coniine was supposed to occur alone, is best procured by the action of iodide of ethyle on coniine. It is a basic oil, very like coniine. Its salts also are analogous to those of coniine and methyloconiine.

\section{Hydrated oxide of ethylomethyloconium $\left.\begin{array}{ll}\mathrm{N}_{16} & \stackrel{\mathrm{C}_{2}}{\mathrm{C}_{24}} \\ \mathrm{C}_{4} & \mathrm{H}_{3} \\ \mathrm{H}_{5}\end{array}\right\} 0, \mathrm{HO}=$}

$\mathrm{C}_{22} \mathrm{H}_{23} \mathrm{~N} \mathrm{O}_{2}$. This ammonium base is formed when iodide of ethyle acts on methyloconiine. The iodide of ethylomethyloconium thus formed, yields splendid crystals, and the solution of these acted on by oxide of silver, gives the hydrated oxide, which does not crystallise readily, but is very alkaline, caustic, and bitter. When heated, it yields water, olefiant gas, and methyloconiine.

\section{Hydrated oxide of diethyloconium, $\left.\begin{array}{ccc}\mathrm{N}_{16}^{\mathrm{C}_{4}} & \mathrm{H}_{14}^{\mathrm{H}_{5}} \\ \mathrm{C}_{4} & \mathrm{H}_{5}\end{array}\right\} \mathrm{H}, \mathrm{H} \mathrm{O}=\mathrm{C}_{24}$}

$\mathrm{H}_{25} \mathrm{NO}_{2}$. This base is formed like the last, by acting on ethyloconiine by iodide of ethyle, and decomposing the iodide with oxide of silver. The hydrate is very alkaline, caustic, and bitter, and is resolved by heat into olefiant gas, water, and ethyloconiine.

We see that in nicotine, the group $\mathrm{C}_{10} \mathrm{H}_{7}$ replaces $\mathrm{H}_{3}$, whether $\mathrm{C}_{10} \mathrm{H}_{7}$ be a single or a triple group, as for example $\left(\mathrm{C}_{2} \mathrm{H}, \mathrm{C}_{4}\right.$ $\left.\mathrm{H}_{4}, \mathrm{C}_{4} \mathrm{H}_{2}\right)$; while in coniine, the group $\mathrm{C}_{16} \mathrm{H}_{14}$ replaces $\mathrm{H}_{2}$, whether $\mathrm{C}_{16} \mathrm{H}_{14}$ be single, or double, as $\left(\mathrm{C}_{10} \mathrm{H}_{7}, \mathrm{C}_{6} \mathrm{H}_{7}\right)$ or some other combination. Consequently nicotine is a nitryle base, like trimethylamine, and coniine an imide base, like dimethylamine.

Sparteine, $\mathrm{C}_{16} \mathrm{H}_{13} \mathrm{~N}$. This base occurs in broom (Spartium scoparium). It is a rather rancid oil, of a very bitter taste. It is decomposed by hot nitric acid, yielding when chloride of lime is added to the product, chloropicrine, Boiling hydrochloric acid decomposes it with an odour of mice. Its salts are little known. (Stenhouse.)

The three bases just described are the only natural bases belonging to the strongly-marked group of volatile oily bases. But several artificial compounds are known, which belong to this group. These have been described in their proper place, and it will be seen that their analogy to nicotine and coniine is perfect.

\section{b. Bases of Cinchona Bark.}

a. Quinine. $\mathrm{C}_{20} \mathrm{H}_{12} \mathrm{~N} \mathrm{O}_{2}$, or $\mathrm{C}_{40} \mathrm{H}_{24} \mathrm{~N}_{2} \mathrm{O}_{4}+n$ aq. SrN. Chinine. This important alkaloid is found along with cincho- 
nine, in most species of cinchona bark. It predominates in yellow bark, Cinchona fava, China regia, or C. calisaya; and is obtained by boiling with an excess of milk of lime the decoction in diluted hydrochloric acid of the bark, and treating the precipitate with hot alcohol, which dissolves cinchonine and quinine. On evaporation, the cinchonine is deposited in crystals and the quinine remains dissolved. Water is added, which causes the quinine to separate as a resinous mass. It may be obtained in crystals as a hydrate with 6 aq. There is another crystalline hydrate with 2 aq., by the spontaneous evaporation of its solution in absolute alcohol. It is very sparingly soluble in water, but very soluble in alcohol and in acids. Its solutions are very bitter. When heated with hydrate of potash, it yields carbonate of potash, hydrogen gas, and quinoline or leucoline (see p. 440 et seq.)

Quinine is decidedly alkaline, and neutralises the acids. Its salts, especially the sulphate, are very much used in medicine, especially as febrifuge and tonic remedies, in most cases very superior to the bark in substance. The sulphate of quinine used in medicine is $2\left(\mathrm{C}_{40} \mathrm{H}_{24} \mathrm{~N}_{2} \mathrm{O}_{4}\right), 2 \mathrm{~S} \mathrm{O}_{3}, 2 \mathrm{HO}+14$ aq. The acid sulphate, $\mathrm{C}_{40} \mathrm{H}_{24} \mathrm{~N}_{2} \mathrm{O}_{4}, 2 \mathrm{~S} \mathrm{O}_{3}, 2 \mathrm{H} \mathrm{O}+14$ aq., is much more soluble in water; hence, in draughts, sulphate of quinine is generally dissolved in diluted sulphuric acid. The hydrochlorate, phosphate, citrate, and ferrocyanate of quinine have also been employed in medicine.

Methyloquinium, $\left.\mathrm{C}_{40} \underset{\mathrm{C}_{2} \mathrm{H}_{3}}{\mathrm{H}_{24}}\right\} \mathrm{N}_{2} \mathrm{O}_{4}$. This compound, homologous with ammonium, is obtained as iodide, when iodide of methyle acts on quinine. From the iodide, by the usual means of oxide of silver, the hydrated oxide is obtained, which is probably a strong base, but has not been fully described.

Ethyloquinium, $\left.\mathrm{C}_{40} \mathrm{C}_{4} \mathrm{H}_{5}\right\} \mathrm{N}_{2} \mathrm{O}_{4}$ is formed when iodide of ethyle acts on quinine. The hydrated oxide, prepared from the iodide by oxide of silver, is an energetic base, which attracts carbonic acid from the air. The preceding base is said to resemble it, which is no doubt true.

We may here mention the remarkable salt discovered by $\mathbf{M r}$. Herapath, which is formed when bisulphate of quinine is dissolved in strong acetic acid, warming the solution, and an alcoholic solution of iodine added drop by drop. The mixture being allowed to stand in a quiet place, deposits large hexagonal plates, which by reflected light are emerald green, with metallic lustre like the elytra of the golden beetle. By transmitted light, they have only a faint olive colour. If two of these plates be super- 
posed, so that their larger diameters are at right angles, no light passes through, even when the crystals are no thicker than $\frac{1}{500}$ th of an inch. This is exactly what happens with two tourmalines of which the axes are crossed. If polarised light be used, it passes through, giving to the uncovered part of each plate, complementary colours, such as green and rose-colour, while the double or covered part appears of a deep chocolate brown. Hence these crystals may be used as polarisers and depolarisers, and they have the advantage of allowing far more light to pass through than tourmalines.

The composition of this salt is $\mathrm{C}_{40} \mathrm{H}_{24} \mathrm{~N}_{2} \mathrm{O}_{4}, \mathrm{I}_{2}, 2 \mathrm{~S} \mathrm{O}_{3}, 2 \mathrm{H} \mathrm{O}$ +10 aq., and it may be considered as the bisulphate of iodoquinine, a base composed of quinine +2 eqs. of iodine. Indeed such a compound is formed when iodine is triturated with quinine, as an amorphous brown mass, very similar to iodocinchonine.

b. Quinidine, $\mathrm{C}_{40} \mathrm{H}_{24} \mathrm{~N}_{2} \mathrm{O}_{4}+4$ aq. This base, isomeric with quinine, is obtained from what is called in commerce quinoidine, which is an amorphous basic substance, found in the mother liquors of quinine. This is a mixture of quinine and quinidine. The latter is much less soluble in ether than quinine, and crystallises when pure with great facility, in large rhombic prisms, which effloresce in the air. It differs from quinine also, in its action on polarised light, for quinine causes deviation to the left, quinidine to the right. The neutral sulphate of quinidine is like that of quinine, and may be used for the same purposes.

c. Quinicine, $\mathrm{C}_{40} \mathrm{H}_{24} \mathrm{~N}_{2} \mathrm{O}_{4}$. This base, also isomerio with dry quinine, is formed when the salts of quinine or of quinidine are heated for some time to about $248^{\circ} \mathrm{F}$. It is bitter and febrifuge, insoluble in water, very soluble in alcohol, and causes deviation of the plane of polarisation to the right. It does not appear to crystallise.

d. Cinchonine, $\mathrm{C}_{40} \mathrm{H}_{24} \mathrm{~N}_{2} \mathrm{O}_{2}$. This base predominates in the grey bark Cinchona condaminea, or C. rubiginosa, and is also found in large quantity, as well as quinine, in red bark, $C$. oblongifolia. Its preparation has been above described. It crystallises very readily, and is not so bitter as quinine, although highly febrifuge. When heated, a considerable part is sublimed. When distilled with potash, it yields quinoline. It neutralises the acids, forming crystallisable salts, which may be substituted for those of quinine.

It is very important to observe that cinchonine only. differs from quinine by 2 eq. oxygen; and although hitherto no one has succeeded in converting one into the other, little doubt can be entertained that this will be accomplished in process of time. 
The fact that both yield quinoline is very interesting. Cinchonine yields with chlorine and bromine substitution-bases, chlorocinchonine and bromocinchonine, \&c., in which 1 eq. of hydrogen is replaced by chlorine or bromine.

Quinine and Cinchonine may be distinguished in solutions by adding first chlorine water, so as to make the liquid yellow, and then a little ammonia, which strikes a green colour with quinine, but not with cinchonine. If too much ammonia have not been added, the green colour changes to violet on the addition of a little more chlorine.

e. Cinchonidine, $\mathrm{C}_{40} \mathrm{H}_{24} \mathrm{~N}_{2} \mathrm{O}_{2}$. This base, isomeric with cinchonine, occurs in a bark resembling the Huamalies cinchona bark, also in the Cinchona bark of Maracaibo, and in that called of Bogota. It is extracted by the same process. It forms hard, brilliant, rhomboidal prisms, striated on the faces. They are soluble in alcohol, very sparingly soluble in water or ether. Both this base and cinchonine cause the plane of polarisation to deviate to the right.

f. Cinchinicine, $\mathrm{C}_{40} \mathrm{H}_{24} \mathrm{~N}_{2} \mathrm{O}_{2}$. This base, isomeric with the two preceding, is formed by the action of heat on the sulphate of cinchonine. It is insoluble in water, soluble in alcohol and amorphous, bitter and febrifuge, causing deviation of the plane of polarisation to the right.

Bichlorocinchonine, $\left.\mathrm{C}_{40} \underset{\mathrm{Cl}_{2}}{\mathrm{H}_{22}}\right\} \mathrm{N}_{2} \mathrm{O}_{2}$. This base in which chlorine replaces 2 eqs. of hydrogen in cinchonine, is formed when chlorine is passed through a solution of the acid hydrochlorate of cinchonine. It is precipitated by ammonia, and erystallises from alcohol in minute prisms. It forms salts with acids.

Bromocinchonine, $\left.\mathrm{C}_{40} \underset{\mathrm{Br}}{\mathrm{H}_{23}}\right\} \mathrm{N}_{2} \mathrm{O}_{2}$, is formed by the action of bromine on the acid hydrochlorate of cinchonine. It forms scaly crystals and produces salts with some acids.

Sesquibromocinchonine, $\mathrm{C}_{40} \mathrm{H}_{\frac{4}{2}} \mathrm{Br}_{\frac{2}{3}}^{3} \mathrm{~N}_{2} \mathrm{O}_{2}$, is formed along with the preceding base. It may be obtained in slender acicular crystals, and has a feeble bitter taste, but is alkaline. Its salts crystallise readily. The formula appears strange, but it is intended to show that the $24 \mathrm{eqs}$. of hydrogen in cinchonine are here made up in the proportion of $22 \frac{1}{2}$ to $1 \frac{1}{2}$ of hydrogen and bromine. $\mathrm{H}_{\frac{4}{2}}$ is $=\mathrm{H}_{22} \frac{1}{2}$ and $\mathrm{Br} \frac{3}{2}$ is $=\mathrm{Br} 1 \frac{1}{2}$; the latter expressions, however, are never used, as being inconsistent with the very notion of atoms. It is not easy, however, to see how the fractions $\frac{45}{2}$ and $\frac{3}{2}$ are less inconsistent with that notion. The fact is, that equivalents are probably never single atoms, but groups, 
and such groups may be supposed to be divided, which atoms cannot be.

Bibromocinchonine, $\left.\mathrm{C}_{40} \underset{\mathrm{Br}_{2}}{\mathrm{H}_{22}}\right\} \mathrm{N}_{2} \mathrm{O}_{2}$ is formed like the two preceding, using an excess of bromine. It is separated by ammonia, and forms pearly needles, and sometimes octohedral crystals, the latter being a hydrate with $4 \mathrm{aq}$. It is also basic.

Iodocinchonine, $2\left(\mathrm{C}_{40} \mathrm{H}_{24} \mathrm{~N}_{2} \mathrm{O}_{2}\right)+\mathrm{I}_{2}$, is not a substitutionproduct like the preceding four bases, but is cinchonine plus iodine. It is formed when the two are triturated together. Alcohol dissolves the mass, and by spontaneous evaporation deposits, first, saffron-coloured tabular crystals of iodocinchonine, and then hydriodate of cinchonine. The former is insoluble in barley-water, which dissolves the latter. It is slightly bitter, soluble in alcohol and ether.

Methylocinchonium, $\left.\mathrm{C}_{40} \underset{\mathrm{C}_{2} \mathrm{H}_{3}}{\mathrm{H}_{24}}\right\} \mathrm{N}_{2} \mathrm{O}_{2}$, is obtained as iodide when iodide of methyle acts on cinchonine. Treated with oxide of silver, this salt yields the hydrated oxide, which is a strong base, soluble in water, homologous with hydrated oxide of tetramethylium. Its salts are very soluble and do not readily crystallise.

Methylocinchonidium. This is the radical of another strong base isomeric with the preceding, and its iodide and hydrated oxide are obtained in the same way, from cinchonidine, and resemble those of methylocinchonium.

Quinine, cinchonine, quinidine, cinchonidine, quinicine, and cinchonicine, all, when heated with potash, yield quinoline, a volatile oily base, already alluded to.

g. Aricine, $\mathrm{C}_{46} \mathrm{H}_{26} \mathrm{~N}_{2} \mathrm{O}_{8}$. This base was found in 1828 , in a cinchona bark from Arica, in Peru, and has not since occurred. It is very similar to cinchonine, from which it differs in being soluble in ether. Aricine forms prismatic crystals larger than those of cinchonine. It is sparingly soluble in water, very soluble in alcohol, soluble in ether. It has a bitter taste. Nitrio acid dissolves it with an intense green colour, but decomposes it. Its salts are very soluble, and crystallise readily. The neutral sulphate, however, forms, when its hot solution is cooled, a gelatinous mass.

Besides the above bases, other alkaloids are said to have been found in different species of cinchona; as pitayine, in the China pitaya, chinovine in the China nova, another alkaloid in the China of Carthagena, blanquinine in the China blanca, which is the bark of Cinchona ovifolia and C. macrocarpa; and cinchovatine in Cinchona ovata. This last crystallises well, and forms crystallisable 
salts. It has been analysed, and the results lead to the formula $\mathrm{C}_{46} \mathrm{H}_{26} \mathrm{~N}_{2} \mathrm{O}_{8}$, so that it is the same as aricine.

\section{c. Bases of the Papaveracea.}

a. Morphine. $\mathrm{C}_{34} \mathrm{H}_{19} \mathrm{NO}_{6}+2$ aq. This alkaloid occurs in opium, which is the inspissated juice of Papaver somniferum. Perhaps the easiest method of extracting it is the following. The soluble part of opium is extracted by water, and the concentrated infusion is mixed with solution of chloride of calcium, this salt being added in slight excess. On standing, especially if warmed, the mixture deposits a copious brownish-grey precipitate of mixed meconate and sulphate of lime (the morphine being in the opium partly as meconate, partly as sulphate), while hydrochlorate of morphine remains in solution with a very large proportion of dark brown colouring matter. The brown solution is evaporated till, on cooling, the hydrochlorate crystallises, forming a nearly solid mass, which is subjected to very strong pressure in flannel. A thick, viscid, nearly black mother-liquor is thus expressed, which contains all the narcotine and colouring matter. The squeezed mass or cake of hydrochlorate of morphine is of a fawn colour. It is redissolved in hot water, filtered if necessary, and recrystallised, so much water being used, that on cooling a semisolid mass is obtained. This is again squeezed out, and if the squeezed cake is not quite white, it in only necessary to repeat the operation. A little animal charcoal in the second, or better still in the third, crystallisation, assists in removing the last traces of colour. The second and third mother liquids, although coloured, are not to be thrown away, but should be added to the solntion of a fresh portion of opium, so that the small quantity of hydrochlorate which is retained in solution shall not be lost. In crystallising hydrochlorate of morphine, the liquid should always be acidulated with hydrochloric acid (after the animal charcoal is separated), because in this way very little indeed is retained in solution.

The purified hydrochlorate, which still retains about $\frac{1}{30}$ of codeine, is now dissolved in hot water, and supersaturated with ammonia; on cooling, the morphine is deposited as a snow-white crystalline powder, which may be crystallised by means of hot alcohol. The codeine, with some of the morphine, remains in the mother-liquor.

Morphine forms hard transparent brilliant crystals, almost insoluble in water, soluble in hot alcohol, insoluble in ether. It is decidedly alkaline, neutralising acids, and forming crystallisable 
salts. All its solutions are bitter, and act as narcotie poisons. It is coloured red by nitric acid, and brownish red by iodic acid; it also strikes a deep blue with perchloride of iron.

The salts of morphine are much used in medicine, especially the hydrochlorate, the acetate, and the sulphate. A solution of any of these salts, of five grains to the ounce, may be administered in the same dose as tincture of opium (laudanum). The hydrochlorate or muriate is prepared as above described, and is used in the state in which it is obtained by repeated crystallisation, containing $\frac{1}{15}$ of its weight of a mixed hydrochlorate of morphine and codeine, which has much the same action. The acetate and sulphate are best made directly by dissolving in acetic and sulphuric acids the precipitated morphine till they are neutralised, and then evaporating. $1 \mathrm{lb}$. of good opium yields $1 \frac{1}{2}$ oz. of hydrochlorate of morphine. These salts are most valuable anodynes, and do not derange the stomach nearly so much as an equivalent dose of laudanum; but they do not act so decidedly in producing sleep as in allaying pain and irritation; at least the sleep they induce does not come on so soon as in the case of laudanum. The patient, however, even when he does not sleep, feels refreshed, almost as if he had slept, and on the whole the preparations of morphine are preferred by the physician, and have, in this country at least, nearly banished the use of laudanum. The black drop contains impure citrate of morphine. When morphine is acted on by sulphuric acid, it yields a compound, sulphomorphide, said to be analogous to sulphamide, but not yet fully studied. When heated with potash, morphine yields some of the volatile bases of the series of ethylamine, at all events one of these, methylamine, and, it is said, also propylamine.

When boiled with iodine, morphine is converted into a brown amorphous substance, soluble in hot acids or alkalies, and deposited as the solutions cool. This is Iodomorphine, which is said to consist of 4 eqs. morphine and 3 eqs. iodine, a very doubtful formula. The analysis gave 4.5 per cent. too little iodine. Iodomorphine, acted on by mercury, yields protoiodide of mercury, and a buff amorphous body, containing iodine, the nature of which is uncertain.

With the iodides of methyle and ethyle, morphine yields ammonium bases.

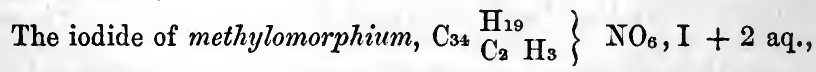
is a crystalline power, very soluble in hot water, which deposits it on cooling in rectangular prisms. With oxide of silver, the salt yields iodide of silver and a brown amorphous mass, probably 
hydrated oxide of methylomorphium. It is, however, acted on by iodide of methyle, which we should hardly expect. Perhaps some of the hydrogen in the morphine it contains is replaced by methyle.

The iodide of ethylomorphium, $\left.\mathrm{C}_{34} \underset{\mathrm{C}_{4} \mathrm{H}_{5}}{\mathrm{H}_{19}}\right\} \mathrm{NO}, \mathrm{I}+$ aq. resembles the preceding compound. With oxide of silver it yields iodide of silver, and a brown caustic alkaline solution, probably of the hydrated oxide of ethylomorphium, which dries up into a brown amorphous mass.

b. Codeine, $\mathrm{C}_{36} \mathrm{H}_{21} \mathrm{NO}_{6}+2$ aq. - This alkaloid is obtained as above described, from the mother-liquor of the precipitated morphine, which, being evaporated, deposits a mixed hydrochlorate of morphine and codeine. This salt being purified, is acted on by potash, which dissolves the morphine, while the codeine is left as a viscid mass, which soon becomes hard and crystalline. It is purified by solution in ether or in water, both of which solvents leave the morphine, which may be mixed with it, undissolved. The ethereal solution, by spontaneous evaporation, deposits it, especially if a little water be added, in fine anhydrous prisms; the aqueous solution gives large octohedral crystals, which are a hydrate, with 2 eqs. of water.

Codeine is a powerful base, forming neutral salts with acids. Its solutions are bitter, and would seem to have an anodyne action on the system; but in some circumstances they appear to excite intolerable itching on the whole skin. It is therefore possible, that the itching caused in some persons by opium, and by the commercial muriate of morphine, proceeds from codeine.

Codeine, when dissolved in excess of moderately strong sulphuric acid, is converted into amorphous codeine, without any change in composition. If the action be longer continued, there is found a new body, sulphocodeide, possibly analogous to an amide.

With diluted nitric acid, codeine yields the substitution base, nitrocodeine, $\left.\mathrm{C}_{36} \mathrm{~N}_{\mathrm{N}_{4}}^{\mathrm{N}_{4}}\right\} \mathrm{NO}_{6}$. It forms shining yellowish or fawn-coloured crystals, and gives crystalline salts with acids. With sulphuret of ammonium, nitrocodeine yields a new base, probably $\mathrm{C}_{36} \mathrm{H}_{22} \mathrm{~N}_{2} \mathrm{O}_{6}$. With bromine, codeine yields the base bromocodeine, Сs6 $\mathrm{H}_{20} \mathrm{Br} \mathrm{NO}_{6}$, in small white crystals; and another compound, tribromocodeine, $\mathrm{C}_{36} \mathrm{H}_{18} \mathrm{Br}_{3} \mathrm{~N} \mathrm{O}_{6}$, which seems to be amorphous, and feebly basic. With chlorine, codeine

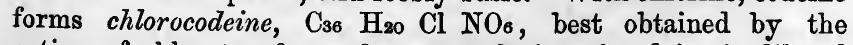
action of chlorate of potash on a solution of codeine in diluted 
hydrochloric acid. It much resembles bromocodeine, and its salts also resemble those of that base. With iodine, oodeine forms a very remarkable compound, which is not a substitution-product, but resembles iodoquinine in composition, and contains iodine and codeine. It contains 1 eq. codeine and 3 eqs. iodine, and its crystals belong to the doubly oblique system. They are very large, exhibiting two colours, and have lately been fully described. With cyanogen, codeine yields the compound dicyanocodeine, in shining crystals. Its formula is $\mathrm{C}_{40} \mathrm{H}_{21} \mathrm{~N}_{3}$ $\mathrm{O}_{6}=\mathrm{C}_{36} \mathrm{H}_{21} \mathrm{NO}_{6}+2 \mathrm{C}_{2} \mathrm{~N}$. It belongs, therefore, to the same elass of bodies as cyaniline, cyanotoluidine, and cyanocumidine, and is not a substitution-product.

Heated with bases, codeine yields ammonia, methylamine, propylamine, and a volatile crystalline base, not yet examined, but probably one of the bases of the methylamine series higher in the scale. When codeine is acted on by strong nitric acid, a resinous-looking acid is obtained, which, when heated with a solution of potash, yields volatile bases in abundance. For all the above details concerning the products derived from codeine, we are indebted to Dr. Anderson. It will be seen that they establish a great resemblance to, and connection with, the artificial bases, more particularly in the action of cyanogen, and in the fact that codeine, like the oxygenised artificial bases, yields volatile non-oxygenised bases when decomposed by heat.

The iodide of ethylocodium, $\left.\mathrm{C}_{36} \mathrm{H}_{22} \mathrm{H}_{3}\right\} \mathrm{NO}_{6}, \mathrm{I}$, is obtained by the action of iodide of ethyle on codeine, aided by heat. It is soluble, and crystallises in fine needles. Acted on by oxide of silver, it yields a caustic alkaline solution, which attracts carbonic acid from the air. This is probably the hydrated oxide of ethylocodium, and like the corresponding base of morphine, it is acted on by iodide of ethyle, the action being apparently complex.

The resemblance of codeine to morphine, in its chemical relations, is very great, and a glance at the formulæ here given for these bases will explain this; for these formulæ differ only by $\mathrm{C}_{2} \mathrm{H}_{2}$, or, in other words, are homologous. It appears, however, that morphine is not converted into codeine by the action of iodide of methyle, as methylamine is into dimethylamine. It is therefore probable that the homology exists in the group or groups replacing the 3 eq. of hydrogen, and that methyle is not, directly at least, one of these groups, in codeine.

c. Paparerine, $\mathrm{C}_{40} \mathrm{H}_{21} \mathrm{NO}$. This is a new erystalline base, discovered by Merck in opium. It is little known, but seems to be related to Thebaine, (which see). 
Porphyroxine, also discovered by Merck in opium, has been already mentioned as a neutral compound.

d. Thebaine. This base also occurs in opium. It is nearly insoluble in water, soluble in alcohol and ether. Its solutions are alkaline, and have an acrid metallic taste. It forms crystallisable salts with acids. According to Kane, its formula is $\mathrm{C}_{25} \mathrm{H}_{14} \mathrm{NO}_{3}$, but Anderson has shown it to be $\mathrm{C}_{38} \mathrm{H}_{21} \mathrm{NO}_{6}$.

e. Pseudomorphine, $\mathrm{C}_{27} \mathrm{H}_{18} \mathrm{NO}_{14}$ ?-This base is occasionally found in opium. It forms shining scales. It is sparingly soluble in water and weak alcohol, insoluble in absolute alcohol and in ether. It is readily dissolved by caustic potash or soda. It is coloured blue by perchloride of iron. It forms salts with acids, which are as yet little known.

f. Narceine, $\mathrm{C}_{46} \mathrm{H}_{29} \mathrm{NO}_{18}$ (Anderson). -This, which is a feeble base, also occurs in opium. It is sparingly soluble in water, soluble in alcohol, insoluble in ether. It melts at $197^{\circ}$. It is coloured blue by hydrochloric acid, but not by perchloride of iron. Acids dissolve it, and form definite salts with it, and it appears probable that two, perhaps three, substances, have been described under this name.

g. Narcctine, $\mathrm{C}_{46} \mathrm{H}_{25} \mathrm{NO}_{14 .}$ - This is another weak base, found in opium in larger proportion than any other, except morphine. It may be obtained either from the mother-liquor of muriate of morphia by adding ammonia, or by digesting the insoluble part of opium in diluted acetic acid, and precipitating by ammonia. The impure na. cotine is purified by solution in hot alcohol, with the aid of animal charcoal. On cooling, narcotine is deposited in crystals, which are insoluble in water and alkalies, soluble in alcohol, ether, and acids. Its salts are bitter, and crystallise with great difficulty.

The recent researches of Liebig, Wöhler, and Blyth, and still more lately those of Anderson, have made known a series of products of decomposition derived from narcotine, when acted on by peroxide of manganese and sulphuric acid, and also by bichloride of platinum. These our space will only permit us briefly to mention.

1. Opianic acid, $\mathrm{C}_{20} \mathrm{H}_{10} \mathrm{O}_{10}=\mathrm{C}_{20} \mathrm{H}_{9} \mathrm{O}_{9}, \mathrm{H} \mathrm{O}$. This acid crystallises in slender prisms, and forms soluble and crystallisable salts with baryta and the oxides of lead and silver, and with oxide of ethyle. When melted, opianic acid passes into an insoluble state, its composition remaining the same. Anderson has obtained the opianate of oxide of ethyle, or opianic ether.

2. Opiammon, $\mathrm{C}_{40} \mathrm{H}_{19} \mathrm{NO}_{16}$. This compound is derived from 2 eq. opianate of ammonia by the loss of $1 \mathrm{eq}$. ammonia and $4 \mathrm{eqs}$. 
water. It is a pale yellow powder, which, by boiling with water, is converted into opianic acid and opianate of ammonia.

3. Xanthopenic Acid.-When opiammon is acted on by alkalies, it gives off ammonia and yields opianate and xanthopenate of potash. An acid separates the xanthopenic acid as a yellow Hocculent precipitate. It forms salts of a fine yellow colour, but has not been fully examined. It contains nitrogen.

4. Opiano-sulphurous Acid, $\mathrm{C}_{20} \mathrm{H}_{9} \mathrm{O}_{11} \mathrm{~S}_{2}, \mathrm{H} \mathrm{O}$, is formed by the action of sulphurous acid on opianic acid, and is produced by the substitution of 2 eqs. sulphurous acid for 2 eqs. water. It has a bitter taste, and forms crystallisable salts.

5. Sulphopianic Acid, $\mathrm{C}_{20} \mathrm{H}_{9}\left\{\begin{array}{l}\mathrm{O}_{7} \\ \mathrm{~S}_{2}\end{array}\right\}, \mathrm{H} O$. This acid is formed by the action of sulphuretted hydrogen on opianic acid, and is, in fact, opianic acid, in which 2 eqs. of oxygen are replaced by 2 eqs. sulphur. It is an amorphous yellow powder, which crystallises from alcohol. Its salts are soon decomposed, yielding sulphurets of the metals.

6. Hemipinic Acid, $\mathrm{C}_{20} \mathrm{H}_{8} \mathrm{O}_{10}, 2 \mathrm{H} \mathrm{O}$. It is a bibasic acid, and, according to Anderson, forms an acid ether. This is a product of oxidation of opianic acid. The hemipinic acid crystallises in regular four-sided prisms. It forms insoluble salts with the oxides of lead and silver.

7. Cotarnine, $\mathrm{C}_{26} \mathrm{H}_{13} \mathrm{~N}$ O. This is a base formed along with opianic acid. 1 eq. narcotine, $\mathrm{C}_{46} \mathrm{H}_{25} \mathrm{~N} \mathrm{O}_{14}$, and 2 eqs. oxygen yield 1 eq. cotarnine, $\mathrm{C}_{25} \mathrm{H}_{13} \mathrm{~N} \mathrm{O}_{6}+1$ eq. opianyle, $\mathrm{C}_{20} \mathrm{H}_{10} \mathrm{O}_{8}$ +2 eqs. water, $2 \mathrm{H} \mathrm{O}$.

Cotarnine forms a deep yellow radiated mass, soluble in alcohol and in water. It is bitter and alkaline, and forms crystallisable double salts with the bichlorides of mercury and platinum.

8. Opianyle, $\mathrm{C}_{20} \mathrm{H}_{10} \mathrm{O}_{\mathrm{s}}$ (Anderson). This compound is formed along with cotarnine, when narcotine is acted on by nitric acid so as to be gently oxidised. It forms fine needles, soluble in alcohol and ether. When its solution in oil of vitriol is heated, it becomes deep purple. It is related to opianic acid and hemipinic acid, thus :-

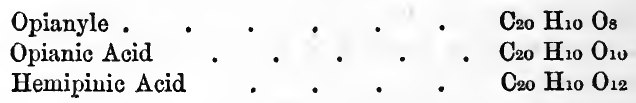

Anderson has also described a hydrate of opianylc, $\mathrm{C}_{20} \mathrm{H}_{11} \mathrm{O}_{9}$ $=\mathrm{C}_{20} \mathrm{H}_{10} \mathrm{O}_{8}, \mathrm{H} \mathrm{O}$.

Narcotine may be regarded as formed of cotarnine plus a body $\mathrm{C}_{20} \mathrm{H}_{12} \mathrm{O}_{8}$, which would be a hyduret of opianyle, $\mathrm{C}_{20} \mathrm{H}_{10} \mathrm{Os}_{8}$, 
$\mathrm{H}_{2}$. This very easily explains the action of oxidising agents in producing from narcotine, opianic acid, hemipinio acid, opianyle, and cotarnine.

9. Teropiammon, $\mathrm{C}_{60} \mathrm{~N} \mathrm{H}_{22} \mathrm{O}_{26}$ (Anderson). This compound is formed when narcotine is oxidised by nitric acid of moderate strength. It is derived from 3 eqs. of opianio acid and 1 of ammonia, 4 of water being separated. It forms small white needles, sparingly soluble in any solvent. Its solution in oil of vitriol becomes crimson red when heated.

10. Humopinic Acid is a dark brown humus-like acid formed by the action of heat on narcotine. Its composition is not established with certainty, but resembles that of other similar bodies. It contains no nitrogen.

11. Apophyllic Acid, $\mathrm{C}_{26} \mathrm{~N} \mathrm{H}_{7} \mathrm{O}_{8}$ (Anderson). This is a product of decomposition of cotarnine. It forms crystals very like those of apophyllite and equally cleavable. When heated it yields an oily liquid, said by Wöhler to be quinoline, but according to Anderson, isomeric either with aniline, or with anthranilie acid, as it cannot be quinoline.

When nitric acid acts on cotarnine so as to form apophyllic acid, several other substances are produced, which are not yet fully known. Among them, however, Anderson has found ethylamine and methylamine, and has reason to think that other bases of the same series, but higher in the scale, also occur.

12. Narcogenine, $\mathrm{C}_{36} \mathrm{H}_{19} \mathrm{~N} \mathrm{O}_{10}$, is formed, along with opianic acid, when narcotine is not so far oxidised as to yield cotarnine. 2 eqs. narcotine with 5 eqs. oxygen yield 2 eqs. narcogenine, 1 eq. opianic acid, and 3 eqs. water. It forms a crystallisable double salt with bichloride of platinum, but when separated, it is resolved into narcotine and eotarnine. 2 eqs. narcogenine with 2 eqs. oxygen contain the elements of 1 eq. narcotine, 1 eq. cotarnine, and 1 eq. carbonic acid.

13. Narcotinic Acid. When nareotine is heated with potash it forms a soluble compound which contains an acid, apparently isomerio with narcotine, or differing from it only by 1 or 2 eqs. water. When this acid, which is called narcotinic acid, is separated from its salts, it rapidly passes into narcotine, so that it is unknown in a separate form. Its atomic weight seems to be half that of narcotine, so that 1 eq. narcotine probably forms 2 eqs. of the acid.

Such is a very brief and imperfect account of the results of the recent researches of Wöhler, Blyth, and Anderson, on narcotine. They are of very great importance as indicating a method which may lead to the discovery of the true constitution of the alkaloids. 
Wertheim has recently observed the occurrence in opium of three narcotines, homologous with one another, as follows:-

Methylonarcotine
Ethylonarcotine
Propylonarcotine $\cdot \cdot \cdot \cdot \cdot \cdot \begin{aligned} & \mathrm{C}_{44} \mathrm{~N} \mathrm{H}_{43} \mathrm{O}_{14} \\ & \mathrm{C}_{48} \mathrm{O}_{14} \\ & \mathrm{C}_{48} \mathrm{~N}_{27} \mathrm{O}_{14}\end{aligned}$

The difference at each step is $\mathrm{C}_{2} \mathrm{H}_{2}$, and as we have seen that methylamine, ethylamine, and perhaps propylamine, may be obtained from narcotine, it is probable that these bases are constituents of these three narcotines. Ethylonarcotine, it will be seen, is the substance we have described as narcotine, but like all contiguous homologous compounds, these nareotines resemble each other so much as not to be easily distinguished or separated from one another.

h. Chelidonine, $\mathrm{C}_{40} \mathrm{H}_{20} \mathrm{~N}_{2} \mathrm{O}_{6}$. This alkaloid occurs in Chelidonium majus, along with chelerythrine. It is bitter, insoluble in water, and alkaline, forming crystallisable salts.

$i$. Chelerythrine, found in the same plant, forms a grey powder, which excites violent sneezing. With acids it forms red salts, which are narcotic and poisonous.

$k$. Glaucine. This alkaloid occurs in the leaves and stem of Glaucium luteum. It may be obtained in pearly scales; its taste is bitter, and acrid, and it forms salts with acids. Its composition is not yet ascertained.

l. Glaucopicrine is found in the root of the same plant. It is bitter and forms salts of a bitter and nauseous taste. Its composition is unknown.

\section{d. Alkaloids of the Solanacece, the Strychnece, and other Vegetable} Families.

a. Hyoscyamine.-This base is found in Hyoscyamus niger and other species of hyoscyamus. Its composition is not yet known. It is extracted from the seeds by a difficult and tedious process, and may also be obtained, although with much loss, by distillation with potash, like conicine. It is very prone to decomposition when in contact with mineral alkalies. It crystallises, when pure, in radiated groups of needles, but sometimes forms a viscid amorphous mass. When moist it has a stupefying smell like that of tobacco. It is very poisonous, causing, like coniine, tetanic spasms. It dilates the pupil powerfully. It is fusible and volatile, but is partially decomposed when distilled. It dissolves in water, alcohol, and ether. It neutralises the acids, forming crystallisable salts, which are very poisonous. The fact that it is volatile, and 
has a smell analogous to that of nicotine, suggest the idea that it may be a true volatile base, of the class of nicotine, representing ammonia, but high in the scale, and probably containing no oxygen.

b. Daturine. This base is obtained from the seeds of Datura Stramonium. It is, in preparation and properties, very analogous to hyoscyamine. It is, however, less soluble in water, and crystallises in fine brilliant prisms, from its alcoholic solution. It is fusible, volatile, and very poisonous, dilating the pupil. Its salts are crystallisable and very poisonous. Its precise composition is unknown, but it is now said to be identical with atropine. Possibly it may be only homologous with that base.

c. Stramonine.-This is another crystalline compound found in Stramonium. It is crystallisable, volatile, soluble in alcohol and ether, insoluble in water. Its nature is uncertain, and its composition unknown.

d. Atropine, $\mathrm{C}_{34} \mathrm{H}_{23} \mathrm{NO}_{6}$. This alkaloid is the active principle of Atropa Belladonna. It is obtained like daturine, and being equally prone to decomposition, much is always lost. It is sparingly soluble in water and ether, more soluble in alcohol. It crystallises in white silky prisms, and sometimes forms an amorphous mass like glass. It is very bitter, acrid, and poisonous, dilating the pupil like hyoscyamine and daturine. It is fusible and volatile, and neutralises acids, forming salts which are bitter, acrid, and poisonous, and which crystallise. These salts, from their very poweriul action in permanently dilating the pupil, are very well adapted for medical use, being much more uniform than the extract.

e. Solanine, $\mathrm{C}_{84} \mathrm{H}_{68} \mathrm{~N}_{28}$ ? This alkaloid occurs in many species of Solunum, as in S. nigrum, S. dulcamara, and in the potato, S. tuberosum. In the latter it is found in large quantity, especially in the shoots, when the tubers have germinated in dark cellars. The shoots are extracted with dilute sulphuric acid, and the solution precipitated while hot by ammonia. The precipitate is puritied by solution in alcohol. It forms a crystalline powder, very bitter and acrid, and highly poisonous, but not dilating the pupil. Its salts do not crystallise readily. There is some reason to suspect that the alkaloid of the shoots of potatoes may be distinct from that of the bitter-sweet, Solanum dulcamara.

f. Veratrine, $\mathrm{C}_{34} \mathrm{H}_{21} \mathrm{NO}_{6}$ ? This alkaloid is found in Veratrum Sabadilla, $\boldsymbol{V}$. album, \&c. It is extracted as atropine is, and is generally obtained as a crystalline powder, nearly white, very acrid and poisunous, exciting, when introduced into the nostril, violent and even dangerous sneezing. It is insoluble in water, 
but very soluble in alcohol, and may be obtained by the spontaneous evaporation of its alcoholic solution, in prismatic erystals several lines in length. It is coloured red both by nitric and sulphuric acid.

Veratrine, in the form of tincture, and still more in that of ointment ( $1 \mathrm{drachm}$, or $\frac{1}{2} \mathrm{drachm}$ to $1 \mathrm{oz}$. of lard), is now much used as an external application in neuralgia and obstinate rheumatio pains. Its effects in many cases are highly beneficial. In making the ointment, the veratrine should first be rubbed with a few drops of alcohol to an impalpable powder, and the lard then added. If this be not done, the gritty particles of veratrine in the ointment cause so much irritation when rubbed into the skin as to prevent its use for any length of time. We are indebted chiefly to Dr. Turnbull for our knowledge of the valuable properties of this alkaloid.

g. Sabadilline. This name has been given by Couerbe to a second crystalline body found by him along with veratrine. It is alkaline, soluble in hot water, insoluble in ether, and forms crystallisable salts with acids. Couerbe states its formula to be $\mathrm{C}_{20} \mathrm{H}_{13} \mathrm{NO}_{5}$; but, according to Simon, it is a compound of veratrine with resin, containing also resinate of soda.

h. Colchicine. This alkaloid is similar to veratrine, for which it was formerly taken. It is found in Colchicum autumnale. It is crystallisable, bitter, and very poisonous. Nitric acid colours it. blue or violet. It is soluble in water, alcohol and ether. Its salts are crystallisable, bitter, acrid, and poisonous. They might probably be used in medicine advantageously, instead of the very uncertain preparations of colchicum which are at present employed. In a very small dose, colchicine causes purging and vomiting. Its composition is unknown.

i. Aconitine, $\mathrm{C}_{60} \mathrm{H}^{47} \mathrm{~N} \mathrm{O}_{14}$ ? This alkaloid, the exact composition of which is unknown, is found in Aconitum Napellus, and, probably, also in $A$. ferox and other species. It is obtained by the usual method, but, being very prone to suffer change, much is lost. It forms a crystalline powder, or occasionally a vitreous amorphous mass. It is in the highest degree bitter, acrid, and poisonous, and is said by Geiger to dilate the pupil. On the other hand, the plant contracts the pupil and causes numbness of the parts to which it is applied; and Dr. Turnbull has obtained an aconitine possessing these properties in a very high degree. Either, therefore, there are two bases in the aconite, or, as is much more probable, the aconitine of Geiger, having an action different from that of the plant, is a product of decomposition, while that of 'Turnbull is unchanged.

Turnbull's aconitine is an invaluable remedy in the same painful 
diseases in which veratrine is employed. It is, unfortunately, obtained in small proportion, and as yet is very expensive. A cheaper and more productive method of preparing it is a very great desideratum.

k. Delphine, $\mathrm{C}_{27} \mathrm{H}_{19} \mathrm{~N} \mathrm{O}_{2}$ ? This alkaloid, analogous to veratrine, is found in Stavesacre, Delphinium Staphysagria. It has only been obtained hitherto as a yellowish-white powder, not crystallised, very acrid, and poisonous. It forms neutral salts, hitherto little examined. It may be used in the same affections and in the same manner as veratrine.

l. Staphisine, This is a substance found along with delphine, and said to be $\mathrm{C}_{32} \mathrm{H}_{23} \mathrm{~N} \mathrm{O}_{4}$. It is acrid and poisonous, but is probably only a compound of delphine.

m. Emetine, $\mathrm{C}_{37} \mathrm{H}_{27} \mathrm{NO}_{10}$ ? This is the active principle of ipecacuanha, the root of Cepleaelis Ipecacuanha. When pure, it is a white powder, alkaline, soluble in alcohol and in hot water, insoluble in ether. $\frac{1}{16}$ of a grain acts as an emetic. In a dose of from 2 to 4 grains it is poisonous. Its salts do not crystallise.

n. Chiococcine, and o. Violine are two very similar alkaloids, found in Chiococca ramosa and Viola odorata. They are supposed by some to be emetine disguised by a little foreign matter.

p. Strychnine, $\mathrm{C}_{42} \mathrm{H}_{22} \mathrm{~N}_{2} \mathrm{O}_{4}$. This alkaloid is found in nux vomica, the seeds of Strychnos nux vomica, in St. Ignatius's bean, the seed of S. Ignatii, in the wood of $S$. colubrina, and in the poison called Upas Tieuté, derived from $S$. Tieuté. It is extracted by decoction with dilute sulphuric acid, precipitating the decoction with milk of lime, and acting on the precipitate after washing it with cold alcohol, by boiling alcohol, which on cooling deposits the strychnine in very regular transparent brilliant crystals. If brucine is present, it remains chiefly in the mother liquid, but the two bases may be separated by converting both into nitrates, and crystallising; the nitrate of strychnine crystallises readily, while the nitrate of brucine remains dissolved.

Strychnine is very insoluble, requiring 7000 parts of water. It is so bitter, that 1 part gives a very strong and persistent bitter taste to 40,000 parts of water. It dissolves in hot alcohol, although sparingly if the alcohol be pure, and is insoluble in ether. When pure it is only coloured yellow by nitric acid; a trace of brucine causes it to be reddened by that acid. It forms crystallisable salts, which are intensely bitter. Their solutions are precipitated white by alkalies, by tincture of galls, and by iodide of potassium, in white crystals by sulphocyanide of potassium, and as yellow powders by solutions of gold and 
platinum. When heated with bases, strychnine yields the volatile base quinoline already described.

Strychnine and its salts, especially the latter, from their solubility, are most energetic poisons. They produce spasmodic motions, and are used in very small doses as remedies in paralysis; they seem to have a specific action on the lower part of the spinal column. The average dose is $\frac{1}{12}$ of a grain. In the event of an overdose, the best antidote is infusion of galls or strong tea, which also contains tannine.

With chlorine and bromine, strychnine yields the substitution products, chlorostrychnine, a base ; trichlorostrychnine, a neutral substance; and bromostrychnine, a base. With iodine it forms, like quinine or codeine, a peculiar compound in which 4 eqs. of strychnine seem to be united to 6 of iodine. It forms orangecoloured scaly crystals, which are decomposed by acids, strychnine being reproduced.

q. Brucine, $\mathrm{C}_{46} \mathrm{H}_{26} \mathrm{~N}_{2} \mathrm{O}_{8}$. This alkaloid occurs along with strychnine in nux vomica, and also in the false angustura bark, the bark of Brucia antidysenterica. It is prepared as strychnine. Besides the methods above mentioned for separating the two bases, there is another, which is, to boil the mixture with water as long as it dissolves brucine, or till the strychnine is no longer reddened by nitric acid. Brucine forms large transparent crystals, which I have found to become opaque in closely stopped phials. It is very bitter and poisonous, but much less so than strychnine. It may be used for the same purposes in a rather larger dose. It is reddened strongly by nitric acid, and the red solution becomes violet on the addition of solution of tin. It is thus distinguished from strychnine and morphine. Its salts, for the most part, crystallise with facility.

With bromine, brucine forms a substitution base, bromobrucine. With iodine it appears to yield two compounds, one of which contains 4 eqs. of brucine and 6 eqs. of iodine, the other 2 eqs. of brucine and 6 eqs. of iodine.

Nitric acid decomposes it, producing a new compound, cacotheline, $\left.\mathrm{C}_{40} \underset{2}{2} \mathrm{~N}_{22} \mathrm{~N}_{4}\right\} \mathrm{N}_{2} \mathrm{O}_{10}$, along with nitrite or hyponitrite of oxide of methyle, oxalic acid, water and deutoxide of nitrogen. The production of oxide of methyle in this action, would seem to render it probable that methyle exists, as such, in brucine.

$r$. Jervine, $\mathrm{C}_{60} \mathrm{H}_{45} \mathrm{~N}_{2} \mathrm{O}_{5}$. This alkaloid is found in white hellebore, Veratrum album, along with veratrine, from which it is separated easily, as it crystallises first from the alcoholic solution; and its sulphate is far less soluble than that of veratrine. It forms a crystalline powder, fusible, insoluble in water,. 
soluble in alcohol, and forming with sulphuric, nitric, and hydrochloric acids, very sparingly soluble salts, so that the solution of the acetate is precipitated by these three acids.

s. Curarine. This alkaloid is obtained from the South American poison called curari, which is derived from some plant of the family Strychneæ. It is a deadly poison when introduced into a wound, but may be swallowed with impunity. The curarine forms a yellowish amorphous bitter mass, which is more poisonous than the curari which yields it. Its salts are bitter, but do not crystallise.

t. Corydaline, $\mathrm{C}_{34} \mathrm{H}_{22} \mathrm{~N} \mathrm{O}_{10}$ ? Found in the root of Corydalis bulbosa and $C$. fabacea. It forms a light grey powder, very soluble in alcohol, which deposits it in crystals. It is reddened by nitric acid, and forms crystallisable salts with acetic and sulphuric acids.

u. Carapine. Found in Carapus Guianensis. It is a white pearly fusible powder, very bitter, soluble in water and alcohol, insoluble in ether, forming crystallisable salts with hydrochloric and acetic acids.

v. Cusparine. Found in the true angustura bark, that of Bonplandia trifoliata or Cusparia febrifuga. It forms fusible octohedral crystals, sparingly soluble in water, very soluble in alcohol.

w. Daphnine occurs in the bark of Daphne gnidium and $D$. mezereon. It is obtained by distilling the infusion with magnesia. It is alkaline and acrid, and forms crystallisable salts with nitric and sulphuric acids, according to Vauqueline. Baer and Gmelin could not obtain it.

$x$. Bebeerine is the active principle of the bark of the bebeeru tree of Guiana, which seems to be analogous to quinine. It has not been obtained crystallised, but Von Planta has obtained it as a snow-white powder, strongly basic, the salts of which do not crystallise. Bebeerine and its salts are bitter and highly febrifuge. Von Planta has shown that its formula is $\mathrm{C}_{38} \mathrm{H}_{21} \mathrm{~N} \mathrm{O6}$, and that it is not, as was supposed, isomeric with morphine.

y. Sanguinarine is found in Sanguinaria Canadensis. It forms a grey powder, which is alkaline and yields red salts. It excites sneezing, and is possibly identical with chelerythrine.

z. Azadirine, found in Malea azadirachta, is alkaline, forms a crystallisable salt with sulphuric acid, and is powerfully febrifuge.

aa. Capsicine is the active principle of the capsules of Capsicum annuum or cayenne pepper. It has a resinous aspect and a burning taste, but when quite pure may be crystallised. It forms crystallisable salts with acetic, nitric, and sulphuric acids. - It is soluble in alcohol, insoluble (when pure) in ether and in water. 
bb. Crotonine occurs in the seeds of Croton tiglium, and may be obtained from croton oil by boiling it with water and magnesia. It forms crystals, which are fusible, soluble in alcohol, insoluble in water. It forms crystallisable salts with sulphuric and phosphoric acids.

cc. Buxine occurs in boxwood bark. It forms a bitter, brown, amorphous mass, soluble in alcohol, alkaline, and forming a crystalline sulphate. It excites sneezing.

dd. Apyrine. Found in Cocos lapidea. It is a white alkaline powder, forming crystalline salts with acids.

ee. Cynapine. From Athusa cynapium. It is crystallisable, soluble in water and alcohol, and forms a crystalline sulphate.

ff. Cissampeline, or Pelosine, $\mathrm{C}_{36} \mathrm{H}_{21} \mathrm{NO}_{6}$, from Cissampelos Pareira, is a white powder, soluble in alcohol and ether; alkaline, forming soluble salts, of which the hydrochlorate crystallises. Its formula, as given by Bœdeker, is the same as that of codeine. It is probably, however, isomeric, not identical, with that base. It forms a hydrate with 3 eqs. of water. When exposed to light and air, the hydrate is changed into a yellow base, pelluteine, $\mathrm{C}_{42} \mathrm{H}_{21} \mathrm{NO}_{7}$.

gg. Oxyacanthine and Berberine are two bitter substances found in the barberry, Berberis vulgaris. The former is decidedly alkaline, and forms crystallisable salts. The latter is bitter, yellow, and feebly, if at all alkaline. It crystallises, and is used in dyeing. Its formula is $\mathrm{C}_{48} \mathrm{H}_{19} \mathrm{NO}_{10}$.

hh. Surinamine and Jamaicine are two alkaloids, found in Geoffroa Surinamensis and $G$. inermis. Both are crystallisable, and form crystallisable salts; those of the latter are precipitated by tannine and corrosive sublimate.

ii. Piperine, $\mathrm{C}_{68} \mathrm{H}_{38} \mathrm{~N}_{2} \mathrm{O}_{12}$. This compound is found in pepper, Piper nigrum and $P$. longum. It is very crystallisable, soluble in alcohol, very pungent. It is a feeble base, but does form salts, especially double chlorides, containing hydrochlorate of piperine.

Piperidine, $\mathrm{C}_{10} \mathrm{H}_{11} \mathrm{~N}$. When piperine is heated with potashlime, or acted on by nitric acid, it yields an oily volatile base, which is piperidine. It is an imide base, 2 of the 3 eq. of hydrogen in ammonia being replaced by $\mathrm{C}_{10} \mathrm{H}_{10}$, thus, $\mathrm{N} \underset{\mathrm{C}_{10} \mathrm{H}_{10}}{\mathrm{H}}$ \}.

It is probable that the group $\mathrm{C}_{10} \mathrm{H}_{10}$ is itself made up of two groups, each replacing 1 eq. of hydrogen. Piperidine is a strong base, analogous to ammonia. Its salts crystallise perfectly. With cyanio acid it yields a compound homologous with urea, in which 2 eq. of hydrogen is replaced by the group $\mathrm{C}_{10} \mathrm{H}_{10}$, or, as it may be called, piperyle. This compound is called piperylurea. With 
the cyanate of methyle and ethyle, piperidine yields two other ureas, methylopiperylurea and ethylopiperylurea. All these ureas erystallise beautifully. With the iodides of methyle, ethyle, and amyle, piperidine yields nitryle bases, which are methylopiperi-

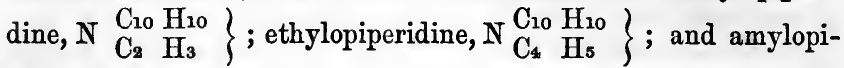
peridine, $\left.\mathrm{N}_{\mathrm{C}_{10} \mathrm{H}_{11}}^{\mathrm{C}_{10} \mathrm{H}_{10}}\right\}$. These all resemble piperidine more or less.

The two first of these three new bases acted on by iodide of methyle and iodide of ethyle, give rise to two ammonium bases; hydrated oxide of dimethylopiperidium, $\left.\begin{array}{ccc}\mathrm{N}_{2} & \mathrm{C}_{2} & \mathrm{H}_{10} \\ \mathrm{C}_{2} & \mathrm{H}_{3}\end{array}\right\} \mathrm{H}, \mathrm{HO}$; and

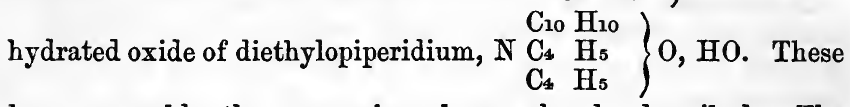
bases resemble the ammonium bases already described. The iodide of dimethylopiperidium forms brilliant crystals; that of the ethyle compound has not been obtained crystallised.

Piperidine also forms other compounds, such as piperylosulphocarbamic acid, which is obtained united to piperidine in the form of very fine crystals, $\left.\mathrm{C}_{22} \mathrm{H}_{22} \mathrm{~N}_{2} \mathrm{~S}_{4}=\stackrel{\mathrm{N}(\mathrm{CS})_{2} \mathrm{C}_{10} \mathrm{H}_{10} \mathrm{~S}}{\mathrm{~N} \mathrm{H} \mathrm{C}_{10} \mathrm{H}_{10} \mathrm{HS}}\right\}$.

Cahours. has also obtained piperylbenzamide and piperylcuminamide, the former of which is amide, in which 1 eq. of hydrogen is replaced by benzoyle, the other by piperyle, $\left.\mathrm{N}_{\mathrm{C}_{10} \mathrm{H}_{10}}^{\mathrm{C}_{14} \mathrm{H}_{5} \mathrm{O}_{2}}\right\}=$ $\mathrm{C}_{24} \mathrm{H}_{15} \mathrm{NO}_{2}$. The latter is analogous in composition, cumyle

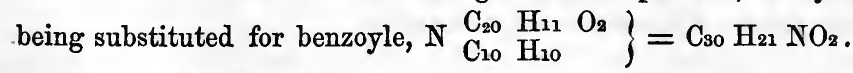
Both form fine crystals.

$k k$. Menispermine and Paramenispermine are found in Cocculus indicus, the seed of Menispermum cocculus. Menispermine is white, fusible, crystallisable, and forms salts, of which the sulphate crystallises. Its formula is $\mathrm{C}_{18} \mathrm{H}_{12} \mathrm{NO}_{2}$.

Paramenispermine has the same composition. It is less fusible, but sublimes at a high temperature. It does not appear to form definite salts. Both are insoluble in water, and soluble in alcohol; and paramenispermine is insoluble in ether.

ll. Harmaline, $\mathrm{C}_{22} \mathrm{H}_{16} \mathrm{~N}_{2} \mathrm{O}_{2}$, and Harmine, $\mathrm{C}_{26} \mathrm{H}_{12} \mathrm{~N}_{2} \mathrm{O}_{2}$.These alkaloids occur united with phosphoric acid in the seeds of Peganum Harmala. The first forms brownish-yellow prisms, bitter, astringent, and acrid, very soluble in alcohol, little soluble in water or ether. It is fusible, and partly volatile. It forms, with acids, yellow crystallisable salts. By oxidising agents, 
harmaline is transformed into a red matter, which forms red salts with acids. The harmala red of commerce is the powder of the seeds already transformed into the phosphate of the red harmaline. It is used in dyeing, especially in giving to silk every shade of red, rose-colour, and pink. It is produced abundantly in the steppes of southern Russia, and is little known out of that country. Fritzsche states that by oxidation harmaline yields two new bases, leucoharmine and chrysoharmine, the composition of which is not given. Besides these, harmaline yields a variety of substitution-products, such as nitroharmaline, nitroharmine, \&c. With 1 eq. of hydrocyanic acid, it forms a new base, cyanharmaline, $\mathrm{C}_{28} \mathrm{H}_{15} \mathrm{~N}_{3} \mathrm{O}_{2}$.

mm. Caffeine, $\mathrm{C}_{16} \mathrm{H}_{10} \mathrm{~N}_{4} \mathrm{O}_{4}+2$ eq. Sxw. Theine. Guaranine. This remarkable compound is found in coffee, in tea, in Guarana officinalis, or Paullinia sorbilis, and in Ilex Paraguayensis. It is best obtained by adding to a decoction of tea a slight excess of acetate of lead, and evaporating to dryness the filtered liquid. The dry mass mixed with sand, is heated in the apparatus described for benzoic acid, when caffeine is obtained in crystals. Tea yields more than 1 per cent.

Caffeine forms fine white prisms, of a silky lustre, which are soluble in water, alcohol, and ether, bitter, fusible, and volatile.

It is a feeble base, but forms, with hydrochloric acid and sulphuric acid, salts which yield very large crystals.

The most interesting circumstance in relation to caffeine is, that while, in some form, such as coffee, tea, Paraguay tea, or Guarana tea, plants containing it are used by nearly all nations as a necessary of life, and never relinquished when they can by any means be obtained: and while the only other substance much used for the same purpose, namely, cocoa or chocolate, contains a body closely allied to, and indeed homologous with, caffeine, there is a very remarkable analogy and connection between the composition of caffeine and its derivatives, and certain products of the animal functions, those, namely, connected with uric acid. This is not the proper place to enter on the formation and destruction of uric acid in the body, which will be done hereafter, but we shall here mention briefly the results of Rochleder's investigation of the derivatives of caffeine, and show their analogy with those of uric acid. Caffeine when acted on by chlorine, yields a weak acid, which resembles alloxantine, and it is, in fact, homologous with alloxantine. This is amalic acid, $\mathrm{C}_{12} \mathrm{~N}_{2} \mathrm{H}_{7} \mathrm{O}_{8}$, differing from anhydrous alloxantine, $\mathrm{C}_{8} \mathrm{~N}_{2} \mathrm{H}_{3} \mathrm{Os}$, by $2\left(\mathrm{C}_{2} \mathrm{H}_{2}\right)$. When this, the alloxantine of the caffeine series, is oxidised, it yields cholestrophane, a compound homologous with parabanic acid, the product of the oxidation of alloxantine; and lastly, when amalic acid acts on ammonia, there 
is formed caffeomurexide, a compound having the same striking external characters as murexide, with which it is homologous. Thus we have :-
Alloxantine
\begin{tabular}{lllll}
$\mathrm{C}_{8}$ & $\mathrm{~N}_{2}$ & $\mathrm{H}_{3}$ & $\mathrm{O}_{8}$ \\
\hline
\end{tabular}
Parabanic Acid $\mathrm{C}_{6} \mathrm{~N}_{2} \mathrm{H}_{2} \mathrm{O}_{8}$
Amalic Acid $\quad \mathrm{C}_{12} \mathrm{~N}_{2} \mathrm{H}_{7} \mathrm{O}_{8}$
Murexide
$\mathrm{C}_{24} \mathrm{~N}_{10} \mathrm{H} \quad \mathrm{O}_{18}$
Cholestrophane $\mathrm{C}_{10} \mathrm{~N}_{2} \mathrm{H}_{8} \mathrm{O}_{6}$
Caffeomurexide $\mathrm{C}_{36} \mathrm{~N}_{10} \mathrm{H}_{24} \mathrm{O}_{16}$

The difference between the two murexides is not $\left.2 \mathrm{C}_{2} \mathrm{H}_{2}\right)$ or $\mathrm{C}_{4} \mathrm{H}_{4}$, as in the other two cases, but $\mathrm{C}_{12} \mathrm{H}_{12}$ or $3\left(\mathrm{C}_{4} \mathrm{H}_{4}\right)$, because in murexide three atoms of alloxantine are grouped to yield one of murexide, $\mathrm{C}_{24}$, and by analogy, 3 atoms of amalic acid must be grouped to yield one of the new murexide, C36. The analogy between the two series cannot be overlooked, and will, no doubt, be found to extend to many other points. We may also regard amalic acid as dimethylalloxantine, and cholestrophane as dimethyloparabanic acid, 2 eq. of methyle in both, replacing 2 eq. of hydrogen.

Caffeine, when acted on by chlorine, yields, in addition to amalic acid, chloride of cyanogen and hydrochlorate of methylamine; and we may suppose caffeine to be composed of $\mathrm{C}_{2} \mathrm{~N}+$ $\mathrm{C}_{2} \mathrm{H}_{5} \mathrm{~N}+\mathrm{C}_{12} \mathrm{~N}_{2} \mathrm{H}_{2} \mathrm{O}_{4}$. It is the latter group, with 2 eqs. of water and 2 of oxygen, which forms amalic acid. It is analogous to the group uryle, which, with oxygen and water, forms alloxantine. Uryle is $\mathrm{C}_{8} \mathrm{~N}_{2} \mathrm{O}_{4}$, but to be homologous with the above group in caffeine, it ought to be $\mathrm{C}_{8} \mathrm{~N}_{2} \mathrm{H} \mathrm{O}_{4}$, and as neither group is known in the separate form, it is quite possible that the true uryle, or radical of alloxantine, is $\mathrm{C}_{8} \mathrm{~N}_{2} \mathrm{H} \mathrm{O} \mathrm{O}_{4}$, which, with 2 of water and 2 of oxygen, will form alloxantine, instead of requiring 1 of oxygen and 3 of water, as the formula $\mathrm{C}_{8} \mathrm{~N}_{2} \mathrm{O}_{4}$ does. Since caffeine yields cyanogen and methylamine to chlorine, it is probable that both are ready formed in it.

When cholestrophane, $\mathrm{C}_{10} \mathrm{~N}_{2} \mathrm{H}_{6} \mathrm{O}_{6}$, is boiled with potash, it yields ethylamine, $\mathrm{C}_{4} \mathrm{H}_{7} \mathrm{~N}$, water being decomposed. Along with this, it yields oxalic and carbonic acids and ammonia, while parabanic acid, heated with potash, yields only ammonia, oxalic acid and carbonic, but 2 eqs. of ammonia. The intermediate homologous compound, $\mathrm{C}_{8} \mathrm{~N}_{2} \mathrm{H}_{4} \mathrm{O}_{6}$, if obtained, would yield methylamine, oxalic and carbonic acids, and ammonia.

nn. Theobromine, $\mathrm{C}_{14} \mathrm{H}_{8} \mathrm{~N}_{4} \mathrm{O}_{4}$ ? This substance, which is a weak base, homologous with caffeine, is found in the seeds of Theobroma cacao, or chocolate. Its constitution is still obscure. But Rochleder has obtained from it, by oxidation, a compound $\mathrm{C}_{22} \mathrm{~N}_{2} \mathrm{H}_{8} \mathrm{O}_{16}$, and a salt of ammonia, $\mathrm{N} \mathrm{H}_{4} \mathrm{O}, \mathrm{C}_{22} \mathrm{~N}_{2} \mathrm{H}_{8} \mathrm{O}_{10}$. 
The anhydrous acid of this salt, $\mathrm{C}_{12} \mathrm{~N}_{2} \mathrm{H}_{8} \mathrm{O}_{10}$, is homologous with alloxan and inosinic acid. We have :-

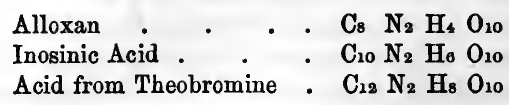

It will be seen that this last acid has the same relation to alloxan that amalic acid has to alloxantine, and that both caffeine and theobromine, when oxidised, yield products analogous to substances formed by the oxidation of uric acid.

\section{e. Alkaloids found in the Animal Organism.}

Only two such bases are yet known, namely: urea, already described as occurring in the urine: and kreatinine, which Liebig has detected both in the juice of flesh and in urine, and which has been described as an artificial base, it having been first observed as a product of the action of acids on kreatine.

The following substances have been noticed as alkaloids, but are very little known: Castine in Vitex agnus castus; Cicutine in Cicuta virosa; Charophylline in Charophyllum bulbosum; Esenbeckine in Esenbeckia febrifuga; Digitaline in Digitalis purpurea ; Eupatorine in Eupatorium cannabinum; Euphorbine in Euphorbium; Convolvuline in Convolvulus scammonium; Pereirine in Pereira bark; Lobeline in Lobelia inflata ; Carapine in Carapa bark; Fagine in the beech nut or mast; Fumarine in Fumaria officinalis; Hederine in ivy seeds ; and others.

We now come to a class of compounds, very widely distributed in the vegetable kingdom, but not exhibiting the same varieties as the class hitherto described, and not characterised in the same marked properties. This is a class of neutral or indifferent non-azotised bodies, which are never poisonous, hardly even possessed of medicinal properties, and exhibit no striking chemical characters. It includes sugar, starch, gum, or mucilage, pectine or vegetable jelly, and woody fibre or lignine, with their derivatives. We can only describe them briefly.

\section{INDIFFERENT NON-AZOTISED COMPOUNDS.}

\section{Sugar.}

There are various substances to which this name is given. These are, cane sugar, glucose, (grape sugar, starch sugar, diabetic sugar, or sugar of honey,) sugar of milk, and amorphous or uncrystallisable sugar. Besides these, there are one or two 
substances resembling sugar, such as mannite or mushroom sugar, and inosite or sugar of muscle.

1. Cane Sugar, $\mathrm{C}_{12} \mathrm{H}_{11} \mathrm{O}_{11}=\mathrm{C}_{12} \mathrm{H}_{9} \mathrm{O}_{9}+2 \mathrm{H} 0$, occurs in great abundance in the sugar-cane, the beet-root, the maple, besides many other vegetables. It is extracted from the juice of these plants by crystallisation, the evaporation being conducted at as low a temperature as possible. It crystallises with great facility, either in small grains by rapid cooling of a strong syrup, as in loaf sugar; or in large aistinct crystals by a slow process, as in sugar-candy. The above formula represents the composition of pure crystallised sugar.

Sugar forms large transparent hard crystals, which melt at $302^{\circ}$, or, according to Peligot, at $356^{\circ}$, forming a viscid liquid, which on cooling forms a transparent amorphous mass, barley sugar. This, when kept, gradually becomes crystalline, opaque, and friable. About $420^{\circ}$ sugar is converted into a brown tasteless mass, caramel, losing 3 eqs. of water.

Sugar dissolves in $\frac{1}{3}$ of its weight of cold, and in any quantity in boiling water; a solution saturated at $230^{\circ}$ becomes a solid crystalline mass on cooling (tablet): a solution saturated in the cold is viscid, and is called syrup. Syrup, when long boiled, loses the property of crystallising. The crystallisation of sugar from syrup is also prevented by the addition of $\frac{1}{20}$. of oxalic, citric, or malic acids. When boiled with diluted sulphuric acid, cane sugar is converted into grape sugar. With strong sulphuric acid it produces a dark brown liquid, containing a new acid, sulphosaccharic acid. Nitric acid converts it into saccharic acid, oxalio acid, and carbonic acid.

When boiled with very diluted sulphuric acid, sugar absorbs oxygen from the air, and produces formic acid, and a brown matter identical with ulmine, formed by the decay of wood. Sugar prevents the precipitation of many metallic solutions by alkalies; and when mixed with oxide of copper and potash, the oxide of copper is dissolved, forming a purple solution, which, on boiling, deposits a red suboxide of copper. It reduces partially the oxides of many metals, when boiled with their solutions.

Sugar forms crystallisable compounds with the alkalies, oxide of lead, and chloride of sodium. When in contact with the lining membrane of the stomach of a calf, or with the caseine of milk, sugar is transformed into lactic and butyric acids.

Sugar, if taken along with nitrogenised food, may be called nutritious: it would appear, however, to act ohiefly in contributing to the support of respiration, and thus keeping up the animal heat. An animal, confined to sugar as food, soon dies from want of nitrogenised or albuminous matter, with the 
symptoms of starvation. It has lately been proved that sugar, that is, grape sugar, exists in the blood, especially that of certain parts.

When a solution of sugar is examined by polarised light, it gives rise to a series of rings of the prismatio colours, but the plane of polarisation is made to deviate to the right.

\section{SUGAR WITH BASES AND SATTS.}

With lime sugar forms a sparingly soluble compound, $\mathrm{C}_{22} \mathrm{H}_{9}$ $\mathrm{O}_{\ominus}+\left\{\begin{array}{ll}\mathrm{Ca} & \mathrm{O} \\ \mathrm{H} & \mathrm{O}^{\circ}\end{array}\right.$ With baryta, it forms a crystallisable compound, $\mathrm{C}_{12} \mathrm{H}_{\theta} \mathrm{O}_{\theta}\left\{\begin{array}{ll}\mathrm{Ba} \mathrm{O} \\ \mathrm{H} & \mathrm{O}^{*}\end{array}\right.$ With oxide of lead it yields an insoluble compound, $\mathrm{C}_{12} \mathrm{H}_{9} \mathrm{O}_{9}, 2 \mathrm{~Pb} \mathrm{O}$; and with common salt it yields a crystalline compound, $2 \mathrm{C}_{12} \mathrm{H}_{9} \mathrm{O}_{9}+\left\{\begin{array}{l}3 \mathrm{HO} \\ \mathrm{Na} \mathrm{Cl}\end{array}\right.$.

2. Glucose. Syn. Grape Sugar. Diabetic Sugar. Starch Sugar, $\mathrm{C}_{12} \mathrm{H}_{14} \mathrm{O}_{14}$. This sugar occurs in the juice of many fruits, and is besides a product of the metamorphosis of starch, cane sugar, woody fibre, sugar of milk, \&c., when boiled with diluted acids. It may also be obtained from starch by the action of infusion of malt, or of diastase. It occurs in the urine of those affected with diabetes mellitus. The crystals which form in honey are likewise glucose.

It is best extracted from dried grapes, or honey, and is also prepared on the large scale from starch. 1 part of starch is boiled with 4 of water, and from $\frac{1}{100}$ to $\frac{1}{10}$ of sulphuric acid, during 36 or 40 hours; or an infusion of malt is added to jelly of starch, which soon becomes liquid, and, in a few hours, is converted into sugar. When acid is used, it is neutralised by chalk, the solution of sugar tiltered, and evaporated to a syrup, or, if required, to a dry mass. In this process, starch, $\mathrm{C}_{12} \mathrm{H}_{10} \mathrm{O}_{10}$, takes up 4 eqs. water, and produces glucose, $\mathrm{C}_{12} \mathrm{H}_{14} \mathrm{O}_{14}$; so that 100 parts of pure starch yield, or ought to yield, 122 of glucose. The same explanation, only varying the quantity of water, applies to the conversion into glucose of cane sugar, $\mathrm{C}_{12} \mathrm{H}_{11} \mathrm{O}_{11}$; woody tibre, $\mathrm{C}_{12} \mathrm{H}_{8} \mathrm{O}_{8}$; and sugar of milk, $\mathrm{C}_{12} \mathrm{H}_{12} \mathrm{O}_{12}$, these compounds requiring 3,6 , and 2 eqs. of water respectively to form glucose.

The action of infusion of malt is not explained; all that we know is, that this infusion, or a solution of diastase, a substance contained in it, does actually cause starch to take the form of glucose. It is probable that the diastase is in a state of 
decomposition, and may act as a ferment. The action of the acid would seem to be equally obscure; but there is some reason to think that there is first formed, as in the case of ether, a coupled acid, or acid salt, which, like sulphovinic acid, is decomposed by boiling. According to De Saussure, sulphuric acid and starch actually form a crystallisable compound.

Glucose crystallises from alcohol in square tables or cubes; a concentrated syrup of it yields only a mass formed of crystalline grains. It is much less soluble, requiring $1 \frac{1}{2}$ part of cold water, and less sweet to the taste, than cane sugar : in fact, 1 part of cane sugar sweetens as much as $2 \frac{1}{2}$ of glucose. It is much more soluble in cold alcohol than cane sugar. At $212^{\circ}$ glucose loses 2 eqs. of water; when heated beyond $284^{\circ}$ it becomes caramel. Hot water dissolves any quantity of glucose, but the syrup is not nearly so viscid as that of cane sugar. Solution of glucose exhibits the prismatic rays with polarised light, and causes the plane of polarisation to deviate to the right.

Glucose is easily distinguished from cane sugar by the action of acids and bases. With pure bile and a little sulphuric acid, it strikes a violet colour, and probably cane sugar will do the same. Strong sulphuric acid dissolves without charring it, forming sulphosaccharic acid; and the alkalies and alkaline earths, which do not decompose cane sugar unless very concentrated, rapidly convert glucose into a brown matter. Peroxide of lead converts it, at $212^{\circ}$, into basic formiate of lead, carbonate of lead, and water.

The best and most delicate test for glucose is Trommsdorff's or the copper test. If to a solution of glucose we add a little solution of sulphate of copper, and then caustic potash in proper quantity, a blue solution is formed, which, on standing a little, deposits a red precipitate of suboxide of copper, and becomes finally colourless. This change is accelerated by boiling, and if there be no excess of the copper salt, the whole copper is precipitated. By this means we can easily detect 1 part of glucose in 1,000,000 of water, a perceptible red precipitate being formed. Even a solution ten times more diluted, and containing therefore only 1 part in $10,000,000$, is seen to be tinged red in certain positions, or when we look through a considerable depth of it. Cane sugar does not produce the same effect unless long boiled, and then it is probably converted, in part at least, into glucose.

By this test, glucose has been detected by Bernard as a normal constituent of the blood in certain parts of the body.

The same process may be applied to the determination of the amount of sugar in any liquid. The liquid is first boiled with acid, to convert all cane sugar into glucose, and then a solu- 
tion of a salt of copper in potash, of a known strength, is added as long as it is decolorised by the saccharine solution.

With baryta and lime, and oxide of lead, glucose forms compounds which it is difficult to obtain pure. That with baryta appears to contain 3 eqs. baryta for 2 eqs. of gluoose, and as the baryta replaces water in such compounds, it is probably $\mathrm{C}_{24} \mathrm{H}_{25} \mathrm{O}_{25}, 3 \mathrm{Ba} \mathrm{O}=\mathrm{C}_{24} \mathrm{H}_{22} \mathrm{O}_{22}\left\{\begin{array}{ll}3 \mathrm{BaO} \\ 3 \mathrm{H} \mathrm{O}\end{array}\right.$. The compound with lime appears to be $\mathrm{C}_{12} \mathrm{H}_{12} \mathrm{O}_{12}, 2 \mathrm{Ca} \mathrm{O}$; and that with lead $\mathrm{C}_{12} \mathrm{H}_{11} \mathrm{O}_{11}, 3 \mathrm{~Pb} \mathrm{O}$. If we suppose the dry glucose to be $\mathrm{C}_{12} \mathrm{H}_{11} \mathrm{O}_{11}$, and to combine with 3 eqs. water and 3 eqs. base, then we should have

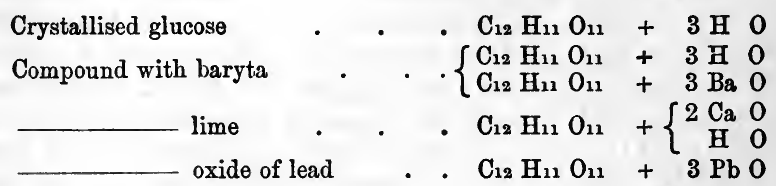

But the compound which grape sugar forms with common salt, and which crystallises very readily, does not agree exactly with this view. The crystals are $2\left(\mathrm{Cl}_{12} \mathrm{H}_{12} \mathrm{O}_{12}\right)+\mathrm{NaCl}+2 \mathrm{H} \mathrm{O}$; and at $212^{\circ}$ they lose the two equivalents of water.

The sulphosaccharic acid, above mentioned as being formed when grape sugar is acted on by oil of vitriol, has not been fully examined. It forms a soluble salt with baryta.

With organic acids glucose forms compounds, the sugar in which cannot be brought to crystallise. Hence organio acids in vegetable juices act injuriously by first converting cane sugar into glucose, and then forming with it uncrystallisable compounds.

When sugar is boiled with hydrochloric acid, it yields different brown products, according to the strength of the acid. With equal parts of acid and water, it yields a body, $\mathrm{C}_{24} \mathrm{H}_{11} \mathrm{O}_{9}$ : with a weaker acid, two brown compounds; a soluble one, $\mathrm{C}_{40} \mathrm{H}_{14} \mathrm{O}_{10}$, and an insoluble one, $\mathrm{C}_{46} \mathrm{H}_{16} \mathrm{O}_{14}$. When boiled with diluted sulphuric acid, two substances are formed, which are nearly black; one, sacchulmine, insoluble, and the other sacchulmic acid, soluble, in ammonia. The latter is $\mathrm{C}_{35}$ $\mathrm{H}_{10} \mathrm{O}_{15}$.

When boiled with alkalies, cane sugar is first converted into grape sugar, and then into formic acid, and two new acids, the glucic acid and the melassic acid. Glucic acid is very soluble, and its formula is either $\mathrm{C}_{12} \mathrm{H}_{9} \mathrm{O}_{9}$, or $\mathrm{C}_{24} \mathrm{H}_{18} \mathrm{O}_{18}$, It is chietly 
formed before the application of heat, which converts it into melassic acid. This latter acid is formed from sugar by the joint action of heat and alkalies. It has a very dark colour, and when separated by hydrochloric acid, appears as a black flocculent deposit. Its formula is $\mathrm{C}_{24} \mathrm{H}_{13} \mathrm{O}_{13}$.

Caramel, the black matter formed by heating sugar to about $400^{\circ}$, has the formula of anhydrous cane sugar, $\mathrm{C}_{22} \mathrm{H}_{9} \mathrm{O}_{9}$, or $\mathrm{C}_{24}$ $\mathrm{H}_{18} \mathrm{O}_{18}$. It dissolves readily in water, forming a solution like sepia, which is tasteless when pure. The caramel of commerce contains a good deal of undecomposed sugar.

When sugar is distilled with 3 parts of lime, it yields a liquid which is a mixture of acetone and propione. Propione is a colourless liquid, of an agreeable odour, boiling at $183^{\circ}$, and insoluble in water. Its formula is $\mathrm{C}_{10} \mathrm{H}^{10} \mathrm{O}_{2}$, or $\mathrm{C}_{5} \mathrm{H}_{5} \mathrm{O}$. The formation of these products is therefore easily accounted for.

When sugar is heated with hydrate of potash, several products are formed, but among them is an acid, $\mathrm{C}_{6} \mathrm{H}_{6} \mathrm{O}_{4}=$ $\mathrm{C}_{6} \mathrm{H}_{5} \mathrm{O}_{3}, \mathrm{H} \mathrm{O}$, which is propylic acid, evidently derived from propione by oxidation at the expense of the hydrate. It is very similar to acetic acid, and, like it, belongs to the series of volatile acids of the general formula $\left(\mathrm{C}_{n} \mathrm{H}_{n}\right)_{2}+\mathrm{O}_{4}$.

\section{ACTION OF NITRIC ACID ON SUGAR.}

Saccharic Acid, $\mathrm{C}_{12} \mathrm{H}_{8} \mathrm{O}_{14}, 2 \mathrm{H} \mathrm{O}$, is one of the products of the action of diluted nitric acid on cane or grape sugar. When stronger acid is used, oxalic and carbonic acids are the chief products. When sugar has been heated with 2 parts of nitric acid and 10 of water, the acid liquid gives, with basic acetate of lead, an insoluble saccharate of lead, which is decomposed by sulphuretted hydrogen, and the acid solution so far neutralised with potash, that on evaporation it yields crystals of the acid saccharate of potash. This salt is purified, again converted into saccharate of lead, and again the lead salt is decomposed by sulphuretted hydrogen. The acid this time is pure. It crystallises with difficulty.

This acid has been supposed to be quintibasic, and to form five series of salts; but the latest researches of Heintz lead to the conclusion that it is $\mathrm{C}_{22} \mathrm{H}_{8} \mathrm{O}_{14}, 2 \mathrm{H} \mathrm{O}$. Saccharic acid forms a crystallisable acid salt with potash, and definite salts with many other bases. It is isomeric with mucic acid, for $\mathrm{C}_{12} \mathrm{H}_{8} \mathrm{O}_{14}, 2 \mathrm{H} \mathrm{O}$ is the formula of mucic acid.

The saccharate of silver, when gently heated under water, is decomposed, the silver being reduced; and as this occurs with- 
out effervescence, the reduced metal adheres to the glass, and forms a bright mirror surface. The other saccharates are only interesting in respect to their composition. On the whole, saccharic acid is a compound of high theoretical interest, and the formation of two isomeric acids, saccharic and mucie, by the action of nitric acid on cane and grape sugar on the one hand, and on gum and sugar of milk on the other, is a fact which may hereafter lead to a knowledge of the true constitution of the different kinds of sugar.

Notwithstanding the fact that cane sugar is easily converted into glucose, and that the formulæ differ only by 3 eqs. water, it is evident that these two kinds of sugar differ more than if they were merely different hydrates of the same compound. Strong mineral acids instantly decompose cane sugar, but have little action on grape sugar; while alkalies, which combine with cane sugar to form crystalline compounds, rapidly convert grape sugar into dark compounds, glucic and melassic acids. And although both sugars agree in undergoing the same (vinous) fermentation, yet this is because cane sugar, before fermenting, becomes grape sugar.

When vegetable juices containing cane sugar are evaporated, the presence of organic acids causes its conversion into grape sugar; and when lime is added, to clarify the juice, the action of the lime on grape sugar, when evaporated, produces glucio and melassic acid; in other words, renders much sugar dark and uncrystallisable, converting it into molasses. A great part of the loss owing to this cause has, of late years, been avoided by carefully neutralising with sulphurio acid as soon as the lime has effected the clarification.

3. Sugar of Milk, or Lactine, $\mathrm{C}_{12} \mathrm{H}_{10} \mathrm{O}_{10}, 2 \mathrm{H} \mathrm{O}=\mathrm{Cl}_{12} \mathrm{H}_{12} \mathrm{O}_{12}$, is obtained by evaporating clarified whey till it crystallises. When pure, it forms hard white crystals, soluble in 5 or 6 parts of cold and $2 \frac{1}{2}$ of hot water. The taste of the crystals is feeble, but a concentrated solution tastes very sweet. It is insoluble in ether and alcohol. It is isomeric with glucose or grape sugar. By boiling with diluted acids, it is converted into grape sugar. By the action of nitrio acid, it yields mucic or saccholactic acid. It combines with ammonia and with oxide of lead. Its presence prevents the precipitation of many metallic solutions. Sugar of milk is susceptible of the vinous, lactic, and butyric fermentations; and it is well known that some nations prepare an intoxicating liquor from milk by fermentation. There is reason to think that, previous to fermentation, it is, like cane sugar, converted into grape sugar; and, at all events, milk does not 
ferment until an acid has been formed in it, which acid converts lactine into grape sugar.

Sugar of milk forms two compounds with oxide of lead, the composition of which is not fully ascertained.

Mucic Acid, $\mathrm{C}_{22} \mathrm{H}_{8} \mathrm{O}_{14}+2 \mathrm{H} \mathrm{O}$, is formed when diluted nitric acid acts on sugar of milk, gum, or mannite. It is a white crystalline powder, of a feebly acid taste, soluble in 6 parts of boiling water, which deposits nearly the whole on cooling. Its solution, when long heated and evaporated, yields the modified mucic acid. Mucic acid dissolves oil of vitriol with a crimson colour. When heated, it blackens, and yields among other products, pyromucic acid.

Mucic acid is bibasic, and forms two series of salts, one with 2 eqs. fixed base, the other with 1 eq. fixed base, and 1 eq. water. These salts have little interest. The mucate of oxyde of ethyle or mucic ether, crystallises in 4-sided prisms, soluble in hot water. When boiled with a base it yields alcohol, and mucate of the base. Its formula is $\mathrm{C}_{12} \mathrm{H}_{8} \mathrm{O}_{14}+2 \mathrm{Ae} \mathrm{O}$.

Modified Mucic Acid is more soluble in water, soluble in alcohol, from which solution it is deposited in square tables. Its aqueous solution, saturated at the boiling point, deposits on cooling, ordinary mucic acid. Its salts are more soluble than the mucates, but the acid in them easily passes into the ordinary acid. It is probable that the modified acid contains 1 eq. of water more than the other.

Pyromucic Acid, $\mathrm{C}_{10} \mathrm{H}_{3} \mathrm{O}_{5}+\mathrm{H} \mathrm{O}$, is formed by the dry distillation of mucic acid. 1 eq. of mucic acid, $\mathrm{C}_{12} \mathrm{H}_{10} \mathrm{O}_{16}$, contains the elements of 1 eq. pyromucic acid, $\mathrm{C}_{10} \mathrm{H}_{4} \mathrm{O}_{6}, 6$ eqs. water, $\mathrm{H}_{6}$ $\mathrm{O}_{6}$; and 2 eqs. carbonic acid, $\mathrm{C}_{2} \mathrm{O}_{4}$. Pyromucic acid forms brilliant white scales, fusible at $266^{\circ}$, and volatilises completely at a temperature somewhat higher. It is soluble in water and alcohol. Its salts are not important. It is isomeric with pyromeconic acid, but quite distinct in properties. Pyromucate of oxide of ethyle, $\mathrm{C}_{10} \mathrm{H}_{3} \mathrm{O}_{5}$, Ae $\mathrm{O}$, is a solid crystallisable compound, fusible at $93^{\circ}$, volatile at $410^{\circ}$. Chlorine acts on this ether, forming a new compound, $\mathrm{C}_{14} \mathrm{H}_{8} \mathrm{Cl}_{4} \mathrm{O}_{6}$, the constitution of which is quite uncertain.

4. Sugar of Mushrooms. - Wiggers obtained from ergot of rye a saccharine compound, crystallising in transparent rhombic prisms, soluble in water and alcohol, and susceptible of the vinous fermentation. An analysis of this sugar gave the formula $\mathrm{C}_{12}$ $\mathrm{H}_{13} \mathrm{O}_{13}$, that is, grape sugar, minus 1 eq. water. This may be a distinct kind of sugar; but the mushroom sugar of Braconnot is mannite or manna sugar.

5. Sorbine, $\mathrm{C}_{12} \mathrm{H}_{12} \mathrm{O}_{12}$. This is another form of sugar, isomeric 
with glucose. It is found in the berries of the mountain ash. It forms colourless crystals, of a sweet taste, less soluble in alcohol than the other forms of sugar. By heat it is decomposed, yielding a red acid, sortinic acid, said to be $\mathrm{C}_{32} \mathrm{H}_{18} \mathrm{O}_{15}$. This formula is very doubtful. It reduces the salts of copper, especially when potash is added.

6. Inosite, $\mathrm{C}_{12} \mathrm{H}_{12} \mathrm{O}_{12}+4$ aq. This is a sugar found in the juice of flesh. It forms mammellated groups of minute crystals, which are sweet and very soluble in water, sparingly in strong alcohol, insoluble in absolute alcohol and ether. It does not appear to undergo the vinous fermentation, but is capable of the lactic and butyric fermentations.

If a very small portion of inosite be added to a drop or two of nitric acid, dried up, and the residue moistened with a little ammonia and chloride of calcium, and again evaporated to dryness, all on a platinum spatula, a fine rose colour is developed. $\frac{1}{50}$ th part of a grain may be thus detected. Inosite does not reduce copper to the suboxide when mixed or boiled with the sulphate of copper and caustic potash.

7. Uncrystallisable Sugar. Sugar of fruits, $\mathrm{Cl}_{12} \mathrm{H}_{12} \mathrm{O}_{12}$. This is another isomeric form of glucose. It is found in most vegetable juices, especially if acid, also in honey along with grape sugar. Cane sugar is converted into it by boiling with diluted sulphuric or hydrochloric acid. It causes the plane of polarisation to deviate to the left. It acts on the copper test exactly as glucose does, and undergoes the vinous fermentation like that sugar. Indeed in the fermentation of cane sugar, it is into this form of grape sugar that the cane sugar is converted, before it ferments, and this change is seen in the action on polarised light.

We have seen that starch and woody fibre may be converted into grape sugar by boiling with dilute sulphuric acid; in like manner, salicine and phloridzine, boiled with the same acid, yield saliretine and phloretine in each case, along with grape sugar. But the action of the infusion of malt is still more singular; we have seen that starch, by contact with infusion of malt, is rapidly converted into grape sugar. This action is ascribed to the presence of diastase, a nitrogenised body which exists in malt, and which, while it causes the conversion of starch into sugar, itself disappears. The action is evidently due to the state of decomposition of the diastase, which is an albuminous body in a state of change; and there is no doubt that when seeds germinate, the starch they contain is in this manner rendered soluble, and conveyed, as sugar, to all parts of the plant, there to be converted into woody fibre by a process the inverse of that by which woody fibre is converted into sugar. 
This latter is seen in the ripening of fleshy fruits, where a quantity of cellular matter (lignine) disappears, and the proportion of sugar very much increases.

\section{VINOUS OR ALCOHOLIC FERMENTATION.}

This name is given to that change by which sugar is resolved into alcohol and carbonic acid, by contact with a ferment. The sugar must be dissolved in water, and the solution must be exposed to a temperature of from $40^{\circ}$ to $86^{\circ}$. If a ferment, such as yeast, be added, the sugar soon disappears, carbonic acid is given off in large quantity, and the liquid is found to contain alcohol, which may be separated by distillation. Now, grape sugar $\mathrm{C}_{12} \mathrm{H}_{14} \mathrm{O}_{14}$, contains the elements of 2 eqs. alcohol, 4 eqs. carbonic acid, and 2 eqs. water, $2\left(\mathrm{C}_{4} \mathrm{H}_{6} \mathrm{O}_{2}\right)+4 \mathrm{C} \mathrm{O}_{2}+2 \mathrm{H} \mathrm{O}$; and, by very exact experiments, it has been proved that 100 parts of grape sugar yield only $47 \cdot 12$ of alcohol, 44.84 of carbonio acid, together 91.96 parts, the loss, 9.04 parts being the 2 eqs. of water separated. On the other hand, cane sugar, $\mathrm{C}_{12} \mathrm{H}_{11}$ $\mathrm{O}_{11}$, requires the addition of $1 \mathrm{eq}$. of water to yield 2 eqs. alcohol and 4 eqs. carbonic acid, $=2\left(\mathrm{C}_{4} \mathrm{H}_{6} \mathrm{O}_{7}\right)+4 \mathrm{C} \mathrm{O}_{2}$ : and here also experiment has demonstrated that 100 parts of cane sugar yield 53.727 parts of alcohol, and 51.298 of carbonic acid, together 105.025 ; the increase, or 5.025 parts, being due to the 1 eq. of water taken up to form dry grape sugar, $\mathrm{C}_{12} \mathrm{H}_{12} \mathrm{O}_{12}$, into which cane sugar is converted before it undergoes fermentation. These facts prove that the ferment takes no direct part in the reaction, but only acts by inducing a state of change.

A considerable number of substances, if in a state of decomposition, act as ferments on a solution of sugar; among these are, besides yeast, vegetable gluten, albumen, caseine or fibrine, and the corresponding animal substances; also animal matter generally, if in a state of putrefaction.

It appears that when a vegetable juice, such as grape juice, or an infusion of malt, ferments, the admission of the air is necessary to the commencement of the change, which then goes on, even if air be afterwards excluded. It was stated by Gay Lussac, that the oxygen of the air acted by inducing decomposition of the ferment, but recent experiments have shown that air may be admitted to such solutions, without fermentation occurring, provided the air be passed through a tube filled with cotton wool. The reason seems to be that the germs of fungi are thus arrested, which would otherwise cause fermentation by being developed in the ferment, which is decomposed during their growth. It is certain that yeast consists in great part of the cells of a minute fungus, the 
germs of which must have found admission. Some liquids, however, ferment or putrefy even when supplied only with air filtered as above. This is the case with infusion of malt. However this may be, we are sure that, with or without fungi, the ferment is only capable of acting as a ferment when it is in a state of decomposition, and the only explanation we can give of the fermentation of sugar is, that the particles of these bodies, being in a state of decomposition, are in motion, and by communicating, mechanically, an impulse or motion to the particles of the sugar, destroy the balance of affinities to which the existence of sugar is owing; and thus give rise to a new balance or equilibrium, more stable under the given circumstances. The elementary particles of the sugar being disturbed in their previous arrangement, group themselves according to their individual affinities: and while the carbon forms, on one side, a compound containing all the hydrogen (alcohol), it yields, on the other, a compound containing the greater part of the oxygen (carbonic acid).

When a natural juice, as that of the grape, ferments, some of the various substances it contains undergo a decomposition, probably of an analogous kind, giving rise to other new products, which are important in regard to the flavour of the liquid (wine, beer, or spirits) produced in the fermentation. Thus all wine contains œnanthic ether, the higher-flavoured wines contain acetic, propylic, butyric, and caproic ethers, possibly also others, but all in very small proportion; potato spirit contains the oil of potato spirit (Fuseloel. German); grain spirit contains a similar oil. It is not improbable, that besides the vinous fermentation which takes place in the greater part of the sugar, a peculiar reaction occurs, between a portion of the sugar and the ferment (or some other nitrogenised compound present), the result of which is the production of these peculiar oily liquids. The bouquet, or so much prized flavour of the finer wines, is doubtless owing to some ethereal compound produced in a similar way, but the origin, properties, and composition of which are as yet altogether unknown. It may be mentioned, here, however, that onanthic ether, which is the cause of that peculiar smell which belongs to all wine, and is so marked that we can at once tell, after many weeks or months, that an empty bottle has formerly contained wine, is a compound of oxide of ethyle with a fatty acid; and the oil of potato spirit is a compound analogous to alcohol; the hydrated oxide of a radical amyle, $\mathrm{Cro}_{11}$.

In the fermentation of the marc of the grape, or the expressed residue, in which much sugar is left, and probably because of the presence of a peculiar ferment in the skins or seeds, there occur 
other alcoholic fermentations, yielding besides ethylic alcohol, the propylic, butylic, amylic, and caproic alcohols. These may be easily explained :-

$$
\begin{aligned}
& 2 \mathrm{C}_{12} \mathrm{H}_{12} \mathrm{O}_{12}=8 \mathrm{CO}_{2}+ \begin{cases}\mathrm{C}_{4} \mathrm{H}_{6} \mathrm{O}_{2} & \begin{array}{c}
\text { Alcohols. } \\
2\left(\mathrm{C}_{6} \mathrm{H}_{8} \mathrm{O}_{2}\right)
\end{array} \\
2 \text { Ethylic } \\
2 \mathrm{H} \mathrm{O} \text { Propylic }\end{cases} \\
& \text { or }=8 \mathrm{CO}_{2}+\left\{\begin{array}{l}
2\left(\mathrm{C}_{8} \mathrm{H}_{10} \mathrm{O}_{2}\right) \\
4 \mathrm{H} \mathrm{O}
\end{array}\right. \text { Butylic } \\
& \text { or }=8 \mathrm{CO}_{2}+ \begin{cases}\mathrm{C}_{6} \mathrm{H}_{8} \mathrm{O}_{2} \\
\mathrm{C}_{10} \mathrm{H}_{12} \mathrm{O}_{2} \\
4 \mathrm{H} \mathrm{O} & \text { Propylic } \\
\mathrm{H} \text { mylic }\end{cases} \\
& \text { or }=8 \mathrm{CO}_{2}+ \begin{cases}\mathrm{C}_{12} \mathrm{H}_{14} \mathrm{O}_{2} & \text { Caproic } \\
\mathrm{C}_{4} \mathrm{H}_{6} \mathrm{O}_{2} & \text { Ethylic } \\
4 \mathrm{H} \mathrm{O} & \end{cases}
\end{aligned}
$$

\section{LACTIC FERMENTATION.}

When a solution of sugar is placed in contact with pressed curd, or unsalted skimmed-milk cheese, which has previously been exposed to the atmosphere for some time, and the mixture is kept at a temperature from about $75^{\circ}$ to $90^{\circ}$, a peculiar change takes place, which has been called a fermentation, but which differs from the vinous fermentation in this, that the escape of gas does not appear to be essential to it. The liquid soon becomes acid, from the conversion of sugar into lactic acid; but when the amount of free acid reaches a certain point, the fermentation is thereby checked. If now the free acid be neutralised by the addition of carbonate of soda, or carbonate of lime (chalk), lactate of soda or of lime is formed, carbonic acid is disengaged, and the fermentation recommences. By repeating the addition of soda or chalk as often as free acid appears, the whole of the sugar may be at last converted into lactic acid, and obtained in the form of lactate of soda or of lime.

The best method is to dissolve 1 part of cane sugar or sugar of milk in about 5 of water, to add at the commencement $\frac{1}{2}$ part of prepared chalk, and a proper quantity of curd or cheese, and to place the whole in a temperature of $77^{\circ}$ to $90^{\circ}$. Effervescence occurs, owing to the decomposition of the chalk, and in process of time the lactate of lime is deposited in small round masses of minute crystals, in such quantities as to solidify the whole mixture. This result sometimes takes place in ten days; at other times, according to the precise temperature or the state of the curd, not till after several weeks or even months. But when it is complete, which is known by the cessation of the disengagement of gas and the solidification of the mass, the sugar 
appears to be entirely converted into lactic acid, without the formation of any other product. This is the true lactic fermenttation; but, as we shall see, it is apt to be mixed and complicated with other forms of decomposition.

The conversion of sugar, $\mathrm{C}_{12} \mathrm{H}_{12} \mathrm{O}_{11}$, or of lactine, $\mathrm{C}_{22}$, $\mathrm{H}_{12} \mathrm{O}_{12}$, into lactic acid, $\mathrm{C}_{22} \mathrm{H}_{10} \mathrm{O}_{10}, 2 \mathrm{H} \mathrm{O}$, is very simple; for lactine already contains the elements in the necessary proportion, and cane sugar requires only the addition of 1 eq. of water. It is therefore probable, a priori, that no other product should be formed along with the lactic acid; and in several of my own experiments I have obtained so large a quantity of lactate of lime as to lead to that conclusion, while $\bar{I}$ could detect no other product, except some colouring matter and a little undecomposed sugar.

It is this fermentation which occurs in milk when exposed to the air. The caseine enters into the state in which it becomes capable of exciting the lactic fermentation in the sugar of milk (lactine); but as soon as the liquid becomes very acid, the fermentation is checked, unless an alkali be added. Hence sour milk contains both lactic acid and undecomposed lactine; but by the occasional addition of alkali the whole lactine may be converted into lactic acid, or rather, lactate of soda.

\section{VISCOUS FERMENTATION.}

When certain saccharine juices, such as those of beet-root, carrots, onions, \&c., are exposed to a temperature of from $86^{\circ}$ to $104^{\circ}$, a peculiar fermentation takes place. The sugar disappears, but instead of alcohol and carbonic acid, there are obtained mannite, lactic acid, and a mucilaginous substance, having the composition of gum; this latter renders the liquid ropy and viscid, hence the name given to the process.

$\begin{gathered}\text { The composition of mannite is } \\ \text { That of lactic acid is }\end{gathered}$
Together

It is evident, therefore, that 2 eqs. of dry grape sugar, $\mathrm{C}_{24} \mathbf{H}_{24}$ $\mathrm{O}_{24}$, gaining 2 eqs. hydrogen, might give rise to mannite and lactic acid. The gum has the same composition as sugar, so that we are led to believe that the nitrogenised constituents of the juice acts on the sugar, to which they yield hydrogen; and that these compounds are themselves decomposed by the loss of hydrogen, mannite, and lactic acid, which are very permanent, being produced from the sugar. 
There is every reason to believe that the viscous fermentation is a mixed process, consisting of the lactic fermentation and of another, the true viscous fermentation, the products of which are gum and mannite. In the preparation of lactio acid from sugar, with the aid of a curd above described, I have sometimes obtained a little mannite, but always in so small a proportion as to indicate that its production was not essentially connected with that of the lactio acid, while in other experiments no mannite appeared. Where mannite did occur, I am inclined to suppose that the lactic fermentation was more or less complicated with the viscous.

\section{BUTYRIC FERMENTATION.}

After the sugar, in the lactic fermentation, has been converted as above described, into lactate of lime, if the mixture, still containing the caseine or curd, be kept for some time in a temperature of from $90^{\circ}$ to $105^{\circ}$, the nearly solid mass of lactate dissolves by degrees, while a mixture of hydrogen and carbonio acid gases is given off, and at last the whole mass, except the cheese and any excess of chalk, becomes liquid. When the disengagement of gas has ceased, the liquid is found to contain no lactate, but only butyrate of lime; and this salt may be thus easily obtained in any quantity. The conversion of lactic into butyric acid is easily explained; for $\mathrm{C}_{22} \mathrm{H}_{22} \mathrm{O}_{12}=\mathrm{Cs}_{8} \mathrm{H}_{8} \mathrm{O}_{4}+$

\section{Lactic Acid. Butyric Acid.}

$\mathrm{H}_{4}+4 \mathrm{C} \mathrm{O}_{2}$; and it is probable that no other product is formed; for in some experiments $I$ have seen the lactate converted entirely into pure butyrate of lime, which crystallised to the last drop.

It is evident that under certain circumstances, the three kinds of fermentation just described may occur simultaneously, so that the liquid may contain lactic acid, butyric acid, mannite, gum, and unchanged sugar.

Mannite, $\mathrm{C}_{12} \mathrm{H}_{14} \mathrm{O}_{12}$, occurs as the chief ingredient of manna. It is also found in certain juices, in mushrooms, in roots, such as that of celery, and is formed artificially as above described. It is easily purified by solution in alcohol, and crystallisation. It forms, when crystallised in water, large prisms, of a weak sweet taste. It is not susceptible of the vinous fermentation. Nitric acid and permanganate of potash act on it as on sugar. By a mixture of nitric and sulphuric acids, it is converted into nitromannite, $\left.\mathrm{C}_{22} \underset{(\mathrm{N} \mathrm{O}}{\left.\mathrm{H}_{8}\right)_{6}}\right\} \mathrm{O}_{12}$. This compound forms silky crystals, which explode by heat or by percussion. 
Sulphuric acid forms with mannite sulphomannitic acid, $\mathrm{C}_{12}$ $\mathrm{H}_{14} \mathrm{O}_{12}, 6 \mathrm{~S} \mathrm{O}_{3}$.

Dulcose, $\mathrm{C}_{12} \mathrm{H}_{14} \mathrm{O}_{12}$, is isomeric with mannite. It is a substance resembling mannite, sent from Madagascar, and produced from some plant not yet known. It forms brilliant crystals. It differs from mannite in several points.

Phycite, $\mathrm{C}_{12} \mathrm{H}_{14} \mathrm{O}_{12}$ ? This seems to be also isomeric with mannite. It is found in Protococcus vulgaris. It forms rectangular prisms of a decided sweet taste. It differs in various properties from the two preceding compounds.

Quercite, $\mathrm{C}_{12} \mathrm{H}_{12} \mathrm{C}_{10}$. This substance, found in acorns, forms hard crystals, slightly sweet. Its formula is that of mannite minus 2 eq. of water. Its action on polarised light is not mentioned, but the three preceding compounds have no such action.

Lactic Acid, $\mathrm{C}_{12} \mathrm{H}_{10} \mathrm{O}_{10}, 2 \mathrm{H} \mathrm{O}$, so called because it occurs in sour milk, is also formed abundantly, as above described, in a peculiar fermentation of certain saccharine juices at a high temperature. In milk it is derived from the sugar of milk; and by neutralising sour milk with carbonate of soda, adding sugar of milk, allowing it again to become acid, again neutralising, and so on in succession, as long as the caseine causes the peculiar change to take place, it may be obtained in large quantity. A still easier process is to dissolve 14 parts of cane sugar in 60 of water, and to add 4 of moist cheese and 7 of prepared chalk. The mixture being kept some time at from $77^{\circ}$ to $86^{\circ} \mathrm{F}$. will at last become quite thick with crystals of lactate of lime. If the action of the caseous ferment be pushed further, and at a higher temperature, the lactate of lime is not obtained, but in its place, butyrate of lime in large quantity. (See Butyric Fermentation.) The above quantities will yield about 13 parts of lactate after it has been purified by crystallisation; besides from $1 \frac{1}{2}$ to 2 parts mannite. The acid of sauerkraut is lactic acid, and by boiling the juice of sauerkraut with chalk or carbonate of zinc, lactate of zinc or of lime may be obtained.

Liebig has lately shown that lactic acid exists abundantly in the juice of flesh; and as abundantly in the flesh of carnivorous as in that of herbivorous animals. This is remarkable, as the food of carnivorous animals contains no saccharine or amylaceous matter, so that the lactic acid must be formed from albuminous compounds. The same remark applies to the occurrence of lactine in the milk of carnivora, and to that of glucose in dogs fed on flesh.

It has been stated by Cap and Henry, that lactic acid exists in normal urine, as lactate of urea; but I have never been able by their process to obtain from urine a trace of lactic acid, nor 
anything but pure urea. Pelouze and others were equally unsuccessful; and Liebig has proved by experiments on a very large scale, that urine contains no lactic acid in the normal state; and further, that lactic acid, taken internally, cannot be recognised in the urine.

From the lactate of lime, lactic acid may be obtained by the action of oxalic acid, which removes the lime as oxalate. The filtered solution is lactic acid, which is concentrated by evaporation, and purified by solution in ether. From the lactate of soda, lactate of zinc may be obtained by adding chloride of zinc to the hot saturated solution; on cooling, lactate of zinc, being sparingly soluble in cold water, crystallises. This salt, acted on by barytio water, yields lactate of baryta, from which sulphuric acid removes the baryta, and the filtered liquid is pure diluted lactic acid.

In its most concentrated form, hydrated lactic acid is a syrupy liquid, of a very strong but pleasant acid taste. Its Sp. G. is $1 \cdot 215$. Its formula, according to Engelhardt and Maddrell, is bibasic, $\mathrm{C}_{12} \mathrm{H}_{10} \mathrm{O}_{10}, 2 \mathrm{H} \mathrm{O}=\mathrm{C}_{12} \mathrm{H}_{12} \mathrm{O}_{12}$. It is therefore isomeric with dry grape sugar and with lactine, or sugar of milk, both of which are $\mathrm{C}_{12} \mathrm{H}_{12} \mathrm{O}_{12}$. At $482^{\circ}$ the hydrate is decomposed, and yields anhydrous lactic acid, $\mathrm{C}_{12} \mathrm{H}_{10} \mathrm{O}_{10}$, as an amorphous mass, which, with dry ammonia, forms lactamate of ammonia. Water converts it into the hydrated acid. Heated beyond $482^{\circ}$, the anhydrous acid yields a solid crystalline sublimate, $\mathrm{C}_{2} \mathrm{H}_{8} \mathrm{O}_{8}$, which has been called anhydrous lactic acid, or sublimed lactic acid, better lactide. This compound dissolves readily in hot water, and the solution if evaporated yields the original hydrate. But when the acid is neutralised by bases, only one of the 2 eqs. of water taken up by the sublimed acid is replaced by a base; and consequently we cannot look on lactide, that is, the sublimed acid as the true anhydrous acid. The anhydrous acid, as it exists in the lactates, is $\mathrm{C}_{12} \mathrm{H}_{10} \mathrm{O}_{10}$; and lactide is not lactic acid, but is converted into lactic acid when boiled with water.

The general formula for the lactates is $\mathrm{C}_{12} \mathrm{H}_{10} \mathrm{O}_{10}, 2 \mathrm{M} \mathrm{O}$. The lactates of the alkalies are very soluble and deliquescent: that of lime is less soluble in cold water and crystallises readily. The lactate of zinc is sparingly soluble in cold water, and is hence well adapted for the extraction and purification of the acid.

The salts of the lactic acid from flesh differ in the amount of water of crystallisation from those of the acid from milk.

Lactic acid may be regarded as a coupled formic acid, the copula being aldehyde; for $\mathrm{C}_{6} \mathrm{H} \quad \mathrm{O}_{6}$ (the half of lactic acid) = $\mathrm{C}_{2} \mathrm{H}_{2} \mathrm{O}_{4}+\mathrm{C}_{4} \mathrm{H}_{4} \mathrm{O}_{2}$. This constitution has been rendered probable, by the discovery that lactic acid may be artificially formed 
from alanine, a base obtained by the action of hydrocyanio and hydrochloric acids on aldehydammonia. It is well known that hydrocyanic acid with hydrochloric acid yields formic acid and ammonia. In the above reaction, therefore, we have formic acid and aldehyde in statu nascenti. They probably combine to form lactic acid, but ammonia being also present, another reaction ensues, and alanine, a body isomeric with lactamide, is obtained. We have thus, first, $\mathrm{C}_{2} \mathrm{H}_{2} \mathrm{O}_{4}+\mathrm{C}_{4} \mathrm{H}_{4} \mathrm{O}_{2}=\mathrm{C}_{6} \mathrm{H}_{6} \mathrm{O}_{6}$; and then $\mathrm{C}_{6} \mathrm{H}_{6} \mathrm{O}_{6}+\mathrm{N} \mathrm{H}_{3}=2 \mathrm{H} \mathrm{O}+\mathrm{C}_{6} \mathrm{H}_{7} \mathrm{NO}_{4}$. The latter group is alanine, which, when acted on by hyponitrous acid, yields lactic acid, thus; $\mathrm{C}_{6} \mathrm{NH}_{7} \mathrm{O}_{4}+\mathrm{NO}_{3}=\mathrm{C}_{6} \mathrm{H}_{6} \mathrm{O}_{6}+\mathrm{HO}+\mathrm{N}_{2}$.

If such be the true constitution of lactic acid, we should expect it, as well as alanine, to be one of a homologous series. And, in fact, this is the case. An acid has been discovered, glycolic acid, $\mathrm{C}_{4} \mathrm{H}_{4} \mathrm{O}_{6}$, which is formio acid, $\mathrm{C}_{2} \mathrm{H}_{2} \mathrm{O}_{4}$, coupled with formic aldehyde, $\mathrm{C}_{2} \mathrm{H}_{2} \mathrm{O}_{2}$; and glycocine, $\mathrm{C}_{4} \mathrm{~N} \mathrm{H}_{5} \mathrm{O}_{4}$, is the compound homologous with alanine, and corresponding to the new acid. The only other known members of these series are leucine, $\mathrm{C}_{22} \mathrm{~N} \mathrm{H}_{13} \mathrm{O}_{4}$, and the acid derived from it, $\mathrm{C}_{22} \mathrm{H}_{12} \mathrm{O}_{6}$; the latter, however, is but little studied. But glycolic acid, as will be mentioned under hippuric acid, is perfectly analogous in properties to lactic acid. The above facts also explain how it is that lactic acid, when distilled, yields so much aldehyde.

Lactamide, $\mathrm{C}_{12} \mathrm{H}_{14} \mathrm{~N}_{2} \mathrm{O}_{8}$, is obtained when ammonia is made to act on lactic acid. It is only remarkable from the fact that it is one of four isomeric or polymeric compounds, namely, lactamide, alanine, sarcosine, and urethane (carbamate of oxide of ethyle), all of which have perfectly distinct properties. It is called lactamide, because, like anides in general, it yields lactic acid and ammonia when acted on by acids and bases, which the other three do not.

Socoloff and Strecker have shown that an acid probably exists containing lactic and benzoio acids, the benzolactic acid, $\mathrm{C}_{40}$ $\mathrm{H}_{20} \mathrm{O}_{24}=\mathrm{C}_{12} \mathrm{H}_{12} \mathrm{O}_{12}+2\left(\mathrm{C}_{14} \mathrm{H}_{6} \mathrm{O}_{4}\right)$. 'This is homologous with benzoglycolic acid, an acid apparently pre-existing in hippuric acid, or of which hippuric acid is the amide. The formulæ here given are double those in the section referred to ; but the latter are easily doubled, and the relations are the same, both ways. (See Hippuric Acid.)

\section{Starch. $\mathrm{C}_{12} \mathrm{H}_{10} \mathrm{O}_{10}$.}

This very important compound is universally diffused in the vegetable kingdom. It occurs in seeds, as in those of wheat and other cerealia, and also in the leguminosæ; in roots, as in the 
tubers of the potato; in the stem or pith of many plants, as in Sagus Rumphii; in some barks, as that of cinnamon; and in pulpy fruits, such as the apple. Finally, it is contained in the expressed juice of most vegetables, such as the carrot, in a state of supension, being deposited on standing. Starch has been recently detected in the blood, in the brain, and in other parts of the animal body.

It is chiefly extracted from wheat flour (common starch); from potatoes (potato starch); from the root of Jatropha manihot (tapioca); from that of Maranta arundinacea (arrow-root); from the stem and pith of Sagus farinifera Rumphii (sago); the substances known by these different names being all essentially the same.

When flour is kneaded with water in a cloth, the water carries off the starch in suspension, and deposits it on standing, leaving behind the gluten. By a similar process starch is purified from the cellular substance and other matters mixed with it in potatoes, which are rasped, and then treated with water as above. Sago, being finally dried at a somewhat high temperature, acquires a horny and translucent appearance.

Pure starch is a snow-white powder, of a glistening aspect, which makes a crackling noise when pressed with the finger. It is composed of transparent rounded grains, the size of which varies in different plants. Those of the potato are the largest; those of the leguminosæ, as peas, are very small; and those of wheat and rice are smaller still. According to Payen, the largest starch granules, those of certain potatoes, have a length of 185 thousandths of a millimetre. The length of those of arrowroot is 140 thousandths of a millimetre. Those of the pea and of wheat measure 50 thousandths of a millimetre; while those of the seeds of beet and of Chenopodium quinoa have the length of 4 and 2 thousandths of a millimetre respectively. As 1 thousandth of a millimetre is equal to 4 one hundred thousandths of an inch, or 0.00004 , the above measurements in decimals of an inch, will be:-

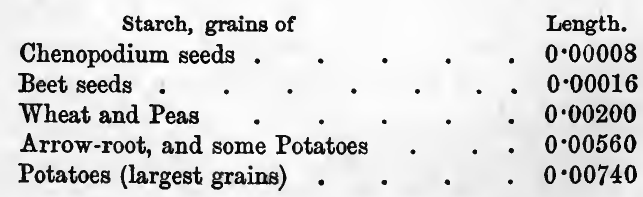

Starch is insoluble in cold water, alcohol, and ether; but when heated with water, it first becomes viscid, and is then converted into a kind of solution, which, however, is not complete, but 
is rather formed by the swelling of the grains of starch into a mucilaginous mass. On cooling. the whole forms a stiff, semiopaque jelly, starch paste. If dried up, this yields a translucent mass, which softens and swells into a jelly with water, like tragacanth. The solution, or mixture of starch and water, has the remarkable property of striking a deep blue colour with free iodine. This appears to be owing not so much to a shemical and definite combination, as to the mechanical division of the iodine ; there is even reason to think that the blue colour is that of iodine finely divided, adhering to the starch as a dye does to the fibres of cloth.

When starch is warmed with water, to which has been added either some infusion of malt or some diluted acid, the viscidity of the mixture disappears, and the fluid solution is no longer coloured blue by iodine. As soon as this is the case, the whole of the starch has disappeared, and has been converted into a soluble gum called dextrine, from its power of causing the plane of polarisation to deviate to the right. This change may be completely effected by heating starch to a certain point in sealed tubes, with water containing only $\frac{1}{500}$ th part of oxalic acid. The action of malt, in converting starch into dextrine, depends, as has been mentioned, on the presence of a nitrogenised or albuminous body, diastase. According to the proportion of malt or of acid, and the temperature employed, the change is more or less rapid; and when the action is continued, the dextrine is in its turn converted into glucose, or grape sugar, which from this circumstance is also called starch sugar.

In contact with oil of vitriol, starch appears to form a compound or coupled acid, sulphoamidic acid. Strong nitric acid, rubbed up with potato starch, dissolves it, forming a viscid liquid, from which water precipitates a white explosive compound, called xyloidine. This compound has some of the properties of gum tragacanth, but it contains the elements of nitric acid, and has not yet been fully investigated. According to Pelouze, its formula is $\mathrm{C}_{6} \mathrm{H}_{4} \mathrm{O}_{4}+\mathrm{NO}_{5}$; according to Ballot, it is $\mathrm{C}_{15} \mathrm{H}_{12} \mathrm{NO}_{16}$; but more recent researches tend to show that the formula of xyloidine is $\mathrm{C}_{12}\left\{\begin{array}{l}\mathrm{H}_{8} \\ 2 \mathrm{NO}_{4}\end{array}\right\} \mathrm{O}_{10}$; that is, starch in which 2 eqs. of hydrogen are replaced by 2 eqs. of nitrous acid. When starch is distilled with moderately strong sulphuric acid, it yields carbonic acid, formio acid, and a pungent volatile oil, hitherto very little examined.

The blue compound of iodine and starch is best prepared by adding to the liquid filtered from the viscid paste obtained by boiling starch with water, first iodide of potassium, and then 
solution of chlorine, as long as it causes a blue precipitate, which is to be washed till the water passes deep blue, and to be dried in vacuo. Its colour is so intense as to be nearly black. It does not appear to be a compound in definite proportions. The best method of using starch as a test for iodine in mineral waters, \&c., is to add to the water some starch paste, and then a little nitric acid or chlorine. The latter is best added in the form of gas, its weight allowing it to be poured like water; while in this way we are less likely to add an excess, which would destroy the blue colour. Or we may place in the bottom of a phial the liquid to be tested, adding a little oil of vitriol, and suspending from the stopper a slip of paper moistened with starch paste. After a time, if iodine be present, the paper will exhibit a tinge of blue. By these tests $\frac{1}{40000}$ th part of iodine in a liquid may be detected.

With bromine, starch forms an orange-yellow precipitate, which cannot be dried without decomposition.

Dextrine is obtained by heating to about $120^{\circ}$ a mixture of 20 parts of starch paste and 1 part of strong infusion of malt, until iodine no longer colours the mixture blue. The addition of strong alcohol now precipitates the dextrine as a thick syrup, while any sugar remains dissolved. Payen recommends the use of nitric acid. The starch is first mixed with $\frac{1}{500}$ th of its weight of nitric acid at $40^{\circ}$ of Beaumé, that is, ordinary but not the most concentrated acid; and in order to distribute this small proportion equally, so much water is added that the starch can absorb the whole without being rendered in any degree liquid. It is well kneaded together, cut into blocks, which are dried in a stove, in a current of air, after having been broken into small fragments ; the temperature being gradually increased to $140^{\circ}$, or not beyond $176^{\circ} \mathrm{F}$., till at the end of the process it is kept for some time at a constant temperature of $230^{\circ}$. In this way a gum is obtained much less coloured than that made with malt. When dried, dextrine much resembles gum, from which, however, it differs in the extreme facility with which it is converted into sugar when warmed with dilute sulphuric acid or infusion of malt, and by not yielding mucic acid when acted on by nitric acid. The composition of dextrine is the same as that of starch. In fact, dextrine is supposed by some to be the substance which is contained in the grains of starch, inclosed in an insoluble membrane, which is burst in the process of conversion of starch into dextrine, or solution of starch, by means of acids and infusion of malt. The substance present in the malt which has the property of effecting this change is called diastase. It contains nitrogen.

According to other observers, the grains of starch are composed 
of concentric layers of one and the same substance (dextrine?), the outer layer being insoluble in water. As starch is found to contain a small proportion of a matter analogous to wax or to caoutchouc, we may suppose that the presence of this matter in the outer layer is the cause of its insolubility, or that the whole mass of the grains is, by its means, rendered insoluble, and endowed with the property of swelling up with water to a paste or jelly. Dextrine will then be the purified, and consequently soluble, matter of starch. If the outer coat be different from the contents of the grains, it has still the same composition; for analysis shows no difference between starch, dextrine, and the insoluble matter left on the filter when starch is boiled with diluted acids.

Leiocome.-This name is given to a substance having the properties of gum, which is prepared by simply roasting or torrefying starch at about $300^{\circ}$. It is, in fact, capable of being used instead of gum in calico-printing, and is made on the large scale. It has a yellowish-brown colour. It is probably dextrine, more or less pure, generally containing some undecomposed starch. When well made it dissolves in cold water like gum.

Inuline, dahline, alantine, datiscine, menyanthine, $\mathrm{C}_{12} \mathrm{H}_{10} \mathrm{O}_{10}$ ? $\mathrm{C}_{24} \mathrm{H}_{18} \mathrm{O}_{18}$ ? $\mathrm{C}_{32} \mathrm{H}_{24} \mathrm{O}_{24}$ ? $\mathrm{C}_{24} \mathrm{H}_{21} \mathrm{O}_{21}$ ?-This is a substance analogous to starch in the roots and tubers of Inula helenium, Dahlia variabilis, Helianthus tuberosus, and many other synantherous plants, which do not yield ordinary starch.

It is extracted from the roots by boiling water, and is deposited by the concentrated decoction as a brittle white mass, formed of crystalline grains, or as a fine powder. It is tasteless, insoluble in cold, very soluble in hot water. Diluted sulphuric acid, with the aid of heat, rapidly converts it into grape sugar, from which, like starch, it differs only by a certain amount of the elements of water. The best analyses make it isomeric with starch. It would appear to differ in different vegetables, but always retaining the character of this class of bodies, namely, the presence of hydrogen and oxygen in the proportions to form water. Iodine colours it slightly brown.

Lichenine, $\mathrm{C}_{12} \mathrm{H}_{10} \mathrm{O}_{10}$.-This is a variety of starch found in Lichen islandicus, or Iceland moss. It forms, when pure, a nearly colourless, tasteless mass, which swells up into a transparent jelly with cold water, and dissolves entirely in hot water. When its solution is boiled, it forms pellicles, like milk, which adhere to the vessel. Its solution is not coloured by iodine, but the jelly is rendered blue by that test. By diluted and boiling sulphuric acid it is converted into sugar; by nitric acid, into oxalic and saccharic acids. It has the composition of starch. 
Saponine is the name given to a variety of starch obtained from the root of Saponaria officinalis. Its properties are little known.

\section{Gum.}

This name was formerly given to almost all exudations from plants. It is now limited to certain rather abundant substances, which are solid, uncrystallisable, transparent or translucent, colourless or nearly so ; tasteless, inodorous, soluble in water, or at least softening in it, and insoluble in alcohol, ether, fat, and volatile oils. They yield mucic acid when acted on by nitric acid. They may be divided into gums which dissolve in cold water (arabine, mucilage), and gums which only swell up to a jelly (tragacanth or bassorine, cerasine, pectine). Arabine and cerasine contain oxygen and hydrogen in the proportion to form water: the other gums are nearly analogous in composition.

Arabine, or gum arabic, is found as an exudation from several species of acacia. What is called gum senegal is essentially the same. It is nearly colourless, transparent, hard, and brittle, and has a mild taste. It is very soluble in cold water, and forms a viscid mucilage, from which alcohol precipitates the gum. The diluted solution is precipitated by silicate of potash, subacetate of lead and protonitrate of mercury. When a mixture of gum, water, and sulphuric acid is kept for some time at a temperature near boiling, it is converted into grape sugar. The composition of gum is $\mathrm{C}_{12} \mathrm{H}_{10} \mathrm{O}_{10}$, that is, the same as that of starch, which accounts for the trausformation. Arabine yields 2 or 3 per cent. of ashes, containing a good deal of lime.

Mucilage is the name given to a substance resembling gum, found in many vegetables, such as linseed, althea, and others. It differs from arabine in being less hard when dry, and less transparent. It would appear, however, that the mucilage of althæaroot is essentially starch inclosed in cells formed of woody fibre or cellulose. The different mucilages are resolved into grape sugar by being heated with dilute sulphuric acid, and therefore may be considered as containing, like starch and arabine, water plus carbon. Since all these mucilages contain much mineral matter, the mucilage of linseed, for example, leaving 11 per cent. of ashes rich in lime, it is probable that their peculiar qualities depend on the presence of phosphate of lime or other salts of lime, disguising either starch or arabine.

Bassorine is the name given to a substance which forms the ohief part of gum tragacanth and of gum bassora, and also, according to some, of salep, a mucilaginous substance, obtained from the 
bulbs of Orchis mascula. According to Schmidt, however, salep is really formed of swelled-up grains of starch.

Pure bassorine resembles arabine in appearance, but is less transparent, and instead of dissolving in cold water, only swells up to a very great extent, forming a viscid mass. Its composition is analogous to that of arabine, and by digestion with diluted sulphuric acid, it is transformed, like salep, into grape sugar and cellulose. Cerasine is the name given to that part of the gum of the cherry, plum, or almond trees, which is insoluble in cold water. It probably contains a mixture of gum arabic with bassorine, or with salep.

During what is called the viscous fermentation, which takes place in certain sweet vegetable juices, as that of beet-root, there is formed, along with lactic acid and mannite, a mucilaginous compound, which causes the viscidity. When dried it has nearly the characters and composition of arabine.

Pectine is the substance which causes the juice of some pulpy fruits, as apples and pears, to coagulate or gelatinise when mixed with alcohol, by which the pectine is precipitated. When dried, it resembles gum or isinglass, and forms a jelly with water. By the action of nitric acid it yields oxalic and mucic acids. It generally yields about 8 per cent. of ashes, containing much phosphate of lime. In contact with alkalies, it is transformed into pectic acid. Pectic acid is easily obtained from many vegetables, as, for example, rasped carrots, by washing them well with distilled water, and then boiling 50 parts of the squeezed residue with 300 of water and 1 of potash. The pectate of potash is deposited as a jelly in the filtered liquid on cooling. Either this salt or the pectate of lime may be decomposed by diluted hydrochloric acid, which leaves the pectic acid as a jelly, which dries up into transparent lamina, insoluble in water but very soluble in alkalies. From these solutions acids precipitate it as a jelly. In this form it is slightly soluble in boiling water, but the solution gelatinises on the addition of acids, salts, alcohol, or sugar. It is supposed not to exist readyformed in the plants, but to be produced by the action of alkalies on pectine.

The alkaline pectates, when dry, form gummy solids, soluble in water. Alcohol causes the solution to gelatinise, and even an excess of potash or soda has the same effect. The earthy and metallic pectates are gelatinous and insoluble. When dried, pectine, pectic acid, and all the pectates, assume a cellular structure, so to speak.

The jelly formed in currant juice, as well as other juices by the addition of sugar, is pectine or pectic acid. The boiling of such 
juices probably promotes the formation of jelly ; for it has been shown that when the insoluble part of unripe currants, after being washed, is boiled with water acidulated with a vegetable acid, a considerable quantity of pectine is formed, probably by a transformation of the oellular tissue.

The composition of pectic acid is not fully ascertained. According to Regnault it is $\mathrm{C}_{12} \mathrm{H}_{8} \mathrm{O}_{11}$, or $\mathrm{C}_{12} \mathrm{H}_{7} \mathrm{O}_{10}$, $\mathrm{HO}$. According to Mulder, it is $\mathrm{C}_{22} \mathrm{H}_{8} \mathrm{O}_{10}$. But the researches of Chodnew have led him to adopt the formula $\mathrm{C}_{28} \mathrm{H}_{18} \mathrm{O}_{24}, 2 \mathrm{H} \mathrm{O}=\mathrm{C}_{28} \mathrm{H}_{20}$ $0_{26}$. In all the formulæ there is an excess of oxygen over hydrogen.

The whole subject of the mucilaginous compounds, including pectine and pectic acid, is still very obscure and requires renewed investigations.

Apiine is a substance analogous to pectine, found in parsley, Apium graveolens. Glycyrrhizine is the name given to a substance resembling both sugar and gum, which is the chief ingredient in liquorice, the juice of the root of Glycyrrhiza glabra. It is soluble in hot water, and gelatinises on cooling. Its taste is sweet and also acrid; but it does not, like sugar, undergo the vinous fermentation. Its formula is said to be $\mathrm{C}_{16} \mathrm{H}_{12} \mathrm{O}_{6}$.

Sarcocolline is a gummy matter found in the sarcocolla of commerce, which is the dried juice of Penæa mucronata. It is soluble in alcohol and water, and has a taste both sweet and bitter. Formula $\mathrm{C}_{22} \mathrm{H}_{19} \mathrm{O}_{10}$ ? or $\mathrm{C}_{40} \mathrm{H}_{32} \mathrm{O}_{14}$ ?

\section{Woody Fibre.}

The skeleton of plants, after everything soluble in water, alcohol, ether, diluted acids, aud diluted alkalies has been removed, is called woody fibre. It varies in aspect and in composition as obtained from different plants. That of box or willow, when dried, is $\mathrm{C}_{22} \mathrm{H}_{9} \mathrm{O}_{9}$; that of oak is said to be $\mathrm{C}_{36} \mathrm{H}_{22} \mathrm{O}_{22}$; and that of beech is intermediate between these two. All varieties, however, may be represented as composed of carbon plus water.

Recent researches have shown that wood is composed of two parts : 1. cellulose, which forms the parietes of the vegetable cells, and which is found in its purest form in Swedish paper. Its formula, according to Mitscherlich, is $\mathrm{C}_{12} \mathrm{H}_{10} \mathrm{O}_{10}$, which accounts for its being convertible into starch and sugar. If paper be moistened, and sulphuric acid dropped on it, and allowed to remain till it forms a jelly; this jelly, when washed, is coloured blue by iodine. The application of heat with the acid converts 
the starch into sugar. Cellulose is found in the lower animals, as in the mantle of the ascidia, and others, as well as in the muscles of invertebrata. And 2. lignine, which fills those cells, or forms an incrustation on their walls. The latter dissolves in strong nitric acid, the former is left undissolved. Again, oil of vitriol dissolves cellulose without blackening, and it appears to convert it into dextrine, with which it agrees in composition; while lignine separated from cellulose is said to contain $\mathrm{C}_{35} \mathrm{H}_{24} \mathrm{O}_{20}$.

There can be little doubt that cellulose and even lignine are essentially isomeric with starch, dextrine, gum, and glucose, or differ only from the last in the proportion of water. In plants we see constantly wood produced from the soluble forms, and in germination, maturation of fruits, and other vegetative changes, we also see woody fibre dissolving in the forms of dextrine, gum, and sugar, or transformed into starch. Nay, by the mere action of boiling water, we can so change many kinds of woody fibre or cellulose, as to render it entirely soluble in water ; in other words, we can artificially convert it into dextrine. And all forms of it, when boiled with diluted acids, pass into glucose or grape sugar.

In its usual state of aggregation, cellulose is not coloured by iodine, but if it be first acted on by strong sulphuric and oily alkalies, so that disintegration has commenced, it is then coloured blue by iodine. In some cellular plants, the cellular tissue is at once coloured violet by solution of iodine.

Linen, cotton, and paper are woody fibre, more or less pure. All these substances, and others of similar nature, but less pure, such as tow and sawdust, yield, when acted on by nitric acid, explosive compounds, analogous to that obtained from starch, already described under the name of xyloidine.

Gun Cotton is best made by immersing for two minutes 1 part of clean dry cotton wool in about 10 parts of an acid composed of equal volumes of oil of vitriol, Sp. G. $1 \cdot 840$, and nitric acid, Sp. G. 1.510. It is then gently pressed, to expel as much as possible of the acid, rapidly washed with water, till the water runs off tasteless, and dried by a gentle water heat. 100 parts of dry cotton are found, when thus treated, although little changed in appearance, to have increased in weight to 169.5 parts. Of this weight 102.5 parts are nitric acid, and 67, or two-thirds, of the original weight, are derived from the cotton, and represent what may be called anhydrous cotton, the remaining one-third, or 33 parts, having been lost in the shape of water, which has combined with the acid.

According to Porret and Teschemacher, anhydrous cotton is $\mathrm{C}_{12} \mathrm{H}_{8} \mathrm{O}_{8}$, and gun cotton is $\mathrm{C}_{12} \mathrm{H}_{8} \mathrm{O}_{8}+4 \mathrm{~N} \mathrm{O}$, which 
corresponds to the above proportions. But the gun cotton may also be $\mathrm{C}_{12} \mathrm{H}_{8} \mathrm{O}_{12}+4 \mathrm{~N} \mathrm{O}_{4}$, or $\mathrm{C}_{12} \mathrm{H}_{8} \mathrm{O}_{16}+4 \mathrm{~N} \mathrm{O}_{3}$; while xyloidine, whether from starch or from woody fibre, is $\mathrm{C}_{12} \mathrm{H}_{8}$ $\mathrm{O}_{8}+2 \mathrm{~N} \mathrm{O}_{5}, \mathrm{Cl}_{12} \mathrm{H}_{8} \mathrm{O}_{10}+2 \mathrm{~N} \mathrm{O}_{4}$, or $\mathrm{C}_{12} \mathrm{H}_{8} \mathrm{O}_{12}+2 \mathrm{~N} \mathrm{O}_{3}$. This explains the superior explosive force of gun cotton, the combustion of which must be more complete. In fact, when exploded, it leaves no visible residue.

It is not probable that gun cotton will supersede gunpowder for fire-arms, as it is expensive, and its preparation is somewhat uncertain. But when well made, it explodes so rapidly that it will probably be found advantageous in the blasting of rocks.

By the continued action of acids or of hot alkalies, woody fibre yields a substance which is coloured blue by iodine. Linen, cotton, or paper, all of them different forms of woody fibre, when moistened with pretty strong sulphuric acid, are converted apparently first into dextrine, and afterwards into grape sugar. When heated with a more diluted acid, linen yields an amylaceous pulp hardly soluble in water, the composition of which is $\mathrm{C}_{2} 2$ $\mathrm{H}_{10} \mathrm{O}_{10}$, that of starch and cellulose.

When exposed to air and moisture, wood undergoes eremacausis, being slowly converted into a friable mass, which contains a larger proportion of carbon than the original wond. It would appear that the oxygen of the atmosphere combines with the hydrogen, and that carbon and oxygen are given off from the residue as carbonic acid, $\mathrm{C} \mathrm{O}_{2}$. As the residue is found still to consist of carbon and water, it is evident that for every equivalent of carbon removed, there are separated 2 eqs. of oxygen and hydrogen, so that the proportion of carbon to water in the residue is constantly increasing. Woody fibre, $\mathrm{C}_{12} \mathrm{H}_{10} \mathrm{Ofo}$, will thus yield first a residue of $\mathrm{C}_{11} \mathrm{H}_{8} \mathrm{O}_{8}$; then $\mathrm{C}_{10} \mathrm{H}_{6} \mathrm{O}_{6}, \mathrm{C}_{8} \mathrm{H}_{4} \mathrm{O}_{4}$, and so on. When air is left in contact with moist wood, its oxygen is removed and replaced by an equal volume of carbonic acid. This is one chief source of the insalubrity of marshy districts; and the effect is seen still more strikingly in the case of houses which have been submerged in an inundation, which are very unwholesome as long as the wood is moist.

The tendency of wood to decay is checked or destroyed by acids and many salts, especially corrosive sublimate. Out of contact of air, moist wood putrefies, yielding a white friable residue, containing less carbon than the wood. Such products have been analysed, but the results are not such as to lead to any certain formula. They were probably impure.

The composition of brown coal is analogous to that of wood partially decayed, but subjected to changes of the nature of putrefaction, as well as to eremacausis. Two specimens of brown 
eoal yielded $\mathrm{C}_{33} \mathrm{H}_{21} \mathrm{O}_{16}$ and $\mathrm{C}_{32} \mathrm{H}_{15} \mathrm{O}_{9}$, formulæ which are doubtful.

All the products of the decomposition of wood might be derived from oak wood, $\mathrm{C}_{36} \mathrm{H}_{22} \mathrm{O}_{22}$, if that were its true formula, by the fixation of oxygen, and the separation of water and carbonio acid.

When the substance called mould, which contains the debris of decayed vegetable matter, is boiled with alkalies, the filtered solution deposits, on the addition of acids, a brown precipitate, which has been called ulmine, humus, humine, geine, ulmic acid, humic acid, and geic acid. It is generally admitted that this precipitate is a product of the action of the alkali on the decayed vegetable matter, and the name of humus, humine, or geine is given to the substance which is believed to yield the humic acid. But this humus has not been isolated, and is not known.

Mulder examined the precipitates obtained from a variety of different sources, decayed wood, turf, peat, mould, \&c. With one exception, he found all to contain nitrogen, varying from 2.5 to 7 per cent. It is evident that these substances are vegetable matter in different stages of decay. Mulder considers these precipitates as compounds of water, or water and ammonia, with three different acids : 1. acid of mould, $\mathrm{C}_{40} \mathrm{H}_{12} \mathrm{O}_{14}$ : 2. humic acid, $\mathrm{C}_{40} \mathrm{H}_{12} \mathrm{O}_{12}$ : 3. ulmic acid, $\mathrm{C}_{40} \mathrm{H}_{14} \mathrm{O}_{12}$.

When sugar is boiled with diluted acids, it yields brown substances analogous to, if not identical with, these acids of Mulder.

It is important to observe the general presence of ammonia in mould, \&c. This ammonia has no doubt been absorbed from the air in great part ; and this will explain the favourable influence which these substances exert on vegetation. They act also in furnishing, by their slow decay, a continual supply of carbonic acid.

Crenic Acid and Aprocrenic Acid are two brown extractive matters, analogous to the preceding, and derived from decaying vegetable matter, which are found in certain mineral waters. They both appear to contain nitrogen.

\section{PRODUCTS OF THE DISTILLATION OF WOOD.}

When wood is heated in close vessels, it gives rise to an immense variety of products, according to the kind of wood and to the presence or absence of resinous or oily matters. In all cases there are formed gaseous, liquid, and solid products, with a residue of charcoal.

The gases are carbonic acid, carbonic oxide, olefiant gas and 
marsh gas. The liquids are partly soluble in water, partly insoluble. The latter constitute the tar, and are of a semifluid consistence.

The substances soluble in water are, besides water itself, acetic acid, acetone, pyroxylic spirit (hydrate of oxide of methyle), acetate of oxide of methyle, lignone, xylite, and mesite.

The oily substances, insoluble in water, are very numerous, including creosote, picamar, eupion, capnomor, \&c. Along with these are the compounds which at the ordinary temperature are solid, such as paraffine, naphthaline, oedriret, pittacall, pyréne, chryséne, and pyroxanthine. The last mentioned, being very volatile, chiefly accompanies the acetio or pyroligneous acid.

\section{Volatile Products, soluble in water.}

Acetic Acid. This is one of the chief products of the distillation of woods. Its mode of purification and its properties have been already described. As prepared from this source, it is often called pyroligneous acid. The crude or impure acid is highly antiseptic; not only because vinegar, like most acids, is so, but also because it contains much creosute dissolved. Hence it not only preserves meat, but gives to it a powerful and agreeable smoked flavour.

Pyroxylic Spirit. This name is given to the spirituous liquid, distilled from the crude pyroligneous acid before the latter is purified. It is a mixed fluid, the chief component being hydrated oxide of methyle, which is accompanied by acetate of oxide of methyle, unless it has been rectified with quicklime, which decomposes the latter. Lignone is the name given to a volatile liquid, somewhat resembling alcohol, observed in pyroxylio spirit by Gmelin and Liebig. Its formula is not ascertained, since it does not, as far as we know, form definite compounds from which its equivalent might be deduced. Xylite is another similar volatile liquid, which, according to Schweitzer, is $\mathrm{C}_{\mathbf{2 2}}$, $\mathrm{H}_{12} \mathrm{O}_{5}=2\left(\mathrm{C}_{2} \mathrm{H}_{3} \mathrm{O}\right)+\left(\mathrm{C}_{8} \mathrm{H}_{6} \mathrm{O}_{3}\right)=2 \mathrm{MeO}+\mathrm{Ac}_{2} \mathrm{O}_{3}$; that is a compound of two eqs. oxide of methyle and 1 eq. of a sesquiacetylic acid. When acted on by potash, it yields a crystalline salt $\mathrm{C}_{18} \mathrm{H}_{15} \mathrm{O}_{7}, \mathrm{~K} \mathrm{0}$, while hydrated oxide of methyle separates. An excess of potash causes the formation of three products: xylitic naphtha, $\mathrm{C}_{12} \mathrm{H}_{12} \mathrm{O}_{3} ;$ xylitic oil, $\mathrm{C}_{12} \mathrm{H}_{9} \mathrm{O}$; and xylitic resin, $\mathrm{C}_{8} \mathrm{H}_{6} \mathrm{O}$. When distilled with sulphuric acid, xylite, if moist, yields a new compound, a volatile liquid, mesitene, $\mathrm{C}_{6} \mathrm{H}_{6} \mathrm{O}_{3}$. If anhydrous, it yields besides another compound, methol, a less volatile liquid, which appears to be a carbohydrogen, $\mathrm{C}_{4} \mathrm{H}_{3}$; isomeric with acetyle, if it be not that radical. Mesite is another 
volatile ethereal liquid found in pyroxylic spirit, which, according to Schweitzer, is $\mathrm{C}_{6} \mathrm{H}_{6} \mathrm{O}_{2}$; isomeric with acetone. He considers it as composed of oxide of methyle and oxide of acetyle, $\mathrm{C}_{2} \mathrm{H}_{3} \mathrm{O}$ $+\mathrm{C}_{4} \mathrm{H}_{3} \mathrm{O}=\mathrm{Me} \mathrm{O}+\mathrm{Ac} \mathrm{O}$. The liquid called Mesite by Reichenbach would appear to be acetate of oxide of methyle, Mt $\mathrm{O}+\mathrm{Ac}_{3} \mathrm{O}_{3}$, mixed with a more highly carbonised body, apparently composed of $\mathrm{C}_{26} \mathrm{H}_{25} \mathrm{O}_{15}$. This latter is resolved by the action of lime into 3 eqs. acetio acid $\left(\mathrm{C}_{12} \mathrm{H}_{9} \mathrm{O}_{9}\right)$, and a volatile liquid, $\mathrm{C}_{14} \mathrm{H}_{16} \mathrm{O}_{6}$. Along with the above, another liquid appears to occur in the mesite of Reichenbach, the composition of which is $\mathrm{C}_{21} \mathrm{H}_{23} \mathrm{O}_{10}$. In addition to all the liquids above mentioned, as occurring in pyroxylio spirit, acetone is frequently found.

The very great similarity in properties of so many substances, namely, hydrated oxide of methyle, acetate of oxide of methyle, lignone, xylite, mesite, and acetone, is worthy of notice. Most of these liquids have nearly the same density and boiling point; they are all inflammable, and their solubility in water is nearly equal. Hence they all occur mixed, and are with great difficulty separated, so as to obtain each in a state of purity; indeed, in most of them we cannot be sure that this has yet been accomplished. It is highly probable that, like the two first, all the rest will be found to be compounds of methyle. Our knowledge on the subject is still very limited.

The purified pyroxylic spirit, or hydrated oxide of methyle, has been already fully described, along with its chief derivatives.

\section{Volatile Oily Products, insoluble or sparingly soluble in Water.}

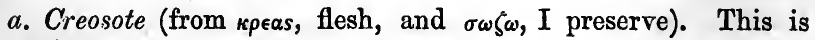
one of the most important products of the distillation of wood. It is found, partly dissolved, in the pyroligneous acid, partly along with other oils, in the tar. When the crude pyroligneous acid is saturated at $167^{\circ}$ with dry sulphate of soda, an oil separates, which contains much creosote. In like manner, by the rectification of tar, an oil of tar is obtained, the heavier portions of which contain a good deal of creosote. These oils are neutralised with carbonate of potash, and the fluid thus deprived of acid is distilled with water. The distilled oil is acted on by diluted phosphoric acid to remove ammonia, and, probably, traces of oily bases, again distilled, and dissolved in aqua potassæ, Sp. G. 1.12, which dissolves the creosote, along with portions of other oils, but separates a good deal of eupion, \&c. The alkaline solution is now supersaturated with dilute sulphuric acid (after having been boiled in the air till it has become dark brown), when the impure 
creosote separates. It is again rectified, and the treatment with potash, boiling, addition of sulphuric acid, and rectification, repeated till the rectified oil dissolves entirely in weak potash, and this alkaline solution, on being boiled, acquires only a slight tinge of colour. It is then finally rectified, and is pure when it continues colourless on being kept. The tar of peat appears to be very rich in creosote, and it also occurs in coal-tar. Good tar, from beech wood, is said to contain from 20 to 25 per cent.

Pure creosote is a colourless transparent liquid, of a high refractive and dispersive power, of a tolerably fluid but oily consistence. Its Sp. G. is 1.037, according to Reichenbach, its discoverer, and other chemists; but there is some discrepancy on this point, Dr. Christison having always found it as high as 1.060 and upwards. Its boiling point is $397^{\circ}$. It gradually becomes coloured brown when kept, unless absolutely pure. Creosote has a very strong, peculiar, persistent smell of smoke, analogous also to that of castoreum, not fetid, but unpleasant when concentrated. Its taste is burning, with a sweetish after-taste.. It disorganises the skin, causing a white spot, where the cuticle soon peels off, without inflammation. When applied to the interior of the mouth and to the tongue it smarts strongly, whitening and disorganising the cuticle.

Internally it is a powerful poison, but in a small dose may be employed advantageously in some cases of vomiting and disease of the mucous membrane. It is given much diluted with water. Externally, it may be employed, either in the form of aqueous solution, of ointment, or pure, as a styptic, and is a valuable application to indolent ulcers, and to many chronic cutaneous affections. Pure creosote, applied to the hollow of a decayed tooth, so as to touch the exposed nerve, instantly relieves, in many cases, the most violent toothache. It acts apparently by coagulating the secretions, and thus forming a covering to the nerve.

Creosote dissolves in about 80 or 100 parts of water, and is exceedingly soluble in alcohol and in acetic acid. These solutions have the smell, taste, and antiseptic power of the creosote.

Creosote possesses a singular antiseptic power. Flesh of all kinds, if steeped for a few hours in a weak solution of creosote, becomes insusceptible of putrefaction: and the same effect is produced when the flesh is exposed to the vapour of creosote. This is the reason why the smoke of wood possesses antiseptic properties; smoked meat or fish is merely meat or fish which has absorbed the vapour of creosote from the smoke in which it has been suspended. The creosote appears to act on flesh, \&c., in virtue of its remarkable power of coagulating albumen, which 
also accounts for its styptio action. Tongues and hams may be smoked and effectually cured by immersing them for 24 hours in a mixture of 1 part of pure creosote and 100 of water or brine; and when thus prepared, they have the delicate smoked flavour observed in reindeer tongues, as usually cured by smoking.

Owing to the difficulty of obtaining creosote quite pure, its composition is hardly ascertained with certainty. According to Deville, whose researches are the most recent, it may be regarded as having the composition of the alcohol, so to speak, of the series of benzoyle. His analyses lead to the formula $\mathrm{C}_{14} \mathrm{H}_{8} \mathrm{O}_{3}=$ $\mathrm{C}_{14} \mathrm{H}_{7} \mathrm{O}_{2}, \mathrm{H} \mathrm{O}$; but $\mathrm{I}$ cannot ascertain whether this be the formula he adopts. The alcohol he speaks of would be $\mathrm{C}_{14} \mathrm{H}_{8} \mathrm{O}_{2}$; and this, if not creosote, is probably isomerio with it, as will be explained further on.

It is particularly to be noticed, that there is a very great resemblance between creosote and carbolic acid (or hydrated oxide of phenyle, $\mathrm{C}_{12} \mathrm{H}_{5} \mathrm{O}, \mathrm{HO}$ ), a substance obtained from coal-tar, and which will soon be described. So great is this resemblance, that I am inclined to consider creosote as a somewhat impure carbolic acid, the impurities being substances homologous with carbolic acid; or rather the carbolic acid is the impurity, in a body, belonging to the same homologous series; and others of the series may be present. The taste, smell, density (according to some), boiling point, solubility in water, \&c., poisonous and antiseptic action, of these two bodies, are the same. Both combine with alkalies, forming crystallisable compounds, and, what is more important, their composition in 100 parts is almost identical. The chief differences seem to be, that carbolic acid may be obtained in crystals, which, howerer, on contact with the air, instantly liquefy and retain the liquid form, without any appreciable change of composition, apparently from the effect of a trace of moisture. Also, the salts of carbolic acid with bases are more easily formed and more permanent than those of creosote. A splinter or shaving of fir wood, dipped into carbolio acid, and then into nitric or muriatic acid, becomes first blue and then brown; which does not appear to be the case with creosote. But Laurent has recently shown, that creosote, when acted on by a mixture of hydrochlorio acid and chlorate of potash, yields abundance of chloranile, a character in which it agrees with carbolic acid. Both substances also yield nitropicric acid, when acted on by nitric acid, although in the case of creosote this acid is accompanied by others not yet examined. These results I have myself also obtained; and it would appear, that if creosote be not carbolic acid, contaminated with some foreign matter, these two bodies are at least closely connected, and belong apparently to the same series, which is 
either that of benzoyle or that of phenyle. It is not impossible that creosote may be a definite compound of carbolio acid with some substance of closely allied composition, but of basic properties.

The recent investigations of Gorup-Besanez have thrown some light on this subject. Commercial creosote is rarely anything else than carbolic acid, which so much resembles it. But genuine creosote does not yield with hydrochloric acid and chlorate of potash, chloranile, but a body somewhat resembling it, the formula of which, according to Gorup-Besanez, is $\mathrm{C}_{26} \mathrm{H}_{6} \mathrm{Cl}_{6} \mathrm{O}_{6}$. Gerhardt suggests the much more probable formula $\mathrm{C}_{16} \mathrm{H}_{4} \mathrm{Cl}_{4} \mathrm{O}_{4}$, which makes the compound homologous with chloranile, $\mathrm{C}_{22} \mathrm{Cl}_{4} \mathrm{O}_{4}$. In the latter, no hydrogen is left, because the whole 4 eq. has been replaced by chlorine, in forming it from quinone (kinone), $\mathrm{C}_{12} \mathrm{H}_{4} \mathrm{O}_{4}$. We may therefore suppose that, as chloranile is quadrichlorinised quinone, so the chlorine compound from creosote is derived from a body $\mathrm{C}_{16} \mathrm{H}_{8} \mathrm{O}_{4}$, homologous with quinone. And as chloranile is also to be obtained from carbolic or phenylic acid, $\mathrm{C}_{12} \mathrm{H}_{6} \mathrm{O}_{2}$, so we may conceive the chlorine compound from creosote to be derivable also from the body $\mathrm{C}_{36} \mathrm{H}_{10} \mathrm{O}_{2}$, homologous with carbolic acid. Now creosote may perhaps be the body $\mathrm{C}_{16} \mathrm{H}_{8} \mathrm{O}_{4}$, homologous with quinone; or it may be the body $\mathrm{C}_{16} \mathrm{H}_{10} \mathrm{O}_{2}$, homologous with carbolic acid. Its great resemblance to carbolic acid renders the latter the more probable view, and it also agrees much better with the analyses of creosote, which have led some to adopt the formula $\mathrm{C}_{14} \mathrm{H}_{8} \mathrm{O}_{2}$; but these analyses are not sufficiently accordant to decide between $\mathrm{C}_{14} \mathrm{H}_{8} \mathrm{O}_{2}$ and $\mathrm{C}_{16} \mathrm{H}_{10} \mathrm{O}_{2}$, both of which, be it observed, are homologous with carbolic acid. It is probable that both compounds exist, and that they resemble each other as well as carbolic acid. This reminds us of the homologues of nicotine in tobacco, and of narcotine in opium; and leads to the notion of the following series :-

$\begin{array}{llll}\text { Carbolic acid . } & \cdot & \mathrm{C}_{12} \mathrm{H}_{6} \mathrm{O}_{2} \\ \text { Methylocarbolic acid } & \cdot & \mathrm{C}_{14} \mathrm{H}_{8} \mathrm{O}_{2} \\ \text { Ethylocarbolic acid } & \cdot & \mathrm{C}_{16} \mathrm{H}_{10} \mathrm{O}_{2} \\ \text { Amylocarbolic acid } & \cdot & \cdot & \mathrm{C}_{22} \mathrm{H}_{16} \mathrm{O}_{2}\end{array}$

The second and third acids would, of course, be very similar to each other and to carbolic acid, and would, in fact, be two of the acids represented in the 7 th line of the table, p. 141. Probably all these acids are creosotes, and the three first of those above named probably occur all together, in variable proportions, in the creosote of commerce, even if none of those higher in the scale, $\mathrm{C}_{18} \mathrm{H}_{12} \mathrm{O}_{2}, \mathrm{C}_{20} \mathrm{H}_{14} \mathrm{O}_{2}$, or $\mathrm{C}_{22} \mathrm{H}_{16} \mathrm{O}_{2}$, should accompany them, which is not probable. The fact that creosote has the acid 
character, as well as the smell of carbolic acid, is favourable to this view; but we must not forget, that there is another series of homologues, in a certain sense, of earbolic acid ; namely, carbolate of methyle or phenometole, carbolate of ethyle or phenetole, and carbolate of amyle or phenamylole, already mentioned, having the same formulæ, empirically, as our supposed methylocarbolic, ethylocarbolic, and amylocarbolio acids. These, however, phenometole, \&c., are neutral ethers, and not acids.

There is still another series, homologous with earbolic acid, empirically, the first of which is benzoic alcohol, $\mathrm{C}_{14} \mathrm{H}_{s} \mathrm{O}_{2}$. The cuminic alcohol is also known, its formula being $\mathrm{C}_{18} \mathrm{H}_{12} \mathrm{O}_{2}$. These are oils, quite distinet from the creosotes, as well as from the series last named. In all these series methyle, ethyle, \&c., are substituted for hydrogen; but sometimes for part of the hydrogen in the acid radical; at other times, for the hydrogen of the water combined with that radical, or for part of the hydrogen in the basic substance combined with the acid.

Such is the view we are disposed to take of the real nature of creosote, by which the creosote of commerce will be a mixture of several homologous acids, carbolic acid being the first.

Creosote dissolves many organic substances, such as indigo, camphor, fats, essential oils, and resins, and undergoes numerous changes by the action of acids, alkalies, and other reagents, such as chlorine, potassium, and others, With oil of vitriol it is coloured purple, and appears to form a coupled acid. None of these reactions or products have been properly investigated, and we shall, therefore, not confuse the reader by a deseription of them, more especially as the composition of creosote itself is doubtful.

$b$, Picamar is the name given by Reichenbach to another oil discovered by him along with creosote in the heavy oil of tar. It is purified by a tedious process, by the aid of potash, with which it forms a crystalline compound. When pure, it is a colourless oil, of Sp, G. 1·10, of a burning and very bitter taste (hence its name from pix and amarus), and a slight smell. It boils at about $510^{\circ}$. It combines with alkalies, forming crystallisable salts, and may therefore be viewed as an acid in some sense, although it is quite neutral to test paper. Its composition is unknown. As it is a feeble acid, it may be, as we have supposed creosote to be, a homologue of carbolic acid, and one of those high in the scale, possibly amylocarbolic acid, $\mathrm{C}_{22} \mathrm{H}_{16} \mathrm{O}_{2}$.

c. Capnomor (from $\kappa a \pi \nu d s$, smoke, and $\mu \hat{i} \rho \alpha$, part) is another oil discovered by Reichenbach, in the heavy oil of tar, along with creosote and picamar. When the creosote is purified by a solution of weak potash, the oil left undissolved contains a good deal of HI H 2 
capnomor, which is purified by a tedious process. It is a limpid, colourless oil, of a high refracting power, with an aromatic odour of ginger, and a somewhat styptic after-taste. Its Sp. G. is 0.9775 ; it is quite neutral, and boils at $365^{\circ}$. With sulphuric acid it is coloured red, and yields a coupled acid. Nitric acid converts it into oxalic acid, nitropicrio acid, and another crystalline substance not yet examined.

d. Eupion (from $\epsilon \hat{v}$, fine, and $\pi i \delta \nu$, oil or fat) is a fourth oily liquid discovered by Reichenbach in oil of tar. Being more volatile than the rest, it is purified chiefly by rectification. When pure it is colourless, very fluid, not greasy to the feel, but less soft than water, tasteless, and of a somewhat agreeable odour, like that of some flowers, such as narcissus. Its Sp. G. is 0.740 , and Reichenbach states that he has even obtained it so low as 0.633 , being the lightest known liquid. It is volatile, boiling at $117^{\circ}$ or lower. It is in the highest degree indifferent, resisting the action of the strongest acids and alkalies. In fact, as it is prepared from the oil of tar by rectification and the action of potash, sulphuric and nitric acids, alternately on the rectified oil, it is evident that it must resist these agents. There is good reason to believe that several, even many, different liquids have been described under this name, and that most of these are not readyformed in the tar, but products of the action of acids, \&c., on the oil of tar. Reichenbach, however, by simple rectifications of the oil obtained by distilling rape oil, obtained a liquid having the characters of eupion. So muoh is certain, that similar liquids are formed by the action of oil of vitriol on oil of tar. The whole of the liquids called eupion are carbohydrogens, and their formula is either $\mathrm{CH}$, or some multiple of this, or else one nearly approaching to such a multiple, as $\mathrm{C}_{10} \mathrm{H}_{12}$, \&c. It is very remarkable that some of them are very volatile, while others, apparently of the same composition, require a strong heat, from $400^{\circ}$ to $500^{\circ}$ for example, to boil them. This, however, is just what we might anticipate in a series, or a mixture of two series of homologous compounds. Indeed, the most recent researches indicate that the name eupion includes many of the lower members of a homologous series of carbohydrogens, possibly the hydurets of the acetylic series of radicals, possibly also hydurets of the ethylic or electropositive radicals.

The purest varieties of eupion burn with the aid of a wick, yielding a very brilliant, luminous white flame, free from smoke, and may hereafter be turned to account.

3. Solid Products of the Distillation of Wood.

a. Paraffine. This name is given (from parum and affinis, 
because its affinities are feeble) to a white solid rolatile substance very similar to wax, discovered by Reichenbach in tar. It occurs in the last portions of the rectification of the tar, which are semisolid. It is squeezed out, and purified by one or two crystallisations in ether, which dissolves it when boiling, and deposits it on cooling in beautiful silvery scales. These, when melted, assume, on cooling, the aspect of pure white wax.

Paraffine exists in large proportion in the Rangoon petroleum, and some other bituminous mineral products. It is formed in large quantity in the distillation of wax and of peat. It melts at $110^{\circ}$, and distils unchanged at a high temperature. Its Sp. G. is 0.870 . It burns, in a wick, with a beautiful clear white light, free from smoke, fully equal to that of the finest wax, if not superior to it. Like eupion, it is highly indifferent, and it is, like eupion, a carbohydrogen, containing either $\mathrm{CH}$, or some multiple of it, or a near approach to such a multiple. According to Lewy, it is $\mathrm{C}_{20} \mathrm{H}_{21}$, or rather $\mathrm{C}_{40} \mathrm{H}_{42}$; but there is reason to believe that many compounds have been confounded under the name of paraffine, and that it includes all the higher members of the series, $\mathrm{Cn}_{\mathrm{n}}$ ( $\mathrm{n}$ being 2 or 4, 6, 8, 10, \&c.), which are derived from the acids $\left(\mathrm{C}_{n} \mathrm{H}_{n}\right)_{2} \mathrm{O}_{4}$, and of which several have been described, as methylene, ethylene, propylene, butylene, \&o., and which are really the hydurets of the radicals of these acids. Under the vague term paraffine may also be included the higher members of the series, $\mathrm{C}_{n} \mathrm{H}_{n}+2$, the hydurets of the ethylic or electropositive radicals. Brodie gives reasons for thinking that the paraffine of wax contains the two compounds $\mathrm{C}_{54} \mathrm{H}_{54}$ and $\mathrm{C}_{60} \mathrm{H}_{60}$, the former derived from cerotic, the latter from melissic acid. There is also reason to believe that the compounds $\mathrm{C}_{54} \mathrm{H}_{66}$ and $\mathrm{C}_{60} \mathrm{H}_{62}$ occur in what is called paraffine, along with others of the same series, lower in the scale, such as that analysed by Lewy, which is either $\mathrm{C}_{20} \mathrm{H}_{20}, \mathrm{C}_{20} \mathrm{H}_{22}, \mathrm{C}_{40} \mathrm{H}_{40}$, or $\mathrm{C}_{40} \mathrm{H}_{42}$. It is acted on by chlorine with the aid of heat, but the reaction is not yet studied. The strongest acids and alkalies do not act on it even with the aid of heat, if we except fuming sulphuric acid.

By the distillation of the most bituminous kinds of cannel coal, such as the Boghead cannel coal, or Torbanehill mineral, as it has been called (which, so far as I can judge, appears to be a true oannel coal, containing a larger proportion of the bituminous constituent of coal, and consequently a larger proportion of hydrogen, than any other), there is obtained, especially when as moderate a heat as possible is employed, a very large proportion of oils, containing some solid carbohydrogens. By rectification there may be obtained oils which are as volatile as camphine or nearly 
so; then, oils which are less volatile, but do not deposit any crystals, even in cold weather, and lastly, oils, still less volatile, which in cold weather deposit crystals, and lastly, such as are solid or semisolid at the ordinary temperature.

The less volatile liquid oils of this process are sent into commerce as paraffine oil, and they are no doubt homologous with paraffine, whatever may be the true formula of that substance, or rather series of substances. Paraffine oil is fluid, but not too volatile, and it is not oxidised nor thickened by exposure to air. It is therefore most valuable for oiling machinery, and all the large quantity of it made is instantly purchased for this important use. When thus used, it slowly evaporates. The more volatile portions, which would evaporate too fast to be used in machinery, may be used in lamps as camphine, and are probably a mixture of camphine and oils homologous with it.

All these substances have the same or very nearly the same composition in 100 parts, and do not differ in any cases more than homologous compounds do. In the series $\mathrm{C}_{n} \mathrm{H}_{n}$, all have the same composition; in the series $\mathrm{C}_{n} \mathrm{H}_{n}+2$, the lower members differ somewhat from the higher, but the higher come very near to those of the other series, as well as to each other. Such is a general view of what is known concerning eupions, paraffines, and paraffine oils. It will be seen that the gaseous, liquid, and solid products of the distillation of wood or the bituminous matter of coal, derived from woody matter or cellulose, pass into one another by insensible gradations, and that the carbohydrogens thus formed are astonishingly numerous, but all possess analogous properties.

b. Cedriret. This is another compound discovered by Reichenbach in oil of tar. When impure creosote is dissolved in potash, and acetic acid added, an oil separates, which contains the creosote and other oils; but a certain quantity of oily matter remains dissolved in the acetate of potash. This is distilled, until what passes over causes a red precipitate in a solution of sulphate of iron. It is then collected separately, being pure cedriret. It is a volatile solid, which crystallises in a solution of sulphate of iron, forming a net-work of orange-red crystals, which dissolve in oil of vitriol with a blue colour. . Much of the colour of oil of tar is probably owing to this substance.

c. Pittacal. This is yet another compound obtained by Reichenbach from the heavy oil of tar. When the heaviest portions are nearly neutralised by potash, the addition of barytic water gives rise to a deep blue colour. This belongs to pittacal, but the mode of its purification is not yet published. When pure, it is a solid, like indigo, of a very fine deep blue colour, exhibiting on the 
polished surface the aspect of gold. It admits of being fixed on cloth, and would make a valuable dye-stuff. Its composition is unknown, but it appears to contain nitrogen. Its name is derived from $\pi \iota \tau \tau \alpha$, pitch, and $\kappa a \lambda o s$, beautiful. It is a compound of very great interest, although most probably a product of decomposition of the oil of tar, and not ready formed in it. It is very desirable that it should be further investigated.

The recent experiments of Greville Williams on the salts of ethyloquinoline, alluded to under the volatile artificial bases, suggest the idea that the beautiful blue compound, with golden surface, obtained by him, may be identical or homologous with pittacal. The blue compound of Williams seems to be basic, and contains nitrogen. It is therefore possible that pittacal may be derived from quinoline existing in the oil of tar used by Reichenbach, although it has not yet been shown to exist in wood tar. But wood, though it contains little nitrogen, must contain some, and therefore basic oils are to be looked for in wood tar.

d. Pyroxanthine. This is a volatile crystalline solid, first observed by Scanlan in the crude pyroligneous spirit. When this is rectified with lime, the lime becomes dark-brown; and when this coloured mass is acted on by hydrochloric acid, there is left undissolved a dark-brown matter, which is a mixture of pyroxanthine and a resinous matter. The mass is boiled with hot alcohol, which, on cooling, deposits the pyroxanthine in crystals, which are purified by recrystallisation. They are of an intense yellow colour, fusible, and volatile in a current of air, or with the vapour of other substances, but partly decomposed when heated alone in a dry tube. Pyroxanthine dissolves in sulphuric acid with a deep bluish-red, and in strong hydrochloric acid with a splendid purple colour, which soon passes to dark-brown. I found its composition to be very nearly $\mathrm{C}_{21} \mathrm{H}_{9} \mathrm{O}_{4}$; but as it forms no definite compounds, I could not control the analysis.

Such are the chief products of the distillation of wood, as far as they are yet known. Their importance is very great, and will be still greater when they shall have been better studied, as most of them will admit of useful applications. But no doubt can be entertained that the above numerous list is far from being complete, and that more compounds remain to be discovered in tar. Indeed, several or most of the carbohydrogens characterising coal-tar occur also, although in smaller quantity, in woodtar. Such substances are naphthaline, anthracene, and others. It is to be borne in mind that the composition of wood-tar varies, according to the kind of wood, the presence or absence of oily or resinous substances, the comparative abundance of nitrogenised 
matter, and finally the temperature at which the distillation is carried on.

Wood, coal, and brown coal or lignite, yield, when distilled, an oil of the consistence of butter, in which creosote, paraffine, paraffine oils, and eupion, are found, along with other products not yet examined.

\section{PRODUCTS OF THE DISTILLATION OF COAL.}

Coal differs from wood in several points, although it is unquestionably derived from the decay, under pressure, of woody fibre and the other substances which make up the mass of the early vegetation of which our coal-beds are the remains. Coal, when examined by the microscope, is found to be a mixture, in which we can generally detect the remains of vegetable cells and vessels. But the cellulose has been converted into a peculiar substance, which contains very little oxygen, and is dark in mass, but in thin slices yellow. It is easily melted and burns with much luminous flame, and by its melting, causes the caking of coal. Some kinds of coal have been so far altered that none of this matter is present, and the coal burns, like coke, without flame, that is, without the yellow flame of coal or coal gas. Such coal is called anthracite or blind coal. Ordinary coal contains more or less of the yellow fusible matter, which is called bituminous, but is quite different from natural bitumen, for the latter dissolves easily in ether, naphtha, or oil of turpentine, which have no solvent action on the yellow substance of coal. In cannel coal, this yellow matter predominates, so that such coal takes fire at a candle and burns with much flame and light. It also yields the most and by far the best gas. The best cannel coal is that of Boghead, or that of Torbanehill, which are from the same bed, and which contain more of the yellow or bituminous matter, and fewer remains of woody cells, than any other kind. This coal is much prized as a gas coal, and also as yielding paraffine oil, when distilled at a moderate heat, as has been explained under paraffine. All coals, between anthracite and the Boghead cannel coal, are mixtures, of the yellow matter with more or less of coke, or a substance closely resembling it, which in anthracite is nearly pure. In the Boghead coal, on the contrary, the fusible matter predominates entirely, while the coke is absent or nearly so, and woody cells are rare, apparently because the vegetable matter which yielded that coal was either not wood, or wood and other vegetable tissues first reduced to a pulp. The rounded masses of the vellow matter seen in the Boghead coal under the microscope 
are supposed to derive their form from cells which had existed. The amount of ash or earthy matter in coals varies from 3 or 4 per cent. to 30 or 40 per cent.; because clay and sand have often been mixed with the vegetable matters in the process of carbonisation, to a greater or less extent, by the action of water.

Coal contains much less water, and a much larger per-centage both of carbon and nitrogen, than wood. Hence it is decomposed at a higher temperature, and yields much ammonia, cyanogen, and other nitrogenised products. We shall not here dwell on ammonia and cyanogen, further than to mention that out of the aqueous products of the coal gas-works large quantities of ammonia are obtained; and that so much hydrocyanic acid is also present, that a patent was taken out some years since for the preparation of Prussian blue from the gas liquor. We proceed to describe the chief ingredients of coal-tar.

a. Carbolic Acid. Srn. Hydrated Oxide of Phenyle. $\mathrm{C}_{12} \mathrm{H}_{5} \mathrm{O}$, $\mathrm{H} 0$. This remarkable acid is found in that portion of the oil of coal-tar which boils between $300^{\circ}$ and $400^{\circ}$. This is agitated with twice its volume of potash ley, and the aqueous solution, on the addition of an acid, yields hydrated carbolic acid (impure) as a heavy oil. It is purified by rectification with a very little solid potash.

When pure, carbolic acid generally appears as an oily liquid, colourless, and of a high refracting power, neutral to test paper, of Sp. G. 1.062 to 1.065. It has a burning taste, and the odour of creosote, to which it has a very great resemblance. In certain circumstances it forms long, needle-shaped crystals, which very readily lose the solid form by exposure to the atmosphere, and which also liquefy in sealed tubes, without any obvious cause. The crystals melt at $94^{\circ}$, and boil at $368^{\circ}$. The extraordinary resemblance between carbolic acid and creosote has been noticed above; and there can be little doubt that, if not essentially the same, they are closely connected and belong to the same series, or are homologous compounds. Indeed, Städeler has extracted from the urine of the cow not only carbolic acid, but also an acid homologous with it, which he calls taurylic acid, $\mathrm{C}_{14} \mathrm{H}_{8} \mathrm{O}_{2}$. It is probable that this acid will prove to be identical with creosote.

A splinter of pine-wood, if dipped, first in carbolic acid, and then in moderately strong nitric acid, becomes of a deep blue, which soon passes into brown.

According to Laurent, carbolic acid is the hydrated oxide of phenyle, $\mathrm{C}_{12} \mathrm{H}_{5}$, and its formula is $\left(\mathrm{C}_{12} \mathrm{H}_{5}\right) \mathrm{O}, \mathrm{H} \mathrm{O}$. This radical, phenyle, gives rise to a series of derived compounds which may be represented as follows:- 
Hydrate of Phenyle, or Carbolic Acid $\mathrm{C}_{12} \mathrm{H}_{5}, \mathrm{O}+\mathrm{HO}$

$\left.\begin{array}{c}\begin{array}{c}\text { Sulphocarbolic Acid } \\ \text { Acid) }\end{array} \\ \cdot\end{array} \begin{array}{c}\text { (Sulphophenic } \\ \cdot\end{array}\right\} \mathrm{C}_{12} \quad \mathrm{H}_{5} \quad \mathrm{O}, \mathrm{HO}+2 \mathrm{~S} \mathrm{O}_{3}$

Chlorophenesic Acid . • . . $\mathrm{C}_{12}\left\{\begin{array}{l}\mathrm{H}_{3} \\ \mathrm{Cl}_{2}\end{array}\right\} \mathrm{O}, \mathrm{H} \mathrm{O}$ $\left.\begin{array}{c}\text { Chlorophenisic Acid, identical with } \\ \text { the Chlorindoptenic Acid of } \\ \text { Erdmann . . . . . }\end{array}\right\} \mathrm{C}_{12}\left\{\begin{array}{l}\mathrm{H}_{2} \\ \mathrm{Cl}_{3}\end{array}\right\}$, Н O

Chlorophenusic Acid, Chlorinised

Chlorindoptenic Acid of Erd- $\mathrm{C}_{12}$

$\mathrm{O}, \mathrm{H} \mathrm{O}$

Bromophenisic Acid: $: \mathrm{C}_{12}\left\{\begin{array}{l}\mathrm{H}_{2} \\ \mathrm{Br}_{3}\end{array}\right\}$ O, Н О

Nitrophenesic Acid . . . . $\mathrm{C}_{12}\left\{\begin{array}{l}\mathrm{H}_{3} \\ 2 \mathrm{~N} \mathrm{O}_{4}\end{array}\right\} \mathrm{O}, \mathrm{H} \mathrm{O}$

$\begin{gathered}\text { Nitrophenisic Acid, identical with } \\ \text { Nitropicric Acid } \\ .\end{gathered} \mathrm{C}_{12}\left\{\begin{array}{l}\mathrm{H}_{2} \\ 3 \mathrm{~N} \mathrm{O}_{4}\end{array}\right\} \mathrm{O}, \mathrm{H} \mathrm{O}$

Phenylamide (Aniline) . . . $\quad \mathrm{C}_{12} \mathrm{H}_{5}$ Ad.

Thus the carbolic acid is connected with the derivatives of indigo, of salicyle, and other bodies, which yield nitropicric acid. This connection is also shown in the formation of chloranile, from carbolic acid, by the action of chlorate of potash and hydrochloric acid. (See, under Indigo, the formation of chloranile from aniline.) It is also shown, by the fact, that salicylic acid, $\mathrm{C}_{14} \mathrm{H}_{6} \mathrm{G}_{6}$, when distilled alone, with lime, or with pounded glass, is resolved into carbonic acid, $2 \mathrm{C} \mathrm{O}_{2}$, and carbolic acid, $\mathrm{C}_{12} \mathrm{H}_{6} \mathrm{O}_{2}$. The action of carbolic acid, on organic compounds, is the same as that of creosote. Thus it dissolves indigo, \&c. and coagulates albumen, preventing the putrefaction of animal substances.

With bases, it forms salts, some of which crystallise, but which retain an alkaline reaction. With oil of vitriol, it yields a coupled acid, sulphocarbolic, or sulphophenic acid, which forms a soluble salt with baryta.

The formulæ in the above table illustrate the formation, by substitution, of the chlorophenesic, chlorophenisic, and chlorophenusic, of the nitrophenesic and nitrophenisic acids. It is not necessary here to do more than point out their relation to carbolic acid and phenyle. The chlorobenzide of Mitscherlich, $\mathrm{C}_{22} \mathrm{H}_{6} \mathrm{Cl}_{6}$ is, according to Laurent, hydrochlorate of chlorophenise, $3 \mathrm{H} \mathrm{Cl}+\mathrm{Cl2}_{2}\left\{\begin{array}{l}\mathrm{H}_{3} \\ \mathrm{Cl}_{3}\end{array}\right.$. This last body, chlorophenise, which is obtained by the action of potash on chlorobenzide, would appear to be derived by substitution, not from phenyle $\mathrm{C}_{22} \mathrm{H}_{5}$, but from benzole $\mathrm{C}_{12} \mathrm{H}_{6}$; although it may be derived also from oxide of phenyle, $\mathrm{C}_{12}\left\{\begin{array}{l}\mathrm{H}_{5} \\ \mathrm{O}\end{array} ;\right.$ as may benzole itself. Chlorophenise cannot be obtained directly 
from hydrate of phenyle, or its derivatives: but, on the other hand, the series of benzole has an obvious relation to that of phenyle. In fact, Laurent considers benzole as in some measure the fundamental compound, or nucleus, and calls it phene = $\mathrm{C}_{12} \mathrm{H}_{6}$. It is probably rather the hyduret of phenyle, $\mathrm{C}_{22} \mathrm{H}_{5}$, $\mathrm{H},=\mathrm{C}_{12} \mathrm{H}_{6}$.

It has already been stated that nitrophenisic acid is identical with nitropicric acid. Nitrophenesio acid is somewhat similar, and forms salts which crystallise with facility, and detonate when heated. The nitrophenesate of baryta is a beautiful salt, like bichromate of potash.

Oxyphenic Acid. Sxn. Pyrocatechine. Pyromorintannic Acid. $\mathrm{C}_{12} \mathrm{H}_{6} \mathrm{O}_{4}$. This acid is formed in the destructive distillation of catechu, or of morintannic acid, as well as in that of gum-ammoniac and pencedanine. It is crystalline, soluble in water, and has a smell like that of carbolic acid, from which it differs only by 2 eq. of oxygen. It is also volatile.

By the action of chlorine it is converted into chloroxyphenic or chloroniceic acid, $\mathrm{C}_{22} \mathrm{Cl}_{5} \mathrm{H}_{5}$. This acid is best obtained by the action of chlorine on a solution of benzoate of potash. It forms small crystals, grouped in radiate masses. With nitrio

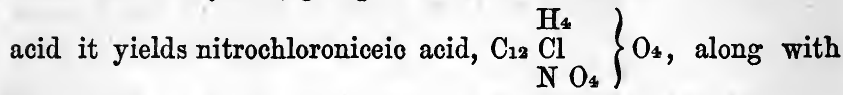
an acid, which is citraconic acid, in which 1 eq. of hydrogen is replaced by chlorine, and another by $\mathrm{N} \mathrm{O}_{4}$.

By the action of heat on chloroniceic acid, there is formed a carbohydrogen, paranicene $=\mathrm{C}_{20} \mathrm{H}_{12}$, which is a yellow, crystalline, volatile solid. Nitric acid converts it into nitroparanicene, $\left.\mathrm{C}_{20} \underset{\mathrm{NO}_{11}}{\mathrm{NO}_{4}}\right\}$.

Along with paranicene there is formed a liquid, which is bichloronicene, $\left.\mathrm{C}_{20} \underset{\mathrm{Cl}_{2}}{\mathrm{H}_{10}}\right\}$ Nitric acid converts this into a new compound, $\left.\mathrm{C}_{20} \underset{\mathrm{Cl}_{2}}{\stackrel{\mathrm{H}_{8}}{2} \mathrm{O}_{4}}\right\}$.

When the two nitro compounds last named are acted on by sulphuret of ammonium, they yield two bases; paranicine, from paranicene, which is $\mathrm{C}_{20} \mathrm{H}_{13} \mathrm{~N}$, forming white flocks, soluble in ether; and chloronicine, $\mathrm{C}_{20} \mathrm{H}_{12} \mathrm{Cl}_{2} \mathrm{~N}$. Both these bases form crystalline salts with acids.

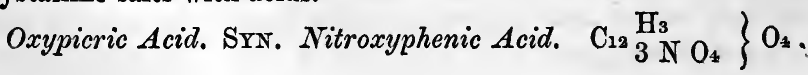


This acid, which has also been named styphnic acid, and artificial bitter, or the artificial tannin of Brazil wood, is evidently derived from oxyphenio acid; but is formed by the action of nitric acid on various gum resins, as ammoniac, assafotida, galbanum, sagapenum ; or on the watery extracts of Brazil wood, Campenety wood, or sandal wood; on euxanthic acid, or on peucedanine.

It forms minute erystals like fern leaves, or large yellow prisms. It is sparingly soluble, and has an astringent taste. It explodes slightly when suddenly heated. Its salts explode, when heated, much as the picrates do.

\section{b. Volatile Bases of Coal Tar.}

Besides carbolic acid, Runge found in coal-tar two other acids, rosolic and brunolic acids (of which little is known), and three basic volatile oils, kyanol, leukol, and pyrrol. The last has been little examined; but the recent researches of Hofmann have confirmed Runge's statements as to the two first, which have also been identitied with bases produced from different quarters, namely, aniline and quinoline or leucoline. These bases have already been fully described under the head of volatile artificial alkaloids.

According to Anderson, there exists a series of bases called pyrrole bases, which are bases of the aniline or of the picoline series, coupled with a red compound.

That there is a relation between aniline and carbolic acid appears from their formulæ; for aniline is the amidide of carbolic acid, or phenamide, being thus deduced from carbolate of ammonia. $\mathrm{C}_{12} \mathrm{H}_{5} \mathrm{O}, \mathrm{NH}_{3}=\mathrm{H}^{\mathrm{O}}+\mathrm{C}_{12} \mathrm{H}_{5}, \mathrm{NH}_{2}$. The relation may be better exhibited thus:

Carbolic Acid (anhydrous), or Oxide of Phenyle $=\mathrm{C}_{12} \mathrm{H}_{5} \mathrm{O}$ Aniline, amaphenase, or phenylamide . . $=\mathrm{C}_{12} \mathrm{H}_{5} \mathrm{Ad}$

As another experimental proof of this relation, it may be mentioned that salicylamide, $\mathrm{C}_{14} \mathrm{H}_{5} \mathrm{O}_{4}, \mathrm{~N} \mathrm{H}_{2}=\mathrm{C}_{14} \mathrm{H}_{7} \mathrm{~N} \mathrm{O}_{4}$, which has the same composition as nitrotoluole and anthranilic acid, both of which yield aniline, when heated with lime gives not aniline but carbolic acid, ammonia, and probably a carbohydrogen.

It will be remembered that picoline is isomerio with aniline. This isomerism extends to toluidine and lutidine, $\mathrm{C}_{14} \mathrm{H}_{\theta} \mathrm{N}$, and also to the higher members of the aniline and picoline series. It depends on the fact, that while aniline is an amide base, picoline is a nitryle base, and so on. 


\section{c. Volatile Carbohydrogens in Coal Tar.}

a. Naphthaline, $\mathrm{C}_{10} \mathrm{H}_{4}$, or $\mathrm{C}_{20} \mathrm{H}_{8}$. This remarkable compound occurs in all kinds of tar, but most abundantly in coal-tar, as being formed at a very high temperature. It is formed in additional quantity when any of the elements of oil of tar, such as creosote, carbolic acid, \&c., or even alcohol and ether, are passed through tubes heated to a strong red-heat. It is easily obtained by redistilling coal-tar, when the latter portions are so full of naphthaline as to be semisolid. It is well squeezed out, and purified by sublimation and crystallisation in hot alcohol. Or the oil of coal-tar is saturated with chlorine gas, which, by destroying some of the oils, allows the naphthaline to crystallise.

Pure naphthaline is colourless and volatile, and forms large tabular, transparent crystals, of a very peculiar smell, and an acrid aromatic taste. It boils at $414^{\circ}$, but distils easily with the vapours of water, and is dissipated, like camphor, if left exposed at the ordinary temperature.

It is acted on by chlorine and bromine, which combine with it in the first instance, and also give rise to a large number of compounds formed by substitution; and by sulphuric and nitric acids, each of which produces a number of new compounds with it. These changes have been studied with singular perseverance and remarkable sagacity, by Laurent, more especially the action of chlorine, bromine, and nitric acid. His researches have been attended with unusual success, and he may be said to have originated and established, by these researches, the now received doctrine of substitutions. I cannot hope to give even an accurate outline of all that Laurent has done in this department, because I have nowhere seen a complete account of these curious results as they now stand : but I shall place", before the reader such a general account of them as may show the great importance of the subject, and some of the interesting facts already ascertained.

\section{Action of Chlorine and Bromine on Naphthaline.}

(It is necessary here to explain the principle of nomenclature provisionally adopted by Laurent, especially for cases, like this, of substitutions, where the ordinary nomenclature is entirely inapplicable. The nomenclature of Laurent may be thought by some, uncouth ; but it is simple, systematic, and consistent with itself. Beginning with naphthaline, he gives to the compounds formed by the successive substitution of chlorine for hydrogen, names beginning with chlo, and ending with a syllable 
in which the vowels $a, e, i, o$, and $u$, are employed to designate the replacement of $1,2,3,4$, and 5 eqs. of hydrogen. Thus, if in naphthaline, $\mathrm{C}_{20} \mathrm{H}_{8}, 1$ eq. of hydrogen is replaced by chlorine, we have the compound $\mathrm{C}_{20}\left\{\begin{array}{l}\mathrm{H}_{7} \\ \mathrm{Cl}\end{array}\right.$. This is called chlonaphtase. The compound $\mathrm{C}_{20}\left\{\begin{array}{l}\mathrm{H}_{6} \\ \mathrm{Cl}_{2}\end{array}\right.$ is chlonaphtese; $\mathrm{C}_{20}\left\{\begin{array}{l}\mathrm{H}_{5} \\ \mathrm{Cl}_{3}\end{array}\right.$ is chlonaphtise, \&c. The corresponding compounds of bromine are bronaphtase, bronaphtese, and bronaphtise. When we get as far as chlonaphtuse $\mathrm{C}_{20}\left\{\begin{array}{l}\mathrm{H}_{3} \\ \mathrm{Cl}_{5}\end{array}\right.$, as there are no more vowels, we begin again with $a$, adding a syllable to the word. Thus $\mathrm{C}_{20}\left\{\begin{array}{l}\mathrm{H}_{2} \\ \mathrm{Cl}_{6}\end{array}\right.$ is chlonaphtalase ; $\mathrm{C}_{20}\left\{\begin{array}{l}\mathrm{H} \\ \mathrm{Cl}_{7}\end{array}\right.$ is chlonaphtalese, and $\mathrm{C}_{20} \mathrm{Cl}_{8}$ is chlonaphtalise, and so on with bromine. When hydrogen is replaced, partly by chlorine, partly by bromine, then the name is so constructed that the final syllable indicates the sum of the equivalents of chlorine and bromine, while both chlorine and bromine are prefixed. Thus, chlonaphtose is $\mathrm{C}_{20}\left\{\begin{array}{l}\mathrm{H}_{4} \\ \mathrm{Cl}_{4}\end{array}\right.$; and the compound $\mathrm{C}_{20}\left\{\begin{array}{l}\mathrm{H}_{4} \\ \mathrm{Cl}_{3} \\ \mathrm{Br}\end{array}\right.$

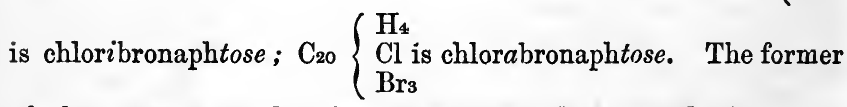
of these two may also be bromachlonaphtose, and the latter bromichlonaphtose. We shall see, hereafter, that it may. be necessary to use both forms to distinguish different compounds which are isomeric. Again, where hydrogen is replaced by $\mathrm{N} \mathrm{O}_{4}$, we have $\left(\mathrm{N} \mathrm{O}_{4}\right.$ being represented by $\left.\mathrm{X}\right), \mathrm{C}_{20}\left\{\begin{array}{l}\mathrm{H}_{7} \\ \mathrm{X}\end{array}\right.$ nitronaphtase, and so forth. In like manner, if $\mathrm{H}$ were replaced by Ad $\left(\mathrm{Ad}=\mathrm{NH}_{2}\right)$, we should have $\mathrm{C}_{20}\left\{\begin{array}{l}\mathrm{H}_{7} \\ \mathrm{Ad}\end{array}=\mathrm{C}_{20} \mathrm{H}_{9} \mathrm{~N}\right.$. This would be called amanaphtase; and such a compound has been already described under the name of naphthalidine, or naphtylamine. It is evident that this nomenclature, althongh it gives rise to words of a singular aspect, is yet easily understood, and may even, in many cases, serve as well as a formula to remind us of the composition. Laurent has applied it to many other series, as we have seen in the series of phenyle, where we have chlorophenesic, chlorophenisic, and chlorophenusic acids, \&c. In previous sections we have used another nomenclature for substitution compounds; e. g., chloraniline, dichloraniline, trichloraniline, \&c. This might also be used here. 
When chlorine is brought in contact with naphthaline, the latter melts, and there are formed at once two compounds of chlorine and naphthaline; the chloride of naphthaline, $\mathrm{C}_{20} \mathrm{H}_{8}+$ $\mathrm{Cl}_{4}$; and the subchloride of naphthaline, $\mathrm{C}_{20} \mathrm{H}_{8}+\mathrm{Cl}_{2}$. At the same time hydrochloric acid is disengaged, arising from the action of chlorine on one of these chlorides. The former is a solid, which is best purified by solution in hot oil of petroleum, which deposits it, on cooling, in crystals. It may also be purified by means of boiling ether, which dissolves it to a certain extent, and deposits it on cooling. Chloride of $\mathrm{N}$ is decomposed by heat, yielding no less than four different isomeric forms of chlonaphtese. An alcoholic solution of potash converts it into two more forms of chlonaphtese. It is also acted on by chlorine, bromine, nitric acid, and sulphuret of ammonium, yielding many new compounds.

Subchloride of $\mathrm{N}$ is an oily liquid, which by the action of heat is partially changed into hydrochloric acid, and one form of chlonaphtase. An alcoholic solution of potash also converts it into chlonaphtase and chloride of potassium. Chlorine converts it into two chlorides of chlonaphtase, isomeric but distinct; if the heat is too strong, there is formed one kind of chlonaphtise.

When bromine is made to act on naphthaline, no bromide of $\mathrm{N}$ is formed, but hydrobromic acid is separated and bronaphtase is produced.

Chlonaphtase, $\mathrm{C}_{20}\left(\mathrm{H}_{7} \mathrm{Cl}\right)$, is obtained by acting on subchloride of $\mathrm{N}$ by alcoholic solution of potash. On the addition of water, an oily liquid separates, which is purified by rectification, and is then chlonaphtase. No isomeric modification of it is yet known, but the existence of such is extremely probable. Bromine acts on it, converting it into bromide of chlorabronaphtese, $\mathrm{Br}_{4}+\mathrm{C}_{2} \mathrm{O}$ $\mathrm{H}_{6} \mathrm{Cl} \mathrm{Br}$.

Bronaphtase, $\mathrm{C}_{20}\left(\mathrm{H}_{7} \mathrm{Br}\right)$, is formed by the direct action of bromine on naphthaline, care being taken to avoid excess of bromine, which would form bronaphtese, and excess of naphthaline, which would remain unchanged. Bronaphtase is a colourless oil, decomposed by chlorine and bromine, the latter converting it into bronaphtese and the products of the further action of bromine on bronaphtese. As yet only one form of bronaphtase is known.

Chlonaphtese, $\mathrm{C}_{20}\left(\mathrm{H}_{6} \mathrm{Cl}_{2}\right)$, occurs in no less than seven different isomeric forms. These are distinguished by Laurent as modifications $a, c, a d, e, f, x$ and $y$, but $I$ am not aware of the principle on which these letters are selected. $a, c, f$, and $x$, are obtained by the action of heat on the chloride of $\mathrm{N}$; $a d$ and $e$ by boiling chloride of $\mathrm{N}$ with tincture of potash ; and $y$ by the 
action of chlorine on nitronaphtese. $a$ and $x$ are liquid; all the rest crystallise easily. The solid forms have each a different point of fusion, and the whole seven give different results when acted on by chlorine and bromine. Since, therefore, the composition of all seven is the same, we are compelled to adopt the conclusion that it is not the same 2 eqs. of hydrogen which are replaced by chlorine, and that it is not indifferent which equivalents of hydrogen are thus replaced, but that, on the contrary, the properties of these compounds depend on the particular equivalents or moleoules of hydrogen replaced by chlorine, and that consequently the arrangement, or relative as well as absolute position of these molecules in the compound molecule, is a matter of far greater importance (at least, in this and other analogous cases), in reference to chemical characters, than the properties of the elements, or their place in the electrochemical arrangement.

Referring to what I have said on the subject of types, at pp. 12-19, I would here point out that naphthaline is a type, the molecule of which is made up of 20 equivalents (not single atoms, but molecules) of carbon, and 8 equivalent molecules of hydrogen; and that chlonaphtese is a subtype, in which the 20 molecules of carbon are associated, as in the fundamental type, with 8 other molecules, not all as before of hydrogen, but 6 of hydrogen and 2 of chlorine, a body usually considered as entirely opposed to hydrogen, chlorine being, strongly negative, and hydrogen strongly positive. Yet the type remains unchanged, and we cannot help seeing that the two molecules of chlorine, in virtue of their position in reference to the 20 of carbon, are playing the part of 2 molecules of hydrogen.

Further, if we conceive the 8 molecules of hydrogen in the fundamental type to occupy each a fixed position, in relation to the 20 of carbon, we see from the wonderful phenomena just indicated, namely, from the existence of seven distinct forms of chlonaphtese, that, in each of these, a different pair of molecules of hydrogen has been replaced by chlorine. If the 8 molecules of hydrogen be supposed to be numbered, according to the fixed position of each in the compound molecule of the type, then we san see that in chlonaphtese $a$, the molecules 1 and 2 may be those replaced, while in $c$ the molecules 6 and 7 may be those replaced by chlorine, and so on. It is easy to calculate that in this way at least 28 different isomeric forms of chlonaphtese may exist, and of these seven are already known.

Our space will not admit of details on the different forms of chlonaphtese, but I have thought it indispensable to explain the view now taken of these singular compounds, in a general way. 
It is evident that we may expect much light to be thrown on the obscure subject of the molecular arrangement of compounds by continued researches in the same direction. The recent progress of those departments of physics which are most closely allied to chemistry, has established the existence of certain relations between the atomic weight and constitution of compounds and their physical properties, such as volume or density, volatility, state in regard to cohesion, solidity, fluidity, \&c., and crystalline form. We now see a prospect of tracing the connection between the molecular arrangement of compound bodies and their chemical properties; and we may even hope hereafter to be enabled, simply by accurate observation of the external properties of a body, physical and chemical, to ascertain its composition and constitution; and also to predict with accuracy the properties of compounds yet unformed, the formation of which will probably become a problem, solvable by a few rules of universal application. An excellent illustration of this has lately been given in the prediction of Liebig in 1839, of the properties of volatile bases, discovered in 1849 and 1850-51, by Würtz and Hofmann. The prediction was fully verified, and the processes employed were such as admit of very wide application. (See Ethyle, Methyle, and Artificial Organic Bases).

For the present, we have only the distant prospect of these results; but we have only assiduously to pursue the study of nature on true inductive principles, in order to be hereafter enabled to bring into order the chaos, so to speak, of interesting and important observations, the number of which is hourly increasing, while a large proportion of them have not yet found a use or an application. We must now return to the derivatives of naphthaline, which we have only space briefly to name.

Bronaphtese, $\mathrm{C}_{20}\left(\mathrm{H}_{6} \mathrm{Br}_{2}\right)$, is easily formed by the action of bromine on naphthaline or on bronaphtase. It is a crystallisable solid, and probably corresponds to chlonaphtese, $c$. Only one bronaphtese is yet known. It forms several compounds with bromine.

Chlonaphtise, $\mathrm{C}_{20}\left(\mathrm{H}_{5} \mathrm{Cl}_{3}\right)$, occurs in six different forms (out of 55 which are possible), $a, a c, c, g, d$, and $a d$, all of which are crystallisable solids. They are obtained in different ways : $a$ by boiling with tincture of potash the oily modification of chloride of chlonaphtase; $a c$ by the action of chlorine on chlonaphtese $a d$, melted; and $g$ along with some of $a$, by boiling with tincture of potash the crystallised chloride of chlonaphtase; $d$ by distilling the crystallised chloride of chlonaphtase; and $a d$ by boiling with tincture of potash the double chloride of naphthaline and of 
chlonaphtase; $a$ is converted by chlorine into chlonaphtose $a$; and bromine converts it into chloribronaphtose $a$.

Bronaphtise, $\mathrm{C}_{20}\left(\mathrm{H}_{5} \mathrm{Br}_{3}\right)$, is obtained by heating the bromide of bronaphtise, when bromine is given off. It is crystallisable. Only one form is yet known.

Chlonaphtose, $\mathrm{C}_{20}\left(\mathrm{H}_{4} \mathrm{Cl}_{4}\right)$, occurs in four isomeric forms, $a, b, e$, and $k$, out of a very large number which are possible. They are all crystallisable. It is unnecessary to mention the methods employed to obtain them, which are analogous to those already described for chlonaphtise or chlonaphtese.

Bronaphtose, $\mathrm{C}_{20}\left(\mathrm{H}_{4} \mathrm{Br}_{4}\right)$, appears to exist in two forms $a$ and $b$, both crystallisable.

Chlonaphtuse, $\mathrm{C}_{20}\left(\mathrm{H}_{3} \mathrm{Cl}_{3}\right)$, and Bronaphtuse, $\mathrm{C}_{20}\left(\mathrm{H}_{3} \mathrm{Br}_{5}\right)$, are not yet known.

Chlonaphtalase, $\mathrm{C}_{20}\left(\mathrm{H}_{2} \mathrm{Cl} 6\right)$, is obtained by the action of chlorine on chlonaphtise $a$. It forms soft flexible prisms.

Chlonaphtalese, $\mathrm{C}_{20}\left(\mathrm{H} \mathrm{Cl}_{7}\right)$, is not yet known.

Chlonaphtalise, $\mathrm{C}_{20} \mathrm{Cls}$, the compound in which all the hydrogen of naphthaline is replaced by chlorine, is obtained by continuing the action of chlorine on chlonaphtise $a$. It is also crystallisable. Laurent, apparently from its crystalline form, considers it to correspond with the modifications $c$ of chlonaphtese and chlonaphtise ; and for the same reason he considers the only chlonaphtalase known, as chlonaphtalase $a$. In the case of chlonaphtalise, if different modifications can occur, they must depend on a different principle from that which regulates the modifications of those compounds in which both chlorine and hydrogen are concerned. But until a complete account of Laurent's views be published, it is not easy to ascertain exactly what those views are. I suspect some error in the only account of those researches to which I could refer.

Besides the above, there are a number of compounds derived from naphthaline, in which the hydrogen is replaced by bromine and chlorine at once.

Chlorebronaphtise a, $\mathrm{C}_{20}\left(\mathrm{H}_{5} \mathrm{Cl}_{2} \mathrm{Br}\right)$, is a crystalline solid very similar to chlonaphtise $a$.

Chlorcbronaphtose $b, \mathrm{C}_{20}\left(\mathrm{H}_{4} \mathrm{Cl}_{2} \mathrm{Br}_{2}\right)$, is obtained by the action of bromine on chlonaphtese $f$.

Chloribronaphtose a, Bromachlonaphtose $a$, and Bromachlonaphtose $b$, are three isomeric compounds. The first is obtained by the action of bromine on chlonaphtise $a$, and the bromine is therefore placed second in the name. The two others are both formed when chlorine acts on bronaphtese. These are the compounds alluded to at page 18. They furnish a very beautiful proof of the truth that the position of the 
replaced or replacing molecule is all-important. They are all crystallisable.

Broneclonaphtose $b, \mathrm{C}_{20}\left(\mathrm{H}_{4} \mathrm{Cl}_{2} \mathrm{Br}_{2}\right)$, is a crystalline solid, obtained by boiling chloride of bromechlonaphtise with tincture of potash.

Chloribronaphtuse, $\mathrm{C}_{20}\left(\mathrm{H}_{3} \mathrm{Cl}_{3} \mathrm{Br}_{2}\right)$, is a crystalline solid, formed by the action of bromine on chloride of naphthaline.

There remain to be described some compounds, analogous to the chlorides of naphthaline, and containing consequently chlorine or bromine, in addition to the type or subtype.

Chloride of chlonaphtase, $\mathrm{Cl}_{4}+\mathrm{C}_{20}\left(\mathrm{H}_{7} \mathrm{Cl}\right)$, is obtained by the action of chlorine on the subchloride of naphthaline. It is the most remarkable of the whole series from the great size and beauty of its crystals. It occurs in an isomeric form as an oily liquid. When distilled, these compounds yield different forms of chlonaphtise mixed together.

Chloride of chlonaphtese, $\mathrm{Cl}_{4}+\mathrm{C}_{20}\left(\mathrm{H}_{6} \mathrm{Cl}_{2}\right)$, occurs in three isomerio forms. $a$ and $x$ are oily liquids, derived respectively from chlonaphtese $a$ and $x$ by the action of chlorine, and $c$ is derived from chlonaphtese $c$ in the same way, and is crystalline. They all yield chlonaphtose when heated, but in different forms.

Bromide of chlonaphtese, $\mathrm{Br}_{4}+\mathrm{C}_{20}\left(\mathrm{H}_{6} \mathrm{Cl}_{2}\right)$, is obtained by the action of bromine on chlonaphtese $c$. It is crystalline. An excess of bromine produces at least five different compounds.

Bromide of chlorabronaphtese, $\mathrm{Br}_{4}+\mathrm{C}_{20}\left(\mathrm{H}_{6} \mathrm{Br} \mathrm{Cl}\right)$, is formed when bromine acts on chlonaphtese. It is crystalline.

Bromide of bronaphtese, $\mathrm{Br} 4+\mathrm{C}_{20}\left(\mathrm{H}_{6} \mathrm{Br}_{2}\right)$, is formed by the action of bromine on bronaphtese. It is crystalline, and when distilled yields hydrobromic acid and bronaphtose.

Subbromide of bronaphtise, $\mathrm{Br}_{2}+\mathrm{C}_{20}\left(\mathrm{H}_{5} \mathrm{Br}_{3}\right)$, is formed along with the preceding. It is also crystalline.

Bromide of bronaphtise, $\mathrm{Br}_{4}+\mathrm{C}_{20}\left(\mathrm{H}_{5} \mathrm{Br} 3\right)$, is also a highly crystalline solid.

Subchloride of bronaphtase, $\mathrm{Cl}_{2}+\mathrm{C}_{20}\left(\mathrm{H}_{7} \mathrm{Br}\right)$, formed by the action of chlorine on bronaphtase, crystallises in regular rhomboidal plates.

Chloride of bronaphtese, $\mathrm{Cl}_{4}+\mathrm{C}_{20}\left(\mathrm{H}_{6} \mathrm{Br}_{2}\right)$, crystallises in long prisms.

Perchloride of bronaphtise, $\mathrm{Cl}_{5}+\mathrm{C}_{20}\left(\mathrm{H}_{5} \mathrm{Br}_{3}\right)$, crystallises in right prisms with rhombic base.

Chloride of bromechlonaphtise, $\mathrm{Cl}_{4}+\mathrm{C}_{20}\left(\mathrm{H}_{5} \mathrm{Br}_{2} \mathrm{Cl}\right)$, crystallises in oblique rhombic prisms. When boiled with tincture of potash it yields bromechlonaphtuse, $\mathrm{C}_{20}\left(\mathrm{H}_{3} \mathrm{Br}_{2} \mathrm{Cl}_{3}\right)$.

All the preceding compounds have been discovered, studied, 
analysed, and described by Laurent, besides a large additional number of derivatives of naphthaline, under the agency of chlorine and bromine, which he has not so fully examined. Those here named are sufficient to illustrate the endless variety of compounds attainable. Every subtype of the original type of naphthaline admits of numerous permutations; and where both chlorine and bromine are present, the number of possible permutations is enormously increased. In short, these remarkable researches have only made us acquainted with a very small selection of the possible products of the action of chlorine and bromine on naphthaline, the type remaining unchanged.

Thus the subtype chlonaphtose, $\mathrm{C}_{20}\left(\mathrm{H}_{4} \mathrm{Cl}_{4}\right)$, admits of 65 isomeric modifications, all different, as does, of course, bronaphtose also. But these subtypes may yield the modified subtypes chlorabronaphtose, chlorebronaphtose, chloribronaphtose, bromachlonaphtose, bromechlonaphtose, and bromichlonaphtose, and others, difficult to name, depending on the relative proportions and positions, in the molecule of the subtype, and of the 4 eqs. chlorine and bromine. It is easy to imagine 14 such modified subtypes, and there appears no reason why each of them, with the two subtypes, should not admit of at least 65 isomeric forms. This would give 1040 isomeric forms, all included under the two subtypes chlonaphtose and bronaphtose, or under one subtype which may be called naphtose, $\mathrm{C}_{20}\left(\mathrm{H}_{4} \mathrm{X}_{4}\right) . \mathrm{X}$ is here put for chlorine or bromine.

Gerhardt has given the following table of the compounds obtained from naphthaline, with chlorine and bromine :-

\section{Chlorides and Bromides.}

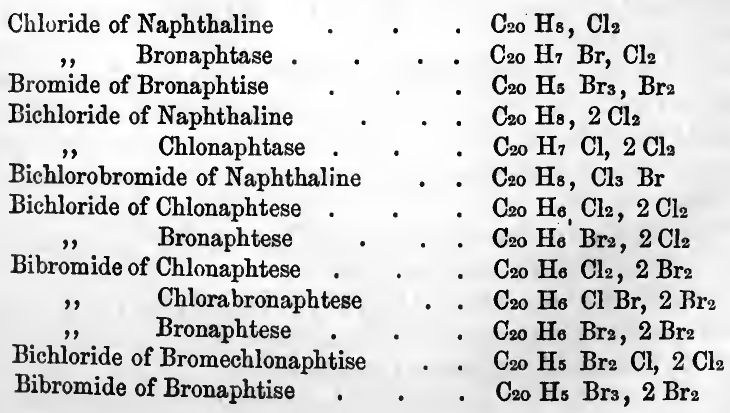


2. Naphthalines chlorinised and brominised.

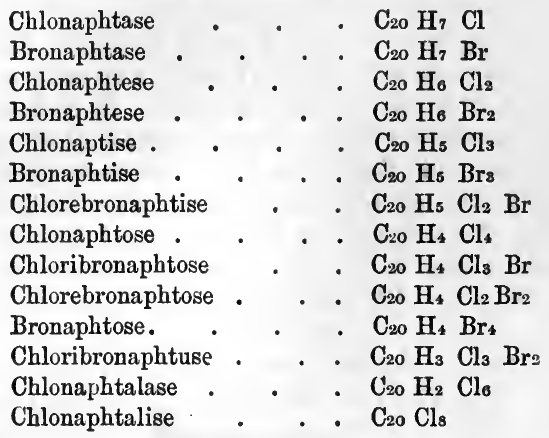

I have retained Laurent's names, as those of Gerhardt do not suit our language. It will be seen by this table that naphthaline and its products by substitution form one group, the members of which, combining with chlorine or bromine, form another group.

\section{Action of Sulphuric Acid on Naphthaline.}

When naphthaline is dissolved in warm oil of vitriol to saturation, the solution, if left exposed to the air, becomes a semisolid mass of a dirty purplish colour. This, dried on a porous brick, leaves a quantity of scales, very soluble in water and alcohol, which are a mixture of two acids.

a. Sulphonaphthalic acid, $\mathrm{C}_{20} \mathrm{H}_{8} \mathrm{~S}_{2} \mathrm{O}_{6}+2$ aq. The above mixture, being dissolved in water, is saturated with carbonate of lead, which forms insoluble sulphate of lead, and two soluble salts, one of which is soluble in alcohol and contains this acid, and yields it when decomposed by sulphuretted hydrogen. The acid forms a hard crystalline fusible mass, of an acid and bitter taste. Its salts are soluble and crystallisable. Their formula is $\mathrm{C}_{20} \mathrm{H}_{7} \mathrm{M} \mathrm{S}_{2} \mathrm{O}_{6}$. Their acid is formed from 1 eq. of napthaline and 2 eq. of oil of vitriol, $\mathrm{SO}_{3}, \mathrm{HO}$, by the separation of 2 eqs. of water, which enter into the acid, however, as water of crystallisation, and are expelled by bases.

The same amount of naphthaline, with twice as much oil of vitriol, forms, by the separation of 4 eqs. of water, the next acid, $\mathrm{C}_{20} \mathrm{H}_{8}+4\left(\mathrm{HO}_{1} \mathrm{SO}_{3}\right)-4 \mathrm{HO}=\mathrm{C}_{20} \mathrm{H}_{8} \mathrm{~S}_{4} \mathrm{O}_{12}$.

Sulphonaphthalic acid forms with chlorine four substitution acids, in which $1,2,3$, and 4 eqs. of hydrogen are replaced by chlorine. With bromine it yields two such acids, with 1 and 2 eq. of bromine. It also yields, with nitric acid, an acid, the 
nitrosulphonaphthalic acid, in which 1 eq. of hydrogen is replaced by $\mathrm{NO}_{4}$.

b. Disulphonaphthalic acid, $\mathrm{C}_{20} \mathrm{H}_{8} \mathrm{~S}_{4} \mathrm{O}_{12}$ ? The lead salt, insoluble in alcohol, contains this acid, the salts of which are soluble, bitter, and hardly crystallisable. It is possible that a third acid accompanies these two; for Faraday obtained a third salt of baryta, and Berzelius found a third salt of lead in the mother liquid of the other two. Faraday's salt, which remains with the sulphate of baryta formed in the process by the free sulphuric acid, and may be extracted by boiling water, yields about 42 per cent. of sulphate of baryta when calcined.

The vapours of anhydrous sulphuric acid, passed over fused naphthaline, form with it a red liquid. If the acid be in excess, there is formed a new acid, the hyposulphoglutinic acid, besides small quantities of the preceding acids; if there be excess of naphthaline, there are formed two neutral bodies, sulphonaphthaline and sulphonaphthalide.

Hyposulphoglutinic acid, when pure and dry, is a hard glassy mass. When precipitated from its salts by stronger acids, it forms a viscid hydrate like turpentine. Its salts are generally soluble and do not crystallise. Its composition is unknown.

Sulphonaphthaline is a crystalline fusible solid, formed from 2 eq. of naphthaline and 2 eq. of oil of vitriol, by the separation of 4 eq. of water. Its formula is $\mathrm{C}_{40} \mathrm{H}_{14} \mathrm{~S}_{2} \mathrm{O}_{4}$, or it may be written $\left.\mathrm{C}_{40} \mathrm{H}_{2 \mathrm{SO}_{2}}^{\mathrm{H}_{14}}\right\}$; that is, 2 eq. of naphthaline, in which 2 eq. of hydrogen are replaced by 2 eq. of sulphurous acid $\mathrm{SO}_{2}$.

3. Action of Nitric Acid on Naphthaline, and its Derivatives.

Nitric acid acts on naphthaline, and gives rise to a whole series of compounds in which $\mathrm{N} \mathrm{O}_{4}$ is substituted for hydrogen. The same principles apply here as in the action of chlorine and bromine on naphthaline. It is to Laurent that we are indebted for our knowledge of these compounds, which our space will only allow us to name.

Nitronaphtase, or nitronaphthaline, $\mathrm{C}_{20} \mathrm{H}_{7} \mathrm{X}$ ( $\mathrm{X}$ is here put for $\mathrm{NO}_{4}$ ), is best formed by causing nitrous acid to pass through melted naphthaline. It is purified by means of alcohol, and forms long prisms of a sulphur-yellow colour, fusible at $110^{\circ}$. Chlorine decomposes it, producing chlonaphtose. Nitric acid converts it into nitronaphtese. By the action of sulphuretted hydrogen and ammonia, it yields, as has been already explained, a base, naphtalidine or naphtylamine, $\mathrm{C}_{20} \mathrm{Ad} \mathrm{H}_{7}=\mathrm{C}_{20} \mathrm{H}_{9} \mathrm{~N}$.

Nitronaphthaline, according to Piria, when its alcoholic solution 
is boiled with solution of sulphite of ammonia in excess, until a drop no longer becomes turbid when mixed with water, yields two strata of liquid. The lighter is an alcoholic solution of new products, the heavier an aqueous solution of sulphate and sulphite of ammonia. The former is evaporated to a small bulk and deposits on standing crystals of the ammonia salt of a new acid, thionaphtamic acid. The mother liquid of these crystals contains the ammonia salt of another new acid, isomeric with the first, naphtionic acid.

Naphtionic acid, $\mathrm{C}_{20} \mathrm{H}_{3} \mathrm{~N} \mathrm{~S}_{2} \mathrm{O}_{5}, \mathrm{H} \mathrm{O}+\mathrm{aq}$. The salt last mentioned is dissolved in hot water, and on the addition of hydrochloric acid, the new acid falls as a reddish-white crystalline powder. When pure, it is colourless. It neutralises all bases, and forms salts which crystallise in large and regular crystals. It is very permanent, except that it is decomposed by oxidising agents.

Thionaphtamic acid, $\mathrm{C}_{20} \mathrm{H}_{8} \mathrm{~N} \mathrm{~S}_{2} \mathrm{O}_{5}, \mathrm{HO}$. This acid forms also crystallisable salts, but cannot be obtained in a separate or free state, because on adding acids to its salts it is at once decomposed, yielding sulphuric acid, and naphtalidine or naphtylamine, the base already mentioned as formed by the action of sulphuret of ammonium on nitronaphthaline. Its salts form red or purple micaceous scales, but are probably colourless when pure; the colour arising from the action of the air, which produces a purple resin with naphtalidine. These salts are easily decomposed. When heated with dilute sulphuric acid, they yield abundant scaly crystals of sulphate of naphtalidine, and this is the best method of obtaining that base. Piria finds that naphtalidine and its salts yield with oxidising agents a violet amorphous compound, which he calls naphtamine. Its composition is not yet known. When nitronaphtaline is acted on by sulphurous acid, we have $\mathrm{C}_{20} \mathrm{H}_{7} \mathrm{NO}_{4}+2 \mathrm{~S} \mathrm{O}_{2}+2 \mathrm{H} \mathrm{O}=$ $2 \mathrm{~S} \mathrm{O}_{3}+\mathrm{C}_{20} \mathrm{H}_{9} \mathrm{~N}$; and the naphtalidine, $\mathrm{C}_{20} \mathrm{H}_{\theta} \mathrm{N}$, combines with 2 eqs. of sulphurie acid to yield the two new acids; thus, $\mathrm{C}_{20} \mathrm{H}_{9} \mathrm{~N}+2 \mathrm{~S} \mathrm{O}_{3}=\mathrm{C}_{20} \mathrm{H}_{8} \mathrm{~N} \mathrm{~S}_{2} \mathrm{O}_{5}, \mathrm{HO}$. This at once explains the easy resolution of one of these acids into naphtalidine and sulphuric acid; the other acid, which is so permanent, must have a different arrangement of the same elements.

Naphtalase, $\mathrm{C}_{20}\left(\mathrm{H}_{7} \mathrm{O}\right)$, is a yellow crystalline solid, formed by gently heating nitronaphtase with 10 parts of lime slightly moistened. It communicates to oil of vitriol a magnificent blue colour. It is insoluble in alcohol as well as in water, otherwise it would recall pyroxanthine, which is yellow and volatile, and colours sulphuric acid purple.

Nitronaphtese, $\mathrm{C}_{20}\left(\mathrm{H}_{6} \mathrm{X}_{2}\right)$, forms a crystalline powder fusible 
at $365^{\circ}$, insoluble in water, very sparingly soluble in alcohol. It is converted, by the action of sulphuretted hydrogen and ammonia, into the new artificial base, seminaphtalidine or azonaphtylamine $=\mathrm{C}_{20} \mathrm{H}_{10} \mathrm{~N}_{2}$.

Nitronaphteise, $\mathrm{C}_{20}\left(\mathrm{H}_{5 \mathrm{t}} \mathrm{X}_{2 \mathrm{t}}\right)$ ? is a crystalline compound formed when naphthaline is added in small quantities to a large mass of hot nitric acid. Nitronaphtese is produced along with it. It is pale-yellow and very fusible, becoming liquid even in boiling alcohol. This great fusibility prevents us, notwithstanding its strange formula, from considering it as a mixture of nitronaphtese and nitronaphtise, the former of which melts at $365^{\circ}$, the latter at $410^{\circ}$, and which are very insoluble in ether, in which liquid nitronaphteise readily dissolves.

Nitronaphtise, $\mathrm{C}_{20}\left(\mathrm{H}_{5} \mathrm{X}_{3}\right)$, is formed along with the preceding, and crystallises in rhomboidal plates of a pale-yellow colour, fusible at $410^{\circ}$.

Nitronaphtale is a crystalline substance, formed by the longcontinued action of nitrio acid on the mother liquor of all the preceding. It melts at $420^{\circ}$, and sometimes solidifies in an amorphous state; a slight heat, or touching the melted substance with a point, causes it to crystallise. It seems to be an isomeric form of the preceding compound, and a third is said to exist.

Nitronaphtesic acid, $\mathrm{C}_{16} \mathrm{H}_{43} \mathrm{~N}_{1 \frac{1}{3}} \mathrm{O}_{4}$ ? $\mathrm{C}_{32} \mathrm{H}_{9} \mathrm{~N}_{3} \mathrm{O}_{8}$ ? This acid is formed when nitronaphtalese is boiled with tincture of potash, and is separated from the potash by nitric acid. When dry it is brownish-black, and forms brown salts, which are soluble and uncrystallisable. Nitronaphtaléisic acid, $\mathrm{C}_{12} \mathrm{H}_{4} \mathrm{O}_{6} \mathrm{~N}_{\frac{1}{2}}$, or $\mathrm{C}_{24} \mathrm{H}_{8} \mathrm{O}_{12} \mathrm{~N}_{3}$, is a similar acid, formed from nitronaphtaléise. Nitronaphtisic acid is another brown acid, formed in the same way from nitronaphtalise.

Oxide of chloroxénaphtose, $\mathrm{O}_{2}+\mathrm{C}_{20}\left(\mathrm{H}_{4} \mathrm{Cl}_{2} \mathrm{O}_{2}\right)$, is formed when nitric acid acts on crystallised chloride of chlonaphtose. It appears as a yellow crystalline solid. It is accompanied by chloranaphtisic acid, into which it is also converted by boiling with tincture of potash.

Chloranaphtisic acid, $\mathrm{C}_{20} \mathrm{H}_{5} \mathrm{Cl} \mathrm{O}_{6}$, is formed from the preceding compound, as follows: $\mathrm{C}_{20} \mathrm{H}_{4} \mathrm{Cl}_{2} \mathrm{O}_{4}+\mathrm{HO}+2 \mathrm{KO}$ $=\mathrm{H} \mathrm{Cl}+\left(\mathrm{C}_{20} \mathrm{H}_{5} \mathrm{Cl} \mathrm{O}_{8}+\mathrm{K} \mathrm{O}\right)$. It is separated from the potash by adding an acid, and forms yellow crystals, fusible at $392^{\circ}$. Its salts are for the most part insoluble, and exhibit the most beautiful colours, including yellow, orange, red, and carmine.

Oxide of chloroxénaphtalise, $\mathrm{O}_{2}+\mathrm{C}_{20} \mathrm{Cl}_{6} \mathrm{O}_{2}$, is obtained along with chlophtalisic acid and other compounds, when nitric acid is boiled with chlonaphtalase, $\mathrm{C}_{20} \mathrm{H}_{2} \mathrm{Cl}_{6}$. It forms golden scales. 
Chloroxénaphtalesic acid, $\mathrm{C}_{20} \mathrm{H} \mathrm{Cl}_{5} \mathrm{O}_{6}$, is formed when the preceding compound is acted on by potash, which at once changes it into a fine carmine-red substance, from which acids separate the new acid as a yellow crystalline powder. It forms beautiful red salts with potash and ammonia. It is formed as follows: $\mathrm{C}_{20} \mathrm{Cl}_{6} \mathrm{O}_{4}+\mathrm{HO}+2 \mathrm{~K} \mathrm{O}=\left(\mathrm{C}_{20} \mathrm{H}_{\mathrm{Cl}} \mathrm{O}_{6}+\mathrm{KO}\right)+$ $\mathrm{K} \mathrm{Cl}$.

The existence of the two chlorinised acids, just described, leads to the idea of the existence of an acid $\mathrm{C}_{20} \mathrm{H}_{6} \mathrm{O}_{6}$, from which they are derived by substitution. Now this acid has not yet been obtained by the oxidation of naphthaline, though that will probably be done sooner or later. But alizarine, the colouring principle of madder, is now believed to have this formula, and is, perhaps, the compound in question. If so, and if it could be formed artificially from naphthaline, the discovery would prove a most profitable one, from the importance of madder as a dye, and the small amount of colouring matter it contains.

Phtalic or naphtalic acid, $\mathrm{C}_{16} \mathrm{H}_{4} \mathrm{O}_{6}, 2 \mathrm{H} \mathrm{O}=\mathrm{C}_{16} \mathrm{H}_{6} \mathrm{O}_{8}$, is formed by the action of nitric acid on chloride of naphthaline. It forms rounded groups of lamellar crystals, and yields crystallisable salts. When distilled with lime, it yields benzole (phéne), and carbonic acid, $\mathrm{C}_{16} \mathrm{H}_{6} \mathrm{O}_{8}=4 \mathrm{C} \mathrm{O}_{2}+\mathrm{C}_{12} \mathrm{H}_{6}$. When the hydrated acid is distilled, it yields the anhydrous acid in fine elastic needles.

The acid phtalate of ammonia, $\mathrm{C}_{66} \mathrm{H}_{4} \mathrm{O}_{6}, \mathrm{NH}_{4} \mathrm{O}, \mathrm{HO}$, when heated yields water, $4 \mathrm{H} 0$, and phtalimide, $\mathrm{C}_{16} \mathrm{H}_{5} \mathrm{~N} \mathrm{O}_{4}$.

Phtalamide, $\mathrm{C}_{16} \mathrm{H}_{6} \mathrm{~N} \mathrm{O}_{5}$, is obtained by acting on anhydrous phtalic acid with ammonia. It appears that phtalimide is acid phtalate of ammonia, minus 4 eqs. water, while phtalamide is anhydrous phtalate of ammonia, $\mathrm{C}_{16} \mathrm{H}_{4} \mathrm{O}_{6}+\mathrm{NH}_{3}$, minus 1 eq. water. Both are crystalline solids, and both appear to form definite compounds with oxide of silver.

Nitrophtalic acid, $\mathrm{C}_{16} \mathrm{H}_{3} \mathrm{~N} \mathrm{O}_{10}, 2 \mathrm{H} 0$, is derived from phtalic acid by the substitution of 1 eq. $\mathrm{NO}_{4}$ for 1 eq. hydrogen. $\mathrm{C}_{16} \mathrm{H}_{4} \mathrm{O}_{6}-\mathrm{H}+\mathrm{N} \mathrm{O}_{4}=\mathrm{C}_{16} \mathrm{H}_{3} \mathrm{~N}_{10}$. It forms beautiful paleyellow crystals ; and when gently heated, it yields the anhydrous acid in fine white needles.

Chlophtalisic acid, $\mathrm{C}_{16} \mathrm{H} \mathrm{Cl}_{3} \mathrm{O}_{6}$, is formed along with the oxide of chloroxenaphtose when chlonaphtalase is boiled with nitric acid. It is crystallisable and represents anhydrous phtalic acid, in which 3 eqs. hydrogen have been replaced by 3 eqs. chlorine.

To judge from the action of ehlorine unaided by heat, and of nitric acid, on naphthaline, that body, $\mathrm{C}_{20} \mathrm{H}_{8}$, is composed of two earbohydrogens, $\mathrm{C}_{16} \mathrm{H}_{4}$ and $\mathrm{C}_{4} \mathrm{H}_{4}$, the latter of which is 
more easily altered. The former, plus 6 eqs. oxygen, yields phtalic acid, $\mathrm{C}_{16} \mathrm{H}_{4}+\mathrm{O}_{6}$; and the substitution in phtalic acid of $\mathrm{NO}_{4}(=\mathrm{X})$ for $\mathrm{H}$ yields nitrophtalic acid, $\mathrm{C}_{16}\left\{\begin{array}{l}\mathrm{H} \\ \mathrm{X}\end{array}\right.$

B. Anthracene, $\mathrm{C}_{30} \mathrm{H}_{12}$. This compound, which is polymeric with naphthaline, is also found in coal-tar, and is sometimes called paranaphthaline. It melts at $356^{\circ}$, and distils at $392^{\circ}$, yielding foliated plates. When acted on by nitric acid, it gives rise to a series of compounds, in which oxygen is substituted for hydrogen, while the compounds thus formed combine with hyponitrous acid. Thus, we have

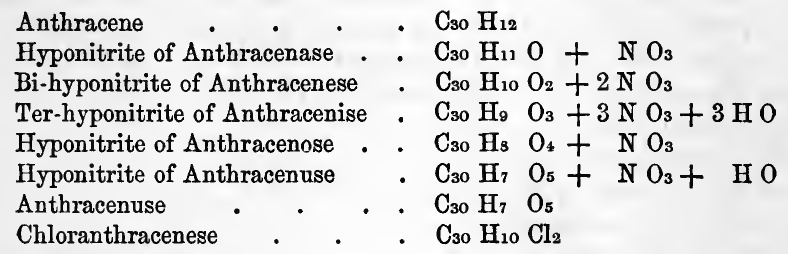

The second compound of the above list is not known; but its existence is probable. In all the works to which I have access, there appear to be errors, probably of the press, in the table, which I have ventured to correct, so as to bring the formulæ into correspondence with the systematic names devised by Laurent, who discovered all these substances, on the same principle as in the case of naphthaline.

$\gamma$. Metanaphthaline. SrN. Retisterene. This compound is formed in the destructive distillation of resin, in the last stages of the process. It forms fatty scales, fusible at $158^{\circ} \mathrm{F}$., and boiling at $617^{\circ} \mathrm{F}$. Its analysis seems to show that it is isomeric or polymeric with naphthaline.

$\delta$. In the distillation of benzoate of lime, there are formed, along with benzophenone, two carbohydrogens, isomeric or polymeric with naphthaline. One of these forms fine prisms, fusible at $197^{\circ} \mathrm{F}$, the other appears in mammellated groups of minute crystals, fusible at $149^{\circ} \mathrm{F}$. If we suppose one to be isomeric with naphthaline, $\mathrm{C}_{20} \mathrm{H}_{8}$, and the other polymeric, $\mathrm{C}_{30} \mathrm{H}_{12}$, it is easy to see how they may be formed from benzophenone, which is phenyle, $\mathrm{C}_{12} \mathrm{H}_{5}$, plus benzoyle, $\mathrm{C}_{14} \mathrm{H}_{5} \mathrm{O}_{2}$, and is therefore = $\mathrm{C}_{26} \mathrm{H}_{10} \mathrm{O}_{2}$. For

$$
\begin{aligned}
& 2\left(\mathrm{C}_{26} \mathrm{H}_{10} \mathrm{O}_{2}\right)=2 \mathrm{C} \mathrm{O}_{2}+\mathrm{C}_{20} \mathrm{H}_{3}+\mathrm{C}_{30} \mathrm{H}_{12} \\
& \text { Benzophenone. Carbohydrogen, Carbohydrogen, } \\
& \begin{array}{l}
\text { No. 1. } \\
\text { No.2. }
\end{array}
\end{aligned}
$$


But these compounds have not jet been fully studied.

e. Chrysene, $\mathrm{C}_{12} \mathrm{H}_{4}$, is found among the last portions of the rectification of coal-tar. It is a yellow crystalline solid, insoluble in most liquids. It melts at $455^{\circ}$. With nitric acid it forms nitro-chrysene, $\left.\mathrm{C}_{12} \mathrm{H}_{3} \mathrm{~N} \mathrm{O}_{4}\right\}$

5. Pyrene, $\mathrm{C}_{30} \mathrm{H}_{12}$, is found accompanying the preceding. It is a good deal more fusible. When acted on by nitric acid, it yields bi-nitropyrene, $\left.\mathrm{C}_{30} \mathrm{H}_{2 \mathrm{~N}_{4}}^{\mathrm{N}_{4}}\right\}$. Pyrene seems to be polymeric with naphthaline.

The occurrence of so many compounds as have been already mentioned, isomeric or polymeric with naphthaline, all produced at a high temperature, is remarkable. For we should rather expect to obtain these solids of high atomic weight at lower temperatures, and the liquids and gases of lower atomic weight at the higher temperatures. There must be some cause which produces the formation of bodies containing $\mathrm{C}_{20}$, possibly in some $\mathrm{C}_{10}$, or $\mathrm{C}_{30}$, or $\mathrm{C}_{40}$, or even more than this. But we do not at present know what this cause is. On the other hand, it is possible, that, as we have two homologous series of hydrocarbons among the products of destructive distillation, namely, the series of marsh gas, $\mathrm{C}_{2} \mathrm{H}_{4}$, or, in general terms, $\mathrm{Cn}_{n} \mathrm{H}_{n}+2$, and that of olefiant gas, $\mathrm{C}_{4} \mathrm{H}_{4}$, that is, $\mathrm{C}_{\mathrm{n}} \mathrm{H}_{\mathrm{n}}$, the former being hydurets of methyle, \&c., the latter, hydurets of formyle, acetyle, \&c.; so, there may also be a third homologous series, to which naphthaline belongs. Thus we might have $\mathrm{C}_{14} \mathrm{H}_{2}, \mathrm{C}_{16} \mathrm{H}_{4}, \mathrm{C}_{18} \mathrm{H}_{6}$, $\mathrm{C}_{20} \mathrm{H}_{8}$ (naphthaline), $\mathrm{C}_{22} \mathrm{H}_{10}, \mathrm{C}_{24} \mathrm{H}_{12}, \mathrm{C}_{26} \mathrm{H}_{14}$, and so on. Contiguous members of such a series will differ very little in composition or in properties; and it is so difficult to purify the individual compounds in such mixtures, that the existing analyses might in many cases agree with such formulæ. This does not exclude the existence of isomeric and polymeric modifications, which we know to be very frequent in carbohydrogens.

When bituminous shale is distilled, it yields a thick empyreumatic oil, composed of several products. Among these is an oil apparently identical with eupion, and another very peculiar oil, called ampeline. This oil has neither taste nor smell, but is in some points more analogous to creosote than to any other substance. It dissolves in water, but a few drops of acid cause it to separate. Its composition is still unknown. It may possibly be a product of the action of oil of vitriol, which is used in its preparation, on some other substance.

Ampelic Acid is formed by the action of nitrio acid on that part of the oil of schist which distils at $300^{\circ}$. It is oily, soluble in hot 
water, and forms very soluble salts. Its composition is not known with certainty. A similar oil, obtained from coal-tar, was found to contain $\mathrm{C}_{14} \mathrm{H}_{6} \mathrm{O}_{6}$. This would be isomerio with hydrated salicylic acid. Williams, as mentioned elsewhere, has found many basic oils in the oil of bituminous shales.

FOSSIL RESINS, WAX, OIL, NAPHTHA, \&c.

Retinite, or Retinasphalt, is a fossil resin found in lignite or wood-coal. It is fusible and combustible, and almost entirely soluble in alcohol. Retinic Acid, $\mathrm{C}_{21} \mathrm{H}_{9} \mathrm{O}_{3}$, was found by Johnston in the retinasphalt of Bovey.

Hatchetine is another fossil resin found in the lignites of Wales. It is colourless, or slightly yellow, fusible and volatile.

Scheererite is a colourless, translucent substance, of a pearly lustre, found in the Swiss lignites. Both Hatchetine and Scheererite appear to be carbohydrogens, and much resemble paraffine not quite pure. They are, perhaps, mixtures of homologous paraffines, or when pure, each may represent a different paraffine.

Middletonite is a fossil resin, found near Leeds in coal.

Idrialine is a remarkable solid carbohydrogen, found in the quicksilver mines of Idria. Its composition was said to be $\mathrm{Cs}_{s} \mathrm{H}$, or perhaps $\mathrm{C}_{12} \mathrm{H}_{7}$; and therefore polymerio with chrysone. But it has lately been shown to contain oxygen, and to be, perhaps, $\mathrm{C}_{84} \mathrm{H}_{28} \mathrm{O}_{2}$. It forms nitroidrialine, with nitric acid, which is $\left.\mathrm{C}_{84} \mathrm{H}_{5 \mathrm{NO}_{4}}^{\mathrm{H}_{23}}\right\} \mathrm{O}_{2}$. It also yields, when heated, a carbohydrogen idryle, in two isomerio or polymeric forms; ${ }_{n} \mathrm{C}_{6} \mathrm{H}_{2}$. It colours oil of vitriol intensely blue, forming a coupled acid. Succisterene, a solid body obtained by Pelletier and Walter in the distillation of amber, has the same composition, and colours sulphuric acid blue. It is, therefore, in all probability, identical with idrialine.

Ozokerite, or Fossil Wax, is found in large masses in the bituminous schist of Slamick in Moldavia. When distilled, it yields a substance like wax, and also a good deal of paraffine. Ozokerite is very fusible, and burns with a bright flame. A similar substance occurs in the coal mines of England, and it has been shown to be a mixture of at least four compounds, probably most of them paraffines.

Fichtelite is a fusible, volatile, crystallisable solid, found in branches of pine-trees, in the peat of the turbaries in the Fichtelgebirge. It appears to have the formula $\mathrm{C}_{20} \mathrm{H}_{15}$, and is probably derived from essence of turpentine, $\mathrm{C}_{20} \mathrm{H}_{16}$.

Tekoretine, Phylloretine, Xyloretine, and Boloretine, are the 
names of four resinous compounds, found in the peat of Denmark, on the remains of pine-trees. Tekoretine and Phylloretine are both fusible, volatile, and crystallisable. The former appears to be $\mathrm{C}_{20} \mathrm{H}_{9}$, the latter $\mathrm{C}_{5} \mathrm{H}_{3}$. Xyloretine is less fusible and is decomposed by heat. It crystallises, and its formula is said to be $\mathrm{C}_{40} \mathrm{H}_{33} \mathrm{O}_{4}$, which only differs from that of sylvic acid by 3 eq. hydrogen. Boloretine is fusible, but does not orystallise. Its formula is $\mathrm{C}_{40} \mathrm{H}_{32}+3 \mathrm{H} 0$, but it occurs also with 5 or 6 eqs. of water.

Asphaltum, Mineral Pitch, Pitch of Judea. These are the names of certain substances of similar characters, found in different parts of the world, as in Trinidad, in Hanover, and at the Dead Sea in Palestine. They all resemble pitch in aspect, and are composed of a dark-brown resin, mixed with more or less of a brilliant black matter, asphaltene, $\mathrm{C}_{20} \mathrm{H}_{16} \mathrm{O}_{3}$, or of a liquid volatile oil, petrolene, $\mathrm{C}_{20} \mathrm{H}_{16}$. The former of these is probably an oxide of the latter. The different kinds of asphaltum are much used for waterproof cements, and for pavements, or roofs. Naphteine is a somewhat analogous substance, found in the limestones of the Maine et Loire.

Petroleum and Naphtha. In certain spots, in the neigbourhood of the Caspian, in Ava, at the Tegernsee in Bavaria, at Amiano in Italy, and near Neufchatel, as well as in other places, pits dug in the earth become filled with water, on which floats, more or less abundantly, an oily matter, formerly called rock-oil. The purer kinds are little coloured and very fluid, and when distilled with water, leave hardly any residue. These are called naphtha. Other kinds, as the petroleum of Rangoon in Ava, are dark-coloured, and semi-solid, but become liquid at $80^{\circ}$ or $90^{\circ}$. These yield by distillation, first, much naphtha, nearly colourless, and then much paraffine, which is easily purified. Naphtha, when pure, has the Sp. G. $0 \cdot 775$, and its formula is $\mathrm{C}_{n} \mathrm{H}_{n}$. At least, many analyses give exactly this result, namely, 85.7 per cent. of carbon, and 14.3 per cent. of hydrogen. But all who have examined naphtha find it to be a mixture of many oils, agreeing in composition, or nearly so, but differing in boiling point, density, \&c. This is just what we find in a mixture of homologous compounds, and we may infer that a great part of naphtha consists of carbohydrogens of the formula $\mathrm{C}_{n} \mathrm{H}_{n}$, about the middle of the scale, as the lowest are gaseous, the higher solid. They may, perhaps, be $\mathrm{C}_{12} \mathrm{H}_{12}$, $\mathrm{C}_{14} \mathrm{H}_{14}, \mathrm{C}_{16} \mathrm{H}_{16}, \mathrm{C}_{20} \mathrm{H}_{20}, \mathrm{C}_{24} \mathrm{H}_{24}$, \&c. But as some oils in naphtha contain less hydrogen, there is, probably, another series present in which the hydrogen is less than the carbon, as, for example, $\mathrm{C}_{12} \mathrm{H}_{10}, \mathrm{C}_{14} \mathrm{H}_{12}$. Rectified naphtha is used for the 
purpose of preserving potassium and sodium, which have no action on it if water be not present. In many places the native naphtha is used to give light.

Reichenbach found that coal, distilled with water, yielded a little of an oil very similar to petroleum.

All the above substances are formed by the decay or destruction of organic matter, chiefly wood. It is not altogether improbable that those kinds which, like the Rangoon petroleum, contain paraffine ready formed, may have been formed by the action of heat on beds of vegetable remains, situated pretty deep in the crust of the earth.

Soot and Lamp-black are produced by the imperfect combustion or organic matters. They contain much carbon, mixed, in soot with an acid resinous matter, and with a substance analogous to humus, but containing nitrogen, and called asboline. Lamp-black, besides a little resin and oily matter, often contains naphthaline, which may be extracted by alcohol.

\section{COMPOUNDS, VEGETABLE AND ANIMAL, CONTAINING NITROGEN AND SULPHUR, ALBUMINOUS OR SANGUIGENOUS COMIPOUNDS.}

Besides the few oils which contain sulphur, there is a very important class of compounds, common to plants and animals, in which nitrogen and sulphur are essential constituents. These are called albuminous, because they all have a resemblance to albumen, which is one of them ; and sanguigenous, because they are capable of forming blood in the animal body, and are the only compounds capable of this. Since from blood the animal body derives its whole nutrition, while the blood is formed from these compounds, they are called also nutritious compounds; and since they alone can form organised animal tissues, they are called plastic elements of nutrition, to distinguish them from sugar, starch, fat, \&c., which are the respiratory elements of food, and furnish, by their oxidation, the animal heat.

These compounds, which we shall call the sanguigenous bodies, are three in number: namely, Albumen, vegetable and animal; Fibrine, vegetable and animal; and Caseine, vegetable and animal; and under these heads are included certain modified forms of them, as emulsine, a form of albumen, and others.

They all agree in the total absence of crystalline form, and in having a very complex constitution. They agree, moreover, in certain chemical characters; they dissolve with the aid of heat in potash, and the solution blackens the salts of lead, proving that part at least of the sulphur is present in an unoxidised 
state. They all dissolve, with the aid of heat, in strong hydrochloric acid, yielding, if air be admitted, not otherwise, a purple solution, which after a time changes to a dark brown. The very acid liquid which is obtained by dissolving mercury in its own weight of nitric acid, gives a very intense red colour to all these substances. This character is so well marked, that we can in this way detect the presence of one part of albumen in 100,000 of water. When oxidised by sulphuric acid and peroxide of manganese, or by sulphuric acid and bichromate of potash, or by fusion with caustic potash, they all yield similar products; namely, compounds of the series of aldehyde, of that of acetic acid, of the nitryle series, benzoic acid, and oil of bitter almonds, leucine, and tyrosine. All these things indicate a very great similarity in constitution. Their composition, in 100 parts, is so nearly the same, according to the best analyses we have, that they have been supposed to be isomeric, and probably are so. It is evident, from the products of their decomposition, and from the fact that any one of them, in the animal body, may yield the others, that they are very closely related, and may even be identical in composition, so far as concerns their combustible elements. Mulder thought he had obtained from them a compound free from sulphur, $\mathrm{C}_{40}$ $\mathrm{N}_{5} \mathrm{H}_{31} \mathrm{O}_{12}$, which he called proteine, and which he supposed to combine with sulphur and phosphorus, to yield the sanguigenous bodies, thus :-

Crystalline humour $=15$ eq. Proteine +1 eq. Sulphur

Caseine . $=10$ eq. , + 1 eq. ,

Vegetable Gelatine $=10$ eq.,$"+2$ eq. ,

Egg Albumen $=10$ eq. ", +1 eq. ", +1 eq. Phosphorus

Fibrine . $=10 \mathrm{eq} ., \quad+1 \mathrm{eq},,+1 \mathrm{eq}$. ,

Blood Albumen . =10 eq. ", +2 eq. , +1 eq. ",

But the theory of proteine cannot be maintained; for not only does no such compound, free from sulphur, exist; but the amount of sulphur in sanguigenous matter is two or three times greater than indicated by Mulder's formulæ: and that sulphur is present in two states. Moreover, his formula for blood albumen, gives twice as much sulphur as in egg albumen, while in fact the latter contains most sulphur; and, lastly, we have no proof that phosphorus is present in any other form than that of the salts of phosphoric acid. The best analyses yet made yield the formulæ given further on, on the supposition that the molecule of sanguigenous matter contains not less than 2 eqs. of sulphur, an assumption justified by the existence of sulphur in two forms of combination.

There is yet another and a very remarkable character, 
common to all sanguigenous bodies. When exposed to air, moisture, and a certain temperature, they all absorb oxygen, enter into decay, with offensive products, and in this state are ferments, the motion of their particles, according to the theory of Liebig, being communicable by contact to those of fermentescible bodies, such as sugar, which, when pure, do not ferment or decay. Hence the sanguigenous bodies are putrescible, or ferments. Lastly, no sanguigenous compound can exist without containing a certain small but essential proportion of alkalies, and alkaline or earthy phosphates.

If we bear in mind, not only that any one of these substances, in the animal body, can yield the two others, but also that, by digestion with acidulated water, or with a solution of a salt of potash or soda, coagulated or insoluble fibrine and caseine may be rendered soluble, and the solution will exhibit all the properties of a solution of albumen, coagulating when heated, and acting like albumen on polarised light ; that, by means of caustic alkalies, we can give to insoluble fibrine and albumen the characters of soluble caseine; it certainly appears most probable that these substances are entirely alike both in composition and constitution, and only differ in their physical state, or in the mineral matter with which they are united.

This would lead us to admit a common principle, not indeed proteine, but what may be called albumine, which can exist in different isomeric, polymeric, or allotropic forms, becoming thus soluble or insoluble. Soluble albumen, that of eggs and of blood, is perhaps the bi-albuminate of soda; soluble caseine is the neutral albuminate of potash; and fibrine is probably insoluble albumen, combined with earthy phosphates. This is perhaps the most probable view that we can take at present.

1. Albumen. This compound is found in vegetable juices dissolved, and in some, indeed in most seeds, in the solid form, as in nuts, almonds, mustard-seed ; the emulsine of almonds and of mustard being a form of albumen. It is never found, however, without some of the other sanguigenous compounds, and in many seeds the proportion of albumen is small, as in the cereals and leguminous seeds. It is also found dissolved in the blood, in the juice of flesh, and in other animal fluids. On the supposition that one molecule of albumen contains 2 eqs. of sulphur, (and it may contain more, but cannot contain less,) the latest analyses lead to the empyrical formula, $\mathrm{C}_{216} \mathrm{~N}_{27} \mathrm{~S}_{2} \mathrm{H}_{169} \mathrm{O}_{68}$, besides phosphates, though how these are arranged we know not. The albumen of eggs, or white of eggs, has the same formula, with 3 eqs. of sulphur instead of 2 , and 36 eqs. of nitrogen instead of 27 , or $\mathrm{C}_{216} \mathrm{~N}_{36} \mathrm{~S}_{3} \mathrm{H}_{169} \mathrm{O}_{68}$, and phosphates. 
Albumen, in its natural state, is soluble in cold water, and white of egg or serum of blood is simply a strong solution of it, which in the former case is ropy and viscid. Its distinguishing character is coagulability by heat. A heat of $140^{\circ}$ to $160^{\circ}$ continued for a short time, causes it to coagulate, and serum or white of egg becomes a solid mass. Weaker solutions deposit the coagulated albumen in flocculi. Coagulated albumen is no longer soluble in water, but with this exception it is unchanged in its combustible elements, and its coagulation probably depends on the same cause as the conversion of the fluid styrole by heat into solid metastyrole. It is an isomeric transformation. Albumen is further coagulated by acids, by creosote, by corrosive sublimate, by ferrocyanide of potassium, and by infusion of galls. The purest albumen is obtained from white of egg or the serum of blood, by neutralising carefully the alkali they contain with acetic acid, and then adding cold water, which causes the deposition of translucent flocculi, which, when washed with water, have the appearance of flour paste. This, dried at a gentle heat, is albumen, as pure as we know it. But even in this state it contains earthy and alkaline phosphates, without which it never exists in any organic fluid or tissue. If white of egg, mixed with twice its bulk of water, be pressed through a cloth, to break the cells, and a little subacetate of lead added to the liquid, an abundant precipitate is formed. This is to be washed, mixed with water, and decomposed by a current of carbonic acid. The whole is filtered, and to the filtered liquid there is added a few drops of sulphuretted hydrogen, and it is warmed to about $140^{\circ} \mathrm{F}$., not more, when the first flocks of albumen which coagulate carry down with them the whole of the sulphuret of lead. The liquid is now again filtered, and evaporated to dryness at $104^{\circ} \mathrm{F}$., when the dry residue is soluble albumen, said to be free from mineral matter.

To obtain pure coagulated albumen, mix white of egg with its bulk of water, filter and evaporate at $104^{\circ} \mathrm{F}$. to the original bulk. Then add a concentrated solution of caustic potash : the whole soon forms a translucid yellowish elastic mass. This is broken up, exhausted by cold water, avoiding the contact of air. It is then dissolved in boiling water, or in boiling alcohol, and the solution precipitated by acetic or phosphoric acid. The precipitate, if well washed, is pure insoluble albumen, and leaves no ash when burnt.

Soluble albumen thus purified is transparent, amorphous, almost colourless, and tasteless. It leaves, at most, a very trifling ash, which is neutral. It dissolves slowly in water at a very moderate heat, but not entirely, some part becoming insoluble. Its concentrated solution is ropy, like the white of egg. White of egg, dried 
in vacuo, leaves thirteen per cent. of ash, chiefly phosphate of lime.

Pure coagulated or insoluble albumen is, when dry, yellow, transparent like horn, and brittle. The solution of soluble albumen coagulates entirely when heated to $150^{\circ} \mathrm{F}$., or a little more; coagulated albumen is quite insoluble in cold water; boiling water dissolves it in part, but alters it. If kept in a sealed tube for some time in contact with water at $302^{\circ} \mathrm{F}$., it dissolves by degrees to a clear liquid, which has lost the power of coagulating by heat.

Solution of albumen causes the plane of polarisation to deviate to the left.

When it putrifies, albumen yields, among other products, sulphuretted hydrogen or sulphuret of ammonium, as is observed in the putrefaction of eggs. Among the other products are valerianic and butyric acids, a crystalline body of a pungent odour; an oily acid, and a peculiar substance, which dissolves in hydrochloric acid with a fine violet colour, yielding tyrosine with other substances. When heated it burns with the odour of burnt horn or feathers. When heated with dry caustic potash, till hydrogen begins to come off, it is partially oxidised, producing leucine and tyrosine, and in the addition of an acid, yields offensive volatile products not yet examined, the odour of which is stercoraceous. When more fully oxidised by sulphurie acid and peroxide of manganese or bichromate of potash, it yields oil of bitter almonds, benzoic acid, various aldehydes, $\mathrm{Cn}_{n} \mathrm{H}_{n}-1$, $\mathrm{O}, \mathrm{H} \mathrm{O}$; various volatile acids, $\left(\mathrm{Cn} \mathrm{Hn}_{2} \mathrm{H}_{4}\right.$; and various nitryles, such as hydrocyanic acid or formonitryle, $\mathrm{H} \mathrm{Cy}$ or $\mathrm{C}_{2}$ $\mathrm{H} \mathrm{N}$; acctonitryle or cyanide of methyle, $\mathrm{C}_{4} \mathrm{H}_{3} \mathrm{~N}$, or $\mathrm{C}_{2} \mathrm{H}_{3}$, $\mathrm{C}_{2} \mathrm{~N}$; valeronitryle, \&c., \&c. ; besides other products not yet investigated, by the study of all of which we shall in time unravel its very complex constitution.

The emulsine of almonds is a variety of albumen, with which it agrees closely in properties. Its chief peculiarity is that it is capable of causing that peculiar fermentation in amygdaline, by which the oil of bitter almonds is formed. It also acts as a ferment to salicine. In doing this, it, like other ferments, acts in virtue of a state of decomposition, commenced by the oxygen of the atmosphere, but it is the only ferment yet known which acts on amygdaline. The emulsine of sweet almonds is the same as that of bitter almonds. Diastase, the ferment in malt, is also albumen in a state of decomposition.

The emulsive or albuminous matter of mustard seed is another variety of albumen, capable of acting as a ferment on that compound which yields the oil of mustard. 
The composition of albumen is not positively known. We have given the formula which appears best to represent the results of analysis. But it may be mentioned here, that Lieberkühn proposes the formula $\mathrm{C}_{144} \mathrm{H}_{112} \mathrm{~N}_{18} \mathrm{~S}_{2} \mathrm{O}_{44}$; while Hunt thinks that the sulphur in it replaces part of the oxygen in a hypothetical compound $\mathrm{C}_{24} \mathrm{H}_{17} \mathrm{~N}_{3} \mathrm{O}_{8}$ which is supposed to be derived from 2 eqs. of cellulose or of starch, $2\left(\mathrm{C}_{12} \mathrm{H}_{10} \mathrm{O}_{10}\right)$ with 3 eqs. of ammonia, $3 \mathrm{~N} \mathrm{H}_{3}$, by the separation of 12 eqs. of water. Thus, $2\left(\mathrm{C}_{12} \mathrm{H}_{10} \mathrm{O}_{10}\right)+$ $3 \mathrm{NH}_{3}-12 \mathrm{H} \mathrm{O}=\mathrm{C}_{24} \mathrm{H}_{17} \mathrm{~N}_{3} \mathrm{O}_{8}$. If 1 eq. of oxygen be replaced. by sulphur, then we have $\left.\begin{array}{ccc}\mathrm{C}_{24} \mathrm{H}_{17} & \mathrm{~N}_{3} \mathrm{O}_{7} \\ \mathrm{~S}\end{array}\right\}$. This multiplied by 6, would give $\mathrm{C}_{144} \mathrm{H}_{102} \mathrm{~N}_{18} \mathrm{~S}_{6} \mathrm{O}_{42}$, which agrees well enough with the preceding in the carbon, nitrogen, and oxygen, but has too little hydrogen, and too much sulphur by far. On this account Hunt's idea must be regarded as very doubtful, since we can hardly suppose sulphur to replace less than 1 eq. of oxygen in the supposed compound. All chemists agree, however, in giving to albumen a complex formula.

While white of egg is very carefully neutralised by acetic acid, and then much diluted, it deposits, on standing, albumen in a flocculent shape. This precipitate dissolves readily in solution of salt or of nitre, and the solution coagulates with heat. When we add to serum or white of egg a certain amount of a neutral salt, it becomes precipitable by those acids, such as phosphoric, acetic, oxalic, \&c., which had previously no effect. And if to a solution of albumen in acetic acid we add a neutral salt, the albumen becomes more easily coagulable by heat. So that elevation of temperature and increase of saline matter, both contribute to the formation of insoluble albumen, and probably to the construction of tissues in the animal body.

Albumen seems to be a weak acid, and is supposed to be bibasic. Caustic potash, added to white of egg, converts it into a gelatinous mass. This, if washed with water, is soluble in boiling water and boiling alcohol. Its solution is not coagulated by heat, but acids cause an abundant coagulum, soluble in excess of acid. These are the characters of caseine, and it is quite possible that caseine may be this albuminate of potash. If the gelatinous mass be washed with cold water, dissolved in hot alcohol, precipitated by ether, the precipitate dried, powdered, and thoroughly washed with water, we obtain an insoluble albuminate of potash, which contains $5 \cdot 5$ per cent. of potash, that is, 2 eqs. for $\mathrm{C}_{144}$ in the albumen.

Albuminate of soda appears to be the compound present in serum and white of egg. It is now thought probable that it is an acid albuminate, that is, a salt with 1 eq. fixed base, to 1 eq. of a bibasic acid. When boiled, it is resolved into albumen, which 
coagulates and is found to contain no alkali, and neutral albuminate of soda, which remains dissolved. Caustic soda forms with white of egg a gelatinous albuminate, soluble like that of potash, which seems to be the neutral salt just mentioned, with 2 eqs. of soda. White of egg contains 1.6 per cent., the soluble or neutral albuminate contains 3.7 per cent. of soda, and these numbers agree with the calculation from 1 eq. and 2 eqs. of soda for 1 of albumen, $\mathrm{C}_{144} \mathrm{H}_{110} \mathrm{Na}_{2} \mathrm{~N}_{18} \mathrm{~S}_{2} \mathrm{O}_{44}+2$ aq. We have already stated that this formula for albumen is not certain, but yet it is not far from the truth as to the relative proportions. The albuminate of mercury is insoluble as well as that of copper. Hence, white of egg is used as an antidote in cases of poisoning by salts of these metals.

Fibrine. This compound is found in vegetable juices, which deposit it spontaneously on standing; that is, it spontaneously coagulates. It is found also in seeds, especially those of the cereals, and in smaller quantity in other seeds. It occurs dissolved in blood, and is spontaneously coagulated on standing, when it includes the red globules, and forms the clot; but if the blood be stirred when drawn, it is obtained free from the globules, and white. It constitutes also the chief part of muscular fibre. Fibrine is best obtained from blood, by beating with a bundle of rods, to which it adheres in strings. These are placed in a current of water for some time to wash away the blood globules. Those parts which still appear red are picked out, and the residue placed in water to which a few drops of acetic acid have been added. Here it swells up into a translucent mass, in which impurities are easily seen and removed. The clean portion is again washed to remove the acid, when it shrinks to its former bulk, and acquires its fibrous aspect. It still contains fatty matter and water; the fat is removed by drying it and boiling with alcohol and ether. It is also found dissolved in chyle and lymph. It is characterised by its spontaneous coagulation from its natural solutions, after which it is quite insoluble, and resembles coagulated albumen; and the composition of the pure fibrine of flesh and of flour is the same as that of blood albumen as nearly as can be ascertained, although we cannot yet say that they are absolutely isomeric, because the amount of phosphates may differ. The composition of blood fibrine is said to be different in the phosphates. Fibrine from wheat-flour, and fibrine of muscle, both dissolve in weak hydrochlorio acid (1 drop to an ounce of.water), forming a turbid fluid, in which common salt causes coagulation. Fibrine of blood, in the same acid, swells up to a beautiful jelly, but does not dissolve, probably on account of some difference in the phosphates. Coagulated fibrine, whether vegetable or animal, 
when covered with water and left to itself, undergoes a change; a small part of it putrifies, and the rest is dissolved. The liquid, freed from fat by filtration, now contains albumen, coagulable by heat and acids; and this is accounted for by the fact, that the proportion of the organic elements is the same in both.

It must be remembered, that fibrine, as obtained from blood, or flesh, or in any other way, is always in the insoluble or coagulated form; for we cannot preserve it in the soluble form in which it exists in blood, in vegetable juices, and probably also in flesh. It is supposed that the rigidity which occurs in the muscles soon after death, depends on the fibrine of the muscles passing into the insoluble state. At all events, the fibrine of flesh, as soon as we can obtain it, is insoluble; nor can we at all explain why the dissolved fibrine becomes insoluble.

It appears that fibrine is not, or at least not always, homogeneous; for it may be divided into two parts by crystallisable acetic acid, and the microscope shows both fibres and a granular matter, the latter being soluble in the acetic acid, the former only swelling up to a jelly. But as some specimens are entirely dissolved, and others hardly at all, it is probable that these two substances are mixed in variable proportions. They must be nearly alike in composition, for the analysis of fibrine gives numbers almost exactly the same as those of albumen, but varying, within certain limits, less or more.

By the action of neutral salts, aided by a little alkali, fibrine is dissolved, and the solution has the properties of albumen.

Gluten is the name given to the azotised matter of grain, which appears to be a mixture of fibrine with glutine, an azotised substance soluble in alcohol. Gluten is distinguished for the facility with which it passes, as in malt, into the state of a soluble ferment, called diastase, and this again for its energetic action on starch, of which 2000 parts may be converted into glucose by 1 of diastase. But as the same effect is produced by contactwith putrid flesh, yeast, gastric juice, and animal membranes, it is probable that diastase is not a distinct compound, but only soluble fibrine in a state of change.

The products of decomposition of fibrine resemble those of albumen; but it appears that fibrine more readily undergoes that action, when boiled with strong potash, or heated to a certain point with dry potash, by which tyrosine and leucine are formed. The volatile products of oxidation are the same as those of albumen. The products of the putrefaction of fibrine are precisely the same as in the case of albumen.

Leucine, $\mathrm{C}_{12} \mathrm{~N} \mathrm{H}_{13} \mathrm{O}_{4}$, has been already mentioned, as homologous with glycocine and alanine, and has the same relation to valerianic acid as they have to formic and acetic acids, or rather 
the same relation to valeraldehyde as they have to formaldehyde and aldehyde, $\mathrm{C}_{22} \mathrm{~N} \mathrm{H}_{13} \mathrm{O}_{4}=\mathrm{C}_{2} \mathrm{NH}+\mathrm{C}_{10} \mathrm{H}_{10} \mathrm{O}_{2}+2 \mathrm{H} \mathrm{O}$. It crystallises in scales, which are volatile, and when heated with potash yields valerianic acid, carbonic acid, and hydrogen.

$\underbrace{\mathrm{C}_{12} \mathrm{~N} \mathrm{H}_{13} \mathrm{O}_{4}}_{\text {Leucine. }}+3(\mathrm{KO}, \mathrm{HO})=\underbrace{\mathrm{KO}, \mathrm{C}_{10} \mathrm{H}_{9} \mathrm{O}_{3}}_{\text {Valerianate of Potash. }}+\mathrm{N} \mathrm{H}_{3}+2\left(\mathrm{KO}, \mathrm{CO}_{2}\right)+\mathrm{H}_{4}$

Leucine has been detected in the liver of the calf, as a natural product.

Leucine is isomeric with such a compound as would be formed by the combination of 1 eq. cyanic acid, 1 eq. oxide of amyle, and 2 eqs. water, $\mathrm{C}_{12} \mathrm{~N} \mathrm{H}_{13} \mathrm{O}_{4}=\mathrm{C}_{2} \mathrm{NO}+\mathrm{C}_{10} \mathrm{H}_{11} \mathrm{O}+2 \mathrm{H} \mathrm{O}$. But when cyanic acid acts on hydrated oxide of amyle, it yields allophanate of oxide of amyle, $\mathrm{C}_{14} \mathrm{~N}_{2} \mathrm{H}_{14} \mathrm{O}_{6}=\mathrm{C}_{10} \mathrm{H}_{11} \mathrm{O}+$ $\mathrm{C}_{4} \mathrm{~N}_{2} \mathrm{H}_{3} \mathrm{O}_{5}$, which is a crystalline compound quite distinct from leucine. And when cyanate of potash is distilled with sulphamylate of potash, there are formed cyanate of oxide of amyle, and its polymeric cyanurate of oxide of amyle, $\mathrm{C}_{10} \mathrm{H}_{12}$ $0, \mathrm{C}_{2} \mathrm{NO}$, and $3\left(\mathrm{C}_{10} \mathrm{H}_{11} \mathrm{O}\right)+\mathrm{C}_{6} \mathrm{~N}_{3} \mathrm{O}_{3}$; both also distinct from leucine, the former being $\mathrm{C}_{12} \mathrm{NH}_{11} \mathrm{O}_{2}$, and the latter three times that formula. There can be little doubt, therefore, that leucine is really the homologue of glycocine and alanine, and that with hyponitrous acid it will yield the leucic acid, $\mathrm{C}_{22} \mathrm{H}_{22} \mathrm{O}_{e}$, homologous with glycolic and lactie acids; as also that it will form a benzoleucic acid, $\mathrm{C}_{26} \mathrm{H}_{18} \mathrm{O}_{10}=\mathrm{C}_{26} \mathrm{H}_{16} \mathrm{O}_{8}, 2 \mathrm{H} \mathrm{O}$, homologous with benzoglycolic and benzolactic acids. The best method of preparing leucine will be presently described, along with that for tyrosine. Leucine is found among the products of the putrefaction of fibrine, as well as of albumen. Leucine is also isomeric with amylo-urethane, or carbamate of oxide of amyle.

Tyrosine, $\mathrm{C}_{18} \mathrm{~N} \mathrm{H}_{11} \mathrm{O}_{6}$, is formed along with leucine and other compounds not yet investigated, when to fused caustic potash an equal weight of dry fibrine is added, and the mixture, which froths much, is heated till the colour changes from dark brown to yellow. The mass is then dissolved in water, neutralised with acetic acid, and, after filtration, allowed to cool. Tyrosine is deposited in concentric groups of needles. The mother liquid, on further evaporation, yields leucine, which is purified by means of alcohol, in which it dissolves along with potash, which is separated by adding sulphuric acid. The alcoholic solution is evaporated, the residue dissolved in water, and then treated with acetate of lead to remore sulphuric acid, and with sulphuretted hydrogen to remove lead, and the liquid on evaporation yields crystals of leucine. Both leucine and tyrosine are also formed 
when albumen or fibrine is boiled for six or eight hours with 4 or 5 parts of strong hydrochloric acid, or with sulphuric acid diluted with 4 parts of water. The solution is evaporated in the water-bath, the residue dissolved in water, mixed with excess of lime, boiled to expel ammonia, and filtered. The liquid on evaporation yields a syrupy mass with crystals. Alcohol of 86 per cent. dissolves the syrup and leaves the crystals, consisting of leucine and tyrosine, which are separated by their different solubility in water. It is to be observed that acids produce a third crystalline body, resembling leucine and tyrosine, but different from both. These two substances are also formed in the putrefaction of fibrine, \&c., along with a peculiar volatile crystalline body, having a penetrating and putrid smell, but not yet analysed. When leucine putrifies, it yields valerianic acid and ammonia, with other products. The formation of leucine appears to precede that of tyrosine (Bopp). Hinterberger has recently shown that tyrosine is also obtained by the action of potash or sulphuric acid on horn, and in a larger proportion than from fibrine, with less leucine. Horn yields almost 1 per cent. of its weight of pure dry tyrosine. Warren de la Rue has found tyrosine in cochineal, so that it is a nataral product of animal life.

Tyrosine forms long fibrous cystals, sparingly soluble in cold water and alcohol, more soluble in hot water. It is evidently a substance of a very peculiar nature, and must be thoroughly investigated. In composition, tyrosine, $\mathrm{C}_{18} \mathrm{~N} \mathrm{H} \mathrm{H}_{11} \mathrm{O}_{6}$, comes nearest to hippurio acid, $\mathrm{C}_{18} \mathrm{~N} \mathrm{H}_{9} \mathrm{O}_{6}$; but we have, as yet, no experiments to show any practical relation between them. Tyrosine is neutral, but it is capable of uniting with bases. When acted on by diluted nitric acid, it yields a new compound, $\mathrm{C}_{18} \mathrm{~N}_{3} \mathrm{H}_{11} \mathrm{O}_{1}=\mathrm{C}_{18} \mathrm{NH}_{11} \mathrm{O}_{6}+2 \mathrm{NO}_{5}$. It is not, however, a compound of tyrosine and nitric acid, but of nitric acid $1 \mathrm{eq}$.

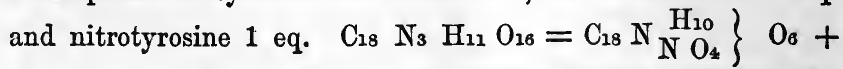
$\mathrm{NO}_{5}, \mathrm{H} \mathrm{O}$. The first group of the last formula, nitrotyrosine, is tyrosine, in which 1 eq. of hydrogen is replaced by 1 eq. of nitrous acid. It has, in a higher degree than tyrosine itself, feeble basic properties, and combines not only with nitric, but with sulphuric and hydrochloric acids. But it also combines with oxide of silver, in the proportion of 2 eqs. of nitrotyrosine to 3 of oxide of silver. Nitrotyrosine forms yellow crystals.

Leucine and tyrosine have here been described as products of the decomposition of fibrine; but it must be remembered, that they are also obtained in the decomposition of albumen, cascine, and horn, by the action of alkalies and of acids, and by 
putrefaction. They are interesting, because the fact that they occur in so many ways, is a proof either that they form two of the proximate constituents of sanguigenous matter, or that they enter into the composition of such proximate constituents, which are not yet known. They are certainly some of the intermediate products of the destruction of animal tissues, between the sanguigenous bodies at one extreme, and the still less complex excreted compounds, such as uric acid, hippuric acid, urea, kreatine, kreatinine, \&c., and are rather too near the latter to be the true proximate constituents of sanguigenous matter.

Caseine. This, the third great form of sanguigenous matter, is, like the two already described, found in vegetables, chiefly in seeds, and in largest proportion in leguminous seeds; hence it is sometimes called legumine. In the animal kingdom it is chiefly found dissolved in milk, as it is in smaller proportion in some vegetable juices. It is distinguished from fibrine and albumen by its not coagulating either spontaneously or by heat, and by forming a skin when its solution is evaporated. Its formula (on the supposition of 2 eqs. of sulphur) is said by Liebig to be $C_{288}$ $\mathrm{N}_{36} \mathrm{~S}_{2} \mathrm{H}_{228} \mathrm{O}_{90}$, with phosphates, and there is no difference between vegetable and animal caseine. Gerhardt and other chemists consider caseine as the albuminate of potash, and insoluble or coagulated caseine as isomeric with albumen. The latter view explains very easily the ready conversion of one into the other in plants or animals; but Liebig's view, as we shall see, may also be so applied as to explain this remarkable fact. Caseine is not coagulated by heat, but is coagulated by dilute acids, which are unable to coagulate albumen. It is easily redissolved by alkalies.

In milk, which is alkaline, caseine is dissolved, along with sugar of milk, salts, and suspended oil or butter. When milk turns sour, its alkali is gradually neutralised and overpowered by lactic acid, produced by the fermentation of sugar of milk, and the caseine at last coagulates from the presence of free acid. It is absolutely certain that caseine, in the animal body, can yield albumen and fibrine, because young animals, fed on milk alone, produce blood and muscle, and imilk contains no other sanguigenous compound than caseine. Cheese is coagulated and pressed caseine, and when made from well-skimmed milk, is nearly pure; but that made from sweet milk or cream contains also much butter. The infusion of the lining membrane of a calf's fourth stomach, or rennet, as it is called, contains albumen or some other substance of a like nature, in a state of decay, that is, of decomposition. It acts on the sugar in milk, converts a part of it into lactic acid, and thus causes coagulation; 
but as curd is formed before the milk has become sour, we must infer either that the caseine coagulates as soon as the milk becomes neutral, or that the ferment or rennet coagulates it by an action of contact. Perhaps both are true. Nay, it has been found that milk, even when made distinctly alkaline, coagulates with rennet if warmed rather more than without the alkaii. Indeed, it would appear that the curd, a coagulated caseine, is a compound of caseine with phosphate of lime. For if an acid be cautiously added, so as just to neutralise the alkali, the milk remains liquid, but coagulates on boiling, and this curd is like that from rennet, insoluble in alkaline solutions, in which pure caseine is soluble. Caseine is the proper ferment for the lactic and butyric fermentation, as fibrine or gluten is for the vinous fermentation. The caseine of peas and beans is obtained by rubbing up the seeds with water, and allowing the starch to settle. We have then a solution of caseine, turbid, and, but for the absence of butter and sugar, hardly to be distinguished from skimmed milk. The Chinese make cheese from peas and beans, and coagulate it by rennet. This cheese, when it decays or putrifies, acquires the peculiar smell and taste which characterise the cheese from milk in the same states. Fresh cheese is tasteless, save for the butter in it; but on kceping, oily acids, such as butyric and valerianic acids, and ammonia, are slowly formed, and hence the strong flavour of old cheese. (See Milk and Cheese.)

The products of the decomposition of caseine are almost exactly the same as those of albumen and fibrine, whether it be decomposed by alkalies, by acids, or by putrefaction; for it yields leucine, tyrosine, benzoic acid, hyduret of benzoyle, various aldehydes and volatile acids, and several nitryles, such as hydrocyanic acid, or formonitryle, acetonitryle, and valeronitryle. Besides all these, there are, as in albumen and fibrine, many others not yet fully studied.

Such is a brief account of the chief sanguigenous compounds, and of the products of their decomposition. Some other substances, occasionally described with these, appear to be simply impure albumen, fibrine, or caseine. Such are Fungine, from mushrooms; Gliadine, the viscid portion of the gluten of wheatflour; and Diastase, the supposed active principle of malt, which converts starch into sugar. It is fibrine in a state of change, and its action on sugar depends on that state, which is induced when grain is moistened and allowed to germinate, as in malting. The substance of horn, feathers, and hair, seems to be a modification of coagulated albumen. It is no longer fit for sanguification perhaps from its insolubility ; but the products of its decomposition are similar to those of sanguigenous matter. Aninal 
mucus much resembles albumen, when dried. It then swells up if moistened, like gum tragacanth. It forms a precipitate with picric acid. Like albumen and fibrine it contains sulphur, and it is a powerful ferment, as is seen in bile and urine, both of which putrify when left in contact with the mucus of the gallbladder or of the urinary bladder, but do not undergo putrefaction or fermentation, if the mucus be removed. Globuline, the colourless azotised matter of the blood globules, also found in the crystalline humour of the eye, is another albuminous compound, very nearly related to albumen. Fibroine is the name given to an albuminous substance found in silk and in sponge. Vitelline is the albuminous matter of the yolk of eggs. Ichthine is that of the yolk of the ova of cartilaginous fishes. Emydine is that of the yolk of the turtle's eggs. Crystallohematine is a crystalline substance, generally red, but colourless when pure, found in the blood of certain animals, especially of rodentia and carnivora. It is an azotised compound, but its exact composition is not yet known. It is very easily altered, and cannot be recrystallised from its solution.

The most interesting fact connected with sanguigenous bodies is, their mutual convertibility. Any one of the three is capable of forming the two others in the body, for animals can live on milk, on eggs or blood, and on flour or muscle, which respectively contain, almost no sanguigenous matter, but caseine, albumen, and fibrine. The apparent isomerism of the fibrine of flesh and flour, with the albumen of blood, is also very important, especially as we can now convert that fibrine into albumen artificially.

When a vegetable juice, containing one or more of these compounds along with sugar, is exposed to the air, oxygen is absorbed, and a change is commenced in the albumen, fibrine, caseine, gluten, \&c., which is soon communicated to the sugar, causing it to undergo the vinous fermentation. The temperature rises, and occasionally the viscous fermentation takes place, producing lactic acid, gum, and mannite. During fermentation, a grey deposit is formed; this is yeast or ferment. When the whole of the azotised principles have not been decomposed or rendered insoluble, the liquid, if excluded from air, remains without further change; but if air be admitted, the alcohol is converted into acetic acid, oxygen being absorbed by the azotised matters, the contact of which causes the alcohol also to absorb oxygen. When the sugar is in excess, only part of it is converted into alcohol, and part of the azotised matter takes the form of insoluble yeast, the rest being decomposed. The saccharine and spirituous liquid undergoes no further alteration. All the above 
statements apply to the juice of the grape, and to the formation of wines and vinegar. It has been already mentioned that grape juice does not ferment if air, filtered through cotton or asbestos, be supplied to it; and that when unfiltered air is admitted, fermentation occurs, accompanied by the development of minute cellular plants or fungi. It would appear that the germ of these obtain access and find their proper pabulum in the juice. Some infusions, as that of meat, however, ferment even when supplied with filtered air. The presence of the germs is not perhaps essential, but it may assist in commencing the change, which, when once begun, continues till either the ferment or the sugar is decomposed.

When the juice, as that of the grape, contains tartario acid, ethers are formed, which give the liquid a peculiar smell and flavour, such as œnanthic ether, which is characteristic of all wines. When a juice contains sugar and caseine, it is most apt to undergo the lactic fermentation, or at least the caseine favours the production of lactic acid from the sugar. But at a high temperature, such as $100^{\circ}$, butyric acid is formed, instead of lactic acid.

Vegetable fibrine, as it is found in wheat-flour, is subject to continual alteration by contact with water; and in this state it has the singular property of converting starch into dextrine, a soluble gum, and then into sugar. This remarkable power is best seen in germinating grain, as in malt, of which a small part mixed with a large quantity of starch in a thick paste, and warmed to $150^{\circ}$ or $160^{\circ}$, very soon renders the whole quite fluid and dissolved, and finally converts it into grape sugar. That part of the fibrine which acts on the starch has become soluble in water. It is called diastase.

Diastase is made by rubbing up malt with a little water, expressing the mixture, adding just enough alcohol to separate the albumen, and to allow the liquid to filter. The filtered liquid mixed with more alcohol, deposits the diastase. It is purified by being repeatedly dissolved in water and precipitated by alcohol. It is finally dried at a temperature of $100^{\circ}$ or $110^{\circ}$. Thus prepared, diastase cannot be a pure compound, but it possesses in a high degree the power of promoting the solution of starch, that is, its conversion into dextrine and sugar. Ono part of diastase can convert into dextrine, with a little sugar, no less than 2000 parts of starch. Diastase is evidently fibrine altered, and still more prone to change. Its solution cannot be kept, it becomes acid, and loses its action on starch.

Malt is made by softening barley in water, and then exposing it to the air in moderately thick layers, at a moderate 
temperature, turning it frequently. In about four days the seeds germinate, if they have not been allowed to become too hot, and if the air has had free access. As soon as the germ has acquired the length of the seed, the operation is checked by drying the seeds in a current of warm air. They now constitute malt. In this operation much carbonic acid is given off, oxygen being no doubt absorbed; the azotised matter in the seeds has undergone a change, and has acquired the properties of diastase; and the starch has in part disappeared, its place being supplied by grape sugar and dextrine.

When the malt is infused in warm water, the metamorphosis of the starch is completed, and the whole dextrine passes into sugar, which dissolves, along with extractive matter and salts. The solution is called must. When sufficiently concentrated, hops and yeast are added, and fermentation being carried on, the result is beer or ale, according to the strength of the must. When the malt has been in part roasted, the beer becomes very darkcoloured, as in the case of porter.

To obtain grain spirit, the meal, either of barley, oats, or rye, or a mixture, is digested in warm water along .with 1 part of malt for 4 of meal, till the mass, at first thick, becomes fluid, a proof that all the starch has been metamorphosed. Yeast is then added, and, after fermentation, the must, or wort as it is called, is distilled and rectified. Potato spirit is obtained in the same way, only using potato starch, instead of barley meal or rye flour.

Many other vegetable matters, and many fruits, may be made to yield spirit, malt or diastase being used in all cases where starch is to be converted into sugar.

Potato spirit is accompanied by the hydrated oxide of amyle, or oil of potato spirit, or amylic alcohol, with some butylic alcohol, and probably other alcohols : grain spirit by an oily matter, consisting chiefly of margaric, capric, and œnanthic acids, probably in part as margaric and cnanthic ether, and of a volatile oil, called by Mulder oleum siticum, that is, oil of grain. Wine spirit, that is, brandy, contains cnanthic ether, and the spirit of molasses, or rum, owes its flavour to butyric ether. Brandy, but especially the coarse brandy made from the expressed grapes, contains besides alcohol, methylic, propylic, butylic, much amylic, and some caproic alcohol. The oils which contaminate potato and grain spirit are offensive, and even injurious to health : they are included by the Germans under the general term Fuseloel, and, as we have seen, are chiefly composed of several alcohols, among which the amylic predominates, an alcohol of a very unpleasant odour. There are also compounds of uxide of ethyle, 
and perhaps some of its homologues, with oily acids, some of which are fragrant, others unpleasant.

Yeast, or Ferment, is the mass which rises to the surface during the vinous fermentation of grape juice, infusion of malt or other similar liquids. If yeast have been added, as is usual in the case of malt, and absolutely necessary when we wish to ferment a solution of pure sugar, then at the end of the process, in those liquids which contain, as grape juice and infusion of malt do, much soluble albuminous matter (fibrine), we find in the scum carried up by the carbonic acid gas, a quantity of yeast much larger than we have added to the liquid.

This yeast is a mixture of starch or dextrine, sugar, insoluble fibrine, and hordeine, a form of cellulose or woody fibre. If kept it continues to decompose, giving off carbonic acid, till at last all the fibrine has been rendered soluble and decomposed, and all the starch, dextrine, or sugar, converted into alcohol, or, if air be freely admitted, into acetic acid. Finally, an insoluble residue is left which is cellulose. It is a disputed point, whether this cellulose was at first mechanically suspended in the juice, infusion, or yeast, or whether it has been produced, possibly as a result of the growth of minute fungi, from dextrine or sugar previously dissolved. It is difficult to decide, as yeast always contains much insoluble matter, and we must add yeast in order to reproduce it. Still it is probable, since we obtain much more yeast from a nearly or quite clear infusion of malt, than we added to it, and of much the same or exactly the same quality, that cellulose is here reproduced from dextrine or sugar.

A given weight of yeast can only convert into alcohol and carbonic acid a limited amount of sugar, because the yeast acts in virtue of its decomposition, and as soon as it is all decomposed, and cellulose alone is left, the action of course ceases.

The ferment of grape juice is called the lees of wine.

Panification. Bread may be made from any flour containing, as all good flour does, vegetable fibrine, sugar, and starch. The flour being made into a paste with warm water, and yeast being added, it is set aside in a warm place. After a time, more flour is kneaded into the mass, which has begun to rise, and the whole is now heated in the oven, or baked. The yeast induces the vinous fermentation in the sugar of the flour, and the alcohol and carbonic acid escaping, raise the bread and render it porous. The starch in general is little changed, but the sugar disappears, as well as a part of the gluten or fibrine. To avoid this loss, bread is now raised by means of carbonate of soda or ammonia and a diluted acid, which are added to the dough, and the effect is perfectly satisfactory. Equally good or better bread is obtained, 
and the quantity of flour which will yield 1500 loaves by fermentation, furnishes 1600 by the new method, the sugar and fibrine being saved. It has been stated that the increased weight is due to water merely, but it is not easy to see why the sugar, retained in the new method, and decomposed in the old, for alcohol may be collected from bread during its formation by the old method, should not add to the weight of the solid residue. It is at all events certain that excellent bread is made without the use of yeast. The addition of a little alum to the dough is useful in arresting that decomposition in the flour which is apt to occur if it have been kept in a moist place. Too much alum cannot be introduced, as it would prevent the fermentation. The best addition to flour which has suffered from moisture, is lime-water, which neutralises the acid formed, and destroys the musty flavour, restoring the sweetness of the flour. It is also quite wholesome, and is even a most desirable addition in the case of children, who consume much lime in their growth.

Mucus, the semi-fluid matter that is found on the surface of the mucous membranes, and also in the urinary and gall bladders, is another form of albuminous matter. Its analysis comes very near to that of albumen, and the precise difference, if any, between them, is not known.

We have already mentioned that sanguigenous bodies can alone yield blood, and that they also form tissues; but they are not, strictly speaking, the only compound of which tissues may be formed. The membranes and vessels, as well as some other parts, are composed of gelatine, and are called the gelatigenous tissues. But gelatine cannot yield blood, and the reason is very obvious, in its composition; for it contains no sulphur. Chondrine, the substance of which cartilage is formed, agrees in this respect with gelatine.

Gelatine is found in the skin, the cellular membranes, and membranes in general. Its distinguishing character is that of dissolving in hot water, and forming a jelly on cooling. The impure gelatine obtained by boiling hoofs and hides is called glue. The purest gelatine is isinglass, from the air-bladder of fishes. But the gelatine of bones, hoofs, and skins may be purified so as to be equal to isinglass. It is often called osseine, and the name of gelatine is now given to the product of boiling it with water. When dry, it is quite transparent, and soluble in water; it is likewise very adhesive. Its formula, according to the latest analysis, is $\mathrm{C}_{82} \mathrm{~N}_{13} \mathrm{H}_{67} \mathrm{O}_{32}$. But the property of gelatinising depends on the presence of phosphates; for when gelatine is long boiled with water alone, or with a little alkali, phosphate of lime is deposited, and the solution no longer forms a 
jelly on cooling. The distinguishing character of gelatine or rather of osseine, is that of being slowly dissolved by boiling with water, and then forming a jelly on cooling. In this change the weight of the osseine, whether from bones or otherwise, is not altered, and its composition remains the same.

Tannine, or tannic acid, converts osseine into leather. But the finer kinds of skins are not tanned, but prepared by means of hydrochlorate of alumina, made by adding common salt to solution of alum. The alumina combines with the osseine, forming an imputrescible substance, in which the structure of the original membrane is not injured. The soft supple kid leather, and what is called Hungary leather, for saddles and harness, which are remarkably supple, are prepared in this way.

In the bones of certain palmipedes, the vertebræ of fishes, and some shells, there is found an insoluble form of osseine, not converted into gelatine by boiling with water.

Chondrine, the gelatine of cartilages, is similar to gelatine, but its composition is best represented by the formula, $\mathrm{C}_{72} \mathrm{~N}_{9} \mathrm{H}_{50} \mathrm{O}_{32}$, and phosphates. It is precipitated, when in solution, by acids, alum, and salts of lead, which do not precipitate gelatine. Both gelatine and chondrine are precipitated by infusion of galls; and leather is a compound of tannic acid with gelatine. The matter of the elastic tissue, such as that of the yellow ligaments of the vertebral column, differs both from osseine and chondrine.

When osseine or gelatine is acted on by potash, it yields, among other products, glycocine or glycocoll, $\mathrm{C}_{4} \mathrm{~N} \mathrm{H}_{5} \mathrm{O}_{4}$, and leucine, homologous with it, $\mathrm{C}_{12} \mathrm{~N} \mathrm{H}_{13} \mathrm{O}_{4}$. With acids, similar results are obtained. When oxidised by means of sulphuric acid and bichromate of potash, gelatine yields many, if not all, of the products derived from sanguigenous bodies, except such as contain sulphur; such as the aldehydes, the volatile acids, and the nitryles, besides benzoic acid and hyduret of benzoyle, and an oil with the odour of oil of cinnamon, not yet analysed, possibly a homologue of the oil of bitter almonds or hyduret of benzoyle, $\mathrm{C}_{16} \mathrm{H}_{8} \mathrm{O}_{2}$, or $\mathrm{C}_{18} \mathrm{H}_{10} \mathrm{O}_{2}$.

It is most important to remember that gelatine is not capable. of forming blood, and hence cannot be the chief constituent of food. If available at all, it can only be to form membranes. As there is no reason why the above formulæ should be doubled or multiplied, and they cannot be halved, because the eqs. of nitrogen are 13 and 9 , it would appear that gelatine and chondrine are both much less complex than albumen, fibrine, or caseine, and in fact products, perhaps among the first products, of their destruction by oxidation. (See the processes going on in the animal body.) They are themselves resolved, by further 
oxidation, into products still less complex. But it is evident that along with gelatine or chondrine, some product or products must be formed, containing the sulphur of the sanguigenous compounds. Such a body actually occurs in the bile.

Bone contains from 37 to 44 per cent. of gelatine with vessels, nerres, \&c. ; the rest are earthy matter, phosphate of lime chiefly. This proportion varies in disease, mollities ossium being attended with a deficiency of earthy matter. In young animals also, there is less than the average proportion of earthy matter, while in old age there is more of the earthy and less of the animal matter in the bones.

The mineral ingredients of bone are, phosphate of lime, phosphate of magnesia, common salt, and at all events in burnt or calcined bones, carbonate of lime. Besides these, bones always contain fluoride of calcium, which has been said to be more abundant, and even to reach 12 or 15 per cent. in fossil bones. If so, the cause probably is, that a great part of the phosphate has been carried away by water containing carbonic acid, in which it dissolves, while the fluoride, being much more insoluble, is left. In recent bones, the amount of fluoride of calcium varies, but does not in all probability ever exceed 3 or 4 per cent., and is often much less. It has been stated that recent bones properly treated may be made to yield as much fluoride as fossil bones; but it is probable, from the known action of water containing carbonic acid on bones, that there is generally less phosphate, and consequently more fluoride, in the latter, than in the former. Its use would seem to be to render the bones tougher, by destroying all tendency in the phosphate to crystallise. For pure phosphate of lime is much disposed to crystallise, and it is often observed that the presence of a small proportion of foreign matter deprives substances of the power of crystallising.

Bone Black or Ivory Black is the black mass left when bones or teeth are calcined in close vessels. It consists of bone earth and charcoal, and the latter, being finely divided, has much decolorising and deodorising power. It is possible that all the varieties of animal charcoal owe some of their properties in part to the phosphates which they all contain in less proportion than bone black, and in part to the presence of some solid compound of carbon and nitrogen, such as paracyanogen. The oily matters obtained in the distillation of bones, known as Dippel's animal oil, are rich in volatile oily bases. Anderson has found in them all the known bases of the aniline and pyridine series, as well as others not fully studied. They also contain a good many of the bases of the methylamine series.

Gerhardt has observed that gelatine dissolves in oil of vitriol, 
and that the solution, if diluted and boiled, yields sulphate of ammonia, and a fermentescible saccharine matter (probably glucose, or a body isomeric with it).

This fact is important, for Hunt considers gelatine to be formed directly from cellulose and ammonia by the separation of water or of its elements. Thus :-

$$
\underbrace{\mathrm{C}_{12} \mathrm{H}_{10} \mathrm{O}_{10}}_{\text {Cellulose. }}+2 \mathrm{~N} \mathrm{H}_{3}=6 \mathrm{HO}+\underbrace{\mathrm{C}_{12} \mathrm{H}_{10} \mathrm{~N}_{2} \mathrm{O}_{4}}_{\text {Gelatine. }} \text {. }
$$

If this be the true formula of gelatine, then it is easy to see how, by taking up 6 or 8 eqs. of water, it may yield glucose and ammonia. The analysis of gelatine, however, does not yield so much nitrogen, by nearly 2 per cent., as Hunt's formula requires. Still these relations must be borne in mind. We have, with Liebig, and also with Hunt, supposed gelatine to contain no sulphur. But some chemists state that it does contain about $0 \cdot 6$ to 0.7 per cent. This may possibly be essential, but may also depend on the presence of albuminous matter, which would also diminish the percentage of nitrogen.

It may here be mentioned that some chemists regard osseine, or gelatine, chondrine, and elastic yellow tissue, to be isomeric, at least so far as the combustible elements are concerned.

\section{THE BILE.}

This important animal fluid is separated from the blood, in which it is always present in small proportion, by the liver, and collected in the gall bladder. Thence it passes into the small intestine, and on its way downwards, is entirely reabsorbed, and expelled from the system, not, as was formerly supposed, in the fres, but in the form of various oxidised products, chiefly carbonic acid, water, ammonia, common salt, and sulphuric acid, by the lungs, the skin, and the kidneys. The colouring matter of the fæces, formerly regarded as bile, is not bile at all, and is moreover, even if it were bile, but a small fraction of the whole hepatic secretion. It may be one of the products derived from bile, but even this is doubtful, and it can represent at most a very small part of that secretion.

Bile is a viscid oily liquid, of a greenish yellow colour, and very bitter taste, followed by a sweetish after-taste. It is entirely miscible with water, and its solution froths like one of soap. In the gall-bladder bile is mixed with mucus. This is got rid of by dissolving the bile in alcohol, filtering the solution from the mucus, and drying it up in the water-bath. 
The dried bile has a dark-green colour in mass, but in powder is much paler. It may be obtained nearly white by decolorising the alcoholic solution with animal charcoal. It still retains, besides the true bile, traces of cholesterine and of fats, which may be removed by ether.

The bile; which, according to some of the best chemists, appeared to be a complex and variable mixture of a multitude of products differing little in properties, has now become, by the admirable researches of Strecker, one of the most striking examples of the insight gained into the most complex organic compounds by the study of the products of their decomposition, and turns out to have a constitution very far from being so complex as was imagined.

It is, in fact, a mixture, in certain proportions which vary in different species of animals, but are permanent in each species, of two salts, of which the base is soda (with small and variable portions of potash and ammonia), and of which the acids are two remarkable products of the destruction of sanguigenous matter, and perhaps, one of them, of gelatigenous matter, by the oxygen of the blood. One of these acids, cholic acid, contains nitrogen; the other, choleic acid, contains nitrogen and sulphur, the latter element being derived from the sanguigenous compounds.

Cholic Acid, $\mathrm{C}_{52} \mathrm{~N} \mathrm{H}_{45} \mathrm{O}_{12}=\mathrm{C}_{52} \mathrm{~N} \mathrm{H}_{44} \mathrm{O}_{11}, \mathrm{H} \mathrm{O}$, is the first of these. It was discovered by Gmelin, but its nature and composition were first ascertained by Strecker. When the solution of dry bile in absolute alcohol is mixed with ether, a deposit is formed, which on standing is converted into a mass of small crystals. These are chiefly cholate of soda, with a little choleate. When decomposed by sulphuric acid, radiated groups of crystals are gradually deposited, which are cholic acid. They are sparingly soluble in water and ether, very soluble in alcohol. Cholic acid is decomposed when heated with mineral acids, and also when heated with potash. Its salts are bitter and sweet, and resemble soaps. They crystallise if in contact with ether.

Paracholic Acid, isomeric with cholic acid, is formed with it, and left behind when the crystalline deposit caused by sulphuric acid is boiled with water, which dissolves the cholic acid. Paracholic acid appears in pearly scales, insoluble in water. It dissolves in alcohol, and on the addition of water is deposited almost entirely in the form of cholic acid; so that each acid is capable of passing into the other. There exists also an amorphous form of cholic acid.

When cholic acid is boiled with potash, it yields two products, namely, a basic compound, glycocine, already described, and a 
new acid, which forms very fine and regular crystals, which are either oblique rhombic tables, tetrahedra, or quadratic octahedra. This is the cholalic acid. This acid, the cholic acid of Demarçay, contains no nitrogen, and its formula is $\mathrm{C}_{48} \mathrm{H}_{40} \mathrm{O}_{10}=\mathrm{C}_{48} \mathrm{H}_{39}$ $\mathrm{O}_{8}, \mathrm{H} O$. Its salts are somewhat like soaps, as itself resembles fatty acids. Like cholic acid, cholalic acid appears in three isomeric modifications. The production of this acid and of glycocine from cholic acid is easily explained.

To cholic acid .
Add 2 eqs. water
$\cdot \frac{\mathrm{C}_{52} \mathrm{~N} \mathrm{H}_{43} \mathrm{O}_{12}}{\mathrm{C}_{52} \mathrm{~N} \mathrm{H}_{45} \mathrm{O}_{14}}$

Deduct glycocine . $\mathrm{C}_{4} \mathrm{NH}_{5} \mathrm{O}_{4}$

Remain . - $\mathrm{C}_{48} \mathrm{H}_{40} \mathrm{O}_{10}=$ Cholalic acid, $\mathrm{C}_{48} \mathrm{H}_{39} \mathrm{O}_{9}, \mathrm{H} \mathrm{O}$.

When cholic acid is boiled with acids, it undergoes the same decomposition. Glycocine is found in combination with the acid used, and there is deposited, not cholalic acid, but choloidic acid, $\mathrm{C}_{48} \mathrm{H}_{39} \mathrm{O}_{9}$, that is cholalic acid, minus 1 eq. of water. This new acid is isomerio with anhydrous cholalic acid, and when it combines with bases, choloidio acid loses no water. Its salts have, therefore, the same composition as those of cholalic acid, but different properties. The choloidic acid is resinous, and cannot crystallise. Its salts are also amorphous. The acid is insoluble in water, soluble in alcohol. It was formerly called resin of bile.

When choloidic acid is further boiled with hydrochloric acid, it loses 3 eqs. more water, and at last leaves an indifferent neutral resinous substance, called Dyslysine, the formula of which is $\mathrm{C}_{48}$ $\mathrm{H}_{36} \mathrm{O}_{6}$.

Such is the history of the first salt in bile, cholate of soda. Its acid is resolved into cholalic acid and glycocine, and the cholalic acid is further converted by acids into choloidic acid and dyslysine. But in the bile these last compounds are not present; they are products of decomposition out of the body. Even cholalic acid is not known to exist in the body ; but as glycocine possibly does, in that case it may also be found.

Choleic acid, $\mathrm{C}_{52} \mathrm{~N} \mathrm{~S}_{2} \mathrm{H}_{45} \mathrm{O}_{14}=\mathrm{C}_{52} \mathrm{~N} \mathrm{~S}_{2} \mathrm{H}_{44} \mathrm{O}_{13}, \mathrm{H} \mathrm{O}$. The second salt of bile, choleate of soda, contains this acid, which, however, it is difficult to obtain in a state of purity. Its salts

- do not so readily crystallise as those of cholic acid, and its composition is known by the products of its decomposition. The acid itself resembles cholic acid in taste and other properties. When boiled with potash, it yields cholalio acid, and a new body, taurine, in which all the sulphur of the acid, as well as its 
nitrogen, are found. When boiled with acids the same change occurs, but the cholalic acid, as before, is changed into choloidic acid, and if the process be continued, into dyslysine.

This at once shows us, that the two acids of bile are coupled compounds, in which the same acid, anhydrous cholalic acid, is coupled with two different compounds; the copula in cholic acid being anhydrous glycocine; in cholic acid, anhydrous taurine. The latter change is thus represented:-

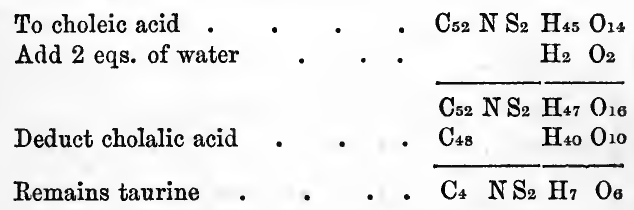

Taurine, $\mathrm{C}_{4} \mathrm{~N} \mathrm{~S}_{2} \mathrm{H}_{7} \mathrm{O}_{6}$, forms large and fine prisms, which are neutral, and have a cooling taste. It is very permanent, and is a very remarkable compound, from the large amount of sulphur, upwards of 24 per cent., it contains. The formula of taurine is such, that it may be made up of the elements of 1 eq. aldehyde, $\mathrm{C}_{4} \mathrm{H}_{4} \mathrm{O}_{2}, 1$ eq. ammonia, $\mathrm{NH}_{3}$, and 2 eqs. sulphurous acid, $\mathrm{S}_{2} \mathrm{O}_{4}$; but Redtenbacher found that sulphurous acid, acting on aldehydammonia, produced not taurine, but a crystalline compound isomeric with it.

We now see that the bile is a mixture of choleate of soda with cholate of soda, and that when decomposed it yields in the first instance cholalic acid from both the acids of bile, glycocine from cholic, and taurine from choleic acid. This actually occurs when the bile putrifies or ferments. It is only when boiled long with acids that choloidic acid and dyslysine appear.

The bile of serpents and of fishes is almost pure choleate of potash and soda, and contains above 6 per cent. of sulphur. The bile of the pig is chiefly formed of the soda salt of a peculiar biliary acid, hyocholic acid, $\mathrm{C}_{54} \mathrm{~N} \mathrm{H}_{43} \mathrm{O}_{10}=\mathrm{C}_{54} \mathrm{~N} \mathrm{H}_{42} \mathrm{O}_{9}, \mathrm{H} \mathrm{O}$, along with a little hyocholeate of soda. Hence the proportion of sulphur in pig's bile is much less than in ox bile. Hyocholic acid contains, like cholic acid, glycocine, coupled with an acid containing no nitrogen. There is a relation between hyocholic acid and the acids of ox bile, for when cholic acid is boiled with water, there is formed an acid, cholonic acid, $\mathrm{C}_{52} \mathrm{~N} \mathrm{H}_{41} \mathrm{O}_{10}$, similar to cholic acid, and this acid is homologous with hyocholic acid, differing from it by $\mathrm{C}_{2} \mathrm{H}_{2}$. Indeed the cholonic acid differs from cholic acid by only 2 eqs of water. The hyocholeic acid of pig's bile, that is, the sulphurised acid, appears to be $\mathrm{C}_{54} \mathrm{~N} \mathrm{~S}_{2}$ $\mathrm{H}_{44} \mathrm{O}_{21}, \mathrm{H} \mathrm{O}$, and to bear the same proportion to choleic acid 
that hyocholic acid does to cholic acid. Both the acids of pig's bile are decomposed by boiling with bases and with acids. With bases hyocholic acid yields glycocine and hyocholalio acid, Cso $\mathrm{H}_{40} \mathrm{O}_{8}=\mathrm{C}_{50} \mathrm{H}_{39} \mathrm{O}_{7}, \mathrm{H} \mathrm{O}$, analogous to cholalic acid. Hyocholeic acid, treated in the same way, yields taurine, and hyocholalio aoid. With acids, they yield taurine and glycocine, while the hyocholalic acid, like cholalic acid, yields a product of a resinous nature, corresponding to choloidic acid, or rather to dyslysine. This is hyodyslysine. It is $\mathrm{C}_{50} \mathrm{H}_{38} \mathrm{O}_{6}$. It has the same relation to dyslysine as hyocholic acid has to cholonic acid, differing from it by $\mathrm{C}_{2} \mathrm{H}_{2}$. The compound corresponding to choloidic acid, which would be $\mathrm{C}_{50} \mathrm{H}_{39} \mathrm{O}_{7}$, is not yet known.

The following table will convey a clearer notion of the biliary products above stated :-

\begin{tabular}{|c|c|c|c|c|c|}
\hline & & 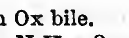 & & & \\
\hline & $=\mathrm{C}$ & & Нyo & & \\
\hline & $\mathrm{C}_{52}$ & $\mathrm{~N} \mathrm{H}_{41} \mathrm{O}_{10}$ & $?$ & $=\mathrm{C}_{54}$ & NI \\
\hline & - & & $\mathrm{Hy}$ & & \\
\hline & $=\mathbf{C}_{48}$ & $\mathrm{H}_{39}$ & & $=\mathrm{C}_{50}$ & \\
\hline & $=\mathrm{C}_{48}$ & & & $=$ Cso & \\
\hline & $\mathrm{C}_{4}$ & 4 & & $\sigma_{4}$ & $\mathrm{O}_{4}$ \\
\hline & $=\mathrm{C}$ & $D_{14}$ & cid & $=\mathrm{C}_{5}$ & 50 \\
\hline & & & & & \\
\hline
\end{tabular}

Of these, however, only the two acids, cholio and choleic acids of ox bile, and hyocholio and hyocholeio acids of pig's bile, are found in the bile, combined with soda, and, in fishes especially, also with potash. The rest are all products of decomposition, and the four acids of $o x$ and pig bile are to be regarded as coupled compounds of anhydrous cholalic and hyocholalic acids with anhydrous glycocine, and of anhydrous cholalic and hyocholalic acids with anhydrous taurine. Hence, when decomposed, each ingredient takes up 1 eq. of water to form the hydrated acid and hydrated glycocine and taurine. Human bile resembles ox bile. Sheep bile is more like that of fishes, rich in choleate of soda, and consequently in sulphur.

The presence of bile may be recognised by two tests: one is the action of nitrio acid on it, which turns it first green, then blue, violet, red, and finally yellow. These changes, which are supposed to depend on the action of the acid as the colouring matter of the bile, enable us to detect the presence of bile in urine, serum of blood or chyle, when from disease it is too abundant. But a more delicate test, which applies to the purified bile, is the fine but transient purple colour produced when we add a little sulphuric acid to a solution containing bile and grape sugar or diabetic sugar. By the addition, therefore, 
of a little pure bile to urine, followed by that of sulphuric acid, we may also employ this test for the detection of diabetic or grape sugar in urine.

Bile, as drawn from the gall-bladder, is strongly coloured, and the colouring matter, which is undoubtedly also a product of the destruction of sanguigenous matter, possibly a secondary product from bile itself, has not been yet fully investigated, from the difficulty of purifying it, since it is not crystallisable. Its composition, when known, will certainly throw much light on the changes connected with the formation or destruction of the bile. Bile may be decolorised by dissolving it in absolute alcohol to separate mucus, and digesting the alcoholic solution with animal charcoal till the colour is removed. The addition of pure ether in successive portions to the colourless solution causes the deposition of an amorphous mass, which, on standing in contact with ether, changes into crystals, formerly called crystallised bile, which are chiefly cholate of soda in the earlier deposits, and choleate in the latter ones, the choleate crystallising less readily, and part of it remaining amorphous. The first deposit contains, therefore, less sulphur than the later ones, and sometimes hardly any, that is, very little choleate, and it is the whole of the deposits, taken together, which represent pure bile, that is, bile free from its colouring matter - a compound that does not occur in the animal body, for there the bile is always strongly coloured.

Cholesterine, $\mathrm{C}_{52} \mathrm{H}_{44} \mathrm{O}_{2}+2$ aq. This is a peculiar crystallisable fatty substance, found in the bile in small quantity. It occurs, however, more abundantly in biliary calculi, usually along with more or less of the colouring matter of the bile, but sometimes nearly pure. It is purified by solution in hot alcohol, which deposits it in brilliant silvery scales. The formula above given is, to a certain extent, empirical, but is chosen because, while it represents the results of analysis, it exhibits a near relation to the biliary acids.

That there is some relation between cholesterine and the acids of bile is evident from the occurrence of cholesterine in the bile, and also from the fact, that both cholesterine and bile, when oxidised by nitric acid, yield a new acid, cholesteric acid, $\mathrm{C}_{16} \mathrm{H}_{8} \mathrm{O}_{8}, 2 \mathrm{HO}$. The products of the action of nitric acid on choloidic acid, which is simply cholalic acid minus water, are very numerous. There are first a whole series of the volatile acids $\left(\mathrm{Cn}_{\mathrm{n}} \mathrm{H}_{n}\right)_{2} \mathrm{O}_{4}$, namely, formic? acetic, propylic ? butyric, valerianic, caproic? caprylic, and capric acids. Then there are found also among the volatile portions an oil, whioh, with bases, yields a new nitro-acid, nitrocholic acid, $\mathrm{C}_{2} \mathrm{~N}_{4} \mathrm{H} \mathrm{O}, \mathrm{HO}$, and 
a neutral pungent liquid, cholacrole, $\left.\mathrm{C}_{16} 4 \mathrm{H}_{4}^{\mathrm{H}_{4}}\right\} \mathrm{O}_{10}$ ? the vapour of which has a stupifying power. Among the non-volatile products there are found two acids, choloidanic acid, $\mathrm{C}_{32} \mathrm{H}_{24} \mathrm{O}_{14}$, which crystallises, and the above-named cholesteric acid, which forms a mass like gum. Cholesterine, when acted on by nitric acid, yields volatile acids, chiefly the acetic, in small proportion, while cholesterio acid is the chief product. When heated with strong sulphuric and phosphorio acid, cholesterine yields to them all its oxygen as water, and a number of carbohydrogens are formed, which are said to be isomeric or polymeric, but which may be perhaps homologous. These are solid, and hence have probably a high atomic weight. The carbon is in greater proportion than the hydrogen.

With sulphuric acid are formed three carbohydrogens, cholestereline $a, b$, and $c$. With phosphoric acid there are obtained two, cholesterone $a$ and $b$. By the analyses of Zwenger, these all appear to be isomeric or polymeric. Cholestereline $a$ is earthy; but may be obtained in small crystals from oil of turpentine, which dissolves it. It melts at $464^{\circ} \mathrm{F} .: \quad b$ crystallises from ether, and melts at $491^{\circ}$; and $c$, which is soluble in ether, is resinous and not crystalline, and fusible at $260^{\circ}$. Cholesterone $a$ forms fine brilliant rectangular prisms, fusible at $154^{\circ} ; b$ yields minute silky prisms, fusible at $338^{\circ}$,

If we admit that these compounds are isomeric, their analysis agrees well with the formula $\mathrm{C}_{53} \mathrm{H}_{42}$. Now this is simply the formula we have adopted for cholesterine, $\mathrm{C}_{52} \mathrm{H}_{44} \mathrm{O}_{2}$, minus $2 \mathrm{HO}$. We may suppose cholesterone $a$, the most fusible, to be $\mathrm{C}_{26} \mathrm{H}_{22}$; cholesterone $b$ and cholestereline $c$ to be $\mathrm{C}_{52} \mathrm{H}_{42}$; and cholesterelines $a$ and $b$ to be $\mathrm{C}_{104} \mathrm{H}_{84}$. All these formulæ are easily deduced, along with water, from a new grouping of the elements of cholesterine. It is, however, possible that some of these compounds may be homologous and not isomeric or polymeric; for two such compounds, for example, as $\mathrm{C}_{52} \mathrm{H}_{42}$ and $\mathrm{C}_{50} \mathrm{H}_{40}$ would not materially differ in their analyses, and would differ but little in fusibility and other properties.

In the preceding paragraphs we have given briefly all that is known concerning the chemistry of the bile with any accuracy; and we gladly strike out a long list of supposed biliary constituents, which are now seen to have been mixtures of those described, and which were the results of imperfect methods. Much remains to be done, but what is above described has been so well ascertained, that the greater part of it appears likely to remain.

It remains to be mentioned that cholesterine is found in large 
proportion in brain and nervous matter. To extract it from brain the ethereal extract of brain is dried up, and boiled with alcohol rendered strongly alkaline by caustic potash. By this means we form cerebrate of potash, oleate of potash, phosphate of potash, glycerine, and cholesterine. On cooling, the cerebrate, the phosphate, and the cholesterine are deposited; and from the deposit cold ether dissolves the cholesterine, which is easily purified by a few crystallisations. It is also present in the blood, and one part of the function of the liver may be to prepare it for the production of nervous tissue.

Biliary calculi are, in man, usually composed of cholesterine and a deep yellow colouring matter, not unlike choloidic acid. In the ox the concretions are generally composed of colouring matter alone. Bezoar stones, which are obviously biliary concretions, and said to be those of antelopes, are chiefly composed of a new acid, lithofellic acid, insoluble in water, soluble in alcohol and crystallisable. Its formula is $\mathrm{C}_{40} \mathrm{H}_{36} \mathrm{O}_{8}=\mathrm{C}_{40} \mathrm{H}_{35} \mathrm{O}_{7}, \mathrm{HO}$ which shows it to be of the same class as choloidic acid or dyslysine. With nitric acid it yields a substitution compound, $\mathrm{C}_{40} \mathrm{NH}_{28} \mathrm{O}_{11}$, according to Wöhler. Probably its true formula will be found

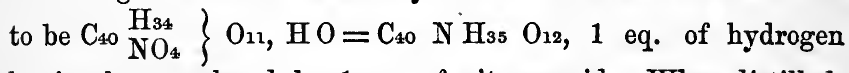
having been replaced by 1 eq. of nitrous acid. When distilled, lithofellic acid loses 2 eqs. of water, and yields pyrolithofellic acid, $\mathrm{C}_{40} \mathrm{H}_{34} \mathrm{O}_{6}$, probably the dyslysine of the antelope bile.

\section{BRAIN AND NERVOUS MATTER.}

The chemistry of brain is so little advanced, that hardly anything can be said of it. It contains nearly 80 per cent. of water, 7 per cent. of albumen, or of a substance like albumen, and several fatty bodies, one of which is cholesterine, the mode of extracting which has just been given. It is said also to contain two peculiar acids, one of which is cerebric acid, which has a composition intermediate between that of a fatty acid and that of albumen, probably a coupled compound. The other is an oily acid, containing, as it is said, a considerable amount of phosphoric acid, united to a neutral oil, cerebroleine. This acid is called oleophosphorio acid. A similar acid occurs in yolk of egg. All this is very vague, but still there can be no doubt that brain does contain either free phosphoric acid or acid phosphates in large quantity compared to other animal products, in which the ashes are neutral or alkaline, whereas the ash of brain is always strongly acid. But there is no proof in favour of the opinion of some that the brain contains phosphorus unoxidised, or in any form but that of phosphorio acid and phosphates. The large pra- 
portion of phosphoric acid leads us to suspect the presence of some peculiar form of albuminous or sanguigenous matter, since all sanguigenous bodies differ in regard to the phosphates they contain. According to Liebig, baryta water extracts from brain two distinct acids, forming soluble salts with baryta, and apparently of a fatty or quasi-fatty nature. These, however, have not yet been investigated, and we do not know with certainty the formula or even the nature of any of the proximate constituents of brain. But we may conjecture that this remarkable tissue, performing the highest functions, those of thought and emotion, must have a constitution still more complex than the other tissues of the body.

We do not yet know where the nervous matter is formed, but we must suppose that it is conveyed to the nerves in the blood. Now the blood does contain fatty matters, among which is cholesterine, one of the constituents of the brain. If this be so, then perhaps the liver assists in producing the cerebral compounds, which, being formed, are conveyed to the blood dissolved or suspended in the bile. The pancreatic juice, which has been said to aid in dissolving fat, may also possibly have a share in the formation of nervous matter. We must look now to the chemical study of the brain for further knowledge on this important subject. From what is known, it would seem as if fat and albumen, two of the chief animal products, unite to form nervous matter, which in construction is more complex : but in composition, that is, in 100 parts, is intermediate between fat and albumen.

MILK.

This important secretion, as has been already mentioned, consists chiefly of water, holding in solution caseine, sugar of milk, and salts, chiefly of potash. In this solution, which is naturally only translucent or semi-opaque, there is suspended an oily matter, butter, which consists chiefly of margarine, or margarate of oxide of glyceryle, oleine or oleate of the same base, and small quantities of the neutral oils, butyrine, caproine, capryline, and caprine, which contain oxide of glyceryle combined with the butyric, caproic, caprylic, and capric acids. To these last oils the flavour of milk is owing. It is remarkable that the volatile vily acids in milk, except the margaric acid, are only such as contain $8,12,16$, and 20 eqs. of carbon. Those with 10, 14, and $18 \mathrm{eqs}$, namely, valerianic, œnanthylic, and pelargonic acids, are never found in milk. Their compounds with oxide of glyceryle are not known, except in the case of valerianic acid; and that compound would hardly do for milk, because it is the cause of 
the unpleasant odour of train-oil in which it occurs in small quantity. Probably the other two are also offensive. According to Heintz, who does not admit margaric acid, the chief fats in butter are stearine, palmitine and oleine. As margaric acid stands between palmitic and stearic acids, the ultimate result is much the same. We have given elsewhere the reasons for admitting the existence of all these acids.

Milk, or any other solution of caseine, when evaporated in the air, forms a pellicle, which is renewed as fast as it is removed. This is insoluble, and yields ashes containing lime and phosphate of lime.

Milk may be analysed by drying it up in vacuo, dissolving the butter by a mixture of ether and alcohol, and the sugar of milk and salts by cold water. The caseine remains in this way undissolved, the salts having been first removed.

When exposed to the air, milk undergoes a peculiar change. The caseine enters into decomposition, and this decomposition passes to the sugar of milk, which yields a little lactic acid, and this causes the caseine not yet decomposed to coagulate. But the decomposition continues : the sugar of milk is at last entirely converted into lactic acid, mannite, and gum; and if the acid be neutralised, and fresh sugar added, it will undergo the same change as long as any caseine remains. This is one method followed for obtaining lactio acid and lactates. The coagulum, separated from the whey when first formed, and pressed out, forms cheese. In making the better kinds of cheese, the milk, instead of being allowed to coagulate spontaneously, is coagulated by contact with water in which part of the lining membrane of a stomach has been infused. This infusion is called rennet, and it acts, as we have already explained under the lactic fermentation, by virtue of containing albumen or gelatine in a state of decomposition, which is at once communicated to the sugar.

When milk, spontaneously coagulated, is exposed to a heat of from $75^{\circ}$ to $85^{\circ}$, without any addition, the sugar of milk passes into grape sugar, and vinous fermentation ensues. The fermented milk distilled, yields a spirit containing traces of butyric ether.

If sugar is made to ferment with caseine at about $100^{\circ}$, carbonic acid and hydrogen gases are disengaged, and butyric acid is formed in large quantity.

The chief mineral substances in milk are potash and phosphate of lime, which are found in its ashes. The ashes also contain sulphates, although milk does not. The sulphurio acid in the ashes is derived from the oxidation of the sulphur of the caseine. We have already mentioned, under caseine, that it is regarded as probable that caseine is the albuminate of potash in a soluble form, 
The proportions of water, caseine, sugar of milk, butter, and salts, are very variable in milk. It generally contains about 86 per cent. of water, 4 to 7 of caseine, 3.5 to 5.5 of butter, and 3 to 5.5 of sugar of milk and salts. For the best method of analysing milk, proposed by Haidlen, I must refer to the "Annalen der Chemie und Pharmacie," xlv., 274. By this method Haidlen obtained as follows :-

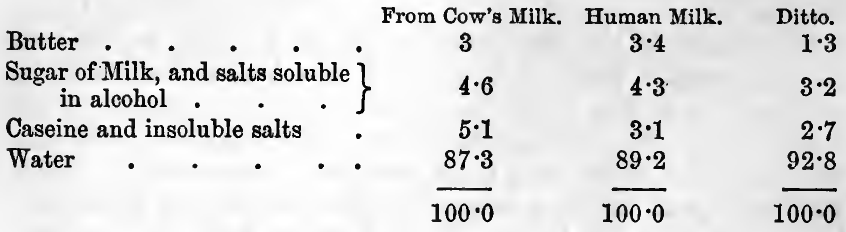

The colostrum, or milk given immediately after parturition, differs from normal milk in containing 15 to 25 per cent. of albumen, with less caseine, butter, and sugar of milk.

The milk of bitches, according to Simon, contains from $14 \cdot 6$ to $17 \cdot 4$ per cent. of caseine, $16 \cdot 2$ to $13 \cdot 3$ of butter, and no sugar.

Cheese is caseine in a state of incipient or progressive decomposition or putrefaction. In the finer kinds of cheese, there is a large proportion of butter, and to the volatile acids of the butter may be ascribed the flavour of cheese. Some kinds of cheese are full of what are called eyes, that is, hollows, caused by the formation of bubbles of gas; and in these hollows, a liquid is sometimes found, containing free ammonia, a product of decomposition, which greatly heightens the flavour, and also, to judge by the flavour, some of the volatile acids, such as butyric acid, probably as salts of ammonia. When the blue mould appears in cheese, it is in a state of rapid decay or eremacausis, and much ammonia is given off. Little is yet known of the chemical differences in the making of different kinds of cheese. The richest are made almost entirely from cream, as Stilton and Parmesan. Others, as Gruyère, Gloucester, Cheshire, and Dutch cheese, are made with fresh uncreamed milk, or mixtures of this with cream; and more or less salt is used, as well as different methods of coagulating, in different places.

It is probable that a careful study of these processes wonld lead to the discovery of the means of giving to cheese any desired flavour, although it is commonly believed that much more depends on the nature of the pasture. This, no doubt, has some influence ; for in Gruyère cheese we often perceive a garlic flavour, derived from wild garlic on the hills. But since the chief causes 
of the flavour are products of decomposition, we may infer that the method of manufacture will have a great influence in this respect.

\section{GASTRIC JUICE.}

This juice, as extracted from the stomach of executed criminals, is colourless or slightly yellow, turbid, and distinctly acid. It contains free acids along with chlorides of potassium and sodium.

The nature of the free acid present in the gastric juice has been disputed. When it is distilled, free hydrochloric acid is obtained, and this is often, perhaps always, accompanied by butyric acid. But it must be remembered that these acids are volatile, and that therefore their presence in the distilled liquid affords no proof of their existence in the free state in the gastric juice. On the other hand, Lehmann obtained from the gastric juice, by a peculiar process, a salt of magnesia, which he analysed, and which liebig has shown to be lactate of magnesia. There can be no doubt of the presence of phosphoric acid, free or combined; and it is most probable that in the normal juice, the fixed acids, phosphoric and lactic, are, in part at least, free, while the volatile acids, hydrochlorio and butyric, are present in the form of salts. In the distillation, the latter are expelled in the free state, the fixed acids taking their place. This view is confirmed by the phenomena exhibited by the juice of flesh (see Liebig's Researches on the Chemistry of Food, and the next section of this work), which undoubtedly contains free lactio and phosphoric acids, (or, what is the same thing, acid phosphates and acid lactates,) along with chlorides, and appears to have a very close resemblance to the gastric juice.

The property of dissolving or digesting food such as albumen, fibrine, caseine, \&c., is owing in part to the presence of free acid, and in part to the presence of a portion of the lining membrane of the stomach dissolved, and in a state of change. The gastrio juice converts into chyme or digests, albumen, fibrine, \&c., out of the body as well as in it, if the temperature of the stomach be kept up ; and water acidulated with a trace of hydrochloric acid, and afterwards left for 24 hours in contact with the lining membrane of the stomach, acquires in a very high degree the solvent power of the gastric juice. Water thus prepared dissolves in 8 to 12 hours, at the temperature of from $86^{\circ}$ to $104^{\circ}$, hardboiled white of egg, \&c., which requires 4 days at a temperature of $158^{\circ}$ to $176^{\circ}$ to be dissolved by water merely acidulated with the same proportion of acid, but not placed in contact with the stomach. This latter fluid, however, dissolves meat better than it does albumen, because the meat supplies some membranous 
matter in a state of change, by which the solution of the fibrine is much promoted.

All attempts to isolate the supposed principle-pepsine, as it was called, which is supposed by some to be the solvent of food in the stomach-have failed. The gastric juice has only yielded traces of animal matter, and we have not yet any proof that its solvent action depends on a peculiar compound, and is not rather the effect of a kind of fermentation induced in the food by contact with the particles of the dissolved epithelium, themselves in a state of change, and consequently of motion. On the whole, then, taking into account the facts of artificial digestion, it appears most probable that digestion is a process analogous to fermentation in the conditions under which it takes place, namely, a certain temperature, and contact with azotised matter in a state of decomposition; but differing from the usual forms of fermentation in its phenomena, no gas being disengaged, and its chief result being the solution of an originally insoluble matter, similar to the conversion of starch into sugar, by contact with diastase or infusion of malt. But the gastric juice cannot effect the conversion of starch into sugar, and therefore digests only sanguigenous matter.

\section{JUICE OF FLESH.}

The muscular mass of animal bodies is chiefly composed of muscular fibre (fibrine), coloured by blood, contained in innumerable small vessels, of those vessels, of nerves, of tendinous matter, and of cellular tissue. More than $\frac{3}{4}$ ths of its weight is water, which, holding in solution a great variety of substances, penetrates every part of the mass, and constitutes the juice of flesh, as it is termed. This fluid is contained either in peculiar minute vessels or in the cells of the cellular tissue, and when the flesh is finely chopped and pressed, the juice is obtained more or less mixed with blood. The best method is to mix the chopped flesh with water, to squeeze out the mass, and to repeat this process if necessary. In this way, the juice of flesh is obtained in a somewhat diluted state, and, as already stated, not free from blood.

This fluid has recently been studied by Liebig (Researches on the Chemistry of Food ${ }^{*}$ ) with very interesting results. It is uniformly neutral or acid, more frequently acid, and when neutral immediately after death, it soon becomes acid; and the acids present are lactic acid, in large quantity, phosphoric acid, a new azotised acid, inosinic acid, and in smaller quantity some

* London : Taylor and Walton. 1847. 
other organic acid, not yet examined. No sulphuric acid is present, save a trace from the blood. The bases are potash in large proportions, both as phosphate, lactate, inosinate, \&c., and as chloride of potassium ; soda in much smaller quantity, chiefly as chloride of sodium, (and probably derived from the blood, at least for the most part); magnesia; lime; the latter in much smaller proportion; and kreatine. The juice contains besides, albumen dissolved, which is coagulated by heat, and amounts to from 2 to 3 per cent. of the weight of the flesh. The insoluble residue of fibrine, \&c., amounts when dry to 17 or 18 per cent., so that the average composition of flesh will be nearly as follows :-

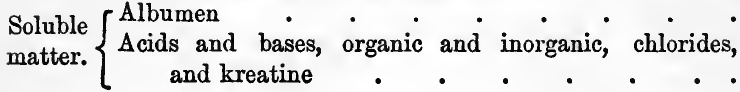

Water $. \quad . \quad . \quad . \quad . \quad . \quad . \quad . \quad .78$

Fibrine, vessels, nerves, cells, \&c. $\quad$ - . . . 17

and kreatine . . . . . . .

It is obvious that in order to study with success the soluble matters not coagulated by heat, we must operate on large quantities of flesh, since, for example, $10 \mathrm{lb}$. of flesh will not yield more than $4 \mathrm{oz}$. of these substances, among which are five or six acids, and as many bases, besides chlorine and kreatine. Thus, from $10 \mathrm{lb}$. of beef, only about 50 grains of kreatine can be obtained.

Kreatine. This substance was first noticed by Chevreul in the soup or extract of meat, but as he obtained it in very small quantity, and other chemists did not succeed in procuring it, its composition was unknown, and its existence was even doubted, as an ingredient of Hesh, Berzelius having suggested that it might be an accidental product of decomposition. Liebig showed that it is always present in all kinds of flesh, at least in all those tried by him, namely, in the flesh of the horse, ox, ox-heart, sheep, pig, calf, roe-deer, hare, marten, red-deer, fox, fowl, and pike. Schlossberger had shortly before obtained a trace of it from the flesh of an alligator, although too little for analysis, and I have since found it in the flesh of the turkey, pigeon, skate, cod, haddock, salmon, herring, and turbot. From a large amount of the flesh of the dog-fish I obtained a mere trace of it, but it has since been found in the flesh of cetacea. Liebig observed that when the juice containing it is evaporated, the kreatine is destroyed or rendered uncrystallisable by the free acid. By neutralising, therefore, the juice, obtained as above mentioned, (after heating it so as to coagulate the albumen, and 
separating the coagulated albumen by filtration), with baryta water, and evaporating to the consistence of syrup at $130^{\circ}$ or $140^{\circ}$, he obtained the kreatine in quantity amply sufficient to allow him to study its composition and characters. The following table contains his results and my own, in regard to the proportion of kreatine obtained from several kinds of flesh. It will be seen that in all it is small, varying from 0.607 to 3.21 per 1000.

Liebig. Gregory.

1000 parts of the flesh of fowl yielded of kreatine $\quad 3 \cdot 20\left\{\begin{array}{l}3 \cdot 21 \\ 2 \cdot 90\end{array}\right.$

" $\quad$ ox-heart $, \quad, \quad-\left\{\begin{array}{l}1.375 \\ 1.418\end{array}\right.$

1000 parts of the flesh of cod yielded of kreatine,$-\left\{\begin{array}{l}0 \cdot 935 \\ 1 \cdot 710 \\ 2 \cdot 105 \\ 2 \cdot 516 \\ 3 \cdot 012\end{array}\right.$

\begin{tabular}{|c|c|c|c|c|c|c|}
\hline "' & $"$ & pigeon & $"$ & $"$ & - & 0.825 \\
\hline 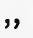 & , & horse & " & ", & 0.72 & - \\
\hline$"$ & ", & $0 x$ & ", & " & $0 \cdot 697$ & - \\
\hline$"$ & $"$ & kate & $"$ & $"$ & - & 0.607 \\
\hline$"$ & " & haddock & " & $"$ & - & 1.504 \\
\hline$"$ & $"$ & Ealmon & $"$ & " & 一 & 1.321 \\
\hline & $"$ & herring & ", & $"$ & -\{ & $\left\{\begin{array}{l}1.45 \\
1.86\end{array}\right.$ \\
\hline & ", & turbot & ", & ", & 一 & 1.51 \\
\hline
\end{tabular}

Fowl yields the largest proportion, but kreatine is obtained much more cheaply from cod. I find that the chopped cod, well mixed with little more than its own weight of water, and pressed out, yields a fluid, which, when neutralised (after the coagulation of the albumen) by baryta, filtered to separate the phosphate of baryta, and gently evaporated till, on cooling, it forms a thin jelly, deposits, on standing, kreatine in large crystals nearly pure. In one experiment, $25 \mathrm{lb}$. of cod yielded 164 grains; in another, $30 \mathrm{lb}$. yielded 356 grains. In recent experiments, I have obtained from cod 2 to $2 \cdot 5$, and once even 3 per 1000 , so that cod may yield about as much as fowl, and from its cheapness, and the facility of extracting it, is by far the best source of kreatine. The variations in the amount of kreatine in cod, may depend on the season or on the age of the fish. The crystals, by one recrystallisation from 6 or 7 parts of boiling water, are rendered quite pure. The kreatine obtained from haddock was less pure than that from skate or cod, and was evidently mixed with a less soluble matter, the nature of which I have not had time as yet to ascertain.

Kreatine forms brilliant hard prismatic crystals, efflorescing at $212^{\circ}$, very soluble in hot water, less so in cold water, sparingly 
soluble in alcohol. It is neutral, and its formula is $\mathrm{C}_{8} \mathrm{~N}_{3} \mathrm{H}_{11} \mathrm{O}_{6}$ $=\mathrm{C}_{8} \mathrm{~N}_{3} \mathrm{H}_{9} \mathrm{O}_{4}+2 \mathrm{H} \mathrm{O}$.

By the action of acids, kreatine is resolved into the new base kreatinine, and water; and by that of bases into the new base sarcosine, and urea, as already explained.

Kreatine has been discovered, along with kreatinine, in urine. Pettenkofer obtained, by the addition of chloride of zine to a concentrated extract of urine, a crystalline precipitate, containing chloride of zinc and an organio matter, the composition of which he found to be $\mathrm{C}_{8} \mathrm{~N}_{3} \mathrm{H}_{8} \mathrm{O}_{3}$. Liebig has proved that this substance is resolved by the action of alcohol into kreatine, $\mathrm{C}_{8} \mathrm{~N}_{3} \mathrm{H}_{21} \mathrm{O}_{6}$, and kreatinine, $\mathrm{C}_{8} \mathrm{~N}_{3} \mathrm{H}_{7} \mathrm{O}_{2}$; and that it was, in fact, as analysed by Pettenkofer, a mixture of those substances, in the proportion of $3 \mathrm{eqs}$. of the latter to 1 of the former. The original crystals of Pettenkofer were a mixture of free kreatine, with a compound of chloride of zinc with kreatinine.

The addition of chloride of zinc to the mother liquor which has deposited the kreatine from the juice of flesh, yields the compound of Pettenkofer, so that kreatinine exists ready formed in the juice of flesh. It is probable that kreatinine is the substance really present in urine, and that when separated it takes up water, and is in part reconverted into kreatine.

Lactic Acid. This acid, as an ingredient of the juice of flesh, is very interesting; and it is remarkable that Liebig, who demonstrated its absence in normal urine (in which, according to Cap and Heury, it exists in combination with urea), should have been the person who detected it in flesh. It is present in large quantity, and occurs as abundantly in the flesh of carnivorous as in that of herbivorous animals. Its origin and formation in the former is not yet explained, and is a problem of much interest. The presence of lactic acid in the juice of flesh is also important, as readily accounting for its presence in the gastric juice, which, as we have seen, is also acid. The lactic acid in the juice of flesh is obviously consumed in respiration, as will be explained hereafter.

Inosinic acid. This acid has been little studied. Its formula is $\mathrm{C}_{10} \mathrm{~N}_{2} \mathrm{H}_{6} \mathrm{O}_{10}, \mathrm{H} \mathrm{O}$, and it forms, with baryta, a salt which crystallises in silvery scales. I have only been able to obtain it from the flesh of fowl and turkey, and it is probably absent in the other kinds of flesh.

Phosphoric Acid. This acid exists in large proportion in the juice of flesh, in the form of acid tribasic phosphate of potash, $\mathrm{POs}\left\{\begin{array}{l}\mathrm{K} O \\ 2 \mathrm{HO}\end{array}\right\}$, a salt which is always produced when phosphoric acid is made to act on chloride of potassium or salts of 
potash. This salt is strongly acid, and chiefly contributes to the acidity of the juice. It is most important and worthy of remark, that the blood, which is separated from the juice of flesh only by the finest membranes, cell-walls, or parietes of vessels, is invariably alkaline, containing the common phosphate of soda, $\mathrm{PO}_{5}\left\{\begin{array}{l}2 \mathrm{NaO} \\ \mathrm{H} \mathrm{O}\end{array}\right\}$, a salt which is always formed when phosphorio acid acts on chloride of sodium or salts of soda, and which is strongly alkaline.

Here we observe, first, that while soda is essential to blood, potash is equally essential to the juice of flesh ; secondly, that in these two fluids, soda and potash cannot, as in so many other cases they can, replace each other; thirdly, that by this arrangement, an acid and an alkaline fluid are constantly in close vicinity to each other, separated only by membrane, at all parts of the body; fourthly, that being at the same time in contact with muscle and nerve, the conditions of electric ourrents are present; while such currents, as is known, have recently been proved by Matteucci to exist. Lastly, that animals cannot form blood or bile, unless their food contain, along with phosphates, salts of soda, or at least, chloride of sodium, so that when they are fed on the land-plants of certain soils, in which sodium is nearly absent, common salt must be given to them.

The function of the phosphate of magnesia, which occurs in large quantity in the juice of flesh, is not yet even conjectured ; but it cannot be doubted that all the substances present, organio as well as inorganic, have each a special part to perform.

\section{SALIVA.}

The chief use of the saliva is to assist in digestion, whether by itself containing animal matter in a state of change, or by its remarkable power of inclosing and retaining bubbles of air, the oxygen of which commences the change necessary to digestion, on coming in contact with the food or the stomach and gastric juice. To serve this purpose, the saliva has a very great degree of viscidity, so that it froths up easily, and the froth does not fall readily. It is alkaline, and contains hardly more than 1 or 2 per cent. of solid matter, partly mucus, partly the usual salts, partly a peculiar soluble matter, ptyaline, which acts as a ferment, and readily converts starch into sugar. As it cannot dissolve albuminous matter, while the gastrio juice cannot convert starch into sugar, the saliva would seem to be intended for the latter purpose. The salts of the saliva are the chlorides of potassium, sodium, and calcium, some potash, and soda, with 
a large proportion of bone-earth. It appears also to contain a trace of sulphocyanide of potassium: at least it reddens with persalts of iron; and although acetates do this, there is some reason to ascribe the effect here to sulphocyanides.

The pancreatic juice resembles saliva, but appears to be slightly acid, and contains 8 or 9 per cent. of solid matter, including ptyaline and a matter like caseine. This juice is added to the chyme in its passage through the duodenum, along with the bile and intestinal mucus. Bernard has shown that the pancreatic juice has the property of decomposing fats, and setting free the acids, and that by its means fat is rendered capable of entering the absorbent vessels. The latter statement is doubtful.

\section{EXCREMENTS.- URINE.}

The chyme, after receiving the pancreatic juice, the bile, and mucus, passes along the intestine, where the absorbents or lacteals take up the fluid part, leaving the insoluble portions. The chyle or absorbed fluid is partly conveyed into the abdominal veins, and partly made to pass through numerous glands (in which process it loses its acid reaction, becoming alkaline), from which it proceeds to the thoracic duct, and is then, with the lymph, poured into the vena cava to mix with the renous blood.

In the mean time, the insoluble parts of the chyme are rejected, and accumulate in the large intestines, various gases being disengaged, such as carbonic acid, hydrogen, carburetted hydrogen, nitrogen, and sulphuretted hydrogen.

The solid excrements of man contain very little matter soluble in water, and consist of woody fibre, with fatty, resinous, and waxy substances, and finally the insoluble salts of the food, namely, phosphates of lime and magnesia, with traces of soluble salts, and some silica.

The urine of man contains urea and uric acid, also hippuric acid, kreatine, and kreatinine, and other organic oompounds, very imperfectly known. Its acid reaction, according to Liebig, depends on the fact that phosphate of soda dissolves uric and hippuric acids, forming an acid solution. If 40 grains of dry phosphate of soda $\mathrm{P} \mathrm{O}_{5}\left\{\begin{array}{l}2 \mathrm{Na} O \\ \mathrm{H} \mathrm{O}\end{array}\right\}, 15$ grains of uric acid, and 15 grains of hippuric acid, be dissolved in $1 \mathrm{lb}$. of hot water, a solution is formed which on cooling deposits $7 \frac{1}{2}$ grains of uric acid, and the remaining liquid forms an artificial urine, which, like the natural, is acid. Urine contains also phosphoric acid, magnesia, often ammonia, soda, phosphate of soda, common salt, 
sulphuric acid, or sulphate of soda, in short, the soluble salts of the food, along with sulphuric acid formed by the oxidation of the sulphur of the tissues. The addition of ammonia to urine causes a precipitate of phosphate of lime.

Fresh urine, filtered (to separate mucus) into a perfectly clean vessel, keeps unchanged for weeks or even months; but if in contact with decomposing animal matter, the urea is speedily transformed by putrefaction into carbonate of ammonia, while phosphate of lime is precipitated, the urine becoming strongly alkaline.

The urine of the herbivora contains much uric acid, also hippuric acid: that of the carnivora contains more urea, and is strongly acid : uric acid predominates very greatly in the urine of birds, and that of reptiles is nearly pure acid urate of ammonia.

When benzoic acid is administered internally it appears in the urine as hippuric acid, which latter acid is generally present in small quantity in urine. It has also been found that nitrobenzoic acid is changed in the body into nitrohippuric acid, a new acid, in which 1 eq. of hydrogen in hippuric acid is replaced by nitrous acid. The acid reaction of human urine is not owing to lactic acid, as was formerly supposed, but to free urio acid dissolved by the phosphate of soda. The urine is sometimes neutral, and always becomes alkaline, when the food contains salts of potash or soda with organic acids, because, the acids being oxidised in the body, yield carbonates of the alkalies. It is evident, therefore, that by attention to diet, the urine may be brought into any desired condition, as will be more fully explained hereafter.

When water, or a very weak saline solution, is taken into the stomach in successive large quantities, it is rapidly absorbed by. endosmosis, and the urine becomes more and more diluted, till at last it resembles the water that has been taken. But if a solution containing as much saline matter as the blood, or a little more, is taken into the stomach, it is not absorbed, and the stomach becomes so loaded that the experiment cannot be continued. If, again, the solution be much stronger, the water of the blood passes outwards by exosmosis, and produces diarrhœe. This explains the purgative action of neutral salt, which is well exemplified by sprinkling salt on a portion of fresh membrane such as bladder, or on meat, both of which yield water and are soon swimming in brine.

The salts of the urine and of the excrements, being derived directly from the food, vary according to its nature, the soluble inorganic salts of the food being found in the urine, the insoluble salts in the excrements. Thus, the ashes of the food of the 
carnivora contain no carbonates, but are rich in phosphates, and such also is the case with the salts (or ashes) of their excreta, liquid or solid. In fact, if we know the nature and composition of the ashes of the food, we can tell at once the salts of the urine. In an adult animal, the quantity of salts excreted is precisely equal to that contained in the ingesta, and therefore, by altering the food, we can alter at pleasure the nature of the salts in the urine.

As an example, we may here adduce the case of the horse, which animal consumes, in his food, a certain quantity of mineral substances, derived ultimately from the soil :-

THE HORSE.

\begin{tabular}{|c|c|c|c|}
\hline \multicolumn{2}{|c|}{ Consumes of Mineral Substances. } & \multicolumn{2}{|c|}{ Excretes of Mineral Substances. } \\
\hline $\begin{array}{l}\text { In } 15 \mathrm{lb} \text {. of hay, } \\
\text { In } 4.54 \mathrm{lb} \text {. of oats, } \\
\text { In water }\end{array}$ & $\left.\begin{array}{r}\text { oz. } \\
18 \cdot 61 \\
2 \cdot 46 \\
0 \cdot 42\end{array}\right\} 21 \cdot 49$ & $\begin{array}{l}\text { In the urine } \\
\text { In the fæces }\end{array}$ & 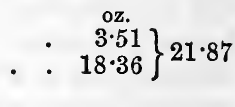 \\
\hline
\end{tabular}

The above result is one obtained by actual and very careful experiment, and the nature of the salts is found to be the same, as indeed must obviously be the case, as long as the animal does not change its wejght. A growing animal will retain the phosphates in part to aid in forming bone, and an old or wasting animal will give out more salts than are taken in.

It is obvious that analyses of urine or excrement are unnecessary if we can examine the food; and that in general they must be useless, since we can never expect the same result twice, unless where the food is not varied.

The excrements, according to Liebig, represent the incombustible and unburned, or partially burned ingredients of the food, and, if we view the body as a furnace, in which the animal heat is produced by the oxidation or combustion of the food, they correspond to the soot and ashes of a common fire. That they contain partially oxidised matter is proved by the fact, recently observed by Liebig, that the albuminous compounds, when partially oxidised by fusion with potash, yield, on the subsequent addition of an acid, volatile matters possessing, according to the albuminous substance employed, the peculiar odours which characterise the fæces.

It is plain from this, that the offensive odour of excrements depends on the presence of intermediate products of oxidation, which, when duly examined, will throw much light on the nature of the sanguigenous compounds which yield them. Some analogous 
compounds exist in urine, and give to it its peculiar, and in some animals, very offensive smell. According to Städeler, the urine of the cow contains an offensive product, which is resolved into a peculiar nitrogenised product, and one or two peculiar acids. It contains, besides, salts of three acids. The acids which he observed in the volatile products are, first, taurylic acid, $\mathrm{C}_{14}$ $\mathrm{H}_{8} \mathrm{O}_{2}$, isomerio with anisole, and perhaps identical with creosote. In properties it resembles carbolic acid, and is probably its true homologue, anisole being a neutral body. Secondly, carbolic acid itself, well known as a product of destructive distillation, $\mathrm{C}_{12} \mathrm{H}_{6} \mathrm{O}_{2}$. These two acids, taurylic and carbolic, are very similar. Thirdly, damaluric acid, $\mathrm{C}_{14} \mathrm{H}_{12} \mathrm{O}_{4}=\mathrm{C}_{14} \mathrm{H}_{11} \mathrm{O}_{3}, \mathrm{HO}$, which is a volatile acid, with a smell analogous to that of valerianic acid. It has the same relation to cenanthylio acid, $\mathrm{C}_{14} \mathrm{H}_{14} \mathrm{O}_{4}$, as angelicic acid has to valerianic acid, and acrylio acid to propylic acid. Fourthly, damolic acid, also volatile, and probably $\mathrm{C}_{26} \mathrm{H}_{24} \mathrm{O}_{4}$, that is, standing in the same relation to cocinic acid, $\mathrm{C}_{26} \mathrm{H}_{26} \mathrm{O}_{4}$, as damalurio acid does to œnanthylic acid. There are several other products, not yet examined; but the above are clearly products of incomplete oxidation, from their resemblance to products of destructive distillation, which is only a process of incomplete oxidation. And it is a very singular confirmation of Liebig's views, that urine should be found to contain carbolic acid, one of the ingredients of smoke, an acid isomeric with creosote, another constituent of smoke, and others of the same class. There can be little doubt that the fetid products in the fæces will contain the same substances as those here described from urine, or others homologous with them, and corresponding with the bodies found in smoke and tar. This would prove the comparison of the process going on in the body to combustion in a furnace with a limited supply of air to be more than an illustration, and to be a real truth.

Guano, so highly prized as a manure, is the decajed excrement of sea fowls, which was originally, like that of reptiles, and indeed also of birds in general, mixed urine and fæces, the urine being solid or semisolid, and consisting of urate of ammonia. It varies much in the proportions of its ingredients, both because the original excrement must have varied according to the food of the birds in different places, and also because some specimens have not been so long exposed to air and moisture as others, and some are almost fresh. Thus some guano contains upwards of 30 per cent. of uric acid, while in other specimens hardly a trace of that acid is left. The better qualities of guano contain much ammonia, partly free or as carbonate, as proved by its odour, partly combined, as sal ammoniac, oxalate, urate, and phosphate of 
ammonia and magnesia. They also contain phosphate of soda and much phosphate of lime, the latter being derived from the bones of the fish on which the birds fed. There are also found sulphate of potash and soda, and oxalate of lime, in guano. The remainder is water, and a brown matter like humus.

It is easy to see that guano must act chiefly as a source of ammonia and of earthy and alkaline phosphates, so valuable to growing plants, especially to those cultivated for food; and that its value depends very much on the amount of phosphates it contains. But although the value of guano is unquestionable, let us not overlook the fact, that while we are ransacking the most remote islands for guano, that substance supplies us with nothing but the mineral salts and the ammonia which have formed crops of vegetables and races of animals at some former period, and that it differs in no essential point from the fresh or modern excreta of man and animals nearer home, which excreta, at least those of man, the most valuable of all, we allow to be carried into the sea in quantities which may be measured by the food we consume. In fact we take out of the sea, in the shape of guano, only part of what we throw into it in the contents of our common sewers. These valuable matters, instead of being carefully collected and preserved, as in China, are sent to form the food of sea plants: on these plants animals feed, which animals serve as food to fish. The fish are consumed by sea fowl, and we recover in their excrement a small part of what we are constantly throwing away. Another part of what we lose we recover in this country, at a great expense, in the shape of bone-earth, which, however, must be taken from other countries. We shall return to this subject: meanwhile, let us express a hope that Europe will at length follow generally, as in some districts it has done, the rational example set by the eminently practical Chinese, of restoring to the soil, as nearly as possible, in the shape of excreta, what we take from it in our crops and cattle, and thus keeping up its fertility.

\section{URINARY CALCULI.}

These are of various kinds, according to the peculiar condition of the urine.

Uric Acid Calculus is the most frequent, being the usual deposit when the urine is acid. Its origin, as a calculus or deposit, that is, in abnormal quantity, is owing to deficient aeration, much oxygen being required to resolve it into soluble compounds, such as urea, carbonate of ammonia, or even oxalic acid. Hence sedentary habits, highly carbonised food, and indulgence in 
strong wine, all favour its production: the first by diminishing the supply of oxygen, the two latter causes by seizing on the oxygen to the exclusion of the uric acid. It is easily recognised by the action of potash, which dissolves it, and forms a solution from which acids precipitate uric acid: or by nitric acid, which dissolves it with effervescence, and yields, on evaporation of the solution, a deep red stain, becoming purple with potash. Uric acid calculus is commonly tinged more or less red or brown. When pure it is entirely dissipated before the blowpipe.

Urate of Ammonia also occurs, and is distinguished from uric acid by disengaging ammonia when dissolved in potash.

Phosphate of Lime is very frequent when the urine is neutral or alkaline. It is white and earthy, soluble in nitric acid, and precipitated by ammonia. It is fixed in the fire.

Phosphate of Ammonia and Magnesia is also pretty frequent. It dissolves easily in acetic acid, and when heated gives off ammonia, leaving a solid mass soluble in acids.

Fusible Calculus is a mixture of the two preceding. It melts readily before the blowpipe.

Oxalate of Lime constitutes the mulberry calculus, and often appears as minute crystals in the urine. When heated, it leaves carbonate of lime; or if heated in a tube with oil of vitriol, it gives off carbonic oxide. It dissolves in acids, and is precipitated by alkalies.

Carbonate of Lime occasionally, but very rarely, constitutes a urinary calculus, easily recognised by the action of hydrochlorio acid, which dissolves it with effervescence, and by a red heat, which leaves quicklime.

Cystic Oxide or Cystine, and Xanthic Oxide, are very rare calouli. Their characters and composition have been given under Uric Acid.

\section{LYMPH.}

This fluid may be looked on as blood devoid of its colouring matter. When drawn from the vessels, it coagulates like blood, from the separation of fibrine; and the liquid in which the coagulum has formed itself, coagulates when heated, like the serum of the blood. Human lymph contains about 96 per cent. of water, and variable proportions of albumen, fibrine, and salts, the salts amounting to nearly 2 per cent.

BLOOD.

This important fluid, from which the whole animal body is formed, and by which it is supplied and nourished, is a thick, 
somewhat viscid, alkaline liquid, of a slight saline taste, and a peculiar faint odour. It is deep red and opaque, and has a density of 1.0527 to 1.057 .

It is made up of an immense number of globules or flattened disks, floating in a limpid yellowish fluid. When drawn, it soon coagulates, forming a trembling jelly, which gradually contracts, expressing a yellowish liquid, the serum, which is occasionally turbid, and is always alkaline to test paper, and saline to the taste.

The coagulation consists in the separation of the fibrine previously dissolved, which, owing to some unknown cause, assumes the insoluble state, forming a fine network or jelly, in which the globules are inclosed. If the blood be beaten with a rod, the fibrine separates perfectly and adheres to the rod; but it is in the form of white filaments, and the globules remain suspended in the serum, no jelly whatever being formed in this case. Or if the fresh blood be mixed with 8 times its bulk of solution of sulphate of soda, no coagulum is formed, the fibrine remains dissolved, and a sediment is deposited which contains the globules unaltered.

The red globules thus prepared may be collected in a filter. Pure water added to them, or to the coagulum of blood, rapidly alters their form, and in fact dissolves them into an opaque liquid. This action of water is thus explained : the globules are formed of a thin, colourless, and transparent coat, inclosing a very soluble colouring matter. They float in a saline liquid, in which there is equilibrium between the contents of the globules and the fluid surrounding them. But when the latter is diluted with water, the equilibrium is disturbed, and endosmosis takes place, by which the contents of the globules acquire so greatly increased a volume, that the globules burst and their contents are dissolved in the water. The torn membranes of the globules may be detected by the microscope.

In saline solutions, the globules do not absorb water any more than in the serum. When collected in a filter, the globules form a red mass of the consistence of honey, consisting of fibrine and albumen, the latter in combination with the colouring matter. In a concentrated solution of chloride of calcium, the globules lose water by exosmosis, and contract in volume. If now placed in pure water, the globules again swell, and burst, forming a jelly which dissolves in water. The solution, on standing, deposits fibrine in white membranous masses, and the supernatant liquid, when boiled, is coagulated, indicating the presence of albumen.

The colouring matter of the blood is contained in the globules 
in combination with albumen, but is unknown in a state of purity. The compound of albumen and colouring matter is of a deep-red colour, becoming bright in contact with air or oxygen, and being rendered nearly black by carbonic and sulphurous acids, sulphuretted hydrogen and sulphurets. Protoxide of nitrogen gives it a purple colour.

The red compound gives 2 per cent. of ashes, of which $\frac{1}{3}$ is peroxide of iron ; and iron is uniformly present in red blood, which is the only animal product in which it occurs. This iron cannot be detected in the globules or their contents by the usual tests, but after passing chlorine through the red solution till the colour is destroyed, the iron may be detected by ferrocyanide of potassium.

When the red compound of albumen and colouring matter above mentioned is moistened with oil of vitriol, so gradually as not to become warm, a pasty mass is obtained, which attracts moisture from the air and forms a red jelly. If this be very gradually rubbed up with pure water, it contracts into a dark-red matter, which is surrounded with a colourless or yellowish liquid. This liquid is found to contain all the iron, and the dark matter, when calcined, leaves a white ash entirely free from iron, if the operation has been well performed. I have repeated this interesting experiment, first devised, I believe, by Sanson, which proves that, although the red compound contains iron, yet the colour does not necessarily depend on that metal; for the colour is altogether uninjured by the complete removal of the iron just described, although the colouring matter actually obtained in this experiment is not the original colouring matter of the blood, but modified.

The Hematosine of Lecanu is also a product of decomposition. It is prepared by means of diluted sulphurio acid, alcohol, and ammonia, by a tedious process. It is dark-brown, and forms red solutions with the alkalies, being insoluble in water, alcohol, and ether. It contains part of the iron of the blood, but as some kinds of hematosine contain $\frac{1}{4}$ or $\frac{1}{3}$ more iron than others, while its properties continue the same, it is obvious that the iron does not contribute essentially to those properties, such as the colour. Hematosine contains 6 to 8 per cent. of iron.

But the iron serves an important purpose in the blood; and we have reason to think that it is present in the form of oxide, for sulphuretted hydrogen and soluble sulphurets cause the blood to become first green and then black, owing to the formation of sulphuret of iron-a character indicating either the oxide or some corresponding compound, and not a compound like ferrocyanogen, in which sulphurets cannot detect the iron. 
Moreover, we see that oil of vitriol dissolves out oxide of iron; and although alkalies and ferrocyanide of potassium do not detect it, this is owing to the blood being an alkaline liquid, and to the presence of so much animal matter.

It is from the blood that are formed the tissues, the cells, muscular fibre, nervous matter, \&c. \&c. ; and we may, therefore, expect to find some relation, between their composition and that of the blood. In fact, flesh, or muscular fibre, as it exists in the body, including vessels, nerves, fat, \&c., has exactly the same composition as the blood has on an average of venous and arterial, or a mixture of both. We may, therefore, look on muscular fibre, or animal flesh, as simply blood more highly organised.

In addition to the substances mentioned above, namely, albumen, fibrine, colouring matter, and salts, blood also contains fat, apparently cholesterine, along with fatty acids and a peculiar fat, called seroline.

Glucose or grape sugar has also been detected in the blood, more especially in that of the liver, by Bernard. It seems to be converted, by isomeric transmutation, into lactic acid, which combines with the soda of the blood, and sets free carbonic acid, the lactic acid being itself burned off in the course of the circulation. The crystalline substance formed in the blood of some animals, which has been called hematocrystalline, has been already alluded to as a nitrogenised compound.

The normal proportions of serum and clot are 87 per cent. of serum to 13 of clot. 1000 parts of human blood contain $869 \cdot 15$ of serum, of which 790.37 are water, 67.8 albumen, and 10.98 are salts and fatty matter : along with 130.85 of clot, containing 125.63 albumen and fibrine of the globules, and $2 \cdot 27$ hematosine (a little fatty matter and traces of salts being present in all three), also $2 \cdot 95$ of fibrine, separate from the globules.

Venous blood contains more water and fewer globules than arterial blood.

The blood contains gases, chiefly carbonic acid and nitrogen, which it gives off in vacuo, or in a current of hydrogen. It is said to contain free oxygen; but this seems very improbable, when we reflect that fibrine absorbs oxygen, transforming it into carbonic acid, and that blood is instantly altered by contact with oxygen. The change from venous to arterial blood, from dark to florid, depends on the presence of oxygen, but also requires the presence of a saline solution. Indeed, a similar change of colour takes place in vacuo if the clot of venous blood be there covered with a pretty strong solution of various salts.

One chief function of the blood is, after conveying oxygen to 
all parts of the system, to carry to the lungs, there to be exhaled, the carbonic acid formed in the extreme vessels. For this purpose it is admirably adapted, from the fact that it is alkaline, and that its alkalinity depends in herbivora on the carbonate of soda, and in carnivora on the phosphate of soda, $\mathrm{P} \mathrm{Os}\left\{\begin{array}{l}2 \mathrm{Na} O \\ \mathrm{H} \mathrm{O}\end{array}\right\}$, a salt, the solution of which absorbs carbonio acid better than a solution of carbonate, and, when in contact with air or oxygen, gives off the whole of it ; whereas a carbonate, if it absorbed as much, would give up only half of the quantity absorbed. This important property of phosphate of soda has recently been demonstrated by Liebig; and we can now see why potash cannot replace soda in the blood, since the tendency of potash is to form an acid phosphate, $\mathrm{P}_{5} \mathrm{O}_{5}\left\{\begin{array}{ll}\mathrm{K} & \mathrm{O} \\ 2 & \mathrm{H}\end{array}\right\}$. Liebig and Enderlin have also proved that the blood of carnivora does not, as had been asserted, contain carbonate of soda.

\section{THE NUTRITION OF PLANTS AND ANIMALS.}

The animal and vegetable kingdoms of nature are connected together in a beautiful system of mutual dependence, exhibiting a perpetual circulation of certain elements through both, the mineral kingdom being the point of departure, and that also where the circulation terminates, to recommence unceasingly.

Plants derive their nourishment exclusively from the mineral world. It is clear that the first plants must have done so ; and although the decaying remains of former plants now contribute to vegetation, we shall see that they do so under mineral forms, and not essentially ; they promote vegetation, but are not indispensable to it.

The mineral food of plants, then, consists of carbonic acid, water, and ammonia, all of which are obtained from the atmosphere, and of sulphur (sulphuric acid), phosphorus (phosphoric acid), alkalies, earths, salts, and metals, all derived from the soil. Without the aid of the matters derived from the soil, the most abundant supply of carbonic acid, water, and ammonia, is of no use. But if a soil contain these necessary substances, plants will thrive in it, even if they have no carbonio acid nor ammonia furnished in the shape of manure beyond the usual atmospheric supply, provided time be given.

During germination-a process which goes on best in the dark - oxygen is absorbed, and carbonic acid given out. Heat is also generated. It cannot be doubted that a very essential part is performed also by endosmosis, that is, the tendency of water or of 
weak saline solutions to pass through a membrane or cell-wall, on the other side of which is a strong saline or saccharine solution. By this means, the first supply of water penetrates the cell-walls of the seed and its embryo, and there forms a strong solution ; for the presence of moisture and oxygen induces putrefaction of a portion of the albuminous matter always present in the cells ; this putrescent matter becomes a ferment, and converts the insoluble starch, stored up in the cells, into soluble sugar, as happens in an infusion of malt. But it likewise renders soluble the whole remaining albuminous matter, as happens when fibrine is left under water. Thus the cells become filled with a strong solution of sugar, albumen (fibrine or caseine) and salts, and now endosmosis rapidly goes on. The cells become distended, and those of the embryo are developed according to the vital law impressed on them at their formation, producing leaves by a constant formation of new cells. Such is the result of germination; but it must be noticed that already certain new compounds appear, apparently formed in the cells out of the carbonic acid, water, and ammonia supplied from the atmosphere either directly to the first leaves, or indirectly, through the water percolating the soil, to the cells of the radical fibrils, along with phosphates, alkalies, and common salt.

Here first comes into play that amazing chemical force, by which, in the vegetable cell, carbonic acid is decomposed, and its oxygen given out; while water, ammonia, and the mineral salts are all rendered available. This demands the aid of light, but, as already mentioned, even in germination, and in plants which are etiolated or grown in the dark, certain new compounds have been formed, such as occur under the influence of light also. Whether these are formed in the same way without as with light, is not known with certainty, but as in the dark oxygen is absorbed, not given out, we may suppose that they are produced in germination by a process of putrefaction or destruction of more complex molecules, such as those of albumen. The substances here alluded to are certain vegetable acids, found in the juice of germinating seeds and etiolated plants, and especially malic acid and malamide or asparagine, formed from malate of ammonia by the loss of water. This body is very abundant in etiolated plants, such as asparagus and vetches grown in the dark ; it is also found naturally in Althaa officinalis. Now we may suppose malic acid to be formed by the decomposition of albumen, along with ammonia, by a kind of fermentation, so long as light is absent; or it may be formed from the sugar by oxidation, a process active in germination. Thus 2 eqs. of dry grape sugar, $\mathrm{C}_{24} \mathrm{H}_{24} \mathrm{O}_{24}$, with 12 eqs. of oxygen, may yield 
3 eqs. of hydrated malic acid, $3\left(\mathrm{C}_{8} \mathrm{H}_{6} \mathrm{O}_{10}\right)=\mathrm{C}_{24} \mathrm{H}_{18} \mathrm{O}_{30}$, and 6 eqs. of water. The malic acid thus formed, in contact with ammonia, derived from the atmosphere or from albumen, will yield malamide (asparagine).

$\mathrm{C}_{8} \mathrm{H}_{4} \mathrm{O}_{8}, 2 \mathrm{NH}_{4} \mathrm{O}=2 \mathrm{H} \mathrm{O}_{+}$

$\mathrm{Cs}_{8} \mathrm{~N}_{2} \mathrm{H}_{10} \mathrm{O}_{8}$.

Malate of Ammonia.

Malamide.

As soon as light, however, comes into play, the true vegetative process begins: carbonic acid is decomposed and oxygen given out, and the carbon of the carbonic acid, with varying proportions of oxygen and hydrogen from water, of nitrogen from ammonia, and of sulphur from sulphuric acid, produces with the aid of phosphates, alkalies and salts,.in the vegetable cell all the vast variety of vegetable products. It is here that the prodigious chemical power of the vegetable cell comes into play, and this is so great, that it is able to perform, gradually, at .the ordinary temperature, what the most powerful agencies of heat and electricity cannot do in the laboratory, namely, to decompose carbonic acid, setting free its oxygen. But we are not to suppose that the carbonic acid is totally decomposed into carbon and oxygen, and that the carbon, thus set free, combines with hydrogen, oxygen, nitrogen, \&c., to yield vegetable products, for the insolubility of carbon would prevent this. Of a number of associated molecules of carbonic acid, some lose part of their oxygen, and the residue of these, with the remaining carbonic acid and water, \&c., enters, at the moment of separation, into new forms of combination. It is, therefore, necessary to consider the effect of vegetation as it is manifested, first, on carbonic acid and water together; secondly, on these, with the addition of ammonia; and lastly, on all these, with the aid of sulphuric acid. The first yields the non-nitrogenous compounds; the second, the nitrogenised compounds; and the third, those which contain both nitrogen and sulphir.

Even when carbonic acid and water are brought together in the cell, this is not enough. There must be present, first, albuminous matter, without which no active cell can exist; secondly, mineral matter, especially alkalies, phosphates, and salts. All these conditions being fulfilled, and light being admitted, we may suppose the first organic acid formed to be oxalic acid, the least complex of all. Crystallised oxalic acid is $\mathrm{C}_{4} \mathrm{H}_{6} \mathrm{O}_{12}=\mathrm{C}_{4} \mathrm{O}_{6}, 2 \mathrm{H} \mathrm{O}+4 \mathrm{aq}$. Now this is evidently formed from 4 eqs. of carbonic acid, $\mathrm{C}_{4} \mathrm{O}_{8}$, and 6 eqs. of water, $6 \mathrm{H} \mathrm{O}$, together, $\mathrm{C}_{4} \mathrm{H}_{6} \mathrm{O}_{14}$, by the separation of 2 eqs. of oxygen. And this, which is performed with ease in the vegetable cell, we 
cannot do in the laboratory, although we can produce oxalic acid from more complex organic products, such as sugar, starch, and many others. It is found abundantly in plants of the genera Rumex, Rheum, Oxalis, Sedum, Sempervivum, in many lichens and others. The reader will observe that in the crystallised acid, 4 eqs. of water are merely water of crystallisation, and that the driest oxalic acid is only $\mathrm{C}_{4} \mathrm{H}_{2} \mathrm{O}_{8}$ or $\mathrm{C}_{4} \mathrm{O}_{6}, 2 \mathrm{HO}$, the 2 eqs. of water being basic. Of course we may leave out 4 eqs. of water of crystallisation, and suppose dry oxalic acid to be derived from 4 eqs. of carbonic acid and 2 of water, $=\mathrm{C}_{4} \mathrm{H}_{2} \mathrm{O}_{10}$, by the separation of 2 eqs. of oxygen as before.

Let us now ascend a step higher in the organic scale; to the formation, namely, of malic acid, which is more complex than oxalic acid. Now this acid may be formed, either from oxalio acid itself, or, like oxalic acid, from carbonic acid and water, but in both cases by the separation of oxygen. In the former case, 2 eqs. of crystallised oxalic acid, $2\left(\mathrm{C}_{6} \mathrm{O}_{12}\right)=\mathrm{C}_{8} \mathrm{H}_{12} \mathrm{O}_{24}$, by losing 6 eqs. of water and 8 eqs. of oxygen, yield $\mathrm{C}_{8} \mathrm{H}_{6} \mathrm{O}_{10}=$ $\mathrm{C}_{8} \mathrm{H}_{4} \mathrm{O}_{8}, 2 \mathrm{H} \mathrm{O}$, or hydrated malic acid, which is bibasic. Or again, 2 eqs. of dry oxalic acid, $2\left(\mathrm{C}_{4} \mathrm{H}_{2} \mathrm{O} 8\right)=\mathrm{C}_{8} \mathrm{H}_{4} \mathrm{O}_{16}$, taking up 2 eqs. of water, and losing 8 eqs. of oxygen, yield $\mathrm{C}_{8} \mathrm{H}_{6} \mathrm{O}_{10}$, or hydrated malic acid. In the latter case, 8 eqs. of carbonic acid and 6 of water, together $\mathrm{C}_{8} \mathrm{H}_{6} \mathrm{O}_{22}$, losing 12 of oxygen, yield $\mathrm{C}_{8} \mathrm{H}_{6} \mathrm{O}_{10}$, or hydrated malic acid.

Tartaric and citric acids are easily shown to be producible in the same way. 2 eqs. of dry oxalic acid and 2 of water, together $\mathrm{C}_{8} \mathrm{H}_{6} \mathrm{O}_{18}$, losing 6 of oxygen, yield $\mathrm{C}_{8} \mathrm{H}_{6} \mathrm{O}_{12}=\mathrm{C}_{8} \mathrm{H}_{4} \mathrm{O}_{10}$, $2 \mathrm{H} 0$, hydrated tartaric acid. Or, 8 eqs. of carbonic acid and 6 of water, together $\mathrm{C}_{8} \mathrm{H}_{6} \mathrm{O}_{22}$, losing 10 of oxygen, give $\mathrm{C}_{8} \mathrm{H}_{6}$ $\mathrm{O}_{12}$, hydrated tartaric acid. Again, 3 eqs. of dry oxalic acid and 2 of water, together $\mathrm{C}_{22} \mathrm{H}_{8} \mathrm{O}_{26}$, losing 12 of oxygen, yield $\mathrm{C}_{12}$ $\mathrm{H}_{8} \mathrm{O}_{14}=\mathrm{C}_{12} \mathrm{H}_{5} \mathrm{O}_{11}, 3 \mathrm{H} \mathrm{O}$, or hydrated citric acid, which is tribasic. Or 12 eqs. $\mathrm{C} \mathrm{O}_{2}$, plus $8 \mathrm{H} \mathrm{O}$, together $\mathrm{C}_{12} \mathrm{H}_{8} \mathrm{O}_{32}$, losing 18 of oxygen, give $\mathrm{C}_{12} \mathrm{H}_{8} \mathrm{O}_{14}$, or hydrated citric acid.

In like manner, every vegetable acid, and every one of the neutral compounds of carbon, hydrogen, and oxygen, may be derived, first from some less complex acid or neutral compound, containing more hydrogen than itself; secondly, from a certain proportion of carbonic acid, to be determined by the number of eqs. of carbon in the compound, and of water; oxygen, in all cases, being given off. As the proportion of oxygen to carbon and hydrogen diminishes, the acids become weaker, till the oxygen exactly suffices to form water with the hydrogen, when we have either very feeble acids, or neutral bodies, such as sugar, gum, and starch. As the oxygen is still further diminished, 
we have neutral, bitter, and acrid compounds, or coloured bodies, or such as yield colouring matters, with ammonia and oxygen: further on still, we have aromatic oils and volatile quasi-resinous crystallisable acids; then resins ; and lastly, when all the oxygen is expelled, certain oils, which are carbohydrogens. The following tabular view will furnish a comparative statement of the amount of carbonic acid and water required, and of oxygen given off, on the supposition that each compound is formed directly from carbonic acid and water. But, as we have seen, each may also be formed from any of those below it in the scale, that is more highly oxidised, as citric acid from oxalic acid, or sugar from citric acid. To illustrate this, if to citrio acid, $\mathrm{C}_{22} \mathrm{H}_{8} \mathrm{O}_{14}$, we add 6 eqs. of water, we have $\mathrm{C}_{12} \mathrm{H}_{14} \mathrm{O}_{20}$, and this, losing 6 eqs. of oxygen, yields $\mathrm{C}_{12} \mathrm{H}_{14} \mathrm{O}_{14}$, or crytallised glucose. In like manner, 3 eqs. of malic acid and 16 of water, losing 18 of oxygen, yield the same sugar; or 3 eqs. of tartaric acid, with 10 of water, losing 18 of oxygen, likewise yield grape sugar. These are changes which occur every day in ripening fruits, and it is probable that sugar, starch, and other compounds are really formed in the plant, not directly from carbonic acid and water, as in the table, but from some intermediate compound, as sugar is here shown to be from the three acids just mentioned.

Acid employed. Water added. Oxygen separated. Glucose.

$\begin{array}{llll}\text { Citric } & \mathrm{C}_{12} \mathrm{H}_{8} \mathrm{O}_{14}+6 \mathrm{H} & \mathrm{O}_{6} & =\mathrm{C}_{12} \mathrm{H}_{14} \mathrm{O}_{14} \\ \text { Malic } 3\left(\mathrm{C}_{8} \mathrm{H}_{4} \mathrm{O}_{10}\right)+16 \mathrm{H} \mathrm{O} & \mathrm{O}_{18} & =2\left(\mathrm{C}_{12} \mathrm{H}_{14} \mathrm{O}_{14}\right) \\ \text { Tartaric } 3\left(\mathrm{C}_{8} \mathrm{H}_{6} \mathrm{O}_{12}\right)+10 \mathrm{H} \mathrm{O} & \mathrm{O}_{18} & =2\left(\mathrm{C}_{12} \mathrm{H}_{14} \mathrm{O}_{14}\right)\end{array}$

But as these are merely intermediate steps in the whole process of deoxidation, by which sugar, starch, fat, oils, \&c. are formed from carbonic acid and water, and the final result is the same whether these bodies be formed directly or indirectly, we shall confine ourselves, in the tabular view, to their direct formation from carbonic acid and water.

TABULAR VIEW OF THE PRODUCTION OF VEGETABLE COMPOUNDS FROM CARBONIC ACID AND WATER,

BY DEOXIDATION.

Group 1.-Stronger Vegetable Acids soluble in Water.

Substance formed.

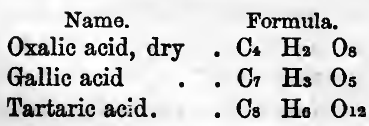

Carbonic acid used. in eqs. 4 7

8

Water
used.
in eqs.
2
3
6

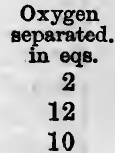




\begin{tabular}{|c|c|c|c|c|}
\hline \multicolumn{2}{|c|}{ Substance formed. } & \multirow{3}{*}{$\begin{array}{l}\text { Carbonic acid } \\
\text { used. } \\
\text { in eqs. } \\
8\end{array}$} & \multirow{3}{*}{$\begin{array}{c}\text { Water } \\
\text { used. } \\
\text { in eqs. } \\
6\end{array}$} & \multirow{3}{*}{$\begin{array}{c}\text { Oxygen } \\
\text { separated. } \\
\text { in eqs. }\end{array}$} \\
\hline Name. & Formula. & & & \\
\hline lic Acid & $\mathrm{C}_{8} \mathrm{H}_{6} \mathrm{O}_{10}$. & & & \\
\hline onitic acid & . $\mathrm{C}_{12} \mathrm{H}_{6} \mathrm{O}_{12}$ & 12 & 6 & 18 \\
\hline tric acid & . $\mathrm{C}_{12} \mathrm{H}_{8} \mathrm{O}_{14}$ & 12 & 8 & 18 \\
\hline econic acid & . . $\mathrm{C}_{14} \mathrm{H}_{4} \mathrm{O}_{14}$ & 14 & 4 & 18 \\
\hline annic acid & . $\mathrm{C}_{54} \mathrm{H}_{22} \mathrm{O}_{34}$ & 54 & 22 & 96 \\
\hline inic acid & . . $\mathrm{C}_{28} \mathrm{H}_{22} \mathrm{O}_{22}$ & 28 & 22 & 56 \\
\hline
\end{tabular}

The above are all acids, and in all but kinic acid the oxygen exceeds the hydrogen. Kinic acid is but a feeble acid. All other vegetable acids of similar properties to the above belong to this group, but those here given are sufficient to characterise it.

\section{Group II. - Indifferént Neutral Compounds.}

\section{Substance formed.}

Name.

Cellulose . . $\mathrm{C}_{12} \mathrm{H}_{10} \mathrm{O}_{20}$

Starch. - . $\mathrm{C}_{12} \mathrm{H}_{10} \mathrm{O}_{10}$

Cane Sugar . . $\mathrm{C}_{12} \mathrm{H}_{11} \mathrm{O}_{11}$

Gum . . $\mathrm{C}_{12} \mathrm{H}_{11} \mathrm{O}_{11}$

Grape sugar, dry . $\mathrm{C}_{12} \mathrm{H}_{12} \mathrm{O}_{12}$

Do. crystals $\mathrm{C}_{12} \mathrm{H}_{14} \mathrm{O}_{14}$

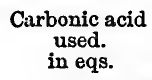

12

12

12

12

12

12

\section{Water used. in eqs.}

10

10

11

11

12

14
Oxygen separated. in eqs.

24

24

24

24

24

24

This important group contains, in every instance, hydrogen and oxygen in the proportion to form water, so that the whole of the oxygen of the carbonic acid, but not that of the water, has been separated. They may be viewed, theoretically, as formed of carbon, plus water; thus starch may be $\mathrm{Cl}_{22}+10 \mathrm{H} \mathrm{O}$.

Grovp III.-Neutral Bodies, chiefly Bitter, Acrid, Coloured, or yielding Colours with Ammonia.

\begin{tabular}{|c|c|c|c|c|c|}
\hline \multicolumn{3}{|c|}{ Substance formed. } & Carbonic acid & Water & Oxygen \\
\hline Name. & & Formula. & $\begin{array}{l}\text { used. } \\
\text { in eqs. }\end{array}$ & $\begin{array}{l}\text { used. } \\
\text { in eqs. }\end{array}$ & $\begin{array}{l}\text { separated. } \\
\text { in eqs. }\end{array}$ \\
\hline Mannite & & . $\mathrm{C}_{12} \mathrm{H}_{14} \mathrm{O}_{12}$ & 12 & 14 & 26 \\
\hline Orcine & & - $\mathrm{C}_{14} \mathrm{H}_{8} \mathrm{O}_{4}$ & 14 & 8 & 32 \\
\hline Smilacine & & - $\mathrm{C}_{18} \mathrm{H}_{15} \mathrm{O}_{6}$ & 18 & 15 & 45 \\
\hline Meconine & & - $\mathrm{C}_{20} \mathrm{H}_{10} \mathrm{O}_{8}$ & 20 & 10 & 42 \\
\hline Quassiine & & - $\mathrm{C}_{20} \mathrm{H}_{12} \mathrm{O}_{6}$ & 20 & 12 & 46 \\
\hline Peucedanine & . & - $\mathrm{C}_{24} \mathrm{H}_{12} \mathrm{O}_{6}$ & 24 & 12 & 54 \\
\hline Salicine & & . $\mathrm{C}_{26} \mathrm{H}_{18} \mathrm{O}_{14}$ & 26 & 18 & 56 \\
\hline Antiarine & & - $\mathrm{C}_{28} \mathrm{H}_{20} \mathrm{O}_{10}$ & 28 & 20 & 66 \\
\hline Pectine & & . $\mathrm{C}_{28} \mathrm{H}_{21} \mathrm{O}_{24}$ & 28 & 21 & 53 \\
\hline Hæmatoxylin & e & - $\mathrm{C}_{32} \mathrm{H}_{14} \mathrm{O}_{12}$ & 32 & 14 & 66 \\
\hline Populine & & - $\mathrm{C}_{40} \mathrm{H}_{22} \mathrm{O}_{26}$ & 40 & 22 & 86 \\
\hline
\end{tabular}




\section{PRODUCTION OF VEGETABLE COMPOUNDS. 545}

\begin{tabular}{|c|c|c|c|c|}
\hline \multicolumn{2}{|c|}{ Substance formed. } & Carbonic acid & Water & Oxygen \\
\hline $\begin{array}{l}\text { Name. } \\
\text { hloridzine }\end{array}$ & $\begin{array}{c}\text { Formula. } \\
\mathrm{C}_{42} \mathrm{H}_{24} \mathrm{O}_{2 \mathrm{O}}\end{array}$ & $\begin{array}{c}\text { used. } \\
\text { in eqs. } \\
42\end{array}$ & $\begin{array}{c}\text { used. } \\
\text { in eqs. } \\
24\end{array}$ & $\begin{array}{c}\text { separated } \\
\text { in eqs. } \\
88\end{array}$ \\
\hline Limonine & . $\mathrm{C}_{42} \mathrm{H}_{25} \mathrm{O}_{13}$ & 42 & 25 & 96 \\
\hline Hellenine & . . $\mathrm{C}_{42} \mathrm{H}_{28} \mathrm{O}_{8}$ & 42 & 28 & 106 \\
\hline Cnicine & - $\mathrm{C}_{52} \mathrm{H}_{34} \mathrm{O}_{18}$ & 52 & 34 & 120 \\
\hline Elaterine. & - . $\mathrm{C}_{60} \mathrm{H}_{25} \mathrm{O}_{18}$ & 60 & 25 & 127 \\
\hline
\end{tabular}

In this group, which is a very numerous one, various kinds of compounds appear. Mannite closely approaches to sugar; antiarine and elaterine are acrid poisons : salicine, phloridzine, populine, and quassiine, pure bitters ; pectine, a gelatinising substance; hæmatoxyline, a coloured body : orcine forms a fine colour with ammonia; and others are indifferent. It has lately been shown that populine, from poplar bark, is related to salicine, from willow bark. It is salicine, in which 1 eq. of hydrogen is replaced by 1 eq. of benzoyle, $\mathrm{C}_{24} \mathrm{H}_{5} \mathrm{O}_{2}$. This is proved by the fact that it may be made to yield salicine and compounds of the benzoic series. The fact is most interesting, as showing that the law of substitution prevails among very complex compounds, and explaining how similar compounds may be formed. The above are only examples. In the whole, not only the oxygen of the carbonic acid, but also part of that of the water, has been separated, so that the hydrogen remaining exceeds the oxygen.

Group IV.-Oxygenated Volatile Oils, and Aromatic Acids related to them.

\begin{tabular}{|c|c|c|c|c|c|}
\hline \multicolumn{3}{|c|}{ Substance formed. } & Carbonic acid & Water & Oxygen \\
\hline $\begin{array}{c}\text { Name. } \\
\text { Oil of bitter alm }\end{array}$ & londs & . $\mathrm{C}_{14} \mathrm{H}_{a} \mathrm{O}_{2}$ & $\begin{array}{c}\text { in eqs. } \\
14\end{array}$ & $\begin{array}{c}\text { in eqs. } \\
6\end{array}$ & $\begin{array}{l}\text { in eqs. } \\
32\end{array}$ \\
\hline Benzoic acid & & - $\mathrm{C}_{14} \mathrm{H}_{6} \mathrm{O}_{4}$ & 14 & 6 & 30 \\
\hline Oil of spiræa & & - $\mathrm{C}_{14} \mathrm{H}_{8} \mathrm{O}_{4}$ & 14 & 6 & 30 \\
\hline Salicylic acid & & - $\mathrm{C}_{14} \mathrm{H}_{6} \mathrm{O}_{6}$ & 14 & 6 & 28 \\
\hline Oil of Anise & & - $\mathrm{C}_{16} \mathrm{H}_{8} \mathrm{O}_{4}$ & 16 & 8 & 36 \\
\hline Anisic acid & & - $\mathrm{C}_{16} \mathrm{H}_{8} \mathrm{O}_{8}$ & 16 & 8 & 34 \\
\hline Cumarine & & - $\mathrm{C}_{18} \mathrm{H}_{4} \mathrm{O}_{4}$ & 18 & 4 & 36 \\
\hline Cumaric acid & & - $\mathrm{C}_{18} \mathrm{H}_{4} \mathrm{O}_{8}$ & 18 & 4 & 34 \\
\hline Oil of cinnamon & & - $\begin{array}{ll}\mathrm{C}_{18} \mathrm{H}_{8} & \mathrm{O}_{2}\end{array}$ & 18 & 8 & 42 \\
\hline Cinnamic acid . & & - $\mathrm{C}_{18} \mathrm{H}_{3} \mathrm{O}_{4}$ & 18 & 8 & 40 \\
\hline Oil of cumine & & - $\mathrm{C}_{20} \mathrm{H}_{12} \mathrm{O}_{2}$ & 20 & 12 & 50 \\
\hline Cuminic acid & & . $\mathrm{C}_{20} \mathrm{H}_{12} \mathrm{O}_{4}$ & 20 & 12 & 48 \\
\hline
\end{tabular}

In this group the oxygen is still further diminished. All the compounds are volatile, and the acids approaoh to resins in composition. The oils are fragrant and yield the acids by simple oxidation ; in fact, most of these oils are aldehydes. 
Grotp V. - Volatile Oily and Fatty Acids, of the Formula $\left(\mathrm{C}_{n} \mathrm{H}_{n}\right)_{2} \mathrm{O}_{4}$.

Substance formed.

\begin{tabular}{|c|c|c|c|}
\hline \multicolumn{2}{|l|}{ icia } & & $\begin{array}{c}\text { Formula. } \\
\text {. } \mathrm{C}_{2} \mathrm{H}_{2} \mathrm{O}_{4}\end{array}$ \\
\hline xide of Methyl & & & - $\mathrm{C}_{2} \mathrm{H}_{3} \mathrm{O}$ \\
\hline Acetic acid & & • & . $\mathrm{C}_{4} \mathrm{H}_{4} \mathrm{O}_{4}$ \\
\hline Oxide of Ethyle & & & - $\mathrm{C}_{4} \mathrm{H}_{5} \mathrm{O}$ \\
\hline Glycerine, dry & & - & . $\mathrm{C}_{6} \mathrm{H}_{4} \mathrm{O}_{2}$ \\
\hline Propylic acid & 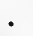 & & - $\mathrm{C}_{6} \mathrm{H}_{6} \mathrm{O}_{4}$ \\
\hline Butyric acid & & - & . $\mathrm{C}_{8} \mathrm{H}_{8} \mathrm{O}_{4}$ \\
\hline Valerianic acid & • & & - $\mathrm{C}_{10} \mathrm{H}_{10} \mathrm{O}_{4}$ \\
\hline Oxide of amyle & & & - $\mathrm{C}_{10} \mathrm{H}_{11} \mathrm{O}$ \\
\hline Caproic acid & - & & - $\mathrm{C}_{12} \mathrm{H}_{12} \mathrm{O}_{4}$ \\
\hline Enanthylic acid & & & . $\mathrm{C}_{14} \mathrm{H}_{14} \mathrm{O}_{4}$ \\
\hline Caprylic acid & - & & - $\mathrm{C}_{16} \mathrm{H}_{10} \mathrm{O}_{4}$ \\
\hline Pelargonic acid & & & - $\mathrm{C}_{18} \mathrm{H}_{18} \mathrm{O}_{4}$ \\
\hline Rutic acid & • & & - $\mathrm{C}_{20} \mathrm{H}_{20} \mathrm{O}_{4}$ \\
\hline Margaritic acid & & - & - $\mathrm{C}_{22} \mathrm{H}_{22} \mathrm{O}_{4}$ \\
\hline Laurostearic acid & & & - $\mathrm{C}_{24} \mathrm{H}_{24} \mathrm{O}_{4}$ \\
\hline Cocinic acid & & - & - $\mathrm{C}_{26} \mathrm{H}_{26} \mathrm{O}_{4}$ \\
\hline Myristic acid & $\theta^{\circ}$ & & - $\mathrm{C}_{28} \mathrm{H}_{28} \mathrm{O}_{4}$ \\
\hline Benic acid & & 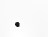 & - $\mathrm{C}_{30} \mathrm{H}_{30} \mathrm{O}_{4}$ \\
\hline Cetylic or Palmit & & acid & $\mathrm{C}_{32} \mathrm{H}_{32} \mathrm{O}_{4}$ \\
\hline Oxide of Cetyle & - & & - $\mathrm{C}_{32} \mathrm{H}_{33} \mathrm{O}$ \\
\hline Margaric acid . & & - & - $\mathrm{C}_{34} \mathrm{H}_{34} \mathrm{O}_{4}$ \\
\hline Stearic acid & - & & - $\mathrm{C}_{30} \mathrm{H}_{30} \mathrm{O}_{4}$ \\
\hline Balenic acid & & - & - $\mathrm{C}_{38} \mathrm{H}_{38} \mathrm{O}_{4}$; \\
\hline Arachidic acid & . & & - $\mathrm{C}_{40} \mathrm{H}_{40} \mathrm{O}_{4}$ \\
\hline Behenic acid & & - & - $\mathrm{C}_{42} \mathrm{H}_{42} \mathrm{O}_{4}$ \\
\hline Cerotic acid & - & & $\mathrm{C}_{54} \mathrm{H}_{54} \mathrm{O}_{4}$ \\
\hline Oxide of Ceryle & & • & . $\mathrm{C}_{54} \mathrm{H}_{55} \mathrm{O}$ \\
\hline Melissic acid & $\therefore$ & & - $\mathrm{C}_{60} \mathrm{H}_{60} \mathrm{O}_{4}$ \\
\hline Oxide of Melissy & & • & . $\mathrm{C}_{60} \mathrm{H}_{61} \mathrm{O}$ \\
\hline
\end{tabular}

Carbonic acid Water Oxygen used. used. separated. in eqs. in eqs. in eqs.

$\begin{array}{rrr}2 & 2 & 2 \\ 2 & 3 & 6 \\ 4 & 4 & 8 \\ 4 & 5 & 12 \\ 6 & 4 & 14 \\ 6 & 6 & 14 \\ 8 & 8 & 20 \\ 10 & 10 & 26 \\ 10 & 11 & 30 \\ 12 & 12 & 32 \\ 14 & 14 & 38 \\ 16 & 16 & 44 \\ 18 & 18 & 50 \\ 20 & 20 & 56 \\ 22 & 22 & 62 \\ 24 & 24 & 68 \\ 26 & 26 & 74 \\ 28 & 28 & 80 \\ 30 & 30 & 86 \\ 32 & 32 & 92 \\ 32 & 33 & 96 \\ 34 & 34 & 98 \\ 36 & 36 & 104 \\ 38 & 38 & 110 \\ 40 & 40 & 116 \\ 42 & 42 & 122 \\ 54 & 54 & 158 \\ 54 & 55 & 162 \\ 60 & 60 & 176 \\ 60 & 61 & 180\end{array}$

This group includes the chief oily and fatty acids, as well as their aldehydes, which are omitted, and some oxides of the ethylic series. Glycerine is added, because these acids are always found in nature combined with it. The only two acids in which either part only of the oxygen of the carbonic acid, or the whole of it, without any from the water, has been separated, are the formic and acetic acids, which, accordingly, are powerful acids, soluble in water, and, though volatile, not oily. The rest are all oily or fatty, and become weaker as we rise in the scale, and in proportion as the oxygen of the water is more completely separated. The oxides of ethyle, methyle, amyle, cetyle, ceryle and melissyle occur 
combined with acids of this series, forming compound ethers, to some of which the flavour of many fruits is to be ascribed. The acetate, butyrate, caproate, and caprylate of ethyle are found in the pineapple, melon, strawberry, \&c. A similar salt of oxide of amyle gives flavour to the pear. Oil of gaultheria is the salicylate of methyle. Spermaceti is the cetylate of cetyle; and one kind of wax is the palmitate, another the cerotate, of oxide of ceryle.

\section{GrovP VI.-Resins and Camphors.}

Substance formed.

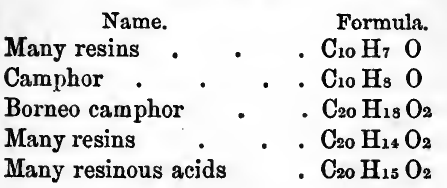

$\begin{array}{ccc}\begin{array}{c}\text { Carbonic acid Water } \\ \text { used. } \\ \text { in eqs. }\end{array} & \begin{array}{c}\text { Oxygen } \\ \text { us eqs. } \\ \text { in eq. }\end{array} & \begin{array}{c}\text { separated. } \\ \text { in eqs. }\end{array} \\ 10 & 7 & 26 \\ 10 & 8 & 27 \\ 20 & 18 & 56 \\ 20 & 14 & 52 \\ 20 & 15 & 53\end{array}$

In this widely diffused group, very little oxygen is left. It includes an immense number of isomeric and polymeric resins and resinous acids. All are highly combustible.

\section{Grovp VII.-Carbohydrogens.}

$\begin{array}{ccc}\text { Substance formed. } & \begin{array}{c}\text { Carbonic acid } \\ \text { used. } \\ \text { Name. }\end{array} \\ \text { in eqs. }\end{array}$

\section{Water used. in eqs.}

$\begin{array}{rr}5 & 4 \\ 10 & 8 \\ 14 & 8 \\ 16 & 8 \\ 14 & 7 \\ 15 & 12 \\ 18 & 12 \\ 20 & 14\end{array}$

\begin{tabular}{|c|}
\hline $\begin{array}{l}\text { Oxygen } \\
\text { separated } \\
\text { in eqs. }\end{array}$ \\
\hline 14 \\
\hline 28 \\
\hline 36 \\
\hline 40 \\
\hline 35 \\
\hline 42 \\
\hline 48 \\
\hline 54 \\
\hline
\end{tabular}

In this group, which is very widely diffused, the whole of the oxygen, both of the carbonic acid and of the water, has been separated, and consequently no further deoxidation can take place. Hence these compounds are very permanent, and are chiefly altered by their natural tendency to absorb oxygen under favourable circumstances.

It will be seen that these seven groups are so many successive steps in the scale of deoxidation, at one end of which stand carbonic acid and water, at the other the non-oxygenated essential oils. And it is evident, also, that the members of one group may be converted into those of the next higher by 
deoxidation, with or without the addition of water. This is probably what takes place, leaving out of view, for the present, nitrogen and sulphur. First, oxalic acid is formed; then malic, tartaric, and citric acids, \&c., from it, or from each other; then sugar, starch, \&c., from the acids; bitter, acrid, and coloured compounds from sugar, starch, \&c.; then oxygenated volatile oils; and then acids, perhaps also from sugar, \&c. ; then the oily and fatty acids, either from the preceding oils and acids or from sugar; then the resins, from the fats or from sugar; and, lastly, the carbohydrogens. Thus we have a picture of the whole process of vegetation, as far as concerns compounds devoid of nitrogen and sulphur; and we find it to be uniformly one of deoxidation. We shall find, also, that the same law holds in regard to all those products in which nitrogen and sulphur are present. But before examining that subject, it may be remarked that the chief mass of plants is made up of woody fibre or cellulose, $\mathrm{C}_{12} \mathrm{H}_{10} \mathrm{O}_{10}$, with starch, sugar, and gum, oils, resins, and fats, as well as vegetable acids, and that all of these are destitute of nitrogen. Hence the chief part of the food of plants is that which supplies carbon, hydrogen, and oxygen; in other words, carbonic acid and water.

These are supplied by the atmosphere. The various processes, constantly going on, of the decay of dead animals and vegetables, the respiration of animals, and combustion, are at every moment pouring carbonic acid into the air, and yet, in the free open atmosphere, the proportion of carbonic acid never increases, as it would do in a closed space, beyond the average of $\frac{1}{2000}$ th, or a little more, but never exceeding ${ }_{10}^{1}$ th part of the volume of the air. Now these processes not only produce carbonic acid, but also consume oxygen, and that in the same proportion, the oxygen they take up being equal in volume to the carbonic acid which it forms. And yet, not only does the proportion of carbonic acid in the air not increase, but that of the oxygen does not diminish. Evidently, therefore, some cause must be in operation, directly opposed to, and exactly balancing the processes of respiration, decay, and combustion. And such a process is that of vegetation, or the action of growing plants on carbonic acid and water under the influence of light, by which, as we have seen, these are deoxidised, vegetable products are formed, and oxygen is given out. Thus the air is kept in a state of purity, and yet is constantly undergoing change; for as fast as respiration, decay, and combustion consume oxygen and form carbonic aoid, vegetation consumes carbonic acid and produoes oxygen. Any excess of carbonic acid instantly causes an increase of vegetation, and therefore of oxygen, as well as of food for animals. When animals, by this 
food, increasc, they produce more carbonic acid, and so on, the oxygen circulating from the air to carbonic acid in the animal processes, and from carbonic acid, by means of plants, back to the air again.

It is quite conceivable, that in the earlier geological periods; when, as it appears, warm-blooded animals did not exist, the air may have been unfit for them, by reason of its containing too much carbonic acid. But this, within certain limits, would be favourable to vegetation, and especially, as there is reason to believe, to that of cryptogamous plants, such as ferns and lycopodiaceæ, and also the cycadaceæ. The action of such plants growing with enormous luxuriance, and not balanced by animal life, would in time diminish the amount of carbonic acid, increasing at the same time that of oxygen in the air, till it became fit for the respiration of warm-blooded animals, and the carbon, thus removed from the air, would be stored up in the form of remains of these plants, protected from decay by being covered with some rocky mass. In accordance with this idea, while the animals of the carbonaceous and other early periods seem to have been reptiles or fish, that is, such as required little oxygen, the vegetation, as found, partly decayed, in our coal-beds, seems to have been prodigiously luxuriant; and it is said that ferns and other similar plants, which abound in coal, really do grow most luxuriantly in an atmosphere charged with carbonic acid, to a much greater degree than air is. Be this as it may, vegetation would purify such an atmosphere, till warm-blooded animals and man could live in it; and then, the balance, once attained, would continue undisturbed as at the present day. In fact, air, taken from closed vessels, from the tombs of Egypt, 3000 years old, or the ruins of Herculaneum, 2000 years old, has been found as rich in oxygen as that of the present time.

Plants, then, obtain all their carbon, directly or indirectly; from the air: directly, by absorption through the leaves; indirectly, through the absorption of water by the roots, this water having dissolved some carbonic acid in passing through the air, and more in filtering through the soil, in which carbonic acid is constantly formed by the decay of organic matter. But this solution of carbonic acid has another and very important function to perform, namely, to dissolve earthy and alkaline phosphates and carbonates, and thus to supply the plant with its mineral food. Although, therefore, part of the wood, \&c., of a plant may be formed from the carbonic acid entering by the roots, yet as plants give out from the roots a certain amount of carbon in the form of excretions, we find that the whole increase in the weight of carbon in a growing plant is really derived 
from the air by the leaves. The soil becomes richer in carbon rather than poorer, and thus the carbon of all crops, as far as its weight is concerned, comes from the air. There is no evidence that mould or humus ever enters the plant as such; but it is converted into carbonic acid, which enters by the roots and acts as a solvent for mineral salts. This, as we shall see, is the true reason why the presence of humus in the soil or in manure is advantageous. But direct experiments have proved, that plants can grow in perfection, and produce fertile seeds, as the first plants must have done, in a soil destitute of humus or mould, provided it contain the necessary alkalies, phosphates, and other mineral salts, in a form adapted for entrance into the plant. In this case, the atmosphere easily supplies the whole of the carbon required, as well as the ammonia, if the necessary time be given.

Let us now attend to the nitrogen of plants. This, as already stated, is supplied to wild plants entirely by the air, and, so far as we know, only in the form of ammonia. Some authors have held that nitric acid furnishes nitrogen to plants, and that this acid is formed in the air by thunder-storms, and carried down by the rain, and they point to the occurrence of nitric acid in springs in proof of this. Now, it is true that nitric acid is formed in thunder-storms, but in very minute quantity, whereas ammonia is, and must be, present in the air at all times. When electric sparks are passed through air, nitric acid is formed, but a continuance of the sparks again decomposes it. Indeed, there is reason to believe that the nitric acid of storms is produced by the oxidation of ammonia of the air, as in nitrification, where ammonia is oxidised into nitric acid and water, $\mathrm{N} \mathrm{H}_{3}+\mathrm{O}_{8}=$ $\mathrm{N} \mathrm{O}_{3}, 3 \mathrm{H} \mathrm{O}$; so that, even if nitric acid did yield nitrogen to plants, that nitrogen might be derived from ammonia. This would account, too, for the small amount of nitrio acid formed. For if it were produced by the action of electricity on the nitrogen and oxygen of the air, there seems to be no reason why it should not be formed in very large quantity; while ammonia forms less than $\frac{1}{10000}$ th of the air, perhaps much less. Nitric acid is chiefly found in springs where decaying organic matter is near them, as in towns, and is formed from the ammonia produced in their decay, by the same process as in nitrification. Besides, although plants probably possess the power of decomposing nitrie acid, we know that many plants, such as tobacoo and sunflower, actually produce nitrio acid, or, at least, do not destroy that which enters them. On the whole, we may admit that both ammonia and nitrio acid may yield nitrogen to plants, but we must not forget that nitric acid is most commonly formed from ammonia.

The origin of the ammonia in the air is obvious. It is 
contained in the excreta of animals, chiefly in their urine, and is also produced from the decay and putrefaction of dead animals and vegetables, the whole of their nitrogen rising into the air as carbonate of ammonia. The combustion of coal also yields it, and it is said that some ammonia is given out in the respiration or transpiration of animals. It is stated, that if the air of a crowded theatre be allowed to escape by a narrow opening above, while fresh air enters below, the air passing out is pungent from ammonia; but whether this be derived from the lungs or the skin is not known. But it is evident that the air must be continually receiving supplies of ammonia ; and as plants cannot grow without it, and fix large quantities of it, they must be continually removing it from the air. Here, then, is a balance between vegetable life and animal life, supported by plants on the one hand, and decay on the other, similar to that above explained of the carbonic acid and oxygen; only the balance of nitrogen is single, while that of carbonic acid and oxygen is double. Ammonia, that is, its nitrogen, is taken up by plants, by them supplied to herbivorous animals, and by these to carnivorous animals; so that in this case vegetation and animal life act on the same side. And the decay or putrefaction or combustion of both vegetables and animals sends the whole of their nitrogen, in the form of ammonia, back to the atmosphere. This balance is as perfect as the former, and both combined keep the composition of the air perfectly uniform within certain limits.

The proportion of ammonia in the air is so small that it cannot be detected by direct tests applied to a small portion of air ; but it is easily detected in rain water by acidulating it with sulphuric acid, and evaporating it to $\frac{1}{300}$ th or $\frac{1}{400}$ th of its bulk. The addition of lime or potash will then set free ammonia, which may be recognised by its smell, and by forming white vapours with hydrochloric acid; or the addition of bichloride of platinum will cause a yellow precipitate of ammonio-chloride of platinum. It is evident that the ammonia of the air is brought to the soil by the rain, and enters plants partly by the roots, partly by the leaves; and it has been proved by experiment that the air is capable of supplying to plants, in the form of ammonia, all the nitrogen they require, provided only the soil contain the necessary mineral food in available forms, and time be allowed. When we come to treat of manures, we shall speak of the uses of ammonia in them.

It will be as well to mention here, that the only remaining combustible or organic element of the food of plants, namely, sulphur (for the phosphorus is always in the incombustible form of phosphoric acid), is obtained from sulphates in the soil, chiefly 
sulphate of lime or gypsum, which serves to explain the value of gypsum as a manure.

We have seen how, in a general way, plants form, from carbonic acid and water, all those vegetable products which contain neither nitrogen nor sulphur, and invariably by the separation of oxygen. Now, to produce nitrogenised compounds, such as malamide or asparagine, indigo, and the vegetable bases, morphine, quinine, strychnine, caffeine, \&c., it is only necessary that, to the conditions formerly mentioned, namely, an actire cell, containing albuminous matter, and the presence of phosphates, alkalies and salts, carbonic acid and water, sugar, \&c., ammonia should be added; and the plant then, by the same process of deoxidation, gives rise to nitrogenised products. That these conditions are fulfilled is proved by the fact, that the juice of plants contains sugar or gum, albuminous matter, alkalies, phosphates, and salts, and among these, salts of ammonia. And it is easy to illustrate the formation of the compounds here alluded to. It must first, however, be mentioned, that ammonia, from its nature, is a very remarkable substance. It cannot be formed by the direct union of its elements, but only when they meet in the nascent state; and, from the strong attractions of both its elements for other elements, it is particularly liable to transformations. Of these, the oxidation of both its elements, which occurs best in contact with bases, as in nitrification, has been mentioned. When ammonia is in contact with lime, air, and moisture, nitrate of lime is formed, and from this the nitrate of potash, $\mathrm{N} \mathrm{H}_{3}+\mathrm{CaO}+\mathrm{O}_{8}=\mathrm{CaO}, \mathrm{N} \mathrm{O}_{5}+3 \mathrm{H} \mathrm{O}$. But in organic chemistry we meet with another peculiar transformation or series of transformations of ammonia, those, namely, in which one, two, or all three of its atoms of hydrogen are removed, in union with oxygen, derived from an acid; and both residues, the ammonia minus hydrogen, and the acid minus oxygen, combine to form new compounds, which are called amides, imides, and nitryles, according as the ammonia has lost one, two, or three atoms of hydrogen, and the acid, of course, one, two, or three atoms of oxygen. The simplest example of an amide is oxamide, formed as follows: $\underbrace{\mathrm{NH}_{3}, \mathrm{H} \mathrm{O}, \mathrm{C}_{2} \mathrm{O}_{3}}=\underbrace{\mathrm{N} \mathrm{H}_{2}, \mathrm{C}_{2} \mathrm{O}_{2}}+2 \mathrm{H} \mathrm{O}$.

Oxalate of Ammonia.

Oxamide.

All amides are formed in the same way, by the separation of 2 eqs. of water from the neutral salt. If the acid be bibasic, the neutral salt contains of course 2 eqs. of ammonia; but here also, 2 eqs. of water are separated to yield the amide, as in asparagine or malamide.

$$
\underbrace{\mathrm{C}_{8} \mathrm{H}_{4} \mathrm{O}_{8}, 2 \mathrm{~N} \mathrm{H}_{4} \mathrm{O}}_{\text {Malate of Ammonia. }}=\underbrace{\mathrm{C}_{8} \mathrm{~N}_{2} \mathrm{H}_{10} \mathrm{O}_{8}}_{\text {Mulamide. }}+2 \mathrm{H} \mathrm{O} \text {. }
$$


When the same change takes place in the acid salts of ammonia, with a monobasic or bibasic acid, the result is an acid amide. Thus binoxalate of ammonia yields oxamic acid.

$$
\begin{aligned}
& \mathrm{C}_{2} \mathrm{O}_{3}, \mathrm{HO}, \mathrm{C}_{2} \mathrm{O}_{3}, \mathrm{~N} \mathrm{H} \mathrm{H}_{4} \mathrm{O}=\mathrm{C}_{4} \mathrm{NH}_{3} \mathrm{O}_{6}+2 \mathrm{H} \mathrm{O} \\
& \text { Binoxalate of Ammonia. }
\end{aligned}
$$

and

$$
\underbrace{\mathrm{C}_{8} \mathrm{H}_{4} \mathrm{O}_{8}, \mathrm{H} \mathrm{O}, \mathrm{N} \mathrm{H} \mathrm{H}_{4} \mathrm{O}}_{\text {Acid Malate of Ammonia. }}=\underbrace{\mathrm{C}_{8} \mathrm{~N} \mathrm{H}_{7} \mathrm{O}_{8}}_{\text {Malamic Acid (Aspartic Acid). }}+2 \mathrm{H} \mathrm{O} .
$$

Thus the general law for amides, neutral and acid, is the elimination of 2 eqs. of water from a neutral or acid salt of ammonia. These amides are very numerous as artificial products, and one at least, malamide, or asparagine, is an important natural product. They all, when boiled with acids or bases, yield ammonia, and the acid from which they were formed. It is probable that amides have an important part to play in vegetation, and that they may contribute to the formation of vegetable bases. For in several instances, artificial amides are converted into bases, isomeric or polymeric with themselves. Thus, oil of bitter almonds, with ammonia, yields a neutral crystalline compound, hydrobenzamide,

$$
3(\underbrace{\mathrm{C}_{14} \mathrm{H}_{6} \mathrm{O}_{2}}_{\text {Oil of bitter almonds. }})+2 \mathrm{~N} \mathrm{H} 3=6 \mathrm{H} \mathrm{O}+\underbrace{\mathrm{C}_{42} \mathrm{~N}_{2} \mathrm{H}_{18}}_{\text {Hydrobenzamide. }}
$$

and this compound, when boiled with potash, is transformed into the isomeric base, amarine. In like manner, oil of bran, or furfurole, $\mathrm{C}_{15} \mathrm{H}_{6} \mathrm{O}_{6}$, with 1 eq. of ammonia, yields 3 eqs. of water, and 1 eq. furfuramide, $\mathrm{C}_{15} \mathrm{~N} \mathrm{H}_{6} \mathrm{O}_{3}$; and this, boiled with potash, is converted into the base furfurine, C3o $\mathrm{N}_{2} \mathrm{H}_{12} \mathrm{O}_{6}$, polymeric with it.

Under Indigo we have described colourless indigo, as it exists in the plant. This is probably an amide of an acid not yet known, $\mathrm{C}_{16} \mathrm{H}_{4} \mathrm{O}_{3}$. For

$$
\underbrace{\mathrm{C}_{16} \mathrm{H}_{4} \mathrm{O}_{3}}_{\text {Supposed Acid. }}+\mathrm{N} \mathrm{H}_{4} \mathrm{O}=2 \mathrm{HO}+\underbrace{\mathrm{C}_{16} \mathrm{~N} \mathrm{H}_{6} \mathrm{O}_{2}}_{\text {White Indigo. }} .
$$

And this, when exposed to air, passes by the simple removal by oxidation of 1 eq. of hydrogen into blue indigo, $\mathrm{C}_{16} \mathrm{~N} \mathrm{H}_{5} \mathrm{O}_{2}$.

Some nitrogenous colours are produced by the combined action of ammonia and oxygen on colourless substances from plants, both 
processes going on outside of the plant, whereas in indigo the action of ammonia has taken place in the plant, and oxygen alone is wanting to produce the colour. The beautiful dye archil is produced by the action of ammonia and air on the colourless orcine. Now, in this case, an amide is probably first formed, and afterwards oxidised.

$$
\underbrace{\mathrm{C}_{14} \mathrm{H}_{8} \mathrm{O}_{4}}_{\text {Orcine. }}+\mathrm{N} \mathrm{H}_{3}=\underbrace{\mathrm{C}_{14} \mathrm{H}_{9} \mathrm{~N} \mathrm{O}_{2}}_{\text {Orcinamide. }}+2 \mathrm{HO}
$$

and

$$
\underbrace{\mathrm{C}_{14} \mathrm{H}_{9} \mathrm{~N} \mathrm{O}_{2}}_{\text {Orcinamide. }}+\mathrm{O}_{6}=\underbrace{\mathrm{C}_{14} \mathrm{H}_{7} \mathrm{~N} \mathrm{O} \mathrm{O}_{6}}_{\text {Blue Orceine. }}+2 \mathrm{HO}
$$

Amygdaline, the peculiar bitter compound which yields the oil of bitter almonds, seems to be an amide of amygdalinic acid, an acid which may be obtained from it. Thus,

$$
\underbrace{\mathrm{C}_{40} \mathrm{H}_{27} \mathrm{O}_{25}}_{\text {Amygdalinic acid. }}+\mathrm{N} \mathrm{H}_{4} \mathrm{O}=\underbrace{\mathrm{C}_{40} \mathrm{~N} \mathrm{H}_{27} \mathrm{O}_{22}}_{\text {Amygdaline. }}+4 \mathrm{HO} \text {. }
$$

We may conclude, therefore, that all those compounds found in plants, which contain nitrogen without sulphur, are either amides, or derived from such compounds.

When a salt of ammonia loses so much water, that 1 eq. of ammonia has lost 2 eqs. of hydrogen, and 1 eq. of the acid 2 eqs. of oxygen, the resulting compound is called an Imide, Imide being the name of the compound $\mathrm{N} \mathrm{H}$ which is left, as Amide is of $\mathrm{NH}_{2}$. A few imides are known, but all are artificial, and, besides, they agree with amides in yielding the original acid and ammonia, when boiled with acids and bases. If they exist, therefore, in plants, they will be formed in the same way as amides, and yield analogous compounds. We need not, therefore, dwell on them. Were neutral oxalate of ammonia to lose 3 eqs. of water, instead of 2 , it would yield oximide, $\mathrm{N} \mathrm{H}_{4} \mathrm{O}$, $\mathrm{C}_{2} \mathrm{O}_{3}=3 \mathrm{H} \mathrm{O}+\mathrm{C}_{2} \mathrm{NH} \mathrm{H}$, which last would be oximide, differing from oxamide by 1 eq. of water.

The nitryles, which are compounds in which all the hydrogen of ammonia has separated as water with 3 eqs. of oxygen from the acid, are, for the most part, artificial products. Thus, benzoate of ammonia, $\mathrm{N} \cdot \mathrm{H}_{4} \mathrm{O}, \mathrm{C}_{14} \mathrm{H}_{5} \mathrm{O}_{3}$, if deprived of all its oxygen, that is, of the 3 eqs. in the anhydrous acid, and of the 1 eq. in the oxide of ammonium, along with 4 eqs. of hydrogen, 3 from the ammonia, and the fourth from the water which converts ammonia 
into the oxide of ammonium, and is necessary to the existence of all salts of ammonia with oxygen acids, yields Benzonitryle, $\mathrm{C}_{24}$ $\mathrm{N} \mathrm{H}_{5}$. It would appear that nitryles, which contain no oxygen so far as known, are derived from such acids as contain only 3 eqs. of oxygen when anhydrous, or 4 in the form of hydrate. Hence the acids of the Formic and Acetic series $\left(\mathrm{Cn}_{n} \mathrm{H}_{n}\right)_{2} \mathrm{O}_{4}$, readily yield nitryles, as well as those of the Benzoic series. The reader will observe, that in the equations above given for amides, the ammoniacal salts are represented with $\mathrm{N} \mathrm{H}_{4} 0$, when the acid is represented as anhydrous, and with $\mathrm{N} \mathrm{H}_{3}$, when the acid is given as hydrate. In all cases, 1 eq. of water must be present, beyond the anhydrous acid and dry ammonia, $\mathrm{N} \mathrm{H}_{3}$, and it is of no consequence whether this be written along with the anhydrous acid, or with the ammonia, which it converts into

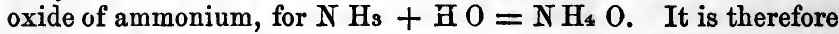
quite indifferent whether oxalate of ammonia be written $\mathrm{N} \mathrm{H}_{3}$, $\mathrm{C}_{2} \mathrm{O}_{3}, \mathrm{H} \mathrm{O}$, or $\mathrm{N} \mathrm{H}_{4} \mathrm{O}, \mathrm{C}_{2} \mathrm{O}_{3}$, and so for all similar cases. The fourth atom of hydrogen in $\mathrm{N} \mathrm{H}_{4} \mathrm{O}$, is always separated as water along with the other three, or with one or two of them. This is the reason why in the formation of amides 2 eqs., of imides 3 eqs., and of nitryles 4 eqs. of water are expelled from the neutral dry salt of ammonia. The oxalate of ammonia, as we have seen, yields oxamide by losing 2 eqs., and oximide, $\mathrm{C}_{2} \mathrm{~N} \mathrm{H} \mathrm{O}$, which would probably appear as cyanogen and water, by losing 3 eqs. of water; and if it lost 4 eqs., it would yield cyanogen $\mathrm{C}_{2} \mathrm{~N}$, which is the oxalonitryle. Now there is a curious relation between the nitryles and cyanogen. For Formonitryle, $\mathrm{C}_{2} \mathrm{~N} \mathrm{H}$, is hydrocyanic acid; Acetonitryle, $\mathrm{C}_{4} \mathrm{~N} \mathrm{H}_{3}$, is the cyanide of methyle, and so on. Benzonitryle, $\mathrm{C}_{14} \mathrm{~N} \mathrm{H}_{5}$, is probably the cyanide of phenyle, $\mathrm{C}_{2} \mathrm{~N}, \mathrm{Cl}_{12} \mathrm{H}_{5}$. It is not known whether all nitryles are cyanides, or whether some may not be isomerio with cyanides. We may conclude, therefore, that cyanogen and hydrocyanic acid, which occur in plants, are nitryles formed, the former from oxalate, the latter from formiate of ammonia. And it now appears, that some of the non-oxygenated volatile bases, such as nicotine and coniine, are basic nitryles.

Having now explained the reaction of ammonia to form amides, imides or nitryles, let us consider the formation of such compounds as contain nitrogen without sulphur, supposing them, as before, to be produced immediately, as we know they are mediately, from the food of plants, namely, carbonic acid, water, and ammonia. This will be best illustrated by a tabular arrangement. 


\begin{tabular}{|c|c|c|c|c|c|}
\hline \multicolumn{2}{|c|}{ Substance formed. } & \multirow{2}{*}{$\begin{array}{l}\mathrm{CO}_{2} \\
\text { used. } \\
\text { in eqs. }\end{array}$} & \multirow{2}{*}{$\begin{array}{c}\text { H O } \\
\text { used. } \\
\text { in eqs. }\end{array}$} & \multirow{2}{*}{$\begin{array}{l}\mathrm{NH}_{3} \\
\text { used. } \\
\text { in eqs. }\end{array}$} & \multirow{2}{*}{$\begin{array}{l}0 \text { sepa- } \\
\text { rated. } \\
\text { in eqs. }\end{array}$} \\
\hline Name. & Formula. & & & & \\
\hline Asparagine & - $\mathrm{C}_{8} \mathrm{~N}_{2} \mathrm{H}_{10} \mathrm{O}_{8}$ & 8 & 4 & 2 & 12 \\
\hline White indigo & . $\mathrm{C}_{16} \mathrm{~N} \quad \mathrm{H}_{6} \mathrm{O}_{2}$ & 16 & 3 & 1 & 33 \\
\hline Amygdaline & - $\mathrm{C}_{40} \mathrm{~N} \quad \mathrm{H}_{27} \mathrm{O}_{22}$ & 40 & 24 & 1 & 82 \\
\hline Nicotine. & . $\mathrm{C}_{20} \mathrm{~N}_{2} \mathrm{H}_{14}$ & 20 & 8 & 2 & 48 \\
\hline Coniine & . $\mathrm{C}_{16 \mathrm{~N}} \mathrm{H}_{15}$ & 16 & 12 & 1 & 44 \\
\hline Morphine & - $\mathrm{C}_{34} \mathrm{~N} \quad \mathrm{H}_{19} \mathrm{O}_{6}$ & 35 & 16 & 1 & 80 \\
\hline Quinine & - $\mathrm{C}_{40} \mathrm{~N}_{2} \mathrm{H}_{24} \mathrm{O}_{4}$ & 40 & 18 & 2 & 94 \\
\hline Strychnine & . $\mathrm{C}_{42} \mathrm{~N}_{2} \mathrm{H}_{22} \mathrm{O}_{4}$ & 42 & 16 & 2 & 96 \\
\hline Caffeine . & . $\mathrm{C}_{16} \mathrm{~N}_{4} \mathrm{H}_{10} \mathrm{O}_{4}$ & 16 & 0 & 4 & 28 \\
\hline
\end{tabular}

The table will sufficiently illustrate the formation of the different classes of nitrogenised compounds which it embraces, namely, indifferent bodies, bitter compounds, colouring matters, volatile and fixed organic bases. They all contain very much less oxygen than the carbonic acid and water from which they have been derived, and some of them none at all. But there is no doubt that they are formed mediately, not immediately, and possibly from acids or sugar, acting on ammonia with deoxidation, the acids or sugar having been previously formed from carbonic acid and water by deoxidation.

There is a small but pretty widely diffused group of compounds containing sulphur, but no nitrogen. These are oils, and, like almost all organic compounds of sulphur, they have a fetid alliaceous odour. Such are the oils of garlic, and probably of hops, of Polygonum hydropiper, and of Arum maculatum. The only one yet analysed is the oil of garlic, $\mathrm{C}_{6} \mathrm{H}_{5} \mathrm{~S}$, which may be viewed as the sulphuret of the radical allyle, or acryle, $\mathrm{C}_{6} \mathrm{H}_{5}$, isomeric, but not identical, with propionyle, $\mathrm{C}_{6} \mathrm{H}_{5}$, the radical of propionic or propylic acid. It is of course easily derived from 6 eqs. $\mathrm{C} \mathrm{O}_{2}, 5$ eqs. $\mathrm{H} \mathrm{O}$, and 1 eq. $\mathrm{S} \mathrm{O}_{3}$, for these, losing 20 eqs. of oxygen, would yield $\mathrm{C}_{6} \mathrm{H}_{5} \mathrm{~S}$. It is very probable that the oils of onions and leeks contain similar compounds, perhaps homologous ones, such as sulphuret of formyle, $\mathrm{C}_{2} \mathrm{H}_{3} \mathrm{~S}$, or sulphuret of acetyle, $\mathrm{C}_{4} \mathrm{H}_{3} \mathrm{~S}$, or sulphuret of butyryle, $\mathrm{C}_{8} \mathrm{H}_{7} \mathrm{~S}$.

There is one remarkable oil, that of mustard, which contains both sulphur and nitrogen. It is, in fact, as has been already fully explained, the sulphocyanide of allyle or acryle, $\mathrm{C}_{6} \mathrm{H}_{5}+\mathrm{C}_{2}$ $\mathrm{N} \mathrm{S}_{2}=\mathrm{C}_{8} \mathrm{~N} \mathrm{H}_{5} \mathrm{~S}_{2}$, and it may be deduced from 8 eqs. $\mathrm{C} \mathrm{O}_{2}, 2$ eqs. $\mathrm{H} \mathrm{O}, 1$ eq. $\mathrm{N} \mathrm{H}_{3}$, and 2 eqs. $\mathrm{S} \mathrm{O}_{3}$, simply by the loss of 24 eqs. of oxygen, or the whole oxygen they contain.

We now come to the most complex of all the organic products, namely, the albuminous or sanguigenous compounds, albumen, fibrine, and caseine, which are the highest in the vegetable scale, 
and the production of which, both as essential to the seeds, and as furnishing sanguigenous food for man and animals, is the chief object of vegetation. They contain, as we have seen, five combustible elements, carbon, hydrogen, nitrogen, oxygen, and sulphur, along with phosphates, essential to their existence. They cannot, therefore, be formed without a supply of phosphates, and as their presence is indispensable both to the cell and to its contents, plants cannot grow in a soil devoid of phosphates. In such a soil seeds will germinate, and produce a few leaves at the expense of the' seed, or of the first leaves, which die off. But here they stop, and however abundant the supply of carbonic acid, water, ammonia, sulphuric acid, silica, potash, common salt, lime, \&c., yet if phosphoric acid be absent, the plant soon withers, without having produced seeds. When supplied with phosphates, plants grow vigorously and produce abundant fruit, and having done so, either die, or become torpid for the winter, after losing their leaves. So close is the connection between phosphates and sanguigenous matter, that the seeds, where the latter accumulate, yield, when burned, ashes formed chiefly, sometimes entirely, of phosphates; while the ashes of wood, straw, or leaves, contain chiefly carbonates and silica.

We have seen that these compounds are very complex, not only containing five organic elements, but containing a very large number of these in one molecule. Thus the smallest molecule indicated by analysis * contains of

\begin{tabular}{|c|c|c|c|c|c|c|}
\hline & Carbon. & $\begin{array}{l}\text { Hydrogen. } \\
\text { Atoms. }\end{array}$ & $\begin{array}{c}\text { Nitrogen. } \\
\text { Atoms. }\end{array}$ & Sulphur. & $\begin{array}{l}\text { Oxygen. } \\
\text { Atoms. }\end{array}$ & $\begin{array}{l}\text { Total. } \\
\text { Atoms. } \\
\end{array}$ \\
\hline Alb & . 216 & 169 & 27 & 2 & 68 & 482 \\
\hline Case & - 288 & 228 & 36 & 2 & 90 & 544 \\
\hline Fibrine . & - 216 & 169 & 27 & 2 & 68 & 482 \\
\hline
\end{tabular}

The fibrine in the table is vegetable fibrine, which agrees with that of flesh or sarcofibrine, in being apparently isomeric with

* The formulæ here given to the sanguigenous bodies are not given as certain, but only as a near expression of the results of the best analyses. We have already, in speaking of albumen, fibrine, \&c. mentioned not only these formulæ, but more simple ones proposed by Hunt and others. The latter, however, agree less closely with analysis. It is certain only that the true formulæ are not yet known, but that they are very complex, and in the tables which follow, as well as others farther on, we merely use these formulæ for the purpose of illustration. Any others corresponding equally well with analysis, might be employed; but as no additional certainty has been obtained in regard to the true formulæ, those used in the last edition of this work are retained.

It is, however, necessary to remind the reader that caseine, which is here supposed to differ materially in composition from albumen and fibrine, is now believed by many to be isomeric with them in the combustible or organic part; in fact to be albuminate of potash. But as this is still uncertain, the formula here given to caseine is left as it was. 
vegetable albumen. (Blood fibrine or hematofibrine is said to be a different substance.) Now, we can easily suppose these bodies to be formed from sugar, $\mathrm{C}_{12} \mathrm{H}_{12} \mathrm{O}_{12}$, that is, dry grape sugar, along with ammonia and sulphuric acid. Thus,

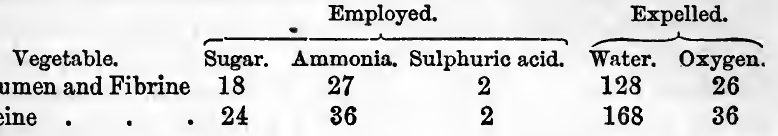

Blood fibrine is not formed in vegetables, and is therefore omitted here. We can also easily deduce the formulæ of vegetable albumen and fibrine, and that of caseine, from carbonic acid, water, ammonia, and sulphuric acid, by deoxidation. In fact, the formation of sugar is only the first or early part of the process, supposed to occur before ammonia is concerned.

\begin{tabular}{|c|c|c|c|c|c|}
\hline \multirow[b]{2}{*}{$\begin{array}{l}\text { Vegetable. } \\
\text { Albumen and Fibrine }\end{array}$} & \multicolumn{3}{|c|}{ Employed. } & \multirow[b]{2}{*}{$\begin{array}{c}\mathrm{SO}_{3} \\
2\end{array}$} & Expelled \\
\hline & $\begin{array}{l}\mathrm{CO}_{2} \\
216\end{array}$ & $\begin{array}{r}\text { 프 } \\
88\end{array}$ & $\mathrm{~N} \mathrm{H}_{27}$ & & $\begin{array}{l}\text { Oxygen. } \\
454\end{array}$ \\
\hline Caseine & 288 & 120 & 36 & 2 & 612 \\
\hline
\end{tabular}

The following diagram will illustrate the process, viewed in two successive stages, the first being the formation of sugar.

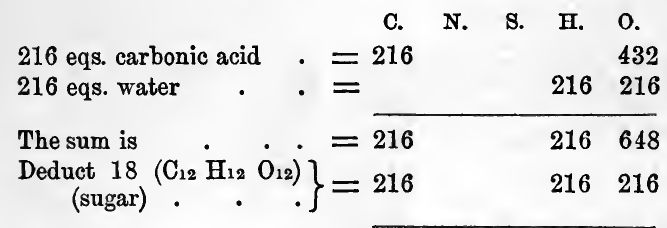

The difference is . .

432 eqs. of oxygen expelled in producing 18 egs. of sugar.

c. N. S. H. 0 .

18 eqs. sugar $\mathrm{C}_{12} \mathrm{H}_{12} \mathrm{O}_{12} \quad \cdot=216$

Add 27 eqs. ammonia $\mathrm{N} \mathrm{H}_{3}=$

And 2 eqs. sulphuric acid $\mathrm{S}_{3}=$

$216 \quad 216$

And 2 eqs. sulphuric acids $\mathrm{O}_{3}=$

276
The sum is
Deduct 128 eqs. water $\mathrm{H} \mathrm{O} .=$

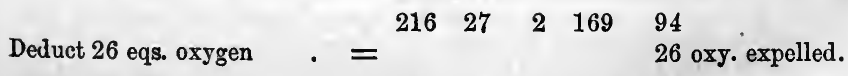
Remains Albumen $\quad . \quad=\quad \begin{array}{lllll}216 & 27 & 2 & 169 & 68\end{array}$


Thus we see that if albumen be formed from sugar, there must be expelled 128 eqs. of water and 26 of oxygen; whereas, if it were formed immediately from carbonic acid and water, as explained in the second table, $454 \mathrm{eqs}$. of oxygen and no water would be given off. The reason of this is that albumen contains much less of bydrogen than of carbon, whereas sugar contains an equal amount of both, as well as of oxygen, and the excess of hydrogen in sugar must be given out in the form of water, while, if carbonic acid and water could directly yield albumen (with ammonia, \&c.) only 88 eqs. of water would be necessary to 216 of carbonic acid and 27 of ammonia.

We have now traced the formation of all classes of vegetable products, and in doing so, two points are specially worthy of notice; first, that plants possess the power of building up, from comparatively simple molecules, such as are more complex, and that this complexity increases as we rise in the scale of vegetable products, till we come to those substances which form the vegetable tissue, such as woody fibre or cellulose (which is $\mathrm{C}_{12} \mathrm{H}_{10} \mathrm{O}_{10}$, or perhaps a multiple of these numbers. The composition of starch, which is semi-organised, is the same as that of cellulose, $\mathrm{C}_{12} \mathrm{H}_{10}$ $\mathrm{O}_{10}$ ), and albumen, caseine or fibrine, which are not only essential to the vegetable cell, but destined to form animal tissues, are greatly more complex even than this. Secondly, that in every part of the vegetable process, in the formation of every form of vegetable products, of acids, neutral bodies, cellulose, starch, fats, oils, resins, bases and sanguigenous matters, one character universally prevails, that, namely, of deoxidation of the materials and liberation of the oxygen. When we deoxidise any substance in the laboratory, we do it by causing the oxygen to combine with another body; but vegetables, while they produce all matters essential to their own development and to the life of animals, give out, in doing so, the oxygen which is removed, and thus plants cannot grow without, as a necessity of their life, restoring to the air the oxygen withdrawn from it by the life of animals. We shall see that the chief processes going on in the animal body, which produce results equally varied and wonderful, are, in both the above points, of an opposite nature.

It would seem as if deoxidation were somehow favourable to the coalescence of smaller molecules to form larger and more complex ones, for in almost all of those cases in which we have succeeded in building up, as the plant does, complex atoms from simpler, it is by deoxidation. But these cases are still few, and we have not yet learned to produce in this way natural vegetable products, apparently because we cannot set imitate either the vegetable cell or the peculiar vegetable process of deoxidation; 
but are compelled to use coarser methods. We are more fortunate in the destruction or decomposition of complex organic products, for in this way, and especially by processes of oxidation, we have produced many substances identical with, and many others perfectly analogous to, vegetable products, but invariably less complex than the substances from which they are obtained. In this we imitate the destructive animal processes, while we fail to imitate the constructive process, whether of vegetable or animal life, the latter requiring the aid of the vital force to modify chemical action; the former depending chiefly, if not entirely, on chemical causes within our reach.

In vegetation, water acts, not only by its elements, which are required, along with those of carbonic acid, ammonia, and sulphuric acid, to yield vegetable products, but also as a solvent for the essential mineral matters derived from the soil, all of which enter the plant, dissolved in water, or rather in a solution of carbonic acid in water.

of these mineral substances, the most important are the alkalies, potash, and soda ; the alkaline earths, lime and magnesia ; oxide of iron; silicic acid; chlorides of sodium and potassium; in sea plants also the iodides of some metals; and the phosphates of lime and magnesia. It is as a solvent for lime, magnesia, oxide of iron, and the earthy phosphates and carbonates, that the carbonic acid taken up by the rain in passing through the air and the soil is so important; for all the other substances are soluble in pure water. And it is as a constant source of carbonic acid for this purpose in the soil that the presence of organic matter in the soil, or in the manure added to it, is so advantageous as experience shows it to be.

The alkalies and earth, oxide of iron, and silicic acid, found in fertile soils, are derived from the decay of rocks, and chiefly of felspar, the chief ingredient of granite, gneiss, mica slate, clay slate, hornblende rock, porphyry, greywacke, all slaty rocks, and all varieties of trap and volcanic formations. Many of these are very rapidly disintegrated, as some granites and slates, and the lava beds of Vesuvius, and yield at once a fertile soil. Others being more slowly acted on by the weather, yield soils of inferior quality, full of undecomposed felspar, which is quite insoluble. In such cases, the addition of quick or slaked lime has the effect of hastening the decomposition of the felspar, and rendering its elements soluble. This is the true explanation of the action of lime on stiff clay soils, which contain disintegrated, but not much decomposed felspar. There is found on the sides of Aitna and Hecla a voleanic felspar rock, or one closely allied to felspar, which is called Palagonite. This is so readily decomposed, 
yielding abundance of soluble silica and of potash, the most essential elements (except the phosphates) of cereal crops, that it is actually used as a manure for poor soils, and will ere long be imported into other countries for that purpose. The fertility of soils, cateris paribus, depends on the proportion of decomposed felspar, or of such as is annually brought by the weather into the soluble form. The chief benefit derived from frequent ploughing, especially in stiff soils, is that it promotes the access of air and moisture, and thus hastens the decomposition of the felspar. The burning or calcination of stiff clay has the same effect; for calcined felspar is much more easily acted on by the air than it would have been previous to calcination.

Common salt is found everywhere, in every spring, and in every soil. Sulphate of lime or gypsum, which yields to plants their sulphur, is also very generally diffused in soils and in water, in which it is soluble. The quantity of gypsum in many soils is so large, that no benefit can be derived from the addition of it as manure, and the farmer who adds it in such cases, simply wastes the price of the gypsum. But where it is absent, or present only in small proportion, then the addition of gypsum produces wonderful results.

The phosphates, which are absolutely indispensable to the growth of all vegetables, are found, fortunately for mankind, universally diffused both in rocks and in soils. There are few rocks, such as granite, greywacke, \&c., in which minute scattered crystals of apatite or phosphate of lime may not be seen with the aid of a microscope; and the phosphates are present in every soil on which vegetation is found. The volcanic beds, which yield such fertile soils, are particularly rich in phosphates, and these salts also occur in many limestones (all of which have had an animal origin, as seen in shell marble, shell limestone, and chalk, which are often entirely formed of shells), in considerable quantity. Such limestones are of course the best for the farmer. But, on an average, the proportion of phosphates in any soil is so small, that one, or at most a few good crops, exhaust it, or nearly so ; that is, they exhaust the available supply of phosphates, \&c. There are soils, such as those of Vesuvius, of certain districts on the lower Danube, and of great part of the United States, which either have not been exhausted by centuries of cropping of wheat or tobacco, or have only become exhausted after 100 or 200 years of annual crops of the richest kind. But ordinary soils are comparatively poor, and hence the necessity for manure, that is, for restoring to the soil what has been removed in the crop, especially the phosphates and alkalies, the ingredients most easily exhausted. The excreta of animals, solid and liquid, being 
rich in these substances, are the best manure; because they contain the phosphates and alkalies in the proportions required for plants, and also in the proper forms, since they are derived from the plants on which the animals have fed, and are, indeed, nothing else than the ashes of those plants. But farm-yard manure is not valuable only as containing phosphates, but also as containing ammonia or sources of ammonia, and organic matter, which serves as a source of carbonic acid. The most essential elements of manure are the phosphates and carbonaceous matters ; for the latter supply a solvent, carbonic acid, for the phosphates both of the manure and of the soil; and if this solvent be supplied, and the soil or manure supply also phosphates, alkalies, and sulphates, the air will furnish ammonia, provided time be given. This has been practically demonstrated on a large scale by Liebig, who, by manuring a barren and worthless soil with phosphates, alkalies, silica, and sulphates alone, obtained excellent results with wood and other perennial crops; but found the result less favourable with cereals and other annual crops, which require a large supply of phosphates in a short time. He next addaed to the mineral manure sawdust, to yield carbonic acid, and now he obtained excellent results even for annual crops, although no ammonia was added, and the atmosphere supplied all the nitrogen. But it is certain that when the supply of phosphates is rapid, as it was here from the solvent action of the decaying sawdust, the air can supply any amount of ammonia, which cannot be assimilated without abundance of phosphates. The further addition of ammonia to the mineral manure and sawdust brought it to the state of farm-yard manure, and then the same result is obtained in a still shorter time-a point of much importance in temperate or cold climates. Of course, to render, as in this experiment, barren soil fertile, is an expensive process, from the necessity of adding to it so great an amount of mineral matter. But wherever a sufficient quantity can be added to it, without raising the cost of the improved barren soil to or above that of fertile soil, then, by the judicious cropping and manuring of the improved land, its artificial fertility, like that of a soil originally fertile, may be kept up from its own produce, aided by the action of the weather, and if economically procurable, by liming.

Farm-yard manure, solid and liquid together, is the standard or model manure, and the object of all artificial manures is, in part or in whole, to imitate or replace it. The best sources of phosphates, next to farm-yard manure, are bone-dust and guano. Bone-dust is composed of phosphates and gelatine. Guano is the decaying residue of the excrements of sea-fowl fed on fish, 
and contains phosphates and salts of ammonia, with some carbonaceous matter. It is evident that both resemble farm-yard manure, more or less, and that both are deduced, like it, from the vegetable food of animals; for the fish consumed by the seafowl fed on other fish, which fed on anjmalculæ, which fed on sea-plants. Another valuable source of phosphates is that of beds of phosphate of lime, derived from extinct animals. There are such beds, of vast extent, in Spain : others have been found near the Rhine, and in Canada ; and beds more or less entirely composed of coprolites, the fossil excrements of fish and reptiles, fed on other animals, are abundant in England, as well as in other countries: These may be called fossil guano, and only differ from recent guano in having lost their ammonia and carbon. All these are extremely valuable as manures.

But we must never forget that the best and most economical of all manures is farm-yard manure, or, what is the same thing, the night-soil and urine of inhabited places. In large cities, the amount of this invaluable matter is prodigious ; it represents, in fact, as far as phosphates go, the whole food of the inhabitants; and yet, from ignorance or carelessness, the greater part of it is wantonly cast into the sea, from which a mere fraction is, at a great expense, recovered as fish and guano. There are, no doubt, some practical difficulties in the way of saving the. whole of the contents of our sewers in large cities; the ehief of which is, the enormous amount of water added to them in order to wash them away from our habitations. But the subject has at length been taken up in earnest, and there are no difficulties which may not be overcome. The bone-dust we import, at a high cost, from other countries, is lost to them, and is to us only a circuitous mode of recovering a small part of what we have thrown away. In process of time, no nation will consent to sell bone-dust, save at a price which will render its use impossible; and we must come to that to which the Chinese, with their practical sagacity, have come many centuries ago, namely, the principle of restoring to the land everything that is taken from it in the crops; the only principle on which agriculture can be permanently or profitably conducted on average soils. The amount of phosphates in our edible crops is far beyond anything ever seen in natural wild plants; and, therefore, the supply required by a dense population, and obtained in the excessive development of seeds and roots in cultivated plants, must be given to the soil in the shape of manure, the best being that derived from man, who consumes the crops. The rotation of crops, which takes advantage of the fact that one crop requires more of one ingredient and less of some other than another crop 
does, and the occasional use of fallow, which allows the weather to act and to render soluble a fresh supply of mineral matter, are only different branches of the best method of following out the great principle of agriculture above enunciated. The cereals and grass require more silica; turnips and potatoes more of the alkalies; peas, beans, clover, and other leguminosæ, more lime and sulphates; and thus may be alternated with great advantage, although all require a full supply of phosphates. For details on these important subjects, which our space forbids us to enter upon more fully, but the principle of which we have endeavoured to explain, the reader is referred to Liebig's Agricultural Chemistry, to the third edition of his Letters on Chemistry, and to a small work recently published by him, the "Principles of Agricultural Chemistry."

A mistake is often made in the use of artificial manures, which is caused by ignorance of the principles on which they act. The farmer has added guano, bone-dust, or gypsum, and has obtained a very heavy crop. He repeats the dose, and a failure ensues. The reason is, that the first addition had enabled the crop to assimilate all the available alkalies, silica, or sulphates present in the soil, or the greater part of them, so that, on the second addition of phosphates, there was a great deficiency of one, or another, or of all these matters ; and as all are equally essential, the deficiency of any will cause failure. On restoring the balance, success again will be the result. Good farm-yard manure is the only one not liable to this cause of failure, because it is the only complete manure; the others being more or less partial, or confined to one substance. Of course the same risk of failure exists, if the artificial manure added at first with success has been potash, or salts of ammonia; for the addition of any of these, if previously deficient on the soil, will enable the crop to take up a large proportion of the others, or of phosphates, and may exhaust it of any one for the time. This ought particularly to be attended to, as when a special manure acts, it does so by exhausting the soil, pro tanto, of all the other necessary mineral ingredients.

The ashes of wood, peat, or coal, are valuable as manure, containing the mineral elements of wood, and of those plants from which peat and coal have been formed; mixed, in coal, with clay and sand. The ashes of straw are very valuable, especially for grass or the straw of corn. In general, the ashes of any crop are the best mineral manure for that plant. The burning of heath, or of the turf pared from moor-land soil, is useful in two ways; first, by the action of heat on the felspar in the soil;

* Walton and Maberly, Loudon. 
secondly, by supplying the ashes. By examining the ashes of the plants which grow naturally on any soil, we can easily discover what are the most valuable constituents of that soil, and thus judge of the proper addition to it. The ashes of any crop are identical with the ashes of the excreta of the animals fed on that crop.

The addition of ammonia to any soil, in the manure, can only be of use in so far as that soil contains, in an available form, a due proportion of alkalies, silica, phosphates, common salt, oxide of iron, and sulphates; and the same is true of every special manure. A soil, if totally deficient in any one necessary ingredient, will be absolutely barren. Thus, if all the other matters be present, and phosphates, or sulphates, or alkalies be alone absent, no plant can grow there; but the addition of the ingredient which is wanting will convert the soil into a very fertile one. If a soil be fertile for turnips or potatoes, and will not yield corn or hay, it is deficient in soluble silica, essential to straw. If a soil yield grass and straw, but not corn, as sometimes happens, this may arise from deficiency of magnesia, which, in the form of phosphate, is essential to the husk of grain, so that in its absence no perfect seed is formed. Bran and urine, which both contain phosphate of magnesia, are on that account, and on many others, very valuable for corn. A soil on which corn grows, while clover or peas do not, is probably deficient in available lime or in gypsum. But in some plants the absence of one base may be supplied by another, potash by soda, lime by magnesia. When a soil is deficient in most, or in all, of the necessary mineral matters so often mentioned, it is hopelessly barren. Such is the case with the soil of districts where quartz rock prevails, or where the soil has been derived from that rock, as is seen in part of the north-west of Ireland. But happily such soils are rare, because such rocks are rare in any great extent. The deserts of Asia and Africa are barren, not because the soil is devoid of the mineral food of plants, but because of the want of water. Even in the desert, every spring is surrounded by an oasis of vegetation.

We have said that the presence of decaying organic matter in the soil is useful in two ways; as a source of carbonic acid, which principally acts, dissolved in water, as a solvent for lime, magnesia, and phosphates; and as a source of ammonia. Both of these enable the plant to assimilate the necessary mineral matter in a far shorter time than it could without them; and this is a point of very great importance in our climate, where the summer is short, compared to that of tropical regions. To illustrate the power of water containing carbonic acid to dissolve phosphate of lime, insoluble in pure water, I may state that I found the 
carcase of a pig, buried on the slope of a hill, which was moist and undrained, when taken up after fourteen years, to have shrunk into a flat cake (the change, most probably, had been effected in a much shorter time), which was entirely composed of fatty acids from the fat of the animal. The muscles and membranes, nerves and vessels, had putrified and disappeared, and not a trace of bone-earth was to be found. The whole had been dissolved by the water percolating through the body, and had thus been carried down to the lower ground. I found the water, like all water that passes through soils, to contain carbonic acid, and this had been the solvent. Hence, if cemeteries are placed in a high situation, the bone-earth is rapidly carried down to the vallies in a moist climate. In a dry situation, bones remain unaltered for eenturies, as in the sandy soil of the field of Cannæ, on which the bones of those killed in that battle are often found to this day entire, though having lost much of their animal matter. The solvent power of carbonic acid must act in every soil, and hence the value of humus on the soil and of decaying organic matter in manure.

We have now examined the process of vegetation, its results, and its indispensable conditions. It is perhaps worth while to remind the reader of the very important part played by the solar rays in vegetation. That essential process, by which the plant decomposes carbonic acid and water, liberating their oxygen, can only take place under the influence of luminous rays; for although, on the prevalent undulation theory, heat and light are both regarded as vibrations of the same kind, only differing in velocity, light only appearing when the vibrations amount to from 400 to 800 billions in a second, and non-luminous heat when they fall short of 400 billions in a second,-although, therefore, light and heat are both motion, yet non-luminous heat cannot enable a plant to decompose carbonic acid and water. Now, if we consider that the ehemical effect of light, light being motion, is a mechanical effect, and that the mechanical power necessary to decompose carbonic acid and water is enormous, we see that the amount of mechanical effect produced in growing vegetables by the solar rays must be very great indeed. In fact, it has been calculated that the mechanical foree derived from the sun by the amount of wood growing in a year on one square foot of surface corresponds to that which would be required to raise a weight of $486,000 \mathrm{lbs}$. to the height of one foot; and this is only $\frac{1}{12}$ th of the whole effect of the sun's rays, of which only $\frac{1}{5}$ th reaches the plant, and half of that is lost. The recent investigations of Carnot, Joule, W. Thomson, and others on the relations of heat, light, and mechanical effect, tend, indeed, to show 
that all the mechanical effect (except in a few cases, such as water and tide mills, volcanic action, and a few more), which is available to man, is derived from the solar rays, directly or indirectly. We have seen that a vast amount is expended in producing vegetables, and it is obvious that the whole mechanical power of the animal body is drawn, mediately or immediately, from that which has been stored up in plants; while the power of the steamengine is derived from the conversion into heat of the mechanical force stored up in the fuel, whether wood or coal. This force, as we have seen, proceeds directly from the solar rays.

Vegetables, as we have seen, cannot possibly grow and form seeds, without at the same time producing, as parts of their own structure, the food of animals in its two great forms, of non-nitrogenous or respiratory food, namely, starch, sugar, gum, and oils; and nitrogenous, plastic, or sanguigenous food, namely, albumen, fibrine, and caseine. The former, which do not enter into the formation of blood, save, to a small extent, oils or fats, may exist free from ashes or mineral matter, although these are necessary to their production; but the latter cannot exist, in the organism, at least, without containing at all events phosphates. And thus, by the beautiful arrangement which renders albumen, fibrine, and caseine indispensable to the development of plants, and to the production even of starch, sugar, and fat; and which has rendered the presence of phosphates indispensable to the existence of albumen, fibrine, and caseine ; vegetables cannot grow, nor produce the plastic food of animals, or that which yields blood, without at the same time producing, and supplying to animals, the earthy matter required for their bones, and in a smaller proportion, for the blood and for all the tissues. If albumen, \&c., could be formed without phosphates, or if even blood and muscle could exist without phosphates, still animals could not exist or be formed without bone-earth. As it has been arranged with perfect wisdom however, the mere fact that a plant has grown, necessarily implies that it contains the materials required to support animal life, provided, of course, it be not a poisonous plant, and probably there is no plant which may not serve as food for some animal.

The food of animals, then, consists of respiratory and sanguigenous matter. It is first masticated and mixed with the saliva, which includes in its viscid substance a quantity of air. It is then introduced into the stomach, where digestion takes place. The saliva, which is alkaline, and contains a peculiar modification of albumen, called ptyaline, has a peculiar power, not possessed by the gastric juice, of converting starch into sugar, which is an essential part of digestion. It also conveys oxygen, in the form 
of inclosed air, to the stomach, where the oxygen probably assists in producing the peculiar change in the gastric juice, or in the epithelial matter suspended in it, by which the gastric juice dissolves albumen, fibrine, or caseine. The gastric juice, which is acid, and contains phosphoric, lactic, and hydrochloric acids, has the very remarkable property of dissolving coagulated albumen, as well as fibrine and caseine, at the temperature of the body, but has no action on starch. Since it acts as well after filtration as before, it is probable that the ferment it contains is a dissolved one, and not, as Frerichs states, suspended epithelial cells.

When the food has been digested to a certain extent, it leaves the stomach, and enters the intestines, where it is soon after mixed with the bile. It is also continually mixed with the intestinal secretion, a fluid which is viscid, alkaline, and contains no albumen. It possesses, however, both the power of the saliva to dissolve starch as sugar, and that of the gastric juice to dissolve sanguigenous matter, and it evidently contributes to the completion of the digestion of the starch and albumen, \&c., of the food. The bile, as has been proved, is nearly all reabsorbed in its passage through the intestines, and the pancreatic juice, which also mixes with the food, has been said by Bernard to be the agent of the solution of fat or oil, which it is said to convert into an emulsion, capable of entering the absorbents. It is certain that the pancreatic juice can resolve neutral fat oils into glycerine and fatty acids, and that this action is prevented by the gastric juice, and favoured by bile. But the latest researches tend to prove that the means by which fat is absorbed are not yet known, although the bile and pancreatic juice, especially the bile, in some way contribute to it. Oil is not absorbed by diffusion or endosmosis, nor are the fatty acids, when separated; and the absorbed fat is neutral. Whatever be the means, the fat, if not exceeding a certain amount, is certainly absorbed, and thus, when the food has passed through the stomach and intestines, all the starch, albumen, \&c., and fat, has been taken up, as well as nearly the whole of the bile. The insoluble residue, or fæces, consists of woody fibre, a little colouring matter, supposed to come from bile, and the insoluble earthy salts of the food, as well as of the effete tissues, while the soluble salts, entering the circulation, are expelled in the urine.

The fibrine and caseine of the food appear in the dissolved food, or chyle, almost entirely as albumen, along with the albumen of the food; and it would seem that albumen, in the blood, as in the egg, is the true source of the tissues. Chyle contains very little fibrine, and blood only about 1 or 2 parts in 1000 , and there is reason to think that fibrine is rather, in 
the blood, a product of the destruction of the tissues, than a substance from which they are formed. The chyle, along with the lymph, is mixed with the blood and passes through the lungs, where it probably acquires its colour. The red corpuscles of the blood are nucleated cells, or rather the nuclei of such cells, which abound, in a colourless form, in lymph, and are even found without colour in the blood, especially in a diseased state. The blood is the most complex of all fluids, containing not only the food of all the tissues, but the products of their oxidation. For in the lungs the venous blood takes up oxygen, and gives out carbonic acid. It then goes, as arterial blood, to the heart, whence it is sent to every part of the body, carrying with it the oxygen, which it gradually exchanges for carbonio acid, formed by the oxidation of the tissues, but, as the products of oxidation are constantly removed from it by the excretory and secretory organs, they are always in small proportion. In this brief sketch we have not alluded to the presence of glucose in the blood and liver, because the subject is still but little understood. But under the blood we have mentioned that the glucose is transformed into lactic acid, which is oxidised and burnt off in the blood.

Let us now endeavour to follow some of the changes, first in digestion, and then in the circulation. The reader will bear in mind that

$\left.\begin{array}{l}\text { Vegetable albumen, } \\ \text { and vegetable } \\ \text { fibrine }\end{array}\right\} \begin{aligned} & \text { are isomeric } \\ & \text { with }\end{aligned}\left\{\begin{array}{l}\text { Albumen of blood } \\ \text { Albumen of flesh } \\ \text { Fibrine of flesh }\end{array}\right\} \begin{gathered}\text { all being } \mathrm{C}_{216} \\ \mathrm{~N}_{27} \mathrm{~S}_{2} \mathrm{H}_{160} \\ \mathrm{O}_{68} .\end{gathered}$

Consequently, when vegetable albumen and fibrine are digested into animal albumen, we may call it an isomeric transmutation. But when caseine, which is $\mathrm{C}_{288} \mathrm{~N}_{36} \mathrm{~S}_{2} \mathrm{H}_{228} \mathrm{O}_{90}$, is digested, it yields albumen and chondrine. Thus :

$\left.\begin{array}{r}1 \text { eq. Caseine } \\ +10 \text { eqs. Water }\end{array}\right\}$ may yield $\left\{\begin{array}{l}1 \text { eq. Blood albumen } \\ 1 \text {, Chondrine } \mathrm{C}_{72} \mathrm{~N}_{9} \mathrm{H}_{59} \mathrm{O}_{32}\end{array}\right.$

Thus all the sanguigenous vegetable food is converted into albumen. This albumen, if employed to form muscular fibre, has only to be again converted into the isomeric fibrine of flesh. But if albumen is to yield the fibrine of blood, the change may be as follows :

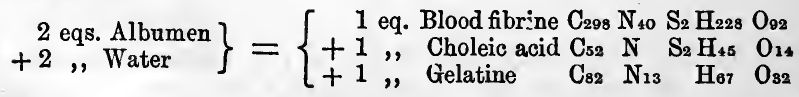

Here we see the blood-fibrine, which is most probably a product of the destruction of albumen or of fibrine of flesh, is 
produced along with gelatine and one of the acids of bile. Another similar change, yielding the other acid of bile, may be

$$
\left.\begin{array}{l}
2 \text { eqs. Albumen } \\
+6 \text { eqs. Water }
\end{array}\right\}=\left\{\begin{array}{l}
1 \text { eq. Blood fibrine } \\
+1 \text { eq. Cholic acid } \mathrm{C}_{52} \mathrm{~N} \mathrm{H}_{43} \mathrm{O}_{12} \\
+1 \text { eq. Gelatine } \\
+2 \text { eqs. Sulphuric acid. }
\end{array}\right.
$$

We may suppose these two processes to be joined; and then 4 eqs. of albumen with 8 of water, will yield 2 eqs. of bloodfibrine, 2 of gelatine, 1 of choleic acid, 1 of cholic acid, and 2 of sulphuric acid. Indeed, blood-fibrine, or hematofibrine, is probably only formed in consequence of the production of gelatine and bile, and it may be regarded as albumen, half converted into gelatine and bile; for we have seen it formed with gelatine and choleic acid from albumen and water, and

$$
\left.\begin{array}{c}
1 \text { eq. Hematofibrine } \\
+18 \text { eqs. Water }
\end{array}\right\}=\left\{\begin{array}{c}
3 \text { eqs. Gelatine } \\
+1 \text { eq. Choleic acid. }
\end{array}\right.
$$

Consequently, whenever gelatine and bile have been formed, hematofibrine is to be looked for. We see, then, first, that when a young animal is fed on caseine (milk), the formation of albumen, necessary for the tissues, is accompanied by that of chondrine, the substance of which cartilages and the soft part of bones are formed; secondly, that when gelatine, the matter required for membranes, is formed from albumen, we obtain along with it the acids of bile, essential for another purpose. If chondrine be required from albumen, it may be obtained as follows :

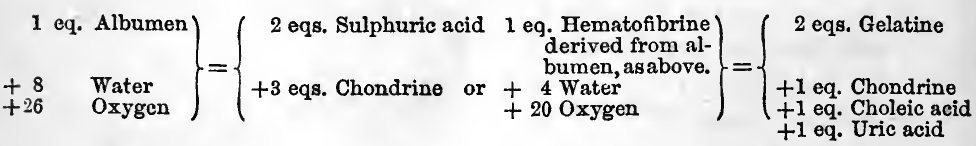

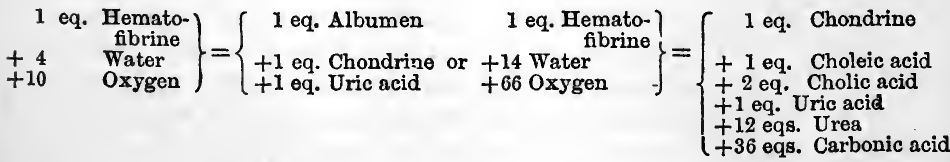

Here we find that every one of the above changes is one of oxidation, and that, as it proceeds farther, we come to the constituents of the urine, and to carbonic acid. This, then, is the characteristic feature of the changes in the animal body. As 
long as albumen is to be formed, and even, to a certain extent, in the formation of gelatine and chondrine, these three bodies being the chief materials of the tissues, the addition of water alone suffices; but when oxygen comes into play, the changes strike deeper, and we obtain the constituents of bile and of urine, as well as carbonic acid, to be excreted by the lungs. When gelatine and chondrine have formed part of tissues, they, like albumen, become effete, and must be decomposed and removed. We have then:

1 eq. Chondrine $\} \quad 1$ eq. Cholic acid 1 eq. Gelatine $) \quad(1$ eq. Cholic acid $\left.\begin{array}{c}\text { probably by a } \\ \text { fermentation }\end{array}\right\}=\left\{\begin{array}{l}+2 \text { eqs. Uric acid and }+10 \text { eqs. Water } \\ +8 \text { " Water }\end{array}\right\}=\left\{\begin{array}{c}1 \text { eqs. Uric acid } \\ +12 \text { " Water }\end{array}\right.$

And we may also have, with albumen,

$$
\left.\begin{array}{rl}
1 \text { eq. } & \text { Albumen } \\
+10 \text { eqs. Water } \\
+56 \text { ", Oxygen }
\end{array}\right\}=\left\{\begin{aligned}
6 \text { eqs. } & \text { Choleic acid. } \\
+2 & \text { Cholic acid. } \\
+12 & \text { "rea. } \\
+36 & \text { Oarbonic acid. }
\end{aligned}\right.
$$

There are certainly many intermediate stages, but of these little is known. One, however, may be mentioned as probable, namely, the production from gelatine of kreatine, hippuric acid, water, and carbonic acid. Thus,

$\left.\begin{array}{c}1 \text { eq. Gelatine } \\ +58 \text { eqs. Oxygen. }\end{array}\right\}=\left\{\begin{array}{cl}3 \text { eqs. } & \text { Kreatine } \\ +2 & \text { Hippuric acid } \\ +12, & \text { Water, excreted by lungs and } \\ \text { kidneys. } & \begin{array}{ll}\text { excreted in the } \\ \text { urine. }\end{array} \\ +22, & \text { Carbonic acid, excreted by lungs. }\end{array}\right.$

Again, choleic acid fully oxidised will yield as follows :-

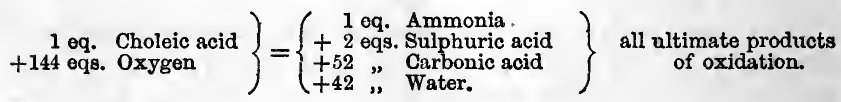

Cholic acid, which contains no sulphur, will yield

$$
\left.\begin{array}{c}
1 \text { eq. Cholic acid } \\
+122 \text { eqs. Oxygen. }
\end{array}\right\}=\left\{\begin{array}{c}
1 \text { eq. Ammonia. } \\
+52 \text { eqs. Carbonic acid. } \\
+40 \text {,. Water. }
\end{array}\right.
$$

When uric acid is produced, in warm-blooded animals, but a small part of it reaches the urine; because, from their structure, only soluble compounds can be excreted through the kidneys, 
ureters, bladder, and urethra. The uric acid is therefore further oxidised ; and

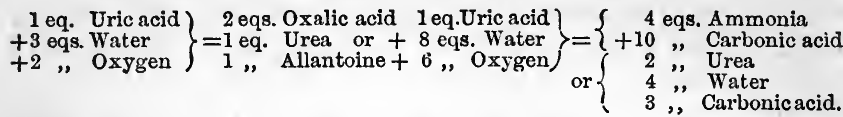

In this manner the insoluble uric acid is converted, by oxidation, into soluble products. Oxalic acid is obviously the result of imperfect oxidation, and meeting with lime in the bladder, forms the oxalate of lime or mulberry calculus. Allantoine, also a product of imperfect oxidation, is found in the allantoic fluid, the urine of the fetal animal, and in that of very young animals. With full oxidation of the uric acid, only urea, water and carbonic acid, or ammonia and carbonic acid, are formed. The ammonia, if in excess, throws down earthy phosphates in the bladder, and thus gives rise to the phosphatic calculi. The uric acid calculi, the commonest of all, depend on the deficiency of oxygen, or what is the same thing, excess of sanguigenous, and particularly of gelatinous food, as well as on sedentary habits, which diminish the amount of inspired air ; and lastly, on deficiency of alkalies, the presence of which is necessary to promote the oxidation of the uric acid.

In the changes we have thus briefly described, by which the tissues are wasted, the nitrogen of these tissues takes ultimately the form of urea, and thus the quantity of urea secreted in a certain time is a measure of the amount of change or waste of matter in the system. An adult man secretes, on an average, about 463 grains of urea, or rather more than 1 ounce avoirdupois in 24 hours. The quantity of uric acid in normal urine is so small in comparison, that it hardly amounts to $\frac{1}{1000}$ of the weight of the urea, and may therefore be neglected.

It has been proposed to observe and record the daily secretion of urea in health and disease, as an important fact in physiology and pathology, and for this purpose various methods have been proposed for determining the percentage of urea in urine directly and quickly. Perhaps the best is that of Liebig, which is founded on the fact, that urea forms with peroxide of mercury a white compound, insoluble in water. He employs a neutral and diluted solution of pernitrate of mercury, of known strength. This is added to a known volume of urine as long as it causes a white precipitate. But as the nitric acid set free prevents the complete precipitation, the liquid is now neutralised exactly by baryta water, and the pernitrate now causes a further white precipitate. 
These operations are repeated; until at last a yellow precipitate begins to appear, which is a proof that all the urea is precipitated. The volume of pernitrate employed being known, and previous experiments having ascertained how much urea is precipitated by 100 volumes of it, it is easy to calculate the urea present in the urine under examination.

Another form of this process is as follows. To the urine is added half its volume of a mixture of equal parts of the saturated cold solutions of hydrate of baryta and nitrate of baryta, which precipitates the whole of the sulphuric and phosphoric acids present. To the filtered liquid the neutral and diluted solution of pernitrate of mercury of known strength is added by degrees until a drop of the liquid, tested with carbonate of soda, gives' a yellow precipitate, which shows that a slight excess of pernitrate has been added.

In either case if any given volume of urine, say 10 cubic inches, requires 30 cubic inches of the normal solution of pernitrate, and if we have made this normal solution of such strength that 1 cubic inch of it corresponds to 1 grain of urea, then the 30 cubic inches employed will indicate 30 grains of urea in the 10 cubio inches of urine.

We have now to attend to the formation of caseine (in milk) from albumen or fibrine, isomeric with it, in the animal body. This change, like the others, depends in some degree on oxidation.

$$
\left.\begin{array}{rl}
2 \text { eqs. } & \text { Albumen } \\
+4 & \text { Water } \\
+6, & \text { Oxygen }
\end{array}\right\} \text { may yield }\left\{\begin{array}{l}
1 \text { eq. Caseine* } \\
1,, \text { Gelatine } \\
1, \text { Cholic acid } \\
1,, \text { Uric acid } \\
2 \text { eqs. Sulphuric acid. }
\end{array}\right.
$$

2 eqs. of albumen are necessary to yield one of caseine, because the latter compound contains more carbon, hydrogen, nitrogen, and oxygen than the former. We have already seen that when caseine, in the young animal, yields albumen, it also yields chondrine, these being the two substances most necessary for the young animal. Here we see that when milk is formed in the mother's body, it is accompanied by gelatine, cholic acid, uric acid, and sulphuric acid, to be employed in her own organism.

Let us now consider the albumen of eggs, which differs from that of blood, as follows :-

* On the supposition that caseine is as represented on p. 474. But it has been stated, that caseine is possibly the albuminate of potash. 


$\begin{array}{llll}\text { Blood albumen is } & \cdot & - & \mathrm{C}_{216} \mathrm{~N}_{27} \mathrm{~S}_{2} \mathrm{H}_{169} \mathrm{O}_{68} \\ \text { Egg albumen is } & \cdot & - & \mathrm{C}_{216} \mathrm{~N}_{36} \mathrm{~S}_{3} \mathrm{H}_{169} \mathrm{O}_{68}\end{array}$

The difference is 9 eqs. of nitrogen and 1 of sulphur more in egg albumen than in blood albumen. In the body of the fowl we may suppose that

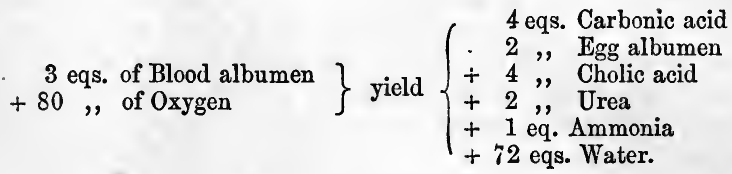

And during incubation, or in the stomach or circulation of an animal,

$$
\left.\begin{array}{l}
1 \text { eq. Egg albumen } \\
+1 \text {,. Sugar } \\
+10 \text { eqs. Water }
\end{array}\right\} \text { may yield }\left\{\begin{array}{l}
1 \text { eq. Blood albumen } \\
+4 \text { eqs. Urea } \\
+1 \text { eq. Ammonia } \\
+4 \text { eqs. Carbonic acid } \\
+3,, \text { Water. }
\end{array}\right.
$$

Of course fat and oxygen may supply the place of sugar, since, as we shall see, fat is sugar minus oxygen. Now, the yolk of egg is rich in fat, and is said also to contain sugar; while the food of animals, in addition to eggs, when they are used, always contains starch, sugar, or fat. And it is quite certain, that all the tissues are formed in the egg from egg albumen and the oil or sugar of the yolk, aided by oxygen; as also, that in the animal body, all the tissues may be formed, or blood produced, from eggs, especially with starch, sugar, or fat.

We have now considered, as fully as our space permits, the relations of the sanguigenous compounds to each other, and to the tissues, and we have seen that these compounds are formed in plants, and that in animals they are mutually convertible, sometimes by an isomeric transmutation, as when fibrine is dissolved into albumen-a process capable of being imitated out of the body, and at other times with the aid of water, or of water and oxygen. In no case is free oxygen given out, as in plants; but the final result is, that the oxygen taken up in the lungs is given out as carbonic acid and water.

We have not entered into details as to the colouring matter of the blood or hematosine. This is albuminous, and contains iron. Hence the use of iron in the food. We do not know its exact composition, nor where it is formed. But we do know that the purest and whitest blood fibrine also contains iron, and hence 
possibly one function of this hematofibrine is to yield the material for hematosine.

There remains one more substance, which certainly assists mechanically in the formation of tissues, and probably is essential to the production of cells and of blood corpuscles. This is fat, which we have seen to be non-nitrogenous, and to be formed abundantly in the vegetable kingdom. Many kinds of food contain fat or oil ready formed, and, as we have seen, it enters the blood in some obscure way, to which the bile and probably the pancreatio juice contribute. It is never absent from blood. But fat, which is not a sanguigenous compound, can also be produced from starch and sugar in the animal body. The bee forms wax, a true fat, from honey ; and animals abundantly fed with grain, which contains only traces of fat, and so situated as to suffer from deficiency of oxygen, become rapidly loaded with fat. Thus ortolans, when kept in confinement, which reduces their respiration, and in the dark, which leads them to feed voraciously, by a natural instinct, on the admission of light, may thus be led to feed many times a day on grain, and rapidly become absolute balls of fat. When geese are prevented from moving, and crammed with maize or wheat, their livers become so charged with fat as finally to be unfit for their proper functions, and greatly enlarged. And stall-fed cattle are notoriously much fatter than such as have to travel far in search of food, while wild animals are almost always lean.

These facts prove that fat is formed from starch, which first becomes sugar, in the animal body. In this particular the animal resembles the plant; but in the animal, the oxygen separated from sugar to convert it into fat, is not given out, as in the plant. It is either at once converted into carbonic acid and water, or added to the oxygen taken up from the air, and used for the same purposes. Thus we see, that the power of the animal to deoxidise sugar, which he employs when oxygen from the air is deficient, supplies that deficiency. To illustrate this, the reader must be reminded that fats and fat oils are compounds of oxide of glyceryle or oxide of lipyle, $\mathrm{C}_{6} \mathrm{H}_{4} \mathrm{O}_{2}$, or $\mathrm{C}_{3} \mathrm{H}_{2} \mathrm{O}$, with fatty or oily acids, chiefly of the series $\left(\mathrm{C}_{n} \mathrm{H}_{n}\right)_{2}$, $\mathrm{O}_{4}$. Such a neutral fat, consisting of margaric acid and oxide of lipyle, is the chief ingredient of human fat, and is called margarine. - If the acid be oleic acid, the fat is called oleine; if the acid be butyric acid, butyrine, and so on. Now every fatty or oily acid, as well as oxide of lipyle, may be derived from sugar by deoxidation, and in the case of oleic acid, $\mathrm{C}_{36} \mathrm{H}_{34} \mathrm{O}_{4}$, and oxide of lipyle, $\mathrm{C}_{6} \mathrm{H}_{4} \mathrm{O}_{2}$, both of which contain an excess of carbon over hydrogen, by deoxidation and loss of water. Thus, 
1 eq. Sugar $\mathrm{C}_{12} \mathrm{H}_{12} \mathrm{O}_{12}$, losing 4 eqs. of oxygen, yields 2 eqs. propylic acid $=2\left(\begin{array}{lll}\mathrm{C}_{8} \mathrm{H}_{6} \mathrm{O}_{4} \\ )\end{array}\right)$.

1 ," Sugar $\mathrm{C}_{12} \mathrm{H}_{12} \mathrm{O}_{12}$, losing 4 eqs. of oxygen and 4 eqs. of water, yields 2 eqs. oxide of lipyle $=2\left(\mathrm{C}_{8} \mathrm{H}_{4} \mathrm{O}_{2}\right)$.

3 eqs. Sugar $\mathrm{C}_{36} \mathrm{H}_{3 e} \mathrm{O}_{3 e}$, losing 32 eqs. of oxygen, yield 1 eq. stearic acid $=\mathrm{C}_{36} \mathrm{H}_{30} \mathrm{O}_{4}$.

3 ," Sugar $\mathrm{C}_{30} \mathrm{H}_{36} \mathrm{O}_{3 e}$, losing 30 eqs. of oxygen and 2 eqs. of water, yield 1 eq. oleic acid $=\mathrm{C}_{36} \mathrm{H}_{34} \mathrm{O}_{4}$.

17 , Sugar $17\left(\mathrm{C}_{12} \mathrm{H}_{12} \mathrm{O}_{12}\right)$, losing 180 eqs. of oxygen, yield 6 eqs. margaric acid $=6\left(\mathrm{C}_{34} \mathrm{H}_{34} \mathrm{O}_{4}\right)$.

This table suffices to show that all fatty acids of the acetic series, such as propylic, margaric and stearic acids, are derived from sugar by simple deoxidation, and that oleic acid and oxide of lipyle are derived from sugar by the loss of oxygen and water. Let us now take one fat, margarine, which is composed of margaric acid and oxide of lipyle.

\begin{tabular}{|c|c|c|c|c|c|}
\hline & $\begin{array}{l}\text { Sugar. } \\
17 \text { eqs. yield }\end{array}$ & $\underset{6}{\text { Margaric acid. }}$ & Oxide of lipyle. & $\begin{array}{c}\text { Oxygen. } \\
180\end{array}$ & Water. \\
\hline & 3, , yield & & 6 & 12 & 12 \\
\hline Consequently & $20 \quad$ yield & 6 & 6 & 192 & 12 \\
\hline
\end{tabular}

that is, 6 eqs. of margarine, 12 of water, and 192 of oxygen.

Let us now revert to the oxidation of albumen; and we find that

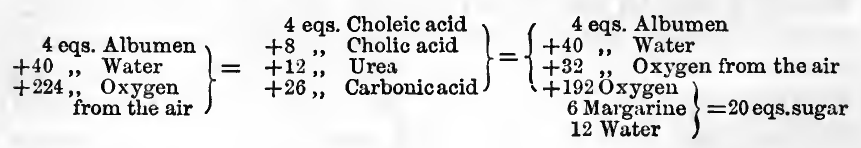

Here we see that on the left hand the albumen is oxidised by the air alone, but on the right hand is represented as unable to obtain more than 32 eqs. of oxygen, out of 224 that it requires from the air, and as procuring the remainder from 20 eqs. of sugar, which, yielding 192 of oxygen, form, at the same time, 6 eqs. of margarine or fat, and 12 of water. This proves that the formation of fat from sugar in the animal body, which certainly takes place, is a source whence the blood may obtain oxygen when respiration is impeded. Were it not for this, an animal kept from free motion would soon die from the accumulation of unoxidised matters in the blood. Instead of dying, the animal lives; but, if supplied with starch in his food, obtains 
the oxygen necessary for the change of matter in the vital processes from the starch or sugar, and in doing so necessarily becomes fat.

There is another process by which fat may be formed in the body, and which does not, like this one, depend on a want of oxygen. It is, probably, in this way that the normal fat is formed, when there is a full supply of oxygen, and it appears to be a species of fermentation, in which the liver seems to be somehow concerned, and hydrogen gas is given off, which of course is immediately oxidised into water in the body. Calf's liver, when placed in water, gives off hydrogen by a species of fermentation, and fat, the same as occurs in blood, is found at all times in bile. The supposed fatty fermentation of sugar actually occurs out of the body, as in the butyric fermentation, butyric acid being a true fatty acid, and being found in butter and fat, and in the fermentation of starch sugar, where rutylic and margario acids, of the same series, are produced, and are found, along with hydrated oxide of amyle, in the oil of grain or potato spirit.

The following table will show the derivation of fatty acids from sugar by fermentation. It will be seen that acetic acid, which, although belonging to this series, is not oily, may be formed from sugar by a polymeric transmutation, but that all the rest are obtained by the separation of carbonio acid and hydrogen, always in equal proportions. Most of the acids require so much sugar as will yield 3 eqs. of acid, and therefore we represent all as formed in that quantity; but, obviously, where the number of the equivalents of sugar is divisible by 3 , we may trace the formation of 1 eq. of acid.

\begin{tabular}{|c|c|c|c|c|c|}
\hline \multicolumn{2}{|c|}{ Acids. } & \multirow{2}{*}{$\begin{array}{l}\text { Eqs. of } \\
\text { Sugar } \\
\text { yield. }\end{array}$} & \multirow{2}{*}{$\begin{array}{l}\text { Eqs. of } \\
\text { Acid. }\end{array}$} & \multirow{2}{*}{$\begin{array}{c}\text { Eqs. of } \\
\text { Carbonic } \\
\text { Acid. }\end{array}$} & \multirow{2}{*}{$\begin{array}{l}\text { Eqs. of } \\
\text { Hydrogen }\end{array}$} \\
\hline Name. & Formula. & & & & \\
\hline Acetic. & - $\mathrm{C}_{4} \cdot \mathrm{H}_{4} \mathrm{O}_{4}$ & 1 & 3 & 0 & 0 \\
\hline Propylic . & - $\mathrm{C}_{6} \mathrm{H}_{6} \mathrm{O}_{4}$ & 2 & 3 & 6 & 6 \\
\hline Butyric & . $\mathrm{C}_{8} \mathrm{H}_{8} \mathrm{O}_{4}$ & 3 & 3 & 12 & 12 \\
\hline Valerianic & . $\mathrm{C}_{10} \mathrm{H}_{10} \mathrm{O}_{4}$ & 4 & 3 & 18 & 18 \\
\hline Caproic & - $\mathrm{C}_{12} \mathrm{H}_{12} \mathrm{O}_{4}$ & 5 & 3 & 24 & 24 \\
\hline Enanthylic & - $\mathrm{C}_{14} \mathrm{H}_{14} \mathrm{O}_{4}$ & 6 & 3 & 30 & 30 \\
\hline Caprylic & - $\mathrm{C}_{16} \mathrm{H}_{16} \mathrm{O}_{4}$ & 7 & ' 3 & 36 & 36 \\
\hline Pelargonic & . $\mathrm{C}_{18} \mathrm{H}_{18} \mathrm{O}_{4}$ & 8 & 3 & 42 & 42 \\
\hline Rutylic or rut & ic $\mathrm{C}_{2 \mathrm{O}} \mathrm{H}_{20} \mathrm{O}_{4}$ & 9 & 3 & 48 & 48 \\
\hline Margaritic & . $\mathrm{C}_{22} \mathrm{H}_{22} \mathrm{O}_{4}$ & 10 & 3 & 54 & 54 \\
\hline Laurostearic & - $\mathrm{C}_{24} \mathrm{H}_{24} \mathrm{O}_{4}$ & 11 & 3 & 60 & 60 \\
\hline Cocinic . & - $\mathrm{C}_{26} \mathrm{H}_{26} \mathrm{O}_{4}$ & 12 & 3 & 66 & 66 \\
\hline Myristic & . $\mathrm{C}_{28} \mathrm{H}_{28} \mathrm{O}_{4}$ & 13 & 3 & 72 & 72 \\
\hline
\end{tabular}


BY FERMENTATION.

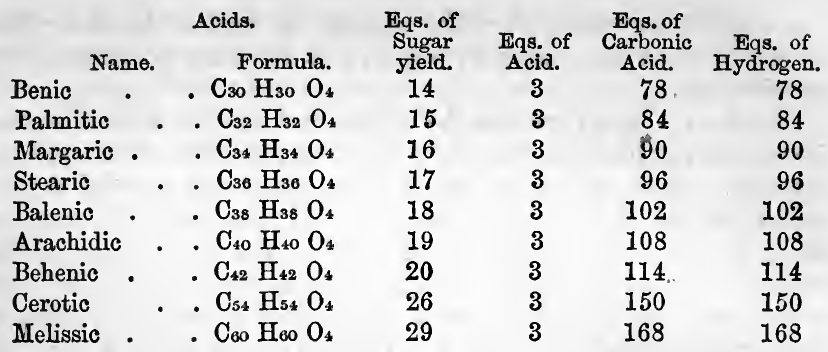

It will be seen that 1 eq. of each of the acids-butyric, œnanthylic, rutylic, cocinic, palmitic, and balenio acids-might be derived from $1,2,3,4,5,6$, and 9 eqs. of sugar respectively. Also, that the number of eqs. of sugar required to yield 3 eqs. of any acid is always exactly 1 less than half that of the eqs. of carbon in the acid. The derivation of oxide of lipyle and of oleic acid, both of which contain more carbon than hydrogen, from sugar by fermentation is probably not so simple as that of the volatile oily acids; but it can hardly be doubted that they may be thus formed, as they are abundant in many animal fats.

We conclude, therefore, that the origin of fat in the animal body is threefold. First, it is derived, ready formed, from plants : secondly, it is formed, in the absence of oxygen, or when oxygen is deficient, by the deoxidation of sugar, which thos supplies the oxygen wanted; and thirdly, it is also formed by a species of fermentation, along with carbonic acid and hydrogen, the latter being converted into water by the oxygen of the blood.

With reference to the tissues, fat is of great importance, as it seems to aid in the formation of cells, and it exists, accordingly, like water in a wet sponge, mechanically in the substance of most tissues, from which it may be pressed out. It has an important share in producing nervous matter; but perhaps its chief function is to aid in yielding the animal heat by its oxidation in the course of the circulation. To this we shall return hereafter.

Let us now briefly consider the process of respiration. The venous blood, loaded with the carbonic acid which it has brought from the remotest capillaries, where every change leads finally to the formation of carbonic acid, is sent to the lungs, and in the pulmonary cells exposed to the action of inspired air, separated from it by a membrane of extreme tenuity. Here the laws of diffusion of gases, in contact with an absorbent liquid, come into 
play. The blood gives off carbonic acid, and takes up hydrogen in its stead. The separation of the carbonic acid from the venous blood, which is absolutely essential to life, cannot take place in an atmosphere containing much carbonic acid. Hence, if the inspired air contain 10 per cent., or upwards, of carbonic acid, and only 10 per cent. of oxygen, the blood does not get rid of its carbonic acid, and death ensues. This happens when animals are forced to breathe the same air for a long time, because every respiration adds to the carbonic acid and diminishes the oxygen. Even with 1 per cent. of carbonic acid, and 19 or 20 per cent. of oxygen, oppression is felt. But if, while carbonic acid be added, oxygen be also added, the air will still admit of the diffusion of carbonio acid from the blood. It has been shown that animals can live in air containing from 17 to 23 per cent. of carbonic acid, provided it contain 35 or 40 per cent. of oxygen; so that the poisonous action of carbonic acid depends on its preventing the diffusion outwards of the carbonic acid of the blood. Hence the importance of ventilation. The air in a crowded, ill-ventilated room, soon becomes unfit for diffusion, because, while the oxygen is diminished, the carbonic acid is increased. The expired air, with normal respiration, instead of at most $\frac{1}{1000}$ th of carbonic acid, the quantity in the atmosphere, contains $3 \cdot 5$ to 5 per cent. ; that is, from 35 to 50 times as much as the air inspired. With very deep respiration, the carbonic acid in expired air increases to 85 or 90 times that of the inspired air. The oxygen in the former case is diminished from 20 to 15 or $16 \frac{1}{2}$ per cent.; in the latter, to 11 or $11 \frac{1}{2}$ per cent. In the blood, these two changes balance each other, for it absorbs oxygen, and gives out carbonic acid nearly, but not quite, in the same volume, a part of the oxygen absorbed being converted into water. The blood has the very remarkable property of absorbing both oxygen and carbonic acid, the former by means not yet fully understood, the latter by the presence of alkaline salts, which means enable it to absorb far more of both gases than an equal bulk of water could do. Blood can absorb 13 or 14 times more oxygen than water. Whichever be the gas present in largest quantity, is readily given out in an air containing little of that gas, but is not given out in an air in which much of the gas is present. The density or rarity of air is a matter of far less consequence, than that it should contain little carbonic acid, in order to allow the venous blood to give off its carbonic acid. When it can do so, it takes up oxygen, and becomes arterial, and is then sent to the left auricle, and, entering the left ventricle, is sent to carry its oxygen to all parts, and exchange it for carbonic acid, probably produced by previous supplies of oxygen, 
Verdeil has recently discovered, in the parenchyma of the lungs, a peculiar acid, which he has named pneumic acid. The acid liquid obtained by chopping the lungs small and mixing them with distilled water, is strained, and coagulated by heat, to separate albumen. It is then neutralised with baryta water, and evaporated to $\frac{3}{4}$ of its bulk in the water bath. Sulphate of copper is now added to separate the last traces of albumen, with fatty matter, \&c., and any excess of the sulphate is precipitated by a little sulphuret of barium. The filtered solution is now evaporated till crystals of sulphate of soda appear, a little diluted sulphuric acid is added, and the whole is boiled with absolute alcohol, which dissolves the pneumic acid, leaving the sulphate of soda undissolved.

The new acid crystallises from alcohol in brilliant needles, forming radiate groups. At $212^{\circ}$ it loses no water. Heated more strongly, it melts, and is decomposed. It is very soluble in water, insoluble in cold, but soluble in boiling alcohol, insoluble in ether. It forms crystalline saits with bases, and expels carbonic acid from the carbonates.

Its precise formula is not known, but it is said to contain carbon, hydrogen, nitrogen, sulphur and oxygen.

The function of pneumic acid is as yet unknown, but, like lactic acid, it probably exists in the blood or tissue combined with some base.

The way in which the oxygen and carbonio acid are absorbed by the blood, and yet so easily given off when wanted, may be illustrated by other chemical changes. From the change in colour of the blood-corpuscles, they are probably the substance to which the oxygen is attached, when absorbed. If an engraving be exposed to the vapour or gas of iodine, the ink will take up iodine, in proportion to its thickness; and if the engraving be now pressed on paper covered with starch and moistened by an acid, a blue impression is obtained. Here the iodine leaves the ink for the starch. If the blue impression be now placed in contact with silver or copper, the iodine leaves the starch for the metal, and if the silver or eopper plate be now exposed to the vapour or gas of mercury, the mercury will attach itself to the parts not acted on by the iodine, as in the daguerreotype, and produce a daguerreotype copy of the engraving without the aid of light. Now we may suppose the blood-corpuscles to take up oxygen, to yield that oxygen to the effete tissues, oxidising them, and then to take up the carbonic acid, and convey it to the lungs, where it is given off by diffusion. An animal dies instantly in pure carbonic acid, because in that gas no diffusion of carbonic acid can take place; 
but it lives for a time in hydrogen, because in that gas the carbonic acid can leave the venous blood, and the oxygen already in the lungs can support for a time the vital process.

We see, then, that the arterial blood conveys oxygen to all parts of the system, and that this oxygen is given out by the venous blood as carbonic acid, and as water by the lungs, skin, and kidneys. This oxidation of carbon and hydrogen not only carries on the vital chemical changes, but also yields the animal heat, which is therefore produced not in the lungs, but everywhere, chiefly in the capillaries. Now a working man consumes nearly $14 \mathrm{oz}$. of carbon, and produces about 25 cubic feet of carbonic acid in 24 hours, containing about $37 \mathrm{oz}$. of oxygen. The quantity of water produced is also ascertained, for all the oxygen is not converted into carbonic acid. And if we calculate the amount of oxygen consumed in $\mathbf{2 4}$ hours, and knowing how much of it becomes carbonic acid, consider the remainder as forming water; we can then deduce, from the known amount of heat given out in the conversion of so much oxygen into carbonic acid and water by combustion, the amount of heat which ought to be given out in the slow oxidation of carbon and hydrogen in the body, which is the same as in combustion, only spread over a longer period. This calculation is found to agree closely with the amount of animal heat, as directly observed, and therefore we are satisfied that the animal heat is entirely due to the chemical changes, all depending on oxidation, which go on in the body. It is evident that the animal heat is promoted by everything that increases the supply of oxygen, as increased respiration, or cold, which, rendering the air denser, supplies its own antidote, within certain limits, provided the food be abundant; and that clothing, which prevents loss of heat by cooling, renders a less amount of oxygen and of food necessary. Hot weather, or a hot climate, has the same effect, and reduces the appetite for food, which is the fuel to be burned in the animal furnace to produce heat. In cold climates, much more food, and especially more respiratory food, such as fat, oil, blubber, and the like, is instinctively consumed.

It is obvious also, that since carbon and hydrogen are the chief sources of animal heat in their oxidation, while nitrogen and its compounds are hardly combustible, the chief portion of the animal heat must come from the non-nitrogenous food, starch, sugar, and fat, hence called respiratory food. Indeed, so low is the combustibility of the sanguigenous food, that we may say that all the heat comes from the respiratory matter; for we find the proportion of carbon to nitrogen in the solid and liquid excreta to be as great, or greater, as in albumen, \&c. Now, 
since the whole nitrogen is found in the excreta, it is evident that these contain also the whole carbon of the albuminous matters, or, at least, a quantity equal to it; for while the tissues contain, for every equivalent of nitrogen, 8 eqs. of carbon, urine contains, in man, only about 1.8 or 2 eqs. of carbon for 1 of nitrogen. Here 6 eqs. of carbon have disappeared for every eq. of nitrogen. But in the horse, the eqs. of carbon are to those of nitrogen in the urine as 6.6 to 1 ; in the cow, as 8 , or even as 16 to 1 ; in the pig, as 10 to 1 ; so that in these latter cases the tissues cannot be supposed to have contributed much, if anything, to the animal heat.

Hence the food of animals should contain a due admixture of sanguigenous and respiratory food, the former to supply the waste of the tissues, the latter to supply the animal heat. The sanguigenous food is albumen, fibrine, or caseine; the respiratory, starch, sugar, or fat. The following table, from Liebig's Letters, to which the reader is referred for full details, shows the relative proportions of sanguigenous and respiratory matter in various kinds of food, the respiratory food, for the sake of comparison, being calculated as starch.

TABLE OF THE RELATIVE PROPORTIONS OF THE SANGUIGENOUS TO THE RESPIRATORY CONSTITUENTS IN DIFFERENT KINDS OF FOOD.

Sanguigenous.

Cow's milk contains, for $\quad 10$

Human milk

Lentils

Horse beans

Peas

Fat mutton

Fat pork

Beef

Hare

Veal

Wheat flour

Oatmeal

Rye floor

Barley

Potatoes, white

Potatoes, blue ,"

Rice

Buckwheat
10

10

10

10

10

10

10

10

10

10

10

10

10

10

10

10

10
Respiratory (as Starch). $30=\left\{\begin{array}{l}8 \cdot 8 \text { fat, and } \\ 10 \cdot 4 \text { milk sugar. }\end{array}\right.$ 40

21

22

23

$27=11 \cdot 25$ fat.

$30=12 \cdot 5$, ,

$17=7 \cdot 08$,

$2=0.03$,

$1=0.41$,

46

50

57

57

86

115

123

130

Here we see that milk and grain, the two best forms of natural 
food, contain, for one of sanguigenous, 3 to $5 \cdot 7$ of respiratory matter. Fat meat has also 1 to 3 or 1 to $2 \cdot 7$, and agrees with peas and beans very nearly. Lean beef, hare, and veal, have far too little respiratory matter, only $1 \cdot 7,0.2$, and 0.1 , to 1 of plastic or sanguigenous food; while potatoes, rice, and buckwheat, have $8 \cdot 6,11.5,12 \cdot 3$, and 13 of respiratory, for 1 of sanguigenous matter. For this reason, respiratory matter should be added to lean meat, and also to peas and beans; but sanguigenous matter should be added to potatoes and rice. This explains the instinctive and universal use of beans with bacon, pork with pease pudding (bacon containing very little sanguigenous matter), veal with ham, potatoes and rice with lean meat, flour and butter with eggs (which are very rich in albumen). The best proportion for a working man is about 5 of respiratory for 1 of albuminous food, and for a young animal, 3 or 4 of respiratory to one of sanguigenous matter. These proportions, which are those of grain and milk, are easily obtained in the mixtures above named.

When the proportion of respiratory matter is too small, then a large amount of sanguigenous matter must be used to supply heat, which is a great waste, since such matter is the worst source of heat. Thus, to obtain the same amount of heat, we must use:-

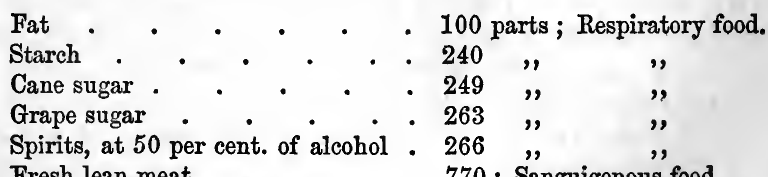

Alcohol belongs to the respiratory class; and, therefore, if properly diluted, and used as an addition to sanguigenous food, such as lean meat, cheese, eggs, or pease and beans, it is useful, rather than hurtful. But if added to food already containing $5,6,10$, or 15 parts of respiratory, for 1 of sanguigenous matter, it is hurtful, as reducing the proportion of the latter still further. Now when the food contains too little sanguigenous food, enormous quantities of it are required to supply the waste of matter. Thus it will take 123 parts of rice to supply as much tissue as 33 parts of fat pork, or 125 of blue potatoes to yield as much tissue as 27 of lean beef. But the quantity of rice or potatoes which may be used is limited by their price, and also by their bulk; for the necessary supply may be too bulky for the stomach which has to digest it, and of course a large amount of respiratery matter is wasted in the use of such food. If the 
peasantry attended, or were taught to attend, to the due admixture of their food, they might cause their limited wages to go much further than at present. But for details on this subject I must again refer to Liebig's Animal Chemistry, and to the third edition of his "Letters on Chemistry."

The amount of bodily or mental work done is measured by the daily waste of tissues, muscular or nervous, \&c., and the daily food must contain, if health is to continue, exactly as much sanguigenous matter, and in an available form, as will supply the daily waste. With less, the body is wasted. With more, the blood is loaded with products of change beyond the normal standard, and disease ensues, if this go beyond certain limits. The respiratory food not only gives the animal heat by its oxidation, but protects against oxidation the blood and tissues. A full supply of it is equivalent to so much clothing; and vice versâ, warm clothing enables men to do with less respiratory food.

Such is the relation of respiration to digestion and nutrition, and it will be seen that they are mutually indispensable. In digestion the food is rendered soluble, and prepared for assimilation and for oxidation. In respiration the blood is oxidised, and carries oxygen to assist in the waste of tissue everywhere, while it gives off the carbonic acid formed by previous changes.

We have now to consider the mineral elements of food, and the share they take in the vital process. All food, capable of sustaining animal life, must contain mineral salts. These are alkaline and earthy phosphates and sulphates, common salt, and oxide of iron, with, in a few cases, iodides, and in all a minute proportion of fluorides.

The blood, in order to perform its functions, must be alkaline, and it is rendered alkaline either by carbonate of soda or by phosphate of soda, $\mathrm{PO}_{5}, 2 \mathrm{Na} \mathrm{O}, \mathrm{H} \mathrm{O}$, or by both. The first occurs in herbivora; the second in carnivora, and in animals fed on grain, peas, \&c., alone; the third in omnivora. It is very remarkable that two salts, so different in composition as carbonate of soda, $\mathrm{Na} \mathrm{O}, \mathrm{C} \mathrm{O}_{2}$, and phosphate of soda, $2 \mathrm{NaO}, \mathrm{H} \mathrm{O}$, $\mathrm{PO}_{5}$, should yet closely agree in being alkaline, and in their action on carbonis acid in the blood. They both readily absorb it, and as readily give it out in another gas.

But while the blood, the saliva, the intestinal secretion, the bile, and milk are alkaline, and the pancreatic juice either alkaline or neutral, the juice of the muscles, the gastric juice, and the urine of carnivora and omnivora are acid. Even the urine of herbivora, although permanently alkaline, is acid from excess of carbonic acid as it leaves the body. This soon evapo- 
rates, and the urine becomes alkaline from the presence of alkaline carbonates. The acidity depends, in the juice of flesh, on the presence of phosphate of potash, $\mathrm{P} \mathrm{Os}, \mathrm{K} \mathrm{O}, 2 \mathrm{H} \mathrm{O}$, which is an acid salt, and yet is formed in close proximity to the alkaline phosphate of soda, the blood and juice of flesh being separated only by very fine membranes. An alkaline state of the blood is absolutely indispensable to its functions; acid blood is inconsistent with life. Hence every acid that enters the blood must be at once neutralised. And yet it is in close proximity to, and communicates by endosmosis and exosmosis with, an acid liquid, the juice of flesh, or gastric juice, or urine, and is constantly receiving uric acid and other acids. Vegetable acids, taken in the food, are speedily oxidised in the blood into carbonic acid and water, and only appear as alkaline carbonates. It would appear that the formation of tissues cannot go on without the presence of free phosphoric acid, or at least acid phosphates, while their destruction demands the presence of free alkali, which we know to be favourable to oxidation. Thus, in the egg, the phosphoric acid is in great excess. As the lymph and chyle are also alkaline, the formation of the blood itself appears to depend on the presence of excess of alkali. The flesh of all animals is the same in respect to mineral matter; but the blood, and of course the urine, of the herbivora contains less phosphates and more carbonates.

The ashes of the food are either phosphates and sulphates alone, as in flesh, grain, peas, \&c., or phosphates, sulphates; silicates, and carbonates, as in grass and green vegetables. They are partly soluble in water, partly insoluble. Now, in the body, the soluble parts of the ash are found in the urine, the insoluble in the solid excreta. When the phosphoric acid is not present in sufficient quantity to unite with all the bases, it forms insoluble salts with lime and magnesia, while the alkalies appear as sulphates and carbonates. But where more phosphoric acid is present, the insoluble salts are, as before, phosphates and silicates of lime, magnesia, and oxide of iron, while the soluble ones are phosphates and sulphates of the alkalies. Hence, knowing the nature of the ashes of the food, we can tell the salts of the urine and fæces; and by altering the food, we can change these at pleasure. The uric and hippuric acids formed in the change of matter, are found, so far as not oxidised, in the urine, and the following table shows the composition of the urine with food composed of flesh, grain, and leguminous seeds, and also with grass, clover, turnips, potatoes, and fruit. The earthy phosphates and carbonates, it must be remembered, are soluble in excess of acid. 
With fond consisting of Flesh, Bread,

Peas, Beans, \&c., the urine contains :-

Free Phosphoric acid

Phosphates of $\left\{\begin{array}{l}\text { Lime, } \\ \text { Magnesia, }\end{array}\right\} \begin{aligned} & \text { dissolved by } \\ & \text { excess of acid }\end{aligned}$

Phosphates

Sulphates

Urates

Hippurates

This urine is permanently acid.

Acid urine usually contains uric acid.
With food, such as Hay, Clover, Turnips, Potatoes, Fruit, \&c., the urine contains :-

\section{Free Carbonic acid}

Carbonates of $\left\{\begin{array}{l}\text { Lime, } \\ \text { Magnesia, }\end{array}\right\}$ dissolved in

Carbonates

Sulphates $\}$ of the alkalies.

Hippurates

This urine has a transient acid reaction, and a permanent alkaline one.

It contains no phosphoric, and no uric acid.

Since the soluble salts of the ashes of the blood are the same as those of the urine, and since we can affect the nature of the urine by change of diet, we may not only draw conclusions as to the soluble salts of the blood from the urine, but also modify these by diet.

When we consider the absolute necessity of phosphoric acid and the phosphates, as well as of the other mineral ingredients of food, to the vital changes, we see how important it is that the food should contain these in due proportion and admixture. This is the reason that fibrine alone will not support life; that yolk of egg, cheese, and similar matters, also fail, when used alone, to support life. The fibre of meat is rich in phosphoric acid, but has lost the alkalies of the juice: the yolk of egg is still richer in phosphoric acid, but wants the alkali of the white. Cheese is also rich in phosphoric acid, but requires the alkalies of the whey, and so on. Salt is useful, both with eggs and cheese, to supply soda for the blood and bile; indeed it is useful with all food, but still more with those substances. It appears generally to promote the secretions, probably from its adding to the solvent powers of water. Meat, boiled to rags, is fibrine, and is only nutritious with the soup. Hence the continental habit, of eating the boiled meat after the soup made from it, is rational. All food, to be fully nutritive, must contain iron; and those kinds of animal food, which are found by experience to yield less blood, are deficient in iron as well as alkalies. Such are milk, eggs, and fish, all of which are allowed to form part of the fasting or meagre diet of Catholic countries.

The reader will find, in Liebig's "Letters on Chemistry,' numerous analyses of the ashes of blood, flesh, milk, cheese, grain, fish, eggs, urine, and fæces, establishing the general principles here laid down, and the great importance of the mineral constituents. Our space is too limited to allow more than a statement of the principle, that phosphoric acid in excess is required for the production of the tissues, and excess of alkali is required to form the blood, and enable it to perform its functions of destroying the tissues by oxidation. The blood, indeed, conveys to all parts 
the materials for the formation of tissues, but as that formation requires free phosphoric aoid, it probably takes place out of the blood.

We have already stated that food ought to contain a due proportion of sanguigenous and respiratory matter. In preparing it care should be taken to lose nothing of the mineral elements. Hence the salting of meat, which expels by exosmosis a great part of the juice with its salts, is injurious, unless the salts are replaced by a free use of green vegetables, which contain much saline matter, sometimes 10,15 , or 20 per cent. of the dried plant, or by preserving the juice, boiling it down to get rid of the salt, and using it as sauce to the meat, or by adding the soluble extract of meat. The system, so common in England, of boiling food of any kind in a large quantity of water, which is thrown away, is also very bad. Vegetables ought to be stewed, with very little water, and the juice eaten with them. Meat ought either to be roasted, stewed, or if boiled, for eating, boiled as follows: The meat is thrown into boiling water, and kept at $212^{\circ}$ for a minute or two; this coagulates the albumen of the juice near the surface, so as to form a kind of crust or coat. Cold water is now added so as to reduce the temperature to $165^{\circ}$, at which it is kept till fully dressed in the centre. The crust of albumen keeps in the juice, and meat thus boiled is not only tender, but has all the flavour and nutritive power of roast meat. But if the soup be wanted, then the meat is put on with a little cold water, and slowly heated to boiling. The water enters and the juice is expelled, before the albumen of the latter is coagulated; and after boiling for a little, the best possible soup is obtained.

If, in countries like Australia, Buenos Ayres, and others, where cattle are killed for their hides alone, or for the hide and tallow, the flesh were thus exhausted, and the soup, using only about as much water as meat, evaporated to a soft extract, this, the true extract of meat or portable soup, would be obtained at a cheap rate, and furnish a valuable article of food, 1 part of which is equal to nearly 30 of fresh meat. This is to be carefully distinguished from the portable soup in hard gelatinous cakes, which consists chiefly of gelatine extracted, by long boiling, from meat and bones, and sometimes does not contain even $\frac{1}{10}$ th of true extract. Gelatine cannot yield blood, and is worthless as food, save perhaps in a very small proportion, to yield membrane. The true extract of meat is always soft, and does not gelatinise, and would be invaluable for convalescents and wounded soldiers. It is slightly deficient, compared with meat, in phosphorio acid, but that is easily supplied by grain or peas. 
It is hardly necessary to point out the importance of salt as a chief source of sodium or soda for the blood and bile. The absence of salt is sure to be injurious, and in countries where it is scarce, it fetches a price equal to that of gold or silver. Animals instinctively take it, and search for it, and its effects on them, on their general health and all their secretions, are most marked.

Of all food, perhaps good bread, made from the whole meal of wheat, oats, or rye, is the most economical, since nature supplies in it the due proportion of sanguigenous, respiratory, and mineral matter. But since the fibrine of flour is identical with that of flesh, and fat corresponds to a certain amount of starch, fat meat agrees closely with bread, and has a better proportion of ingredients than peas, beans, and lentils. Hence the doctrine of the vegetarians is founded on a mistaken assumption, that there is a radical difference between meat and vegetables. It is certain that the structure of man fits him for the use, though not the exclusive use, of animal food; and even the vegetarians do not exclude milk, cheese, and eggs, all of which are of animal origin; the first about equal to meat in sanguigenous value; the two last greatly exceeding it. A man may feed as fully, nay more fully, and form more blood on a vegetable diet, one of peas for example, than on one of very fat meat. There is no known difference in the power of forming blood, between flesh and bread, if the flesh be mixed with sufficient fat or some starch; and there is one reason why flesh should form part of man's food, besides that derived from the structure of his teeth and digestive organs, namely, that since the chief use of food is to supply the waste of muscle, the best substance for this must be the muscle of animals. The great error in diet is not that of eating flesh, but of eating too much flesh, or too much sanguigenous matter, whether animal or vegetable. And the only true principle of diet is to obtain the necessary amount of sanguigenous matter, of respiratory matter, and of mineral salts, no matter from what sources. In applying this rule to man, a mixed animal and vegetable diet is obviously the best; both as being in the end most economical, because flesh is nearest of all to what it has to supply; and because, although fat can replace the stareh of vegetables as a source of heat, the ashes of vegetables yield a more abundant supply of alkalies. The instinct of man agrees entirely with this view, and, like all one-sided and exclusive ideas, vegetarianism is inconsistent with nature. Still, it is certain that men can over-eat themselves more easily with animal than with vegetable food, and that they can live on vegetables alone. But they are not intended, by their structure, 
to do so, and even on vegetables may easily live too high, especially if milk, cheese, and eggs be added.

The abuse of fermented liquors is hurtful in two ways ; first, by the peculiar stimulus which alcohol, unless much diluted, exerts on the nervous system in some way unknown; and, secondly, by increasing the proportion of respiratory matter to sanguigenous far beyond the proper standard. By virtue of its strong attraction for oxygen, alcohol is first oxidised, while the food and tissues are imperfectly oxidised, and disease is thus induced. If the food already contain a full or large proportion of starch or fat, every drop of alcohol is hurtful; but when the food is too rich in sanguigenous matter, wine and beer are wholesome. In proof of the fact, that alcohol supplements the other respiratory food, it is observed that those who drink no wine consume far more bread, vegetables, rice, or puddings, than wine drinkers; and the good health enjoyed by the natives of wine and beer countries who use these liquors freely but not to excess, proves that alcohol is not essentially hurtful, when properly diluted, but acts as respiratory food. Those who take much fat, butter, or oil, cannot take wine, and feel no desire for it.

If $w_{c}$ wish to fatten an animal, its food ought to contain a large proportion of respiratory food, the excess of which, beyond what is required for the animal heat, is converted into fat, and stored up in cells. But as sanguigenous matter is required for the change of the tissues and also to form the fat cells, an animal cannot be fattened, nor even live, on starch alone.

We have now seen that the food is digested, enters the circulation, restores the wasted tissues, and supplies during its oxidation the secretions, the excretions, and the animal heat. It is, in other words, burned in the body, as in the furnace. The products of the complete combustion of the respiratory food, namely, carbonic acid, water, and ammonia, escape by the lungs and kidneys. The products of incomplete combustion chiefly derived from sanguigenous food are partly found in the bile, partly in the urine and fæces, where they represent the soot of the furnace, and in fact are, to a great extent, identical with some of the compounds found in tar, which is liquid soot. Carbolic acid, for example, is found both in urine and in tar; and urine also contains another acid, homologous with carbolio acid. The ashes are divided by water into the soluble, which appear in the urine, and the insoluble, which are found in the fæces, along with indigestible woody fibre, undigested starch, fetid products, which can be imitated by the partial oxidation of albuminous matter out of the body; 
and, lastly, tarry products, originating in the same way. The ashes of the liquid and solid excreta are identical with those of the food, if that be uniform, but change with the diet.

So long as the vital force prevails, the oxidation in the body affects only the respiratory food and the effete or worn-out tissues. It produces, however, as it advances, products which no longer obey the vital force, and, yielding to physical forces, crystallise. And these, such as hippuric acid, kreatine, uric acid, and urea, are destined to be excreted. After death, the oxygen of the air, no longer opposed by the vital force, attacks the blood and tissues. The first effect is a degree of decay, and by contact of the decaying albuminous matter, now a ferment, with the entire albuminous compounds remaining, these putrify, and are finally, by the process of decay and putrefaction, resolved into the organic food of plants, carbonic acid, water, ammonia, and sulphuric acid, while the ashes are restored to the soil.

Now the circuit begins again, which we have already traced, The plant grows, and from carbonic acid, water, ammonia, sulphuric acid, and the mineral salts, forms its tissues and products, ready to become the food of a new generation of animals, which at death are again resolved into food for plants. And thus the balance is kept between the animal and vegetable kingdoms, the atmosphere, water, and soil, or mineral kingdom, supplying the field of contest, and the necessary media.

Although, as we have seen, animals can form blood and tissues either from albumen, fibrine, or caseine, provided the necessary salts be present, and therefore can convert those compounds into one another, there is no evidence that they can form either albumen, fibrine, or caseine, as the plant does, from any substance not containing one of them. On the contrary, the processes of animal life, being processes of oxidisation and destruction, are opposed to those of vegetation. The chief regetable products formed by animals are fat, benzoic acid, which is found in the urine of herbivora, and oxalic acid. Starch has lately been detected in various parts of the body, but its origin is still doubtful. Glucose appears to be a normal constituent of the blood in some parts, and is probably formed in the body; there is reason to believe that it is produced by the decomposition of albuminous matter. But all of these may be formed by oxidation or by fermentation. Plants are characterised chemically by construction of complex molecules and deoxidation, animals by destruction of complex molecules and oxidation, and the products of each form of life are the food of the other.

The food of carnivora, being identical with their blood and muscle, the digestive apparatus is in them very simple; and the 
fæces, consisting chiefly of insoluble phosphates, much less bulky than in herbivora. The respiratory part of their food is fat, present even in lean flesh. But as it is often deficient in quantity, they are compelled to burn a portion of sanguigenous matter for heat, a wasteful expenditure of force. This is done by muscular motion, which rapidly wastes the tissues. Hence the restlessness and constant activity of carnivorous animals, even in confinement.

The remarkable fact already noticed, of the existence in all parts of the body of an alkaline liquid, the blood, and an acid liquid, the juice of flesh, separated by a very thin membrane, and in contact with muscles and nerve, seems to have some relation to the fact now established of the existence of electric currents in the body, and particularly to those which occur when muscles contract. The animal body may be regarded as a galvanic engine for the production of mechanical force. This force is derived from the food, and with the food has been derived, as we have seen, from the solar rays. A working man, it has been calculated, produces in 24 hours an amount of heating or thermal effect equal to raising nearly 14 millions of lbs. to the height of 1 foot, heat being one form of mechanical effect. But, from causes connected with the range of temperature, he can only produce, in the form of actual work done, about as much mechanical effect as would raise $3,600,000 \mathrm{lbs}$. to the height of 1 foot, and that in 24 hours. Even this is a prodigious amount of force, and whether we regard it as derived from heat, electricity, or ohemical action, it is ultimately derived from the luminous solar rays, on which vegetation depends.

Our space will not permit us to enter into detail on the chemical nature of the causes of disease, which are still very imperfectly understood. But some things are evident. Thus, if the digestion be so far impaired, by any cause, as not to dissolve the necessary amount of respiratory matter, the tissues, no longer protected, are wasted by oxidation, and the products of this, such as uric acid, load the urine, as is seen in all febrile diseases. If this state continues, the lungs, where oxygen is absorbed, become affected. When oxygen is deficient, combustible matter accumulates in the blood beyond the due proportion, and the liver is called on to work beyond its powers, in secreting bile or forming fat, and hepatic disease ensues, as we see in hot climates, when people indulge in full feeding, and respiration is languid. Should the food be too abundant and too rich in sanguigenous matter this amounts to a deficiency of oxygen; and, if, at the same time, there be a deficiency of alkali, necessary to promote the oxidation of effete matter in the blood, we have the uric acid 
diathesis, or with a little more oxygen, the oxalic acid diathesis. Gelatinous food, which cannot form blood, and is deficient in alkali, and strong wines which contain no tartar, greatly promote this diathesis; light wines, which contain tartar, vegetables which are rich in alkali, exercise in the open air, and moderation in sanguigenous diet, are the best remedies. On the Rhine, where light wines are freely used, uric acid calculus is unknown as a native disease, but with drinkers of port and sherry, this calculus, as well as its concomitant gout, are very frequent. A change of diet and mode of life, on the principles we have endeavoured to explain, will alter entirely the character of the urine, and of course of the blood. Hence the supreme value of diet and regimen as means of cure, acknowledged by all experienced physicians. It is probably in this direction, in the study of the secretions, and the means of changing their nature, that we may hope for the greatest practical improvement in medicine.

One question is still very obscure, namely, how that portion of insoluble phosphate which is derived from the effete tissues reaches the intestinal canal. The earthy phosphates are insoluble in water and alkaline solutions; hence they are insoluble in the blood. But yet they are conveyed, probably in some form of combination soluble in water, to the intestines. They are soluble in excess of phosphoric or carbonic acid; but the blood is never acid. But as the soluble forms of sanguigenous matter contain earthy phosphates, so it is possible that when such matter is destroyed, some of the products contain these phosphates in a soluble form. Such products, however, are still unknown. Morbid ossification probably depends on the absence or deficiency of such products; and mollities ossium, in all probability, is caused by a morbid excess of phosphoric acid in some part. In chlorosis, the number of red blood globules is diminished, and as these contain much iron, the disease is connected with a defect in the quantity of iron in the food, or perhaps with a want of its proper solvent. This explains the benefit derived from the use of iron, under which the red globules rapidly increase in number in many cases. In all acute febrile diseases, and in all chronic diseases, which affect the digestive or nutritive powers, emaciation occurs, because the oxygen of the air, no longer opposed, burns off the tissues and wastes them. This explains the good effects of cod-liver oil, and other oily matters, such as olive oil, butter, and cream, in many chronic wasting diseases: it protects the tissues till the disease has run its course, if curable, and retards the fatal termination when the disease is incurable; provided the stomach have still the power of taking up the oil. The 
absence of salt deranges the whole vital process, and particularly the secretion of bile, which requires soda, that is, oxide of sodium. But salt certainly acts as salt, and has, for example, a wonderful power of combining with other substances, such as sugar and urea, with both of which it forms soluble crystalline compounds. It is hardly necessary to mention that, if alkali, instead of free acid, should prevail in the urine-for healthy urine is always acid-the earthy phosphates are thrown down, and give rise to phosphatic calculus.

When children are injudiciously fed on diet composed chiefly of starch, such as sago, arrow-root, and the like, there occurs a great deficiency of blood and of bone-forming matter; fat accumulates, but the frame is weak and ricketty, from small muscles and soft bones. Children ought to have a full supply of sanguigenous food, and even bread contains too little for them; milk or flesh should be added. But whether fed on bread and milk, or meat and bread, there is apt to occur a deficiency of phosphate of lime, from the rapid formation of bone. But as meat, eggs, and milk, contain an excess of phosphoric, that is not enough of lime to convert the whole acid into phosphate of lime, $\mathrm{P} \mathrm{O}_{5}, 3 \mathrm{Ca}$, lime is a good addition to the food of young children. It may be given in the form of lime-water, which the peasants of Germany give to their children with the best results, while the children, guided by instinct, greedily take it.

Since the products of the waste of tissues are sent out of the body by the lungs, the skin, the kidneys, and the intestines, we can see how the occurrence of an impediment, in any of these quarters, calls into increased action the others. Checked perspiration causes an accumulation of effete matter in the urine, which becomes loaded with products of imperfect oxidation. When, from any cause, such a check to perspiration, or to the due action of the lungs, this loading of the urine goes very far, it begins to deposit a sediment, even in the bladder or kidneys. As Prout pointed out, the effect of a purgative in this case, is greatly to increase the action of the intestines, and thus to relieve the blood and the urine, the latter acquiring its natural colour, and normal composition.

The use of tea, coffee, and chocolate has become, in some form, to all nations, as a necessary of life. Coffee, tea, and Guarana and Paraguay tea, used for the same purposes, all contain caffeine; while chocolate or cocoa contains theobromine, a body closely allied to caffeine. It would therefore appear, that caffeine has a decided action on the system, promoting, in some way, the natural vital changes. Under caffeine, we have seen the very curious relation existing between caffeine and its derivatives, 
and urio acid and its derivatives. Besides this, caffeine closely approaches, in composition, to kreatine and glycocine. We may therefore conjecture that it aids the change going on in some parts; that it gives a sense of refreshment and new vigour is certain, but we cannot yet trace its action, although it may very possibly promote the secretion of bile. One thing is remarkable, that no people, after once learning to use tea or coffee, ever gives it up; and that the chief effects of tea, coffee, and chocolate are the same, and that tea and coffee contain the same base, and chocolate an allied one. They are also rich in mineral salts.

On all of the subjects which have been here so briefly touched upon in connection with the growth and nutrition of plants and animals, much more might be said, whether in reference to physiology, to agriculture, or to medicine. But this would be out of place in an elementary work, and the reader who wishes for more detailed information will find it in the works of Liebig, especially in the last editions of his "Agricultural Chemistry," and of his "Letters on Chemistry," and in a condensed form, in a small work which he has lately published, under the title of "Principles of Agricultural Chemistry." These works are rich in sagacious and comprehensive views on the most interesting questions. But the reader must remember, that all such views, as well as those indicated in the present work, are not to be regarded as ascertained truth, but are only the best conclusions we can draw, in the present state of our knowledge, from the best-ascertained facts in our possession. They must infallibly be modified, and in some cases perhaps refuted, by increasing knowledge; but in that case they will be replaced by others corresponding to the state of knowledge at the time. 


\section{SUPPLEMENT.}

CONTAINING MATTER WHICH COULD NOT BE INTRODUCRD IN ITS FROPER PLACE IN THE TEXT.

\section{A NEW CLASS OF ALCOHOLS.}

WE have already described acroleine, or the hydrated oxide of acryle, $\mathrm{C}_{6} \mathrm{H}_{4} \mathrm{O}_{2}$, and acrylic acid, $\mathrm{C}_{6} \mathrm{H}_{4} \mathrm{O}_{4}$. As these compounds have to each other the same relations as aldehyde has to acetic acid, it was natural to look for the alcohol of the series.

Will had shown that oil of mustard is the sulphooyanide of the radical allyle, $\mathrm{C}_{6} \mathrm{H}_{5}$, which is isomeric, but not identical with propionyle.

Berthelot and De Luca, by studying the action of iodide of phosphorus on glycerine (the substance which yields acroleine), obtained the compound $\mathrm{C}_{6} \mathrm{H}_{5} \mathrm{I}$, which they called iodised propylene. And they further proved that when this iodide is acted on by sulphocyanide of potassium, there are formed iodide of potassium and oil of mustard, or sulphocyanide of allyle. Oil of mustard had already been artificially produced from oil of garlic, which is the sulphuret of allyle, but the recent experiments confirm Will's view by forming oil of mustard by means of a different compound. It is now evident that the radical in the iodised propylene of Berthelot and De Luca is identical with the allyle of Will and Wertheim.

Hofmann and Cahours have now succeeded in obtaining not only the alcohol of this series, but many other compounds, and have shown that there exists a series of alcohols, of which we are about to describe one, running parallel to the two series of alcohols already known, the methylic and the benzoic series. They have named the radical of the new alcohol acryle, which is somewhat unfortunate, as that name was already given to the derived radical of acroleine and acrylic acid, corresponding to acetyle. It is not easy to see why they did not adopt the name allyle, already given to the same radical in oil of mustard and oil of garlic.

When iodide of allyle (for, to avoid confusing the reader, we Q. Q 2 
shall retain the old name) is acted on by oxalate of silver, we obtain iodide of silver and oxalate of allyle, $\mathrm{C}_{16} \mathrm{H}_{10} \mathrm{O}_{8}=\mathrm{C}_{4} \mathrm{O}_{6}$, $2\left(\mathrm{C}_{6} \mathrm{H}_{5} \mathrm{O}\right)$. This, acted on by ammonia, yields oxamide and allylic alcohol, exactly as oxalate of ethyle yields oxamide and ethylic alcohol.

Allylic (or acrylic) alcohol, $\mathrm{C}_{6} \mathrm{H}_{6} \mathrm{O}_{2}=\mathrm{C}_{6} \mathrm{H}_{5} \mathrm{O}, \mathrm{H} \mathrm{O}$, is isomeric with acetone and with propylaldehyde, but is quite distinct from these two substances. It burns with a luminous flame, and mif es with water in all proportions. It has a peculiar pungent odour, resembling that of mustard, and this odour characterises, more or less, all the compounds of this series. When acted on by potassium, hydrogen is given off, and a transparent gelatinous mass is formed, corresponding to the substance formed when potassium acts on alcohol. The latter contains potassium, ethyle, and oxygen. The new body contains potassium, allyle, and oxygen. When this is acted on by iodide of allyle, iodide of potassium is formed, and oxide of allyle, or allylic ether, is separated, as a liquid lighter than water, and insoluble in it. The action is as follows :

$$
\underset{\mathrm{K}}{\mathrm{C}_{6} \mathrm{H}_{5}}\left\{\begin{array}{l}
\mathrm{O} \\
\mathrm{O}
\end{array}+\mathrm{C}_{6} \mathrm{H}_{5} \mathrm{I}=\mathrm{KI}+\underset{\mathrm{C}_{6} \mathrm{H}_{5}}{\mathrm{C}_{6} \mathrm{H}_{5}}\right\} \begin{aligned}
& \mathrm{O} \\
& \mathrm{O}
\end{aligned}
$$

By distilling allylic alcohol with chloride, bromide or iodide of phosphorus, the chloride, bromide, and iodide of allyle are easily obtained. With potash and bisulphuret of carbon allylic alcohol yields a splendid yellow salt, corresponding to the xanthate of potassium.

With sulphuric acid, allylic alcohol forms an acid corresponding to sulphuric acid. The new acid forms a soluble salt with baryta

$$
\left.\underset{\mathrm{C}_{6} \mathrm{H}_{5}}{\mathrm{Ba}}\right\} \stackrel{\mathrm{S} \mathrm{O}_{4}}{\mathrm{~S} \mathrm{O}_{4}}
$$

The carbonate, acetate, benzoate, oxalate, oxamate, and cyanate of oxide of allyle have all been formed and analysed.

The cyanate of allyle, with ammonia, yields fine crystals of allylurea.

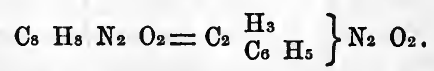

If we suppose the 2 eq. of oxygen in this were to be replaced by sulphur, we have thiosinnamine, already described under oil of mustard, the formula of which is

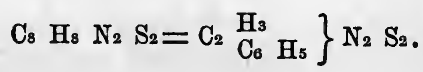


Aniline forms with cyanate of allyle a corresponding compound, analogous to allylurea, which crystallises beautifully.

With water, cyanate of allyle yields diallylurea, $\mathrm{C}_{14} \mathrm{H}_{12} \mathrm{~N}_{2} \mathrm{O}_{2}=$ $\left.\mathrm{C}_{2} \underset{2}{\stackrel{\mathrm{H}_{2}}{\left(\mathrm{C}_{6} \mathrm{H}_{5}\right)}}\right\} \mathrm{N}_{2} \mathrm{O}_{2}$, which has been already mentioned as sinapoline, and represented as diallylic urea.

When cyanate of allyle is heated with potash, sinapoline rises to the surface, and an alkaline liquid distils over, which is a mixture of volatile base's. Among these have been already detected Methylamine, Propylamine, and Allylumine, oi Acrylamine, $\left.\mathrm{C}_{6} \mathrm{H}_{7} \mathrm{~N}=\mathrm{N}_{\mathrm{C}_{6} \mathrm{H}_{5}}^{\mathrm{H}_{2}}\right\}$. This new base boils between $180^{\circ}$ and $190^{\circ}$; but it has not yet been found possible to obtain its platinum salt crystallised.

These results prove incontestably the existence of a new series of alcohols, running parallel to that of the methylic and ethylio alcohols, and of a number of other series connected with this one, and parallel to the corresponding series connected with alcohol and acetic acid, so often referred to. The following tablecontains the formulæ of the compounds of the allylic or acrylic series hitherto obtained, along with those of the ethylic series.

Allylic or Acrylic Series.

Ethylic Series.

$\mathrm{C}_{6} \mathrm{H}_{6} \mathrm{O}_{2}$. . Alcohol . . C4 $\mathrm{H}_{6} \mathrm{O}_{2}$

$\left.\begin{array}{lll}\mathrm{C}_{6} & \mathrm{H}_{5} & \mathrm{O} \text {, or } \\ \mathrm{C}_{12} & \mathrm{H}_{10} & \mathrm{O}_{2}\end{array}\right\}$. . Oxide or Ether . $\quad\left\{\begin{array}{lll}\mathrm{C}_{4} & \mathrm{H}_{5} & \mathrm{O} \text {, or } \\ \mathrm{C}_{8} & \mathrm{H}_{10} & \mathrm{O}_{2}\end{array}\right.$

$\mathrm{C}_{6} \mathrm{H}_{5} \mathrm{Cl}$. . . Chloride . . $\mathrm{C}_{4} \mathrm{H}_{5} \mathrm{Cl}$

$\mathrm{C}_{6} \mathrm{H}_{5} \mathrm{Br}$. . . Bromide . . $\mathrm{C}_{4} \mathrm{H}_{5} \mathrm{Br}$

$\mathrm{C}_{6} \mathrm{H}_{5} \mathrm{I}$. . . Iodide . . $\mathrm{C}_{4} \mathrm{H}_{5} \mathrm{I}$

C6 $\mathrm{H}_{5} \mathrm{~S}$, or $\} \quad \mathrm{C}_{4} \mathrm{H}_{5} \mathrm{~S}$, or

$\left.\mathrm{C}_{12} \mathrm{H}_{10} \mathrm{~S}_{2}\right\}$ Oil of Garlic. Sulphide of ethyle $\begin{cases}\mathrm{Cs}_{8} \mathrm{H}_{10} \mathrm{~S}_{2} \\ \mathrm{C}_{6}\end{cases}$

$\mathrm{C}_{6} \mathrm{H}_{5}, \mathrm{C}_{2} \mathrm{~N} \mathrm{~S}_{2}\left\{\begin{array}{c}\text { Sulphocyanide. } \\ \text { Oil of Mustard. Sulphocyanide of ethyle }\end{array}\right\} \mathrm{C}_{4} \mathrm{H}_{5}, \mathrm{C}_{2} \mathrm{NS}_{2}$

$\mathrm{C}_{2}\left(\mathrm{~K}, \mathrm{C}_{6} \mathrm{H}_{5}\right) \mathrm{S}_{4} \mathrm{O}_{2}$. Xanthate of potassium . $\mathrm{C}_{2}\left(\mathrm{~K}, \mathrm{C}_{4} \mathrm{H}_{5}\right) \mathrm{S}_{4} \mathrm{O}_{2}$

$\mathrm{C}_{6} \mathrm{H}_{5} \mathrm{O}, \mathrm{C}_{2} \mathrm{NO}$. Cyanate . . $\mathrm{C}_{4} \mathrm{H}_{3} \mathrm{O}, \mathrm{C}_{2} \mathrm{NO}$

$\mathrm{C}_{2}\left(\mathrm{H}_{3}, \mathrm{C}_{6} \mathrm{H}_{5}\right) \mathrm{N}_{2} \mathrm{O}_{2}\left\{\begin{array}{l}\text { Urea. } \\ \text { allylic and ethylic }\end{array} \mathrm{C}_{2}\left(\mathrm{H}_{3}, \mathrm{C}_{4} \mathrm{H}_{5}\right) \mathrm{N}_{2} \mathrm{O}_{2}\right.$

$\mathrm{C}_{2}\left(\mathrm{H}_{3}, \mathrm{C}_{6} \mathrm{H}_{5}\right) \mathrm{N}_{2} \mathrm{~S}_{2}$ Sulphuretted Urea. Thiosinnamine. ?

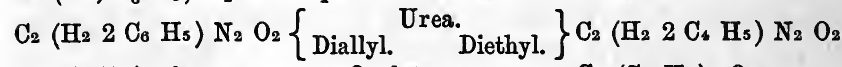

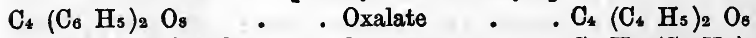

$\mathrm{C}_{4} \mathrm{H}_{2}\left(\mathrm{C}_{6} \mathrm{H}_{5}\right) \mathrm{NO}_{6}$. . Oxamate . . . $\mathrm{C}_{4} \mathrm{H}_{2}\left(\mathrm{C}_{4} \mathrm{H}_{5}\right) \mathrm{N} \mathrm{O}_{6}$

$\mathrm{C}_{6} \mathrm{H}_{5} \mathrm{O}, \mathrm{CO}_{2}$. . . Carbonate . . $\mathrm{C}_{4} \mathrm{H}_{5} \mathrm{O}, \mathrm{C} \mathrm{O}_{2}$

$\mathrm{C}_{8} \mathrm{H}_{5}, \mathrm{O}, \mathrm{C}_{4} \mathrm{H}_{3} \mathrm{O}_{3}$. . Acetate . . . $\mathrm{C}_{4} \mathrm{H}_{5} \mathrm{O}, \mathrm{C}_{4} \mathrm{H}_{3} \mathrm{O}_{3}$

$\mathrm{C}_{6} \mathrm{H}_{5} \mathrm{O}, \mathrm{C}_{14} \mathrm{H}_{5} \mathrm{O}_{3}$. . Benzoate . . $\mathrm{C}_{4} \mathrm{H}_{5} \mathrm{O}, \mathrm{C}_{14} \mathrm{H}_{5} \mathrm{O}_{3}$

$\mathrm{C}_{6} \mathrm{H}_{5} \mathrm{O}, \mathrm{HO}, 2 \mathrm{~S} \mathrm{O}_{3}$. . Sulpho-acid . . $\mathrm{C}_{4} \mathrm{H}_{5} \mathrm{O}, \mathrm{H} \mathrm{O}, 2 \mathrm{~S} \mathrm{O}_{3}$

$\mathrm{NH}_{2}, \mathrm{C}_{6} \mathrm{H}_{5}$. . . Amide base . . $\mathrm{NH}_{2}, \mathrm{C}_{4} \mathrm{H}_{5}$ 
Allylio or Acrylic Series.

Ethylio Series.

$\left.\mathrm{C}_{6} \mathrm{H}_{6} \mathrm{O}_{2} \cdot{ }^{2} \begin{array}{c}\text { Aldehyde. } \\ \text { Acrylic. Acetylic }\end{array}\right\} \mathrm{C}_{4} \mathrm{H}_{4} \mathrm{O}_{2}$

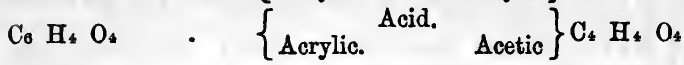

Co $\mathrm{H}_{6} \quad \cdot\left\{\begin{array}{c}\text { Hydrocarbon. } \\ \text { Propylene. Marsh gas }\end{array}\right\} \mathrm{C}_{4} \mathrm{H}_{6}$

It will be seen that the analogy is perfect. The aldehyde of the new series is acroleine, or the hydrated oxide of the radical $\mathrm{C}_{6} \mathrm{H}_{3}$, already known by the name of acryle, and corresponding to acetyle, C4 Hs. Acrylic acid, also long known, is the hydrated teroxide of the radical $\mathrm{C}_{6} \mathrm{H}_{3}$.

Now we have already seen that acrylic acid is one of the homologous series (see pp. 293 tt seq.), of which we have named six members, namely, acrylic acid, the third: angelic acid, the fifth ; moringic acid, the fifteenth ; oleic acid, the eighteenth; dœglinio acid, the nineteenth; and erucic acid, the twenty-second in the series. We cannot now doubt that, like acrylic acid, each of these, as well as each of the unknown members of the series, will be found to have a corresponding alcohol. These alcohols, whose existence implies, in each case, that of compounds corresponding to those in the table just given and many others, do not appear to occur in nature; but it seems likely that they may be obtained from bodies like the iodide of allyle, $\mathrm{C}_{6} \mathrm{H}_{5} \mathrm{I}$, as the allylic alcohol has been from that compound. Indeed, Hofmann and Cahours have already found that bromide of valeryle, $\mathrm{C}_{10} \mathrm{H}_{9} \mathrm{Br}$, undergoes changes similar to those seen in the iodide of allyle; and also that some of the compounds from olefiant gas, probably iodide or bromide of acetyle, $\mathrm{C}_{4} \mathrm{H}_{3} \mathrm{I}$, or $\mathrm{C}_{4} \mathrm{H}_{3} \mathrm{Br}$, appear to yield analogous results.

We may here subjoin a short table, showing the relations of the new alcohols and their derived acids to the old alcohols and their derived acids :-

Group of Alcohols.

$\begin{array}{llll} & & \text { New. } \\ \mathrm{C}_{2} & \mathrm{H}_{2} & \mathrm{O}_{2} \\ \mathrm{C}_{4} & \mathrm{H}_{4} & \mathrm{O}_{2} \\ \mathrm{C}_{6} & \mathrm{H}_{6} & \mathrm{O}_{2} & \text { Allylic } \\ \mathrm{C} & \mathrm{H}_{8} & \mathrm{O}_{2} \\ \mathrm{C}_{10} & \mathrm{H}_{10} & \mathrm{O}_{2} \\ \mathrm{C}_{12} & \mathrm{H}_{12} & \mathrm{O}_{2} \\ \mathrm{C}_{16} & \mathrm{H}_{14} & \mathrm{O}_{2} \\ \mathrm{C}_{16} & \mathrm{H}_{16} & \mathrm{O}_{2}\end{array}$

$\mathrm{C}_{80} \mathrm{H}_{30} \mathrm{O}_{2}$

$\mathrm{C}_{36} \mathrm{H}_{38} \mathrm{O}_{2}$ old.

$\mathrm{C}_{2} \mathrm{H}_{4} \mathrm{O}_{2}$ Methylic $\mathrm{C}_{2} \mathrm{O}_{4}$

C. $\mathrm{H}_{6} \mathrm{O}_{2}$ Ethylic $\mathrm{C}_{4} \mathrm{H}_{2} \mathrm{O}_{4}$

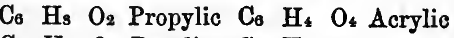

Cs $\mathrm{H}_{10} \mathrm{O}_{2}$ Butylic $\mathrm{C}_{8} \mathrm{H}_{6} \mathrm{O}_{4}$

$\mathrm{C}_{10} \mathrm{H}_{12} \mathrm{O}_{2}$ Amylic $\mathrm{C}_{10} \mathrm{H}_{8} \mathrm{O}_{4}$ Angelic

$\mathrm{C}_{12} \mathrm{H}_{14} \mathrm{O}_{2}$ Hexylio $\mathrm{C}_{12} \mathrm{H}_{10} \mathrm{O}_{4}$

$\mathrm{C}_{14} \mathrm{H}_{10} \mathrm{O}_{2}$ Heptylic $\mathrm{C}_{14} \mathrm{H}_{12} \mathrm{O}_{4}$

$\mathrm{C}_{16} \mathrm{H}_{18} \mathrm{O}_{2}$ Octylic $\mathrm{C}_{16} \mathrm{H}_{14} \mathrm{O}_{4}$

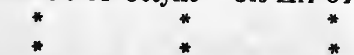

Group of Acids.

New. Old.

Carbonic ? $\mathrm{C}_{2} \mathrm{H}_{2} \mathrm{O}_{4}$ Formic

$\mathrm{C}_{4} \mathrm{H}_{4} \mathrm{O}_{4}$ Acetic

C6 $\mathrm{H}_{6} \mathrm{O}_{4}$ Propylic

C8 $\mathrm{H}_{8} \mathrm{O}_{4}$ Butyric

$\mathrm{C}_{10} \mathrm{H}_{10} \mathrm{O}_{4}$ Valeric

$\mathrm{C}_{12} \mathrm{H}_{12} \mathrm{O}_{4}$ Caproic

$\mathrm{C}_{14} \mathrm{H}_{14} \mathrm{O}_{4}$ Enanthylic

$\mathrm{C}_{10} \mathrm{H}_{10} \mathrm{O}_{4}$ Caprylic

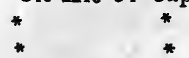

$\mathrm{C}_{30} \mathrm{H}_{30} \mathrm{O}$. Stearic 
There are many blanks in this small extract, as it were, from the entire table. But these will rapidly be filled up.

\section{A NEW CLASS OF ACIDS.}

The reader will remember, that we have described (pp. 288-293) a series of bibasic acids, running parallel to the monobasic volatile acids of the formylic series. He will also recollect, that in the benzoic group there is a series of monobasic volatile acids, of which benzoic acid is the type, and which, like the acids of the formylic series, contain, in the hydrated state, 4 eqs. of oxygen.

Now, Hofmann has found that cuminic acid, one of the acids of the benzoic series, the formula of which is $\mathrm{C}_{20} \mathrm{H}_{12} \mathrm{O}_{4}$, when boiled with chromic acid, is converted into a new acid, which he calls

Insolinic Acid, $\mathrm{C}_{18} \mathrm{H}_{8} \mathrm{O}_{8}=\mathrm{C}_{18} \mathrm{H}_{6} \mathrm{O}_{6}, 2 \mathrm{H} \mathrm{O}$. This acid is insoluble in alcohol and ether, and it bears to one of the acids of the benzoic series a relation similar to that which exists between oxalic acid and formic acid. For insolinic acid is bibasic, like oxalic acid, and it will be seen, by the following table, that it contains 2 eqs. of carbonic acid more than the corresponding monobasic acid, just as oxalic acid is formic acid plus 2 eqs. of carbonic acid. The series of the monobasic aromatic acids is itself very imperfect, and of the corresponding bibasic acids two only are known, namely, insolinic acid, and phtalic acid, the latter having been recognised as a member of this series, in consequence of the discovery of insolinic acid.

\section{Mouobasic Aromatic Acids. \\ $\mathrm{C}_{14} \mathrm{H}_{6} \mathrm{O}_{4}$ Benzoic \\ $\mathrm{C}_{16} \mathrm{H}_{8} \mathrm{O}_{4}$ Toluylic \\ $\mathrm{C}_{18} \mathrm{H}_{10} \mathrm{O}_{4}$ ? \\ $\mathrm{C}_{20} \mathrm{H}_{12} \mathrm{O}_{4}$ Cuminic}

Bibasic Acids.

$\mathrm{C}_{16} \mathrm{H}_{6} \mathrm{O}_{8}$ Phtalic

$\mathrm{C}_{18} \mathrm{H}_{8} \mathrm{O}_{8}$ Insolinic

$\mathrm{C}_{20} \mathrm{H}_{10} \mathrm{O}_{8}$ ?

$\begin{array}{lll}\mathrm{C}_{22} & \mathrm{H}_{12} & \mathrm{O}_{8}\end{array}$

Thus we see, that in another quarter a new homologous series of acids has been detected. We have now the formylic series of acids, the oxalic series of bibasic acids, the acrylic series monobasic, the benzoic series monobasic, and the phtalic series bibasic. It will be observed, that while the two monobasic series, the formylic and benzoic, have each a parallel bibasic series, the monobasic acrylic series has, as yet, no such bibasic series parallel to it. But if to acrylic acid, $\mathrm{C}_{6} \mathrm{H}_{4} \mathrm{O}_{4}$, we add $2 \mathrm{C} \mathrm{O}_{2}$, which is the difference between formic and oxalic acids, we obtain the formula $\mathrm{C}_{8} \mathrm{H}_{4} \mathrm{O}_{8}$, which is that of maleic and fumaric acids, wellknown bibasic acids, $\mathrm{C}_{8} \mathrm{H}_{2} \mathrm{O}_{6}, 2 \mathrm{H} \mathrm{O}$. The next acid in this 
series will be $\mathrm{C}_{10} \mathrm{H}_{6} \mathrm{O}_{8}=\mathrm{C}_{10} \mathrm{H}_{4} \mathrm{O}_{6}, 2 \mathrm{H} \mathrm{O}$; and this is the formula of itaconic and citraconic acids, both bibasic. I think it very probable, therefore, that some of the acids just named will prove to belong to a bibasio series, parallel to the acrylic series, and differing from it by 2 eqs. of carbonic acid. And thus we see how every discovery in organic chemistry tends to reduce all known compounds to homologous series. The homology of maleic and fumaric acids, with itaconic and citraconic acids, has not, till now, attracted notice, but the discovery of insolinic acid has not only found a place for phtalic acid, but enabled us to perceive that the vegetable acids just named may also have their place in a series not remotely connected with that of alcohol, or rather with that of acrylic alcohol, and with that of the glycerides.

\section{NEW PHOSPHORISED BASES.}

Thenard, as already mentioned, has shown that methyle can replace the hydrogen in phosphuretted hydrogen, $\mathrm{PH}_{3}$, and thus give rise to new bases.

Hofmann and Cahours have recently, by adopting a new method, greatly extended our knowledge of these. The process consists in the action of terchloride of phosphorus on zincomethyle, zincethyle, zincamyle, \&c., when chloride of zinc is formed, along with the base.

The bases thus obtained are volatile, and correspond to ammonia, in which the nitrogen is replaced by phosphorus, the hydrogen by methyle, \&c. The compounds $\mathrm{P} \mathrm{Mez}$, analogous to kakodyle, As $\mathrm{Mez}_{\text {, }}$ and $\mathrm{P} \mathrm{Mes}$, analogous to ammonia, were obtained by Thenard.

Hofmann and Cahours have not only obtained the compounds $\mathrm{P} \mathrm{Aes}_{3}$ and $\mathrm{PAyl} 3$, but by the action of the iodides of methyle, ethyle, and amyle on these, have formed a number of ammonium bases, analogous to tetramethylium. These last are obtained as iodides, from which, by the action of oxide of silver, the hydrated oxides are obtained, which in properties are analogous to Hofmann's hydrated oxides of tetramethylium and tetrethylium, \&c. The following table contains the formulæ of the radicals, bases, or iodides of radicals, which they have obtained.

$$
\begin{aligned}
& \mathrm{P} \mathrm{Me}_{2} \text { * } \\
& \mathrm{P} \mathrm{Ne}_{3} \\
& \mathrm{P} \mathrm{Me}_{4} \mathrm{I} \\
& \left.\mathrm{P} \underset{\mathrm{Ae}+}{\mathrm{Me}_{3}}\right\} \mathrm{I} \\
& \mathrm{PAe}_{4} \mathrm{I} \\
& \mathrm{P} \underset{\mathrm{Me}}{\mathrm{Aes}}\}
\end{aligned}
$$




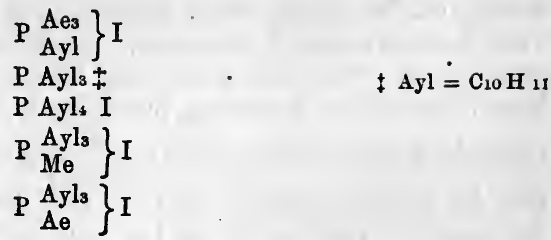

The iodides in this table, acted on by the oxide of silver, yield the hydrated oxides of the several ammonium radicals, and from these the other salts are obtained. At present we have only the general results. The details will soon be published.

It appears, as might be anticipated from the existence of kakodyle and arsenethyle, that arsenic yields a series of similar compounds, and doubtless the other metals which have been shown to combine with radicals of the ethylic series will be found to yield compounds, analogous to some, if not all the above.

\section{TRIGENIC ACID.}

This acid is formed, along with other substances, when the vapour of hydrated cyanic acid is brought in contact with aldehyde. It is first obtained mixed with cyamelide and aldehydammonia, but the whole mass is dissolved in hydrochloric acid of moderate strength, and boiled as long as aldehyde is given off. On cooling, trigenic acid is deposited in small prisms, sparingly soluble in water, nearly insoluble in alcohol. Its formula is $\mathrm{C}_{6} \mathrm{H}_{7} \mathrm{~N}_{3} \mathrm{O}_{4}=\mathrm{C}_{6} \mathrm{H}_{6} \mathrm{~N}_{3} \mathrm{O}_{3}, \mathrm{HO}$, and it is monobasic. The silver salt is somewhat soluble in hot water, and is deposited on cooling in very minute crystalline spherical concretions, which become violet in the light. Leaving out of view the other products, which are secondary, the formation of the acid is explained as follows :-

$$
\underbrace{\mathrm{C}_{4} \mathrm{H}_{4} \mathrm{O}_{2}}_{\text {Aldehyde }}+\underbrace{3\left(\mathrm{C}_{2} \mathrm{~N} \mathrm{O}, \mathrm{HO}\right.}_{\text {Cyanic Acid }})=\underbrace{\mathrm{C}_{3} \mathrm{H}_{7} \mathrm{~N}_{3} \mathrm{O}_{4}}_{\text {Trigenic Acid. }}+4 \mathrm{CO}
$$

This acid contains the elements of anhydrous cyanic acid and of aldehydammonia.

$$
\left.\begin{array}{lllllll}
\mathrm{C}_{6} & \mathrm{H}_{7} & \mathrm{~N}_{3} & \mathrm{O}_{4}
\end{array}=\begin{array}{llll}
\mathrm{C}_{2} & \mathrm{~N} & 0 \\
\mathrm{C}_{2} & -\mathrm{N} & 0
\end{array}\right\} \begin{array}{llll}
\mathrm{N} & \mathrm{H}_{4} & 0 \\
\mathrm{C}_{4} & \mathrm{H}_{3} & 0
\end{array}
$$

v. SUDORIC ACID.

According to Favre, this acid exists in combination with potash and soda in the sweat. It is not yet known in a state of perfect 
purity, but its concentrated solution does not crystallise, and, when heated, gives off ammonia. Favre analysed a mixture of sudorate of silver with ohloride of silver, and obtained results from which, after deducting the chloride, he calculated for the sudorate of silver the formula $\left.\mathrm{C}_{10} \frac{\mathrm{H}_{8}}{\mathrm{Ag}}\right\} \mathrm{NO}_{14}$; so that the acid will be $\mathrm{C}_{10} \mathrm{H}_{9} \mathrm{NO}_{14}=\mathrm{C}_{10} \mathrm{H}_{8} \mathrm{NO}_{13}, \mathrm{HO}$. If this be confirmed, it is interesting as indicating some relation between sudoric acid and uric acid, as well as xanthic oxide and inosinic acid, all of which contain 10 eq. of carbon.

The salts of this acid with bases are all very soluble, but do not crystallise. This circumstance makes their purity somewhat doubtful. These salts are also soluble in absolute alcohol, except the silver salt.

\section{THYMINE.}

This name has been given by Gorup Besanez to a new base, found by him in the thymus gland of the calf. This is extracted by cold water, after removing as much fat as possible, and yields a reddish, very acid, liquid, which when evaporated deposits a brown coagulum. The supernatant liquid is quite limpid; baryta precipitates from it the phosphoric acid. The filtered liquid, when evaporated, forms caseous pellicles, consisting of carbonate of baryta and organic matter, and leaves a brown syrupy liquid, with an odour of soup. The addition of alcohol or ether causes slowly a deposit of impure thymine; which is purified by solution in boiling alcohol, and is deposited on cooling, as a shining powder, formed of minute crystals. $25 \mathrm{lbs}$. of the thymus gland yielded by this process only 3 or 4 grains of thymine.

It has neither taste nor smell ; is very soluble in water, soluble in hot alcohol, insoluble in cold alcohol, in absolute alcohol, or in ether. Heated in a tube, it melts, yielding a crystalline sublimate and alkaline vapours with a smell like that of hydrocyanic acid. Its salts are crystallisable. The hydrochlorate and sulphate are both efflorescent; the chloroplatinate forms octohedra, soluble in water, insoluble in alcohol. 


\section{N D E X.}

PAGE

Absinthine . . . . 353

Absolute alcohol . . . 206

Acetal . . . . . 231

Acetamide . . . . 239

Acetates . . . . 235

products of decomposition of 249

Acetic acid : . . 10, 235

Acetines . . . . 306

Acetochlorhydirine . . . 307

Acetone : : . . . 249

Acetonitryle . . . $\quad .177$

Acetylammonium . . 240

" hydrated oxide of $i b$.

hydrated oxide of $\quad \cdot \quad \cdot 228$

hyduret of . . . . 247

oxychloride of . . 242, 246

oxychlorosulphuret of . 243

oxysulphuret of . $\quad . i b$.

perchloride of . . . 245

Acetylic acid, see Acetic acid .

Acetylous acid . $\quad . \quad 232$

Acid, Aceric . . . . 337

Acetic, acetous, or acetylic . 10, $235,546,577$

" anhydrous . . 234

Achilleic . . . . 337

Aconitic or equisetic . . 312

Acrylic . . . . 292

Adipic . . . . . 290

Aldehydic . . . . 232

Alituric . . . 123

Allophanic . . 181, 220

Alloxanic . . . 115

Alloxano-sulphurous . . 119

Aloeretinic and aloetic $\quad .379$

Althionic
Acid, Amalic . . . . 433

Ambreic . . . . . 301

Ampelic . . . . 491

Amygdalinic . . . 152

Anacardic . . . . 337

Angelic . . . . 293

Anilic, nitrosalicylic, or indigotic . . . 160, 374

Anisic . . . . . 164

Anthranilic . . . .375

Apocrenic . . . .461

Apophyllic . . . . 424

Arachidic . . . 285

Aspartic or malamidic $\quad .322$

Atropic . . . . 337

Balenic . $10,285,546,578$

Behenic - 10, 286, 546, 578

Benic . : 10, 277, 546, 578

Benzoglycolic . . . 137

Benzoic . : . . 129

Benzolactic . . . . 137

Bibromisatinic . $\quad .369$

Bichlorisatinic . . 368

Biethylomeconic . . . 331

Boheic . . . . 328

Boletic . . . . 337

Brassic . . . . . 295

Bromisatinic . $\quad .369$

Bromobenzoic. . . . 138

Bromocomenic : . . 331

Bromosalicylic . . 160

Brunolic . . . 476

Butyric - 10, 258, 448, 546, 577

Caffeic, Caffeotannic . . 328

Caincic . : . . . 337

Camphamic :. . . 335

Campholic . . . . 335 
PAGE Camphovinic . . . . ib. Capric or rutylic $275,546,577$ Caproic . $\quad 10,268,546,577$ Caprylic . 10, 273, 546, 577 Carbazotic, Picric, or nitrophenisic . . . 374 Carbolic . . 170, 180, 473 Carminic . . . . 378 Carmufellic . ., 337 Cerebric . . . . 520 Cerotic - 10, 286, 546, 578 Cetylic or palmitic . : - 10, Chelidonic $279,546,578$

Chinic or kinic . . 332

Chinovic . . . . 337

Chlophtalisic . . . . 489

Chloracetic . . . . 245

Chloranaphtisic . . . 488

Chloranilic . . . . 372

Chlorisatinic . . .368

Chlorocomenic . . . 331

Chloroniceic . . . .475

Chlorophenesic . . . 474

Chlorophenisic . 375,474

Chlorophenusic . . . 474

Chlorosalicylic . . . 160

Chloroxenaphtalesic . . 489

Cholalic . . . . 515

Choleic . . . . ib.

Cholesteric . . . . 518

Cholic. . . . . 514

Choloidanic . . . . 519

Choloidic . . . . 515

Cholonic . . . . 516

Chrysammic . . . 379

Chrysanilic . . . . 375

Chrysolepic . . . 380

Chrysophanic . . . 350

Cinnamic . . . . 166

Citraconic . . . . 313

Citric . . . . . 310

Cobaltocyanic . • . 96

Cocinic - 10, 277, 546, 577

Cocognidic . . . 337

Colopholic . . . $\quad .350$

Comenamic . . . . 331

Comenic . . . . $i b$.

Coneic . . . . . 337

Crameric . . . $i b$.

Crenic . . . . . 461

Croconic . . . $\quad .67$
Acid, Cumaric

Cuminic . . . 131,156

Cyameluric . . . 106

Cyanic . . . . 75

Cyanilic . . . . 107

Cyanoxalic . . . . 127

Cyanuric . . . . 81

Damaluric . . . . 533

Damolic . . . . . $i b$.

Dialuric . . . 122

Dilituric . . . . . 123

Dinitranisic . . . 165

Disulphonaphtalic . . . 486

Dœglinic . . . . 295

Draconic or anisic . $\quad .165$

Elaidic . . . . 294

Ellagic . • • . . 327

Equisetic or aconitic . $\quad .312$

Erythric . . . . . 358

Erucic . . . . 295

Ethalic or palmitic . . 10 ,

$279,546,578$

Ethionic . . . . 227

Ethylocomenic . . . 331

Ethylomeconic . . . 330

Euchronic . . . . 68

Evernic . . . . 360

Eugenic . . . . . 336

Ferridcyanic . . . 94

Ferrocyanic - . . 90

Formic . $10,196,546$

Formobenzoilic . . 134

Fulminic . . . . 79

Fulminuric . . . . 83

Fumaric or paramaleic $\quad .322$

Fungic . . . . . 337

Gallic . . . . . 326

Geic . . . . . 461

Glucic . . . . . 439

Glycolic . : . . 137

Guaiacic . . . . 168

Hemipinic . . . . 423

Hidantoic . . . 126

Hippuric . . . 135

Humopinic . . . 424

Hydrocyanic . . . 71

" anhydrous . $i b$.

Hydrocobaltocyanic • . 96

Hydroferridcyanic • 94

Hydroferrocyanic . . . 90

Hydroleic . . . . 294

Hydromellonic . . . 102

Hydrosulphomellonic . . 108

Hydurilic " . . . 116 
PAGE

Acid, Hyocholalic . . . 517

Hyocholeic . . . . ib.

Hyocholic . . . $\quad i b$.

Hyperuric . . . 128

Hyposulphobenzidic . 139

Hyposulphobenzoic . . 138

Hyposulphoglutinic . . 486

Hyposulphoindigotic . . 366

Hyposulphosuccinic . . 289

Igasuric . . . . . 337

Imasatinic . . . . 373

Indigotic $\quad . \quad$. 169,374

Indinic . . . . 371

Inosinic . . . . .528

Insolinic . $\quad . \quad$. 599

Iodosalicylic . . . . 160

Isatinic . $\quad . \quad .367$

Isatiuosulphurous . . . $i b$.

Isethionic . $\quad . \quad .227$

Isotartaric $\quad . \quad . \quad . \quad .319$

Itaconic $\quad . \quad$. $\quad .313$

Japonic . . . . . 328

Kakodylic . . . . 192

Kinic . . . . . 332

Lactic . . . 137, 446

Lactucic . . . . 337

Lævotartaric . . . 318

Lampic . . . . 232

Lantanuric . . . 125

Laurostearic $\quad 10,27 \dot{7}, 546,578$

Lecanoric . . . 357

Leucic . . . . . 295

Leucoturic . . . . 115

Linoleic . . . . . 295

Lipic . . . . . 290

Lithic or uric . . . 109

Lithofellic . . . . 520

Malamidic or aspartic . . 322

Maleic . . . $i b$.

Malic . . . . . 520

Margaric - $10,280,546,578$

Margaritic . . . 10, 277

Mechloic . $\quad$ • . 356

Meconamidic . . . 331

Meconic . . . 329

Meconoethylomeconic . . 331

Melanic . . . . 157

Melanuric . . . . 106

Melassic . . . . 439

Melissic . 10, 287, 546, 578

Mellitic . . . . 68

Menispermic . . $\quad .337$

Mesaconic . . . 314

Mesoxalic . : . 117
Acid, Metacetonic or propylic 10 ,

253,546, 577

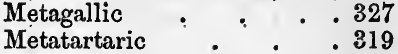

Methionic . . . . 227

Metoleic . . . . 294

Mimotannic . . . 328

Moringic . . . $\quad 293$

Moroxylic . . . . 337

Mucic . . . 442

" modified. . . ib.

Mykomelinic . , .118

Myriospermic . . . 167

Myristic - $\quad 10,277,546,578$

Naphtalic . . . .489

Naphtionic . . . 487

Narcotinic . . . . 424

Nitranisic . . . . 165

Nitrobenzoic . . . . 138

Nitrobutyric $\quad$. $\quad . \quad 259$

Nitrocholic . . : . E18

Nitrocinnamic . . . 166

Nitrococcusic . . . . 378

Nitrohippuric . . . 136

Nitrohydurilic . . . 117

Nitromeconic . . . 356

Nitronaphtesic, \&c. . . . 488

Nitrophenesic, \&c. $\quad . \quad 474$

Nitrophloretic. . . . 164

Nitrophtalic . . . 489

Nitropicric, picric, or carbazotic . . . 374,474

Nitropropylic . . . 228

Nitrosalicylic, anilic, or indigotic . . . 160, 374

Nitrotartaric . . . 314

Nitrovalerianic . 264, 269

Enanthic . . . 336

Enanthylic $\quad \dot{10,270,546,577}$

Oleic . . . . 293

" action of heat on . . ib.

" action of nitric acid on . 294

Oleophosphoric . . . . 520

Opianic . . . . 422

Opianosulphurous . . . 423

Oxalic . . . 62

Oxalovinic . . . 218

Oxaluric . . . . 118

Oxamic . . . . 65

Oxyphenic . . . . 475

Palmitic . $\quad 10,279,546,578$

Parabanic . . . . 118

Paracholic . . . .514

Paracomenic . . . 331 
Acid Paramaleic or fumaric PAGE

Paratartaric or racemic . 317

Parellic . . . . 358

Pectic . . . . 457

Pelargonic 10, 274, 546, 578

Persulphocyanic . . . 101

Phosphovinic . . . 214

Phtalic . . . . . 489

Picric, nitropicric, or carba-

Pimelic $\quad \cdots \quad 374,474$

Pimelic
Pinic . . . . $\quad . \quad .348$

Pneumic . . . 580

Propylic - 10, 253, 546, 577

Prussic or hydrocyanic . . 61

Pyrogallic . • . . 326

Pyromeconic . . . . 331

Pyromucic . . . 442

Pyrotartaric . . . . 320

Quercitannic . . . 324

Quinic or kinic . . . 332

Racemic . . . . 317

Rhodizonic . . . 67

Ricinolic . . . . 303

Rocellic . . . . 336

Rosolic . . . . 476

Rubinic . . . . 328

Rutylic . . 275, 546, 577

Saccharic. . . . .440

$\begin{array}{lllll}\text { Sachulmic } & \text {. } & . & . & 439\end{array}$

Salicylic . . . . . 158

Salicylous . . . . 156

Sebacic . . . . . 291

Solanic . . . . 337

Sorbinic . . . . 443

Stearic . . . . . 283

Styphuic . . . . 475

Suberic . . . . . 290

Succinic . . . . . 289

Sudoric . . . . 601

Sulphacetic . $\quad . \quad .235$

Sulphacetylic . $\quad . \quad 249$

Sulphallylic . . . . 596

Sulphamylic . . . 261

Sulphethamic . . . . 222

Sulphoamidic . . . 453

Sulphocamphic . . . 336

Sulphocamphoric . $\quad .335$

Sulphocarbolic . . . 474

Sulphocetylic . . . 278

Sulphoctylic .. . . . 272

Sulphocyanic or hydrosulphocyanic

Sulphoglyceric
Acid, Sulphoindigotic . $\quad .366$

Sulphomannitic . . . 449

Sulphomesitylic . . . 251

Sulphomethylic . . 179

Sulphophenic . . . 474

Sulphopianic . . . . 423

Sulphopurpuric . . . $\quad$. 366

Sulphosaccharic . . . 439

Sulphotoluylic . $\quad 167$

Sulphovinic . $\quad .213$

Sylvic . . . . 348

Tanacetic . . . . 337

Tannic . . . . 324

Tanningenic . . . . 328

Tartaric . . . 314

" action of heat on $\quad .317$

" anhydrous . . . $i b$.

Tartrovinic . . . . 315

Thionaphtamic . . . 487

Thionuric . . . . 119

Toluylic . . . 141, 170

Trigenic . . . .601

Uramilic . . . 120

Uric . . . . 109

Uroxanic . . . 126

Usnic . . . . 361

Valerianic $\quad$. 10, 264, 546, 577

Viridic . . . . 328

Xanthic . . . . . 218

Xanthopenic . . . 423

Acids, organic, theory of . . 47

hydrogen, theory of . . 49

anhydrous . . . . $i b$.

monobasic . . . . 48

bibasic . . . . . $i b$.

tribasic . . . . $i b$.

polybasic . . . . $i b$.

coupled . . . . $\quad .50$

fatty . . 276-287

volatile oily, series of, $10,546,577$

Aconitic acid . . . . 312

Aconitine . . . . . 427

Acroleine . . . $\quad 309$

Acryle . . 309, 595

hydrated oxide of . $\quad i b$.

Acrylic acid - . 309,595

Action of acids on organic compounds . . . 34

Action of bases on organic compounds - : . . 36

Action of ferments on organic compounds . . . 38

Action of heat on organic compounds 
PAGE Adipic acid
Agriculture, chemistry of, $^{\cdot 297}$ Air, its functions in vegetation $i \vec{b}$. Alanine - . 136, 230, 406 Albumen, animal : . 4496 vegetable . . . . $i b$. its composition : . 499,557 formation in plants . . .558 products of oxidation of .570 produced from fibrine .569

Albuminous compounds 499,557 their origin . . . . 558 their destruction . . 570 Alcohol . . . . 205 absolute . . . . . 206 action of, on the s5stem . 589 products of oxidation of . 228 may replace fat or starch in food action of ihlorine $\therefore{ }^{\circ} \cdot 589$ Alcoholate of potash $\quad . \quad 221$ Alcohols, series of . $\quad 19,22$ Aldehydammonia . $\quad .229$ Aldehyde . . . . . ib. acetic . . . $\cdot \bullet^{-} i b$. butyric . . . . . 257 caprylic . . . . 273 formic . . . . 195 isomeric modifications of .231 œnanthylic . . . 270 propionic . . . . 253 resin of . . . . 231 valerianic . . . . 264 Aldehydes, the series of . $\quad 23$ Aldehydic acid . . . 232 Alituric acid . . . 123 Alizarine . . . . . 351 Alkaloids, artificial . $\quad$. 381 different classes of $\quad 381-408$ natural

Allantoine.

Allophanic acid ${ }^{\circ} \quad$. 181,220 Alloxan . . . . . 113 Alloxanic acid . . $\quad .115$ Alloxantine . . . .120 Allylamine . . . . 597 Allyle . . . 343, 595 benzoate of . . .596 cyanate of . . . . 597 hydrated oxide of . . . 596 iodide, \&c., of . . . 595 other compounds of . 595-598 sulphocyanide of
Allyle, sulphuret of $\quad$ - $\quad 343,595$ series . . . 595 Almonds, oil of . $\quad . \quad+295$ oil of bitter . . 132-156 Aloes, action of nitric acid on . 379 Aloeretinic acid . . . . $i b$. Aloetic acid . . . . $i b$. Alpha resins . . . .350 orceine . . . . 362 orsellesic acid . . . . 360 orsellic acid. . . . . $i b$. Althionic acid . . . .227 Amalic acid . . . 433 Amaphenase or Aniline, 364,375, 389 Amarine . . . . 145, 404 Arnarythrine . . . 359 Amasatine . . . . 373 Amber, and oil of . . 290 Ambreic acid . . . . 301 Ambreine . . . . $i b$. Amide or Amidogen . 52 bases 15, 61, 184 et seq., 383 et seq. Ammelide . . 103 Ammeline . . . 104, 404 Ammonia . . 15,53 acetate of $\quad \therefore \quad .236$ anomalous cyanate of . 78 bases . . . 182, 383 benzoate of . . . 130 malate of, acid . . . 321 cyanate of, basic . : . 77 dialurate of . . . 122 mellitate of . . . 68 oxalate of . . . 64 oxalurate of . . $\quad .119$ phosphate of, and magnesia . 535 thionurate of . . 119 use of, as a manure . 551,562 always present in the atmosphere . . . . 551

Ammonium . . . 53 bases . . . . 187, 384 oxide of . . . . 53 Ampeline . . . . 491 Amygdaline . . . . 152 Amygdalinic acid . . . $i b$. Amylamine . . . 262

Amyle . . . . 260 acetate, \&c., of oxide of . 262 chloride, \&c., of . . . 264 hydrated oxide of . . . 261 Amylophenylamine . 396 Amylotriethylium or Triethylamylium 
PAGE

Amylo-urea $\quad$. $\quad .262$

Anchusine . . . . 351

Anemonine . . . 346

Angelicine . . . . 356

Anilic, indigotic or nitropicric acid .

Aniline 36.374 analogy of, with ammonia 391 Anilocyanic acid . . 394

Anilo-mellone, \&c. . . . 395

Anilo-urea . . . . . 391

Animal heat, origin of $\quad .581$

Animals, nutrition of . . . 568

Anime, resin of . . . 348

Anise, oil of . . 164, 341

Anisic acid . . . . 164

Anisidine . . . . 165

Anisoine . . . . 341

Anisole . . 165, 171, 180

Anisyle . . . . 164

Annotto or Anatto . . . 351

Anthracene . . . . 490

Anthranilic acid . . . . 375

Antiarine . . . . 354

Apiine . . . . . 458

Aporetine . . . . 350

Apyrine . . . . . 431

Arabine . . . . . 456

Arachidic acid . . . . 285

Archil . . . . . 362

Aricine . . . . . 417

Arrow root . . . . 452

its unfitness for food . . 506

Arsenethyle . . . . 211

Arsenethylium . . . . $i b$.

Arsenodiethyle . . $\quad i b$.

Artificial bases - $\quad 381$ et seq.

Arum, oil of . . . . 345

Asarone . . . . . 346

Asparagine or malamide . $\quad .322$

Aspartic or malamidic acid . $i b$.

Asphaltum . . . . 493

Assafœetida, oil of . . . 345

Athamantine . . . . 301

Atmosphere, functions of, in animal life

functions of, in vegetation

contains ammonia et seq.

contains ammonia : 551

proportion of oxygen and carbonic acid in, always the same.
Atoms, arrangement of, very important . . 16-18

Atropine . . . . . 426

Azadirine . . . . 430

Azobenzide . . . 140

Azobenzoide . . . . 147

Azobenzoyle . . . 143

Azolitmine . . . . 362

Azotised animal and vegetable

products . . $356-435$ origin of . - . 552 et seq. changes of, in the body, 569 et seq.

Balentc acid . . . 10, 285

Balsam of Peru . . 166 of Tolu . 1 167, 348

Bases, organic or alkaloids $\quad 381$ artificial . . . $381-408$ " formation of . . $i b$. from volatile oils . $\quad . \quad 389$ of animal oil . . . . . 397 of cinchona bark . . . 413 of coal tar . . 389, 396 of the Papareracer . . 418 of the Solanaceæ, \&c. $\quad . \quad 425$ derived from aniline $\quad 375-378$

, from animal products 406

" from naphthaline . 402

" from narcotine . . 423 from oil of bitter al-

monds . . . 145, 404 derived from oil of bran .405 " from oil of mustard 402 amide . . . . 182, 383 ammonia . . 184, 384 ammonium . . . 187, 386 containing chlorine, \&c. . $i b$.

" platinum $55-60$ nitryle $\cdot{ }^{-} \quad 185,384$

Basil, oil of $\quad$. $\quad . \quad 342$

Bassic acid . $\quad$. $\quad 10,285$

Bassorine . . . 456

Bebeerine . . . 430

Beer . . . . 508

Behenic acid . . 10, 286

Benic acid . . . 10,277

Benzamide . . . . 134

Benzène or benzole, 139, 141, 392

Benzhydramide . . . 143

Benzile . . . . 146

hydrocyanate of . . 149

Benzilic acid . . . . 147

Benzimide . . . . 145 
Benzoates

Benzoic acid anhydrous

131

Benzoic alcohol

. 151 series

131

Benzoicines

307

Benzoene or toluole $\quad$ 141, 167

Benzoglycolic acid

. 137

Benzolactic acid

ib.

Benzoine

145

hydrocyanate of . $\quad .149$

Benzoinam . . . . . 146

Benzoinamide . . . $\quad i b$.

Benzole . . 139, 141, 168

chloride of . . . 140

Benzolone . . . . 144

Benzone, or benzophenone $\quad .142$

Benzostilbine . . . . 144

Benzoyle . • . . 128

chloride, \&c., of . . . 133

hyduret of . . . . 132

formation of hyduret of .153

benzoate of hyduret of . . 135

Berberine . . . . 431

Bergamot . . . . . 342

Beta orceine . $. \quad . \quad .362$

orsellic acid . . . . 360

resins . . . . . 350

Bezoar stones . . . . 520

Bibromisatine . . . . 369

Bibromisatyde . . . .370

Bichlorisatine . . . .368

Bichlorisatinic acid . . . $i b$.

Bichlorisatyde . $\quad .370$

Bichloricinchonine . . .416

Bichloronicene . . . 475

Bicyanide of mercury . . $\quad$. 88

Biethylomeconic acid . $\quad 331$

Bile . . . . . 513 its constitution . . ib. et seq.

ox . . . . . 514

pig . . . . . . 517

products of decomposition of $i b$.

serpents'. . . . 516

function of . . . . 489

Biliary calculi . . . 520

Binitrobenzophenone . 142

Bismethyles . . . . 218

Bitartrate of potash . $\quad 315$

Bitter almonds, oil of . . 132

thenry of its formation $\quad .153$

Bituminous shale . . .491

Blanquinine . . . .417

Blood, coagulation of . $\quad .536$
Blood, composition of absorbs more oxygen than water . . . 579 is always alkaline . $\quad .539$ uses of the globules, the phosphate of soda, and the iron of . 578 et seq.

Blue, Prussian, its formula $\quad 92$

Blue colouring matters . 352, 356

Blue, Indigo . . . 362

Boiling of meat and vegetables 587

Boa constrictor, urine of . 109

Boletic acid . . . . 337

Boloretine . . . . .493

Bone earth . . . . 563

in guano . . . 533, 563

dust as a manure $\quad . \quad 563$

Bones . . . . . . 512

gelatine of . . . 511

Borneene . . . . . 341

Borneo camphor . . . 335

Brain and nervous matter . 520

Brandy . . . . . 508

Brazil wood . . . 352

Bread and flesh compared . 587

Bromachlonaphtose, \&c. . . 482

Bromal . . . . 246

Bromaniline . . . . 375

Bromide of benzole. $\quad .149$

Bromide of chlonaphtese, \&c. . 483

Bromisatine . $\quad$. $\quad .369$

Bromobenzoic acid . . .138

Bromocinchonine . . . 416

Bromocodeine . . . 420

Bromocomenic acid . . 331

Bromohelicine . . 163

Bromophenisic acid . . 474

Bromosalicylic acid . . 160

Bromostyrole . . . 349

Bronaphtase, \&c. . . .479

Browu coal . . . . 472

Brucine . . . . 429

Bryonine . . . . 354

Butter, volatile oily acids of, 268

proportion of in milk et., 521

Butylamine $. \quad . \quad 256$

Butyle . . . . . 255

compounds of . . . 256

Butylene . . . . . 255

Butylic mercaptan . . . 256

Butyral, or butyric aldehyde . 257

Butyramide . . . 260

Butyrate of oxide of ethyle $\quad 259$ 


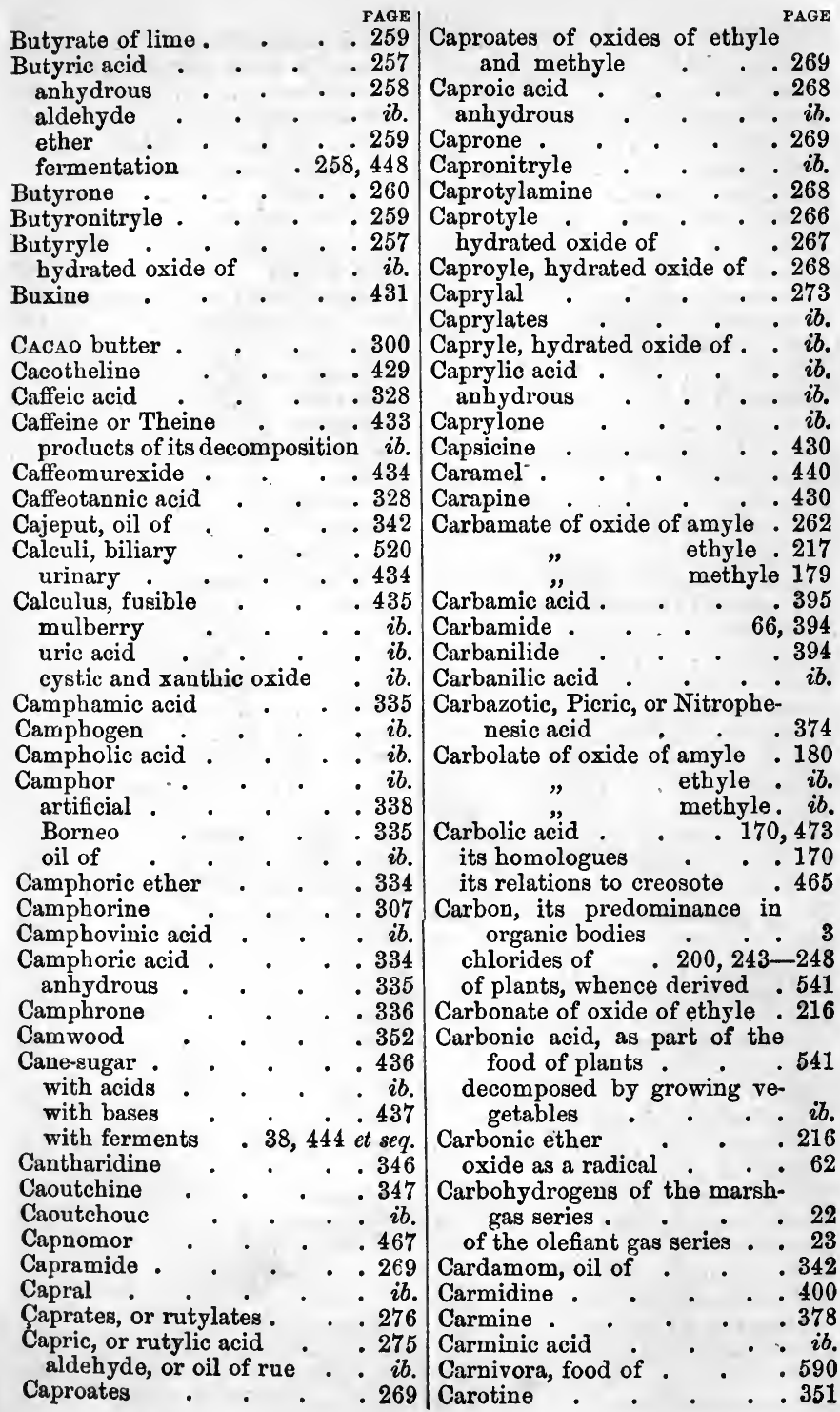


PAQE

Carthamine . $\quad . \quad .351$

Carraway, oil of . . . . 34z

Carvacrole . . . . ib.

Carvene . . . . . $i b$.

Carvole . . . . . ib.

Caryophylline . . . . 336

Cascarilla, oil of . . . 342

Caseine, animal and vegetable. 504

products of decomposition of 505

produces lactic fermentation 446

Castorine . . . 301

Castor oil . . . . 302

Catalysis, doctrine of . $\quad 40$

Catechine . . . . 328

Catechu . . . . . ib.

Cathartine . . . . . 354

Cedriret . . . . . 470

Cellulose . . . . . 458

Cerasine . . . . . 457

Cerebric acid . . . . 520

Cerebroleine . . . . $i b$.

Cerosine . . . . 301

Cerotate of oxide of ceryle . 286

Cerene . . . . . $i b$.

Cerotic acid . $. \quad . \quad i b$.

Ceric alcohol . . . . $i b$.

Ceryle, cerotate of oxide of $\quad i b$.

hydrated oxide of . . . $i b$.

Cetine . . . . 280

Cetrarine . . . . . 353

Cetyle . . . . . 277

oxide of . . . . . $i b$.

oxide of, and sodium . $\quad 278$

palmitate of oxide of . .280

hydrated oxide of, or ethal . 277

Cetylic, or palmitic acid . 279

alcohol, or ethal . . . 277

mercaptan . . . . 279

Chærophylline . . . 435

Chamomile, oil of . . . 342

Charcoal, animal . . 512

Cheese . . . . . 523

flavour of . . . . $i b$.

from peas and beans $\quad .505$

Chelerythrine . . . . 425

Chelidonine . . . . ib.

Chemistry, organic . . . 1 of animal and vegetable life 494

Chinovic acid . . . . 329

Chinovine . . . . 417

Chiococcine . . . . 428

Chlonaphtase . . . . 479

Chlonaphtese . . . . ib.

Chlonaphtise . . . . 481

Chlonaphtose . . . . 482

Chlonaphtuse . . . . $i b$.

Chlonaphtalase . . . . ib.

Chlonaphtalese . . . ib.

Chlonaphtalise . . . . ib.

Chlophtalisic acid . . . $i b$.

Chloracetic acid . . . 246

Chloral . . . . 231, 245

Chloranaphtisic acid . . 488

Chloranilam . . . 378

Chloranilammon . . . $i b$.

Chloranile . . . . 372

Chloranilic acid . . . . $i b$.

Chloraniline . . . . 376

Chloranisole . . . 165

Chloranthacenese . . . 490

Chlorebronaphtise . . . 482

Chlorebronaphtose . . . $i b$.

Chlorhydrine . . . 306

Chloretheral .. . . . 248

Cinloribronaphtose . . .482

Chloribronaphtuse . . .483

Chloride of acetyle . . 245

" benzole . 140

, benzoyle . . . 134

, bronaphtese . . 483

" carbon . 243, 248

." chlonaphtase .483

" chlonaphtese . . $i b$.

" cyanogen . . 86

" ethyle . . . 206

" methyle . . 177

" napthaline . . 484

" tetramethylium. $\mathbf{1 8 7}$

" tetrethylium . . 225

Chlorindopten . . . 372

Chlorindine . . . . 371

Chlorisatine . . . . 367

Chlorisatyde . .. . . 370

Chlorobenzine . . 140

Chlorocarbonic acid . . 66

ether . . 216

Chlorocodeine . . 420

Chlorodibromaniline . . . 377

Chloroform . . . 198

Chlorohelicine . . . 163

Chloronicine . . . .475

Chlorophenesic, chlorophenisic,

and chlorophenusic acids . 474

Chlorophyll . . . . 352

Chlorosalicine . . . 162

Chlorosalicylimide . . . 160

Chloroxenaphtalise, oxide of . 488 


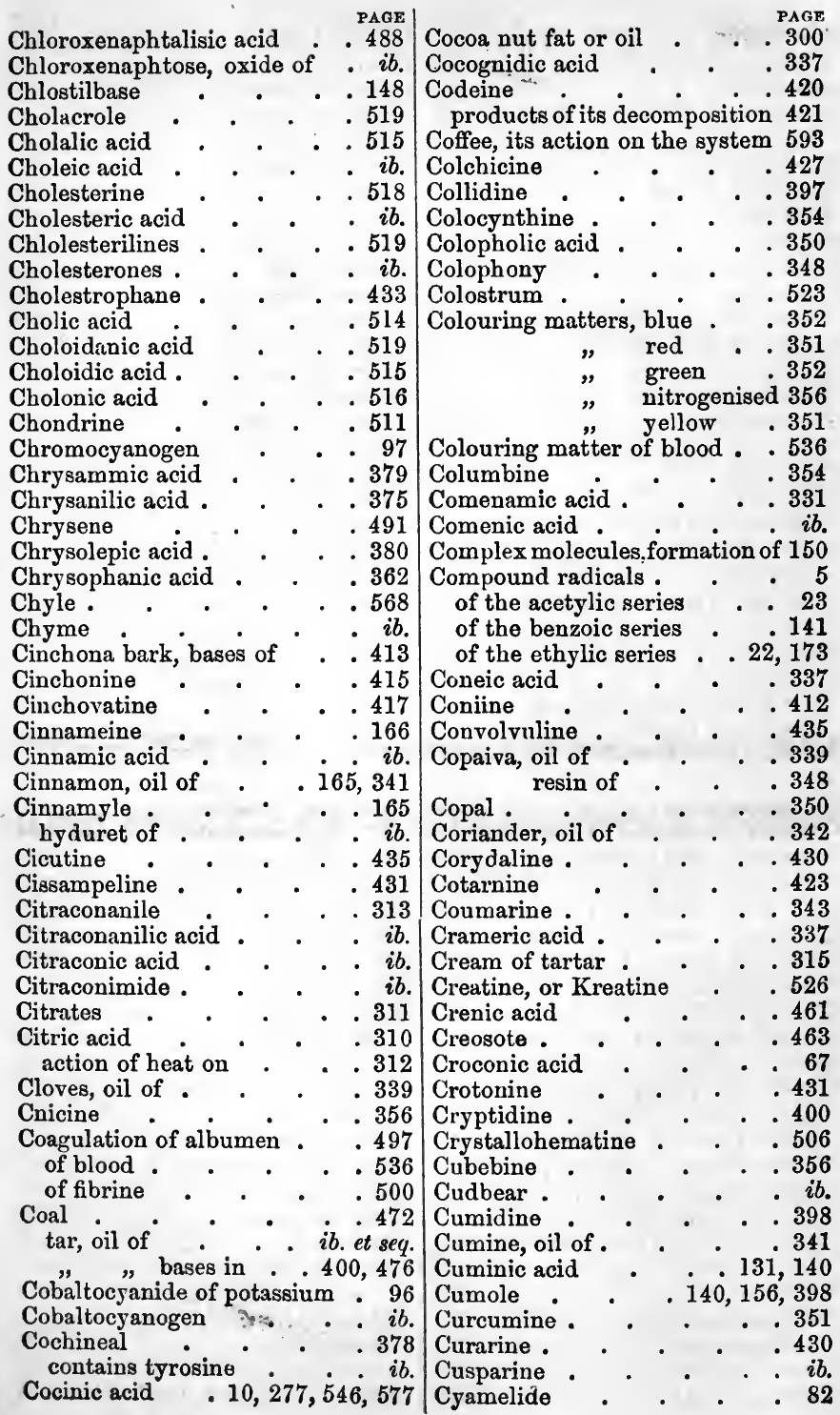




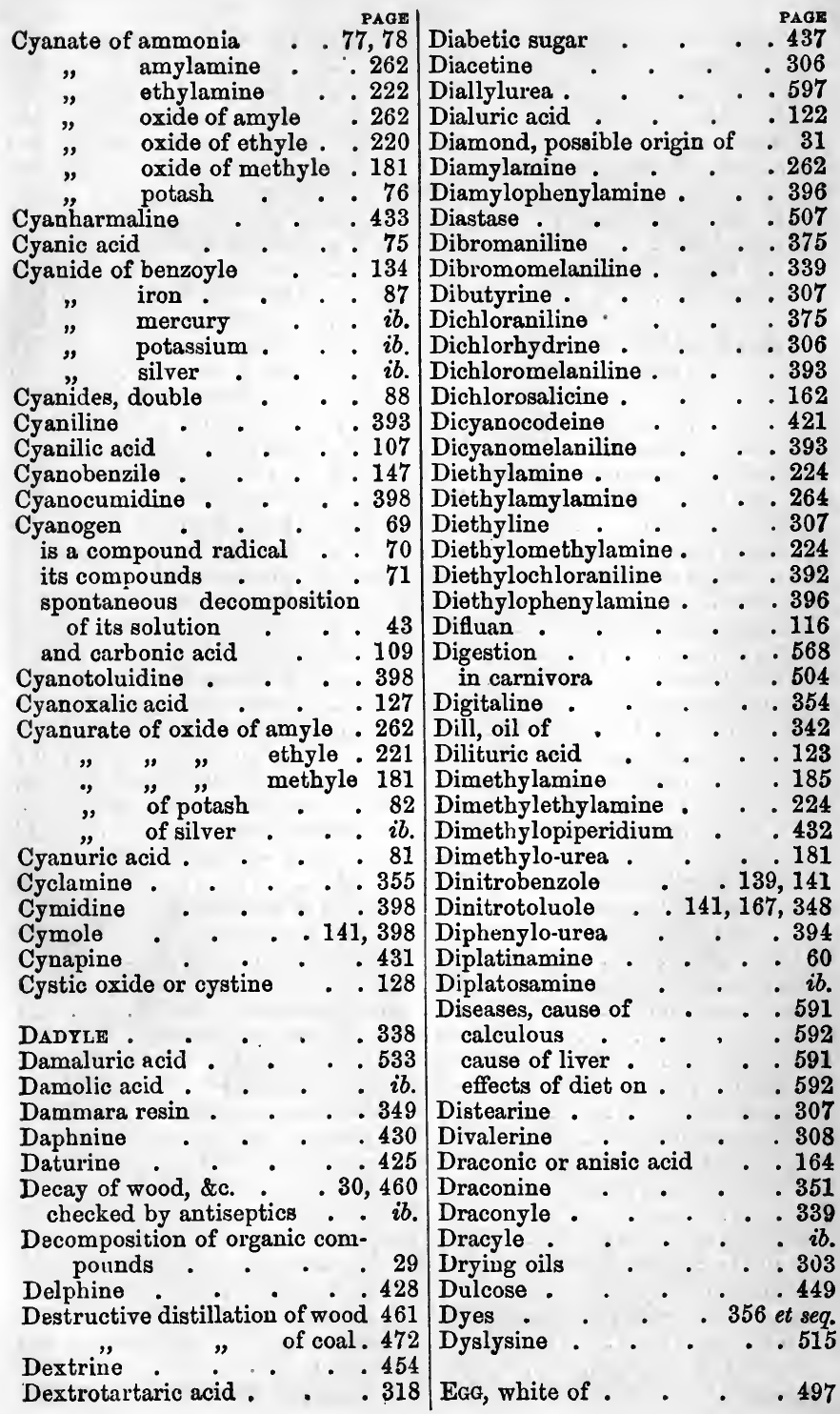


Fog, yolk of

Elaidic acid . . . . 294

Elaidine . . . . 308

Elaldehyde . . . . 231

Elaterine . . . . . 354

Elements of inorganic com-

pounds . . . . 2

of organic compounds $\quad$. 1,2

Elemi, oil of . . . . 338

resin of . .348

Ellagic acid . . . . . 327

Emetine . . . . 428

Emulsine of bitter almonds . 153 sweet almonds is

the same . $i b$.

its action on amygdaline

154

Enamel of teeth contains flu-

oride of calcium . $\quad 512$

Epichlorhydrine . . . 306

Equisetic acid . $\quad .312$

Eremacausis or decay . . $\quad 30$

Ergotine . . . . . 355

Erythric acid . . . . 358

Erythroleine . . . . 362

Erythrolitmine . . . . $i b$.

Erythromannite . . . 359

Essential or volatile oils . 337

$\begin{array}{ll}\text { non-oxygenated } & \text { ib. } \\ \text { oxygenated } & .340 \\ \text { sulphurised } & .343 \\ \text { concreteprinciples } \\ \text { allied to . }\end{array}$

Ethal or cetylic alcohol . .277

Ethalic or cetylic acid, 10, 279, 546,

578

Ether • • • . 201

its formation explained . 202

Eeries of the .
s

Etherine . . . . 204

Etherole - . . . $i b$.

Ethionic acid . . $+\bullet_{i b}$

Ethylacetamide . . . . 239

Ethylamine . . . . 221

Ethylamylophenylamine . .396

Ethyle . . . . 201

analogy with metals . . 14

bromide and iodide of . $\quad 206$

chloride of

$i b$.

compounds with phosphorus 220

cyanide of . . . 208

fluoride of . oxide of . . . $\quad .201$
Ethyle, oxide of, and potassium 221

PAGE

hydrated . . 204

acetate of . . 235

acid sulphate of . 213

allophanate of . 220

benzoate of . . 219

borates of . . . 216

butyrate of . . 259

carbamate of . . 217

carbonates of . . 216

cyanate of . . 220

caproate of . . 269

formiate of . . . 197

hippurate of . . 219

hyponitrite of . . 214

nitrate of..$\quad i b$.

œnanthate of . 336

oxalates of . . 217

oxamate of . . . 218

perchlorate of . . 215

acid phosphate of . 214

propylate of . . 254

rutylate of . . . 276

sulphate of . . 213

salicylate of . .219

silicates of $\quad . \quad 216$

sulphocarbonate of . 218

seleniuret of . . 208

sulphuret of . . 207

hydrosulphuret of . ib.

Ethyle, compounds of, of un-

certain constitution . . 247

products of the oxidation

of . . . . . 228

action of chlorine, \&c., on the

compounds of .. . 242

Ethylocodium . . 421

Ethylomeconic acid . . . 330

Ethylomethylamine . . 224

Ethylomethyloconium . .413

Ethylonarcotine . . . 425

Ethylonitraniline . . . 392

Ethylophenylamine . . 395

Ethylopicolium . . . . 397

Ethylopropylamine . . . 262

Ethylopropylamylamine . . 263

Ethylopyridium . . . 397

Ethyloquinolium.$\quad .401$

Ethylo-urea . . . . 220

Ethyloxamide . ... . 223

Euchronic acid . . 68

Eugenic acid . . . 336

Eugenine . $. \quad . \quad .337$

Euphorbium, resin of . $\quad .348$ 
PAGE

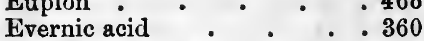

Excrements . . . .530

Excreta, importance of, as manures

Extract of meat, how made .587 $" \quad$ " its value . . $i b$.

Fat, animal, its origin . $\quad .575$ oils, natural . . . 300 " action of bases on . $\quad .304$ " $\quad$ " neat on . $\quad 309$ " " sulphuric acid

Fennel, oil of ${ }^{\text {on }} \cdot$. $^{3} 342$ Ferments . . 38, 444 Fermentation, butyric $\quad 258,448$ lactic .446 theory of 38,444 vinous 205,444

Ferridcyanides 94 Ferridcyanogen . . . $i b$. Ferrocyanic acid . . . 90 Ferrocyanide of potassium . $i b$. Ferrocyanogen iron $\cdot \quad 92$ Fibrine, animal . . . 500 vegetable . . . . $i b$. of blood . . . . $i b$. of flesh and of flour . . ib. transformed into albumen . 501

Fichtelite . . . . . 492

Fixed or ammonium bases, 137, 225, oils . . . 300,307 Flesh, juice of . $\quad . \quad 525$ Fluoride of calcium in bones .512 Food of plants . . $539 \mathrm{et} \mathrm{seq.}$ of animals . . .567 et seq. digestion of . $.568 \mathrm{et} \mathrm{seq}$. respiratory . . . 578 et seq. sanguigenous . . 567, 582 proportions of these in .582

Formanilide . . . . 391

Formiates . . . . 197

Formic acid . . . . 196 aldehyde . . . . 195

Formobenzoilic acid . . . 134

Formomethylal . . . 176

Formonitryle . . . . 61

Formyle
Formyle, perchloride of, or chloroform . . 198 series . . . . 10

Fossil resins, wax, \&c. $\quad . \quad 492$

Fraxinine . . . . 355

Fulminates . . . . 79

Fulminating mercury . $\quad i b$.

Fulminic acid . . . . $i b$.

Fulminurates . . . 84

Fulminuric acid . . . 83

Fumaramide . . . $\quad .322$

Fumaric acid . . . . $i b$.

Fumarimide . . . . 323

Fungic acid . . . . 337

Furfurine. . . .405

Furfurolamide . . . . $i b$.

Furfurole . . . . $i b$.

Fuseloel . . . . 445

Galam butter . . $\quad .300$

Gallic acid and gallates . . 326

Galls, nut . . . . 324

Gamboge . . . . . 351

Gamma resin, of benzoin . 348

Garlic, oil of . . . 345

Gas, marsh, series of $\quad \cdot 22,175$

olefiant, series of $\quad \cdot \quad 23,247$

phosgene . . . 66

Gastric juice . . . 524

Gaultheria procumbens, oil of 158 ,

Geine 180

Geic acid . . . . . ib.

Gelatigenous tissues . $\quad .510$

Gelatine . . . . $i b$. products of oxidation of .511

sugar of, or glycocine 136,406

Gentianine . . . 353

Germination, theory of $\quad .540$

products of . . . . 541

Glaucine . . . . 425

Glaucopicrine $\quad$. $\quad i b$.

Gliadine . . . .505

Globules of the blood . . 536

Glucic acid . . . . 439

Glue . . . . .510

Gluten . . . . . 501

Glycerides . . . 302, 306

Glycerine . . . . 305

Glyceryle and its compounds - $i b$.

Glycocine, or Glycocoll 136, 406

Glycolic acid . . . 137, 406

Glycyrrhizine . . . 458

Goulard's extract . . . 237 
Grape juice, fermentation of $\mathbf{4 4 4}$ Grape sugar . . . 437 action of acids and bases on 439 derived from cane sugar, starch, and woody fibre . 437 Green hydrokinone . $\quad 333$ Gros, the platinised radical of 55 Growth of auimals . 568 et seq. plants . . 540 et seq. Guaiacene : • • . 168 Guaiacine • $\quad \cdot \quad \cdot \quad \cdot 355$ Guaiacum . . . 168 Guaiacyle . . . . . $i b$. hyduret of . . . . $i b$.

Guauine . . . . 128 Guano, composition and origin of . . . . 533, 563 explanation of the action of it $i b$. Guaranine, Theine or Caffeine 433 Gum arabic . . . . 456 benzoin . . 129, 348 Bassora . . . . 456 tragacanth . . . . $i b$. Gun-cotton . . . $\quad$. 459

Hamatoxyline . . . . 352 Hair. . . . . 505 Harmaline . . . . 432 Harmine . . . . $i b$. Hatchetine . . . . 492

Heat, animal, origin of . $\quad .581$ Heavy oil of wine . . . 226 Helicine $. \quad . \quad . \quad 163$ Helicoidine . - . . $i b$. Hellenine . . . . 346 Hemateine . . . . 352 Hematosine . . . . 537 Hemipinic acid . . . . 423 Heptyle . . . . 270 Herapathite, or sulphate of iodoquinine . . . 414

Herbivora, nutrition and respiration in . $\quad . \quad .568$ urine and excrements of . . 532

Hesperidine . . . . 354 Hidantoic acid _ . 126

Hippuric acid . . 135 exists in human urine . . ib. products of its decomposition . . . . 136

Homologous series . . 19 tables of $1,10,22,141,296$, \&c. Honey, sugar of, is grape sugar 437
Hops, oil of $\quad 345$

Hordeine . . . . 509 Horn, products of its decomposition . . . 505

Humic acid . . . . 461

Humine . . . . $i b$.

Humopinic acid . . . 424

Humus . . . . .461

true function of, in vegetation . . . 539,565

Hydrated oxide of acetyle . 229

$$
\text { acryle . } 309
$$

allylo $\quad 595$

amyle . 261

butyle . 256

butyryle . 257

caprotyle .267

caproyle . 268

capryle . . 273

ceryle . 286

cetyle . . 277

doùecatyle. 295

ethyle. . 205

formyle . 195

myricyle . 287

methyle . 176

octyle . . 271

œnanthyle . 270

palmityle . 279

propionyle. 253

propyle . 252

rutyle . . 275

tetramy-

lium, \&c. . 263 tetramethy-

lium, \&c. . 187 tetrethyli-

um, \&c. . 225

valeryle . 264

Hydrindine

. . 371

Hydrobenzamide . . 143

Hydrobenzanilide . . . $i b$.

Hydrobenzoinamide . . 146

Hydrocobaltocyanic acid . $\quad 96$

Hydrocyanic acid . . $\quad 71$

Hydroferridcyanic acid . $\quad .94$

Hydroferrocyanic acid . .90

Hydrokinone, green and white 333

Hydromellonic acid . 102

Hydrosulphomellonic acid . 108

Hydrosulphocyanic acid . . 100

Hydrosulpburet of sulphuret of

amyle . . 264

, butyle . . . 256 
Hydrosulphuret, \&c., of ethyle 207 " methyle.

Hydurets of acetyle, formyle,

\&c. . . . 23, 247

of ethyle, methyle, \&c. $\quad 22,175$

Hyduret of benzoyle . . . 132

" cumple $\quad$ cumale .165

" phenyle, or benzole . . 139, 141

" $\quad \begin{aligned} & \text { salicyle } \\ & \text { sulphobenzoyle }: 156\end{aligned}$

" sulphazobenzoyle. $i b$.

" toluyle, or tuluole 140 , 392,397

Hydurilic acidyle, or xylole $\cdot i b$.

Hyoscyamine . . . . 425

Hyperuric acid . . . . 128

Hyposulphobenzidic acid $\quad .139$

Hyposulphobenzoic acid . . 138

Hyposulphoindigotic acid $\quad .366$

IDRIALINE . . . . . 492

Idryle . . . . $\quad i b$.

Ilicine. . . . . 353

Imabromisatinese . . . 374

Imachlorisatinase, \&c. $\quad . \quad . \quad i b$.

Imasatine . . . . 373

Imasatinic acid . . . . ib.

Imesatine . . . . $i b$.

Imperatorine $. \quad . \quad .355$

Importance of phosphates in food

Indigo, blue white . . . 364

action of sulphuric acid on 366 " potash and heat on . . . 375

$"$ nitric acid on .367

" chlorine on . . $i b$.

" oxidation of . . $i b$.

Indigotic, or Nitrosalicylic acid 160 ,

Indigotine, or pure indigo . $\quad .364$

Indine $. \quad . \quad . \quad .370$

Indinic acid $\quad . \quad . \quad . \quad 371$

Inosinic acid . . . .528

Inosite . . . . .443

Inuline . . . . 455

Iodide of cyanogen . . . 86 of tetramethylium . $\quad .187$ of tetramylium . . . 263 of tetrethylium . . . 225
Iodide of amylotriethylium PAGE of triethylophenylium . . 386 of methylethylamylophenylium

Iodocinchonine . . . 417

Iodocodeine . . . . 421

Iodonicotine $. \quad . \quad . \quad 411$

Iodoquinine, sulphate of $\quad 414$

Iodosalicylic acid . . . 160

Ipecacuanha, contains emetine 428

Iridiocyanogen . • . 98

Iron always present in blood . 537 not essential to the colour of

the globules . . . ib.

Isatan . . . . . 372

Isatine . . . . . 367

Isatinic acid . . . . . 367

Isatinosulphites $\quad . \quad . \quad i b$.

Isatyde . . . . $\quad .370$

Isethionic acid . . . . 227

Isotartaric acid $\quad . \quad . \quad .319$

Isoterebenthene . . . 340

Itaconic acid . . . . 313

Itaconanilide . . . . $i b$.

Ivory black . . . . 512

J JLAP, resin of $\quad . \quad . \quad .349$

Jamaicine . . . . .431

Jervine • • . . . 429

Juniper, oil of . . . . 338

KAKODYLE . . . . . 191

compounds of . . . 192

Kakodylic acid . . . . $i b$.

Kakoplatyle . . . $\quad i b$. compounds of . . . 193

Kermes . . . 379

Kidneys, function of .593 et seq.

Kinic acid and kiuates . $\quad 332$

Kinone . . . . . $i b$.

Kinovic, or Chivonic acid 329,337

Kreatine, preparation of . . 526 proportion of, in different kinds of flesh products of its decomposition . . . 407, 528

Kreatinine . . . .407

is found in urine . . 528

Kyanole, or Aniline 139, 364, 375,

LAC, resins of .

Lactamide, isomeric with alanine, sarcosine and urethane $\quad 136,231,451$ 
Lactic acid . . 258, 448, 528 anhydrous $\quad$. $\quad . \quad 456$ artificially produced $\quad 258,449$ exists in juice of flesh and gastric juice . $\quad 449,528$ is not present in normal urine 449 Lactic fermentation . . . $i b$. Lactine, or Sugar of Milk . 441 Lactucic acid . . . . 337 Lactucine . . . . 355 Lævotartaric acid . . . 318 Lake, carmine _. . . 378 Lamp black . . . . 494 Lampic acid . . . . 232

Lantanuric acid . . . 125 Lard . . . . . 300 Laurel-water . . . 153

Laurostearic or Lauric acid, 10, 277,

Lavender, oil of . _ . 342

Leather . . . 325, 511

Lecanorate of oxide of ethyle . 357 Lecanoric acid . . $i b$.

Legumine, or vegetable Caseine 504

Leiocome . . . .455

Lemons, oil of . . . 339

Lepidine . . . . 400

Leucic acid . . 136, 502

Leucine, preparation of . . 502 is homologous with glycocine and alanine, 137, 230, 407,

(230, 0101

Leucoharmine . . . .433

Leucoturic acid . . 115

Lichenine . . . . .455

Lichens . . . . 356

Light, essential to vegetation . 540 its relations to heat, motion,

and chemical force . 566, 590

Lignine, or woody fibre $\quad .459$

Lignone . . . . . 462

Limonine, or limone . . . 316

Lipic acid . . . . 290

Lipyle, oxide of . . . . 575

Liriodendrine . . . . 353

Lithic acid, or Uric acid . 109

Lithofellic acid . . . 520

Litmus . . . . . 362

Liver, functions of . . 513

Lophine

145,404
PAGE

. .351

Lutidine . . . . 397

Lymph . . . . . 535

MADDER, constituents of . . 351

Magnesia, ammoniaphosphate of . . . .535

lactate of . . . . 525

MagnUs, salt of . $\quad . \quad 55$

Malamide, or Asparagine $\quad 322$

Malamidic acid, or Aspartic acid . $i b$.

Malates . . . . 321

Maleates . . . . . 322

Maleic acid . . . . $i b$.

Malic acid . . . 320

Malt . 507

its action on starch, 444,453 , $50 \%$

its action on sugar 444,507

Manganocyanogen . . 98

Manna . . . .448

Mannite . . . $i b$.

Manures, theory of . 562 et seq.

Margaramide . . . 282

Margarates . . . 281

Margaric acid . 10, 280, 546, 578

Margarine . . . 282, 575

Margaritic acid . . . 277

Margarone . . . . . 282

Marjoram, oil of - . $\quad .342$

Marsh-gas, its homologues . . 22

Mastic . . . . . 349

Mechloic acid . . . . 356

Meconamidic acid . . 331

Meconates . . . . 330

Meconic acid . . . 329

Meconoethylomeconic acid . . 331

Meconine . . . . 356

Melam . . . 104

Melamine . . . 104, 404

Melampyrine . . . . 356

Melaniline . . . .393

Melanoximide . . . . 394

Melassic acid . . . . 439

Melene . . . . . 287

Melissic acid . . 287, 546

Mellite . . . . 68

Mellitic acid . . . . $i b$.

Mellone . . . 85

Mellonides . . . . $i b$.

Membranes . . . .510

Menispermine . . . . 432

Menyanthine . . . . 353 
PAGE

" series of . . 25

. . . . 211

Mesite . . . . . 462

Mesitene . . . . . $i b$.

Mesitylole and its derivatives . 251

Mesaconic acid . . . 314

Mesoxalic acid . . . . 117

Metacetone, or Propione . . 254

Metacetonic acid, or Propylic

Metagallic acid

253

Metaldehyde

327

231

Metals, radicals containing, 190, 209

Metanaphthaline

. 490

Metastyrole

167,339

Metatartaric acid . . 319

Metaterebenthene . . . 340

Methionic acid . . . 227

Methol . . . . 462

Methylamime . . 15, 182, 383

Methyle . . . . .173

" and phosphorus . . 193

chloride, bromide, and iodide

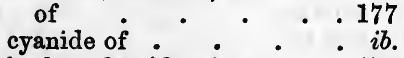

hydrated oxide of . . . 175

oxide of . . $\quad i b$.

, acetate of .. . 180

" sulphate . . . 178

" acid sulphate of . . 179

" allophanate of . . 181

" benzoate of. . . 180

" carbamate of . . 179

" carbolate of . . 180

" cyanate of . . 181

" cyanurate of . $\quad$. $i b$.

" nitrate of . 179

" oxalate of . . . ib.

" salicylate of . 180

products of oxidation of . 194

sulphuret of, hydrosulphu-

retted

177

Methylethylamine . . . 224

Methylethylamylamine . . 264

Methylethylamylophenylium . 396

Methylethylophenylamine . ib.

Methylobutylamine . . 262

Methylocinchonidium . .417

Methylocinchonium . . . $i b$.

Methyloconiine . . .412

Methylodiethylamine . . . 224

Methylodiethylamylium . . 264

Methylomorphium .. . . 419
PAGE

Methylonarcotine . . . 425

Methylonicotium . . .411

Methylophenylamine . . 396

Methylopiperidine . . . 432

Methylopropylamine . . 256

Methyloquinium . . .414

Methyloquinolium . . . 401

Methylotriethylium . . . 225

Metbylo-urea . . . . 181

Methyloxamic acid . . . 184

Methyloxamide . . . 183

Middletonite . . . . 492

Milk, composition . . . 521

fermentations of . 441, 522

sugar of, or lactine $\quad . \quad .441$

Mimotannic acid . . . . 328

Mineral food of plants . $\quad .560$

Mollities ossium . . . . 512

Monacetine . . . . 306

Morindine . . . . . 351

Morine . . . . . $i b$.

Moroxylic acid . . . 337

Morphine, preparation of . 418 salts, and tests of $\quad .419$

Mould . . . . . 461

Mouldering, or decay of wood 460

Mucic acid . . . 442

Mucilage . . . . 456

Mucus . . . . 510

Mudarine . . . . 354

Mulberry calculus . . . 435

Murexan . . . . . 125

Murexide . . . . 123

of caffeine $\quad . \quad . \quad .434$

Muscular fibre . . . 510

Mushroom sugar. . . . 442

Must of wine . . . 508

Mustard, oil of . . 255, 402

bases from . . . . $i b$.

Mykomelinic acid . . 118

Myricine . . . . . 287

Myricyle, hydrated oxide of . ib.

Myriospermine . . . 167

Myriospermic acid . . . $i b$.

Myristic acid . . 10, 277, 577

Myristine . . . . 307

Myronic acid . . . 345

Myrosine . . . . 344

Myroxyline . . . 167

NAPBTHA . . . . 493

Naphtalase, \&c. . • . . 487

Naphtalic acid. . . 489

Naphtalidine . . 402,487 
Naphthaline . action of chlorine on . . ib. bases from . . . . 402 " nitric acid on . . 486 Naphtëne sulphuric acid on. 485

Narceine . . . . . . 422

Narcogenine . . . . 424

Narcotine . • • . . 422

Narcotinic acid . . . 424

Neroli, oil of $\quad$. $\quad . \quad 339$

Nervous matter . . . 520

Nicotine . . . . 410

Nitraniline . . . 378,392

Nitranisic acid . . . 165

Nitranisole . . . 165

Nitranthracenase, \&c. . $\quad$. . 490

Nitrindine . . . . . 371

Nitrobenzanisidine . . . 165

Nitrobenzoic acid . . . 138

Nitrobenzole . . . . . 139

Nitrocodeine . . . . 420

Nitrocumidine $\quad . \quad . \quad .398$

Nitrodibromaniline . . . 378

Nitrogen, compounds of, not combustible . . . 581

Nitroglycerine . • . . 306

Nitrobarmaline . . . 433

Nitrohippuric acid - 136,531

Nitrohydrobenzamide . . 143

Nitrohydurilic acid . . . 117

Nitromannite . . . . 448

Nitromeconic acid . . . 356

Nitromesidine . . . . 257

Nitromesitylole, \&c. . . . $i b$.

Nitronaphtale $\quad . \quad .488$

Nitronaphtase, \&c. $\quad$. . . .486

Nitronaphtesic acid, \&c. . $\quad .488$

Nitrophenesic acid . . . 474

Nitrophenisic or picric acid 374,

Nitrophtalic acid 474

Nitrophtalic acid . $\quad . \quad 489$

Nitropicric or nitrophenisic acid . . 374,474

Nitroprussides . $\quad .95$

Nitrosalicylamide . $\quad 181,219$

Nitrosalicylate of oxide of ethyle . . . . 219 methyle . . . 181

Nitrosalicylic or indigotic acii

Nitrometastyrole $\quad$ • $\begin{array}{r}160,374 \\ .339\end{array}$

Nitrostyrole . . . . 338

Nitrostilbase, \&c. . $\quad . \quad 149$

Nitrotartaric acid . .314

Nitrotoluole . . 140, 167, 348

Nitrotyrosine . . . . 503

Nonyle . . . . . 278

Nutgalls . . . . . 324

Nutmeg butter . . .300

Nutrition of plants and animals 539

et seq.

Octylamine $. \quad . \quad .272$

Octyle . . . . . 271

$"$ hydrated oxide of . . $i b$.

Odorine . . . . . 396

Enanthic acid . . . 336

ether. . . . . $i b$.

Enanthole . . . . .270

Enanthylamine . . . 271

Enanthyle . . . . 270

Enanthylic acid . 10,270, 577

Oil " anhydrous 270

Oil of anise . . 163, 341

asafœida . . . . 345

bitter almonds . . 132,153

cinnamon . . . 165,341

cloves . . . 336, 341

coal tar . . . 396, 472

copaiva . . . . 339

cumin . . . . 156,341

Dippel . . . . . 397

estragon . . . . 342

garlic . . . . 345

gaultheria or wintergreen

juniper 158, 169, 219

lemons . $\cdot{ }^{\circ} \cdot{ }^{\cdot} \quad . \quad 339$

mustard . . . . 343

neroli or orange flower . 339

rose . . . . 342

rue . . . 275,342

spiræa . . . . 156, 341

tar . . . 463, 472

turpentine . . . . 337

valerian . . . . . 341

wine . . . . 226

Oils, fat or fixed . . . 300

replace alcohol in food $\quad .589$

action of heat on $\quad .309$

sulphuric acid on 302

nitric acid on . 290

nitrous acid on 294, 302

bases on . . 304

Oleates .

Olefiant gas, and its series 23,247

Oleic acid . . . . 293 
PAOE

Oleic acid,action of nitric acid on 294 series

Oleines

Olivile

Olivine

Opiammon

Opianic acid

Opianine .

308

. 356

Opianosulphurous acid . ib

Opianyle . $. \quad . \quad i b$.

Opium . . . . 418

bases and other principles of 418 ,

Orceine

Orcine $.{ }^{-}+{ }^{-} i b$.

Oreoselone . . . . 301

Organic acids . . . 47

bases, artificial, formation, and theory of . . 388

bases, action of hyponitrous acid on

408

bases, natural . . . 410

Organic compounds, action of acids on

action of bases on $\quad$. 36

action of ferments on . . 38

action of heat on . . . 37

characters of . . . . 3

elements of . $\quad$. $\quad$. 2

formation of, artificial 46,388

metamorphoses of . 42

oxidation of . . . . 30

Oxalate of lime calculus . $\quad .535$

Oxalates . . . . 62

Oxalic acid ..$\bullet_{-} \quad{ }_{b}$.

origin of, in urine $\quad 572,592$

series of bibasic acids . $\quad 288$

tests of . . . . 62

Oxalovinic acid . . 218

Oxamate of oxide of ethyle : ib.

of oxide of methyle. . . 179

Oxamic acid . . . 65

Oxamide . . . . 64

Oxanilamide . . . . 394

Oxanilide . . . . 393

Oxidation of the compounds of ethyle

of the compounds of methyle 194

of dead organic matter $\quad 30$

of indigo. . . . . 367

of sanguigenous compounds 501 of uric acid . . . 111

Oxide of acetyle, hydrated $\quad .229$ of allyle.
Oxide of amyle

PAGE

" hydrated . $i b$.

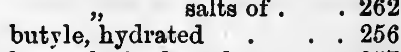

butyryle, hydrated . . $\quad .257$

caprotyle, hydrated $\quad$. $\quad 267$

caproyle, hydrated . . $\quad .268$

capryle, hydrated . . . 273

cetyle. . . . 277

" hydrated . . . ib.

ceryle, hydrated . . . 286

ethyle . . . . . 201

ethyle, hydrated . . . 205

" salts of . . . 212

formyle, hydrated . $\quad$. 195

methyle . . . . . 175

" hydrated $\quad$ ib.

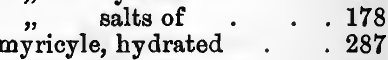

octyle, hydrated . . . 271

cnantbyle, hydrated . $\quad 270$

palmityle, hydrated . 279

pelargyle, hydrated . . 274

propionyle, hydrated . . 254

propyle, hydrated . . 252

rutyle, hydrated . . . 275

valeryle, hydrated . . 266

Oxyacanthine . . . . 431

Oxycarburet of potassium . . 67

Oxychloride of acetyle . . 243

" formyle . . 200

Oxygen," action of, on dead

organic matter . . 30

Ozokerite . . . . 492

Palladiocyanogen a 98

Palm oil . . . 279, 308

Palmitic acid . 10, 279, 546, 577

Palmitines . . . . 307

Palmitone . . $\quad 280$

Palmityle, hydrated oxide of .279

$"$ hyduret of . 280

Pancreatic juice, supposed

function of . . . 530

Panification . . . . 509

Papaverine . . . . . 421

Parabanic acid . . 118

Paracyanogen . . . 99

Paraffine . . . . . 468

different kinds of . . 469

Paramenispermine . . . 432

Paramide . . . . . 68

Paranaphthaline . . 490

Paranicene . . . . 475 
PAOE

Parasalicyle . . . $\begin{array}{r}475 \\ 158\end{array}$

Paratartaric acid, or Racemic acid

Parellic acid

. 317

$\cdot 358$

Parsley, oil of . . . 342

Parietine, or chrysophanic acid . . 351,362

Parvoline . . . . 398

Pectic acid . . . . . 457

Pectine..$i b$.

Pelargonic acid . 10, 274, 577

" $\quad \begin{aligned} & \text { anhydrous } . \quad .273 \\ & \text { ether . . }\end{aligned}$

Pelargone

Pelargyle, hyduret of . . . ib.

Peppermint, oil of . . . 342

Pepsine . • . . 525

Perbromide of formyle . . 200

Perchloride of formyle, or chloroform . . . . 198

Pereirine . . . . 435

Periodide of formyle . . . 200

Persulphocyanic acid . . 101

Peru, balsam of . . . . 166

Peruvine . . . . . $i b$.

Petroleum . . . . .493

Peucedanine . . . . . 355

Peucyle . • . . . 338

Peyrone, salt of . . . 57

Phene, or Benzole $\quad$ 139, 168

Phenole. . . . 170,180

Phenetole . . $i b . \quad i b$.

Phenomethole. . . ib. $i b$.

Phenamylole . • 171, 180

Phenylamine or Aniline . $\quad 364$,

375, 389

Phenyle . . . . .473

hyduret of . . . . 475

hydrated oxide of or carbolic acid

Phenylo-urea . . . ${ }^{\circ} 394$

Phenylo-urethane . . . 395

Phillyrine . . . 355

Phloretine . . . 163,354

Phloridzéine . . . $i b$. $i b$.

Phloridzine . . ib. ib.

Phosgene gas . . . . 66

Phosphates of potash and soda in the animal fluids.

of lime and magnesia in

bones . . . . 512

of magnesia in juice of flesh. 526 Phosphorised bases.
Phosphorus, organic radica PAaE containing - . 193, 600

Phosphorus of the tissues always present as phosphoric acid

Phtalamide . . . . 489

Phtalic acid . . . . $i b$.

„, series of bibasic acids . 599

Phtalimide . . . . 489

Phycite . . . . . 449

Phylloretine . . . . 492

Picamar . . . . .467

Picoline . . . . . 396

series of bases . . 396 et seq.

Picric or Nitrophenisic acid . 374

Picroerythrine . . . 354

Picrolichenine . . . 353

Picrotoxine . . . . 354

Picryle or picrine . . .405

Pimelic acid . . . . 290

Pimpernel, oil of . . . 342

Pinic acid . . . . . 348

Piperidine . . . . 431

Piperine . . . . . $i b$.

Piberylbenzamide . . . 432

Piperylosulphocarbamic acid . $i b$.

Piperylurea . . . . $i b$.

Pitch, mineral . . . .493

of Judea $\quad . \quad . \quad . \quad i b$.

Pittacal . . . 470

Plants, nutrition of $\quad 540$ et seq. ashes of, as manure . $\quad .564$

Platinamine . . . . 60

Platinocyanogen . . . 97

Platinopyridine . . . 398

Platosamine . . . 60

Plasters . . . . . 305

Platinised bases, with ammonia 55

with ethylamine and methylamine with arsenic $\cdot . \cdot 223$

With arsenic $\cdot{ }^{\circ} \cdot 192$

Plumbagine . . . . 355

Plumbethyle . . . . 211

Polychroite . . . . 351

Polychrome . . . . 352

Polymerism . . . 9

Populine. . . . . 353

Porphyroxine . . . . 355

Porter . . . . 508

Potash, found in the ashes of plants. . . 2,560 acetate of . . . . 236 cyanate of . . . 76 


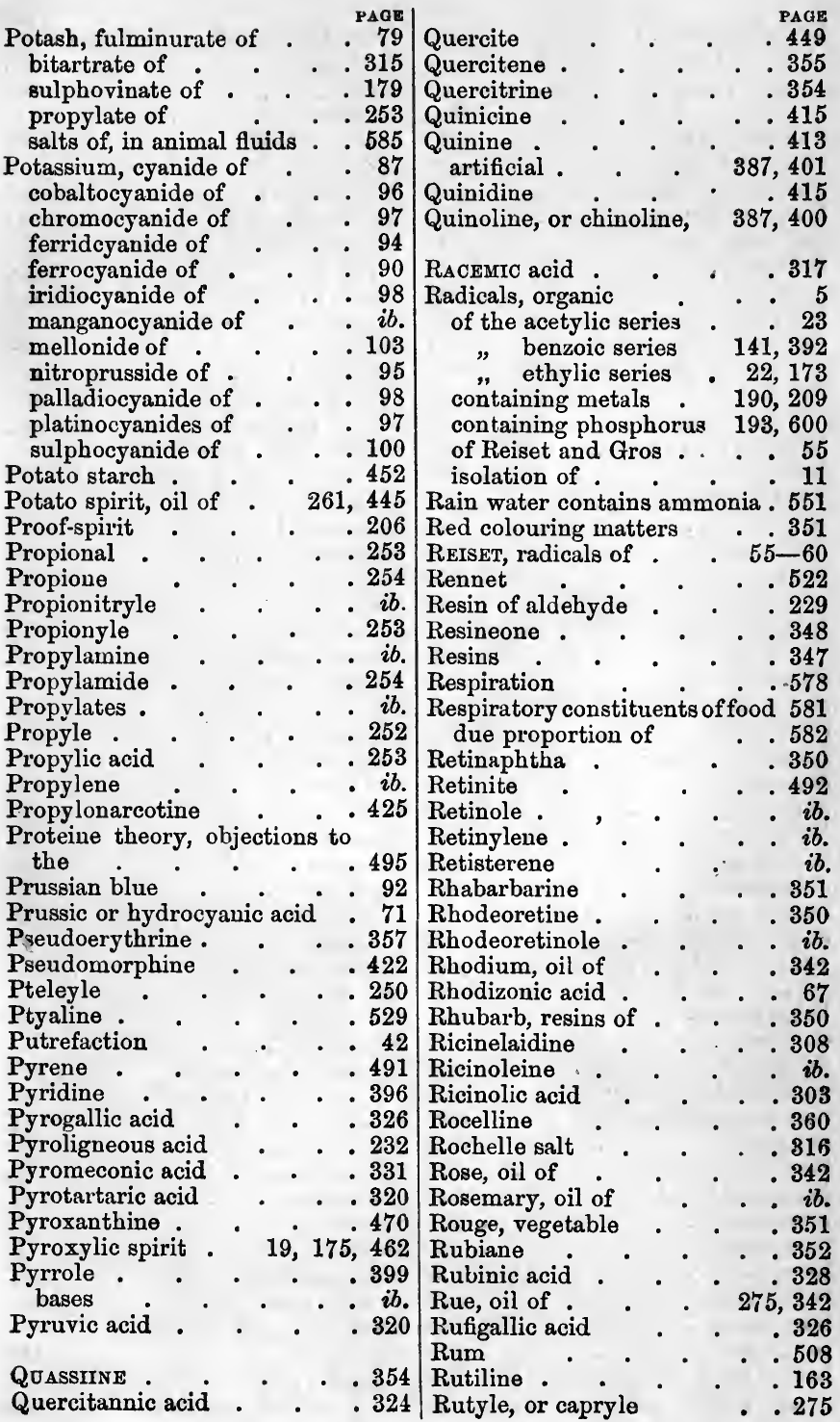




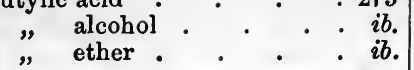

Sabadilline

Saccharic acid

Sacchulmic acid . . . . 439

Sacchulmine . . . . ib.

Safflower . . . . . 351

Saffron . . . . . $i b$.

Sago oil of . . . . . 342

Salicine . . . . . 161 products of decomposition of $i b$.

Salicyle • • • • 156 hyduret of $. \quad . \quad . i b$.

Salicylic acid . . . . 158

Salicylimide $\quad$ • . 157

Salicylous acid $\quad$ • . . 156

Saligenine . . . . . 161

Saliretine $. \quad . \quad . \quad 162$

Saliva . • . $\quad 529,567$

its principal function . . $i b$.

Salts of the urine, with different diet

531,586

Sanguinarine . . . . 430

Santaline . . . . . 351

Santonine . . . . 353

Sassafras, oil of . . . . 342

Saponine . . . 355,456

Sarcocolline . . . .458

Sarcosine . . . . 407

Savine, oil of . . . . 338

Scheererite . . . . 492

Scillitine . . . . . 354

Schweinfurth green $\quad . \quad .238$

Sebacic acid . . . . 291

" ether . . . . $i b$.

Sebamide . . . . . 292

Sebine . . . . . 308

Selenaldine . . 230, 404

Seleniuret of ethyle $\quad . \quad 208$

Seminaphtalidine . . .402

Seneguine . . . . .355

Seroline . . . . . 538

Serum of blood . . . 536

Sinapine . . . . . 345

Sinapisine . . . . $i b$.

Sinapoline . . . 344,403

Sinkaline . . . 346,403

Sinnamine . . . 343,403

Smilacine

Soaps, hard

355

304
Sorbine . . . . . 442

Spaniolitmine • . . . 362

Sparteine . . . . 413

Spermaceti . . . 280,301

Spiræa, oil of . . . 156, 341

Spirit of wine . . . . 205

proof . . . . 206

Spirit, pyroxylic . 175, 462

Spontaneous combustion $\quad \cdot 33$

Stannethyle . . . . 210

Staphisine . . . . 428

Starch, common . . . .451

potato .. . . 452

Starch, its conversion into

dextrine and sugar . 453

Stearates . . . . 284

Stearic acid . . . . 283

Stearine . . . . . 284

Stearoptenes . . . . 337

Stilbene . . . . . 148

Stibethyle . . . . . 209

compounds of . . . $i b$.

Stibiomethyle . . . . 190

Stibiomethylium . . . $i b$.

Storax . . . 338,349

Strychnine . . . . . 428

Stramonine . . . . 426

Styracine . . . . 349

Styrole . . . . . ib.

Styrone . . . . . $i b$.

Styryle . . . . . $i b$.

oxide of . . . . $i b$.

cinnamate of . . . . $i b$.

Suberamide . . . . 291

Suberanilide . . . . $i b$.

Suberic acid . . . $\quad 290$

Suberyle . . . . . $i b$.

Subacetate of lead . . . . 237

Substitution copper - • - ib.

Succinamide $\quad . \quad . \quad 289$

Succinamic acid $. \quad . \quad . \quad i b$.

Succinates . . . . . $i b$.

Succinic acid . . . . $i b$.

Succinimide . . . . ib.

Succisteren $\Theta$. . . 492

Sudoric acid . . . . 601

Suet . . . . . 300

Sugar . . . . .435 
PAGE

Sugar, action of nitric acid on 440 grape $. \quad . \quad 437$ Ai with acids, bases, \&c. . 439

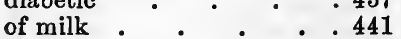
mushroom . . . .442 uncrystallisable . . 443 fermentation of . . 38, 444 butyric fermentation of . .448 lactic fermentation of . $\quad .446$ viscous fermentation of. .447 vinous fermentation, $38,205,444$ of gelatine, or glycocine $\quad .406$ of lead produced from starch, woody

fibre, gum, \&c., 437, 453, 507

Sulphamethylane . . . 178

Sulphamylic acid . . . 261

Sulphasatyde . . . $\quad .370$

Sulphazobenzoyle, hyduret of 149

Sulphesatyde . . . . ib.

Sulphessale . . . . 148

Sulphethamic acid . . . 222

Sulphoamidic acid . . . 453

Sulphobenzide . . . 139

Sulphobenzoyle, hyduret of $\quad .147$

Sulphocamphic acid . . 336

Sulphocetylic acid . . . 278

Sulphocyanide of ammonium . 100

" potassium . 104

Sulphocyanic acid . $\quad . \quad 100$

Sulphocyanogen . $\quad \cdot \quad i b$.

Sulphoglyceric acid . $\quad .305$

Sulphohydrokinone . . . 333

Sulphoindigotic acid $\quad . \quad 366$

Sulphomellone . . . . 107

Sulphomesitylic acid . . 251

Sulphomethylic acid . . . . 179

Sulphonaphtalide . . . 486

Sulphonaphtaline . . . $i b$.

Sulphopianic acid . . . 423

Sulphophenic acid . . . 474

Sulphovinic acid . . . . 213

Sulphuret of ethyle . . . 207

" methyle . . 177

Surinamine . . . . .431

Sweet bay, oil of . . $\quad .342$

Sylvic acid . . . . . 348

Synaptase . . . . . 154

Syringine . . . . . 353

TABLE of homologous compounds, related to ethyle and acetyle .
Table of benzoic series of compounds of ethyle and methyle . . . 296 Tabular view of the origin of vegetable products . 543-547 view of the changes in animal

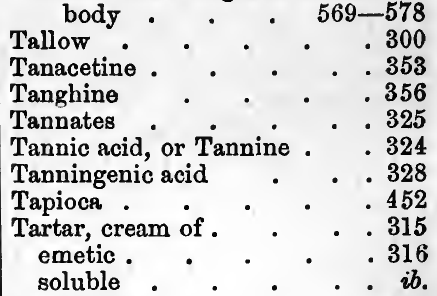

Tartaric acid . . . . 314

$\begin{array}{ll}\prime & \text { action of heat on } .317 \\ & \text { anhydrous }\end{array}$

Tartramide . . . . 319

Tartrates . . . . 315

„ double . . . . 316

Tartrovinic acid . . . 315

Taurine . . . . 516

Taurylic acid . . . 533

Tea, contains caffeine . $\quad 433,593$ oil of . . . . . $\quad .342$ use of . . . . . 593

Tekoretine . . . . 495

Tellurethyle . . . . 211

Terebene . . . . 340

Teropiammon . . . . 424

Terpine . . . . 340

Tetramethylium . . . 187

Tetramylium . . . . 263

Tetrastearine . . . . 308

Tetrethylium . . . . 225

Tetrethylo-urea . . . . 226

Thebaine . . . . 422

Theine, the same as Caffeine . 433

Theobromine . . . . 434

Thialdine . . . 230, 403

Thionaphtamic acid . . 487

Thionurate of ammonia . $\quad .119$

Thionuric acid . . . . $i b$.

Thiosinnamine . . 343, 402

Thyme, oil of . . . . 342

Thymine . . . . .602

Thymoile . . . . 342

Thymoilole . . . . $i b$.

Toluidine . . . 141, 397

22 Toluole . . . . 141, 167 
Tcluylic acid

Tribromaniline . . $\quad .372$

Tribenzoicine • . . . 307

Tricetylamine . . . . 278

Trichloraniline . . . . 372

Trichlorosalicine . . . . 162

Triethylamine . . . . 226

Trigenic acid . . . . 601

Trimetliylamine . . . . 185

Triamylamine . . . . 263

Triethylophenylium . . . 396

Trinitrolophyle • . . .405

Trinitropicryle $\cdot \quad \cdot \quad \cdot i b$.

Turkey-red . . . . . 351

Turmeric . . . . . 351

Turpentine $. \quad . \quad . \quad .337$

" $\quad$ isomers of $\quad . \quad+340$

Turpentinic acid $\quad . \quad$. 338

Types, chemical theory of $\quad 12$

Tyrosine . . . . . 502

UPAS poison or antiarine $\quad .354$

Uramile . . . . . 120

Uramilic acid . . . $\quad i b$.

Urate of ammonia . . . 110

Urea . . . . . 78

artificial . . . 47,78

amplic . . . . 262

aniline . . . . . 391

determination of, in urine . 572

ethylic . . . . 220

methylic . . . . 181

nitrate of . . . . 78

oxalate of . . . . $i b$.

Urethane . . . . . 217

Urethylane . . . $\quad .179$

Uric acid . . 109

its derivatives 114 et $s \in q$.

Uric oxide, or xanthic oxide .127

Urinary calculi $\quad . \quad 127,535$

Urine of man . . . . 585

U $"$ constituents of . $i b$.

Urine of birds . . 533, 563

$"$ carnivora . . . 531

" serpents . . . 109

Urine, use of, as manure . $\quad .563$

Uroxanic acid . . . . 126

Uroxile • . . . $i b$.

Uryle . . . . . . 109

Urylic acid . . . . $i b$.

Usnic acid . . . . . 361

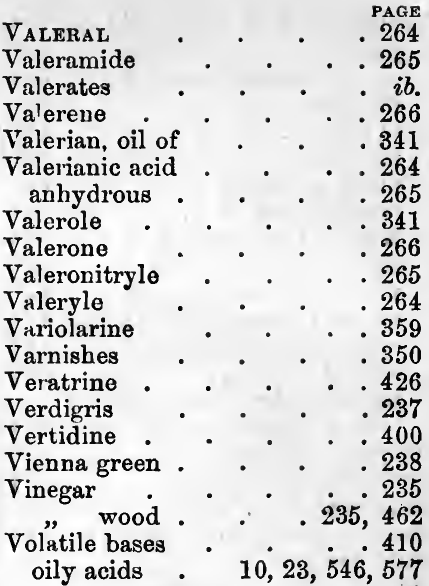

. $\quad$ table of . . . 10

table of formation

of, from sugar . 577

WATER, uses of, in vegetation, 541,

,,$\quad$ in blood $\quad .579$ pepper, oil of . . . 345

raiv, contaius ammonia . 551

Wax, bees', its constituents 279 ,

286

Chinese, its constituents $\quad .286$

fossil . . . . . 492

White indigo $. \quad . \quad . \quad 364$

Wine, its preparation . . 445

its bouquet . . . . $i b$.

its peculiar smell $\quad . \quad i b$.

oil of $\quad . \quad . \quad . \quad .226$

Wood, distillation of $\quad . \quad 461$

decay of . $\quad . \quad 30,460$

coal or brown coal $\quad . \quad 472$

vinegar . . . . .462

Wood-spirit or pyroxylic spirit

$161,175,462$

Woody fibre, or cellulose . 458

$" \quad, \quad$ its formation . 557

Wormwood, oil of . . . 342

Wort . . . . . 508

XANTHIC acid . . . . 218

" oxide . . . 127

Xanthopenic acid . . . 423

Xylidine . . . 397, 453 


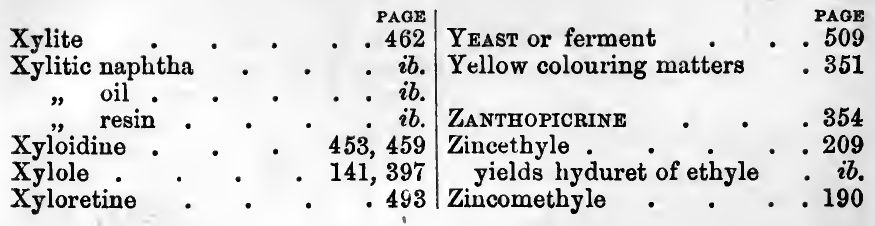

THE END. 


\section{DOUBLE VOLUMES NOW READY.}

* * Double Volumes may now be had strongly and handsomely bound in cloth, with Indexes, price 3s. 6d. each Double Volume.

\section{DR. LARDNER'S \\ MUSEUIM OF SCIENCE \& ART.}

\section{A MISCELLANT OF}

INSTRUCTIVE AND AMUSING TRACTS ON THE PHYSICAL SCIENCES, AND ON THEIR APPLICATION TO THE USES OF LIFE.

ILLUSTRATED BY ENGRAVINGS ON WOOD.

THE purpose of the publishers of this series is to supply a collection of instructive essays and tracts, composed in a popular and amusing style, and in easy language, on the leading discoveries in the Physical Sciences, and on their most important and interesting applications to the Arts which contribute to the convenience and ornament of life. Such papers may be read with pleasure even by those who have been disciplined in the study of the sciences. Many interesting details and incidents which are usually excluded from systematic treatises, will be suitably placed among such illustrations. Persons, whose occupations exclude the possibility of systematic study, will thus be enabled to collect, without the expenditure of more time and thought than they can easily spare, the flowers and the fruits of the garden of knowledge, and may in their hours of leisure obtain a considerable amount of information on subjects of the highest interest.

To place this Series within the reach of all classes, it is published at ONe Penny, in weekly numbers of 16 pages, large $12 \mathrm{mo}$, printed on good paper, in a clear type, and illustrated when necessary by engravings on wood; also in Monthly Parts, price 5d., in a wrapper; in Quarterly Volumes, price 1s. $6 d$; ; and in Half-yearly Volumes at 3s. $6 d$.

\section{LONDON: WALTON \& MABERLY,} UPPER GOWER STREET, AND IVY LANE, PATERNOSTER ROW. 
Contents of Vols. I. and II. (double), 3s. $6 \mathrm{~d}$. cloth.

VOL. I., price 1s. 6d., in handsome boards.

Part I., price $5 d$.

1. The Planets; Are they Inhabited Worlds? Chap. I.

2. Weather Prognostics.

3. The Planets. Chap. II.

4. Popular Fallacies in Questions of Physical Science.

PART II., price $5 d$.

5. Latitudes and Longitudes.

6. The Planets. Chap. III.

7. Lunar Influences.

8. Meteoric Stones and Shooting Stars. Chap. I.

Part III., price $6 d$.

9. Railway Accidents. Chap. I.

10. The Planets. Chap. IV.

11. Meteoric Stones and Shooting Stars. Chap. II.

12. Railway Accidents. Chap. II.

13. Light.
VOL. II., price 1s. 6d., in handsome boards.

PART IV., price 5d.

14. Common Things.-Air.

15. Locomotion in the United States. Chap. I.

16. Cometary Influences. Chap. I.

17. Locomotion in the United States. Chap. II.

PART V., price $5 d$.

18. Common Things.-Water.

19. The Potter's Art. Chap. I.

20. Locomotion in the United States. Chap. III.

21. The Potter's Art. Chap. II.

PART VI., price $6 d$.

22. Common Things.-Fire.

23. The Potter's Art. Chap. III.

24. Cometary Influences. Chap. II.

25. The Potter's Art. Chap. IV.

26. The Potter's Art. Chap. v.

\section{Contents of Vols. III. and IV. (double), 3s. 6d. cloth.}

\section{VOL. III., price 1s. 6d., in handsome boards.}

ParT VII., price $5 d$.

27. Locomotion and Transport, their Influence and Progress. Chap. I.

28. The Moon.

29. Common Things. - The Earth.

30. Locomotion and Transport, their Influence and Progress. Chap. II.

Part VIII., price 5d.

31. The Electric Telegraph. Chap. I.
32. Terrestrial Heat. Chap. I.

33. The Electric Telegraph. Chap. II. 34. The Sun.

PART IX., price $6 d$.

35. The Electric Telegraph. Chap. III.

36. Terrestrial Heat. Chap. II.

37. The Electric Telegraph. Chap. IV.

38. The Electric Telegraph. Chap. V.

39. The Electric Telegraph. Chap. VI.

VOL. IV., price 18 6d., in handsome boards.

PART X., price $5 d$.

40. Earthquakes and Volcanoes. Chap. I.

41. The Electric Telegraph. Chap. VII.

42. The Electric Telegraph. Chap. VIII.

43. The Electric Telegraph. Chap. IX.

ParT XI., price 5d.

44. Barometer, Safety Lamp, and Whitworth's Micrometric Apparatus.
45. The Electric Telerraph. Chap. $X$.

46. Earthquakes and Volcanoes. Chap. I1.

47. The Electric Telegraph. Chap. XI.

48. Steam.

$$
\text { PART XII., price 6d. }
$$

49. The Electric Telegraph. Chap. XII.

50. The Electric Telegraph. Chap. XIII.

51. The Electric Telegraph. Chap. XIV.

52. The Electric Telegraph. Chap. XV.

\section{Contents of Vols. V. and VI. (double), 3s. 6d. cloth.}

\section{VOL. V., price 18. 6d., in handsome boards.}

Part XIII., price $5 d$.

53. The Steam Engine. Chap. I.

54. The Eye. Chap. I.

55. The Atmosphere.

56. Time. Chap. I.

PART XIV., price $5 d$.

57. The Steam Engine. Chap. II.

58. Common Things,-Time. Chap. II.
59. The Eye. Chap. II.

60. Common Things.-Pumps.

PART XV., price $6 d$.

61. The Steam Engine. Chap. III.

62. Common Things.-Time. Chap. III.

63. The Eye. Chap. III.

64. Common Things.-Time. Chap. IV.

65. Common Things.-Spectacles-The Kaleidoscope. 
VOL. VI., price 18. 6d., in handsome boards.

ParT XVI., price $5 d$.

66. Clocks and Watches. Chap. I.

67. Microscopic Drawing and Engraving. Chap. I.

68. Locomotive. Chap. I.

69. Microscopic Drawing and Engraving. Chap. II.

PART XVII., price $5 d$.

70. Clocks and Watches. Chap. II.

71. Microscopic Drawing and Engraving. Chap. III.
72. Locomotive. Chap. II.

73. Microscopic Drawing and Engraving. Chap. IV.

PART XVIII., price 6d.

74. Clocks and Watches. Chap. III.

75. Thermometer.

76. New Planets.-Leverrier and Adams' Planet.

77. Leverrier and Adams' Planet, concluded.

78. Magnitude and Minuteness.

Contents of Vols. VII. and VIII. (double), 3s. 6d. cloth.

VOL. VII., price 1s. 6d., in handsome boards.

PART XIX., price $5 d$.

79. Common Things. - The Almanack. Chap. I.

80. Optical Images. Chap. I.

81. Common Things. - The Almanack. Chap. II.

82. Optical Images. Chap. II.

Part XX., price 5d.

S3. How to Observethe Heavens. Chap. I.

84. Optical Images. Chap. III. Common Things.-The Looking-Glass.
85. Common Things. - The Almanack. Chap. III.

86. How to Observe the Heavens. Chap. II. Stellar Universe. Chap. I. PART XXI., price $6 d$.

87. The Tides.

88. Stellar Universe. Chap. II.

89. Common Things. - The Almanack. Chap. IV.-Colour. Chap. I.

90. Stellar Universe. Chap. III.

91. Colour. Chap. II.

VOL. VIII., price 1s. 6d., in handsome boards.

PART XXII., price 5d.

92. Common Things.-Man. Chap. I.

93. The Stellar Universe. Chap. IV.

94. Magnifying glasses.

95. Common Things.-Man. Chap. II.

PART XXIII., price $5 d$.

96. Instinct and Intelligence. Chap. I.

97. The Stellar Universe. Chap. V.

98. Common Things.-Man. Chap. III.
99. Instinct and Intelligence. Chap. II. PART XXIV., price 6d.

100. Instinct and Intelligence. Chap. III. 101. The Solar Microscope. - The Camera Lucida.

102. The Stellar Universe. Chap. VI.

103. Instinct and Intelligence. Chap. IV.

104. The Magic Lantern.-The Camera Obscura.

\section{Contents of Vols. IX. and X. (double), 3s. 6d. cloth.}

VOL. IX., price 1s. $6 d$., in handsome boards.

Part XXV., price 5d.

105. The Microscope. Chap. I.

106. The White Ants-Their Manners and Habits. Chap. I.

107. The Microscope. Chap. II.

108. The White Ants-Their Manners and Habits. Chap. II.

PART XXVI., price 5d.

109. The Surface of the Earth, or First Notions of Geography. Chap. I.

110. The Microscope. Chap. III.
111. The Surface of the Earth, or First Notions of Geography. Chap. II.

112. The Microscope. Chap. IV.

Part XXVII., price $6 d$.

113. Science and Poetry.

114. The Microscope. Chap. V.

115. The Surface of the Earth, or First

Notions of Geography. Chap. III.

116. The Microscope. Chap. VI.

117. The Surface of the Earth, or First Notions of Geography. Chap. IV.

VOL. X., price 1s. 6d., in handsome boards.

PART XXVIII., price 5d.

118. The Bee. Chap. I.

119. The Bee. Chap. II.

120. Steam Navigation. Chap. I.

121. The Bee. Chap. III.

PART XXIX., price 5d.

122. Steam Navigation. Clap. II.

123. The Bee. Chap. IV.
124. Electro-Motive Power. Chap. I.

125. The Bee. Chap. V.

PART XXX., price $6 d$.

126. Steam Navigation. Chap. III.

127. Tho Bee. Chap. VI.

128. Steam Navigation. Chap. IV.

129. The Bee. Chap. VII. [Borealis.

130. Thunder, Lightning, and the Aurori 
1 Volume $12 \mathrm{mo}$, 250 pages, price $2 \mathrm{~s}$. cloth lettered,

\title{
THE ELECTRIC TELEGRAPH POPULARISED.
}

\section{WITH ONE HUNDRED ILLUSTRATIONS.}

BY DIONYSIUS LARDNER, D.C.L.

(From the " Museum of Science and Art.")

" The reader will find the most complete and intelligible descrintion of Telegraphic Apparatus in Dr. Larduer's admirable chapters on the subject."-North British Review.

\section{THE MICROSCOPE.}

BY DIONYSIUS LARDNER, D.C.L.

(From the " Museum of Science and Art.")

1 volume, with 147 Engravings, 2s. cloth lettered.

\section{COMMON THINGS EXPLAINED.}

\section{BY DIONYSIUS LARDNER, D.C.L.}

CONTAINING

AIR.-EARTH.-FIRE.-WATER.-TIME.-THE ALMANAC.-CLOCKS AND

WATCHES.-SPECT ACLES.-COLOUR.-KALEIDOSCOPE.-PUMPS.

(From the " Museum of Science and Art.")

1 volume, with 114 Engravings. 12mo, 2s. 6d. cloth lettered.

\section{POPULAR ASTRONOMY.}

BY DIONYSIUS LARDNER, D.C.L.

\author{
Containing
}

HOW TO OBSERVE THE HEAVENS.-IATITUDE AND LONGITUDE.-THE EARTH.-THE SUN.-THE MOON.-THE PLANETS, ARE THEY INHABITED?-THE NEW PLANETS.-LEVERRIER AND ADAMS' PLANET.LUNAR INFLUENCES.-THE TIDES.-THE STELLAR UNIVERSE.

(From the "Museum of Science and Art.")

1 volume, with 119 Engravings, 12mo, 2s. 6d. cloth lettered.

LONDON : WALTON AND MABERLY,

UPPER GOWER STREET, AND IVY LANE, PATERNOSTER ROW. 


\title{
PRINTED FOR WALTON AND MABERLY,
}

\author{
UPPER GOWER STREET, AND IVY LANE, PATERNOSTER ROW.
}

\section{HANDBOOK OF INORGANIC ANALISIS;}

One Hundred and Twenty-two Examples, illustrating the most important processes for determining the Elementary composition of Mineral substances.

\section{BY FRIEDRICH WOHLER,}

Professor of Chemistry in the University of Göttingen.

Edited by Dr. A. W. Hofmann, Professor in the Royal College of Chemistry.

$$
\text { Iarge } 12 \mathrm{mo} \text {. 6s. } 6 \% \text {. }
$$

This collection of examples for practice in Chemical Analysis, is designed chiefly for the use of the laboratory. It is drawn up under the impression thet it is easier for most minds to obtain a cleir insight into gen ral relations and laws by the study of special cases, than inversely to acquire a knowledge of individual cases by first directing the attention to general Rules.

\section{HANDBOOK OF ORGANIC ANALYSIS;}

Containing a detailed Account of the various methods used in determining the Elementary Composition of Organic substances.

\section{BY JUSTUS LIEBIG.}

Edited by Dr. Hofmins. Illustrated by 85 Woodcuts. 12mo. 58. cloth.

This work is a second edition of BA RON LIEBIG's "Instructions in Organic Analysis." In preparing it he was assisted by Dr. STRECKER of Christimia, and DR. HOFMANN of London. Since the publication of the first edition a great variety of new apparatus, and many new merhods have beel proposed; such as have stood the test of vigorous experimental examination have been adopted and described in the present work. The Editor's convictions of the want of such a handbook by the laboratory student who wishes to engage in organic investigation, led him to undertake the publication of the English Edition.

\section{ELEMENTS OF CIIEMICAL ANALYSIS. QUALITATIVE AND QUANTITATIVE.}

\section{BY EDWARD ANDREW PARNELL.}

Second Edition. Svo. 98. cloth.

This work embraces both qualitative and quantitative analysis : it is intended for laboratory use, and for those who are not altogetler unacquainted with chemistry. It opeus with instructions for performing the ordinary manipulations of analytical processes, embracing an account of the necessary ntensils, and also tle modes of preparing and applying the varions reagents. A considerable portion of $t^{\prime} \mathrm{e}$ work is then devoted to the statement (partly in the tabular form), of the appearnnces prodnced hy the principal reagents when applied to chemical subst:ınces. These appearances constitute the gromudwork of a systematic course of operations. next described, to be followed in the qualitative examination of all kinds of substances, illustrated by appropriate examples.

In the divi-ion of the work which treats of quantitative analysis, processes are described by which the constituents of substances are separated from each other, and their weights determined. Prominence is here given to all articles of commercial importance, as alkalies, manganese, and all metallic ores, bleaching powder, etc. In an Appendix, processes are introduced for the complete analysis of crude potashes, ores of nuanganese. saltpetre, guano, the asbes of vegetables, tcc., and copious tables to facilitate calculations in analysis. 


\section{OUTLINES OF THE COURSE OF QUALITATIVE ANALYSIS,}

FOLLOWED IN THE GIESSEN LABORATORY.

BY HENRY WILL, PH. D.,

Professor Extraordinary of Chemistry in the University of Giessen.

With a Preface by Baron Liebig.

8vo, 6s., or with the Tables mounted on linen, $7 s$.

\section{FAMILIAR LETTERS ON CHEMISTRY,}

\section{IN ITS RELATIONS TO PHYSIOLOGY, DIETETICS, AGRICULTURE,} COMMERCE, AND POLITICAL ECONOMY.

\section{BY JUSTUS VON LIEBIG.}

Third Edition. Complete in 1 vol. Foolscap 8vo. 6s. cloth.

The object of the author has been to present a sort of bird's-eye view of Chemistry in all its various aspects and relations; to show its importance as a means of unfolding the secrets of nature; and also to show the influence which it exerts, through its various applications in manufactures, agriculture, and medicine, on the present social condition of mankind.

After introductory remarks on the importance of chemistry, and on the principles on which a judicious application of chemistry must be founded, an historical sketch is given of the progress of this science from the time of the alchemists. The general principles of chemistry are then discussed, as chemical affinity, chemical equivalents, the atomic theory, etc.; also the subjects which are related both to chemistry and natural philosophy, as crystallisation, isomorphism, isomerism, condensation of gases, etc. The principles on which some important chemical mauufactures, that of soda in particular, are founded, are explained, and the influence on commerce of such applications pointed out.

A large portion of this work is devoted to a consideration of the various changes to which bodies of organic origin are subject, as fermentation, putrefaction, \&c.; the origin of organic bodies; the connectiou of chemistry with animal physiology, comprising the subjects of nutrition and respiration, with results of the author's researches on dietetics, and observations on the comparative nutritive value of different articles of food: also the connection of chemistry with vegetablo physiology, conuprising the principles by which the use of manures should be guided, and other applications of chemistry to agriculture.

\section{CHEMISTRY IN ITS APPLICATIONS TO AGRICULTURE AND PHYSIOLOGY. \\ BY JUSTUS LIEBIG, M.D.}

Edited by Lyon Playfair, Ph. D., and WM. Gregory, M.D.

Fourth Edition, revised. 8vo. 68. 6d. cloth.

The farmer who now carries on his operations without reference to the recent applications of chemistry to his calling, as developed in this work, is certainly not in a position to compete successfully with his more scientific neighbour.

This work is divided into two principal sections. In the first, the derivation of the various constituents of vegetables is traced to the atmosphere and the soil. $\Delta$ pplication of the principles thus developed is then made to the art of culture. Among the subjects here treated of are, the causes of fertility and barrenness; the means of improving indifferent soils; the reasons why the practice of rotation of crops and fallow is found advantageous, and the principles on which the judicious application of manures should be founded. This first part concludes with a full account of the results of various analyses of the ashes of plants, which are useful as a guide to the choice of efficient manures.

In the second part of this work, the author discusses the subjects of fermentation, decay, and putrefaction, and makes application to the processes of making wine, beer, and vinegar. The natural processes of nitrification, and the conversion of wood into coal, and, lastly, the action of poisons, contagions, and miasms are here elucidated. 


\section{ANIMAL CHEMISTRY.}

\section{OR, CHEMISTRY IN ITS APPLICATION TO PHYSIOLOGY \& PATHOLOGY.}

\section{BY JUSTUS LIEBIG, M.D.}

Edited, from the Author's Manuscript, by WM. GREGoRY, M.D. Third Edition. Part I. (the first half of the work). 8vo. 6s. $6 d$. cloth.

A general idea of the scope of this treatise may be inferred from the following list of the principal subjects treated of in Part I. - Animal Heat, which is shown to result from the slow combustion of carbon and hydrogen; the respiratory process; the nutrition of carnivora and herbivora, comprising the change which food undergoes in the process of assimilation, and the relation of the vegetable to the animal kingdom; the origin of urine and bile, and the use of bile as an element of respiration. as well as in the digestive process ; the classification of articles of food into two classes, elements of nutrition, and elements of respiration, and their relative value for those purposes; the formation of fat from starch and sugar; the manner in which living vegetables may derive their non-uitrogenized constituents, as sugar, starch, acids, oils, \&c., from carbonic acid and water; the function of the intestinal canal, and the origin and nature of fæces.

The subject of the second division of this work is, " the Metamorphoses of Animal Tissues." Of this subject, the part already published contains the first section, which is devoted to the consideration of the principles which ought to direct, and the method which ought to be pursued in, the investigation of this interesting and important subject.

\section{RESEARCHES INTO THE MOTION OF THE JUICES IN THE ANIMAL BODY.}

BY JUSTUS VON LIEBIG.

8vo. 5 s.

\section{DYEING AND CALICO PRINTING.}

\section{BY EDWARD ANDREW PARNELL,}

Author of "Elements of Chemical Analysis."

(Reprinted from Parnell's " Applied Chemistry in Manufactures, Arts, and Domestic Economy, 1844.")

With Illustrations. 8 vo. 78. cloth.

NOW ON SALE, IN ARTIFICIAL IVORY,

\section{A SMALL BUST OF PROFESSOR LIEBIG,}

HEIGHT 10 INCHES.

Price 15s., or Packed in a Box, 168. 


\section{COMPLETE COURSE OF NATURAL PHILUSOPHY.}

4 Vols. 20s. cloth.

\section{HANDBOOK OF NATURAL PHILOSOPHY.}

BY DIONYSIUS LARDNER, D.C.L.,

Formerly Professor of Natural Philosophy and Astronomy in University College, London.

New Edition, Revised and greatly Enlarged, with several hundred additional illustrations. Large $12 \mathrm{mo}$. 4 Vols., each 5s., cloth lettered.

This work is intended for the general reader who desires to attain accurate knowledge of the various departments of physical science, without pursuing them according to the more profound methods of Mathematical investigation. Hence the style of the explanations is studiously popular, and the graver matter is everywhere accompanied by diversified elucidations and examples, derived from common objects, wherein the principles of science are applied to the purposes of practical life.

It has also, specifically, been the author's aim to supply a manual of such physical and mechanical knowledge as is required by the Medical and Law Student, the Engineer, the Artisan, the superior classes in Schools, and those Students who, before entering on a course of mathematical studies, may wish to take the widest and most commanding survey of the field of inquiry upon which they are about to enter.

Great pains have been taken to render this work complete in all respects, and co-extensive with the actual state of the sciences, according to the latest discoveries.

- Although the principles of the sciences are here, in the main, developed and demonstrated in ordinary and popular language, a few mathematical symbols are occasionally used throughout the work, for the purpose of expressing results more clearly and concisely. These, however, are never employed without a previous ample expl:anation of their signification.

The present edition bas been enlarged by the interpolation of a great number of illustrations of the general principles of Physics, taken from their various applications in the Arts, such examples being in all cases elucidated by appropriate engraved figures of the instruments and machines described. Many improvements have also been introduced in the Diagrams for the illustration of Physical Principles, the number of which has been greatly augmented.

The series consists of Four Treatises, which are independent of each other, and may be purchased separately.

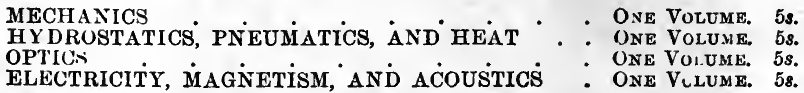

The Four Volumes taken together form a complete course of Natural Philusophy, sufficient not only for the highest degree of School Education, but for that numerous class of University Students who, without aspiring to the attainment of Academic honours, desire to acquire that general knowledge of these Sciences which is necessary to entitle them to graduate, and, in the present state of society, expected in all well-educated persons. 


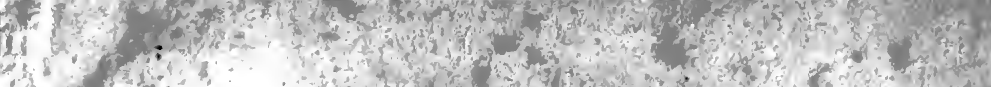

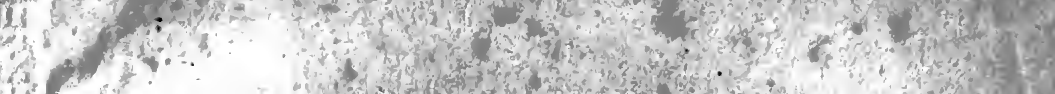

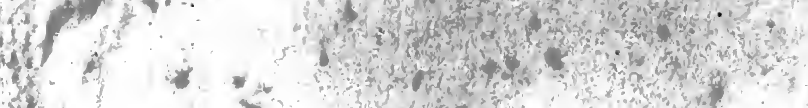

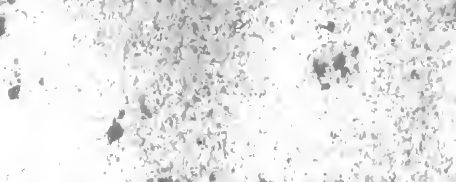
$\therefore$ a

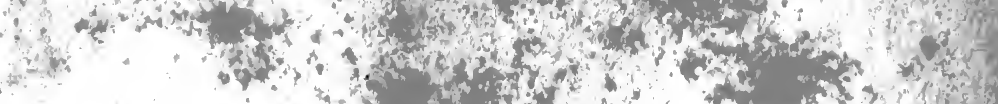

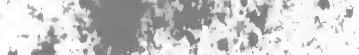

(4):

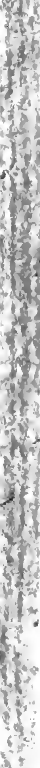

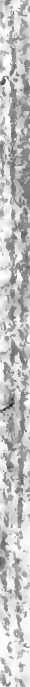
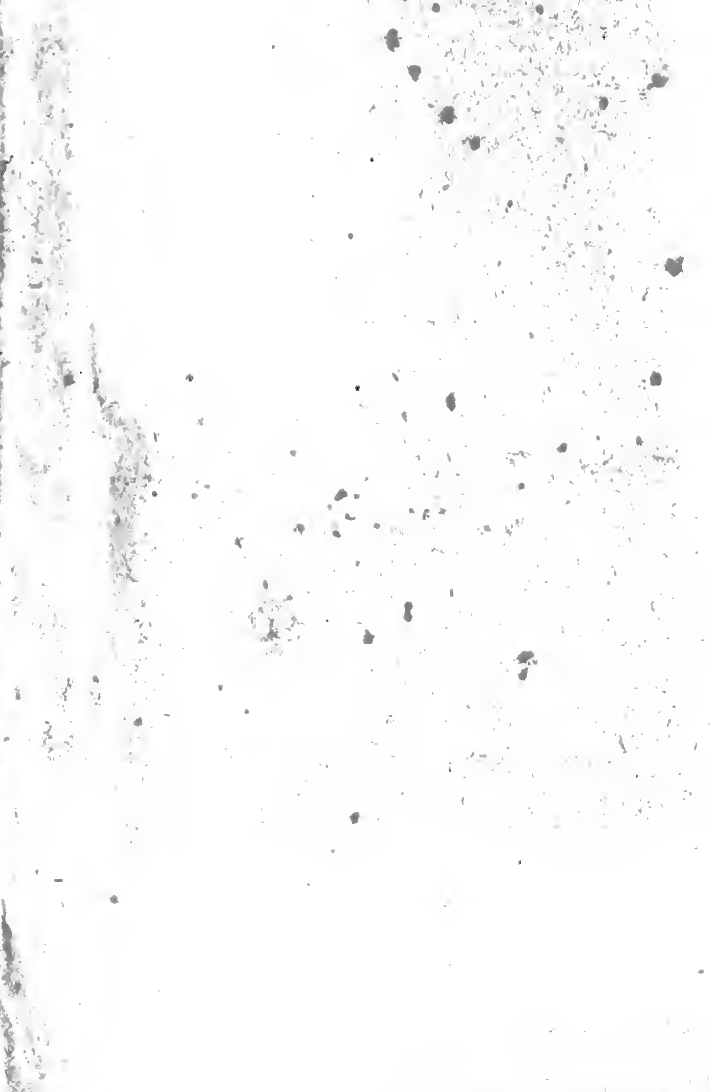

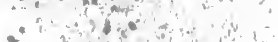

a

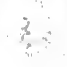

$\lim ^{2}$

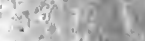

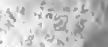

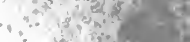
की $x=18$ y-y yxy $\therefore x_{1}+\frac{1}{4}$ $\left(\begin{array}{l}1 \\ -1\end{array}\right.$ $x^{2}+f^{2} x^{2}+l^{2}$
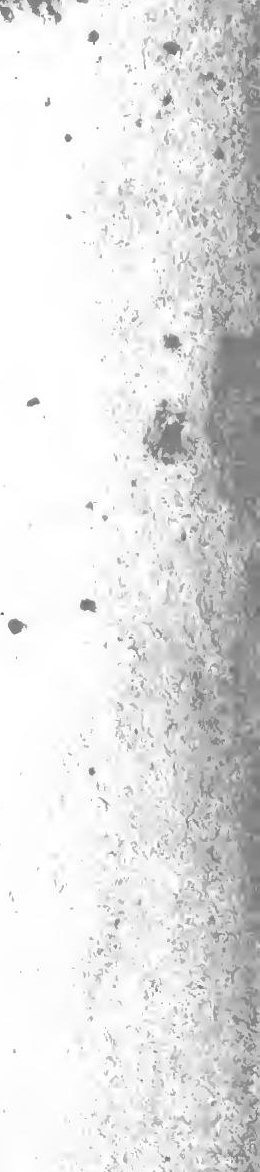
QH ${ }^{\circ}: 2,82$

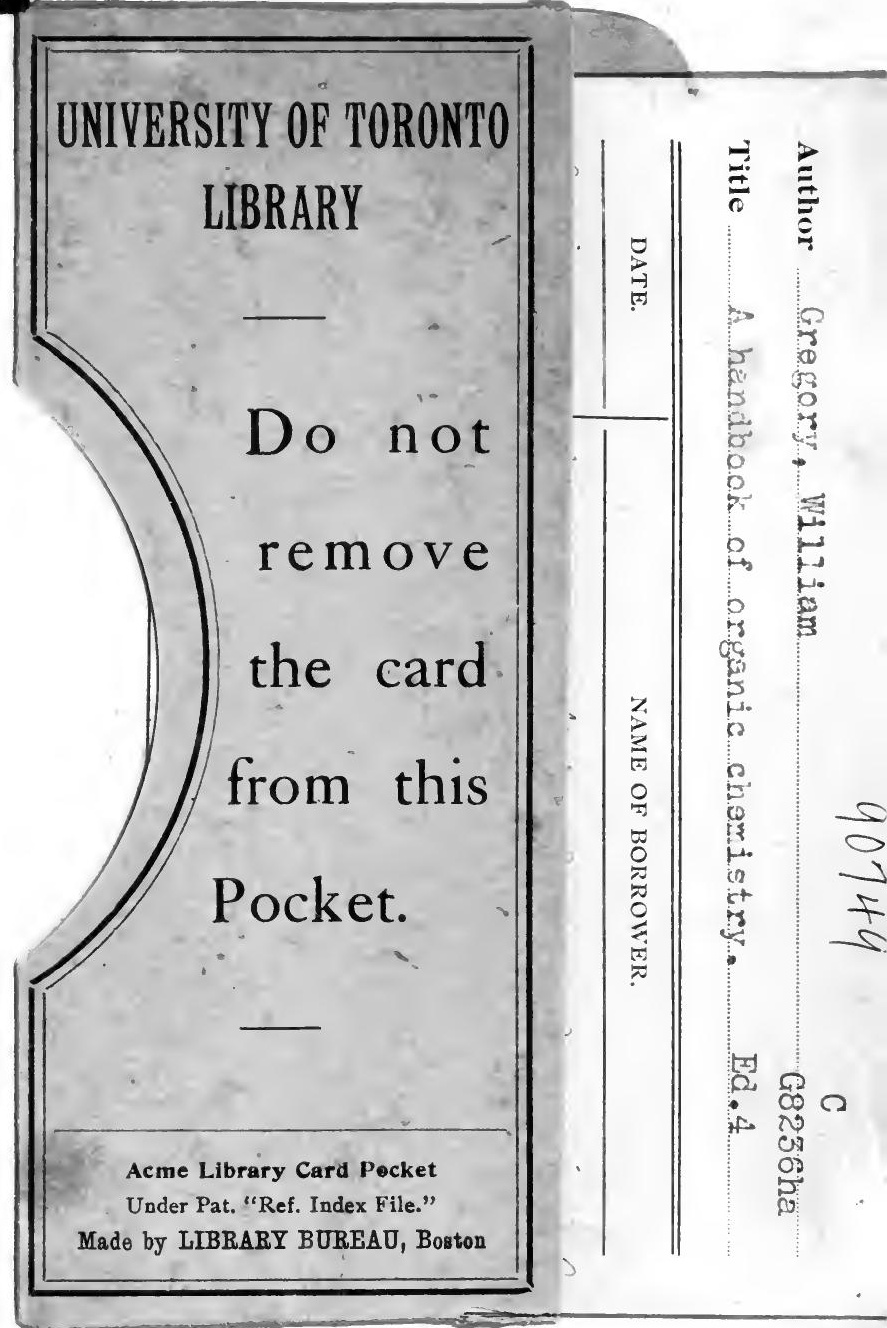


ondij

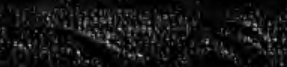

